\title{
Eighteenth Meeting of the European Neurological Society 7-11 June 2008, Nice, France
}

Symposia and Free Communications

The abstracts have been reviewed by:

Z. Argov, O.A. Bajenaru, R. Baringer, C. Bassetti, K. Bhatia, T. Brandt, H. Cock, G. Comi, C. Desnuelle, V. Dietz, A. Ducros, C. Elger, M. Eraksoy, J. Ferro, M. Filippi, O. Hardiman, H-P. Hartung, L. Kappos, J. Kesselring, C. Krarup, P. Landrieu, G.L. Lenzi, R. Lewis, D. Leys, H. Manji, D.H. Miller, I. Milonas, G. Moonen, D. Pareyson, Y. Parman, V. Planté-Bordeneuve, P. Pollak, H. Reichmann, M. Rousseaux, G. Said, E. Scarpini, E. Schmutzhard, A. Sena, V. Silani, R. Soffietti, C. Sommer, A. Steck, J. Valls-Solé, M.J.D. Vidailhet, J. Volkmann 


\section{Contents}

\section{Presidential symposium}

Coma and locked-in syndrome II/3

\section{Symposia}

Behavioural disorders and dementia II/4

Autoimmune disorders of the nervous system II/5

Multiple sclerosis: when to start a treatment and which treatment II/6

Transient ischaemic attack (TIA): imaging and management II/7

\section{FREE COMMUNICATIONS \\ Oral Sessions}

Session 1: Multiple sclerosis $1 \quad$ II/8

Session 2: Cerebrovascular disorders 1 II/11

Session 3: Peripheral neuropathy II/12

Session 4: Epilepsy 1 II/14

Session 5: Neurogenetics II/16

Session 6: Neuro-oncology II/18

Session 7: Multiple sclerosis 2 II/20

Session 8: Infection II/21

Session 9: Neurorehabilitation II/23

Session 10: Cerebrovascular disorders 2 II/25

Session 11: Pain II/26

Session 12: Epilepsy 2 II/28

Session 13: Clinical neurophysiology II/30

Session 14: Extrapyramidal disorders $1 \quad$ II/31

Session 15: Muscle disorders II/33

Session 16: General neurology 1 II/34

Session 17: Multiple sclerosis 3 II/36

Session 18: Higher function disorders II/38

Session 19: Cerebrovascular disorders 3 II/39

Session 20: Neuro-ophthalmology II/41

Session 21: Coma and sleep disorders II/42

Session 22: Multiple sclerosis 4 II/38

Session 23: Higher function disorders 2 II/39

Session 24: Cerebrovascular disorders 4 II/48

Session 25: Motor neuron disease II/49

Session 26: Extrapyramidal disorders 2 II/51

Session 27: New insights into MS disease mechanisms II/52

Session 28: General neurology 2 II/53

Session 29: Child neurology II/54

\section{POSTER SESSIONS}

Poster Session 1

Cerebrovascular disorders II/55

Clinical neurophysiology II/60

Neurogenetics II/65

Child neurology II/71

Peripheral neuropathy II/74

Multiple sclerosis II/78

Neuro-imaging II/83

Poster session 2

Cerebrovascular disorders II/89

Clinical neurophysiology II/93

Extrapyramidal disorders II/97

Epilepsy II/101

Peripheral neuropathy II/104

Multiple sclerosis II/109

Motor neuron disease II/114

Neurorehabilitation II/119

Poster session 3

Peripheral neuropathy II/123

Dementia II/125

Infection II/129

Epilepsy II/134

General neurology II/137

Multiple sclerosis II/141

Muscle disorders II/145

Neuro-oncology II/151

Poster session 4

Cerebrovascular disorders II/156

Higher function disorders II/161

Extrapyramidial disorders: Parkinson's disease II/165

Epilepsy II/168

General neurology II/172

Multiple sclerosis II/176

Muscle disorders: neuromuscular junction disorders II/182

Neuro-opththalmology II/185

Poster session 5

Cerebrovascular disorders II/189

Dementia II/193

Extrapyramidial disorders II/197

Preclinical neurology II/199

General neurology II/200

Multiple sclerosis II/204

Pain and headache II/212

Sleep disorders II/218

Author index II/221 


\section{Presidential symposium}

\section{Coma and locked-in syndrome}

Coma and locked in syndrome - Disorders of consciousness

G. Moonen

University of Liège (Liège, BE)

Physiological and pathological alterations of consciousness can be classified on a two axis diagram wakefulness $\mathrm{x}$ awareness. We will focus on the definition, diagnosis and prognosis of vegetative (VS) and of minimally conscious states (MCS) pointing out the frequent misdiagnosis. Currently, clinical evaluation, electrophysiology and neuroimaging should be combined to characterize as precisely as possible "the clinical phenotype" of these patients.

We will point out 1) the urgent need for objective markers available at the bedside, 2) impaired fronto-parietal global workspace and disconnection syndrome in VS while 3) preserved pain and emotional perception in MCS.

Finally, self evaluation of quality of life by chronic locked-in syndrome patients gave "unexpected" results when compared with matched controls pointing out their right to die but also and more often to communicate and to receive appropriate care.

Multimodal neuroimaging studies of recovery of consciousness after severe brain injury

N. Schiff

Cornell University Medical College (New York, US)

Despite major advances in neuroscience, recovery of consciousness after brain injury remains poorly understood. At the origin of this challenge is the surprising degree of uncertainty of underlying brain function that may be present when confronted with patient at the bedside with very limited or even no overt signs of behavioral responsiveness. Across the range of behaviorally defined states from vegetative state (no evidence of self or environmental awareness), minimally conscious state (at least some evidence of awareness), and up but not including patients in locked-in state (full consciousness with no motor control) there are many patients whose level of consciousness we cannot at present confidently assess.

This lecture will focus on multi-modal neuroimaging studies that provide emerging insight into possible biological mechanisms at a 'circuit-level' underlying recovery of consciousness after severe brain injury. Standard clinical imaging modalities and behavioral assessments provide very limited guides for prognosis. Moreover, current neurological practice relies on probability estimates derived from these standard measures without consideration of a variety of possible underlying mechanisms producing impaired brain function. The discussion will review recent studies of severely braininjured patients in vegetative state and minimally conscious state. We will examine multi-modal neuroimaging evidence of residual functional capacity and possible changes in brain structural connectivity in some patients remaining in prolonged minimally conscious state. Rare patients within this subgroup have demonstrated spontaneous recoveries of consistent communication. Possible common underlying 'circuit-level' mechanisms of brain dysfunction will be discussed based on measurements obtained from functional magnetic resonance imaging, quantitative electroencephalography, positron emission tomography, and diffusion tensor imaging techniques. We will also discuss the clinical and neuroscientific foundations underpinning a recent study that demonstrated behavioral improvements with central thalamic stimulation in a single severely brain-injured human subject who had remained in the minimally conscious state (MCS) for 6 years.

Collectively, experimental and clinical data support further research to develop diagnostic and investigational therapeutic approaches at the 'circuit-level' to address the problem of recovery of consciousness after severe brain injury. The long-range goal of these studies is to establish methods to track, predict, and assist recovery across a heterogenous pool of brain-injured patients. However, such development will require stringent effort to develop patient assessment strategies, study designs and ethical frameworks to ensure that this line of enquiry remains aimed at achieveable and desirable patient-centered goals.
Detecting awareness in non-communicative patients using fMRI

A.M. Owen

MRC Cognition and Brain Sciences Unit (Cambridge, UK)

The vegetative state is one of the least understood and most challenging conditions in modern medicine. The term describes a condition in which patients who emerge from a coma appear to be awake, but show no signs of awareness. It is extremely difficult to assess cognitive function in these patients, because their movements may be minimal or inconsistent, or because no cognitive output is possible. This factor, coupled with limited experience of this complex condition and closely related disorders such as the minimally conscious state, has been linked to an alarmingly high rate of misdiagnosis on clinical audits. In a series of recent studies, we have demonstrated that functional neuroimaging can be used to identify residual cognitive function, and even conscious awareness, in some patients who are assumed to vegetative state or minimally conscious state, but still have abilities that cannot be detected by standard clinical means2-13. I will describe a series of paradigms that systematically increase in complexity with respect to the cognitive processes required, and therefore allow us to infer how much cognition remains based on 'normal' patterns of brain activation. At the lowest level, we examine responses to various sound and speech stimuli. A significant minority of vegetative and minimally conscious patients produce speech-related responses in the superior temporal-lobe region that are indistinguishable from those in a healthy brain. More complex linguistic stimuli, which produce distinct patterns of activation associated with comprehension, also elicit normal responses in some, but fewer, patients. However, an appropriate neural response to the meaning of spoken sentences is not unequivocal evidence of awareness. For example, we have recently demonstrated that light sedation in healthy volunteers disrupts the 'normal' neural response to speech comprehension and is associated with a marked reduction in performance. We have therefore developed a new approach in which patients assumed to be vegetative or minimally conscious are instructed to perform mental imagery tasks at specific points during the fMRI scan. Results from a small group confirm that some patients, diagnosed as vegetative, retain the ability to understand spoken commands and to respond via their brain activity rather than speech or movement. Reproducible and robust task-dependent responses to commands without the need for practice or training could be a novel way for some vegetative, minimally conscious, or locked in patients to use their residual cognitive capacities to communicate thoughts by modulating their own neural activity. Our pilot results in healthy volunteers confirm that this method can be used to communicate real-time 'yes' and 'no' responses, without the need for any overt behavior, with $100 \%$ reliability.

4

Brain-computer interfaces in the locked-in syndrome

A. Kübler, F. Nijboer, A. Furdea, S. Halder, N. Birbaumer University of Tubingen (Tubingen, DE)

Brain-computer interfaces (BCI) provide connections between the brain and a computer. Voluntary regulation of neuro-electrical activity or brain activity as a response to sensory stimulation are used to control cursor movements or switches on a computer. BCIs are aiming at restoring communication particularly in locked-in patients. With more than 35 severely paralysed patients, some of them in the locked-in state, it could be shown that BCI control and communication is possible in all stages of paralysis. BCI on the basis of neurofeedback and those which use evoked potentials (P300) also operate in the auditory mode rendering this technology feasible for patients with impaired vision; time needed for training, however, exceeds by far that of visual BCI. New applications for the P300-BCI are Internet browser and Painting. Instead of letters, weblinks and graphic icons are presented in a matrix for selection. Healthy volunteers and patients are highly motivated to use a BCI and BCI performance is influenced by motivation, but not by current mood. Specifically, mastery confidence increases with training whereas fear to fail decreases with training. At the same time fear to fail is positively related to performance confirming the well-established Yerkes-Dodson law that a medium level of arousal promotes optimal performance. Future development is aiming at merging different EEG and muscular signals, different sensory presentation modes, and a variety of applications into one system for the benefit of severely impaired patients. 


\section{Symposia}

\section{Behavioural disorders and dementia}

25

Pathophysiological basis of behaviour: relevance to dementia

T.W. Robbins

University of Cambridge (Cambridge, UK)

The study of dementia requires careful neuropsychological assessment for the monitoring of progress of disease and of effects of any putative treatments. In this presentation, I will describe the use of the CANTAB automated test battery which has been validated in brain damaged patients with discrete cortical lesions and by functional neuroimaging where feasible. I will describe the design and use of a visuospatial paired associates learning test, which has been shown to have utility in the detection of Alzheimer's disease, both in patients with questionable dementia and in the normal healthy population. This test is sensitive to damage to the hippocampus and to the prefrontal cortex, and I will present data from functional neuroimaging studies in the normal population. I will contrast this with a neuropsychological assessment of basal ganglia diseases such as Parkinson's and Huntington's disease, where the accent is on assessment of fronto-striatal executive function, in tests of planning, working memory, inhibition, decision-making and cognitive flexibility. Many of these tests are based on procedures used in experimental animals to enable a translational approach and this will also be demonstrated, using novel data that also indicates possible sensitivity to candidate pharmacological treatments. The utility with respect to functional genomics will also be illustrated in the context of the COMT polymorphism in Parkinson's disease. The theoretical significance as well as the application of this neuropsychological approach will also be described.

\section{6}

\section{Behavioural disorders in the synucleinopathies}

S.F. Cappa

Vita-Salute University and San Raffaele Scientific Institute (Milan, IT)

The careful definition of clinical phenotypes maintains its importance in the era of molecular neurology. In the field of neurodegenerative disorders, an important aim is the definition of specific profiles of cognitive and behavioural dysfunction, and the assessment of their relationship to the topography of brain pathology and to the underlying molecular mechanisms of disease. The spectrum of behavioural manifestations which can be observed in the synucleinopathies (SNP) is wide, and presents an important overlap with the behavioural disorders found in the tauopathy spectrum. This is not unexpected, for a number of reasons. In the first place, many behavioural disorders are manifestations of the multiple facets of executive dysfunction, which actually reflects the pathological involvement of fronto- subcortical mechanisms, rather than the specific mechanism of disease. Second, other symptoms, such as depression or agitation, may be due to environmental interactions, or represent side effects of symptomatic pharmacological treatments. However, it is likely that some features of cognitive and behavioural dysfunction may be relatively specific for the SNP, reflecting underlying neurobiological differences. In the case of Parkinson's disease (PD), depression is an important clinical issue, which may be more prevalent than in the case of other neurodegenerative disorders, reflecting the complex combination of neurotransmitter abnormalities, involving dopamine, serotonin and norepinephrine. Another clinically relevant aspect in the management of PD are the impulse control disorders, such as pathological gambling and hypersexuality, which appear however to be related to dopaminergic medications. It has been proposed that a form of parasomnia, i.e. REM sleep behaviour disorder (RBD), may represent a relatively specific behavioural marker of the SNP. RBD is characterised by the loss of the physiological muscle atonia during REM sleep, and by prominent dream enactment behaviour. RBD has been found to precede the onset of PD, multiple system atrophy (MSA) or dementia with Lewy bodies (DLB) by years or even decades. In particular, the observation that subclinical neuropsychological impairments are present in patients with the idiopathic form of RBD has led to the suggestion that the condition may represent a risk factor for evolution to dementia, an hypothesis that needs to be evaluated with longitudinal studies. The high frequency of RBD in the SNP probably reflects the pathological involvement of specific brainstem nuclei in these conditions. Similarly, it has been suggested that visual hallucinations, which are one of the diagnostic hallmarks of DLB, may be attributed to a disease-specific physiopathological mechanism of cortical functional imbalance.
27

MCI and Alzheimer's disease

H. Feldman

Clinic for Alzheimer's Disease and Related Disorders, University of British Columbia (Vancouver, CA)

The broad spectrum of neuropsychiatric symptoms (NPS) that occurs in Alzheimer's disease $(\mathrm{AD})$ can be considered along both the stages of disease and its sub-classification into type of symptoms (e.g. psychosis, affect and behavior). NPS have a complex genesis that includes neurobiological, social and psychological factors which interplay and which must be taken into consideration. Together, NPS represent one of the strongest predictors of the future need for nursing home placement and are associated with caregiver distress including increased their increased use of psychoactive medications, poorer self-rated health and depression.

Recently proposed $\mathrm{AD}$ research criteria require that there be a gradual and progressive impairment in episodic memory with supportive biomarkers of medial temporal lobe atrophy on MRI, abnormal temporal parietal FDG PET and abnormal A 1-42, total tau and p-tau in CSF. This case definition allows that patients can be diagnosed in the prodromal stage of AD before there is significant multidomain cognitive impairment with significant functional disability. It is important in turn to characterize the NPS within this prodromal stage. While there is no specification around the presence and type of NPS symptoms in prodromal AD studies indicate that the most frequent symptoms on the Neuropsychiatric Inventory are depression, apathy, irritability and anxiety. Their presence is associated with a significantly increased risk of progression from defined Mild Cognitive Impairment to $\mathrm{AD}$ and they are associated with increased levels of cognitive impairment and functional disability.

In the mild to moderate stages of $\mathrm{AD}$, agitation and aberrant motor activity become particularly prevalent as they affect $50-66 \%$ of patients and begin to negatively impact social function. Current recommendations for care of NPS in mild to moderate AD include both routine evaluation for NPS as well as a multidimensional plan that includes community programs, nonpharmacological and pharmacological treatments.

The moderate to severe stages of AD include the highest rates of prevalence of NPS and the time when management is most challenging as semantic memory, and language abilities become most impaired. Maintaining circadian rhythms, addressing psychosis and managing agitation are amongst the challenges clinicians face at a time when pharmacological options with psychoactive medications are used with increasing caution around their risks and benefits. The utility of acetylcholinesterase inhibitors and memantine are a treatment option with potential benefit on NPS and can represent a useful treatment consideration at these stages.

28

Behavioural disturbances in frontotemporal lobar degeneration: recent advances in molecular genetics and neurobiology

E. Scarpini, Daniela Galimberti

University of Milan, Fondazione Ospedale Maggiore Policlinico (Milan, IT)

Behavioural symptoms, including apathy, dishinibition, irritability, are typical features of neurodegenerative dementias. Among these disorders, Frontotemporal Lobar Degeneration (FTLD) represents the second most common type of early-onset degenerative dementia after Alzheimer's disease. FTLD is the clinical term used to describe a complex and heterogeneous syndrome that includes Frontotemporal Dementia (FTD), semantic dementia (SD), and Progressive Aphasia (PA). More recently, Progressive Supranuclear Palsy (PSP) and Corticobasal Degeneration Syndrome (CBDS) have been considered under the same label of FTLD, because they overlap both clinically and neuropathologically. The presenting clinical features consist of behavioral dysfunction and personality changes, often with language impairment, loss of social awareness, overeating and impulsiveness, whereas memory is relatively spared. Concerning FTLD, in 1994 an autosomal dominantly inherited form of FTD with parkinsonism was linked to chromosome 17q21.2. Subsequently, other familial forms of FTD were found to be linked to the same region, resulting in the denomination "frontotemporal dementia and parkinsonism linked to chromosome 17" (FTDP-17) for this class of diseases. All cases of FTDP-17 have so far shown a filamentous pathology made of hyperphosphorylated tau protein. Although many FTD families exhibit MAPT mutations, in some cases these mutations did not occur suggesting that other, related genes on chromosome 17 also cause FTD in an autosomal-dominant manner.

Subsequently, the region within the 3.53 centimorgan critical region defined by haplotype analysis in reported families was examined. Several pathogenic mutations were found in progranulin gene (PGRN), harbouring a new causative gene in FTLD pathology. Progranulin is a widely expressed growth factor, which plays a role in development, wound repair and inflam- 
mation by activating signalling cascades that control cell cycle progression. Its role in neurodegeneration is at present under investigation. Neuropathology analysis revealed that ubiquitin immunoreactive neuronal cytoplasmatic and intranuclear inclusions were associated with PGRN mutations. Additional studies demonstrated that mutations in PGRN are associated with highly variable clinical phenotypes, including PSP and CBDS, suggesting that other unidentified environmental and genetic factors might produce considerable phenotypic variability even in patients carrying the same mutations.

\section{Autoimmune disorders of the nervous system}

29

Latest developments in multiple sclerosis

G. Comi

Università Vita Salute San Raffaele (Milan, IT)

Some recent epidemiological studies suggest that the prevalence of multiple sclerosis (MS) in increasing. The modification of the risk has been attributed to the changes in lifestyles or to a more strict control of infections in early life because of the ameliorated hygienic conditions. The increase of prevalence seem to be predominantly present in females, a finding in line with the first hypothesis. Last year has been a break through in genetic analysis of MS: besides the well known role of two other genes codifying for the interleukin 7 receptor and the interleukin 2 receptor alpha have been discovered to ply a significan role in MS. A great contribution to the discovery came from whole genome analysis, thank to the contribution of the concerted action of many centres. Ongoing studies on subgroup of patients may contribute to clarify the other genetic factors determining the risk of MS and influencing the disease course. Genomic and proteomic studies are also largely used to evaluate pathways involved in MS pathogenesis and are expected to contribute to a more individualised treatment. The role of environmental factors has been recognised since a long time, nevertheless the ascertainment of toxic or infective factors involved remain questionable at the best. A great attention has been arised about the potential role of Epstein Barr virus: a recent study found EBV genome in the majority of a group of MS brain and exceptionally in control brain. These findings have not been replicated yet from other laboratories.

There are growing evidences that the immunoloical abnormalities characterising MS change along the disease evolution. In the early phases there is a predominant role of the acute inflammatory lesions, inflammation in MS is dominated by class I MHC restricted CD8+ T-cells, with a potential role for CD4+ T cells in initiating the lesions. On the progressive phases of MS the immunological derangement is compartimentalized with evidence of lymphatic like tissue in subependimal space. Moreover grey matter involvement, rare in early MS become relevant. The correlation between early and late immunological changes remain elusive.

Therapy of MS is substantially improved; availability of different drugs with specific efficacy/safety profile call for the individualisation of treatments based on the prognostic factors and the disease phase. Early treatment is perhaps the most important strategy to follow, recently supported by the evidence that the control of inflammation in early MS allow to significantly modify the short and long term disease evolution.

\section{0}

Autoimmune diseases of the neuromuscular junction

A. Vincent

John Radcliffe Hospital (Oxford, UK)

\section{Myasthenia gravis}

Most patient with myasthenia gravis have AChR antibodies (AChR-MG) that cause morphological damage, by cross-linking the AChR divalently resulting in increased internalization and degradation, and by direct inhibition of the ACh binding site causing a pharmacological block of function. Patients with MG can be divided into subgroups based on age at onset, HLA association, thymic involvement, and AChR antibody status. Thymoma occurs in up to $10 \%$ of MG patients, mostly presenting between the ages of 30 and 60 years. Ocular MG occurs in about $20 \%$ of patients, and only $50 \%$ have AChR antibodies.

About $10 \%$ to $15 \%$ of all patients with MG and generalized symptoms do not have anti-AChR antibodies detectable by the radioimmunoprecipitation test. A proportion of patients without AChR antibodies have antibodies to MuSK which is a receptor tyrosine kinase restricted to the neuromuscular junction in mature muscle. Interestingly, the prevalence of MuSK anti- bodies among patients without $\mathrm{AChR}$ antibodies is highly variable between different centres around the world suggesting a possible environmental stimulus. They are mainly IgG4, and are almost never found in patients with AChR antibodies or with thymoma. The distinctive features of MuSK-MG are often marked ocular, bulbar, neck or respiratory symptoms and, in contrast to AChR-MG, the patients may have normal electrophysiology in limb muscles with evidence of neuromuscular defects in facial muscles (eg. Orbicularis occuli). They respond to immunosuppression with prednisolone and azathioprine but often alternative immunosuppressive treatments are required. It is not yet clear how the antibodies cause the neuromuscular junction defect.

There remaining patient usually have less severe symptoms and are more responsive to standard treatments than the MuSK-antibody positive patients. Recently we proposed that they have AChR antibodies undetectable by current laboratory tests. At least a proportion of these patients have AChR antibodies detectable by an immunofluorescent method using human embryonic kidney cells to express AChRs at high density (Leite, Willcox and Vincent unpublished observations).

\section{Other neuromuscular junction disorders}

Two other autoimmune diseases of the neuromuscular junction, the Lambert Eaton syndromes and acquired neuromyotonia, are associated with antibodies to voltage-gated calcium and potassium channels respectively. In LEMS the antibodies are found equally in cases with or without small cell lung cancer (or rarely other tumours) but those with cancer may also develop cerebellar ataxia. The peripheral symptoms tend to improve with treatment whereas the central symptoms rarely show a good response. About $20 \%$ of cases of acquired neuromyotonia are associated with thymoma, and/or myasthenia. Most patients can be treated with anti-epileptic drugs and do not need immunosuppression. However, Morvan's syndrome (severe neuromyotonia, autonomic and central disturbance) often requires immunotherapies, although some cases show spontaneous improvement over time.

31

Pathogenesis and treatment of the Guillain-Barré syndrome

$H$-P. Hartung

Heinrich-Heine-University (Dusseldorf, DE)

Guillain-Barré syndrome (GBS) continues to be a challenge as an emergency condition associated with significant mortality and disability. Recent years have witnessed enormous advances in deciphering the pathogenesis of GBS. It is now widely accepted that GBS represents a spectrum including AIDP, AMAN, AMSAN, Fisher syndrome (FS), and regional variants. Most are preceded by an infective illness and the leading bacterial agent recorded is C. jejuni. The detection in sera of antibodies to gangliosides and related glycosphingolipids of protean specificities and to ganglioside complexes has prompted efforts to establish a link through the process of molecular mimicry.The case has been proven most convincingly for the AMAN variant of GBS. Epidemiologically, there is a clear relationship between antecedent $C$. jejuni infection and the presence of ganglisode IgG antibodies, mostly against GM1, GD1a anfd GalNAc-GD1a. Parenteral administration of ganglsiodemixtures generated in few patients an anti-GM1 IgG response and AMAN type neuropyhsiology and neuropathology. It has been possible to induce a replica of AMAN in rabbits by immunizing with GM1 and similarly by injectinmg an isolated lipoologosaccharide fraction from a C. jejuni strain associated with development of GBS that contained GM1-like epitopes. In FS, a GT1a-like lipooligosaccharide mimicking GQ1b was purified from a $C$. jejuni isolate emphasizing that epitopes shared between the microbial agent and peripheral nerve drive an antibody response that underlies the neuropathy. The clinical manifestation within the spectrum of GBS may be largely determined by lipooligosaccharide biosynthesis genes and their polymorphisms. Ganglioside antibodies may perturb neuromuscular transmission and in a clearly complement-dependent fashion disrupt the molecular topography of nodal and paranodal proteins, induces motor axonal degeneration and perisynaptic Schwann cell injury.

Supportive and intensive care management had already greatly improved the guarded prognosis of GBS when in the 1980s plasma exchange and later IVIG were introduced as equally effective treatments. Some patients however still show a poor recovery, in particular when axonal damage occurs during the course of the disease.

Given the key impact of complement activation with assembly of the membrane attack complex, complement targeted therapies have successfully been studied in animal models.Most recently the monoclonal C5 convertase antibody eculizumab, approved for paroxysmal nocturnal haemoglobinuria, was shown to prevent functional and pathological changes in an in vitro and an in vivo model of FS.

In view of the pathological heterogeneity of the disease, other immunointerventions directed at $\mathrm{T}$ cells may also be worth assessing. 


\section{References}

Halstead SK et al. (2008) Brain epubahead

Kieseier BC et al. (2004) Muscle Nerve 30:1131-156

Kiesier BC et al. (2006) Curr Opin Neurol 19:433-436 and 437-445

Meyer zu Hörste G et al. (2007) Nat Clin Pract Neurol 3:198-211

Meyer zu Hörste G et al. (2008) Muscle Nerve 37:3-13

Yuki N (2007) Muscle Nerve 35:691-711

32

Immunopathogenesis of inflammatory myopathies

R. Hohlfeld

Ludwig Maximilians University (Munich, DE)

Three types of inflammatory myopathy are traditionally distinguished: polymyositis (PM), inclusion body myositis (IBM), and dermatomyositis (DM). IBM is the most common inflammatory myopathy in adults, whereas "pure" PM is much less common than was previously thought.

In PM and especially IBM, clonally expanded, CD8-positive T cells invade muscle non-necrotic fibers expressing MHC class I antigens. The causative target antigen(s) have not been defined. Recently it became possible to "revive" putatively pathogenic, autoimmune CD8+ T cells from frozen muscle biopsy tissue. These "resurrected" T cells can be used to search for the target antigen(s). Apart from T cells, the inflammatory infiltrates in PM and IBM muscle contain B cells, macrophages, and myeloid dendritic cells.

In DM the mechanisms of muscle fiber injury seem to be different. The infiltrates contain plasma cells and plasmacytoid dendritic cells. According to one hypothesis, anti-endothelial antibodies cause capillary injury and subsequent peri-fascicular atrophy. According to another hypothesis, antiDNA and/or anti-RNA antibodies play a major role, triggering a cascade of pathogenic changes amplified by interferon-alpha, as has been proposed for systemic lupus erythematosus.

\section{References:}

Hohlfeld, Engel (1994) Immunol Today 15:269-274

Wiendl, Hohlfeld, Kieseier (2005) Trends Immunol 26:373-380

Dalakas, Hohlfeld (2003) Lancet 362:971-982

Greenberg (2007) Neurology 69:2008-2019

Hohlfeld, Engel (2007) Neurology 69:1966-67

\section{Multiple sclerosis: when to start a treatment and which treatment}

\section{6}

The rationale for early treatment in multiple sclerosis

A. Compston

University of Cambridge (Cambridge, UK)

The arrival of therapies that are considered to modify the course of multiple sclerosis, and the licensing of the first wave of medications, marks the beginning of an era that will close with the discovery of fully effective and safe medicines for this complex and potentially disabling disease. Taken together, the results of clinical trials support the hypothesis that inflammation is necessary for new lesion formation and conditions axon degeneration. The implication is that immunological therapies will best prevent sustained accumulation of disability and disease progression if given early in the course and before the cascade of events leading to axon degeneration is irretrievably established. This may explain the present limitations of immunotherapy in patients with secondary progressive multiple sclerosis. But it raises the dilemma of exposing individuals who may never develop disabilities from multiple sclerosis to the unpredictable hazards of prolonged immunosuppression as the price paid for stabilising the disease process in those who are destined to do badly. The goals of future therapies in multiple sclerosis must confront the main unmet need of how to limit the neurodegenerative aspect of disease progression. One analysis of the interplay between inflammation and neurodegeneration is that areas of focal damage, constituting plaques, are driven by inflammation and microglial activation. Regions of the central nervous system or the brain and spinal cord as a whole may be genetically susceptible to neurodegeneration but this vulnerability nevertheless has to be exposed by inflammation. Early treatment aims to inhibit the establishment of immunological chronicity manifesting as diffuse microglial activation; protect intact axons from acute injury using anti-excitotoxic and membrane stabilizing agents; provide trophic support for persistently demyelinated axons using growth factors and strategies that might include pharmaceutical strategies for enhancing remyelination; and promote plasticity and axon regeneration by manipulation of extracellular matrix molecules and manipulation of inhibitory environments. Together, it is hoped that these interacting processes will effectively and safely limit and repair the damage.

47

Starting treatment after a CIS

G. Comi

Università Vita Salute San Raffaele (Milan, IT)

There are converging evidences that axonal damage starts very early in multiple sclerosis (MS). This early nervous damage occurring inside the demyelinating lesions is due to inflammation, which is predominant in early stages of MS. In order to prevent irreversible nervous damage, the underlying cause of disability, it is essential to start disease modifying treatment as early as possible.

Patients with a Clinically Isolated Syndrome (CIS) and exclusion of other diseases, have a high risk of developing MS, particularly if they show abnormalities on the initial magnetic resonance imaging (MRI) scan of the brain and/or evidences of intrathecal synthesis of immunoglobulins. Epidemiological studies and clinical trials performed in CIS patients demonstrated that, whitin 2 years, about $90 \%$ of these patients can be diagnosed as MS according the McDonald criteria. Moreover patients with a high number of lesions and/or enhancing lesions in the bran MRI performed at first presentation, have a very high risk of experiencing a second attack shortly after the first. Baseline MRI feature in patients with a first event seem not only to determine the risk of conversion to clinically definite MS, but also correlate with later disability.

All the three clinical trials of beta interferons conducted in CIS patients - the BENEFIT study, the Controlled High-risk Avonex multiple sclerosis Prevention study (CHAMPS) and the Early Treatment of MS study (ETOMS) - have shown that treatments can reduce the rate of conversion to clinically definite MS, prolong the time to the second attack and reduce brain MRI activity. Extension of these studies confirmed that even after 5 years of follow up patients who received immediate treatment still maintain a significant benefit on the prevention of a second attack. Moreover the 3-year data analysis of the BENEFIT trial revealed a significant $(40 \%)$ reduction of the risk of confirmed EDSS progression in those who received immediate treatment compared to those who had a delayed interferon beta $1 \mathrm{~b}$ treatment (after the 2 years of the double blind phase), a definite evidence that early treatment protects from irreversible nervous damage. More recently the PRECISE clinical trial demonstrated that also Glatiramer Acetate significantly reduce in CIS patients the risk of conversion to clinically definite MS by $44 \%$.

Based on the uniform positive results of all these clinical trials treatment of CIS with beta interferons is now widely recognized and both EMEA and FDA approved this indication; unfortunately some countries still refuse to reimburse such treatment to CIS patients for economic reasons. Nevertheless not all CIS patients should receive an immediate treatment. Patients without brain/spinal cord lesions have a low risk to develop a second attack. The same reservation against an immediate treatment should be applied also to CIS patients without an adequate exclusion of alternative diagnoses.

48

Starting treatment when definite MS is diagnosed

C. Confavreux

Hôpital Neurologique (Lyon, FR)

The therapeutic armamentarium in multiple sclerosis (MS) have greatly developed over the last years, notably for relapsing forms of MS. Moreover, new therapies are continuously emerging. Since 1993, several pivotal studies have shown that beta-interferons and glatiramer acetate, at the stage of clinically definite MS (CDMS), are able to reduce relapse frequency and MRI activity by about $30 \%$ and $50 \%$, respectively. However, their impact on the accumulation of disability in the mid and long run remains uncertain. In the late nineties, several studies suggested that efficacy among interferons could differ depending on the unit dose per intake and the frequency of administration. That said, none of these studies was definitely convincing, especially when blinding was considered. Last months have introduced some clarification and simplification in the area. Indeed, different studies (BEYOND, REGARD, BECOME) have allowed an head-to-head comparison between several drugs. The conclusion is that the different beta-interferons and glatiramer acetate exhibit a similar level of efficacy in relapsing MS. In the meantime, natalizumab has emerged as a new therapy with a much greater efficacy as it decreases the relapse rate by $60 \%$ and MRI activity by $80 \%$. Unfortunately, it is also likely to induce serious infectious and, possibly, neoplastic complications requiring the closest monitoring while administrating 
the drug. Last, but not least, since early 1990's, several studies have shown the main advantage of intensive immunosuppressive interventions with mitoxantrone and, to a lesser extent, with cyclophosphamide, in aggressive forms of MS.

Considering the different treatments available and current evidence of their efficiency, what are the therapeutic choices to the physician in 2008 in definite MS? Official guidelines for the approved therapies may prove quite useful as a first guide. In the relapsing forms of MS with a low relapse rate, the choice to treat can be dismissed according to many criteria including the patient's wish. In more active forms with, at least, one or two relapse(s) in the one or two preceding year(s), it is logical to propose interferons or glatiramer acetate. In some cases, and for various reasons, oral immunosuppressants such as mycophenolate mofetil, or even azathioprine, can also be suggested. These treatments are the first-line of interventions. In the cases with a more severe clinical activity and those showing resistance to interferons or glatiramer acetate, it is logical to prescribe natalizumab with a close monitoring. This therapy is proposed in the second place. Last, in the rare forms of aggressive MS right from onset or during a disease breakthrough, one should propose third place therapies like mitoxantrone, or even cyclophosphamide.

This apparently simple therapeutic design hides many uncertainties though. When to start a treatment? Which is the place of MRI in therapeutic decisions? Which first-line therapy is to be proposed? What are the criterias to assess the efficacy of a treatment? When to stop a treatment? When to adjust a treatment or to switch to another one? Should one look for therapeutic windows after a given period, let us say 24 months, of continuous treatment with natalizumab? Should one propose a treatment to take over from mitoxantrone or cyclophosphamide and which treatment ? This list is not limitative ... Despite these many uncertainties, it is clear that MS has entered the therapeutic area and that it is nowadays possible to tailor the immune intervention for controlling the acute focal recurrent inflammatory process which is the first edge of MS. Are we able to control in parallel the diffuse and progressive neurodegenerative process which is the other edge of the disease? This is another story. We may expect however that concentrating on molecules which are able to penetrate in the central nervous system beyond the blood-brain barrier and on early and intensive immune intervention could represent the next significant steps in the long route of the cure of this often devastating disease.

\section{9}

Immunosuppresents as first choice, followed by immunomodulatory agents R.P. Lisak

Wayne State University School of Medicine (Detroit, US)

After 140 years of no therapies that successfully modify the course relapsing remitting multiple sclerosis (MS), the past 15 years have seen the approval of three types of therapy (interferon-beta, glatiramer acetate and natalizumab) that have been demonstrated to reduce relapses and have a positive effect on progression to disability, at least over the periods of time that are feasible for clinical trials. There are new agents being tested that also hold promise and studies of combinations of approved agents. Suggestions have been made that intensive immunosuppression can be of benefit for patients who are clearly having frequent relapses despite treatment with one or more of the approved agents. All of this supports the clinical observation that the current therapeutic approaches are imperfect. This has lead to the suggestion of inducing disease control with immunosuppressive agents given simultaneously or for several months before initiating treatment with an immunomodulatory agent that is know to have acceptable side effects, limited toxicity and tolerability. Most published studies combining immunosuppression with immunomodulatory agents are, as noted, for treatment failures and there are very few studies of any size or with controls demonstrating the efficacy or even the long-term safety of such an approach. As we have seen one cannot assume that combinations are safe, even short-term induction therapy. This argues against routine use of induction therapy with immunosuppressive therapy followed by immunomodulation. Two questions need to be considered. One is how would you organize such a study including patient selection, selection of agents and outcome measures. The related question is that in the absence of such available data, are there patients in whom the theoretical benefits and their clinical course at the time of diagnosis or prior to any treatment with disease modifying agents, should be offered such therapy.

\section{Transient ischaemic attack (TIA): imaging and management}

50

Diagnosis and risk assessment for TIA

P.M. Rothwell

University of Oxford (Oxford, UK)

Approximately $30 \%$ of ischaemic strokes are preceded by a transient ischaemic attack (TIA). However, due to methodological problems in early studies of prognosis, the immediate risk of stroke after a TIA was underestimated. Recent hospital-based and population-based cohort studies have reported 7-day risks of stroke of up to $10 \%$. Yet, there is considerable international variation in how patients with suspected TIA are managed in the acute phase, with some healthcare systems providing immediate emergency inpatient care and others providing non-emergency outpatient clinic assessment. Clinical guidelines state that all patients in whom a diagnosis of TIA is suspected should be assessed and investigated quickly, although the time limit set varies from 24 hours to 14 days. However, the key question is not, in fact, whether emergency in-patient care or non-emergency outpatient care is most appropriate. Rather, it is for which patients is emergency assessment required and which patients can be appropriately managed in a non-emergency outpatient setting? Only about $50 \%$ of patients referred for specialist assessment with suspected TIA have the diagnosis confirmed and so even if the 7-day stroke risk after a TIA is as high as $10 \%, 95 \%$ of referrals will not have a stroke in that time period.

Validated scores are now available to predict the short-term risk of stroke after TIA. These scores have several uses. Firstly, they allow primary care and other front-line physicians to identify which of the patients in whom they suspect a diagnosis of TIA should be referred-on for specialist assessment as an emergency. Secondly, they allow secondary care physicians to determine which patients with probable or definite TIA require emergency investigation and treatment. Thirdly, they allow public education about the need for medical attention after a TIA to focus on the specific symptoms and characteristics that identify high-risk individuals.

The relationships between clinical risk scores and findings on brain and vascular imaging and other investigations are now being determined and composite scores are being derived and validated. It is likely that these models will allow very accurate prediction of risk in individual patients, which will facilitate decisions about the urgency and intensity of treatment and the need for hospitalisation, as well as the design of treatment trials in the acute phase after TIA.

Reliable data on prognosis, risk factors, and correlates with findings on brain and vascular imaging is also leading to a better understanding of the mechanisms of early recurrence, which is vital if effective new treatments are to be developed. Studies of biomarkers, particularly markers of an increased tendency to thrombosis, are also leading to potential new treatment targets.

There are, in fact, few other areas of clinical practice in neurology that have seen such a rapid increase in understanding of prognosis, risk factors, investigation, mechanisms and treatment than TIA and minor stroke.

51

Ultrasound and MRI in the diagnosis of transient ischaemic attacks (TIA) M.G. Hennerici

University Clinic Mannheim (Mannheim, DE)

The most intense neuroimaging stroke research focuses upon expanding neuroimaging to provide information to the treating physican about the acute state of the ischaemic brain, and the risks and benefits that may be associated with reperfusion therapy in a given individual. Clinically useful information is likely to result from rapid, widely accessible and accepted technologies that can distinguish brain tissue likely to die unless reperfused (= true penumbral tissue) from tissue likely to die despite reperfusion (= core tissue). Less developed but equally important are techniques to predict which patients are most likely to be at risk from reperfusion treatment e.g. from haemorrhage due to an apparent leakage of the blood-brain barrier. Decisions about returning blood flow to the ischaemic brain will be made on biologic data regarding the amount and location (topographic aspects) as well as regeneration/remodelling capacities (functional aspects) of brain tissue that will die unless there is reperfusion. This cannot be achieved by means of the traditional definition of cerebral ischaemia ("TIA" vs. stroke subtypes based on the time course of relapsing/persistent clinical signs or symptoms), which disregard the aforementioned instantaneous brain tissue changes plus their underlying, often overlapping and rapidly changing vascular mechanisms (hemodynamic/embolic/collateral blood flow/small vessel perfusion/partial reperfusion and distal embolism from thrombotic fragmentation etc).Short lasting, relapsing and remitting symptoms ("TIA") 
in the acute phase are highly specific predictors of a poor short-term prognosis but are still most difficult to assess with presently available neuroimaging techniques (MRI/US) because of the obscure presentation and heterogeneity of associated oligaemic tissue, that will eventually survive without reperfusion (and without clinical deficit), but represents the area of highest vulnerability to deteriorate early within hours/days. While such areas can be identified with current PET in highly selected patients recruited in research studies only, areas of different degrees of abnormal perfusion cannot yet be discriminated by means of MRI/US. This is the most promising area of research to develop algorithms for brain maps presumed to characterise core/penumbra/oligaemic areas and display changes during the spontaneous and treatment-related course in real-time. Pilot clinical studies are on their way and will improve from currently developed hard- and software technologies. They will replace the already outdated concept of TIA in acute brain ischaemia.

53

TIA clinic concept

P. Canhão

Hospital de Santa Maria (Lisbon, PT)

Patients with a transient ischemic attack (TIA) need urgent and expert evaluation soon after their event, as they are at high risk of suffering a stroke. It is controversial what the optimal setting for their first assessment is. Patients with a suspected TIA are frequently seen at emergency department or by their family doctor as outpatient. Comprehensive testing and diagnosis often cannot be organized at those places, and there is a long delay between the TIA and completeness of the diagnostic evaluation and start of treatment. Because the risk of stroke is higher in the first days after TIA, those types of care are currently considered unsatisfactory to prevent stroke. On the other hand, some patients are directly referred and admitted to hospital, but this approach may be not cost-effective and carry unnecessary hospitalization risks.

TIA clinic is an alternative setting to evaluate patients with suspected TIA, providing it can be of easy access, may be able to perform investigations urgently, and initiate appropriate treatment soon after the TIA.

To be effective, TIA clinic should be immediately available. Long delays to assessment to TIA clinic are not adequate. Audits usually identify that long delays to assessment are associated with high rates of recurrent stroke before the clinical appointment.

TIA clinic should be organized to: 1) provide a standardized assessment by vascular neurologists or specialist stroke services, able to distinguish between TIA or minor stroke and other stroke mimics;2) make available auxiliary tests to clarify the etiology of the TIA, and exclude other conditions; 3) Identify patients at high risk for subsequent stroke, for example accordingly to the $\mathrm{ABCD} 2$ score and the results of further ancillary tests; 4 ) make clinical decisions, deciding whether the patient could be discharged home or be admitted to a stroke unit; 5) Start immediately secondary prevention.

At the TIA clinic, immediate work-up diagnostic should be organized to provide: brain imaging (either MRI or a CT scan); electrocardiography to exclude a high-risk source of cardiac embolism; carotid duplex ultrasonography and transcranial Doppler of brain arteries, to exclude a high-grade stenosis; routine blood tests; individual patients may need transthoracic or transoesophageal echocardiography when a cardiac source for embolism is suspected.

Several reports confirmed the feasibility of urgent investigation and early treatment of patients seen at TIA clinics. Some reports further suggest that evaluation at TIA clinics and immediate treatment reduces stroke risk after TIA. It is also likely to involve lower costs than those of hospitalization, and greater patient satisfaction because most of patients can be discharged home.

Improvement of the articulation between TIA clinics and primary care physicians, emergency departments, and other clinics or hospital departments, may increase the access of patients with suspected TIA, and their potential for preventing or treating stroke.
FREE COMMUNICATIONS

Oral Sessions

\section{Oral session 1}

\section{Multiple sclerosis 1}

\section{4}

Abnormal connectivity inside the motor network in patients with multiple sclerosis: a multicentre fMRI study

M. Rocca, M. Absinta, P. Valsasina, O. Ciccarelli, A.J. Thompson, L. Mancini, S. Marino, N. De Stefano, A. Rovira, X. Montalban, A. Gass, L. Kappos, C. Wegner, C. Enzinger, S. Ropele, T. Korteweg, F. Barkhof, P.M. Matthews, M. Filippi Scientific Institute San Raffaele (Milan, IT); University College London (London, UK); University of Siena (Siena, IT); Hospital Vall d'Hebron (Barcelona, ES); University Hospital (Basel, CH); University of Oxford (Oxford, UK); Medical University Graz (Graz, AT); VU University Medical Centre (Amsterdam, NL); Imperial College and Hammersmith Hospital (London, UK)

Objectives: In small groups of multiple sclerosis (MS) patients, abnormal activations of areas of the sensorimotor network and, more recently, abnormal connectivities between these areas have been shown. The aims of this study were to characterize abnormalities of functional connectivity within the motor network in a large number of MS patients and their correlation with structural MRI parameters. We also assessed the feasibility of a multi-centre fMRI studies of brain connectivity.

Methods: Structural and functional MRI scans during right hand movement were acquired in 61 right-handed MS patients without hand impairment and 74 age-matched controls at eight European sites. T2 lesion load (LL) and corpus callosum area (CCA) were measured. In a subgroup of subjects, diffusion tensor (DT) MRI metrics inside the CC and the corticospinal tracts (CST) were also assessed.

Results: CCA was reduced in MS patients compared to controls $(\mathrm{p}<0.0001)$. DT MRI metrics inside the CC and the left CST were significantly abnormal in MS patients compared to controls. Dynamic causal modelling optimisation showed that in both patients and controls a model with the left primary sensorimotor cortex (SMC) as the seed region to compute correlation maps was the best predictor of system behaviour. In this model, compared to controls, MS patients had increased functional connectivity between: the left primary SMC and the left prefrontal cortex (PFC), the left PFC and the supplementary motor areas (SMA) and vice versa, the left secondary sensorimotor cortex (SII) and the SMA and vice versa, the right SII and the SMA and vice versa, the left SII and the right SII, and the right SMC and the SMA. They also showed reduced functional connectivity between the left SMC and the right cerebellum. No interaction was found between disease group and centre. Coefficients of altered connectivity were weekly correlated with brain T2 LL and moderately correlated with left CST damage.

Conclusions: Multi-centre fMRI studies of functional connectivity changes in diseased people are feasible. This large fMRI study shows consistent changes of measures of abnormal functional connectivity in MS patients in comparison with controls, which might have an adaptive role in limiting the clinical consequences of structural damage. The analysis of connectivity shows that short-range connectivities are enhanced as long as long-distance connectivities might be impaired by progressive pathology.

055

Functional sensory motor connectivity is preserved in patients with paediatric multiple sclerosis

M. Rocca, M. Absinta, A. Ghezzi, L. Moiola, M. Sormani, G. Comi, M. Filippi Scientific Institute San Raffaele (Milan, IT); Department of Neurology (Gallarate, IT)

Objectives: The occurrence and effectiveness of adaptive cortical mechanisms have been considered among the factors contributing to limit the clinical manifestations of multiple sclerosis (MS). To further elucidate the role of brain plasticity in MS, we investigated changes of measures of functional connectivity inside the motor network in patients with pediatric MS, who are usually considered to have a more favourable clinical course, in comparison with those with the adult-form of the disease. We also assessed the correlation between changes of measures of connectivity and structural damage within the corpus callosum (CC) and the corticospinal tract (CST). 
Methods: Dual-echo, diffusion tensor MRI and functional MRI scans during right hand movement were acquired in 17 patients with pediatric MS, 16 adult patients with clinically isolated syndromes (CIS) suggestive of MS, 14 adult patients with relapsing-remitting (RR) MS and no clinical disability, and 10 healthy controls. Whole brain, CC and CST T2 lesion load (LL) as well as diffusivity metrics within the CC and the CST were measured. Analysis of connectivity was performed using a dynamic causal modelling (DCM).

Results: DCM analysis showed that coefficients of connectivity of the sensorimotor network were similar between healthy controls and pediatric MS patients, while in adult patients with CIS and in those with RRMS there was a progressive increase of functional connectivity between the left and the right primary sensorimotor cortex (SMC) and vice versa, the right cerebellum and the left SMC, the right cerebellum and the right SMC, the left secondary sensorimotor cortex (SII) and the left SMC, and the supplementary motor area and the left SMC. All these connectivity changes were more pronounced in patients with RRMS. Increase of coefficients of connectivity was correlated with disease duration and regional damage inside the $\mathrm{CC}$ and the CST.

Conclusions: The preservation of brain adaptive properties might contribute to explain the more favourable clinical outcome of pediatric MS patients. The progressive recruitment of cortical networks over time in patients with the adult forms of the disease, partially related to the accumulation of irreversible structural damage in selected white matter regions, might result in a more precocious exhaustion of the their plastic reservoir, thus contributing to the clinical progression of the disease.

The study was supported by a grant from the Fondazione Mariani (contract $\mathrm{R}-07-62)$.

056

Detection of EBV antigens in germinal centres in the brain of people with multiple sclerosis

C. Maggiore, G. Trillo-Pazos, R. Reynolds, G. Giovannoni, D. Miller Institute of Neurology (London, UK); Imperial College (London, UK)

Objectives: Understanding the neuropathology of multiple sclerosis (MS) is essential to understand mechanisms of damage and to develop new therapies. MS lesions have distinct histological and immunopathological characteristics depending on disease activity. However, the aetiology of the immune response in this disease is still unknown.

Importantly, in a significant proportion of people with MS (PwMS) affected by a secondary progressive disease ectopic B-cells follicles with germinal centers can be detected in the meninges. These data have been already confirmed by Prineas et al. and Serafini and al. and our preliminary data. In this study, we tested for the presence of germinal centres and EBV infection in MS, as recent studies have involved EBV as a possible trigger agent of disease activity.

Methods: Using snap frozen brain blocks from PwMS provided by MS tissue bank UK, we identified by immunohistopathology lesions and lymphoid infiltrates.

We used different markers for lymphoid tissues and in particular CD79a for the B-lineage, CD68 for macrophages, $\mathrm{CD} 19$ for B lymphocytes and CD20 for mature B cells and follicular dendritic cells; for lesions we used GFAP, MAP2, PLP and CNPase. We stained for EBV involvement in the lesions and in the tertiary lymphoid organs (TLOs) using antibodies targeting antigens of EBV latent phase such as EBNA-1, LMP-1 and LMP-2a.

Results: We confirmed the presence of germinal centres that are CD20+, $\mathrm{CD} 21+, \mathrm{CD} 3+$ with expression of EBV antigens (EBNA1, LMP-1 LMP-2) in brains of PwMS. These germinal centres were microdissected using laser capture microdissection to test for the presence of viral gene expression using The Human Panviral Diagnostic Array.

Conclusion: In contrast with classic germinal centers, TLOs are found in non-lymphoid tissue and are defined by chronic inflammation, the local production of immunoglobulin, and the chronic upregulation of chemokines normally absent in these tissues. EGCs are a feature of several chronic infectious and inflammatory diseases, including rheumatoid arthritis, ulcerative colitis, Sjögren's syndrome, and Helicobacter gastritis. Our preliminary data suggest that MS can follow the same pathogenic mechanism and that EBV could be the trigger agent in EGCs of SPMS. Currently we are testing if other viruses are also present within MS at different stages of disease using a microgenomics approach.
057

Structural and functional MRI abnormalities of the language network in primary progressive multiple sclerosis: a combined functional MRI and tractography study

M. Rocca, A. Ceccarelli, G. Riccitelli, M. Rodegher, G. Comi, M. Filippi

Scientific Institute San Raffaele (Milan, IT)

Objectives: Impairment of several cognitive domains, including attention, memory, reasoning and verbal fluency, is frequently encountered in patients with primary progressive multiple sclerosis (PPMS). In this study, we combined functional magnetic resonance imaging (fMRI) and diffusion tensor (DT) MRI tractography to explore functional and structural abnormalities of the language network in PPMS patients.

Methods: Using a 3 Tesla scanner, dual-echo, DT MRI and fMRI during the performance of a verbal fluency task were acquired from 15 righthanded PPMS patients and 17 sex- and age-matched healthy volunteers. Neuropsychological tests (NPT) exploring memory, attention, verbal fluency and reasoning were administered. DT MRI tractography was used to calculate DT derived metrics inside the left and right arcuate fasciculus. The corticospinal tracts (CST) were studied as "control" white matter fiber bundles. fMRI analysis was performed using statistical parametric mapping.

Results: None of the patients showed abnormal performance in NPT exploring verbal fluency. Compared to controls, PPMS patients had increased MD values in the arcuate fasciculus and CST, bilaterally. During fMRI, compared to controls, PPMS patients had significantly reduced activation of the left caudate nucleus and the left inferior frontal gyrus (IFG). They also showed increased activation of the left precuneus, and the inferior parietal lobule, bilaterally. In PPMS patients, MD increase in the left arcuate fasciculus was significantly related to increased activation of the left precuneus $(\mathrm{r}=0.87)$ and decreased activation of the left caudate nucleus $(\mathrm{r}=-0.83)$ and the left IFG $(r=-0.86)$, while no correlations were found with CST damage.

Conclusions: In PPMS patients without verbal fluency deficits, abnormal recruitment of language-related network occurs. The correlation found between measures of abnormal activation and selective damage of the left arcuate fasciculus suggests that functional cortical changes in patients with PPMS might represent an adaptive response driven by damage of specific WM structures.

This study was supported by a grant from FISM (Fondazione Italiana Sclerosi Multipla) - contract n. 2003/R/48.

\section{8}

Magnetic resonance imaging findings of a phase III trial comparing Betaferon $^{\circledR}$ with Copaxone ${ }^{\circledR}$ treatments in relapsing-remitting multiple sclerosis

M. Filippi, B.G.W. Arnason, G. Comi, S. Cook, D. Goodin, H-P. Hartung, D. Jeffery, L. Kappos, P. O'Connor, T. Bogumil, B. Stemper, V. Filipov, M. Groth, F. Boateng

Scientific Institute University Hospital San Raffaele (Milan, IT); Surgery Brain Research Institutes (Chicago, US); UMD New Jersey Medical School (Newark, US); University of California (San Francisco, US); Heinrich-HeineUniversitat (Dusseldorf, DE); Wake Forest University School of Medicine (Winston-Salem, US); University Hospital (Basel, CH); St Michael's Hospital (Toronto, CA); Bayer Schering Pharma AG (Montville, US); Bayer Schering Pharma AG (Berlin, DE)

Objective: To directly compare magnetic resonance imaging (MRI) outcomes in relapsing-remitting multiple sclerosis (RRMS) patients treated with interferon beta-1b (IFNB-1b; Betaferon $\left.{ }^{\circledR}\right)$ or glatiramer acetate $(\mathrm{GA}$ Copaxone $\left.{ }^{\circledR}\right)$.

Methods: This Phase III, randomised, multicentre study was a large-scale head-to-head comparison of the efficacy, safety and tolerability of IFNB-1b $500 \mathrm{mcg}$, IFNB-1b $250 \mathrm{mcg}$ and GA $20 \mathrm{mg}$. Treatment-naïve patients with RRMS were randomised to subcutaneous IFNB-1b $500 \mathrm{mcg}$ or $250 \mathrm{mcg}$ every-other-day, or GA $20 \mathrm{mg}$ daily for ?104 weeks. Changes in MRI parameters, including T2-weighted, and T1-gadolinium (Gd)-enhancing lesions, volume of T1-weighted hypointense, and total brain volume, were secondary or exploratory endpoints. MRI scans were performed at screening and annually.

Results: The clinical efficacy and tolerability results of this study have been presented elsewhere. A total of 2244 patients from 198 centres worldwide were randomised; 899 patients to IFNB-1b $500 \mathrm{mcg}, 897$ to IFNB-1b 250 $\mathrm{mcg}$ and 448 to GA $20 \mathrm{mg}$. At the end of the study, there were no significant differences between the groups in number or volume of T1 Gd-enhancing lesions. Similarly, there were no significant differences between groups in change in volume of T1-hypointense lesions ('black holes') and brain volume from screening. The cumulative number of T2 lesions at the end of study, however, was lower in the IFNB-1b groups compared with GA 
( $p=0.001$ and 0.017 for $500 \mathrm{mcg}$ and $250 \mathrm{mcg}$ vs GA). T2 lesion volume change between screening and study end was also lower in the IFNB-1b groups compared with GA $(p=0.001$ and $p<0.001)$.

Conclusions: This was the first large-scale study to directly compare IFNB-1b with GA and included MRI monitoring of all patients. There were no differences between treatment groups in terms of T1-hypointense lesions,T1 Gd-enhancing lesions and brain volume. This may indicate that the effects of the two treatments on MRI-derived surrogates, potentially reflecting neuronal or axonal loss, are similar. IFNB-1b-treated patients however, experienced greater reductions in T2 lesion numbers and volumes than those treated with GA, suggesting differences in treatment effects on the overall burden of disease.

This study was supported by Bayer Schering Pharma AG, Berlin, Germany

059

Treatment with glatiramer acetate delays conversion to clinically definite multiple sclerosis in patients with clinically isolated syndrome suggestive of MS

G. Comi, M. Filippi on behalf of the PreCISe Study Group

Objective: To evaluate the efficacy of early treatment with glatiramer acetate (GA, COPAXONE $\left.{ }^{\circledR}\right)$ in delaying progression to clinically definite multiple sclerosis (CDMS) in patients presenting with clinically isolated syndrome (CIS) suggestive of MS.

Background: GA reduced relapse rate and MRI-monitored disease activity in relapsing-remitting MS patients. This is the first prospectively planned, randomized, controlled, multicenter trial, which assessed the efficacy of GA therapy initiated shortly after the first clinical event suggestive of MS.

Design/methods: Patients presenting with a first clinical event and $>2$ T2-weighted brain lesions $>6 \mathrm{~mm}$ were enrolled. Only patients with unifocal manifestation were randomized to receive either sc $20 \mathrm{mg} /$ day GA or placebo. The primary outcome was time to CDMS, based on a second clinical attack. MRI metrics served as secondary and exploratory endpoints. Treatment continued for up to 36 months, unless a conversion to CDMS occurred. A preplanned interim analysis was performed on data accumulated from approximately $80 \%$ of the three-year study exposure.

Results: A total of 481 patients were randomized to receive GA $(n=243)$ or placebo $(n=238)$. Key baseline characteristics were: age (31.1 \pm 6.9 years), time from first event to randomization $(74.0 \pm 14.9$ days), corticosteroid use for first attack ( $64 \%$ of patients), EDSS $(1.0 \pm 1.0)$, number $(31.5 \pm 30.7)$ and volume $(6.0 \pm 6.2 \mathrm{ml})$ of T2 weighted lesions, and number $(1.5 \pm 2.9)$ and volume $(0.3 \pm 0.6 \mathrm{ml})$ of Gd-enhanced lesions; no difference between the study arms. Results from interim analysis showed that GA reduced the risk of developing CDMS by $45 \%$ compared to placebo. The 25 th percentile time to CDMS was prolonged by $115 \%$ for GA (to 722 days from 336 days for placebo; hazard ratio $0.55, \mathrm{p}=0.0005$ ). The proportion of patients converted to CDMS was reduced from $43 \%$ in the placebo to $25 \%$ in the GA group ( $\mathrm{p}<0.0001)$.GA was well tolerated, with $16 \%$ overall withdrawals to the time of the interim analysis, and a safety profile similar to that observed in RRMS. Following the results of the interim analysis all subjects were offered open label therapy with GA and continued follow-up as planned in the original protocol.

Conclusions/relevance: The results establish efficacy of early treatment with GA on disease evolution in CIS patients and a positive scan at screening MRI.

Study supported by TEVA Pharmaceutical Industries Ltd

060

The relationship between brain NAWM and GM damage is localised to specific, clinically relevant areas in early primary progressive multiple sclerosis B. Bodini, Z. Khaleeli, M. Cercignani, D.H. Miller, A.J. Thompson, O. Ciccarelli

Institute of Neurology, UCL (London, UK)

Objectives: Magnetic Resonance Imaging (MRI) investigation of the early, most clinically dynamic stages of primary progressive multiple sclerosis (PPMS), allows examination in vivo of the pathological mechanisms underlying progression. Diffuse MRI abnormalities have been demonstrated in the normal appearing white matter (NAWM) and grey matter (GM) of patients with PPMS. Aim of this study was to investigate whether and to what extent the NAWM damage correlates with the connected abnormal GM, and how their relationship contributes to disability.

Methods: We studied 35 patients with PPMS within five years of clinical onset. All patients were assessed using the Expanded Disability Status Scale
(EDSS) and the Multiple Sclerosis Functional Composite (MSFC) subtests. The recently developed method of tract-based spatial statistics (TBSS) was used to localise regions of reduced fractional anisotropy in the NAWM. An optimised voxel-based morphometry (VBM) approach was employed to identify regions of GM atrophy in patients. Anatomical correspondence between abnormalities in the NAWM and atrophy in the GM was identified, and quantitative correlation between the two compartments was then estimated using the Spearman's correlation coefficient. In the quantitatively linked areas, the relative contribution of NAWM and GM damage to disability, as measured by the EDSS and MSFC subtests, was assessed using multiple regression analyses.

Results: Patients showed widespread NAWM damage and diffuse GM atrophy. Anatomical correspondence between reduced NAWM FA and reduced GM atrophy was found in 11 regions. Four of these areas showed a quantitative correlation, namely the right sensory-motor region with the adjacent cortico-spinal tract, the left and right thalamus with the corresponding thalamic radiations and the left insula with the immediately adjacent white matter. All four areas were clinically eloquent, showing an independent and distinct contribution of NAWM and GM to disability.

Conclusions: NAWM damage and GM atrophy in early PPMS are interdependent in specific, clinically relevant brain regions. This suggests that there may be a link between the pathological processes occurring in the two compartments in these areas. Longitudinal studies are needed to determine whether GM damage is secondary to damage in connected NAWM tracts or, alternatively, it represents the primary target of the disease process leading to secondary axonal degeneration and demyelination in WM.

B. Bodini is funded by the European Neurological Society.

061

Therapy-related headache in multiple sclerosis patients under interferonbeta: a prospective study

R. Tanasescu, M. Ticmeanu, E. Moraru, I. Cojocaru, A. Oprisan, E. Constantinescu, D. Luca, A. Frasineanu, C. Burcin, A. Gitman, A. Nicolau, C. Baicus Colentina Hospital (Bucharest, RO); University of Medicine and Pharmacy Carol Davila (Bucharest, RO)

Headache $(\mathrm{H})$ can occur with $58 \%$ life-time prevalence in multiple sclerosis (MS) patients. Initially, $\mathrm{H}$ frequency during interferon beta (IFNB) therapy was not considered significantly higher vs placebo, but an increase was later reported. In order to describe $\mathrm{H}$ prevalence, characters and changes in MS patients under IFNB, we performed a prospective study on 71 MS patients.

71 patients with relapsing-remitting MS (mean age $36+/-6,34$ ) treated with IFNB (52 IFNB1a, 24 IFNB1b) were followed for a mean time of 20 (18-38) months, under a protocol including 3 month-visits, special-designed interview including McGill pain questionnaires and individual prospective diary. No $\mathrm{H}$ syndrome was considered if part of flu-like syndrome. We defined the $\mathrm{H}$ therapy-related (TRH) if occurred in the first 24 hours after IFNB injection. Chi-square and Fisher exact tests were used to observe association between categorical variables (Graphpad Instat 3).

$63,4 \%(45)$ of patients developed TRH, occurring at $1-20 \mathrm{~h}$ (mean 6)from injection and during for $1-24 \mathrm{~h}$ (mean 8,5). $\mathrm{H}$ was unilateral in $35 \%$, bilateral $65 \%$, throbbing $28 \%$, pressing $64 \%$, and was classified as mild in $43 \%$, moderate $45 \%$, severe $12 \%$. Nausea and photophobia where encountered in $25 \%$ and $28 \%$, respectively. TRH generally started later than flu-like syndrome (mean $4 \mathrm{~h}$ ). $56 \%$ of $\mathrm{H}$ fulfilled the ICHD criteria for tension headache, $18 \%$ for common migraine, the remaining unclassified.

We didn't find any association between $\mathrm{H}$ and age, sex, gender, age at MS onset, IFNB type.

In 31 patients $(43,7 \%)$, there was a history of $\mathrm{H}$ prior to IFNB treatment (primary headache, $\mathrm{PH}$ ). 27 of them (87\% of $\mathrm{PH}$ patients) developed TRH, $20(64,5 \%)$ in the first 6 months of treatment, and 21 of PH patients $(67,7 \%)$ noticed changes in $\mathrm{H}$ intensity/duration, and $5(16,1 \%)$ in $\mathrm{H}$ type. There was a significant presence of TRH among MS patients with $\mathrm{PH}(\mathrm{p}=0,0004)$, with a relative risk of 1.935 (95\% Confidence Interval 1.339 to 2.798 ) to develop TRH mainly in the first 6 months of therapy.

Concluding, TRH is more frequent in our patients than already reported. Patients with PH have a higher risk to develop TRH. We suggest a thorough follow-up of MS patients under IFNB, especially those with PH, to optimize early attack and prophylactic therapy of $\mathrm{H}$. Even that $\mathrm{H}$ existence is not at all a contraindication for IFNB therapy, it is important to determine the presence and extent of $\mathrm{H}$ before the start of IFNB and closely monitor them, in order to adjust therapy if $\mathrm{H}$ increases. 


\section{Oral session 2}

\section{Cerebrovascular disorders 1}

\section{2}

The effect of antiplatelets during or after endovascular coiling for aneurysmal subarachnoid haemorrhage

W.M. van den Bergh, R.S.C. Kerr, A. Algra, G.J.E. Rinkel, A.J. Molyneux on behalf of the International Subarachnoid Aneurysm Trial (ISAT) Collaborative Group

Objective: Antiplatelets (APTs) are often used during or after endovascular coiling of aneurysms in patients with subarachnoid haemorrhage (SAH). There is, however, no evidence that APTs improve outcome in these patients. Methods: All 43 centres in the International Subarachnoid Aneurysm Trial (ISAT), comparing endovascular coiling with neurosurgical clipping in patients with ruptured intracranial aneurysms, were sent a questionnaire whether they never, sometimes or always used APTs during or after coiling. Risk ratios (RR) for coiling compared with clipping for poor outcome after 2 months and 1 year were calculated. These RRs were calculated separately for patients treated in hospitals that always used APTs during or after coiling and in patients treated in hospitals that used APTs never or sometimes.

Results: Nineteen centres responded, representing $1422(66 \%)$ of the 2143 patients randomised in ISAT. Standard prescription of APTs during coiling was done in two responding centres ( $8 \%$ of coiled patients), whereas it was done after coiling in 6 centres $(24 \%)$. The overall RR for poor outcome of coiling versus clipping was 0.67 (95\% CI $0.57-0.79)$ after two months and $0.74(0.62-0.89)$ after one year. For two months' outcome RR was 0.82 $(0.45-1.49)$ in hospitals that always used APTs during coiling versus 0.66 $(0.55-0.78)$ in those that never or sometimes used APTs (ratio of RR's 1.24, $\mathrm{p}=0.56$ ). Similar observations were made for APT use after coiling. The ratio of RRs for one-year outcome was $1.01(\mathrm{p}=0.89)$ for APT use during coiling and $1.00(\mathrm{p}=0.77)$ for use after coiling.

Conclusion: The results of this study do not support the assumption that APTs during or after endovascular coiling improve outcome in patients with aneurysmal SAH.

\section{3}

Polymorphisms and mutational analysis of the NOTCH3 gene in a large cohort of patients affected by leukoencephalopathy

C. Ungaro, F.L. Conforti, T. Sprovieri, P. Servillo, M. Liguori, L. Citrigno, A.L. Gabriele, A. Magariello, A. Patitucci, M. Muglia, R. Mazzei

ISN National Research Council (Mangone, IT)

Objective: The aim of the present study was to analyse the coding region and intron-exon boundaries of the NOTCH3 gene in a large cohort of patients affected by leukoencephalopathy to investigate the presence of genetic variants.

Patients and methods: We analyzed the exons $2-23$, exons $3-4$, exons 3-4-6-8 respectively in 157, 684 and 542 patients affected by leukoencephalopathy. Genomic DNA was extracted from peripheral-blood leucocytes using the salting out method. Twenty-two exons (2-23) out of 33 of the NOTCH3 gene and their intronic flanking sequences were amplified by PCR with sets of oligonucleotide primers specific for NOTCH3. The amplicons were then analyzed by Denaturing High Performance Liquid Chromatography; patients' chromatograms for each exon were compared with corresponding normal controls and the exons showing an abnormal DHPLC eluition profile were directly sequenced in both forward and reverse directions on an ABI Prism 3130XL genetic analyzer.

Results: The molecular analysis revealed several nucleotide alterations in comparison to the wild type sequence. In particular we identified 20 different mutations in 45 subjects from 30 families, 23 polymorphisms, 11 of them were novel, and seven genetic variants of unknown pathological meaning never been reported previously.

Discussion and conclusion: Cerebral Autosomal Dominant Arteriopathy with Subcortical Infarcts and Leukoencephalopathy (CADASIL) is a cerebrovascular disease caused by mutations in the NOTCH3 gene. Most CADASIL associated mutations result in a gain or loss of a cysteine residue in one of the 34 EGF-like repeats in the extracellular domain of the Notch3 protein, thus sparing the number of cysteine residues within a domain. To date, more than 130 different mutations in the NOTCH3 gene have been reported in CADASIL patients, the $95 \%$ being missense point mutations. Many polymorphisms have also been identified in the NOTCH3 coding sequence some of them leading to amino acids substitutions.

Here, we report a summary of 20 mutations, 23 polymorphisms and
7 new nucleotide variations in a cohort of 684 patients affected by leukoencephalopathy and we hope this NOTCH3 gene mutational analysis in a so significant number of unrelated and related patients may help in molecular screening for NOTCH3 gene and it may contribute to enlarge the NOTCH3 gene variations database.

The work was in part supported by the Italian Ministero dell'Istruzione, dell'Università e della Ricerca Grants MIUR-FIRB2006-RBIP06PMF2_006.

\section{4}

Prothrombotic gene variation and new vascular events after cerebral ischaemia of arterial origin

D.M.O. Pruissen, F.R. Rosendaal, C.J.M. Frijns, L.J. Kappelle, H.L. Vos, A. Algra on behalf of the SMART Study Group

Objectives: Several genetic variants involved in haemostasis have been associated with ischaemic stroke or myocardial infarction. Stroke patients who carry a prothrombotic genotype may also be at increased risk for new vascular events.

Methods: We included 887 patients with nondisabling cerebral ischaemia of arterial origin, who were referred to a university medical center in The Netherlands between 1995 and 2005 and followed them for the occurrence of ischaemic stroke, myocardial infarction or death. The primary outcome was a composite of death from all vascular causes, nonfatal ischaemic stroke, nonfatal myocardial infarction, whichever happened first. Through a systematic literature search we identified 22 prothrombotic variants in 14 genes that were previously associated with ischaemic stroke or myocardial infarction.

Results: During a 4.6-year follow-up period new vascular events occurred in 135 patients (annual event rate $3.3 \%$ ). None of the 22 variants was associated with the occurrence of new vascular events. Eight additional analyses with secondary outcomes or among subgroups revealed four associations that were likely to be false positive after accounting for multiple testing.

Conclusion: In this cohort, prothrombotic genetic variants do not affect the risk of new vascular events after cerebral ischaemia of arterial origin. This study does not support the use of prothrombotic genetic variants to identify stroke patients at increased risk for new vascular events or to guide antithrombotic treatment.

This study was funded by a grant from the Medical and Health Research Programme of the Netherlands Organisation for Scientific Research (NWO), grant No. 904-61-190.

\section{5}

Prothrombotic genetic variants and atherosclerosis in patients with cerebral ischaemia of arterial origin

D.M.O. Pruissen, L.J. Kappelle, F.R. Rosendaal, A. Algra on behalf of the SMART Study Group

Objectives: Several prothrombotic genetic variants have been associated with an increased risk of arterial disease. These variants probably affect arterial thrombus formation but may also promote atherosclerosis. We hypothesized that specific prothrombotic variants lead to advanced atherosclerosis in patients with cerebral ischaemia of arterial origin.

Methods: We included 689 patients with nondisabling cerebral ischaemia of arterial origin. Twenty-two variants in 14 genes that were previously associated with ischaemic stroke or myocardial infarction, were genotyped. Carotid intima-media thickness, the presence of symptomatic carotid stenosis and age at the occurrence of cerebral ischaemia were documented.

Results: None of the variants was associated with carotid intima-media thickness or younger age at the occurrence of cerebral ischaemia. Factor V Leiden (mean prevalence difference $25 \%$; $95 \%$ CI 11-40) and the glycoprotein 1b-alpha Thr145Met variant (mean prevalence difference $12 \%$; $95 \% \mathrm{CI}$ 2.3-22) were associated with symptomatic carotid stenosis. The factor XIII subunit B His95Arg variant was marginally associated with older age at the occurrence of cerebral ischaemia (mean age difference 2.3 years; $95 \% \mathrm{CI}$ 0.03-4.5). After accounting for multiple testing by determination of the false discovery rate, only the association between factor V Leiden and symptomatic carotid stenosis remained present.

Conclusion: Prothrombotic genetic variants showed no consistent association with three markers of advanced atherosclerosis in patients with cerebral ischaemia of arterial origin. This study does not support the hypothesis that prothrombotic genetic variants have a direct role in the pathogenesis of atherosclerosis.

This study was supported by a grant from the Medical and Health Research 
Programme of the Netherlands Organisation for Scientific Research (NWO), grant No. 904-61-190.

\section{6}

Contribution of obesity and abdominal fat mass to risk of stroke: the Mannheim-Heidelberg Stroke Study

Y. Winter, S. Rohrmann, J. Linseisen, O. Lanczik, A. Ringleb, J. Hebebrand, T. Back

Center for Mental Health (Stuttgart, DE); German Cancer Research Center (Heidelberg, DE); Klinikum Mannheim (Mannheim, DE); University Heidelberg (Heidelberg, DE); University Duisburg-Essen (Essen, DE); Saxon Hospital (Arnsdorf, DE)

Objectives: Obesity has become one of the most prevalent conditions that pose a large impact on public health worldwide. We investigated markers of obesity and abdominal fat mass in a case-control study in cerebrovascular patients to evaluate the risk contribution to stroke and transient ischemic attacks (TIA) in central Western Europe.

Methods: 1137 participants were included in the study, 379 cases and 758 regional controls matched for age and sex. Consecutive cases of ischemic stroke $(n=301,79 \%)$, intracerebral hemorrhage (ICH) $(n=37,10 \%)$ or TIAs ( $\mathrm{n}=41,11 \%$ ) were recruited at the University Depts. of Neurology in Mannheim and Heidelberg between February 1, 2005 and January 31, 2006. Associations were assessed between different markers of obesity (body mass index [BMI], waist-to-hip ratio [WHR], waist circumference and waist-tostature ratio [WSR]) and risk of stroke or TIA by using conditional logistic regression adjusted for other risk factors.

Results: BMI showed a moderate association with cerebrovascular risk which was non-significant after adjustment for physical inactivity, smoking, hypertension, and diabetes (odds ratio [OR] 1.37, $95 \%$ confidence interval [CI] $0.87-2.17$, top quartile vs. bottom quartile). Markers of abdominal fat mass were strongly associated with risk of stroke and TIA. For the waist-tohip ratio, adjusted OR for every successive quartile were greater than that of the previous one (2nd quartile: OR 1.34, CI 0.68-2.64; 3rd quartile: OR 3.22, CI 1.75-5.91; 4th quartile: OR 8.44, CI 4.73-15.08). Significant associations with risk of stroke/TIA were also found for waist circumference and WSR (OR 4.53, CI 2.77-7.39 and OR 3.99, CI 2.42-6.58, top vs. bottom quartile after risk adjustment, respectively).

Conclusion: Markers of abdominal adiposity (waist circumference, WHR) showed a graded and significant association with the risk of stroke and TIA that is independent of other vascular risk factors. Waist circumference and related ratios can better predict stroke than BMI.

The study was supported by the German Ministry of Education and Research, research grant 01GS0491.

\section{7}

Copeptin, the C-terminal part of the vasopressin pro-hormone to predict outcome in patients with stroke

M. Katan, N. Morgenthaler, F. Fluri, C. Zweifel, P. Schuetz, J. Struck, A. Bergamnn, S. Engelter, A.J. Steck, B. Mueller, M. Christ-Crain

Universitiy Hospital Basel (Basel, CH); B.R.A.H.M.S AG, Biotechnology Centre (Henningsdorf-Berlin, DE)

Objectives: Early prognostic factors to predict outcome in stroke patients would be helpful to guide decisions on treatment and interventions to optimize neurological outcome. Vasopressin (AVP) levels were found to be elevated in patients with stroke correlating with disease severity; however, its measurement is cumbersome. Copeptin is more stable, released in an aequimolar ratio with AVP and can be assayed readily in plasma.

Methods: From a prospective observational study, data of the first 249 patients who were admitted (from Nov. 2006 to Nov. 2008) to the emergency department with a cerebrovascular event were analyzed. On admission severity of ischemic stroke was assessed by the National Institute of Health Stroke Scale (NIHSS). In all patients, copeptin levels were determined with a new sandwich immunoassay and compared with functional outcome after 3 months as assessed with the modified Ranking score (mRS).

Results: Of the first 249 patients $43 \%$ patients were female, $75 \%$ had an ischemic stroke, $19 \%$ had a transient ischemic attack (TIA) and $6 \%$ had a hemorrhagic stroke. The median age was 75 (36-95) years. On admission the median NIHSS was 5 (1-42). Copeptin levels on admission were higher in patients with a worse outcome (i.e., mRS ?3) compared to patients (overall population and subgroup of ischemic stroke) with a good outcome (i.e., mRS $<3, \mathrm{p}<0.0001)$. In the multivariate regression analysis copeptin was an independent marker from traditional risk factors (i.e., age, blood pressure, gender, diabetes; Odds ratio 1.2, $<<0.05$ ) and also independent of the NIHSS (odds ratio 1.1; $\mathrm{p}<0.05$ ). Copeptin had an area under the receiver operating curve (AUC) of 0.72 to predict functional outcome, within the range of the NIHSS (0.75), the combination of the NIHSS and copeptin tended to be even better with an AUC of 0.78 . Copeptin levels did not correlate with plasma sodium or osmolality values.

Conclusion: Elevated copeptin levels measured on admission were associated with poor functional outcome after 3 months. Thus, copeptin is a novel predictor of outcome in patients with ischemic stroke. The prognostic value of copeptin is independent from traditional risk factors and the NIHSS, and as such adding new information for risk-stratification.

068

Triple and quadruple spontaneous cervical artery dissection: presenting characteristics and long-term outcome in 12 patients

M. Arnold, G.M. De Marchis, C. Stapf, R. Baumgartner, K. Nedeltchev, F. Buffon, H.P. Mattle, M.G. Bousser

University of Berne (Berne, $\mathrm{CH}$ ); University Hospital Lariboisière (Paris, FR); University Hospital (Zurich, $\mathrm{CH}$ )

Background: Spontaneous cervicocephalic artery dissection (sCAD) of more than two cervical arteries is a rare event.

Patients and methods: We studied vascular and potential SCAD risk factors, triggering events, clinical and neuroimaging findings, and outcome of patients with multiple sCAD. Patients were drawn from prospective hospital-based $\mathrm{SCAD}$ registries.

Results: Of 740 consecutive patients with sCAD, 11 (1.5\%) had 3 and one $4(0.1 \%)$ sCAD. Eight of these 12 patients were women. One patient had additional dissections of the celiac trunk and hepatic artery. Vascular risk factors included hypertension $(\mathrm{n}=1)$, hypercholesterolemia $(\mathrm{n}=6)$, current smoking $(n=5)$ and migraine $(n=6)$. No patient had family history of sCAD, fibromuscular dysplasia (FMD) or connective tissue disease. SCAD was preceded by a minor trauma in 5 and an infection in 4 patients. Clinical manifestations included ischemic stroke $(n=8)$, transient ischemic attack $(n=3)$, headache $(n=9)$, neck pain $(n=4)$, Horner syndrome $(n=5)$, pulsatile tinnitus $(n=2)$ and dysgeusia $(n=1)$. Brain MRI revealed ischemic infarcts that affected one vessel territory in 7 and two territories in 2 patients. Threemonth outcome was favorable (modified Rankin scale score $0-1$ ) in $10 \mathrm{pa}-$ tients $(83 \%)$. No recurrent stroke or SCAD occurred during a mean followup of $50 \pm 29$ months.

Conclusion: Multiple sCAD occurred preferentially in women and caused clinical symptoms and signs mainly in one vascular territory. In none of the patients FMD or any underlying arteriopathy was apparent. The majority of multiple SCAD was preceded by a minor trauma or infection. Clinical outcome was favorable in most patients and long-term prognosis benign. The data suggest transient vasculopathy as major mechanism for multiple SCAD.

\section{Oral session 3}

\section{Peripheral neuropathy}

069

Aetiology of small- and mixed-fibre neuropathy

E. Vlckova, C. Sommer, J. Bednarik

Masaryk University (Brno, CZ); Bavarian University Wurzburg (Wurzburg, $\mathrm{DE})$

Objectives: Small-fibre neuropathy (SFN) is reported to be associated with a range of diseases or conditions that are believed to be at least partly different from those associated with large- or mixed fibre neuropathy. The aim of the study is to evaluate etiology in a group of SFN patients in comparison with mixed (i.e., small- and large-) fibre neuropathy (MFN) group and sex and age-matched group of healthy volunteers.

Methods: A detailed medical history was taken and exhaustive blood tests were performed in a group of 84 patients with "burning feet" syndrome and clearly proven affection of small nerve fibres verified by abnormal thermal thresholds and reduced intra-epidermal nerve fiber densities in the distal calf (54 men and 30 women, median: 58 ; range: $25-83$ years) and in a group of 47 healthy controls (30 men and 17 women, median: 59; range: 29-85 years). Thirty-three patients exhibited additional nerve conduction abnormalities as signs of large-fibre involvement (MFN group) while 51 patients showed isolated small-fibre involvement (SFN group). 
Results: Univariate analysis disclosed several clinical and laboratory parameters that were significantly associated with both small- and mixedfibre neuropathy in comparison with the control group. None of them, however, significantly discriminated between the MFN and SFN subgroups. The multivariate regression model disclosed that diabetes mellitus (odds ratio $[\mathrm{OR}]=4.08)$, chronic alcoholism ( $\mathrm{OR}=5.31$ - more significantly in men than women) and cholesterol serum levels $(\mathrm{OR}=4.51)$ were the only parameters independently associated with small-fibre involvement. The other parameter significantly associated with neuropathy in an univariate model intercorrelated with either diabetes mellitus (glycaemia, glycosylated hemoglobin, triglycerides), alcoholism (carbohydrate deficient transferrin, transaminases, mean corpuscular volume of erythrocytes) or both (uric acid).

Conclusions: Small-fibre neuropathy is probably not an independent entity, at least from an aetiological point of view. The main aetiological factors of both small- and mixed-fibre polyneuropathies in developed countries are probably diabetes mellitus, chronic alcohol abuse, and, surprisingly, serum cholesterol levels.

Supported by Research Plan of the Czech MInistry of Education MSM0021622404

070

Sensory loss, pains, motor deficit and axonal regeneration in length dependent diabetic polyneuropathy

G. Said, D. Beaudoin, K. Toyooka

Hôpital de la Salpétrière (Paris, FR); CHR d'Aix en Provence (Aix en Provence, FR); Osaka University (Osaka, JP)

In order to learn more on the occurrence of pains and motor deficit in severe diabetic polyneuropathy we have reviewed the clinical data of a series of 30 diabetic patients with an uncommonly severe length-dependent diabetic polyneuropathy (LDDP), including 10 patients who had a sural nerve biopsy.

Extensive sensory loss predominated on pain and temperature sensations and affected all four limb extremities, anterior trunk in all, plus the top of the scalp in 9 patients, and the cauda equina territory in 2 . Twenty patients complained of neuropathic pains. Symptomatic autonomic dysfunction was present in 28/30 patients; mild distal motor deficit in 12 patients, severe distal weakness in only one. Vibratory sensation was impaired in the lower limbs in 18 patients; position sense in 8 .

In the 10 nerve biopsy specimens, the density of myelinated axons was reduced to $23 \%$ and that of unmyelinated axons to $8.5 \%$ of control values. Regenerating axons accounted for $32.4 \pm 19.8 \%$ of the myelinated fibres. On teased fibre preparations $13.9 \%$ of fibres were undergoing axonal degeneration, while $29.4 \%$ of fibres showed segmental abnormalities of the myelin sheath.

We conclude that: 1) distal motor deficit occurs only after major loss of sensory fibres in LDDP; 2 ) the unmyelinated axons are predominantly affected; 3) absence of clinical improvement contrasts with the high proportion of regenerating axons; 4 ) detection of alteration of pain and temperature sensations in the feet seems the best method for neuropathy screening in diabetic patients.

\section{1}

Small fibre neuropathy in heterozygous females with Fabry's disease

R. Liguori, V. Di Stasi, A. Burlina, R. Mignani, F. Carubbi, E. Bugiardini, P. Montagna, V. Donadio

University of Bologna (Bologna, IT); S. Bassiano Hospital (Bassano del Grappa, IT); Degli Infermi Hospital (Rimini, IT); University of Modena (Modena, IT)

Objectives: Fabry disease is a rare $\mathrm{X}$ linked lysosomal storage disorder resulting from deficiency of á-galactosidase A activity. Although the severity of clinical features including a small fiber neuropathy in male patients is well described, only recently the high prevalence of a multisystemic disease in heterozygous females showing Fabry mutation has been reported. This study sets out to examine the presence of a small fiber neuropathy in females with Fabry disease.

Methods: We studied 10 females patients with Fabry disease (mean age $35 \pm 4$ ) four of them in enzyme replacement therapy (ERT) because of renal and/or cerebral involvement. In all patients the following examinations were performed: neurological examination; visual analogue scale (VAS) to quantify pain sensation (from $0=$ no pain to $100=$ maximal pain experienced); motor and sensory conduction studies to evaluate large mylinated nerve fiber; thermoregulatory sweating test (TST); microneurography from peroneal nerve to analyze skin (SSNA) and muscle (MSNA) sympathetic nerve activity; skin biopsy to study epidermal nerve fiber (ENF) density and autonomic skin innervation which was graded by a semiquantitave scale from $0=$ normal to $4=$ absent innervation .

Results: Five patients complained distal acroparaesthesiae (mean VAS $69 \pm 8$ ) whereas the other patients were asymptomatic. Neurological examination showed slight reduced deep tendon reflex in lower limbs in two symptomatic patients; TST detected lower limb hypoidrosis and microneurography disclosed absent SSNA in one symptomatic patient. Skin biopsy disclosed reduced ENF density and morphological abnormalities of the nerve fibers, predominantly in the leg in all patients (mean ENF density $7 \pm 1$; n.v. 17.4 \pm 3 ) with a moderate dysfunction of the autonomic innervation (mean rate $2 \pm 0.5$ ). The pain VAS scale was unrelated to ENF density and to skin autonomic innervation rate.

Conclusion: Heterozygous females with Fabry disease although asymptomatic may presented a small fiber neuropathy involving both sensory and autonomic nerve fibers. This finding highlights the importance of a careful assessment of these patients to identify early a peripheral nerve dysfunction which may represent a marker to start the ERT. Furthermore pain scale was unrelated to the skin innervation abnormalities suggesting that pain mechanisms were not a direct effect of the skin denervation.

\section{2}

Investigation of ion channel abnormalities in $\mathrm{P0}$-deficient knockout mice by threshold-tracking

M. Moldovan, S. Alvarez, R. Martini, C. Krarup

University of Copenhagen (Copenhagen, DK); University of Wurzburg (Wurzburg, DE); Rigshospitalet (Copenhagen, DK)

Objectives: Mice deficient in the peripheral myelin component P0 (P0-/-) mimic severe forms of inherited peripheral neuropathies in humans, with defective myelin formation and consequent axonal loss. From birth P0-deficient mice show a progressive slowing of conduction velocity and an increase in threshold for action potentials originally attributed to alterations in the electrical properties of the myelin. Recent immunohistological studies revealed that P0-deficient mice have an ectopic nodal expression of voltage-gated sodium channel 1.8 isoform (Nav 1.8) and disorganization of paranodal potassium channels. The functional consequences of these abnormalities are unclear.

The aim of this study was to investigate the ion channel abnormalities of P0-deficient mice by "threshold-tracking", a clinically applicable, electrophysiological technique that can offer clues about the nodal and internodal membrane function of peripheral nerves in vivo.

Methods: Electrophysiological studies were carried out under anesthesia in 2-4 months old P0-deficient mutant mice. Tibial nerves were stimulated at the ankle and the evoked motor responses were recorded from the plantar muscles using subcutaneous needle electrodes. Multiple measures of axonal excitability were recorded by "threshold-tracking".

Results: All mice had severely reduced motor performance. Motor responses were at least $200 \%$ delayed and had amplitudes below $10 \%$. There was evidence of spontaneous activity. The largest abnormality in excitability was increased accommodation during depolarizing threshold electrotonus. Furthermore, the recovery cycle appeared shifted upwards with markedly increased refractoriness at the expense of the superexcitable period that normally follows a single impulse. These abnormalities were aggravated by cooling. P0-deficient nerves also showed decreased accommodation to hyperpolarization and increased strength-duration time constant.

Conclusion: Our data suggest that $\mathrm{P} 0$-defficent nerves show marked abnormalities in response to prolonged depolarization and prolonged refractoriness after a single impulse that were aggravated by cooling consistent with ectopic nodal expression of Nav 1.8. Furthermore, we also found evidence of $\mathrm{K}+$ channel dysfunction that confirm previous immunohistological studies. Thus changes in molecular architecture of the node of Ranvier lead to marked abnormalities of excitability in P0-defficent nerves.

\section{3}

Multifocal motor neuropathy, a population-based study in the Netherlands E.A. Cats, W. van der Pol, H. Franssen, J.H.J. Wokke, R.M. van den Berg-Vos, L.H. van den Berg

University Medical Center Utrecht (Utrecht, NL)

Objectives: Multifocal motor neuropathy (MMN) is an immune-mediated neuropathy characterized by slowly progressive, asymmetric weakness of the limbs without sensory loss. Treatment with intravenous immunoglobulin (IVIg) can lead to improvement of muscle strength. Maintenance treatment is needed to maintain the effect. We documented phenotype and treatment effects of patients with MMN in the Netherlands.

Methods: Population-based study during 1 year. All neurologists in the 
Netherlands were asked to refer their MMN patients, a national neuromuscular database was screened for MMN patients and patients were informed by the Dutch Neuromuscular Patient Association. All patients underwent neurological examination and nerve conduction studies.

Results: We identified ninety-five MMN patients, 88 participated in the study, 64 (73\%) were men. Mean age at onset was 40 years (range 22-66); at time of study 53 years (range 27-78).

Electrophysiological findings were definite motor conduction block in $70(79,5 \%)$ and probable in $18(20,5 \%)$ patients. Site of onset was hand/forearm in $53(60 \%)$, lower leg in $31(35 \%)$ and upper arm in $4(5 \%)$ patients. Mean number of affected regions (upper arm, lower arm, upper leg, lower leg, both sides) at time of study was 4 (range 1-8). Upper legs were spared in most cases (76 patients, $86 \%$ ). Sixty-seven patients (76\%) improved with IVIg and received maintenance treatment. Mean IVIg starting dose was $12 \mathrm{~g} /$ week (range 3-35), mean frequency once per 4 weeks (range 1-13). Mean time from start IVIg to study was 7 years (range $0-17$ ). At time of study mean IVIg dose was $17 \mathrm{~g} /$ week (range 5-100), mean frequency once per 3 weeks (range 1-8). A slight decrease in muscle strength over time was documented in all patients.

Conclusion: MMN is a slowly progressive asymmetric disease with onse of muscle weakness in hand/forearm or lower leg. Most patients improve after IVIg treatment, but a slight decrease in muscle strength is present in all patients despite maintenance treatment.

This study was supported by an unconditional grant from Baxter Healthcare B.V.

\section{4}

Phenotypic characterisation of Charcot-Marie-Tooth disease type 2 associated to a novel dynamin 2 mutation

J. Berciano, E. Gallardo, K. Claeys, A. García, A. Canga, O. Combarros, V. Timmerman, P. De Jonghe

University Hospital Marqués Valdecilla (Santander, ES); University of Antwerp (Antwerp, BE)

Objective: To characterize a Charcot-Marie-Tooth disease type 2 (CMT2) phenotype associated to a novel missense dynamin 2 mutation.

Patients and methods: Our pedigree comprises a proband patient aged 55 years, and two affected daughters aged 32 and 23 years. Electrophysiological study was performed according to the requirements of the CMT neuropathy score. MR imaging study included T1- and fat suppressed T2weighted spin-echo sequences of thigh, lower leg and foot musculature. After discarding mutations in known genes causing CMT2, mutation analysis of dynamin 2 was performed by PCR sequencing of the coding exons on genomic DNA of the patients, two unaffected relatives and 192 controls.

Results: All three patients showed progressive distal leg paresis and pes cavus with infantile onset, gait only being possible with support in the proband. Hand atrophy was noted just in the proband. Ankle reflexes were absent. Neither proximal weakness nor external ophthalmoplegia was noted. There was stocking hypoesthesia. Lower leg nerves were inexcitable. Upper limb motor conduction velocities were in the "intermediate" range $(32 \mathrm{~m} / \mathrm{s})$ just in the proband who showed severe attenuated distal CMAP $(0.1 \mathrm{mV})$, whereas both her affected daughters exhibited preserved motor conduction velocities and CMAPs. MR imaging study demonstrated preservation of thigh musculature, extensive fatty atrophy of all four lower leg muscle compartments with lesser involvement of the deep posterior one, and massive fatty atrophy of foot musculature. Fatty muscle infiltration increased distally in 19 out of $66(25 \%)$ visualized calf muscles in the three patients, but this percentage increased to $64 \%$ in the youngest and least severe patient. Molecular study revealed a disease causing mutation in the dynamin 2 gene; the sequence variation is a c.1072G $>$ A nucleotide mutation in exon 7 of the middle domain of dynamin 2 resulting in a Gly358Arg change.

Conclusions: The reported missense mutation of the middle domain of the dynamin 2 gene may cause a distinctive form of CMT2 with no overlapping with centronuclear myopathy caused by mutations in the same domain. Motor conduction velocities slowed down to the "intermediate" range only in nerves with severely attenuated CMAP amplitudes. MR imaging study accurately depicts lower limb muscle involvement; distal predominance of fatty atrophy observed here suggests that in this form of CMT2 axonal degeneration is length-dependent.

Supported by CIBERNED (ISCIII, Madrid, Spain)
075

Mutations in the periaxin gene cause early-onset demyelinating neuropathy with variable severity of disease course

M. Milani, C. Marchesi, G.M. Fabrizi, T. Cavallaro, G. Piccolo, M.R. Balestrini, G. Lauria, D. Pareyson, F. Taroni

Fondazione IRCCS Istituto Neurologico C. Besta (Milan, IT); University of Verona (Verona, IT); Fondazione IRCCS Ist Neurologico Mondino (Pavia, IT)

Mutations in the periaxin gene (PRX,19q) are a rare cause of autosomal recessive demyelinating CMT (CMT4F) and Déjérine-Sottas disease (DSD). The gene encodes two protein isoforms, L(ong)-periaxin and S(hort)-periaxin, which are required for the maintenance of peripheral nerve myelin. To date, 11 nonsense or frameshift mutations have been reported in $<10$ CMT/DSD families.

Objectives: To screen the PRX gene in CMT1A/MPZ/PMP22/Cx32-negative patients with early-onset severe demyelinating sensory-motor neuropathy.

Methods: We analysed 20 patients by DHPLC and direct sequencing of the 4.9-kb PRX coding region. RESULTS: Five novel frameshift or nonsense mutations were found in 4 index cases. Four of the 5 mutations affect the long (L) but not the short (S) variant of the protein. In family 1, 2 siblings, aged 45 and 43, compound heterozygotes for 2 null mutations (Q547stop and P807fs), developed an early-onset demyelinating neuropathy of different disease severity. Both of them had delayed motor milestones and pes cavus. The youngest patient was diagnosed as DSD on the basis of severe scoliosis, sensory ataxia, motor NCVs $<9 \mathrm{~m} / \mathrm{s}$, and almost complete loss of myelinated fibers at nerve biopsy. His brother had a milder disease with slight skeletal deformities and was classified as demyelinating CMT. In Family 2, 2 siblings exhibited a clinical phenotype of different severity with a profound NCV reduction. Only one heterozygous mutation was found (K118fs) affecting both forms of the protein. In Family 3, the 34-yr-old proband was homozygous for the nonsense mutation E682stop. She presented with early-onset severe neuropathy $(\mathrm{MCV}<10 \mathrm{~m} / \mathrm{s})$ and sensory ataxia, and was diagnosed as DSD. Her younger brother is reported to have a milder phenotype. In Family 4, a 33-yr-old woman carried a homozygous frameshift mutation (P258fsX313). The disease manifested in infancy with distal weakness, ataxic gait, profound deep sensory impairment, and signs of severe hypertrophic demyelinating neuropathy at biopsy.

Conclusions: The PRX mutations thus far reported, including ours, cause early-onset neuropathy, but the severity of subsequent course may significantly differ, raising doubts about the utility of differentiating DSD and CMT. Interestingly, in the majority of cases, the mutations affect the long (L) but not the short $(S)$ variant of the protein, suggesting that S-periaxin expression may account for clinical variability in these patients.

Partly supported by Telethon-UILDM grants to FT and GMF

\section{Oral session 4}

\section{Epilepsy 1}

\section{6}

Nonconvulsive status epilepticus: seizures or acute neurological injuries? J. Choi, G. Krauss, P. Kaplan Johns Hopkins University School of Medicine (Baltimore, US)

Objective: It is often difficult to identify seizures and nonconvulsive status epilepticus (NCSE) in patients with acute cerebral insults, since disorders such as meningitis and hemorrhage, may also cause both confusion and continuous EEG epileptiform discharges. We identified clinical and EEG features which distinguished seizures and acute cerebral disturbances as causes of confusional states.

Methods: We studied patients with confusion and whose EEG suggested possible NCSE. Patients were diagnosed as having seizures, severe acute cerebral insults, or both. Patients responding immediately to AEDs or with intermixed overt seizures were classified as having seizures. Patients' clinical diagnosis and EEG features were correlated and evaluated using decision tree analysis.

Results: Fifty adults had confusion and original EEGs suggesting NCSE: 11 were diagnosed with seizures, 19 had acute cerebral disorders only, 20 had both. AEDs reduced epileptiform discharges acutely in $90 \%$. EEG discharge 
morphology, focality and rhythmicity did not correlate with clinical diagnosis. Most $(86 \%)$ patients with EEG epileptiform discharge frequencies $>2.1 \mathrm{cps}$ had seizures; lower frequency discharges were associated with acute disorders without seizures (Chi2 $11, \mathrm{p}=0.001$ ). Decision tree analysis accurately classified patients' diagnosis ( $64 \%$ sensitivity, $100 \%$ specificity): patients with seizures had either no history of an acute injury or EEG discharge frequencies $>2.6 \mathrm{cps}$; remaining patients with rhythmic or quasirhythmic 1.3 to 2.1 cps discharges had acute injuries only.

Conclusion: A simple classification system based on discharge frequency and the clinical context may distinguish NCSE from encephalopathies and acute cerebral disturbances in patients with confusional states.

\section{7}

Demographics and management of epilepsy patients presenting to the emergency department: can such presentations be reduced?

G.P. Winston

National Hospital for Neurology and Neurosurgery (London, UK)

Objective: UK guidelines suggest that patients with epilepsy should have regular review at least yearly. However, some patients present via the emergency department (ED) with seizures and receive their ongoing care here rather than through formal follow up. This study was designed to identify patients with known epilepsy presenting to the ED to determine if better care could minimise such presentations.

Methods: All patients presenting to the ED in a large teaching hospital in the East Midlands, UK, with a seizure over a 2 month period were identified from the ED database. Medical and computer records for those patients with known epilepsy were reviewed to study their demographics and management.

Results: 57 patients were identified with an age ranging from 16 to 85 (mean 41 , median 38 ), with the majority in the age range $20-49.56 \%$ were male, $21 \%$ had a learning disability and $4 \%$ were care home residents. $60 \%$ were discharged home from the ED and only a single patient ( $2 \%)$ was admitted to a neurology ward.

$67 \%$ of patients had a documented neurology review prior but this was in the preceding year in only $44 \% .19 \%$ of patients were reviewed by neurology during admission and none was reviewed by an epilepsy specialist nurse. $58 \%$ of patents either had no followup arranged or were asked to see their GP. The other $42 \%$ were followed up by neurology.

Patients attended with a seizure on average 0.90 times in the prior year (range 0-4) and 1.22 times in the subsequent year (range 0-6). Those with learning disabilities were overrepresented with $50 \%$ more ED presentations. Around half of the patients did not attend in the year before or after the index seizure.

Conclusions: In this study, around half of the patients attending the ED with a seizure had done so in the preceding year and/or will do so in the following year. The majority of patients seen in the emergency department were discharged home, and only a minority had formal neurology followup (around 2 in 5) or input during admission (around 1 in 5). This represents a lost opportunity for review which could help to reduce the number of patients presenting to the ED with a seizure. Those with a learning disability attend more frequently and whilst this may represent epilepsy that is harder to control, this is a particular group to target as they may not seek healthcare input themselves for poor control.

\section{8}

Assessment of autoimmunity in the pathogenesis of epilepsies in young women with long-standing intractable drug-resistant epilepsy

Y. Lekomtseva, B. Lang, A. Vincent

Institute of Neurology and Psychiatry (Kharkiv, UA); University of Oxford (Oxford, UK)

Objectives: Antibody-mediated autoimmunity underlies a diverse range of disorders in the nervous and endocrine systems and is relatively common in young women in whom the autoimmune diseases have a tendency to start after puberty. There is increasing evidence that autoimmunity is a factor in some forms of epilepsy, where autoantibodies (Abs) to receptors, ion channels and other proteins involved in transmitter release have been detected. We have therefore investigated for occurrence of Abs to glutamic acid decarboxylase (GAD), a presynaptic enzyme responsible for synthesis of the neurotransmitter GABA; voltage-gated calcium channels (VGCCs) and voltage-gated potassium channels (VGKCs) at different phases of the ovarian cycle (OC) in the sera of women with different types of epilepsies.

Methods: Blood samples, from 19 women with intractable epilepsy (WWE), were taken on day 7 of their OC (midfollicular/"oestrogen" phase) and day 21/OC (midluteal/"progesterone" phase). Radioimmunoprecipita- tion assays were used for the detection of GAD, VGKCs, and VGCCs Abs. Results were compared to sera from healthy controls.

Results: All women (mean age $31.89 \pm 7.69$ years) had long-standing severe intractable epilepsy (seizure onset $9.95 \pm 9.68$; duration $20.68 \pm 9.46$ years); with $31.6 \%$ having $1-3$ seizures/month whilst $36.8 \%$ were having greater than 4 seizures/month. All were receiving more than one antiepileptic drug; etiology: infection (31.6\%), history of epilepsy (26.3\%) and immunization/allergia $(21 \%)$, brain trauma $(15.8 \%)$, pathology of delivery $(10.5 \%)$, none $(21 \%)$. VGCCs Abs were not detected in any of WWE. Increased titres of Abs to VGKCs (>100pM) were detected in 2 women $(10.5 \%)$ with drug-resistant localization-related catamenial epilepsy, whilst increased titre of Abs to GAD (> 1unit/ml) was found in one woman $(5.3 \%)$. They had drug-resistant epilepsy with the onset in early childhood, generalized EEG epileptic abnormalities with the prevalence on left side. There was no significant difference in Abs titres on day 7th or day 21st of their OC. There is no evidence from this study that changes in oestrogen/progesterone levels during the $\mathrm{OC}$ alter Abs levels.

Conclusion: The presence of Abs to VGKCs and GAD in young WWE suggests that there are some immune forms of acquired epilepsies. An immunological predisposition may play a role in catamenial epilepsy and may be associated with therapy-resistant epilepsy; future studies are needed to confirm these relationships.

Y. Lekomtseva was supported by an ENS Fellowship 2007

079

Identification of the epiphysis system in women with catamenial and/or circadian-dependent epileptic seizures during the different phases of the menstrual cycle

Y. Lekomtseva, B. Lang, H. Alexopoulos, T. James, A. Vincent

Institute of Neurology and Psychiatry (Kharkiv, UA); University of Oxford (Oxford, UK); John Radcliffe Hospital (Oxford, UK)

Objectives: Catamenial epilepsy occurs in women whose seizures predominantly occur around menses or at ovulation1. It is thought that the ratio of cyclical steroid hormones estrogen and progesterone may be responsible for the increase in seizure frequency, as estrogen is thought to be proconvulsive, increasing neuronal excitability, whilst progesterone is anticonvulsive. In some patients with epilepsy a causal relationship has also been suggested with the circadian rhythm, seizures of these patients clustering at certain points of day. We tested this hypothesis by measuring both the steroid hormone changes and the diurnal changes in melatonin levels in the plasma of patients with catamenial epilepsy throughout the menstrual cycle (MC).

Methods: Ten women with catamenial seizures (CE, mean age $\pm S D$, $31.32 \pm 7.53$ years), 9 women with long standing epilepsy, not considered to be catamenial (LSE, $30.7 \pm 8.32$ ), 10 healthy controls (HC, 31.6 \pm 5.36 ) were bled $(8.00 \mathrm{am}$ and $8.00 \mathrm{pm})$ on the 1st day $\pm 1-2$ days (IA, IB), 7th day (midfollicular phase, IIA, IIB), 21st day (midluteal phase, IIIA, IIIB) of their MC. The serum melatonin levels were measured by radioimmunoassay (Phoenix Pharmaceuticals, USA); estradiol, progesterone, luteal hormone (LH), follicular stimulating hormone (FSH) levels were all measured using a chemiluminescence immunoassay. None of women was taking oral contraceptive.

Results: In LSE and controls, the day-time melatonin levels did not show significant changes during different MC phases. However, women with $\mathrm{CE}$ had higher melatonin levels than LSE women or HC in both morning and evening levels during the early part of the cycle (IA, IB) and midfollicular phase, although levels returned to normal at the midluteal phase (IIIA, IIIB). The full hormone profile was tested in 8 women: 3 with CE; 5 LSE. CE showed a significant decrease in progesterone levels $(22 \pm 18 \mathrm{vs} 47.7 \pm 15.32 \mathrm{nmol} / \mathrm{L}$, $\mathrm{p}<0.05)$ and increased level of FSH during the midluteal phase $(9.7 \pm 5.27$ vs $4.02 \pm 1.31 \mathrm{IU} / \mathrm{L}, \mathrm{p}<0.05)$ compared to controls. LSE had high levels of LH at both day 1 and midluteal phase whilst FSH was only raised during the midluteal phase.

Conclusion: Reduction in melatonin levels during the midluteal MC phase may play role in provoking catamenial seizures. In patients with CE there was a suggestion that at day 1 and 7 of the MC cycle high melatonin levels were associated with normal hypophysis hormone levels; conversely, normal melatonin levels at the midluteal phase were associated with increasing FSH.

This work was supported by ENS Fellowship 2007 
080

Public perception and attitude towards epilepsy in northeastern Nigeria

A. Ibrahim, Y. Nyandaiti, S. Bwala

Federal Medical Centre. (Azare, NG); University of Maiduguri Teaching Hospital (Maiduguri, NG)

Objectives: Epilepsy is a common neurological problem in Nigeria and some people believe it is caused by evil spirits, offending oracles or contact with secretions from patients. This study determined the perception of the public as to the cause and treatment of epilepsy, and their attitude towards patients with epilepsy.

Methods: A cross-sectional study was conducted, using a face-to-face questionnaire interview at three different locations in Maiduguri, a NorthEastern Nigeria city.

Results: Four hundred and fifty-three out of 500 subjects (267 males and 186 females aged 16 to 60 years) responded; $53 \%$ had tertiary education and $6.8 \%$ had medical education. Two hundred and twenty-two (49\%) thought individuals with epilepsy have a normal personality and intellect, although $375(82.8 \%)$ would not marry an individual with epilepsy; $345(76.2 \%)$ agreed to continue with the relationship should their spouse or friend develop epilepsy. Two hundred and thirty-one (51\%) believed epilepsy is caused by brain disease and should be treated with drugs by a doctor, 177 (39\%) believed it is caused by either an evil spirit, offending oracles or contact with secretions from patients and should be treated by prayers/herbs from a spiritualist/herbalist and $45(10 \%)$ mentioned both brain disease and evil spirits as the cause and drugs/prayers as treatment. Three hundred and eighty-four $(84.2 \%)$ recommended free treatment for epilepsy and 348 $(76.8 \%)$ would not elect an individual with epilepsy into a political office.

Conclusion: Public knowledge, perception and attitude towards epilepsy in North-Eastern Nigeria are unfavourable and public enlightenment strategies should be adopted for improvement and to reduce social stigma associated with epilepsy.

\section{1}

Ketogenic diet has no negative effects on neurogenesis in the rat dentate gyrus

D. Kondziella, J. Strandberg, T. Thorlin, F. Asztely

University Hospital Sahlgrenska (Gothenburg, SE); Sahlgrenska Academy (Gothenburg, SE)

Objectives: Ketogenic diet is a therapeutic alternative in the treatment of refractory epilepsy in children. However, there are concerns that a high-fat diet may influence cognition and the normal development of the brain. The hippocampus is of special interest in this regard due to two reasons. Firstly, it has a pivotal role in learning and memory and secondly, in the hippocampal dentate gyrus neurogenesis continues throughout life. Several external factors such as stressful events and lack of physical exercise have been shown to decrease juvenile and adult hippocampal neurogenesis. The present study therefore investigated whether ketogenic diet has negative effects on neurogenesis in the dentate gyrus.

Methods: Twenty-one days after birth 30 rats received a high-fat diet ( $90 \%$ fat by energy) or a standard rat diet ad libitum for four weeks. Food texture and housing conditions were identical in both groups. To detect the proliferation of newborn cells all animals received four intraperitoneal injections of bromodeoxyuridine (BrdU; $75 \mathrm{mg} / \mathrm{kg}$ ) on the last day of the experiment. Hippocampal slices were incubated with primary monoclonal mouse anti-BrdU IgG antiserum and secondary horse anti-mouse IgG antiserum.

Results: Gain in body weight during the experiment was similar in both groups. Quantification of BrdU-labeled cells in the granule cell layer of the dentate gyrus revealed no differences between the ketogenic diet group and controls.

Conclusion: Ketogenic diet has no negative effects on neurogenesis in the rat dentate gyrus.

\section{2}

Risk of seizure recurrence after withdrawal of antiepileptic drugs in multiply handicapped Egyptian children with epilepsy

N. Kitchener, S.S. Aziz, N. Michael

General Organization for Teaching Hospital (Cairo, EG); Ain Shams Univerity (Cairo, EG)

Objectives: To determine the incidence and risk factors of seizure recurrence in multihandicapped Egyptian children with epilepsy after withdrawal of antiepileptic drugs (AEDs).

Methods: This was a prospective study from January 1995 to January 2006. The study population was multihandicapped children with epilepsy followed up in the out patient clinic of Centre for Children with Special Needs; Egyptian Integrated Care Association (CCSN; EICA), Cairo, Egypt. The eligible children were less than 13 years, seizure-free for at least 24 months, with two normal EEG records, separated by 6 months. The AEDs were withdrawn slowly. The patients were then followed up to look for seizure recurrence.

Results: One hundred sixty two children were recruited in the study. The average age of onset of epilepsy was 14 months. Most patients had partial seizures $(49 \%)$. Most were controlled on monotherapy $(64.2 \%)$, and were treated with Phenytoin (24\%), Carbamazepine $20.4 \%$, and Sodium valproate $16 \%$. The seizure free duration before starting to withdraw AED was $48.3 \pm 9$ months. None of the patients had seizure recurrence while the AEDs were being tapered. There were 77 patients $(47.5 \%)$ with seizure recurrence. The average duration of follow-up for the patients who did not have seizure recurrence was $45 \pm 3$ months. Most of recurrences occurred in the first twelve months, remainder during next twelve months. The risk factors of seizure recurrence were abnormal neurological examination, the need for use of polytherapy, presence of abnormal findings on brain imaging (CT and/or MRI).

Conclusions: Risk of seizure recurrence after AED withdrawal in multiply handicapped Egyptian children was high at $47.5 \%$.

\section{Oral session 5}

\section{Neurogenetics}

083

Episodic ataxia type 1 in identical twins: genotype-phenotype correlation T.D. Graves, S.M. Zuberi, H. Morris, S. Schorge, D.M. Kullmann, M.G. Hanna Institute of Neurology (London, UK); Royal Hospital for Sick Children (Glasgow, UK); Medical School (Cardiff, UK)

Episodic ataxia type 1 (EA1) is a rare autosomal dominant disorder characterised by brief attacks of ataxia and is due to mutations in the voltage-gated potassium channel Kv1.1, encoded by KCNA1. Attacks generally improve with age and unlike episodic ataxia type 2 (EA2), are not associated with progressive ataxia. There are no reports of EA1 occurring in twins.

Objectives: To provide phenotypic data in two sets of identical twins with EA1; to identify KCNA1 mutations in these families and to characterise physiological basis of their disease.

Methods: All available members of the pedigrees were examined clinically by one investigator. The entire KCNA1 gene was directly sequenced. The mutations identified were inserted into a cDNA construct of KCNA1 contained within the mammalian expression vector, pcDNA3, using site-directed mutagenesis. Human embryonic kidney (HEK-293) cells were transiently transfected using standard techniques. Potassium currents were measured using whole-cell patch clamp recordings with potassium gluconate-based internal solution and standard mammalian Ringer's solution.

Results: The first pedigree has severe EA1. Attacks increase in both frequency and severity with age and the probands' mother developed a progressive cerebellar syndrome. The proband is one of identical twins, both of whom are affected to differing degrees. Another large pedigree was identified with classical EA1. The probands' mother is also one of affected identical twins. They have different attack frequency, severity and duration

Two novel missense mutations, R307C and F414S were identified, which were not present in 228 control chromosomes. Both mutations are in highly conserved regions of the gene and affect functionally important protein domains. Functional analysis revealed a reduction in current density and a depolarising shift in the voltage dependence of activation, leading to loss of function.

Conclusions: These cases highlight the fact that differences in phenotype, more usually seen in siblings or between generations must be due to environmental factors, as modifier genes cannot be implicated when there is no genetic variation between individuals. 
084

\section{Spinocerebellar ataxia type 17. Case series}

A. Gonzalez-Aguilar, M.E. Alonso, P. Yescas, I. De Biase, S. Bidichandani, A. Rasmussen

National Institute of Neurology \& Neurosurgery (Mexico City, MX); University of Oklahoma Health (Oklahoma, US)

Spinocerebellar ataxia type 17 is caused by CAG/CAA triplet repeat in the gene for TATA-binding protein (TBP). Clinically, the phenotype is ataxia, dementia, and chorea, parkinsonism, and psychiatric symptoms. In contrast with the other ataxias caused by CAG repeat expansion, anticipation is rare in SCA17.

Patients and methods: We identified a total of 19 patients with SCA17. All patients were Mexican, and whereas 17 belonged to 3 ADCA pedigrees, 2 were sporadic cases. Clinical information was available for all 19 patients, and DNA samples were available from both sporadic and 10 of the familial patients.PCR and direct sequencing was performed to identify the precise length and repeat configuration of the CAG/CAA alleles in the TBP gene. Information of age at onset of disease, clinical manifestation, radiological findings and neuroconduction test was obtained.

Results and discussion: By direct sequencing, we analyzed the expanded CAG/CAA repeats in 12 patients from whom DNA samples were available. All familial cases $(n=10)$ were found to carry the "uninterrupted" configuration of CAG/CAA alleles, and both sporadic cases showed the more common "interrupted" configuration. The uninterrupted alleles are uncommon; accounting for only 10 of 90 previously reported. A highly significant correlation was alleles noted between repeat length and the age of onset for our 10 patients $(\mathrm{r}=0.8 ; \mathrm{p}=0.003)$. The clinical features were consistent with SCA17: ataxia $(100 \%)$, dementia $(78 \%)$, chorea $(21 \%)$, parkinsonism(16\%), and psychiatric symptoms $(50 \%)$. Four patients had mild sensorimotor axonal neuropathy, mainly in lower limbs. Magnetic resonance imaging showed cerebellar atrophy $(40 \%)$ with variable degrees of brainstem and cerebral atrophy $(60 \%)$. We compared age of onset intergenerational showed significant increases on intergenerational transmission. Anticipation correlated with parental age $(r=0.75 ; \mathrm{p}=0.007)$. However, 7 of our 17 ADCA patients $(37 \%)$ had an age of onset of younger than 20 years. In conclusion, we have shown that intergenerational transmission of the uninterrupted mutant alleles in SCA17 almost always results in further expansion and genetic anticipation. Spinocerebellar ataxia 17 the diagnosis can be inferred dominant autosomal ataxia or sporadic, ataxia with cognitive deterioration, ataxia with psychiatric manifestations, dementia of early beginning, dominant Huntington like, parkinson familiar and must be considered in these sufferings when they make debut in the childhood.

\section{5}

ALS genes: a mutational screening in a large cohort of Italian patients

C. Gellera, N. Ticozzi, B. Castellotti, C. Colombrita, M. Plumari, C. Bragato, L. Morandi, D. Testa, A. Bellino, A. Ratti, V. Silani

IRCCS-Istituto Neurologico Carlo Besta (Milan, IT); IRCCS Istituto Auxologico Italiano - Università degli Studi di Milano (Milan, IT)

Amyotrophic Lateral Sclerosis (ALS) is an adult-onset and rapidly progressive neurodegenerative disorder, caused by the selective loss of motor neurons in the cerebral cortex, brainstem and spinal cord. Although most ALS cases are sporadic (SALS), approx. $10 \%$ of them are familial (FALS). At least 12 distinct loci linked to the disease have been identified. Mutations in 4 pathogenetic genes: SOD1, Alsin, SETX, VAPB and in 3 susceptibility genes: VEGF, DCTN1, ANG1 have been described.

Mutations in SOD1 gene account for about $20 \%$ of familial cases with more than one hundred different mutations described so far. Mutations in Alsin, VAPB, SETX, and DCTN1 genes are rare. Angiogenin (ANG1) is a novel candidate gene for the pathogenesis of ALS. It is an angiogenic factor up-regulated by hypoxia and it is highly expressed in motor neurons. Missense mutations in ANG gene have been identified in Northern Europe patients.

We have screened for SOD1 and ANG1 mutations a large cohort of Italian patients from two Neurological referral centres: 762 index cases (149 FALS and 613 SALS).

We have found SOD1 mutations in 18/149 (12\%) unrelated FALS and in 7/613 (1\%) SALS patients. 10/18 (55\%) FALS carried mutations frequently described in different ethnic backgrounds (A4V, L84F, G93D and L144F). Interestingly, we identified 4 new SOD1 mutations.

ANG1 screening in the same cohort of patients and 517 controls allowed us to identify 7 different mutations (five novel) in 14 patients. Three mutations are located in the signal peptide region, three in the coding sequence and one in the 3' untranslated region. No mutations have been found in controls. The observed mutational frequency of ANG gene accounts for about $1.2 \%$ with an over-representation of FALS $(2.3 \%)$ compared to SALS $(1 \%)$ cases.
Other candidate genes have also been screened in a subset of patients: alsin gene in 75 patients with a juvenile onset and SETX gene in 44 patients with a dominant inheritance. No mutations were identified in exons where mutations were previously described to occur.

A subset of sporadic patients $(n=95)$ were also screened for deletions in SMN2 gene, as a possible candidate ALS susceptibility gene. We did not find a significant association of SMN2 deletions in patients respect to controls.

Our results indicate that SOD1 still remain the main determinant in the pathogenesis of hereditary ALS and ANG1 gene represents a strong susceptibility factor for both SALS and FALS cases in the Italian population.

\section{6}

Molecular analysis of spastin gene (SPG4) mutations in patients with familial and sporadic spastic paraplegia

S. Baratta, D. Di Bella, C. Mariotti, R. Fancellu, S. Di Donato, C. Gellera, F. Taroni

IRCCS Istituto Neurologico Carlo Besta (Milan, IT)

Hereditary spastic paraplegias (HSP) are a clinically and genetically heterogeneous group of neurodegenerative disorders. Mutations in the SPG4 gene are responsible for $\sim 15-40 \%$ of autosomal dominant (AD) HSP cases; HSP due to SPG4 mutations is generally described as a pure form of the disease, but has also occasionally been described in association with additional clinical features. This gene encodes spastin, a member of the AAA family of ATPases.

Objectives: To screen a large cohort of patients with spastic paraparesis for SPG4 mutations, including both point mutations and micro-rearrangements.

Methods: The spastin gene was analysed by dHPLC and/or direct sequencing in 222 unrelated index cases, including $107 \mathrm{AD}$ cases, 19 cases compatible with an autosomal recessive (AR) pattern of inheritance (ie, $\geq 2$ affected individuals in one generation only) and 96 sporadic cases. Moreover, a subgroup of patients was screened for micro-rearrangements in the SPG4 gene by multiplex ligation-dependent probe amplification (MLPA) to assess the frequency of deletions/duplications.

Results: Thirty-two different pathogenic mutations (15 missense, 8 frameshift, 5 nonsense, 5 splicing) were found, 21 of which are novel. The mutations were not found in a large control population. Twenty-eight mutated patients were identified in the AD group (28/107, $26 \%$ ), whereas only 4 in the sporadic group $(4 / 96,4.2 \%)$, and 1 in the possibly-AR group (1/19, $5.2 \%)$ with an overall frequency of $14.9 \%(33 / 222)$. In the subgroup of patients screened by MLPA, we identified 3 patients $(2 \mathrm{AD}, 1 \mathrm{~S})$ with different microdeletions in the SPG4 gene, corresponding to a frequency of $18.8 \%$. The majority of mutated patients exhibited a pure spastic paraparesis phenotype.

Conclusions: Overall, in our study, SPG4 point mutations account for $14,9 \%$ of spastic paraparesis patients, a frequency which raises to $26 \%$ if only $\mathrm{AD}$ families are considered, consistent with other data reported in the literature. MLPA study demonstrate that a relevant proportion of mutationnegative HSP patients in fact carry SPG4 microdeletions, confirming that haploinsufficiency of SPG4 is a major cause of AD-HSP. Exon deletions are almost as frequent as point mutations, thus indicating that gene dosage experiments should always be performed when screening SPG4 in HSP patients, in order to assess the real frequency of SPG4-HSP.

Supported by an Italian Ministry of Health grant (RF Neurodegenerazione ex art 56) to FT

\section{7}

Dynamin gene (DNM2) mutation in Czech Charcot-Marie-Tooth type 2 family with variable phenotype

R. Mazanec, P. Ridzon, C. Stengel, O. Keller, E. Vyhnalkova, K. Hühne, J. Haberlova, M. Bojar, B. Rautenstrauss, P. Seeman

University Hospital Motol (Prague, CZ); Thomayer University Hospital (Prague, CZ); Friedrich-Alexander University (Erlangen, DE)

Background: The dynamin 2 (DNM2) is a member of large GTPases and is a part of the cellular fusion-fission apparatus. DNM2 plays a crucial role in cellular transport and in the stability of microtubules network. The mutations in the DNM2 can cause axonal or intermediate types of CMT disease.

Objective: To perform genotype-phenotype clinical and electrophysiological correlations in patients with detected DNM2 mutations.

Methods: 25 members out of large Czech CMT2 family were genetically tested for DNM2 mutations. Family members carrying DNM2 mutation were clinically and electrophysiologically examined.

Results: Causative missense mutation Met580Thr of DNM2 gene was found in 8 subjects $(3 \mathrm{M}+5 \mathrm{~F})$ out of 25 family members. The main clinical 
features of this genotype showed wide ranged disease onset between 2 nd and 4th decade, distal muscle atrophies, absent reflexes and sensory disturbances at lower limbs in $5 / 8$ subjects, muscle atrophies at upper limbs in $6 / 8$, pes cavus in $2 / 8$ subjects only. The wide phenotype variability and slowly progressive course of the disease were noticed. Electrophysiological examination confirmed pure axonal type of conduction abnormality in $7 / 8$ and intermediate type in $1 / 8$. Median nerves were more severely affected than ulnar nerves and sensory fibres were more affected than motor nerves.

Conclusions: DNM2mutation Met580Thr can cause mainly axonal type of CMT disease. DNM2 gene should be tested in CMT2 families with a large variability of the phenotype severity and adult onset of the disease.

Supported by IGA NR/9517-3 and VZ 00064203/6506

\section{8}

Autosomal recessive Pelizaeus-Merzbacher-like disease- 1 with a milder phenotype in a family harbouring a novel GJA12 gene mutation

E. Salsano, J. Orthmann-Murphy, S.S. Scherer, G. Uziel, E. Lamantea, C. Abrams, A. Bizzi, M. Savoiardo, M.Zeviani, D. Pareyson

C.Besta Neurological Institute (Milan, IT); University of Pennsylvania School of Medicine (Philadelphia, US); SUNY Downstate Medical Center (New York, US)

Objective: To describe a kindred with autosomal recessive PelizaeusMerzbacher-like disease-1 (PMLDAR1) caused by a novel mutation in the gap junction protein alpha-12 gene (GJA12, encoding connexin47, Cx47), and showing a phenotype milder than previously reported. X-linked Pelizaeus-Merzbacher disease (PMD) and PMLDAR1 are hypomyelinating leukoencephalopathies caused by PLP1 (Proteolipid Protein 1 gene) and GJA12 mutations, respectively. PMD shows high phenotypic variability, whereas PMLDAR1 is homogeneous and characterized by psychomotor delay, progressive spasticity, inability to walk or early loss of walking ability, nystagmus and cerebellar signs.

Patients/results: Two brothers and their female cousin developed slowly progressive gait difficulty during childhood or adolescence. They acquired ability to walk independently, talk and write, despite minimal motor disturbances had been noted since infancy in two subjects. Patient 1 (age 39) had a relatively pure mild spastic paraplegia and was able to walk unaided; patient 2 (age 36) had moderate complicated spastic paraplegia, and walked with unilateral support; patient 3, a 53-year-old female, had more severe course, became chairbound at age 30 , had sphincter disturbances and severe scoliosis. Dysarthria, reduced manual dexterity, mild cerebellar signs, pes cavus, and sensory disturbances were variably present. Nystagmus and peripheral neuropathy were not observed. No clearcut mental retardation was evident at WAIS test. MRI showed white matter abnormalities consistent with a hypomyelinating leukoencephalopathy; MR-Spectroscopy showed a near-normal metabolite profile. Genetic analysis revealed a novel homozygous GJA12 missense mutation (I33M). I33M appears to form gap junction plaques, similar to wild-type Cx47, when expressed in HeLa cells; further functional studies are in progress.

Conclusions: PMLDAR1 encompasses a spectrum of clinical phenotypes wider than previously thought, including a late-onset, slowly progressive near-pure spastic paraplegia. All family members had a normal or near-normal psychomotor development, preserved walking capability until adulthood, and no nystagmus. The I33M is not a drastic amino acid change but I33 is invariant in mammalian Cx47 species. These features may explain the milder phenotype associated with the I33M mutation, as compared with the early-onset severe presentations associated with other GJA12 mutations.

\section{9}

Voxel-based morphometry study of atrophy's patterns in spinocerebellar ataxia type $2,6,7$ and 8

P. Caroppo, L. Orsi, F. D'Agata, A. Boghi, R. Mutani, M. Bergui, G.B. Bradac, P. Mortara

University of Turin (Turin, IT); Hospital Molinette (Turin, IT); Hospital S. Croce (Cuneo, IT)

The aim of this study was to examine the different patterns of cerebellar and/or brainstem atrophy in spinocerebellar ataxia (SCA) type 2, type 6, type 7 and type 8. Twenty four patients and twenty four healthy volunteers were studied. Voxel-Based Morphometry (VBM) was applied to segment Grey Matter (GM), White Matter (WM) and Cerebro-Spinal fluid (CSF) of highresolution T1-weighted brain volumes of each group. Our results demonstrate different patterns of GM and WM affection detected by magnetic resonance imaging (MRI). SCA2 have a diffuse cortical supra and infratentorial atrophy with the whole brainstem affected; SCA6 have a nearly pure cerebellar atrophy with minimal GM atrophy in the cingulum; SCA7 have a dif- fuse cortical supra and infra tentorial atrophy with WM in brainstem affected but with GM relatively preserved; SCA8 have a relatively pure cerebellar atrophy with small scattered cortical GM atrophy and pure cerebellar WM atrophy.

The SCA2 subgroup was also tested with a neuropsychological battery, covering the principal cognitive domains, to explore the possibility of a correlation with some disease features.

Anterior lobe cerebellar atrophy correlates with the disease progression, the precocity of the onset with the gravity of the clinical signs (ICARS), the general memory and the phonological fluency with posterior cerebellar volume, the planning ability with the global GM, the tests requiring sharp motor performance with anterior cerebellum. While the cognitive deficits are evident in SCA2 the skills seem to have a global impairment linked to the presence of atrophy and not to its extension or severity.

\section{Oral session 6}

\section{Neuro-oncology}

\section{0}

PPAR-gamma activators: off-target against TGF-beta mediated glioma cell migration and brain invasion

S. Seufert, J. Hauke, E. Hahnen

University of Cologne (Cologne, DE)

Gliomas are the most common primary tumours of the central nervous system, with glioblastomas as the most malignant entity. Rapid proliferation and diffuse brain invasion of these tumours are likely to determine the unfavourable prognosis. Considering its pro-migratory properties, the transforming growth factor beta (TGF-,) signaling pathway has become a major therapeutic target. Analyses of resected glioma tissues revealed an intriguing correlation between tumour grade and the expression of TGF-, 1-3. Using an organotypic glioma invasion model, we identified the peroxisome proliferator-activated receptor gamma (PPAR-gamma) agonist troglitazone (TRO), which has been developed for the treatment of type II diabetes mellitus, as a potent inhibitor of TGF-, mediated glioma migration and brain invasion. The anti-migratory property of TRO occurred at clinically achievable levels and was mediated by transcriptional repression of TGF-,1-3, resulting in dramatically reduced TGF-, secretion. To date, TRO appears to be the most potent inhibitor of TGF-, expression and may thus represent a promising drug for adjuvant glioma therapy. Here, we show that the PPARgamma inactive TRO derivative delta2-TRO antagonized TGF-, signaling and ex vivo glioma invasion similar to TRO, giving clear evidence that the anti-glioma properties of TRO occur in a PPAR-gamma independent manner. The identification of the yet unknown anti-migratory target protein of (delta2)-TRO may provide new therapeutic strategies for adjuvant therapy of glioma and other highly migratory tumour entities.

\section{1}

Unmasking the face behind recurrence: mechanisms underlying drug resistance of brain tumour stem cells

V. Martin, M.M. Alonso, J. Xu, D. Liu, H. Colman, W.K.A. Yung, J. Fueyo, C. Gomez-Manzano

M.D. Anderson Cancer Center (Houston, US)

The identification of brain tumor stem cells (BTSCs) has been a defining moment in cancer research. According to the BTSC model, those cells exhibit the capacity to repopulate tumors after treatment due to their chemoresistant phenotype. Eradication of the stem-cell compartment of a tumor may therefore be essential to achieve stable, long-lasting remission, and even a cure, of cancer. In order to efficiently target BTSCs the mechanisms of drug resistance need to be elucidated. We have generated a panel of BTSCs from surgical human glioblastoma specimens that were characterized by examining the expression of stem-cell markers, and by analyzing the capacity for self-renewal, multilineage differentiation and re-establishment of tumors after transplantation. Of interest, BTSCs displayed a more chemoresistant phenotype to a panel of several drugs with different mechanisms of action, when compared to established glioma cultures. Several lines of evidence indicate that Tie2 promotes chemoresistance in normal stem cells, mediating the repopulation of the bone marrow after genotoxic stress. Recently, our group identified Tie2 expression in BTSCs obtained from malignant 
gliomas, suggesting a role of Tie 2 in mediating chemoresistance. Treatment of sorted of Tie2+ and Tie2- BTSCs with several chemotherapeutic agents showed that activation of Tie2 is related to a significant increase of the ICD50s. Viability studies were performed by using MTT and annexin assays. The chemoresistance of those cells were Tie2-dependent, as demonstrated by experiments consisting in downmodulation of Tie2 by siRNA, or by transferring Tie2 cDNA to Tie2-negative cells. Thus, our preliminary data show an increase, up to $400 \%$, in the IC50 dose of several drugs in Tie2-expressing cells versus Tie2-negative isogenic counterparts. We further observed that Tie2 pathway activation regulates the expression of ATP-binding cassette transporters - $\mathrm{ABC}$ transporters - by using quantitative real time PCR. Functional studies showed that Tie2 active pathway was related with drug efflux, assessed by using selected substrates and inhibitors of the $\mathrm{ABC}$ transporters. In vivo studies to further confirm the role of Tie2 in the chemoresistance of gliomas are in progress. Taken together our results indicate that Tie2 activation in BTSCs may play an important role in multidrug resistance, and open a new field for the search of more effective therapies to avoid the - until now - inevitable brain tumor recurrence.

092

Meningioma responding to temozolomide: report of two cases

E. Laguzzi, D. Guarneri, I. Morra, R. Rudà, E. Trevisan, A. Ducati, R. Soffietti University of Turin (Turin, IT)

Objectives: Resection remains the main modality of treatment of meningiomas and can be curative in some cases. Conventional radiotherapy and radiosurgery may have a role in meningiomas incompletely resected, anaplastic or recurrent. When other treatments have failed, hormonal therapy or chemotherapy may be considered. Few data are available regarding chemotherapy in meningiomas.

We report two cases of patients with meningioma responding to temozolomide chemotherapy, standard schedule $\left(200 \mathrm{mg} / \mathrm{m}^{2}\right.$ administered orally on the first 5 days of a 28 -day cycle).

Case 1. A 59 years-old man had a left frontal meningioma surgically removed in 2002, 2003 and 2004. The last histological diagnosis was meningotelial atypical meningioma (MIB-1 $12 \%$, progestinic receptors positive in $20 \%$ of tumour cells) and the patient was treated with radiotherapy (59.4 Gy) and chemotherapy with idroxyurea. Due to a progressive disease, 24 cycles of temozolomide chemotherapy were administered achieving radiological partial response (60-70\%) and complete seizure control, without serious side effects. To present, patient has been in follow up with stable disease for eight months.

Case 2. A 25 years-old woman had a left parietal meningioma surgically removed in 1981, 1999 and 2005, with radiotherapy after second surgery. The last histological diagnosis was transitional meningioma with secretory areas. In 2007 she had surgery and radiotherapy for a right parietal microcystic meningioma (MIB-1 20\%). Patient was treated with 6 cycles temozolomide. The residual tumour showed a partial response $(50 \%)$ and patient improved clinically. Temozolomide chemotherapy is ongoing and well tolerated.

Conclusions: We report two cases of response of meningiomas to temozolomide, both with a radiological and clinical response. Further clinical trials are needed to confirm the activity of temozolomide in meningioma patients.

093

Safety and efficacy of fotemustine in recurrent or progressive gliomas

E. Trevisan, E. Laguzzi, R. Rudà, D. Guarneri, R. Soffietti

S.Giovanni Battista Hospital (Turin, IT)

Objective: The aim of this study was to evaluate safety and efficacy of fotemustine in patients with progressive or recurrent gliomas after surgery, radiotherapy and one or more lines of chemotherapy.

Patients and Methods: From September 2004 to May 2007 we treated 82 patients ( 25 female, 57 male) with a median age of 52 years (range: $19-78 \mathrm{yrs)}$ and Karnofsky Performance Status (KPS) $\geq 50$. Fotemustine was administered intravenously at the dose of $100 \mathrm{mg} / \mathrm{m}^{2}\left(75 \mathrm{mg} / \mathrm{m}^{2}\right.$ if previous myelotoxicity) on days $1,8,15$ (induction phase). After a $4-5$ weeks rest period, fotemustine was given every 3 weeks until progression or unacceptable toxicity. MRI was performed at baseline, after one month from induction and every 3-4 cycles. Response on MRI was evaluated according to Macdonald criteria. Histological diagnosis was as follows: $47 / 82(57.3 \%)$ glioblastoma, $13 / 82$ $(16 \%)$ astrocytoma grade III, $8 / 82(9.7 \%)$ oligodendroglomas grade III, 7/82 ( $8.5 \%)$ oligoastrocy toma grade III, $7 / 82(8.5 \%)$ oligodendroglioma grade II. Results: Median survival from start of fotemustine is 5 months (range: 1-26): 5 months in astrocytic gliomas, 6 months in oligodendroglial gliomas. The median TTP is 3 months (range: 1-19+): 2 months in astrocytic gliomas,
4 months in oligodendroglial gliomas. Median PFS-3 months of $48 \%$; PFS- 6 months of $15 \%$; PFS- 12 months of $3 \%$. An objective response (CR+PR) was observed in $15.5 \%$ of patients, with $50.6 \%$ of stable disease $(\mathrm{mR}+\mathrm{SD})$. Myelosuppression was the most common adverse event ( $84.8 \%$ of patients) and occurred mainly after the induction phase. Grade 3-4 leucopenia was observed in $50.6 \%$ of patients, grade 3-4 thrombocytopenia was observed in $55.6 \%$, and grade $3-4$ anemia was observed in $6.3 \%$. Only 1 case of fatal midollary aplasia after the induction phase. A moderate reversible increase in hepatic enzymes was noted in $5 \%$ of patients. Other adverse events were diarrhea, rash, thromboembolic events.

Conclusion: Fotemustine as second-line chemotherapy and sometimes as third or fourth-line in patients with recurrent/progressive gliomas is a relatively safe treatment. A correlation between methilation of MGMT and response to fotemustine is possible. The analysis of MGMT status is ongoing.

\section{4}

Temozolomide in gliomatosis cerebri: final results of an Italian study R. Rudà, E. Laguzzi, E. Trevisan, A. Pace, A. Silvani, R. Soffietti on behalf of the Italian Association of Neuro-Oncology (AINO)

Background: Gliomatosis cerebri is a diffuse glioma, usually astrocytic, growth pattern consisting of exceptionally extensive infiltration of large regions of CNS (involvement of at least three lobes). GC is usually an aggressive tumor (Grade III,WHO 2007). The best treatment of GC is unknown; patients are rarely offered surgery, and radiotherapy may result in a non negligeable risk of neurotoxicity. The role of chemotherapy is unclear.

Aim of the study: Objective of this retrospective, multicentric study was to assess the efficacy and toxicity of Temozolomide in patients with histologically verified GC.

Design/methods: Since 1999, 51 patients with GC were treated with Temozolomide either at progression after prior radiotherapy/chemotherapy or upfront. Tissue specimens were diagnostic for astrocytoma in 17, oligoastrocytoma in 3,oligodendroglioma in 5 , gemistocytic astrocytoma in 2 , anaplastic astrocytoma in 9, anaplastic oligoastrocytoma in 2, anaplastic glioma in 6,glial proliferation consistent of gliomatosis cerebri in 6, focal area of GBM in 1. Patients characteristics were as follows: median age 51 years (range 18-75 years), 29 males and 22 females, median KPS at diagnosis 80 (range 50-100). Presenting symptoms were as follows: seizures (25 patients), intracranial hypertension (9), motor deficits (7), mental status changes (5), visual disturbances (4), ataxia (1). Thirty out of 51 pre-treatment MRI scans demonstrated some contrast enhancement. All patients were treated with Temozolomide $200 \mathrm{mg} / \mathrm{m} 2$ per day for 5 days every 4 weeks until progression or unacceptable toxicity. Response was evaluated, according to Macdonald criteria, on MRI using both T1-weighted with Gadolinium and FLAIR images.

Results: The median number of cycles was 6 (range 2-26). Overall response rate (CR + PR + "minor response") was $23 \%$. Median time to tumor progression (TTP) was 11.1 months and the overall median survival was 14.2 months. A clinical benefit, consisting in a reduction of seizures or improvement of intracranial hypertension, was observed in $29 \%$ of patients. Haematological toxicity grade III-IV was mild ( $14 \%$ thrombocytopenia).Multivariate analysis showed contrast enhancement on MRI as the strongest independent prognostic factor.

Conclusions: Temozolomide has activity in GC with a clinical benefit in some patients and is well tolerated. Dose-dense schedules of temozolomide could improve the results. A phase II study is ongoing,with molecular analysis included.

095

Prospective randomised study comparing first-line nitrosourea-based chemotherapy/radiotherapy versus radiotherapy alone in non-resectable glioblastomas

M. Frenay, J. Simon, L. Benbouker, L. Taillandier, D. Castera, A. Busson, M. Campone, C. Lebrun on behalf of the FOTEGLIO Group

Objective: To compare time to progression in patients with non resectable glioblastomas (GBL) treated with either radiotherapy (RT) or a nitroso-urea based chemotherapy (CT) with platinium salts.

Methods: Phase III multicentric prospective randomized study comparing time to progression (TTP) in patients diagnosed for GBL confirmed with histological diagnosis according to WHO classification. All patients had non resectable tumor because of location or callosal extension. CT (F/CDDP/ VP16) consisted with 4 monthly cycles of Fotemustine $\left(100 \mathrm{mg} / \mathrm{m}^{2}, \mathrm{~d} 1\right)$; Cisplatinium $\left(33 \mathrm{mg} / \mathrm{m}^{2} / \mathrm{d}, \mathrm{d} 1-3\right)$; Etoposide $\left(75 \mathrm{mg} / \mathrm{m}^{2} / \mathrm{d} 1-3\right)$ followed with conventional fractionated radiotherapy (55-60Gy). MRI was performed at baseline before and after surgery and every 8 weeks. Clinical and QOL evaluation was monthly reported. 
Results: 50 patients were randomized (26 CT-RT and 24 RT alone) with mean age: 58 yrs (33-71); Mean Karnofsky score: $86.4+/-11$; Mean time between biopsy and NU-CT or RT was less than 6 weeks. $70 \%(35 / 50)$ had only stereotaxic biopsy and others had partial resection. Time to progression was 242 days (IC95:99-314) for CT-RT versus $131 \mathrm{~d}$ (IC95:92-208) for RT alone; $\mathrm{p}=0.0085$. Median survival was $375.5 \mathrm{~d}$ (IC95:323-427) for CT-RT vs 325 (IC95:200-504) $\mathrm{p}=0,36$ for $\mathrm{RT}$ alone. The best tumoral response was $23 \%$ for CT-RT vs $8 \%$ with RT. X \% of patients had grade III or IV side effects (CTRT:62\% hematological toxicity; RT: $17 \%$ neurological toxicity). Nine patients died during the follow-up ( 6 tumor progression, 2 sepsis, 1 unknown).

Conclusions: Time to progression and median survival are increased with the association F/CDDP/VP16-RT compared with RT alone.

This work was support by Servier

\section{Oral session 7}

\section{Multiple sclerosis 2}

\section{6}

Investigation of role of peripheral EBV reactivation in driving multiple sclerosis disease activity as measured by gadolinium enhanced MRI R. Farrell, D. Antony, D. Clark, J. Swanton, L. Fisniku, Z. Khaleeli, A. Thompson, D.H. Miller, G. Giovannoni

Institute of Neurology (London, UK); Institute of Cell and Molecular Science (London, UK); Barts and the London NHS Trust (London, UK)

Objective: To measure Epstein Barr virus activity and serological responses in peripheral blood of subjects with Multiple Sclerosis and correlate this with new gadolinium-enhancing lesions on MRI.

Background: Multiple sclerosis is thought to be triggered in susceptible individuals by an environmental agent/infection. A substantial body of evidence links EBV with MS. Epidemiological studies have shown elevated levels of anti-EBV antibodies predate development of MS. Peripheral EBV reactivation has been linked to disease activity and recently EBV infected B cells have been described in postmortem brain tissue of subjects with MS.

Design/methods: 100 subjects involved in serial imaging studies were identified - 50 CIS, 25 RRMS, 25 PPMS. Subjects had serial Gd-enhanced MRIs over a 5-year period, at which time blood was stored. EBV DNA in blood was quantified by real-time qPCR and EBV serology for anti-EBNA1 IgG, anti-VCA IgG and anti-EBV IgM using commercial kits. Serology for other common viruses was also performed. Statistical analysis was performed using Anova, t-test and logistic regression models.

Results: All subjects had evidence of past EBV infection. EBV DNA was found in 5 subjects, IgM response to EBV was detected in 13 subjects all associated with Gd-enhancing lesions (ns). Significant differences in level of antibodies to EBNA1 and ratio of anti-EBNA1:VCA IgG antibodies were found between subgroups, highest in RRMS group compared with PPMS and CIS. EBNA1:VCA ratio $(\mathrm{R}=0.36, \mathrm{p}<0.001)$ and EBNA1 $(\mathrm{R}=0.33$, $\mathrm{p}<0.001)$ correlated with development of new Gd-enhancing lesions.

Conclusions/relevance: Subjects with active disease have elevated levels of EBNA-1 IgG specifically which may reflect the severity of the initial antigenic challenge (primary infection/infectious mononucleosis). No acute peripheral reactivation was found to be associated with new Gd lesions. This suggests the primary EBV infection has a role in triggering the disease. In other EBV related disorders specific antibody patterns have been described as risk factors for subsequent disease. Whether these antibodies have a pathogenic role or reflect the underlying immune dysregulation in MS remains unclear and requires further investigation.

This study was supported by MS Society of Great Britain and Northern Ireland

097

Cytokine regulation of gene expression for osteoprotegrin in central nervous system mixed glial cell cultures

R.P. Lisak, J. Benjamins, B. Bealmear, D. Studzinski, L. Nedelkoska

Wayne State University (Detroit, US)

Objectives: We observed differential effects of Th1, Th2 and monocyte/ macrophage (M/M) cytokine (Cyt) mixtures on gene expression for osteo- protegrin (OPG), a member of the tumor necrosis factor receptor superfamily (TNFRSF). We have employed microarray technology as a screening technique in mixed central nervous system (CNS) mixed glial cell cultures at 6 hours to search for potential genes and proteins of interest involved in inflammatory responses in the CNS (Lisak et al 2006). OPG is known to be involved in bone development but as a secreted decoy receptor has the potential to modulate several proinflammatory and proapoptotic molecules. Our objective was to confirm and extend the results of the screening study by examining the effects of the different Cyt on the gene for OPG at different time points employing Quantitative Real Time-Polymerase Chain Reaction (QRT-PCR).

Methods: Mixed rat CNS glial cell cultures were incubated with mixtures of Cyt typical of Th1 and Th2 lymphocytes and M/M; levels of gene expession were examined at 6 and 24 hours and 3 and 5 days employing QRT-PCR. Levels of expression of a transcript for OPG were compared to that of GAPDH, a housekeeping gene.

Results: Th1 Cyt had a minor upregulatory effect on OPG gene expression at 6 hours, followed by a decline to essentially control levels, compared to GADPH, at all later time points, paralleling the lack of any significant effect of Th1 Cyt at 6 hours using microarray technology. Th2 Cyt caused significant upregulation of gene expression for OPG at 6 hours with sustained upegulation at 24 hours and 3 days and a modest increase in expression still noted at 5 days. M/M Cyt induced upregulation of gene expression at 6 hours with a decline to lesser levels of upregulation by 24 hours, which were sustained at those levels at later time points. Both Th2 and M/M Cyt had induced upregulation of gene expression for OPG at 6 hours using microarray technology, in agreement with the QRT-PCR results.

Conclusion: We have confirmed our earlier screening studies and demonstrated for the first time that expression of the gene for OPG is regulated by Cyt in endogenous CNS glial cells. If this regulation is accompanied by changes in total or differential expression of OPG at the level of protein, this could result in downregulation of proinflammatory effects to glial cells.

Funded by Teva Neuroscience and the Parker Webber Neurology Endowment of Wayne State University/Detroit Medical Center 
099

Identification of novel potential susceptibility genes: results of a wholegenome study in primary progressive multiple sclerosis

F. Martinelli Boneschi, F. Esposito, M.E. Rodegher, V. Martinelli, B. Colombo, L. Moiola, P. Rossi, A. Ghezzi, G. Coniglio, R. Capra, L. Collimedaglia, T. Wells, J. Wojcik, H. Abderrahim, G. Comi

Scientific Institute San Raffaele (Milan, IT); Ospedale Gallarate (Gallarate, IT); Ospedale of Potenza (Potenza, IT); University of Brescia (Brescia, IT); MS Clinics of Novara (Novara, IT); Merck-Serono Research Center (Geneva, $\mathrm{CH})$

Objective: PP represents a very rare clinically homogeneous disease course of MS. The genetic component of MS susceptibility is believed to result from the action of allelic variants in several genes. Each gene is expected to have a small individual effect, the largest influence on disease risk being carried by the presence of the HLA DRB ${ }^{\star} 1501$ allele in the Caucasian population. We decided to perform a Whole Genome Scan single nucleotide polymorphisms (SNP) analysis on a sample of MSPP cases and controls using the Affymetrix Genchip 500k ${ }^{\circledR}$.

Methods: We have enrolled 197 patients affected by PPMS according to Poser criteria, and 234 age- and sex-matched controls. PPMS cases have been selected as part of a multicentric Italian study. Statistical analysis included a Quality Control step involving the filtering of SNPs based on minor allele frequency, missing genotypes and absence of Hardy Weinberg disequilibrium in controls. A total of 277.866 SNPs $(55.6 \%$ of the total) were retained for statistical analyses. Genotypes were analyzed through univariate processes, combining genotypic and allelic tests.

Results: By using a p-value of 10-4 at either allelic or genotypic Pearson exact test, 20 SNPs have been selected, of whom 16 were located within 10 different gene populated regions including HLA, and 4 into desert regions. Among them, two were located in the intronic region of the DPP6 gene on chromosome 7q36. The DPP6 protein is predominantly expressed in the brain; it binds specific voltage-gated potassium channels and alters their expression and biophysical properties. We are currently in the process of confirming the hits detected in the whole genome scan using high-density genotyping on the genes found associated.

Conclusion: We have performed the first whole genome scan analysis on a PPMS collection. We confirmed the role of HLA in the susceptibility to MS, and we identified novel susceptibility genes which needs to be confirmed in replication samples. Among them, DPP6 has been recently demonstrated to be associated to another neurological disease, the amiotrophic lateral sclerosis, which share some features with PPMS. We propose that the strategy of collecting rare homogeneous variant of a complex disease might represent an alternative effective strategy in finding disease associated genes in complex genetic disease.

This work has been performed in collaboration with the genetic laboratories of Merck-Serono

\section{0}

Magnetic resonance imaging in MOG-induced EAE: relation between leakage of the blood-brain barrier as assessed by gadofluorine $M$ enhancement and infiltration by SPIO-labelled macrophages

G. Ladewig, L. Jestaedt, K.V. Toyka, M. Bendszus, G. Stoll

University of Wurzburg (Wurzburg, DE)

Background: Magnetic resonance imaging (MRI) plays a central role as the most important paraclinical parameter to assess disease activity in multiple sclerosis (MS). Contrast agents such as Gadolinium (Gd)-DTPA indicate blood brain barrier (BBB) disruption thereby characterizing only one feature of inflammation. In experimental autoimmune encephalomyelitis (EAE), a common animal model of MS, we could recently show that the novel contrast agent Gadofluorine M (Gf) (Bayer Schering Pharma AG, Germany) (Misselwitz et al. (2004) Radiology 231:682-688) is much more sensitive in detecting active lesions than Gd-DTPA (Bendszus et al., submitted). In addition, iron-based contrast media such as superparamagnetic iron oxide nanoparticles (SPIO) allow in vivo tracking of infiltrating macrophages (Bendszus \& Stoll (2003) J Neurosci 23:10892-10896).

Objectives: To disclose the spatiotemporal relationship between macrophage infiltration and breakdown of the BBB as revealed by Gf enhancement in chronic relapsing EAE.

Methods: EAE was induced by immunization with myelin oligodendrocyte protein (MOG) in 17 Dark Agouti rats. When the first clinical symptoms appeared SPIO was administered intravenously. Cranial and spinal MRI was performed using T1-w and T2-w high resolution sequences on a 1.5T MR scanner $24 \mathrm{~h}$ later. To allow for direct comparison $\mathrm{Gf}$ was injected into the same animals thereafter and MRI was repeated $24 \mathrm{~h}$ later.

Results: On T1-w MRI, Gf-enhancement indicating leakage of the BBB was consistently found in numerous areas of the spinal cord, brain stem, cortex and optic nerves. Gf-uptake closely corresponded to lesions with macrophage infiltration and albumin extravasation on tissue sections. Notably, SPIO accumulation was only observed in a small portion of the Gf enhancing lesions in 4 of 17 animals. Vice versa, many areas showing signal loss on $\mathrm{T} 2{ }^{*}-\mathrm{w}$ MRI indicative of macrophage entry did not exhibit Gf enhancement.

Conclusion: We used Gf, a more sensitive contrast agent for detection of BBB disruption than Gd-DTPA and could reinforce previous observations that macrophage infiltration and $\mathrm{BBB}$ leakage occur independently. Macrophages can obviously infiltrate the CNS in areas with an intact BBB. This may have implications for future MRI monitoring of disease activity in MS.

This work was supported by the Gemeinnützige Hertie-Stiftung, Frankfurt/Main and a professorship for neuroimaging to M.B. donated by BayerSchering Pharma AG to the University of Wurzburg

\section{1}

Gait and balance parameters as clinical markers of disease progression in early multiple sclerosis

C.L. Martin, M.P. Galea, B.A. Phillips, T.J. Kilpatrick, H. Butzkueven

The University of Melbourne (Victoria, AU); La Trobe University (Victoria, AU)

Objectives: This study investigated changes in the gait and balance performance of newly diagnosed, non-disabled people with multiple sclerosis, compared to change in clinical status measured with the Expanded Disability Status Scale (EDSS) over a 12-month period.

Methods: Thirty-eight participants with relapsing remitting multiple sclerosis and minimal neurological signs without clinical disability (EDSS $0-3.0)$ participated in the study. They attended laboratory-based gait and balance assessments, and clinical neurological examinations at baseline and one year.

Results: During the follow-up period three participants experienced a clinical relapse, while 35 participants remained clinically stable, presenting with minimal change in EDSS scores rated by an experienced neurologist. At the one year assessment, each of the relapsing participants demonstrated a reduction in self-selected speed and stride length and increased double limb support time during gait, and a decreased Functional Reach Test score, compared to baseline. Comparison of the baseline and one-year gait and balance measures for the non-relapsing participants indicated that these individuals also experienced a deterioration in performance, represented by a significant decrease in self-selected speed $(\mathrm{p}<0.001)$ and stride length $(\mathrm{p}<0.001)$ and increased double limb support time during gait $(\mathrm{p}=0.01)$, and a decreased Functional Reach Test score $(\mathrm{p}<0.001)$. The non-relapsing participants also demonstrated reduced ankle motion throughout the gait cycle which corresponded to a decrease in the amplitude of gastrocnemius activity recorded during the stance phase of gait.

Conclusion: These results provide evidence of functional deterioration in people newly diagnosed with multiple sclerosis, even in the absence of acute clinical relapse and change in clinical status measured by the EDSS. The outcomes of this study therefore suggest that gait and balance parameters may be useful clinical markers of disease progression in people with multiple sclerosis in the early stages of the disease.

\section{Oral session 8}

\section{Infection}

\section{2}

Bacterial meningitis is adults: temporal association between initiation of parenteral antibiotics and cerebrospinal fluid sterilisation

B. Menezes, G. Francis, T. Solomon

University Hospital Aintree (Liverpool, UK); Wirral University Teaching Hospital (Wirral, UK); The Walton Centre for Neurology and Neurosurgery (Liverpool, UK)

Introduction: We evaluated cerebrospinal fluid (CSF) sterilisation on initiating parenteral antibiotics in adult meningitis, especially in those with likely bacterial meningitis (BM), and to ascertain causes of delayed lumbar puncture (LP). 
Methods: Records of patients diagnosed as meningitis in a hospital over 3 years and fitting the following case definitions were retrospectively reviewed:-

Adult meningitis: Clinically meningitic patients aged $\geq 15$ years with CSF pleocytosis (> 4leucocytes $/ \mathrm{ml}$ )

Likely bacterial meningitis: Adult meningitis cases fitting Meningitest criteria (Chavanet et al) for likely bacterial aetiology

Culture positive BM: Adult meningitis cases with positive CSF cultures

Results: 92 cases had adult meningitis, 16 being culture positive BM. All culture positive cases were detected when LP was performed $<8$ hours after commencing parenteral antibiotics and none ?8 hours later. Of the 24 cases of likely bacterial meningitis, 8 had sterile CSF. 6 of these had LP $\geq 8$ hours after initiating parenteral antibiotics (statistically significant).

Commonest cause delaying LP was performance of computerised tomography (CT). However, CT was not indicated in $68 \%$. CT was normal in nearly all undergoing inappropriate scanning.

Conclusions: When meningitis is suspected, LP should be performed $<8$ hours after initiating parenteral antibiotics to prevent missed diagnosis due to early CSF sterilisation. Inappropriately requested CT delays LP and is invariably normal.

\section{3}

Neurological features of HIV-seropositive Nigerians: a retrospective analysis of 184 patients

F. Salawu, A. Danburam, U. Kirawa, B. Isa, O. Martins, A. Adamu, B. Gundiri, J. Dikka, P. Thomas, A. Olokoba, S. Bwala

Federal Medical Centre (Yola, NG); University of Ilorin Teaching Hospital (Ilorin, NG); University of Maiduguri (Maiduguri, NG)

The Human Immunodeficiency Virus (HIV) is one of the most challenging health problems of this era and Nigeria is one of the sub-Saharan countries worst hit by the infection.

Objectives: Little is known about the prevalence of neurological complications of human immunodeficiency virus (HIV) infection in northeast Nigeria. A retrospective study of the neurological problems arising in Nigerians with HIV-1, HIV-2 and dual infections in a single defined geographi$\mathrm{cal}$ area in northeast Nigeria was therefore undertaken.

Methods: The case notes of 184 treatment-naïve HIV-seropositive Nigerian patients referred for a neurological opinion from a total known unselected HIV-seropositive population of 527 attending outpatient clinic were reviewed.

Results: HIV-seropositive Nigerians comprising 102 females (mean age 25 years) and 82 males (mean age 31 years) had neurological manifestation, with minor problems frequently encountered early in the course of disease (11 at Centre for Disease Control (CDC) stage II, 47 at CDC stage III), and including seizures related to ingestion of traditional concoctions in seven. Many of the patients had simultaneous occurrence of two or more syndromes; these were included as separate cases. The most frequent neurological problem in those patients in CDC group IV (126 patients) was multi dermatomal herpes zoster including ophthalmic zoster (57 patients), tuberculous meningitis (35 patients), Bell's palsy including facial diplegia (18 patients), vacuolar myelopathy (10 patients), subacute combined degeneration of the cord (10 patients), AIDS dementia complex (8 patients), distal sensory polyneuropathy ( 8 patients), and cerebral toxoplasmosis (7 patients). All patients with a structural lesion had appropriate focal signs on examination. Patients suffering from cerebral toxoplasmosis developed mental impairment significantly more often than patients with central nervous symptoms of other aetiologies did.

Conclusion: The results of our retrospective investigation indicate unselected series of Nigerian HIV-infected patients have an increased risk of developing lesions of the central and peripheral nervous system at all stages of the disease. Herpes zoster was the commonest neurological manifestation among HIV-infected Nigerians. The range of neurological disorders associated with HIV/AIDS could increase further as use of highly active antiretroviral therapy increases,survival lenghtens and there are more Nigerians living with HIV.

\section{4}

Immunoendocrinological factors involved in the heterogeneity of human neurocysticercosis

G. Cárdenas, E. Sciutto, M. Romano, A. Fleury

National Institute of Neurology (Mexico City, MX)

Introduction: Neurocysticercosis (NCC) is one of the most frequent parasitosis of central nervous system. Most of the countries of Asia, Africa and Latin America are endemic and an increase incidence has been reported in industrialized country like United States. Inflammation is one of the most important pathogenic mechanisms. Clear data of sexual dimorphism have been reported in NCC as, in swine cysticercosis and in experimental cysticercosis (Taenia crassiceps). The mechanisms involved in this dimorphism are unknown.

Objective: To determine the relevance of immunoendocrinological status in the pathogeny of neurocysticercosis.

Methods: 52 patients were included before the administration of specific treatment (Albendazole and steroids). Phase of the menstrual cycle was determined in women by interrogatory. Levels of progesterone in women and levels of estradiol (E2) in both men and women were determined in sera by radioimmunoanalysis (RIA). Levels of interleukin (IL) 6 and 10 in sera and cerebrospinal fluid (CSF) were determined by ELISA in all the patients.

Results: Patients with benign NCC showed higher levels of E2 than patients with severe forms of NCC (for men $\mathrm{P}=0.03$ and for women $\mathrm{P}=0.047$ ). In sera of women in postovulatory phase there were correlations between: 1) high levels of E2 and progesterone and low levels of CSF cells $(P=0.007$ and $\mathrm{P}=0.03$, respectively), and 2) high levels of progesterone and low levels of IL 6 in CSF $(\mathrm{P}<0.0001)$.

Conclusions: In our knowledge this is the first study that determines relationships between immunoendocrinological status and pathology in patients with neurocysticercosis. Our results point to a possible protectoranti-inflammatory effect of progesterone and E2. The interrelation of these hormones with the pro-inflammatory IL- 6 could be involved in this effect. These results are very relevant as they could represent the base for the research of new therapies to better control the inflammation in subarachnoidal NCC.

\section{5}

Clinical presentations of neurocysticercosis in children. A series of 139 paediatric cases

L. Rodriguez

National Institute of Neurological Scien (Lima, PE)

Objective: Neurocysticercosis is a common causes of seizures and secondary epilepsy in children in developing countries. It is increasingly diagnosed and considered endemic in most of the world. We aimed to assess the clinical profile of presentation of neurocysticercosis in children.

Methods: We systematically assessed the clinical presentation in 139 children with a probable or definitive diagnosis of Neurocysticercosis who were admitted to a neurological ward in Lima, Peru, between years 2000 to 2004

Results: There was a mean of 27 new cases per year, most of which belonged to the 11 to 15 years age group. There were similar proportions of male and female patients ( $51 \%$ versus $49 \%$ ). Sixty-three percent of the cases presented with a single parenchymal brain enhancing lesion ("cysticercal granuloma"), and only $44 \%$ of these had a positive serology on enzymelinked immunoelectro transfer blot (EITB, Western Blot). Eleven (8\%) patients presented with massive brain infestations, and another $11(8 \%)$ with calcified lesions only.

Conclusions: Cysticercosis is frequent in children with neurological symptoms in Peru, and it presents mostly as a single brain enhancing nodule associated to seizures.

\section{6}

Late onset neuropathy after treatment of leprosy

M. de Freitas, O.J.M. Nascimento, F. Cardoso, V. dos Santos, M. Hahn

University Federal Fluminense (Niterói-RJ, BR)

Leprosy is one of the most common peripheral nerve diseases in the world. It usually affects the skin and the nerves. Although the prevalence of the disease is decreasing, leprosy continues to be a major cause of infective neuropathy in tropical and subtropical countries. Sometimes after the treatment with the three drugs recommended by WHO, some patients develops peripheral neuropathy that appears to be of autoimmune origin.

We described 14 patients ( 8 men and 6 women) with different types of peripheral neuropathy that appears during the treatment and others different times after it. The middle age was 54 years. We perform electrophysiological studies and nerve biopsy in all of them.

Paresthesias was the most important complaint, present in ten patients (71\%). Other symptoms included: pain $(6=42 \%)$ and weakness $(3=21 \%)$. All they had superficial anesthesia or hypoesthesia (thermal and pain). Six had polyneuropathy, three mononeuropathy and five multiple mononeuropathy. The nerve biopsy showed in all cases inflammatory infiltrates mainly perivascular, fibrosis of endoneurium, perineurium and epineurium, total or partial loss of nerve fibres, without bacilli. The majority of the patients were treated with corticosteroids having relief of their symptoms.

We concluded that late onset peripheral neuropathy during and after leprosy therapy is common in our country. It is probably of immune mecha- 
nism, with antibody against an unknown antigen of the bacilli. Although we had few cases of this kind of neuropathy, some of them may become better using corticosteroids.

\section{7}

Neurocysticercosis: review of a hospital series of 63 patients

N.M. Inácio, R. Simões, V. Silva, A.N. Pinto, E. Parreira, A.V. Salgado Fernando Fonseca Hospital (Amadora, PT); IPOFG (Lisbon, PT)

Objectives: Neurocysticercosis (NCC) is considered to be the most common parasitic disease of the central nervous system (CNS) and is an emerging infection in developed countries. Its clinical manifestations, therapy and prognosis depend on many factors, mainly the number, location and the stage of the lesions. Our goal was to characterize the demographic aspects of the population with the diagnosis of NCC, the clinical manifestations, correlation between clinical and imagiological aspects, and response to treatment.

Methods: A review of the clinical charts of all patients admitted to our hospital with a diagnosis of NCC, between January 1996 and February 2007, was performed by independent observers. For the statistical analysis, the Kruskal Wallis test was used. P values of $<0.05$ were considered to be statistically significant.

Results: 63 patients were included with the diagnosis of NCC (mean age of $35,8 \pm 14.8$ years, $57 \%$ males), the majority of which was born in Cape Verde $(71 \%)$. Epilepsy was the most frequent clinical manifestation (73\%). Intracranial hypertension was present in $6 \%$ of patients, with isolated headache and focal signs having the same frequency $(5 \%)$. Intraparenchymal cysts were present in $83 \%$ and in the other $17 \%$ there was also involvement of CSF containing spaces. In the parenchymal NCC, the most common clinical manifestation was epilepsy (79\%), followed by focal signs and headache (6\% each). After treatment with cysticidal drugs and corticoids there was clinical resolution in $71 \%$ of patients. Imagiologic resolution was higher in patients with a single cyst (complete in $55 \%$ and partial in $36 \%$ ) compared with patients who presented two or more lesions (complete in $29 \%$ and partial in $53 \%$ ). In patients who also presented extraparenchymal form of NCC, the most common clinical manifestation was also epilepsy (present in $64 \%$ of cases), the remaining of the patients presenting signs of intracranial hypertension (36\%). After treatment these patients had a clinical resolution in $71 \%$ of cases and none of the patients presented a complete imagiologic resolution during follow up.

Conclusions: In our series we can conclude that NCC affects mainly young adults, mainly from endemic areas, reflecting the demographic characteristics of part of the population who attends our hospital. Epilepsy is the most common clinical manifestation and imagiologic resolution was mainly affected by the number of lesions present at the time of diagnosis.

\section{Oral session 9}

\section{Neurorehabilitation}

\section{8}

Motor-evoked potential in presymptomatic spondylotic cervical cord compression

I. Shaheen, W. Saadany, G. Abd El Hady

Alexandria University (Alexandria, EG)

Objective: Is to investigate whether electrophysiological parameters of Motor Evoked potential (MEP) could detect clinically asymptomatic (preclinical) spondylotic cervical cord compression before progression to symptomatic myelopathy and to compare between MEP and Somatosensory Evoked Potential (SEP) in this regard.

Methods: Twenty patients with clinical and radiological features of cervical spondylsis without clinical or radiological evidence of cervical myelopathy were included in the study. Fifteen healthy subjects, represented a control group. The following procedures were carried out bilaterally for patients and unilaterally for control subjects. Percutaneous transcranial magnetic stimulation (TMS) to elicit MEP; to evaluate the neurofunctional integrity of the central motor pathway. SEP of both median and posterior tibial nerves was recorded to evaluate the neurofunctional integrity of the central sensory pathway.

Results: The mean age of patients was $52.7 \pm 6.2$ years; the majority of them were men $(60 \%)$. The mean duration of illness was $3.36 \pm 1.5$ years.
There were 6 patients ( $30 \%$ ) with mJOA score of 17 and 14 patients $(70 \%)$ with $\mathrm{mJOA}$ score of 18 . The mean score of mJOA scale was $17.73 \pm 0.45$. The mean age of control subjects was $48.8 \pm 4.5$ years; the majority of them were men $(60 \%)$. Both plain X-ray and MRI revealed cervical spondylotic changes from $\mathrm{C} 4$ to $\mathrm{C} 7$ in the form of intervertebral disc degeneration and osteophytosis of vertebral bodies. Indentations of the dural sac on MRI were observed at C5/6, C6/7 and C4/ 5 intervertebral levels in order of incidence. However the cord was reportedly normal. The C4-5 intervertebral level was involved in 3 patients, who were 60 year old and older. Central motor conduction time (CMCT) was prolonged in 6 patients. Three of those patients had bilaterally prolonged CMCT; they were the same elderly patients with C4-5 involvement. In the other 3 patients CMCT was unilaterally prolonged. MEP amplitude didn't show significant abnormality among patients. There was no significant difference in patients' SEP latencies or amplitudes when compared to control subjects.

Conclusion: MEP provides an objective and quantitative approach to assess the motor functional integrity of the corticospinal pathways. Measurement of CMCT could serve as a detector of subclinical spondylotic cervical cord involvement before progression to symptomatic mylopathy even earlier than SEP can do.

\section{9}

The comparison of two different rehabilitation programmes in patients with unilateral peripheral vestibulopathy

B. Dönmez, G. Akdal

Dokuz Eylül University (Izmir, TR)

We aimed to investigate the effects of two different balance training programs in patients with unilateral peripheral vestibulopathy (UPV). Fourteen subjects were enrolled into the study. None of the subjects in the study had Meniere's disease, bening paroxysmal positional vertigo, acute vestibular neuronitis or labyrinthitis. None of the subjects were on vestibular suppesant medication. The mean age of subjects was 55,93 $\pm 16,33$ years and there were 6 males, 8 females in the groups. Clinical and objective balance abilities were assessed. Berg Balance Scale, subjective Dizziness Handicap Inventory, Dynamic Gait Index and static posturography for objective balance functions were assessed before and after the treatment sessions. Modified clinical test of sensory interaction on balance limits of stability (LOS), walk across, tandem walk were the parameters assessed by the static posturography. After completing the pretest assessment the subjects were assigned in a ranked order, first subject to static posturography group and second subject to home program group. The subjects were treated with two different rehabilitation techniques by static posturography training or supervised home program throughout 6 weeks. Supervised home treatment group received exercises to improve gaze stability, postural stability and habituation. In static posturography group, the physiotherapist provided the treatment.

For statistical analysis; Mann-/Whitney U-test was used to compare differences between pre- and post-treatment balance parameters. The Wilcoxon paired rank test was used to compare differences among balance outcomes within each group. The results were accepted as statistically significant at $\mathrm{P}<0.05$. Both groups had improved their balance performances significantly. In posturography group, reaction time and max excursion in LOS parameters were found significantly different. In conclusion; vestibular rehabilitation improves balance in UPV patients. Static posturography rehabilitation program significantly improves LOS parameters which reflect the balance functions in subjects' daily activities.

\section{0}

Usefulness of oropharyngoesophageal scintigraphy in evaluation of tracheobronchial aspiration in neurological patients

F. Pesella, M. de Scisciolo, E. Biggi, P. Bongioanni, A. Nacci, B. Dell'Anno, $R$. Cantini, R. Raschillà, M. Grosso, B. Fattori, G. Mariani

Regional Center of Nuclear Medicine (Pisa, IT); Neurorehabilitation Unit (Pisa, IT); ENT Unit (Pisa, IT)

Objectives: Dysphagia and bolus aspiration are two of the most frequent and invalidating symptoms of various neurological diseases. Swallowing diseases are the most important cause of tracheobronchial inhalation, which causes pneumonia ab-ingestis, with a mortality rate ranging from 20 to $62 \%$. Oropharyngoesophageal scintigraphy (OPES) permits a functional and semi-quantitative study of the various stages of swallowing, reveals and estimates bolus aspiration presence.

In the present study, we used OPES to achieve a dynamic study of deglutition in neurological patients (Amyotrophic Lateral Sclerosis, Parkinson's disease, ictus cerebri, etc.) to diagnose and quantify bolus aspiration.

Methods: We enroled 21 neurological patients (13 females and 8 males; mean age $64.3 \pm 11.4 \mathrm{SD}$ ) with dysphagia. All patients underwent OPES with 
99mTc-nanocolloid using both liquid and semi-solid bolus. Parameters analysed were: Oral, Pharyngeal and Esophageal Transit Time, Oro-Pharyngeal Retention Index, Esophageal Retention Index, Esophageal Emptying Rate and Aspiration Rate (AR). AR was calculated by the following formula: $\mathrm{AR}=(\mathrm{IA} / \mathrm{AT} 0-\mathrm{AT} 1) \mathrm{x}$ 100, where IA stands for Inhaled Activity, while AT0 and AT1 stand for Oral Activity before and after radioactive bolus swallowing.

Results: OPES revealed tracheobronchial inhalation in 15 patients: 4 patients showed laryngeal aspiration with an AR mean percentage of $6 \%, 6 \mathrm{pa}-$ tients showed tracheal aspiration (AR mean percentage: $15 \%$ ) and the last 5 patients bilaterally bronchial-pulmonary aspiration (AR mean percentage: $45 \%$ ). In one patient with bilaterally massive bronchial-pulmonary aspiration caused by attynical damage of various cranial nerves, OPES lead surgical treatment to functionally laryngectomy.

Conclusions: OPES allows an objective and quantitative evaluation of inhaled bolus into tracheobronchial tract.

It is easy to perform, well tolerable, repeatable and inexpensive.

Although this technique has a low resolution to define anatomic structure, OPES is useful in determining the exact percentage of the inhaled bolus into tracheobronchial branch in neurological patients with dysphagia and represents an useful and accurate tool to monitoring the response to medical and/or surgery therapy.

\section{1}

\section{Overall quality of life in chronic locked-in syndrome}

M-A. Bruno, J. Berheim, F. Pellas, D. Ledoux, C. Schnakers, A. Vanhaudenhuyse, S. Goldman, G. Moonen, S. Laureys

Coma Science Group (Liege, BE); Caremeau Hospital (Nîmes, FR); Erasme Hospital (Brussels, BE); University of Liège (Liege, BE)

Objectives: The locked-in syndrome (LIS) is a characterized by quadriplegia and anarthria. Classically, communication is only possible by means of vertical eye movements and/or blinking. LIS is most often caused by a ventropontine vascular lesion. Healthy individuals and medical professionals sometimes assume that the quality of life of a LIS patient is so poor that it is not worth living. In collaboration with the French Association for Locked-In Syndrome (ALIS- http://alis-asso.fr/), we here present data on chronic LIS patients' self-rated overall wellbeing.

Method: 97 patients with LIS were asked to respond to a questionnaire dealing with diagnosis, quality of life and end-of-life issues. Quality of life was assessed using the Anamnestic Comparative Self Assessment scale (Bernheim 1999)

Results: Responses were obtained in 65 LIS patients. Mean duration of LIS: $9 \pm 5$ years. In $41 \%$ of the cases, it was a relative of the LIS patient and not the treating physician who first realized that the patient was conscious and could communicate via eye movements. The vast majority (92\%) of LIS patients never or only occasionally had suicidal thoughts and more than half $(54 \%)$ would like resuscitation to be tempted in case of cardiac arrest. More than half of the patients $(55 \%)$ never considered or discussed euthanasia and a small but existing minority $(5 \%)$ wanted euthanasia at the moment of the study. Quality of life in LIS was not significantly different from healthy matched controls $(1,02 \pm 3,05$ and $1,69 \pm 1,82$ respectively).

Conclusion: Our results stress the need for physicians who are confronted to acute LIS to recognize this infrequent syndrome as early as possible. Health care workers need a better understanding of the long-term outcome of LIS. Opposite to the beliefs of many physicians, LIS patients self-report a meaningful quality of life and the demand of euthanasia is existing but uncommon. Ethical guidelines emphasize LIS patients' right to autonomy and to demonstrate their ability to exercise it, including taking end-of-life decisions. Clinicians should realize that quality of life often equates with social rather than physical capacity. It's important to emphasize that only the medically stabilized, informed LIS patient is able to accept or to refuse life sustaining treatment. LIS patients should not be denied the right to die but also they should not be denied the right to live with the best possible care.
0112

Outcome prediction in brain-injured patients

D. Ledoux, M-A. Bruno, C. Schnakers, J. Giacino, M. Ventura, L. Vanopdenbosch, E. Peeters, E. Lannoo, T. Willemart, S. Laureys

Coma Science Group (Liege, BE); JFK Johnson Rehabilitation Institute (New Jersey, US); Centre de Traumatologie et de Réadaptation (CTR) (Brussels, $\mathrm{BE}$ ); Department of Neurology AZ (Brugge, BE); Ziekenhuis Inkendaal, Koninklijke Instelling (Vlezenbeek, BE); Centrum Locomot. \& Neurologische Revalidatie UZ (Ghent, BE); Centre Neurologique William Lennox (Ottignies, $\mathrm{BE}$ )

Objectives: Progress of intensive care has increased the number of patients surviving severe acute brain damage. These patients classically progress through different stages before fully or partly recovering consciousness. Following severe acute brain damage, most of these patients recover from their coma to a vegetative state (VS; ie wakefulness without awareness) within four weeks after the injury. VS patients who recover, typically transit through minimally conscious state (MCS. There is however no predictive model of recovery from the VS and the MCS. A better understanding of patients' outcome would help in decisions about care, rehabilitation or end-of-life decisions. In the present study, we tried to establish reliable guidelines to predict outcome in these patients.

Method: The Belgian Federal Public Service Health, Food Chain Safety and Environment build a web-based electronic database allowing standardized online recording which include demographic information, injury acute care history and longitudinal multicentric following of VS and MCS patients. In this database, data were available for 200 patients. 116 VS patients $(52$ traumatic and 64 non-traumatic) and 84 MCS patients (35 traumatic and 49 non traumatic). These patients were evaluated at $1,3,6$ et 12 months post injury with the Coma Recovery scale (Giacino, Kalmar et al. 2004.

Result: Traumatic patients had a better outcome than nontraumatic: $72 \%$ of traumatic versus $24 \%$ nontraumatic patients recovered consciousness. After a traumatic or nontraumatic brain injury, patients who were in MCS one month after insult were more likely than patients in VS to recover sustained consciousness, both. Among traumatic brain injured patients, $48 \%$ of patients MCS at one month recovered versus $23 \%$ of VS patients $(p=)$. For patients who suffered from nontraumatic brain damage, $26 \%$ of patients MCS one month after injury recovered versus $1 \%$ of VS patients.

Conclusion: These results confirm that patients with traumatic etiology have better prognosis than patients with nontraumatic causes. Moreover, our study highlights that outcome is better for MCS than for VS patients.

Giacino, J. T., K. Kalmar, et al. (2004). “The JFK Coma Recovery Scale-Revised: measurement characteristics and diagnostic utility." Arch Phys Med Rehabil 85(12):2020-2029.

\section{3}

Blinking to visual threat does not herald consciousness

A. Vanhaudenhuyse, J. Giacino, C. Schnakers, K. Kalmar, M-A. Bruno, O. Gosseries, S. Brédart, G. Moonen, S. Laureys

Coma Science Group (Liege, BE); JFK Johnson Rehabilitation Institute (New Jersey, US); University of Liège (Liege, BE)

Objectives: In vegetative state patients, we are limited to observe motor responses and to interpret these behaviors as signs of consciousness or not. The blink response to threat is a usual bedside method for testing visual processing. At present, there is no consensus on whether blinking to visual threat heralds consciousness (i.e., is incompatible with the diagnosis of VS) or is a purely automatic reflex. The aim of our study was to test how frequent blinking to visual threat is observed in vegetative patients and to evaluate the prognostic value of blinking to threat.

Method: We studied 91 VS patients who were evaluated using the Coma Recovery Scale Revised (CRS-R, Giacino et al., 2004) (60 in New Jersey and 31 in Liège; mean age was $43 \pm 20$ years SD). Etiology was traumatic in 41 and non-traumatic in 50 patients (i.e., anoxic encephalopathy $(n=27)$, ischemic or hemorrhagic stroke $(n=12)$, metabolic encephalopathy $(n=8)$, and tumor $(n=3)$ ). Nineteen patients were studied in the acute (mean interval $15 \pm 6$ days post-insult), and 72 in the chronic setting ( $3 \pm 5$ months post-insult). Blink to visual threat was defined as a blink following presentation of visual threat on at least 2 trials with either eye (as described in the CRS-R). Outcome at one year for traumatic and three months for non-traumatic cases was assessed using the Glasgow Outcome Scale (GOS)

Results: Forty-six out of the 91vegetative patients showed blink to visual threat: 9 died ( 2 traumatic, 7 non-traumatic), 20 remained in VS ( 8 traumatic, 12 non-traumatic) and 17 emerged from their VS (10 traumatic, 7 non-traumatic). Out of the other 45 patients, 6 died ( 1 traumatic, 5 nontraumatic), 28 remains in VS (13 traumatic, 15 non-traumatic) and 11 emerged from their VS (7 traumatic, 4 non-traumatic).

Conclusion: Blinking to visual threat is not at all exceptional in vegeta- 
tive patients meeting all clinical signs of VS. Moreover, its presence does not foretell consciousness nor recovery of consciousness in severely brain-injured patients.

\section{Oral session 10}

\section{Cerebrovascular disorders 2}

\section{4}

Non-stroke patients admitted in acute stroke unit

E. Giralt, A. Rodriguez, A. Ois, R.M. Vivanco, C. Pont, G. Cucurella, M. Sepulveda, J. Roquer

Hospital Del Mar (Barcelona, ES)

Objectives: Clinical studies have shown that acute stroke unit (ASU) care is effective in reducing morbidity and mortality compared to conventional unit in ischemic stroke. Early evaluation of patients and more availability of complementary tests in ASU provide more accuracy in the diagnosis of stroke. Many non-cerebrovascular diseases presents with a strokelike clinical picture. Some hospital-based studies reported high proportion of stroke mimics (5-29\%). The assessment of the patient in ASU allows rapid diagnosis and treatment. The aim of our study is to evaluate the patients who are admitted in a ASU but do not suffer from stroke.

Methods: We prospectively evaluated patients admitted with suspected ischemic stroke in our hospital and allocated to the ASU in a 17-month period (June 2005-November 2007). Diagnostic assessment procedures and medical treatments for acute stroke in ASU are standardized, with dedicated stroke staff who works exclusively in the care of stroke patients. Neurovascular study includes blood sample, cardiac monitoring, CT scan or MRI and carotid and transcranial ultrasonographic study. Other studies were performed individually. After a complete study, final diagnosis was non-stroke (NSP) in patients with initial suspicious neurological symptoms but negative study. We recorded demographic data, vascular risk factors, code stroke activation (extrahospitalary/hospitalary), clinical data, severity (NIHSS) and length of stay, and compared with stroke patients (SP).

Results: 539 patients were evaluated. 38 patients were NSP $(7.1 \%) .65 .8 \%$ were men. Mean age was $68.1 \pm 14.2$ vs $74.2 \pm 12.7(\mathrm{p}=0.005)$. Extrahospitalary code stroke was activated only in $8 \%$ patients and hospitalary code stroke in $21 \%$, without differences with SP. Median initial NIHSS was lower in NSP $(2(\mathrm{q} 1, \mathrm{q} 3: 1,3)$ vs $5(\mathrm{q} 1, \mathrm{q} 3: 2,12) \mathrm{P}=0.000)$ with mild brainstem symptoms as a main clinical feature (isolated dysarthria or facial palsy, diplopia and vertigo). 3 patients received rtPA ( $4 \%$ of total thrombolytic therapies). The most common non-stroke diagnoses were seizure $(15.8 \%)$, toxic/metabolic $(13.2 \%)$, peripheral vertigo $(10.5 \%)$, cranial nerve palsies $(10.5 \%)$, and medically unexplained symptoms $(8 \%)$. No acute lesions were identified in CT scan or MRI.

Conclusion: ASU provides specialized diagnosis in stroke patients, but in our setting $7.1 \%$ of patients were non-stroke. These patients are younger, with mild clinical symptoms and negative neuroimaging studies compared with stroke patients.

\section{5}

Anterior versus posterior circulation strokes: comparison of clinical, radiological, and outcome characteristics

G.M. De Marchis, N. Renz, A. Kohler, M. Arnold, J. Gralla, G. Schroth, H.P. Mattle, K. Nedeltchev

University of Berne (Berne, $\mathrm{CH}$ )

Objective: Ischemic stroke in the posterior circulation (PS) has received less attention than stroke in the anterior circulation. It is unclear whether demographic, clinical, radiological factors and treatments exert different effects on the clinical outcome in PS and in AS. We set out to compare demographic characteristics, prevalence of vascular risk factors, stroke etiology, imaging data, acute treatment and clinical outcome of AS and PS.

Subjects and methods: From 10/2003 to 07/2006, 312 patients with AS and 93 patients with PS were admitted to our university-based stroke center. All patients underwent a complete stroke workup including clinical examination, blood laboratory tests, brain CT and/or MRI, neurovascular imaging (CT- or MR-angiography, neurosonography). Clinical outcome was assessed at 3 months using the modified Rankin scale (mRS).

Results: Patients with AS and PS did not differ in terms of demographic characteristics, prevalence of vascular risk factors, and stroke etiology. Newly diagnosed atrial fibrillation was more frequent in AS (17.2\%) than in PS $(2.2 \%, \mathrm{P}<0.01)$. The median National Institutes of Health Stroke Scale (NIHSS) score was 8 in both AS and PS. Patients with AS presented more often abnormalities on diffusion weighted imaging $(97.1 \%$ vs. $86.3 \%$, $\mathrm{p}<0.01)$, a diffusion/perfusion-mismatch $(55.2 \%$ vs. $17.2 \%, \mathrm{p}<0.001)$, a vessel's occlusion $(53.2 \%$ vs. $35.8 \%, \mathrm{p}<0.01)$, and early ischemic CT signs $(62.8 \%$ VS $34.3 \%, \mathrm{p}<0.01)$ than patients with PS. The proportion of patients with favourable clinical outcome (mRS 0 to 2) was similar in AS and PS (60\% vs. $72 \%$ ). In the multivariate analysis of patients with AS, advanced age and NIHSS score were independent predictors of unfavourable outcome (OR $1.04,95 \% \mathrm{CI}, 1.0-1.1, \mathrm{p}<0.001$ and $\mathrm{OR} 1.2,95 \% \mathrm{CI}, 1.15-1.3, \mathrm{p}<0.001)$. In PS, NIHSS score, coronary heart disease, atrial fibrillation, and vessel occlusion as seen on MRA increased the odds of unfavourable outcome independently (OR 1.16, $95 \%$ CI $1.1-1.22$, p $<0.001$; OR 9.6, $95 \%$ CI, 2.2-41.2, p $<0.01$, and OR $8.5,95 \%$ CI, $1.6-43.5, \mathrm{p}<0.01)$.

Conclusion: Patients with AS and PS did not differ in terms of demographic characteristics, vascular risk factors, etiology, stroke severity and clinical outcome. AS presented more often with abnormal imaging findings. Stroke severity predicted outcome in both AS and PS, whilst coronary heart disease, atrial fibrillation and vessel occlusion predicted unfavourable outcome in PS but not in AS.

0116

Stent-assisted coil embolisation of wide neck intracranial aneurysms: midterm follow-up

J. Sedat, Y. Chau Huu, M.H. Mahagne, L. Suissa, S. Lachaud, J. Vargas, J. Szapiro

Hopital St Roch (Nice, FR)

Objective: To evaluate the midterm results of intracranial stent-assisted coil embolization in the treatment of wide-necked cerebral aneurysms.

Methods: Between 2004 and 2007,41 patients with 43 cerebral aneurysms were treated with stenting and coiling. Every lesion had a wide neck (more than $4 \mathrm{~mm}$ or with a dome-neck ratio $<1.5$ ). 6 aneurysms were treated with an intracranial balloon-expandable stent (INX; Medtronic) and 37 with a flexible self-expanding nitinol stent (Neuroform stent Boston Scientific). 31 aneurysms were unruptured aneurysms; 5 were recanalized aneurysms, 7 were acutely ruptured. 37 aneurysms were located in the anterior and 6 in the posterior circulation. Angiographic follow up was performed 6 and 18 months following the endovascular procedures. The patients were evaluated clinically at six months, 18 months, and then annually after treatment, with the Glasgow Outcome Score (GOS), and the Modified Rankin Score (mRS).

Results: Successful deployment of stent was obtained in 42 of 43 . In one patient the deployment of an intracranial balloon-expandable stent failed, but a new procedure performed 6 months later with a self-expanding stent succeed. Thrombo-embolic events during the procedure were observed in 5 patients. In three of them, in situ thrombolysis was carried out during the procedure and was effective. 2 symptomatic periprocedural thromboembolic complications were observed: in one patient the neurological deficit was transient; the other presented a serious and permanent deficit. One patient presented a transitory deficit due to osmotic disruption of the bloodbrain barrier after procedure using non-ionic contrast material.

Of the 43 embolized aneurysms, angiography showed $22(51,2 \%)$ aneurysm occlusions, $11(25.5 \%)$ neck remnant, $7(16.3 \%)$ residual aneurysms, and $3(7 \%)$ recanalized aneurysms for which a new endovascular coiling was performed. For the 7 patients with ruptured aneurysm, 3 were classified GOS 1,2 were classified GOS 2, and 2 patients died during hospitalization.

For the 36 patients with unruptured aneurysm, clinical follow-up one year after treatment: one patient was mRS 4, one was mRS 2, and $34(94,4 \%)$ were mRS 0.

Conclusion: Stent-assisted coil embolization is a safe and effective technique in the treatment of wide-necked cerebral aneurysms. Further studies are needed to evaluate the long-term stability of aneurysmal occlusion and tolerance to the stent

0117

Course of stroke due to paroxysmal atrial fibrillation is different from that due to permanent atrial fibrillation

J. Staszewski, B. Brodacki, K. Tomczykiewicz, J. Kotowicz, A. Stepien Military Medical Institute (Warsaw, PL)

Permanent (ptAF) and paroxysmal (pxAF) atrial fibrillation carry similar risk of ischemic stroke (IS), however the impact of pxAF on stroke prognosis has not been determined yet. Our aim was to compare the clinical picture of IS due to ptAF and pxAF. Prospective, single centre study in patients with 
AF and acute IS with 6-month follow-up was conducted. The severity of stroke was assessed in mRS (non-dependence: $\mathrm{mRS} \leq 2$ points, dependence $\mathrm{mRS} \geq 3<=5$ points). The course of IS was categorized as favorable ( $\mathrm{mRS} \leq 2$ points) or unfavorable ( $\mathrm{mRS} \geq 3$ points). Stroke type was categorized as cardioembolic or noncardioembolic using a clinical classification scheme. Of 838 consecutive patients admitted due to IS within 24 months, 178 patients ( $21 \%)$ were included: 70 (39\%) with pxAF, 108 (61\%) with ptAF. The frequency of vascular risk factors and concomitant antithrombotic treatment were similar in study groups. Comparing with ptAF patients with pxAF more often presented with subcortical, lacunar strokes $(21 \%$ vs $8 \%$, $\mathrm{p}=0.01$ ), and were less often dependent at admission ( $50 \%$ vs $81 \%$, $\mathrm{p}<0.001)$, discharge $(9 \%$ vs $53 \%, \mathrm{p}<0.001)$ and after 6 months $(29 \%$ vs $52 \%, \mathrm{p}<0.001)$. Strokes in pxAF were usually categorized as noncardioembolic $(35 \%$ vs $18 \%, p=0.01)$. The multivariate analysis revealed that ptAF was an important risk factor for unfavorable short- $(\mathrm{OR} 3,73 ; \mathrm{p}=0.005)$ and long-term outcome (OR 2,05; $p=0.01)$ of IS. The presented results suggest different course of stroke due to pxAF and ptAF. Patients with pxAF had more favorable short- and long-term outcome of stroke. That can possibly be explained by more common noncardioembolic strokes in that population.

\section{8}

The pattern of anterior cerebral artery infarction: a study of 48 patients A. Pfundstein, S. Lee, H. Sorgenfrei, P. Berlit

Alfried Krupp Hospital (Essen, DE)

Objectives: In order to evaluate the pattern of anterior cerebral artery (ACA) territory infarction, we studied 48 consecutive patients who were admitted to our stroke unit over a 5 year period (2003 through 2007).

Methods: We performed initial CCT and ultrasound studies of the craniocerebral vessels in all patients. Magnetic resonance imaging (MRI) studies including diffusion weighted imaging (DWI) and time of flight (TOF) magnetic resonance angiography (MRA) was done within 1 week. The pathogenesis of the infarction was evaluated using transesophageal echocardiography, $24 \mathrm{~h}-\mathrm{ECG}$ and laboratory tests for coagulation disorders.

Results: With a total number of 48 , ACA infarction represented $2.1 \%$ of all 2325 patients with ischemic stroke in this 5 year period. Isolated ACA infarctions occurred in 14 circumstances. There was a clear predominance of female gender (women 33, men 15), especially in isolated ACA infarctions (women 13, men 1). The clinical spectrum of right sided infarctions $(n=30)$ included acute confusional state, hemiparesis and hemineglect; left sided infarctions $(n=15)$ presented with mutism, hemiparesis with lower limb predominance and transcortical aphasia. Akinetic mutism was the leading symptom of bilateral ACA infarction $(n=3)$. A combination of ACA and MCA infarction was seen most frequently due to cardiogenic embolism, while isolated ACA infarctions occurred more often in large artery disease. We found no gender differences regarding the etiology. In women, anomalies of the circulus arteriosus Willisii like A1-hypoplasia or -aplasia were more frequent.

Conclusions: We found an absolute predominance of female gender in ACA infarctions, especially in isolated ACA infarctions. Right sided infarctions were more common than left sided ACA infarctions. While there were no differences in the pathogenesis, anatomic variants of the Willis circle may play a causative role.

\section{9}

Cerebral ultrasound perfusion imaging: comparability to perfusion-MRI and introducing a new mismatch model

W. Marouf, A. Hetzel, W.D. Niesen

Hanse-Klinikum Stralsund (Stralsund, DE); University Hospital of Freiburg (Freiburg, DE)

Background: Cerebral Ultrasound Perfusion Imaging (CUPI)is a new, relatively simple and affordable bedside technique for the illustration of the regional cerebral perfusion. However, this method is not part of the diagnostic battery of acute stroke patients. Our aim was to evaluate its comparability to PWI-MRI in the settings of acute stroke in thrombolysis time window and the diagnostic and prognostic value of the sonographic mismatch concept.

Methods: Thirty seven patients ( 13 women, 24 men) presented to our stroke unit in the first 6 hours after developing acute focal neurological deficits referring to the middle cerebral artery (MCA) were included in the study. CPUI was done in the acute settings as part of the basic neurosonographic evaluation. Phase inversion harmonic techniauqe was used. Patients with insufficient bone window were excluded. The acute MRI stroke-protocol was performed directly after the initial clinical and neurosonographic assessment. PWI-MRI was performed by 24 Patients. A follow-up neurosonographic assessment, in order to detect the potential recanalizaton, was performed within 3-6 hours. The clinical outcome was evaluated by modified rankin scale at discharge.

By analyzing the bolus kinetics the hypoperfused areas (measured in $\mathrm{cm} 2$ ) were evaluated by constructing a summation-image of the consequentially acquired images from each loop up to peak intensity image.

The areas of DWI and PWI (measured in $\mathrm{cm} 2$ ) were measured at the corresponding thalamic plane. The mismatch between the hypoperfused (PWI or CPUI) and the infarcted areas (DWI) was determined.

Results: The areas of perfusion deficits according to the CPUI correlated significantly with areas measured according to the PWI-MRI (spearman correlation coefficient $(r=0,85, \mathrm{P}<0.01)$. The new Sonographic mismatch model could detect the MR-mismatch with a specificity of $80 \%$ and sensitivity of $94 \%$. The patients were divided into two groups. Group A included patients with a sonographic mismatch with an early recanalization. Group B included patients who did not demonstrate a mismatch as well as patients without early recanalization. $\mathrm{mRS}$ at discharge of group $\mathrm{A}$ and group $\mathrm{B}$ were 1,7 and 3,7 respectively $(\mathrm{P}<0,05)$.

Conclusion: CPUI showed an acceptable correlation with PWI-MRI. The new sonographic mismatch has a a prognostic value. This method, using the contrast bolus kinetics has anyway its own limitations. With each study the regional cerebral perfusion at only one plane could be depicted.

\section{0}

Serum magnesium and early stroke severity: influence on stroke syndromes P. Milia, K. Nardi, K. Lees, M. Paciaroni, F. Palmerini, G. Agnelli University of Perugia (Perugia, IT); University of Glasgow (Glasgow, UK)

Background: Magnesium revealed to be benefit in animal models of stroke and recently the IMAGES trial revealed significant benefit of intravenous magnesium in patients with non cortical stroke particularly in lacunars clinical syndromes (LACS)

Purpose and methods: We aimed to test whether serum magnesium collected at admission on acute stroke patients can play a role on early neurological clinical pattern and if there is a difference between the various clinical syndromes and/or subtypes of stroke

We studied patients consecutively admitted to our Stroke Unit. Serum Magnesium was collected at admission: values were divided in four subgroups $(\mathrm{Mg}<1.6, \mathrm{Mg} 1.7, \mathrm{Mg} 1.8-1.9, \mathrm{Mg}>2)$. Severity of stroke was assessed according to NIHSS, respectively at admission (NIHSSO) and after 72 hours (NIHSS72). Outcome was valuated using mRS.

Results: One thousand consecutive acute stroke patients were studied (832 Ischaemics, mean age $75 \pm 11,168$ Haemorrhagics mean age $73 \pm 13$ ). Among the overall patients the mean NIHSS score difference between the four $\mathrm{Mg}$ groups showed a worst clinical pattern in patient with $\mathrm{Mg}<1.6$ at NIHSSO (10 $\pm 7 \mathrm{p} 0.01)$. Focusing on ischemic stroke the same results was obtained for $\mathrm{Mg}<1.6$ group at NIHSSO ( $10 \pm 7 \mathrm{p} 0.02$ ) showing also a less probability to be alive at 72 hours ( $p 0.02)$. There were no differences in hemorrhagic strokes. The stroke syndromes had similar frequencies among the four subgroups although LACS showed a worst clinical pattern at NIHSS72 $(6 \pm 5, \mathrm{p} 0.01)$ within the $\mathrm{Mg}<1.6$ group. After binomial logistic regression analysis Lacs group showed a more probability of bad outcome $(\mathrm{mRS}>2)$ having a Mg 1.7 at admission (p 0.03 CI 1.07-7.3).

Conclusions: Low serum magnesium at admission seems to be related with bad clinical presentation in ischaemic stroke patients rather than haemorrhagics. Particularly LACS syndrome seems to be more sensible to low serum magnesium, thus justifying the recent results of clinical trials and underlying the opportunity of MG treatment in LACS.

\section{Oral session 11}

\section{Pain}

\section{1}

Spinal cord stimulation versus conventional medical management for failed back surgery syndrome: long-term results from the PROCESS study S. Eldabe, R.S. Taylor, S. Thomson, E. Buchser on behalf of the PROCESS Study Group

Objectives: Patients with FBSS experience chronic pain, disability, and reduced health-related quality of life (HRQoL). Few studies report long term results of treatment. This trial investigates the long-term effectiveness of the 
addition of spinal cord stimulation (SCS) to conventional medical management (CMM) in failed back surgery syndrome (FBSS) patients.

Methods: The PROCESS trial randomized 100 FBSS patients with predominant radicular leg pain to receive SCS $(n=52)$ plus CMM or CMM alone $(\mathrm{n}=48)$. After 6 months, patients were allowed to cross-over. The primary outcome was $\geq 50 \%$ leg pain relief at 6 months (VAS). Secondary outcomes were health-related quality of life (Short Form-36), functional capacity (Oswestry Disability Index), patient satisfaction, and adverse effects. At 6 months, compared with CMM, significantly more patients randomized to SCS achieved the primary outcome $(p=0.0001)$. Compared with CMM alone, SCS patients reported improved HRQoL and function. Cross-over was requested by $32(73 \%)$ patients randomized to CMM versus $5(10 \%)$ randomized to SCS. We examined the outcomes of SCS treatment in patients randomized to SCS continuing stimulation at 24 months. We also compared the primary outcome between SCS and CMM groups in 2 ways: 1) "Treated as intended" where patients are analysed as randomised, and crossovers who received alternative therapy are considered not to have achieved $50 \%$ pain relief; 2) "As treated" method where patients are analysed based on the final treatment received.

Results: The group of 42 patients randomized to SCS who continued SCS and were followed for 24 months reported statistically significant improvement in leg pain $(p<0.0001)$, HRQoL ( $p \leq 0.01$ for $7 / 8$ domains of the Short Form-36), and function $(\mathrm{p}=0.0002)$ compared with baseline. Thirteen patients $(31 \%)$ experienced device-related complications which required a surgical procedure. At 24 months, $50 \%$ or more leg pain relief was achieved by $17 / 46$ patients randomized to SCS $(37 \%)$ versus $1 / / 41$ randomized to CMM ( $2 \%)(p=0.003$ according to the "treated as intended" analysis. Similarly, in an "as treated" analysis $1 / 15(7 \%)$ of patients receiving CMM at 24 months achieved $50 \%$ or more leg pain relief, compared to $34 / 72(47 \%)$ for patients on SCS $(p=0.02)$.

Conclusion: In selected FBSS patients, pain relief with SCS is sustained at 24 months and is associated with clinically important improvements in health-related quality of life, functional capacity, and patient satisfaction.

All logistical aspects of the study were managed and funded by Medtronic Europe sàrl.

0122

Numbness in clinical and experimental pain: a cross-sectional study exploring the mechanisms of reduced tactile function

C. Geber, W. Magerl, R. Fondel, M. Fechir, T. Vogt, M. Dieterich, R.-D. Treede, F. Birklein

Johannes-Gutenberg Universität (Mainz, DE)

Background: Pain patients often report distinct numbness of the painful skin although no structural peripheral or central nerve lesion is obvious. In this cross-sectional study we assessed the reduction of tactile function and studied underlying mechanisms in patients with chronic pain and in healthy participants exposed to phasic and tonic experimental nociceptive stimulation.

Methods: Mechanical detection (MDT) and pain thresholds (MPT) were assessed in the painful area and the non-painful contralateral side in $10 \mathrm{pa}-$ tients with unilateral musculoskeletal pain. Additionally, 10 healthy participants were exposed to nociceptive stimulation applied to the volar forearms (capsaicin; electrical stimulation, twice each). Areas of tactile hypaesthesia and mechanical hyperalgesia were assessed. MDT and MPT were quantified adjacent to the stimulation site.

Results: Tactile hypaesthesia in pain patients and in experimental pain (MDT-z-scores: $0.66 \pm 0.30$ and $-0.42 \pm 0.15$, respectively, both $\mathrm{p}<0.01$ ) was paralleled by mechanical hyperalgesia (MPT-z-scores: $+0.51+0.27, \mathrm{p}<0.05$; and $+0.48 \pm 0.10, p<0.001)$. However, hypaesthesia and hyperalgesia were not correlated. Although 9 patients reported numbness, only 3 of them were able to delineate circumscript areas of tactile hypaesthesia. In experimental pain, the area of tactile hypaesthesia could be mapped in 31/40 experiments (78\%). Irrespective of the mode of nociceptive stimulation (phasic vs. tonic) tactile hypaesthesia and hyperalgesia developed with a similar time course and disappeared within approximately one day.

Conclusions: Hypaesthesia (numbness) often encountered in clinical pain can be reproduced by experimental nociceptive stimulation. The time course of effects suggests a mechanism involving central plasticity.

Supported by the Deutsche Forschungsgemeinschaft (BI579/1 and 579/4; Tr236/16-2), the Bundesministerium für Bildung und Forschung (DFNS; grant: 01EM0506).
0123

Assessing pain in disorders of consciousness: the Coma Pain Scale

C. Schnakers, A. Vanhaudenhuyse, S. Majerus, D. Ledoux, M. Boly, M-A. Bruno, P. Boveroux, A. Demertzi, G. Moonen, S. Laureys

University of Liege (Liège, BE)

Objectives: Assessing behavioral responses to pain is difficult in severely brain-injured patients recovering from coma as no tool was specifically developed until now. We decided to develop and validate a behavioral scale adapted for monitoring pain in patients with altered states of consciousness, the Coma Pain Scale (CPS).

Methods: We assessed patients diagnosed as being in a vegetative (MSTF, 1994) or in a minimally conscious state (Giacino et al., 2002) in the intensive care and in the neurology unit of the university hospital of Liège (Belgium). After a noxious stimulation (pressure applied to the middle right and left fingernail), behavioral responses were scored using five behavioral scales administered in a randomized order, the CPS and four other scales employed in non-communicative patients as the elderly (PAINAD; Lane et al., 2003 and CNPI; Feldt, 2000) and newborns (CRIES; Krechel et al., 1995 and FLACC; Merkel et al., 1997).

Results: Twenty four patients ( 9 women; 10 traumatic; $50 \pm 15$ years-old; studied 15 days to 3 years after acute brain injury) diagnosed as being in a vegetative $(n=11)$ or in a minimally conscious state $(n=13)$ were included. According to Spearman correlation, the concurrent validity was good (between 0,71 and 0,82 ). Moreover, ANOVA revealed that the total score was significantly different according to the level of consciousness (vegetative or minimally conscious state) only with the CPS ( $\mathrm{F}=8.2 ; \mathrm{p}<0.01)$.

Discussion: Our preliminary results show that the CPS may be an interesting tool to evaluate pain in severely brain injured patients with altered states of consciousness.

\section{4}

Central representation of cold-evoked pain relief in capsaicin induced pain: an event-related fMRI study

C. Mohr, S. Leyendecker, I. Mangels, C. Helmchen

University of Lubeck (Lubeck, DE)

Termination of a painful or unpleasant condition can be rewarding, even when the stimulus, which elicits pain relief, is painful under normal circumstances. We aimed to identify central neural mechanisms of pain relief from capsaicin-elicited heat hyperalgesia by administering cold stimuli. We hypothesized that cooling might facilitate endogenous descending inhibitory mechanisms.

We compared intraindividual neural responses of 15 healthy male volunteers to cold $\left(20^{\circ}, 0^{\circ} \mathrm{C}\right)$, intermediate $\left(30^{\circ} \mathrm{C}\right)$ and heat stimuli $\left(43^{\circ} \mathrm{C}\right)$ on untreated vs. capsaicin-treated skin using event-related fMRI in a $2 \times 4$ factorial design. Thermal stimuli were applied at the right hand in two separate imaging sessions using a Peltier-element. Psychophysical ratings of the perceived valence and intensity (VAS: 1-100) were obtained after each stimulus. The $43^{\circ} \mathrm{C}$-stimulus was perceived as excessively painful on the capsaicintreated skin as opposed to an unpleasant sensation on the normal skin. In contrast, the $0^{\circ} \mathrm{C}$ stimulus was perceived unpleasant when applied on untreated skin while subjects rated the same stimulus pleasant in the capsaicintreated condition.

When neural responses to the $0^{\circ} \mathrm{C}$ stimulus were compared between the untreated and capsaicin-treated skin condition there were stronger BOLD responses in prefrontal cortex (PFC) and periaqueductal grey (PAG) which correlated with increasing perceived pleasantness (VAS). Based on a connectivity analysis which identified cold dependent contributions of PFC activity with PAG in heat hyperalgesia we propose that cold-induced pain relief partly results from activation of endogenous descending inhibition of nociception. The data illustrate that perception of nociceptive input may largely be determined by competing aversive-appetitive motivational states.

Supported by a University grant

0125

Which stimulation could trigger the release of TNF alpha?

T. Eberle, H. Krämer, M. Fechir, C. Sommer, F. Birklein

University of Mainz (Mainz, DE); University of Wurzburg (Wurzburg, DE)

Recent studies showed that inflammatory cytokines play an important role in the development of the complex regional pain syndrome (CRPS). Especially TNF alpha is known to be responsible for some clinical symptoms like hyperalgesia. In this study we aim to determine the mechanisms which trigger the release of TNF alpha.

We investigated 10 healthy subjects with a dermal microdialysis under 
three different conditions. First, in the absence of any stimulation we applied phosophoramidon, a potent NEP (neutral endopepdidase) blocker. In the second experimental protocol, after one hour of phosphoramidon treatment, we applied electric current stimulation $(1 \mathrm{~Hz}, 20 \mathrm{~mA})$ to the skin for 30 minutes. Finally in the third experimental protokol stimulus using deep impact stimulator was applied for 30 minutes one hour after phosophoramidon treatment.

Our results revealed a slight increase of TNF alpha concentration compared to baseline levels for all three conditions. Moreover in comparison to the electric current stimulation we saw a significant increase of TNF alpha concentration after stimulus with impact stimulator ( $p<0,01 ;$ t-test). trauma.

In conclusion, our results verify release of TNF alpha after minimal deep

\section{6}

Histamine-iontophoresis for differentiation of $\mathrm{C}$-fibre function in post-herpetic neuralgia

T. Schlereth, A. Schreiber, F. Birklein

Johannes Gutenberg-University (Mainz, DE)

Objectives: Two different types of postherpetic neuralgia (PHN) are described. 1. Irritable nociceptor type: characterized by spontaneous pain and preserved sensory function. 2 . Deafferentation type: characterized by spontaneous pain with sensory deficits due to degeneration of C-fibres.

Methods: In this study 22 patients (mean: $74 \pm 1$ years) with PHN were examined with quantitative sensory testing (QST) and laser-Doppler imaging. Flare size was evaluated after iontophoresis of histamine. Histamine induces an axon reflex flare. For statistical evaluation students paired t-test was used.

Results: In 13 patients histamine flare sizes could be obtained. In 9 patients flare sizes were smaller on the affected side $(p<0.05)$, while flare sizes were unchanged or even increased in 4.

Flare size depends on the innervation density of histamine-sensitive Cfibres. Therefore diminished flare sizes should indicate C-fibre loss. Accordingly in patients with reduced flare temperature sensitivity, which is related to C-fibre function, was reduced (cold detection, warm detection, thermal sensory limen; all $\mathrm{p}<0.05)$, while the functions of myelinated fibres were preserved (mechanical detection threshold, mechanical pain threshold, vibration, pressure pain; all n.s.). Mechanical allodynia occurred in five patients with deafferentation pain.

Conclusion: In patients with bigger flare sizes temperature sensation was not significantly impaired, while mechanical pain threshold $(\mathrm{p}=0.06)$ was reduced and mechanical allodynia occurred in all of them. This indicates A-fibre sensitisation corresponding with the irritable nociceptor type. PHN.

Histamine iontophoresis seems useful for differentiation of subtypes of

Supported by DFG (Bi 579/1, Bi 579/4) and the GRNNP.

\section{7}

Clinical characteristics of neuropathic pain patients diagnosed with failed back surgery syndrome (PROCESS study)

S. Thomson, L. Jacques

Basildon and Thurrock University Hospital (Orsett, UK); Montreal Neurological Institute and Hospital (Montreal, CA)

Objectives: Neuropathic pain (NeuP) commonly affects the back and legs and is associated with severe disability and psychological illness. It is unclear how patients with predominantly NeuP due to failed back surgery syndrome (FBSS) compare to patients with other chronic pain conditions. The current study presents data on characteristics associated with FBSS patients compared to those with complex regional pain syndrome, rheumatoid and osteoarthritis, and fibromyalgia.

Methods: The PROCESS trial randomized 100 patients from 12 centres to spinal cord stimulation (SCS, $\mathrm{n}=52$ ) plus conventional medical management $(\mathrm{CMM})$ or CMM alone $(\mathrm{n}=48)$. Patient parameters collected at baseline included age, sex, previous surgeries, time since last surgery, employment, primary source of pain, severity of back and leg pain (VAS), health-related quality of life (HRQoL), level of disability, medication and non-drug therapies. Reference population data was drawn from the literature.

Results: At baseline, patients in the PROCESS Study had a similar age and gender profile compared to other conditions. Back pain severity was similar across conditions, but PROCESS patients suffered from greater leg pain and had lower HRQOL. PROCESS patients commonly took opioids, while antidepressants and NSAIDs were more often used for other conditions. Prior to baseline, $87 \%$ of patients had tried at least 4 different treatment modalities.
Conclusion: Patients suffering from chronic pain of neuropathic origin following FBSS, often fail to obtain adequate relief with conventional therapies and suffer greater pain compared to patients with other chronic pain conditions. Alternative treatments such as SCS should be considered earlier in the treatment continuum.

The PROCESS study was sponsored by Medtronic Europe sàrl.

0128

Efficacy and safety of motor cortex stimulation for chronic neuropathic pain: critical review of the literature

D. Fontaine, M. Lanteri-Minet, P. Paquis

Centre Hospitalier Universitaire de Nice (Nice, FR)

In order to clarify the utility of Motor Cortex Stimulation (MCS) in the treatment of intractable neuropathic pain, we performed a critical review of the literature and a global analysis of previous reported patients. A computerised search of PubMed databases from 1991 to 2006 was conducted for publications using the key words motor cortex, stimulation, and pain. Inclusion criteria for the review were based on publications' and patients' characteristics and clarity of the pain relief evaluation. According to these criteria, 14 studies remained selected for analysis, including 210 patients. All these studies were series of cases and none was controlled. The percentages of patients who experienced a pain relief $>30 \%,>40 \%,>50 \%$ and $>70 \%$ were respectively $60,2 \%, 55,4 \%, 42,5 \%$ and $29,3 \%$. Out of the 210 patients, 119 $(56,7 \%)$ had a "good outcome" (pain relief $>40 \%$ or $>50 \%)$. Out of $152 \mathrm{pa}$ tients with a follow-up superior than 1 year, $69(45,4 \%)$ had a "good outcome". In responders, mean VAS score decrease was $56,6 \%$. Pain relief $>40-50 \%$ was achieved in $53,8 \%$ of the 117 patients with central pain, and $68,1 \%$ of the 44 patients trigeminal neuropathic pain, reported in the 14 selected series. Adverse effects were systematically reported in 10 studies, accounting for 157 patients. Infections $(5,7 \%)$, and wound problems/hardware dysfunction $(5,1 \%)$ were the most common complications. Seizures occurred in 19 patients, in the early postoperative period, but no chronic epilepsy were reported. Although MCS appeared to be relatively safe and efficient, these results have to be considered with caution, as the reported studies had numerous biases, including absence of control, low number of patients with long follow up, variability of surgical techniques and stimulation parameters, absence of postoperative localisation of the electrodes. Despite these biases, our review suggests that MCS could improve significantly some patients with severe and medically resistant pain, for who no other treatment is currently available. Studies with a better design are mandatory to confirm and evaluate the efficacy of MCS for chronic neuropathic pain

\section{Oral session 12}

\section{Epilepsy 2}

\section{9}

Cognitive functions in children with frontal and temporal epilepsy M. Mazurkiewicz-Beldzinska, A. Matheisel, A. Olszewska, M. Szmuda Medical University of Gdansk (Gdansk, PL)

Aim of the study: In order to compare the cognitive functions in children with newly diagnosed temporal (TLE) versus frontal lobe epilepsy (FLE) and control group which consisted of healthy children the following study was performed.

Material and methods: 39 children with TLE 24 children with FLE and 24 healthy subject were included in study. Each child had neuropsychology assessment using age-normed and validated instruments. The applied test battery consisted of measures assessing both intelligence as well as executive and motor skills.

Results: In all epilepsy patients there was no evidence of anatomical brain damage. There were no differences in mean age and gender between the groups. There was no significant difference in global IQ scores between the groups. Children with FLE had significantly lower scores than the other two groups in non-verbal memory tasks, presented higher attention deficit and had slower performance speed. The TLE group performed significantly worse as compared with control group in verbal learning and performance speed with no differences in attention. The correlations between the results of test and the localization of epileptic foci were performed. 
Conclusion: The children with new onset FLE present with more severe cognitive and attention problems compared with TLE group, however the TLE group differ significantly in some measures with control group. That can lead to development of school problems. Early recognition of those deficits should lead to appropriate treatment procedures.

\section{0}

Pregabalin in patients with primary brain tumours and epilepsy

J. Novy, R. Stupp, A.O. Rossetti

CHUV (Lausanne, $\mathrm{CH}$ )

Objectives: Patients with brain tumors and symptomatic epilepsy should be treated with antiepileptic drugs (AED) without enzyme inducing properties, due to interactions with most novel chemotherapy agents; some new AED seem particularly interesting from this point of view. We describe our experience with Pregabalin (PGB), as to our knowledge its use in this setting has not been reported yet.

Methods: Retrospective assessment of 9 consecutive patients with epilepsy and primary brain tumors followed at our outpatient clinic, who received PGB as add-on or as second-intention monotherapy. This medication was introduced between June 2006 - September 2007. We recorded diagnosis, seizure type, indication of PGB introduction and dosage, concomitant treatment, efficacy and tolerance. Follow-up was carried out until discontinuation of PGB treatment, death, or January 2008 for the other subjects.

Results: Seven men and 2 women with a median age 58 yrs (range 43 to 75 ) were treated for a median duration of 5 months (2-19). Six patients had glioblastoma, 2 low grade glioma and 1 primary CNS lymphoma. Seven subjects had a prior surgical resection, 6 received chemotherapy (temozolomide or irinotecan), and 4 underwent radiotherapy; only one subject had no specific oncologic treatment. PGB was introduced before specific oncologic treatment in 4, after it in 3 , and concomitantly in one. Six patients suffered from secondarily generalized seizures and 3 from simple-partial seizures. PGB was used to replace an enzyme-inducing AED in 3 patients, because of intolerance of previous AED in 5; whereas in 2 patients it was added because of insufficient seizure control. PGB was used as monotherapy in 3 subjects, associated with levetiracetam in 5, and with lamotrigine in one. Median daily dosage was $450 \mathrm{mg}(150-600)$. All patients experienced at least a $50 \%$ seizure reduction; 2 became seizure free, and 4 remained seizure free. Five patients reported side-effects: fatigue, weight gain, peripheral oedema, and erectile dysfunction; medication had to be discontinued in 2 cases after 2-3 months. Three patients died from their oncologic disease 3-5 months after PGB introduction.

Conclusion: $\mathrm{PGB}$ seems to be promising for seizure treatment in patients with brain tumors, even as a monotherapy. The possibility of rapid titration may represent an important advantage. This experience is now being investigated in a prospective controlled study.

\section{1}

Epilepsy and neurocysticercosis in Qatar

B. Mesraoua, D. Deleu, H. Al hail, N. Akhtar, A. D'souza

Hamad Medical Corporation (Doha, QA)

Purpose: Taenia solium neurocysticercosis (NCC) is increasingly being reported in patients suffering from epilepsy. However, its true prevalence and association with late onset epilepsy is largely unknown in the Middle East particularly in Qatar. Our study will demonstrate that NCC is also a major cause of first seizures in an increasingly young population in Qatar

Methods: This is a retrospective and prospective study, based on hospital populations.Neurocysticercosis was diagnosed on the basis of the following: consistent radiographic findings and a compatible epidemiologic and clinical history (without diagnostic serological findings). All individuals with suspicion of NCC at the emergency department were admitted for further investigations and treatment.The complementary examinations included an awake EEG, a CT SCAN or MRI of the brain and serum and CSF studies for some of them.

Results: Fifty patients were diagnosed as having NCC on the basis of the above criteria. The large majority of patients were male $(98 \%$, most of them expatriate from the Asian Subcontinent).At first presentation, most of the patients $(80 \%)$ were older than 20 years $96 \%$ of them presented with seizures. Neurological findings, Brain CT and MRI abnormalities, EEG as well as cysticidal therapy and seizures presentations and outcome will be discussed.

Conclusions: This study confirms that NCC is a major cause of epilepsy in an hospital based (increasingly young) population in Qatar.NCC is a relatively new disease in this country, imported recently from areas where NCC is endemic by a growing Indian subcontinent working force. However, the prognosis of NCC related seizures is good.
0132

The quality of life of chronic epileptic patients in a sub-Saharan hospital F. Salawu, A. Danburam, M. Wakil

Federal Medical Centre (Yola, NG); Federal Neuropsychiatry Hospital (Yola, NG)

Epilepsy can be a lifelong problem complicated by recurrent seizures, intolerable side effects, and devastating effects on the individual and his or her loved ones.

Objectives: To evaluate the aspects of health related quality of life (QOL) of Nigerians with epilepsy.

Methods: We consecutively identified 42 Nigerians ( 29 males and 13 females) with epilepsy attending the neurology clinic of the State Specialist Hospital. Each Patient had complete physical examination and electroencephalography. A brief screening tool, the 10-item questionnaire (QOLIE10) to assess QOL was administered to the subjects during routine clinic visits.

Results: The commonest aspect of QOL affected in the subjects was feeling of energy, which was impaired in $19(45 \%)$ subjects. This was followed by memory problems in $16(38 \%)$. Ten $(23 \%)$ subjects reported fear of experiencing next seizure. Only seven $(16 \%)$ and five $(11.9 \%)$ of subjects reported work and social limitations respectively. None reported difficulty with driving or operating machinery, or mental disturbances.

Conclusion: Energy and memory deficits seem to be the major problems affecting the QOL of Nigerians with epilepsy in this study. Most subjects did not consider physical and social limitations important. This screening tool could provide potentially useful information for initial assessment or follow-up of problem area that are not commonly evaluated during routine clinical visits with patients with epilepsy, even in a busy clinical practice.

\section{3}

Anticonvulsant activity of perampanel, a selective AMPA receptor antagonist, in rodent models of epileptic seizure

Y. Hashizume, T. Hanada, A. Ogasawara, M. Ueno, Y. Nishizawa

Eisai Tsukuba Research Laboratories (Tsukuba, JP)

Objective: To determine the anticonvulsant activity spectrum of perampanel, a non-competitive selective AMPA receptor antagonist, using rodent models of generalized and partial seizures.

Background: AMPA receptor-mediated glutamate signaling plays an important role in the pathophysiology of epileptic seizures. Previous use of AMPA receptor antagonists in animal seizure models has suggested their potential for the treatment of epilepsy.

Methods: Electroshock seizures were induced in mice by electrical stimulation to both corneas ( $80 \mathrm{~V}, 0.4 \mathrm{~s}$ for maximal electroshock seizure [MES] in ddY mice). Audiogenic seizures were induced in DBA/2J mice by sound stimulation $(11 \mathrm{kHz}, 115 \mathrm{db})$. Pentylenetetrazole (PTZ)-induced seizures were generated in ICR mice by subcutaneous injection of $90 \mathrm{mg} / \mathrm{kg}$ PTZ. Seizure protection (ED50 for tonic extension inhibition) was measured in animals treated with perampanel $(0.3$ to $3 \mathrm{mg} / \mathrm{kg}$ ) or vehicle alone administered 0.5 to 1 hour before seizure induction. Changes in afterdischarge threshold current (using an ascending stimulation schedule) and afterdischarge duration, motor seizure duration, and seizure severity were measured as a function of perampanel dose $(1,1.25,2.5,5$, or $10 \mathrm{mg} / \mathrm{kg})$ or vehicle alone in amygdala-kindled Sprague-Dawley rats.

Results: Perampanel protected mice from audiogenic seizures, MES, and PTZ-induced seizures (ED50 $=0.47,1.6$, and $0.94 \mathrm{mg} / \mathrm{kg}$, respectively). In amygdala-kindled rats, perampanel 5 and $10 \mathrm{mg} / \mathrm{kg}$ significantly reduced motor seizure duration, afterdischarge duration, and seizure severity compared with vehicle alone $(\mathrm{P}<0.05$ for all measures, ANCOVA then Dunnett type test). Perampanel $10 \mathrm{mg} / \mathrm{kg}$ significantly elevated afterdischarge threshold in amygdala-kindled rats compared with vehicle alone $(\mathrm{P}<0.05$, ANCOVA then Dunnett type test).

Conclusions: Glutamate signaling plays a central role in seizure initiation and spread. Results from this study suggest that perampanel, a non-competitive selective AMPA receptor antagonist, attenuates a spectrum of seizure types and may be an effective treatment for epilepsy.

Authors are employed by Eisai Co., Ltd. 
0134

Testing superiority of oxcarbazepine in comparison to carbamazepine with respect to driving ability in healthy volunteers: a double-blind, crossover trial

R. Kenntner-Mabiala, Y. Kaussner, S. Hoffmann, J. Klatt, F. Tracik, H.P. Krüger

University of Wurzburg (Wurzburg, DE); Novartis Pharma GmbH (Nuremberg, DE)

Objectives: The commonly used antiepileptic drug (AED) carbamazepine (CBZ) causes cognitive side effects being at least partly relevant for driving safety. In contrast to CBZ, the effects of oxcarbazepine (OXC) on cognitive functions are by far less. Up to now, there is no study comparing the effects of OXC and CBZ on driving performance. The present study aimed to show superiority of OXC as compared to CBZ with respect to driving ability as assessed by a driving simulator test.

Methods: Driving performance of 30 healthy volunteers under the treatment of OXC and CBZ was measured in a high-fidelity driving simulator of the Würzburg Institute for Traffic Sciences (http://www.wivw.de) with a double blind, crossover design. The test course contained a representative range of scenarios and comprised a length of about $100 \mathrm{~km}$ of simulated distance entailing a mean driving time between 80 and 90 minutes. Lane keeping performance, mean and standard deviation of speed, as well as mean and standard deviation of distance to cars ahead were continuously recorded by means of the driving simulation software. Driving mistakes and collisions were registered by two experts. Eyelid closure index was recorded as behavioral measure of sleepiness.

Results: Both drugs had negative effects on driving performance which are reflected in poorer lane keeping performance, higher rate of driving mistakes, and increased sleepiness. These effects were most obvious in monotonous nocturnal situations but they could be compensated in situations being challenging on cognitive and motoric driving skills. Drug effects on driving performance and sleepiness were more pronounced for CBZ than for OXC.

Conclusions: Since both drugs caused impairment on driving performance, warning about driving a car on the package insert is indispensable. However, for some subjects, effects of drugs on driving performance were negligible. Hence, for individual patients in whom cognitive AED side effects do not manifest distinctly, the ability to drive may be acceptable. Since negative effects on driving performance were less pronounced for OXC than for CBZ, OXC should be preferred over CBZ for patients with epilepsy who need to drive.

This study was supported by Novartis Pharma GmbH

\section{5}

Predictors of mortality in status epilepticus: preliminary result of a hospital based study in northeast Nigeria

F. Salawu, B. Isa, A. Danburam, P. Thomas-Sukur, B. Gundiri, U. Kirawa, A. Adiel

Federal Medical Centre (Yola, NG)

Objectives: We evaluated the clinical profile and predictors of poor outcome in a cohort of Nigerian patients with status epilepticus admitted to the intensive care unit.

Methods: A prospective study was carried out on 84 patients with status epilepticus between May 2005 and April 2007 including sociodemographic data, aetiology, detailed analysis of clinical and laboratory data, duration, and type of seizure. Factors for poor outcome, defined as death or morbidity as measured by deterioration in functional status were analysed in a multivariate logistic regression analysis.

Results: The study population comprised of $32(38.1 \%)$ males and 52 $(61.9 \%)$ females with age range of 21 to 53 years $(32.7+/-8.7)$. Majority of the females were pregnant in their third trimester. Generalized tonic-clonic status epilepticus was present in $71 \%, 12 \%$ had epilepsia partialis continua and $17 \%$ had complex partial seizure. The underlying causes for status in the majority were non-compliance to antiepileptic treatment, neuroinfection (2 HIV positive individuals had multiple cerebral abscesses), cerebrovascular disease, anticonvulsant withdrawal, alcohol, and other drug withdrawal in younger males. No cause could be found in $15.5 \%$ of patients. An overall mortality was over $30 \%$, and most patients died of uncontrolled seizures of generalized tonic-clonic type, complicated by the effects of the underlying illness or an injury sustained because of a seizure. The predictors of mortality were delay in treatment, female sex, pregnancy, hypotension, increased age, prolonged status epilepticus and acute symptomatic seizures. Functional outcome at discharge deteriorated in $34 \%(20 / 58)$ of nonfatal cases. Increased length of hospitalization and acute symptomatic seizures were predictors of functional disability.
Conclusion: Mortality and morbidity is high in Nigerians with status epilepticus. The results suggest that non-compliance to antiepileptic treatment and early withdrawal of anticonvulsants are the most important reasons that could be improved through social and educational rehabilitation.

\section{6}

Insulinoma leading to transient status epilecticus in reversible brain damage: a neurophysiological and neuroradiological study G. Pavan, D. Dalla Libera, B. Colombo, V. Martinelli, F. Minicucci, A. Zerbi, G. Comi

San Raffaele Hospital (Milan, IT)

Objectives: To report an unusual case of insulinoma leading to an epileptic status which results in reversible cortical MRI lesions.

Methods: Observational case report.

Results: A 42-year-old man without past medical history except cocaine abuse presented with epileptic status successfully treated with a loading dose of i.v. Phenytoin (DPH). Blood analyses were normal except for low levels of venous blood sugar $(40 \mathrm{mg} / \mathrm{dl})$. No previous symptoms referring to hypoglycemia were described. Ictal EEG showed continuous fronto-temporal spike and wave activity with a prevalence in the right side. CT scan was normal. A cerebral MRI showed T2 diffuse hyperintensity of the cortex in girus recti, corpus callosum and girus cinguli with a symmetrical bilateral distribution while a slight, diffuse cortical tumefaction was seen over the cortex. DT images outlined a slight positive signal and post-contrast MRI showed enhancement of cortical mantle. Repeated glycemia were below the normal values and normoglycemia was difficult to achieve despite therapy (oral Diazosside and 33\% Glucose solutions i.v.). A significant improvement in neurological examination (with normal cognitive status and no other pathological signs) was observed in 5 days. Normalization in EEG features was achieved in 2 weeks of oral DPH. An abdominal MRI revealed a $2 \mathrm{~cm}$ round nodular mass located over the pancreatic tail. Radical surgical treatment was performed and histological findings on the lesion confirmed the diagnosis of insulinoma (synaptophysin-positive; insulin $70 \%$; proliferative index $\mathrm{Ki} 67 / \mathrm{MIB1}=1 \%$; mitotic index $=0$; no necrosis or angioinvasion). No seizures have occurred since then. Four month-follow up cerebral MRI revealed the disappearance of the described cortical lesions, the normalization of the apparent diffusion coefficient and no enhancement after gadolinium, while a slight enlargement of subarachnoid spaces was evident.

Conclusions: Insulinoma leading to hypoglicemia must be taken into account for differential diagnosis with seizures, even when no further systemic or neurological symptoms are associated. Interestingly, this case suggests that a prolonged hypoglicemia, together with the cellular consequence of status epilepticus, may increase neuronal vulnerability to damage. These findings may induce acute cortical abnormalities which may be reversible if the causes are promptly removed.

\section{Oral session 13}

\section{Clinical neurophysiology}

\section{7}

Cortical changes in the follow-up to a vestibular neuritis: a voxel-based morphometry study

P. Schlindwein, B. Baier, P. Stoeter, M. Dieterich

Gutenberg University Hospital (Mainz, DE)

Vestibular neuritis (VN) is defined to be a sudden partial, unilateral labyrinthine failure. VN is probably due to reactivation of herpes simplex virus 1 in the geniculate ganglion or other infectious diseases of the inner ear. The aim of our study was to examine the cortical morphological changes in the follow-up of $\mathrm{VN}$ patients with the help of voxel-based morphometry (VBM) and magnetic resonance imaging (MRI).

Twenty-one patients ( 9 females, average age $56,7 \pm 10,4$ years) with a unilateral VN (8 rightsided) had a follow-up examination that included a highresolution sagittal T1-weighted image (MPRAGE sequence, 180 slices, slice thickness $=1 \mathrm{~mm}$, image matrix $\left.=256^{2}, \mathrm{TR}=9.7 \mathrm{~ms}, \mathrm{TE}=4 \mathrm{~ms}\right)$ in a clinicial $1.5 \mathrm{~T}$ scanner (Siemens Vision, Erlangen, Germany) more than 6 months after the disease onset (mean $2.5 \pm 1.6$ years). The diagnosis of $\mathrm{VN}$ had been based on the acute occurrence of the characteristic symptoms, a careful neurological and neuro-otological examination as well as a canal paresis found during caloric irrigation of the horizontal semicircular canal. 
An age- and gender-matched healthy control group underwent an identical MRI-protocol in the same scanner. Statistical analysis in a paired t-test setup was done with SPM5 ( $\mathrm{p}<0.001$, uncorrected, cluster size $>5$ voxels) after standardized preprocessing.

In contrast to the healthy controls, all VN patients showed a significant increase in gray matter intensities of the MT/V5 in the left hemisphere. The same result was found bilaterally when our analysis was restricted to the patients with residual deficits in caloric irrigation during the follow-up. The latter patients also exhibited significant decreases in white matter intensities in the posterior insular vestibular cortex (PIVC) bilaterally. Furthermore, all VN patients showed a significant loss in white matter intensities in the right superior temporal gyrus and the left hippocampus. Correlation analysis disclosed that gray matter intensities in MT/V5 bilaterally and the right cerebellar semilunar lobe correlated negatively with the deviation of the dynamic SVV.

This is the first demonstration by means of VBM to show structural changes within the cortex in the follow-up of patients with a peripheral cranial nerve lesion. Our study gives further evidence that the loss of vestibular function may well be compensated by a volumetric increase in neurons of visual motion processing such as the MT/V5 and the semilunar lobe of the cerebellum.

Supported by the DFG (German Research Foundation)

\section{8}

Evaluation of sympathetic vasomotor control in patients with neurally mediated syncope

J.V. Gutierrez Gil, E. González, A. Olivares, R. Andrade, A. Voustianiouk, H. Kaufmann

Cuban Institute of Neurology (La Habana, CU); Center for Surgical and Clinical Research (La Habana, CU); New York University (New York, US)

Objectives: Previous papers have demonstrated that sympathetic vasomotor control (SVC) disorders play a key role in the pathophysiology of neurogenic syncope (NS). This study is aimed to evaluate whether these SVC disorders occur only during acute fainting episodes or persist as a permanent trait of patients with NS. The temporal profile of SVC features in NS could be useful in the differential diagnosis of these syndrome.

Methods: Electrocardiogram and beat to beat tonometric blood pressure (BP) (Colin Pilot 9200) were evaluated in 32 patients (age: $22.1 \pm 7.2$ years) with clinical diagnosis of NS (at least 3 episodes per year) and 40 agematched controls (age: $24.3 \pm 6.5$ years). Recordings were made at rest and during the Valsalva maneuver (VM). The following indexes were derived: Percentage of change of systolic BP during phases IIA (BP-IIA); IIB (BP-IIB) and IV (BP-IV) of the VM, referred to average baseline BP before the strain; Duration of phase II (DUR-IIA, ms) and low-frequency/ high-frequency in$\operatorname{dex}(\mathrm{LF} / \mathrm{HF})$ of heart rate variability spectral power.

Results: Patients showed a significantly lower decrement \{BP-IIA: -7.9 (5.5) vs. $-11.5(8.6), \mathrm{p}<0.03$ \}and a faster recovery \{DUR-IIA: 5.64 (2.6) vs. 7.9 (1.7), $\mathrm{p}<0.04\}$ of $\mathrm{BP}$ during phase IIA than controls. Both groups reached similar blood pressure recovery during phases IIB [BP-IIB: 4.3 (9.7) vs. 5.5 (12.3) $\}$ and IV $\{17.5$ (14.6) vs. 20.6 (10.1)\}. The index LF/HF was higher in patients with NS than in normal subjects $\{2.8(2.9)$ vs. $1.3(1.4)\}$ but this difference was not statistically significant.

Conclusions: These findings suggest: first, that patients with NS, outside the fainting episode, are able to develop a normal regulation of $\mathrm{BP}$ during $\mathrm{VM}$ and second, that the process of BP recovery during early phase II starts faster in these patients than in normal controls. These results emphasize the transient nature and NS, with normal autonomic function regulation between the fainting attacks. The faster recovery of BP could imply that patients with NS have a mildly increased, rather than decreased, SVC tone as a permanent feature. This pattern could be useful to differentiate NS with other disorders such as pure autonomic failure, autonomic neuropathies and multisystemic atrophy.

This paper is sponsored by the Cuban Ministry of Pulic Health

\section{9}

Age-related decline in motor cortical specialisation: a TMS study

R. Chieffo, L. Straffi, A. Inuggi, F. Spagnolo, G. Comi, L. Leocani

H. San Raffaele (Milan, IT)

Objectives: To evaluate aging effect on motor cortical representation and inter-hemispheric inhibition using focal transcranial magnetic stimulation (TMS)

Methods: Motor cortical mapping of the two hemispheres using TMS, with simultaneous recording of four upper limb muscles (abductor pollicis
brevis-APB; abductor digiti minimi-ADM; extensor carpi radialis; biceps) was performed in 16 right-handers subjects subdivided in two groups: 7 younger $(4 \mathrm{M}$, aged $25.5 \pm 2.3)$ and 9 older $(6 \mathrm{M}$, aged $61.3 \pm 11)$. Ipsilateral silent period (iSP) was measured, as the amount of suppression of maximal voluntary EMG in APB to focal TMS of the ipsilateral hand motor cortex, in 7 younger $(4 \mathrm{M}$, aged $26 \pm 4)$ and in 6 adults $(3 \mathrm{M}$, aged $62 \pm 10)$. Contraction of ipsi and contra-lateral resting muscles was also measured

Results: The older group had a significantly higher MEPs threshold of the right compared to the left hemisphere ( $\mathrm{p}=0.01$ pared $\mathrm{T}$ test $)$ and a significantly higher inter-hemispheric threshold difference compared to the young group $(\mathrm{P}=0,03$ pared $\mathrm{T}$ test). Map density was also more asymmetric in the older compared to the younger group ( $\mathrm{p}=0.021$ ANOVA) being smaller in non-dominant hemisphere. In the younger iSP over the dominant hemisphere was higher both compare to their non-dominant hemisphere and to the dominant hemisphere of older subjects ( $\mathrm{p}<0.019$ paired T test), who had symmetric iSP. During voluntary maximal contraction of right-APB, older show a greater coactivation of ipsilateral $\mathrm{ADM}$ and more mirroring activity $(\mathrm{P}<0,025$ paired $\mathrm{T}$ test $)$.

Conclusions: Normal aging is associated to a relative decrease in cortical excitability of non dominant hemisphere leading to a more evident interhemispheric difference in motor cortical representation of muscles of the upper limb. Greater tendency to mirroring and loss of movement selectivity with aging could be the expression of a reduced efficiency of intra-cortical inhibitory circuits.

0140

Detecting consciousness in coma survivors: an active event-related paradigm

C. Schnakers, F. Perrin, M. Schabus, S. Majerus, D. Ledoux, P. Damas, M. Boly, A. Vanhaudenhuyse, M-A. Bruno, G. Moonen, S. Laureys

University of Liege (Liège, BE); University of Lyon (Lyon, FR); University of Salzburg, AT

Objectives: Disentangling vegetative from minimally conscious patients is often difficult when relying only on behavioral observation. In this study, we explored an event related potential (ERP) paradigm as an alternative method for the detection of voluntary brain activity.

Methods: The participants were twenty-two right-handed patients (10 traumatic) diagnosed as being in a vegetative (VS) $(n=8)$ or in a minimally conscious state (MCS) $(\mathrm{n}=14)$. They were presented sequences of names containing the patients' own name or other names, in both passive and active conditions. In the active condition, the patient was instructed to (1) count her/his own name or (2) to count another target name.

Results: Like controls, MCS patients presented a larger P3 response to the patient's own name, in the passive and in the active conditions. Moreover, the P3 to target stimuli was higher in the active than in the passive condition, suggesting voluntary compliance to task instructions like observed in controls. In contrast, no $\mathrm{P} 3$ differences between passive and active conditions were observed for any of the VS patients.

Conclusion: Our results suggest that the presented "active" ERP paradigm may permit detection of voluntary brain function in severely brain damaged patients, and this in cases with very limited external behavioral responses such as MCS patients only showing visual fixation or eye tracking.

\section{Oral session 14}

\section{Extrapyramidal disorders 1}

\section{1}

Neuropathologic findings in patients with Parkinson's disease and mild cognitive impairment

C. Adler, D. Connor, M. Sabbagh, T. Beach, H. Shill, L. Vedders, L. Sue, J. Caviness

Mayo Clinic (Scottsdale, US); Sun Health Research Institute (Sun City, US)

Objective: Cognitive decline in Parkinson's disease (PD) is common. PD-dementia prevalence varies by study, but reports are rare for PD in terms of mild cognitive impairment (MCI) and autopsy findings for PD-MCI have not been reported. We previously reported that of $86 \mathrm{PD}$ cases in our cohort, $21 \%$ had MCI, and now report the neuropathologic findings in 6 of our PDMCI cases. 
Methods: Subjects in our brain and body donation program are diagnosed as having PD based on UK brain bank criteria. Cognitive impairment was assessed by a neuropsychological test battery that included digits forward and backward, Rey AVLT, controlled oral word association, category fluency, clock drawing, Judgment of Line Orientation, Trails parts A and B, Stroop, and MMSE. The diagnosis of PD-MCI was made if a subject with PD had a subjective cognitive complaint but the cognitive deficit did not result in functional decline. The subject needed to have a deficit of $>1.5 \mathrm{SD}$ below the expected age corrected mean score for one of the five cognitive domains assessed (frontal/executive, amnestic, visuospatial, attention, language). Of our 63 autopsied PD cases 6 had MCI (2 women, 4 men), 16 were PD-cognitively normal, and 41 were PD-dementia. Standard neuropathological methods were used including immunohistochemical analysis with alphasynuclein antibodies for Lewy bodies (LB) and LB-related neurites, and both CERAD and Braak Alzheimer's disease (AD) staging for neuritic plaques and neurofibrillary tangles.

Results: The PD-MCI cases had a mean age of 83.2 years and mean PD duration of 9.5 years. Five of the 6 PD-MCI cases were levodopa-responsive and 3 had motor fluctuations. One PD-MCI (PD for 2 years) had not been treated. Pathology in one case showed brainstem-predominant LB stage (the early, untreated case), 3 had limbic LB stage, and 2 had neocortical LB stage. Two cases were Braak stage IV for $A D$ with CERAD moderate neuritic plaque density, 1 was Braak stage III for AD with CERAD moderate neuritic plaque density, and the 3 were Braak stage II or III with sparse or no neuritic plaques. There was mild amyloid angiopathy in 2 cases.

Conclusions: Neuropathologic findings in PD-MCI revealed predominantly Lewy bodies alone in 3 cases and significant AD changes in 3 cases. The extent of Lewy body pathology varied widely. The underlying pathophysiology for MCI in PD is likely multifactorial, consistent with what is found in PD-dementia, but may occur with Lewy body pathology alone.

This work was funded by Federal Grants P30 AG019610, the Arizona Biomedical Research Commission, Michael J. Fox Foundation for Parkinson \& iexcl; \& brvbar; s Research (Prescott Family Initiative), Mayo Foundation for Medical Research, and Sun Health Research I

\section{2}

Executive functions after deep brain stimulation for Parkinson's disease who worsens?

C. Daniels, K. Witt, J. Reiff, P. Krack, M. Krause, K. Boetzel, A. Schnitzler, L. Wojtecki, R. Hilker, E. Kalbe, G.-H. Schneider, A. Kupsch, G. Deuschl for the German Parkinson Study Group, Neurostimulation Section

Objectives: Deterioration of executive function after subthalamic deep brain stimulation (STN-DBS) has been described frequently. However, risk factors for executive dysfunction are unknown. High age or preexisting cognitive decline are supposed to have a major impact on executive performance after STN-DBS.

In this study we evaluate factors influencing the outcome on executive functioning tests after STN-DBS.

Methods: The study was conducted as a substudy of the "Randomized trial of deep brain stimulation for Parkinson's disease". We investigated verbal fluency and Stroop test performance in 60 patients with Parkinson's disease before and after STN-DBS operation compared to a control group receiving an optimized medical treatment. Verbal fluency and Stroop test results and the corresponding change scores (scores at 6 month - scores at baseline) were correlated to age, motor function and neuropsychological test scores at baseline.

Results: Patients with STN-DBS showed a significant decline in the verbal fluency test and the Stroop test compared to the medical treatment group. This worsening showed no correlation with age, motor function or global cognitive function at baseline. Decline in executive function was inversely correlated to baseline scores: Those patients, who scored high on executive scores at baseline, experienced a most prominent decline in the follow-up examination.

Conclusions: Patients with STN-DBS showed a significant decline in the verbal fluency test and the Stroop test. This decline of executive functions is in line with the data from the literature. Interestingly, those patients, who scored high on executive tests at baseline, experienced a most prominent decline in the follow-up examination. This finding argues against a preexisting cognitive impairment as a major factor for executive worsening after STNDBS.

Supported by a grant from the German Federal Ministry of Education and Research
0143

The genotype and phenotype of chorea-acanthocytosis

B. Bader, C. Dobson-Stone, A. Monaco, R. Walker, A. Danek

Ludwig-Maximilians Universität (Munich, DE); Garvan Institute of Medical Research (Darlinghurst, AU); The Wellcome Trust Centre for Human Genetics (Oxford, UK); VA Medical Center (New York, US)

Objective: To investigate the clinical and genetic findings, course of disease and epidemiology of the main neuroacanthocytosis syndrome chorea-acanthocytosis (OMIM \#200150) in a large cohort of 106 international patients.

Methods: We retrospectively analyzed clinical records of patients and standardized physicians' questionnaires. Cases suffering of this rare disorder were recruited by literature search as well as direct approach by patients. Clinical diagnosis was confirmed by genetic sequencing of the VPS13A gene. Results: Among these 106 patients from 18 different countries, we found 63 unique exon mutations without a specific hot spot within the VPS13A gene. Typical course of disease starts at the mean age of 29 years. Among the initial symptoms we found seizures $(42 \%)$ to be the most common, next are orofacial dyskinesia $(25 \%)$ and psychiatric disorders $(23 \%)$, mostly described as obsessive-compulsive disorder. A set of 13 different symptoms was analyzed for the time of appearance during the course of disease. The disease develops as a progressive neurodegenerative disorder showing hyperkinetic movement disorder in the beginning and a more hypokinetic pronounced movement disorder in the later stages. In average, the disease ends fatally 12 years after onset of symptoms.

Conclusion: This study provides an extensive insight into the genotype and clinical phenotype of chorea-acanthocytosis. Furthermore, it helps to classify the disease into three different phases, frequently found in typical chorea-acanthocytosis patients: Initial phase I consists of seizures and orofacial motor-tics, phase II describes symptoms like chorea and tongue dystonia, and finally phase III comprises parkinsonism.

\section{4}

Cerebral adaptation strategies in cervical dystonia

P.M. de Vries, B.M. de Jong, D.E. Bohning, V.K. Hinson, M.S. George, K.L. Leenders

University Medical Center (Groningen, NL); Medical University of South Carolina (Charleston, US)

Objectives: In cervical dystonia (CD), neuroimaging has shown changed cerebral activation patterns related to abnormal movement in dystonic body parts. Our group demonstrated that movement in non-dystonic body parts showed similar activation changes (1). It was puzzling how clinically normal movements were generated by altered cerebral activation. We hypothesized that the brain adapts to abnormal cortical function by recruiting other cortical areas to correctly perform desired actions. To test this assumption, an interleaved method of Transcranial Magnetic Stimulation (TMS) and functional MRI was applied to identify effects of local functional disruption on remote cerebral networks.

Methods: Ten healthy controls and eight CD patients were scanned on a 3T MRI with built-in TMS. They executed and imagined right hand flexion/ extension movement. Half of the conditions were preceded by 10 pulses of 1 $\mathrm{Hz}$ TMS applied to the left superior parietal cortex. This target was chosen since it consistently showed underactivation in dystonia (1) which is thought to reflect impaired sensorimotor integration.

Results: In healthy controls, left superior parietal TMS induced task-related activation patterns similar to that in dystonia comprising increased bilateral prefrontal and posterior parietal activation and decreased premotor and anterior parietal activation during movement execution and imagery. TMS in CD yielded even larger increases in prefrontal and posterior parietal regions while a larger decrease was seen in right anterior parietal cortex during movement imagery. Movement execution showed a reversed pattern with right posterior parietal decrease and left anterior parietal increase.

Conclusion: Superior parietal TMS appeared to mimic activation patterns seen in CD. We inferred that decreased premotor and anterior parietal activations reflected a disturbed ability for sensorimotor integration in regions connected with the sensorimotor cortex. Prefrontal and posterior parietal increases, related to clinically normal hand function, indicate a mechanism of compensation. Top-hierarchy centres in motor control trigger alternative regions to participate in sensorimotor integration. Fragility of this strategy is shown in movement execution: sensory feedback from the limb overrules reduced parietal function and its adaptive consequences so that an overactive anterior parietal cortex may lead to incorrect movements.

1. P.M. de Vries et al. (2007) Clin Neurol Neurosurg 10:120-128 


\section{Oral session 15}

\section{Muscle disorders}

\section{5}

FHL1 Gen mutation associated myopathy: a novel class of inherited myopathies

S. Quasthoff, C. Windpassinger, B. Schoser, S. Hochmeister, V. Straub, B. Lohberger, E. Petek, T. Schwarzbraun, K. Wagner, W.N. Löscher, H. Lochmüller, N. Farra, L. Ofner, A. Mikhailov, J.B. Vincent

Medical University Graz (Graz, AT); Ludwig Maximilians University (Munich, DE); University of Newcastle upon Tyne (Newcastle, UK); Medical University Innsbruck (Innsbruck, AT); University of Toronto (Toronto, CA)

Objectives: Myopathies are inherited muscle disorders characterized by weakness and atrophy. Several myopathies exhibit an X recessive mode of transmission. Duchenne muscular dystrophy and the less severe Becker MD are characterized by reduced levels of functional dystrophin. Emery-Dreifuss MD is another form of adult-onset X recessive MD caused by deficiencies in the emerin protein. In some cases where an X recessive mode of transmission is suspected, no candidate gene has been found. We have identified a large multigenerational Austrian and a smaller family from the U.K. displaying an novel form of X-linked recessive myopathy.

Methods: Clinical assessment of muscle and heart, muscle biopsy, magnetic resonance imaging of skeletal and heart muscle, electron microscopy, muscle immunocytochemistry, STRP analysis, genome-wide SNP analysis, sequencing and mutation analysis of candidate genes, immunohistology, western analysis have been performed.

Results: Affected individuals develop an adult-onset scapulo-axio-peroneal myopathy with rigid-spine syndrome with specific atrophy of postural muscles and pseudo-athleticism/hypertrophy, and cardiac involvement. Known X-linked myopathies were excluded by simple tandem repeat polymorphism and single nucleotide polymorphism analysis, direct gene sequencing and immunohistochemical analysis. STRP analysis revealed significant linkage at Xq25-q27.1. Haplotype analysis based on SNP microarray data from selected family members confirmed this linkage region on the distal arm of the X chromosome. Sequencing of functional candidate genes led to the identification of a missense mutation within the four-and-a-half LIM domain 1 gene (FHL1), which putatively disrupts the 4th LIM domain of the protein. Mutation screening of FHL1 in a myopathy family from the U.K. exhibiting an almost identical phenotype revealed a 3 bp insertion mutation within the 2nd LIM domain. FHL1 on Xq26.3 is highly expressed in skeletal and cardiac muscles. Western blot analysis and imunohistology of muscle biopsies showed a marked decrease in protein expression of FHL1 in patients, in concordance with the genetic data.

Conclusion: We have found a new disorder, X-linked myopathy with postural muscle atrophy (XMPMA) and identified FHL1 as the causative gene. It supports the role of FHL proteins in the development and maintenance of muscle tissue. Mutation screening of FHL1 should be considered for patients with uncharacterized myopathies and cardiomyopathies.

\section{6}

Selective muscle involvement in facioscapulo humeral muscular dystrophy: the role of myoblasts and $4 \mathrm{q} 35$ gene overexpression

S. Sacconi, J.T. Vilquin, R. Tupler, J. Leger, M. Le Cunff, C. Desnuelle

Fédération des Maladies Neuromusculaires (Nice, FR); Institut de Myologie (Paris, FR); MIOGEN LAB- Università di Modena e Reggio Emilia (Modena, IT); INSERM U533 (Nantes, FR)

Background: Facioscapulo humeral muscular dystrophy (FSHD) is an autosomal dominant myopathy characterized by selective muscle involvement. We previously demonstrated that, in contrast with myoblastes issued from non affected territories, FSHD myoblastes from affected territories display defect in myogenic differentiation.

This study is aimed to correlate the extent of muscle involvement to the alteration in differentiation observed in FSHD myoblastes issued from differentially affected territories and gain more inside in FSHD physiopathological process.

Methods: We analyzed 4 myoblasts cultures issued from non dystrophic FSHD vastus lateralis (VL) muscle, 4 from subscapularis (SS) FSHD affected muscle with different level of dystrophy and 4 matched controls. Dynamic gene expression profile was performed on a specific chip containing 6682 genes. 5 timepoints were analyzed: the cell cycle withdrawal (D0), and 24, 48, 72 and 144 hours after inducing myogenic differentiation. The results were analyzed using SAM multiclass analysis and expressed in term of variant or invariant gene during differentiation correlated or not correlated with vari- ation in control myoblasts gene expression. Pathway connectivity analysis and visualisation of different pathways was done using a specific metasearch tool.

The expression of 4q35 gene FRG1, ANT1 was analyzed both by microarray and Taqman real time PCR.

Results: Controls myoblasts issued from VL and SS muscles display clear differences in gene expression at D0 and during differentiation. The dynamic gene expression profile in FSHD myoblasts during differentiation confirm the defect in both cell cycle withdrawal and differentiation mostly in FSHD cell culture from highly dystrophic muscles. 4q35 genes overexpression were seen only in dystrophic SS derived muscle cultures after differentiation suggesting a role of these gene in differentiation defect.

Conclusions: Our results suggest that selective muscle involvement in FSHD patients can be due to different alteration in the ability of derived myoblasts to regenerate muscle. The role of $4 \mathrm{q} 35$ gene overexpression will be discussed as well as the implications for autologous cell therapy.

AFM grant

0147

Autosomal dominant distal myopathy with a myotilin S55F mutation: sorting out the phenotype

J. Berciano, E. Gallardo, R. Domínguez-Perles, E. Gallardo, A. García, R. García-Barredo, O. Combarros, I. Illa

University Hospital Marqués Valdecilla (Santander, ES); Hospital de la Santa Creu i Sant Pau (Barcelona, ES)

Objective: To describe the clinical phenotype of an autosomal dominant pedigree with myotilinopathy.

Methods: Two symptomatic patients and six asymptomatic gene mutation carriers were examined. We performed serum chemistry, electrophysiological study, magnetic resonance imaging (MRI) of lower limb musculature, histochemical and immunohistochemical study on a muscle biopsy, and mutation analysis of the myotilin gene.

Results: Both symptomatic patients, aged 76 and 61, presented with late onset distal lower limb weakness involving ankle and toe flexo-extensor muscles extending up to thigh muscles; there was just mild weakness of intrinsic hand musculature in the elder patient. Electromyography revealed a myopathic pattern. Serum creatin kinase levels were mildly elevated. Muscle biopsy revealed myopathic changes with myotilin- and desmin-positive aggregates. Gene sequencing identified a myotilin S55F mutation. In both patients MRI showed moderate to severe fatty atrophy of all four leg muscle compartments extending up to thigh musculature mainly involving biceps femoris, semimembranosus, vasti and glutei; intrinsic foot musculature was involved but to a lesser degree. In all six gene mutation carriers, aged from 21 to 63 , clinical examinations showed no myopathic signs. MRI was normal in the youngest individual, whereas in the remaining five the outstanding finding was fatty infiltration of the soleus muscles.

Conclusions: Myotilin S55F mutation may cause a clinically distinct autosomal dominant late onset and lower limb onset distal myopathic syndrome involving all four leg muscle compartments. MRI helps reliably depict the topography of muscle fatty atrophy and detect early leg muscle changes in asymptomatic gene mutation carriers.

Supported by CIBERNED (ISCIII, Madrid, Spain)

0148

Central core disease with adult presentation: case reports and review of the literature

S. Duarte, I. Conceição, C. Barroso, R. Cerqueira, A. Fernandes, P. Tavares, T. Evangelista

Garcia de Orta Hospital (Almada, PT); Santa Maria Hospital (Lisbon, PT); Clinical Genetics Center (Oporto, PT)

Objectives: Central core disease is an inherited congenital myopathy, allelic to malignant hyperthermia, usually caused by mutations of the RYR 1 gene, on chromosome 19q13.1. Multiminicore/multicore disease is a distinguishable entity that has some clinical, histological and genetic overlap with central core miopathy, but is classically related with mutations of the SEPN 1 gene. Adult onset is atypical and there is some variability on clinical symptoms that could be explained by genetic heterogeneity. We aimed to report 6 patients, in whom the disease was detected in adulthood, describing clinical, histological and genetic data and to review the previously reported cases.

Methods: We reanalysed muscular biopsies with suspected central core/multiminicore disease features, studied the respective clinical files and performed genetic studies. A Pubmed search was done, in order to find reports of patients who were diagnosed during adulthood. 
Results: 4 patients had high creatine phosphokinase (CPK) levels, between 270 and $601 \mathrm{UI} / \mathrm{L} .3$ patients complained of myalgia and 1 patient had proximal lower limb weakness. 3 patients had personal or familiar history of serious adverse reactions during anaesthetic procedures, leading to death in one case. Both cases with a documented mutation had associated rheumatologic complaints: generalized arthralgia and fatigue in one patient, systemic lupus in another.

Muscular biopsy showed unspecific findings in most patients (4/6), namely variation in fiber size, nuclear centralizations and type 1 fiber predominance. Enzymatic reactions disclosed areas of absent activity, corresponding to the cores and/or multiminicores. Additionally, desmin immunocytochemical study revealed antibody deposition co-localized with cores. Two mutations of RYR 1 gene were found: c.14228G $>$ A (p.Gly4743Asp), exon 6 and c.479A>G (p.Glu160Gly) exon 98. RYR1 and SEPN1 genes sequenciation is now in course, in those patients without positive genetic study. Regarding the literature review, the most frequent symptom was proximal muscular weakness, with myalgia and CPK elevation irregularly detected. 3 cases were associated with scoliosis and in 1 case, generalized muscular hypertrophy was present.

Conclusions: Although the adult clinical presentation of central core disease is usually mild, diagnosis should be suspected, and confirmed, in order to prevent potentially life-threatening reactions to general anaesthesia, affecting the patient or direct siblings.

\section{Oral session 16}

\section{General neurology 1}

\section{9}

Lumbar punctures in emergency medical departments: what are the diagnoses?

B. Majed, K. Senouci, H. Zephir, V. Pichonnier-Cassagne

Arras Hospital (Arras, FR); WHO (Lyon, FR); Roger Salengro Hospital (Lille, FR)

Introduction: The use of a lumbar puncture in Emergency Medical Departments (EMD) represents a necessary tool in several medical care situations. It notably permits the research of meningitis, subarachnoid hemorrhage or polyradiculoneuropathy. In literature, few data are available on the lumbar puncture effectiveness and the diagnoses found among patients benefiting from a lumbar puncture in EMD.

Material and methods: We conducted a retrospective study including all patients who benefited from a lumbar puncture in the adult emergency department of a general hospital (Arras, France) in 2004 and 2005. We included 247 patients. Demographic, clinical, biological and medical imagery data were collected and confronted to the final diagnosis retained.

Results: Median of patients' age was $52[\mathrm{q} 1=36-\mathrm{q} 3=72]$, sex ratio was 1.1. The lumbar puncture incidence, in relation to the number of admitted patients in the EMD in 2004 and 2005, was: 5.3 [6 - 4.7] for 1000 admissions per year. On 247 patients, the use of a lumbar puncture permitted to diagnose several lymphocytary meningitis $(8.5 \%)$, a few bacterial meningitis $(2.5 \%)$, a few acute inflammatory polyradiculoneuropathy ( $2 \%)$, one carcinomatous meningitis and one subarachnoid hemorrhage. Lymphocytary meningitis were more frequently observed among young patients (age under 35 ). When the lumbar puncture results were not contributive, the final diagnoses were essentially neurological diseases $(28 \%)$, infections other than in the nervous system (30\%) and benign headaches (14\%). Differential neurological diseases were more often observed among older patients (age above 35 ) and were mostly epilepsies (44\%) and strokes (24\%). Pulmonary $(34 \%)$ and urinary $(24 \%)$ infections represented the two main sites of infections outside from the nervous system. These infections were overrepresented among oldest patients (age above 65).

Conclusions: Lumbar punctures realized in EMD are contributive in less than $15 \%$ of the cases. Nonetheless, they permit to identify diseases that may compromise the vital prognosis. Regarding the frequency and the seriousness of the differential diagnoses, their research should not be neglected in order to improve lumbar punctures justifications and indications.
0150

Brainstem syndrome prevalence in recurrent neuromyelitis optica

M. Alvarenga, R. Papais-Alvarenga, S. Alves-Leon, O. Fernandez

UNIRIO (Rio de Janeiro, BR)

Neuromyelitis optica is characterized by optic neuritis and transverse myelitis separated by days, months or years with monophasic or recurrent clinical course. New diagnostic criteria (2006) allow clinical findings out of optic nerve and spinal cord. Very few data were published about the association of brainstem syndrome in this condition.

Objectives: To analyze clinical and radiological involvement of the brainstem in a cohort of recurrent neuromyelitis optica.

Methods: From 640 medical records of patients with primary demyelinating disease assisted in Hospital da Lagoa from 1985 to 2006, cases with NMO with recurrent course were selected. Demographics, clinical and radiological data were reviewed. Brainstem signs and symptoms were analyzed. The diagnostic criteria proposed by Mayo Clinic $(1999,2006)$ were applied. Patients with NMOR restricted to optic nerve and the spinal cord were compared to patients with NMOR with brainstem syndrome.

Results: 95 patients were included in the study $89,5 \%$ women and 56,9\% Afro Brazilians. Brainstem involvement occurred in 18 patients and were characterized by vertigo (7), trigeminal syndrome (6), ophthalmoplegia (6) bulbar syndrome (4), hypoacusia (2), facial palsy (2), vomiting (5), hiccups (3), hemi hypoestesia (3), pyramidal syndrome (1) and ataxia (1). Patients with brainstem syndrome were almost similar to those with the disease restricted to the optic nerve and spinal. In a median time of ten years 634 acute events occurred, 36 were brainstem syndrome. Fifty eight patients (61\%) full filled both Mayo Clinic criteria $(1999,2006)$.

Conclusion: Brainstem syndrome occurred in $18,95 \%$ in the cohort. Cranial magnetic resonance showed brainstem lesions in the majority of these cases.

\section{1}

Acute transverse myelitis - Clinical course and associated diseases M. Papais-Alvarenga, L. Thuler, R. Papais-Alvarenga, O. Fernandez UNIRIO (Rio de Janeiro, BR)

Objectives: To analyze the natural history of acute transverse myelitis (ATM) and to evaluate the prevalence of the syndrome among the idiopathic demyelinating diseases of the CNS; to compare monophasic and recurrent ATM; to analyze the value of supplementary tests for the diagnosis of this condition; and to list the comorbidities and factors associated with ATM.

Patients and methods: A retrospective, descriptive, population-based study was carried out, using the medical charts of 460 patients with demyelinating diseases who had received care between 2000 and 2006 at a referral center for the treatment of multiple sclerosis in Rio de Janeiro, Brazil. Patients with monophasic or recurrent ATM were selected for the study and their demographic, clinical and laboratory data were analyzed, particularly MRI of the brain and spine, evaluation of CSF, visual evoked response and NMO-IgG antibodies. Dysfunctions and disabilities were staged according to Kurtzke.

Results: Of the 45 cases of ATM, the majority were female ( $87.5 \%$ ), white $(63.2 \%)$ with the recurrent form of the disease $(58.4 \%)$ and a median age of 34.5 years (range 17-70 years) at the time of the first episode. Over a $30-$ month observation time of the disease (range 6-268 months), 95 episodes of ATM were identified, the majority partially affecting the spinal cord $(56.9 \%)$ at cervical level $(47 \%)$. At the last follow up most of the patients $(66.6 \%)$ were mildly disabled and their gait was unaffected. Monophasic forms are characterized by more severe motor dysfunction in the acute phase of initial myelitis and more severe sequelae; however, the long-term monophasic and recurrent forms evolve with mild disability. MRI of the spine during the acute phase was the most common method used for confirmation of diagnosis. The principal finding was an extensive lesion in T2 (52.3\%) with edema and enhancement $(45.5 \%)$ or extensive T2 lesion without edema $(6.8 \%)$. In three cases, acute myelitis mimicked neoplasia at MRI, resulting in spinal cord biopsy being carried out by neurosurgery, an erroneous and unnecessary procedure. NMO-IgG positivity was found in $25 \%$ of the cases tested, suggesting partial forms of neuromyelitis optica. Sjogren's syndrome and systemic lupus erythematosus (SLE) were diagnosed in two patients. Infections (respiratory infection and herpes zoster), vaccination and cocaine use were reported by four patients as having occurred in the recent past. 


\section{2}

Limbic encephalitis and VGKC-antibodies: the case for widening the clinical spectrum?

L. Zuliani, R. Vitaliani, M. Zoccarato, M. Spinazzi, V. Argentiero, F. Dainese, G.L. Gigli, D. Inzitari, B. Tavolato, A. Vincent, B. Giometto

University of Padua (Padua, IT); Hospital of Treviso (Treviso, IT); Hospital of Venice (Venice, IT); University of Udine (Udine, IT); University of Florence (Florence, IT); Oxford University (Oxford, UK)

Objectives: Limbic encephalitis (LE) is typically characterized by subacute development of disorientation, amnesia and seizures. LE is traditionally considered a paraneoplastic syndrome but in recent years voltage-gated potassium channel antibodies (VGKC-Abs) have been identified in cases of LE without tumour and with a good response to immunosuppressive treatments. The range of clinical presentations of VGKC-Ab-associated encephalitis is not well defined, and there may be under-recognition. Here we describe the clinical profile of 9 patients with LE associated with VGKC-Abs with a broad range of clinical features and additional signs.

Methods: We retrospectively collected clinical and immunological features in patients with possible autoimmune encephalopathy referred to our centre between 2005 and 2007 or whose sera and/or CSF were sent to our laboratory for the detection of onconeural antibodies in the same period, and who had raised VGKC-Abs $(>100 \mathrm{pM})$, detected by radioimmunoassay at Oxford University laboratory.

Results: The 9 collected patients ( 5 male, 4 female; age range 43-71 years) presented with a subacute history of memory loss, confusion and seizures. Behavioural changes were present in 5 out of 9 cases. Additional clinical features included: excessive secretions (2 cases), urinary incontinence (2), and gait disturbances (3). In 1 case there was dysautonomia, neuro-hormonal abnormalities and neuromuscular hyperexcitability. Brain MRI showed signal changes in the medial temporal lobes in 7 out of 9 cases while mild signs of inflammation in the CSF were present in 3 out of the 6 cases available. VGKC antibody titres were raised at onset (range 136-2460pM), in 3 cases with a low titre (100-400pM). Five patients clearly improved after immunosuppression, 3 patients presented poor improvement and 1 patient died due to lung cancer.

Conclusion: VGKC-Ab-associated LE is probably much more common than paraneoplastic LE but is still unrecognized. The full range of clinical presentations has not been established and can include additional features like gait disturbances and, frequently, signs of dysautonomia or hypothalamic involvement, which have not previously been clearly highlighted and may resemble those seen in Morvan's syndrome suggesting overlapping phenotypes. Subacute development of a subacute encephalopathy with or without typical features of LE must prompt the search for VGKC-Abs as many of the positive cases respond well to immunotherapies.

\section{3}

SOX1 antibodies in patients with paraneoplastic neuropathy

M. Tschernatsch, O. Gross, J. Hosch, T. Gerriets, N. Kneifel, C. Probst, I. Krasenbrink, S. Malas, M. Kaps, F. Blaes

Justus-Liebig-Universtiy (Giessen, DE); EUROIMMUN (Lubeck, DE); The Cyprus Institute of Neurology and Genetics (Nicosia, CY)

Objectives: Paraneoplastic neurological syndromes (PNS) are tumour-associated disorders, which are not caused by the tumour itself or its metastases. Since antineuronal autoantibodies can be detected in these patients, an autoimmune pathogenesis is suspected. Recently, a new anti-glial nuclear autoantibody (AGNA) directed against SOX1 has been described in PNS patients. This antibody shows a high specificity for paraneoplastic Lambert-Eaton-myasthenic-syndrome, and can be found in a lower amount in patients with anti-Hu positive PNS and small-cell lung cancer (SCLC) alone, respectively.

Methods: We tested sera from patients with paraneoplastic neuropathy, inflammatory neuropathy, neuropathy of unknown origin and healthy controls for the presence of anti-SOX1-antibodies using a Western Blot with recombinant SOX1 protein.

Results: 5/32 patients with paraneoplastic neuropathy and 4/22 patients with neuropathy of unknown origin showed reactivity against SOX1, whereas no patients with inflammatory neuropathy $(n=39)$ and no healthy controls $(n=27)$ showed any reactivity $(p=0.007)$. Four patients with paraneoplastic neuropathy had underlying SCLC, one patient had bronchial carcinoid. All patients with neuropathy of unknown origin where followed up for several years without diagnosis of a tumour so far, but with signs of a consuming or malignant process in one of them.

Conclusion: Therefore, anti-SOX1 antibodies are not only associated with paraneoplastic LEMS and SCLC, but as well with paraneoplastic neuropathies and may define another group of non-paraneoplastic immune neuropathies.
Since SOX1 has been proposed to be a key factor for maintaining the neural stem cell state, it is tempting to speculate that the underlying cause of PNS could be an auto-immune response against adult neural stem cells with glial properties, such as certain astrocytes.

\section{4}

The Syrinx protocol: preoperative diagnostics and surgical management in syringomyelia

F. Roser, F.H. Ebner, M. Tatagiba

University of Tubingen (Tubingen, DE)

Objective: Minimal spinal trauma as the cause of syringomyelia is commonly neglected in the management of this potentially disabling disease. However the restoration of adequate CSF-circulation can lead to cessation of disease progression. We present an optimized preoperative diagnostic protocol for syringomyelia patients in order to detect the underlying pathology.

Methods and Results: Syringomyelia patients undergo a standardized workup including electrophysiological measurements (SEP and MEP) as well as silent-period testing for detection of alteration in A delta-pain fibres. Beside routine MRI-diagnostics sagittal cardiac-gated sequences (CINE) and sagittal 3D-constructive-interference-in-steady-state (CISS) sequences, were performed. An indication for neurosurgical intervention emerges if a focal adhesion is detected. The operation aims to decompress the subarachnoid space including an enlarging duroplasty.

Results: 57 patients with syringomyelia prospectively underwent the diagnostic protocol. With the expanded electrophysiological workup, a differentiation between hydromyelia (dilated central canal) and syringomyelia with alteration of crossing spinal cord tracts can be made. The sensitivities/specificities of the silent-periods for several syringomyelia symptoms (e.g. dysaesthesia, pain) are significantly higher than of SEP/MEP recordings. With the specialized MRI-diagnostics $24 \%$ more patients could be diagnosed with the syringomyelia-causing pathology: A spinal arachnoid web, cyst or scar developed through a former spinal trauma. All treated patients showed an immediate collapse of the syrinx, neurological symptoms did improve; however in most cases long-standing neurological deficits remain stable.

Conclusion: Syringomyelia should be seen as a symptom of an underlying arachnoid process, most often caused by a minor spinal trauma with scar formation causing CSF-flow obstruction. Neurosurgical detection and restoration of adequate CSF-flow can stop the progredient course of the disease.

This study was supported by a grant from Else-Kröner Fresenius Stiftung, Bad Homburg, Germany (P47/06//A34/06).

\section{5}

Outcome of vegetative and minimally conscious states: results from the Belgian Federal expertise network

D. Ledoux, M-A. Bruno, C. Schnakers, J. Giacino, M. Ventura, L. Vanopdenbosch, E. Peeters, E. Lannoo, T. Willemart, S. Laureys

Coma Science Group (Liege, BE)

Objectives: Progress of intensive care has increased the number of patients surviving severe acute brain damage. These patients classically progress through different stages before fully or partly recovering consciousness. Following severe acute brain damage, some of these patients recover from their coma to a vegetative state (VS; ie wakefulness without awareness) within four weeks after the injury.VS patients who recover, typically transit through minimally conscious state (MCS). There is however no predictive model of recovery from the VS and the MCS. A better understanding of patients' outcome would help in decisions about care, rehabilitation and end-of-life decisions. In the present study, we tried to establish reliable guidelines to predict outcome in these patients.

Methods: The Belgian Federal Public Service Health, Food Chain Safety and Environment build a centralized database allowing standardized recording which include demographic information, injury acute care history and longitudinal following of VS and MCS patients admitted to 14 expert centers. In this database, data were available from 200 patients. $116 \mathrm{VS}$ patients (52 traumatic and 64 non-traumatic) and 84 MCS patients ( 35 traumatic and 49 non-traumatic). These patients were evaluated at 1, 3, 6 et 12 months post injury and the diagnosis was based on internationally accepted criteria of VS and MCS [1].

Results: Traumatic patients had a better outcome than non-traumatic: $72 \%$ of traumatic versus $24 \%$ non-traumatic patients recovered consciousness. After a traumatic or non-traumatic brain injury, patients who were in MCS one month after insult were more likely than patients in VS to recover consciousness and functional communication. Among traumatic brain in- 
jured patients, $48 \%$ of patients MCS at one month recovered versus $23 \%$ of VS patients. For patients who suffered from non-traumatic brain damage, $26 \%$ of patients MCS one month after injury recovered versus $1 \%$ of VS patients.

Conclusion: These results confirm that patients with traumatic etiology have better prognosis than patients with non-traumatic causes. Moreover, our study highlights that outcome is significantly better for patients who are MCS at one month post-acute injury as compared to patients remaining VS at that time.

1. Giacino, J.T., et al. (2002). The minimally conscious state: Definition and diagnostic criteria. Neurology 58:349-353.

Belgian Federal Public Service Health, Food Chain Safety and Environment

\section{Oral session 17}

\section{Multiple sclerosis 3}

\section{6}

Cognitive dysfunction in benign multiple sclerosis is associated with increased severity of corpus callosum damage

S. Mesaros, M. Rovaris, E. Pagani, D. Caputo, A. Ghezzi, R. Capra, A. Bertolotto, G. Riccitelli, V. Martinelli, G. Comi, M. Filippi

Scientific Institute and University HSR (Milan, IT); Scientific Institute Don Gnocchi (Milan, IT); Ospedale di Gallarate (Gallarate, IT); Ospedale Spedali Civili (Brescia, IT); Ospedale di Orbassano (Orbassano, IT)

Objective: In benign MS (BMS), cognitive dysfunction is associated with an increased severity of conventional MRI-occult white and grey matter damage. Based on the directional information provided by the proton self-diffusion measured with diffusion tensor (DT) MRI, we have developed a method which enables us to track and investigate clinically eloquent pathways involved in cognitive performances, such as corpus callosum (CC). The aim of the study was to investigate the relationship between the cognitive profile of BMS patients and the extent of tissue damage in the CC.

Methods: Conventional and DT MRI scans of the brain were acquired from 54 BMS patients (defined as having a disease duration of 15 years or more and an Expanded Disability Status Scale (EDSS) score of 3.0 or less). Neuropsychological tests (NPT) exploring memory, attention and frontal lobe cognitive domains were administered. DT MRI tractography was used to calculate the mean diffusivity (MD) and fractional anisotropy (FA) values from the CC. An index of CC atrophy was also computed.

Results: Nine BMS patients (17\%) had an abnormal performance in three or more NPT, thus fulfilling pre-defined criteria for cognitive impairment (CI). There was no differences in CC atrophy index between cognitively impaired and unimpaired BMS patients. Patients with CI had significantly higher CC lesion load, and more pronounced MD abnormalities, than those without. There were significant correlations between the results of NPT exploring executive functions (mainly the PASAT test), and measures of FA and $\mathrm{MD}$ features from the $\mathrm{CC}$ ( $\mathrm{r}$ values ranged from 0.31 to 0.52 ).

Conclusions: In BMS, cognitive dysfunction is associated with an increased severity of CC damage, in terms of both discrete lesions and fibre bundle disruption. Assessing regional damage with DT MRI may represent a rewarding strategy for a better understanding of BMS profile.

Acknowledgment: This work was supported by ENS Fellowship (Dr S. Mesaros).

\section{7}

Anti-CD20 B cell depletion in immune intervention of central nervous system autoimmune disease

M.S. Weber, T. Prod'homme, T. Karnezis, J.C. Patarroyo, B. Hemmer, C. Linington, C.C.A.. Bernard, F. Martin, S.S. Zamvil

University of California (San Francisco, US); Monash University (Melbourne, AU); Technical University (Munich, DE); University of Aberdeen (Aberdeen, UK); Genentech (South San Francisco, US)

Objective: Based upon findings indicating that both B cells and myelin-specific antibodies $(\mathrm{ab})$ have important pathogenic roles in multiple sclerosis (MS), substantial interest has developed for testing anti-CD20-mediated B cell depletion in MS. However, some data indicate that B cells may also have a regulatory, protective role in CNS autoimmunity. We investigated anti-
CD20 treatment in two distinct murine models of MS: 1) in experimental autoimmune encephalomyelitis (EAE) induced by myelin oligodendrocyte glycoprotein (MOG) p35-55, which does not require B cells for its induction, and 2) in recombinant (r) mouse MOG 1-117-induced EAE, in which B cells are activated.

Methods: EAE was induced in human (h)CD20-transgenic C57BL/6 mice in which anti-hCD20 depletes B cells. Mice received weekly intraperitoneal injections of anti-hCD20 or isotype starting either 21 days prior to immunization, or after onset of paralysis. Clinical and histopathological parameters as well as myelin-specific T cell responses and ab titers were evaluated in all mice.

Results: B cell depletion had beneficial effects in prevention and reversal of EAE induced by rMOG protein which was associated with a decrease in CNS inflammation, frequency of CNS B cells and myelin-specific ab. The frequency of Th1 and Th17 cells, as well as FoxP3+ regulatory T cells (Treg), was also reduced. Remaining antigen-presenting cells (APC) from B cell-depleted mice were incapable to promote Th1 and Th17 differentiation of myelin-specific $\mathrm{T}$ cells in vitro.

In contrast, B-cell depletion exacerbated MOG p35-55-induced EAE with enhanced CNS-infiltration and demyelination despite decreased numbers of CNS-infiltrating B cells. While the number of Th1 and Th17 cells remained unchanged, the frequency of Treg was reduced in $B$ cell-depleted mice. When used as APC, B cells from MOG p35-55-immunized mice, which had remained naïve, could not present MOG protein. $B$ cells from mice immunized with MOG protein however, displayed a proinflammatory phenotype and stimulated naive MOG-specific T cells to proliferate to MOG protein.

Conclusions: These findings indicate that myelin-specific B cells have an important role in processing and presentation of intact myelin to encephalitogenic $\mathrm{T}$ cells whereas naïve $\mathrm{B}$ cells may support $\mathrm{T}$ cell regulation. Taken together, our data support the therapeutic potential of anti-CD20-mediated depletion of antigen-activated B cells in CNS autoimmune disease.

0158

High proportion of patients free from disease activity in all 3 arms of the high-dose Betaferon ${ }^{\circledR}$ trial

L. Kappos, B.G.W. Arnason, G. Comi, S. Cook, M. Filippi, D.S. Goodin, H-P. Hartung, D.R. Jeffery, P. O'Connor, T. Bogumil, B. Stemper, V. Filipov, M. Groth, F. Boateng

University Hospital (Basel, CH); Surgery Brain Research Institutes (Chicago, US); Hospital San Raffaele (Milan, IT); UMD New Jersey Medical School (Newark, US); University of California at San Francisco (San Francisco, US); Heinrich-Heine-Universität (Dusseldorf, DE); Wake Forest University School of Medicine (Winston-Salem, US); St Michael's Hospital (Toronto, CA); Bayer Schering Pharma AG (Montville, US); Bayer Schering Pharma AG (Berlin, DE)

Objective: To investigate the number of patients with relapsing-remitting multiple sclerosis (RRMS) who were free from relapse and magnetic resonance imaging (MRI) activity over a period of $\geq 104$ weeks while treated with interferon beta-1b (IFNB-1b; Betaferon(R)) $500 \mathrm{mcg}$, IFNB-1b $250 \mathrm{mcg}$ or glatiramer acetate $(\mathrm{GA}$; Copaxone $(\mathrm{R}))$.

Methods: The Betaferon(R)/Betaseron(R) Efficacy Yielding Outcomes of a New Dose study examined the safety, tolerability and efficacy of subcutaneous IFNB-1b $500 \mathrm{mcg}, 250 \mathrm{mcg}$ or GA in the treatment of RRMS. RRMS patients with Expanded Disability Status Scale (EDSS) scores $\leq 5.0$ were randomised in a 2:2:1 ratio to subcutaneous IFNB-1b $500 \mathrm{mcg}$ or $250 \mathrm{mcg}$ every other day, or GA $20 \mathrm{mg}$ daily for ?104 weeks. The primary efficacy variable was relapse risk; other pre-defined endpoints included the proportion of patients free from disease activity, which was defined as being relapse-free and having no new T2 lesions or no new Gd-enhancing lesions post baseline.

Results: 2244 patients from 198 study centres worldwide were allocated to IFNB-1b $500 \mathrm{mcg}(\mathrm{n}=899)$, IFNB-1b $250 \mathrm{mcg}(\mathrm{n}=897)$ or GA $(\mathrm{n}=448)$. Mean baseline characteristics were comparable in all groups. No significant differences were found between the three groups for the primary endpoint. At study termination the proportions of patients from each treatment arm who were free from disease activity for at least 104 weeks were $31.5 \%$ in the IFNB- $1 \mathrm{~b} 500 \mathrm{mcg}$ group ( $\mathrm{p}=0.007$ vs $250 \mathrm{mcg}$; $\mathrm{p}=0.031$ vs $\mathrm{GA}$ ), $25.6 \%$ in the IFNB- $1 \mathrm{~b} 250 \mathrm{mcg}$ group ( $\mathrm{p}=1.000$ vs GA) and $25.7 \%$ in the GA group.

Conclusions: In this study a similarly high proportion of patients in each treatment arm was free from disease activity, with some advantage for the higher IFNB-1b dose. The difference favouring the $500 \mathrm{mcg}$ group was likely mainly due to a more pronounced effect on gadolinium-enhancing lesions and new or enlarging T2 lesions. The clinical relevance of this finding was not substantiated during the observation period of this study.

Study supported by: Bayer Schering Pharma AG, Berlin, Germany 


\section{9}

Complete clinical and magnetic resonance imaging response with natalizumab in multiple sclerosis

C. Confavreux, S.L. Galetta, G. Giovannoni, E. Havrdova, M. Hutchinson, F.D. Lublin, P.W. O'Connor, J.T. Phillips, C.H. Polman, R.A. Rudick, A. Pace, R. Hyde, M.A. Panzara

Hopital Neurologique (Lyon, FR); University of Pennsylvania School of Medicine (Philadelphia, US); Barts and The London Queen Mary's School of Medicine and Dentistry (London, UK); Charles University (Prague, CZ); St. Vincent's University Hospital (Dublin, IE); Mt. Sinai School of Medicine (New York, US); St. Michael's Hospital (Toronto, CA); The Multiple Sclerosis Center at Texas Neurology (Dallas, US); VU Medical Centre (Amsterdam, NL); Cleveland Clinic (Cleveland, US); Biogen Idec, Inc. (Cambridge, US)

Objectives: Patients with multiple sclerosis (MS) may continue to experience disease activity despite treatment with traditional disease-modifying therapies. As more effective therapies become available, complete response to treatment becomes a realistic possibility. Natalizumab (TYSABRI ${ }^{\circledR}$ ) reduced relapse rates by up to $68 \%$ and the risk of sustained disability progression by up to $54 \%$ in phase 3 clinical studies in patients with relapsing MS. The objective of this analysis was to assess the ability of natalizumab to increase the proportion of patients free of clinical and MRI disease activity over 2 years.

Methods: Disease activity according to clinical and magnetic resonance imaging (MRI) criteria was evaluated in patients treated with natalizumab or placebo in the phase 3 studies of natalizumab monotherapy (AFFIRM) or natalizumab/interferon beta-1a combination therapy (SENTINEL). Patients with no relapses or disability progression sustained for 12 weeks were classified as having no clinical disease activity; those with no gadolinium-enhancing or new/enlarging T2-hyperintense lesions were classified as having no MRI disease activity; those with neither clinical nor MRI disease activity fulfilled the more stringent combination criteria. The percentage of patients with no disease activity was also analysed in subgroups of patients with highly active (ie, $\geq 2$ relapses in the year before study entry and $\geq 1$ baseline gadolinium-enhancing lesion) and non-highly active disease.

Results: Natalizumab resulted in a significantly higher proportion of patients free of disease activity over 2 years compared with placebo on clinical (AFFIRM: $64.3 \%$ vs $38.9 \%$; SENTINEL: $47.4 \%$ vs $28.0 \%$ ), MRI (AFFIRM: $57.7 \%$ vs $14.2 \%$; SENTINEL: $65.5 \%$ vs $27.6 \%$ ), and combined clinical and MRI (AFFIRM: $36.7 \%$ vs $7.2 \%$; SENTINEL: $31.7 \%$ vs $10.9 \%$ ) measures $(\mathrm{P}<0.0001$ for all comparisons). Highly active and non-highly active disease subgroups demonstrated similar results (all $\mathrm{P}<0.005$ )

Conclusion: Natalizumab significantly increased the proportions of patients free of clinical and MRI disease activity over 2 years compared with placebo regardless of baseline disease activity. Assessments of complete clinical and MRI response in clinical studies of MS therapies, similar to those used in studies of other immune disorders, will provide additional insight into treatment efficacy.

Study supported by Biogen Idec, Inc. and Elan Pharmaceuticals, Inc.

\section{0}

Prevention of conversion of clinically isolated syndromes to clinically defined multiple sclerosis. Evidence from a Cochrane meta-analysis M. Clerico, F. Faggiano, M. Tintore' Subirana, J. Palace, G. Rice, L. Durelli University of Turin (Orbassano, IT); Public Health Department (Novara, IT); Neuroimmunology Unit (Barcelona, ES); Radcliff Infirmary Hospital (Oxford, UK); Neurology Clinic (London, CA)

Objective: Interferon (IFN) beta therapy has been shown by a Cochrane metanalysis. to have a limited efficacy, not extended over the first year of followup, when administered in relapsing remitting multiple sclerosis (RRMS) patients years after the diagnosis. The aim of this metanalsys is to assess the efficacy of very early IFN beta therapy, given at the time of the first episode suggestive of MS

Design/method: We applied the Cochrane criteria for considering studies: types of studies (including only RCT studies), types of participants, interventions, outcome measures. We performed the search strategies for identifying studies through medical literature, data bases and abstracts books. Two primary outcomes were defined: the number of patients converting to clinically defined MS (CDMS), defined by the occurrence of a second clinical episode, and the number of those with side effects.

Results: 454 papers identified; 430 not eligible; 7 referred to three studies: CHAMPS and ETOMS (6 papers) both using once-weekly low-dose (22 or $30 \mathrm{mcg}$ ) IFN beta 1a; BENEFIT (1 papers) using multiple weekly $250 \mathrm{mcg}$ IFN beta 1b. The three studies had different outcomes (occurrence of a second clinical episode or of disease progression in CHAMPS; occurrence of a second clinical episode in ETOMS; time to CDMS in BENEFIT). Per protocol analyses: IFN beta treatment significantly prevented conversion to CDMS in all studies. The metanalysis at 1 year, made taking off the 5 CHAMPS patients whose conversion was due to progression, was significant in per protocol analysis and in all sensitivity analyses but the "worst scenario". The metanalysis at 2 years, which was possible only for ETOMS and BENEFIT and which was made taking off the 2 BENEFIT patients, whose conversion was due to progression, was significant both in per protocol as well as all sensitivity analyses. The metanalysis on the number of patients with serious side effects did not show any significant difference comparing IFN-and placebotreated patients.

Conclusion: Our analysis showed that the efficacy of IFN beta treatment extended both over the first as well as over the second year of treatment. The persistency of IFN beta treatment over time has been shown for early starting IFN beta treatment. IFN beta treatment in the earliest stage of MS seems to have a more persistent effect compared to treatment started later.

\section{1}

Phase I safety study update of RTL1000, a recombinant T-cell receptor ligand specific for an immunodominant MOG peptide, in multiple sclerosis A. Vandenbark, G. Burrows, E. Ravey, H. Offner Portland VA Medical Center (Portland, US)

Objectives: A Recombinant T-Cell Receptor Ligand (RTL1000 = covalently linked HLA-DR2 alpha-1 \& beta-1 domains with attached myelin oligodendrocyte protein (MOG)-35-55 peptide) was developed as a T-cell specific therapy for multiple sclerosis (MS). The primary objective of this study is to evaluate the safety profile of a single intravenous (IV) dose of RTL1000, as assessed by clinical examinations \& magnetic resonance imaging (MRI). Additionally, the study will evaluate its biological activity, specificity \& pharmacokinetics.

Methods: RTL is currently being evaluated in a first-in-human doubleblind, placebo-controlled, Phase 1, dose escalation study of the safety of a single dose of RTL 1000 in relapsing and progressive MS subjects. The study design includes five cohorts (escalating doses of $2 \mathrm{mg}, 6 \mathrm{mg}, 20 \mathrm{mg}, 60 \mathrm{mg}$ and $200 \mathrm{mg}$ ), with six subjects per cohort (4 receiving drug, 2 receiving placebo). All subjects will be followed over 90 days for clinical and MRI changes. Blood samples collected over 28 days will be evaluated for RTL1000 pharmacokinetics, immunogenicity, and for changes in T cell responses to MOG peptide, other neuroantigen peptides and recall antigens. Safety data from each cohort will be reviewed by an independent Data Safety Monitoring Board (DSMB) prior to enrollment of the next cohort. Clinical sites: University of Maryland, College Park, OHSU, Portland, OR, Kansas University, Kansas City, Indiana University, Indianapolis, Yale University, New Haven, CT and Seattle Evergreen, Seattle, WA.

Results: The first two cohorts have been completed as of Jan, 2008, with the next cohort set to be enrolled in Feb, 2008 pending DSMB approval. RTL1000 was detectable in plasma in subjects receiving $6 \mathrm{mg}$ drug. Immunological studies indicate sufficient reactivity in a subset of subjects to MOG peptide at entry to allow assessment of RTL1000 effects to be ascertained when the study is unblinded at its completion. An update of unblinded data on characteristics of enrolled subjects, and pharmacokinetics, immunogenicity and immunological changes induced by RTL1000 will be presented and discussed.

Conclusions: Enrollment is continuing, subject to DSMB review and approval of each cohort. Assays have been developed that can evaluate presence of RTL1000 in plasma, changes in antibodies to RTL1000 and changes in MOG-specific T cells in blood after injection, thus enhancing likelihood of achieving safety, pharmacokinetic, and immunological objectives.

Dr. Vandenbark, Dr. Burrows and Dr. Offner have a significant financial interest in Artielle ImmunoTherapeutics, Inc., a company that has a commercial interest in the results of this research. 


\section{Oral session 18}

\section{Higher function disorders 1}

\section{2}

Spatial correlation between aerobic glycolysis and beta-amyloid deposition A.G. Vlassenko, S.N. Vaishnavi, M.M. Rundle, L.E. Couture, A.Z. Snyder, J.C. Morris, M.E. Raichle, M.A. Mintun

Washington University School of Medicine (St. Louis, US)

Objectives: The distribution of beta-amyloid plaques in patients with dementia of the Alzheimer type (DAT), visualized using positron emission tomography (PET) and the tracer [11C]PIB, has great spatial overlap with the "default network", which is a constellation of brain regions that is more active at rest than during goal-directed task performance and may be selectively affected in DAT. Metabolism in the default network is characterized by elevated levels of aerobic glycolysis at rest. We suggest that chronic elevation of glycolysis throughout life may be associated with higher regional vulnerability resulting in increased beta-amyloid deposition. In this study, we compared the distribution of beta-amyloid plaques in patients with DAT with the distribution of aerobic glycolysis derived by metabolic imaging of young adults.

Methods: PET images were acquired in 11 DAT patients (mean age $79 \pm 5$ yrs) 30 to 60 minutes after injection of [11C]PIB. The distribution of aerobic glycolysis was imaged in 33 healthy young adults (mean age $25 \pm 3$ years) by combining relative estimates of oxygen utilization (CMRO2) and glucose utilization (CMRGlc). Regional CMRO2 was computed based on the acquisition of three PET scans using three [15O] tracers ( $\mathrm{CO}, \mathrm{O} 2$ and $\mathrm{H} 2 \mathrm{O})$. The relative distribution CMRGlc was estimated using [18F]FDG PET. Images of aerobic glycolysis expressed as the glycolytic index (GI) were generated by calculating pixel-by-pixel the residual after regression of CMRGlc on CMRO2, which corresponds to the amount of glucose utilization that differs from what is predicted by the amount of oxygen utilization. For correlation analyses between [11C]PIB and GI, CMRGlc and CMRO2, the entire brain was sampled using adjacent non-overlapping $14 \mathrm{~mm}$ cubes $(\mathrm{n}=194)$.

Results: GI was low in areas with low PIB binding (e.g., cerebellum) and high in areas of high PIB binding (e.g., posterior cingulate and prefrontal cortex). The mean images of [11C]PIB binding and GI were highly correlated $(\mathrm{r}=0.73, \mathrm{p}<0.0001)$, while correlation of [11C]PIB to CMRGlc and CMRO2 was much lower $(r=0.34$ and 0.036 , respectively).

Conclusion: The close spatial relationship between resting aerobic glycolysis in young adults and deposition of beta-amyloid plaques in DAT patients suggests that brain regions shown to have elevation of aerobic glycolysis at rest may spend more time in this state over the lifetime and it may be related to the later development of Alzheimer's pathology.

NIH Grants P50 AG05681; P50 NS006833; P01 AG003991; P30 NS048056

\section{3}

Stutterers' way out of dysfluency: effects of stuttering therapy on brain activity during production of prosodic speech

C.A. Kell, K. Neumann, A.L. Giraud

Johann Wolfgang Goethe University (Frankfurt, DE); Ecole Normale Superieure (Paris, FR)

Objectives: Stuttering most probably rests upon a cortical mal-development of the left inferior frontal gyrus (IFG) during early childhood [1] asking for behavioural fluency-inducing therapy. A relevant therapeutic aspect is modification of the stutterers' prosody, which enhances fluency. The underlying mechanisms of this beneficial effect have not been studied. We set out to delineate the networks involved in production of prosodic speech in normal controls and stutterers before, during and after an intensive fluency-shaping program using functional magnetic resonance imaging (fMRI).

Methods: 13 fluent controls were matched to 13 male German stutterers who underwent the Kassel Stuttering therapy program. Subjects were scanned before therapy, after the three weeks intensive course, and one year later after attending three refresher courses, and performing bio-feedback training at home, on a 3T MRI scanner by acquiring a standard echoplanar imaging sequence. Written syntactically balanced sentences were presented for three seconds in a pseudo-randomised order, preceded by an auditory instruction that indicated whether the sentence had to be read aloud with a normal voice, happy intonation (affective prosody), or pronounced as a question (linguistic prosody). fMRI data underwent a standard SPM5 whole brain analysis. We report interactions between group and task with a threshold of $\mathrm{p}<0.001$, uncorrected.
Results: Stutterers improved prosody and fluency over the year. In controls, production of linguistic and affective prosody produced a stronger activation of the left IFG, anterior insula, and right cerebellum compared to normal speech. Before therapy, stutterers activated the left anterior insula and IFG significantly less than fluent controls for both production of linguistic and affective prosody. After the intensive course, stutterers still hypoactivated this region during production of linguistic prosody, while activation during production of affective prosody normalised. One year later, also activation during production of linguistic prosody normalised. Potentially compensatory regions were the bilateral ventral striatum for production of linguistic and left cerebellum for production of affective prosody.

Conclusion: Stutterers activate only part of the left anterior insula/IFG mobilised by fluent controls during generation of prosody. Therapy might normalise this deficit by subcortical neural adaptation.

1 Chang et al. (2008) NeuroImage 39

This study was supported by the German Research couincil and the German Ministry for Education and Research

\section{4}

Genetic variability in tau phosphorylation-related genes and the risk of Alzheimer's disease

I. Mateo, J.L. Vazquez, E. Rodriguez-Rodriguez, J. Infante, P. Sanchez-Juan, J.M. Polo, J. Berciano, O. Combarros

University Hospital "Marqués Valdecilla" (Santander, ES)

Background: Tau is a microtubule-associated protein that stabilizes neuronal microtubules. Abnormal tau hyperphosphorylation has been suggested as being one of the central events in the development of neurofibrillary tangles, a hallmark lesion in Alzheimer's disease (AD) brains. Although different kinases may phosphorylate tau, a major role has been assigned to GSK3b and CDK5.14-3-3 zeta is a tau chaperone which stimulates tau phosphorylation by connecting GSK3b to tau. Moreover, CDK5 and its activator p35 (CDK5R1 gene) could act like a priming kinase for GSK3b. Saitohin (STH) is a polymorphic gene nested within an intron of the human tau gene, and it encodes a protein with a tissue expression very similar to tau.

Objective: We investigated 12 polymorphisms across 6 genes involved in tau phosphorylation (Tau, GSK3b, CDK5, CDK5R1, 14-3-3 zeta and STH genes). Seven of these polymorphisms have been reported to be associated with $\mathrm{AD}$ in previous single-gene studies.

Methods: The study included $333 \mathrm{AD}$ patients (67\% women; mean age at onset 72.06; SD 8.62; range 40-95 years) who met NINCDS/ADRDA criteria for probable AD. Control subjects were 307 unrelated individuals $(70 \%$ women; mean age 80.48; SD 7.82; range 63-100 years) with Mini-Mental State Examination scores of 28 or more, which were verified by at least one subsequent annual follow-up assessment.

Results: GSK3b (-50) TT and STH (Q7R) RR genotypes are associated with an increased risk (OR 1.99, $\mathrm{p}=0.003$ and OR $2.17, \mathrm{p}=0.04$, respectively) for late onset $\mathrm{AD}$. There is a two and a half-fold decrease in $\mathrm{AD}$ risk associated with both homozygosity for 14-3-3 zeta (rs983583) A allele and homozygosity for tau (rs2471738) C allele. However, genetic variants of the tau (rs242557, rs242559, rs7521, H1/H2), CDK5 (rs9278, rs2069454), CDK5R1 (rs735555) and 14-3-3 zeta (rs964917) genes are not associated with AD.

Conclusions: Genetic variability in the tau phosphorylation pathway may contribute to the risk of $\mathrm{AD}$.

This work was supported by CIBERNED (ISC III, Madrid, Spain)

\section{5}

Apolipoprotein E genotype and grey matter atrophy in frontotemporal dementia and Alzheimer's disease: a disease-specific regional effect F. Agosta, K. Vossel, A. Karydas, A. Boxer, S. Bonasera, B.L. Miller, M.L. Gorno-Tempini

Memory and Aging Center, UCSF (San Francisco, US)

Objectives: The effect of Apolipoprotein E (ApoE) genotype on brain atrophy in frontotemporal dementia (FTD) has not been clarified. In this study, we compare the effect of the e4 allele of ApoE on the pattern of gray matter (GM) atrophy in clinical behavioral variant FTD and Alzheimer's disease (AD) at presentation.

Methods: T1-weighted images were obtained from 32 patients with behavioral variant FTD (mean age $=59$ years, median Clinical Dementia Rating $[\mathrm{CDR}]$ rating $=1.0), 56$ patients with $\mathrm{AD}$ (mean age $=66$ years, median $\mathrm{CDR}=1.0$ ), and 62 healthy controls (mean age $=65$ years). ApoE genotyping was obtained using standard procedures. Voxel-based morphometry, in Statistical Parametric Mapping (SPM5), was used to assess GM atrophy in patients carrying e4 compared to controls and non-carriers in each patient's 
group. Age, gender, total intracranial volume, and disease severity were entered into the statistical analysis as nuisance variables.

Results: ApoE e4 allele carriers were $8(25 \%)$ within the FTD group, 30 (53.6\%) within the $\mathrm{AD}$ group, and 12 (19.3\%) within controls. There was no difference in age, gender, and disease severity between carriers and non-carriers in each group. Compared to controls and FTD non-carriers, FTD patients carrying e4 allele showed greater atrophy in a large area including medial and dorsolateral frontal cortex and anterior insula bilaterally. When compared to controls and $\mathrm{AD}$ non-carriers, $\mathrm{AD}$ patients carrying e4 showed greater atrophy in the left hippocampus and precuneus, and in the bilateral parietal cortex.

Conclusions: Our study showed that ApoE e4 is associated with a more severe pattern of GM loss in disease-specific regions in patients with both behavioral variant FTD and AD at presentation. These findings are consistent with the hypothesis that ApoE may have a role in modulating the morphologic expression of all neurodegenerative disease.

\section{6}

A neuronal migration regulator Reelin pathway may be involved in the white matter pathology in Alzheimer's disease

K. Deguchi, B. Antalffy, H. Takei, S. Powell, R. Yamamoto, G. D'Arcangelo, K. Inoue

Deguchi Pediatric Clinic (Nagasaki, JP); Baylor College of Medicine (Houston, US); The Methodist Hospital (Houston, US); National Center of Neurology \& Psychiatry (Tokyo, JP); Rutgers, State University of New Jersey (New Jersey, US)

Objectives: Reelin and its signaling pathway are not only crucial during embryonic brain development for cellular organization of cortical structures, but are also important for the maturation of neuronal processes and synaptic function in the postnatal and adult brain. Loss of Reelin activity in the adult brain leads to defects in hippocampal-dependent learning and memory. We sought to determine if the Reelin pathway play significant role in the neuropathology of Alzheimer disease (AD).

Methods: We employed a tissue microarray composed of $28 \mathrm{AD}$ and 6 disease control brains and examined the expression of Reelin, Amyloid beta (Ab), and Tau proteins by immunohistochemistry. Additionally, we examined sections of $12 \mathrm{AD}$ and 8 control brains by immunohistochemistry. mRNA levels of Reelin and its signaling molecules were also measured by quantitative PCR in $8 \mathrm{AD}$ and 8 control brains.

Results: In normal adult brains Reelin protein expression was prominent in white matter axonal tracts. However, all AD samples showed reduced immunoreactivity (IR) for Reelin, whereas Ab and Tau expression appeared to be increased in the tissue microarray. Extensive neuropathological examination confirmed reduced IR of Reelin in AD brains. In contrast, we found that mRNA levels of Reelin and its signaling adaptor molecule DAB1 were increased in $\mathrm{AD}$ brains.

Discussion: Recent studies reported controversial results regarding the expression of Reelin in AD. Our study analyzed the expression of Reelin mRNA and protein in AD brain in a larger number of samples. Our data indicate that the transport of Reelin protein in axonal tracts may be reduced in $\mathrm{AD}$ while the mRNA levels increase suggested a potential disruption in the activity of this signaling pathway in AD pathology

\section{7}

Progressive cognitive syndromes associated with posterior atrophy

R. Migliaccio, F. Agosta, K. Rascovsky, B.L. Miller, M.L. Gorno-Tempini

Memory and Aging Center, UCSF (San Francisco, US); Second University of

Naples (Naples, IT); Scientific Institute and University HSR (Milan, IT)

Objective: To identify similarities and differences in the pattern of anatomical involvement at presentation in three progressive cognitive syndromes previously associated with posterior brain atrophy: posterior cortical atrophy (PCA), logopenic/phonological progressive aphasia (LPA), and early age-of-onset Alzheimer's disease (AD-EO) (without LPA or PCA).

Methods: Voxel-based morphometry was used to assess grey (GM) and white (WM) matter atrophy in $14 \mathrm{PCA}, 10 \mathrm{LPA}$, and $16 \mathrm{AD}$-EO patients, and 65 matched controls. A level of significance of $p<0.05$ corrected for multiple comparisons was accepted when comparing patients to controls, and of $\mathrm{p}<0.001$ uncorrected for direct patient-group comparisons.

Results: Each patient-group showed a large area of significant GM atrophy in largely overlapping bilateral parietal, occipital, posterior cingulate, precuneus, posterior temporal, and hippocampal regions, when compared to controls. Small areas of GM loss were also found in the frontal lobes and in the thalami bilaterally. A shared large area of WM atrophy including splenium of corpus callosum, and parietal and temporal lobes has been found. Direct group comparisons showed that each clinical group was also associ- ated with greater atrophy in symptom-specific regions: PCA in right ventraloccipital and superior parietal regions; LPA in left temporo-parietal junction; AD-EO in prefrontal cortex. Each group showed a specific pattern of WM atrophy strictly related to specific GM loss.

Conclusions: These three clinical syndromes were all associated with GM and WM atrophy in bilateral temporo-parietal-occipital cortices. Asymmetric left or right involvement of this network was associated with the aphasic or visuospatial presentations of PCA and LPA, respectively. The multiple cognitive domain involvement typical of AD-EO was associated with more symmetrical, bilateral posterior atrophy and greater involvement of prefrontal regions. It remains unknown whether the overlapping but distinctive patterns of posterior atrophy associated with these clinical syndromes are indicative of similar etiology.

\section{Oral session 19}

\section{Cerebrovascular disorders 3}

\section{8}

Women have a worse stroke outcome after thrombolysis

P. Martinez-Sanchez, B. Fuentes, M. Alonso de Leciñana, J. Masjuan, J. Egido, P. Simal, F. Diaz-Otero, A. Gil-Nuñez, E. Diez-Tejedor

Hospital Universitario La Paz (Madrid, ES); Hospital Universitario Ramon y Cajal (Madrid, ES); Hospital Universitario Clinico San Carlos (Madrid, ES); Hospital Universitario Gregorio Marañon (Madrid, ES)

Background: There are few studies analysing sex differences in outcome after thrombolitic treatment for acute ischemic stroke and they show contradictory results.

Methods: Multicentric observational study with acute stroke patients treated with endovenous rtPA in three university hospitals (2003-2006). We analysed the following variables by gender: vascular risk factors, stroke severity (NIHSS), glycaemia and systolic blood pressure (SBP) previous to rtPA infusion; etiological stroke subtype, in-hospital complication (haemorrhagic transformation, brain oedema), mortality and 3-moths outcome by the modified Rankin Scale (mRS) score.

Results: 325 patients, 144 women. Mean age 65.6 years (SD 13.3). Women had a lower onset to treatment time $(\mathrm{P}=0.018)$, lower frequency of smoking $(\mathrm{P}<0.0001)$ as well as arterial origin strokes $(\mathrm{P}=0.001)$, but higher frequency of atrial fibrillation $(\mathrm{P}=0.007)$ and cardioembolic strokes $(\mathrm{P}=0.008)$ than men. At 3 -months, the $44.2 \%$ of women and the $28.8 \%$ of men had a mRS score $>3(\mathrm{P}=0.008)$. The logistic regression analysis showed that female (OR, $2.048 ; 95 \% \mathrm{CI}, 1.027$ to 4.081$)$ was a predictor of 3 -moths poor outcome; after adjustment for age, sex, previous vascular risk factors, glycaemia, SPB, stroke subtype, stroke severity and in-hospital complications.

Discussion: Female gender is a predictor of poor outcome after thrombolytic treatment for acute ischemic stroke.

0169

Anti-Hsp70 antibodies and serum lipids peroxidation in alcohol-dependent patients

D. Lenart-Jankowska, S. Michalak, W. Kozubski

Poznan University of Medical Sciences (Poznan, PL)

Objectives: The effects of alcohol intake are, among others, related to increased lipids peroxidation, which may further lead to elevated risk of stroke. On the other hand the very early period of abstinence is associated with higher risk of ischemic stroke. Both anti-Hsp antibodies and ox-LDL levels are recognized as new risk factors of cerebral ischemia. However, on the other side evidence has been forthcoming to suggest that some of oxidized lipids could elicit antioxidant and antiatherogenic responses from cells, as well. Among those effects induction of nitric oxide synthase, increased glutathione production and superoxide dismutase activation are emphasized. The aim of this study was to investigate the levels of anti-Hsp70 antibodies and indicators of plasma lipids peroxidation in alcohol-dependent patients.

Methods: Fifty adults, aged $47 \pm 9$ (39 males, 11 females) were recruited from Alcohol Dependence Policlinic in Poznan, Poland and all satisfied DSMIV criteria for alcohol-dependence syndrome. Five subgroups were examined depending on duration of abstinence: I-up to 1 month, II-2 to 3 
months, III- 4 months to 1 year, IV- 2 to 5 years, V-over 5 years. Serum antiHsp70 antibodies (Stressgen, USA) and ox-LDL levels (Mercodia, Sweden) were estimated by means of ELISA. Conjugated dienes (CD), malonyldialdehyde (MDA) and sulfhydryl groups (SG) concentrations in plasma was estimated by means of spectrophotometric methods.

Results: In alcohol-dependent patients anti-Hsp70 levels were below the cut-off value in all groups studied. Anti-Hsp70 levels correlated positively with ox-LDL concentration during 1 st month of abstinency $(\mathrm{rS}=-0,942857$, $\mathrm{p}=0,0048)$ and total cholesterol concentration $(\mathrm{rS}=-0,885714, \mathrm{p}=0,0188)$. In the II group anti-Hsp70 correlated negatively with HDL-cholesterol concentration ( $\mathrm{rS}=-0,684848, \mathrm{p}=0,0289)$. There was no difference in $\mathrm{CD}$ and SG concentration between groups studied. The concentration of MDA increased significantly $(\mathrm{p}<0.05)$ with progression of abstinence $(\mathrm{I}-2.77 \pm$ 0.25 , IV $-3.27 \pm 0.54 \mathrm{umol} / \mathrm{l})$. Early period of abstinence (II group) was associated with increased $(\mathrm{p}<0.05)$ oxo-LDL level.

Conclusion: Alcohol - dependence and abstinence duration have no effect on anti-Hsp70 levels. Lipids peroxidation in early periods of abstinence differ in underlying pathomechanisms from those associated with longer alcohol withdraw. Increased oxidation of LDL's in early abstinence period may form a risk factor for ischemic stroke.

\section{0}

\section{Long-term use of statins in secondary prevention of stroke}

L. Castilla-Guerra, M.C. Fernández-Moreno, C. Castillo-Aguilar, J. MarínMartín, J. Alvarez-Suero, E. Carmona-Nimo, M.D. Jiménez, J.M. LópezChozas

Hospital de la Merced (Osuna. Seville, ES); Hospital de Valme (Seville, ES); Hospital Virgen del Rocio (Seville, ES)

Introduction: In the SPARCL trial, patients with a recent stroke or transient ischemic attack (TIA) randomized to atorvastatin $80 \mathrm{mg} /$ day had a significant $16 \%$ relative risk reduction of stroke, with a mean low-density lipoprotein (LDL) levels of $73 \mathrm{mg} / \mathrm{dL}$, after a mean follow-up of 4.9 years. But, can this be translated to clinical practice?

Objective: To determine the adherence to statin treatment and achievement of NCEP-ATPIII guideline target LDL goals 2 years after discharge

Method: We studied all the patients with IS or TIA admitted to the Hospital de la Merced, Osuna, Spain for one year.After two years follow-up, lipidlowering therapy and cholesterol levels were again assessed.

Results: We included 155 patients, 134 IS and 21 TIA.There were 103 $(66.4 \%)$ men and $32(34.6 \%)$ women, mean age was 71.7 (SD 11.8) years. Statin therapy was used in 83 individual at discharge $(53.5 \%)$. After two years, forty seven patients died, and sixteen patients were lost. From the 91 patients left, 59 (64.8\%) were taking statins. The $89.8 \%$ (44 from 49 ) of patients treated with statins at discharge were on lipid-lowering therapy after two years. Mean LDL after two years in patients in treatment was 101 $( \pm 30.7)$, and approximately one-third of patients were over the target LDL goals. Statin therapy after two years was related to statin treatment at discharge $(p=0.0001)$, carotid atherosclerotic disease $(p=0.001)$, but not with previous stroke, coronary heart disease nor hyperlipidemia ( $\mathrm{p}$ non-significant).

Conclusions: Statin initiation during hospitalization for an ischemic cerebrovascular event is associated with high rates of adherence to treatment, however many patients with previous ischemic stroke/TIA have measured LDL that is higher than SPARCL trial levels and NCEP-ATPIII guidelines recommendations.

\section{1}

Prevalence of restless legs syndrome in population of post-stroke patients J. Urbanska, S. Kobierowski, M. Sieminski

Medical University of Gdansk (Gdansk, PL)

Restless Legs Syndrome (RLS) is a common sleep and movement disorders with prevalence between 5 and $15 \%$ in general population. As it has a significant impact on quality of life its coexistence may interfere with the course of other diseases. The aim of this study was to assess the prevalence of RLS in population of patients after stroke and its correlation with selected cardiovascular risk.

We have performed a screening study in a representative random sample of population of patients who underwent stroke in years 1999 to 2005 . Neurological examination and laboratory tests (blood levels of glucose, HDL, LDL, cholesterole and triglicerides) were performed in patients. Screening for RLS was done with a diagnostic questionnaire based upon the diagnostic criteria developed by International Restless Legs Syndrome Study Group.

We have examined 68 post-stroke patients ( 34 males, age range $42-80$; mean age 68.2 years). According to IRLSSG criteria RLS was found in 16 sub- jects (23,5\%; 11 females and 5 males). There was twofold higher prevalence of RLS among women than in men ( $32.3 \%$ vs. $14.7 \%)$. There was no age difference between RLS-positive and RLS-negative group. The mean laboratory results in the RLS-positive population were as follows: fasting blood glucose - 106,7 mg\%; HDL - 56.2 mg/dl; LDL - $128 \mathrm{mg} / \mathrm{dl}$; cholesterole $-209.7 \mathrm{mg} / \mathrm{dl}$; triglicerides $-138.6 \mathrm{mg} / \mathrm{dl}$. There was no significant difference between laboratory results between RLS-positive and RLS-negative group.

RLS is more common in post-stroke patients than in described samples of general population. There is no evidence that RLS may influence the tested in this study vascular risk factors.

As we have only examined a relation between RLS and some selected vascular risk factors a wider study with more detailed assessment of vascular risk factors in the population of RLS patients is planned.

\section{2}

Gender differences in undetected vascular risk factors after an acute ischaemic stroke

P. Martinez-Sanchez, B. Fuentes, M.A. Ortega-Casarrubios, E. Diez-Tejedor Hospital Universitario La Paz (Madrid, ES)

Background: The prevalence of vascular risk factors are different in men an women, however, the knowledge of vascular risk factors that are diagnosed in the days following an AIS is scarce. Our aim is to analyze possible sex differences in vascular risk factors diagnosed after an acute ischemic stroke.

Methods: Observational study using data from the Stroke Unit Data Bank from consecutive patients with first-ever cerebral infarction (2000-2006). We analyzed: demographic data, previous diagnosed vascular risk factors, stroke severity (Canadian Stroke Scale), stroke subtype, in-hospital complications, functional status at discharge (modified Rankin Scale [mRS]) and vascular risk factors diagnosed after the AIS (atrial fibrillation, arterial hypertension, diabetes mellitus and hyperlipidemia).

Results: 1,673 patients, 941 men. Mean age: 66.96 years (SD 12.25) in men and 70.42 years $(S D 13.03)$ in women $(\mathrm{P}<0.0001)$. Previous vascular risk factors more frequent in men than in women: peripheral arterial disease, coronary arterial disease, current smoking and alcohol abuse $(\mathrm{P}<0.0001)$. Women had a higher frequency of previous arterial hypertension and atrial fibrillation $(\mathrm{P}<0.0001)$. Men showed a higher frequency of post-stroke diagnosed arterial hypertension than women $(9.6 \%$ vs. $4.5 \%, \mathrm{P}<0.0001)$ as well as diabetes mellitus ( $4.4 \%$ vs. $1.5 \%, \mathrm{P}=0.001)$. The logistic regression analysis showed that male (OR, $1.665 ; 95 \% \mathrm{CI}, 1.001$ to 2.748 ) and previous hyperlipidemia (OR, 1.786; $95 \% \mathrm{CI}, 1.026$ to 3.111 ) were the factors associated with post-stroke diagnosis of arterial hypertension, and male (OR, $3.168 ; 95 \% \mathrm{CI}, 1.419$ to 7.070 ) the only factor associated to post-stroke diagnosis of diabetes mellitus; after adjustment for age, sex, previous vascular risk factors, stroke subtype and severity and in-hospital complications.

Discussion: Male is associated with a higher post-stroke diagnosis of diabetes mellitus and arterial hypertension than female. These results suggest the existence of sex-differences in undetected vascular risk factors and wide scope for stroke prevention.

\section{3}

Actigraphic measurement of motor deficits in acute ischaemic stroke N. Gebruers, S. Truijen, G. Nagels, R. Brouns, S. Engelborghs, P. P. De Deyn University College of Antwerp (Merksem, BE); University College of Antwerp (Antwerp, BE)

Objectives: The aim of this study was to investigate the use of actigraphy (accelerometry) to measure motor deficits of the impaired arm in acute stroke patients. We used the National Institute of Health Stroke Scale (NIHSS) and the Fugl-Meyer arm section (FMA) to correlate with the actigraphic data as a measure of validity.

Methods: Thirty-nine patients with acute ischemic stroke were included within one week after stroke onset. At inclusion, motor deficits were assessed by the NIHSS; FMA and actigraphic recordings of both wrists for $48 \mathrm{~h}$ were started.

Results: Moderate but highly significant correlations between ratio (activity impaired arm/ activity unimpaired arm), mean activity data and the total NIHSS (ratio $r=0.59$ and mean activity $r=0.75 ; p<0.001$ ) and FMA (ratio $r=0.54$ and mean activity $r=0.69 ; p<0.001$ ) scores were found. Based on actigraphic motor activity scores, ROC curves were calculated following dichotomisation of the population based on the median scores of NIHSS and FMA, showing good sensitivity (0.90 and 1.0$)$ and specificity $(0.75$ and 0.89$)$ values, resulting in a negative predictive value of $100 \%$ and positive predictive value of $91 \%$. The general linear model showed significantly less activity for the paretic arm in patients with a NIHSS score above seven $(\mathrm{p}<0.0001)$ or FMA scores less than 44

Conclusion: Moderate but highly significant correlations were calculated 
between actigraphy and validated, frequently used stroke scales like NIHSS and FMA. Actigraphy was able to reliably discriminate less impaired from more impaired stroke patients with excellent sensitivity and specificity values. Actigraphy is a valid and reliable clinical research tool that can be used to determine motor impairment of the upper limb in stroke patients.

\section{Oral session 20}

\section{Neuro-ophthalmology}

\section{4}

\section{Vergence deficits in cerebellar lesions}

T. Sander, A. Sprenger, G. Neumann, S. Gottschalk, B. Machner, C. Helmchen, H. Rambold

University of Lubeck (Lubeck, DE); The National Eye Institute (Bethesda, US)

The cerebellum is part of the cortico-ponto-cerebellar circuit for conjugate eye movements. Accordingly, cerebellar lesions cause smooth pursuit deficits and saccadic dysmetria. In contrast, the role of the cerebellum for disconjugate, i.e. vergence eye movements in humans is still unknown. The aim of this study was to investigate whether cerebellar lesions affect vergence eye movements.

Similar to conjugate eye movements, vergence can be separated into two different components: vergence to step targets (fast vergence) and vergence to ramp and sinusoidal targets (slow vergence). Eye movements (fast and slow vergence, smooth pursuit, saccades) were binocularly recorded with the scleral search coil system in 20 patients with acute cerebellar lesions (ischemic strokes) and age-matched healthy controls. Small dim laser stimuli were presented on an earth horizontal platform at the level of the subject's nose. Smooth pursuit always had a constant vergence angle (iso-vergence).

The majority of patients had unilateral lesions in the territory of the posterior inferior cerebellar artery ( 3 patients with infarctions in the superior cerebellar artery territory, one cavernoma patient). Group analysis revealed impairment of both conjugate and disconjugate eye movements as well as reduced saccadic gain.

The gain of slow vergence to sinusoidal targets was reduced in the patients. Patients with vermal lesions (subgroup, $\mathrm{n}=10$ ) had a significant lower gain compared to controls while the other patients did not differ. In contrast, fast vergence to step targets remained unimpaired (acceleration). Smooth pursuit to step-ramp targets was impaired, with increased latency, decreased initial acceleration and reduced gain. Smooth pursuit eye movement gain to sinusoidal targets was significantly reduced. Similar to vergence eye movements to sinusoidal targets, patients with vermal lesions had a lower smooth pursuit gain while the other patients did not differ from controls.

We propose that (1) the cerebellum is involved in the processing of vergence eye movements, (2) the cerebellar vermis plays an important role in executing vergence eye movements and (3) neural control of fast and slow vergence appears to be separated in the cerebellum.

0175

Vestibular and psychiatric symptoms in patients with organic vertigo syndromes: results from a prospective one-year follow-up

C. Best, A. Eckhardt-Henn, R. Tschan, S. Bense, M. Dieterich

Johannes Gutenberg University (Mainz, DE); Bürgerhospital (Stuttgart, DE)

Objectives: Patients with primary vestibular vertigo syndromes often also suffer from anxiety and depressive symptoms, whereas patients with psychiatric disorders often express subjective perceived symptoms of unsteadiness, dizziness or vertigo. Therefore the vestibular system was postulated to be interlinked closely to emotion processing systems. However, the interlinkage of vestibular and psychiatric symptoms in vertigo patients has not been understood in detail. Aim of the current study was to evaluate and correlate subjective perceived vestibular and psychiatric symptoms with the course of the underlying disease as well as with the vestibular function itself.

Methods: This interdisciplinary prospective longitudinal study over 1 year included a total of 68 patients with acute vestibular vertigo syndromes. Four subgroups compared benign paroxysmal peripheral vertigo (BPPV, $n=19)$, vestibular neuritis $(V N, n=14)$, vestibular migraine $(V M, n=27)$, and Menière's disease $(M D, n=8)$. All patients underwent standardized neurological and neuro-otological examinations (including electro-oculogra- phy with caloric and rotatory testing, measurements of the subjective visual vertical, and ocular torsion for otolith testing) as well as a psychometric examination battery, including the Vertigo Symptom Scale (VSS) and the Vertigo Handicap Questionnaire (VHQ), screening for subjective perceived vestibular and psychiatric symptoms at five different times (T0-T4) over 1 year.

Results: VM patients perceived a vertigo induced handicap in daily life, the severity of vestibular symptoms and the vertigo induced anxiety significantly higher than all other patients $(p<0.001-p=0.006)$. The occurrence of a reactive anxiety disorder strongly correlated with subjective perceived vertigo attacks, whilst the occurrence of a reactive depressive disorder correlated with a permanent vertigo sensation. Finally, the amount of fluctuations of the vestibular excitability on caloric testing positively correlated with the subjective perceived severity of vertigo in VM patients.

Conclusion: VM patients are significantly higher handicapped by the subjective perceived vertigo symptoms. These patients show a significantly elevated fluctuation of vestibular excitability on caloric testing, which correlated with the severity of vertigo symptoms. Finally, reactive anxiety disorders correlated with subjective vertigo attacks whilst depressive disorders correlated with a permanent vertigo sensation.

\section{6}

An ipsilateral vestibulo-thalamic tract in the medial lemniscus A. Zwergal, T. Brandt, M. Strupp University of Munich (Munich, DE)

Objectives: Information processing in the vestibular system is organized as a multilevel sensorimotor network to subserve postural, ocular motor and perceptual function. Unilateral damage of vestibular input to the cortex mainly results in pathological deviations of the perceived visual vertical. So far it is unclear, how vestibular input is transmitted from the vestibular nuclei to the cortex. A crossed graviceptive pathway within the MLF has been postulated, which has multisynaptic projections running via the integration centres for eye movements in the roll plane to the thalamus. An uncrossed graviceptive pathway with a direct thalamic projection has been described in animal experiments (vestibulo-thalamic tract). The existance of a human homolog was suggested by functional imaging studies, which show a predominantly ipsilateral ascending projection of vestibular information to the cortex, but the anatomical course of this projection was unknown.

Methods: In a prospective study we analysed 14 patients with acute anteromedial pontomesencephalic infarctions for signs of vestibular and ocular motor dysfunction. All patients underwent a neuro-ophthalmological examination including measurement of subjective visual vertical (psychophysical), ocular torsion (scanning laser ophthalmoscope) and skew deviation (cover test, prisms). Lesion localization was drawn from thin-slice MRI.

Results: In all patients an isolated ipsilateral deviation of the subjective visual vertical (mean: 4.1, range: 2.7 - 6.6) was found without further neuroophthalmological dysfunctions. Distinct lesions in thin-slice brainstem MRI showed an overlap zone in the medial portion of the medial lemniscus.

Conclusions: In the present study it could be shown, that vestibular projections for graviception run in the ipsilateral medial lemniscus to the posterolateral thalamus. This ipsilateral vestibular-thalamic tract is distinct from crossing graviceptive pathways within the MLF and may represent the human homolog of the three-neuron sensory vestibulo-cortical system described in primates.

0177

Acute visual loss: clinically blinded by atypical lymphoma

M. O'Brien, S. Murphy, M. Hutchinson, M. Farrell, N. Tubridy

St Vincent's University Hospital (Dublin, IE); Beaumont Hospital (Dublin, IE)

Primary central nervous system (CNS) lymphoma, once rare, now represents $4-7 \%$ of newly diagnosed primary CNS tumors. Prevalence peaks in the fourth and fifth decade in immunocompetent individuals. Clinical symptoms and signs are often non-specific, patients often having neuropsychological deficits and personality change followed by focal neurological deficits and seizures.

Radiologically, lesions usually occur centrally, with a predilection for the cerebral hemispheres, basal ganglia and corpus callosum. The majority of immunocompetent patients have solitary lesions and ependymal or leptomeningeal involvement is common. 'Classical' magnetic resonance imaging (MRI) appearances include an isointense-to-hypointense mass on T1weighted MRI with a predominantly hyperintense or isointense pattern on T2-weighted images. Gadolinium typically causes marked, uniform enhancement with surrounding oedema.

We report the case of a 17-year-old boy who presented with symptoms 
and signs of optic neuritis. Initial MRI showed diffuse confluent white matter and brainstem abnormalities as well as enhancing lesions in the corpus callosum and periventricular white matter without oedema. CSF showed a lymphocytic pleocytosis. The radiological differential diagnosis included acute disseminated encephalomyelitis (ADEM), leukodystrophy, mitochondrial disease, while lymphoma was considered less likely.

An unusual family history, raised lactate and abnormal muscle biopsy raised the possibility of mitochondrial disorder. He initially responded to steroids but re-presented within weeks. MRI now showed disappearance of the initial enhancing lesions, but worsening of the confluent white matter abnormalities. A brain biopsy confirmed primary CNS B-cell lymphoma.

The unusually young age of onset along with the family history, appearances on imaging and muscle biopsy led to a delay in diagnosis of this primary CNS lymphoma. Progressive leukoencephalopathy has been reported as occurring very rarely in primary CNS lymphoma, $<1 \%$, and often causes a delay in diagnosis. Brain biopsy should be considered in patients with progressive leukoencephalopthy when other investigations fail to reveal a cause.

\section{8}

Positional convergence nystagmus - a neurological disease ?

W. Heide

Allgemeines Krankenhaus Celle (Celle, DE)

Objective: Disorders of vergence eye movements are commonly caused by midbrain lesions. E.g. the dorsal midbrain syndrome manifests with upgaze paralysis and often convergence-retraction nystagmus or at least with an exaggerated convergence tone. Here we present a case with position-dependent convergence nystagmus and convergence spasm, mimicking a mesencephalic disorder.

Case report and results: For a few days a man aged 33 experienced position-dependent vertigo attacks when turning to the left, with spontaneous remission after a few days. Since that time he was afraid to lie down on the left ear. When doing this he progressively noticed vertiginous feelings, as if he was falling to the ground, associated with blurred vision, heart hammering, profuse sweating, tingling of the fingers and panic attacks. Previous investigators had reported convergence nystagmus when positioned on the left ear. They assumed a midbrain lesion, but all diagnostic procedures such as MRI, evoked potentials, electro 7 nstagmography, ultrasound of cerebral vessels and lumbar puncture rendered normal results. In our vertigo unit, positioning the patient on his left ear provoked a panic attack with tremor and the other symptoms mentioned above. The eyes exhibited irregular spasms of convergence, associated with pupillary constriction and accommodation to the near, sometimes also with voluntary nystagmus.

Conclusion: Because of this physiological activation of the near triad and the intact ocular motility, the disorder can be classified as convergence spasm: This is a functional or psychogenic disorder which in the present case was associated with phobic postural vertigo. As it could be provoked by positioning manoeuvres, it was obviously a phobic reaction on a previously experienced benign paroxysmal positional vertigo. Phobic postural vertigo is often preceded by an organic vestibular disorder. This is the first reported case where it was associated with convergence spasm and voluntary nystagmus, thus mimicking convergence-retraction nystagmus.

\section{Oral session 21}

\section{Coma and sleep disorders}

\section{9}

Default network resting state connectivity integrity reflects the level of consciousness impairment in brain-injured patients. An fMRI study in brain death, coma, vegetative state, minimally conscious state and locked-in syndrome

M. Boly, A. Vanhaudenhuyse, L. Tshibanda, M-A. Bruno, P. Boveroux, Q. Noirhomme, C. Schnakers, A. Demertzi, D. Ledoux, B. Lambermont, G. Moonen, R. Dondelinger, C. Phillips, P. Maquet, S. Laureys

University of Liège and CHU Hospital (Liège, BE); CHU Hospital (Liège, BE)

The 'default network' is defined as a set of areas, encompassing posteriorcingulate/precuneus, anterior cingulate/mesiofrontal cortex and temporoparietal junctions, showing more activity at rest than during attentiondemanding tasks. This network has been involved in higher cognitive functions like self-related processes, emotion, and memory. Recent studies have shown that it is possible to reliably identify this network in healthy volunteers in the absence of any task, by resting state connectivity analyses. The aim of this study was to test if the integrity of resting-state connectivity in the default network would differ in different pathological alterations of consciousness. Thirteen acutely brain damaged patients in brain death $(\mathrm{BD}$ $\mathrm{n}=1$ ), coma (5), vegetative state (VS; 3 ), minimally conscious state (MCS; 3 ) and locked-in syndrome (LIS; 1)) and 12 healthy controls participated to the study. Patients were assessed using the Coma Recovery-Scale Revised. For each subject, a 10 minutes resting-state acquisition was performed. Data were analysed using independent component analysis (ICA) and statistical parametric mapping (SPM). A goodness-of-fit comparison was performed (Greicius et al. PNAS 2004; 101:4637-4642) on individual subjects' Z maps, taking the control group's default network as a template. Our analysis searched for differences in the selected components between controls and patients groups. Mean goodness-of-fit scores for individual default network components were respectively 1.25 for controls, 1.22 for LIS, 1.15 for MCS patients, 0.79 for VS and 0.77 for coma. In the BD patient, no cortical spatial map could be identified. Significantly less activity was observed within all areas of the default network for coma and vegetative state patients as compared to controls. Locked-in and minimally conscious patients were not significantly different from controls. Finally, all default network areas connectivity was shown to be linearly correlated with the degree of clinical consciousness impairment of the patients, ranging from coma, to vegetative state, minimally conscious state and locked-in syndrome. Our data show that default network connectivity is decreased in severely brain-damaged patients, in proportion to their degree of consciousness impairment. Ongoing work on a larger patient cohort is currently performed, aiming to evaluate the prognostic value of the presented methodology.

\section{0}

Loss of hypocretin (orexin) neurons with severe traumatic brain injury

C.R. Baumann, C.L. Bassetti, P.O. Valko, J. Haybäck, M. Keller, E. Clark, S. Ludwig, M. Tolnay, T.E. Scammell

Harvard Institutes of Medicine (Boston, US); University Hospital (Zurich, $\mathrm{CH}$ ); University of Zurich (Zurich, $\mathrm{CH}$ )

Objective: To better understand the causes of sleep-wake disturbances in TBI, we aimed to test whether hypothalamic hypocretin neurons are lost after severe traumatic brain injury.

Background: Traumatic brain injury (TBI) frequently results in excessive daytime sleepiness and hypersomnia, but the underlying causes of posttraumatic sleep-wake disturbances are unknown. Narcolepsy is caused by a loss of the hypocretin-producing neurons in the hypothalamus, and in the first days after TBI, cerebrospinal fluid levels of hypocretin-1 are often very low, suggesting injury to the hypocretin system. Similarly, six months after TBI, there is an association between low cerebrospinal fluid hypocretin levels and excessive daytime sleepiness.

Methods: We immunostained hypothalamic sections and counted hypocretin neurons from 4 deceased patients with severe TBI and from 4 control subjects. Control hypothalami contained an average of $44,838 \pm$ 3,988 hypocretin neurons (range 40,700-49,625). In TBI patients, the number of hypocretin neurons ranged from 23,800 to 47,600 (mean $32,106 \pm$ $7,618)$, representing an approximately $30 \%$ cell loss $(\mathrm{p}<0.001)$. Cell densities in TBI patients were reduced by $27 \%$, and perivascular spaces frequently contained hypocretin-immunoreactive debris. In controls, the number of hypocretin neurons differed by no more than $2-4 \%$ between the right and left hypothalami, but in TBI brains, cell counts varied by $10-29 \%$ between sides, possibly reflecting asymmetric trauma.

Conclusion: The loss of hypocretin neurons in patients with severe TBI suggests that disturbed hypocretin signaling may contribute to posttraumatic sleep-wake disturbances.

\section{1}

Working memory in obstructive sleep apnoea patients: a functional MRI study

L. Ferini Strambi, A. Falini, A. Bruschi, M. Aloia, S. Cappa, D. Perani, P. Scifo, N. Canessa, V. Castronovo

Università Vita-Salute S. Raffaele (Milan, IT); National Jewish Medical and Research (Denver, US)

Objective: Obstructive Sleep Apnea syndrome (OSA) is associated with cognitive (attention, memory and executive-functions) and functional (work performance, daytime sleepiness and quality-of-life) deficits. The main contributing factors proposed are daytime-somnolence caused by sleep fragmentation and intermittent hypoxemia that may affect brain functioning. We assessed cognitive performance and cerebral activity during cognitive 
challenge in OSA, using neuropsychological tests and functional-magneticresonance-imaging (fMRI).

Methods: 15 male untreated severe OSA patients (mean age $43.7 \pm 7.5$; AHI > 30) and 15 age-education matched healthy controls. They were assessed with neuropsychological tests, rating scales (ESS, BDI) and qualityof-life questionnaires. During fMRI-scanning participants performed a 2back working-memory task.

Results: Patients scored significantly below controls in short and longterm-memory, executive-functioning. Behavioral results during fMRI-scanning did not show significant differences between groups. Imaging results showed bilateral activations related to task difficulty in both groups in the posterior parietal cortex, insula, pre-supplementary motor area, left ventrolateral and dorso-lateral frontal cortex and cerebellum. Significant differences were also observed. Increased activations in patients, compared to controls, were observed in the left superior, middle and inferior frontal gyri, dorsal medial precuneus, putamen and cerebellum. Decreased activations in patients were observed in the middle-occipital-gyrus and in the pontine reticular formation.

Conclusion: Neuropsychological tests confirmed the cognitive impairments already shown in OSA. Stronger activations in patients were observed in frontal regions associated with sub-vocal verbal rehearsal and strategic organization of information, suggesting a compensatory-recruitment response to support normal performance. The normal behavioral performance during scanning fits with this interpretation, which is also supported by differential fMRI activations in the two groups. Weaker activations in patients were observed in pontine structures involved in respiratory control. The results extend previous reports on the cognitive impairments and the altered functional neurocircuity associated with OSA.

Research supported by Respironics Foundation, USA

\section{2}

Sleep disorders in adolescence

J. Urbanska, S. Kobierowski, G. Bereda, M. Sieminski

Medical University of Gdansk (Gdansk, PL)

Objectives: Sleep and emotional disorders are frequently overlooked in modern society. The aim of research was to determine the frequency and the quality of sleep disorders in adolescence.

Material and methods: To achieve the aim we have used in this study: Soldatos Insomnia Scale - SIS (cut-off 10 points), Epworth Sleepiness Scale - ESS (1-6 considered normal, 7-8 score is average, 9 and over sleepy) and Sleep Disorders Questionnaire (complex scale concerning sleep habits and conditions).

We also took into consideration risk factors like allergy, asthma, diabetes mellitus, hypercholesterolemia, arterial hypertension, hyperthyroidism, hypothyroidism, depression, anxiety and habits like coffee, cigarettes, alcohol, drugs.

Results: 501 people have completed the questionnaires, 196 men and 305 women. The age was $16(24.75 \%), 17(41.72 \%)$ and $18(33,53 \%)$ years. The results in Soldatos Insomnia Scale - SIS - was $13.55 \pm 3.14$ (46.6\% patients were over 10 points) - there was significant difference between women $13.91 \pm 3.02$ and men $13.06 \pm 3.11(\mathrm{p}<0.05)$; in Epworth Sleepiness ScaleESS $-7.09 \pm 4.05$ (33,93\% patients were over 9 points) - there was significant difference between women $7.52 \pm 3.93$ and men $6.41 \pm 4.16(\mathrm{p}<0.05)$. The answers of Sleep Disorders Questionnaire have shown that $18.56 \%$ patients wake up during the night, $20.36 \%$ have uncontrolled head and limbs movements during sleep, $6.19 \%$ feel unpleasant and uncomfortable sensations in the legs or have periodic irresistible urges to move the legs - symptoms of Restless Legs Syndrome, $29.34 \%$ after the sudden awakening feel disorientated or aggressive, 33,93\% feel tiredness during the day, 37,33\% sleep during the day. The other presented symptoms: headache, bruxism, excessive drooling and sweating during the sleep. There was no statistically significant difference between SIS, ESS people with allergy or asthma and healthy population $(p<0.05)$. There was correlation between smoking cigarettes and sleep disorders - smokers SIS $14.27 \pm 3.35$, ESS 7.56 \pm 4.01 . The difference between smokers and non- smokers was statistically significant $(p<0.05)$.

Conclusions: Sleep disorders are frequent problem in adolescence and more intense in women. This fact should be taken in the consideration because the diagnosis and treatment can improve daily functioning this young people.
0183

Time structure analysis of leg movements during sleep

L. Ferini Strambi, M. Manconi, M. Zucconi, M.L. Fantini, S. Marelli, R. Ferri Università Vita-Salute S. Raffaele (Milan, IT); OASI IRCCS (Troina, IT)

Objective: To compare the time structure of leg movements (LM) during sleep among patients with REM sleep behavior disorder (RBD), restless legs syndrome (RLS), and healthy subjects.

Methods: The polysomnographically recorded tibialis anterior (TA) activity during sleep was analyzed by means of a new computed approach able to consider duration, inter movements interval, distribution per sleep stages and across the night, and periodicity. We evaluated 20 drug-free patients with idiopathic RBD, 37 with idiopathic RLS and 14 age-matched controls.

Results: Most of RBD patients ( $85 \%$ ) presented periodic leg movements during sleep (PLMS). PLMS occurred more frequently during NREM sleep in RLS and during REM sleep in RBD. PLMS resulted shorter in duration, less often bilateral, and with a higher inter-movement interval in RBD compared to RLS. Number of PLMS per hour of sleep decreased across the night in both RBD and RLS, but not in controls. In all subjects LM periodicity clearly depended on sleep state, with higher values during NREM than during REM sleep. RBD showed a lower LM periodicity compared to RLS in each of the sleep states.

Conclusions: Significant difference in LM time structure was observed between RBD and RLS. This heterogeneity in muscular TA activity may reflect a different LM pathogenesis in terms of neurotransmitter and central nervous pace-maker implicated in the two disorders. Alternatively, LM may share the same pathogenesis in both disturbances, but their phenotype could be disease and/or sleep state related.

\section{4}

The effects of music background on sleep efficiency, anxiety and depression in schizophrenia patients

L. Vadas, B. Bloch, Y. Haliba, N. Ziv, I. Haimov, I. Kremer

The Max Stern Academic College of Emek Yizreel (Emeak Yisreal, IL); Emek Medical Center (Afula, IL)

Objectives: Disturbed sleep is a common complaint among Schizophrenia patients. Since previous studies have demonstrated treatment resistance for sleep-medication treatment for insomnia in these patients, the aim of the present study was to examine the effects of music background as treatment for insomnia in Schizophrenia patients.

Methods: Twenty four Schizophrenia patients, who had no other major psychiatric, sleep, or neurological disorders, participated in the study (mean age $=45.67, \mathrm{SD}=9.6 ; 13$ males and 11 women). The study comprised one 7day, running-in period, followed by one 7-day experimental period. The treatment was music background at desired bedtime. During each of these two experimental periods, subjects' sleep was continuously monitored with a wrist actigraph (Ambulatory Monitoring, Inc.) and subjects were asked to fill out several questionnaires concerned with a wide spectrum of issues, such as depression, anxiety, and life satisfaction.

Results: A paired-sample t-test was conducted, comparing objective sleep parameters manifested by patients with music background at desired bedtime and without music exposure. A significant difference was found in sleep latency $(\mathrm{t}(23)=3.01, \mathrm{p}<0.006)$, showing shorter sleep latency when music background was played $(21.04 \pm 14.6)$ than when it was not $(37.01 \pm 32.4)$. A significant difference was also found in sleep efficiency $(\mathrm{t}(23)=3.35, \mathrm{p}<0.003)$, showing higher sleep efficiency when music background was played $(86.0 \pm 15.5)$ than when it was not $(82.44 \pm 18.3)$. A significant difference was found in depression level $(\mathrm{t}(23)=2.96, \mathrm{p}<0.007)$, showing lower depression level after background music was played $(12.29 \pm 12.1)$ than when it was not $(15.25 \pm 10.6)$. No significant differences were found in anxiety level $(\mathrm{t}(23)<1, \mathrm{p}>0.05)$, nor in life satisfaction $(\mathrm{t}(23)=1.28, \mathrm{p}>0.05)$.

Conclusion: Overall, the findings imply the beneficial effect of music background as treatment for insomnia in Schizophrenia patients.

\section{5}

Hierarchical differences in peripheral arterial tone during night as measured by the WATCH-PAT device, during PLM, apnea and spontaneous activation

A. Sokolovsky, M. Gorochov, K. Sheffy, G. Pillar, S. Herscovici Ben Gourion University (Beersheva, IL); Itamar Medical (Cesarea, IL); Technion (Haifa, IL)

Introduction: The Watch_PAT (WP) is an ambulatory device validated for the diagnosis of obstructive sleep apnea (OSA) in the home, utilizing monitoring of autonomic activation. One of the potential criticisms of using it in- 
stead of polysomnography may be the lack of tibialis-anterior recording and therefore inability to quantify periodic leg movements (PLM). However, PLM have been shown to affect autonomic activation during sleep. Thus we hypothesized that PLM, apneas and basic autonomic index (spontaneous changes in the autonomic tone) may perform differently in the channels recorded by the WP (actigraphy, PAT, pulse rate), and therefore may be detected by this device.

Methods: We prospectively studied 22 patients ( 21 males), aged $51 \pm 10$ with BMI of $31 \pm 6$ undergoing full night evaluation for suspected OSA in the sleep lab. All patients had a standard PSG with a simultaneous WP recording. Each file was manually scored for PLM and apneas, and then the related changes in signals recorded by the WP were quantified. The data was then compared between apnea, PLM and spontaneous autonomic events.

Results: for PLM, apnea, and spontaneous autonomic activation events, the following changes in signals were found, respectively: significance of Apnea and Spontaneous was tested compared to PLM.

PAT Amplitude Reduction is 1. (SE 0.022), 1.64 (SE 0.006)*, 0.76 (SE $0.018)^{\star}$

Increase in heart rate: 1 (SE 0.018), $1.17($ SE 0.015)* $0.73($ SE 0.005)*

Decrease in heart rate: 1 (SE 0.026), $1.67($ SE 0.021)* $0.77($ SE 0.007)

The values above are normalized to PLM events

Time Difference between Peak pulse Rate and Peak PAT Amplitude [Sec]: -4.7 (SE 0.1998), $-2.69($ SE 0.1635)*, $7.62($ SE 0.0565)*

Time between Max PAT Amplitude and Movement [Sec]: 2.1 (SE 0.4358), -0.65 (SE 0.3565)*, 1.88 (SE 0.1232).

All values are for PLM apnea and spontaneous respectively.

(*stands for significance $\mathrm{P}<0.05$ compared to PLM).

$\mathrm{SE}$ is the mean Standard Error provided by Matlab Multcompare (ANOVA)

Conclusions: using indirect physiological signals such as general movement (actigraphy), pulse rate and (PAT), we found that apnea and spontaneous autonomic activation generate significantly different morphological events than PLM. These differences may be potentially utilized to detect these events with the WP.

S. Herscovici is an employee of Itamar Medical, the developer of the Watch_PAT device. Giora Pillar is a consultant for ITAMAR medical.

\section{Oral session 22}

\section{Multiple sclerosis 4}

\section{6}

Clinical and conventional MRI features of non-disabling MS. A large-scale, multicentre, multinational, cross-sectional study

A. Pulizzi, M. Rovaris, L. Fisniku, M. Calabrese, M.L. Stromillo, M. Sombekke, A. Rovira, D.H. Miller, P. Gallo, N. De Stefano, F. Barkhof, C. Polman, X. Montalban, M. Filippi

Scientific Institute and University HSR (Milan, IT); University College London (London, UK); University Hospital (Padua, IT); University of Siena (Siena, IT); VU University Medical Centre (Amsterdam, NL); Hospital Vall d'Hebron (Barcelona, ES)

Objectives: Although it is well known that multiple sclerosis (MS) may have a benign course (BMS), which is characterized by absent or mild neurological impairment after a long-lasting duration, the structural features of nondisabling MS remains poorly defined. Conventional MRI studies of BMS have achieved conflicting results and failed to find consistent correlates of BMS to be used for a reliable identification of these patients during therapeutic counselling. To re-address this issue, the present study was performed to evaluate the clinical and conventional MRI features of a large and heterogeneous population of patients with non-disabling MS.

Methods: We studied 167 patients with BMS (i.e., having expanded disability status scale [EDSS] score 3.0 or less and a disease duration of at least 15 years) and 173 patients with non-disabling relapsing-remitting MS (NDRRMS) (i.e., having EDSS score 3.0 or less and a disease duration between 5 and 14 years), who were retrospectively identified from the MS clinics in 6 centers (Amsterdam, Barcelona, London, Milan, Padua, Siena). For all patients, demographic and clinical data (including EDSS rating) had to have been collected within two weeks from a brain MRI scan including high resolution (i.e., with 3-5 mm thick contiguous axial slices) T2-weighted sequences. Centralized image analysis consisted of T2-hyperintense lesion volume (LV) measurement, performed by a single observer using a semi-automated local thresholding technique. A cubic root transform was applied to normalize the distribution of T2 LV before ANOVA group analysis, which was adjusted for patients' age and enrollment center.

Results: Patients with BMS were older than those with NDRRMS (mean age: 47.2 and 39.4 years, $p<0.001$ ). The mean disease duration was 21.6 years for BMS and 8.2 years for NDRRMS. Median EDSS did not differ between the two groups of patients. The mean T2 LV was higher in BMS than in NDRRMS patients (12.99 vs. $8.48 \mathrm{ml}, \mathrm{p}<0.001)$. T2 LV did not differ between those 61 BMS patients with low disability (EDSS $\leq 2.0$ )/long disease duration ( $\geq 20$ years) and the remaining 106 with higher disability/shorter disease duration. T2 LV was correlated with MS duration $(\mathrm{r}=0.29, \mathrm{p}<0.001)$.

Conclusions: The burden of T2-visible lesions does not seem to be a distinctive feature of non-disabling MS, but rather a mere reflection of disease duration.

\section{7}

Evidence for relative cortical sparing in benign multiple sclerosis: a longitudinal MRI study

M. Calabrese, M. Filippi, M. Rovaris, V. Bernardi, M. Atzori, I. Mattisi, A. Favaretto, F. Rinaldi, L. Rinaldi, P. Perini, P. Gallo

Multiple Sclerosis Center of Veneto Region (Padua, IT); San Raffaele Scientific Institute (Milan, IT)

Objectives: A significant inflammatory damage in the cortex of multiple sclerosis (MS) patients has been disclosed by ex vivo and in vivo studies. The aim of our study is to investigate whether the extent of cortical damage in benign MS (BMS) patients might contribute to explain their favourable clinical status.

Methods: Forty-eight patients with BMS (Expanded Disability Status Scale (EDSS) score $\leq 3.0$ and disease duration $\leq 15$ years) and 96 patients with early relapsing-remitting (RR) MS (EDSS score $\leq 3.0$ and disease duration $\leq 5$ years) were included in the study. Brain MRI, including a double inversion recovery (DIR) and a fluid-attenuated inversion recovery (FLAIR) sequence, was acquired at baseline and after 12 months. On DIR images, intracortical (ICL) and cortical-subcortical lesions (CSL) were identified and counted. Total white matter (WM) lesion volume was quantified on FLAIR images.

Results: Compared to early RRMS, patients with BMS had a lower number of ICL at both study time-points ( $\mathrm{p} \leq 0.001$ for both comparisons). At one-year follow-up, a significant increase of ICL and CSL number was observed only in early RRMS patients. The number of ICL was not correlated with WM lesion volume.

Conclusions: In BMS patients, the selective sparing of the cortex from disease-related focal pathology might be one of the factors associated to their favourable clinical status, independently of the (possible) accrual of WM lesions.

0188

Antibodies to native myelin-oligodendrocyte glycoprotein do not predict progression from CIS to MS: further analysis of the BEtaferon ${ }^{\circledR}$ in Newly Emerging multiple sclerosis For Initial Treatment (BENEFIT) study

B. Hemmer, G. Edan, M. Freedman, H-P. Hartung, L. Kappos, D. Miller, X. Montalban, C. Polman, J. Kuhle, S. Nessler, D. Zhou, L. Bauer, V. Lanius, C. Metzig, C. Pohl, R. Sandbrink

Technische Universität (Munich, DE); Centre Hospitalier Universitaire (Rennes, FR); Ottawa Hospital (Ottawa, CA); Heinrich-Heine-Universitat (Dusseldorf, DE); University Hospital (Basel, CH); National Hospital for Neurology and Neurosurgery (London, UK); Hospital Vall d'Hebron (Barcelona, ES); Vrije Universiteit Medical Centre (Amsterdam, NL); Bayer Schering Pharma AG (Berlin, DE)

Objective: To determine whether the presence of antibodies against native myelin oligodendrocyte glycoprotein (MOG) measured using a high-sensitivity bioassay is related to earlier progression to clinically definite MS (CDMS) or advanced disability in patients with a first event suggestive of multiple sclerosis (MS) in the BENEFIT study. Previously, anti-MOG antibody testing was done by Western blot (WB) under denaturating conditions using a recombinant, extracellular MOG domain as target antigen. Here we employed an alternative high-sensitivity cell based assay that allows detection of autoantibodies binding to the extracellular part of the native human MOG (nMOG) protein.

Methods: The ongoing BENEFIT study is designed to evaluate the impact of early versus delayed initiation of IFNB-1b treatment on disease evolution in patients with a first event suggestive of MS. In the placebo-controlled phase of BENEFIT, patients were randomised to IFNB-1b $250 \mathrm{mcg}$ or placebo subcutaneously every other day for 2 years or until developing CDMS. Pa- 
tients who completed this phase were eligible for enrolment in a follow-up phase, in which all patients were offered IFNB- $1 \mathrm{~b} 250 \mathrm{mcg}$ for up to 5 years after randomisation. Primary outcomes include time to CDMS and time to confirmed disability progression. Samples for anti-MOG antibody testing were taken at baseline and analysed as described by Zhou et al. (2007) to search for an association of nMOG antibodies with the occurrence of CDMS and confirmed EDSS progression over 3 years.

Results: Baseline samples of 410 patients were analysed. Antibody titres did not correlate with time to CDMS or time to confirmed disability progression after three years. Given the only weak correlation of antibody titres measured by WB, the nMOG assay appears to detect a different repertoire of antibodies than the conventional solid phase assay.

Conclusions: Antibodies to nMOG at baseline do not predict progression to CDMS and/or time to confirmed disability progression. Further studies are underway to determine whether changes of antibody status to nMOG are associated with clinical parameters during the course of MS.

Study supported by: Bayer Schering Pharma AG, Berlin, Germany

\section{9}

Structural MRI correlates of benign multiple sclerosis. A voxel-based morphometry study of regional grey matter atrophy

S. Mesaros, M. Rovaris, E. Pagani, A. Pulizzi, D. Caputo, M. Zaffaroni, R. Capra, A. Bertolotto, V. Martinelli, G. Comi, M. Filippi

Scientific Institute and University HSR (Milan, IT); Scientific Institute Don Gnocchi (Milan, IT); Ospedale di Gallarate (Gallarate, IT); Spedali Civili, Brescia (Brescia, IT); Ospedale di Orbassano (Orbassano, IT)

Objectives: Using voxel-based morphometry (VBM), previous studies have shown GM atrophy in several cortical and subcortical structures in patients with multiple sclerosis (MS). The aim of this study was to investigate the patterns of regional distribution of grey matter (GM) atrophy in benign multiple sclerosis (BMS) vs. secondary progressive MS (SPMS) patients, in order to better elucidate the mechanisms underlying a favorable clinical evolution of the disease.

Methods: Sixty patients with BMS, 35 with SPMS and 21 healthy volunteers were studied. Neuropsychological tests exploring memory, attention and frontal lobe cognitive domains were administered to BMS patients. Using a 1.5 Tesla scanner, a dual-echo turbo spin-echo and a 3D T1-weighted magnetization prepared rapid acquisition gradient echo (MP-RAGE) sequence were acquired in all subjects. An optimized VBM analysis was performed using statistical parametric mapping and a threshold of 0.05 , corrected for multiple comparisons. Intracranial volume, sex and age were used as nuisance variables in the analysis.

Results: Twelve BMS patients $(20 \%)$ had an abnormal performance in three or more neuropsychological tests (the pre-defined cut-off for cognitive impairment). Compared to healthy subjects, BMS patients had a reduced GM volume in the subcortical and frontoparietal regions. In comparison with BMS patients, those with SPMS had significant GM loss in the cerebellum. No group differences between BMS and SPMS patients were found when only those BMS patients with cognitive impairment or those with shorter disease duration (15-19 years) and higher EDSS scores $(>2.0)$ were considered for the analysis.

Conclusions: Cerebellar atrophy seems to be a major determinant of irreversible locomotor disability in MS. The absence of cognitive impairment and a longer disease duration/lower EDSS score may better identify those BMS patients with the potential for a favourable disease evolution.

Acknowledgements. This study was supported by a grant from "Fondazione Italiana Sclerosi Multipla" (FISM; contract n. 2005/R/18). Dr. Mesaros is supported by a Fellowship of the European Neurological Society (ENS).

\section{0}

Analysis of prevalence, risk factors and prognosis of acute myeloid leukaemia secondary to mitoxantrone therapy in multiple sclerosis patients C. Cordioli, N. De rossi, S. Rasia, E. Cottini, C. Cattaneo, G. Rossi, R. Capra Multiple Sclerosis Center (Brescia, IT); UO Ematology (Brescia, IT)

Objective: To evaluate prevalence and clinical outcome of acute myeloid leukaemia (AML) occurring in mitoxantrone-treated MS patients.

Methods: Clinical records of 1200 patients affected with MS followed at our centre were reviewed to clarify the prevalence, clinical characteristics, influence of therapeutic schedule and outcome of secondary AML.

Results: Among 176 MS patients treated with mitoxantrone from 1991, five (M/F: 1/4) developed AML. At therapy start 3/5 of the APL patients were naive for immunomodulatory drugs against MS, $1 / 5$ had been treated with IFN beta $1 \mathrm{~b}$ for 4 months before mitoxantrone and $1 / 5$ had been treated with glatiramer acetate for 7 months. In AML patients mean age at therapy start was 49,6 y, mean age at AML onset was 53,4 y (range 45-59) at mean time after the first drug course of 41,4 months (range 35-50). In AML patients mean cumulative dose of mitoxantrone was $90,5 \mathrm{mg} / \mathrm{mq}$ (range 22,5-130, median of $110 \mathrm{mg} / \mathrm{mq}$ ) administered over a median of 9,4 courses. In non AML patients mean age at therapy start was 42,5 y (range 18-68); mean cumulative dose of the drug was $83,4 \mathrm{mg} / \mathrm{mq}$ (range 10-190) and median of $80 \mathrm{mg} / \mathrm{mq}$. Our cohort was followed for a mean time of 61,5 months $(5,11$ y) (range 1-199 months). The prevalence of AML in our patients treated with mitoxantrone was $2,76 \%$ and $0,4 \%$ in the whole cohort. $4 / 5$ AML patient showed morphological and cytogenetic features of acute promyelocitic leukaemia (typical t $15 ; 17$ ) while $1 / 5$ was diagnosed as acute monomyelocitic leukaemia (t 11;19).

Complete haematologic and cytogenetic remission was obtained after induction in $4 / 5$ patients. However two of them relapsed (one had a molecular relapse needing chemotherapy treatment, one had two morphological relapses treated with arsenic trioxide and subsequent autologous peripheral stem cell transplantation). In one patient the disease course is not yet available because of recent diagnosis and treatment.

Conclusions: AML prevalence is significantly increased among mitoxantrone-treated MS patients and seems to occur at younger age than in general population ( $75 \%$ of non secondary AML occurs above 60 years of age). Previous treatments for MS and cumulative dose of mitoxantrone does not correlate with the risk of AML development. The clinical outcome of this subset of patients seems worse than in de-novo AML.

\section{1}

MN-166 reduces conversion of new lesions to persistent black holes in multiple sclerosis patients

R. Gammans, F. Barkhof, H. Hulst, R. Landin for the MN-166-CL-01 Investigators

Objective: To determine if MN-166 (ibudilast) treatment at 30 or $60 \mathrm{mg} / \mathrm{d}$ orally reduced evolution of new inflammatory lesions(NL) to persistent black holes (PBH) or increased evolution to remyelinated lesions (RL) as compared to placebo $(\mathrm{P})$ treatment.

Methods: A retrospective, double-blind evaluation of MRI data collected during year 1 of the MN-166-CL-001 study was performed. Patients with relapsing forms of multiple sclerosis (MS) were randomly assigned to daily oral treatment with $\mathrm{P}$ or 30 or $60 \mathrm{mg} / \mathrm{d}$ of MN-166. MRIs were collected bimonthly for $1 \mathrm{yr}$ of treatment (a year 2 active-treatment-only extension of the study is ongoing). Predefined endpoints for this evaluation were the rate of evolution of NL to PBH and RL. New T1 gadolinium-enhancing or new T2 lesions were defined as NL in the first on-study MRI at month 2 and tracked in the month 4 and 10 MRIs. Lesions that were hypointense and inactive at month 10 were PBH. Hypointense lesions at month 2 or 4 that were isointense at month 10 were RL. The relative risk (RR) of NL evolution to PBH and RL per patient was analyzed using a general linear model with the error term from the Poisson distribution.

Results: Of 100 P, $9430 \mathrm{mg} / \mathrm{d}$ and $9860 \mathrm{mg} / \mathrm{d}$ treated patients enrolled 72 $\mathrm{P}, 6430 \mathrm{mg} / \mathrm{d}$ and $5660 \mathrm{mg} / \mathrm{d}$ treated patients had NL in month 2 . The proportions of NL evolving to $\mathrm{PBH}$ were 0.24 for $\mathrm{P}, 0.20$ for $30 \mathrm{mg} / \mathrm{d}$ and 0.16 $60 \mathrm{mg} / \mathrm{d}$ treated patients. The RR for NL evolution to PBH was significantly lower (RR 0.63 , CI $0.44-0.90, \mathrm{p}=0.011$ ) for $60 \mathrm{mg} / \mathrm{d}$ treated patients, and tended to be lower for $30 \mathrm{mg} / \mathrm{d}$ treated patients (RR 0.735 , CI $0.52-1.03$, $\mathrm{p}=0.074$ ) as compared to $\mathrm{P}$ treatment. The RR for NL evolution to RL did not differ significantly among treatments.

Conclusions: Drulovic et al. (ECTRIMS 2007) previously reported that $60 \mathrm{mg} / \mathrm{d}$ of MN-166 significantly attenuated percent brain volume loss $(0.79$ vs. $1.21, p=0.04)$, increased the proportion of patients relapse-free and prolonged the time to first relapse $(\mathrm{p}<0.04)$ but did not significantly reduce cumulative unique lesion count during the first year of MN-166-CL-001. The current findings suggest that $\mathrm{MN}-166$ treatment protects neurons from persistent damage following acute lesion formation, a finding consistent with the observed attenuation of percent brain volume loss. Further studies on the effects of MN-166 treatment on PBH formation and disease progression in patients with relapsing or progressive forms of MS are warranted.

MN-166-CL-01 was supported by MediciNova Inc.

\section{2}

Consistent efficacy with early use of alemtuzumab in relapsing-remitting multiple sclerosis across major demographic subgroups A. Coles on behalf of the CAMMS223 International Study Group

Objectives: Preliminary studies suggest that alemtuzumab, a CD52-specific monoclonal antibody that targets lymphocytes markedly suppresses disease 
activity when administered during the early stages of relapsing-remitting multiple sclerosis (RRMS). The randomized, multi-center, rater-blinded, Phase 2 study, CAMMS223, compares alemtuzumab with high dose interferon beta-1a (IFNB-1a; Rebif ${ }^{\circledR}$ ) in patients with RRMS. A subgroup analysis of the Year 3 data from this study evaluates the role of baseline demography in the efficacy of alemtuzumab.

Methods: 334 treatment-naïve patients with RRMS were randomized 1:1:1 to $44 \mu \mathrm{g}$ SC IFNB-1a 3 times/week or annual cycles of low- ( $12 \mathrm{mg} /$ day) or high-dose ( $24 \mathrm{mg} /$ day) IV alemtuzumab. Alemtuzumab was given for 5 days at Month 0 , for 3 days at Month 12 , and in some patients, 3 days at Month 24. Key entry criteria included MS onset within 3 years of screening, Expanded Disability Status Score (EDSS) 0.0 to 3.0 (inclusive), at least 2 clinical events in the previous 2 years, and at least 1 Gadolinium-enhancing lesion on a screening cranial MRI. Co-primary efficacy outcomes were time to 6-month sustained accumulation of disability (SAD) and relapse rate.

Results: Compared to patients treated with IFNB-1a, alemtuzumabtreated patients overall demonstrated a $73 \%$ reduction in risk for relapse $(\mathrm{p}<0.0001)$ and a $70 \%$ reduction in risk for SAD $(\mathrm{p}<0.0001)$ after 3 years of follow-up. Subgroup analyses of the co-primary outcomes indicate that the treatment effects of alemtuzumab are consistent across sex, age $(<30 /$ $\geq 30$ years), race (Caucasian/non-Caucasian), and country (US/UK/Central and East EU)

Conclusion: Alemtuzumab is significantly more effective than IFNB-1a at suppressing relapses and disability in patients with RRMS through 3 years of follow-up regardless of patient sex, age, race, or country. A comprehensive safety monitoring program has effectively minimized morbidity due to side effects of alemtuzumab such as thyroid disorders, infection, and immune thrombocytopenic purpura.

Rebif $^{\circledR}$ is a registered trademark of EMD Serono, Inc.

Several members of the study group received from Genzyme Corporation: compensation for participating in a Speaker's Bureau or acting in an advisory capacity; financial support for their MS research studies; or compensation (incl. stock) as employees.

\section{3}

The effect of laquinimod on MRI-monitored disease activity in patients with relapsing-remitting multiple sclerosis: a double-blind active extension of the multicentre, randomised, double-blind, parallel-group placebo-controlled study

G. Comi, O. Abramsky, T. Arbizu, A. Boiko, R. Gold, E. Havrdova, S. Komoly, K.W. Selmaj, B. Sharrack, M. Filippi for the LAQ/5062 Clinical Advisory Board and Study Group

Objective: To determine whether the effects observed during the first phase of the double-blind(DB), randomized, placebo-controlled(PC) study with oral daily 0.3 and $0.6 \mathrm{mg}$ laquinimod, on magnetic resonance imaging (MRI)-monitored disease activity are sustained and those seen in the group originally randomized to placebo are reproducible when patients switched to either laquinimod $0.3 \mathrm{mg}$ or $0.6 \mathrm{mg} / \mathrm{d}$ in the 36 -week DB active extension.

Background: The ITT analysis of the primary efficacy endpoint during the 36 weeks of the PC phase of this study showed that patients who received $0.6 \mathrm{mg} / \mathrm{d}$ laquinimod had a $40 \%$ and $55 \%$ reduction in the mean and median number of enhancing lesions on T1-weighted images taken on the last trimester (weeks 24-36), compared to placebo. The annualized relapse favored laquinimod $0.6 \mathrm{mg} / \mathrm{d}$.

Design/methods: Subjects originally assigned to placebo were equally randomized to receive either 0.3 or $0.6 \mathrm{mg} / \mathrm{d}$, while others continued their original treatment, for 36 weeks. MRI was performed at the beginning and at the end of the active extension phase.

Results: Out of the 283 patients who completed the PC phase, 257 (91\%) entered the extension phase to receive laquinimod 0.3 (119 patients) or $0.6 \mathrm{mg} / \mathrm{d}$ (138 patients). 235 patients had an MRI measurement at the end of the active extension phase.

In patients who switched from placebo group to active treatment, mean number of enhancing lesions was reduced by $52 \%$ (from $4.46 \pm 6.55$ to $2.12 \pm 3.73, p<0.0007)$. The reduction was significant for both patients switching to either high or to low dose laquinimod $(\mathrm{p}<0.009$ and $\mathrm{p}<0.03)$. The proportion of enhancing lesion-free patients increased from $31 \%$ at the onset of to $47 \%$ at the end of the extension phase $(p<0.012)$. A significant reduction of the mean number of enhancing lesions was also observed in patients initially treated with $0.6(\mathrm{p}=0.0062)$ and $0.3 \mathrm{mg} / \mathrm{d}(\mathrm{p}=0.0013)$, with no further increase in the proportion enhancing lesion-free patients.

No significant differences in the annualized relapse rate in patients switching from placebo to either high or low dose laquinimod.

Neither new safety signals nor increase in incidence rate of AEs and laboratory abnormalities have emerged following new or prolonged exposure to laquinimod.
Conclusion: The treatment effects on MRI activity seen during the PC phase were reproduced when placebo patients switched to laquinimod. The positive effects persisted in those patients initially randomized to high dose laquinimod.

Supported by Teva Pharmaceutical Industries, Ltd

\section{Oral session 23}

\section{Higher function disorders 2}

\section{4}

Misperception of gravity direction and body orientation in spatial neglect A. Saj, J. Honoré, T. Bernati, M. Rousseaux

Université de Lille (Lille, FR)

Objectives: Hemispheric brain lesions cause a counter-clockwise deviation of the subjective vertical (SV) which is amplified in case of spatial neglect. Besides, neglect patients often show an ipsilesional deviation of the subjective straight ahead (SSA), thought to reflect a lateral shift of the representation of the body. The relations between both phenomena and with the severity of neglect in paper and pencil tests are debated. This study aimed at investigating these relations.

Methods: Forty patients with a right hemispheric stroke were included. Clinical tests (bell test, line bisection, Ogden test) revealed spatial neglect in 24 cases. The participants had to place a luminous rod straight ahead of their trunk or to adjust it to the geographical vertical, in darkness. Postural disorders were evaluated with the Postural Assessment Scale for Stroke patients. The lesions were analysed on MRI or CT pictures.

Results: In patients with neglect, the SV was significantly tilted counterclockwise $\left(-6.6^{\circ}\right)$, and the SSA was deviated rightward $(4.6 \mathrm{~cm})$. In the whole patient group, we found significant correlations between these two errors, and between the SSA deviation and the scores in paper and pencil tests. The SV tilt also correlated with the rightward deviation in line bisection. Furthermore, the SV tilt correlated with the severity of postural disorders, and a damage of the right parietal cortex predicted SSA deviation.

Conclusion: We report for the first time a link between gravity centred and body centred representations (SV and SSA) in patients with a right hemispheric stroke. This suggests that both are related to partially common mechanisms. Furthermore, we confirmed that the SV tilt is related to the postural bias characterizing these patients, and that the SSA shift is strongly associated with (and might contribute to explain) the severity of neglect signs.

0195

An analysis of communication in patients with dementia

M. Rousseaux, A. Sève, M. Vallet, F. Pasquier, M.A. Mackowiak Cordoliani CHRU (Lille, FR); Université de Lille (Lille, FR)

Objectives: Patients with degenerative dementia often present with language disorders. In these patients, we have little information about verbal (VC) and especially non-verbal (gestual) communication (NVC).

Objectives: To analyse VC and NVC in patients with dementia of various origins.

Methods: We included patients with classical criteria of Alzheimer disease (AD: 29), fronto-temporal dementia (FTD: 16), Lewy body disease (LBD: 13), and mild cognitive impairment (MCI: 11). Age: 50-80; MMS > 10/30; no vascular lesion. They were evaluated with the Lille Communication Test (LCT), which is composed of 3 parts: motivation to communication (salutation, attention, participation), VC (verbal comprehension, expressivity, intelligibility, informativity and pertinence of discourse and feedback), and NVC (gesture comprehension, affective expressivity, informativity and pertinence of non-verbal communication and feedback). They were also presented with the MMS, Mattis scale, and neuropsychological tests of executive functioning, gesture production, language, and a frontal behavioural rating scale. Their performance was compared with that of 47 matched control subjects.

Results: AD and FTD patients showed significant impairment $(\mathrm{p}<0.05)$ in motivation (FTD), VC (AD; FTD) and NVC (FTD). LBD and MCI patients were relatively preserved. Subtests analysis revealed that VC was mostly impaired by difficulties in verbal comprehension (AD, FTD), expressivity 
(word finding: AD, FTD; using syntax: FTD), informativity and pertinence (answering open questions: AD, FTD; theme maintenance: FTD; giving new information: AD, FTD; discourse organisation: FTD; discourse adaptation: FTD), and feedback emission (FTD). NVC was mostly impaired (FTD) by difficulties in comprehension of mimes, expressivity (gestures, prosody), and feedback. Furthermore, motivation, VC and NVC correlated with performance in the Mattis scale, MMS, gesture production and language tests.

Conclusions: Most severe communication disorders resulted from FTD and at a lesser degree from AD. Furthermore, the first population was impaired in all the aspects of communication, and this can have severe consequences on social relations. The severity of communicative disorders was likely related with their pre-frontal lesions.

\section{6}

"Tomato and tuna" for testing action understanding after middle cerebral artery infarcts

A. Faber, M. Gade, G. Negri, A. Lunardelli, A. Baumgaertner, F. Binkofski, A. Danek, R.I. Rumiat

LMU (Munich, DE); SISSA (Trieste, IT); NeuroImage-Nord (Hamburg, DE); Neurology (Lubeck, DE)

Objectives: For humans as social beings it is fundamental to understand the actions of others. The underlying mechanisms are still poorly understood, yet one correlate is sought in some kind of "mirroring system" in the brain.

Methods: Since the middle cerebral artery (MCA) territory includes most of the putative mirror neuron system regions, we tested eleven patients ( $72 \pm 6$ years) with chronic brain lesions after MCA infarcts with a newly developed non-verbal test for action understanding. In our "Tomato and Tuna Test" (TTT), three pictures are displayed on a PC screen with "Presentation" (www.nbs.com). An accomplished action goal (e.g. an open tin of tuna) has to be matched with one of two possibly preceding actions (opening tin with a fork or the appropriate instrument, respectively). The TTT emphasises two aspects of action - goals and the sequential nature of actions - that so far have been rarely addressed.

We validated the TTT against two measures (Kissing and Dancing Test KDT of Bak 2003, and Action Videos of Baumgaertner 2007) and compared patients' performance to that of an age-, sex- and education-matched control group as well as fifteen younger controls ( $24 \pm 3$ years). Lesion sites were analysed from patients' CT scans using MRIcro (www.mricro.com).

Results: Patients were less accurate on the TTT than their matched controls, the latter again performing worse than the young controls. In comparison to the KDT and the Action Videos, we confirmed a higher sensitivity of the TTT for differentiating between patients and controls. Lesion analysis disclosed the anterior insula as a brain region possibly associated with deficits in the KDT. Double dissociations were found between the TTT and the KDT, with two patients each being more impaired in the TTT than in the KDT, and vice versa. Their lesions showed almost no overlap.

Conclusion: The only minimal lesion overlap with impaired TTT and KDT performance suggests that distinct brain regions may subserve different aspects of action understanding. Action understanding deficits detected by the TTT are present in patients with lesions to brain areas that belong to the hypothetical mirror neuron system. The double dissociations indicate that the TTT and the KDT measure different aspects of action understanding. Because of its higher sensitivity, we suggest that the "Tomato and Tuna Test" assesses action understanding in an ecologically more valid manner than previous tasks and is more apt to detect deficits.

\section{7}

Genetic and functional analyses of progranulin gene variants in Alzheimer's disease

C. Fenoglio, D. Galimberti, F. Cortini, J.S.K. Kauwe, E. Venturelli, I. Guidi, D. Scalabrini, C. Villa, K. Mayo, E. Dalla Valle, C. Lovati, C. Mariani, N. Bresolin, A.M. Goate, E. Scarpini

University of Milan, IRCCS Ospedale Maggiore (Milan, IT); Washington University School of Medicine (St. Louis, US); University of Milan, Ospedale L. Sacco (Milan, IT)

Objective: To examine Progranulin (PGRN) genetic variability and the effect of the genetic background on PGRN expression rate in patients with sporadic Alzheimer's disease $(\mathrm{AD})$

Background: Null mutations in PGRN cause ubiquitin-positive frontotemporal dementia linked to chromosome 17q21. Although its physiological function is not completely understood, some evidences suggest that PGRN might be implicated in the susceptibility of a wider spectrum of neurodegenerative diseases including $\mathrm{AD}$

Methods: Coding region for PGRN was screened by direct sequencing in $50 \mathrm{AD}$ patients. Subsequently a case-control study was performed in 355 Ital- ian AD patients and 322 controls for rs 9897526 and rs5848 SNPs by allelic discrimination. An independent American set of patients consisting of 329 $\mathrm{AD}$ patients and 302 controls was genotyped by Sequenom genotyping technology to replicate results. The effect of rs5848 on transcription was evaluated by Real-Time PCR in Peripheral Blood Mononuclear Cells (PBMC). Haploview 3.1 software was used to test for association and Sigma Stat v3.1 employed for correlations with clinical variables.

Results: Two SNPs occurring with a frequency higher than $1 \%$ were detected by direct sequence in the Italian series: rs9897526, located in the flanking regions of exons 2 and rs5848 located in the 3'UTR region. A subsequent case-control study revealed a significant increased frequency of rs5848 in patients compared with controls ( $35 \%$ vs $29 \%$; $P<0.024$ ). However, these data failed to be replicated in the American series $(\mathrm{P}>0.05)$. A decreased expression rate of PGRN was found in PBMC from carriers of the polymorphic allele $(\mathrm{P}<0.007)$. Rs 9897526 was not associated with $\mathrm{AD}$ although patients carrying at least one polymorphic allele showed a significant earlier age at onset than non-carriers $(\mathrm{P}<0.004)$.

Conclusion: This is the first attempt to investigate PGRN genetic variations in $\mathrm{AD}$ patients. The rs5848 likely acts as susceptibility factor for $\mathrm{AD}$, and reduces PGRN mRNA levels, possibly contributing to the failure of neuronal survival. However replication of the association findings for rs5848 failed considering the American set of samples, suggesting that these results need to be confirmed in a wider population. In addition, rs9897526 anticipates the onset of the disease.

\section{8}

Osteopontin is increased in the cerebrospinal fluid of patients with early Alzheimer's disease and its levels correlate with cognitive deterioration M. Carecchio, C. Comi, S. Nicola, D. Galimberti, A. Chiocchetti, C. Fenoglio, G. Cappellano, E. Scarpini, F. Monaco, U. Dianzani

Amedeo Avogadro University (Novara, IT); University of Milan (Milan, IT)

Objectives: This work is aimed at comparing Osteopontin (OPN) serum and cerebrospinal (CSF) levels in Alzheimer disease (AD) patients and healthy controls, in order to assess a possible role for this molecule as a predictive factor for the development of the disease. It is known that prominent activation of inflammatory processes and the innate immune response take place in the brains of $\mathrm{AD}$ patients. OPN is a cytokine abundantly expressed by activated T-cells that has been shown to enhance the TH1 and inhibit the TH2 responses. Recent work by Wung et al. shows that OPN is highly expressed by pyramidal neurons in AD brains. Using SELDI-TOF-MS analysis, Simonsen et al. report that a fragment of OPN is increased in the CSF of patients with mild cognitive impairment progressing to $\mathrm{AD}$.

Methods: 48 patients with a diagnosis of probable AD and 31 ethnically matched healthy controls were enrolled from the Department of Neurology of Amedeo Avogadro University, Novara and Department of Neurological Sciences, Dino Ferrari Centre, University of Milan, Italy. Age at onset, disease duration and cognitive state assessed with Mini Mental State Examination (MMSE) were evaluated. Serum and CSF levels of OPN were assessed by ELISA technique.

Results: Patients with AD showed significantly higher OPN levels in the CSF compared to controls (median value: $7672 \mathrm{vs} 2867 \mathrm{ng} / \mathrm{ml}$ ), whereas no difference was found in OPN serum levels between patients and controls (median value: $168 \mathrm{vs} 225 \mathrm{ng} / \mathrm{ml}$ ). A second analysis of OPN CSF levels was performed dividing AD patients into groups according to disease duration $(\leq 3$ years = early $\mathrm{AD} ;>3$ years = moderate-advanced $\mathrm{AD})$ and level of cognitive impairment (MMSE $>15$ and $\leq 25=$ mild $\mathrm{AD}$; MMSE $\leq 15=$ moderate-severe $\mathrm{AD}$ )

Intriguingly, patients with early AD showed significantly higher OPN levels than moderate-advanced patients (median value $8700 \mathrm{vs} 4300 \mathrm{ng} / \mathrm{ml}$ ). Moreover, patients with mild AD showed increased OPN levels in the CSF compared to moderate-severe patients (median value $7960 \mathrm{vs} 4520 \mathrm{ng} / \mathrm{ml}$ ).

Conclusions: These data suggest that OPN is a promising biomarker for $\mathrm{AD}$. The assessment of the CSF protein levels during disease course may provide information on both disease progression and treatment of $\mathrm{AD}$.

\section{9}

Brain activation and deactivation are associated with changes in aerobic glycolysis

A.G. Vlassenko, S.N. Vaishnavi, M.M. Rundle, L.E. Couture, A.Z. Snyder, M.E. Raichle, M.A. Mintun

Washington University School of Medicine (St. Louis, US)

Objectives: The effects of physiological brain stimulation have been studied using positron emission tomography (PET) and functional magnetic resonance imaging showing dramatic increase in cerebral blood flow and glucose utilization but much less change in oxygen utilization in activated brain 
regions, which reflects an increase in aerobic glycolysis. In this study, we have assessed regional PET measures of brain aerobic glycolysis at rest and in response to physiological stimulation with particular attention to activity decreases ("deactivation"). The Word Stem Completion Task (WSCT) was used to provide strong increases in activity of some brain regions including visual cortex and decreases in activity of the regions of the "default network", which is chronically active at rest but decreases its activity in response to various mental tasks.

Methods: Eight healthy young adults (mean age $25 \pm 3$ years) were studied at rest (eyes closed) and during the WSCT. The distribution of aerobic glycolysis was imaged by combining relative estimates of oxygen utilization $\left(\mathrm{CMRO}_{2}\right)$ and glucose utilization (CMRGlc). Regional $\mathrm{CMRO}_{2}$ was computed based on the acquisition of three PET scans using three different [15O] tracers $\left(\mathrm{CO}, \mathrm{O}_{2}\right.$ and $\left.\mathrm{H}_{2} \mathrm{O}\right)$. The relative distribution CMRGlc was estimated using [18F]FDG PET. Images of aerobic glycolysis expressed as the glycolytic index (GI) were generated by calculating a pixel-by-pixel residual after regression of the estimates of CMRGlc to $\mathrm{CMRO}_{2}$, which corresponds to the amount of glucose utilization that differs from what is predicted by the amount of oxygen utilization.

Results: GI increased in regions known to respond positively to the WSCT including visual and motor cortex and cerebellar hemispheres ( $p<0.001$, two-tailed paired t-test). GI remained high and did not change in anterior cingulate cortex and dorsolateral prefrontal regions. GI decreased in response to the WSCT in regions within "default network" including posterior cingulate cortex, ventromedial and dorsal prefrontal cortex, lateral parietal cortex, as well as amygdala and cerebellar vermis $(\mathrm{p}<0.001$, twotailed paired t-test). Most of the regions where GI decreased in response to the WSCT had high levels of resting GI, however some regions had low resting GI and showed further decreases in response to the WSCT.

Conclusion: Regional brain activation and deactivation in response to physiological stimulation are associated with the changes in aerobic glycolysis.

NIH Grants P50 NS006833; P30 NS048056

\section{Oral session 24}

\section{Cerebrovascular disorders 4}

\section{0}

Brain swelling extensively aggravates infarct formation in large territorial stroke: a target for neuroprotection?

T. Gerriets, M. Walberer, N. Ritschel, K. Volk, C. Mueller, M. Tschernatsch, E. Stolz, F. Blaes, G. Bachmann, M. Nedelmann

University Giessen (Giessen, DE); Kerckhoff Clinic (Bad Nauheim, DE)

Object: In territorial stroke vasogenic edema formation leads to raised intracranial pressure and can cause herniation and death. Brain swelling furthermore impairs collateral blood flow to the ischemic penumbra and causes mechanical damage to adjacent brain structures. With the present study we sought to quantify the impact of the space-occupying effect onto ischemic lesion formation.

Methods: Wistar rats were assigned to bilateral craniectomy or to sham operation and then subjected to temporary middle cerebral artery occlusion (MCAO) for 90 minutes. Clinical evaluation and 7T-magnetic resonanceimaging (MRI) were performed after $5 \mathrm{~h}$ and $24 \mathrm{~h}$. Absolute brain water content was determined after $24 \mathrm{~h}$ using the wet/dry method.

Results: Bilateral craniectomy prior to MCAO led to a drastic reduction of lesion volume at both imaging time-points $(\mathrm{p}<0.0001)$. Ischemic lesion volume was 2.7 -fold and 2.3-fold larger in sham-operated animals after $5 \mathrm{~h}$ and $24 \mathrm{~h}$, respectively. Clinical scores were likewise better in craniectomized rats $(\mathrm{p}<0.05)$. After $24 \mathrm{~h}$ midline-shift differed significantly between both groups $(p<0.001)$, but not after $5 \mathrm{~h}$. The relation between brain water content and ischemic lesion volume, as well as T2-relaxation time within the infracted area was not different between the groups $(\mathrm{p}>0.05)$.

Conclusions: The present data indicate that collateral damage caused by the space-occupying effect of large MCA-territory stroke contributes to ischemic lesion formation extensively. Elimination of intracranial pressure thus can be regarded as a highly neuroprotective measure, rather than only a life-saving procedure to prevent cerebral herniation. Further clinical trials are needed to determine the neuroprotective potential of surgical and pharmacological anti-oedematous therapeutic approaches.
0201

Factors affecting the length of stay in the neurological intensive care unit in patients with intracranial haaemorrhage

Z. Haneef, M. Vendrame, A. Bayrlee, A. Azizi

Temple University Hospital (Philadelphia, US)

Objective: To define factors leading to prolonged stay in the Neurological ICU in Intracranial Hemorrahge (ICH). Knowledge of this will help define predictors of complications and help reduce health-care costs.

Methods: Medical data of 159 patients discharged from Neurology ICU in a tertiary care hospital were retrospectively analyzed. Data was gathered on demographics, vitals and GCS at admission, ICU length of stay (ICULOS) in days, anti-epileptic drug (AED) administration, presence of seizures, volume of ICH, proximity of bleed to the cortex, presence of ventriculostomy, seizures, blood hemogram and chemistry during ICU stay. T tests and Pearson correlations were used for univariate analysis with LOS. A multi-step linear forward regression was also performed.

Results: The mean age \pm SD was $64.3 \pm 14.3$ years (female: male $=88: 71$ ) and the median ICU stay was 5 days (range $0-79$ ). There was significant correlation between longer ICU-LOS and higher WBC count $(\mathrm{R}=0.224$, $\mathrm{p}=0.005)$, glucose $(\mathrm{R}=0.343, \mathrm{p}=0.000), \mathrm{CO} 2(\mathrm{R}=-0.165, \mathrm{p}=0.038)$ and bleed volume $(R=0.25, p=0.002)$ at admission. There was no significant correlation with age, admitting temperature, systolic/diastolic BP, hemoglobin, platelet count, sodium, potassium, chloride, creatinine or magnesium. The correlation of ICU-LOS to proximity of bleed to cortex did not reach statistical significance $(\mathrm{R}=0.161, \mathrm{p}=0.053)$.

$T$ test found significant association between the ICU-LOS and the presence of tracheostomy $(\mathrm{p}<0.001)$, gastrostomy $(\mathrm{p}<0.001)$, intra-ventricular haemorrhage $(\mathrm{p}<0.001)$ or ventriculostomy $(\mathrm{p}<0.001)$ and total GCS score at presentation $(\mathrm{R}=-0.487, \mathrm{p}<0.001)$. There was no association of ICU LOS with the presence of seizures $(p=0.94)$ AED in the hospital $(p=0.313)$ or statin use $(\mathrm{p}=0.102)$.

Conclusions: Higher WBC count, blood glucose and $\mathrm{CO}_{2}$ at admission, which could reflect co-morbidity or may be secondary to severe bleed, resulted in longer ICU stay as has been shown previously in non-neurologic critical care literature. Presence of intra-ventricular haemorrhage, lower GCS at presentation and procedures such as gastrostomy and venticulostomy also resulted in longer ICU stay.

\section{2}

Development and implementation of a care pathway for acute stroke P. Martinez-Sanchez, J. Medina, B. Fuentes, M. Grande, C. Llorente, P. Parrilla, A. Fuster, A. Gil, J. Garcia-Caballero, E. Diez-Tejedor

Hospital Universitario La Paz (Madrid, ES)

Background: In-hospital stroke care pathways were implemented in departments of Neurology, General Medicine or Rehabilitation, however there are few studies evaluating the development and the effects of introducing and integrated care pathway (ICP) in hospitals with an Acute Stroke Unit (ASU). Our aim was to develop an evidence-based multidisciplinary ICP for acute ischemic and hemorrhagic stroke care in an ASU.

Methods: A schedule of 1-h fortnightly meetings between the Department of Neurology, Emergency Services, Preventive Medicine and Rehabilitation was established for the ICP design. The evidence base was established through a systematic literature review. Following development, performance was assessed against standards in a before-and-after study. The 'after' (intervention) group comprised 36 consecutive stroke patients and the 'before (control) group comprised 36 stroke patients admitted to the same ACU paired for age, sex, stroke subtype and stroke severity.

Results: The documents that compose the ICP are: clinical flowsheet; treatment sheets; patient and family information document; rehabilitation information document; patient/family satisfaction questionnaire and a didactic unit for patient/family education in stroke management and prevention. The patients mean age was 73.10 years (SD 9.5), $47.2 \%$ were male. The antiplatelet and fibrinolytic treatment and the evaluation of the dysphagia followed the standard in both groups $(100 \%)$; the patient/family satisfaction level was high $(90 \%)$ but the intervention group showed a better quality of documentation. The intervention group tended to a higher adjustment to the standard in laboratory results, first cerebral-TC and peripheral venous line removal times than the control group (51.6 vs. $40 \%, 42.9$ vs. $32.2 \%$ and 60 vs. $39.3 \%$ respectively, P NS), as well as in the neurosonology tests and second cerebral-TC absolute times ( 0.87 vs. 13.3 days and 1.37 vs. 2.06 days respectively, $\mathrm{P}$ NS).

Discussion: Introducing and ICP for acute stroke tends to improve the quality of documentation and process of care. 
0203

Thrombophilic disorders: the link between migraine, atrial septal abnormalities and stroke?

P. Martinez-Sanchez, B. Fuentes, M. Cuesta, M.A. Ortega-Casarrubios, J. Fernandez, E. Diez-Tejedor

Hospital Universitario La Paz (Madrid, ES)

Background: Migraine has been associated with patent foramen ovale (PFO) and more recently, with inherited thrombophilic disorders. Our goal was to assess the relationship between migraine, PFO \pm atrial septal aneurism (ASA) and thrombophilic disorders in young patients with cryptogenic stroke.

Methods: Observational study with inclusion of consecutive patients from the Stroke Unit Data Bank (January 1996-January 2007). We investigated patients under 55 years of age with a cryptogenic acute ischemic stroke. Variables analyzed: gender, vascular risk factors, previous migraine and the presence of hypercoagulable states (HS) by an extensive battery of hematological tests. The presence of PFO \pm ASA was assessed by transesophageal echocardiography \pm transcranial Doppler sonography.

Results: 188 patients were included. Median age [intercualtile range]: 43.5 years [12], $38.2 \%$ women. Previous migraine was present in $9 \%$ (17 cases) of patients. Patients with migraine were more commonly females ( $76.5 \%$ vs. $35.1 \% \mathrm{P}=0.001$ ) and tended to have fewer classic vascular risk factors. Migraine was associated with a higher frequency of HS (crude OR, $3.33 ; 95 \%$ CI, 1.06 to 10.50 ) and the presence of PFO + ASA (crude OR, 7.50; $95 \%$ CI, 2.17 to 25.93). Patients with migraine and PFO had the highest frequency of HS (crude OR, 16.2; $95 \%$ CI, 2.78 to 94.14 ).

Discussion: In acute stroke patients under 55 years of age, thrombophilic disorders are diagnosed more frequently in patients with migraine, especially if they have atrial septal abnormalities. Some types of migraine might be an expression of an underlying HS and could predispose patients to ischemic stroke if they are associated with atrial septal abnormalities.

\section{O204}

Blockade of bradykinin receptor B1 but not bradykinin receptor B2 provides protection from brain oedema, postischaemic inflammation and cerebral infarction

M.L. Austinat, S. Braeuninger, G. Stoll, T. Renné, C. Kleinschnitz

Universität Wurzburg (Wurzburg, DE)

Background: Inflammation and edema formation are critically involved in the pathophysiology of ischemic stroke and are a frequent cause of secondary infarct growth. Kinins are highly active proinflammatory peptides which are released during inflammation and tissue injury including cerebral infarction. The effects of kinins are mediated by two receptors (B1 and B2 receptor [B1R, B2R]) and comprise induction of edema formation and release of proinflammatory mediators. Previous studies could show that the blockade of the B1R protects from ischemic tissue damage, e. g. in models of myocardial infarction.

Objectives: To analyze the effect of B1R and B2R deficiency or blockade on infarct size, edema formation and inflammatory processes after transient middle cerebral artery occlusion (tMCAO) in mice.

Methods: B1R knockout (KO), B2R KO and wildtype (WT) mice were subjected to 60 min tMCAO and infarct volumes were determined by 2,3,5triphenyltetrazolium chloride (TTC) staining at day 1 . To analyze the effect of pharmacological B1R inhibition in WT mice, the selective B1R inhibitor R-175 was administered i. v. 1 hour after tMCAO. The extent of brain edema was determined by injecting Evan's Blue tracer $2 \mathrm{~h}$ after tMCAO. The postischemic inflammatory response was quantified by qRT-PCR against IL-1b, $\mathrm{TGFb}-1 \mathrm{a}$ and TNFa and the amount of infiltrating immune cells was determined by immunohistochemistry.

Results: B1R null mice developed significantly smaller brain infarctions and less neurological deficits compared to WT controls $\left(16.8+4.7 \mathrm{~mm}^{3} \mathrm{ver}-\right.$ sus $50.1 \pm 9.1 \mathrm{~mm}^{3}$, respectively; $\mathrm{p}<0.0001$ ). This was accompanied by a dramatic reduction of brain edema in the ischemic hemisphere as measured by extravasation of Evan's blue tracer $\left(6.7 \pm 1.6 \mathrm{~mm}^{3}\right.$ versus $81.7 \pm 17.8 \mathrm{~mm}^{3}$, respectively; $p<0.0001$ ) and less postischemic inflammation within the infarcted tissue. Pharmacological blockade of B1R likewise salvaged ischemic brain tissue $\left(18.7 \pm 11.6 \mathrm{~mm}^{3}\right.$ versus $50.1 \pm 9.1 \mathrm{~mm}^{3}$, respectively; $\left.\mathrm{p}<0.01\right)$ and improved neurological outcome in a dose-dependent manner even when B1R inhibitor was applied 1 hour after tMCAO. In contrast, B2R deficiency or inhibition did not confer neuroprotection and had no effect on the development of tissue edema or postischemic inflammation.

Conclusion: These data demonstrate that blocking of B1R can diminish brain infarction in mice by reducing edema formation and inflammation and may open new avenues for acute stroke treatment in humans.
0205

tPA and MMP-9 interactions in focal cerebral ischaemia: distinct mechanisms of gene regulation

J. Lee, P. Zickler, S. Jander

Heinrich-Heine University (Dusseldorf, DE)

Objectives: Tissue plaminogen activator (tPA) is broadly used for thrombolysis in acute ischemic stroke, but recent data suggest additional pleiotropic actions including cytokine-like features and activation of matrixmetalloproteinases (MMP). tPA augments MMP-9 activity thereby leading to neurovascular proteolysis, infarct enlargement, and hemorrhage. On the other hand MMP-9 may also promote beneficial tissue repair in the subacute phase after stroke. Aim of the present study was to elucidate spatiotemporal patterns and mechanisms of TPA and MMP-9 induction in focal brain ischemia with the perspective of more specific therapeutic targeting.

Methods: Focal cerebral ischemia was induced in adult male Wistar rats $(230-300 \mathrm{~g})$ by cortical photothrombosis. Subgroups of the rats $(n=4)$ were pretreated with either MK-801 $(2 \mathrm{mg} / \mathrm{kg}$ body weight i.v. $30 \mathrm{~min}$ before the onset of ischemia) or with Memantine $(20 \mathrm{mg} / \mathrm{kg}$ body weight i.p. $30 \mathrm{~min}$ before the onset of ischemia). Tissue samples were prepared from the stroke lesion and frontal nonischemic cortex ipsi- and contralateral to the lesion at $4 \mathrm{~h}, 16 \mathrm{~h}, 7$ days and 14 days after the onset of ischemia. Total RNA was extracted and quantitative real-time RT-PCR was performed using tPA- and MMP-9-specific primer sets.

Results: Two waves of TPA and MMP-9 gene expression were found after focal cortical ischemia. tPA mRNA was first upregulated at 4 hours and reached a second peak at 7 days. MMP-9 expression showed a slightly delayed, but otherwise similar pattern with an early peak at 16 hours and a second peak at 7 days. The early induction of tPA and MMP-9 occured to a similar extent in the lesion and nonischemic ipsilateral cortex whereas the second peak was restricted to the lesion. The NMDA receptor antagonists MK-801 and Memantine blocked early gene induction in ipsilateral remote cortex. In contrast, gene expression in the lesion was only slightly reduced at the early time point and unaffected at day 7.

Conclusions: The spatially distinct biphasic pattern of gene expression suggests different underlying mechanisms of gene induction. Our data reveal cortical spreading depression (CSD) as a potential mechanism of early MMP-9 induction in nonischemic remote cortex. Via their effect on CSD-dependent gene induction, NMDA receptor antagonists like Memantine may selectively reduce harmful MMP-9 effects at early stages after cerebral ischemia while not influencing the late induction probably contributing to beneficial tissue repair.

Work was supported partly by the Deutsche Forschungsgemeinschaft (Ja 690/5-2).

\section{Oral session 25}

\section{Motor neuron disease}

\section{6}

Mechanisms of cold paralysis

H. Franssen, L.H. Van den Berg, L.J. Van Schelven

University Medical Centre Utrecht (Utrecht, NL)

Objectives: The development of weakness in cold (cold paralysis) was reported in lower motor neurone disorders. The following mechanism was proposed: cooling induces slowing of the $\mathrm{Na} / \mathrm{K}$ pump which leads to axonal depolarization; this may yield a depolarizing block in diseased axons in which impulse propagation is at risk. Our objective was to obtain evidence for this hypothesis by assessing if cold induces weakness and axonal depolarization.

Methods: The right arm of 10 normal subjects was investigated at 37,25, 20 and $15^{\circ} \mathrm{C}$. These temperatures were attained by prolonged warming or cooling in water. At each temperature were assessed: thenar muscle strength, excitability in median nerve motor axons at the wrist, peak systolic velocity of a wrist artery and neuromuscular transmission.

Results: Weakness occurred at 20 and $15^{\circ} \mathrm{C}$. Excitability tests showed progressive changes from 37 to $15^{\circ} \mathrm{C}$ (fanning in of threshold electrotonus, steepening of current threshold relation, increase of refractory period, decrease of superexcitability), consistent with progressive axonal depolarization. Peak systolic velocity decreased, consistent with progressive arterial 
vasoconstriction. Neuromuscular transmission and compound muscle action potential amplitude were unaffected.

As cooling induced vasoconstriction and as the excitability changes were similar to those known to be induced by ischemia, a control experiment was performed. At $37^{\circ} \mathrm{C}$, a cuff was inflated around the upper arm until peak systolic velocity had the value previously measured at $15^{\circ} \mathrm{C}$. Excitability tests in this condition showed no axonal depolarization and cuff release induced no axonal hyperpolarization. This indicates that the depolarization induced by cooling cannot be explained by ischemia but is due to cooling induced $\mathrm{Na} / \mathrm{K}$ pump slowing.

As excitability studies depend on muscle recording and as slow muscle relaxation occurs at low temperatures, another control experiment was performed. At $15^{\circ} \mathrm{C}$ excitability was tested with a slow stimulus rate, enabling full muscle relaxation after each stimulus. This showed no differences with testing at a normal stimulus rate, indicating that the excitability changes with cooling could not be attributed to slow muscle relaxation.

Conclusion: Cooling induces weakness, and axonal depolarization due to temperature related $\mathrm{Na} / \mathrm{K}$ pump slowing. The depolarization may contribute to the cold paralysis that is observed in motor neurone disorders.

\section{7}

Percutaneous nocturnal oximetry in amyotrophic lateral sclerosis: periodical desaturation

J. Costa, S. Pinto, A. Pinto, M. de Carvalho

Institute of Molecular Medicine, Lisbon Faculty of Medicine (Lisbon, PT); Santa Maria Hospital. (Lisbon, PT)

Introduction and objectives: Respiratory function is critical in the prognosis of amyotrophic lateral sclerosis (ALS). Percutaneous nocturnal oximetry (PNO) is a useful screening tool of the respiratory function in ALS. PNO recordings of some ALS patients disclose a periodical pattern of $\mathrm{O} 2$ desaturation (PP), the clinical meaning of which is not fully understood. In this study we aimed to further characterize these changes, in particular the relations with the respiratory muscles status.

Methods: Prospective study enrolling 261 consecutive ALS patients. Clinical, pulmonary and neurophysiological tests performed were: ALS functional rating scale (ALSFRS), forced vital capacity (FVC), maximal inspiratory pressure (Pimax), mouth occlusion pressure (MOP), phrenic nerve motor response, needle electromyography of the diaphragm, and PNO. A subgroup of patients underwent polysomnography. Patients with PP were selected for further analysis.

Results: A total of 837 PNO recordings were analyzed independently by two of the authors (mean number of recordings per patient of 3.2). Fortyfive patients $(17.2 \%)$ showed a typical PP. Four patients were excluded, 13 had normal diaphragm (Group 1), and in 28 the diaphragm was abnormal (Group 2). Group 1 and 2 were comparable, except for respiratory functional score, FVC and PImax which were significantly lower in Group 2. In Group 1 , REM sleep was absent and hypoventilation occurred at slow-wave sleep. Five patients in Group 1 were very spastic, had low MOP/FVC and a short survival.

Conclusion: This study identified a subgroup of ALS patients (Group 1) with marked signs of upper motor neuron lesion, strong respiratory muscles, periodical hypoventilation, low MOP/FVC ratio and poor prognosis. We speculate that they have a central respiratory dysfunction and deserve special care.

Susana Pinto has a grant from the "Fundação para a Ciência e Tecnologia" SFRH/BD/30714/2006.

\section{8}

Ocular fixation and saccades in motor neurone disease: markers of frontal lobe dysfunction?

C. Donaghy, R. Pinnock, S. Abrahams, C. Cardwell, O. Hardiman, V. Patterson, R.C. McGivern, J.M. Gibson

Royal Victoria Hospital (Belfast, UK); University of Edinburgh (Edinburgh, UK); Beaumont Hospital (Dublin, IE)

Objective: Studies of eye movements in Motor Neurone Disease (MND) to date have been conflicting and studies have been small. Although disturbances of ocular fixation have been noted in MND, ocular fixation has not been formally examined. With the recent characterization of ocular fixation using saccadic intrusion (SI) amplitude and fixation periods, we performed a cross-sectional study to examine ocular fixation and saccades in patients with MND with a view to creating a surrogate and objective marker for disease.

Methods: Forty-four patients and forty-five controls were recruited. Ocular fixation, reflexive saccades and antisaccades were examined using infra- red oculography and all subjects then underwent a neuropsychological evaluation.

Results: SI amplitude, antisaccade latency and antisaccade errors were found to be greater in patients compared to controls. Reflexive saccades were similar between the groups. SI amplitude and antisaccade errors correlated with neuropsychological measures sensitive to lesions of the frontal lobes.

Conclusions: These results indicate that abnormalities in ocular fixation and saccades may be a marker of the sub-clinical frontal lobe dysfunction in MND. A longitudinal study to examine if ocular fixation and saccades deteriorate with time would be of great interest as this could provide a quantifiable objective marker of disease progression.

\section{9}

Diffusion tensor MRI of the cervical cord as a tool to monitor disease progression in amyotrophic lateral sclerosis

F. Agosta, P. Valsasina, S. Sala, D. Caputo, M. Perini, A. Prelle, M.E. Fruguglietti, F. Salvi, I. Bartolomei, M. Filippi

Neuroimaging Research Unit (Milan, IT); Fondazione Don Gnocchi (Milan, IT); Ospedale di Gallarate (Gallarate, IT); Fondazione Policlinico Mangiagalli (Milan, IT); University of Bologna (Bologna, IT)

Objectives: To define the progression of intrinsic tissue damage and atrophy in the cervical cord from patients with sporadic amyotrophic lateral sclerosis (ALS).

Methods: Conventional and diffusion tensor (DT) MRI of the cervical cord were obtained from 17 ALS patients and 11 controls, at baseline and after a median follow up of 9 months. Brain conventional MRI was also acquired. At each time point, cervical cord cross-sectional area, average mean diffusivity (MD) and average fractional anisotropy (FA), as well as the presence of T2 hyperintensities along the brain portion of the CST were assessed. Cord conventional and DT MRI metrics changes were analyzed using a mixed effect linear model (accounting for repeated measures within each subject non equally spaced in time), including follow up duration, group (controls vs. ALS patients) and a follow up duration $\mathrm{x}$ group interaction term, as independent variables.

Results: Mean ALS functional rating scale scores were 26 (range $=7-33$ ) at baseline and 22 (range $=6-33$ ) at follow up. During follow up, ALS patients showed a significant decrease of cord area $(\mathrm{p}=0.003)$ and cord average FA $(p=0.001)$. Similarly, the increase of cord average MD at follow up was significantly higher in ALS patients compared to controls $(\mathrm{p}=0.01)$. In ALS patients, changes of cord area were not associated with changes of diffusivity metrics over time.

Conclusions: Progressive tissue loss and injury to the remaining tissue, which are not strictly interrelated, occur in the cervical cord of ALS patients. This suggests that imaging the cervical cord may be a useful tool to monitor ALS evolution, either natural or modified by treatment.

\section{0}

Cognitive assessment and auditory event-related potentials in patients with motor neuron disease

M. Consonni, S. Iannaccone, M. Lacerenza, M. Zamboni, A. Raggi, S.F. Cappa San Raffaele Scientific Institute and Vita-Salute University (Milan, IT); San Raffaele Turro Hospital (Milan, IT)

Objective: To investigate the presence of sub-clinical cognitive dysfunction in Motor Neuron Disease (MND) using auditory event-related potentials (ERPs). To differentiate the pattern of cognitive dysfunction in patients suffering from either upper or lower motor neuron impairment.

Methods: The presence of subclinical cognitive impairments was investigated using neurophysiological assessment and ERPs recordings in $12 \mathrm{pa}-$ tients with Amyotrophic Lateral Sclerosis (ALS), 5 patients with Lower Motor Neuron Disease (LMND) and 14 age- and education-matched healthy controls. N1, Mismatch Negativity (MMN), and novelty P300 (P3a) were recorded by a Passive Oddball Paradigm. An extensive battery of neuropsychological tests assessing the domains of attention, memory, language, executive functioning and reasoning were administered to patients and controls. Patient's Clinical features, disability, cognitive status and depression were evaluated with standardised scales.

Results: ALS patients had a shorter disease duration and greater motor impairment than LMND patients. There were no differences between patients and controls for depression scale scores. ALS patients had lower performances on working memory and fluency tasks compared with LMND patients and controls. In ERP recording, ALS patients showed N1 and MMN amplitudes reduction over frontal sites and P3a was of lower amplitude compared with the controls. No significant differences were found in ALS-LMND and LMND-controls comparisons for ERP measures. There were no significant differences between groups for latencies. In the ALS group, the N1 and 
P3a latencies correlated with symptom severity and N1 latency increases with longer disease duration.

Conclusion: In ALS, and not in LMND, our ERPs findings confirm the hypothesis of a sub-clinical cognitive impairment. Modifications in ALS ERPresponses suggest a dysfunction in switching attention and novelty detection mechanisms. The pattern of cognitive impairment support the dysfunction of frontal network in ALS. In contrast, LMND patients did not differ from healthy controls in ERP parameters and neuropsychological performances. N1 and P3a latencies are related to the severity of illness, and could be effectively used for the psychophysiological follow-up of ALS patients.

0211

Alexithymia in amyotrophic lateral sclerosis. A neuropsychological approach

S. Roy-Bellina, H. Brunel, C. Almohsen, M.C. Gély-Nargeot, S. Carton, W. Camu

Hôpital Gui de Chauliac (Montpellier, FR)

Objectives: The domain of neuropsychology is not studied enough in amyotrophic lateral sclerosis (SLA). Nowdays, ALS patients are not discribed as very depressed, anxious, and seem to accept without great ditress this diagnostic. So, it appears interesting to study interactions between the psychology of emotions and the ALS patients's cognitive operation, more precisely, between alexithymia, characterized by an incapacity to report emotions, and the cognitive impairment observed in ALS. The aim of this research is to answerd two questions: are ALS patients alexitymic? Is the cognitive impairment observed in ALS responsible for this alexithymic operation?

Methods: There are 14 ALS patients (11 men et 3 women, aged from 47 to $81 \mathrm{yrs}$ (mean $=64.43, \pm 9.33$ ), developing since 45 month $( \pm=36)$ and an ALSFRS score from 26 to 48 (mean $=39.8)$ and 9 controls subjects $(2$ men and 7 woman, aged from 50 to $79 \mathrm{yrs}($ mean $=61.89, \pm=9.1$ ). They answer to the following questionnaires and tests: Alexithymia scale from Toronto at 20 items (TAS 20), Beck's scale for depression (BDI II), Apathy scale from Robert et al., (2002) and a battery of tests to assess executive functions.

Results: The TAS 20 score is more higher $(\mathrm{p}=0.0198)$ in ALS patient than MS patients. There is one positive correlation between depression and alexithymia $(p=0.0214)$. Reach statistical significance was observed on the Stroop Test $(p=0.0024)$ and verbal fluency for both literal form $(p=0.004)$ and categoriel $(\mathrm{p}=0.0012)$. ALS patients present a difficulty to stand their selective attention for inhibition tasks, a lack of research strategies in memory and a bad capacity of lexical initiation.

Conclusion: The main results reveal assessment of slight cognitif deficits and an alexithymia symptomatology, without any link allowing to conclude at common physiopathologic process.

Therefore, it's possible to consider the alexithymic operation in ALS patients like a defensive and adaptative process, to cope with the anguish and stress, and the reality of death.

\section{Oral session 26}

\section{Extrapyramidal disorders 2}

0212

Dystonia in the Woodhouse Sakati syndrome - A new family and literature review

S.A. Schneider, K.P. Bhatia

Institute of Neurology (London, UK)

Objectives: Woodhouse-Sakati-syndrome is a rare autosomal recessive neuroendocrine disorder characterized by the combination of alopecia, hypogonadism, diabetes mellitus, mental retardation, sensory neural deafness and extrapyramidal features.

Methods: Here we present clinical details of two affected siblings from a new Middle East family and draw attention of movement disorder specialists to this entity. We also review previous reports with particular focus on neurological and movement disorder features.

Results: 55 cases from 23 families have been reported in 12 case reports between 1973 and 2007. Most of the reported families are from the Middle Eastern countries although rare Caucasian cases have been described.

The following features (and their frequency) were identified: Autoim- mune disorders (Alopecia (100\%), Hypogonadism (98\%), Thyroid dysfunction (30\%), Diabetes mellitus: $(60 \%)$ ); Neurological features: Cognitive impairment/mental retardation: $(75 \%)$, Deafness $(74 \%)$, Extrapyramidal features $(50 \%)$. Among the Movement disorders, dystonia and chorea of the limbs were most common with onset in adolescence. Facial muscles were usually spared.

Other features included neurological features like dysarthria, seizures, pyramidal signs, polyneuropathy, incontinence; Keratoconus, Camptodactyly and Acanthosis nigrans; ECG changes (T-wave abnormalities) and white matter changes on MRI.

Conclusion: Woodhouse Sakati syndrome is one cause of autosomal recessive dystonia and should be considered particularly in patients from the Middle East.

\section{3}

Inflammation-related genes and the risk of Parkinson's disease: a multilocus approach

J. Infante, I. García-Gorostiaga, P. Sánchez-Juan, C. Sánchez-Quintana, J.L. Gurpegui, E. Rodríguez-Rodríguez, I. Mateo, J. Berciano, O. Combarros

University Hospital "Marqués de Valdecilla" (Santander, ES); Institute for Formation and Research of the Foundation "Marqués de Valdecilla" (IFIMAV) (Santander, ES); Laredo Hospital (Laredo, ES)

Objective: Inflammation has been increasingly recognized to play an important role in the pathogenesis of Parkinson's disease (PD). In this study we analyzed the effect of polymorphisms in a cluster of 5 inflammatory genes (TNF-alfa, IL-6, IL-8, IL-1a, and IL-10) on the risk of PD using a multilocus approach by the set-association method.

Methods: Study population consisted of 197 PD patients and 173 controls, all of them Caucasians originating from a limited geographic area in Northern Spain. Genotyping of the TNF-alfa (-1031), IL-6 (-174), IL-8 (-251), IL-1a (-889), and IL-10 (-1082) polymorphisms was performed by a Taq-Man single-nucleotide polymorphism assay (Applied Biosystems, Warrington, UK) and an ABI PRISM 7000 sequence detection system (Applied Biosystems). Association between dichotomous variables was analyzed with odds ratio, and $95 \%$ confidence intervals were estimated by the Cornfield method. P-values were estimated by chi-square or Fisher exact tests. Multivariate analyses were performed adjusting for age and gender using logistic regression. We performed multilocus analyses using set-association approach that has been implemented in Sumstat software (http://linkage. rockefeller.edu/ott/sumstat.html).

Results: No significant differences in genotype distribution were found between patients and controls for any of the SNPs. Crude odds ratios were very similar to odds ratios adjusted by sex and age by logistic regression. Setassociation analysis did not reveal a significant association between the set of inflammatory gene SNPs and PD risk. The SNP which presented the lowest $\mathrm{p}$-value for association with PD was TNF-alfa $(\mathrm{p}=0.19)$; the addition of the other tested SNPs gradually increased the $p$-value of the sum statistic and therefore did not add any contribution to the model.

Conclusions: Set-association analysis did not reveal an independent nor an interactive effect of these inflammatory genes on the risk of Parkinson's disease.

\section{4}

Limb-kinetic apraxia in Parkinson's disease: evidence from diminished response of manual dexterity to dopaminergic treatment

A. Gebhardt, T. Vanbellingen, B. Kersten, S. Bohlhalter

Parkinson Centre (Tschugg, CH); University of Berne (Berne, $\mathrm{CH}$ ); Inselspital, University of Berne (Berne, $\mathrm{CH}$ )

Objectives: Patients with Parkinson's disease (PD) often show impaired dexterity although being only minimally bradykinetic pointing to a distinct underlying disorder called limb kinetic apraxia (LKA). The goal of the present study was to investigate the differential dopaminergic response of manual dexterity and bradykinesia supporting this concept.

Methods: 11 patients ( 4 women, age $65.3+8.5$, mean + SD) with PD and wearing off fluctuations were tested in ON and OFF separately. For the assessment of dexterity the patients were instructed to rotate a Swiss 50-Rappen coin between their thumb, index and middle finger, for bradykinesia to tap between index finger and thumb, each task as fast as possible. Performance was measured by video analysis of coin rotation (CR) and finger tapping (FT) frequency during three 10 second periods. For each patient's hand the arithmetic mean of the respective task performances was calculated and proved to be reliable. For statistical analysis, we used a repeated measures factorial design $(2 \times 2 \times 2)$ with the factors medication (ON-vs. OFF-state), task (FT vs. CR) and handedness (dominant vs. non-dominant hand).

Results: Although dopaminergic treatment improved dexterity (CR 
$7.0+0.7$ in ON vs. $5.8+0.6$ in OFF, mean $+\mathrm{SD}$ ), the effect was considerably less strong than for bradykinesia (FT $24.6+2.4$ vs. $17.3+1.9$ ), as indicated by a significant interaction effect $(\mathrm{p}=0.002)$ between CR and FT performances. The dissociation was present in all but one patient who showed similar response in both tasks. By contrast, for handedness no interaction effect could be detected $(\mathrm{p}=0.598)$.

Conclusion: Diminished response of manual dexterity to dopaminergic treatment supports the notion that patients with PD suffer from LKA. The findings are relevant for more targeted rehabilitation strategies.

\section{5}

"One day" switch from cabergoline to transdermal rotigotine patch: well tolerated but at higher dose

M.T. Pérez-Saldaña, M. Bosca, J.C. López-Poma, J.A. Burguera

Hospital la Fe (Valencia, ES)

Objective: Benefits of long acting ergotic dopamine agonist cabergoline in advanced Parkinson disease and in motor complications of levodopa are well known. There is a novel concern about its increased risk of fibrotic valvular disease. At the same time, new transdermal delivery of dopaminergic drugs with constant dopaminergic stimulation appeared: rotigotine. The objective of the study is to assess the efficacy and tolerability of one day switch from cabergoline to transdermal rotigotine patch.

Methods: We followed Parkinson's disease patients who were 'one day' changed from cabergoline to the recommended rotigotine doses. Patients were offered to do the change. If they accepted, they were included in the study. Patients were titrated to the optimal rotigotine dose during the each of the successive visits according with one movement disorders specialist neurologist experience.

Tolerability was measured with the clinical global impression scale of the patients. We registered secondary effect and drops outs.

Results: A total of 22 Parkinson disease patients were followed during a mean of 7'64 \pm 1'96 moths since the switch was done. Three patients were excluded because the change was not direct.

They were 11 men and 8 women. The mean age was 63'05 $\pm 11^{\prime} 909$ years. The mean duration of the symptoms was $12^{\prime} 26 \pm 8^{\prime} 62$ years. The mean daily levodopa equivalent dose was $836^{\prime} 88 \pm 31^{\prime} 189 \mathrm{mg}$. Their mean Hoehn-Yahr punctuation at their last visit was 2'57 $\pm 0^{\prime} 96$.

The mean dose of cabergoline was 3' $84 \pm 2$ ' $24 \mathrm{mg}$. The mean initial rotigotine dose was $6^{\prime} 10 \pm 2$ ' $05 \mathrm{mg}$. The patch was well tolerated in $12 / 19(8 / 12$ improved, and $4 / 12$ remain without changes).The doses of rotigotine were increased in $12 / 19$ patients $(2,4,6$ and $8 \mathrm{mg}$ in $4,5,1$ and 2 patients respectively). This increment was at the first visit in 10/19 patients. Two patients were drop out because of adverse effects.

The most common complaint of the patients was poor adhesion in 11/19 patients. Other secondary effects were applications site reactions in 7/19 patients, ankle edema and dizziness in one patient respectively.

Discussion: Done to the fact of cabergoline dangerous secondary effects, switch to other non-ergotic DA agonist is recommended. The change to rotigotine was generally well tolerated. There was a need of increasing doses in $12 / 19$ patients. We suggest that a higher dose than that of the initial recommended is needed, especially for those with more than $3 \mathrm{mg} /$ daily of cabergoline.

\section{6}

Epidemiology of musculoskeletal disorders in Parkinson's disease and their impact on health-related quality of life

A. Natalwala, P. Davies, C. Buckley, C.E. Clarke

University of Birmingham (Birmingham, UK); Birmingham Children's Hospital (Birmingham, UK); City Hospital (Birmingham, UK)

Objectives: To examine the frequency, characteristics and management of joint disorders as well as their impact on health-related quality of life in patients with Parkinson's disease.

Methods: We sent a postal questionnaire to 136 non-demented Parkinson's disease patients.

Results: Of $113(83 \%)$ respondents, 74 (66\%) had seen a doctor regarding joint problems and 48 of these $(65 \%)$ had received a rheumatological diagnosis. The most commonly affected joints were: lower back $(46 \%)$, shoulders $(43 \%)$, and knees (43\%). There was no effect on EuroQol EQ-5D summary score, but PDQ 39 summary score was increased in patients with diagnosed joint disorders compared to those without a rheumatological diagnosis and those who had not consulted about joint disorders ( $38 \mathrm{v} 27 \mathrm{v} 25$; $\mathrm{p}<0.015)$. This was due to worse scores in the PDQ 39 domains of bodily discomfort ( 58 v 50 v $25 ; p<0.001)$, mobility ( 58 v 30 v $40 ; p=0.007)$, and activities of daily living $(63 \mathrm{v} 21 \mathrm{v} 29 ; \mathrm{p}=0.001)$ in patients with diagnosed joint disease.
Conclusion: There was evidence that patients with joint disorders received inadequate medical, surgical and allied health professional treatments. This preliminary evidence suggests that joint disorders are common in Parkinson's disease and cause additional deterioration in health-related quality of life.

\section{7}

Pitx3-transfected astrocytes secret neurotrophic factors and protect against dopamine neuron degeneration in mesencephalon cultures

D. Yang, C. Peng, W. Le

Institute of Health Sciences (Shanghai, CN)

The transcription factor Pitx3 is crucial for the development and differentiation of dopamine neurons. Our previous work has shown the Pitx3 can upregulate the expression of brain-derived neurotrophic factor (BDNF) and glial cell line-derived neurotrophic factor (GDNF) in neuroblastoma cell line SH-SY5Y. Since both BDNF and GDNF originate from astrocytes, we therefore investigated whether Pitx3 can increase the expression and secretion of BDNF and GDNF in primary astrocyte cultures. We first transfected primary rat astrocytes with Pitx3 plasmid and collected the conditioned medium (CM) from the Pitx3-transfected cultures. Then we measured the BDNF and GDNF levels from the CM and tested the protective effect of the CM against rotenone-induced dopamine neuron injury in primary ventral mesencephalon (VM) cultures. We found that the BDNF and GDNF levels were 1.4fold and 1.5-fold higher in the CM from Pitx3-transfected astrocytes than in controls. Incubation with the CM from Pitx3-transfected astrocytes significantly reduced the rotenone-induced dopamine neuron injury in VM cultures, and such protection can be significantly blocked by pre-incubation with antibodies against either BDNF or GDNF. These results demonstrate that Pitx3-transfection in astrocytes can up-regulate BDNF and GDNF expression and produce protective benefit to dopamine neurons, which might be a promising therapeutic alternative for Parkinson's disease.

\section{Oral session 27}

\section{New insights into MS disease mechanisms}

\section{8}

IL2RA and IL7RA genes confer susceptibility for multiple sclerosis in two independent European populations

F. Weber, B. Fontaine, I. Cournu-Rebeix, A. Kroner, M. Knop, S. Lutz, F. Müller-Sarnowski, M. Uhr, T. Bettecken, M. Kohli, S. Ripke, M. Ising, P. Rieckmann, D. Brassat, G. Semana, M-C. Babron, S. Mrejen, C. Gout, O. Lyon-Caen, J. Yaouanq, G. Edan, M. Clanet, F. Holsboer, F. Clerget-Darpoux, B. MüllerMyhsok

MPI of Psychiatry (Munich, DE); Pitié-Salpêtrière, INSERM (Paris, FR); University of Wurzburg (Wurzburg, DE); UBC Hospital (Vancouver, CA); University of Toulouse (Toulouse, FR); CHU Pontchaillou (Rennes, FR); INSERM (Ville Juif, FR)

Objectives: Multiple Sclerosis (MS) is the most common chronic inflammatory neurologic disorder diagnosed in young adults. Several twin and familial aggregation studies clearly demonstrate that there is a genetic impact on susceptibility. MS, however, is considered to be a complex genetic disorder The well established HLA-association does not completely explain the genetic impact. Identification and validation of non-HLA-genes conferring susceptibility to MS has proven to be very difficult due to the small individual contribution of each of these genes. Recent work render IL2RA and IL7RA highly attractive candidates for the first validated MS susceptibility genes outside the HLA region. The effects of both genes, however, were modest at best, so that only replications in a large number of different samples will make their involvement in MS susceptibility convincing.

Methods: Two single nucleotide polymorphisms (SNPs) in the IL2RA gene (rs12722489, rs2104286) and one SNP in the IL7RA gene (rs6897932), were genotyped in a German sample, consisting of 206 MS patients and 605 age and sex matched controls, and in a French sample, consisting of $540 \mathrm{MS}$ trios using the hME Assay by the MALDI-TOF technology (Sequenom, San Diego). Statistical analysis was performed using the UNPHASED Software V 3.0.10. which allows to jointly analyse data from trios and unrelated individuals.

Results: The two SNPs in the IL2RA and the SNP in the IL7RA gene, all 
three recently published, do contribute to the non-HLA genetic risk in two unrelated European MS patient populations with odds ratios varying from 1.1 to 1.5 . Allele frequencies of the associated SNPs ranged from $0.74-0.87$ in the French and from 0.77-0.84 in the German sample.

Conclusion: By genotyping three SNPs within the IL2RA and the IL7RA genes in two independent European samples, we confirmed the association of both genes with MS susceptibility. The odds ratios and allele frequencies were similar to those previously published. Therefore our findings convincingly corroborate the recently published results.

Although the genetic risk conferred by both genes is small, the discovery and the validation of new genetic risk factors in independent populations may help to understand MS pathogenesis by providing us valuable information of as yet unsuspected biological pathways.

The study was supported by REFGENSEP (French Multiple Sclerosis Genetics group) which received grants from INSERM, AFM, ARSEP, Groupe Malakoff, Biogen-Idec, and help from Genethon and CIC Pitié-Salpêtrière and the DNA bank from Würzburg, which is support

\section{9}

Detection of viruses in multiple sclerosis using the human panviral diagnostic array

G. Trillo-Pazos, A. Khan, P. Kellam, R. Reynolds, J. Bell, R. Weiss, D.H. Miller Institute of Neurology, UCL (London, UK); Windeyer Institute, UCL (London, UK); Imperial College (London, UK); Edinburgh University (Edinburgh, UK); Windeyer, UCL (London, UK)

Objective: To determine if MS has a viral aetiology, we designed and developed a novel Human Panviral Diagnostic Array (HVDA) to test for the presence of viruses in human post-mortem brain tissue samples.

Methods: The HVDA was designed using a biological approach to detect latent and lytic infection by 210 viruses in human clinical samples. Total RNA was extracted from 6 post-mortem MS cases; 4 control cases with dementia; 4 samples with clinical viral HIV or Hepatitis B infection and hybridised to our array using standard Agilent array protocols. Array data was analysed using the SPSS v12 WIN statistics platform up-regulated viral probes were identified with ANOVA at $\mathrm{p}<0.05$ compared to negative controls.

Results: With the HVDA we detected a "common" viral pattern of infection consisting of endogenous retroviruses, latent herpesvirus infection and other viruses across the tissue samples tested. We were able to detect HIV, hepatitis B and EBV infection in those cases with clinically diagnosed viral infection prior to death. No single virus has being exclusively associated with MS in our samples so far. These results have being verified by RT-PCR. These findings suggest that there is an apparent "viral ecosystem" present in human tissues independent of disease.

Conclusion: This HVDA can detect clinically diagnosed viral diseases in human tissue samples and an apparent background "viral ecosystem". However, specific detection of viruses depends on issues of levels of infection (i.e. copy number) and the brain is a complex cellular system that is refractive to widespread viral infection. To address this, we are currently testing the "hit and run" mechanism of viral infection by using a microgenomics approach in MS.

\section{0}

Morphology and evolution of cortical lesions in multiple sclerosis. A longitudinal MRI study

M. Calabrese, M. Filippi, M. Rovaris, I. Mattisi, V. Bernardi, M. Atzori, A. Favaretto, L. Rinaldi, P. Perini, P. Gallo

University of Padua (Padua, IT); San Raffaele Institute and University (Milan, IT)

Objectives: Cortical lesions (CLs) can be detected in the majority of patients with established multiple sclerosis (MS), but little is known about the features of their evolution over time. This study was performed to investigate the short-term MRI evolution of CLs, with the ultimate aim to achieve a better in vivo understanding of their nature.

Methods: Seven hundred and sixty-eight CLs from 107 MS patients (76 with relapsing-remitting $[R R]$ and 31 with secondary progressive [SP] MS) were followed with brain MR examinations, including a double inversion recovery (DIR) sequence, every six months for at least 1 year. CLs number, volume and morphological features were assessed at each time-point.

Results: Six hundred and eighty CLs $(88.5 \%)$ remained morphologically unchanged during the follow-up period, while $74(9.6 \%)$ showed an increase in their individual size. Only $6(0.8 \%)$ CLs present at baseline (all in RRMS patients) disappeared at follow-up MRI scans. No enlarging CLs spread into the subcortical white matter. No CLs ever showed gadolinium enhancement.
At baseline, the mean number of CLs was higher in SPMS than in RRMS patients $(p<0.001)$, whereas the mean number of new CLs per patient after one year did not differ between the two groups.

Conclusion: Over a one-year period, CLs can increase their number and size in a relevant proportion of MS patients, without ever spreading into the subcortical white matter or showing inflammatory features similar to those of white matter lesions. The short-term evolution of CLs seems to be influenced by the disease duration rather than by the MS stage.

0221

A diffusion-tensor MRI study of the cervical cord damage in benign and secondary progressive multiple sclerosis patients

M. Rovaris, B. Benedetti, A. Pulizzi, D. Caputo, M. Zaffaroni, R. Capra, A. Bertolotto, V. Martinelli, G. Comi, M. Filippi

Scientific Institute San Raffaele (Milan, IT); Scientific Institute Don Gnocchi (Milan, IT); Ospedale di Gallarate (Gallarate, IT); Spedali Civili (Brescia, IT); Ospedale San Luigi (Orbassano, IT)

Objectives: Diffusion tensor (DT) MRI is able to quantify the severity of brain and cervical cord pathology in multiple sclerosis (MS), thus providing in vivo pieces of information about the extent of tissue damage that can go undetected when using conventional MRI. Aim of the study was to investigate the DT MRI patterns of cervical cord damage in benign MS (BMS) and secondary progressive MS (SPMS) patients, in order to achieve a better understanding of the nature of disability in MS.

Methods: Conventional and DT MRI scans of the cervical cord were acquired from 61 patients with BMS, 49 with SPMS and 18 healthy controls. After the creation of mean diffusivity (MD) and fractional anisotropy (FA) maps, histograms of MD and FA values were produced for the cervical cord tissue. Cross-sectional cord area (CSA) at C2 level was also computed.

Results: The median expanded disability status scale (EDSS) score was 2.0 (range: 0.0-3.0) for BMS and 6.0 (range: 4.0-8.0) for SPMS patients. Compared to healthy controls, BMS patients had higher average cord MD $(\mathrm{p}<0.001)$ and lower average CSA $(\mathrm{p}<0.03)$. When compared to BMS patients, SPMS patients had lower cord average FA $(p=0.003)$ and lower average CSA $(p<0.001)$. In MS patients, EDSS was significantly correlated with CSA $(r=-0.45, p<0.001)$ and average cord FA $(r=-0.37, p=0.002)$. Both CSA and average cord FA entered a multivariable model as independent predictors of EDSS in MS patients $(\mathrm{p}=0.001)$.

Conclusion: Our results are consistent with the presence of a milder tissue damage in the cervical cord of MS patients without locomotor disability, which might be a hallmark of the benign course of the disease. DT MRI may complement atrophy measurement in a comprehensive MRI assessment of cervical cord abnormalities.

The study was supported by a grant from FISM (Fondazione Italiana Sclerosi Multipla) - contract n. 2005/R/18.

\section{Oral session 28}

\section{General neurology 2}

\section{2}

Immune responses to GAD65 in stiff-person syndrome: clonality, avidity and persistence T. Holmøy, G. Skorstad, B. Vandvik, A. Hestvik, P. Torjesen, K. Alvik, F. Vartdal

Institute of Immunology (Oslo, NO); Ullevål University Hospital (Oslo, NO) Aker University Hospital (Oslo, NO); Rikshospitalet University Hospital (Oslo, NO)

Objectives: To characterize the immune response against the autoantigen glutamic acid decarboxylase (GAD) 65 in stiff person syndrome (SPS).

Methods: Clonal patterns and binding characteristics of the anti-GAD65 IgG antibody response in serum and cerebrospinal fluid (CSF) were studied with isoelectric focusing-immunoblot and radioimmunoassay (RIA)in five SPS patients. GAD65 specific T cells were cloned from blood and CSF, and T cell epitopes were charted by overlapping synthetic peptides.

Results: Anti-GAD65 IgG antibodies in CSF and serum were oligoclonal, predominantly IgG1, with high avidity for GAD65. Isoelectric focusing-immunoblot displayed intrathecal synthesis of oligoclonal anti-GAD65 IgG in 
all five SPS patients, while RIA demonstrated intrathecal synthesis of antiGAD65 IgG in four. The binding avidity of anti-GAD65 IgG from CSF was more than 10 times higher than in serum in two of the patients, but did not differ substantially in the remaining three. These differences were not related to symptom severity. The clonal patterns of anti-GAD65 IgG in CSF and serum remained unchanged for up to 7 years after symptom debut. Proliferative responses against GAD65 were detected in peripheral blood mononuclear cells in all five SPS patients, but only in two of eight healthy controls. GAD65 reactive T cells could be cloned from either blood or CSF of all four SPS patients available for further T cell assays (blood only in one, blood and CSF in two, CSF only in one). We were able to map the fine specificity of GAD65 reactive T cell clones from the CSF of all three patients, whereas this was only possible with $\mathrm{T}$ cell clones from the blood of one patient. GAD65 reactive T cells from blood were restricted by HLA-DR, whereas GAD65 specific T cells from CSF were restricted by either DR or DP. The T cells recognized epitopes within all parts of the GAD65 molecule, and no shared T cell epitope was detected.

Conclusion: The intrathecal humoral immune response against GAD65 in SPS appears to be mediated by a stable population of affinity maturated and clonally expanded B cells, and may be driven by GAD65 specific Thelper cells. Thus, T cells may contribute to the pathogenesis of SPS.

\section{3}

Health-related quality of life in myasthenia gravis

D. McKee, R. Lee, E. Whetton, C. Roberts, J. Sussman

Centre for Clinical Neurosciences (Manchester, UK); University of Manchester (Manchester, UK)

Objectives: 1) To study the impact of myasthenia gravis (MG) on health-related quality of life (HRQoL) in a large group of unselected patients with a full spectrum of disease severity. 2) To study the correlation between disease severity as measured by the Myasthenia Gravis Activities of Daily Living profile (MG-ADL) and HRQoL.3) To determine which elements of the myasthenic symptom complex have most impact on HRQoL.

Methods: Data was taken from an epidemiological study of MG in which multiple overlapping sources of case ascertainment were used in an attempt to identify all MG patients in a defined geographical region. HRQoL was measured using the Medical Outcomes Survey Short Form 36 (SF-36), a widely used and validated scale for which there is published data for the general UK population. The SF-36 was self-completed by post. Patients were interviewed and examined to collect clinical data and current MG-ADL score.

Results: Data from 177 patients was analysed. Significant differences were seen between patients and general population data in all subdivisions of the SF-36, with reductions more apparent in physical than psychological indices. The physical functioning (PF) subscore of SF-36 was most affected, with an average reduction in 18.19 SF-36 points in MG patients compared to population normative data $(\mathrm{p}=<0.005)$. Physical composite scores (PCS) and mental composite scores (MCS) of MG patients were on average 9.83 and 5.34 SF-36 points respectively less than those of the general population $(p=<0.005)$. There was a correlation between increasing severity of MG measured by MG-ADL and reduction of HRQoL measured by SF-36. Spearman's rank correlation of total MG-ADL scores against PCS and MCS demonstrated correlation coefficients of 0.521 and 0.299 respectively $(p=<0.005)$. Subanalysis of each element of MG-ADL suggests that limb impairment has a more profound impact than oculobulbar symptoms on HRQoL.

Conclusion: This is the largest study of HRQoL in MG yet performed. Using an unselected population-based MG cohort, it allows for comparison between normative population data and also that of other chronic medical conditions. MG clearly has an impact on HRQoL, the magnitude of which is related to MG severity. There is a correlation between MG-ADL and HRQoL, which adds validity to the MG-ADL as a tool for assessment in the clinical setting. The observation that limb impairment has most impact on HRQoL may be useful in weighting a disease-specific score.

\section{4}

Pathogenicity of autoantibodies in stiff-person syndrome

T. Chang, B. Lang, P. Brown, A. Vincent

University of Oxford (Oxford, UK); Institute of Neurology (London, UK)

Objectives: Stiff person syndrome (SPS) is a highly disabling disorder of the central nervous system (CNS) characterised by progressive muscular rigidity and spasms. An autoimmune aetiology in SPS has been suggested because of its association with autoantibodies, autoimmune diseases and response to immunotherapy. Autoantibodies (Abs) to glutamic acid decarboxylase (GAD, the enzyme that synthesises the inhibitory neurotransmitter GABA) are detected in approximately 60 to $80 \%$ of patients with SPS.
However, because GAD is intracellular and inaccessible to circulating Abs, it is not clear whether GADAbs are themselves pathogenic or are merely a marker for another humoral or cellular immune mechanism. In general, pathogenic Abs in neurological disorders are disease-specific, high affinity, IgG1 or IgG3, and bind to the surface of the target cells. In principle, the disease should be reproduced in animal models by passive transfer of patient IgG, although there are not well-established methods for passive transfer of CNS disorders.

Methods: Sera of 12 classical SPS patients and 13 SPS variants were studied. GADAb titres, affinity and subclasses were evaluated. Patient sera were tested for IgG binding to murine primary neuronal cultures. Clinical phenotype, inflammatory markers, apoptosis and neuronal loss were compared between mice injected with purified IgG from two patients and mice injected with control IgG.

Results: GADAbs at high titre (median $7500 \mathrm{U} / \mathrm{ml}$ ) showed $76 \%$ sensitivity in the total SPS cohort with $100 \%$ sensitivity in clinically well-defined classical SPS. GADAbs were high affinity (KD 0.06 to $0.78 \mathrm{nM}$ ), predominantly IgG1 subclass, and bound to permeabilised GABAergic cultured neurons. There was also evidence of Abs binding to the cell surface of GABAergic neurons, and absorption studies suggested that these Abs were not directed against GAD. However, passive transfer of GADAb-positive IgG for five days did not succeed in inducing any signs of disease in mice.

Conclusions: The results show that GADAbs are similar in immunological characteristics to those of other pathogenic Abs and that SPS sera also contain IgG Abs, other than GADAbs, that bind to the surface of GABAergic neurons. Although a short trial of passive transfer failed to demonstrate that Abs by themselves are causative in SPS, this could be explained by the limitations in the experimental model due to limited availability of IgG for passive transfer and further attempts should be made.

\section{Oral session 29}

\section{Child neurology}

\section{5}

Serum and cerebrospinal fluid levels of the B-cell activating factor BAFF in paediatric opsoclonus-myoclonus syndrome - correlation with neuronal surface-binding autoantibodies

V. Fühlhuber, S. Altenkämper, S. Bick, A. Kirsten, T. Gerriets, J. Hosch, M. Tschernatsch, M. Kaps, F. Blaes

Justus-Liebig-Universität (Giessen, DE)

Objective: Childhood opsoclonus-myoclonus syndrome (OMS) occurs idiopathic or, in association with a neuroblastoma, as a paraneoplastic syndrome. Since the disease responds to immunosuppressants and autoantibodies were identified in some patients, an autoimmune pathogenesis has been suspected. While the newly discovered B-cell activating factor (BAFF) is obviously involved in systemic autoimmune diseases, its association with neuroimmunological diseases is hardly understood. We here investigated, whether OMS children have elevated BAFF levels in serum or cerebrospinal fluid (CSF) and whether BAFF correlates with surface-binding autoantibodies.

Methods: BAFF levels in serum and CSF were determined by ELISA, and surface-binding autoantibodies to cerebellar granular neurons have been investigated by flow cy tometry in 17 OMS patients, 16 neuroblastoma (NB) patients and 13 healthy controls (HC).

Results: $83.3 \%$ of OMS children, but only $12.5 \%$ of NB patients and none of the controls had autoantibodies to the surface of cerebellar granular cells $(\mathrm{p}<0.001)$. Although the mean BAFF serum level was not elevated in OMS, there was a correlation of BAFF level and CGN autoantibodies $(\mathrm{r} 2=0.62$, $\mathrm{p}<0.01$ ). Moreover, CSF/serum ratio was significantly increased in OMS patients compared to controls (OMS $0.39 \pm 0.32$ vs. controls $0.08 \pm 0.06$, $\mathrm{p}<0.01)$ and this was not caused by a blood-brain barrier disturbance.

Conclusion: These data indicate, that an activated B-cell system in the cerebrospinal fluid is involved in the pathogenesis of OMS, and BAFF may be a candidate parameter for measuring activation of B-cell immune system. 


\section{6}

Children (<10yrs) with clinically isolated syndrome: long term follow-up fora second clinical event

M. Eraksoy, Z. Yapici, G. Akman-Demir, M. Kürtüncü, H. Özcan

Istanbul University (Istanbul, TR)

Multiple sclerosis (MS) under 10 years of age is rare $(<1 \%)$. According to accumulating data, interval between first and a second clinical event has been reported longer than adult-onset patients (AOMS) with MS. However, there has been some debate on this issue in the recent publications.

This study was designed to investigate the interval between first and second relapse in patients developed clinically isolated syndrome under 10 years of age. The patients' first and second clinical events were seen first and the last authors, the other authors joined to follow-up.

Childhood-onsetMS frequency is $4.2 \%$ in our cohort. Eighteen children $(11.6 \%)(<10 y r s)(15 F, 3 M)$ developed the first clinical event such as: Acute disseminated encephalomyelitis $(\operatorname{ADEM})(n=1), B O N / O N(n=5)$, sensorymotor $(4)$, brainstem $(n=4)$, $\operatorname{motor}(n=2)$, serebellar $(n=1)$ signs and one child developed MRMS.

Seventeen patients developed clinically definite MS median 96 months later. One patient has not developed clinical relapse but his MRI findings were consistent with Barkof/Tintoré criteria.

Median disease duration was 6 yrs;median follow up duration 6 yrs. Most of the children under 10 years of age $(n=17 / 18)$ had a second clinical event mean 96 months.

In conclusion, development of a second clinical event in children under 10 years of age was found longer than patients with AOMS (24 months in our AOMS cohort). MRI abnormalities have important diagnostic value.

\section{7}

Verbal memory in Egyptian temporal lobe epileptic children

N. Kitchener, N. Nagy, S.S. Aziz

General Organization for Teaching Hospital (Cairo, EG); Ain Shams Univerity (Cairo, EG)

Objectives: Temporal lobe epilepsy in childhood may present with great clinical, electroencephalographic and etiological diversity. The relationship between the lateralization of the epileptic focus and performance on memory tests is not well-established. Aim of the study was to assess verbal memory in children with newly diagnosed left temporal lobe epilepsy (LTLE), and to study the effects of its course on verbal memory (VM) by comparing the memory performance of newly diagnosed patients with that of control group and chronic patients with LTLE. VM was also evaluated as a prognostic factor for seizure control. Effects of antiepileptic drugs (AEDs) on VM were assessed after one year of treatment.

Methods: The study sample consisted of 50 newly diagnosed and 50 chronic patients with EEG confirmed LTLE, and normal imaging studies. A group of 50 healthy volunteers served as controls for neuropsychological evaluation.

Results: The study showed that VM may be affected already at the time of the diagnosis of LTLE. Mild memory dysfunction (test performance one standard deviation below the mean performance of controls) was found in one third of the patients with newly diagnosed LTLE. Clinically meaningful, moderate memory impairment (test scores two standard deviations below the control mean) was present in a minority of these patients. Memory dysfunction was shown mainly in retrieval of previously learned unrelated words after delay, and in the percentage of words retained. The VM performance of patients with newly diagnosed LTLE was almost the same as that of patients with chronic LTLE. The groups differed in delayed recognition of words, patients with chronic uncontrolled LTLE having poorer performance. Clinically meaningful, moderate verbal memory impairment at the time of the diagnosis of LTLE was one of the predictors of seizure outcome. The VM of newly diagnosed patients did not decline during one year of treatment with different AEDs, and preliminary five-year follow-up of newly diagnosed LTLE patients also showed no statistically significant deterioration of VM.

Conclusions: These findings suggest that memory dysfunction becomes evident already in the early course of epilepsy, and memory performance remains relatively stable, and even improved in patients with controlled epilepsy. In addition, moderate memory impairment at the time of diagnosis is one of the predictors of epilepsy intractability.
0228

Day, night and seasonal variations in febrile seizure, Tehran Iran

F. Khodapanahandeh

Iran University of Medical Sciences (Tehran, IR)

Objective: Febrile seizures are common in childhood, affecting $2 \%$ to $5 \%$ of children between 6 months and 5 years. We performed this study to determine any circadian or seasonal pattern of the occurrence of febrile seizures.

Methods: In a retrospective cross sectional study files of all children with febrile seizure aged between 6 months and 5 years admitted between Apri 2000 and Mars 2005 to the pediatric ward of Rasool Akram hospital were reviewed.

Results: 221 children entered to the study. Mean age of children were $29 / 09 \pm 18 / 64$. Cases were considered in four different times of the day (morning 6 to $11: 59 \mathrm{AM}$, afternoon 12 to $5: 59 \mathrm{MD}$, evening 6 to $11.59 \mathrm{PM}$, night 12 to 5:59 AM). There was a significant difference $(\mathrm{p}<0.03)$ in occurrence of seizure in the evening and afternoon. The percentage of occurrence of febrile seizure in different seasons were as follows: $17.6 \%$ in spring, $26.2 \%$ in summer, $21.3 \%$ autumn, $34.8 \%$ in winter. Considering seasonal variation seizures occurred significantly $(\mathrm{p}<0.015)$ in winters.

Conclusions: Our study showed that febrile seizures occur more commonly in the evening and afternoon. Winter was the most prominent season for occurrence of seizures.

\section{POSTER SESSIONS Poster session 1}

\section{Cerebrovascular disorders}

P229

Clinical characteristics of nonagenerian stroke

D.I. Chang, M.K. Kim, S.H. Heo, S.H. Kim

Kyunghee Medical Center (Seoul, KR); Seoul Medical Center (Seoul, KR); Hanyang University Hospital (Seoul, KR)

Background: As the elderly population is fast growing, the incidence of stroke is also increasing. We studied the clinical characteristics of nonagenarian stroke compared to a population of patients under the age of ninety.

Methods: Subjects included 44 nonagenarian stroke patients and 22,227 control patients aged under ninety. Clinical characteristics including age, sex, risk factors, stroke subtype, and outcome (one-year prognosis, evaluated by a modified Rankin scale) were analyzed.

Results: The proportion of nonagenarian stroke accounted for $0.2 \%$ of all cases of stroke. Ischemic stroke was more common than hemorrhagic stroke in the nonagenarian group. In addition, the female gender was more frequent $(p<0.01)$. As the patients were older, their admission period was shorter, the discharge against medical advice was increased, and the mortality was higher. Hypertension and atrial fibrillation were significantly higher ( $\mathrm{p}<0.05, \mathrm{p}<0.001$, respectively) in the nonagenarian ischemic stroke patients.

Conclusions: Nonagenarian stroke patients have unique clinical characteristics compared with stroke patients under the age of ninety.

\section{P230}

Citizens and phsysicians awareness of stroke: a community screening programme in northern Portugal

E. Moreira, T. Pimentel, A. Tuna, R. Magalhães, M. Correia, C. Silva Instituto de Biologia Molecular e Celular (Porto, PT); Hospital Geral de Santo António (Porto, PT); Instituto de Ciências Biomédicas Abel Salazar (Porto, PT)

Objectives: Portugal has a high stroke incidence both in urban and rural areas. For designing an intervention program for the community population and GPs, a survey was conducted to evaluate the recognition of warning signs for stroke and transient focal neurological attacks (TFNA).

Methods: In the first semester of 2007, a community screening program involved the GPs $(n=64)$ and $1 \%$ of the population registered at 3 Health Centres in Northern Portugal $(n=685)$. The program included a questionnaire filled by the HC users on stroke lesion location, stroke signs/symptoms and health services attendance, risk factors and a checklist of experience of 
stroke symptoms in the last year by himself/family member. Case scenarios were used to assess GPs knowledge of neurological attacks and the "gold standard" for comparison was given by consensus of neurologists.

Results: Overall $663(97 \%)$ users were contacted and just more than a half of GPs participated (53\%). Respondents were mainly women $(70 \%)$ and median age was 47 years, with $25 \%$ older than 62 years and education was 4 years, with $25 \%$ over 9 years. The correct lesion localization was mentioned by $53 \%$, more often more educated $(69 \%$ vs. $27 \%)$ and younger $(65 \%$ vs. $38 \%$ ) (chi-square $=37, \mathrm{df}=3, \mathrm{p}<0.001)$. Symptoms more frequently recognized were hemiparesis $(64 \%)$ and disartria $(61 \%)$ and monocular blindness less recognized (34\%). Almost all users identified HTA as a risk factor $(86 \%)$ but diabetes and cardiac pathology was mentioned by just more than half of them $(56 \%)$ and more frequently by less educated users (chisquare $=13, \mathrm{df}=3, \mathrm{p}<0.005)$

Half of the GPs identified correctly 9 out of the 16 case scenarios presented. Compared with the group of 10 neurologists, failures were more frequent in two out of ten stroke/TIA cases and three out of five TFNA cases (chi-square $<9, \mathrm{df}=1, \mathrm{p}>0.003$ ).

Conclusions: Recognition and action towards warning signs of stroke is poor, mainly for at risk persons, older and less educated, and risk factors were predominantly acknowledged by persons more likely to have a previous experience. Moreover GPs failures to recognize "cases", may contribute to an overall under recognition, especially when dealing with TIAs and TFNAs, both considered risk factors for stroke.

Fundação para a Ciência e Tecnologia- FCT/FEDER project POCI/SAUESP/59885/2004

\section{P231}

Transcranial direct current stimulation improves naming in aphasic patients F. Cogiamanian, A. Monti, S. Marceglia, R. Ferrucci, F. Mameli, S. MrakicSposta, M. Vergari, S. Zago, S. Barbieri, A. Priori

Fondazione IRCCS Ospedale Maggiore Policlinico (Milan, IT); Università di Trento (Milan, IT)

Objective: Transcranial direct current stimulation (tDCS) is a non invasive technique for focal modulation of brain that induces persisting excitability changes of human cerebral cortex.

This brain stimulation technique can be also adjuvant strategy to improve functional recovery after ischemic stroke. Nonetheless, although the ability to generate lists of words increases after tDCS in normal subjects, the effects of tDCS in patients with aphasia are unknown. This study aimed to assess the effect of tDCS over left fronto-temporal areas in chronic non-fluent post-stroke aphasia.

Methods: Eight patients with chronic non-fluent aphasia were studied before and immediately after tDCS (2mA, 10 minutes) over the damaged left fronto-temporal areas. The naming function was measured by a computercontrolled task. To assess the specificity of our findings, in additional control experiments patients were evaluated before and after cathodal tDCS over the occipital cortex.

Results: Whereas anodal tDCS and sham tDCS over the left fronto-temporal areas failed to induce significant changes ([mean \pm SE] anodal tDCS, pre $12.0 \pm 2.29$ vs post $11.83 \pm 2.29, \mathrm{p}=0.20$; sham tDCS, pre $11.70 \pm 2.14$ vs post $11.70 \pm 2.15, \mathrm{p}=0.20$ ), cathodal tDCS significantly increased the accuracy of the naming task (cathodal tDCS, pre $11.70 \pm 1.96$ vs post $14.50 \pm 1.69$, $\mathrm{p}=0.002$ ). Cathodal tDCS over occipital cortex did not induce any effect on naming function.

Conclusions: Cathodal tDCS over the damaged left fronto-temporal areas in patients with chronic non-fluent aphasia improves naming. Because tDCS is simple, safe and inexpensive, this technique could be complementary to speech rehabilitation in the management of aphasic patients.

\section{P232}

Influence of smoking on cerebral flow

J. Kochanowicz, G. Turek, R. Rutkowski, Z. Mariak

Medical University of Bialystok (Bialystok, PL)

Objectives: Cigarette smoking is a well-known risk factor of atherosclerosis as well as a risk factor of stroke and cerebral aneurysms. On the other hand many authors reported decreased incidence of certain neurodegenerative conditions in smokers, such as Alzheimer or Parkinson disease. Certainly smoking influences cerebral circulation, however little is known about cerebral blood flow parameters directly after smoking a cigarette. The aim of this study is to explore the direct effect of smoking on blood flow velocity and impedance indexes in the carotid and vertebral arteries and their main intracranial branches.

Methods: We examined 30 young healthy men (age range, $20-28$ years) with color coded duplex sonography using a $2.5 \mathrm{MHz}$ transcranial probe, via the temporal acoustic window. Blood flow velocities and impedance indexes (PI and RI) were studied on both sides in the middle, anterior, posterior cerebral artery. Additionally the internal carotid artery was examined using a 7.5 $\mathrm{MHz}$ probe in order to calculate the Lindegaard index (which express a ratio between blood velocity in the middle cerebral and in the internal carotid artery). Each artery was examined before and after smoking a cigarette. Differences between blood flow parameters were tested with paired t-test. Probability less than 0.05 indicated significance.

Results: Blood flow velocity in each artery invariably increased after smoking of a cigarette. Nevertheless the increase in peak-systolic, mean and end-diastolic velocity reached statistical significance $(\mathrm{p}<0.01)$ only in the middle cerebral artery. In the same time the impedance indexes decreased, though the decrease was statistically significant only in the middle cerebral artery. In the anterior cerebral artery only RI decreased statistically significantly $(\mathrm{p}<0.05)$. Lindegaard indexes did not change after smoking.

Conclusion: Even one smoked cigarette causes significant increase in blood flow velocities and decrease of impedance indexes (RI, PI) in the middle cerebral artery. Smoking cigarettes promotes cerebral blood flow by decreasing peripheral vascular impedance, but resultant increase in blood flow velocity in the major cerebral arteries can add to shear stress resulting in atherosclerosis.

P233

Prevalence of patent foramen ovale in stroke patients less than 60 years of age

K. Savio, L. Coppo, L. Bolamperti, M. Ravagnani, M. Reggiani, F. Monaco Amedeo Avogadro University (Novara, IT)

Introduction: Right to left shunt usually due to patent foramen ovale (PFO) is a well established risk factor for ischemic stroke, especially in young patients. Atrial septal aneurysm and hypercoagulable state increase the risk of stroke in patients with PFO. Contrast transcranial Doppler (TCCD) is a non invasive tool for detection of PFO. In patients with cryptogenic stroke, the prevalence of PFO is about $45 \%$ versus $25 \%$ of the general healthy population.

Objectives: 1) To evaluate PFO prevalence and the distribution of vascular risk factors in stroke patients (pts) with less than 60 years of age.

Methods: Consecutive stroke out and inpatients Department of Neurology - Novara, that underwent (TCCD) to detect PFO from 2005 to 2007. Structured interview to evaluate cerebrovascular risk factors, neuroimaging findings and other diagnostic tools to detect PFO.

Results: We enrolled 96 consecutive cryptogenetic stroke pts $(52 \mathrm{M}, 44 \mathrm{~F}$, mean age 47.5 years): $90 \mathrm{pts}$ (93\%) with single ischemic lesion (SL) and $6 \mathrm{pts}$ $(6 \%)$ with multiple ischemic lesions (ML) at the neuroimaging. 41 pts ( $42 \%)$ were positive for hypertension, $2(2 \%)$ for diabete, $21(21 \%)$ for smoke, 26 $(27 \%)$ for dislypidemia, $2(2 \%)$ for ischemic cardiopathy, $3(3 \%)$ for atrial fibrillation, $30(31 \%)$ for atheromasic lesions of epiaortic vessels, without emodinamic effects. 5 pts $(5 \%)$ had a previous stroke and $9(9 \%)$ a previous transient ischemic attack. 45 pts underwent to homocysteine dosage, that was positive in 18 pts $(40 \%) .45$ pts underwent to thrombophilia laboratory testing, that was positive in 12 pts $(26 \%)$. Only 6 women $(13 \%)$ used estoral contraceptive. 21 pts $(21 \%)$ suffered for headache ( 3 for migraine and 18 for tension type headache).

52 pts ( $54 \% ; 32 \mathrm{M}, 20 \mathrm{~F}$ ) was positive for PFO with TCCD: $92 \%$ with SL (48 pts), $8 \%$ with ML (4 pts). 44 pts $(45.8 \% ; 25 \mathrm{M}, 19 \mathrm{~F})$ was negative: $95 \%$ with SL (42 pts); $5 \%$ with ML (2 pts). Shunt was small in $50 \%$ of pts, moderate in $28 \%$ and large in $21 \%$. These data were confirmed by echocardiography.

Conclusion: In our population the prevalence of PFO was comparable to that of other reported series. We did not find a correlation between PFO and migraine in our stroke patients. TCCD is an effective diagnostic tool.

\section{P234}

Juvenile cerebellar infarction in bilateral superior cerebellar artery territory caused by drug-induced vasospasm

M. Kobari, N. Yagi, T. Konishi, N. Imai, M. Serizawa

Shizuoka Red Cross Hospital (Shizuoka City, JP)

Objective: Although we frequently encounter cases of cerebellar infarction, infarcts occurring simultaneously in both cerebellar hemispheres, especially in young adults, are rare. We report two cases of infarction in the bilateral superior cerebellar artery (SCA) territory, presumably due to arterial vasospasm induced by medication.

Case 1: Eight hours after delivery of twins, a 33-year-old woman suddenly became comatose without convulsion. Methylergometrine maleate had been injected for uterine contraction. As she became conscious, she 
showed bilateral cerebellar ataxia, right hemiparesis, and dysarthria. MRI demonstrated infarction in the bilateral SCA territory, as well as left thalamus. MR angiography revealed vasospasm of the vertebrobasilar arteries. She was treated with magnesium sulfate and a hyperosmolar agent. Intravenous administration of methylergometrine maleate, a potent vasoconstrictor, was thought to be the cause of vasospasm in the posterior circulation.

Case 2: A 32-year-old women was admitted because of dizziness, occipital headache and vomiting. She exhibited dysarthria and cerebellar ataxia, predominantly on the left side. CT/MRI showed cerebellar infarction in the bilateral SCA territory. Cerebral angiography performed later, however, demonstrated patent SCA. Thorough investigation revealed no definite cause of stroke; however, the OTC cold remedies she had been taking for four days included ephedrine hydrochloride. Since ephedrine is a vasoconstrictor, ephedrine-induced vasospasm was considered to be the cause of infarction.

Discussion: Methylergometrine maleate, an ergot alkaloid, and ephedrine hydrochloride, a sympathomimetic, are vasoconstrictive on the systemic, coronary, and cerebral arteries. Cases of myocardial and cerebral infarction due to vasospasm caused by such vasoconstrictors have occasionally been reported1)2). The affinity of these agents for the vertebrobasilar arteries might be related to differences in the distribution of receptors.

Conclusion: In patients with cerebral infarction with no evident cause or risk factors, especially in young adults, the possibility of arterial vasospasm induced by medication should be considered.

\section{References}

1. Bruno A, et al. (1993) Stroke associated with ephedrine use. Neurology 43:1313

2. Cantu C, et al (2003) Stroke associated with sympathomimetics contained in over-the-counter cough and cold drugs. Stroke 34:1667

\section{P235}

Insulin resistance and dyslipidaemia are strongly associated with ischaemic stroke and asymptomatic carotid artery stenosis in non-diabetics M. Mijajlovic, N. Lalic, A. Jotic, A. Pavlovic, J. Zidverc, Z. Jovanovic, N. Sternic

Institute of Neurology (Belgrade, RS); Institute of Endocrinology (Belgrade, RS)

Background and aims: Decreased insulin sensitivity (IS) with compensatory hyperinsulinemia plays a crucial role in the pathogenesis of atherosclerosis, but their role in ischemic stroke (IST) has not yet been elucidated. This study was aimed to analyze IS as the indicator of atherosclerotic activity, as well as plasma insulin (PI) levels and dyslipidemia pattern in 100 patients with atherothrombotic IST confirmed on brain computerized tomography/magnetic resonance imaging- (group A), 100 patients with asymptomatic stenosis of one or both internal carotid arteries $<50 \%$-(group B), and 50 healthy controls-(group C) Patients with diabetes mellitus and ischemic heart disease were excluded from the study.

Material and methods: IS was determined by Homeostasis Assessment Model (HOMA-IR), PI levels were determined by Radioimunoassay. We measured total cholesterol, LDL and HDL cholesterol and trygliceride levels in all groups.

Results: IS was significantly lower in group A compared to group B and $\mathrm{C}(3.82 \pm 0.27$ vs. $2.69 \pm 0.22, \mathrm{p}<0.05 ; 3.82 \pm 0.27$ vs. $1.510 .22, \mathrm{p}<0.01)$. PI levels were significantly higher in group $\mathrm{A}$ in comparison to group $\mathrm{B}$ and $\mathrm{C}$ $(16.00 \pm 1.2$ vs. $11.95 \pm 0.88, \mathrm{p}<0.05 ; 16.00 \pm 1.2$ vs. $7.12 \pm 0.19, \mathrm{p}<0.01)$. Different patterns of dyslipidemia were observed in ACAS in comparison to IST.

Conclusion: Our results indicate that atherothrombotic IST as well as ACAS are strongly associated with decreased IS and increased PI levels, although all the patients were non diabetics.Also, different patterns of dyslipidemia between ACAS and IST were observed.

\section{P236}

Decompressive hemicraniotomy in acute MCA infarctions: a hospital-based study of 22 patients, Dubai

M. Thakre, J. Inshasi

Rashid Hospital (Dubai, AE)

Objective: Decompressive hemicraniectomy and durotomy for malignant MCA infarction remains a controversial procedure but can be associated with reasonable clinical outcomes in highly selected patients. The selection of appropriate patients for intervention is of the utmost importance, but exact criteria remain to be defined. The study aimed to determine the survival rate and functional outcome, and the associated prognostic factors in patients with malignant MCA infarctions
Methods: This study included 22 patients with malignant MCA infarctions who were admitted in the neurology department at Rashid Hospital in Dubai, UAE from January 2003 to December 2007 and underwent a large ipsilateral decompressive hemicraniotomy. Clinical neurological presentation was evaluated using the NIHSS, Glasgow Coma Scale. Functional outcome was evaluated using the Barthel index (BI) and the Glasgow Outcome Scale (GOS), modified Rankin Score (mRS) at discharge and 6 months later.

Results: 22 patients were included in this study. Mean age was 43.8 years, 21 were males, 1 was female. 13 had Hypertension and 5 had Diabetes, no risk factors identified in 2.Mean GCS on admission was 13.68 and mean time to arrival to hospital from onset was 5.9 hours. Mean NIHSS score on admission was 17.04.14 patients had right MCA infarction. 11 had pupillary dilation before surgery. Mean GCS at surgery was 7.6 hours. Median time to surgery from onset of stroke was 30 hours. 16 were operated before 48 hours and 6 after. Mean ICU stay was 9.8 days. Median time for mechanical ventilation was 7.5 days. Surgical complications included 3 with pneumonia and 1 had seizures. Mean stay in hospital was 50 days. Mean BI was 43.66 on discharge with $7 / 19>70 \%$.Mean mRS was 3 on discharge. 2 patients during hospital stay (3rd day) and 1 died after 4 months. Average Glasgow outcome score was 3.

8 patients were able to walk by 6 months with minimal support, with favourable outcome ( $>60) \mathrm{BI}$ in 11 patients. Only 2 patients were in G2 GOS (persistant vegetative state)who were $>45$ years old with multiple risk factors for stroke.

Conclusion: This is the first study conducted in Dubai and UAE addressing the decompressive hemicraniectomy in MCA infarctions and it showed better survival than previously reported. Functional outcome improved and independence became possible especially in younger patients. Age, time of intervention and multiple vascular risk factors are the main prognostic indicators.

\section{P237}

Seizures after intracerebral haemorrhage: distance of haematoma from cortex but not haematoma size predicts seizure occurrence

M. Vendrame, Z. Haneef, A. Azizi

Temple University (Philadelphia, US)

Objective: To determine the risk factors for epileptic seizures in patients with intracerebral hemorrhage (ICH).

Methods: A retrospective review of 99 patients with ICH was conducted to analyze occurrence of seizure in relation to size and location of the hematoma. Using multivariate non-linear regression analysis with logistic growth, we determined whether factors such as location and size of the hematomas were associated with development of seizures.

Results: Seizures occurred in 14 of the 99 patients. There was no significant difference in the average size of hematoma between patients who developed seizures $\left(10.76 \pm 1.86 \mathrm{~cm}^{3}\right)$ and patients that remained seizures-free $\left(13.94 \pm 2.41 \mathrm{~cm}^{3}\right)$. However, in eleven of the $14(78 \%)$ seizure patients versus 40 of the $85(47 \%)$ seizure-free patients the boundaries of the hematomas were located at less than $15 \mathrm{~mm}$ from the cortex (Odd's Ratio 4.12; CI 1.073-15.84).

Conclusions: The size of hemorrhage was not associated with the increased risk of seizure, but the proximity to the cerebral cortex (distance less than $15 \mathrm{~mm}$ ) was a significant risk factor for seizure development. This data identifies a sub-population of ICH patients that are at risk for seizures and should be targeted for seizure prophylaxis.

\section{P238}

Middle cerebral artery stiffness and carotid atherosclerosis markers: analysis on a population of consecutive stroke patients

S. Galantucci, F. Corea, M.A. Abbas, M. Spinelli, F. Fumagalli, M. Bacigaluppi, G. Comi

INSPE IRCCS S. Raffaele (Milan, IT)

Aim: This study aims to compare Middle Cerebral Artery (MCA) stiffness indexes such as Pourcelot's Resistance index (RI), and Gosling's pulsatility index (PI), obtained trough transcranial color-coded sonography (TCCS) with markers of systemic atherosclerosis calculated by Carotid Duplex Sonography (CDS), like Intima Media Thickness (IMT) and again RI and PI in the extracranial carotid arteries.

Methods: We included 100 patients (Men: $64 \%$. Mean age: 63,76 [range $=$ $20 ; 98, \mathrm{SD} \pm 16,074$.$] ), with cerebral ischemic events (stroke or transitory is-$ chemic attacks [TIA]), consecutively admitted to our Stroke Unit between August 2006 and May 2007.

CDS and TCCS examinations have been performed on all patients at the time of arrival to the Stroke unit and, concerning the TCCS, with particular attention to the Middle Cerebral Arteries (MCA), using the transtemporal 
window. For both CDS and TCCS peak systolic velocity (PSV), end diastolic velocity (EDV), mean velocity (MV), Gosling's pulsatility index (PI), and Pourcelot resistance index (RI) have been calculated for each examined artery. Moreover, for the CDS, measurement of mean carotid intima-media thickness (IMT) has been performed.

Results: The most frequent cerebrovascular risk factors were: hypertension, diabetes mellitus, hyperlipidemia, cigarette smoking, obesity, previous stroke or TIA, atrial fibrillation, previous acute myocardial infarction and family history.

Seventy-six patients were diagnosed as "stroke", 24 as "TIA": $45 \%$ of stroke patients were PACI, $7 \%$ TACI, $26 \%$ LACI, $22 \%$ POCI (OCSP criteria). Concerning the main sonographic parameters for CDS, the mean IMT was $0,84(\mathrm{SD} \pm 0,22)$, with a median of 0,85 . For the Internal Carotid Artery (ICA) the mean RI was $0,66(\mathrm{SD} \pm 0,1)$, PI $1,22(\mathrm{SD} \pm 0,32)$. The MCA parameters were mean PSV 72,5 (SD $\pm 21,4)$, EDV 31,9 (SD $\pm 11,7)$, PI 0,94 (SD $\pm 0,25)$, RI $0,57(\mathrm{SD} \pm 0,09)$. At the bivariate analysis all the markers of MCA stiffness were found to be correlated with extracranial atherosclerosis markers: IMTMCA RI ( $p<0,001)$ ICA RI-MCA RI $(\mathrm{p}<0,001)$.

Discussion: Analyzing the results we found that extracranial carotid atherosclerosis, is associated with TCCS findings suggestive of increased intracranial resistances. These data support the hypothesis that atherosclerotic disease involves in a similar way both extra and intracranial vessels. The TCCS assessment may be an important tool for diagnostic completion in patients with ischemic stroke or TIA.
Our aim was to identify clinical or radiological measures that could assist in predicting a poor clinical outcome in ABCI.

Methods: We studied the clinicoradiological features and functional outcomes of 42 patients with $\mathrm{ABCI}$ ( $>2 \mathrm{~cm}$ and territorial) using diffusionweighted imaging. Functional outcomes were divided into two groups (favorable $(0-2)$ and poor (3-6) prognosis) by modified Rankin Scale. To permit quantitative analysis of the images, basilar artery score (BAS; range: $0-3$ ) was established. Clinical features, BAS, mass effect and radiological findings were compared between the groups.

Results: The clinical outcome was favorable in $57 \%(n=24)$ of patients, and poor in $43 \%(n=18)$.

Clinical symptoms and signs were not different between the groups, however, most patients with poor prognosis had decreased mentality at onset ( $72 \%$ versus $25 \%, \mathrm{p}=0.021$ ). The bilateral posterior inferior cerebellar artery (PICA) territory was the most frequently involved site in favorable group $(42 \%)$ and PICA + superior cerebellar artery (SCA) territory was in poor group (39\%). Patients with poor prognosis had a higher BAS (2.4 versus 1.6, $\mathrm{p}=0.035)$ than those with favorable. Patients with the presence of concomitant lesion outside the cerebellum were more in the poor group ( $78 \%$ versus $33 \%, p=0.02$ ), however, mass effect did not affect their prognosis.

Conclusion: Our study suggests that decreased mentality at onset, severity of basilar artery stenosis and concomitant lesion outside the cerebellum are predictors of poor clinical outcome in the ABCI.

\section{P241}

Cardiac troponin $\mathrm{T}$ elevation after stroke: The relationships between elevated serum troponin $\mathrm{T}$, stroke location, and prognosis

Y.B. Kim, P.W. Chung, H.S. Moon, B.C. Seo, H.S. Song

Kangbuk Samsung Hospital (Seoul, KR)

Background: Serum cardiac troponin $\mathrm{T}(\mathrm{cTnT})$ is regarded as a specific marker of acute coronary syndrome. However, increased cTnT is sometimes identified in patients with acute ischemic stroke. The clinical implications of increased serum cTnT in stroke patients remain unclear. The aim of the present study was to identify the relationships between elevated serum cTnT and stroke severity, location and prognosis.

Method: From JAN 2005 to DEC 2006, 455 consecutive patients who were admitted to Kangbuk Samsung hospital due to acute ischemic stroke were recruited for participation in this study. Diagnosis of acute ischemic stroke was confirmed by brain diffusion-weighted imaging (DWI) MRI within 3 days of stroke onset. Patients who did not undergo echocardiography and electrocardiography were excluded from the study, as were patients for whom there was no data on serum cTnT. Patients with cardiac diseases, such as acute myocardial infarction (ACC/AHA criteria), congestive heart failure and valvular heart disease, and other debilitating medical problems were also excluded. A total of 39 patients were excluded. The remaining $416 \mathrm{pa}-$ tients were divided into 2 groups: elevated $c \operatorname{TnT}$ group $(=>0.01 \mathrm{ng} / \mathrm{ml}$ $\mathrm{N}=45)$ and normal cTnT group $(<0.01 \mathrm{ng} / \mathrm{ml}, \mathrm{N}=371)$. A modified Rankin scale was used to compare the prognosis between the 2 groups.

Results: Serum cTnT was elevated in $10.8 \%$ (45/416) of the acute ischemic stroke patients. The clinical and epidemiologic characteristics were comparable between the 2 groups. Stroke severity, as assessed by the $\mathrm{Na}$ tional Institutes of Health Stroke Scale Score (NIHSSS), was significantly worse in patients with elevated serum $c \operatorname{TnT}$ than in patients with normal serum cTnT $(8.46 \pm 1.98$ vs. $5.29 \pm 2.25, \mathrm{p}=0.035)$. Insular lobe involvement was more common in patients with elevated serum cTnT than in those with normal serum cTnT $(28.9 \%$ vs. $15.1 \%, \mathrm{p}=0.042)$. Prognosis was worse in patients with elevated serum cTnT than in those with normal serum cTnT (the rate of favorable outcome; $17.8 \%$ vs. $27.4 \%, \mathrm{p}=0.027$ ). The results analyzed by univariate and multivariate regression indicated that elevated cTnT was independently related to an insular involvement, cardioembolism and high severity score.

Conclusion: Elevated serum cTnT in acute ischemic stroke was associated with severe neurological deficit at stroke onset and insular lobe damage. Acute ischemic stroke patients with elevated serum cTnT had a worse prognosis than those with normal serum cTnT.

This study was supported by IN-SUNG Foundation for Medical Research

P242

Clinical analysis of 30 patients with cerebral venous sinus thrombosis $J-H$. Chen, N. Li, K. Dong

Beijing Mining Aggregation General Hospital (Beijing, CN); Beijing Tiantan Hospital, Capital Medical University (Beijing, CN)

Background and purpose: Cerebral venous thrombosis is is an uncommon

condition and elusive diagnosis because of its nonspecific presentation and

\section{The prognostic factors of acute bilateral cerebellar infarcts}

S.C. Hong, J.Y. Ryu, C.H. Kim
Hanil Hospital (Seoul, KR)

Objectives: The clinical prognosis of acute bilateral cerebellar infarcts (ABCI) affecting basilar artery is usually poor. There are few reports, however, in which correlations have been established between the clinical and radiological findings. 
its numerous predisposing causes. It often affects young-to-middle-aged patients, and more commonly women and this is partly due to greater awareness among physicians and neurologists, and partly to improved non-invasive imaging techniques. It is more common than previously thought, imaging plays a key role in the diagnosis. In this study, we summarize the clinical and imaging feature of cerebral venous sinus thrombosis in order to give some instruction for decreasing the misdiagnosis rate and mortality about this disease in practice.

Methods: A total of 30 patients with a mean age of 35 years (range, 16-50) hospitalized our Hospital between March, 2005 to March, 2007 were enrolled. We analyzed retrospectively the clinical features, neuroimaging changes, treatment and prognosis of them.

Results: Twenty-two of all 30 patient s were presented nonspecific headache and early presenting symptom, five cases suffered from seizures while three cases got unconsciousness. Ten patients have been misdiagnosed at onset, the rate of misdiagnosis reached $33.3 \%$. Twenty-four out of $30 \mathrm{pa}-$ tients were made a definite diagnosis as cerebral venous sinus thrombosis after neuroimaging, ten patient $s$ accepted digital subtraction angiography examination were all confirmed as cerebral venous sinus thrombosis. All the patient $s$ were accepted anticoagulation and intracranial pressure control. Twenty-four cases improved, one had no change and five died among all those patients during hospitalization.

Conclusions: Cerebral venous sinus thrombosis has no specific clinical feature and a high rate of misdiagnosis. Neuroimaging examination turns out to be valuable for the early diagnosis of cerebral venous sinus thrombosis.

State Key Laboratory of Cognitive Neuroscience and Learning Open Project Grant

P243

Cerebral venous thrombosis: a retrospective study of 31 cases

H. Horozoglu, I. Midi, N. Afsar, S. Aktan

Marmara University Hospital (Istanbul, TR)

Objectives: Thrombosis of the intracranial veins and sinuses, so called cerebral venous thrombosis (CVT), accounts for $0.5 \%$ of all strokes. CVT is typically multifactorial and the extent of thrombosis depends on the predisposing factors. The aims of this study are to describe the age, gender, clinical aspects, cause and predisposing factors for CVT as well as to evaluate the MR-venography findings related to CVT in a series of 31 patients.

Methods: Thirty symptomatic CVT patients, admitted to our hospital between June 2001 and December 2007 were retrospectively reviewed regarding to demographical data, etiology, and clinical presentation. Patients were evaluated by a standard protocol including cranial MR imaging (MRI) and cranial MR-venography (MRV) for the diagnosis of the hemorrhagic infarction, local causes and the localization of the thrombosed sinuses. Patients were asked for condition such as dehydration, pregnancy or postpartum period, use of oral contraceptive. Behcet's disease, infection, malignancies, inflammatory disease, hereditary coagulation disorders were systematically analyzed with laboratory tests.

Results: Thirty one patients (M 9, F 22; age range 17-70, mean 34.5 years), with evidence of CVT on MRI and MRV, were included. The most common symptom on admission was headache (25 patients, 80.6\%). On admission: 9 (29\%) had visual symptoms (as blurred vision, diplopia), 5 (16\%) had nausea and vomiting, $9(29 \%)$ had paresis and paresthetic symptoms and 6 (19\%) presented with seizures. On imaging 14 had venous infarcts and 5 had previous history of lower extremity deep venous thrombosis. The transverse sinus was most frequently involved $(67.7 \%)$. As to the etiology: eight $(25.8 \%)$ had Behcet's disease, five (16\%) had antithrombin III, protein C, or protein S deficiencies, nine $(29 \%)$ had F V Leiden or prothrombin gene mutations, four $(12.9 \%)$ were pregnant or post partum period, two were using oral contraceptives and seven patients had various other predisposing factors ( 2 external pressure occlusion, 2 diarrhea, 2 policytemia, 1 fasting). Some patients were detected with more than one condition.

Conclusions: CVT may be provoked by the increase in blood viscosity secondary to several conditions. Behcet's disease was the most common etiological factors in our series emphasizing once more the contribution of this disease to CVT in the Turkish population and the importance of its systematic screening during the etiological work-up.
P244

Predictors of cerebral venous and sinus thrombosis: clinical and neuroimaging correlations

I. Verulashvili

Tbilisi State Medical University (Tbilisi, GE)

Objectives: Cerebral venous and sinus thrombosis (CVST) for many years have been diagnosed mainly at autopsy. The widespread use of cerebral angiography, CT of the brain and more recently MRI made early diagnosis of CVST possible and completely modified our knowledge of this condition. But still very little is known about the correlations of clinical and neuroimaging predictors of CVST manifestation.

Methods: We performed a multicenter (3 centers) prospective study. A cohort of 61 patients aged 35 to 72 (mean 52) years with CVST, evaluated from 2000 through 2007, were screened from over 1800 patients of acute cerebral stroke. CVST was diagnosed by CT/MRI angiography, MRI venography (MRV), or traditional angiography. The selected patients were followed up at 6 months: information on death, neurological status, recurrent CVST and other thrombotic events were obtained from direct observation (end of 3-rd and 6-th months) or by telephone interviews.

Results: The spectrum of clinical symptoms reflects the degree of venous congestion, which depends not only on the extent of thrombosis in the dural sinuses or deep veins, but also on the territory of the involved vessels and the establishment of venous collaterals. The chief complains during admission were headache $(37.8 \%)$, loss of consciousness $(24.3 \%)$, seizures $(17.2 \%)$, weakness $(16.5 \%)$, and blurred vision $(4.2 \%)$. The average delay time from onset of symptoms to admission was 4 days. MRV performed after diagnosing the thrombosis of the major sinuses (CST) in patients with intensive headache and seizures (in 27 cases from 43 ) revealed the lateral sinus dysplasia.

Conclusions: Deep cerebral venous system thrombosis is an underdiagnosed condition when symptoms are mild, but cases with bilateral thalamic lesions cause cognitive dysfunctioning, coma or death. Isolated thrombosis of the straight sinus is rare; usually there is involvement of the Galenic system or the superior sagittal sinus and it is an additional predictor of poor outcome. The prognosis of CVST in our prospective study was essentially good in cases of long-term anticoagulant treatment. In patients with the lateral sinus dysplasia complicated by CST our data emphasized that low-intensity warfarin therapy (ratio 1.5 to 2.0 ) prevents the recurrent thrombotic events in 18 from 27 patients. In cases of deep cerebral venous system thrombosis anticoagulant therapy usually was prescribed for 3 months, which was enough to decrease the risk of recurrent CVST.

\section{P245}

Cerebral venous thrombosis in Dubai. Clinical, radiological and prognostic study of 25 cases

J. Inshasi, M. Thakre

Rashid Hospital (Dubai, AE)

Introduction: Cerebral venous thrombosis (CVT) is a rare disease. Diagnosis is a challenge because of its variable clinical presentation, high index of suspicion is required.

Objectives: We aimed to Study all cases of CVT admitted to our hospital between 2000-2007demonstrating the clinical, Radiological, demographic data and the functional outcome and comparing this with the International Study on Cerebral Vein and Dural Sinus Thrombosis (ISCVT)

Methods: This is a prospective hospital based study which included 25 patients admitted in the Neurology Department, Rashid Hospital in Dubai.Primary outcome was death or dependence assessed by modified Rankin Scale at end of follow up and Secondary outcome was death or dependence at 6 months.

Results: There were 13 (52\%) males and 12 (48\%) females in the Study. Mean age was 39.1 years. $6(25 \%)$ patients presented with isolated intracranial hypertension. $22(88 \%)$ patients had a GCS between 9-13 on admission and $3(12 \%)$ had GCS $<9$ on admission. Headache was the commonest mode of presentation $92 \%$ followed by seizures $32 \%$, blurring of vision $28 \%$ and weakness $24 \%$. No cause for CVT was identified in $9(36 \%)$, severe anaemia in $3(12 \%), 3(12 \%)$ patients were in postpartum period at presentation, 2 $(8 \%)$ had thyroid function abnormality, $2(8 \%)$ were on oral contraceptive pills. 1 patient each of dehydration, glomus tumor, tuberculous meningitis, thrombocytosis and pregnant state was identified. 22 (88\%) patients were anticoagulated with LMWH in therapeutic doses and were later changed to warfarin for 1 year. $5(20 \%)$ patients required mechanical ventilation. Mean duration of hospital was 18 days.

Modified Rankin Scale was applied to patients on discharge, after 6 months, 1 year or when last seen. $1(4 \%)$ patient was lost to follow up at 6 months and $4(16 \%)$ by 1 year. Events which occurred during follow up included DVT in $1(4 \%)$ and seizures $9(36 \%) .2$ (8\%) patients died during 
hospitalization, 19 (90\%) had complete recovery and $1(4 \%)$ had dependent life when last seen at 3 years follow up.

Conclusion: This is the first study done in Duabi and UAE about CVT. Though the number of patients was small,we demontrated higher percentage of males involved than females as compared with the ISCVT study In $36 \%$ of patients no cause was found. As compared to the ISCVT Study we had significantly lower mortality $8 \%$ VS $27 \%$, with complete recovery in $90.4 \%$. Poor prognostic factors were intracranial infection,coma,ICH. We demonstrated that treatment with anticoagulation was safe and effective.

\section{P246}

Aetiological variability and approachment of cerebral sinus venous thrombosis

Y. Kaya, Z. Ozozen, Ü.S. Benli, U. Can, S. Gülsen

Baskent University (Ankara, TR)

Objectives: Cerebral venous sinus thrombosis is a serious and potentially life-threatening cerebrovascular disorder with a wide spectrum of clinical presentations and prognosis. The risk factors are classified as acquired conditions like nephrotic syndrome, anemia, trauma, surgery, pregnancy, puerperium, malignancy, infections, drugs or hereditary as coagulopathies as factor V Leiden-, methylene tetrahydrofolate reductase or prothrombin gene mutations, protein $\mathrm{C}$ or S deficiency or antithrombin III deficiency.

Headache is the most frequent and often the earliest symptom of cerebral venous sinus thrombosis.The other symptoms can be vomiting, transient or persistent visual obscuration, focal or generalized seizures, focal neurologic signs, lethargy and coma.The venous sinuses that are most frequently thrombosed are lateral, cavernous and superior sagittal sinuses.

Methods: We studied 18 patients, 14 female and 4 male, with a diagnosis of cerebral venous sinus thrombosis whose mean age was 46.61, range 17-74. This retrospective study analsyed to determine etiology and approachment of cerebral sinus thrombosis.

Results: Two patients were diagnosed with Behcet's diseases, two patients had malignancies, two patients were in the postpartum period, two had prothrombin gene mutations, one was a renal transplant recipient, one patient was diagnosed with essential thrombocythemia and the last patient had chronic renal failure. Anemia in four and sinusitis in five patients was determined as the contributing risk factors.

eadache was the most common and initial symptom in twelve patients. Other patients presented with seizures, alteration in consciousness, vomiting, nause, or hemiplegia.

Conclusion: We emphasize with this study that cerebral venous sinus thrombosis has variable etiology that may be revealed with detailed searching. Determining the cause-and-effect relationship is important for planning urgent treatment strategies and secondary prevention.CT angiography and / or venous MRA are sensitive and noninvasive procedure for early diagnosis and follow-up of cerebral venous thrombosis clinical conditions which can cause morbidity or mortalitiy.

\section{Clinical neurophysiology}

P247

Modified wavelet Morlet analysis of EEG in Parkinson patients

A. Gabova, V. Gnezditski, A. Karabanov, G. Kuznetsova, A. Karasev, S. Kachalov, Y. Obukhov

IHNA RAS (Moscow, RU); Neurology RAMS (Moscow, RU); IRE RAS (Moscow, RU)

Objectives: EEG changes are known in Parkinson patients in delta, teta, alpha and beta frequency bands. Wavelet Morlet analysis contrasted with Fourier analysis gives the possibility to observe The EEG time-frequency dynamics during the recording. The objectives of the work is the wavelet Morlet analysis of EEG time-frequency dynamics in Parkinson patients in alpha and beta bands.

Methods: EEG recordings of Parkinson patients were taken from the database of the Institute of Neurology PAMS, Moscow. Sixteen Parkinson patients were investigated with predominant symptoms of tremor-rigidity and rigidity-tremor. Registration was performed using $10 \times 20$ system by Neurocartograph (MBN, Moscow) device. EEGs from 16 electrodes were investigated. Linked ears were used as reference. Wavelet transform modified specially for the time-frequency EEG analysis in the Institute of Radioengineering RAS was applied.

Results: Concerning alpha band our results confirm the data of Stoffers et al (2007) about the decrease of alpha frequency in EEG of Parkinson pa- tients. Beta activity was increased with bursts in the whole diapason. The frequency of the bursts fluctuated during the recording. We could not see the exact division to lower and upper beta ranges in the whole beta band diapason. By the method of modified wavelet Morlet it could be revealed for EEG frequency the noncoincidence in different brain areas and asymmetry between the same areas of two hemispheres.

Conclusion: Progression of Parkinson disease is coupled with the progression in EEG frequensy changes. Modified wavelet transform gives the possibility to investigate EEG dynamics and obtain new data about EEG time-frequency characteristics of Parkinson patients

The project is supported by Program of RAS "Fundamental Science-for Medicine"

\section{P248}

Generalised absence epilepsy and its coexistence with occipital non-convulsion epilepsy: the comparison of time-frequency structure of spike-wave discharges in patients and in animals with absence epilepsy

A. Zharikova, V. Gnezditski

INHA, Russian Academy of Science (Moscow, RU); Institute of Neurology (Moscow, RU)

Objective: Absence epilepsy is characterized by spike-wave discharges (SWDs) of $3 \mathrm{~Hz}$ in patients and 6-7 Hz in WAG/Rij rats (genetic model of absence epilepsy). We investigated two types of SWDs: generalized (SWD-G) and local occipital (SWD-O) ones. Modified wavelet Morlet transform (Bosnyakova, Obukhov, 2005) was used to specify their time-frequency structure.

Methods: EEG of 18 out-patients from Institute of Neurology, Moscow (Childhood absence epilepsy and Juvenile absence epilepsy) and $37 \mathrm{WAG} / \mathrm{Rij}$ rats were analyzed. EEG of patients was registered using the $10 \times 20$ system with linked ears as a reference. Neurocartograph (MBN, Moscow) was used for the registration. In rats EEG was registered with chronic epidural electrodes placed at the frontal, parietal and occipital cortices with Bioscript BST-2000 electroencephalograph. The modified wavelet Morlet transform was used to make graphs of time-frequency dependence in the discharges.

Results: In patients long and short generalized SWDs were analyzed. At the beginning of SWD-G the highest frequency was measured. Then during the whole long SWD-G the main frequency oscillated with periodicity about $1-2$ sec. During short SWD-G the frequency quickly dropped. In 2 patients the coexistence of generalized and occipital SWDs was shown. The frequency of SWD-O was more slowly than that of SWD-G. Occipital SWDs could originate separately or could precede generalized SWDs.

In rats the similar SWD-G time-frequency dynamics were obtained however in another frequency band. In about $70 \%$ of EEG recordings in rats the coexistence of SWD-G and SWD-O was demonstrated. It was shown that SWD-G and SWD-O responded oppositely to apomorphine (Midzianovskaya et al., 2004) and metoprine (Samotaeva et al., 2007). These drugs decreased the SWD-G and increased the SWD-O in rats.

Conclusion: The time-frequency structure of SWD-G and SWD-O was investigated in patients and in WAG/Rij rats. The obtained results extend the validity of WAG/Rij rats for the experimental investigation of non-convulsive epilepsy types. Specific time-frequency structure and different reaction of SWD-G and SWD-O to the listed drugs confirm that they are rather different and non-dependent types of electrical activity of the brain.

\section{P249}

Vestibular evoked myogenic potentials in "vestibular" migraine

B. Baier, M. Dieterich

Uniklinik Mainz (Mainz, DE)

Objectives: Sound-induced vestibular evoked myogenic potentials (VEMPs) are a tool for investigating saccular function, measured from the tonically contracted sternocleidomastoid muscles (SCM) in response to loud sound stimuli. Aim of the present study was to assess VEMPs in patients with vestibular migraine and to explore if saccular function or pathways are affected by vestibular migraine. This is especially interesting for the differentiation from similar symptoms due to Menière's disease.

Methods: Amplitude and latency of VEMPs were measured from the SCM in 66 patients with vestibular migraine (median age: 46 years; range 17-70 years) and compared with 60 age-matched controls (median age: 45 years; range $17-73$ years).

Results: 25 of 66 patients with vestibular migraine $(38 \%)$ had lower bilateral EMG-corrected amplitudes than the controls $(\mathrm{p}<0.01)$, whereas no difference was seen with regard to the latencies.

Conclusion: The present data of patients with vestibular migraine give evidence that the VEMP amplitudes may be bilaterally significantly reduced 
compared to controls. This electrophysiological finding points to an affection of peripheral vestibular structures such as the saccule, probably caused by hypoperfusion-induced ischemia. Therefore, structures in the inner ear seem to contribute to vertigo in vestibular migraine in at least one third of the patients.

\section{P250}

Autonomic neuropathy and gallbladder motility disorders in patients with diabetes mellitus

D. Georgescu, C. Georgescu, M. Simu, L.A. Georgescu

University of Medicine (Timisoara, RO)

Aim: Assessment of gallbladder motility disorders in patients with diabetes mellitus and autonomic neuropathy before and after treatment with gabapentinum.

Patients and methods: 30 patients with type II diabetes mellitus displaying various symptoms of autonomic neuropathy undertook this clinical study. Patients had one or more of these symptoms: sinus rest tachycardia, orthostatic hypotension, urinary disorders with no kidney functional impairment, gastric and bowel disorders: dyspepsia, delaying of gastric emptying, nocturnal diarrhea. They've got no previous gastric surgery, vagal denervation, or gallstones, sex ratio was: $\mathrm{M} / \mathrm{W}=19 / 11$, mean age = $65,32 \pm 9,78$ years, body mass index $=33,44 \pm 5,51 \mathrm{~kg} / \mathrm{m}^{2}$. They also didn't receive associated treatment with prokinetic agents. Patients undertook a clinical trial consisting of treatment with gabapentinum with initial dose of $3 \times 300 \mathrm{mg} /$ day, with increasing doses till $3600 \mathrm{mg} /$ day for 6 months. We 've focus our study on the gallbladder motility response related to this medication. Patients were examined by transabdominal ultrasonography with a 3,5 $\mathrm{MHz}$ convex array probe, before and after the treatment. We calculated the gallbladder initial and residual volume with ellipsoid method and gallbladder ejection fraction. in fasting state and 30 minutes after a cholecystokinetic meal. An ejection fraction bellow $60 \%$ was considered pathological.

Results: Before therapy the group of diabetic patients with neuropathy had a mean gallbladder ejection fraction $=31,10 \% \pm 3,98$, showing a constant low rate of gallbladder emptying. After treatment the mean gallbladder ejection fraction was $49,59 \% \pm 5,11$, representing an elevation of gallbladder ejection fraction with $18,49 \% \pm 1,13 \%$, and an improving of gallbladder motility.

Conclusions: Gallbladder motility disorders related to autonomic neuropathy are represented by delaying of gallbladder emptying and decreasing the gallbladder ejection fraction. These disorders are considered to be risk factors for gallstones and other complications. According to our results after treatment with gabapentinum diabetic patients showed a slightly but visible improvement of gallbladder motility with promising clinical outcome regarding the dyspeptic complains. However there is insufficient data for these conclusions to have statistical significance.

P251

The search for new neurogenic vestibular-evoked potential waveforms: a topographical scalp mapping study using air-conducted tone auditory stimuli

E. Papathanasiou, A. Lemesiou, P. Myrianthopoulou, M. Pantzaris, S. Papacostas

Cyprus Institute of Neurology \& Genetics (Nicosia, CY)

Objective: Interest in finding potentials recorded from the scalp surface, in response to auditory stimuli that are known to stimulate the saccule, has increased over the last few years. Our group discovered a negative potential $5 \mathrm{mec}$ after stimulus onset, arising in the parietal areas. In this study, we carried out an extensive mapping of the scalp, to search for other waveforms that could potentially come from the vestibular system.

Methods: Twenty-two normal volunteers (age range 20-30 years) were recruited for the first part of the study, which involved recording 64 derivations, and comparing potentials recorded at $70 \mathrm{~dB} \mathrm{nHL}$ (known to stimulate the cochlea alone) and $90 \mathrm{~dB}$ nHL (known to simulate both the saccule and cochlea) using a $1 \mathrm{kHz}$ tone-pip auditory stimulus delivered via headphones. The difference between the two recordings at each derivation (presence or absence of a waveform, and not a simple shift in latency) was considered to represent a waveform potentially arising from the vestibular system. In the second part of the study, a separate independent set of ten normal volunteers (age range 28-43 years) were examined to determine if the candidate waveforms appeared consistently amongst the normal individuals.

Results: Six waveforms were discovered from the first part of the study, occurring at 14,12, 10,8,6 and $4 \mathrm{msec}$ after stimulus onset. In the second part of the study, half of these waves $(12,10$ and 8 msecs) were not analyzed further, as they did not appear consistently in the second group of normal subjects. The waveform at $4 \mathrm{msecs}$ was found to be the N5 waveform that we had discovered previously (recorded at P3-Fpz for the left ear and visa versa). The wave at 14 msecs is a "W" shaped wave recorded at T6-O2 for the left ear (and visa-versa), and the wave at 6 msecs is monophasic and recorded at $\mathrm{O} 2$ P3 for the left ear (and visa-versa).

Conclusions: Using air-conducted tone-pip auditory stimuli, three negative waves in total can be recorded that may arise from the vestibular system. The N5 wave is believed to arise from the pons based on its latency. The N6 wave may arise from the midbrain, whereas the N14 wave may arise at the cortex. Future use of these waves may localize vestibular pathway lesions at or between these locations. Also, it appears that the posterior scalp is an area belonging to the vestibular system, whereas the recording of cochlear potentials is located at the anterior half of the scalp.

\section{P252}

Recruitment patterns of spinal motoneurons in human sleep

F. Salih, S. Steinheimer, P. Grosse

Charité-Universitätsmedizin (Berlin, DE)

Objective: This study examines recruitment patterns of spinal motoneurons in human sleep. Though reduced excitability of spinal motoneurons has previously been shown in sleep it still remains open whether inhibition affects the entire spinal motoneuron pool in the same way and to the same extent in NREM and REM sleep.

Methods: To address these questions we assessed F-tacheodispersion alongside conventional F-wave-parameters (F-persistence, F-amplitudes) in 17 healthy subjects in NREM2, NREM3/4, REM and wakefulness. Stimuli were delivered on the ulnar nerve at wrist level with a stimulus intensity of 1.2 times of the sleep-stage specific maximal M-response at a frequency of $0.2 \mathrm{~Hz}$. Compound muscle action potentials were recorded from the $1 \mathrm{DI}$ muscle. For analysis repeated sets of at least 128 stimuli were stored to obtain 15 artefact-free F-waves. F-tacheodispersion was calculated based on Fconduction-velocities using the modified Kimura-formula (Chroni and Panayiotopoulos, 1993).

Results: F-persistence (ratio sleep-stage/wakefulness; NREM 2: 0.46 \pm 0.11 ; NREM3/4: $0.36 \pm 0.09$; REM: $0.14 \pm 0.05$ ) confirmed decreased spinal excitability in all sleep-stages, most markedly in REM as did F-amplitudes (ratio sleep-stage/wakefulness; NREM2: $0.45 \pm 0.08$; NREM3/4 $0.38 \pm 0.06$; REM: $0.35 \pm 0.15$ ). The range of F-tacheodispersion (ratio sleepstage/wakefulness; NREM2: 0.82; NREM3/4: 0.82; REM: 0.64) was reduced in all sleep stages with a shift (difference between medians of sleep-stages and wakefulness; NREM 2: $-1.84 \mathrm{~m} / \mathrm{s}$; NREM 3/4: -2.31 ; REM: -0.05$)$ towards lower conduction-velocities in NREM.

Discussion: Our study shows sleep-stage specific reduced excitability of spinal motoneurons. Variations in shifts of F-tacheodispersion suggest that supraspinal inhibitory mechanisms might act differently on fast-conducting spinal motoneurons in NREM and REM.

\section{P253}

Neurological and neurophysiological examinations of workers occupationally exposed to arsenic

H. Sinczuk-Walczak, T. Halatek

Nofer Institute of Occupational Medicine (Lodz, PL)

Objectives: Several metals have neurotoxic properties, especially heavy metals. Neurological and neurophysiological studies indicate that the central (CNS) and peripheral nervous system (PNS) may be affected by arsenic (As). The aim of the present study was to assess the effects of inorganic arsenic on central and peripheral nervous system.

Methods: The study covered a selected grup of 21 male workers (mean age 41,9, SD 7,6 years; range 31-55y) involved in a copper smelting factory. Their employment duration ranged between 5-33 years (mean 8,1 SD 7,8y). Arsenic concentrations in air in work places amounted on average 0,01003 $\mathrm{mg} / \mathrm{m}^{3}, \mathrm{SD} 0,00866$. Urine arsenic concentrations found in workers ranged from 860 to $453 \mathrm{ug} / \mathrm{L}$ (mean 229,6 ug/L, SD 105).The control group consisted of 16 men non-occupationally exposed to As, matched by gender, age and work shifts. The evaluation was carried out on base of neurological examination, electroencephalography (EEG), visual evoked potentials (VEP) and electroneurography $(\mathrm{ENeG})$.

Results: Clinical symptoms, such as headache (19\%), increased emotional irritability (33,3\%), insomnia $(52,4 \%)$, hypersomnia $(33,3 \%)$, limb pains $(19 \%)$, fatigability of limbs $(47,6 \%)$, myospasms $(28 \%)$, paraesthesia $(14,3 \%)$, predominated among functional disorders of the nervous system in workers chronically exposed to As. The objective neurological examinations did not reveal organic lesions in the CNS or PNS. In EEG classified as abnormal, generalized changes were most common. VEP examinations revealed abnormalities in the latency of the evoked response. Stimulation of motor fibres of peroneal and median nerves showed decreased amlitude of 
motor potential. Stimualtion of sensory fibres of median nerves showed decreased amplitude of sensory potential and lower conduction velocity of sural nerves.

Conclusions: The results of this study suggest that exposure to As at concentrations within MAC values and above, induces subclinical effects in the nervous system, especially subclinical neuropathy.

\section{P254}

Direct intracranial evidence for learning-related modulation of motor response preparation

I. Constantinescu, M. De Lucia, M. Zangl, M. Seeck, G. Pourtois, S. Schwartz University of Geneva (Geneva, $\mathrm{CH}$ ); Center for Biomedical Imaging (Geneva and Lausanne, $\mathrm{CH}$ ); University of Marburg (Marburg, DE); University Hospital of Geneva (Geneva, $\mathrm{CH}$ )

Studies using scalp and intracranial EEG in patients with pharmaco-resistant epilepsy have described different components of movement-related cortical potentials,including the motor response preparation potential (MRPP). Motor skill acquisition influences the magnitude and time course of movement-related potentials, but the respective contributions of cortical regions to the different stages of learning still remain unclear.

Our aim was to assess whether MRPP might be modulated at early and/or later stages of motor skill learning using intracranial EEG recordings. We recorded subdural activity in right motor regions including primary motor (M1), premotor cortex and supplementary motor area (SMA) from a 8-by-8 electrodes grid and 8 medial-frontal electrodes in a patient with pharmaco-resistant epilepsy. During the recordings, the patient was presented with four rectangles displayed horizontally on a computer screen. The patient had to react as quickly and accurately as possible when one of the rectangles changed its color, by pressing the spatially corresponding key on a response pad. A fixed 8-keypress sequence was trained during a first session over blocks of 20 sequences, and retested the next morning after one night of sleep (session 2). The regularity of the sequence remained unknown to the patient.

Behavioral results indicated improvement within session 1 corresponding to a "fast-learning" phase, which persisted on the next day, reflecting an additional "slow-learning" phase. ERP results revealed differential modulations of MRPP: both fast and slow learning changed the magnitude of MRPP in M1, whereas slow learning selectively affected an earlier portion of MRPP in the SMA. These findings provide important insights into brain mechanisms of motor skill learning, here influencing at different latencies two cortical frontal regions involved in motor preparation.

\section{P255}

Painful legs and moving toes: subgroup classification according to movement perception

J. Aparicio, S. Álvarez Blanco, J. Valls-Solé

Hospital Clínic (Barcelona, ES); Hospital de Niños (Caracas, VE)

Background/objectives: The syndrome of painful legs and moving toes (PLMT) is characterized by spontaneous pain in the lower limbs associated with involuntary movements of the toes or the whole foot. However, some patients with PLMT believe that they have some voluntary control of their feet movements. Therefore, we decided to investigate whether there were clinical and electrophysiological differences related to movement perception and voluntary control.

Methods: Twelve consecutive PLMT patients were classified according to whether or not they were consciously perceiving their movements (feeling, yes or not) and whether or not they thought that they had a voluntary control of them (will, yes or not). We carried out a standard clinical neurological examination and a conventional electrophysiological examination, including sensory and motor nerve conduction studies, $\mathrm{F}$ wave latencies and needle electromyography.

Results: Ten patients, who declared no feeling of their movements, showed signs of mild to severe polyneuropathy. Three of them declared that they were voluntarily performing occasional movements with the intention to feel their feet and were able to keep their toes and feet quiet until distraction. The remaining two patients, who declared feeling the movements but no control over them, had predominantly signs of L5 or S1 radiculopathy, with frequent action potentials compatible with axon reflexes in the electrophysiological testing for proximal nerve conduction.

Conclusions: Assessment of perception and control of their movements is an important adjuvant to the classification of patients with PLMT. No feeling of movement is more likely related to polyneuropathy, while no control over the movements is more likely related to ectopic generation of activity in motor (and probably also sensory) axons. A complete clinical and electrophysiological analysis contributes to a better understanding of the patho- physiological mechanisms underlying the neuropathic lesion responsible for pain and movement in PLMT patients.

\section{P256}

Long-term cortical plasticity of interhemispheric inhibition in piano players: a transcranial magnetic stimulation study

L Straffi, R. Chieffo, A. Inuggi, F. Spagnolo, G. Comi, L. Leocani IRCCS Hospital S.Raffaele (Milan, IT)

Objectives: The aim of our study was to characterize the influence of skilled motor practice on interhemispheric inhibition using transcranial magnetic stimulation (TMS)

Methods: Nineteen subjects ( 9 pianists: $4 \mathrm{~F}$, age $27.1 \pm 4$; 9 naïve controls: $5 \mathrm{~F}$, age $26.7 \pm 2$ ) were studied. Ipsilateral silent period (iSP) was measured as the average percent suppression of maximal voluntary EMG in abductor pollicis brevis to focal TMS of the ipsilateral hand motor cortex. Upper limb dexterity was assessed using NineHolePegTest (NHPT). Mirror movements to maximal unilateral voluntary isometric contraction were searched with EMG recordings.

Results: In the control group, iSP was significantly greater on the dominant compared to the non dominant side ( $\mathrm{p}<0.05$; paired $\mathrm{T}$ test), while it did not differ in piano players, being their non dominant iSP higher than in controls $(\mathrm{p}<0.05$; paired $\mathrm{T}$ test). The dominant hand performed significantly better than the non-dominant one in control-group $(\mathrm{p}<0.05$; paired $\mathrm{T}$ test $)$ but not in musicians. Mirrors occurred in 4 control subjects, only to voluntary movement of the non dominant hand, with significantly higher frequency ( $\mathrm{p}<0.05$; sign test) compared to the pianist group ( 1 subject). NHPT score $(\mathrm{sec})$ was negatively correlated to iSP to the same hand (Pearson's $\mathrm{r}=0.431, \mathrm{p}=0.015$ ).

Conclusions: In naïve subjects, we found an asymmetric interhemispheric inhibition, being more efficient from the dominant to the non dominant hemisphere, consistently with an asymmetric occurrence of mirror movements. Compared to naïve subjects, piano players have stronger interhemispheric inhibitory effects from the non dominant hemisphere, and a lower occurrence of mirrors to movement of the non dominant hand. Moreover, the strength of interhemispheric inhibition was related to the speed of motor performance. Taken together, these findings suggest that bimanual motor skilled training may act on the transcallosal inhibitory system by rebalancing the interhemispheric asymmetry in favour of the non dominant side.

\section{P257}

Nerve conduction studies in demyelinating CMT

M. Poyraz, Z. Matur, E. Battaloglu, P. Oflazer, Y. Parman, F. Deymeer Istanbul University (Istanbul, TR); Bosphorous University (Istanbul, TR)

Objective: Nerve conduction studies (NCS), particularly those of the median nerve, are very important in deciding whether patients with Charcot-MarieTooth disease (CMT) have the demyelinating or the axonal form, the knowledge of which guide the molecular biological studies. Within the demyelinating form, certain clues from electrodiagnostic studies have been reported to imply the presence of specific mutations. For example, a slow conduction velocity (CV) in PMP22 duplications in contrast to a higher CV in connexin 32 (Cx32) mutations have been noted. Conduction block has been reported in myelin basic protein (MPZ) mutations. This study was undertaken to assess the relationship between NCSs and genetic defects in patients with demyelinating CMT.

Methods: Motor NCSs of median, ulnar, peroneal and tibial nerves and sensory NCSs of median, ulnar, sural and superficial peroneal nerves were performed in 43 patients. The interest of this study is median nerve motor $\mathrm{CV}$ (MCV) and distal latency (DL).

Results: Duplications (21 patients): MCV of the median nerve was between 9 and $28 \mathrm{~m} / \mathrm{sec}$ except for one patient with a CV of $33 \mathrm{~m} / \mathrm{sec}$, mean CV was 19,4. Median nerve DL was between 7 and 15,3 msec, mean DL was 9,5 msec. Cx32 mutations ( 6 patients): MCV of the median nerve was between 29 and $39 \mathrm{~m} / \mathrm{sec}$, mean CV was 33,2 m/sec. Median nerve DL was between 4,9. and 7,6 msec, mean DL was 5,8 msec. MPZ mutations (5 patients): MCV of the median nerve was between 3,5 and $20,3 \mathrm{~m} / \mathrm{sec}$, mean CV was 10,5 $\mathrm{m} / \mathrm{sec}$. Median nerve DL was between 6 and $11,2 \mathrm{msec}$, mean DL was 7,9 msec. Conduction blocks were seen in three patients. SNAPs were unobtainable in all of the patients with MPZ mutations while they were present in some patients with the other two defects. The parameters of other rare mutations were also noted.

Conclusions: This study suggests that NCSs may be useful not in predicting the mutation type, but rather in excluding some mutation types. Duplication is unlikely with a CV $>30 \mathrm{~m} / \mathrm{sec}$ and DL $<7 \mathrm{msec}$. Cx32 mutation is unlikely with a $\mathrm{CV}<30 \mathrm{~m} / \mathrm{sec}$ and $\mathrm{DL}>7 \mathrm{msec}$. These two genetic defects 
being the most common in demyelinating CMT, molecular studies can be carried out accordingly. The presence of conduction block suggests MPZ mutations. We cannot comment further on the NCSs in MPZ mutations since our group of patients with only demyelinating CMT does not include patients with the axonal form of this mutations.

\section{P258}

The effect of repetitive transcranial magnetic stimulation-induced proprioceptive deafferentation to ipsilateral and contralateral motor-evoked potentials

M.J. Kim, K.M. Lee, K.W. Lee

National Police Hospital (Seoul, KR); Seoul National University Hospital (Seoul, KR)

Objectives: It has been proposed that proprioceptive input can modulate neural excitability in both primary motor cortices (M1) simultaneously, although direct evidence for this is still lacking. Previous studies showed that proprioceptive accuracy of one hand is reduced after the application of one$\mathrm{Hz}$ repetitive transcranial magnetic stimulation (rTMS) for 15 minutes over the contralateral somatosensory cortex. The aim of this study was to investigate the effect of rTMS-induced central proprioceptive deafferentiation to excitability of both M1's as reflected in ipsilateral and contralateral motor evoked potentials (MEP)

Methods: Twelve consecutive MEPs of both abductor pollicis bravis were recorded using single-pulse TMS over right M1 in eleven healthy subjects. Immediately after one-Hz rTMS was applied for 15 minutes over the right somatosensory cortex, the MEP measurement was repeated. The proprioceptive function of the left thumb was assessed, before and after rTMS, using a position-matching task.

Results: There was an increase in ipsilateral MEP after the rTMS: whereas no MEPs were recorded on the ipsilateral hand before the rTMS, MEPs were recorded in both ipsilateral and contralateral hand in three of ten subjects. At the same time, the mean log peak-to-peak amplitude was reduced and the mean latency was prolonged in the contralateral MEP $(\mathrm{P}=0.005$ and $\mathrm{P}=0.016$ respectively). All except one subject showed increased error rates of position-matching task after rTMS.

Conclusion: RTMS-induced central proprioceptive deafferentiation reduces the MEP generation in the contralateral hand, and fascilitates that in the ipsilateral hand. This finding suggests that the excitability of M1 is under an inhibition by contralateral somatosensory cortex, and goes with the excitability of ipsilateral somatosensory cortex. Further study is well worthwhile for elucidating the neurophysiological mechanics of the inhibition and its clinical significance in conditions, such as writer's cramp and focal dystonias.

P259

Baclofen-loaded solid lipid nanoparticles administration in rats: pharmacokinetics and neurophysiological study

N. El Assawy, L. Priano, M. Gasco, A. Mauro

Istituto Auxologico Italiano Turin University (Turin, IT); Nanovector s.r.l. (Turin, IT)

Objective: Aim of our study was to evaluate pharmacokinetic profile and tissue distribution of baclofen (Bac) included in solid lipid nanoparticles (BacSLN) administered intraperitoneally (i.p.) in rats, and to correlate its effects upon the $\mathrm{H}$ reflex excitability curves and the behavioural alterations in rats.

Methods: Experiment was performed in healthy male Wistar rats, according to the following scheme: at 9 a.m. on different days, 3 groups composed by 4 rats each were injected IP with Bac-SLN at increasing dosages up to $10 \mathrm{mg} / \mathrm{kg}$ and, as control, with baclofen solution (Bac-SOL). $\mathrm{H}$ reflex response, behavioural characterization by means of a limb movements scoring scale (LS) and the Wilson Scale for sedation (WS), together with Bac detection in plasma, liver, brain and spinal cord were performed at scheduled times after treatment.

Result: After Bac-SLN and Bac-SOL injections, plasma and liver Bac concentration decreased after 1st hour while brain Bac concentration increased until 2nd hour. Peak Bac concentration were significantly higher for BacSLN. No detectable Bac concentration was found in spinal tissue for all the dose and time points tested.

After Bac-SLN H reflex amplitude was reduced up to abolition from 1st to 2 nd hour, then a rebound effect as an increased amplitude was found after 4th hour. Maximal effect of Bac-SLN on LS and WS was found from 1st to 2nd hour and from 1st to 4th hour respectively. On the contrary Bac-SOL showed significant $\mathrm{H}$ reflex reduction without successive rebound and effect on LS and WS only from 1st to 2nd hour.

Conclusion: Our study showed that Bac-SLN i.p. administration provided higher and more prolonged plasma and tissue Bac concentrations compared to Bac-SOL, correlating with $\mathrm{H}$ reflex response and behavioural scales. $\mathrm{H}$ reflex rebound effect and higher level of sedation of Bac-SLN treated rats compared to Bac-SOL could be the expression of a sustained cortical GABA B receptor activation.

\section{P260}

Does training induce changes in the event-related brain potentials in patients with impaired consciousness due to severe traumatic brain injury? N. Lapitskaya, J.F. Nielsen, A. Fuglsang-Frederiksen, J. Jakobsen

Hammel Neurorehabilitation and Research Centre (Hammel, DK); Aarhus University Hospital (Aarhus, DK)

Objectives: Early rehabilitation is important in severely brain-damaged patients. Clinical assessment of rehabilitation effects is insecure due to comprehensive severe disability. The challenge is to identify para-clinical prognostic markers for improvement. The aim of this study was to determine whether passive gait training induces changes in the event related brain potentials (ERP) in patients with severe traumatic brain injury (TBI).

Methods: Ten patients $43.4 \pm 18.4$ (19-70 years old) were enrolled in the prospective controlled open study. All patients suffered from severe TBI (Glasgow Coma Scale $3.9 \pm 1.3$ on admission to acute hospital) and had a functional level $2.3 \pm 0.5$ score according to Rancho Los Amigos Scale $76.9 \pm 23.9$ days after injury, indicating a severe impaired state of consciousness. Computer tomography showed diffuse axonal injury in 6 patients, and focal lesions with diffuse oedema in 4 patients. No surgery was performed in 4 patients, craniotomy in 4 patients, and application of drainage tube in 2 patients. ERP were recorded before and immediately after a single gait training session (17.1 \pm 1.3 minutes) in the robotic gait orthosis (Lokomat ${ }^{\circledR}$; weight upload $19.2 \pm 6.3 \mathrm{~kg}$; treadmill speed $1.5 \pm 0.1 \mathrm{~km} / \mathrm{h}$; distance $427.3 \pm 38.6 \mathrm{~m})$. We used a simple oddball ERP-paradigm $(85 \%$ frequent, $15 \%$ rare stimuli; 40 rare stimuli were averaged in each run; two runs per session were performed). Latency of the P300-component (largest positive wave after P200) was evaluated.

Results: P300-component could be elicited in $7(70 \%)$ patients. P300 latency tended to increase after a single training session in the gait robot (362 $\pm 28.9 \mathrm{~ms}$ before and $412 \pm 73.8 \mathrm{~ms}$ after training, $\mathrm{p}=0.06$ ).

Conclusion: Our preliminary results indicate that ERP may be a non-invasive method for measuring changes in brain potentials as a result of training. The tendency of the P300 latency to increase might be due to a fatiguing effect of gait training on bedridden patients with severe TBI.

\section{P261}

Effect of noise on the visual-evoked potentials

R. Sawaya, M. Kanso

American University of Beirut (Beirut, LB)

Objectives: The object of this research is to study the effect of meaningful noise on the latencies and amplitudes of the pattern reversal visual evoked potentials.

Methods: We studied 20 healthy subjects, not on any medication, aged between 20 and 47 years.

The subjects were placed in a dark silent room 1 meter in front of a checkerboard computer screen with a 60 degree pattern. Gold cup electrodes were pasted on their scalp at $\mathrm{Oz}(5 \mathrm{~cm}$ above inion: active electrode), $\mathrm{Fz}$ $(12 \mathrm{~cm}$ above the nasion: reference electrode) and the ground electrode on the forehead.

Pattern reversal occurred at a rate of $1.9 \mathrm{~Hz}$. The low frequency was $1 \mathrm{~Hz}$, the high frequency filter was $100 \mathrm{~Hz}$. The number of averaged sweeps was 100. Each eye was tested twice, with the other eye patched. The average latencies and amplitudes of the visual evoked responses were calculated for each eye separately.

The same procedure was performed with high volume meaningful noise produced by earphones placed in the subjects' ears from a portable radio.

Results: The latencies and amplitudes of the visual evoked potentials of 40 eyes were recorded at rest and upon stimulation with meaningful noise. The results were compared for each eye separately. The latencies at rest ranged between 82 and $127 \mathrm{~ms}$ with a mean of $109.3 \mathrm{~ms}$ and a SD of $8.9 \mathrm{~ms}$. The latencies upon noise stimulation ranged between 80 and $127 \mathrm{~ms}$ with a mean of $109.9 \mathrm{~ms}$ and a SD of $9.6 \mathrm{~ms}$.

The amplitudes at rest ranged between 3.8 and 18 microvolts with a mean of 8.1 microvolts and a SD of 3.5 microvolts. The amplitudes upon noise stimulation ranged between 3 and 17 microvolts with a mean of 7.9 microvolts and a SD of 3.2 microvolts.

Comparing the latencies for each eye between the state of rest and noise stimulation using the paired samples $\mathrm{T}$ test analyses did not reveal any statistically significant difference between the 2 groups with a $\mathrm{P}=0.311$.

Comparing the amplitudes for each eye between the state of rest and 
noise stimulation using the paired samples $\mathrm{T}$ test analyses did not reveal any statistically significant difference between the 2 groups with a $\mathrm{P}=0.56$.

Conclusion: We conclude from this study that there is no effect of noise stimulation on the latencies or amplitudes of the visual evoked responses performed in the classical manner described in the literature. Furthermore, any delay in the latency or drop in the amplitude beyond the limits defined in our results can not be attributed to a noisy environment.

P262

Visual- and motor-evoked potentials as predictors of long-term clinical disability in multiple sclerosis

R. Schlaeger, M. D`Souza, C. Schindler, L. Grize, L. Kappos, P. Fuhr

University Hospital Basel (Basel, CH); University Basel (Basel, CH)

Objectives: To prospectively investigate whether combined visual (VEP) and motor evoked potentials (MEP) allow prediction of longterm clinical disability in patients with MS.

Methods: VEP and MEP to upper and lower extremities and Expanded Disability Status Score (EDSS) were measured in 30 patients with relapsingremitting or secondary progressive MS at entry and after 6,12 and 24 months (1). EDSS was again assessed at year 14. Patients who were not able to come to our institute at year 14, were assessed by phone (2). The association between EP data and EDSS was measured using Spearman rank correlation. Multivariate linear regression was performed to predict EDSS at year 14 as a function of z-transformed EP-latencies at baseline. Patients received either no treatment or different immunomodulatory agents between month 25 and year 14, which was taken into account by including a factor "therapy" into the model. Validity of the model was assessed using cross-validation.

Results: Data was complete in 28 patients, one patient died prior to final investigation from a ruptured aortic aneurysma and the other was lost to follow-up. EDSS values at year 14 correlated with the sum of $z$-transformed EP latencies at baseline $(\mathrm{rho}=0.7031, \mathrm{p}<0.0001)$. EDSS as predicted by the formula $2.7818+1.2183 *$ Therapy $+0.0669 *$ Sum of $z$-scores at baseline correlated with the observed values $(\mathrm{rho}=0.7311)$.

Conclusion: Combined evoked potentials allow prediction of long term disability in small groups of patients with MS and warrant further study as cost and time effective predictive markers in clinical trials.

\section{References}

1. Fuhr P, et al. (2001) Visual and motor evoked potentials in the course of multiple sclerosis. Brain. 124(Pt 11):2162-2168

2. Lechner-Scott J, et al. (2003) Can the Expanded Disability Status Scale be assessed by telephone? Mult Scler 9(2):154-159.

\section{P263}

Reduced habituation to repeated nociceptive stimuli in patients with fibromyalgia

S. Álvarez Blanco, P. Schestatsky, J. Casanova-Molla, V. Ruiz, A. Collado, J. Valls-Solé

Hospital de Niños J.M. de los Ríos (Caracas, VE); Hospital Clinic (Barcelona, ES)

Introduction: Chronic pain may result from deffective descending inhibitory actions on afferent inputs. In fibromyalgia, reduced endogenous opioid activity may prevent normal inhibition of nociceptive inputs. Evoked potentials to nociceptive inputs show a decrease of amplitude with successive stimuli, and the same occurs with the reflex response of the sudomotor glands, i.e., the sympathetic skin response amplitude decreases with stimulus repetition. Therefore, we have examined the contact heat evoked potentials (CHEPs) and the sudomotor skin response (SSR) to contact heat stimuli to the dorsum of the hands in 20 patients with fibromyalgia, in comparison to 10 age-matched healthy volunteers.

Methods: Contact heat stimuli were delivered to the dorsum of the hand using a computerized thermal contact stimulator (Medoc, Israel). Peak temperature and rate of temperature rise were constant $\left(52{ }^{\circ} \mathrm{C}\right.$ at a speed of $70 \%$ s). We delivered series of 4 stimuli, separated by periods of at least 5 minutes rest. We recorded the evoked potential at $\mathrm{Cz}$ with reference to the earlobes with a bandpass between 0.1 and $50 \mathrm{~Hz}$, and the sudomotor skin response of the palm of the hand with a bandpass of 0.1 to $100 \mathrm{~Hz}$. We calculated the habituation index by dividing the amplitude of the CHEPs and SSRs obtained to the last stimulus of each series by those obtained to the first stimulus of the corresponding series.

Results: CHEPs and SSRs were induced in all healthy subjects and patients by all stimuli at the beginning of each series. The mean amplitude of the responses to the first stimulus of each series were 39,8+23,6 microV for the N2/P2 of the CHEPs and 2564,3+1287 microV for the SSR, and mean onset latencies were $410,7+56,2 \mathrm{~ms}$ for the N2 peak of the CHEPs and 1728,3
$+163,4 \mathrm{~ms}$ for the SSR. Response amplitude declined by the end of each series in all subjects. The habituation index was not different between groups regarding CHEPs ( 0.54 for fibromyalgia patients vs 0.49 for healthy subjects), but that of the SSR was significantly smaller in patients than in healthy subjects $(0.62$ vs $0.25 ; \mathrm{p}<0.01)$. The habituation index had a positive correlation with the time duratioin of chronic pain $(\mathrm{R} 2=0.49)$

Conclusions: Patients with fibromyalgia have an abnormal control of the SSR to nociceptive inputs.

\section{P264}

The effect of endotracheal suctioning on intracranial pressure in severe head injured patients admitted to a neurosurgical intensive care unit, Shiraz, Iran S. Gholamzadeh, M. Javadi, S. Rahnama

Shiraz University of Medical Sciences (Shiraz, IR)

Introduction: Endotracheal suctioning (ETS) is a routine nursing procedure used to decrease pulmonary complications; however, in severe head injured patients it can result in sudden increase in intracranial pressure (ICP) and may put the patients at risk for further cerebral damages. The purpose of this study was to examine the effect of ETS on ICP in severe head injured patients.

Methods: 21 patients with acute severe head injury (Glasgow coma score $\leq 8$, range 4-8) were studied. Each subject received 4 passes of insertion of standardized suction catheter and application of negative pressure limited to $10-15$ seconds in each procedure of suctioning. ETS procedure consisting of administration of 16 breathes at $135 \%$ of patients' tidal volume, $100 \%$ FIO2 before and after suctioning with standardized catheter (16 French) and duration between $10-15$ seconds. Repeated measure model for ANOVA were used to examine the changes in mean ICP at $1 \mathrm{~min}$ before, during first, 2nd, 3rd and 4th passes of catheter insertion.

Results: ICP significantly increased during suctioning $(P<0.001)$. Change in ICP significantly grater in 4 th pass of catheter insertion than other passes.

Conclusion: Changes in ICP induced by ETS in severe head injured patients are significant. Suction passes should be limited to $2-3$ per procedure. Repeated suctioning may increase ICP.

\section{P265}

Evoked potentials following passive finger movements are influenced by variation of stimulation parameters

T. Winkler, K. Bötzel

Ludwigs-Maximilians-Universität, Klinikum Großhadern (Munich, DE)

Objectives: Active or passive finger movements are known to initiate electroencephalographic (EEG) acitivity in the sensorimotor cortex representing proprioceptive processing. The influence of the eliciting movement speed, duration and range has not been analyzed systematically so far. We aimed to determine the particular influence of these parameters on amplitude and latency of the resulting proprioceptive evoked potentials (PEP).

Methods: Thirteen healthy subjects underwent repetitive proprioceptive stimulation by automated passive extension of the right middle finger. Variation of extension speed, angle and duration resulted in eight different movements, each repeated 200 times. The EEG raw data was recorded from the left hemisphere, using electrodes F3, Fz, C3, Cz, P3 and Pz according to the international 10-20 system. Data were averaged offline and characteristic components of the resulting PEP identified. Statistical analysis was performed with repated-measures ANOVA.

Results: In all subjects, three main components of the PEP could be identified: P1 $(45 \mathrm{~ms}, 2 \mu \mathrm{V}), \mathrm{P} 2(85 \mathrm{~ms}, 2.8 \mu \mathrm{V})$ and $\mathrm{N} 1(150 \mathrm{~ms},-1.8 \mu \mathrm{V})$, each with maximum amplitude in $\mathrm{P} 3-\mathrm{Fz}$, representing a dipole origin in the left sensorimotor cortex. No significant dependency on the PEP could be detected concerning variation in duration and angle of the eliciting movement. However, P1 $(\mathrm{p}<0.01)$ and P2 $(\mathrm{p}<0.05)$ were of significantly larger amplitudes in movements with higher speed of finger displacement.

Conclusions: These findings suggest that the velocity of the passive movement has a major influence on the resulting PEP in the sensorimotor cortex. Apparently, duration and angle of the eliciting movement have only negligible influence on evoked potentials and might be processed in a way not accessible for electrophysiological measuring. Characterization of PEP resulting from different physiological movements might be of value in achieving a more detailed understanding of central proprioceptive processing, required for reafferent functions in neuromotoric prostheses. 
P266

Skin biopsy and microneurography to evaluate the lesion site of dysautonomia

V. Donadio, P. Montagna, P. Cortelli, M. Elam, E. Bugiardini, R. Liguori University of Bologna (Bologna, IT); University of Gothenburg (Gothenburg, SE)

Objectives: The lesion site of dysautonomia is often difficult to establish but could be helpful to identify different types of dysautonomia. Recently we reported that skin biopsy associated with microneurography were effective in detecting the underlying sudomotor lesion site in patients with generalized anhidrosis. Here we extended this approach to other neurological disorders with dysautonomia in order to define the autonomic lesion site.

Methods: We have studied three patients with Multiple System Atrophy (MSA), four patients with Pure Autonomic Failure (PAF), three patients with Ross Syndrome (RS) and one patient with sweat gland dysplasia (SGD). In all patients a skin biopsy was taken and the autonomic and somatic skin innervation was evaluated by immunofluorescence analysis. Microneurography from the peroneal nerve was performed in all patients with the aim to analyse the skin sympathetic nerve activity (SSNA) and the muscle sympathetic nerve activity (MSNA). In addition, in the SGD patient, the histopathologic examination of sweat gland was made.

Results: In patients with MSA, immunofluorescence analysis showed a normal amount of both cholinergic and adrenergic sympathetic skin nerve fibers while sympathetic activity was not recorded during microneurography evaluation suggesting a pre-ganglionic sympathetic dysfunction. In patients with PAF and RS somatic and sympathetic skin innervation was poor and deranged at the immunofluorescence analysis and microneurography did not disclose sympathetic activity supporting a post-ganglionic disorders. In the patient with SGD immunofluorescence analysis showed abundant sympathetic skin nerve fibers and microneurography disclosed a normal MSNA and SSNA suggesting a primary lesion of sweat gland which was found at the microscopic histopathologic examination.

Conclusion: Skin biopsy associated with microneurography proved reliable diagnostic tools in detecting the possible site of the autonomic dysfunction in patients with dysautonomia.

\section{P267}

Effects of unilateral clenching and static handgrip to bilateral cerebral circulation

Y. Hasegawa, J. Sakagami, M. Zhang, K. Hori, T. Ono, Y. Maeda

Osaka University Graduate School of Dentistry (Suita-Shi, JP); The Fourth Military Medical University (Xian, CN)

Objective: Recently mastication induced activation in the cerebral circulation has been paid attention as a possible suppressor for the decline of brain function in the elderly. Bilateral activation in cerebral circulation in unilateral mastication was shown in our previous study. Clenching which is an isometric jaw movement differs a lot from mastication in the muscle contraction mode and strength. Therefore, we aimed to investigate the influence of unilateral clenching on the state of task-induced activation in cerebral circulation.

Methods: 12 healthy right handed male subjects with normal dentition and occlusion were enrolled. Bilateral middle cerebral artery velocities (MCAVs) as an index of cerebral circulation, heart rate and finger-tip arterial blood pressure were measured during unilateral isometric clenching and handgrip exercise. In unilateral clenching, subjects wear $1 \mathrm{~mm}$-thick splint on the working side of maxillary dentition. Electromyography (EMG) of bilateral masseter muscles (in clenching) and brachioradialis muscles (in handgrip) was recorded. Subjects were trained before experiment so that EMG activity of working side was $75 \%$ of maximal voluntary contraction (\% MVC), and then performed each task by two times. Each parameter during the task was compared with that before the task, and the difference between the bilateral MCAVs and that between the bilateral EMG activities were examined using the two-factor analysis of variance with the random effect model $(\mathrm{P}<0.05)$.

Results: During handgrip, the MCAV of non-working side was significantly higher than that of working side, showing the contralateral hemispheric dominance of cerebral circulation. Although there was no significant difference between bilateral MCAVs during clenching when all 24 tasks were analyzed, contralateral hemispheric dominance of cerebral circulation was observed in tasks where the EMG activity of the working side was higher than that of the non-working side. Heart rate and blood pressure during handgrip tasks showed higher than those of clenching tasks.

Conclusion: Isometric muscle contraction during clenching and handgrip did contribute to the promotion of the cerebral blood flow. Our results suggested that difference in bilateral masseter muscles activity might cause the difference in cerebral hemispheric circulation.

\section{Neurogenetics}

\section{P268}

Mutation analysis of the SPG11 gene in Italian patients with spastic paraplegia and thin corpus callosum

B. Castellotti, C. Mariotti, R. Fancellu, E. Salsano, A. Castucci, C. Gellera, D. Pareyson, F. Taroni

Fondazione IRCCS Istituto Neurologico C. Besta (Milan, IT)

Hereditary spastic paraplegias (HSP) are a heterogeneous group of neurodegenerative disorders characterized by progressive spasticity of lower limbs associated, in some cases, with other neurological signs. Autosomal recessive HSP (ARHSP) with thin corpus callosum (TCC) is a distinct form of familial spastic paraplegia linked to the SPG11 locus (15q13-15). Mutations (mostly null) in the SPG11 gene encoding spatacsin have ben identified in approx. $40 \%$ of ARHSP with TCC.

Objectives: To screen patients with ARHSP-TCC for spatacsin mutations.

Methods: We analysed 17 unrelated Italian patients by direct sequencing of the 40 SPG11 exons. All patients had lower limb spasticity associated with TCC documented at MRI.

Results: We identified mutations in 6 index patients. Two patients were from consanguineous parents. Patient 1 , born to healthy consanguineous parents, was homozygous for the novel frameshift mutation c.2278delC in exon 12; both parents were heterozygous for this mutation. Patient 2 and her affected sister were compound heterozygotes for the frameshift mutations c.1203delA (exon 6) and c.2843insG (exon 16). Patient 3, a sporadic case, was compound heterozygous for the novel missense variation K1012E (exon 16) and the novel splicing mutation IVS17-1G $>$ C. Patient 4 carried both the novel splicing mutation IVS1-2A $>C$ and the novel missense variation $\mathrm{N} 168 \mathrm{I}$ (exon 3). Patient 5, a sporadic case too, was compound heterozygous for the paternally-inherited W220X nonsense mutation in exon 3 and the maternally-inherited IVS39-2A $>$ G splicing mutation; genotype of this patient was complex, though, as the maternally-derived allele carried also 2 novel putative rare polymorphisms, E369K in exon 6 and A694T in exon 11. Finally, patient 6 , the only child born to consanguineous parents, carried the V2053M missense mutation in homozygous form. This variation has been previously reported in association with the homozygous c.733_734delAT frameshift mutation, but was not found in $>500$ chromosomes from Italian and French healthy controls (DelBo, Neurogenetics 2007).

Conclusions: We have identified 10 SPG11 mutations, 7 of which were novel. The majority of mutations are predicted to cause absence of the protein. SPG11 was found to be mutated in 35\% (6/17) of ARHSP-TCC Italian patients, which suggests genetic heterogeneity higher than previously reported.

Supported by an Italian Ministry of Health grant (RF Neurodegenerazione ex art 56) to FT

\section{P269}

Randomised placebo-controlled double-blind trial to assess safety and efficacy of erythropoietin in patients with Friedreich's ataxia

C. Mariotti, R. Fancellu, L. Nanetti, A. Solari, A. Martini, D. Di Bella, M. Plumari, G. Lauria, M.D. Cappellini, F. Taroni

Istituto Nazionale Neurologico C.Besta (Milan, IT); Fondazione IRCCS Policlinico, Mangiagalli, Regina Elena (Milan, IT)

Friedreich ataxia (FRDA) is a rare autosomal recessive neurodegenerative disorder caused by a mutation in the FXN gene, which encodes a protein named frataxin. As a result of the mutation, frataxin is quantitatively reduced but qualitatively normal. Thus, any pharmacological agent able to increase frataxin intracellular levels would have a great therapeutic relevance. Recent evidences have demonstrated the efficacy of recombinant human erythropoietin (rhuEPO) in increasing frataxin levels in cultured cells (Sturm B, et al. Eur J Clin Invest, 2005), and also in lymphocytes of FRDA patients receiving recombinant human erythropoietin subcutaneously (Boesch at al. Ann Neurol, 2007). We have started a monocentric, randomized, doubleblind, placebo-controlled, dose-finding study aimed at assessing the efficacy and the toxicity of rhuEPO in FRDA patients. In the protocol proposed, rhuEPO will be administered intravenously and frataxin levels will be determined in the patients' peripheral blood lymphocytes as the primary surrogate endpoint ("proof of principle"). Eighteen adult patients affected by FRDA have been randomly assigned to receive erythropoietin $(n=12)$ or matching placebo $(n=6)$. In order to establish the optimal dose and doseschedule, three consecutive cycles of treatment will be performed on the same patients. The treatment will be continued for at least one year. The primary efficacy endpoint will be the level of frataxin protein in peripheral lymphocytes. The secondary endpoints will include: (a) Scale for the As- 
sessment and Rating of Ataxia (SARA); (b) SF-36 Quality of Life rating scale: (c) Echocardiogram; (d) Skin biopsy: quantitative determination of sensory nerve fibre density. For Safety evaluation we will consider the incidence, severity and type of adverse events; changes in clinical laboratory findings (persistence, during the treatment, of hematological parameters not considered to be associated per se with an increased risk of thrombotic events).

The protocol has been approved by the local Ethical Committee. Preliminary data on screening visits and first dose administrations will be presented.

Supported by AIFA grant to FT

P270

Clinical, genetic and MRI findings in a patient affected by L-2- hydroxyglutaric aciduria

C. Ungaro, P.L. Lanza, F.L. Conforti, V. Blasi, T. Sprovieri, A. Mollo, O. Gallo, R. Mazzei

ISN National Research Council (Mangone, IT); ASP 4 (Cosenza, IT)

Objective: In our report, we describe clinical, genetic and neuroradiological features in an Italian patient with L-2-HGA, in whom L-2-HGA was diagnosed at an adult age.

Material and methods: Genomic DNA was extracted from peripheral blood according to standard procedures, after informed consent was given. PCR was performed with primers, specific for each exons comprising the intron-exon bounderies. The samples were sequenced in both forward and reverse directions on an ABI Prism3130XL genetic analyzer.

Results: Patient's MRI revealed an abnormal pattern with subcortical leukoencephalopathy sparing the deep white matter. The symptoms were represented by mental deficiency, severe cerebellar dysfunctions, mild pyramidal and extrapyramidal signs and progressive macrocephaly.

Molecular analysis showed a sequence variation in the exons 8 , highlighting the presence of a homozygous nucleotide variation at position c. 1082 (c. $1082 \mathrm{C}>\mathrm{T}$ ) that leads to substitution of a CGA codon encoding for an arginine residue with a TGA codon predicting for a stop codon at position 335 (Arg335Stop) in the protein.

Discussion and conclusion: L-2-Hydroxyglutaric aciduria (L-2-HGA) (OMIM \#236792) is an autosomal recessive inherited neurometabolic disease, generally observed in infancy or childhood, belonging to the group of organic acidurias, whose biochemical hallmark is represented by increased levels of 2-hydroxyglutaric acid in urine, blood and cerobrospinal fluid, with $90 \%$ of isoforms representing the L-form.

The gene causing L-2-HGA has been identified by Topçu et al.in 2004 and designed as L2HGDH gene. This gene, located on cromosome 14q22.1, encodes for a L-2-hydroxyglutarate dehydrogenase, a putative mitocondrial protein converting L-2-hydroxyglutarate to alpha-ketoglutarate. The mutations found in patients with this disease abolish the activity of this enzyme, therefore the symptoms of this affection are most likely due to the toxic effects of the accumulating metabolite L-2-hydroxyglutarate; in fact, L-2-hydroxyglutarate has been shown to induce oxidative stress and to inhibit mitochondrial creatine kinase in the cerebellum.

In the current study we report the first evidence of a mutation in the L2HGDH gene in an Italian patient affected by L-2-HGA, reinforcing the previously described phenotype of this rare metabolic disease and reaffirming the data that mutations in the L-2-Hydroxyglutaric dehydrogenase gene cause L-2-Hydroxyglutaric aciduria.

\section{P271}

Phenotype of leukoencephalopathy with brainstem and spinal cord involvement and lactate elevation: report of six cases

D. Pareyson, C. Marelli, M. Bugiani, M.R. Balestrini, F. Cardona, L. Farina, D. Testa, G.C. Scheper, M.S. van der Knaap, M. Zeviani, M. Savoiardo, G. Uziel C.Besta Neurological Institute (Milan, IT); La Sapienza University (Rome, IT); Vrije University Medical Center (Amsterdam, NL)

Objectives: To report clinical and radiological findings of six patients affected by genetically proven Leukoencephalopathy with Brainstem and Spinal cord involvement and Lactate elevation (LBSL). LBSL is a rare disease characterized by spasticity, ataxia, peculiar MRI signal abnormalities in brain, brainstem and spinal cord, and increased lactate in white matter (WM) at MR spectroscopy (MRS). Recently, LBSL has been associated with recessive mutations in the DARS2 gene coding for the mitochondrial aspartyl-tRNA synthetase (Scheper et al. (2007) Nat Genet 39:534-539).

Methods: In six patients ( 3 males, 3 females, aged 16-58 years; two sporadic cases and two couples of siblings), MRI showed features typical of LBSL. All patients underwent clinical, neurophysiological, imaging and genetic studies.
Results: Disease onset occurred in the first or second decade of life. However, two sisters had subtle symptoms in their teens but became clearly affected only after pregnancy in their twenties; another patient had disease onset after an infectious disease. All the patients developed progressive neurological deterioration characterized by pyramidal involvement with cerebellar and sensory ataxia. Other signs and symptoms were often evident: cognitive impairment ( $n=2$ patients), seizures $(n=2)$, bradykinesia or hyperkinesias $(n=3)$. Three patients had mild motor neuropathy and four had abnormal electroretinogram. All the patients presented the typical MRI pattern of signal abnormalities in the WM of the cerebral hemispheres, brainstem (characteristically affecting the corticospinal tracts and the intrapontine fibers of the fifth cranial nerve), cerebellum, and in the lateral and posterior cord columns. In three of four patients, MRS revealed increased lactate in the abnormal WM. All the patients were compound heterozygotes for missense or nonsense DARS2 mutations. Two out of three patients had decreased activity of respiratory chain complexes in muscle.

Conclusions: LBSL is characterised by spasticity, mixed ataxia, with variable presence of cognitive, extrapiramidal, peripheral nerve and retinal involvement. Age of onset is variable and sudden worsening may occur during trivial infections or after pregnancy.

\section{P272}

Clinical features of an adult-onset Leigh syndrome caused by the T9176C mutation in the mitochondrial DNA ATPase 6 gene

D. Ronchi, A. Bordoni, R. Virgilio, E. Fassone, A. Di Fonzo, M. Servida, M. Ronzoni, V. Lucchini, M. Matteoli, N. Bresolin, G.P. Comi

University of Milan (Milan, IT); Hospital of Garbagnate (Garbagnate, IT)

Objective: To describe the clinical features of a new family affected by the rare T9176C mutation of the mitochondrial DNA ATPase 6 gene.

Clinical data: The proband is a 22 year-old female patient. She was first seen at this age for the acute onset of diplopia, followed a week later by bilateral ptosis and respiratory distress. Trunk ataxia became evident in about one month. The neurological examination showed bilateral ptosis, internuclear ophthalmoplegia, gait ataxia, and dysmetria at finger to nose in the right side. Family history was positive: a brother died at age 8 years for autoptically confirmed Leigh Syndrome, the mother had short stature and mild mental retardation, a maternal aunt had mental retardation, a maternal cousin died at age 18 yrs and was reportedly affected by "spina bifida and hydrocephalus".

Proband's serum lactate was 57.4 (n.v.: $4.5-19.8 \mathrm{mg} / \mathrm{dl}$ ), CSF lactate: 70.4 EEG was disorganized and slow. Sequential MRI showed bilateral symmetric lesions at the pontine and mesencephalic tegmentum, followed by occipital subcortical lesions and diencephalic and bilateral dentate nuclei involvement.

Materials and methods: Muscle biopsy was analysed by standard methods. Mitochondrial DNA sequence analysis was evaluated in muscle and blood derived DNA.

Results: Cytochrome c oxidase and succinate dehydrogenase histochemical analysis of muscle tissue was normal. MtDNA sequence analysis showed the T9176C mutation within the ATPase 6 gene. RFLP analysis showed a homoplasmic state in proband's blood, muscle, myoblasts, fibroblasts and urinary epitelium. The proband's mother blood DNA carried the mutation in heteroplasmia.

Discussion: The T9176C mtDNA mutation has been reported in five families worldwide, for a total of 10 affected patients. Disease presentation is relatively homogeneous, being Leigh Syndrome in most of them. The age at disease presentation varies among the described patient and do not correlate with age at onset, suggesting that other environmental or genetic factors modulate disease phenotype.

\section{P273}

A novel case of V363I mutation in MAPT gene presenting with a language disorder of the progressive non-fluent aphasia type E. Salsano, G. Rossi, S. Di Donato, F. Tagliavini, D. Pareyson C.Besta Neurological Institute (Milan, IT)

Objective: To report the second case of V363I mutation in microtubule-associated protein tau (MAPT) gene presenting with a progressive non-fluent aphasia (PNFA)-type language disorder.

Case Report: A Sicilian 55-year-old right-handed woman gradually developed difficulties of speech production over a 1-year period. Family history was unremarkable. Neurological examination revealed hesitant, stuttering, slightly nonfluent speech together with mild bradykinesia, mainly on the right side. Neuropsychological tests demonstrated articulatory difficulties, slight reduction of verbal fluency, and tendency to speak using short sentences without grammatical "functor" words; mild oro-facial apraxia was 
also present. The patient and her husband noticed no other cognitive impairment, and social skills were preserved, although she was referred to be more irritable. Brain MRI demonstrated mild frontal atrophy of the left opercular region, and a cerebral SPECT showed inter-hemispheric asymmetry with decreased flow in the left fronto-temporal cortex. DNA analysis showed a missense mutation (V363I) in the MAPT gene, and the patient was diagnosed as frontotemporal dementia and parkinsonism linked to chromosome 17 associated with MAPT gene mutation (FTDP-17T) presenting with PNFA-type language disorder.

Conclusions: At variance with progranulin gene mutations, a language disorder of the PNFA-type has been only exceptionally described as the dominant presenting sign of FTDP-17T. Recently, Munoz et al. (2007) (Am J Alzheimers Dis Other Demen 22:294-299) have demonstrated in a Castilian 73-year-old woman that the novel mutation V363I in MAPT gene may be linked to late-onset, isolated PNFA. Here we confirmed in an unrelated case the association of the V363I mutation with an almost pure PNFA phenotype. Our patient however is younger than the previously described, implying that the correlation between the mutation and a late onset of disease is not clearly defined, as stated by Munoz et al. The penetrance of this mutation is also until now unclear and deserves further studies. Since the wide intra-mutation phenotypic variability characterizing the FTDP-17T, the genotype-phenotype correlation present in the V363I cases is, in our opinion, very interesting.

Supported by Italian Ministry of Health (PS/04/07)

P274

Molecular profile of autosomal recessive cerebellar ataxias in Tunisia G. El Euch-Fayache, Y. Bouhlal, R. Amouri, M. Zouari, M. Kefi, F. Hentati National Institute of Neurology (La Rabta, TN)

Objective: Autosomal recessive cerebellar ataxias (ARCA) are complex group of disorders with more than 18 genetic reported forms described. The genetic screening of Tunisian patients with clinical findings of ARCA is reported.

Methods: 286 Tunisian patients were selected from records of the National Institute of Neurology-Tunis-Tunisia and classified as ARCA. Blood samples for vitamin E and vitamin A tests and DNA were collected from all patients. 744delA mutation of TTPA gene causing ataxia with vitamin E deficiency (AVED) was carried out 124 patients displaying low vitamin $E$ rate and direct mutation screening for Friedreich ataxia (FRDA) gene was performed in the remaining 162 patients. A linkage study with microsatellites markers covering 14 already known loci of ARCA was performed in 72 patients belonging to families with more than two affected members and not linked to AVED or FRDA genes. Mutations analysis of the corresponding gene was performed in families showing linkage to one locus.

Results: $32 \%$ of patients $(n=90)$ were homozygous for the TTPA 744 delA mutation. This mutation was not found in $12 \%$ of the patients $(n=34)$ with low level of vitamin E suggesting the presence of other causing mutation in AVED in Tunisia. $1 \%$ of patients $(n=4)$ have both vitamin A and vitamin E deficiency and decrease of betalipoproteine. $18 \%$ of patients $(n=52)$ were homozygous for FRDA GAA pathological expansion. $7 \%$ of patients $(n=22)$ carried out autosomal recessive spastic ataxia of CharlevoixSaguenay (ARSACS) pathological mutations in the sacsin gene,13 showed oculomotor apraxia type 1 (AOA1) aprataxin gene mutations, and 37 have no linkage with known ARCA loci.

Conclusion: ARCA showed a particular molecular pattern in Tunisia. There is a high AVED frequency a treatable form which represents more than one third of all ARCA Patients. FRDA mutation represents the second encountered form and ARSACS is the third form of ARCA in Tunisia.

\section{P275}

SMN2 gene promoter methylation: implications for progression and therapy of spinal muscular atrophy

J. Hauke, B. Wirth, E. Hahnen

University of Cologne (Cologne, DE)

Spinal muscular atrophy (SMA) is an autosomal recessively inherited neuromuscular disorder. The disease determining survival motor neuron gene 1 (SMN1) is homozygously deleted in $\sim 96 \%$ of SMA patients. Within the SMA region on chromosome $5 \mathrm{q}$, the SMN genes exist in two almost identical copies (SMN1, SMN2) which are ubiquitously expressed and encode identical proteins. Since all SMA patients lacking SMN1 carry at least one SMN2 copy, transcriptional activation of the SMN2 gene is likely to be clinically beneficial. We have shown that several histone deacetylase inhibitors (HDACis) increase SMN2 expression in fibroblasts derived from SMA patients. Here, we show that the DNA demethylating agent 5-aza-2'-deoxycytidine increases SMN2 transcript- and protein levels in SMN1-deleted fi- broblasts. This finding indicates that both, histone acetylation and DNA methylation, regulate SMN2 gene activity. By DNA methylation analysis we identified a highly conserved SMN2 methylation pattern in fibroblasts and blood samples from SMA patients. Comparison of the methylation patterns of type I and type III SMA patients carrying 2 SMN2 copies revealed significant differences in cytosine methylation at 7 specific sites, suggesting that SMN2 promoter methylation modulates the disease severity. Two of the differentially methylated cytosines are located close to a transcriptional start silenced by DNA methylation. Strikingly, pan-HDACis such as SAHA and FK-228 are able to overcome SMN2 silencing by DNA methylation, whereas other isoenzyme selective HDACis such as MS-275 and VPA do not. We suggest that SMN2 promoter methylation modulates the disease severity which may in part explains the variable phenotypes in the presence of identical SMN2 copy numbers. Moreover, our analyses favour pan-HDACis such as SAHA and FK-228 for SMA therapy due to their propensity to counteract SMN2 gene silencing by DNA methylation. These results may improve the understanding of how the SMN2 gene expression is regulated and might have clinical implications for the SMA treatment with HDACis.

This work was supported by: Center for Molecular Medicine, University of Cologne, Families of SMA (FSMA) and Initiative Forschung und Therapie für SMA

\section{P276}

Translation of SOX10 $3 \mathrm{f}$ untranslated region causes a complex severe neurocristopathy PCWH by generation of a deleterious functional domain

K. Inoue, T. Ohyama, Y. Sakuragi, R. Yamamoto, N. Inoue, L-H. Yu, Y. Goto, M. Wegner, J.R. Lupski

National Center of Neurology \& Psychiatry (Tokyo, JP); Baylor College of Medicine (Houston, US); Erlangen University (Erlangen, DE)

Objectives: Peripheral demyelinating neuropathy, central dysmyelinating leukodystrophy, Waardenburg syndrome and Hirschsprung disease (PCWH) is a complex neurocristopathy caused by SOX10 mutations. Most PCWH-associated SOX10 mutations result in premature termination codons (PTCs), for which the molecular mechanism has recently been delineated. However the first mutation reported to cause PCWH was a disruption of the native stop codon that by conceptual translation extends the protein into the $3 \mathrm{f}$ untranslated region (3fUTR) for an additional 82 residues.

Methods: In this study, we sought to determine the currently unknown molecular pathology for the SOX10 extension mutation using in vitro functional assays.

Results: Despite the wild type SOX10 coding sequence remaining intact, the extension mutation led to severely diminished transcription and DNA binding activities. Nevertheless, it showed no dominant-negative interference with wild type SOX10 in vitro. Within the 82-amino acid tail, an 11 amino acid region (termed the WR domain) was responsible primarily for the deleterious properties of the extension. The WR domain, presumably forming an a-helix structure, inhibited SOX10 transcription activities if inserted in the carboxyl-terminal half of the protein. The WR domain can also affect other transcription factors with a graded effect when fused to the carboxyl termini, suggesting that it probably elicits a toxic functional activity.

Discussion: Together, molecular pathology for the SOX10 extension mutation is distinct from that of more common PTC mutations. Failure to properly terminate SOX10 translation causes the generation of a deleterious functional domain that occurs because of translation of the normal 3 ofUTR; the mutant fusion protein causes a severe neurological disease. Further in vivo assay, such as transgenic expression of mutant protein, will facilitate phenotypic consequence of this molecular pathology.

\section{P277}

Mutations in ATP7B gene on suspected Wilson's disease

M. Al Jumah, I. Al Abdulkareem, M. Al Blawi, R. Majumdar, I. Al Traif, A. Al Zaben, A. Al Jumah

King Abdullah International Medical R C (Riyadh, SA)

Objectives: To investigate the pattern of the ATP7B gene mutations in suspected Wilson's disease patients in Saudi Arabia.

Background: Wilson's disease (WD) is ubiquitous disease with great clinical and genetic heterogeneity. Several mutations of the ATP7B gene on chromosome 13 , the gene underlying WD, have been reported for many ethnic groups. Mutations in Arab and Saudi families have been reported in $56 \mathrm{pa}-$ tients with confirmed WD diagnosis by our group. In this study we report our finding in referred patient with suspected WD including our previously described patients.

Methods: One hundred and ten patients from different families with suspected WD by neurologist or gastroenterologist. Those patients are repre- 
senting different parts of Saudi Arabia and large Arab tribes. Clinical presentation and laboratory studies were diagnostic of WD in majority of those patients $(75 \%)$. In the $25 \%$ of the patients all test failed to confirm WD or any other suspected diagnosis. At least one family member of each family underwent mutation screening. Entire ATP7B coding sequence, including intron-exon boundaries were screened for mutation The one that did not show any mutations are still under going SSCP.

Results: Sixty eight patients (62\%) patients showed mutations in one or more exons of the ATP7B gene. Of the 68 patients $92.5 \%$ showed homozygous mutation and five patients $(6.5 \%)$ showed double heterozygous mutation in two or more different exons. ( $28 \%$ ) showed a frameshift mutation in exon 21 (4193delC) this is a novel mutation. One patient showed another novel missense mutation in exon 19 (1341 Gly à Ser). 24 patients $(35 \%)$ showed mutation in exon 3 . Nine patients $(17.6 \%)$ showed mutation in exon 8 (744 Ser à Pro). Eight patients (12\%) showed mutation in exon 12 and $6 \%$ showed mutation in exon 20. No mutation in the ATP7B gene was detected in the remaining 30 confirmed and 12 suspected patients with WD.

Conclusion: Genetic screening of the ATP7B gene detected disease causing mutations in $62 \%$ of Saudi suspected WD patients. Two of these disease causing mutations in exons 21 and 19 are novel. Limited ATP7B gene screening of six exons has $62 \%$ sensitivity to diagnose WD in Saudi population. Further research is needed to increase the diagnostic yield.

This study was supported by King Abdullah International Medical Research Center and King Abdul Aziz City for Science and Technology (KACST project AT 18-03)

\section{P278}

Cognitive deficits in Polish patients with spinocerebellar ataxia type 1 and type 2

M. Rakowicz, T. Jakubowska, E. Zdzienicka, M. Gugala, R. Rola, W. Szirkowiec, A. Sulek, R. Poniatowska, J. Zaremba Institute of Psychiatry and Neurology (Warsaw, PL)

Objectives: SCA1 and SCA2 belong to autosomal dominant, poliglutamine, neurodegenertaive, multisystem diseases. The atrophy of the cerebellum and lesion of its efferent and afferent connections lead to main symptoms expressed by ataxia, dysarthria and oculomotor abnormalities. There is evidence that the cerebellum also modulates cognition, particularly attention, memory and executive functions.

Aim: To determine the prevalence of neuropsychological deficits in molecularly confirmed SCA1 and SCA2 patients and to analyse the influence of CAG repeats, age at onset and disease duration on cognitive function.

Methods: 49 patients with SCA1 and 26 cases with SCA 2 were examined with a battery of neuropsychological tests to assess: general intellectual abilities using the Wechsler Intelligence Scale or Raven Progressive Matrices (IQ) and Mini- Mental State Examination (MMSE). Attention, memory and executive functions were evaluated using the Serial 7's, Rey Auditory Verbal Learning Test, Test of Associated Learning, Benton Visual Retention Test. The severity of ataxia was assessed with International Cooperative Ataxia Rating Scale (ICARS). Cerebellar and brain atrophy was confirmed by MR images.

Results: The mean age at disease onset in SCA1 was 33.0 $9.3 \mathrm{y}$ and in SCA2 $30.0 \pm 11.8$ and ICARS, $33.6 \pm 13.0$ and $40.5 \pm 20.6$ respectively, did not show statistical differences, while disease duration: $7.4 \pm 4.5$ and $11.5 \pm 7.6-$ differed significantly.The data analysis revealed that SCA1 and SCA2 patients did not differ in general intelligence tests. The performance in neuropsychological tests was on a higher level among SCA1 cases. Significant differences were found in general cognitive functioning $(\mathrm{p}<0.02)$, auditory learning $(\mathrm{p}<0.05)$, verbal recalling $(\mathrm{p}<0.001)$, associated learning $(p<0.002)$ and visuo-spatial recognition $(\mathrm{p}<0.001)$. The number of CAG repeats correlated negatively with IQ and ICARS was related to disease duration in both subtypes. In addition, disease duration was inversely correlated with all verbal memory tests only in SCA2 patients. Similarly, ICARS is negatively related to the vast majority of test used in SCA2 cases. MRI also revealed more pronounced atrophy of the cerebellum and frontal region in SCA2 $(\mathrm{p}<0.01)$, while in SCA1 cases, besides cerebellar atrophy, shrinkage of the pons was observed.

Conclusions: The impairment of cognitive function in SCA2 is related to disease duration and degree of disability, and is more severe than in SCA1.

\section{P279}

Epidemiology of spinocerebellar ataxia in Madrid (Area 5)

M.J. Aguilar-Amat Prior, M.J. Abenza, L. Gabaldon Torres, I. Pascual Pascual, J. Arpa Gutierrez

La Paz University Hospital (Madrid, ES)

Spinocerebellar ataxias are a heterogeneous group of disorders that displays marked geographical and ethnical variability.
We studied 214 patients with progressive cerebellar ataxia thath had been send to our centre between 1994 and December 31st 2006. All patients had clinical, hematological, neurofisiological (otoneurology, EMG+TCC, EPMM), neurorradiologic and genetical studies. Patients were classificated genotipically and fenotipically and the prevalence of the differents ataxic disorders in our area were calculated, we also calculate global prevalence.

We stimated the prevalence of ADCA(Autosomal dominant cerebellar ataxia) in our area in 1,7369 cases per 100.000 habitants, (SCA7 15\%, SCA3 $8 \%$ and remain unknown $77 \%$ ); in 4,4092 cases per 100.000 habitants in the case of ARCA,Autosomal recessive cerebellar ataxia, (1,7369 in FRDA; 0,5344 in ATM) and 6 cases per 100.000 habitants in the case of ILOCA(Idiopathic Late Onset Cerebellar Ataxia); and global prevalence of 11,2235 cases per 100.000 habitants.

Prevalence of ADCA in our area is upper than previous studies. We want to emphasize the absence of SCA1 nor SCA2. Prevalence of FRDA is very similar of shown in other studies in Europe and North Africa. Prevalence of ILOCA is superior that found in previous studies in Spain.

\section{P280}

Alpers syndrome in a young adult with two POLG1 mutations

N.A. Visser, K.P.J. Braun, W.M. van den Bergh, F.S.S. Leijten, C.R.B. Willems, L. Ramos Pereira, J.H.J. Wokke

University Medical Center Utrecht (Utrecht, NL); Wilhelmina Children's Hospital (Utrecht, NL)

Introduction: Polymerase-gamma (POLG1) is essential for mitochondrial DNA replication. Mutations in the POLG1 gene cause a wide variety of phenotypes, one of which is Alpers syndrome. Most patients with Alpers syndrome present in early childhood. Typical clinical features are intractable seizures, episodic psychomotor regression and cortical blindness. Hepatic failure occurs frequently after treatment with valproic acid.

Case report: A previously healthy 20 -year-old woman was admitted after two generalized tonic clonic seizures. After the first seizure, two weeks before admission she complained of seeing black spots in both visual fields. Neurological examination was normal. Brain MRI showed two small hyperintense lesions in both occipital lobes. EEG showed slowed background activity and continuous bilateral occipital irritative activity that was most prominent in the left hemisphere. In a few weeks, she developed refractory focal epileptic seizures and cortical blindness, mental deterioration occurred also. Repeated brain MRI showed an increase of the occipital lesions and newly developed parietal and thalamic lesions. We considered a diagnosis of Alpers syndrome, mitochondrial encephalopathy lactate acidosis and stroke like episodes, cerebral vasculitis, Rasmussen encephalitis and Hashimoto's encephalopathy. DNA analysis showed the homozygous mutation $1399 \mathrm{G}>\mathrm{A}$ (A467T) in both exons 7 of the polymerase-gamma gene. She developed a generalized epileptic status that did not respond to a combination of anti-epileptic drugs. About eight weeks after the initial symptoms she died of multi-organ failure.

Conclusion: This 20-year-old woman developed a late onset POLG1 mutation phenotype that was characterized by an aggressive encephalopathy with epilepsy and cortical blindness resembling Alpers syndrome. However, Alpers syndrome usually presents in early childhood. Other interesting findings are the hyperintense contrast enhancing MRI lesions that progressed over time. The severity of this phenotype could not be fully explained by the coexisting 1399G >A (A467T) mutations, which often induce a milder phenotype compared with compound heterozygous mutations. In addition, rapid progression is uncommon in late onset POLG1 phenotypes. An unidentified external factor is likely to have initiated the cascade of deleterious events. This case report further expands the spectrum of Alpers syndrome and POLG1 mutations.

\section{P281}

A combined approach in the diagnosis of CADASIL: molecular analysis of selected NOTCH3 gene exons and morphological evaluation of skin biopsy R. Fancellu, M. Morbin, S. Baratta, D. Testa, D. Pareyson, L. Chiapparini, O. Bugiani, C. Mariotti, F. Taroni

Fondazione IRCCS Ist Neurologico C.Besta (Milan, IT)

Objectives: CADASIL is a hereditary cerebrovascular disease associated with mutations in the NOTCH3 gene. The disease is characterized by adult onset multiinfarct encephalopathy with headache, multiple stroke and dementia. The pathologic hallmark is the presence of granular osmiophilic material (GOM) in the basal lamina of arterioles that can be identified in peripheral biopsies, but the false-negative rate of this finding is unknown. The aim of the study was the search of the most appropriate procedure for the diagnosis of CADASIL. Ultimate diagnosis is based on DNA analysis and available evidences indicate that approx. $75 \%$ of mutations cluster in exons 3 and 4 . 
However since molecular analysis of all the 33 exons cannot be offered as a routine clinical service, other complementary methods, such as skin biopsy, should be considered for identifying the remaining cases.

Methods: We sequenced exons 3, 4,11, and 19 of 27 index patients with clinical and MRI findings consistent with the diagnosis of CADASIL. In 9 out of 19 cases in whom we had identified mutations in the NOTCH3 gene, we have also performed open skin biopsy.

Results: We identified NOTCH3 mutations in 19 patients. GOM was detected by electron microscopy (EM) in all such patients, who had distinct mutations involving an Arg-to-Cys substitution (2 in ex 3, 3 in ex 4, 1 in ex 2, 1 in ex 8, 1 in ex 11, 1 in ex 19).

Conclusions: The clinical suspect of CADASIL should be first screened for mutations in NOTCH3 exons 3 and 4. If negative, an open skin biopsy with EM analysis for GOM should be carried out; if this is positive, sequencing of the remaining NOTCH3 exons is strongly recommended. (Partly supported by AIFA grant FARM659PTX to FT)

\section{P282}

A review of patients referred to a newly established neurogenetics clinic R. Roxburgh, D. Kerr

Auckland City Hospital (Auckland, NZ)

Objectives: To review the sources of referral, casemix, diagnoses and method of diagnosis in a newly established neurogenetics clinic. The benefits of participating in multidisciplinary cardiorespiratory and rehab clinics was assessed by comparing the numbers of referrals made to these services in a combined setting compared with individual referrals. Particiaption in clinical trials/research was recorded.

Methods: All patients seen in the Auckland City Hospital Neurogenetics Clinic had information regarding these parameters recorded prospectively. Results: Over time patients referral to the clinic became more specific with just over half being referred specifically to the neurogenetics clinic in the first year and over $80 \%$ by the fifth year. The commonest source of referral were other neurologists and geneticists. The largest single diagnosis was Huntington's Disease (HD) (86) and muscle diseases (56) the commonest of which was myotonic dystrophy (21). Other disease groups included phakomatoses (29), inherited neuropathies (27), ataxia (23), mitochondrial (21) and hereditary spastic paraparesis (18). Case histories of patients with paraplegin HSP, late onset Friedreich's ataxia, Very long chain fatty acyl coA dehyrogenase deficiency and POLG1 autosomal recessive mutations giving rise to mitochondrial disease are given to illustrate the diversity of condition even in this subspecialty clinic.

Method of diagnoses vary betweeen diseases with the vast majority of HD patients having a genetic diagnosis whereas neurofibromatosis patients only one proceeding to genetic diagnosis. 42 of 143 diagnostic genetic tests yeilded a positive genetic diagnosis. 135 referrals were made to rehabillation services. 32 of these were made to a combined assessment clinic attended by physiotherapist, occupational therapist, speech and language therapist and clinical psychologist. 116 referrals were made to other specialties and 24 of these were seen in combined cardiorespiratory neurogenetics clinics. 51 of the 86 patients with HD are participating in research. Just 3 other patients are involved.

Conclusion: Seeing patients with genetic diagnoses in a dedicated clinic permits rare disorders to be diagnosed and clear treatment programs to be established. Genetic testing is expensive so it is important to become familiar with these conditions to order appropriate tests. To take part in research, countries with small populations need to take part in international collaborations.

\section{P283}

Mitochondrial DNA G8363A mutation in the tRNA Lys gene: clinical features of a new family

R. Virgilio, D. Ronchi, A. Bordoni, E. Fassone, M. Moggio, S. Bonato, G. Conti, C. Donadoni, L. Barbetta, G. Torgano, S. Corti, N. Bresolin, G.P. Comi University of Milan (Milan, IT)

Objectives: To define the clinical presentation in a family carrying a very rare pathogenic mitochondrial DNA (mtDNA) point mutation within the tRNALys gene.

Methods: We investigated two heterozygous twin sisters affected by mental retardation, neural deafness, hypotonia and myoclonic epilepsy and ataxia, reviewing all their clinical records, brain imaging and neurophysiological data, as well as any relevant clinical information of all the maternallyrelated pedigree members (n: 8 ). In one of the twins, morphologic, biochemical, and genetic analysis of muscle biopsy was performed.

Results: Two 30 year-old female twins were born premature, and were shown to have mental retardation. At age 7 years old, a sensory-neural deaf- ness was recognized and at 20 yeras of age myoclonic epilepsy. One of them had migraine attacks, both reported fatigue. B Neurological examination was similar features: in both sisters:hearing loss, hypotonia with absent deep tendon reflexes, limb girdle muscle weakness and ataxia. At 30 years, one of the two sisters developed a pneumonia complicated by a sepsis, needing invasive ventilation and percutaneous endoscopic gastrostomy. Their mother had mild mental retardation and the maternal uncle had neural deafness. Both sisters and her mother had horse collar lipomas. The proband's muscle biopsy showed a severe cytochrome c oxidase (COX) deficiency and raggedred fibers (RRF). A G8363A mutation in the mtDNA tRNALys gene was found by sequence analysis. The mutation was almost homoplasmic in muscle and blood from the twins and heteroplasmic in symptomatic maternal relatives.

Conclusion: The G8363A mutation has been so far described as pathogenic in five indipendent families presenting with variable phenotypes ranging from MERRF-like syndrome, to cardiomyoapthy or Leigh syndrome. Though the pathogenicity of the G8363A mutation, due to a change in the conformation of the tRNA that severely impairs aminoacylation, is well established, counselling is a difficult task in individual cases reported in few patients. This pedigree contributes further data on the clinical spectrum of G8363A tRNALys mtDNA mutation.

\section{P284}

Survivin is differentially expressed and spliced during the course of regeneration of damaged sciatic nerve in mice

S. Amiri, M. Movahedin, Z. Hajebrahimi, S.J. Mowla

Tarbiat Modares University (Tehran, IR)

Object: Survivin, a unique member of the inhibitor of apoptosis (IAP) protein family, is expressed during embryonal development but is undetectable in terminally differentiated cells and tissues. In addition to inhibiting apoptosis, survivin is also essential for proper cell division. Differential splicing of survivin pre-mRNA generates at least five survivin variants: survivin, survivin-delta-Ex3, survivin-2B, survivin-3B and survivin-2a in human and three variants of: survivin 140 , survivin 40 and survivin 121 in mice.

Because of the vital role of survivin in cellular proliferation and apoptotic cell death, and also the necessity of the treatment of the nervous system injuries, we have monitored survivin gene expression changes during the course of regeneration in injured sciatic nerves.

Methods: We used adult male NMRI mice as a model and after anesthetizing the animals, the right Sciatic nerve was transected and at the indicated times $(3,6,12,24,48,96$ and 144 hours $)$ the animals were sacrificed and both distal and proximal segments of the transected Sciatic nerve, intact left Sciatic nerve and L4-L6 segments of spinal cord were dissected. The total RNA was extracted from each sample and semi-quantitative RT-PCR with specific primers for survivin and also beta-microglobulin gene, as an internal control, was performed. To determine cellular distribution of survivin protein, 6 days (144 hours) after the axotomy, survivin protein expression was also evaluated by specific antibody of survivin using immunohistochemistry technique.

Results: We demonstrated the expression of both survivin 140 and survivin40 in distal and proximal segments of sciatic nerve with different intensity, where the expression of survivin 140 was higher than survivin40. In spinal cord segments, only survivin 140 expression was detected. Also, both the nuclear and cytoplasmic distribution of survivin protein was observed. In contrast, survivin protein has not been detected in either distal or proximal segments of Sciatic nerve.

Conclusion: Our data suggest that survivin is differentially expressed and spliced during the course of regeneration in damaged nerve and spinal cord. The data also suggest that manipulation of expression and/or splicing of survivin could potentially affect the process of regeneration in nerve and/or spinal cord injuries.

This work was supported by a grant from the Tarbiat Modares UniversityTehran-Iran

\section{P285}

Molecular analysis of APTX and SETX genes in families with ataxia with oculomotor apraxia in Saudi Arabia

S. Bohlega, L. Al Sharif, T. Alkhairallah, B. Al Mubarak, D. Bakheet, N. Al Tassan

King Faisal Specialist Hospital (Riyadh, SA)

Objective: Ataxia with Oculomotor Apraxia (AOA) is a rare autosomal recessive disorder with two subtypes. AOA1 presents with cerebellar ataxia and oculomotor apraxia between ages 2 and 18 years of age and is accompanied later in life by sensory-motor neuropathy which maybe associated with choreathetosis or mental retardation, hypoalbuminemia, hypercholes- 
terolemia and normal immuno-globins and alpha-fetoprotein levels. Patients with AOA2 present with gait ataxia, cerebellar atrophy, sensory-motor neuropathy, ocular-motor aparaxia and elevated immuno-globins and alpha-fetoprotein levels with a alter age of onset (10-22 years). Although the two forms are not quite distinctive phenotypically, two different genes on chromosome 9 have been linked to AOA. Mutations in the APTX gene have been reported in AOA1 patients and mutations in SETX gene have been found in several AOA2 families.

Method: Four Saudi families with AOA1 and AOA2 phenotypes ( 2 or more affected individuals) have been identified. Comprehensive screening for the whole open reading frame (ORF) of APTX and SETX genes have been performed.

Results: Several common and novel variants have been identified. Additionally, a novel truncating mutation (c.6859 C > T, R2287X) in the SETX gene was identified as the disease causing mutation in one family. This mutation was typed in all family members to identify affected and carrier status as part of the diagnostic service of the project. While the other families with clear diagnosis have been negative for mutations in these genes indicating the genetics diversity of this disorder and implicating on the role of more genes in the development of this rare disorder. Linkage analysis to identify these genes is required.

Conclusion: One of our families carried a novel truncation in the SETX gene, and the other linkage analyses may lead to a different responsible gene(s).

\section{P286}

Transplanted neural stem cell-derived motor neurons improve SMARD1 disease phenotype

S. Corti, M. Nardini, M. Nizzardo, C. Donadoni, S. Salani, F. Fortunato, R. Del Bo, D. Papadimitriou, F. Locatelli, S. Strazzer, N. Bresolin, G.P. Comi

University of Milan (Milan, IT)

Objective: We explored the therapeutic potential of neural stem cell-derived motor neurons into a Spinal Muscular Atrophy with Respiratory Distress type 1 (SMARD1) model (nmd mice). SMARD1 is an autosomal recessive form of infantile motor neuron disease due to mutation in the IGHMBP2 gene. Cell replacement therapy could be a possible therapy for motoneuron diseases including SMARD1.

Methods: We isolated a neural stem cell poluation (NSCs) from murine spinal cord of a transgenic mouse expressing GFP only in motor neurons (HB9-GFP) by immunomagnetical positive selection for the stem cell marker LewisX ( $\mathrm{Le}+)$. These cells were expanded as adherent monolayer and differentiated into motor neurons with a cocktail of growth factors including sonic hedgehog (Shh) and retinoic acid (RA). Differentiated motor neurons were purified by immunomagnetic selection for p75NTR antigen and grafted into spinal cord in nmd mice, an animal model of SMARD1. We assessed the long-term survival of transplanted motor neurons examining their integration with host tissues by immunohistochemistry, and confocal microscopy. Transplanted mice were evaluated for their motor performance using an accelerating rotarod device and survival was recorded.

Results: In vitro, $\mathrm{Le}+\mathrm{NSCs}$ derived motor neurons were positive for $\mathrm{Hb} 9$ GFP as well as for neuronal and motor neuronal markers. They extended long axons and formed neuromuscular junctions with skeletal myotubes. After transplantation of purified MNs in nmd spinal cord, numerous Hb-9GFP+ MNs were readily identified in the host anterior horns. These cells showed colocalization with neuronal and motor neuronal markers (choline acetyltransferase). Furthermore, donor cell-derived motor axons were observed within the ventral roots of transplanted animals. Treates nmd mice presented an improved phenotype with delayed disease progression and increased life span.

Conclusions: Our data demonstrate that transplantation of purified motor neurons has a positive effect on the SMARD1 disease phenotype and open new possibilities for the development of cell therapy in patients with SMARD1 and other motor neuron disease.

\section{P287}

Molecular analysis of neurological disorders characterised by gene dosage alterations

S. Illarioshkin, P. Slominsky, M. Shadrina, S. Klyushnikov, E. Karpova, N. Miklina, N. Savitskaya, S. Limborska, I. Ivanova-Smolenskaya

Research Center of Neurology (Moscow, RU); Institute of Molecular Genetics (Moscow, RU)

Objectives: To assess the reliability and reproducibility of quantitative realtime polymerase chain reaction (PCR) for analysis of gene dosage in Parkinson's disease (PD), diffuse Lewy body disease (DLBD) and hereditary de- myelinating neuropathies, and to establish respective phenotype-genotype correlations.

Methods: The following cohorts of patients were examined: 1) 359 patients with PD, including 345 sporadic and 14 familial cases; 2) 8 patients with sporadic DLBD; 3) 50 patients with demyelinating neuropathies, including 20 patients with hereditary motor and sensory neuropathy type $1 \mathrm{~A}$ (HMSN1A), 4 patients with hereditary neuropathy with liability to pressure palsies (HNPP) and 26 with sporadic 'compression neuropathy' of unknown origin. Alpha-synuclein, parkin and PMP22 gene exons were analyzed quantitatively using a real-time TaqMan PCR system, which allows evaluating the copy number of exons by comparing the intensity of amplification signals from a gene under study with that from the beta-globin gene.

Results: We specially designed the real-time PCR method based on TaqMan chemistry for the analysis of genes involved in PD, DLBD and hereditary demyelinating neuropathies; it has been shown that deletions or multiplications of these genes are relatively frequent. Using this method, we identified heterozygous rearrangements (deletions or duplications) of the parkin gene exons in $5.8 \%$ cases of $\mathrm{PD}$ (including $10.7 \%$ patients with an early form of PD and $1.7 \%$ patients with a late form of the disease). No alpha-synuclein gene dosage alterations were identified in PD or DLBD patients. We confirmed the duplication or deletion of the PMP gene in HMSN1A and HNPP patients, respectively, and found the PMP22 deletion in $7.7 \%$ of patients with sporadic 'compression neuropathy' (two patients out of 26). No correlation was found between the location of parkin gene deletions/duplications and clinical findings in patients with PD.

Conclusion: Gene dosage alterations represent one of the universal mutational mechanisms characteristic of hereditary neurodegenerative disorders, and reliable DNA testing is important for clinical practice. To our opinion, the real-time TaqMan PCR method have several advantages over other techniques, namely: speed, accuracy of the exon dosage analysis and relatively low cost.

Acknowledgements: This study was supported by the Russian Foundation for Basic Research (grant \# 07-04-01046).

\section{P288}

Whole mitochondrial genome analysis in 64 Korean patients with mitochondrial disorders

S.Y. Cho, S.J. Hwang, J.H. Hwang, K.W. Chung, E.M. Cho, H.R. Yang, J.Y. Kwon, J.S. Shin, J.Y. Yoon, B.O. Choi

Ewha Womans University (Seoul, KR); Kongju National University (Gongju, $\mathrm{KR})$

Objectives: Mitochondrial disorders can be caused by point mutations or large deletions in mitochondrial DNA (mtDNA). About 200 different pathogenic mtDNA mutations have been described since 1988. However, genetic defects of Korean mitochondrial patients were not much analyzed; therefore, this study was performed to identify causative mtDNA mutations in patients of Korean origin.

Methods: We enrolled 64 unrelated Korean patients having mitochondrial disorders, and classified as MELAS ( 25 cases), MERRF ( 5 cases), LHON (2 cases), Leigh ( 3 cases), KSS (10 cases), CPEO (12 cases) and uncertain patients ( 7 cases). In all patients, we have analyzed the whole sequence of mtDNA.

Results: We identified 18 point mutations ( 9 known and 9 novel mutations) and 4 large deletions (1 known and 3 novel deletions) from 30 patients. We found 9 known point mutations from 20 patients. The A3243G mutation in tRNAeu^ $\wedge$ Leu was predominantly observed from 9 MELAS patients, and followed by A8344G mutation in tRNA^Lys from 4 MERRF patients. Others were A856G (12S rRNA) in Leigh patient, T3271C (tRNALeu), T9957C (Phe251Leu, CO3) and T10191C (Ser45Pro, ND3) in each MELAS patient, G5979A (CO1) in CPEO patient, G8363A (tRNA^Lys) in an uncertain patient, and G11778A (Arg340His, ND4) in a LHON patient. In addition, we were able to identify 9 unreported mutations in the MITOMAP database (http://mitomap.org) from 8 patients, and these mutations were not found in 200 controls. Four mutations (A2294G, A3145G, T5553G and C15891T) were distributed in rRNA and tRNA region, and other six mutations (G7119A, T9187C, C9717T, T11204C, C13438T and A13849C) were distributed in coding region. Of them, the A13849C (Asn505His) in ND5 was found in the MT48 MELAS patient who also harbors T9957C (Phe251Leu) in CO3. The T11204C (Phe149Leu) in ND4 was found in the MT64 LHON patient who also harbors G11778A in the same gene. From the large deletion screen, the common deletion (4977bp) was found from three patients (KSS). One CPEO patient showed multiple deletions (5112bp and 9024bp).

Conclusion: We report the whole mitochondrial genome analysis in 64 Korean patients with mitochondrial disorders. Our findings emphasize that the whole analysis of mtDNA is worthwhile in the molecular diagnosis evaluation of patients with clinically suspected mitochondrial disorders. 


\section{Child neurology}

P289

Multiple sclerosis - inaugural signs in teenagers

A. Dumea, C. Iliescu, S. Magureanu

Alexandru Obregia Clinical Hospital (Bucharest, RO)

Multiple sclerosis is the most common demyelinating disorder of the central nervous system of the young adult but the onset before 18 years is often over look.

Objective: Our paper aim is the exemplification of different types of onset in multiple sclerosis in the case of 4 female teenage patients which were admitted in our clinic.

Methods: The results of investigations and the clinical manifestation established the multiple sclerosis diagnosis In order to establish the MS diagnosis a series of lab investigations were executed: lumbar puncture, visual(VEP) and auditory evoked potentials (AEP), cerebral MRI.

Results: The patients were admitted in our clinic for unilateral loss of vision $(n=1)$, paresthesia $(n=2)$, muscular weakness $(n=1)$, balance problems, dizziness, ataxic gait, tremor $(\mathrm{n}=1)$.

Lumbar puncture showed pleiocitosis CSF $(n=2)$, normal protein content and the absence of oligoclonal bands. There were changes in the P100 latency of the visual evoked potential (VEP). Also, the auditive evoked potentials (AEP) were modified.

Cerebral IRM showed multiple demyelinating oval lesions in bilateral periventricular white matter.

Conclusions: The various inaugural sign in multiple sclerosis (loss of vision, diplopia, paresthesia, facial pain, muscular weekness, gait and balance problems, bladder, bowel disorders) determine a correct and careful differential diagnosis, implying an interdisciplinary approach. In case of paresthesia the diagnosis of multiple sclerosis should be taken into consideration.

\section{P290}

\section{Update on management of neonatal seizures}

A. Mahmoud

K.F.M.C. (Riyadh, SA)

Seizures in neonates are relatively common, with variable clinical manifestations. Their presence is often the first sign of neurological dysfunction, and they are powerful predictors of long-term cognitive and developmental impairment. Most neonatal seizures occur over only a few days, and fewer than half of affected infants develop seizures later in life. Such neonatal seizures could be considered acute reactive (acute symptomatic), thus the term "neonatal epilepsy" is not used to describe neonatal seizures. Aim of treatment of neonatal seizures may be variable; but usually it is aimed at ruling out immediately treatable causes, decreasing risk of seizure induced C.N.S. injury as well as decreasing detrimental effects of seizures on other systems.

Here we critically review literature on treatment of neonatal seizures in order to generate established guidelines for anticonvulsant use in neonatal seizures (Birth-1 month of corrected age). We went through relevant trials: 1966-Present, review articles: 1980-Present, Medline, Cochrane, hand search. We selected all R.C.Ts, Cohort Series, Occasional Case reports and Case Series.

We documented the study design, randomization, strategy for allocation concealment, blinding, source and characteristics of subject, number of subjects, treatment details and side effects, duration of the study, and outcome measures. We applied Thomson Criteria for level of evidence and we postulated grading of recommendations. 600 articles and review articles were found under the title: management of neonatal seizures. Search was limited to human, to English Language and to newborns. Manual search was also conducted for age between one and three months. Search was conducted for specific drugs with neonatal seizures as well. 45 original articles and 17 review articles were chosen for analysis. At the end we declared the pitfalls in using the drugs that proved to be not effective. We explained why it is becoming important to change the attitude of using the same conventional drugs.

We threw light on the use of new antiepileptic drugs and suggested guidelines for management of neonatal seizures.
P291

Addison's disease as the first manifestation of X- linked adrenoleukodystrophy

A. Potic, V. Milic Rasic, J. Mladenovic, B. Potic, D. Kozic

Medical Faculty University of Belgrade (Belgrade, RS); Clinical Centre

"Zvezdara" (Belgrade, RS); Institute for Oncology and Radiology "Sremska

Kamenica" (Novi Sad, RS)

Introduction: X- linked adrenoleukodystrophy (X-ALD) is an inherited metabolic disease characterized by the impaired peroxisomal very- longchain fatty acids (VLCFA) oxidation and the mutation in the ABCD1 gene. The pathological process affects central and peripheral myelin, adrenal gland and testicular tissue

Objective: We report an adult male with Addison's disease who presented with the neurological symptoms corresponding to adrenomyeloneuropathy (AMN) in the fifth decade of life. Our aim is to point out the role of AMN disclosure among the patients with idiopathic Addison's disease

Methods: A 43-year-old male patient was referred to the neurologists for the slowly progressive symptoms that had appeared four years earlier. The patient had gait difficulties, stiffness and aching in the legs. He had been treated for the Addison's disease since the age of nine. The AMN became suspected

Results: Neurological examination: spastic paraparesis, sensory disturbance in the legs, absent abdominal reflexes, preserved sphincter functions; Electromyoneurography (EMNG):mild axonal neuropathy in the lower extremities; Somatosensory evoked potentials (SSEP) recorded from the median nerve: affection of both peripheral and central sensory pathways; Spinal magnetic resonance imaging (MRI): increased T2 weighted (T2W) signal in the cervical spine predominantly affecting the posterior columns; Brain MRI: T2W hyperintensity within the splenium of the corpus callosum, absent perilesional contrast enhancement; Brain magnetic resonance spectroscopy (MRS): increase in choline/creatine ratio without a significant $\mathrm{N}$ acetyl aspartate decrease, absence of macromolecules; Neuroophthalmological examination:normal findings; Psychological testing (Wechsler Adult Intelligence Scale- WAIS):mild cognitive impairment; Neuropsychological testing:normal findings; Increased plasma VLCFA; Confirmed mutation in $\mathrm{ABCD} 1$ gene

Conclusion: The findings proved that Addison's disease was the first clinical X- ALD manifestation in the patient who has AMN with the cerebral myelin affection. Apart from a mild cognitive impairment, the brain myelin changes have not been clinically manifested so far. After the AMN proof in this male, his two relatives with a potentially fatal childhood cerebral X-ALD were diagnosed early enough to undergo the proper treatment. The X-ALD recognition among the Addisonian patients is vital for the genetic counseling and monitoring for the development of myelin pathology.

P292

Autoimmune thyroiditis-related encephalopathy in a 14-year-old girl

$J$-P. Stellmann, S. Ott, R. Töpper

Asklepios Klinik Harburg (Hamburg, DE)

Over the last years encephalopathy related to autoimmune thyroiditis has been recognized as an important differential diagnosis of dementia with rapid progression as well as of infectious and paraneoplastic encephalopathy. Encephalopathy due to autoimmune thyroiditis typically manifests in epileptic seizures or neuropsychological symptoms. This disease is characterized by a high sensitivity to cortisone and is associated with increased thyroid anti-bodies as well as MRI changes.

We present the case of a 14-year-old girl, which had been admitted to an adolescent psychiatric department because of acute psychotic episodes following a gradual decrease in cognitive and school performance as well as in general activity. After admission she presented secondary generalised seizures induced from the right hemisphere. Because of right temporal and cerebellar hyperintensities on T2 weighted MRI images an encephalitis was suspected, diagnostic procedures including bacteriology, chemistry and serology did not show any pathologic results except of oligoclonal IgG bands. Under the presumed diagnosis of limbic encephalitis treatment with oxcarbazipine, antibiotics, aciclovir and high-dose cortisone was initiated and the patient stabilized. She was then transferred to another adolescent psychiatric department. There further tests with analysis for onconeural antibodies and voltage gated potassium channels were all also negative. Finally the diagnosis of autoimmune thyroiditis related enzephalopathy - also known as Hashimoto encephalopathy - could be made due to a serum thyrogloblin antibody titre of more then $4.000 \mathrm{kU} / \mathrm{l}$ combined with normal blood levels for fT3, fT4 and TSH. Reviewing the clinical course we could correlate the improvement of symptoms and the regression of MRI changes to high dose corticosteroid treatment.

Autoimmune thyroiditis related encephalopathy is a rare disease in 
paediatric neurology but should be included in the differential diagnosis of encephalitis due to its good response to cortisone treatment.

\section{P293}

Neurofibromatosis 1 associated to spinal muscular atrophy: case report

L.H.C. Dos Santos, R.H. Scola, L.E. Munhoz da Rocha, M.S. Bodanese, E.B. Medeiros, C.S. Kay, P.J. Lorenzoni, S.A. Antoniuk, I. Bruck, L.C. Werneck Universidade Federal do Paraná (Curitiba, BR)

Neurofibromatosis type 1 (NF-1) or von Recklinghausen disease is a progressive autosomal dominant disease which is inherited as a monogenic disease. Spinal muscular atrophy (SMA) is a progressive disease which also is inherited as a monogenic disease. The coinheritance both monogenic syndromes, as NF-1 and SMA, in the same patient has not been previously reported. We described a 17 months-old boy with motor development delay who presented generalized weakness, axial hypotonia, café-au-lait macules, generalized hyporreflexia, kyphoscoliosis and left axillary neurofibroma. At 3 years-old he presented humoral immunodeficiency by hypogammaglobulinemia causing repeated pneumonia which has been treated with immunoglobulin. When he was 8 years-old there was worsening of weakness and hypotrofia causing collapsed spine. Cervical and thoracic MRI was normal. Needle electromyography reveals diffuse active desinervation. Molecular analysis for SMA revealed that both exons 7 and 8 of SMN 1 gene were deleted. The characteristic of disease, specially, clinical, immunological, electrophysiologic and genetic findings are discussed and the relationships between NF-1, hypogammaglobulinemia and SMA is up now unknown.

\section{P294}

A fMRI study of patients with paediatric multiple sclerosis

M. Absinta, M. Rocca, A. Ghezzi, L. Moiola, G. Comi, M. Filippi

Scientific Institute San Raffaele (Milan, IT); Department of Neurology (Gallarate, IT)

Objectives: Using functional magnetic resonance imaging (fMRI), movement-associated cortical changes have been described in patients with adult-onset multiple sclerosis (MS). Although epidemiological studies have shown a more favourable course in patients with pediatric MS (onset before the age of 16), the factors responsible for their positive clinical evolution are not fully elucidated yet. Furthermore, the role of brain plasticity in these patients has never been investigated. In this study we wished to evaluate the pattern of cortical activations during a simple motor task in patients with pediatric MS and its relation with the extent of the T2 lesion load (LL).

Methods: From 17 right-handed patients with pediatric relapsing-remitting MS $(\mathrm{M} / \mathrm{F}=6 / 11$; mean age $=15$ years, median disease duration $=2.0$ years, median EDSS $=1.0$ ) and 9 sex- and age-matched right-handed healthy controls, we obtained fMRI during repetitive flexion-extension of the last four fingers of the right hand and brain dual-echo scan. T2 LL were measured using a local thresholding semi-automated segmentation technique. fMRI data were analyzed using the statistical parametric mapping software. We report activations below a threshold of 0.05 , corrected for multiple comparisons.

Results: In pediatric MS patients, mean brain T2 LL was $9.4 \mathrm{ml}$. Compared to controls, pediatric MS patients showed an increased recruitment of the left primary sensorimotor cortex (L SMC). The activity of the L primary SMC was strongly correlated with T2 LL $(r=0.78, \mathrm{p}<0.001)$.

Conclusions: Movement-associated cortical changes do occur in patients with pediatric MS and are characterized by an increased recruitment of the primary SMC. The correlations found between the extent of fMRI changes and T2 LL suggests an adaptive role of such functional cortical changes in limiting the clinical outcome of structural damage.

The study was supported by a grant from the Fondazione Mariani (contract R-07-62).

\section{P295}

Safety monitoring of miglustat in Niemann Pick-C patients

M. Pineda, J. Wraith, M. Beck, J. Imrie, B. Schwierin, B. Bembi

Hospital Sant Joan de Deu (Barcelona, ES); Royal Manchester Children's Hospital (Manchester, UK); Universitaets Kinderklinik (Mainz, DE); Actelion Pharmaceuticals Ltd (Allschwil, $\mathrm{CH}$ ); Instituto per l'Infanzia Burlo Garofolo (Trieste, IT)

Objectives: Niemann Pick-C (NPC) is a rare but devastating genetic disorder arising from abnormal cellular lipid trafficking. Miglustat was approved for use in Gaucher disease type 1 (GD1) in March 2003, and may have a possible use in the treatment of NPC. IS3 is a post-marketing surveillance pro- gramme, implemented at the time of miglustat marketing authorisation in Europe, collecting clinical information on miglustat use in patients with GD1 and other lysosomal storage diseases. IS3 is conducted in accordance with standard of care practice and miglustat product characteristics, and aims to enhance awareness of appropriate safety precautions and monitoring in miglustat use.

Methods: IS $^{3}$ is formulated as a prospective non-interventional, password-protected web-based programme. Physicians are encouraged to enter data from patients receiving miglustat.

Results: From March 2003 to 19 October 2007, information was available on 64 NPC patients ( $54.7 \%$ female) prescribed miglustat in 10 European countries (24 centres). Mean age (SD) was 14.4 (10.6) years; 29 patients $(45.3 \%)$ were $<12$ years old. As at 19th October, 2007, overall exposure to miglustat represented a cumulative period of 87.7 patient-years; the median exposure (range) was 16.4 (0.4-52.8) months. The majority of patients (approximately $70 \%$ ) received a daily miglustat dose of $\leq 600 \mathrm{mg} /$ day. Safety signals (adverse events that might be associated with the use of miglustat) were reported in 28 patients ( $43.7 \%$ ), with most adverse events due to disease progression. Nine patients $(14.0 \%)$ have developed new or worsened neurocognitive deficits. Diarrhoea was observed in 13 patients $(20.3 \%)$ and body weight decrease $>10 \%$ occurred in 8 patients $(12.5 \%)$. Three patients died because of disease progression. Five patients discontinued; two due to diarrhoea, one after the twin sister died, one due to lack of efficacy, one due to inability to swallow the capsule.

Conclusion: NPC patients in the IS ${ }^{3}$ database exhibit disease signs and symptoms that are characteristic for NPC; thus analyses based on IS ${ }^{3}$ findings should be applicable to the clinical NPC population. Miglustat is well tolerated in NPC patients in clinical practice.

This research was supported by Actelion Pharmaceuticals.

\section{P296}

Paediatric spinal injury: a distinct group

N. Cordeiro, M. Awadh, M.E. O'Regan, S. Zuberi, I. Horrocks, R. Mc Williams Fraser of Allander Neuroscience Unit (Glasgow, UK)

Objectives: Little is written about paediatric spinal injury and expertise in spinal rehabilitation is concentrated in a few specialist units. Spinal injury in adults tends to affect physically fit individuals through trauma or demyelinating disease as the most common aetiological groups. This did not reflect the diversity of cases we were seeing in a tertiary paediatric neurology setting.

Methods: The Royal Hospital for Sick Children in Glasgow (RHSC) serves a population of approximately 3 million. We collected data from September 2004 to July 2007 for children admitted to neurology services, requiring rehabilitation for spinal injuries. We sought to define diversity pathology, as well as documenting length of stay.

Results: 12 patients ( 9 boys and 3 girls) required rehabilitation. Mean age at presentation was 7.58 years (SD 4.21). Mean length of stay was 13.6 months (SD 13.4) and only three cases were admitted for less than six months. One child required long-term ventilation.

Aetiological groups included:

1) Trauma - two road traffic accidents,

2) Oncology - two spinal tumours,

3) Inflammation/Infection - Transverse myelitis; polio-like flaccid paralysis caused by enterovirus in a child found later to have a Combined Variable Immune Deficiency.

4) Vascular - anterior spinal artery dissection followed a vigorous bout of trampolining; and spinal cord ischaemia following coarctation repair.

5) Genetic/Metabolic -C1-2 subluxation in a child with Trisomy 21; C1-2 injury in Morquio syndrome.

7) Congenital - Congenital abnormalities of base of skull and spine presenting with acute decompensation and hypoglossal nerve palsy; Hypoxic-ischaemic injury to spinal cord with associated brain injury

8) Other - Progressive scoliosis associated with diplegic cerebral palsy with suspected cord compression.

Conclusions:

1. Children with spinal injury constitute a distinct group with wide aetiological variability.

2. Although rare, paediatric spinal injury is associated with a significant period of inpatient rehabilitation and morbidity.

3. Interaction with other sources of complex disability(congenital/genetic/metabolic etc) makes this a particularly vulnerable group. 
P297

Neuroimaging abnormalities in infants with congenital hemiparesis

R. Teleanu, F. Buruiana, D.A. Plesca, M. Moiceanu, I. Stancea, L. Cretu, D. Epure, A. Lazar

University of Medicine Carol Davila (Bucharest, RO)

Introduction: We are presenting a study of congenital hemiparesis in children witch included 15 patients, who were hospitalised between feb.2006ian.2007.

Material and methods: The children's ages were between 3 month and 17 years. The most comun grup of age was 3 month- 2 years ( 8 patients). An asociated features with known symptomatology of hemiparesis are epileptic seizures (5 patients) and mental retardation ( 5 patients). The affected hemisphere was often the left side (9 cases). 6 children performed CT scaner and 9 performed Magnetic Resonance Imaging (MRI) cerebral. 7 children present congenital malformation ( 3 with porencephaly cavities, 4 migrational defects (schizencephaly, polymicrogyria, septo-optic dysplasia), 2 infants was presented stroke caused by deficit of S and C protein, 2 had cortical atrophy and 4 with a normal head imaging.

Conclusions: MRI has became the diagnostic and prognostic modality of choise for investigating all forms of hemiparesis in infantis. Migrational defects comprise the majority of congenital malformation causing infantile hemiparesis, frequently associated with seizures and mental retardation.

\section{P298}

Motor focal epilepsy associated with autoimmune polyglandular syndrome type 1-a case report

S. Godeanu, C. Burloiu, S. Magureanu, A. Suta

Alexandru Obregia Clinical Hospital (Bucharest, RO)

Objectives: To present the uncommon association between motor focal epilepsy and autoimmune polyglandular syndrome type 1 .

Methods: A 6 years old girl admitted to our clinic for a partial motor and a tonic-clonic generalized seizures. Her history revealed carpal spasm and muscle cramps during the last two months, presumably caused by hypoparathyroidism. We made clinical assesment, EEG, CT scan, ophtalmological examination, routine blood investigations, the serum cortisol level and we tested thyroid status.

Results: Neurological examination showed a IV and VI cranial nerves palsy and the investigations revealed EEG abnormality with temporal bilateral slow spike-waves discharges, a normal CT scan, low serum PTH level, hypocalcemia, hyperphosfatemia. Endocrinological examination sustained the diagnosis of idiopathic hypoparatyroidism. The child was treated with carbamazepine, calcitriol and calcium salts. She continued having rare partial motor seizures and the carbamazepine was replaced by valproate $500 \mathrm{mg} / \mathrm{zi}$.

In May 2005 the patient was admitted again for symptoms of adrenal crisis preceded by skin hyperpigmentation. New laboratory findings showed hyponatremia, hypochloremia, hyperkaliemia, random cortisol level, hypocalcemia. This time cerebral CT showed calcifications of basal ganglia, frontoparietal cortex and cerebellum. After emergency treatment of adrenal crisis, the maintenance therapy of chronic primary adrenal insufficiency has been initiated: replacement of glucocorticoids and mineralocorticoids with prednisone, respective fludrocortisone. The seizures were controlled by valproate in monotherapy. Along the course of the disease the clinical picture of polyglandular syndrome type 1 was completed by occurrence of adrenal insufficiency and chronic mucocutaneous candidiadis.

Conclusions: The autoimmune polyglandular syndrome type 1 is a rare autosomal recessive disorder characterized by chronic mucocutaneous candidiasis, chronic hypoparathyroidism and autoimmune adrenal component occured in a fairly precise chronological order. The spectrum of associated minor clinical diseases includes other autoimmune endocrinopathies (hipergonadotropic hypogonadism, insulin-dependent diabetes mellitus, autoimmune thyroid disease), immunological defects (cellular and humoral).

Conclusion: the particularity of this case consists in unusual sequence of development of three major components of APS type 1 and the association with motor focal epilepsy.

P299

Transient neonatal myasthenia gravis revealing a myasthenia gravis and a systemic lupus erythematosus in the mother

S. Perelman, M. Saint-Faust, P. Velin, D. Dupont, M. Chatel

Hospital Lenval (Nice, FR); Hospital Pasteur (Nice, FR)

Transient neonatal myasthenia gravis (TNMG) and neonatal lupus are rare conditions due to transplacental passage of maternal antibodies. Diagnoses in neonates are suggested usually in context of well-known maternal disease.
We describe a case of TNMG, revealing a myasthenia gravis (MG)associated with systemic erythematosus lupus (SLE) in the mother.

Observation: At days 8 of age, a normal term infant was admitted to hospital for jaundice. Examination revealed poor sucking, facial weakness with poor expression, bilateral mild ptosis and hypotonicity. Diagnosis of TNMG was confirmed because of high level of anti-Acétylcholine Receptor (AChR) antibodies in the baby and his mother. Clinical history and biological investigations in the mother gave evidence of MG with persistent thymus, but also revealed SLE, with 5 of the ARA criteria. The infant did not have any sign of neonatal lupus. His elder brother has an autistic syndrome and had presented a moderate form of TNMG.

Discussion: Our observation is original by its mistaking presentation; it is very rare that TNMG reveals the maternal disease. In case of previous maternal MG, a low percentage of infants develop TNMG (10-20\%) because of protective effect of alpha-foetoprotein in the amniotic fluid and probably because of involvement of two isoforms of AChR: fetal and adult. Improvement is usually obtained within the first three weeks, but the infants should be first carefully monitored and rapidly treated by anticholinesterase drugs if needed. No correlation has been found between maternal symptoms and anti-AChR antibodies titers, and neonatal clinical signs of TNMG. Pregnancy should not be discouraged in myasthenic women, but the course should require regular monitoring of fetal movements because of the main complication, arthrogryposis multiplex congenita. In case of maternal SLE, most cases of neonatal lupus are associated with presence of anti-SSA or SSB antibodies in the mother, particularly when a congenital heart block is observed. Both conditions, MG and SLE are described but pregnancy in those cases are very few and consequences for infants need tight follow-up. The autistic disease in the brother focuses on its mysterious and unknown relationship with auto-immune diseases.

\section{P300}

mtDNA depletion in a case of fatal infant cytochrome $C$ oxidase deficiency presenting with clinical feature of type 1 spinal muscular atrophy

V. Lucchini, A. Berardinelli, M. Carpanelli, M. Sciacco, A. Bordoni, D. Ronchi, E. Fassone, M. Servida, M. Pluderi, G.P. Comi, M. Moggio, N. Bresolin, C. Lamperti

IRCCS Fondazione Ospedale Maggiore (Milan, IT); Fondazione IRCCS C. Mondino (Pavia, IT); Azienda Ospedaliera di Lecco (Lecco, IT)

Objective: To describe a female newborn with clinical feature of type 1 spinal muscular atrophy (SMA1), who presented a severe cytochrome c oxidase (COX) deficiency at muscle biopsy and mtDNA depletion at the Southern Blot. The baby died at 6 months of age for acute respiratory distress.

Methods: We describe a baby born after an uncomplicated pregnancy and delivery, from healthy unrelated parents. The Apgar score was 10. Both morphometrical parameters and body weight were normal. At age 40 days she developed progressive and severe hypotonia and weakness without cranial nerve impairment and no fever. At 2 months of age the baby presented a worsening of both weakness and hypotonia. Moreover, she developed areflexia and weak cry, sucking and swallowing being preserved. Neuropsychological functions and the other cranial nerve functions were normal. All symptoms worsened in the following months, especially respiratory failure, which became more evident at age five months. The patient died for acute respiratory distress at 6 months of age.

All blood tests were normal including creatine kinase, lactate and urinary organic acids. Electromyography, performed at age five months, showed neurogenic findings and reduction of CMAP.

The genetic analysis for SMN1 showed heterozygous condition for the common mutation for SMA1.

Quadriceps muscle biopsy was performed few days before death and cryostate sections were studied with histological, histochemical and immunohistochemical reactions.

Quantitative Southern blot analysis of mtDNA was performed. The following nuclear genes were analysed: Thymidine Kinase 2 (TK2), MPV17 and SUCLG1.

Results: Muscle biopsy showed panfascicular atrophy with few large type I fibers, but the main feature was a severe and generalized COX deficiency. Severe mtDNA depletion was evident at Southern blot. No mutations were found in TK2 as well in MPV17 and SUCLG1.

Conclusion: mtDNA depletion syndromes (MDS), inherited as autosomal recessive traits, result from defects in nucleus-encoded factors involved in mtDNA maintenance. Three main clinical presentation of MDS are known: myopathic, encephalomyopathic and hepatocerebral.

A few cases of mtDNA depletion presenting with a myopathic pattern and clinical features of SMA 1 are described in literature related to mutations in TK2 nuclear gene (Mancuso et al 2002). The absence of mutations in TK2 in this case suggests that other nuclear genes may cause mtDNA depletion syndrome with this phenotype. 
P301

Acute disseminated encephalomyelitis in children: the first attack of multiple sclerosis?

S. Noorbakhsh, H. Tonekaboni, V. Zarabi

Iran Medical University (Tehran, IR); Shahid Beheshti Medical University (Tehran, IR)

Objective: Acute disseminated encephalomyelitis is an uncommon inflammatory demyelinating disease of the central nervous system. The main goal of this study is reporting the demographic, clinical presentation; possible relation between the disease and previous viral infection or vaccination; clinical course; response to therapy, complication of ADEM patients admitted during 2 years study.

Methods: A 2-years retrospective, descriptive, chart review of children with the final diagnosis of ADEM in Tehran, Iran during 2000-2002. The diagnosis was made upon clinical presentation, physical examination and ruling out of other disease by expert paediatric neurologist. ADEM was documented in all cases by charechteristic MRI changes.

Results: The final diagnosis of ADEM provided in 15 patients less than 15 years of age. $88 \%$ of cases in either winter or spring. $46.4 \%$ of cases had a recent upper respiratory tract illness. Previous vavccination in $13.6 \% ; 40 \%$ had not either URI or vaccination. VZV infection in 1 , and UTI in 2 cases. Recent Mycoplasma pneumoniae infection $(\operatorname{IgM})$ in 1 case. Presentattion signs.: Ataxia $60 \%$; altered consciousness $60 \%$, fever+nausa, vomiting $46 \%$; cranial nerve involvement $33 \%$; dysarthric speech $26 \%$; convulsion $20 \%$; hemiparesia $20 \%$; paresthesia $13 \%$; meninsismus $13 \%$; headache $6 \%$. MRI identified inflammation and demyelination in Subcortical white matter (93\%) cerebral cortex involvement $(80 \%)$ periventicular lesions $(60 \%)$. Twelve patients treated with corticosteroids, and 3with intravenous. immunoglobulins. Only 1 death had occurred.Three patients $(20 \%)$ had long-term neurologic sequelae.

Conclusion: ADEM is common in our children. Due to advances in control of traditional exanthematous diseases (measle, rubella..) most cases of ADEM in this study followed nonspecific upper respiratory infections. Differentiation of ADEM from a single episode of multiple sclerosis is difficult. Diagnosis of multiple sclerosis should be done if new symptoms and signs or imaging abnormalities appear, more than 3 months after the onset of clinical symptoms in ADEM cases. Since multiple sclerosis has low incidence in Iranian children, Probably ADEM is not as a part of the multiple sclerosis spectrum in our patients.

\section{Peripheral neuropathy}

\section{P302}

Autonomic dysfunction in sensory neuron diseases

A. Damasceno, M. França-Jr, H. Cury, A. Nucci

State University of Campinas (UNICAMP) (Campinas, BR)

Objectives: To investigate signs and symptoms of autonomic dysfunction $(\mathrm{AD})$ in patients with chronic acquired sensory neuron diseases (SND) associated with different conditions and to determine if $\mathrm{AD}$ is more prominent regarding the associated disorder.

Methods: Autonomic function was quantified through tests of cardiovagal, adrenergic, sudomotor and pupillomotor function (Autonomic Tests Score, ATS), and through a 40-item questionnaire assessing several domains of autonomic symptoms (Autonomic Questionnaire Score, AQS). We studied twenty patients with chronic acquired SND associated with different conditions (toxic, infective, vitamin related, idiopathic and dysimmune). Forty-six age- and sex-matched healthy subjects were evaluated as a control group for the AQS and 15 for the ATS.

Results: All patients reported symptoms of AD. They scored significantly higher than controls on all individual domains of the questionnaire, especially on orthostatic, secretomotor and gastrointestinal $(\mathrm{p}<0,00001)$, and on the total AQS $(p<0,0001)$. Fifty-five per cent of the patients had one or more abnormal cardiovagal test, $60 \%$ orthostatic hypotension and $50 \%$ absent sympathetic skin response, and their ATS was significantly different from the control group $(p=0,001)$. ATS and AQS were significantly correlated $(\mathrm{p}=0,002)$. Kruskal-Wallis analysis showed no significant difference of ATS or AQS when separating patients in groups of different associated disorders.

Conclusions: $\mathrm{AD}$ is a prevalent and important complication among patients with chronic acquired SND, and should be routinely assessed. It is frequent in SND associated with different conditions and not only with paraneoplastic or dysimmune, as previously thought.
P303

Distal hereditary motor neuropathy in Korean patients with a small heat shock protein 27 mutation

B.O. Choi, S.B. Kim, S.Y. Cho, S.J. Hwang, J.S. Shin, S.H. Kang, K. W. Chung, K.D. Park, K.G. Choi

Ewha Womans University (Seoul, KR); Kyung Hee University (Seoul, KR); Kongju National University (Gongju, KR)

Objectives: Distal hereditary motor neuropathy (dHMN) is a heterogeneous disorder characterized by degeneration of motor nerves in the absence of sensory abnormalities. Recently, mutations in the small heat shock protein 27 (HSP27) gene were found to cause dHMN type II or Charcot-Marie-Tooth disease type $2 \mathrm{~F}$ (CMT2F). This study was performed to find the phenotypic characteristics in Korean dHMN patients with HSP27 mutation.

Methods: The authors studied 151 Korean axonal CMT or dHMN families, and found 12 patients from a large Korean dHMN type II family with the Ser135Phe mutation in HSP27. In addition, 200 healthy controls were recruited. Motor and sensory conduction velocities of median, ulnar, peroneal, tibial, and sural nerves were determined in 12 patients. MRI was performed on all dHMN patients in a supine position using a 1.5-T system.

Results: By mutational screening of the HSP27 gene in the Korean dHMN family, we identified a heterozygous form of a missense mutation, i.e., c.C404T (Ser135Phe) in exon 2, which has been previously reported to be an underlying cause of CMT2F. This mutation was inherited in an autosomal dominant manner, and was well associated with familial members with the dHMN phenotype. This mutation site is located in the --crystallin domain and is highly conserved between different species. The frequency of this HSP27 mutation in Koreans was $0.6 \%$. Electrophysiological findings confirmed that disease duration was well associated with disease severity. MRI findings revealed that fatty infiltrations tended to progressively extend distal to proximal muscles in lower extremities, which were compatible with the notion of length-dependent axonal degeneration. In addition, fatty infiltrations in thigh muscles progressed to affect posterior and anterior compartments but to lesser extents in medial compartment, which differs from CMT1A patients presenting with severe involvements of posterior and medial compartments but less involvement of anterior compartment.

Conclusion: In the present study, we described the clinical, electrophysiological, and neuroimaging findings of Korean dHMN type II patients, and found that the frequency of HSP27 mutation was low. In addition, thigh MRI revealed length-dependent degeneration, and different features from that observed in CMT1A patients.

\section{P304}

A case of pancytopenia following intravenous immunoglobulin therapy in Guillain-Barré syndrome

J.H. Cho, S.H. Kim, H-S. Kim, G.S. Kim, S-A. Choi, J.H. Lee

National Health Insurance Corporation (Goyang-si, Gyeonggi-do, KR); Yonsei University Wonju College of Medicine (Wonju-si, Gangwon-do, KR); Pochon CHA University College of Medicine (Sungnam-si, Gyeonggi-do, $\mathrm{KR})$

Intravenous immunoglobulin (IVIg) therapy is widely used in the treatment of neurological autoimmune diseases. Although IVIg are considered a safe treatment, many side effects have been reported. The hematological side effects associated with IVIg include leukopenia, neutropenia, autoimmune hemolysis and coagulopathy. We report a patient with Guillain-Barre syndrome who developed pancytopenia following IVIg therapy. It may need to monitor complete blood count during and after IVIg therapy.

\section{P305}

Type of cell death in dorsal root ganglion neurons following peripheral nerve injury and surgical repair

M. Atlasi, M. Mehdizadeh, M. Bahadori, M. Joghataei

Kashan University of Medical Sciences (Kashan, IR); Iran University of Medical Sciences (Tehran, IR); Gilan University of Medical Sciences (Rashat, IR)

Objectives: To investigate the morphology of dorsal root ganglion neurons after sciatic nerve transection and surgical repair in adult rat.

Methods: Wistar rats were exposed to models of axotomy, direct epineurial suture, and autograft and after 1 and 12 weeks of the surgery, the number and ultrastructure of the neurons evaluated by fluorescence and electron microscopy respectively.

Results: Nerve transection caused sensory neuronal loss and direct epineurial suture but no autograft repair method decreased it. Evaluation of morphology of the neurons showed classic features of apoptosis as well as destructive changes of cytoplasmic organelles such as mitochondria, rough endoplasmic reticulum and Golgi apparatus in primary sensory neurons. 
These nuclear and cytoplasmic changes in primary sensory neurons were observed after the surgical nerve repair too.

Conclusion: The present study imply that following peripheral nerve transection apoptosis as well as cytoplasmic cell death contribute in neuronal cell death and reconnection of proximal and distal nerve ends dose not prevent these processes.

P306

Nitric oxide induces axonal degeneration of active peripheral mouse nerves by interfering with energy supply

S. Alvarez, M. Moldovan, C. Krarup

Panum Institute, University of Copenhagen (Copenhagen, DK); Rigshospitalet (Copenhagen, DK)

Objectives: Exposure of axons to the free radical Nitric Oxide (NO) triggers acute conduction block and if exposure is prolonged it may cause axonal degeneration. It was hypothesized that the effect of NO may be due primarily to energy restriction by inhibition of mitochondrial respiration. We investigated the in vivo effect of NO exposure on active peripheral mouse nerves and compared this with exposure to the mitochondrial uncoupler 2, 4- dinitrophenol (DNP).

Methods: Investigations were carried out in adult female black mice (C57BL/6J, Harlan) and female mutant mice with delayed Wallerian degeneration (C57BL/WldS, Harlan) under anesthesia. The right tibial nerve was stimulated at the ankle. Compound muscle action potentials (CMAPs) were recorded from plantar muscles using subcutaneous needles. Corresponding compound nerve action potentials (CNAPs) were recorded form the sciatic nerve exposed through a small opening by means of a hook electrode. Trains of supramaximal stimuli at $200 \mathrm{~Hz}$ were delivered every 10 minutes for 3 hours. During the trains the sciatic nerve was immersed in either phosphate buffered saline (PBS), $10 \mathrm{mM}$ (Z)-1-[2-(2-Aminoethyl)-N-(2-ammonioethyl)amino]diazen-1-ium-1,2-diolate (DETA NONOate), or up to $1 \mathrm{mM}$ DNP. Following 3 hours of stimulation, the nerves were washed in PBS for 1 hour, the surgical wounds were closed and the mice were left to recover. At the completion of the experiments, tibial nerves were removed and morphological studies were carried out.

Results: Following the repetitive stimulation sequence in PBS, the CNAP recovered within 1 hour and the CMAP within 1 day. We found that acute exposure to NO or DNP had similar effects: (i) a transient conduction failure that appeared to normalize within one hour of washout and (ii) Wallerian degeneration of some axons confirmed at morphological studies. No signs of Wallerian degeneration were observed in unexposed controls, or in exposed WldS mutant mice

Conclusion: Taken together, these data support the hypothesis that neurotoxicity may be caused by energy restriction. Since the pharmacologic effect of NO and DNP was only transient, our data suggest that even a brief period of energy restriction can trigger Wallerian degeneration.

Lundbeck Foundation, the Novo Nordisk Foundation, the Danish Medical Research Council, the Ludvig and Sarah Elsass Foundation and the Foundation for Research in Neurology

P307

Peripheral neuropathy associated with Crohn's disease: case report and literature review

A. Ariatti, E. Canali, F. Girolami, G. Galassi

Institute of Neurosciences (Modena, IT)

Objective: Peripheral neuropathy (PN) in Crohn's disease (CD) is rarely described either related to vitamin B12 deficiency or to metronidazole treatment. Small or large fibre PN may occur at any time being more common the sensory demyelinating form. We present herein a patient with progressing sensorimotor neuropathy associated with $\mathrm{CD}$, in whom role of vitamin deficiency and metronidazole treatment could be excluded.

Methods: This 63 year old woman acutely experienced distal extremity paresthesias and mild weakness during an episode of severe abdominal pain and rectal haemorrhage. Intestinal biopsy diagnosed CD. Neurologic examination at onset showed distal hypoesthesia, involving all modalities, areflexia, gait unsteadiness. Serum B12 and folate level was normal.Electrophysiology revealed unevokable sensory responses, slowed sensory conduction, normal motor findings. Sural biopsy confirmed fiber loss and demyelination. CSF protein content was normal. Patient was shortly treated with oral steroids and vitamins. In the following sixteen years limb paresthesias and unsteadiness continued to worsen in absence of $\mathrm{CD}$ recurrence. When seen on clinical follow-up sixteen years after onset there were mild distal loss of strength, marked trunk ataxia, dysmethria on finger-nose and heel to shin test. Deep jerks were absent.Vibration, position, touch, pin-prick were impaired distally. Cranial nerves were intact.

Result: Laboratory tests including CK, B12,folate level, thyroid and liver functions, immunological screenings were normal. Serum sample tested for paraneoplastic and anti-ganglioside antibodies gave negative results. Electrophysiology confirmed sensorimotor axonal and demyelinating neuropathy.

Discussion and conclusion: Our patient exhibited an acute neurologic illness with evolving course which could be related at onset to CD. Major clinical signs included early intestinal symptoms. Progressing ataxia due to proprioceptive sensory loss and sensorimotor, length dependent neuropathy evolved in absence of $C D$ recurrence. Previous reports outlined that in PN associated with $\mathrm{CD}$, immunomodulatory therapy led sustained improvement in $60 \%$ of patients, positive response occurring as expected in demyelinating form, but also in cases with axonal features. The latter suggests that neuropathy and CD may share possible common autoimmune pathogenetic mechanism. It is likely that there is a primary immune-mediated PN associated with $\mathrm{CD}$ as an extra-intestinal disorder and not merely co-occurrence.

\section{P308}

POEMS syndrome: clinical presentation and effective treatment with autologous peripheral blood stem cell transplantation. A case report

E. Alexiou, A. Galanopoulos, G. Theologos, C. Vlamis, I. Markakis, P. Repousis, G. Gekas

General State Hospital of Nikaea (Piraeus, GR); Metaxa Anticancer Hospital of Piraeus (Piraeus, GR)

Objectives: We present the case of a woman suffering from POEMS syndrome (Polyneuropathy, organomegaly, endocrinopathy, monoclonal protein and skin changes), who was significantly improved after autologous peripheral blood stem cell transplantation (Auto-PBSCT).

Methods: A 47-year-old woman, who had been previously diagnosed as suffering from chronic inflammatory polyneuropathy (CIDP), came to hospital due to deterioration of gait, difficulties to climb stairs and hold heavy objects, swelling and burning pain below the knees. Clinical examination revealed hyperpigmentation of skin and hypertrichosis, edema of lower legs and palpable liver. Neurological signs included predominantly distal symmetrical weakness of all extremities, excessive response of feet and tibiae to pinprick and absence of tendon reflexes.

Results: Nerve conduction studies confirmed polyneuropathy, more severe in lower limbs, with prominent demyelination, including features of axonal degeneration. CSF examination showed elevation of total protein $(113 \mathrm{mg} / \mathrm{dl})$, while other parameters were normal. CT abdominal scanning revealed hepatosplenomegaly. In electrophoresis, IgA protein was slightly high and immunofixation detected monoclonal IgA-lambda protein. Detailed bone investigation showed osteosclerotic lesions of scull and sacroiliac joints, while bone marrow analysis demonstrated plasma cells of $5 \%$, producing lambda monoclonal protein. VEGF (Vascular endothelial growth factor), a cytokine strongly associated with POEMS, was determined at a very high titer $(2100 \mathrm{pg} / \mathrm{ml})$. Endocrinological tests were normal, except for elevated ACTH and TSH.

Clinical and laboratory features met the diagnostic criteria for POEMS syndrome. Due to the severity of the disease (edema, threatened motor disability, high VEGF), an aggressive therapy with high dose chemotherapy and auto-PBSCT was decided. Outcome of treatment was very encouraging, as, one year after therapy, muscle strength has improved robustly, sensory disturbances have remitted, skin hyperpigmentation, organomegaly and edema have disappeared. VEGF has significantly decreased $(900 \mathrm{pg} / \mathrm{ml})$ and no monoclonal protein is detected.

Conclusion: POEMS is a rare syndrome with multiorgan involvement. Polyneuropathy is a dominant presentation of POEMS, mimicking clinically and electrophysiologically CIDP. Auto-PBSCT is a relatively new and much anticipating therapy for preventing serious motor disability and improving morbidity and mortality of disease.

\section{P309}

Phenotypic characteristics of Korean patients with X-linked dominant Charcot-Marie-Tooth disease

B. O. Choi, O. Kwon, S.J. Hwang, S.Y. Cho, H.R. Yang, J.S. Shin, J.Y. Yoon, J.Y. Kwon, K.W. Chung

Ewha Womans University (Seoul, KR); Eulji University (Seoul, KR); Kongju National University (Gongju, KR)

Objectives: X-linked dominant Charcot-Marie-Tooth disease (CMTX), caused by mutations in the connexin $32(\mathrm{Cx} 32)$ gene, is the second most frequent form of CMT. In Koreans, the clinical and electrophysiological features 
of CMTX have not been reported yet. Therefore, we investigate to find the phenotypic characteristics in Korean CMTX patients with Cx32 mutations.

Method: Mutations in Cx32 were screened in 511 patients from 193 unrelated CMT families of Korean origin. In addition, 200 healthy controls were recruited. We measured the CMT neuropathy score (CMTNS) and the functional disability scale (FDS) to quantify disease severity. Motor and sensory nerve conduction studies were done in 31 CMTX patients with Cx32 mutations. Twenty one CMTX patients performed brain MRI, and two patients were carried out sural nerve biopsies.

Results: Ten different $\mathrm{Cx} 32$ mutations $(9$ missense and 1 frameshift mutations) were identified in 31 CMTX patients ( 12 males and 19 females) from 11 unrelated families $(5.7 \%)$. The age at onset was earlier in males $(15.9 \pm 7.6$ years) than females $(32.6 \pm 10.8$ years $)(p<0.001)$, and males were more severely affected than females by FDS and/or CMTNS $(\mathrm{p}<0.001)$, suggesting more rapid clinical deterioration in males. Muscle atrophy, areflexia, and heel-gait defect were also identified more frequently in males $(p<0.05)$. Most electrophysiological parameters worse in males, and especially, motor nerve conduction velocities (NCVs) in median and ulnar nerves, were significantly reduced $(p<0.05)$. CMTX patients showed the typical patterns of length-dependent affection, and intermediate form of motor NCVs between CMT type 1 and 2. Four patients (19\%) showed several T2 high signal intensities in deep white matter. Myelin sheaths are thin for the axonal diameter, which probably represents remyelination following axonal regeneration. Examination with electronic microscope showed the frequent and marked widening of the periaxonal apace and excessive myelin folding.

Conclusion: We described the clinical, electrophysiological, and neuroimaging findings of Korean CMTX patients, and found that the frequency of CMTX (5.7\%) is similar to that reported in Asians, but lower than those in Europeans. The longitudinal follow-up studies are required to clarify the detailed outlining of clinical features.

\section{P310}

First Val30Met transthyretin mutation in a Swiss patient: a case of familial amyloid polyneuropathy

C. Rosin, M. Morris, S. Frank, P. Fuhr, L. Kappos, S. Renaud

University Hospital Basel (Basel, CH); University Hospital Geneva (Geneva, $\mathrm{CH})$

Backround: We present a case of late onset progressive polyneuropathy caused by an amyloidogenic variant of transthyretin (TTR) in a Swiss patient. This Val30Met mutation causes progressive systemic amyloidosis which, left untreated usually leads to death within 10 years after the first symptoms.

Case report: Our 60 year old male patient initially presented with limb paraesthesia, pain, ataxia and erectile dysfunction. Apart from a prothrombin mutation no hereditary diseases were known. Electroneurography at that time had shown signs of axonal loss and demyelination. CSF protein was elevated. A diagnosis of chronic inflammatory demyelinating polyneuropathy (CIDP) was made and the patient was treated with $70 \mathrm{mg}$ of prednisone daily. This treatment did not have any impact on the relentlessly progressive disease, with the patient developing cardiac involvement, bladder and bowel dysfunction and weight loss of over $20 \mathrm{~kg}$. In the meantime he is dependent on full assistance because of plegic distal arm and leg muscles.

Nerve biopsy showed amyloid deposits which stained positive for transthyretin. Genetic analysis demonstrated a Val30Met mutation in the TTR gene.

Conclusions: To our knowledge this is the first case of Val30Met mutation in a patient of Swiss origin. The Val30Met mutation is common in Portuguese, Swedish and Japanese families. No family history is known in our patient but further family studies will show whether this might be a spontaneous mutation. In cases of progressive neuropathy with autonomic dysfunction nerve biopsy and genetic analysis for familial amyloid neuropathy by sequencing of the TTR gene are warranted despite negative family history.

\section{P311}

Clinical and genetic study of the SPTLC1 gene mutation in patients with hereditary sensory neuropathy type 1

H. Mourad, W. Fael

Tanta University (Tanta, EG)

Background: Hereditary sensory neuropathy type 1 (HSN1) is the most common hereditary disorder of peripheral sensory neurons.

Material and methods: This study was carried out on 15 patients with heriditary sensory neuropathy type-1and 10 healthy individuals as a control group. Detailed family history, thorough clinical and neurological examination were performed to all patients. Nerve conduction velocity of ulnar and lateral popliteal nerve (motor and sensory) as well as audiogram was carried out for all patients. genetic study of the serin palmitoytransferase (SPTLC1) gene mutation was performed to all studied groups.

Results: The results showed male predominance with sever clinical manifestation compared to the female group.The results of the nerve conduction velocity revealed reduced conduction velocity in both motor and sensory fibers only in the lateral popliteal nerve with no significant difference in the ulnar nerve. Audiogram revealed bilateral partial nerve deafness in 6 cases. Genetic study revealed mutation of the SPTLC1 in all studied patient groups. Point mutation $\mathrm{C} 133 \mathrm{~W}$ was observed in patients with younger age group with prominant leg ulceration, while point mutation in the $\mathrm{C} 133 \mathrm{Y}$ was observed in the patients complaining of hearing loss.

Conclusion: The SPTLC1 gene mutation play an important role in the pathogenesis and different clinical presentation of the disease.

\section{P312}

Charcot-Marie-Tooth disease type 1A duplication: refining the minimal adult phenotype

J. Berciano, E. Gallardo, A. García, C. Ramón, I. Mateo, J. Infante, E. Rodríguez-Rodríguez, O. Combarros

University Hospital Marqués Valdecilla (Santander, ES)

Background: Whereas absence of signs on physical examination is relatively common in Charcot-Marie-Tooth disease type 1A (CMT1A) duplication in patients under 5 years of age, this is an extremely rare and not well characterized situation in adult patients.

Objective: To describe clinico-electrophysiological features and MRI patterns of lower limb amyotrophy in sub-clinical CMT1A adult patients.

Patients and methods: After reviewing the clinical records of our cohort of 126 CMT1A cases coming from 25 unrelated pedigrees, we found two subclinical adult patients (Patient 1 and Patient 2) studied as secondary cases. Disease severity in terms of ability to walk and run was established using a nine-point functional disability scale (FDS). We administered the CMT neuropathy score (CMTNS), based on patients' symptoms, neurological examination and neurophysiological testing. MRI study included T1- (without and with contrast enhancement) and T2-weighted spin-echo sequences of thigh, lower-leg and foot musculature.

Results: Patient 1 is aged 48 years and has been serially evaluated since age 17 , her repeated physical examinations revealing just pes cavus with normal FDS score and mild CMTNS. Patient 2 is aged 58 and has been serially evaluated since age 48 , her repeated physical examinations also revealing pes cavus with normal FDS score and mild CMTNS; just at her most recent evaluation was flexor hallucis brevis and lumbrical muscle weakening noted. There was marked and uniform slowing of motor and sensory nerve conduction velocities. Compound muscle action potentials were either preserved or attenuated, whereas sensory nerve action potentials were attenuated or absent. Myoelectric deep tendon reflex (T-reflex) responses from biceps and gastrocnemius muscles were preserved though delayed. MRI showed a variable pattern of intrinsic foot musculature involvement: in $\mathrm{Pa}$ tient 1 fatty infiltration mainly involved the lumbricals, whereas Patient 2 showed extensive fatty atrophy of all foot muscles. There was subtle and predominantly distal fatty infiltration of lower leg anterolateral muscle compartments in both patients. Patient 2 showed sub-clinical but evident fatty infiltration of gastrocnemius muscles. In no muscle was edema or contrast enhancement visualized.

Conclusions: The minimal CMT1A adult phenotype includes pes cavus, uniform slowing of nerve conduction velocity, delayed T-reflex, and fatty atrophy of intrinsic foot musculature.

\section{Supported by CIBERNED (ISCIII, Madrid, Spain)}

\section{P313}

A novel 9-bp duplication in the connexin 32 gene causing X-linked CharcotMarie-Tooth disease

M. Keckarevic-Markovic, V. Milic-Rasic, M. Kecmanovic, D. Keckarevic, S. Todorovic, S. Romac

Faculty of Biology (Belgrade, RS); School of Medicine (Belgrade, RS)

Objectives: Connexin 32 (CX32 or GJB1) is a gap junction protein localized to the paranodal region and incisures of myelinating Schwann cells. Mutations in the gene encoding this protein occur in $10-15 \%$ of patients with Charcot-Marie-Tooth disease (CMT). Because CX32 gene is located on the X chromosome, this form of CMT disease is called CMTX. More than 290 different CX32 mutations have been identified and only few of them were insertion/duplication-type mutations. CMTX. 
Methods: Genomic DNA was isolated from blood samples using QIAamp blood purification kit (Qiagen Inc., Valencia, CA, USA). Promotor region and coding exon of CX32 gene were amplified using polymerase chain reaction (PCR) with primers reported previously, and directly sequenced using $\mathrm{ABI}$ 3130 Genetic Analyzer (Applied Biosystems, CA, USA).

Results: Sequence analysis in the proband revealed a hemizigous 9-bp insertion at nucleotide position 570 from the translation start site (RefSeq NM_000166). Proband's daughter was heterozygous for the same mutation. The mutation could be best described as in-frame duplication (c.561 569dupAACCGTCTT) of the first of two tandem direct imperfect repeats. This mutation results in the insertion of three amino acids: p.Val189_Phe190_insLeuThrVal and lies on the border between the second extracellular domain and the fourth transmembrane domain of CX32, together with previously reported c.571 579dupACCGTCTTC. At the protein level those two mutations differ in only one amino acid (p.Leu190 in our case instead of p. Phe190 in previously reported mutation). Clinical investigations in both cases demonstrated mild phenotype, consistent with protein structure predictions analysis using TMHMM and SPLIT4 software that showed no major changes in overall structure of CX32.

Conclusion: This is the first report of C.561 569dupAACCGTCTT mutation (p.Val189_Phe190_insLeuThrVal) in CX32 gene.

Only $3 \%$ of previously described CX32 mutations are insertion-type mutation. Most of them are single nucleotide insertions causing frame-shift and premature translation termination, and 3 of them are in-frame insertions, causing 2, 3 and 7 amino acids inclusion in the CX32 protein. Mutation reported in this study, together with previously described in-frame insertions, involves short tandem direct repeats that are duplicated or flanking the inserted sequence, implying that they originated through an unequal crossingover.

\section{P314}

Madelung's disease phenotype associated with Charcot-Marie-Tooth neuropathy phenotype

M.M. Cruzeiro, L.A. Pires, O.J.M. Nascimento

Universidade Federal de Juiz de Fora (Rio de Janeiro, BR); Universidade Federal Fluminense (Rio de Janeiro, BR)

Madelung's disease or multiple symmetric lipomatosis is a rare disease, which is characterized by non-encapsulated accumulation of fat, generally located symmetrically around the neck and shoulders. The aetiology is unknown, but is often seen in patients with a history of chronic alcoholism and it is accompanied by metabolic abnormalities. Madelung's cervical lipomatosis associated with a polyneuropathy is a rare clinical presentation.

Objective: To report four patients of the same family presenting an association of Madelung's disease with a Charcot-Marie-Tooth (CMT) neuropathy phenotype.

Patients and Methods: Three men (75,78 and 63 y.o.) and one woman (72 y.o) of the same family presented systemic lipomatosis with cervical Madelung characteristics. Their parents had no neurological problem. All the three patients referred difficult to walk since infancy. Actually they are not able to walk alone. Cervical fat deposits appeared during adult life. The neuropathy phenotype is conspicuous with a previous CMT diagnosis.

Results: Wasting distal muscles and contractures of the hands and feet; absent of tendon reflexes, sensory loss (thermal, pain, vibration and pressure) in distal lower and upper limbs and pes cavus were seen in all three cases. Drop foot was present in all the patients and they needed support to walk. Neuropathic pain was referred by the female patient. EDx showed reduced CMAP and SNAP amplitudes in all cases. NCV was $>38 \mathrm{~m} / \mathrm{s}$. Family history disclosed a case of death due to a complicated mediastinal lipomatosis surgery.

Conclusion: Facing a patient with a family history and presenting a sensory and motor axonal polyneuropathy associated to Madelung's phenotype we should disclose CMT neuropathy and consider familial lipomatosis polyneuropathy.

P315

Chronic inflammatory demyelinating neuropathy overlapping hereditary demyelinating neuropathy patients

M.M. Maroco Cruzeiro, O.J.M. Nascimento, M.R.G. Freitas, T.M. Escada, W. Marques Jr., G. Quintanilha

Universidade Federal Fluminense (Rio de Janeiro, BR); USP-RP (São Paulo, $\mathrm{BR})$

Hereditary demyelinating neuropathy consists of a group of peripheral nerve disorders in which Charcot-Marie-Tooth disease (CMT) and Hereditary Neuropathy Pressure Palsy (HNPP) are the most frequent causes. In such conditions the association of newly developed symptoms, mainly mo- tor and sensory, call attention for the possibility of superimposed chronic inflammatory demyelinating polyneuropathy (CIDP) or multifocal demyelinating neuropathy (Lewis-Sumner syndrome).

Objective: To report a series of patients with hereditary demyelinating neuropathies in association with inflammatory demyelinating features.

Patients and methods: Eight patients were referred as having an acquired newly progressive neuropathy. Electrodiagnostic tests performed in all of them disclosed acquired demyelinating features. Clinical findings suggest phenotypes of CMT and HNPP. DNA test was performed in all patients. Sural nerve biopsy was performed in all of them.

Results: Reduced nerve conduction velocity was observed in all cases (median motor nerve conduction velocity $<38 \mathrm{~m} / \mathrm{s}$ ). Conduction block was disclosed in all cases in almost one nerve. DNA tests revealed four CMT-IA patients with classical phenotype, one CMT-X (Conexin-32) and three with HNPP (two with a polyneuropathy and one with a multifocal presentation phenotype Lewis-Sumner syndrome). Nerve biopsy showed demyelinating features with onion-bulbs, active demyelination and inflammatory findings. All patients improved their superimposed recent symptoms with intravenous methylprednisolone pulsetherapy. Two patients (one CMT-IA and the Lewis-Sumner syndrome) relapsed twice.

Conclusion: Facing a sporadic patient with a recent progressive demyelinating neuropathy with EDX pointing to an acquired neuropathy, and a suspect phenotype for hereditary neuropathy a DNA test should be performed. Corticosteroid or immunemodulatory therapy should be considered.

P316

Swallowing dysfunction in hereditary neuropathy with liability to pressure palsies

R.H.Scola, J. Cardosa, P.J. Lorenzoni, C.S. Kay, E.A. Fugmann, W. Marques Jr., C.E. Silvado, L.C. Werneck

Universidade Federal do Paraná (Curitiba, BR); Universidade de Sao Paulo FMRP (Ribeirao Preto, BR)

We report a 41-year-old-man who presented with a history of repetitive peripheral nerve paralysis since infancy and had started to experience sudden episodes of severe dysphagia during the previous year.Videoendoscopic and fluoroscopic examination revealed pharyngeal dysfunction. Barium esophagography was compatible with symmetrical hypotonia of the esophagus. Hereditary neuropathy with liability to pressure palsies (HNPP) was diagnosed by electrophysiological and molecular genetic testing. We suggest that swallowing problems may be a clinical manifestation of HNPP.

\section{P317}

Clinical and electrophysiological phenotypes in Charcot-Marie-Tooth disease type $1 \mathrm{~A}$ with $17 \mathrm{p} 11.2$-p12 duplication

S.J. Hwang, B.O. Choi, S.Y. Cho, J.Y. Kwon, H.R. Yang, K.H. Kim, J.S. Shin, J.Y. Yoon, S.W. Park

Ewha Womans University (Seoul, KR); Kongju National University (Gongju, $\mathrm{KR})$

Objective: Charcot-Marie-Tooth disease type 1 (CMT1A) with 17p11.2-p12 duplication accounts for about $70 \%$ of all cases of CMT type 1 . Most study groups suggested that weakness and sensory loss were correlated with decreased action potential, but not with slowed NCV; however, a few groups said that the decreased nerve conduction velocity (NCV) is correlated with the clinical severity;. Therefore, we investigate to find the correlation between the clinical disability and motor NCV slowing or decreased amplitudes in Korean CMT1A patients with 17p11.2-p12 duplication.

Methods: We enrolled 234 subjects of 109 unrelated CMT1 families of Korean origin. In addition, 200 healthy controls were recruited. Motor and sensory conduction velocities of median, ulnar, peroneal, tibial, and sural nerves were determined. Disease severities were measured by CMT neuropathy score (CMTNS) and functional disability scale (FDS).

Results: Among CMT type 1 patients, 168 patients from 73 families contained duplication (67\%), which is in accordance with the previous reports. Heel-gait defect was significantly reduced than toe-gait defect $(\mathrm{p}<0.001)$. The age at onset was not correlated with clinical or electrophysiological findings. We found that even siblings within the same families demonstrate the different disease severity and electrophysiological features. One of the most important modifying factors for disease severity demonstrated in this study was disease duration. Median nerve compound muscle action potentials (CMAPs) were well correlated with disease duration while motor NCVs were not related with disease duration. Moreover, CMAPs were found to be significantly correlated with corresponding CMTNS and FDS scales, while the motor NCVs did not correlated. Also, we found that the sensory impairment 
were correlated well with corresponding reductions in SNAPs, but not with slowing of sensory nerve conduction.

Conclusion: In the present study, the Korean CMT1A patients with 17p11.2-p12 duplication demonstrated similar clinical and electrophysiological phenotypes of the previous reports from other ethnic groups. We concluded that weakness and sensory loss were correlated with decreased CMAPs, but not with slowed NCVs.

\section{P318}

Correlation of clinical outcomes and disease burden in patients with transthyretin (TTR) amyloid polyneuropathy: study Fx-005, a landmark clinical trial of Fx-1006A, a novel small molecule TTR stabiliser

T. Coelho, M. Waddington-Cruz, V. Planté-Bordeneuve, D. Cros, D. Roy Grogan, J. Packman

Hospital Santo Antonio (Porto, PT); Hospital Universitário Clementino Fraga Filho (Rio De Janeiro, BR); CHU de Créteil (Paris, FR); Massachusetts General Hospital (Boston, US); FoldRx Pharmaceuticals, Inc. (Cambridge, US)

Objectives: Fx-1006A is a novel, selective and potent stabiliser of wild-type and variant transthyretin (TTR), that may confer disease modification and halt disease progression in patients with TTR amyloidosis. Study Fx-005 is evaluating the safety and efficacy of once-daily $\mathrm{Fx}-1006 \mathrm{~A}$ versus placebo in V30M TTR polyneuropathy (ATTR-PN, also known as familial amyloid polyneuropathy). Primary and secondary endpoints will be correlated and analyzed over time to assess disease progression.

Methods: Fx-005 is a landmark Phase II/III randomised, double-blind, placebo-controlled, international, multicenter 18 month study. Currently there are no validated clinical endpoints for ATTR-PN, therefore, endpoints validated for diabetic neuropathy are being utilized. Co-primary endpoints are the Neuropathy Impairment Score-Lower Limb (NIS-LL), an assessment principally of large fibers, and the Norfolk Quality of Life-Diabetic Neuropathy (QOL-DN), which assesses large fiber, small fiber, and autonomic neuropathy. Secondary assessments include quantitative sensory testing, nerve conduction studies, and heart rate response to breathing. This is the first interventional study to report on these endpoints in ATTR-PN patients, and Baseline data are being analyzed to correlate various clinical endpoints and other measures of disease.

Results: A total of 128 patients (70 women) were randomised. Mean age at Baseline was 39.6 (SD 12.8) years. Mean time from symptom onset was 2.6 years. Preliminary blinded mean Baseline NIS-LL and Norfolk QOL-DN scores were 9.3 (SD 11.8) and 29.3 (SD 25.5), respectively. NIS-LL and Norfolk scores correlate with time from symptom onset $(\mathrm{r} 2=0.1644$ and 0.2844 , respectively; $p>0.0001)$, and with each other $(r 2=0.3268 ; p>0.0001)$. Thus, as Norfolk QOL-DN scores worsen, NIS-LL scores worsen. However, Norfolk QOL-DN only explains $32.6 \%$ of the variability in the NIS-LL, suggesting that each measures different aspects of disease and both are important measures of treatment effect. Correlations between secondary and primary endpoints will also be presented.

Conclusions: This is the first reported evaluation of NIS-LL and Norfolk QOL-DN and their correlation to length of disease in this patient population. Preliminary data indicate that NIS-LL and Norfolk QOL-DN correlate well, assess different important aspects of disease, and will measure disease progression in this interventional trial with Fx-1006A (results expected mid 2009).

This study was fully funded by FoldRx Pharmaceuticals, Inc.

\section{P319}

THAOS - Transthyretin Amyloidoses Outcomes Survey, a new global, webbased registry

V.Planté-Bordeneuve, O. Suhr, T. Coelho, R. Labaudiniere, J. Packman, D. Roy Grogan

CHU de Créteil (Paris, FR); Umeå University Hospital (Umeå, SE); Hospital Santo Antonio (Porto, PT); FoldRx Pharmaceuticals, Inc. (Cambridge, US)

Objectives: Available data suggest variability in the natural history of TTR amyloidosis (ATTR), particularly between genotypes and geographic locations. The principal aims of the survey are to better understand and characterize the natural history of TTR amyloidosis by studying a large and heterogeneous patient population. Survey data may be used to develop new treatment guidelines and recommendations, and to inform and educate clinicians about the management of this disease. The ultimate goal is to better understand, manage and treat patients with TTR amyloidosis.

Methods: THAOS is a new global, multi-center, longitudinal observational survey (registry) designed to characterize the variability, progression, and natural disease history, regional differences in disease expression, and the genotypic-phenotypic relationship in TTR amyloidosis. Both symptomatic and asymptomatic patients with wild-type or variant TTR disease will be enrolled and followed in this registry for up to 10 years. It is anticipated that several thousand patients will be enrolled across 50 or more survey sites. Patients will be followed as long as they are able to participate. The frequency and type of clinical and laboratory assessments will be performed according to the physician's standard of care. Patients will continue to receive their current medications and all other standard care for their disease. Physical assessments will include cardiac and neurologic examinations and renal/bladder function. Quality of life will be assessed by EQ-5D and Norfolk-QOL-DN questionnaires. Hospitalizations and medication use, and transplant history will also be recorded. The registry is web-based, with ongoing data analysis and publications coordinated by the THAOS Scientific Board, an international group of amyloid experts. Data collected in THAOS will be complimentary to data collected in the well-established Familial Amyloid Polyneuropathy World Transplant Registry. The THAOS database, a secure interactive web-based system, is designed to be an intuitive tool for each Investigator to enter and review data for their patients at any time during the survey.

Results: Initiation of sites in a number of countries has begun and patient enrollment has commenced.

Conclusions: Throughout the 10-year duration of this registry, data will be analyzed and reported at least annually. Any physician who cares for these patients is invited to participate. Initial data are expected in late 2008.

This study was fully funded by FoldRx Pharmaceuticals, Inc.

\section{Multiple sclerosis}

\section{P320}

A case-control study of environmental risk factors of multiple sclerosis in Iran

A.A. Divani, H. Pakdaman, M. Asadollahi, M. Haghighat-Shoar, G. Vazquez, M. Etemadifar, K. Gharegozli, S. Karkherian, R. Akbari, S.D. Cook

University of Minnesota (Minneapolis, US); Shahid Beheshti University of Medical Sciences (Tehran, IR); Isfahan University (Isfahan, IR); University of Medicine and Dentistry of New Jersey (Newark, US)

Objective: Multiple sclerosis (MS) is a progressive autoimmune disease of the central nerves system with a high morbidity rate. Both environmental and genetic factors have been shown to be associated with etiology of MS. The objective of this study was to assess the putative risk factors associated with MS among patients in Iran.

Methods: We conducted a retrograde case-control study of 395 MS subjects ( $78.7 \%$ women) and their childhood friends ( $61.4 \%$ women) that were used as controls. The mode of recruitment was either from practicing neurologists or the MS societies established in different cities in Iran. Using a designed questionnaire in an in-person interview, information regarding demographics, family history, ethnicity, place of birth, education, occupation, history of infection diseases (Measles, Mumps, Chicken Pox, Rubella, Hepatitis), vaccination, history of surgical procedures, contact with domestic animals, and daily sunlight exposure. Time of symptoms onset, time of diagnosis, MS subtype, method of diagnosis, and lesions locations were collected from each subject's medical record. The effect of different risk factors on likelihood of MS was studies using a conditional linear regression model.

Results: The average age of MS subjects and controls were $30.9 \pm 8.9$ and $31.0 \pm 10.1$ years, respectively. After adjusting for sex and age, smoking was associated with the risk of acquiring MS $(\mathrm{OR}=1.8$, CI: 1.0,3.2). The rest of the data were adjusted for age, sex, and smoking before comparison. There was a significant association between exposure to domestic animals $(\mathrm{p}=0.003, \mathrm{OR}=3.1, \mathrm{CI}: 1.5,6.6)$ and farm animals $(\mathrm{p}=0.4, \mathrm{OR}=8.2$, CI:1.0, $6.8)$ and incidence of MS. When studied separately, contracting Measles $(\mathrm{OR}=1.6, \mathrm{CI}: 1.1,2.2)$, Mumps (OR $=1.6, \mathrm{CI}: 1.1,2.3)$, and Rubella $(\mathrm{OR}=3.0$, CI: 1.5, 6.0) increased the odds of acquiring MS. Any combination of infectious diseases also increased the odds of acquiring MS ( $p=0.03, \mathrm{OR}=1.4, \mathrm{CI}$ : $1.0,1.9)$.

Conclusion: We performed a case-control study of MS to investigate the role of different putative environmental risk factors to better understand the etiology of MS in Iran. The present study revealed that smoking, exposure to domestic and farm animals, and exposure to different infectious diseases may in crease the risk of acquiring MS among Iranian population. However, further studies with larger sample size are needed to better elicit environmental risk factors associated with MS in this particular population. 
P321

Medication gaps in disease-modifying drug therapy for multiple sclerosis are associated with an increased risk of relapse: findings from a national managed care database

A. AL-Sabbagh, R. Bennett, C. Kozma, M. Dickson, D. Meletiche

EMD Serono, Inc. (Rockland, US); University of South Carolina (Columbia, US)

Objective: Multiple sclerosis (MS) patients may decline treatment or become nonadherent with disease-modifying drugs (DMDs), which may result in a return of their symptoms. This study evaluated the relationship between medication gaps and the incidence of severe MS relapses.

Methods: Patients with at least 1 claim for interferon beta-1a intramuscular (IM), interferon beta-1b, interferon beta-1a subcutaneous (SC), or glatiramer acetate from January 1, 2000 through December 31, 2004 were identified from the PharMetrics (Watertown, MA) database. Patients continuously eligible for 24 months following their first DMD prescription (index data) and 6 months before the index data (preperiod) were included in this study. Patients who were younger than 18 years of age or older than 65 years of age, had received natalizumab after the index date, used a study drug in a healthcare facility, or resided in a long-term care facility were excluded from the study. A severe relapse was defined as an MS-related hospitalization or emergency room visit. The maximum gap in therapy (Maxgap) represented the longest continuous period with no evidence of study drug availability based on dispensing data and days' supply. Maxgap was categorized as 0-10 days, 11-89 days, and 90+ days. Covariates included age, gender, region, treatment status (new or existing), comorbidities, and therapy type (mono- or multi-therapy).

Results: 2388 patients met the study criteria (mean age, 43.9 years; women, $76.4 \%$; new patients, $76.7 \%$ ). During the 24 -month study period, $8.1 \%$ of patients had at least 1 severe MS relapse. A significantly increased risk of severe relapse was found for the $90+$ day Maxgap group compared with the $0-10$ day Maxgap group (odds ratio $[\mathrm{OR}]=1.925 ; \mathrm{P}=0.007$ ). Monotherapy with interferon beta-1a IM, interferon beta-1b, interferon beta-1a SC, or glatiramer acetate was associated with a reduced risk of severe relapse (ORs between 0.450 and 0.552 ). Other significant covariates were comorbidity and East region of the United States (Midwest reference) (ORs $=1.090$ and 1.495, respectively). Age, sex, and the other regions were not significant at alpha $=0.05$.

Conclusion: Gaps in DMD therapy in excess of 90 days were significantly associated with a higher risk of severe MS relapse when compared with shorter or no gaps in DMD therapy.

Study was supported by EMD Serono, Inc. and Pfizer Inc.

P322

A blood-based gene expression assay that can identify patients with multiple sclerosis

B. Greenberg, D. Bigwood, E. Eastman

Johns Hopkins School of Medicine (Baltimore, US); Diogenix (Gaithersburg, US)

Objectives: To determine if a unique gene expression signature exists in patients with multiple sclerosis. Given the variability of clinical presentations and the difficulty of making accurate diagnoses, a blood-based assay would be extremely helpful to clinicians in evaluating patients for possible MS. This study evaluated patients with known MS, other autoimmune diseases and case controls with no known disorders to determine if such agene expression signature could be identified.

Methods: In order to investigate the feasibility of developing a commercially viable blood-based MS molecular diagnostic test, blood samples (PBMCs) from 11 patients with MS, 4 patients with systemic lupus erythematosus (SLE) and 8 case controls were selected for analysis. [The MS patients were selected because they were not receiving any MS-related therapy at the time of sample collection and they had no other major complicating diseases or conditions. Not really true] Likewise, the control subjects had no known diseases. [No autoimmune or related disease but I need to check again to see what other types of diseases they might have had] RNA was isolated and used to examine gene expression profiles on the Affymetrix GeneChip ${ }^{\circledR}$ microarray platform (HGU133 Plus 2.0). A t-test was performed which yielded a number of genes that were significantly differentially expressed between test groups.

Results: A total of 339 gene fragments were significantly differentially expressed between the MS and case control samples at a false discovery rate corrected p-value of 0.001 . When this list was filtered to include only those genes showing a fold change of 2.0 or greater between the two groups, the number of gene fragments dropped to 102 . When an even more conservative p-value correction was applied (the Bonferroni correction), the list was fur- ther reduced to 39. A distinct gene fragment list could also be used to accurately differentiate MS patients from those with SLE.

Conclusions: Based on this introductory study a gene expression signature may exist that would differentiate patients with MS from controls and from those with other autoimmune conditions. A large scale validation study examining patients with CIS and early MS is needed and is currently underway. If validated, this assay would represent a major breakthrough in the diagnosis and management of MS patients.

This research was funded by Genelogic, Inc and Diogenix, Inc. both of whom have commercial interests in the assay.

\section{P323}

Comparison of adherence and persistence in patients initiating diseasemodifying therapy for multiple sclerosis

D. Meletiche, T. Shea, F. Wood, B. Shim, E. Becker, R. Bennett, A. AL-Sabbagh EMD Serono, Inc. (Rockland, US); IMS Health Incorporated (Plymouth Meeting, US); PharMetrics, Inc. (Watertown, US)

Objective: Successful treatment of multiple sclerosis (MS) requires adherence to disease modifying therapy (DMT). This study evaluated adherence and 12-month persistence rates of patients initiating 1 of 4 DMTs for MS in a commercially insured population.

Methods: The study population consisted of patients aged 18-64 years who initiated interferon (IFN) beta-1a subcutaneous (SC), IFN beta-la in tramuscular (IM), IFN beta-1b, or glatiramer acetate (GA) during the period from January 2, 2004 to July 5,2005. Using an administrative claims database (PharMetrics, Watertown, MA), patients were identified who had continuous health-plan enrollment for $\geq 12$ months before and $\geq 15$ months after their first MS prescription. The proportion of patients with a medication possession ratio (MPR) > $85 \%$ (appropriate adherence) and 12-month persistence rates (proportion of patients with drug therapy at month 12 without a lapse of therapy $>90$ days) were evaluated for each of the 4 DMTs. Treatment comparisons were evaluated using Wilcoxon rank sum (continuous variables) and chi-square tests (dichotomous variables).

Results: Between January 2, 2004 and July 5, 2005, a total of 3195 patients initiated treatment with IFN beta-1a SC $(n=799)$, IFN beta-1a IM $(n=905)$ IFN beta-1b $(n=344)$, or GA $(n=1147)$. Gender, geographic region, health plan, and product types were similar across all treatment groups. Mean age was significantly higher in the IFN beta-1a IM group vs the IFN beta-1a SC and GA group ( 44.9 vs 43.5 and $43.8 \mathrm{y}$, respectively; $\mathrm{P}<0.01$ ), but was not significantly different from the IFN beta- 1 b group ( $44.4 \mathrm{y}$ ). Adherence (MPR $\geq 85 \%$ ) was significantly higher in the IFN beta-1 a SC group vs the IFN beta1 broup $(49.7 \%$ vs $39.8 \% ; \mathrm{P}=0.002)$, but was not significantly different vs the GA $(45.7 \%)$ or IFN beta-1a IM (48.1\%) groups. Significantly higher persistence rates were observed in patients receiving IFN beta-1a SC $(60.3 \%)$ compared with IFN beta- 1 a IM and IFN beta- 1 b ( $54.9 \%$ and $52.9 \%$, respectively; $\mathrm{P}<0.03$, for both), but not compared with GA $(60.5 \%$; $\mathrm{P}=0.936)$.

Conclusion: In a commercially insured population, demographic characteristics, with the exception of age, were comparable among patients initiating IFN beta-1a SC, IFN beta-1a IM, IFN beta-1b, and GA. The highest persistent rates to therapy were observed with IFN beta-1a SC and GA, although differences in adherence were less pronounced.

This study was supported by EMD Serono, Inc. and Pfizer Inc.

P324

Rapid onset central motor plasticity is not compromised in multiple sclerosis patients

D. Zeller, K. Aufm Kampe, K. Stefan, R. Gentner, K. V. Toyka, P. Rieckmann, J. Classen

University of Wurzburg (Wurzburg, DE); University of Rostock (Rostock, $\mathrm{DE})$

Multiple sclerosis (MS) is an inflammatory demyelinating disease which also leads to substantial axonal injury. Neuronal plasticity is believed to represent an important substrate for recovery from and compensation of motor deficits resulting from demyelination and axonal lesions. The ability of the central nervous system to undergo plastic changes may have an important influence on the relationship between MS pathology and its clinical expression. Although chronic regional reorganization has been demonstrated in MS patients, it remains unknown if the mechanisms and the capacity to express central motor plasticity in MS patients are compromised in the short term. In the present study, we examined rapid onset central motor plasticity, and its relationship to motor impairment in 22 patients with MS (age $36.6 \pm 10.7$ years; 16 women; EDSS $2.3 \pm 1.2$ ). Motor plasticity was studied both neurophysiologically and behaviourally, at least 6 months after the last 
relapse. We used paired associative stimulation (PAS) which combines repetitive electric stimulation of the median nerve with subsequent transcranial magnetic stimulation (TMS) of the contralateral motor cortex. PAS models long-term potentiation of synaptic efficacy in the human primary motor cortex and thus may tap into an important physiological mechanism underlying neuronal plasticity. Additionally, dynamic motor learning was tested by an isometric force production task. Motor impairment was assessed neurophysiologically by the corticomuscular latency to the abductor pollicis brevis muscle as tested by TMS, and by a battery of five functional motor tests. MS patients had significantly longer corticomuscular latencies and performed significantly worse than healthy controls in a weighted measure of functional motor tests. Changes in corticospinal excitability induced by PAS were comparable between MS patients and controls. Baseline-adjusted training-induced motor performance increased similarly in patients and controls. Neither PAS-induced plasticity nor motor learning performance correlated with motor impairment. Comparing good and poor motor performers within the patient group, there were no significant differences with respect to either PAS-induced increases of cortical excitability or motor learning success. The present findings support the notion that, despite significant motor impairment, rapid motor system plasticity remains intact in MS patients, both neurophysiologically and behaviorally.

Support by Hertie-Foundation is gratefully acknowledged.

\section{P325}

Immunogenicity of Rebif ${ }^{\circledR}$ New Formulation (interferon beta-1a) in patients with relapsing multiple sclerosis: results from a phase IIIb study G. Giovannoni, O. Barbarash, F. Casset-Semanaz, J. King, L. Metz, G. Pardo, J. Simsarian, P. Soelberg Sørensen, B. Stubinski on behalf of the Rebif ${ }^{\circledR}$ New Formulation study group

Objective: Investigate the immunogenicity of Rebif ${ }^{\circledR}$ New Formulation (interferon [IFN] beta-1a) in patients with relapsing multiple sclerosis (MS). Data (neutralizing antibody [NAb] status) were compared with historical data from the EVIDENCE (EVidence of Interferon Dose-response: European North American Comparative Efficacy) study and recent results from the REGARD (REbif versus Glatiramer Acetate in Relapsing MS Disease) study with the current Rebif ${ }^{\circledR}$ formulation.

Methods: A Phase IIIb, 96-week, single-arm, multicentre, open-label study of Rebif ${ }^{\circledR}$ New Formulation ( $44 \mathrm{mcg}$ subcutaneously three times weekly) in patients aged 18-60 years with relapsing MS (McDonald criteria) with an Expanded Disability Status Scale score $<6.0$. Serum anti-IFN NAb status, measured by virus cytopathic effect assay, was determined at baseline, every 12 weeks up to week 72 , and at week 96 . NAb titres of $\geq 20$ neutralizing units $(\mathrm{NU}) / \mathrm{mL}$ were considered positive.

Results: At week 96, the proportion of NAb-positive patients (last observation carried forward [LOCF]; primary endpoint) was $17.4 \%$; corresponding values in the REGARD and EVIDENCE studies were $27.3 \%$ and $21.4 \%$, respectively, and $24.3 \%$ in a pooled estimate of both comparator studies using a fixed-effects model. An a posteriori sensitivity analysis was performed using an alternative imputation method to LOCF for missing values to account for variations between studies in the proportion of patients missing the week-96 assessment: the estimated proportion of patients NAb-positive at week 96 was $18.9 \%$, compared with $28.3 \%$ in the REGARD study and $23.9 \%$ in the EVIDENCE study. These estimates were similar to those from non-LOCF sensitivity analyses at week 96 , which excluded missing values, with estimates of $19.2 \%$ (Rebif ${ }^{\circledR}$ New Formulation), $28.8 \%$ (REGARD) and $23.2 \%$ (EVIDENCE). The proportion of patients anytime NAb positive during the 96-week Rebif ${ }^{\circledR}$ New Formulation study was $18.9 \%$, versus $33.7 \%$ (REGARD) and 27.1\% (EVIDENCE). The pooled estimate for both comparator studies was $30.4 \%$.

Conclusions: Although the full impact of NAbs to IFN in clinical practice is unclear, high titres $(\geq 200 \mathrm{NU} / \mathrm{mL})$ have been associated with decreased IFN beta efficacy. Based on indirect comparisons with data from the EVIDENCE and REGARD studies, Rebif ${ }^{\circledR}$ New Formulation, which is produced without the use of foetal bovine serum and is without human albumin as a formulation excipient, has reduced immunogenicity compared with the current formulation of Rebif ${ }^{\circledR}$.

Study supported by Merck Serono International S.A.
P326

Advanced directives' knowledge and performance-related factors in multiple sclerosis patients

G. Miró Andreu, R. Martínez Poveda, A. Cano Orgaz, J.F. Solsona Durán, M. Solsona Perlasia, J.C. Yébenes Reyes, G. Portugués Martínez, M. Serra Prat Hospital de Mataró (Mataró, ES); Hospital del Mar (Barcelona, ES)

Objectives: Advanced directives (AD) are legal in Spain since 2003. Our goal was to evaluate the knowledge of such AD in Multiple Sclerosis's (ME) patients, and to identify related factors.

Protocol: A multiple choice questionary was submitted to EM patients in two centers. Demographic, clinical, disability (EDSS) and quality of life data were collected. Patient's knowledge of eventual EM evolution, illness severity perception, medical determination involvement, hypothetic poor quality of life tolerance, previous conversations about these topics, $\mathrm{AD}^{\prime}$ s knowledge and related factors were evaluated.

Results: 174 EM's patients were included: mean age 45.1 , female $64.9 \%$, elemental studies $37.4 \%$, labour disabilities $42.5 \%$. Average EM's evolution time was 7.9 years, and $48.9 \%$ of them required hospital attendance. Mean EDSS was 3.5, and quality of life was 66.7 over 100 (the better one). Karnofsky's scale average was 75.1 .

$90.4 \%$ of patients thought that EM's was a chronic illness, and $77.1 \%$ that it was a severe one ( 6.2 over 10 , that it's maximum severity). $79.5 \%$ assumed that won't be able to autoserve themselves, and $21.1 \%$ that EM would be death cause. $75.9 \%$ had a good knowledge of their illness (chronic and high disability risk terms).

$83.1 \%$ like to involve themselves in own health decisions. Just $1.8 \%$ would accept living in a coma, $5.4 \%$ with mechanical ventilation, $9.6 \%$ with tube feeding, and $19.9 \%$ in state of total dependence. $29.5 \%$ have previously expressed their purposes, mostly with family.

$59.6 \%$ of requested know about $\mathrm{AD}$, and $2.2 \%$ have redacted one previously. Opinion concerning $\mathrm{AD}$ is quite good: $64.5 \%$ would redact one for themselves, and $82.6 \%$ would delegate somebody taking decision in case of incompetence. Only $14.5 \%$ showed uncomfortable after finishing questionary.

AD knowledge was related with studies level $(\mathrm{p}<0.001)$, severity perception $(p=0.043)$ and an accurate illness information $(62.5 \%$ vs. $38.2 \%$; $\mathrm{p}=0.004)$. Patients who believed that own health determinations, were assumed by doctors, had less knowledge about $\mathrm{AD}$ (37.4\% vs $62.7 \%$; $\mathrm{p}=0.001$ ).

Conclusions: $59.6 \%$ requested patients know $\mathrm{AD}$, related to formation level, severity perception, own's illness knowledge and medical determinations involvement. There is a high patient's aptitude in own's health determinations, a good acceptance in dialoguing in matters related to disabilitydependence and life ending that doesn't generate uncomfort situations, even thougth daily practice doesn't ocurr.

Fundacio Marato TV3

\section{P327}

Correlation of the chronic pain with clinical characteristics of multiple sclerosis

I. Marjanovic, J. Kostic, N. Stojsavljevic, I. Dujmovic-Basuroski, G. Toncev, M. Gavric, T. Pekmezovic, J. Drulovic

Clinical Centre of Serbia (Belgrade, RS); Clinical Centre Kragujevac (Kragujevac, RS); General Hospital Uzice (Uzice, RS)

Objective: The purpose of the study was to examine possible correlation between the chronic pain and different clinical characteristics in patients with multiple sclerosis (MS).

Methods: A group of 83 consecutive patients from the outpatient settings, who met Revised McDonald criteria (2005) for MS were enrolled into this cross-sectional study. We used a structured interview for registration of pain and sensory symptoms (acute and chronic pain syndromes), Visual Analog Scale (VAS), Hamilton Anxiety Scale (HAS) and Hamilton Depression Rating Scale (HDRS). The Spearman's and Pearson's correlation coefficients were used for data analysis.

Results: A total of 83 MS patients, 22 men and 61 women were included. The mean age at examination was $41.8 \pm 9.1$ years. The duration of the disease was $10.4 \pm 6.6$ years. Mean EDSS was $4.0 \pm 1.9$. The course of the disease was relapsing-remitting in $60(72.3 \%)$ patients, secoundary progressive in $18(21.7 \%)$ and primary progressive in $5(6.0 \%)$. On examination 68 $(81.9 \%)$ patients reported the existence of pain during the course pf the disease. The chronic pain was present in $32(38.6 \%)$ cases. According to the VAS intensity of pain was $4.9 \pm 2.2$ in average. Sixty two percent of the patients reported that pain and sensory complients had important influence on their daily activities. Significantly positive correlations were found between chronic pain and following variables: mean age $(\mathrm{p}<0.01)$, course of disease 
$(p<0.05)$, duration of MS, EDSS score $(p<0.05)$, HDRS and HAS scores $(\mathrm{p}<0.01)$, VAS $(\mathrm{p}<0.01)$.

Conclusion: Our results confirmed the high frequency of chronic pain in patients with MS and its influence on their daily activities.

P328

Development of ICF core sets for multiple sclerosis

J. Kesselring, M. Coenen, A. Cieza, N. Kostanjsek, G. Stucki

Valens Rehabilitation Centre (Valens, CH); ICF Research Branch WHO CC FIC (Munich, DE); World Health Organization, WHO (Geneva, CH); Department for Physical Medicine and Rehabilitation (Munich, DE)

Objectives: With the approval of the WHO's International Classification of Functioning, Disability, and Health (ICF) there is now an universally accepted framework to classify and describe functioning, disability and health in individuals with health conditions. The ICF is a very comprehensive classification with 1424 so-called ICF categories. For its application in clinical practice tools, such as ICF Core Sets, are needed. ICF Core Sets are agreedon lists of ICF categories relevant for the description of functioning of individuals with a specific health condition. ICF Core Sets for Multiple Sclerosis (MS) were developed to link this condition to salient ICF categories. The objective of the project is the development of ICF Core Sets for MS to specify functioning and disability of individuals with MS.

Methods: The ICF Core Sets for MS were decided during an international consensus conference according to a multistage and established decision process which integrates evidence from four preparatory studies: a) an empirical study involving $200 \mathrm{MS}$ patients, b) a systematic review of parameters reported in MS research, c) an expert survey including health professionals involved in the treatment of MS patients and d) a qualitative study performed with individuals with MS.

Results: The ICF categories that constitute the ICF Core Sets for MS were identified in an iterative decision-making and consensus process with discussions and voting. Since the requirements to describe functioning in MS are different for a comprehensive multidisciplinary clinical assessment and a research setting, Comprehensive and Brief ICF Core Sets for MS were developed. The Comprehensive ICF Core Set for MS includes as few categories as possible to be practical, but as many as necessary to be sufficiently comprehensive to describe the prototypical spectrum of limitations of functioning in a multidisciplinary assessment. Instead, the Brief ICF Core Set for MS includes as few ICF categories as possible to be practical, but as many as necessary to be sufficiently comprehensive to describe the prototypical spectrum of limitations of functioning in clinical studies.

Conclusion: With the ICF Core Sets for MS we can now rely on an international standard of ICF categories to be relevant in patients with MS to specify functioning. It is expected that the ICF Core Sets for MS will profoundly influence the treatment and rehabilitation of MS patients, clinical practice and research studies.

The project is being funded by Hertie Foundation.

\section{P329}

\section{MS Register in Germany: update 2007}

K. Stuke, P. Flachenecker, U. Zettl, W. Elias, M. Freidel, J. Haas, D. PitschnauMichel, S. Schimrigk, P. Rieckmann

MS Forschungs-u. Projektentw.-gGmbH (Hannover, DE); Neurological Rehabilitation Center Quellenhof (Bad Wildbad, DE); University of Rostock (Rostock, DE); Neurological Practioner (Hamburg, DE); Neurological Practioner (Kaltenkirchen, DE); Jüdisches Krankenhaus Berlin (Berlin, DE); Klinikum Ludenscheid (Ludenscheid, DE); University of British Columbia and Vancouver Coastal Health (Vancouver, CA)

Objectives: In order to monitor the health care situation of persons with MS in Germany, the German MS Society (DMSG Bundesverband e.V.) initiated a national multiple sclerosis (MS) register in 2001. This project aims at collecting epidemiological data, disease characteristics, and information on the health care situation of MS patients from different levels of health care all over the country.

Methods: The minimal data set has been agreed on in consensus with leading MS experts in Germany and its applicability has been studied in 5 MS centres in a 2-year pilot phase that has resulted in its modification. In the extension phase of the project the number of documenting centres steadily rises from 5 in the pilot phase, 15 in 2005, 57 in 2006 to 86 in 2007. As to 15 . January 200890 centres participate (41 neurological hospitals, 15 rehabilitation units, 17 specialized practitioners, and 17 regional MS centres). At the end of 2007 a total of 10,924 standardised data sets have been recorded. Data sets of 10,108 patients $(92.5 \%$ ) passed a 2 -stage quality check controlling for completeness and inconsistencies.
Results: The demographics were similar to those obtained from other epidemiological studies: $71 \%$ of the patients were female, mean age was $44.2 \pm 11.7$ years, mean disease duration was $13 \pm 9.5$ years, and $54 \%$ suffered from the relapsing-remitting form of the disease. Median EDSS was 3.5 and $26 \%$ of patients had an EDSS $\geq 6.0 .70 \%$ of patients at the age of 50 years and $50 \%$ of patients at the age of 60 years scored less than EDSS 6.0. Mean duration from the onset of the disease until diagnosis was 3.2 years. Nearly one third of the patients were prematurely retired due to MS. Early retirement was associated with physical disability, but a considerable proportion of patients were prematurely retired although they were still able to walk independently. The majority of patients received immunomodulatory drugs, whereas a significant proportion of patients was not treated with symptomatic therapies despite existing impairments.

Conclusion: Within a relatively short time-period the extension of the MS register resulted in a broad registration of MS patients from all over the country and in one of the largest data collections worldwide. The register provides valuable data which allow for a reliable estimate on the health care situation of MS patients in Germany. These data may help to improve service provision and overall quality of life for MS patients.

The project is funded by Deutsche-Multiple-Sklerose Stiftung, the official foundation of the German MS Society. Donations to the foundation, e.g. from individuals, organisations or pharmaceutical industry do not exert influence on the project's contents.

\section{P330}

Changes in endothelial cell activation occur in multiple sclerosis lesion formation

N.J. Gutowski, J.E. Holley, P. Eggleton, J.L. Whatmore

Royal Devon and Exeter Foundation Hospital (Exeter, UK); Peninsula Medical School (Exeter, UK)

Ongoing damage and repair take place in multiple sclerosis (MS). In normal wound healing, angiogenesis (new blood vessel formation from pre-existing vessels) occurs in areas undergoing repair. During angiogenesis, endothelial cells lining blood vessels become activated and secrete factors that affect other cells. Astrocytes directly contact blood vessels and are the main cells in chronic lesions where they have a characteristic scar phenotype. MS lesions are often associated with blood vessels which suggest that lesion formation is related to interactions between astrocytes and blood vessels.

Objective: We have previously shown increased blood vessel density in MS cerebral white matter, this study investigated endothelial cell activation.

Methods: Post-mortem sections of cerebral white matter were taken from normal control $(n=5)$ and MS normal appearing white matter $(n=9)$, acute $(n=9)$, subacute $(n=8)$ and chronic $(n=12)$ lesions. Sections were stained for CD105 (expressed on activated endothelial cells) and Gal C (myelin) by double enzymatic immunohistochemistry. The numbers of blood vessels containing activated endothelial cells were quantified by counting vessels in three random fields for each tissue type.

Results: Statistical analysis indicated that the numbers of blood vessels with activated endothelial cells is altered at the different stages of lesion development.

Conclusion: This suggests that changes in angiogenesis could be an important factor in scar development in MS.

Biogenidec has given a research grant which has supported this work.

\section{P331}

Effects of medication and cognitive training on alertness function in patients with multiple sclerosis

P. Calabrese, U. Schlegel, L. Kappos, K. Opwis, I.K. Penner

University Hospital Bochum Langendreer (Bochum, DE); University Hospital Basel (Basel, $\mathrm{CH}$ ); University of Basel (Basel, $\mathrm{CH}$ )

Objectives: Cognitive deficits belong to the key symptoms in multiple sclerosis (MS). Among these deficits, disturbances of attention and memory have a strong impact on activities of daily living and quality of life. The aim of our study was to evaluate possible cognition-enhancing effects of interferon beta-1a (IFNb-1a) treatment in combination with cognitive training in MS patients for a 3-month period using a computerized tonic and phasic alertness test as primary outcome measure.

Methods: We studied altogether 19 MS-patients (mean age $=41.26 \mathrm{yrs}$; mean EDSS $=3.29$ ). During the study period patients received IFN-beta-1a treatment. While for 12 patients pharmacological treatment started at the beginning of the study seven subjects were already on medication. The latter group received an attention training over four weeks. All patients were 
investigated on tonic and phasic alertness by a computerized paradigm. The alertness performance was evaluated at baseline and again after 12 weeks.

Results: When analyzing the two patient groups separately, it turned out that the patients already on medication had significantly better performance in both alertness components at baseline ( $\mathrm{p}<0.05)$. In this cohort, the cognitive training over four weeks had an additional favourable effect on the phasic alertness performance. This effect remained stable after three months. Patients who started with IFNb-1a therapy showed a significant improvement in phasic alertness after 3 months of medication.

Conclusion: In conclusion, these data support the hypothesis of an early and significant IFNb-1a benefit on basic attention functions with the most powerful effect on the phasic alertness component. In addition, cognitive training has been shown to be a possible intervention method to support and reinforce effects of pharmacological long-term treatment.

\section{P332}

Neuropsychology of primary progressive multiple sclerosis

R. Paes, R. Papais-Alvarenga, C. Vasconcelos, M.A. Negreiros, J. Landeira on behalf of Neuropsicologia da Esclerose Múltipla-Projeto Atlântico Sul

Background: The cognitive alterations are symptoms of multiple sclerosis (MS), even so in form Primary Progressive Multiple Sclerosis (PPMS) the data are contradictory in the prevalence and to the type of cognitive alteration.

Objective: The objective this study was estimate the frequency of the cognitive alterations in Brazilian patients, with PPMS according to criteria of Thompson (2000) in the Center of Reference for Treatment of the MS in Rio de Janeiro (Brazil).

Patients and methods: We apply a Battery of Neuropsychological tests in 26 patients and 26 controls matched to sex, age and education level. The battery evaluated the following cognitive functions: tracking for dementia, attention/concentration, processing of information, fluency verbal, memory and abstract thinking and the Beck Scales for Mood Disturbances. The analysis staistics was according on the basis of the study of Rao (1991).

Results: In comparison to the controls, $50 \%$ of the patients with PPMS showed cognitive alterations. The recent memory $(60 \%)$, Verbal Fluency ( $40 \%)$ and processing of information were the cognitive functions $(40 \%)$ more frequently impaired. They had revealed more depressed, but we do not find association between the cognitive deficit and depression. The prevalence of cognitive alterations in our PPMS patients show to be high and e we conclude the necessity of magnifying of the study with the increase of the patients.

\section{P333}

NAbs effect on cognitive, clinical and quantitative MRI outcome measures in RR-MS IFN beta-1b treated patients

R. Lanzillo, R. Liuzzi, V. Scarano, A. Prinster, C. Florio, P. Salvatore, V. Schiavone, T. Ferrante, C. Russo, A. Brunetti, B. Alfano, G. Orefice, V. Brescia Morra

Hermitage Capodimonte (Naples, IT); CNR (Naples, IT); Federico II University (Naples, IT); AORN Cardarelli (Naples, IT)

Background: Few trials issued the effect of disease modifying medications and the influence of Neutralizing Antibodies (NAbs) on cognitive functions in multiple sclerosis (MS). We designed an uncontrolled longitudinal study to evaluate, during 3 years, cognitive performance and its relationship with NAbs production, MRI and clinical data in a group of relapsing-remitting MS Interferon (IFN) beta-1b treated patients.

Methods: Complete neuropsychological battery was grouped into attention, information learning/memory, language and visuo-spatial functions and performed every 12 months. Quantitative MRI and Nabs analysis was performed every 6 months.

Results: Fifty-two patients were enrolled in the study. Twelve patients (23\%) dropped out, due to poor compliance or pregnancy. At baseline neuropsychological evaluation we found $54 \%$ normal, $42 \%$ mildly impaired and $4 \%$ moderately impaired patients. At three years follow up cognitive status was stable in $70 \%$ and improved in $30 \%$ of patients. No significant relations were found between global cognitive outcome versus EDSS change, clinical disease activity and MRI data over the three years follow up. Twenty-one patients $(46 \%)$ showed active MRI scans throughout the study, without any worsening at the corresponding neuropsychological examination. Abnormal WM decreased during the study. Eighteen patients developed NAbs during the therapy ( $37 \%)$. Antibodies presence did not affect clinical outcome, but they were found to correlate with cognitive performance and lesion load already at baseline, with cognitive deficit being a risk factor for producing NAbs $(\mathrm{OR}=5)$.

Conclusions: These data suggest that cognitive impaired patients might have a different immunological pattern of disease and as a consequence develop more often NAbs. This trial suggests a possible beneficial effect of IFN beta-1b treatment on cognitive functions in RRMS patients, with NAbs being not detrimental on drug efficacy.

P334

Predicting neuropsychological recommendation in a multiple sclerosis population

S. Kirzinger, J. Jones, S. Chand, M. Nichol, A. Burns

University of Louisville (Louisville, US)

Objective: To identify in an MS Center database items that predict the results leading to recommendations made after Neuropsychological assessments.

Methods: Information obtained from a standard multi-disciplinary assessment battery performed at the Baptist Hospital East MS Center was analyzed for a randomly selected cohort of 120 patients. The standard battery obtained every six months included an Expanded Disability Status Scale (EDSS), 25ft. Timed Walk, Nine Hole Peg Test, Box and Blocks, Tinetti Balance Test, Modified Fatigue Impact Scale (MFIS), Beck Depression Inventory (BDI), Epworth Sleepiness Scale (ESS), and the Neuropsychological assessments consisting of the Neurobehavioral Cognitive Status Examination (CogniStat), the Repeatable Battery for Assessment of Neuropsychological Status (RBANS), and the Multi-Score Depression Inventory (MDI).

Results: The Cerebral Functional Subscale of the EDSS (EDSS-Cog) was the best and only statistically significant predictor of whether the Neuropsychological evaluation would determine a significant finding that would require intervention either medically or with compensatory strategies $(\mathrm{p} \leq 0.017)$. The EDSS-Cog is based on a global assessment of depression and cognitive difficulties manifested by the patient. Only cognitive issues are scored $>1$. Having an EDSS-Cog score over 1 resulted in a significant Neuropsychological evaluation finding $97 \%$ of the time; but $29 \%$ of patients with no EDSS-cog finding did have a significant Neuropsychological finding. Fatigue, although the most common presenting symptom in the cohort at $77 \%$, was not considered in the EDSS-Cog score.

Conclusions: No physical assessment performed in the MS patient cohort predicted the Neuropsychological evaluation recommendations except the EDSS-Cog. Specificity of the EDSS-Cog was very high, but the sensitivity was weak. Within the Neuropsychological evaluation, depression was most predictive of whether intervention was necessary. The Neuropsychological Depression finding was also statistically significantly related to the BDI scores $>12(\mathrm{p} \leq 0.017)$. Unless specifically considered by themselves, cognition and depression do not correlate with the physical manifestations of Multiple Sclerosis.

Grant Support through Bayer Healthcare

P335

Degenerative markers in cerebrospinal fluid in multiple sclerosis patients V. Sládková, J. Mareš, B. Lubenová, K. Kollárová, R. Opavsky, P. Kanovsky University Hospital of Palacký (Olomouc, CZ)

Introduction: Multiple sclerosis (MS) is an autoimmune inflammatory disease of the central nervous system (CNS) leads to demyelination and axonal loss. Cerebrospinal fluid (CSF) examination is the basic examination to considerate inflammation aktivity and degree of degeneration in CNS.

Objective: Determinate the difference in value inflammatory and degenerative markers between several types of MS.

The group and methods: We still examinated 181 patients in accordance with "MS protokol". 78 MS-patients and 83 controls. Group of MS patients evolves 32 woman and 15 men 18-62 years old with disease course from 1 month to17 years. 25 patients with relapsing- remmiting(RR)form, 3 patient with primary (PP), 5 with secondary progressive(SP)form and $11 \mathrm{pa}$ tients with clinical isolated syndrom (CIS). According to "MS protokol" was evaluated markers of integrity the blood-brain barierr (BBB): albumin kvocient (Qalb) and prealbumin, inflammatory markers: CRP, transferin, haptoglobin, beta-2-microglobulin, orosomucoid, markers of tissue destruction: ApoA-I, Apo-B, protein14-3-3, cystatinC, neuron-specific enolasa, tau-protein and beta-amyloid. The intrathecal synthesis was considered according to the number of oligoclonal IgG bands in alkaline fraction in IEF and IgGkvocient (QIgG). We also evaluated alfa-1-antitrypsin, which is considered as protective factor in CNS.

Results: In respect to integrity of BBB the highest value of Qalbumin was recorded only in PP form and matches moderate disorder of $\mathrm{BBB}$. The highest intrathecal synthesis in accordance with QIgG was in patient with PPform. Number of the oligo IgG bands in alcaline fraction in IEF was in PPMS. Inflammatory markers: haptoglobin-the highest value was in PP. Value of beta-2-microglobulin was normal. Orosomucoid was increased in all 
forms, transferin in PP only.Degenerative markers: ApoA-I and tau-protein was increased in all groups, Apo-B in RRpatients only. The value of cystatinC and beta-amyloid is normal. Protein14-3-3 as a marker of hard damage of CNS was not found in any evaluated patients. The higher value of alpha1-antitrypsin was recorded only in PP.

Conclusions: Summary we note increase values of inflammatory markers predominantly in RR-SM and after CIS. Increase of degenerative markers was found in all MSforms, what support hypothesis being of degeneration in early stages of MS. However outcomes are still not definite and doesn't correspond with literature because of since small group evaluated SP-and PP-patients.

\section{Neuro-imaging}

\section{P336}

Structural correlates of motor network dysfunction in primary progressive multiple sclerosis: a 3 T study

A. Ceccarelli, M. Rocca, M. Rodegher, A. Falini, G. Scotti, G. Comi, M. Filippi Scientific Institute San Raffaele (Milan, IT)

Objectives: Using $1.5 \mathrm{~T}$ scanners, previous studies of the sensorimotor system showed that, contrary to other disease phenotypes, patients with primary progressive multiple sclerosis (PPMS) have an increased recruitment of several brain areas outside the classical motor network. Aims of this study were to confirm previous findings using a 3 Tesla $(\mathrm{T})$ scanner and to investigate the correlation between movement-associated functional magnetic resonance imaging (fMRI) changes and measures of global and regional brain tissue damage.

Methods: From 15 right-handed PPMS patients and 15 sex- and agematched healthy volunteers (HV), we acquired dual-echo (DE), diffusion tensor (DT) MRI and fMRI during the performance of a simple motor task. DT tractography was used to calculate DT-derived metrics of the corpus callosum (CC) and bilateral corticospinal tracts (CST). fMRI analysis was performed using statistical parametric mapping.

Results: Compared to HV, PPMS patients had more significant activations of the right primary sensorimotor cortex, the right cingulate motor area, the secondary sensorimotor area (SII), bilaterally, the basal ganglia, bilaterally, the insula, bilaterally, the left parahippocampal gyrus, the cuneus, bilaterally, and the left cerebellum. In PPMS, activation of the left cuneus and the left SII were significantly correlated with DT MRI metrics of damage in the CC and the left CST.

Conclusions: This study confirms that in patients with PPMS movementassociated fMRI changes extend beyond the classical motor network. The analysis of correlations suggest that in these patients regional damage of selected WM tracts influences motor function.

This study was supported by a grant from FISM (Fondazione Italiana Sclerosi Multipla) - contract n. 2003/R/48.

P337

Grey matter changes and cognitive learning: a tensor-based morphometry study

A. Ceccarelli, M. Rocca, R. Gatti, M. Filippi

Neuroimaging Research Unit (Milan, IT)

Objectives: To assess the short-term structural brain grey matter (GM) changes associated with learning cognitive skills.

Methods: Using a 3T scanner, a dual-echo scan and a 3D T1-weighted fast field echo (FFE) sequence were acquired from 32 medical student (HV) $(\mathrm{M} / \mathrm{F}=12 / 19$, mean age $=22$ years $)$ at two different time points (baseline and after two weeks). Medical students were separated into two groups: 13 'learners' medical students, who performed a cognitive learning period of two weeks, and 19 'non-learners' medical student. GM changes were assessed using tensor based morphometry (TBM), as implemented in SPM2 and an Ancova model, corrected for age and sex.

Results: After cognitive learning, the "learners" medical student, compared to "non-learners", had a significant GM volume increase in the left dorsomedial frontal cortex, right orbitofrontal cortex and left precuneus ( $p<0.001)$. Compared to "learners" medical student, "non-learners" did not show any region of significant GM volume change.

Conclusions: Learning of cognitive skills might result in structural GM changes in different brain areas which are part of specific neuronal networks, implicated in cognitive control and retrieval.
P338

Monkey sign in central pontine myelinolysis. A new proposed imaging sign E. Soto-Cabrera, A. Gonzalez, R. Carrillo, J. Santos-Zambrano

National Institute of Neurology (Mexico City, MX)

Objective: To report a new image in pontine myelinolisis, that it looks like a monkey. The Monkey Sign.

Methods: Case Report.

A 17 yo male was admitted to National Institute of Neurology and Neurosurgery in Mexico City due to sudden onset of clumsiness and difficulty with talking. Two weeks previously, he had been admitted to a General Hospital for vomiting and diarrhea. On admission, his serum sodium level was $119 \mathrm{mEq} / \mathrm{L}$. The hyponatremia was treated, and within 24 hours it was corrected to 123 $\mathrm{mEq} / \mathrm{L}$ and after 48 hours to $138 \mathrm{mEq} / \mathrm{L}$. Results of other laboratory studies at that time were as follows: white blood cell count, $17 \geq 109 / \mathrm{L}$; serum urea nitrogen, $18 \mathrm{mg} / \mathrm{dL}$; serum creatinine, $1.0 \mathrm{mg} / \mathrm{dL}$; serum potassium, $3.4 \mathrm{mEq} / \mathrm{L}$ serum chloride, $90 \mathrm{mEq} / \mathrm{L}$; bicarbonate, $21 \mathrm{mEq} / \mathrm{L}$. The serum cultures were negative. After intravenous administration of antibiotics, his vomit and diarrhea resolved, but pronounced axial ataxia were noted. The patient was then transferred to the National Institute of Neurology and Neurosurgery for evaluation of these neurologic findings. At admission, awake, oriented to time, place, and person; and comprehended all commands but had dysarthria. Strength was normal in all extremities. He was clumsy while performing finger-nose and heel-knee maneuvers on both sides. His ability to perform rapid alternating movements was impaired in both hands. Mildly reduced vibration sense was noted at his ankles, but sensations were otherwise normal. Deep tendon reflexes were $3+$ and symmetric, and Babinski sign was negative bilaterally.Magnetic resonance imaging (MRI) of the brain showed a central hypointense area due to edema, that delimited the corticospinal tract on negative T2-weighted images and a decreased signal intensity on T1-weighted images. During the following week, the patient's dysarthria improved, but he still experienced ataxia at the time of discharge.

P339

Delayed neurodegeneration in the pulvinar and medial dorsal nuclei of the human thalami on diffusion-weighted MRI after hypoglycaemic injury

A. Kunizawa, S. Suzuki, Y. Kitazawa, A. Asai, K. Kawamoto, G. W. Ickenstein, B.K. Siesjo, M. Fujioka

Kansai Medical University, Osaka (Hirakata-city, JP); Helios General Hospital (Aue, DE); Lund University (Lund, SE)

Object: We reported a specific change of neuronal death revealed by serial MRI studies in the brains of humans and rats after global/focal brain ischemia and hypoglycaemic coma. The MRI change (delayed and long-lasting shortening of T1 and T2 relaxation times) suggested progressive neuronal death and glial proliferation (astrocyte and microglia) with paramagnetic substance accumulation in the certain localized areas (Ann Neurol (2003) 54:732-747, Stroke (1999) 30:1975-1977, Stroke (1999) 30:1038-1042, Stroke (1999) 30:1043-1046, Stroke (1997) 28:584-587, Stroke (1994) 25:2091-2095). The early clinical and experimental studies including ours have shown particular resistance of the thalamus against hypoglycemic injury. In the current study, we investigated if diffusion-weighted MRI (DWI) could detect late-onset neuronal change in the thalamus of a patient after hypoglycaemic coma.

Methods: A 67-year-old woman suffered from consciousness disturbance due to insulin-induced hypoglycaemia $(18 \mathrm{mg} / \mathrm{dl}$ of blood glucose level on admission). She had no preexisting neurological diseases, and no clinical or laboratory evidence of hypotension, hypoxia, hypothermia, acidosis or epilepsy on admission. We repeatedly performed neuroimaging studies in the patient within 3 months after hypoglycaemic coma.

Results: MRI revealed delayed (from 1 week after onset) hyperintensity and hypointensity on T1- and T2-weighted images, respectively, in the bilateral striatum and cerebral cortex but no signal changes in the thalamus within 3 months after hypoglycaemia. However, in the thalamus that is considered resistant against hypoglycaemia, consecutive DWI showed hyperintensity (decreased apparent diffusion coefficient) in the bilateral pulvinar and medial dorsal nuclei of thalami from 38 days after hypoglycaemic injury. This DWI hyperintensity subsided with time. The thalamus appeared consistently normal on CT scans during the study period.

Conclusion: This study revealed an existence of a delayed cellular change even in the thalamus with DWI study after hypoglycaemia. The hypoglycaemic damage on MRI in the thalamus appeared different from that observed in the striatum and cerebral cortex. We suggest that the mechanism of hypoglycaemic neuronal damage in the thalamus differs from that in the striatum and cerebral cortex in the human brain. The thalamic change after hypoglycaemia may represent a delayed cellular oedema caused by secondary remote effects resulting from axonal and/or transsynaptic degeneration. 


\section{P340}

The unrecognised stroke is more common in first-ever stroke patients with cardioembolic cause than in those without: a MRI analysis study

A-H. Cho, T-W. Kim, S-J. Lee, Y-M. Shon, D. W. Yang, B.S. Kim

The Catholic University of Korea (Seoul, KR)

Objectives: With MRI analysis, we aimed to investigate the prevalence and clinical significance of unrecognized stroke among patients with first-ever ischemic stroke, and also the factors related to the presence of unrecognized stroke.

Methods: We consecutively included patients with first-ever stroke (ischemic infarction) within 7 days from onset, and in whom MRI (T2weighted image, FLAIR image, diffusion-weighted image) was performed. Demographics, stroke subtype by modified TOAST classification, modified Rankin scale (mRS) at 3 months and risk factors were obtained. Unrecognized stroke was defined as an ischemic infarction or primary intracerebral hemorrhage on MRI irrelevant to the index stroke, without acute lesion on diffusion-weighted image in patients without previous history of stroke. Unrecognized stroke lesions were divided to lacunar infarction and corticalterritorial (infarcts including cortical or territorial lesion) infarction.

Results: Among 106 patients included, 39 patients (36.8\%) had unrecognized previous stroke (ischemic infarction in 37, ischemic infarction combined with intracerebral hemorrhage in 2). Patients with cardioembolic stroke more frequently had unrecognized stroke lesions than those with other stroke subtypes $(9 / 14$ [64.3\%] vs. 30/92 [32.6\%], p = 0.022). Regarding the lesion type of unrecognized stroke, $14(35.9 \%)$ patients had cortical-territorial infarction, while $25(64.1 \%)$ patients had lacunar infarction. The presence of cortical-territorial infarction as a unrecognized stroke was significantly associated with cardioembolic stroke $(8 / 9[88.9 \%]$ vs. 6/30 [20\%], $\mathrm{p}<0.001)$. In univariate analysis, hypertension and cardioembolic stroke were associated with the presence of unrecognized stroke $(p=0.014,0.022)$. After multivariate logistic regression adjusting age and hypertension, cardioembolic stroke independently increased the risk for the presence of unrecognized stroke $(\mathrm{p}=0.02$, Odds ratio $4.52,95 \%$ Confidence interval 1.26-16.18). However, the presence of unrecognized stroke was not associated with poor clinical outcome (mRS at 3 month)

Conclusion: The cardioembolic stroke was significantly associated with the presence of unrecognized previous stroke, especially cortical-territorial stroke. For the better prevention of stroke in patients with a cardioembolic source, investigation of unrecognized stroke lesion is needed even though the patients do not notify their stroke.

\section{P341}

Analysis of voxel-based morphometry of water diffusivity in early postictal diffusion tensor MRI

B.S. Kim, S-H. Kim, Y-M. Shon, D. W. Yang

St. Mary's Hospital (Seoul, KR)

Introduction: Recently, diffusion tenor image (DTI) is capable of improving detection of structural abnormalities of brain tissues by calculation of microscopic movement of tissue water molecules. Generally, in the irritative brain regions from epileptic condition the mean diffusivity (ADC) is increased and the anisotropy reduced during chronic or interictal period. But, there has been scanty of reports on the acute changes of water diffusion by epileptic seizure. We analyzed the diagnostic efficacy of DTI during early postictal period (within $24 \mathrm{hrs}$ ) by voxel based morphometry using SPM and compared the diagnostic yield to that of EEG findings and clinical findings.

Method: Fourty one epilepsy patients (male 24, age: 10-88) and 30 agematched control subjects (age: 23-74, male 13) was selected for analysis. For reflecting the changes by aging process, we divided them into two groups (young $<50$ years, old $>50$ years, patients and controls respectively). All DTI was performed within 24 hours, and fractional anisotropy (FA) and ADC map was calculated by home-made software (DTIcalc) for SPM analysis. The area with increased ADC and decreased FA was compared to the clinical and EEG findings for evaluation of its localizing value.

Result: The area of increased ADC was well corresponded with EEG findings in 12 patients $(29.3 \%)$. In normal conventional MRI group (15/41, $36.6 \%$ ), 6 patients ( $40 \%$ ) shows EEG matched regions of increased ADC. Also, another $5(12.2 \%)$ patients had the DTI findings not correlated with their EEG features, but the areas with increased ADC of them were well corresponded to the presumptive epileptic areas by seizure semiology. Comparing to old groups, young age group showed better diagnostic yield of DTI findings [young vs. old group: EEG abnormality matched to DTI abnormality $11 / 28(39.3 \%)$ vs. $1 / 13(7.7 \%)$; DTI findings matched to EEG findings with normal conventional MRI $6 / 13(46.2 \%)$ vs. $0 / 2(0 \%)$; DTI findings matched only to clinical semiology $3 / 28(10.7 \%)$ vs. $2 / 13(15.4 \%)]$ But, it showed little diagnostic value of FA measurement of DTI (positive result in only one patient in all patients).
Discussion: By SPM analysis, we can detect the acute change of water diffisivity with early postictal DTI in epileptic patients and it has a considerable correlation with the EEG or clinical findings. We can assume that early postictal DTI analysis may be used as a complementary tool to diagnosis of epileptic foci.

\section{P342}

Optimising diffusion measurements for large-scale, multicentre multiple sclerosis trials: a pan-European study

E. Perego, M. Rovaris, J.G. Hirsch, E. Pagani, A. Gass, P. Pouwels, S. Rosendaal, F. Barkhof, F. Agosta, D. Caputo, A. Giorgio, J. Palace, S. Marino, N. De Stefano, F. Fazekas, S. Ropele, M. Filippi

Scientific Institute and University HSR (Milan, IT); University Hospital Basel (Basel, CH); VU University Medical Centre (Amsterdam, NL); Scientific Institute Fondazione Don Gnocchi (Milan, IT); University of Oxford (Oxford, UK); University of Siena (Siena, IT); Medical University of Graz (Graz, AT)

Objectives: To apply a standardized acquisition scheme for diffusion tensor (DT) MRI of multiple sclerosis (MS) patients using different scanners. To investigate which is the inter-scanner variability of DT-derived metrics and whether the sensitivity to disease-related changes varies accordingly.

Methods: Twenty-nine healthy subjects and twenty-five relapsing-remitting MS patients with low disability (expanded disability status scale [EDSS] $\leq 3.5$ ) were studied in 5 centres. Four $1.5 \mathrm{~T}$ scanners and three $3.0 \mathrm{~T}$ scanners from two different manufacturers were used. A pulsed gradient spin echo single shot echo planar DT MRI sequence was used, with the following scheme: TR[ms]:5000-9000, TE[ms]: $90-125$, pixel size: $2.5 \times 2.5 \mathrm{~mm}$, slices: 50 , slice thickness [mm]: 2.5 ; diffusion-encoding gradients directions: $30, \mathrm{~b}$ value $\left[\mathrm{s} / \mathrm{mm}^{2}\right]$ : 900 ; number of acquisitions with $b=0: 4-6$. A high-resolution dual echo sequence was also acquired. The DT was estimated by linear regression and fractional anisotropy (FA) and mean diffusivity (MD) maps calculated. T2-visible lesions were manually segmented on the dual echo scans. After removing lesions and masking, normalized FA and MD histograms from the normal appearing brain tissue (NABT) were created, and average MD/FA and MD/FA peak height values were derived.

Results: The subjects' age was not significantly heterogeneous among centres. There was a significant interscanner heterogeneity for average $\mathrm{MD} / \mathrm{FA}$ and histogram peak height values in both controls and patients. After correcting for scanner, pooled data analysis showed that average MD was significantly higher, average FA significantly lower and MD peak height significantly lower in patients than in controls $(p<0.001, p=0.038$ and $\mathrm{p}<0.001$, respectively). There was no significant interscanner heterogeneity for the observed differences between patients and controls in any histogram-derived variable.

Conclusions: A careful standardization of the acquisition scheme can make DT MRI data collection in multicentre MS studies (such as clinical trials) feasible and reliable for detecting disease-related changes without major intercentre heterogeneities which would affect the data pooling for centralized analysis.

\section{P343}

Presence of brain white matter hyperintensities correlates with poor cognitive test score

H. D’Onofrio, R. Schiefelbein, H. Videla, J. Maegli, A. Caride, J. Cancino, J. Duarte, A. Bertotti, S. Mascaro

Deutsches Hospital (Ciudad Madero, AR); Borda Hospital (Ciudad Madero, AR); Pinero Hospital (Ciudad Madero, AR)

Objective: To evaluate whether presence and distribution of white matter hyperintesities (WMH) found in brain MRI were correlated with cognitive profile in an outpatient sample of non demented individuals.

Background: WMH are reported in brain images as inespecific incidental findings or may be considered representative of pathological changes. Nevertheless the clinical significance of WMH on neurocognitive test (NCT) performance is a long lasting discussion and still remains unclear.On the other hand fewer works focusing on WMH correlation with detailed NCT have evaluated not handicapped independently functioning people.

Method: 39 outpatient, with no stroke history, 30F-9M (mean age 69,95\% CI: 66-73), (years of education 10.6 95\% CI: 9.04-12.1) evaluated in our hospital for different complaints underwent to NCT:Screening(MMSE), Attention and Memory (RAVLT A and B, digit span, Rey-Osterrieth test,Trail Making $A$ and $B$,)and Language tests (phonologic and semantic fluency).Data were correlated with brain MRI which were visually rated for WMH presence and distribution in Frontal (F), Temporal (T), Occipital (O), Parietal (P), Periventricular (PV), Basal ganglia and Infratentorial area, and scored using Scheltens scale: Deep WMH (Score 0: no WMH to Score 6: confluent WMH) and PV WMH (scored 1-2). 
Results: MMSE(median:28)(95\% CI:27-29).

MRI scored:WMH $=0(n=9), W M H>0(n=30:$ mean score: $1.795 \%$

$\mathrm{CI}: 1.2-2.2)$

Poor NCT correlated with presence of WMH.

WMH score $>0$ in F:(r:-0.44, p: 0.0406)and PV:(r-:0.502, p: 0.0125) correlates with $\mathrm{MMSE}<28$.

Using Multiple Regression Analysis RAVLT A (part referred to delayed recall) emerges as memory strongest correlation to WMH score $>0$ in $\mathrm{T}$ (r:- 0.635, p: 0.0356),O(r:-0.502, p:0.0475)P(r:-0.648, p:0.0009).

Trail Making B was the attention task with strongest and significative correlation(WMH score $>0$ in O r:0.905, p:0.0003), T ( $\mathrm{r}: 0.927, \mathrm{p}:<0.0001$ )and $\mathrm{P}(\mathrm{r}: 0.946, \mathrm{p}:<0.0001)$

Trail Making A was the strongest correlation with WMH scores $>0$ in $\mathrm{F}:(\mathrm{r}: 0,801, \mathrm{p}:<0.0001)$ and PV (r:0.817, p: 0.0001).

Comparing WMH score $=0$ and score $=1(1$ to $5 \mathrm{WMH}$ lesser than $3 \mathrm{~mm}$ )for MMSE $<28$ we found T-test with $\mathrm{p}<0.05$ for T, F and PV areas.

Conclusion: Presence of WMH in brain images were associated to cognitive disfunction on tests. Lower scores on Screening and verbal memory (delayed recall)tasks emerge as related to just presence of WMH. Atention and speed of mental processes showed dependency on higher WMH score in the parietal, temporal, frontal and periventricular area.

P344

Clinical and neuroimaging findings in 20 patients with posterior cortical atrophy. Unilateral dilatation of the occipital horn: a sign for neuroimaging diagnosis?

J. Romero-Imbroda, T. Ojea, M. Gonzalez, O. Fernandez

Hospital Carlos Haya (Malaga, ES)

Objectives: Posterior Cortical Atrophy (PCA) is a distinctive dementia syndrome that presents primarily with cortical visual dysfunction with the relative preservation of memory at the onset with the absence of primary ophthalmologic causes. Several diagnostic criteria have been proposed. We report clinical and neuroimaging findings in 20 patients.

Methods: 20 patients (14 women and 6 men) diagnosed of PCA using clinical criteria. Onset mean age 60,4 years and mean follow up of 3,2 years. ( 1 to 10 years). Serial MRI and SPECT was performed in every patient.

Results: All patients presented contructional dyspraxia and complete or partial Gerstmann syndrome. The principal type of visual impairment at the onset was simultanagnosia $(70 \%)$. In the follow up there was an increase of dressing apraxia ( $70 \%$ vs $95 \%)$. There was descriptive presence of occipitoparietal atrophy on MRI in $90 \%$ of the studies increasing in the follow up to $100 \%$ with a relative preservation of the hippocampus. Functional neuroimaging detected posterior hypoperfusion in every patient. Asymmetry was the rule: 7 patients had right hypoperfusion, 6 left and 7 bilateral. Those unilateral hypoperfusion in SPECT (13) had correlation with ipsilateral dilatation of occipital horn of lateral ventricle that increased in serial MRI.

Conclusion: Clinical and neuropsychological deficits with MRI and SPECT investigations support the diagnosis of PCA. Our clinical results agree with other studies. We relation occipitoparietal atrophy in serial MRI with hypoperfusion of the same area in SPECT. The unilateral dilatation of occipital horn of lateral ventricle may be usefull for the neuroimaging diagnosis of PCA.

P345

MR susceptibility sign of the middle cerebral artery in imaging of acute stroke

K. Maher, A. Ibrahim, G. Gouda, A. Fouad

Ain-Shams University (Cairo, EG)

Purpose: To evaluate the accuracy of echo-planar T2*-weighted imaging (WI), compared to magnetic resonance angiography (MRA) and fluid-attenuated inversion recovery (FLAIR) in the detection of acute middle cerebral artery (MCA) thrombotic occlusion.

Materials and methods: 47 consecutive patients with acute stroke involving the MCA territory underwent magnetic resonance imaging (MRI) within 2 to 76 hours after clinical onset. MRI examination included echoplanar T2*-weighted imaging, FLAIR, diffusion-weighted imaging (DWI) and MRA. The susceptibility sign on echo-planar T2*-weighted images, which is indicative of acute thrombotic occlusion involving the MCA, was assessed and compared to findings on MRA and axial FLAIR in all patients and to computed tomography (CT) in 23 patients. National Institutes of Health Stroke Scale (NIHSS) score, which is a clinical scale assessment, was used for evaluating the neurological status of patients.

Results: Fourty seven (47) patients (29 males; age range 11-86 years \{mean: $58.3 y s \pm 14.7$ \} and 18 females; age range $35-83$ years \{mean: 59.4 ys $\pm 12.7\}$ with acute territorial MCA infarcts were included in this study. Out of these 47 patients, 10 had hyperacute MCA infarction (scanned 2-6 hours after ictus), 7 of those patients presented with very severe stroke (NIHSS score 21 or more) and 3 patients with severe stroke (NIHSS score 15-20). Thirty seven (37) patients were studied within 76 hours from ictus (acute to early subacute). Among this group and according to NIHSS score, 5 patients had mild to moderate stroke, 16 patients severe stroke; and 13 patients had very severe stroke. One patient who had very severe stroke with NIHSS score of 22, died. She had left MCA occlusion, presented 4 hours within onset of right hemiplegia and had a history of Diabetes mellitus, hypertension and Chronic Obstructive Pulmonary Disease.

Conclusion: Presence of the susceptibility sign on T2*WI proximal to the MCA bifurcation provides fast and accurate detection of acute proxima MCA thrombotic occlusion. It is considered a warning sign for rapid and efficient intervention for stroke treatment, including thrombolysis and can be used for follow up of thrombus evolution.

\section{P346}

Betaseron vs. copaxone in multiple sclerosis using triple-dose gadolinium 3 tesla MRI endpoints: comparison of spectroscopic findings in normal appearing white matter on MR scans performed at 12 and 24 months L. Wolansky, A. Jedynak, B. Hubbi, S. Kim, M. Haghighi, J. Batlle i Nadal, R. Turbin, B. Biswal, S. Kamin, S. Cook, D. Cadavid

New Jersey Medical School (Newark, US)

Background and purpose: The BECOME trial (Betaseron vs. Copaxone in MS with triple-dose gadolinium and 3-T MRI Endpoints) is the first headto-head trial focusing on the MRI outcomes of subjects treated with these two medications. The primary outcome measure, for each patient the mean \# of enhancing lesions plus the \# of new T2/FLAIR lesions unassociated with enhancement per scan, has been reported elsewhere. The purpose of this communication is to report on the spectroscopic features of the normal appearing white matter (NAWM) at the 12 and 24 month mark.

Methods: 75 subjects with MS were randomized to one of two treatment arms: $20 \mathrm{mg}$ of Copaxone (R) (glatiramer acetate or GA) injected subcutaneously (sc) on a daily basis vs. $250 \mathrm{mcg}$ of Betaseron (interferon beta-1b or IFN) injected sc every other day for up to 24 months. MR Spectroscopic (MRS) measurements were obtained at 12 and 24 months. A single voxel, measuring 1 to $1.5 \mathrm{~cm}$ cubed, was placed on the NAWM selected by reviewing T1, T2, FLAIR, proton density, and Gadolinium enhanced images so as to avoid visible pathology. The pulse sequence utilized was Point Resolved Spectroscopy (PRESS). The metabolites evaluated included the neuronal marker, n-acetyl aspartate (NAA) and the internal frame of reference metabolite, creatine $(\mathrm{Cr})$. NAA/Cr ratio was calculated.

Results: 27 subjects had 12 and 24 month MR spectroscopic data of acceptable quality on both visits to be included. Analysis revealed that 20 of 27 $(74 \%)$ of all subjects, irrespective of treatment group, demonstrated an increase in $\mathrm{NAA} / \mathrm{Cr}$ ratio (mean increase of $4.4 \%$ ). Analysis by treatment group revealed a mean increase of NAA/Cr in the IFN group of $4.9 \%$, (median: $5.7 \%)$ with an increase being seen in $75 \%$ of subjects $(\mathrm{N}=12)$. The mean increase in the GA group was $2.7 \%$, (median: $3.1 \%$ ) with an increase being seen in $73 \%$ of subjects $(\mathrm{N}=15)$. These differences were not significant $(\mathrm{P}=1)$.

Conclusions: MR Spectroscopic analysis of the NAWM of subjects treated for 2 years with GA and IFN revealed a mean increase in the NAA/Cr between 12 and 24 months suggesting a neuroprotective effect of both medications.

The BECOME study is supported through a contract grant from Bayer Healthcare Pharmaceuticals, however, the intellectual property is solely that of UMDNJ.

\section{P347}

Association of demyelinating lesions in the spinal cord and disc disease M. Quigg, A. Lugo, B. Greenberg

Johns Hopkins School of Medicine (Baltimore, US); Ohio State Universtiy (Columbus, US)

Objectives: There are a variety of demyelinating conditions that can effect the spinal cord. Why lesions occur in certain areas of the spinal cord is unknown. There is no data to support an antigen driven hypothesis for selective lesions in the cord. This preliminary study examines the potential relationship between mechanical forces (herniated discs) and autoimmune processes (demyelinating lesions).

Methods: Patients with demyelinating disease who were evaluated at The Johns Hopkins Hospital with both cervical and thoracic MRIs were included in the study. The MRIs were reviewed for the presence of disc disease and herniated discs were rated on a three point scale - 1 point for minor hernia- 
tions, 2 points for moderate hernations (loss of CSF space) and 3 points for severe herniations (impingement on the ventral aspect of the cord). The MRIs were then separately reviewed for the presence of T2 hyperintense lesions. The rostral-caudal borders of these lesions were recorded in an excel spreadsheet.

Results: Out of patients who had both a T2 bright lesion and the presence of at least one disc herniation there was a correlation between locations. $72 \%$ of patients with both herniations and lesions had them overlap at least at one level. $88.8 \%$ of patients with both lesions and disc herniations had the lesion within 2 vertebral segments of the herniation. When individual lesions are studied (some patients had multifocal disease), $53.3 \%$ had a direct correlation with a herniated disc and $73.3 \%$ occured within 2 vertebral segments of a herniated disc. Of the lesions that occured 3 or more vertebral segments away from a herniated disc, only $6.7 \%(2 / 30)$ where solitary lesions (i.e. not in the presence of a remote lesion that had proximity to a herniated disc).

Conclusions: While this preliminary study of 18 patients (30 lesions) suggests a possible association between disc herniation and the location of demyelinating lesions, a larger confirmatory study is needed. Pathologically, a herniated disc could impair venous outflow, at the level of the epidural venous plexus, thus creating an inviting environment for immune cells to cross the blood brain barrier. Based on this theory, herniated discs do not cause lesions, but rather create appropriate environments for their creation in individuals with autoimmune predispositions.

\section{P348}

Functional MRI correlates of neuropsychological impairment in primary progressive multiple sclerosis

M. Rocca, A. Ceccarelli, M. Falautano, M. Rodegher, G. Comi, M Filippi Scientific Institute San Raffaele (Milan, IT); Neuroimaging Research Unit (Milan, IT)

Objectives: Cognitive impairment has been described in a variable percentage of patients with primary progressive multiple sclerosis (PPMS) and has been related to the extent and severity of brain macroscopic disease burden. Aims of this study were to investigate the functional correlates of cognitive network dysfunction in patients with PPMS and their correlations with structural magnetic resonance imaging (MRI) parameters.

Methods: From 16 right-handed PPMS patients and 17 sex- and agematched healthy volunteers (HV), structural and fMRI during the performance of the n-back task were acquired. Neuropsychological tests (NPT) exploring memory, attention and frontal lobe cognitive domains were administered. T2 lesion load (LL) and corpus callosum area (CCA) were measured. fMRI analysis of corrected answers was performed using statistical parametric mapping.

Results: Six PPMS patients had an abnormal performance in three or more NPT, thus fulfilling pre-defined criteria for cognitive impairment (CI). Structural MRI metrics did not differ between CI and unimpaired PPMS patients. Compared to controls and to CI patients, cognitively preserved patients had more significant activations of several regions in the frontal lobes, bilaterally. Conversely, compared to the other two groups, CI patients had more significant activations of several regions in the parietal lobes, the supplementary motor area, the cingulum and the primary motor cortex, bilaterally. In PPMS patients, significant correlation was found between decrease of activation in the frontal lobes and T2 LL.

Conclusions: Increased activations of cognitive-related networks might represent a functional reserve with the potential to limit the onset of cognitive impairment in PPMS. The progressive exhaustion of frontal lobe function, due to accumulation of T2-visible lesion burden, is likely to be among the factors related to cognitive impairment in these patients.

This study was supported by a grant from FISM (Fondazione Italiana Sclerosi Multipla) - contract n. 2003/R/48.

\section{P349}

A 3 Tesla functional MRI study of the resting state networks in ageing M. Rocca, P. Valsasina, M. Absinta, A. Ceccarelli, A. Falini, G. Scotti, G. Comi, M. Filippi

Scientific Institute San Raffaele (Milan, IT)

Objectives: Previous studies, investigating low-frequency fluctuations in functional magnetic resonance imaging (fMRI) data, have demonstrated a coactivation of distributed cortical networks, which characterizes the default mode, or resting state (RS), of the human brain. Aim of this study was to investigate the influence of normal aging on resting state networks (RSN) identified in a cohort of healthy subjects.

Methods: RS fMRI data were acquired from 49 healthy controls (male/female $=20 / 29$, mean age $=34.7$ years, range $=20-69$ years ) using a 3T scan- ner. After data pre-processing (realignment, normalization and smoothing), independent component analysis (ICA) was used to decompose resting fMRI data into spatially independent maps and time courses using the GIFT software. This analysis produced 44 spatially independent maps. Visual inspection of these maps allowed to eliminate components clearly related to artifacts. Then, a frequency analysis of the time courses was used to identify the components with high (50\% or greater) spectral power at low frequency (between 0.01 and $0.05 \mathrm{~Hz}$ ). One-sample statistics of spatially independent maps and correlation with age were assessed using statistical parametric mapping.

Results: Our analysis detected 10 RSN with potential functional relevance: RSN 1 and 2 included primary and secondary visual cortical areas; RSN 3 and 4 included sensorimotor related areas; RSN 5, 6 and 7 included bilateral fronto-parietal-temporal areas; RSN 8 included fronto-parietal areas lateralized to the left hemisphere; RSN 9 included fronto-parietal areas lateralized to the right hemisphere, and RSN 10 included bilateral parietal areas (including the precuneus) and the posterior cingulum. Significant correlations with aging were found exclusively for RSN 1 and 4 .

Conclusions: ICA was able to detect in our data components which agree well with previous literature and which consist of well-known functional networks. Our results suggest that the different RSN of the healthy brain might respond differently to aging. The absence of a correlation between the majority of the observed RSN and age might help to explain the widespread and sometimes aspecific patterns of over-recruitment frequently observed in several neurological conditions during the performance of different activation tasks.

\section{P350}

Is a cranial computer tomography indicated in all patients suffering from transient ischaemic attack?

S. Ulmer, C. Wiese, O. Jansen, C. Koch

University Hospital Schleswig-Holstein (Kiel, DE); University Hospital Schleswig-Holstein (Lubeck, DE)

Objective: A transient ischemic attack (TIA) is defined as an acute onset of neurological symptoms lasting less than 24 hours without evidence of infarction, however, these patients have an increased risk to develop a major stroke. Usually a cranial computer tomography (CCT) is initially performed to rule out other causes of this neurological deterioration such as bleedings, tumors or major strokes. To justify exposure to radiation by CT it should be sensitive enough to depicted the underlying cause.

Methods: All CCTs that had been requested within one year were reviewed. All studies that were requested for patients presenting with TIA were further analyzed. CT scans (multirow-detector scanners, Toshiba), emergency notes, charts and other performed imaging modalities such as MRI were reviewed. Cases that turned out to be a seizure with Todd paralysis rather than a TIA and other causes that would require immediate emergency imaging (i.e. anticoagulated patients with head trauma or acute hypertensive dysregulation) were excluded.

Results: From 3580 requests for a CCT, 350 (9.8\%) were performed because of transient neurological symptoms. 148 of these patients (4.1\%) presented with a TIA lasting less than one hour. Patients under coagulation without a history of a current trauma were excluded leading to 84 remaining patients. Of these cases 3 pathologies $(3.6 \%)$ were found ( 2 strokes, 1 tumor). One infarction was diagnosed on CCT that turned out to be an artifact. The two infarctions were completely missed on CCT. In patients with regredient but longer lasting symptoms than one hour $(n=57), 49$ had normal CCTs. Of these 25 strokes were only detected by MRI (51\%). The remaining group included patients with strokes $(n=4)$, bleeding $(n=1)$, and tumerous lesions $(\mathrm{n}=2)$, which were all detected by CCT.

Conclusion: Strokes can present as TIA especially when they affect clinically "silent" regions. Duration of the symptoms is crucial in the decision of further performed examinations. $66 \%$ of pathological findings were completely missed on CCT, which is therefore dispensable in TIA lasting less than 1 hour. Imaging of patients with regredient symptoms is mandatory, however, a sufficient cerebrovascular imaging such as MRI should be performed.

\section{P351}

Distortion of deep-brain stimulation electrodes in postoperative MR imaging. Where is the tip of the electrode?

S. Ulmer, M. Helle, J. Herzog, D. Falk, M.-H. Mehdorn, G. Deuschl, O. Jansen University Hospital Schleswig-Holstein (Kiel, DE)

Objective: In advanced parkison's disease deep brain stimulation (DBS) of the subthalamic nucleus (STN) is performed for treatment of rigidity, tremor, and bradykinesia. Postoperatively patients undergo MR imaging to 
document the location of the electrodes. To avoid complications in the scanner the FDA recommended to lower the SAR thresholds from $0.4 \mathrm{~W} / \mathrm{kg}$ to 0.1 $\mathrm{W} / \mathrm{kg}$ in 2005.

Methods: In our institution, implantation of deep brain stimulation electrodes (model 3389, Medtronic, Minneapolis, USA) was performed in 235 cases for advanced Parkinson`s disease. Different scanners and SAR limits had been used over the years. Postoperative images from three different epoches were reviewed by two investigators blinded to the scanner brand and SAR thresholds and image quality was judged. We furthermore reviewed the charts in terms of perioperative complications. To measure and describe the size of the susceptibility-artefact an agarose-phantom was created that harbored a DBS electrode during MRI experiments emulating the clinical setting. This phantom was also examined with CT and images were overlayed.

Results: Using a 6-rank score for image quality we found no difference between scanners or SAR limits (one-way ANOVA). There were no MR related complications. The artefact of the electrodes resembled an arrowheadshaped triangle with a broader base in the phase encoding direction. The artefact was 5.3-fold the real size of the tip of the electrode.

Conclusion: The susceptibility artefact varies according to the angle of the trajectory. This is an important finding in judging the stereotactic result and in understanding why in some patients stimulation might be less effective than in others. Reduction of the SAR was considered to result in lower image quality, however, it turned out to be sufficient enough for postoperative image interpretation. MRI can be performed safely following the manufacturers recommendations with acceptable image quality.

P352

An observational study of diffusion-weighted images in patients with transient ischaemic attacks

V.L. Parisi, G.G. Persi, A.C. Ayarza, D.F. Visconti, C.A. Rugilo, E.M. Gatto

Sanatorio Trinidad Mitre (Buenos Aires, AR)

Objectives: Although a Transient Ischemic Attack (TIA) is defined as a neurologic deficit of sudden onset and vascular origin that resolves in less than 24 hours, the new imaging techniques such as Diffusion-Weighted Images (DWI) are demonstrating that brain infarct can occur even when symptoms resolves in minutes.

We agree with other studies that sustain the need for a redefinition of TIA as a neurological deficit or retinal ischemia, lasting less than one hour and without neuroimaging evidence of brain tissue damage.

Our purpose is to evaluate the presence of DWI abnormalities in patients with transient neurological symptoms of ischemic origin and its relationship with the duration of deficit.

Material and methods: We retrospectively analized the Stroke Registry of our institution on the period between 01/01/2006 and 01/30/2008

We established the incidence of stroke and TIA and determined the presence of DWI abnormalities consistent with acute ischemia on patients with TIA, the duration of symptoms and the probable ethiology based on TOAST calssification.

Analyses were performed with the STATISTICS statistical package version 7.0. Between groups comparison was performed using non parametric test for nonnormally distributed data (Rank Sum Test for continous variables). A p $<0.05$ was considered to be significant.

Results: We registered 182 ischemic events on that period. Of these patients, 25 presented with transient symptoms (13.7\%), with DWI abnormalities suggestive of acute ischemia in 9 of them (36\%). In five of these 9 patients $(55 \%)$ symptoms lasted less than one hour and were only motor deficit and or speech disturbances. Median duration of symptoms was 30 minutes for those cases with DWI lesions, and of 10 minutes in those without abnormalities. There was no relationship between the duration of symptoms and the presence of DWI abnormalities $(\mathrm{p}=0.57)$.

In those with DWI acute ischemic lesions, ethiologies were cardioembolic in 4 patients and undetermined in five.

Conclusions: We could not established a relationship between the duration of symptoms and the presence of acute ischemic lesions on DWI. Moreover, in our population $55 \%$ of patients whose deficit lasted less than one hour had DWI abnormalities, though we have a low number of cases.

Beside these results, the evidence is enough to reconsider the definition of TIA, based on symptoms duration and the absence of brain ischemia, mostly for its therapeutic and prognostic implicances.
P353

High resolution diffusion tensor imaging of brain stem infarction at 3T: correlation with clinical symptoms

W.C. Shin, Y.H. Lee, S.H. Bu, S.J. Byun

Kyung Hee Medical School (Seoul, KR); Icheon Medical Center (Icheon, KR)

Objective: Because of complex anatomy of brain stem, small infarctions in brain stem are difficult to assess with regard to the precise location with conventional imaging. We determined whether high-resolution diffusion-tensor imaging (HR-DTI) at 3T is valuable for evaluation of acute ischemic stroke involving brain stem.

Methods: Consecutive 16 patients with acute brain stem infarction were underwent 3T HR-DTI with SENSE acquisition $(\mathrm{TR} / \mathrm{TE}=4000 / 55 \mathrm{~ms}$; FOV $=240 \mathrm{~mm} ; 32$ direction; in-plane resolution $=0.9 \times 0.9 \mathrm{~mm}$; slice thickness $=$ $3 \mathrm{~mm} ; \mathrm{b}=800 \mathrm{~s} / \mathrm{mm}^{2}, 22-24$ axial sections) in conjunction with routine brain MRI. Color-coded map (CDTI) was reconstructed by using post-processing of DTI. Lesions were identified on CDTI and subsequently compared with clinical presentation. The lesion involving CST was classified into two groups - partial and complete involvement. Lesions were identified on CDTI and subsequently compared with clinical presentation.

Results: In all 16 patients, good quality of HRDTI without distortion of image was acquired. Detailed white matter tract was identified on CDTI. In twelve patients with the lesion involving CST, five of seven patients with complete CST involvement show limb weakness, but three of five patients with partial CST involvement showed no limb weakness. Lesions involving inferior cerebellar peduncle (3), medial lemniscus (4), and trigeminal nerve tract (1) were well localized on CDTI, and presented ataxia, paresthesia, and facial numbness, respectively. In seven patients with lesion in the transverse pontine fiber, three presented ataxia.

Conclusion: The finding of HRDTI was well correlated with clinical presentation. Specific lesion localization on HRDTI may help in prediction of the prognosis for patients with brain stem infarction.

P354

Diffusion-weighted MRI and brain SPECT with 99mTc-ethyl cysteinate dimer as early diagnostic tests for Creutzfeldt-Jakob disease

Y. Harigaya, K. Mizushima

Maebashi Red Cross Hospital (Gunma, JP)

Objectives: To evaluate the usefulness of diffusion-weighted MRI (DWI) and 99mTc ethyl cysteinate dimer (99mTc-ECD) single-photon emission computed tomography (SPECT) for the early diagnosis of Creutzfeldt-Jakob disease (CJD).

Methods: Seven consecutive patients (aged 54 to 71 years) were examined using $99 \mathrm{mTc}$-ECD SPECT images at our hospital. Of those, 6 were also examined by MRI including DWI, while 1 could not been examined because of pacemaker implantation. Genetic analysis demonstrated that 6 of the patients had sporadic CJD with methionine/methionine at codon 129 of the prion protein gene, of whom 4 were definite at autopsy, and 2 were probable based on the World Health Organization criteria, while the other had familial CJD with both methionine/valine at codon 129 and isoleucine substituting for valine at codon 180 of the prion protein gene despite no family history. The percentages of DWI abnormalities, decreased perfusion on ECD-SPECT images, periodic synchronous discharges (PSDs) on EEG, detection of CSF 14-3-3 protein, and increase in CSF neuron-specific enolase $(>25 \mathrm{ng} / \mathrm{mL})$ at the first examination were compared.

Results: DWI revealed abnormal hyperintense lesions particularly in the cerebral cortex, caudate nucleus and putamen more sensitively than conventional MRI. In addition, SPECT imaging with $99 \mathrm{mTc}$-ECD demonstrated severely decreased perfusion, which was shown as high intensity by DWI. The percentage of both DWI abnormalities and decreased perfusion shown on ECD-SPECT images were $100 \%$, while $14-3-3$ protein was detected in $86 \%$, NSE increase in $43 \%$ and PSDs in $29 \%$ of the patients. In 3 patients, DWI abnormalities were detected 1 month earlier than PSDs on EEG were evident. The familial CJD patient never had shown PSDs on serial EEG. Both DWI and SPECT demonstrated the serial changes of abnormal lesions with the progression of neurological findings.

Conclusions: DWI was the most sensitive test for early clinical diagnosis of CJD and appears to be useful to monitor the disease. Changes shown by SPECT were helpful to indicate abnormal lesions associated with CJD shown by DWI. We consider that DWI results should be used to obtain clinical diagnostic criteria findings for CJD. 
P355

The occurrence of "typical" MRI findings in progressive supranuclear palsy and multiple system atrophy: a retrospective study

$Z$. Chovancová, P. Hluštík, P. Kanovský

University Hospital (Olomouc, CZ)

Objectives: Conventional MRI is usually normal in patients with Parkinson's disease, whereas it is believed to show characteristic abnormalities in patients with other parkinsonian syndromes, which provides the potential for using objective neuroradiological criteria in the differential diagnosis.

Methods: MRI examinations of the brain were retrospectively assessed in 7 patients ( 4 females, 3 males: aged 59-71, mean $65 \pm 4.2$ years) suffering from progressive supranuclear palsy (PSP) (4 patients) and multiple system atrophy (MSA) (3 patients), confirmed with clinical, laboratory, electrophysiological and cerebrospinal fluid findings (CSF). The areas of midbrain tegmentum and pons were particularly inspected and the following "typical" MRI signs were sought: "morning glory" sign, "hot cross bun" sign, "panda bear face" sign and "standing penguin silhouette" sign, and also hypo- or hyperintensities in the posterolateral putamen (in T2 and FLAIR images).

Results: In all the patients with PSP, we have found the "standing penguin silhouette", only in one patient with PSP we have observed the "morning glory" sign. In contrast, all patients with MSA had hypo- and hyperintensities in the posterolateral putamen in T2 and FLAIR scans. The "hot cross bun" sign and "panda bear face" sign were not found in any patient.

Conclusion: We confirmed the presence of midbrain atrophy as a typical neuroimaging feature ("standing penguin silhouette") in PSP. The "standing penguin silhouette" seems more sensitive then "morning glory" sign. Patients with MSA instead manifested signal changes in the posterolateral putamen in T2 and FLAIR images.

\section{P356}

Diffusion tensor weighted MR-images and in vivo 1H MRS for differentiation of cerebral ischaemia and early multiple sclerosis

Z. Rozhkova, T. Nehrych, A. Khoma, V. Goryachev

Medical Clinic "BORIS" (Kiev, UA); Lviv Medical University (Lviv, UA)

Objectives: On the basis of the analysis of Diffusion Tensor Weighted MRimages (DTI) and in vivo $1 \mathrm{H}$ MRS data of the brain in patients with clinical symptoms of CI and MS we propose quantitative approach for differentiation CI from early stage of MS.

Methods: DTI and $1 \mathrm{H}$ in vivo MRS of the brain were performed in 20 patients with CI (in the age 36-55y., disease duration from 3 to $6 \mathrm{y}$.) and in 18 patients with clinically diagnosed early stage of MS (in the age 23-40y., disease duration 1-3 y.) on 1.5T SIGNA EXCITE (GE). Axial DTI of the brain using a single shot, multi slice spin-echo echo planar diffusion tensor pulse sequence with following parameters: TR/TE $=10000 / 83.6 \mathrm{~ms}$, number of slices $=35, \mathrm{FoV}=24$, slice thickness $=3 \mathrm{~mm}$, interleaved data acquisition were obtained. $1 \mathrm{H}$ spectra are recorded in both hemispheres with the 2DCSI STEAM method: TR/TE $=3000 / 144 \mathrm{~ms}, 16 \times 16$ phase encoding matrix, NS $=2$.

Results: From DT MR images the values of average fractional anisotropy (FA), and ADC coefficients in the genu, splenium, body of corpus collosum, and in the white matter of the frontal and occipital lobe were calculated. In the MS patients compared with the CI diseased patients the FA values are decreased obviously in the anterior and posterior body and splenium of the corpus collosum, but there is no significant difference at the genu, and at the white matter of the frontal lobe, and of the occipital lobe, respectively, between two groups. Decreased FA values in corpus collosum for patients with MS are characteristic pattern for Wallerian degeneration. The mean ADC value in the "black holes" in the MR imaging of the brain in patients with MS is: $(0.90 \pm 0.1) 10-3 \mathrm{~mm}^{2} / \mathrm{s}$. The mean ADC value in the ischemic foci in the MR imaging of the brain in patients with CI disease is: $(1.46 \pm 0.2) 10-3$ $\mathrm{mm} 2 / \mathrm{s}$. Using DTI we can detect the potential disorder of corpus collosum. Comparison FA with ADC values in black holes provide us very useful diagnostic information for differentiation MS from CI disease. From analysis of MR spectra the mean values of $\mathrm{Cho} / \mathrm{Cr}$ ratios are obtain. In the black holes the mean values of $\mathrm{Cho} / \mathrm{Cr}=3.93$, and in the ischemic foci 2.46 , respectively. We have found out correlation $\mathrm{Cho} / \mathrm{Cr}$ ratios with $\mathrm{FA}$ and $\mathrm{ADC}$ values.

Conclusion: FA and ADC values, and also Cho/Cr ratios in the pathologically matched regions of the brain and also in the various structures of corpus collosum are noninvasive quantitative parameters for differential diagnosis CI disease from MS.
P357

MRI study of the cervical cord abnormalities in patients with various forms of multiple sclerosis

Z. Rozhkova, T. Nehrych, A. Khoma, V. Goryachev

Medical Clinic Boris (Kiev, UA); Lviv Medical University (Lviv, UA); Pharm. Comp. Teva (Kiev, UA)

Objectives: On the basis of the analysis of Diffusion Tensor Weighted MRimages (DTI) of the cervical cord we propose quantitative approach for differentiating of relapsing-remitting (RR) and secondary progressive (SP) forms of MS.

Methods: DTI of the cervical spinal cord was performed in 25 patients (PG) with MS (15 with RR, and 10 with SP form, respectively) in the age from 16 to $43 \mathrm{y}$. and in 30 healthy subjects (CG) in the age from 23 to $40 \mathrm{y}$. on $1.5 \mathrm{~T}$ SIGNA EXCITE (GE). Axial DTI of the spinal cord at C2-3, C3-4, and C4-5 levels (EPI, $\mathrm{b}=700$ and $1000 \mathrm{~s} / \mathrm{mm}^{2}$ ) in the cervical cord were obtained. Average fractional anisotropy (FA), ADC coefficients, and cord-cross sectional areas were calculated.

Results: In the CG the mean values of FAs were: $0.63 \pm 0.03$. In the PG the mean values of FAs were $0.57 \pm 0.05$ in $R R$, and $0.50 \pm 0.03$ in SP. All patients of the PG have had significantly lower average cord FAs than all subjects of the CG. The mean ADC coefficients in the PG were the following: for RR $0.80 \pm 0.12$, for $S P-0.89 \pm 0.15$, and in the CG: $0.77 \pm 0.05$. There was no statistical significant difference between ADC coefficients in the CG, and in the PG for both forms of MS. The mean cervical cord cross sectional areas for subjects of the CG were $77.5 \pm 5.13$, and for patients $73.2 \pm 4.63(\mathrm{RR})$, and $63.2 \pm 6.01(\mathrm{SP})$, respectively.

Conclusion: Our approach gives us new quantitative way for the estimation of spinal cord abnormalities in patients with various forms of MS. The patients with MS demonstrate changes in DTI metrics measured in the cervical spinal cord compared to the healthy subjects. Patients with both RR and SP forms of MS have had significantly lower average cord FA within normal appearing cervical spinal cord than subjects of the CG.

\section{P358}

Imaging studies on primary progressive aphasia: a case analysis Y.M. Zhang, Z.H. Yang, X.Q. Zhao, C.X. Wang, Y.J. Wang Beijing Tian Hospital, Capital Medical University (Beijing, CN)

Background and purpose: Primary Progressive Aphasia (PPA) is a rare dementia syndrome with the only or prominent characteristic of progressive decline in language function. In advanced stage, deficit of cognitive capability and loss of daily living ability would turn up while memory ability would be relatively preserved. We often confused how to make the diagnosis because PPA had so many common with Alzheimer's disease. Neuro-imaging examination is a valuable method in making the differential diagnosis. In this study, we tried to report the intracerebral image features of one case of PPA so as to disclose the general lesion area of the disease, the changes of intracerebral blood perfuse and metabolism.

Methods: The PPA patient was diagnosed with Wernicke's aphasia and fluent oral language, we used 3.0T SIEMENS Trio 2003T and AG 2003 work station- siemens to scanning, data analyzed and imaging post processing. With conventionality MRI, we located the damaged areas, with perfusion weighted imaging (PWI), we studied regional blood volume (rCBV) and regional cerebral blood flow (rCBF), mean transit time (MTT), and time to peak (TTP) of the damaged brain areas and compared with that of the mirror hemisphere, and with magnetic resonance spectroscopy (MRS), we sought to measure the lesions macromolecules and small molecules, such as the metabolic rate of $\mathrm{N}$-acetylaspartate (NAA), choline (Cho), and creatine $(\mathrm{Cr})$, and compared the results with those of the mirror hemisphere.

Results: Conventionality MRI demonstrated atrophy of left temporal lobe and frontal lobe, especially left temporal pole of the case, PWI showed that the rCBV and rCBF were significantly decreased while MTT and TTP notably prolonged of these area, compared with those of the mirror hemisphere, and the content of NAA in these area were reduced than those of the c mirror hemisphere.

Conclusions: Major damaged regions of the PPA case is located at left temporal or frontal lobe, and hypoperfusion and hypometabolism of these area may be the pathologic mechanisms of the PPA case.

State Key Laboratory of Cognitive Neuroscience and Learning Open Project Grant and 20071D0300400078 


\section{Poster session 2}

\section{Cerebrovascular disorders}

P359

Cerebral vasoreactivity in non-insulin-dependent diabetic patients

A. Bal, Z. Kazibutowska, E. Motta, A. Golba, M. Debski

Medical University of Silesia (Katowice, PL)

Objectives: Diabetes mellitus acts as an independent risk factor for a circulatory system. The diabetic microangiopathy concerns all layers of artery wall causing endothelial and vasoactivity impairment. The incidence of cerebral vasoreactivity failure in diabetic patients without stroke history was still not estimated. The aim of this study was to assess cerebral vasoreactivity parameters in non-insulin-dependent-diabetic patients (NIDDP) without arterial hypertension and stroke history.

Methods: 32 NIDDP and 27 healthy volunteers were qualified to this study. Mean blood flow velocity and pulsatility index in extracranial and intracranial arteries by Doppler ultrosonography were measured. Changes of the mean blood flow velocity and pulsatility index in right middle cerebral artery after 3-min hyperventilation and 2-min ventilation of mixed gases $\left(5 \% \mathrm{CO}_{2}+95 \%\right.$ air) were also examined. The cerebral vasoreactivity index in hypocapnia and hypercapnia and the time needed to return mean blood flow velocity in right median cerebral artery to the basic level were measured.

Results: Hypocapnia: In NIDDP there was significantly lower decrease of mean blood flow velocity in right median cerebral artery and significantly lower cerebral vasoreactivity index in comparison with the control group. Significantly longer time was also needed for normalisation of blood flow velocity from hypocapnic value as in the control group. Hypercapnia: there was significantly lower mean blood flow velocity and significantly lower increase of the mean blood flow velocity in right median cerebral artery in diabetics in comparison with the control group. The cerebral vasoreactivity was significantly lower and time needed for normalisation of mean blood flow velocity from hypercapnic was longer comparing with the control group.

Conclusion: 1. There is impairment of cerebral vasoreativity in most of NIDDP without stroke history and without arterial hypertension, which may suggest the incidence of diabetic microangiopathy. 2. The transcranial doppler examination is a useful method of measuring vasoreactivity in cerebral arteries in non-insulin-dependent-diabetic patients.

P360

Severe myelopathy due to a spinal dural arteriovenous fistula of the upper thoracic spine. Rapid development of paraplegia mimicking an anterior spinal artery syndrome. A case report

A. Galanopoulos, M. Dimitraka, M. Xifaras, G. Gekas

General Hospital of Nikaea (Piraeus, GR)

Objevtives: We present the case of a young man who came with acute paraplegia, mimicking an anterior spinal artery syndrome, who was finally diagnosed as suffering from spinal dural arteriovenous fistula (SDAVF).

Methods: A 38-year-old man came to our Department because of gait difficulties, paraesthesias of lower extremities and urinary retention. At initial clinical examination, muscle strength was normal in upper and lower limbs, knee and ankle jerks were increased and plantar reflexes were extensor bilaterally. Hypoesthesia to pinprick was detected in lower limbs distally. Clinical course was rapidly progressive, as, in the first hours of admission, he developed paraplegia and complete loss of pain and temperature sensation below the umbilicus, with sparing of position and vibration sensation, mimicking an anterior spinal artery syndrome.

Results: Emergency MRI revealed an homogenous, T2-hyperintensive central lesion, expanding from T4 level to conus medullaris. Tortuous and dilated veins of dorsal spinal cord were also detected, mainly at lower thoracic spine, representing flow void phenomena. Brain and cervical MRI had no specific findings. Catheter angiography documented the existence of a SDAVF, in the T6-T7 intervertebral foramen, feeding from the right posterior sixth intercostal artery. As this artery was not found to contribute to the anterior spinal artery, embolization was attempted and it was successful. Neurological deficit improved significantly after treatment, as well as MRI findings. Patient could move his legs and perceive painful stimuli, although considerable disability remained in walking.

Conclusion: SDAVF is a rare disorder, usually leading to progressive myelopathy. "Arterialization" of venous drainage, due to the shunt of a radicular artery with its corresponding vein, is believed to be the responsible mechanism, as it leads to intramedullary venous hypertension, diminution of normal arteriovenous pressure gradient and spinal ischaemia. Myelopathy normally progresses rostrally in a period of months or years, when recurrencies are not scarce. However, SDAVF may rarely present as acute paraplegia, mimicking anterior spinal artery syndrome. Early detection of SDAVF is essential, because it is a potentially reversible disorder which may be treated with endovascular or surgical therapy, preventing thus severe disability.

\section{P361}

Arterial thoracic outlet syndrome causing stroke by retrograde embolism to vertebral and carotid arteries

A. Leitão, C. Nunes, C. Santos, I. Luzeiro

Centro Hospitalar de Coimbra (Coimbra, PT)

Background: The thoracic outlet syndrome (TOS) is a group of clinical entities with compression of the neurovascular structures that pass through this tight anatomical place, with neurogenic, arterial or venous symptomatology. When there is compression of the subclavian artery the formation of a local thrombus with anterogade or, rarely, retrograde embolism, is a possible complication.

Clinical case: The patient is a 23 years old female, healthy with the exception of a recent diagnosis of TOS caused by a cervical rib, manifested in the previous 5 months by progressivly worsening episodes of coldness, pallor, cyanosis, paresthesias and pain in the extremity and ulnar side of the right superior limb, triggered by its elevation. The angioMRI of the supraaortic trunks revealed arterial and venous compression after right arm elevation, and the nerve conduction studies did not show signs of lesion of the the brachial plexus.

This patient went to the emergency room because of a sudden vertiginous syndrome. At the clinical examination the right arm was hypotense, colder and with a weaker radial and ulnar pulse than the contralateral one; there was a nystagmus to the left, a right Horner syndrome, a right dysmetria and dysdiadochokinesia, and lateropulsion to the right. The cerebral MRI revealed small recent ischemic lesions in the lateral medulla and in the right cerebelar hemisphere, and no visualization of the right vertebral and carotid arteries. The cerebral angiography showed complete occlusion of three arteries: the right subclavian (after the emergency of cervical ascendant), the distal right vertebral and the distal common carotid. The remaining complete investigation of causes of stroke was negative. The patient was anticoagulated and submitted to a right carotid-umeral bypass with parcial exerisis of the right cervical rib. She had a normalization of the arterial flow in the right superior limb and a gradual recovery from the stroke symptoms.

Discussion: This case shows a preventable and ominous complication of an arterial TOS. In this young female there was evolution to a complete thrombosis of the right subclavian artery, which by a mechanism of retrograde embolization or propagation released embolus to the ipsilateral carotid and vertebral arteries, manifested by small strokes in the posterior circulation. To our knowledge it is the first time that it is described retrograde embolism from an arterial TOS both to the right carotid and vertebral systems.

\section{P362}

Serum soluble vascular cell adhesion molecule-1(s VCAM-1) in acute ischaemic stroke

A. Nassef, N. El-khayat, N. Fahmy

Ain Shams University (Cairo, EG)

Background: Neuroinflammatory mechanisms play an important role in ischemic injury and interruption of these processes can result in improved neurological outcomes. Firm adhesion of leukocytes to the endothelial cells as well as leukocyte activation and transmigration across the endothelium is mediated by adhesion molecules.

Aim of the work: We aimed to assess the levels of serum soluble vascular cell adhesion molecule-1 (sVCAM-1) in acute stroke and their correlation to the extent of neurological deficits and short term clinical outcome.

Subjects and methods: Forty four patients with first ever acute ischemic stroke admitted within 72 hours of stroke onset were enrolled,in addition to eighteen normal controls and twelve patients with vascular risk factors matched with age and sex to the patients group. Blood samples were obtained from the patients at the third day of stroke onset and from the control groups and subjected to the measurement of the level of sVCAM- 1 in the serum.

Results: There is a higher mean sVCAM-1 among patients with ischemic stroke compared to normal controls and the difference is highly significant statistically, $\mathrm{P}<0.01$. There is a higher mean sVCAM among patients with large vessel disease compared to patients with small vessel disease and the 
difference is highly significant statistically, $\mathrm{P}<0.01$. There is a highly significant positive correlation between sVCAM- 1 and NIHSS score, $\mathrm{P}<0.01$. Also we found a statistically significant negative correlation between Barthel Index Score and sVCAM-1, $\mathrm{P}<0.05$.

Conclusion: The results support the prior hypothesis of involvement of the adhesion molecules (VCAM-1) in the pathogenesis of acute cerebral ischemia and their impact on neuronal damage and neurological deficit. Therapeutic trials that attempt to interfere with the function of adhesion molecules could be of benefit in acute stroke management.

(Cerebrovascular disorders)

\section{P363}

Associated risk factors for acquired pneumonia among in-hospital stroke patients

A.A.Divani, R. Pakdaman, M.F.K. Suri, A.I. Qureshi

University of Minnesota (Minneapolis, US)

Objective: Investigating the risk factors related to stroke associated pneumonia (SAP) that can potentially be helpful in predicting SAP for prophylactic treatments.

Method: We reviewed the charts for stroke patients admitted to the University Hospital (Newark, NJ) in 2005. Patients who developed SAP during hospital stay were identified. Univariate and multivariate analyses were performed to evaluate the association between different risk factors and developing SAP among stroke patients.

Results: A total of 236 ischemic stroke $(n=188)$ and TIA $(n=48)$ patients' charts were reviewed for the study. Overall $13.6 \%$ of patients developed pneumonia. These patients were more likely had $>1$ alcoholic drink/day $(\mathrm{p}<0.038$, Odds ratio $[\mathrm{OR}]=5.1)$. Admission respiratory rate $(\mathrm{RR})$ of $>17$ showed a significant association with $\operatorname{SAP}(\mathrm{P}<0.038, \mathrm{OR}=2.9)$. Patients with SAP were more likely to be intubated and mechanically ventilated in the ER on presentation $(\mathrm{p}=0.008, \mathrm{OR}=6.4)$, suggesting admission ventilation as a possible risk factor for SAP. There was no significant association between age, race, gender, cigarette smoking, diabetes mellitus, elevated serum glucose, heart failure, intensive unit care stay duration, and malignancy on admission with SAP. We did not observed a significant difference in baseline National Institutes of Health Stroke Scale (NIHSS) as well as some individual NIHSS components (1: level of consciousness, 4: facial palsy, 10: dysarthria) among stroke patients who did and did not develop SAP.

Conclusion: Intubation and mechanical ventilation as well as admission $\mathrm{RR}>17$ may increase the likelihood of developing pneumonia among stroke patients. More extensive studies are required to further assess and ascertain the risk factors that can help to identify the subgroups of stroke patients who may develop SAP.

\section{P364}

Stroke fast track: a 10-month experience in a new Portuguese stroke unit A.M. Rodrigues

Sao Marcos Hospital (Braga, PT)

Background: The portuguese National Institute of Medical Emergency (NIME) coordinates an emergency prehospitalar Stroke Fast Track (SFT), that combines with intrahospitalar activation of a stroke fast track designed to provide thrombolysis to ischemic stroke patients. Hospital S Marcos stroke unit started providing such care in February 2007.

Objectives: To describe and analyse data regarding intrahospitalar activation of the SFT, and evaluate whether there are significant differences between patients brought by NIME and patients arriving by their own means.

Methods: We prospectively recorded data regarding all patients that were integrated in our hospitals' SFT from 5th February to 5th December 2007. Data were statistically processed using frequency analysis and the chi square test.

Results: In a reference area of 1100000 people, and $4600 \mathrm{~km}^{2}$, our hospital received 279 patients through the SFT. Two thirds of the patients were referenced by the prehospitalar SFT, while $33 \%$ came directly to the emergency department. No difference was registered between genders, and the mean age was 69 years. Stroke was diagnosed in $81 \%$ of patients. Other diagnoses were seizures, syncope and psychogenic disorders. Among patients with stroke, $70 \%$ presented ischemic stroke, $20 \%$ hemorrhagic stroke, $8 \%$ had transient ischemic attacks and $2 \%$ subaracnoid haemorrhage. Sixteen percent of all patients underwent intravenous therapy with rt- PA, corresponding to $33 \%$ of the ischemic stroke patients. In the group of patients who did not meet criteria for thrombolysis, $10 \%$ had an NIHSS below 5; $40 \%$ NIHSS above 22 and $50 \%$ had major contraindications for acute rt- PA therapy, such as unknown duration of the symptoms, symptoms present for more than 3 hours, and INR above 1,4. The only significant statistical difference between patients brought by the prehospital SFT and the other patients was that the time door-to-needle was shorter in the first group ( $46 \mathrm{vs} 62 \mathrm{~min}$ ), but the time from the beginning of the symptoms to treatment was the same. Despite this fact, we retrospectively analysed the distance travelled by the patients and detected that the first group of patients were brought from farther locations.

Conclusion: The creation of an intrahospitalar SFT and its important coordination with the prehospitalar SFT allows that a significant number of patients arrive on time to the Hospital, receive proper medical care, including the administration of rt- PA to an increasing number of patients.

\section{P365}

Phendimetrazine-containing drugs induce transient emiparesis B. Corrà, L. Adobbati, L. Lonati, A. Ciammola, G. Parati, V. Silan Università degli Studi di Milano (Milan, IT); Università Milano Bicocca (Milan, IT)

Introduction: Some commonly used anorexiants, including phendimetrazine associated with clorazepate dipotassium, have been associated with stroke-like episodes. In fact, they are amphetamine like sympathomimetics and cause hypertension, vasoconstriction and, in some case, vasculitis. We present the case of two patients taking these drugs and admitted to the Stroke Unit because of neurological symptoms. In our opinion symptoms were due to unreported effect of these molecules, namely vasospasm.

Case report: The first was a woman, 38 years old, taking appetite suppressant (phendimetrazine) for two months. The clinical history included hypertensive crisis, especially during the first pregnancy (nine years ago), hypothyroidism treated by L-tiroxine with full recovery. She was admitted in the Stroke Unit because of acute sensitive and motor right hemisyndrome. Systolic and diastolic arterial pressure was elevated at admission. CT brain scan (performed one hour after symptom onset) and MRI (1.5 T, including DWI) were normal. The symptoms regressed in a few hours. We concluded for transient ischemic attack.

The second patient was a man 51 years old; he was taking phendimetrazine for two months. No relevant events in his medical history. He was admitted in the Stroke Unit because of a motor deficit localized at the left upper limb. The blood pressure was normal. The neurological sign regressed in a week. CT scan (performed five hours after symptom onset) and MRI (1.5 $\mathrm{T}$, including DWI) were normal. We concluded for a minor stroke. There were no coagulation abnormalities at the thrombophylic screening. No atheromasic disease of the epi-aortic vessels at the Eco-Color-Doppler. The brain circulation was normal (it was investigated by Angio-MRI). There were no detected cardiac abnormalities at the echocardiogram.

At the follow up, both patients showed a complete neurological recovery.

Discussion: The two cases reported indicate the possible association between phendimetrazine and stroke. We have hypothesized vasospasm as the pathogenetic mechanism. The normal MRI with DWI supports this hypothesis. Further studies are needed to substantiate this speculation.

\section{P366}

Stroke features in Romania versus Hungary - a comparative study C. Szekeres, Z. Bajko, K. Fekete, I. Szocs, S. Szatmari, D. Bereczki Mures County Hospital (Tirgu Mures, RO); Semmelweis University (Budapest, $\mathrm{HU})$

Objectives: Establishment of a Two Center Stroke Database in two mediumsized European towns: Debrecen, Hungary (Central Europe) and Tirgu Mures, Romania (Central-Eastern Europe) for the comparison of stroke characteristics.

Methods: We examined all the patients admitted with cerebrovascular event between 1st of November 2004 - 30th of October 2005 in the two centres (1473 patients in Tirgu Mures, 764 men, 709 women and 851 patients in Debrecen, 435 men, 416 women).

We mention that in Tirgu Mures, Romania, approximately 600000 persons pertain to the two Clinics of Neurology. In Debrecen Hungary, 210000 persons belong to the Neurology Department of Health and Science Center this explains the big difference in the number of stroke patients admitted during one year to the two clinics.

Descriptive statistical methods were used in the analysis of our data.

Results: Mean age of stroke patients was similar in the two centres: Tirgu Mures, men: $67 \pm 10,5$, women: $70 \pm 10,9$; Debrecen, men: $67 \pm 12,1$, women: $70 \pm 13,3$.

The same difference between women's and men's mean age appears in both countries (men vs. women's average age: $\mathrm{p}<0,001$ in both cities).

Proportion of stroke subtypes differed between the two centres: Tirgu Mures, TIA: $2 \%$, ischaemic stroke: $79 \%$, haemorrhagic stroke: $19 \%$; Debrecen, TIA: $4 \%$, ischaemic stroke: $87 \%$, haemorrhagic stroke: $9 \%$. 
$26 \%$ of the cases in Tirgu Mures, and $38 \%$ of the patients in Debrecen had at least one cerebrovascular event before.

Blood pressure (BP) at admission: systolic BP Tirgu Mures vs. Debrecen $174 \pm 34,5 \mathrm{mmHg}$ vs. $151 \pm 29,3 \mathrm{mmHg}, \mathrm{p}<0,001$; diastolic $\mathrm{BP}: 97 \pm 17,5$ vs. $87 \pm 14,8, \mathrm{p}<0,001$

Conclusions: Mean age of stroke patients was similar, but percentage of stroke subtypes differed significantly in the two regions.

Blood pressure at admission was significantly higher in Tirgu Mures, Romania.

One explanation for this fact could be that more patients in Debrecen suffered one or more cerebrovascular events before, and had better control of treatable risk factors.

One other possible explanation is that in Hungary the population could have a better understanding and treatment of stroke risk factors. This theory is supported by the bigger percentage of patients treated with TIA in Hungary.

This study was in part supported by the Sapientia Foundation, Institute for Research Programmes (grant no. 1467/2004) and by the Romanian-Hungarian Intergovernmental Cooperation (grant no. Ro-19/2005).

P367

Association of metabolic syndrome with carotid atherosclerosis in the North Indian population

D. Khurana, S. Bal, A. Bhansali, S. Prabhakar

PGIMER (Chandigarh, IN)

Introduction: Metabolic Syndrome(MetS) is a risk factor for subsequent development of diabetes mellitus and cardiovascular disease.

Aims and objectives: 1 .To evaluate MetS patients for carotid atherosclerosis by carotid duplex ultrasound examination in North Indians. 2.To determine the significance of each component of MetS in relation to carotid atherosclerosis in these patients.

Methods: Individuals in the age group of 25-50 years,asymptomatic for cardiac or cerbrovascular disease were screened. 162 patients from a hospital based population fulfilled the criteria for MetS(as per NCEP ATP III criteria). 112 age and sex matched controls were selected for comparison. Duplex Ultrasound (DU) examination of extracranial carotid vessels was performed on all patients using Philips HDI-3500 system with a high resolution broad band linear transducer $(5-12 \mathrm{MHz}$ ).

Results: Among the MetS patients, $58 \%$ were males and $42 \%$ were females. Hypertriglyceridemia was the commonest component seen in $79.6 \%$ of the subjects, followed by central obesity seen in $74.6 \%$. Carotid Atherosclerosis(CAth) was observed in $35(21.6 \%)$ MetS patients. Among these, $60 \%$ were males and $40 \%$ females, $82.8 \%$ had mild atherosclerosis (IMT $>0.09 \mathrm{~cm}$ ) and $17.3 \%$ had plaques with mild stenosis $(<50 \%)$ in the carotid arteries. Among MetS patients with CAth $82.6 \%$ had hypertriglyceridemia and $62.8 \%$ had 4 or more risk factors for MetS. Among controls 5 subjects $(4.46 \%)$ had evidence of mild carotid atherosclerosis (IMT $>0.09 \mathrm{~cm}$ ) on DU. MetS was significantly associated with Carotid DU abnormalities as compares to controls (Fischer's exact test $\mathrm{p}<0.0001$ ). On multivariate regression analysis none of the individual components of MetS contributed significantly to presence of carotid atherosclerosis.

Conclusions: The prevalance of MetS is $58.6 \%$ in aymptomatic subjects in the North Indian population. 2. Hypertriglyceridemia is the commonest component of MetS in this population. 3. In patients of MetS with Carotid atherosclerosis, hypertriglyceridemia is the commonest component. 4. Serum triglyceride testing might serve as a pragmatic test for detection of MetS population at risk. 5. This at risk population could be taken up as a target population for prevention of cerebrovascular disease.

P368

Ischaemic and haemorrhagic stroke in young subjects in the Venezuelan Andes: an study of frequency, atiology and risk factors

G. Molina, H. Araujo

Ospedale Niguarda Cà Granda (Milan, IT); Hospital Universitario de Los Andes (Mérida, VE)

Objectives: To determine frequencies, etiologies and risk factors of ischemic and hemorrhagic stroke in the young adult population. Second, to compare these parameters between two different age groups in which the population was divided.

Methodology: We retrospectively identified cases of ischemic and hemorrhagic stroke in the age range from 15 to 45 years who entered the Hospital Universitario de Los Andes of Mérida - Venezuela between January 1993 and December 2003. Medical records were reviewed for etiology and risk factors and an expert validated questionary was filled for every case. Is- chemic stroke subtypes were clasified on the basis of Trial of ORG 10172 in Acute Stroke Treatment (TOAST)criteria. A further comparison of evaluated parameters was made between two age subgroups (15 to 30 and 31 to 45 years of age)

Results: A total number of 269 cases were found. 107 (39,8\%) of cases corresponded to ischemic stroke and $162(60,2 \%)$ to hemorrhagic stroke. When the total population was divided in two age groups, $23 \%$ of cases were found to be in the age range between 15 and 30 years old and $77 \%$ in the age range between 31 and 45 years old. There was not statistical significative difference in the frequency of hemorrhagic and ischemic stroke

between the two age subgroups. Out of the total of hemorrhagic strokes, $94(58 \%)$ of cases corresponded to intracerebral hemorrhage and $68(42 \%)$ to subarachnoid hemorrhage. When ischemic stroke etiology was studied, the following results were found: $24,1 \%$ were considered to be due to atheroesclerosis or small-vessel occlusion, $21,6 \%$ to cardioembolism, $23,3 \%$ had unusual determined causes and in $31 \%$ etiology was undetermined. All ischemic stroke cases considered to have an atherothrombotic origin occurred in patients older than 30 years. Most important risk factors were arterial hypertension, smoking and cronic alcohol intake, but all of them resulted to be relevant just in the age subgroup from 31 to 45 years old.

Conclusions: Hemorrhagic stroke is more frequent than ischemic stroke in young people in the Venezuelan Andes. Etiology of both kinds of stroke in this population is very variable and may correspond to multiple causes. Traditional risk factors for cerebrovascular disease and atherothrombotic stroke origin are more likely to occur in subjects over 30 years old.

P370

Safety and feasibility in clinical practice of percutaneous closure of patent foramen ovale in patients with ischaemic stroke

M.A. Ortega-Casarrubios, B. Fuentes, B. San José, P. Martínez, M. Lara, L. Calvo, G. Galeote, E. Díez-Tejedor

Hospital Universitario La Paz (Madrid, ES)

Objetives: In patients under 55 years old, the combination of Patent Foramen Ovale (PFO) and Atrial Septal Aneurism (ASA) is associated with increased risk of ischemic stroke (IS).In these cases and those with recurrent stroke despite pharmacological treatment percutaneous closure of PFO could be considered a therapeutic option. We evaluated the safety and feasibility of percutaneous closure.

Methods: Observational study with inclusion of consecutive patients with IS under 55 years old managed on the stroke unit (January 2002-December 2006) that were treated with percutaneous closure (if PFO+ASA or isolated $\mathrm{PFO}+$ recurrent stroke). Percutaneous closure was performed with Amplatzer device $(18,25$, and $35 \mathrm{~mm})$. We evaluated: recurrences, complications, residual shunt with Transcraneal-Doppler and echocardiography.

Results: Thirteen patients $(69.2 \%$ men). Mean age: $43.5 \pm 8.9$ years. Seven with IS and six with transient ischemic attack. Eleven patients with PFO+ASA and 2 with isolated PFO+ recurrent stroke. Average time since stroke until percutaneous closure: 168 days (35-455). The follow-up time after closure was 760.4 days (277-1285). Complications: One patient with peri- 
cardial effusion without cardiovascular signs or symptoms. There has been no recurrence. In two patients remained a residual shunt after a year.

Conclusion: Percutaneous closure of PFO can be a safety and feasibility procedure in order to prevent recurrences in selected patients under 55 years old with PFO-related IS.

P371

Dual antiplatelet therapy with clopidogrel and aspirin for stroke prevention following transient ischaemic attack (TIA) or mild-to-moderate ischaemic stroke

M.R. Motamed, S.M. Fereshtehnejad, N. Sadigh

Iran University of Medical Sciences (Tehran, IR)

Objectives: Patients who have transient ischemic attack (TIA) or ischemic stroke are at a high risk of having a first or recurrent stroke. Secondary prevention includes antithrombotic therapy, treatment of risk factors, and interventional treatment of carotid stenosis. The merits of antiplatelet therapy with different protocols in primary and secondary stroke prevention have been demonstrated across numerous trials which lead to somewhat contradictory findings. Our objective was to compare the effects of aspirin, clopidogrel, and clopidogrel plus aspirin in the prevention of recurrent stroke.

Methods: Ninety-two patients with TIA or minor ischemic stroke were randomly assigned in three groups: Group A (aspirin, $80-100 \mathrm{mg} /$ day; 29 patients), group B (clopidogrel, $75 \mathrm{mg} /$ day; 31 patients) and group C (clopidogrel $75 \mathrm{mg} /$ day plus aspirin $80-100 \mathrm{mg} /$ day; 32 patients). All patients were followed for a period of 18 months. Rather than demographic data, the number of new attacks and the time it occurs were recorded for each patient. Kaplan-Meier analysis was performed to compare the median time to stroke outcome in each group.

Results: The patients were $53(57.6 \%)$ male and $39(42.4 \%)$ female with the mean age of $65.47(\mathrm{SD}=8.36)$ year which was not significantly different in three groups of study $(\mathrm{P}>0.05)$. After 18 months of follow-up, the mortality rate was $13.8 \%$, none and $3.1 \%$ in groups $\mathrm{A}, \mathrm{B}$ and $\mathrm{C}$, respectively. The mean number of attacks was significantly lower in group $\mathrm{C}$ [0.63(standard deviation $(\mathrm{SD})=0.49)]$ than groups $\mathrm{A}[1.59(\mathrm{SD}=0.95)]$ and $\mathrm{B}[0.81(\mathrm{SD}=$ $0.65)](\mathrm{P}<0.001)$. Moreover, the mean time to stroke outcome was significantly longer in group $\mathrm{C}[16.12$ (Standard error $(\mathrm{SE})=0.57$ ) month] than groups A [6.76(SE $=1.21)$ month] and B $[13.71(\mathrm{SD}=1.02)$ month] $($ Log rank $\stackrel{\mathrm{P}}{<0.001) \text {. }}$.

Conclusion: Antiplatelet therapy is effective in secondary stroke prevention. Current recommendations are applied in clinical practice, but great variability between different centers remains. Our results showed that aspirin combined use with clopidogrel have better efficacy and decreased rate of recurrence in patients with TIA or minor ischemic stroke in this singlecenter study.

\section{P372}

Sildenafil and cerebral haemorrhage. A report of two cases

O. Anlar, F. Halacoglu

Ataturk Hospital (Ankara, TR)

Sildenafil is an orally active, potent and selective inhibitor of phosphodiesterase type 5, an important regulator of cyclic guanosine monophosphate in the human corpus cavernosum which has recently been introduced for the treatment of erectile dysfunction.

We report two cases who developed intracerebral hemorrhage after sildenafil consumption. The first patient was a 39 year-old man who admitted to hospital with speech disturbance and right limb weakness. He had taken one tablet of Sildenafi $25 \mathrm{mg}$ one hour before. There was no past history of diabetes mellitus, hypertension, erectile dysfunction or another illness. Brain CT scan showed central pontin hemorrhage. MRI demonstrated a pontine cavernous malformation. The second patient was a 60 year-old man who referred to our clinic with speech disturbance and left hemiparesia. From the history it appeared that the symptoms started after tow hours following ingestion of one tablet of sildenafil $100 \mathrm{mg}$. There was a history of erectile dysfunction, arterial hypertension and diabetes mellitus. Brain CT scan showed right thalamic hemorrhage.

The reported occurrence of adverse effects of sildenafil like headache, dizziness, visual disturbances, retinal dysfunction and pupil-sparing third nerve palsy suggest that sildenafil also affects brain microvasculature regulation. We would like to emphasize that spontaneous cerebral hemorrhage can be aggravated after ingestion of sildenafil preparations.
P373

Is the video pharyngolaryngoscopy using a flexible fibre optic endoscopy a practicable method in the diagnosis of swallowing disorders for stroke patients?

P. Grieshofer

Klinik Judendorf Strassengel (Judendorf Strassengel, AT)

Neurogenic swallowing disorders (dysphagia) are very frequent symptomatic complex of neurological diseases. The incidence and importance, however is often underestimated. Gordon et al.(1987) for example found out that $41 \%$ of acute-care stroke patients are affected by dysphagia within the first two weeks after stroke. Approximately $16 \%$ of patients still have swallowing disorders during their stage of rehabilitation.

Patients with neurogenic dysphagia are more likely to be affected by pulmonary infections and dehydration. The rate of lethality is higher. The swallowing research and treadmend group has tried to find out if the videopharyngolaryngoscopy using a flexible fiber optic endoscopy is practicable and safety diagnostic method for a workaday bedside routine of a rehabilitation clinic.

Within one and a half year we examined 185 patients (3-16 weeks after acute stroke) using flexible fiber optic endoscopy. The examination criteria were anamnestically known, presumed or recurrent pulmonary infections.

65 of all examined 185 patients had normal findings (no swallowing disorders), 35 patients had pathologic findings (swallowing disorders without aspiration), 34 patients had swallowing disorders with aspiration. $44 \mathrm{pa}-$ tients of all 150 examined patients had a percutaneous endoscopic gastrostomy tube (PEG-tube) before the examination in your clinic. 25 of these patients had a PEG tube although they didn't show any sign of aspiration. 23 patients had swallowing disorders with aspiration. All examinations were carried out without complications. 8 patients refused examination.

Conclusion: The video-pharyngolaryngoscopy using a flexible fiber optic endoscopy is a safety and diagnostic method to examine oropharyngeal swallowing disorders after stroke. Esophageal dyshagia, however, cannot be examined.

\section{P374}

Combination of infarctions in the anterior spinal artery and posterior inferior cerebellar artery territories. A case report

S. Ben Sassi, G. El Euch-Fayache, A. Regaieg, T. Mabrouk, F. Hentati

National Institute of Neurology (Tunis, TN)

We report the case of a 53-year-old man with a history of lower limb phlebitis who developed acute pure motor deficit in both upper limbs (manin-the-barrel syndrome) with unsteadiness subsequently to a splenic infarct treated by heparin.

Magnetic resonance imaging (MRI) showed infarcts in the upper cervical cord (anterior spinal artery territory) and the left cerebellar hemisphere (posterior inferior cerebellar artery territory).

The man-in-the-barrel syndrome is as yet a barely described clinical feature attributed to ischemia in the territory of the anterior spinal artery (ASA); the combination of posterior inferior cerebellar artery (PICA) and incomplete anterior spinal artery (ASA) infarcts has not been reported previously. Antiphospholipid syndrome and heparin induced thrombocytopenia are the possible causes of this unusual association.

P375

Role of cystatin $\mathrm{C}$ level in the differential diagnosis of central and peripheral vertigo

W. Kim, D.G. Kim, S. Oh, H. Kim, O. Kim, M. Lee

Bundang CHA Hospital, Pochon CHA University College of Medicine (Seong Nam, KR); Youngdong Severance Hospital, Yonsei University College of Medicine (Seoul, KR)

Objectives: It is always a challenging problem to differentiate cerebrovascular origin of vertigo from peripheral etiology for clinical neurologists. Various serological markers have been challenged for differentiation of different type of vertigo. Recent studies suggested that elevated cystatin $\mathrm{C}(\mathrm{CysC})$ level is associated with a higher prevalence of subclinical cerebral infarction. We evaluated the diagnostic efficacy of CysC level in differential diagnosis of central and peripheral vertigo.

Methods: The clinical records were collected retrospectively to search for the patients who were admitted to Bundang CHA hospital during recent 2 years for the treatment of acute vertigo. Total 64 patients with vertigo were identified and followed as part of an ongoing clinical study. Patients who showed subclinical ischemic lesions of brain and vertebrobasilar insufficiency on brain MRI/MR angiography were classified as central vertigo $(\mathrm{CV})$, and peripheral vertigo (PV) was diagnosed in patients with typical 
symptoms, normal brain MRI and normal arterial patency. We compared the CysC level and other clinical data between CV and PV groups.

Results: Total 37 patients were diagnosed as $\mathrm{CV}$, and 27 patients as PV. Mean age of CV patients $(64.51 \pm 9.35 \mathrm{yrs})$ was higher than PV patients $(50.78 \pm 8.70 \mathrm{yrs}, \mathrm{p}<0.001)$. The CysC level was significantly increased in CV $(0.98 \pm 0.22 \mathrm{mg} / \mathrm{l})$ than PV patients $(0.80 \pm 0.16 \mathrm{mg} / \mathrm{l}, \mathrm{p}=0.002)$. Serum creatinine level was also higher in CV $(0.95 \pm 0.20 \mathrm{mg} / \mathrm{dl})$ than PV patients $(0.84 \pm 0.20 \mathrm{mg} / \mathrm{dl}, \mathrm{p}=0.034)$.

Conclusion: The CysC level was elevated in patients with $\mathrm{CV}$, who had ischemic lesions on brain MRI. We suggest that serum CysC level is a feasible and useful surrogate marker in differentiation of central and peripheral vertigo.

P376

Relationship of handedness and language-dominant hemisphere

Z.H. Yang, X.Q. Zhao, C.X. Wang, Z.H. Han, Y.M. Zhang

Beijing Tian Hospital (Beijing, CN); Ministry of Education (Beijing, CN)

Purpose: With the development of medical, the widespread application of imaging, provides evidences for the view of language-dominant hemisphere. In this study, we try to analysis handedness and brain lateralization using aphasia patients.

Methods: We selected 400 stroke cases who were admitted to the department of neurology of beijing tiantan hospital from 2005 to June 2006, and used MRI conducted their brains and western aphasia battery (WAB) classified their language disorders, on the other hand, we also used handedness criterion judged handedness.

Results: All the 400 cases entered the result analysis. Among all the 400 cases of patients who had single lesion focus, there were 237 cases of left hemisphere lesion and 163 cases of right hemisphere lesion. According to the stroke type, there were 254 cases of cerebral infarction and 146 cases of cerebral hemorrhage.

According to the WAB, there were 178 aphasia cases among 400 patients $(44.5 \% 178 / 400)$, and 39 Broca aphasia cases, 35 Wernicke aphasia cases, 4 conduction aphasia cases, 15 complete aphasia cases, 23 transcortical motor aphasia cases, 14 transcortical sensory aphasia cases, 12 transcortical mixed aphasia cases, and 36 nominal aphasia cases. The lesion positions were mainly located in left cerebral hemisphere, and there were 169 cases of left hemisphere lesion and 9 cases of right hemisphere lesion; among 222 cases of non-aphasia, there were 68 cases of left hemisphere lesion and 154 cases of right hemisphere lesion.

Among 400 cases of patients, there were 366 right-handedness $(91.5 \%$, $366 / 400$ ), in which, there were 158 cases of aphasia out of 218 cases of left hemisphere lesion and 7 cases of aphasia out of 148 cases of right hemisphere lesion (crossed aphasia); among 34 cases of non-right handedness $(8.5 \%, 34 / 400)$, there were 19 cases of left hemisphere lesion (11 aphasia cases) and 15 cases of right hemisphere lesion (2 aphasia cases).

Conclusion: Most dominant hemispheres of right-handed people among patients with stroke are left cerebral hemispheres and few are right cerebral hemispheres of Chinese people; most dominant hemispheres of non-righthanded people are left cerebral hemisphere and few are right cerebral hemisphere, that is language-dominant hemisphere is mainly located in left cerebral hemisphere no matter what the patient is left-handed or right-handed.

Key Laboratory for Cognitive Science and Learning of Ministry of Education and School of Psychology and Nature Science Foundation of Beijing (No. 7052035).

\section{Clinical neurophysiology}

\section{P377}

Bilateral drop foot as an initial manifestation of a primary intramedullary tumour

A. Argyriou, A. Makridou, P. Karanasios, N. Makris

Saint Andrew's General Hospital of Patras (Patras, GR)

Aim: We describe the case of a male patient that developed bilateral drop foot (BDF), as an initial manifestation of a primary tumor in the conus medullaris of the spinal cord.

Case report: A 82-years-old Caucasian male patient presented with BDF. Symptoms of weakened dorsiflexion were developed on the left foot 13 months earlier and progressed slowly. Two months later, the right foot dorsiflexors were also affected. Clinical examination revealed muscle wasting in both TAs, more pronounced on the left side and paresis of both TAs and
EHLs, slightly worst at left. Patellar reflexes were diminished, while ankle reflexes were absent. Plantar reflexes were on flexion. Apart from benign prostatic hypertrophy, his past medical history was otherwise unremarkable. Blood counts, general biochemistry, proteins, tumor markers, and levels of vitamin B12 were either normal or negative. Conduction studies revealed normal velocity and decreased amplitude of motor action potential from the right extensor digitorum brevis, which was absent on the left side. Sensory conduction study on both sides was at the lower limits of normal. Electromyoagraphy revealed fibrillations and positive sharp waves in both TAs and EHLs and a much reduced interference pattern with increased amplitudes of motor potentials in both TAs and EHLs, more pronounced on the left side. Muscle sampling in the hands, tongue and rectus abdominalis was normal. A brain CT scan was normal, while in the lumbar spine MRI a highsignal intensity lesion located in the conus medullaris, measuring approximately $2.1 \times 0.4 \mathrm{~cm}$ was revealed. Following Gadolinium administration, there was an irregular enhancement of the lesion and a cystic component was noted, closely in keeping with an intramedullary astrocytoma. Additional findings were a L5-S1 central disc protrusion and mild stenosis at L2L3 and L4-L5 levels, which were thought to be inconsistent with the degree of clinical presentation. Abdominal and lung CT scans were normal.

Results: Surgical intervention was contradicted due to negative prognostic factors. A year later, the patient denies being followed-up with longitudinal MRIs, while his motor deficit concerning dorsiflexion has been clinically progressed. No additional symptoms occurred and no other muscles were affected in the meantime.

Conclusion: CNS pathology should be considered in the differential diagnosis of BDF, especially when other common causes have been ruled-out.

\section{P378}

The significance of lumbrical-interosseous latency comparison in diagnosing carpal tunnel syndrome

A. Argyriou, P. Karanasios, A. Makridou, N. Makris

Saint Andrew's General Hospital of Patras (Patras, GR)

Aim: We sought to assess the significance of the lumbrical-interosseous latency (2LI-DML) comparison in the diagnosis of carpal tunnel syndrome (CTS).

Patients and methods: We examined 62 consecutive hands referred with suspected CTS, using the 2LI-DML test and other measures of median nerve function, including amplitude of orthodromic SNAP from finger 2 (F2 aSAP), velocity of orthodromic SNAP from F2 (F2-SCV), morphology (single or double pick) of SNAP from F4, orthodromic median-ulnar palmar velocity comparison (MUPV) and median distal motor latency to APB (APBDML).The 2LI-DML test was performed as previously described by Preston and Logigian. A subgroup of 25 control hands from healthy subjects was also assessed to calculate our Labs upper limit of normal (ULN) and furthermore support the established ULN of less than $0.4 \mathrm{msec}$ for the 2LI-DML test. Individual 2LI-DML difference was considered abnormal if exceeded mean + $2 \mathrm{SD}$, recorded from healthy hands. Correlations of the 2LI-DML test with standard tests were examined using the Spearman's rank correlation coefficient (CC).

Results: In the control hands the mean 2LI-DML difference was $0.15 \mathrm{msec}$ (SD, $0.115 \mathrm{msec}$ ), so that the ULN value was set at $0.38 \mathrm{msec}$, corresponding to the widely accepted value of for abnormality $(>0.4 \mathrm{msec})$. Amongst the initially referred 62 hands, 43 (69.3\%) were electrophysiologically confirmed to have CTS. The 2LI-DML was abnormal in $100 \%$ of CTS hands, whereas in 3 hands the 2LI-DML was the only abnormal test. The mean 2LI-DML difference in the CTS hands was $1.85 \mathrm{msec}$ (SD: $1.24 \mathrm{msec}$, range: $0.5-5.0 \mathrm{msec}$ ). The $2 \mathrm{LI}-\mathrm{DML}$ difference was highly abnormal $(5.0 \mathrm{msec})$ in a single hand with severe CTS, in which both motor and sensory action potentials were absent. Among the other measures of median nerve function, the MUPV was the most frequently abnormal test (40/43 hands, $93 \%$ ), followed by F2-SCV (38/43 hands, $88.4 \%$ ), F4 double-pick morphology of waveform (36/43 hands, 83.7 \%), APB-DML (31/43, 72.1\%) and F2 a-SAP (23/43 hands, 53.5\%). The 2LI-DML test was significantly correlated with standard tests. The CCs of the 2LI-DML with MUPV, F2-SCV and APB-DML were $0.750,0.868$ and 0.920 , respectively.

Conclusion: The 2LI-DML comparison is highly sensitive in diagnosing CTS, even in mild cases in which other tests of median nerve function fail to detect abnormalities. Considering that the 2LI-DML test is quickly and easily applied, its routine use in the diagnosis of CTS is recommended. 
P379

Predicting disability in post-traumatic coma: prognostic value of somatosensory-evoked potentials

A. Gagliardo, S. Fossi, R. Carrai, E. Mazzeschi, S. Scarpelli, G. Cianchi, M. Bonizzoli, A. Peris, A. Grippo, A. Amantini, F. Pinto

Careggi Hospital University of Florence (Florence, IT)

Purpose: A large part of comatose patients has a traumatic aetiology; about $60 \%$ of the post-traumatic comatose patients survives, one third of whom has a severe disability. We have evaluated the role of Somatosensory Evoked Potentials (SEPs) for prediction of disability vs. recovery.

Materials and methods: We retrospectively evaluated patients with severe head injury (GCS $<=8$ at the admission in Intensive Care Unit). We identified 120 patients whose SEPs have been recorded in the first two weeks of recovery. Cortical SEPs have been classified in each hemisphere as normal $(\mathrm{N})$, pathological (P) for CTT or amplitude, or absent (A). We grouped the SEPs patterns in three grades: grade I (NN, NP), grade II (PP), and grade III (AN, AP, AA). The outcome has been evaluated at no less than 1 year since trauma, using the Glasgow Outcome Scale (GOS).

Results: $24 \%$ of our patients did not awaken (Death or Vegetative State), $22 \%$ developed a Severe Disability, $54 \%$ resulted in a Moderate Disability or a Good Recovery. Among the patients with a grade I SEP, the $92 \%$ awakened $(60 / 65)$, and $54 / 65(\mathrm{PPV}=83 \%)$ had a good outcome (GOS $4-5 \mathrm{LR}+=4,2)$. All the patients but one, with a grade III SEP (26/27), had an unfavourable outcome (GOS $1-3$ PPV 96,3\% LR+ = 30,7). The patients with a grade II SEP $(28 / 120)$, had a prognostic uncertainty (8 not awakening, 10 with severe disability, 10 with moderate disability or good recovery). The $87 \%$ of the patients with a grade I and III SEP has been correctly classified.

Conclusions: In the post-traumatic coma, the awakening is the most common outcome. Then a useful prognostic index for both functional recovery (grade I) and severe disability (grade III) is needed. Since the absence of a cortical SEP stands for an extended hemispheric injury, we have considered the AN and AP SEPs unfavourable patterns for disability (GOS 1-3), alongside the AA pattern. Furthermore we have highlighted the favourable prognostic role of grade I SEP for the functional recovery.

Taking into account the high positive predictive value of grade I SEP $(\mathrm{NN}, \mathrm{NP})$, in case of discrepancy between the clinical state and the predicted prognosis, we have to consider the presence of confounding factors such as hypoxic/hypoperfusive events, axonal damage in strategic areas, or concurrent peripheral causes of weakness.

An SEP recording in the acute coma allows an early identification of patients subgroups with regard to recovery course, neurorehabilitation approach and final outcome.

P380

High-resolution sonography and electrophysiology in carpal tunnel syndrome

A.K. Meena, B.D. Babu, S. Sitajayalakshmi, V.V.S.R.K. Prasad, R. Borgohain, U. Maheshwar

Nizam's Institute of Medical Sciences (Hyderabad, IN)

Objective: The aim of this study was to compare the diagnostic accuracy of electrodiagnostic testing (EDX) and high-resolution sonography (HRUS) in patients with carpal tunnel syndrome (CTS.

Methods: A prospective cohort of 57 patients with possible CTS underwent high-resolution sonography and EDX. The diagnosis of CTS was based on clinical signs and symptoms. The cross-sectional area of the median nerve at the carpal tunnel inlet was assessed by a radiologist, blinded to the clinical and EDX data. Normal sonographic and EDX values were obtained from 50 controls. Sensory palm-wrist, motor palm-wrist latencies,median nerve cross sectional area compared among CTS patients and control subjects using student " $t$ " test.

Results: Ninety-two hands from 57 patients met the clinical diagnosis for CTS. With the cut off value of 0.4 palm-wrist distal sensory latency difference had a sensitivity of $86.43 \%$ and specificity of $94.12 \%$ and a sensitivity and specificity of $72.32 \% \& 96.36 \%$ respectively with cut off value of 0.6 . Mean cross sectional area of median nerve at the inlet of carpal tunnel was $11 \pm 2.75 \mathrm{~mm}^{2}$. Cross sectional area of median nerve at the inlet of carpal tunnel $\geq 11 \mathrm{~mm}^{2}$ had a sensitivity, specificity, positive predictive value and negative predictive value of $54.8 \%, 72.7 \%$. $49 \%, 50 \%$ respectively $(\mathrm{p}<0.0001)$.

Conclusion: In patients with a clinical diagnosis of CTS, the accuracy of HRUS is comparable to that of EDX. High resolution sonography may be a screening tool because it is painless, easily accessible and preferred by the patients and it detects structural abnormalities which may have therapeutic implications.
P381

A case of false paralysis of upper limb: from placebo effect of the TMS to the reorganisation of the hand motor area

C. Bohotin, V. Bohotin, C.D. Popescu

University of Medicine and Pharmacy (Iasi, RO)

Background: In response to different tasks, in normal and pathological conditions, the cortical organization of sensory-motor area has been shown to be highly plastic. Acquisition of a new task is follow by increases of motor projection of cortical territory which supply the specific movement.

Material and methods: In this report, we describe a 33 years old woman which presents a one year false paralysis of left upper limb (after an emotional stress). During this year the patient does not use at all her left upper limb, all activities being made with contra-lateral limb. Neurological examination was normal but we notice the absence of active movements for left upper limb.

Results: Using transcranial magnetic stimulation with a figure of eight coil we measured the motor threshold (MT), motor evoked potentials (MEP) latency and amplitude. On both sides, the motor threshold was $46 \%$ (i.e. bilateral integrity of motor pathway). The MEP amplitude after single pulse TMS at $120 \%$ MT over motor hot spot was 2546 microV for unaffected side and 284 microV on the affected side. The testing session was enough for the patient to start to move her left upper limb. The fully recovery was obtained in around one week. TMS reevaluation, 2 weeks later, shows an important increase $(660 \%)$ of motor evoked potentials amplitude for the left hand (1873 microV) and no modification for right hand (0\%).

Conclusion: We demonstrate a TMS placebo's therapeutic effects because even that single pulse TMS could not induce long lasting effect the patient start to used her hand soon after testing. In addition, our results are one more prove that the increase activity induce a larger projection of involved cortical area while lack of movement induce a significant decrease of cortical projection with a significant decrease of motor performance of respectively cortical area.

\section{P382}

Concentric needle single-fibre electromyography: normative jitter values on voluntary activated extensor digitorum communis

D. Parissis, N. Taskos

Aristotle University of Thessaloniki (Thessaloniki, GR)

Objectives: Single fiber electromyography (SFEMG) is the most sensitive diagnostic method in the evaluation of suspected neuromuscular transmission abnormalities. The use of a reusable, specially constructed electrode has raised concern regarding the risk of transmission of infectious agents, such as prion disease. Therefore, concentric-needle SFEMG has become increasingly popular and appears to be valid in the diagnosis of myasthenia gravis. However, the normative values of the modified technique are still unknown. The aim of this study was to estimate normal jitter parameters on voluntary activated Extensor Digitorum Communis (EDC) using a concentric needle electrode (CNE).

Methods: We prospectively studied 20 healthy subjects. There were 12 men (mean age $24,2 \pm 2,5$ years) and 8 women (mean age $23,5 \pm 0,9$ years). A portable Keypoint electromyograph with inbuilt SFEMG software was used for recording and analysis. A disposable CNE with a diameter of $0,03 \mathrm{~mm}$ and a recording area of $0,03 \mathrm{~mm}^{2}$ was used in all cases. Filter setting was $2 \mathrm{KHz}$ to $10 \mathrm{KHz}$. At least 20 different single fiber action potential pairs were sampled for each subject. The mean value of consecutive differences (MCD) of individual pairs and the mean MCD of each subject were automatically calculated, whereas the examiner noted any impulse blocking.

Results: The mean MCD $(n=20)$ obtained was 23,3 2,7 sec (range of mean values in each subject was 19-29). The upper normal limit of mean MCD is $31,4 \mathrm{sec}$ (which is $3 \mathrm{SD}$ above the mean).

The mean jitter of all potential pairs ( $n=400$, pooled data) analyzed was $23,2 \pm 7,4 \mathrm{sec}$ (range 9-59). The upper normal limit of MCD for individual potential pairs is set to 45,5 usec (which is $3 \mathrm{SD}$ above the mean). This practically means that if more than 2 (among 20) pairs demonstrate jitter value above 45,5 usec, the study indicates abnormality.

Finally, no blocking was detected.

Conclusion: We obtained normal values of jitter for voluntary activated EDC using CNE in a small sample of young adults. Interestingly, our findings are in agreement with the results of previous observations and can be used as a rapid reference. Large multicentre studies, that will define recording conditions (filters, acceptable signals) and analysis procedures (peak or level trigger) are needed to provide more valid reference material. 
P383

The value of somatosensory-evoked potentials in the diagnosis of subclinical myelopathy caused by cervical spondylosis

D. Tsiptsios, C. Maglaras, D. Fotiou, M. Krommyda, M. Parava, P. Beredimas, A. Karlovasitou

Ahepa University Hospital (Thessaloniki, GR); Petra Psychiatric Hospital (Katerini, GR)

Objectives: Myelopathy caused by cervical spondylosis (CS) is one of the most common neurological conditions of the spinal cord observed in the middle-aged population. However, in many cases the neuroimaging findings of the disease are not accompanied by the expected clinical signs and symptoms.

Somatosensory evoked potentials (SSEP) of the upper and lower limbs can provide a useful prospective to the evaluation of the functional integrity of the peripheral and central somatosensory pathway, which is usually affected in CS.

Methods: In 30 patients with severe radiological alterations suggesting $\mathrm{CS}$, but without or mild clinical symptomatology of CS, the delay and amplitude of the SSEP from the upper limbs (N9, N13 and N19) after stimulation of the median and ulnar nerve and of the SSEP from the lower limbs (N21 and P38) after stimulation of the posterior tibial and peroneal nerve were recorded. The patients included in the study did not suffer from any other disease that could possibly affect the somatosensory pathway, such as lumbar spondylosis or diabetes mellitus.

Results: The alterations of N13 morphology and the prolonged N21-P38 time, indicative of spinal cord impairment, were reported as the most common pathological neurophysiological findings. These findings were particularly characteristic in patients that presented cervical stenosis of the spinal column $<13 \mathrm{~mm}$

Conclusions: The SSEP of the upper and the lower limbs exhibit significant reliability in the assessment of the functional integrity of the somatosensory pathway. Therefore, they can provide a useful tool in the diagnosis of subclinical myelopathy due to CS

\section{P384}

Effect of the vallproate acid on the EEG patterns in epileptic children

I. Khachidze, V. Maloletnev, M. Gugushvili

Beritashvili Institute of Physiology (Tbilisi, GE)

Objectives: The aim of the study: to investigate the alteration of different EEG characteristics in epileptic children during the treatment with Valproate acid (Depakin).

Methods: 56 patients aged 3 to 12 were examined. Generalized tonic, clonic and absence seizures were observed in 9 combined seizures in 8 ,myclonic-7 and partial-32 patients. All the patients underwent the qEEG recording at the first visit prior to the administration of any anticonvulsants, further 3-8 months after the start of D consumption. For the analysis were selected the $20 \mathrm{sec}$.samples recorded at rest, during functional loads and 20 sec after their termination.

The EEG pattern with monopolar, bipolar and common average references montage for the evaluation of the background activity specificity and of the presence of epileptiform elements, Absolute values of the power spectra (AVP) of activities were analyzed.

Results: D appears significantly reduce the total AVP indices practically in all zones recorded from the brain's convexital surface.

The EEG analysis of D to influence on epileptiform graphoelements shows that its effect reveals primarily in the reduction of typical epileptiform complexes peak-wave and sharp waves.Also complete reduction of these elements in the resting EEG was observed practically as early as 3 months after the beginning of the treatment.At the same time, the influence of $\mathrm{D}$ on sharp wave,sponteneous paroxysmal bursts provoked by functional tests was expressed to a less extent: these graphoelements continued to be recorded after 6-8 months after the commencement of the treatment.

Conclusion: The dynamics of quantitative (absolute and relative power spectra) and qualitative (epileptiform grapho-elements) characteristics of EEGs has been studied using the method of computer EEG analysis.The results show that D suppresses the generation of peak-wave complexes and spike discharges. To less extent $\mathrm{D}$ influences the generation of sharp and spontaneous waves as well as the paroxysmal bursts provoked by functional exertion.Besides the action of $\mathrm{D}$ causes significant (32-45\%) decrease in excessive synchronization of resting EEG, which reflects in the changes of absolute values of the power specrta.This decrease was most expressed in frontal,temporal,and occipital regions of the brain cortex.The results of the analysis suggest that $D$ reduces the signs of excessive EEG synchronization which is indicative of the decrease in CNS threshold of seizure readiness.
P385

Electroencephalographic findings in patients with viral herpectic and nonherpetic encephalitis

J.I. Castro-Macias

National Institute of Neurology of Mexico (Mexico City, MX)

Introduction: Viral encephalitis is an acute infection of brain parenchyma. Diagnosis is based in clinics, laboratory and pathologic findings. Until now there is no clear correlation between initial electroencephalographic findings and histopathology.

Objective: To characterize electroencephalographic (EEG) changes in patients with acute viral herpetic and non-herpetic encephalitis at the moment of admission to the hospital and 30 days after.

Methods: 19 patients with a diagnosis confirmed of viral encephalitis were included in this descriptive study. A careful description of clinical manifestations, electroencephalographic findings and cerebrospinal fluid (CSF) analysis were done. Polymerase chain reaction (PCR) in CSF was determined for Herpes virus 1,2 and 6.

Results: CSF analysis was normal in 16 patients. By PCR we found herpetic ethiology in 6 patients $(31.5 \%)$. In this patients EEG findings were generalized theta rhythm in $33.33 \%$, generalized theta-delta rhythm with epileptic focal activity (Temporal) in $50 \%$, Frontal intermittent Delta Activity in $16.66 \%$. In patients with non-herpetic encephalitis generalized encephalopathic patterns with Delta-Theta Rhythm and spikes and waves were observed, in temporal region in 4 patients and frontal in 1; one case of epileptic status was found. At day 30 of the illness evolution we found the following findings: 14 patients were normal, 5 patients showed generalized deltatheta pattern and another 2 besides this pattern also focal epileptic activity (Frontal and temporal), in this group we found PCR negative in 3 patients.

Conclusions: There is a clear relationship between abnormal EEG and clinical manifestations of viral encephalitis. EEG findings in acute stage of herpetic encephalitis showed several abnormalities in basal and interhemispheric cerebral activity. We can conclude that there is not one specific EEG pattern in viral herpetic or non-herpetic encephalitis

P386

Early components of potential P300 in patients with dysarthria after subcortical ischaemic cerebral lesions

K. Slotwinski, R. Podemski, S. Budrewicz, M. Koszewicz

Wroclaw Medical University (Wroclaw, PL)

Introduction: The anatomical basis of the executive functions of speech are subcortical structures, the brainstem and cerebellum. These structures are connected with prefrontal cortex which takes part in coordination and organization of the executive speech functions. Early components of potential P300 (N100, N200, P200) are correspond with the efficiency of processes engaging mainly prefrontal cerebral cortex. Therefore we tried to estimated how the early components of potential P300 change in patients with dysarthria after subcortical ischemic cerebral lesions.

The aim of our study was assessment of N100, N200, P200 components in patients with dysarthia after subcortical ischemic cerebral lesions.

Material and methods: The study comprised 13 patients ( 8 male, 5 female), aged 48-74, with dysarthria and subcortical ischemic cerebral lesions shown by computerized tomography (CT) head scan. Patients with any other structural CNS changes coexisting with ischemic cerebral lesions were excluded from the study. Control group consisted of 15 persons ( 8 male, $7 \mathrm{fe}$ male), aged 50-71, with normal CT head scan and without dysarthria and other speech disorders. In all the patients and controls auditory P300 potential was evoked using basic "oddball paradigm". The superficial recording electrodes were placed in $\mathrm{Fz}, \mathrm{Pz}, \mathrm{Cz}$ (according to 10-20 system), with the reference electrode at the mastoid. Responses were recorded and averaged with the filter bandpass $0,30 / \mathrm{s}-70 \mathrm{~Hz}$, sweep speed $1000 \mathrm{~ms}$ and prestimulus baseline of $250 \mathrm{~ms}$. At least 30 target responses were averaged. We analysed the early responces $\mathrm{N} 100, \mathrm{~N} 200, \mathrm{P} 200$ after stimulus onset.

Results: 1.In the group of patients with dysarthria after subcortical ischemic cerebral lesions, statistically significant prolongation of latency and decrease in amplitude of component N200 were found at electrode $\mathrm{Cz}$, in comparison with the control group (mean results respectively of latency 256 $\mathrm{ms} ; 239 \mathrm{~ms}$ and amplitude $4,2 \mu \mathrm{V} ; 6,8 \mu \mathrm{V})$

2.No statistically significant differences of electrophysiological parameters of N100, P200 between patients with dysarthria and the control group.

Conclusion: The obtained results of electrophysiological analysis of endogenous evoked potentials, confirm involvement of wide, inspecific corti$\mathrm{cal}$ areas in subcortical ischemic cerebral lesions and suggest endogenous evoked potentials may be used in monitoring of motor speech disorders in this group of patients. 
P387

Cortical activation to voluntary movement in motor neuron disease is related to the extent of corticospinal damage: an event-related desynchronisation study

L. Leocani, N. Riva, N. Amato, F. Formaglio, R. Fazio, F. Cerri, U. Del Carro, S. Amadio, A. Falini

H San Raffaele (Milan, IT)

Introduction: The aim of our study was to investigate the relationship between cortical activation to voluntary movement, assessed using Event-related desynchronization (ERD) and synchronization (ERS) of mu and beta bands of the EEG (a correlate of cortical activation and inhibition respectively) and the extent of corticospinal damage in motor neuron disease (MND). Corticospinal integrity was assessed using transcranial magnetic stimulation (TMS) and the apparent diffusion coefficient (ADC) at magnetic resonance imaging (MRI), measuring the extent of tissue damage.

Methods: $\mathrm{Mu}$ and beta ERD/ERS to self-paced right thumb extensions were obtained in $16 \mathrm{MND}$ patients (7F; 9 amyotrophic lateral sclerosis, 6 primary lateral sclerosis, age $62 \pm 9$ years) and 15 healthy controls (11F; age $58 \pm 16$ ) from 32 EEG channels. Patients underwent TMS with measurement of ratio between cortical motor evoked potential (MEP) and peripheral motor action potential (MAP) to the right thumb, and ADC of subcortical frontal white matter and corticospinal tract at the cerebral peduncle from 1.5 Tesla MRI. Group comparisons of ERD/ERS and correlation with ADC/TMS parameters were performed using Wilcoxon rank sum and Spearman correlation.

Results: No significant group differences were found for ERD during movement execution. Post-movement ERS was reduced in ALS patients vs controls $(\mathrm{p}<0.001)$. Contralateral sensorimotor, frontal and parietal ERD was positively correlated with left subcortical frontal ADC $(r=-0.72$; $\mathrm{p}<0.005)$, and negatively with MEP/MAP ratio $(\mathrm{r}=0.69 ; \mathrm{p}<0.05)$. Postmovement beta ERS was negatively correlated with corticospinal ADC $(\mathrm{r}=-0.78 ; \mathrm{p}<0.0005)$ and positively with MEP/MAP ratio $(\mathrm{r}=0.55$; $\mathrm{p}<0.05)$.

Conclusions: The positive relationship, in MND patients, between ERD and TMS/MRI measures, suggests that corticospinal and subcortical frontal damage may be related to increased cortical activation to movement execution, indicating compensation or selectivity loss. Reduced post-movement ERS in MND compared to controls, related to MRI and TMS measures of corticospinal damage, may derive from reafferent feedback or to abnormal cortical inhibitory mechanisms.

\section{P388}

Tendon jerk reflexes during gait in healthy children: age-dependent development

M. Hodapp, C. Klisch, V. Mall, W. Berger, M. Faist

University Hospital of Freiburg (Freiburg, DE)

Objectives: In healthy children and adults Hoffmann (H-) reflexes are profoundly modulated throughout the step cycle in a functionally meaningful way and contribute to the EMG pattern observed during gait. For the soleus muscle the modulation pattern shows a relative maximum in the mid-stance phase and a complete depression during the swing phase.

In children the reflex amplitudes are depressed by supraspinal inhibitory mechanisms in parallel with the maturation of the corticospinal tract. In healthy adults, for the quadriceps muscle a similar pattern of reflex modulation in $\mathrm{H}$ - and tendon jerk (T-) reflexes was shown. The question arises, whether T-reflexes are modulated in a similar manner as H-reflexes in the soleus muscle in children and whether this modulation also underlies an age-dependent development.

Methods: In 24 healthy children aged 10-16 years soleus T-reflexes and background EMG were investigated during walking on a treadmill. To test for the age-dependent development children were divided in two age groups (10-14 and 15-16 years). Reflexes were obtained during 8 different phases of the step cycle and in a control condition with similar voluntary isotonic muscle activity during standing.

Results: In all children soleus T-reflexes were modulated rhythmically with a relative maximum during mid-stance and smaller amplitudes during the swing phase. For the reflex amplitudes no age-dependent changes were shown, but the Yang-modulation index increased significantly.

Conclusion: The present data show that $\mathrm{T}$ - and H-reflexes are modulated in a similar way in healthy children. The significant increase in modulation index is mainly due to the depression during the swing phase with age. This indicates that the soleus tendon jerk reflex underlies an age-dependent development.
P389

Study on brainstem auditory-evoked potentials in diabetes mellitus M. Talebi

Medical Universty of Tabriz (Tabriz, IR)

Objective: Diabetes mellitus is one of the most frequent metabolic diseases which cause different disability in many organs such as brainstem. Previous studies detected controversially association between the results of brainstem evoked activation potential (BEAP) abnormality and diabetes mellitus. The aim of this study is determination of relation between diabetes mellitus and BEAP.

Method: Fifty six diabetic subjects (54.2 yrs \pm 9.9 years) and seventy three healthy subjects ( 50.87 yrs \pm 10.6 years) studied. Diabetic subjects were classified according to type of diabetes (insulin-dependent and non insulindependent), FBS level (above and below $130 \mathrm{mg} / \mathrm{dl}$ ) and HbA1C (more and less than $7 \%$ ). BEAP results from these groups compared with each others and control groups' results.

Results: There was no significant difference from comparison between BEAP findings of FBS level, HbA1C and type of diabetes with each others $(\mathrm{P}=0.683, \mathrm{P}=0.151, \mathrm{P}=0.496$ respectively $)$. The comparison of results of latency of I, II waves between diabetic group and control group do not have significant difference $(\mathrm{P}=0.583, \mathrm{P}=0.910)$ but there was meaningful association between latency of wave III, IV, V and interpeak latency of I-III, III-V in diabetic and control group $(\mathrm{P}=0.012, \mathrm{P}=0.023, \mathrm{P}<0.0001, \mathrm{P}=0.035$, $\mathrm{P}=0.003$ ).

Conclusion: Subjects with diabetes mellitus have abnormalities in auditory brainstem pathway and this study supports the efficacy of BEAP as an early diagnostic and less expansive test for evaluation of brainstem defect in diabetes subjects.

\section{P390}

Entrapment neuropathies in Pes planus

R. Rattanatharn

Faculty of Medicine, Chulalongkorn University(Bangkok, TH)

Background: Pes planus, or flat foot, is a common foot problem and associates with tarsal tunnel syndrome and plantar fasciitis. Some of pes planus subjects complain with paresthesia at the medial side of the foot.

Objective: To compare the function of medial and lateral plantar nerves

between normal feet and pes planus feet

Design: Cross-sectional analytic study

Setting: Department of Rehabilitation Medicine, King Chulalongkorn Memorial Hospital

Method: History, physical examination and foot print of healthy volunteers who are more than 20 years of age were taken, Arch index are then measured and classified as either pes planus or normal foot group. Nerve conduction studies were performed on the sural nerve, sensory and motor of medial and lateral plantar nerves. Needle EMG was performed at abductor hallucis muscle. The findings from both nerve conduction studies and needle EMG were compared between two groups.

Result: There were 35 volunteers; 3 male $\& 32$ female, grouping into pes planus group 33 feet and normal foot 37 feet. The body weight, BMI, impaired pinprick sensation and positive Tinel's sign at ankle were found more in pes planus group significantly $(\mathrm{p}<0.05)$. In pes planus group, there were significantly slower SNCV of sural nerve $(51.3 \mathrm{~m} / \mathrm{s}: 50.0 \mathrm{~m} / \mathrm{s}, \mathrm{p}=0.038)$, more prolonged distal sensory onset latency of medial plantar nerve $(2.3 \mathrm{~ms}: 2.0$ $\mathrm{ms}, \mathrm{p}=0.021$ ), lower CMAP amplitude of medial and lateral plantar nerves ( $9.7 \mathrm{mV}: 13.8 \mathrm{mV}, \mathrm{p}=0.001 ; 3.8 \mathrm{mV}: 6.8 \mathrm{mV}, \mathrm{p}=0.005$ respectively) and more positive sharp wave in needle EMG examination $(93.1 \%: 29.6 \%, p<0.001$.)

Conclusion: The pes planus feet had slower nerve conduction velocities, and denervation of abductor hallucis muscles more than normal feet.

\section{P391}

Conduction nerve study, new findings in amyotrophic lateral sclerosis R. Tejeda, A. Montoya

Lucia Iñiguez Landin Clinical Surgical H (Holguín, CU); Santiago de C. Clinical Surgical Hospital (Santiago de Cuba, CU)

Objectives: To evaluate the changes in peripheral motor nerve conduction study (MNCS) in amyotrophic lateral sclerosis (ALS)patients, to determine electrophysiological patterns comparing them with motor neuropathies that primarily affect peripheral fibres.

Methods: Twenty one patients with confirmed diagnosis of ALS were studied. Exploration accomplishes EMG of four limbs with concentric needle and MNCS in different segments of median, cubital, peroneal and posterior tibial nerves. Motor conduction velocity (MCV) by segments was calculated. The correlation among the EMG findings and quantified data of MNCS 
was estimated. Data were compared through multivariate statistical analysis with a healthy control group and with other motor neuropathies (CIDP, multifocal motor neuropathy and inherit motor neuropathy).

Results: Significant reduction in MCV of proximal segments in median and cubital nerves in patients with ALS was obtained. EMG reinnervation signs were statistically correlated with MCV slowness. The analysis showed significant differences between ALS patients and the healthy subject group and from those with peripheral motor neuropathies.

Conclusions: ALS was associated with significant reduction of MCV in proximal peripheral segments of upper limbs, which enhance with the presence of EMG reinnervation signs. The electrophysiological pattern of ALS could be easily differentiated from healthy patients and other types of motor neuropathies.

Relevance: Pathological findings of MNCS in patients with ALS were confirmed, not frequently accepted in the diagnosis of that disease. In addition its electrophysiological pattern showed no confusion with other processes that typically affect MCV.

P392

Trigeminal somatosensory-evoked potentials in different headache types and facial pain

S. Dempewolf, M. Ahlborn, M. Lanz, S. Bunten, A. Bremerich, S. Happe

Klinikum Bremen-Ost (Bremen, DE); Klinikum Bremen-Mitte (Bremen, DE)

Objective: Somatosensory evoked potentials elicited by trigeminal nerve stimulation (TSEP) allow studying the peripheral and central portion of the trigeminal pathway.

Until now this method is not a routine clinical testing in the diagnostic workup of headache. The aim of our study was to evaluate the yield of the TSEP in different headache types.

Methods: We retrospectively studied all TSEP of patients aged 17 years or older examined in our department during $10 / 1997$ to $2 / 2006$, who complained of headache, facial pain or numbness $(n=311) .94$ patients were suffering from trigeminal neuralgia (TN), 30 from trigeminal neuropathy, and 34 from persistent idiopathic facial pain (PIFP). In 65 patients, headache or facial pain attributed to temporomandibular joint disorder (TMD) and other pain syndromes were diagnosed, and in 88 patients pure facial sensory deficits were present.

Results: In TN patients, pathological latencies of N13 and P19 were seen ipsilaterally in $25.9 \%$ and $8.9 \%$, contralaterally in $13.0 \%$ and $1.2 \%$. Pathological side differences were found in $23.5 \%$ (N13) and $20.5 \%$ (P19). Patients suffering from trigeminal neuropathy showed ipsilateral delayed N13 latencies in $16.7 \%$, P19 was normal in all subjects. A pathological contralateral N13 was measured in $7.4 \%$, a pathological P19 in $4.0 \%$. In $25 \%$ of the patients, the N13- and in $36 \%$ the P19- side differences were abnormal. The percentage of pathological N13 and P19 waves were lower in PIFP patients: ipsilateral $6.1 \%$ and $3.3 \%$, contralateral $3.1 \%$ and $6.3 \% .10 .9 \%(\mathrm{~N} 13)$ and $1.6 \%$ (P19) of patients suffering from TMD showed delayed latencies ipsilaterally and contralaterally in $9.5 \%(\mathrm{~N} 13)$ and $1.6 \%(\mathrm{P} 19)$ of all cases. Abnormal side differences were observed in $11.3 \%(\mathrm{~N} 13)$ and $4.9 \%$ (P19). In patients complaining of a facial sensory loss, latencies of $\mathrm{N} 13$ and P19 on the affected side were increased in $5.1 \%$ and $2.7 \%$, and on the unaffected side in $3.7 \%$ and $2.6 \%$. Pathological side differences were seen in $10.5 \%(\mathrm{~N} 13)$ and $11.3 \%$ (P19)

Comparing the average latencies of $\mathrm{N} 13$ and P19, side differences and amplitudes of each condition, significant differences were found in ipsilateral N13- and P19- latency and N13- side difference. There was a slight significant delay of contralateral N13-latency.

Conclusion: TSEP may offer a valuable tool in patients suffering from headache. In particular, the latencies of N13- and P19- waves are of great interest and vary in their expression regarding to the underlying condition.

P393

Baroreflex sensitivity in untreated essential hypertension

Z. Bajkó, K. Csapó, C. Szekeres, S. Molnár, M. Magyar, P. Soltész, L. Csiba Mures County Emergency Hospital (Targu Mures, RO); University of Debrecen (Debrecen, $\mathrm{HU}$ )

Objectives: Sensitivity of the arterial baroreflex(BRS) has been increasingly used as an index of cardiac autonomic control. Abnormalities in baroreflex control of heart rate may be important in the pathogenesis of essential hypertension. Recently nononvasive techniques have gained increasing popularity. Measurement of BRS as the slope of the relationship between spontaneous changes in SBP and RR interval has proved to be an easy and practical method.

The aim of this study is to investigate the arterial baroreflex in untreated hypertension.
Methods: 28 recently diagnosed, untreated hypertensive patients (mean age: $48,3 \pm 8,1 \mathrm{SD}, \mathrm{M} / \mathrm{F}: 1$ ) and 28 healthy volunteers (mean age: $46,1 \pm 6,5$, $\mathrm{M} / \mathrm{F}: 1,07)$ were included in this study. The diagnosis of hypertension was confirmed with ABPM. Carotid arteries were examined by high resolution B mode ultrasonography. Patients with carotid stenosis were excluded.

We investigated the BRS with complex noninvasive haemodinamic monitor(impedance cardiograph, beat to beat blood pressure measurement device, high resolution 3 -channel-ECG) during tilt table examination $\left(70^{\circ}\right.$ tilt angle). Special software calculated BRS with sequence method. The following parameters were obtained: event count (up and down events, the number of detected baroreceptor reflex events), baroreflex effectiveness index(BEIthe ratio between the total number of baroreflex events and a total number of ramp-like changes in blood pressure, regardless of whether the latter are followed by a change in RR interval or not), slope(the steepness of a regression line within the coordinate axis of RR interval and blood pressure).

Results: The total event count(the number of baroreceptor sequences) was significantly lower in orthostatic position(passive tilt) in hypertension group $(51 \pm 23$ versus $66 \pm 29, p=0,03)$. The BRS slope was also significantly lower in hypertension group in lying position $(9,1 \pm 3,9 \mathrm{~ms} / \mathrm{mmHg}$ vs. $12,2 \pm 6 \mathrm{~ms} / \mathrm{mmHg}, \mathrm{p}=0,031$. The BEI was significantly lower both in lying and orthostatic position $(58 \pm 16$ vs. $67 \pm 14, \mathrm{p}=0,02,54 \pm 15$ vs. $63 \pm 16$, $p=0,03)$. In the hypertension group the event count and BEI was higher in females, the difference was not statistically significant.

Conclusions: There are important abnormalities in baroreflex function also in recently diagnosed, untreated hypertensives. There are important gender differences in baroreflex sensitivity in hypertensive patients. Further analysis and higher patient number are needed to sustain this observations.

\section{P394}

Enhanced sensory cortical excitability in Parkinson's disease $Z$-A. Wu, K-K. Liao Taipei Veterans General Hospital (Taipei, TW)

We studied the somatosensory evoked potentials (SEP) to median nerve stimulation at wrist in 12 patients with Parkinson's disease (PD). We selected patients who did not have tremor but had predominant rigidity with asymmetric body involvement. In PD patients, the N20 amplitudes contralateral to the rigid side were higher than those of another side. High frequency oscillations (HFO) were subtracted from the cortical potentials P15-N20-P25 and also showed higher amplitudes and more numbers of negative peaks. These findings were well correlated with rigidity score, i.e. higher rigidity scores with higher SEP and HFO amplitudes. A comparison between preand post-medications in 3 PD patients, the amplitudes of N20 and HFO decreased after medication with Madopar. Our results indicate that basal ganglia and the related dopaminergic pathway may modulate the sensory processing in the brain of PD patients.

\section{Extrapyramidal disorders}

\section{P395}

Parry Romberg syndrome with hemicorporal dystonia. Report of one case A. Lugo-Pong, G. Cárdenas

National Institute of Neurology (Mexico City, MX)

Introduction: Parry Romberg syndrome is characterized by a progressive but self-limited atrophy of skin and subcutaneous tissue on one side of the face, which may be associated with various neurological, ophthalmic and cutaneous abnormalities.

Objective: To show a clinical case of Parry Romberg syndrome and hemicorporal dystonia.

Case report: A 41 year old woman, with Parry Romberg syndrome since 9 years old, she underwent plastic surgery at 40 year old for this problem and began her illness one year after with sudden dizziness and involuntary- abnormal posture of her left foot, few months later her left arm adopted also a dystonic posture marking difficult to walk. Neurological examination showed slight right facial atrophy and left hemicorporal dystonia. Magnetic resonance showed right cerebral atrophy and T2 signal hyperintensity at the right brachium conjunctivum, electronystamography showed vestibulecerebellar dysfunction. Immunological antibodies and serological HIV were negatives; she received anticholinergic drugs with slight improvement.

Conclusion: Other associations with this syndrome are pigment changes, vitiligo, nevi and hyperpigmented areas on the affected side, alopecia and change from normal hair colour to white. Ophthalmic defects such as ipsilateral Horner's syndrome, blepharophimosis, chronic cyclitis, iritis, 
cataract and secondary glaucoma may occur. Oral manifestations like hemiatrophy of the lips, tongue, jaws and buccal fat pad may be present. Our patient had only progressive facial hemiatrophy with none of this associated abnormalities but hemicorporal dystonia, in our knowledge this is the first report of this association without specific cause; not associated with vegetovascular origin.

\section{P396}

Chorea-acanthocytosis: a diagnosis not to be ruled out in absence of acanthocytes

C. Bayreuther, M. Borg

Hôpital Pasteur (Nice, FR)

Objectives: Chorea-acanthocytosis (ChAc) is one of the Neuroacanthocytosis syndromes, which form a group of disorders characterized by the association of neurological abnormalities and spiculated red blood cells called acanthocytes. ChAc patients show involuntary movements, psychiatric abnormalities and progressive cognitive deterioration.

We report a case of ChAc in which blood smears failed to objectify acanthocytes.

Methods: Case report

A 26-years-old man presented since two years with hyperkinetic movements. There was no family history, parents were consanguineous. Neurological examination revealed choreatic hyperkinesia, predominant in the orofacial region and particularly in the tongue leading to eating and swallowing difficulties and ulcerations of the lip and tongue due to self-biting automutilation. Patient showed postural instability with falls. There was mild cognitive decline and behavior abnormalities with repetitive activities.

Results: Cerebral MRI showed caudate atrophy. Molecular testing for Huntington's disease (HD) was negative. Routine biological screen was normal except CPK and LDH elevation. Copper and ceruloplasmin blood levels were normal, as well as purine metabolism and lipoproteins. Further screening for metabolic diseases showed no significant abnormality. Expression of Kell antigens was normal. In several blood smears no acanthocytes were seen. Electromyographic studies showed slight neuropathic changes. Despite the absence of acanthocytes, we sent blood samples for chorein Western blot (LMU Munich, Prof. Danek), that showed absent/markedly reduced level of chorein in erythrocyte membrane. This makes a mutation in the ChAc gene likely and the diagnosis of ChAc was established. Genetic studies for VPS13A are ongoing.

Conclusion: ChAc is an autosomal recessive disorder due to mutations of the VPS13A gene coding for chorein. A HD like phenotype is common. Classically ChAc patients present with orofacial involuntary movements with tongue and lip-biting and feeding dystonia. CPK is often increased and denervation of peripheral nerves associated. Presence of acanthocytes is usually required for the diagnosis of ChAc. Absence or late-appearing of acanthocytes in ChAc have been described in few case reports. Their presence might be controlled by other factors; alternatively absence may be due to technical problems in detecting acanthocytes. In conclusion ChAc is a rare disorder in which the presence of acanthocytes is not mandatory.

\section{P397}

Neuropathology of primary adult onset non-DYT1 dystonia

J.L. Holton, S.A.Schneider, T. Ganesharajah, S. Gandhi, C. Strand, P. Shashidharan, J. Barreto, N.W. Wood, A.L. Lees, K.P. Bhatia, T. Revesz

Institute of Neurology (London, UK); Mount Sinai School of Medicine (New York, US)

Background: Idiopathic adult-onset primary dystonia usually affects the upper body and remains focal. The underlying mechanisms are not fully understood. Neuropathological studies are limited in the literature. Recently, ubiquitinated perinuclear inclusion bodies in the brainstem of patients with DYT1-related dystonia have been described. [1] In DYT3, causing X-linked recessive dystonia-parkinsonism, neuronal loss in the striosome compartment of the striatum has been reported.[2] However, it was unclear whether these changes are characteristic of these particular disorders or an epiphenomenon of dystonic conditions in general.

Methods: We present pathological data of six cases with primary adultonset dystonia and four controls. We used immunohistochemistry to determine the presence of inclusion bodies immunoreactive for torsinA, ubiquitin and laminA/C in the brainstem. The degree of neuronal loss was determined by the calcineurin expressing neurons in the striosome compartment of the striatum.

Results: In contrast to early-onset DYT1 dystonia, neuronal inclusions immunoreactive for torsin A, ubiquitin and laminA/C were not present in the brainstem nuclei. There was no apparent loss of the striatal striosome compartment.
Conclusion: Our findings suggest that the underlying mechanism in the adult-onset primary torsion dystonia is different from that of early-onset DYT1-related dystonia and also DYT3 X-linked recessive dystonia-parkinsonism. Alternative mechanisms may underlie these two conditions.

\section{References}

1. McNaught KS, Kapustin A, Jackson T, Jengelley TA, Jnobaptiste R, Shashidharan P et al. (2004) Brainstem pathology in DYT1 primary torsion dystonia. Ann Neurol 56(4):540-547

2. Kaji R, Goto S, Tamiya G, Ando S, Makino S, Lee LV (2005) Molecular dissection and anatomical basis of dystonia: X-linked recessive dystoniaparkinsonism (DYT3). J Med Invest 52(Suppl):280-283

\section{P398}

Topiramate-induced restless legs syndrome: two cases

R. Velasco-Calvo, P.E. Bermejo

Puerta de Hierro University Hospital (Madrid, ES); La Paz University Hospital (Madrid, ES)

Restless legs syndrome (RLS) is a disorder characterized by an intense and irresistible urge to move the legs, sensory complaints and motor restlessness. Some antiepileptics have been proposed as a treatment for RLS (gabapentin, valproate, lamotrigine, pregabalin, levetiracetam, carbamazepine and oxcarbazepine) whereas others may induce it such as phenytoin or zonisamide. The role of topiramate remains unknown since it has been proposed as a cause and as a treatment of RLS. We report two cases of topiramate-induced RLS.

A 32-year-old woman affected by chronic migraine started to take topiramate as a prophylactic treatment. It was titrated up to $50 \mathrm{mg}$ bid. Concurrently, she complained of an unpleasant sensation in her legs and urge to move them, specially during inactivity and in the late evening. This discomfort was relieved by movement, resulting in motor restlessness and insomnia.

A 36-year-old woman who suffered from severe episodic migraine started topiramate as a preventive treatment. It was titrated up to $75 \mathrm{mg}$ per day. During titration, she experienced nocturnal leg discomfort during inactivity with an urge to move the legs. what resulted in motor restlessness and insomnia.

Neurological evaluation, serum studies and neurography were normal in both patients and symptoms rapidly disappeared when topiramate was suspended.

To our knowledge, this is the second comunication describing a potential role of topiramate on provoking RLS. It has multiple mechanisms of action including enhancement of GABAA-mediated via, inhibition of glutamate-mediated neurotransmission, blockage of voltage-dependent sodium and calcium currents and inhibition of carbonic anhydrase. Some of them are similar to those of zonisamide, another drugs that may induce RLS suggesting a possible role of these mechanisms in the pathophysiology of this disorder. In a recent study, topiramate has demonstrated to reduce levodopainduced dyskinesias in MPTP-lesioned primates what could be due to the above mechanisms or to a specific dopamine antagonist action that is hitherto unrecognized. Additionally, topiramate has been suggested to modulate cortico-mesolimbic dopamine function and has been effective in managing impulse control disorders, pathologies related to dopamine system.

We alert clinicians of potential dopaminergic effects of topiramate and that RLS should be considered in patients treated with this drug.

\section{P399}

Post-operative medication doses are reduced equally in Parkinson's patients who undergo either bilateral pallidal or subthalamic deep-brain stimulation A. Premkumar, M. Lyons, R. HIllman, B. Falk, S. Lemens, M. Wheeler, J. Caviness, E. Driver-Dunckley, C. Adler, V. Evidente

Mayo Clinic Arizona (Scottsdale, US)

Objective: Deep brain stimulation (DBS) in the subthalamic nucleus (STN) and globus pallidus internus (GpI) are both effective in treating motor symptoms of advanced Parkinson's disease (PD). Although there is no definitive evidence to suggest that one location is superior to the other, the STN has been traditionally chosen due to reports of greater potential medication reduction. This finding may be due to selection bias and differing post operative management. At our institution, STN was the preferred target for PD patients at the inception of the program in 2002. However due to increasing reports of cognitive/psychiatric sequelae wih STN-DBS, we started to perform GPi DBS in 2005. Our objective was to compare post operative medication changes in PD patients who underwent STN versus GPi DBS surgery who had similar baseline characteristics and post operative management. 
Methods: We reviewed records of all PD patients who underwent GPiDBS and matched them to PD STN-DBS cases based on age, pre-op motor UPDRS scores, and pre-op levodopa equivalent dose (LED). We calculated percent LED reduction at time of optimization of programming (2-8 months post DBS). All patients were managed postoperatively by the same neurologist.

Results: We identified 12 GPi-DBS patients and matched them to 12 STN patients who underwent surgery at our institution. Mean age was 65.5 and 66.6 years for GPi and STN respectively. Both groups had 2 women and 10 men. Mean pre-op LEDs were $869.6 \pm 690.4 \mathrm{mg}$ for GPi and $962.5 \pm 662.5 \mathrm{mg}$ for STN ( $p=0.6)$. Mean pre-op "medication off” motor UPDRS scores were $46.6 \pm 24.6$ for GPi and $34.1 \pm 28.9$ for $\operatorname{STN}(\mathrm{p}=0.08)$. There was a similar reduction in post-DBS LEDs at time of optimization in both GPi (36.9\%) and STN $(38.0 \%)$ groups. Two GPi and 1 STN patient completely discontinued PD medications post-DBS at time of optimization. Mean post-op "medication off, stimulation on" motor UPDRS scores did not differ significantly for GPi $(16.4 \pm 15.6)$ and $\operatorname{STN}(19.1 \pm 18.6)$ groups $(\mathrm{p}=0.45)$.

Conclusions: Both GPi-DBS and STN-DBS patients were found postoperatively to have equivalent PD medication dose reductions at the time of optimal programming. Given the greater concern for neuropsychiatric sequelae with STN stimulation, GPi may be a preferred target at least on short term follow up.

P400

The role of co-morbidity on health-related quality of life of Greek patients with Parkinson's disease

E. Andreadou, M. Anagnostouli, E. Kararizou, M. Rentzos, T. Kontaxis, I. Evdokimidis

Athens National University (Athens, GR)

Parkinson's disease (PD) is relatively common among the aged in all countries and has a negative impact on health related quality of life (HRQoL). The increasing prevalence of accompanying disorders in the elderly could further influence the HRQoL. Quality of life assessment contributes to a better understanding of the disease's consequences on the patient, and thus helps in decision-making.

Objectives: To evaluate the impact of other common co-morbid disorders [hypertension (HT), dyslipidemia, ischemic heart disease(IHD), diabetes mellitus (DM), minor stroke, arthritis, low back pain or osteoporosis and depression] on HRQoL of Greek PD patients.

Patients and methods: Data about PD age at onset, treatment, age, gender, marital status, education, occupation, income, social contacts, co-residence, disease severity and concomitant diseases were collected of 139 consecutive patients (68 men and 71 women) in our outpatient clinic. Disease severity was assessed using the unified Parkinson's disease rating scale (UPDRS) including Hoehn and Yahr and Schwab and England (S\&E) activities of daily living (ADL) scale.To assess quality of life, the specific Parkinson's disease questionnaire (PDQ-39) was used. A multivariate multiple regression model with normal errors was used for the statistical analysis.

Results: The mean age of the patient population was 69.61 (SD 9.12). Most patients were married $(70.5 \%)$, retired $(61.9 \%)$ and had social contacts with relatives and/or friends ( $88 \%$ ). 99 patients had at least one other co-morbid disorder: Arthritis etc 59, HT 52, depression 39, dyslipidemia 31, IHD 21, DM 19 and minor stroke 13. The mean total PDQ-39 score was 22.117 (SD 18.21) with Mobility, ADL, Emotional Well-being and Bodily Discomfort being the most affected dimensions. The main negative HRQoL determinants were disease duration $(\mathrm{F}=14.045, \mathrm{p}<0.001)$, social isolation $(\mathrm{F}=12.591, \mathrm{p}<0.001)$, sleep disturbances $(\mathrm{F}=10.434, \mathrm{p}<0.002)$ and low degree of independence measured by $S \& E$ scale $(F=42.896, p<0.001)$. The coexistence of other disorders had no impact on HRQoL in our patients $(\mathrm{F}=1.082, \mathrm{p}=0.384)$

Conclusion: This study is the first report concerning the effect of other co-existing chronic medical conditions on HRQoL of PD patients. Surprisingly, our study did not reveal any impact of the other co-morbid disorders on HRQoL after controlling for sociodemographic variables, a result that might be interesting to be evaluated in other ethnic groups and other disorders.

P401

Characteristics of good-responders to long-term continuous apomorphine infusions in advanced Parkinson's disease

$J$-E. Vanderheyden

CHU Charleroi, Vésale (Montigny/Tilleul, BE)

Objectives: To find out the clinical and social characteristics of the Parkinsonians good-responders to long-term continuous subcutaneous Apomorphine infusions on the basis of our data from 2002.
Patients and method: The features of 22 Parkinsonians treated during more than 3 years (LT group, mean duration $=5.4$ years) by Apomorphine infusions are compared with those of 32 patients who stopped this treatment before 3 years (ST group, mean duration $=10.2$ months).

Results: There is no difference between the two groups regarding the Apomorphine rate $(2.74 \mathrm{vs} 2.66 \mathrm{mg} / \mathrm{h} 24 \mathrm{~h}$ a day), but we observe that $67 \%$ of the males stopped the treatment before 3 years vs only $55 \%$ of the females $(\mathrm{p}<0.05)$. Age at onset of Apomorphine is younger in the LT group $(68.6$ years vs $73.8 ; \mathrm{p}<0.01$ ). Moreover we observe more side effects (especially subcutaneous nodules) in the ST group, but, in the LT group, patients, relatives and/or carers are more implicated in the technical aspects of the pump and collaborate in preventing side effects. Finally, there is no difference at social level thanks to the reimbursement.

Conclusions: Except for allergy or cumbersome side effects, the use of Apomorphine subcutaneous continuous infusions may be efficacious and well tolerated, but younger patients, females and a proactive collaboration of the patient and/or carers are positive criteria for a good response in the longterm.

\section{P402}

Correlation between odour identification and cardiac 123 I-MIBG in Parkinson's disease

M. Iijima, M. Osawa, M. Momose, M. Suzuki, M. Iwata

Tokyo Women's Medical University (Tokyo, JP)

Objective: Olfactory dysfunction and autonomic symptoms are recognized as non-motor symptoms in Parkinson's disease (PD). These functions are markers for a preclinical diagnosis of PD because pathological changes in the olfactory and autonomic systems start before motor symptoms develop. In this study, we investigated an association between olfaction and cardiac 123 I-metaiodobenzylguanidine (MIBG) uptake in patients with PD.

Methods: Subjects were thirty non-demented patients with idiopathic PD (19 men and 11 women, age $64.8 \pm 12.0$ years) with a disease duration of 6-168 months (54.2 \pm 42.0 months). The motor performance was assessed using the Hoehn and Yahr Staging scale (H\&Y), and the motor section (part III) of the Unified Parkinson's Disease Rating Scale (UPDRS): 6 patients were in H\&Y stage I, 22 were in stage II, 7 were in stage III, and 1 was in stage IV. Twenty-two patients were taking anti-parkinsonian medication, and $8 \mathrm{pa}$ tients were not on any medications. The smell identification test included 12 odorants: perfume, a rose, condensed milk, a Japanese orange, curry, roasted garlic, fermented beans/sweaty socks, gas for cooking, menthol, India ink, wood, and Japanese cypress (hinoki). These odors were chosen from different areas of everyday Japanese life, as they are smells familiar to the Japanese population. The subject smelled one scent, then chose 1 of 6 possible answers: 4 pictures of entities associated with the odors labeled with their names, 1 of which was correct, and 2 other ones (unknown and not detected). For the MIBG myocardial scintigraphy, planar scintigraphic imaging in the anterior view was obtained 15 minutes (early) and 4 hours (delayed) after the intravenous injection of MIBG (111 MBq) using a single head gamma camera. To measure MIBG uptake, heart (left ventricle) and mediastinal regions were drawn manually. The heart-to-mediastinum ratio (H/M) for both early and delayed images, and myocardial washout rate (WR) for 4 hours were calculated.

Results: The number of correct answers in the smell identification test correlated significantly with $\mathrm{H} / \mathrm{M}$ ratio in both early and delayed phases, and WR $(p<0.01)$. There is not any correlation between UPDRS scores and the number of correct answers, $\mathrm{H} / \mathrm{M}$ ratio or WR.

Conclusion: These results suggested that the olfactory system degenerated in parallel with the cardiac sympathetic nervous system in idiopathic PD.

\section{P403}

Interference effects of observed movement in Parkinson's disease

N. Albert, G. Cohen, Y. Peiris, R.C. Miall, P. Praamstra

University of Birmingham (Birmingham, UK)

Objective: When a visual stimulus contains both relevant and irrelevant (distracting) information, a response conflict may ensue that interferes with the required action. Previous research has demonstrated that Parkinson patients are more susceptible to response conflict than control subjects. Here, we investigate whether $\mathrm{PD}$ patients have a similar sensitivity to interference from observed movements, resulting from coactivation of action observation and action performance circuitry in the premotor cortex.

Methods: Eight PD patients and age-matched control subjects performed horizontal and vertical arm movements, while they observed a video with similar movements. The observed movements were performed by person or consisted of a moving dot with the same stimulus kinematics. 
Movements were performed in the same plane as the observed movement (congruent condition) and orthogonal to the observed movement (incongruent condition). Movements were recorded by a motion tracking system with a sensor mounted to the participant's index finger. The variance of the movements was measured as an index of interference. PD patients had moderate disease severity and were tested after overnight withdrawal from medication.

Results: Control subjects demonstrated a significant congruency effect with more variable movements in the incongruent relative to the congruent condition. This effect was confined to movements performed in the horizontal plane. Parkinson patients demonstrated greater variability in the horizontal compared to the vertical plane, without modulation by congruency. Comparison between the groups yielded a significant effect of movement plane, due to greater variability in the horizontal plane. This effect was stronger for patients than for controls. There were no effects of movement agency (person vs. dot movement)

Conclusion: Action imitation involves the activation of movement observation and movement execution processes in the ventral premotor cortex. The coactivation of these processes can cause interference from observed movements with performed movements. The present data indicate that PD patients do not have an enhanced sensitivity to this type of interference.

\section{P404}

Sporadic Guam parkinsonian complex or the co-incidence of several neurodegenerative conditions: the study of 4 Czech cases suffering from parkinsonism, amyotrophic lateral sclerosis and dementia

P. Kanovsky, K. Farnikova, I. Nestrasil

University Hospital (Olomouc, CZ)

Study objective: The parkinsonian complex of Guam is an endemic neurodegenerative condition, which has been described only in the islands of Guam archipelago and at the Kii peninsula of Japan. Up to now, only one "sporadic" case has been described (including the autopsy) in Japan. Here we describe the clinical, laboratory and neurophysiological characteristics of the neurodegenerative disorder presenting in 4 patients with the complex syndrome of parkinsonism, amyotrophic lateral sclerosis, and dementia.

Methods: 4 consecutive patients of caucasian and Czech origin, presenting with the complex syndrome of slowly progressive parkinsonism, amyotrophic laterál sclerosis and dementia were completely examined clinically, including neuropsychological examination, and they were assessed using EMG and EP. The blood and CSF samples were also examined, and the levels of inflammatory and neurodegenerative markers (beta-amyloid, cystatin $\mathrm{C}$ and tau-proteins) were established.

Results: The clinical phenotype in all four patiens was corresponding to that one described in the parkinsonian complex of Guam, including the presence of cognitive deficit at the level of mild to severe dementia. The findings of EMG examination in all cases were those typically seen in ALS, and they met the El Escorial criteria. CSF levels of neurodegenerative markers (tau-protein) were elevated in all four patiens. CSF levels of inflammatory markers were normal.

Conclusion: The unique appearance of the syndrome typical for the endemic Guam complex in patients of caucasian origin in Europe raises a question of endemicity and heredity of the Guam complex and deserves further rersearch.

\section{P405}

Dopamine neuron degeneration induced by MPP+ is independent of CED4 pathway in Caenorhabditis elegans

P. Pu, W. Le

Institute of Health Sciences (Shanghai, CN)

Incubation of Caenorhabditis elegans with 1-methyl-4-phenylpyridinium $(\mathrm{MPP}+)$ resulted in dopamine neuron degeneration accompanied by reduced mobility and increased lethality. The mechanisms by which MPP+ induces neurodegeneration remain unclear. We employed apoptosis-related gene mutants and necrosis-related gene mutants to determine the cell death mode(s) involved in MPP+-induced dopamine neuron degeneration in living animal model of C. elegans. Our study showed that dopamine neuron degeneration induced by MPP+ was independent of CED-4 classical programmed cell death pathway while the necrosis-related gene vha-12 mutant strain showed a significant resistance to MPP+ neurotoxicity. Our results suggest that the necrosis machinery may contribute to MPP+-induced dopamine neuron degeneration in C. elegans. Furthermore, our study also documented that mazindol, a dopamine transporter inhibitor, can completely protect dopamine neurons from MPP+-induced degeneration in this in vivo Parkinson's disease model.
P406

Differential diagnostic value of normal uptake but increased 123I-MIBG washout in parkinsonism

Y. Uchiyama, M. Momose, C. Kondo, K. Kusakabe, M. Iwata

Tokyo Women's Medical University (Tokyo, JP)

Objectives: Norepinephrine analogue, 123I-metaiodobenzylguanidine (MIBG) imaging, is useful in differentiating Parkinson's disease (PD) from other Parkinsonism. Decreased cardiac MIBG uptake is one of the characteristics of PD, but some PD patients reveal normal initial uptake, but increased MIBG washout rate (WR). The purpose of the present study is to elucidate whether an increased MIBG WR has an independent differential diagnostic value in patients with PD, and to clarify the clinical significance of WR.

Methods: One-hundred-three patients $(67 \pm 11$ y.o., 57 women $)$ with Parkinsonism except diabetes mellitus and obvious cardiac disease were enrolled. All patients underwent MIBG scintigraphy, and heart-to-mediastinum ratio $(\mathrm{H} / \mathrm{M})$ as MIBG cardiac uptake on both initial $(15 \mathrm{~min})$ and delayed ( 4 hours after injection) images were generated. WR from initial to delayed uptake was also calculated. Decreased initial H/M was defined as $\leq 1.6$ based on our institutional control studies. Final diagnosis was confirmed based on clinical findings during more than 6 months follow-up periods. Clinical diagnosis and findings such as age, tremor, rigidity, bradykinesis, and orthostatic hypotension $(\mathrm{OH})$ were compared to MIBG WR.

Results: In 103 patients, neurologists diagnosed 62 as PD (66 \pm 11 y.o.), 6 as DLB ( $77 \pm 8$ y.o.) and 35 as Parkinson syndrome (PS) ( $68 \pm 12$ y.o). PD and DLB were classified into one group as Lewy body disease (LBD: 68 patients). Initial $\mathrm{H} / \mathrm{M}$, delayed $\mathrm{H} / \mathrm{M}$ and $\mathrm{WR}$ in $\mathrm{LBD}$ vs. PS were $1.79 \pm 0.44$ vs. $2.32 \pm 0.53(\mathrm{p}<0.001), 1.65 \pm 0.58$ vs. $2.34 \pm 0.70(\mathrm{p}<0.001), 50.1 \pm 24.2$ vs. $28.5 \pm 23.1 \%(\mathrm{p}<0.001)$, respectively. When initial $\mathrm{H} / \mathrm{M} \leq 1.6$ was defined as LBD, positive predictive value (PPV) was $91 \%$ (29/32), but negative predictive value (NPV) was $45 \%(32 / 68)$. Among 71 patients with H/M >1.6 (higher H/M group), WR $>30 \%$ has $72 \%$ of sensitivity, $66 \%$ of specificity, $72 \%$ of PPV and $66 \%$ of NPV for the diagnosis of LBD. Multivariate analysis showed that WRs were independently correlated with the diagnosis of LBD (F-value 26), age (F-value 10) and OH (F-value 9) $(\mathrm{r}=0.55, \mathrm{p}<0.001)$ among the clinical parameters.

Conclusions: MIBG washout has an incremental diagnostic value to differentiate PD/DLB from PS among patients with normal or borderline MIBG initial H/M. However, WR is also increased in elderly patients and $\mathrm{OH}$ patients without PD/DLB, which should be considered when the parameter is used.

\section{P407}

Peculiarities of brain metabolism in the region of posterior cingulate gyrus in non-demented patients with Parkinson's disease: diffusion tensor imaging and in vivo $1 \mathrm{H}$ MRS study

Z. Rozhkova, T. Kalishchuk-Slobodin, N. Karaban', I. Karaban', J. Golovtshchenko

Medical Clinic Boris (Kiev, UA); National Medical Academy of Postgraduate Education (Kiev, UA); Academy of Medical Sciences of Ukraine (Kiev, UA)

Objectives: On the basis analysis of Diffusion Weighted MR-images (DTI) and $1 \mathrm{H}$ MRS data in the region of posterior cingulate gyrus in non-demented patients with Parkinson's disease (PD) and in healthy volunteers we propose quantitative approach for characteristics of the axonal disruption in white matter tract in patients with PD.

Methods: Two groups of patients are studied by MRI and 1H MRS with 1.5T SIGNA EXCITE (GE). The 1st group includes 10 non-demented patients with PD in the age from 48 to 70 years. The 2 nd group consists of 15 healthy volunteers in the age from 18 to 73 years. DTI are obtained using a single shot spine echo type of echo planar imaging: TR/TE $=10000 / 38 \mathrm{~ms}$, $\mathrm{b}$ value $=$ $1000 \mathrm{~s} / \mathrm{mm}^{2}$, the ADC values in the region of posterior cingulate gyrus (PCG) are calculated. $1 \mathrm{H}$ spectra are recorded for all subjects in the region of PCG with the SVS STEAM method: TR/TE $=1365,1500 / 135 \mathrm{~ms}$, VOI $=8 \mathrm{~cm}^{3}$, NS $=$ 64 .

Results: From analysis of 1H MRS data the mean values of NAA, and also the mean values of NAA/Cr ratios in both hemispheres in the region of PCG are calculated. Average fractional anisotropy (FA), ADC coefficients, and volume ratio (VR) are calculated. Spearman correlation analysis revealed a positive correlation between NAA/Cr ratio and FA (right hemisphere: $\mathrm{r}=0.410$, $\mathrm{p}=0.018$, left hemisphere: $\mathrm{r}=0.570, \mathrm{p}=0.001$ ), $\mathrm{VR}$ (right hemisphere: $\mathrm{r}=0.441, \mathrm{p}=0.008$, left hemisphere: $\mathrm{r}=0.590, \mathrm{p}=0.001)$, and a negative correlation with mean diffusivity (MD) (right hemisphere: $r=-0.440, p=0.008$, left hemisphere: $r=-0.540, p=0.001$ ).ROC curve revealed better specificity and sensitivity combining NAA/Cr and VR value in the right hemisphere $(\mathrm{AUC}=0.910)$ than using only NAA values $(\mathrm{AUC}=0.770)$ or $\mathrm{VR}(\mathrm{AUC}=$ 0.850 ) values, respectively.

Conclusion: 1H MR spectroscopy gives a new insight into brain bio- 
chemistry in patients with PD and determinates the biochemical basis of DTI alterations. These findings are consistent with axonal loss or dysfunction in patients with PD. It provides evidence that DTI identifies axonal disruption in white matter tracts.

\section{P408}

Combined in vivo $1 \mathrm{H}$ MRS and diffusion tensor imaging study in non-demented patients with Parkinson's disease (PD) and PD patients with neuropsychological deficits

Z. Rozhkova, T. Kalishchuk-Slobodin, N. Karaban', J. Golovtshchenko, I. Karaban'

Medical Clinic Boris (Kiev, UA); National Medical Academy of Postgraduate Education (Kiev, UA); Academy of Medical Sciences of Ukraine (Kiev, UA)

Objectives: On the basis analysis of Diffusion Weighted MR-images (DTI) and $1 \mathrm{H}$ MRS data we propose the quantitative indicators for the characteristics of the local metabolic state of brain in patients with PD and PD patients with neuropsychological deficits.

Methods: Three groups of patients are studied by MRI and 1H MRS with 1.5T SIGNA EXCITE (GE). The 1st group includes 10 non-demented patients with PD (48-70y). The 2nd group consists of 10 patients with PD and with neuropsychological deficits $(40-70 \mathrm{y})$. The 3 rd group consists of 10 healthy volunteers. $1 \mathrm{H}$ spectra are recorded in both hemispheres in the occipital lobe (gray matter), in the frontal lobe (white matter), in the lentiform nucleus, putamen and substantia nigra, and in the temporoparietal cortex with the SVS STEAM method: TR/TE $=1500 / 144 \mathrm{~ms}$, VOI $=8 \mathrm{~cm}^{3}, \mathrm{NS}=64$. DTI are obtained using EPI method:TR/TE $=10000 / 38 \mathrm{~ms}, \mathrm{~b}=1000 \mathrm{~s} / \mathrm{mm}^{2}$, the ADC values in the above mentioned regions are calculated.

Results: From the spectra in all sampled regions of the brain the peak areas of three main metabolites: NAA, $\mathrm{Cr}$ and Cho, and also the metabolite ratios: NAA/Cho, NAA/Cr and Cho/Cr are obtained. For the patients of the 1st group the significant decrease of NAA and $\mathrm{Cr}$ and the increase of Cho peak areas in sampled regions are observed. There is the significant reduction in $\mathrm{NAA} / \mathrm{Cr}$ ratios in the right and left averaged temporoparietal cortex for the patients of the 1st group compared with the patients of the 2 nd group. There is no dependence between NAA/Cr ratios and motor disability or disease duration, and neuropsychological deficits. There are no significant differences in NAA/Cho and Cho/Cr for the patients in the 1 st and in the 2nd group for any of the regions. The values $\mathrm{NAA} / \mathrm{Cr}$ ratios in the 1 st and 2 nd groups are significantly lower than in the 3rd group, and Cho/Cr ratios - higher in substantia nigra, than in patients of the 3rd group. Statistically difference in $\mathrm{ADC}$ values in the 1st and 2nd groups in putamen and insignificant difference in substantia nigra are obtained. There is correlation between ADC value and signal intensity in putamen in non-demented patients with PD (1st, and 2nd group, respectively) and subjects of the 3rd group.

Conclusion: Signal intensity on T2-weighted images, ADC values, and concentration of main cerebral metabolites in putamen and substantia nigra can be use for characteristics of the grade of neuropsychological deficits in patients with PD.

\section{Epilepsy}

\section{P409}

Bowel ischaemia: a rare thiopental-related complication in refractory status epilepticus

C. Cereda, M.M. Berger, A.O. Rossetti

CHUV (Lausanne, $\mathrm{CH}$ )

Objectives: Treatment of Refractory status epilepticus (RSE) aims at EEG burst-suppression with an appropriate anesthetic agent. While propofol may lead to hypotension and rarely to an "infusion syndrome", adverse events with barbiturates are mostly represented by respiratory, cardiovascular or infectious complications. We report here on two subjects who suffered from acute bowel ischemia.

Methods: Description of two patients undergoing thiopental (THP) treatment for RSE between April-June 2007.

Results: Patient 1: a 73-year-old man presented a partial-complex RSE after an acute stroke, resistant to lorazepam, phenytoin and valproate. EEG burst suppression was achieved with THP (total dose: $303 \mathrm{mg} / \mathrm{kg}$ over 48 hours); 36 hours after THP discontinuation, the still comatose patient had an sudden elevation of lactate levels $(10.5 \mathrm{mmol} / \mathrm{l})$, associated with peritonismus. A surgical exploration showed necrosis of the terminal ileum and the colon until the to recto-sigmoid junction. He died 12 hours later. Patient 2: A 21 year-old woman presented a de novo generalized-convulsive RSE of unknown origin despite exhaustive investigations, resistant to clonazepan, phenytoin, valproate, levetiracetam, topiramate, and to 5 courses of EEG burst-suppression attempts (propofol, midazolam, ketamine). During the 6th attempt, THP was used with mild hypothermia $\left(34^{\circ} \mathrm{C}\right)$, targeting a complete EEG suppression. Daily dosage was stepwise reduced after the first 48 hours; she received a total dose of $840 \mathrm{mg} / \mathrm{kg}$ over 150 hours. On the sixth day, she developed an ileus with elevated serum lactate $(5,6 \mathrm{mmol} / \mathrm{l})$. The surgeon described a megacolon with focal caecum necrosis, but no sign of vascular or inflammatory changes was noted on histological examination. After a partial colon resection, the patient recovered moderately following a 7th burst-suppression treatment. Of note, none of the patients experienced significant arterial hypotension (under norepinephrine administration) during THP treatment.

Conclusion: Intestinal infarction complicating oral phenobarbital overdose has been anecdotally reported, but has never been described in the context of RSE treatment. Although the underlying mechanism is unknown, we suggest mechanical ischemia secondary to a drug induced paralytic ileus. To our knowledge, our report is the first to describe this potential dangerous THP side effect, and underlies the importance of serial serum lactate monitoring to detect this potentially fatal entity.

\section{P410}

Ictal cerebral blood flow changes during absence seizures

G. Genc, S. Bek, B. Ozturk, O. Oz, S. Demirkaya, Z. Gokcil, Z. Odabasi Gulhane Military Medical Academy (Ankara, TR)

Background: After epilepsy surgery, mapping lateralization clinically and by laboratory during ictal phase became more valuable. Transcranial Doppler (TCD) is a noninvasive useful technique to assess blood flow variations of main cerebral arteries. In this study we investigated cerebral flow changes in absence seizures.

Methods: Cerebral blood flow velocity analyses has been performed for 3 patients, hospitalized in our Video EEG monitoring unit to assess the seizure semiology. Absence seizure has been diagnosed clinically and by monitoring characteristic 3-3,5 Hz spike-wave discharges. Data of cerebral blood flow velocity variations during total 43 seizures is computed offline.

Results: 1) Ictal mean blood flow velocity increase $(69,2 \pm 7,0)$ and post ictal decrease $(41,7 \pm 8,9)$ were statistically significant $(\mathrm{p}<0,001$, Wilcoxon sign test). Also in left MCA ictal velocity increase $(69,7 \pm 12,8)$ and post ictal decrease $(42,1 \pm 10,4)$ were statistically significant $(\mathrm{p}<0,001$, Wilcoxon sign test).2) Left MCA velocity change from basal to ictal flow $(\% 26,5 \pm 14,6)$ was higher than the mean percentage changes from basal to ictal flow velocity in right MCA $(\% 19,3 \pm 12,4)(\mathrm{p}<0,05$, T-test). 3) There was no significant difference between right $(\%-28,2 \pm 14,6)$ and left $(\%-23,6 \pm 13,6)$ MCA velocity in the mean percentage changes from basal to post ictal flow velocities ( $\mathrm{p}>0,05$, T-test)

Conclusion: The findings in different studies concerning evaluation of cerebral perfusion, in literature are conflicting. It is admitted that the initial increase of cerebral blood flow velocities during absence seizures are reflections of cortical hyperactivity and the decrease of blood flow velocities in a short time under basal values is the sign of suppression. In our study blood flow velocity increase observed more distinctively in dominant hemisphere in all 3 patients statistically despite of generalized spike-wave discharges. Different rates of blood flow variations between dominant and non-dominant hemisphere, despite of generalized spike-wave discharges, may be a sign of lateralization.

\section{P411}

Self-reported quality of sleep and epilepsy

H. D'Onofrio, H. Videla, J. Maegli, J. Ure, D. Filipponi, R. Folcini, J. Ollari, M. Diez, A. Caridi, M. Baña

Pinero Hospital (C.Madero, AR); Borda Hospital (C.Madero, AR)

Objective: The aim of this study was to evaluate differences in self-reported subscales of sleep quality between subjects with controlled epilepsy under therapy and an age-matched non epileptic control group.

Background: A large body of evidence has been built about the linkages between sleep and epilepsy, specially related to effects of sleep deprivation either on seizures or interictal discharges in EEG. On the other hand numerous reports about sleep disrupted organization in epileptic patients suggest that unstable sleep promote seizures and seizures fragment sleep which facilitates the epileptic process however sleep evaluations are usually overlooked in clinical setting.

Method: 45 adult patients (mean age $34.7,(95 \% \mathrm{CI} \cdot 31$ to 37$)$ with documented and controlled epilepsy receiving $1(n=24)$ or $2(n=21)$ antiepileptic drugs (AED)were compared with a non epileptic group $(n=30$, mean age 39 (95\% CI 35 to 44 p:0.066) recruited between outpatients of a psychiatric hospital and evaluated about their sleep quality using Pittsburgh Sleep Qual- 
ity Index.(PSQI). Demographic data, clinical history and medication use was considered as well. Each participant completed the Pittsburgh Sleep Quality Index.(PSQI) and each subtest was scored (0-1 good quality, 2-3 poor quality)

Results: Data were compared based on poor (2-3) scores, using McNemar-chiSquare test for differences between groups. Scores rated as poor were more likely to be found in epilepsy group (Quality of sleep: $46.6 \%$ p: 0.0001 , latency of sleep: $30.6 \%$ p: 0.003 , efficiency of sleep: $49.3 \%$, p: $<0.0001$, perturbation of sleep: $28.8 \%$, p: 0.009 , daytime hypersomnolence: $28 \%$, p: 0.0005 and the total score rated $>625.33 \%$, p: 0.0017 )

The only subscale rated better for epilepsy sample was duration of sleep: $45.3, \mathrm{p}:<0.0001$.

Conclusion: Patients with epilepsy under AED exhibited a poor sleep quality profile and behave worse than control group in all subscales but one (perception of duration of sleep). The recognition that subjective quality of sleep is poorer in epileptic patients is a valued piece of information to design strategies leading to stabilize sleep and the practice of healthy lifestyle behaviors so sleep quality tests should be evaluated routinely in patients with epilepsy.

\section{P412}

Noninvasive presurgical expressive and receptive language investigation in epileptic patients using functional magnetic resonance imaging and nearinfrared spectroscopy

I. Pelletier, A. Gallagher, P. Vannasing, J. Leroux, J. Tremblay, D. Bastien, D. Nguyen, R. Béland, D. Saint-Amour, F. Lepore, M. Lassonde

Université de Montréal (Montreal, CA); Centre de Recherche de l'Hôpital Sainte-Justine (Montreal, CA); Hôpital Notre-Dame du CHUM (Montreal, CA)

The cognitive outcome of the surgical removal of an epileptic focus is closely related to the adequate assessment of the localisation and functional capacity of language and memory areas which need to be spared by the neurosurgeon. Traditionally, presurgical evaluation of epileptic patients has been achieved by means of the intracarotid amobarbital test (IAT) and neuropsychological measures. The advent of neuroimaging techniques has provided new ways of assessing these functions by means of non-invasive methods. Functional Magnetic Resonance Imaging (fMRI) and Near-Infrared Spectroscopy (NIRS) have been used to investigate language lateralization in epileptic patients $[1,2]$, which show good concordance with IAT. Since the IAT procedure has many limitations, especially in children, we propose an fMRI and NIRS combined presurgical assessment to non-invasively identify the critical language areas in patients (aged from 6 to 25) with refractory partial epilepsy. All patients underwent a verbal fluency task to assess expressive speech and a story listening task to assess receptive speech. It is interesting to validate the effectiveness of a story listening protocol since it is easily applicable in young epileptic children who often present an intellectual delay. Indeed, the story listening task requires less intellectual capacities than the other well-known receptive language tasks (i.e. paradigms of syntactic decisions or reading). Results show a clear concordance between fMRI, NIRS and IAT. If replicated in a larger sample, fMRI and NIRS may be good alternative techniques to reduce the need for invasive monitoring in epileptic patients especially in young children and in mentally-challenged patients.

\section{P413}

Fractures, osteoporosis, epilepsy and anti-epileptic drug use: what about the guidelines?

K. Beerhorst, M.C.T.F.M. de Krom, A. Aldenkamp, F. Tan, P. Geusens

University Hospital Maastricht (Maastricht, NL); Epilepsy Centre Kempenhaeghe (Heeze, NL)

Objectives/purpose: We report two patients with osteoporosis after longterm use of anti-epileptic drugs (AEDs) to draw attention to the lack of official (Dutch, European (EFNS, ENS), AAN) guidelines how to deal with this problem in people with epilepsy.

Methods: Case reports.

Results: A 56-year-old woman with cryptogenic temporal lobe epilepsy since the age of 10 (for which she had (had) several AEDs: phenobarbital (PB), phenytoin (PHT), levetiracetam (LEV), and valproic acid (VPA) for 10, 10,2 and 22 years respectively) with low frequent complex partial seizures under VPA monotherapy at that moment broke her left ankle at a seizure. She was found to have had 6 other fractures related to a fall or seizure. A dual-energy-X-ray absorptiometry (DEXA) scan for measurement of bone mineral density (BMD) revealed osteopenia. Besides her perimenopausal status and long-term AEDs use patient had no other risk factors for developing osteopenia.
A 49-year old man with symptomatic temporal lobe epilepsy with complex partial seizures since the age of 14 had been treated with PB, PHT, vigabatrin (VGB) for 18,35 and 15 years respectively. On PHT and VGB he had a low seizure frequency. At the age of 47 years a spontaneous cruris fracture was diagnosed. Because he had had 5 other fractures, most of which fall or seizure related, a DEXA scan was performedwhich revealed moderate osteoporosis. So, because no other causes were detected the long-term use of PHT and PB should have been a major risk factor for osteoporosis. He was started on calcium and alendronic acid as was his AEDs therapy modified: PHT was replaced by LEV, VGB was stopped because of visual field defects and clobazam added. His seizure frequency is stabile.

Conclusion: With these case report we would like to draw attention to the possibly underestimated effect of AEDs on the development of low bone mineral density and osteoporosis with increased fracture risk. In spite of the growing alertness on these adverse effects we were unable to find European or AAN guidelines on the management of osteoporosis in people with epilepsy. Further investigations on this subject are necessary resulting in a protocol for surveillance and treatment of low BMD in people with epilepsy.

\section{P414}

Occult cerebrovascular disease in late-onset epilepsy. A literature review and novel hypothesis

L.M. Gibson, L.M. Parkes, H.C.A. Emsley

University of Edinburgh (Edinburgh, UK); University of Liverpool (Liverpool, UK)

Objectives: This review aims to summarise current understanding of associations between occult cerebrovascular disease (CVD) and late-onset epilepsy (LOE). Ideas from studies in LOE patients and related populations will be combined to form a new hypothesis on the link between LOE and occult CVD.

Methods: MEDLINE, EMBASE, CINAHL and AMED databases were searched in January 2008 for studies which explored associations between occult CVD and LOE. Clinical studies including patients with epilepsy aged over 60 at the time of their first seizure and preclinical studies using adult animal models were eligible for inclusion. Secondary references were identified using Web of Science. Only studies published in English were reviewed.

Results: Seven studies were included. Two found a higher prevalence of occult CVD in patients with adult-onset epilepsy compared to controls $(p<0.05)$. Another reported a prevalence of occult CVD of $10.6 \%$ in LOE patients. Significant reductions in regional cerebral blood flow (rCBF) in LOE patients with leukoaraiosis compared to controls $(\mathrm{p}<0.05)$ were found. A preclinical study demonstrated blood-brain-barrier (BBB) leak leading to paroxysmal activity in brain slices. Cortical cerebral arteriovenous malformations are reportedly associated with an increased risk of epilepsy. One paper provided a hypothesis that occult CVD may cause LOE.

However, most occult CVD is subcortical whereas epilepsy derives from the cortex. A new hypothesis of diffuse cerebral microangiopathy due to hypertension is proposed as a mechanism underlying occult CVD and LOE. Diffuse cerebral microangiopathy due to hypertension may manifest as occult CVD in the subcortex and epilepsy in the cortex by impairing rCBF or BBB function. Both mechanisms are associated with occult CVD and perhaps lead to epileptogenesis via cortical excitotoxicity or extravasation of albumin respectively.

Neurovascular coupling methods assess vasculature ability to respond to neural activity by increasing $\mathrm{CBF}$, and may help assess $\mathrm{rCBF}$ impairments in LOE patients. Animal models of occult CVD and LOE may also help explore the pathological mechanisms linking these diseases.

Conclusions: Evidence suggests that occult CVD is present in LOE patients, but the mechanisms linking LOE and occult CVD are poorly understood. A new hypothesis of diffuse cerebral microangiopathy as an underlying factor in both diseases has been proposed. Neurovascular coupling measures and animal models may help explore this hypothesis.

This review was written as part of an intercalated degree, for which LM Gibson received a Wolfson Intercalated Award.

\section{P415}

Mutations in NHLRC1 gene are predominant cause of Lafora disease in Serbian population

M. Kecmanovic, N. Jovic, M. Keckarevic-Markovic, V. Dobricic, D. Keckarevic, P. Ignjatovic, S. Romac

Faculty of Biology (Belgrade, RS); School of Medicine (Belgrade, RS)

Objectives: Progressive myoclonic epilepsies (PME) are a heterogeneous group of rare inherited disorders, associated with myoclonus, generalized 
epileptic seizures and progressive neurological deterioration. Lafora's disease (LD, OMIM 254780) is an autosomal recessive, fatal disease that is characterized by pathognomic Lafora bodies (LB) in neurons and other cell types. LD is described as symptoms starting in first or second decade of life with epileptic seizures followed by progressive central nervous system degeneration beginning with myoclonic seizures, tonic-clonic seizures, focal occipital seizures, intellectual decline and finally death within 10 years of onset. Despite the clinical homogeneity there is also genetic heterogeneity in $\mathrm{LD}$, characterized by at least two genes that are in the base of this disorder. Namely, LD is caused by mutations or in EPM2A encoding dual-specificity protein phosphatase either in NHLRC1 encoding E2 dependent E3 ubiquitin ligase. There is also at least one other gene that can cause LD.

Here we report genetic findings on eleven patients from seven families whose clinical, electroencephalographic and biopsy data proved the diagnosis of LD, as well as one new NHLRC1 mutation.

Methods: Genomic DNA was extracted from peripheral blood cells using QIAamp blood purification kit (Qiagen Inc., Valencia, CA, USA). Exons 1-4 of the EPM2A gene and single NHLRC1 exon and flanking sequences were amplified by polymerase chain reaction (PCR) using primers reported previously. The amplified fragments were purified and directly sequenced on ABI 3130 Genetic Analyzer (Applied Biosystems, CA, USA).

Results: Our analysis identified one novel mutation and two recurrent mutations in the NHLRC1 gene of probands of 7 LD families. None of these individuals harbored mutation in the EPM2A. These mutations include p.E350fs in homozygous state in nine individuals from five LD families. One individual from one family was compound heterozygote carrying p.E350fs and p.G331fs mutations. P.I343T mutation, identified in this study, was found in one individual in homozygous state. We performed haplotype analysis of LD chromosomes with c.1048-1049delGA (p.E350fs) mutation using two markers flanking NHLRC1 gene (D6S1567 and D6S1688) and one intragenic marker (C332T). We observed founder effect for this mutation.

Conclusion: Mutations in NHLRC1 are the common cause of LD in the Serbian population, what is mostly due to founder effect.

\section{P416}

The course of seizures in pregnant women with epilepsy

O. Oz, S. Bek, G. Koc, G. Genc, O. Arslan, Z. Gokcil, Z. Odabasi

Gulhane Military Medical Academy (Ankara, TR)

Introduction: Women with epilepsy are considered to have "high risk" pregnancies because of the increased incidence of complications and need close monitoring of the disease.

Objective: To evaluate the course of seizures during pregnancy.

Methods: We evaluated 94 pregnant women with epilepsy who was referred to our epilepsy department from 1992 to 2006.75 pregnant women were included in our study. The patients whose pregnancy were terminated and who have not enough information were excluded. 19 patients were followed up without drug treatment. 56 patients used any AEDs during pregnancy. ( 48 monotherapy, 8 combination therapy with two drugs). The frequency of seizures during pregnancy in the preconception period (last one year) was compared.

Results: Totally 75 cases were evaluated, the frequency of seizures increased in 18 patients (24\%), decreased in 17 patients $(23 \%)$ and unchanged in 40 patients $(53 \%)$ during pregnancy. Pregnancies were seperated into two groups by follow-up with or without drug treatment. The course of seizures were evaluated in both groups separately. The frequency of seizures increased in 15 patients, decreased in 13 patients and unchanged in 28 patients among 56 patients who were followed up with drug treatment. The frequency of seizures increased in 4 patients, decreased in 4 patients and unchanged in 11 patients among 19 patients who were followed up without drug treatment. Status epilepticus wasn't observed.

Conclussion: In our study, the changes of fit frequency of the patients without AEDs treatment was found similar to the patients with AEDs treatment. This might be associated with the limitation on number of patients. Emergent techniques in foetal ultrasonography also provide major facilities especially for the follow-up of pregnant women with epilepsy. Hereby foetus with anomaly can be recognised at an early stage and the medical abortus can be performed. Also this tecniques help patients defeate anxiety about conception with AEDs.
P417

Effects of valproic acid and carbamazepine monotherapies on asymmetric dimethylarginine, homocysteine, folate and vitamin B-12 levels: possible links to pathogenesis of epilepsy and complications of medication O. Oz, Z. Gokcil, S. Bek, G. Genc, E. Akgul, E. Cakir, U. Ulas, S. Demirkaya, Y. Kutukcu, Z. Odabasi

Gulhane Military Medical Academy (Ankara, TR)

Objectives: We aimed to assess the effect of valproic acid (VPA) and carbamazepine (CBZ) monotherapies on the homocysteine metabolism and asymmetric dimethylarginine (ADMA), homocysteine ( $\mathrm{pHcy}$ ), folate and vitamin B-12 levels. We also attempted to uncover the relationship between NO and epilepsy via the levels of ADMA.

Methods: Thirty five newly diagnosed epilepsy patients included. Patients formed in two groups VPA $(\mathrm{n}=17)$ and CBZ $(\mathrm{n}=18)$. Before therapy and at 3rd month, fasting blood samples were obtained. pHcy, folate, vitamin B-12 and ADMA levels were measured.

Results: At the 3rd month, patients had significantly higher ADMA levels in VPA $(p=0,002)$ and CBZ $(p=0,024)$ groups, when compared with the basal levels distinctly. In VPA group, $\mathrm{pHcy}(\mathrm{p}=0,005)$ and VitB-12 $(\mathrm{p}=0,001)$ levels were significantly higher at the $3 \mathrm{rd}$ month. In folate levels no difference was observed. In CBZ group folate levels were significantly lower $(p=0,006)$ at the 3rd month. pHcy $(p=0,117)$ and VitB-12 $(p=0,557)$ levels had no significantly difference.

Discussion: The role of NO in epilepsy has been investigated by several authors with various NO synthase inhibitors and contradictory roles for NO in the development and pathogenesis of seizures have been suggested. ADMA is an endogenous NO synthase inhibitor and it has given us an opportunity to design a clinical trial to eveluate the functional interaction between NO, epilepsy and AEDs.

In conclusion, $\mathrm{NO}$ plays a critical role in convulsions. Our data suggest that it is a proconvulsant agent in CNS. VPA and CBZ may exhibit their anticonvulsive effects by lowering NO levels via increasing ADMA levels. This is the first study indicating increased ADMA levels in patients with epilepsy under AED therapy. Increased ADMA and pHcy levels in patients under VPA and CBZ therapy suggests these patients have vascular risk. Decreased folate levels may be associated with cognitive and hematologic complications. Importance of increased vit-B-12 levels is unknown yet.

\section{P418}

Changes of mean diffusivity in temporal lobe epilepsy with hippocampal sclerosis: relationship with clinical features

S-H. Kim, K-S. Lee, Y-M. Shon, Y.I. Kim, D. W. Yang, B.S. Kim

Catholic University (Seoul, KR)

Introduction: Diffusion tensor imaging (DTI) provides information about tissue water diffusion and has been widely used for detecting structural abnormalities of brain tissue.

In mesial temporal lobe epilepsy (mTLE) patients, interictal DTI found increased diffusivities (ADC) in ipsilateral temporal areas.

We analysis the changes of ADC value from mTLE patients by voxel based morphometry (VBM) using SPM and their relationship with clinical features.

Method: Nineteen temporal epilepsy patients (male: 5, age: 17-45) and 20 age matched control subjects (male: 10,21-49 years old) was selected for analysis.

All were mTLE patients confirmed by high-resolution MRI long-term EEG monitoring.

Secondary generalized (SG: 14) vs complex partial seizure (CP: 5), With aura (WA : 10) vs without aura (WOA : 9) Febrile seizure (FS : 5) vs non febrile seizure (NFS : 14) All DTI was performed interictal period, and ADC map of both controls and the patients was calculated by home-made software (DTIcalc) for SPM analysis for comparison.

Result: secondary generalized vs complex partial group

SG(14) patients showed increased ADC in ipsilateral hippocampus and bilateral temporal, frontal, occipital white matter, splenium.

CP(5) patients, increased ADC was only ipsilateral hippocampus and splenium.

SG patients shows more extensive abnormalities than CP patients. SG patients had wider areas of increased ADC in the ipsilateral hippocampus and splenium than CP group did.

With aura vs without aura group

WA(10) patients showed more diffuse ADC regions than WOA(9) patients did.

Unexpectedly, there was conspicuous ADC increase in ipsilateral thalamus in WOA group than the changes in WA patients.

Febrile seizure vs none febrile seizure group

There were no differences between ADC changes of FS(10) and those of 
Discussion: By SPM analysis, we can detect the change of water diffusivity in mTLE patients.

Although we cannot figure out the causal-relationship between the ADC changes and their clinical features, the posterior hippocampus and ipsilateral splenium may be related to the ictal spreading into generalization and also amnesia of aura may be associated with abnormal ADC values in the ipsilateral thalamus.

We can assume that DTI analysis may be used as a complementary tool for assessment of clinical diversities of epilepsy patients.

\section{P419}

Cognitive decline in patients with intractable epilepsy

S. Lazarova I. Barbov, I. Petrov

Clinic for Neurology (Skopje, MK)

Objective: Intractable epilepsy is related to various transient and chronic brain electric and neurochemical disturbances. There is increasing evidence that chronic epilepsy induces secondary neuronal metabolic and structural decline. However, there is no convincing evidence that the cognitive abilities of patients deteriorate with increasing duration of intractable epilepsy.

Methods: To examine whether duration of refractory temporal lobe epilepsy (TLE) is related to generalized cognitive impairment, psychometric intelligence based on the full scale intelligence quotient (FSIQ, WAIS-R) was determined in 54 patients with unilateral TLE. For analyses of variance (ANOVA) patients were grouped into three categories: $<15,15-30$, and $>30$ years of refractory TLE.

Results: An ANOVA and a multiple regression analysis showed that duration of TLE affects FSIQ. Patients with $>30$ years of TLE performed worse than patients with 15 or 30 years of TLE. The factors side of seizure origin and type of lesion on MRI did not reach significance. A second ANOVA including education as factor showed that in patients with higher educational attainment, the mean FSIQ was stable for a longer duration of TLE than in less educated patients. The variables age at epilepsy onset, education, frequency of interictal epileptiform discharges, frequency of habitual and generalized seizures, serum concentration of antiepileptic drugs, and polypharmacy were statistically controlled.

Conclusions: Psychometric intelligence of patients with a longer duration of refractory TLE were most severely impaired. Consequently, refractory TLE seems to be associated with slow but ongoing cognitive deterioration. It is assumed that epilepsy related noxious events and agents exhaust the compensatory capacity of brain functions. However, as in dementia and Alzheimer's disease, higher educational attainment as an indicator of higher brain reserve might delay the cognitive decline.

\section{P420}

Genetic polymorphism of CYP2C19 on phenytoin pharmacokinetics in Indian epileptic patients developing toxicity

S. Vivekanandhan, M. Murali, V. Sneha, M. Tripati, M. Behari, S. Raghavan, D.C. Jain

All India Institute of Medical Sciences (New Delhi, IN); Safdarjung Hospital (New Delhi, IN)

Objectives: Phenytoin, a widely used anti-epileptic drug, exhibits marked inter-individual variation in Pharmacokinetics and attributed to genetic factors such as CYP2C19 polymorphisms. The present study was undertaken to investigate the influence of CYP2C19*2 variant genotypes on Phenytoin Pharmacokinetics in the epileptic patients showing toxicity.

Methods: 30 epileptic individuals who had developed toxicity to phenytoin and 30 control epileptic subjects who had not developed toxicity to phenytoin were genotyped for CYP2C19*2 polymorphisms by Polymerase chain reaction-restriction fragment length polymorphisms (PCR-RFLP Method). Allele frequencies were derived from genotypic data. Case-control comparisons were made using Chi-square tests. Deviations from the Hardy-Weinberg equilibrium were also tested. Phenytoin plasma levels were analyzed by reverse phase HPLC method and Pharmacokinetic parameters such as area under the concentration curve (AUC), maximum concentration $(\mathrm{C} \max )$, time to $\mathrm{C} \max (\mathrm{t} \max )$, drug clearance and half-life $(\mathrm{t} 1 / 2)$ were estimated by non compartmental analysis using PK solutions software.

Results: The CYP2C19*2 polymorphisms was seen to be in Hardy-Weinberg equilibrium and showed significant allelic association but did not show significant genotypic association $(\mathrm{P}=0.301 ; \mathrm{OR}=0.51(0.16-1.61)$. The variant allele showed significant association with the phenytoin toxicity phenotype $(\mathrm{P}=0.01 ; \mathrm{OR}=2.35(1.19-4.67)$.

The pharmacokinetics parameters in heterozygosity group showed longer half life $(\mathrm{t} 1 / 2=35.26 \mathrm{hrs})$ and less clearance rate $(\mathrm{CL}=0.46 \mathrm{~L} / \mathrm{hr})$ when compared to wild type group $(\mathrm{t} ?=21.9 \mathrm{hrs}, \mathrm{CL}=0.9 \mathrm{~L} / \mathrm{hr})$.

Conclusion: Our findings suggest that the $\mathrm{CYP} 2 \mathrm{C} 19{ }^{*} 2$ genetic polymor- phisms may be associated with the individual difference in phenytoin pharmacokinetics. An individualized dosage regimen design incorporating such genetic information would help to increase the clinical efficacy of phenytoin and reducing the toxicity in epileptic patients.

\section{Peripheral neuropathy}

\section{P421}

Neurotrophin receptors expression in skin probes of patients with neuropathic pain

B.O. Popescu, I. Mindruta, O. Bajenaru, R. Colesniuc, G. Chelu, D.F. Muresanu, A.M. Trandafir, M. Sajin

Victor Babes National Institute (Bucharest, RO); University Hospital Bucharest (Bucharest, RO); Iuliu Hatieganu University of Medicine (Cluj Napoca, RO)

Objectives: The pathogenesis of neuropathic pain includes various mechanisms, such as loss of peripheral axons, aberrant neuroplasticity and altered cell signaling in both peripheral and central nervous systems. Different cellular components of the skin have been found to synthesize neurotrophic factors, which signal to the small diameter sensitive nerve fibers. These nerve endings are the most affected fibers in patients with neuropathic pain. The main objective of the present study was to evaluate the expression of neurotrophic factor receptors in skin probes of patients with neuropathic pain.

Methods: Based on informed consent, we obtained skin biopsies from 15 patients (12 patients with neuropathic pain and 3 controls with accidental leg injuries). The skin biopsies were performed from the calf region, using a standard protocol. For the patients with neuropathic pain, diagnosis was based on complains of pain in the lower legs rated more than 40 on visual analogue scale, on nerve conduction studies and on quantitative sensory testing (thermal thresholds using the limits method on Medoc TSA System). Skin tissue was fixed in paraformaldehyde for immunohistochemical assessment.

Results: We analyzed the expression of Trk A and p75NTR receptors in epidermis and dermis of our samples. Protein gene product 9.5 (PGP 9.5) expression analysis was used to identify and quantify the nerve endings. As reported before, we found a depletion of PGP 9.5 positive fibers in the specimens of neuropathic pain patients, regardless of the etiology of neuropathy, as compared to the age-matched controls. Expression of both Trk A and p75NTR was not restricted to nerve endings but was identified instead in the epidermis as well, mainly in the stratum basale. However, the epidermis of neuropathic patients stained more intensely with anti-Trk A antibody as compared to the epidermis of controls. In our samples expression of p75NTR appeared to be unchanged, even though in general the expression of the two receptors is inversely regulated. We will proceed to confirm these results further on by Western blotting.

Conclusions: As compared to controls, in neuropathic pain patients we found an altered expression of neurotrophic receptors in both nerve endings and epidermal basal cells. This change may represent an adaptive effect generated by chronic metabolic stress in sensory neurons.

\section{P422}

Clinical and electrophysiological evaluation of 222 patients with CharcotMarie-Tooth disease type $1 \mathrm{~A}$ recruited in the CMT-TRIAAL (ascorbic acid therapy for Charcot-Marie-Tooth $1 \mathrm{~A}$ disease)

D. Pareyson, A. Schenone, N. Rizzuto, G.M. Fabrizi, L. Santoro, G. Vita, A. Quattrone, L. Padua, F. Gemignani, F. Visioli, D. Calabrese, A. Solari on behalf of the CMT-TRIAAL Group

Objective: To report the clinical and electrophysiological data of the baseline assessment of the 222 patients with Charcot-Marie-Tooth disease type $1 \mathrm{~A}$ (CMT1A) recruited in the CMT-TRIAAL (CMT-TRial Italian with Ascorbic Acid Long term).

Method: The CMT-TRIAAL is a phase III randomized, double blind, placebo-controlled study, involving CMT1A symptomatic adults from 8 centres. Treatment consists of two-year oral AA (1500 mg/day) or placebo. Efficacy endpoint are changes in: CMT Neuropathy Score (CMTNS, primary endpoint); distal maximum voluntary isometric contraction; 10-meter timed walking (T10MW); 9-hole-peg test (9HPT); Overall Neuropathy Limitations Scale (ONLS); pain and fatigue VAS; health-related quality of life (SF-36); electrophysiology. Assessments are performed at baseline and every six months thereafter. PMP22 expression in skin nerves is evaluated in consenting patients from three centres. 
Results: Characteristics of the 222 enrolled patients are: females 134 $(60 \%)$, males 88 , mean age 42 yrs (SD 13; range 18-70). Skin biopsy was performed in 53 consenting patients. Results of baseline evaluation are the following, expressed as mean (SD): CMTNS 13.4 (4.5); ONLS 3 (1.3); T10MW $9.1 \mathrm{sec}$ (5.3); 9HPT (dominant side) $23 \mathrm{sec}$ (6.7). Disease severity, according to the CMTNS, was mild in $28 \%$, moderate in $65 \%$ and severe in $7 \%$ of patients; disease was more severe in females [13.9 (5)] than males [12.5 (3.9)], who, however, were younger. Quality of Life, according to SF36, was significantly lower in CMT1A patients as compared to the Italian population. According to VAS score, pain [3.7 (3.1)] and fatigue [4.8 (2.8)] are a major complaint in CMT1A. Motor and sensory conduction study results were similar to that already reported in the literature. The first year of follow up will be concluded in March 2008 and data will be available for the whole patient series.

Conclusions: The CMT-TRIAAL recruited patients appear to be well representative of the adult CMT1A population. This is the largest series of CMT1A patients studied thus far and the first assessed using several outcome measures.

Supported by Telethon-UILDM Grants GUP04002-GUP05007, and partially by the Italian Medicines Agency (AIFA, independent drug research program, FARM53APAH)

\section{P423}

Diabetic amyotrophy and treatment with intravenous immunoglobulin

E. Kouremenos, A. Kodounis, D. Naoumis, M. Anastasiadou, M. Maltezou 251 General Hospital of Hellenic Air Force (Athens, GR); 1st General Hospital of the Institute of Social Security (Athens, GR)

Objective: The asymmetrical, painful diabetic amyotrophy attacks mainly elderly patients with type II diabetes mellitus, with initially unilateral pain invasion in the lumbar region and the thigh. This is followed by weakness and atrophy of the affected regions, as well as attack of the contralatelar limb in a period of about 3 months. The pathogenic mechanism is probably autoimmune, and the natural course of the disease involves spontaneous improvement and recovery within a period of 2 years. Provided treatments, such as corticosteroids and immunoglobulin, are able to decrease pain or facilitate faster recovery.

In the present study, we investigate the efficacy of treatment with intravenous immunoglobulin (iv Ig) in patients with diabetic amyotrophy, evident by the clinical and laboratory findings.

Method: For this purpose, we studied 8 patients, 5 males and 3 females, aged between 60-70 years old, who suffered from type II diabetes mellitus, treated with insulin for 12-18 months, who presented diabetic amyotrophy, with an average at Neurological Symptom Score (NSS) of 4. The patients were treated with iv $\mathrm{Ig}(0,4 \mathrm{gr} / \mathrm{Kg}$ of body weight, for 5 days, for 6 consecutive months) and we registered the clinical and laboratory findings for this period.

Results: There was full remission of symptoms in $25 \%$ of the patients 2 months after the beginning of treatment. After 4 months of treatment, $75 \%$ of the patients were free of symptoms and after 6 months, the percentage reached $87,5 \%$.

Conclusion: The faster improvement of symptoms is obvious, in comparison to the expected improvement due to the natural course of the disease. This outcome is consonant with recent studies.

\section{P424}

High-resolution ultrasound as an adjunct method for clinical neurophysiology

E. Wilder-Smith, A. Therimadasamy

National University Hospital (Singapore, SG)

Objectives: To provide experience from our Neurophysiological laboratory that High Resolution Ultrasound (HRU) is an increasingly useful adjunct diagnostic method for the clinical neurophysiologist.

Methods: We give examples of how HRU contributed towards attaining a diagnosis in our clinical neurophysiology laboratory.

Results: Two patients with humerus fracture and acute arm weakness could not be satisfactorily examined with nerve conduction due to pain and swelling. HRU demonstrated radial nerve swelling allowing for appropriate treatment measures to be undertaken. In a case of humerus fracture with repair, HRU pinpointed the unusual distal upper arm site of radial nerve lesion. In a young man, ulnar nerve conduction was unable to localise because of partial nerve unexcitability. HRU localised neuropathy to the sulcus ulnaris region. In a patient with partial weakness of the wrist/finger extensors HRU showed swelling of the motor branch of the radial nerve as it penetrated the supinator canal. Other examples of the usefulness of HRU include diagnosis of carpal tunnel syndrome where widespread/severe neuropathy limited nerve conduction, demonstration of sensory neuromas as well as damage to the brachial plexus. For the latter, visualisation was up to the extraforaminal cervical roots.

Conclusion: High Resolution Ultrasound is developing into a useful adjunct diagnostic method in the neurophysiological laboratory.

\section{P425}

Central and peripheral demyelination: clinical and electrophysiological correlations

T. Crani, V. Lisnic, M. Gavriliuc, O. Odainic

Institute of Neurology and Neurosurgery (Chisinau, MD)

Objectives: The goal of the study was to establish the frequency of clinical and electrophysiological manifestation of the central nervous system (CNS) and peripheral nervous system (PNS) impairment in patients with polar demyelinating diseases. Cases of clinical and preclinical impairment of the CNS in chronic inflammatory demyelinating polyneuropathy (CIDP) as well as cases of impairment of the PNS in multiple sclerosis (MS) are reported. So far it has been not clear if the syndrome of central and peripheral demyelination is either a separate nosoligical entity or a variant of CIDP, or SM.

Methods: Sixty consecutive patients with CIDP were diagnosed according to the American Academy of Neurology criteria and 60 patients had definite MS. Clinical and electrophysiological methods of investigation were utilised (nerve conduction studies, electromyography and evoked potentials of different modalities).

Results: Clinical signs of CNS impairment were diagnosed in 7 patients with CIDP (11,66\% cases). The Babinski sign was positive in all the cases. Preclinical signs of CNS impairment were established more frequently $45 \%$ cases, had significant increase of the central conduction time. Clinical signs of neuropathy were revealed in 5 patients with MS ( $8,33 \%$ cases), but far more frequently were established electrophysiological signs of nerve conduction impairment, detected in $43,33 \%$ of cases.

Conclusions: CIDP and MS occupy the opposite poles of the clinical-neurophysiological continuum of central and peripheral demyelination. Multiple intermediary forms with both CNS and PNS impairment exist.

\section{P426}

New $10 \%$ liquid intravenous immunoglobulin for treatment of multifocal motor neuropathy: a prospective study of efficacy, safety and tolerability E.A. Cats, W.L. van der Pol, R.M. van den Berg-Vos, J.H.J. Wokke, H. Franssen, L.H. van den Berg

University Medical Center Utrecht (Utrecht, NL)

Objectives: Multifocal motor neuropathy (MMN) is an immune-mediated motor neuropathy with slowly progressive, asymmetric limb weakness. Maintenance treatment with freeze-dried intravenous immunoglobulin (IVIg) $5 \%$ may attenuate muscle weakness and delay the permanent nerve damage. Treatment with IVIg $5 \%$ can be time consuming, treatment with $10 \%$ ready-to-use liquid IVIg may reduce the infusion time. We evaluated the efficacy, safety and tolerability of a $10 \%$ liquid IVIg preparation in 20 patients with MMN.

Methods: Twelve months monocenter prospective open-label study. Twenty patients with MMN were treated with their regular maintenance dose of IVIg $5 \%$ for a period of 3 months. Treatment was changed into an equal dose of $10 \%$ liquid IVIg for the following 9 months. Muscle strength (MRC sum score of ten muscle groups) was measured at six outpatient clinic visits; functional impairment was assessed using Guy's Neurological Disability and Self Evaluation Scales.

Results: At inclusion, the mean IVIg regimen was $18.8 \mathrm{~g} /$ week (range 10-45 g/week) given every 3 weeks (range 1-4 weeks) and was similar with the $10 \%$ liquid IVIg preparation. Muscle strength did not change after switching IVIg $5 \%$ into $10 \%$ liquid IVIg (Wilcoxon signed-rank test $\mathrm{p}=0,126)$. Guy's Neurological Disability score of one patient worsened one point. Two patients reported one point improvement and one patient one point deterioration on the Self Evaluation Scale. All other patients posted no change. Mild drug related adverse effects were reported in $35 \%$ with $10 \%$ liquid IVIg (four patients headache, two patients mild allergic skin reaction, one patient dizziness) compared to $40 \%$ with IVIg $5 \%$. With $10 \%$ liquid IVIg, infusion time did not change in 3 patients ( $15 \%$ ), was reduced up to twofold in 12 patients $(60 \%)$ and more than twofold in 5 patients $(25 \%)$. Mean infusion time with $10 \%$ liquid IVIg was 2.5 compared to 4.5 hours with IVIg $5 \%$.

Conclusion: Ready-to-use $10 \%$ liquid IVIg was well tolerated in patients with MMN and muscle strength did not change. Treatment with $10 \%$ liquid IVIg was associated with substantial reductions in infusion times in $85 \%$ of patients. 
This study was supported by an unconditional grant from Baxter Healthcare B.V.

\section{P427}

Short-term and long-term efficacy of immune globulin intravenous, $10 \%$ caprylate/chromatography purified for the treatment of chronic inflammatory demyelinating polyradiculoneuropathy

H-P. Hartung, V. Bril, M. Dalakas, C. Deng, P. Donofrio, K. Hanna, R. Hughes, N. Latov, I. Merkies, P. van Doorn

Heinrich-Heine-University (Dusseldorf, DE); University Health Network (Toronto, CA); National Institutes of Health (Bethesda, US); Talecris Biotherapeutics (Research Triangle Park, US); Vanderbilt University (Nashville, US); King's College London, Guy's Hospital (London, UK); Cornell University (New York, US); Erasmus MC (Rotterdam, NL)

Objectives: To evaluate efficacy of $10 \%$ caprylate/chromatography-purified immune globulin intravenous (IGIV-C) in chronic inflammatory demyelinating polyradiculoneuropathy (CIDP) in a randomised, double-blind, placebo-controlled trial.

Methods: Patients (pts) received IGIV-C (Gamunex $\left.{ }^{\circledR} ; \mathrm{n}=59\right)$ or placebo (PLA; $\mathrm{n}=58$ ) every 3 weeks (wk) for up to $24 \mathrm{wk}$ (first period). A blinded, response-conditional crossover period provided rescue therapy as needed. Pts with an INCAT score that worsened by at least 1 point at any visit (day 16 up to wk 24), showed no change starting at wk 6, or improved but returned to baseline levels after wk 6, crossed over to rescue therapy for up to $24 \mathrm{wk}$. During the crossover period, pts were withdrawn if INCAT score did not improve by at least 1 point after the first infusion. Pts who responded (at least 1-point improvement in INCAT score, maintained through last visit) during either the first or crossover periods were eligible for a 24-wk extension phase; pts were re-randomised to receive IGIV-C or PLA every $3 \mathrm{wk}$. Pts who relapsed (ie, INCAT score worsened from extension baseline by at least 1 point) were withdrawn.

Results: During the first period, 32 (54\%) IGIV-C-treated pts vs 12 $(21 \%)$ PLA-treated pts maintained at least a 1-point improvement in INCAT score $(\mathrm{P}=0.0002) ; 23$ IGIV-C-treated pts and 45 PLA-treated pts crossed over to rescue therapy. During the crossover period, 26/45 (58\%) IGIVC-treated pts vs 5/23 (22\%) PLA-treated pts maintained at least a 1-point improvement in INCAT score $(\mathrm{P}=0.005)$. Pts rescued from PLA to IGIV-C also had significantly greater mean improvements in grip strength and MRC sum score vs pts rescued from IGIV-C to PLA. For the extension phase, we evaluated a subset of $57 \mathrm{pts}$ who had responded to IGIV-C during the first or crossover periods: 31 were re-randomised to IGIV-C and 26 to PLA. Pts who continued to receive IGIV-C during the extension phase received long-term benefit with maintenance therapy with a lower probability of relapse, $13 \%$ with IGIV-C vs $45 \%$ with PLA (hazard ratio, 0.19 [95\% CI, 0.05-0.70]), and a significantly longer time to relapse vs PLA-treated pts $(\mathrm{P}=0.011)$.

Conclusion: This study demonstrated both short-term and long-term IGIV-C efficacy. The crossover period data supported first period results, even though the crossover period sample size was smaller. Most importantly, the extension phase demonstrated the continued efficacy benefit of longterm IGIV-C maintenance therapy for CIDP.

Study supported by: Talecris Biotherapeutics, Center for Science and Education.

\section{P428}

Multifocal motor neuropathy with conduction block associated with a nonHodgkin B-cell lymphoma

H. Pérez-Pérez, M.P. Pueyo-Morlans, P.J. Pérez-Lorensu, B. GonzálezGonzález, D. Navajas-Carasa, R. Marrero-Abrante, J.A. Rojo-Aladro, C. Croissier-Elías, P. De Juan-Hernández

Hospital Universitario de Canarias (La Laguna, ES)

Introduction: Multifocal motor neuropathy (MMN) is an immune-mediated demyelinating peripheral neuropathy of unknown aetiology and clinically characterised by slowly progressive asymmetrical weakness of limbs without sensory loss. The diagnosis of MMN is based on clinical, electrophysiological and laboratory characteristics. It has been rarely reported in association with haematological tumours.

Methods: We present the case of a 67 years old woman who developed a non-Hodgkin B-cell lymphoma two years after a MMN diagnosis with progressive clinical worsening and early decreasing response to intravenous immunoglobulin (i.v. Ig) treatment.

Conclusion: We hypothesize that, in this case, $\mathrm{MMN}$ could have appeared as a paraneoplastic syndrome preceding lymphoma symptoms and signs, based on its atypicals clinical progression and response to i.v. Ig treatment, as well as on the close time relationship between the two entities. As far as we know, only three cases associating MMN and lymphoma have been described in the medical literature.

\section{P429}

Multiple cranial neuropathy revealing a final stage of Wegener's granulomatosis

L. Romero, S. Dimas, S. Vargas, R. Vega, I. Horta

National Institut of Neurology (Mexico City, MX)

Wegener granulomatosis (WG) is a necrotizing vasculitis that primarily affects small-caliber vessels of the arterial system. Microvasculitis produces necrotizing granuloma in lungs, focal necrotizing vasculitis and glomerulonephritis. Left untreated, the disease is rapidly fatal.

Purpose: Report a rare case of multiple cranial neuropathy revealing a final stage of Wegener's Granulomatosis.

Case history: A 41-year-old male with symptoms that resemble an upper respiratory tract infection associated with frontal headache. The clinical examination presented a protruded mass in the left nasal mucosa. The lesion was excised and reported as unspecified inflammatory process. Lesion in the nasal mucosa recurred 4 times in a 5 years period, three years later he presented a nodular cutaneous lesion in malar region. Two years later he developed a recurrent sinusitis. He was admitted at the hospital in a first time with fever and vertical diplopia. The diagnosis of Tolosa Hunt syndrome was established and treated with oral prednisone with totally remission. When the oral dose of prednisone was diminished, bilateral ophthalmoplegia showed up again, and develop V, VII, IX, X, XI, and XII cranial nerve affection. No other neurological manifestation was detected. Lung tomography showed a cavitary apical lesion. Axial T1-weighted postcontrast cranial magnetic resonance imaging revealed meningeal enhancing. Lumbar puncture was normal. One month later he develops bilateral optic atrophy, hypocusia, distal symmetrical sensorimotor polyneuropathy and cerebral hemorrhage. The antineutrophil cytoplasmic antibodies directed against protinase 3 were positives. Skin biopsy showed vasculitis granulomas and necrosis. Two days later the patient died of a massive lung hemorrhage.

Comment: Peripheral neuropathy is a frequently neurological feature in Wegener's disease because involve mainly large diameter nerves and this correlate with the typical mononeuritis multiplex presentation. Nevertheless multiple cranial neuropathy as the first and principal neurological affection with rapid progression to the death is less frequent. This is a possible remarkable clinic point for an appropriate and timely treatment of wegener's granulomatosis with other fatal potential vasculitic organ manifestation.

\section{P430}

Clinicopathological analysis of peripheral neuropathy associated with $M$ proteinaemia

M. Kobayashi, M. Takeuchi, M. Suzuki, K. Yamane, S. Uchiyama, M. Iwata Tokyo Women's Medical University (Shinjuku-ku, Tokyo, JP); Ohta-Atami Hospital (Koriyama, Fukushima, JP)

Objective To make a comparative analysis of the clinical, electrophysiologi$\mathrm{cal}$ and nerve biopsy findings of peripheral neuropathy associated with $\mathrm{M}$ proteinemia. Methods A total of 16 patients (aged 44 to 84 years, average 68.9) were examined. Eight patients had monoclonal gammopathies of undetermined significance (MGUS), four patients had Crow-Fukase syndrome (CFS), and four patients had anti-MAG antibody-related neuropathy, malignant lymphoma, polyarteritis nodosa and chronic inflammatory demyelinating polyneuropathy (CIDP)- complicated multiple myeloma. M protein subtypes were IgM in 5 patients, IgG in 6 patients, IgA in 3 patients, and mixed IgG and IgA type (IgG+IgA) in 2 patients. We investigated the type of distribution and classified it as polyneuropathy type (P), asymmetric polyneuropathy type (aP) or multiple mononeuropathy (MM). The type of impairment was categorized on the basis of motor $(\mathrm{M})$ or sensory $(\mathrm{S})$, axonal $(\mathrm{Ax})$ or demyelinating $(\mathrm{D})$ from both clinical and neurophysiological examinations. Nerve biopsy was performed in 12 patients and clinicopathological findings were evaluated. Results Type of distribution: IgM group showed $\mathrm{P}$ type in 3, MM type in 2.IgG group showed $\mathrm{P}$ type in 4, aP type in 3 and $\mathrm{MM}$ type in 1. IgA group showed 1 case each of $\mathrm{P}, \mathrm{PP}$ and $\mathrm{MM}$ type. Both cases in IgG+IgA group showed aP type. As for type of impairment, in the IgG group, $\mathrm{M}$ dominant and S dominant neuropathy were 4 patients for each. On the other hand, all but one patient from the IgM and IgA groups showed sensory dominant neuropathy. The prevalence of axonal and demyelinating neuropathy did not vary between these groups. Some of the patients with axonal neuropathy showed evidence of necrotic vasculitis or cell infiltration on nerve biopsy. Conclusions Axonal neuropathy with $\mathrm{M}$ proteinemia was related to vasculopathy in some patients. We considered the association between $\mathrm{M}$ proteinemia and vasculitis with a review of the literature. 
P431

Clinical variability in patients with chronic inflammatory demyelinating polyradiculoneuropathy

M. Suzuki, M. Takeuchi, M. Kobayashi, S. Uchiyama, M. Iwata

Tokyo Women's Medical University (Tokyo, JP)

Objective: Chronic inflammatory demyelinating polyradiculoneuropathy (CIDP) is an aquired peripheral nerve disorder, presumably of immunological origin, characterized by weakness, areflexia or hyporeflexia, and sensory disturbances that progresses over more than a month, or evolves chronically over many months. Its clinical presentation and course are variable. In order to learn about the clinical variability of CIDP, we reviewed the clinical manifestation of CIDP patients.

Methods: We reviewed medical records of patients in the department of neurology from 1999 to 2007 . About 220 patients were admitted for peripheral neuropathies. Clinical characteristics of CIDP patients among them were reviewed retrospectively. Patients with monoclonal gammopathy were excluded in this study.

Results: Thirty-nine men and nineteen women were included. The patients' ages ranged from 29 to 84. Twelve had diabetes. Four patients developed Guillain-Barre syndrome-like-onset. Two patients were on dialysis for chronic renal failure. Two patients developed CIDP after renal transplantation. Six patients had spinal spondylosis. The times between symptom onset and diagnosis ranged from 3 months to 9 years. Paresthesia or muscle weakness in the lower extremities were the most common symptom for the onset. In the clinical manifestations, twelve were motor dominant neuropathy, six were sensory dominant neuropathy, and the others were sensorimotor neuropathy. Nine patients relapsed more than three times. Thirty-one patients were treated with intravenous immunoglobulin (IVIG). Seven patients were treated with IVIG and corticosteroids. Four were treated with only corticosteroids, four were treated with plasmapheresis. Eight patients were treated with a combination of more than 3 immune therapies. Four patients were improved without treatement.

Discussion: CIDP can occur in patients with diabetes or renal failure, and rarely develops in patients of renal transplantation. One-fourth of patients need combinations of immune therapies.

Conclusions: The present study has demonstrated the clinical variability of CIDP with concurrent illness.

P432

A landmark clinical trial of a novel small molecule transthyretin (TTR) stabiliser, Fx-1006A, in patients with TTR amyloid polyneuropathy: a phase II/III, randomised, double-blind, placebo-controlled study

M. Waddington-Cruz, T. Coelho, L. Maia, A. Martins da Silva, V. Planté-Bordeneuve, O. Suhr, J. Campistol, I. Conceiçao, H. Schmidt, P. Trigo, D. Cros, D. Roy Grogan, J. Packman

Hospital Universitário Clementino Fraga Filho (Rio De Janeiro, BR); Hospital Santo Antonio (Porto, PT); CHU de Créteil (Paris, FR); Umeå University Hospital (Umeå, SE); Hospital Clínic de Barcelona (Barcelona, ES); Hospital de Santa Maria (Lisbon, PT); Universitatsklinikum Münster (Münster, DE); FLENI (Buenos Aires, AR); Massachusetts General Hospital (Boston, US); FoldRx Pharmaceuticals, Inc. (Cambridge, US)

Objectives: Fx-1006A is a novel, selective and potent stabiliser of wild-type and variant TTR, that may confer disease modification and halt disease progression in patients with TTR amyloidosis. Study Fx-005 is evaluating the effects of Fx-1006A in patients with V30M ATTR polyneuropathy (ATTR-PN), also known as familial amyloid polyneuropathy. Study design and Baseline disease characteristics will be presented.

Methods: Fx-005 is a Phase II/III randomised, double-blind, placebocontrolled, multinational study assessing safety and efficacy of Fx-1006A versus placebo for the treatment of ATTR-PN. Patients were randomised in a 1:1 ratio to receive $\mathrm{Fx}-1006 \mathrm{~A} 20 \mathrm{mg}$ or placebo for 18 months. The co-primary endpoints, the Neuropathy Impairment Score-Lower Limb (NIS-LL), and Norfolk Quality of Life-Diabetic Neuropathy total quality of life score (Norfolk) are instruments validated in diabetic neuropathy (DN). Secondary efficacy assessments include quantitative sensory testing, nerve conduction, and heart rate response to breathing, with pharmacodynamics assessed by TTR stabilization. Safety assessments include vital signs, laboratory tests, electrocardiograms, echocardiography, physical examination, and adverse events.

Results: A total of 128 patients (70 women) with ATTR-PN were randomized. Mean age at Baseline was 39.0 (SD 12.7) years, with $18.2 \%$ of patients enrolled with late onset disease (symptom onset $>=50$ ). Average time from symptom onset was 2.7 years overall, and 4.1 years and 2.4 years in the late vs. early onset groups, respectively. The most common symptoms at presentation included paraesthesia/dyaesthesia, muscle weakness, diarrhea, nausea, and vomiting. A notable difference between the late vs. early onset group was a higher frequency of cardiac symptoms ( $40.9 \%$ vs. $21.2 \%$ ). Mean Baseline NIS-LL and Norfolk scores were 9.7 (SD 12.4) and 29.2 (SD 25.3), with worse Norfolk scores in the late onset group (39.7 vs. 27.6).

Conclusions: Preliminary demographic data suggest that patients are representative of the general population with ATTR polyneuropathy, with Baseline NIS-LL and Norfolk scores similar to those reported in patients with Stages $1 \mathrm{~b} / 2 \mathrm{a}$ DN. Differences in the late vs. early onset group include longer time from symptom onset, worse Norfolk but similar NIS-LL scores, and more cardiac symptoms at Baseline. Final study results are expected in mid 2009.

This study was fully funded by FoldRx Pharmaceuticals, Inc.

\section{P433}

The electrophysiological evaluation of 70 Iranian rheumatoid arthritis patients

M. Yazdchi Marandi, H. Mikaeili, A. Ebrahimi

Emam Khomeini Hospital (Azadi, IR)

Objectives: We have conducted an electrophysiological investigation to find out the incidence and patterns of peripheral nerves involvements in rheumatoid arthritis patients and to correlate them with some patients' characteristics.

Patients and methods: Using clinical examination and proper laboratory end electrophysiological tests, seventy unselected rheumatoid arthritis patients ( 50 female and 20 male) were evaluated for evidence of peripheral nervous system involvement. The results were correlated with clinical data (severity and duration of disease) using statistical tests.

Results: Clinical and electrophysiological manifestations of periphera nervous system involvement were found in 33 patients $(47.1 \%)$. Most of cases were asymptomatic. The most common disorder was entrapment neuropathy, which was predominantly carpal tunnel syndrome (17 patients, $24.3 \%)$. Polyneuropathy was detected in 14 patients $(24 \%)$ and was mostly sensorimotor. Also, it was exclusively axonal type and was significantly common in severe cases. $(\mathrm{p}<0.05)$

Conclusion: Electrophysiological findings of peripheral nerve damage, even in the absence of clinical evidences, are common in Rheumatoid arthritis.

P434

Peripheral neuropathy as a presenting form of microscopic polyangiitis M.A. Tejero, A.L. Guerrero, E. Hernández, J. Martín-Polo, J.I. Martín-Serradilla, F. Gutiérrez, F. Iglesias, F. Sánchez-Barranco, E. Laherrán Complejo Asistencial de Palencia (Palencia, ES)

Objectives: Microscopic polyangiitis (MPA) is a systemic vasculitis histologically characterized by small-vessel involvement. Rapidly progressive glomerulonephritis is frequent, but the disease may be also associated with other manifestations as peripheral neuropathy.

Methods: A 43-year-old woman was admitted in our hospital because of subacute numbness and weakness in her legs and arms. Medical history unremarkable. Days before admission, oliguria, nocturia, fatigue, and slight fever. No abnormalities in general examination. Neurological exam disclosed weakness in muscles supplied by single nerves, associated with sensation loss and dysesthesia in those nerves. Laboratory values showed hematuria, moderate proteinuria and progressively increasing serum creatinine. Electrophysiological examination including nerve conduction studies and needle electromyography was performed; it revealed severe axonal motor and sensitive impairment of different individual nerves. Tests for autoantibodies, including antineutrophil cytoplasmic antibodies (ANCA) were negative. Oral prednisone at an initial dosage of $1 \mathrm{mg} / \mathrm{kg} /$ day led to a slight improvement in neuropathy symptoms, but not in kidney function. Renal biopsy sampled was then obtained, and showed extracapillary proliferative glomerulonephritis with no immune deposits. Nerve biopsy revealed severe axonal degeneration. Cyclophosphamide pulse therapy was then added, and neuropathy and renal improvement was achieved and sustained during a two years follow-up. ANCA were repeatedly tested, and turned positive one year after beginning of disease. Diagnosis of MPA was established.

Results: Although peripheral neuropathy is not an uncommon manifestation of MPA, only recently some studies have provided the characteristics of peripheral nervous system involvement in MPA, comparing them with the other two small-vessel systemic vasculitides (Wegener's granulomatosis (WG) and Churg-Strauss syndrome (CSS)), classic polyarteritis nodosa (PAN), and nonsystemic vasculitic neuropathy. In all these diseases, electrophysiological and histopathologic abnormalities consistent with axonal damage are commonly found, and most patients fulfil criteria for the diagnosis of multiple mononeuropathy. 
Conclusion: Abrupt development of multiple mononeuropathy with acute renal insufficiency is clue to underlying systemic arteritis. Although peripheral neuropathy favours PAN or CSS diagnosis, it may be the presenting form of MPA, as this case report shows.

P435

Clinical study of 35 patients with sensory neuropathy and impaired glucose tolerance

O.J.M. Nascimento, G. Quintanilha, M.R.G. Freitas

Universidade Federal Fluminense (Rio de Janeiro, BR)

Peripheral neuropathy due to diabetes has already been studied for several decades. Until recently, we associated the involvement of peripheral nerve with an inappropriate glycemic control in the most advanced stage of the disease. Currently, it is considered that the onset of the neural injury can occur in the initial phase of this metabolic abnormality.

The clinical aspects of the sensory neuropathy associated to the impaired glucose tolerance are analyzed in 35 Brazilian patients.

All patients met the American Diabetes Association (ADA), and the World Health Organization (WHO) criteria for glucose intolerance. We studied 20 male and 15 female, with ages ranging from 30 to 83 years. The importance of signs and symptoms related to small nerve fibers dysfunction was considered taking into account the involvement of the autonomic nervous system, or the prevalence of the neuropathic pain. Other causes of sensory neuropathies have been excluded, such as vitamin B12 deficiency, collagenosis, toxic and infectious neuropathies.

A distal symmetrical lower limb involvement with positive (neuropathic pain) and/or negative (reduced temperature and pinprick sensations) symptoms, and clinical signs of autonomic neuropathy were seen in most patients. Almost all patients presenting autonomic signs also complained of neuropathic pain. These patients had higher fast glucose. We observed that the glycemic levels were not related to the severity of the symptoms or to the presence of any specific symptom. Most patients presented a normal electrophysiological study or a mild axonal injury, pointing to a predominant small fiber involvement.

An investigation of metabolic alterations related to glucose can clarify several peripheral neuropathy cases misdiagnosed as having idiopathic neuropathy. The early diagnosis and the aggressive treatment can be crucial for the control, development and progression of the small fiber neuropathy in glucose intolerant patients.

\section{P436}

CIDP associated with hepatitis $\mathrm{C}$ virus infection responsive to combination antiviral therapy

R. Taipa, T. Coelho, M. Pires, T. Moreira, C. Correia

Hospital Santo António (Porto, PT)

Introduction: Peripheral neuropathy in hepatitis $\mathrm{C}$ virus ( $\mathrm{HCV}$ ) infected patients is mainly described as axonal and usually associated with cryoglobulinemia. We describe a CIDP case with HCV infection that showed marked clinical improvement under antiviral therapy.

Case report: A 52 year-old-man was referred for numbness and progressive weakness of the limbs beginning 2 years ago. He first noticed numbness of both hands that progressively evolved to the feet with associated motor weakness. His medical history, besides past alcoholic abuse, was unremarkable. Neurological examination showed a moderated symmetric distal weakness, with prominent involvement of the upper limbs. Deep tendon reflexes were absent. Plantar responses were both flexor. He had a dysesthesic sensation and severe impairment for position and vibration sensation in a stocking-and-gloves distribution. Cranial nerves were normal, no sphincter or dysautonomic disturbances.

Electromyography showed slow motor nerve velocities, prolonged distal latencies and absent $\mathrm{F}$ waves. Sensory nerves velocities were undetectable. CSF was acellular, with protein concentration of $0.37 \mathrm{~g} / \mathrm{L}$ and glucoses of 0.62 $\mathrm{g} / \mathrm{L}$. Serum hepatic enzymes were slightly increased. Serologic markers were positive HCV (3a genotype, viral load of $479432 \mathrm{UI} / \mathrm{ml}$ ). Cryoglobulins were undetectable and anti-myelin-associated glycoprotein antibodies were negative. Sural nerve biopsy was performed and showed prominent features of demyelisation without onion bulbs.

On November of 2007 antiviral therapy was started, combining peginterferon-alfa 2a (IFN-alfa) $180 \mu \mathrm{g} /$ week and ribavirin $(1000 \mathrm{mg} /$ day). The patient noticed neurological improvement after the 4 th week of therapy with increase of strength and diminished numbness. PCR for HCV became negative on the 8th week. At this time neurological examination revealed slightly decrease vibration sense, no position errors and markedly improvement on motor weakness.

Discussion: The mechanism of peripheral nerve damage in HCV associ- ated polyneuropathy seems to be vasculitic. Immunologically mediated demyelisation mechanism was seldom reported. In our patient, HCV infection was the only possible immunological trigger factor found. The clinical improvement could be attributable either to viral eradication or due to INF-alfa treatment. Taken in account CIDP related to IFN use documented in the literature, this case raises questions about the appropriate management of these patients.

\section{P437}

Chronic inflammatory demyelinating polyneuropathy. A 10 -year retrospective clinical study of a hospital-based population

S. Kojan, M. Al Jumah

King Abdullah International Medical R C (Riyadh, SA)

Objectives: To describe the clinical, electrophysiological findings and prognosis of Chronic inflammatory demyelinating polyneuropathy (CIDP) in tertiary care center in Saudi Arabia.

Background: CIDP is a chronic, acquired disorder of the peripheral nerves system. Several studies have indicated a significant variability of clinical characteristics, electrophysiological findings, and a wide spectrum of evolution and treatment response. This is a retrospective chart review of patients diagnosed and managed for CIDP at King Abdulaziz Medical City over the last 10 years. It describes the clinical variability of CIDP, electrophysiological findings as well as response to different treatment modalities.

Methodology: All patients that had been diagnosed with CIDP over the last 10 years were collected and reviewed. Data related to patients biography, age of onset, presenting symptoms and signs, electrophysiological data, cerebrospinal protein, modality of treatment and outcome measures were reviewed.

Results: We reviewed 15 patients who were diagnosed and treated for CIDP over the last 10 years. There were 10 men and 5 women. Mean age of onset was 41 years (ranged from $13-71$ years). $27 \%$ of the patients had diabetes mellitus type-II. Mean time to diagnosis was 12 months (ranging from 1.5 months to 3 years) however there was one patients who was diagnosed 15 years after symptom onset. $87 \%$ had diffused proximal and distal weakness. Asymmetric weakness documented in $20 \%$ of patients. Most patients $(93 \%)$ had sensory symptoms. And all patients had absent reflexes. Mobility was impaired in the majority of patients and $40 \%$ were wheelchair bound at diagnosis. CSF protein was elevated in all patients ranging from $0.67 \mathrm{gr} / 1$ to $5.7 \mathrm{gr} / 1$.

Only 9patients $(60 \%)$ met the AAN electrophysiological criteria for CIDP and $60 \%$ had mixed axonal and demyelinating features. All patients except one had been treated with at least with one of accepted treatment modalities. 4/15 had no fallow up, one patient was static and all other $10 \mathrm{pa}-$ tients had improved, $50 \%$ of which became normal.

Conclusion: Although our patients sample was small, more than half of our patients had mixed axonal and demyelinating. Neither presence of axonal involvement nor presence of DM had affected the long term outcome. CIDP has favorable prognosis. AAN electrophysiological criteria are not mandatory for diagnosis.

\section{P438}

Isolated peroneal neuropathy due to compression by tumourous sarcoidosis at the fibula head

S. Thomann, M. Tolnay, P. Fuhr, L. Kappos, N. Lüscher, S. Renaud

University Hospital (Basel, CH); Crossklinik Merian Iselin Spital (Basel, $\mathrm{CH}$ )

A 43-year-old woman had a 5-year history of slowly progressive left footdrop. She was admitted to our clinic because of painful dysesthesia along the lateral side of the left leg since 6 months. Her medical history was remarkable for generalized sarcoidosis with affection of lung and heart which required no medical treatment as she was asymptomatic. Clinical examination revealed a peroneal neuropathy with weakness $M 3$ of left foot elevation and M4 of pronation and sensory impairment along the lateral side of the leg and foot. Nerve conduction studies revealed an axonal lesion of the left peroneal nerve and needle EMG acute denervation of the anterior tibial muscle whereas the long peroneal muscle was normal. A CT scan of the left leg revealed a popliteal mass near the fibular head. Surgical resection of the popliteal mass was performed and the tumour turned out to be a large sarcoid granuloma with compression of the peroneal nerve. After removal, pain ceased immediately, while footdrop remained unaffected. A muscle biopsy from the left anterior tibial muscle showed extensive infiltration of muscle with non-necrotizing granulomas with epitheloid and Langerhans-type giant cells, consistent with the diagnosis of sarcoidosis.

Sarcoidosis is a systemic, granulomatous disease of unknown aetiology. Patterns of musculosceletal involvement include asymptomatic microscopic granulomata, acute polymyositis-like syndromes, chronic progressive myopathy and nodular or tumorous sarcoidosis. Asymptomatic involvement of 
muscles is quite common and has been reported in 25 to $75 \%$ of patients, although the true incidence is not clear since muscle biopsy is not performed routinely. Clinically recognizable involvement of the nervous system is rare and occurs in $<10 \%$ of patients. Although neurosarcoidosis has predilection for the base of the brain, any part of the CNS or peripheral nervous system may be affected. Peripheral nervous system affections most commonly present as cranial nerve palsies, polyneuritis or polyneuropathy. In our case, isolated peroneal neuropathy was due to the compressive effect of a tumorous sarcoid granuloma at the fibula head. Interestingly, the patient had no further symptoms although muscle biopsy revealed profuse myositis.

Conclusion: In systemic sarcoidosis, nerve affection due to compression by sarcoid granulomas has to be kept in mind as differential diagnosis to direct nerve infiltration.

\section{P439}

Changes in electrophysiology in patients with chronic inflammatory demyelinating polyneuropathy treated with immune globulin intravenous, $10 \%$ caprylate/chromatography purified versus placebo for up to 24 weeks V. Bril, H. Katzberg, M. Dalakas, C. Deng, P. Donofrio, K. Hanna, H-P. Hartung, R. Hughes, N. Latov, I. Merkies, P. van Doorn

University Health Network (Toronto, CA); Stanford University (Palo Alto, US); National Institutes of Health (Bethesda, US); Talecris Biotherapeutics (Research Triangle Park, US); Vanderbilt University (Nashville, US); Talecris Biotherapeutics (Research Triangle Park, US); Heinrich Heine University (Dusseldorf, DE); King's College London, Guy's Hospital (London, UK); Cornell University (New York, US); Erasmus MC (Rotterdam, NL)

Objectives: Nerve conduction studies (NCS) are an objective tool to diagnose chronic inflammatory demyelinating polyneuropathy (CIDP) and measure treatment response in clinical trials. As secondary and exploratory assessments, we evaluated improvements from baseline in NCS in patients treated with immune globulin intravenous, $10 \%$ caprylate/chromatography purified (IGIV-C) or placebo.

Methods: Patients with CIDP received a baseline loading dose of $2 \mathrm{~g} / \mathrm{kg}$ of IGIV-C (Gamunex $\left.{ }^{\circledR} ; n=59\right)$ or placebo $(n=58)$ and then a 1-g/kg maintenance infusion every 3 weeks for up to 24 weeks (first period) in a randomised double-blind study. Motor and sensory nerves were assessed at baseline and endpoint/week 24 applying standardised procedures. The amplitude measured from the most proximal site of the most severely affected nerve and the amplitudes for each nerve/stimulation site were analyzed by analysis of covariance. All nerve conduction tracings were read in a blinded manner by a centralised neurology laboratory.

Results: A trend toward improvement from baseline in amplitude of the most severely affected motor nerve at the most proximal stimulation site was observed with IGIV-C $(0.69 \pm 1.86 \mathrm{mV})$ versus placebo $(0.47 \pm 2.29 \mathrm{mV}$; $\mathrm{P}=0.542$ ), and a greater trend toward improvement was observed $(1.08 \pm 2.15 \mathrm{mV}$ versus $0.46 \pm 2.03 \mathrm{mV}$, respectively; $\mathrm{P}=0.089)$ when Erb's point was excluded from consideration of the most proximal site. Improvements from baseline favoring IGIV-C versus placebo were observed for 129 of 142 electrophysiological parameters, of which 4 amplitudes, 11 latencies, 4 velocities, $1 \mathrm{~F}$-wave latency, 1 duration, and 1 area reached statistical significance. The averaged amplitudes from all motor nerves significantly improved from baseline $(\mathrm{P}=0.035)$, and conduction block significantly decreased $(\mathrm{P}=0.027)$ with IGIV-C versus placebo, particularly in the lower limbs.

Conclusion: Although the study was not powered to detect significant differences, treatment with IGIV-C for up to 24 weeks improved NCS parameters, with some reaching statistical significance versus placebo. Improvements in electrophysiologic parameters in patients with CIDP further support intravenous immunoglobulin therapy as first-line therapy for CIDP.

Study supported by: Talecris Biotherapeutics, Center for Science and Education.

\section{Multiple sclerosis}

\section{P440}

Severe evolution of multiple sclerosis with childhood onset

A. Constantinescu, C.D. Popescu, V. Constantinescu

Rehabilitation University Hospital (Iasi, RO); University of Medicine and Pharmacy (Iasi, RO)

Background: Multiple sclerosis (MS) with childhood onset is a rare manifestation, being estimated at 0.4 to $10.5 \%$ of cases. The literature presents only a few studies of early onset MS.
Methods: We describe three cases of MS with early onset with peculiar, complex evolution followed up in our clinic.

Case 1 - female patient developed at the age of 14 vestibulo-cerebellar signs, first diagnosed as pseudotumoral viral encephalitis. Criteria for MS diagnostic were: the clinical evolution with exacerbating-remitting signs, magnetic resonance imagery (MRI), cerebrospinal fluid and evoked potentials positive evaluation. The treatment with interferon for 4 years did not stop the evolution. Six years from the onset of MS, the patient developed glomerulosclerosis with chronic interstitial nephritis. Cyclophosphamide puls therapy associated with corticosteroids as background treatment improved the clinical status; present Expanded Disability Status Scale (EDSS) score is 3.5 .

Case 2 - female patient diagnosed at the age of 13 with internuclear ophtalmoparesis and spastic paraparesis, with good results following corticosteroid therapy, diagnosed with MS according to the McDonald criteria. The patient received interferon for 3 years and then azathioprinum, taking into consideration the secondary progression phase of the disease evolution. In 10 years of evolution, the patient EDSS score reached the value of 8.5. Interestingly, the twin brother of the patient concomitently developed Hodgkin Disease.

Case 3 - male patient, at the age of 15 presented with episodic sensory symptoms in the limbs and cognitive difficulties, ignored by his family. At the age of 17, drug use contributes to his clinical deterioration. Difficult differential diagnosis, the MRI investigation describing pseudotumoral aspect of the demyelinated plaques. McDonald criteria for MS were fulfilled. The patient presented also psychotic signs with severe instability which made impossible the use of immunotherapy and lead to suicide.

Conclusion: The differential diagnosis was complex in all three cases. The severe evolution or lack of compliance contributed to supplementary difficulties in treatment and follow-up.

\section{P441}

Neuroaxonal brain viability does not differ between benign and early relapsing-remitting multiple sclerosis patients

B. Benedetti, M. Rovaris, A. Pulizzi, D. Caputo, M. Zaffaroni, R. Capra, A. Bertolotto, V. Martinelli, A. Falini, G. Comi, M. Filippi

Scientific Institute San Raffaele (Milan, IT); Scientific Institute Don Gnocchi (Milan, IT); Ospedale di Gallarate (Gallarate, IT); Center, Spedali Civili (Brescia, IT); Ospedale San Luigi (Orbassano, IT)

Objectives: The term benign multiple sclerosis (BMS) indicates a favourable course of MS, when patients have mild or no disability several years after the clinical onset of the disease. Axonal damage, which is considered a major determinant for the accumulation of disability in MS, can be measured in vivo using proton MR spectroscopy (1H-MRS). Aim of the study was to quantify the severity of "global" axonal damage in BMS and early relapsing-remitting (RR) MS patients, using whole brain $\mathrm{N}$-acetylaspartate (WBNAA) $1 \mathrm{H}-\mathrm{MRS}$, in order to better elucidate the structural correlates of a non-disabling disease evolution.

Methods: WBNAA concentration was measured in 48 patients with BMS and 17 patients with early RRMS, using an unlocalized ad hoc $1 \mathrm{H}$-MRS sequence. Conventional dual echo and T1-weighted scans were also obtained to measure T2-hyperintense lesion load (T2LL) and normalized brain volume (NBV).

Results: T2LL was higher in BMS than in early RRMS patients $(\mathrm{p}=0.018)$, while neither NBV nor WBNAA concentration significantly differed between the two groups of patients. No correlations were found between WBNAA concentration and EDSS, T2LL and NBV.

Conclusion: The similarity in the WBNAA concentration between BMS and early RRMS patients fits with the hypothesis that a non-disabling longterm evolution of MS may be due, at least in part, to the preservation of axonal density and integrity. Such a condition seems to be independent from the burden of MRI-visible lesions.

The study was supported by a grant from FISM (Fondazione Italiana Sclerosi Multipla) - contract n. 2005/R/18.

\section{P442}

Application of De Seze criteria to 14 patients who presented a first severe acute demyelinating event

E. Bozzolo, V. Bourg, S. Chanalet, C. Lebrun-Frenay

Hôpital Pasteur (Nice, FR)

Introduction: The application of De Seze criteria (2007) to patients presenting a first severe acute demyelinating event helps to distinguish an acute disseminated encephalomyelitis (ADEM) from other CNS inflammatory diseases, with a sensitivity of $83 \%$ and a specificity of $95 \%$. We applied these 
criteria to 14 patients who presented a first severe acute demyelinating event and whose later clinical evolution permitted to clearly identify the neurological diagnosis.

Method/Patients: This study concerned 14 patients who presented a first acute demyelinating event. Our series initially included 16 patients but 2 were excluded because of their initial clinical condition in order to respect the exclusion criteria of the original article ( 1 case of isolated acute retrobulbar optic neuritis and 1 case of acute cervical myelitis). We identified 11 women $(78.6 \%)$ and 3 men $(21.4 \%)$ with a mean age of 33.7 years \pm 12.5 years, the follow-up of the patients being from 3 months to 11.5 years after the initial episode (4 years average follow-up). At the end of the follow-up, 7 patients were diagnosed as ADEM (50\%) and 7 as multiple sclerosis (MS) $(50 \%)$. Five patients out of 7 in the MS group have a tumor-like presentation $(71,4 \%)$, this parameter partly explaining the initial discrepancy in diagnosis. After application to our series, De Seze criteria for ADEM have a sensitivity of $85,71 \%$ and a specificity of $71,43 \%$.

Conclusion: The application of the new criteria does not enable us to find the same values as in the original article,in term of sensitivity, specificity, positive and negative predictive values. The lack of specificity comes from the misclassification of MS patients with a tumor-like presentation for which there are 2 out of 5 false positive. One of the explanations is that the clinical criteria used can be part of atypical forms of MS, in particular in its tumorlike presentation. De Seze criteria represent an invaluable help for the clinician in the diagnosis of a first severe demyelinating event. Considering our results, a tumor-like form of MS could be a exclusive situation to the application of these criteria. A prospective study on a more important cohort is necessary to confirm or invalidate these preliminary results.

P443

Decreased postural balance in multiple sclerosis patients with low disability C. Fjeldstad, G. Pardo, D. Bemben, J. Cramer, M. Anderson, A. Knehans, M. Bemben

MS Center of Oklahoma (Oklahoma City, US); University of Oklahoma, Norman (Oklahoma City, US); University of Oklahoma Health Sciences Center (Oklahoma City, US)

Objectives: To evaluate balance in women with Multiple Sclerosis (MS) that have low disability. The secondary purpose was to investigate the relationship between balance and Expanded Disability Status Scores (EDSS).

Methods: Subjects were 18 to 64years old; 66 individuals with MS ( $\mu=$ $43.9 \pm 1.2$ years) and 45 healthy controls $(\mu=40.4 \pm 2.4$ years).All patients had an EDSS $\leq 3.5$. Both groups underwent a series of five balance measures using the Neurocom Balance Master.

Results: The mean EDSS for the MS group was 1.8. For balance, there was a significant difference between the two groups, with the MS group performing worse on: unilateral stance eyes open, unilateral stance eyes closed, tandem walk end sway, and modified clinical test for sensory integration of balance (MCTSIB) measures standing on foam surface with closed eyes $(p<0.01)$. There was a positive correlation $(p<0.01)$ between balance and EDSS scores.

Conclusions: The MS group did significantly worse on four of the five balance measures, indicating greater postural instability despite the low disability measured by EDSS when compared to healthy controls. There were positive,low to moderate relationships between EDSS scores and balance measures, indicating better postural stability with lower EDSS scores.

\section{P444}

The rehabilitation of attention and executive functions in multiple sclerosis: a controlled follow-up study

C. Stampatori, R. Capra, D. Zanotti, C. Sottini, F. Mattioli

Spedali Civili Brescia (Brescia, IT)

Cognitive dysfunction has been demonstrated in patients with multiple sclerosis (MS), correlating with the chronic progressive course, total lesion load, cortical atrophy and the Kurtzke EDSS (Expanded Disability Status Scale) score (Benedict et al., 2004). More recently cognitive impairment, in particular involving attention, memory, information processing and visuospatial abilities has also been shown in patients with benign course and low disability (Bensa et al., 2006) and the efficacy of different rehabilitative procedures have been reported.

Objective: to test the efficacy of a standardized PC supported rehabilitative procedure (Rehacom) of attention, information processing speed and planning in a controlled study design, of patients with relapsing remitting MS, EDSS $<4$ and clinical stability in the previous 12 months. Inclusion criteria were pathological scores in both the Winsconsin Card Sorting Test (WCST) and in the Paced Auditory Serial Addition Test (PASAT), which were submitted as a screening test to a group of $80 \mathrm{MS}$ patients with the clinical characteristics above mentioned.

Methods: Fourteen patients were included and divided in an experimental ( 7 females, mean age 49.14, SD 9.2 years; mean years of schooling 7.85, SD3.8; mean disease duration 11 years) and in a control (7 females, mean age 44,SD 10.42 years; mean years of schooling 11,4,SD 4,6; mean disease duration 15.42 years) group. All the patients underwent a baseline and a follow up extensive neuropsychological and behavioural assessment (Short tale, verbal fluency, Selective Reminding Test, 10/36 Spatial Recall Test, Symbol Digit Modality Test, TEA Divided Attention, MADRS and MSQoL). The experimental group was submitted to a neuropsychological intensive (24 sessions lasting 1 hour, frequency 3/week) training of attention and planning (two subgroups of 4 and 3 patients respectively with an inverse order of training) conducted by a psychologist.

Results: A selective improvement in attention and executive functions tests was obtained after the training in the experimental group, with a generalization to other cognitive domains and to the quality of life measures.

\section{References}

Benedict RH et al. (2004) Arch Neurol 61:226-230

Bensa et al. (2006) Rev Neurol 162:1221-1231

\section{P445}

Exploratory analysis of the effects of alternative definitions on the prevalence of relapses using administrative claims data

D. Meletiche, C. Kozma, R. Bennett, A. AL-Sabbagh

EMD Serono, Inc. (Rockland, US); University of South Carolina (Columbia, US)

Objective: Administrative claim-based information is a potential source of useful data for conducting outcome studies of the effectiveness of multiple sclerosis (MS) therapies. However, consensus criteria to identify patients who experience a relapse from claims data are lacking. Alternative definitions of relapses were applied to claims-based data to explore estimates of relapse prevalence in MS patients.

Methods: Subjects diagnosed with MS or who had at least 1 MS diseasemodifying drug (DMD)-prescription claim (interferon beta-1a, interferon beta-1b, or glatiramer acetate) from July 1,2000 through December 31,2004 were identified from the PharMetrics (Watertown, MA) Patient-Centric database. Relapse occurrence was evaluated for the 24 months following the first DMD prescription claim during the enrolment period in continuously eligible patients. Claims-based variables used to document relapses included hospitalisation, emergency room (ER) use, steroid use, and worsening of symptoms (eg, ataxia, spasticity, tremor, speech problems). The prevalence of relapses during the 24-month follow-up period was assessed using 4 definitions of relapse: (1) MS hospitalisation or ER visit, (2) definition 1 plus injectable methylprednisolone use, (3) definition 1 plus any steroid use, or (4) definition 3 plus presence of a diagnosis indicating worsening symptoms.

Results: 2400 patients met the selection criteria. Patients had a mean (standard deviation) age of 43.9 (9.4) years, and $76.3 \%$ were women. Relapses occurred in $8.2 \%(n=197)$ of patients with application of the most restrictive definition, at least 1 MS hospitalisation or ER visit. When use of injectable methylprednisolone was included in the definition, $19.9 \%(n=454)$ of patients were identified as having experienced a relapse. The occurrence of relapse increased further to $44.3 \%(n=1064)$ when the third definition of relapse, which included the use of any steroid, was applied. $79 \%(n=1897)$ of patients were identified as having experienced a relapse using the fourth and most liberal definition of relapse.

Conclusion: Considerable variation in prevalence estimates of relapse was found. Prevalence estimates varied greatly depending on the claimsbased criteria used to define a relapse. This highlights the need for validation of claims-based criteria with clinical records to accurately identify MS relapses.

This study was supported by EMD Serono, Inc. and Pfizer Inc.

\section{P446}

Factors determining quality of life of Polish subjects with multiple sclerosis E. Papuc, H. Bartosik-Psujek, A. Jamroz-Wisniewska, Z. Stelmasiak Medical University of Lublin (Lublin, PL)

Objective: The aim of the study was to elicit the most important factors determining quality of life of Polish subjects with multiple sclerosis.

Method: 173 patients with a diagnosis of MS according to McDonald criteria participated in the study $(\mathrm{M} / \mathrm{F}=52 / 121$; mean age: $36,9 \pm 8,9$; mean dis- 
ease duration: $8,9 \pm 6,6$ years; mean EDSS: $4,34 \pm 1,84)$. All subjects were assessed with MMSE, Beck Depression Inventory (BDI), Fatigue Severity Scale (FSS), World Health Organization Quality of Life Instrument (WHOQOL100 ) and with EDSS. Socio-demographic, clinical and functional factors were taken into consideration in the study. In the first step correlation analysis was performed. As we found more than one statistically significant variable positively correlated with domains of quality of life, in order to elicit the most important factors determining quality of life of multiple sclerosis patients, regression analysis was performed.

Results: Results of regression analysis revealed that depressive symptoms predicted the highest percentage of variation in overall quality of life $(20 \%)$, quality of life in physical (37\%) and psychological (48\%) health domains and environmental domain $(25 \%)$. Level of disability measured in EDSS contributed most to explaining variation of overall quality of life $(4 \%)$ and quality of life in physical ( $2 \%$ ) and environmental domain (10\%). Quality of life in multiple sclerosis patients was strongly predicted by depression, level of disability, fatigue and marital status.

Conclusions: The strongest predictors of quality of life in multiple sclerosis patients are depression, level of disability, fatigue and marital status. These factors explained $20 \%, 4 \%, 3 \%$ and $3 \%$ of variation of overall quality of life. Altogether these factors explained $30 \%$ of variation of overall quality of life of multiple sclerosis patients.

P447

Tolerability and adherence to Betaferon ${ }^{\circledR}$ and Copaxone ${ }^{\circledR}$ in a phase III clinical trial

G. Comi, B.G.W. Arnason, S. Cook, M. Filippi, D.S. Goodin, H-P. Hartung, D.R. Jeffery, L. Kappos, P. O'Connor, T. Bogumil, C. Pohl, V. Filipov, M. Groth, F. Boateng

Scientific Institute and University Hospital San Raffaele (Milan, IT); Surgery Brain Research Institutes (Chicago, US); UMD New Jersey Medical School (Newark, US); University of California at San Francisco (San Francisco, US); Heinrich-Heine-Universität (Dusseldorf, DE); Wake Forest University School of Medicine (Winston-Salem, UK); University Hospital (Basel, CH); St Michael's Hospital (Toronto, CA); Bayer Schering Pharma AG (Montville, US); Bayer Schering Pharma AG (Berlin, DE)

Objective: To make direct comparisons of tolerability and adherence between interferon beta-1b (IFNB-1b; Betaferon $\left.{ }^{\circledR}\right)$ and glatiramer acetate (GA; Copaxone ${ }^{\circledR}$ ) in patients with relapsing-remitting multiple sclerosis (RRMS).

Methods: The Betaferon ${ }^{\circledR} /$ Betaseron $^{\circledR}$ Efficacy Yielding Outcomes of a New Dose study was a head-to-head trial comparing the safety, tolerability and efficacy of IFNB-1b $500 \mathrm{mcg}$, IFNB-1b $250 \mathrm{mcg}$ and GA. Treatment-naïve patients were randomised to subcutaneous IFNB-1b $500 \mathrm{mcg}$ or $250 \mathrm{mcg}$ every other day, or GA $20 \mathrm{mg}$ daily for ?104 weeks. Tolerability parameters were important secondary endpoints; adherence to treatment and incidence of adverse events were monitored throughout the study.

Results: The overall results of this study $(n=2244)$ have been presented elsewhere; 897 patients were randomised to IFNB-1b $250 \mathrm{mcg}$ and 448 to GA $20 \mathrm{mg}$. Adherence was higher with IFNB-1b $250 \mathrm{mcg}$ group compared with GA; slightly higher proportions of IFNB-1b 250 mcg-treated patients completed treatment and completed the study. Influenza-like symptoms were initially more common in the IFNB-1b $250 \mathrm{mcg}$ than the GA group, but rapidly decreased with time. Injection-site reactions (ISR), particularly pain and pruritus, were more frequent with GA. Incidence of ISR diminished with time in both groups. Other adverse events, including fatigue, depression, arthralgia, and paraesthesia, were similar in both groups. Alterations in liver enzymes, blood lipids and thyroid function were slightly more frequent in the IFNB-1b $250 \mathrm{mcg}$ group as were decreases in leukocytes. The incidence of serious adverse events was slightly lower in the IFNB-1b 250 mcg group compared with the GA group. Further data will be presented.

Conclusions: This was the first large-scale study to directly compare IFNB-1b with GA and confirmed the benign tolerability profile of IFNB-1b $250 \mathrm{mcg}$. Adverse event frequencies were generally similar and as expected. Adherence to IFNB-1b $250 \mathrm{mcg}$ was excellent and was slightly higher than to GA.

Study supported by: Bayer Schering Pharma AG, Berlin, Germany

P448

Supplemental oestrogen may enhance clinical effects of $\mathrm{T}$ cell receptor vaccination in women with multiple sclerosis

H. Offner, D. Bourdette, A. Vandenbark

Oregon Health \& Science University (Portland, US)

Objectives: Vaccination with T cell receptor (TCR) peptides induces regulatory T cells ( $\operatorname{Tr} 3$ and Treg) that can recognize and regulate potentially path- ogenic myelin reactive $\mathrm{T}$ cells expressing the same $\mathrm{V}$ region genes. Estrogens (E2, E3 and EE) can also inhibit myelin reactive T cells by inhibiting macrophage activation and inducing Treg cells. Combination therapy for experimental encephalomyelitis of TCR determinants plus E2 was more effective than either agent alone. Our objective was to determine if supplemental estrogen could enhance effects of TCR vaccination in women with multiple sclerosis (MS).

Methods: Thirty-four subjects with relapsing or progressive MS (25 females and 9 males) treated monthly with NeuroVax (TCR peptides from BV5S2, BV6S5 \& BV13S1 in IFA) in an open-label trial were evaluated clinically over a one year period for changes in Expanded Disability Status Score (EDSS). Clinical outcome (improved, unchanged, worse) was assessed by net changes in EDSS at exit vs. entry after a period of at least 6 months of TCR therapy in women taking supplemental estrogen $(n=13$, mean age $48 \mathrm{y})$, women not taking supplemental estrogen $(\mathrm{n}=12$, mean age $49 \mathrm{y})$, and males $(n=9$, mean age 52y). Differences between groups were evaluated using the Chi Square Test.

Results: Women taking supplemental estrogen plus TCR therapy had a significantly better clinical outcome than women not taking supplemental estrogen $(p=0.02)$, but were not different from males treated only with TCR therapy.

Conclusion: Supplemental estrogen appeared to enhance the therapeutic effect of TCR peptide vaccination in women with MS.

Dr. Offner and Dr. Vandenbark have a significant financial interest in Orchestra Therapeutics and Effective Pharmaceuticals, companies that have a commercial interest in the results of this research.

\section{P449}

Working memory performance in early relapsing-remitting multiple sclerosis is associated with reduced functional adaptation

I.K. Penner, L. Doessegger, M. Amann, J.G. Hirsch, H. Duyar, E.W. Radue, L. Kappos, A. Gass

University of Basel (Basel, CH); University Hospital Basel (Basel, CH)

Objectives: Cognitive deficits in MS are common and mainly affect information processing speed, attention, working memory and flexibility. These disturbances can already be present at the early stages of the disease and substantially influence quality of life. Previous fMRI studies on cognition in MS revealed changes in brain activation patterns. MS patients even at very early stages of the disease were shown to activate additional brain regions. The aim of the present study is to monitor the evolution of functional changes over time related to cognitive performance in patients at early stages of MS

Methods: Six patients with RRMS (mean age: 39, mean EDSS: 2.2, mean disease duration: 4) and six healthy controls (HCs; mean age: 34 ) underwent a comprehensive neuropsychological examination on the cognitive domains mainly affected in MS. In addition, depression, fatigue and quality of life were assessed. During the fMRI investigation, subjects performed an alertness and a working memory task with increasing task complexity (1-back, 2-back, 3-back). fMRI was performed on a 3T head scanner (Allegra, Siemens). Data analysis was done using AFNI software.

Results: The neuropsychological examination revealed significant differences between patients and HCs in information processing speed, verbal and spatial short-term memory. In contrast, performance in working memory measures did not differ significantly between the two cohorts. In addition, patients scored significantly higher on fatigue. Significant differences in brain activation patterns were found during 2-back and 3-back performance, where patients showed increased activation in medial frontal gyrus (MFG). These changes however were not related to decreased task performance. While HCs showed a significant and widely distributed change of activation for the contrast between 3-back and 1-back, only sparse change was found in patients.

Conclusion: Our results strongly indicate an altered functional adaptation to higher demanding working memory tasks (2-back and 3-back) in patients at early stages of RRMS despite the lack of obvious behavioural impairments. In contrast to HCs, patients showed only a signal increase related to task difficulty while decreases of signal were absent. These results might indicate that already at early stages the functional adaptation of the CNS in terms of learning mechanisms is impaired. 
P450

Clinical results of the first-in-man single ascending dose study of PI-2301, a second generation peptide copolymer for the treatment of multiple sclerosis

J. Kovalchin, J. Krieger, I. Dufour, K. Collins, M. Genova, M. Augustyniak, K. Rafuse, T. Avril, G. Gandon, A. Patat, N. Fauchoux, U. Patel, E. Mascioli, E. Zanelli

Peptimmune (Cambridge, US); Centre Eugene Marquis (Rennes, FR); Biotrial (Rennes, FR)

Objectives: PI-2301 is a novel compound in a class of autoimmune therapeutics called peptide copolymers. Copolymers are random mixtures of peptide sequences comprised of limited numbers of amino acids. Copaxone ${ }^{\circledR}$ is a copolymer which has been approved as a primary treatment for Relapsing Remitting-Multiple Sclerosis (RR-MS). PI-2301, like Copaxone, is an immunomodulator which promiscuously binds to MHC Class II molecules and induces a skewed TH2 T-cell response characterized by the activation and expansion of T-cells and monocytes which secrete IL-4, IL-5, IL-10, IL13 and CCL22. This regulatory response is believed to interfere with the expansion of autoreactive TH1/TH17 cells. PI-2301 has shown superior therapeutic efficacy as compared to Copaxone in murine experimental allergic encephalomyelitis (EAE), an animal model which resembles multiple sclerosis, in both daily and weekly subcutaneous (SC) dosing regimens. The purpose of the present study was to evaluate the safety, tolerability and early immunological effect(s) following SC administration of PI-2301 in a Single Ascending Dose, first-in-man study involving healthy, male adult volunteers.

Methods: The clinical study was designed in accordance with recommendations as defined in the Duff report and Committee for Human Medicinal Products (CHMP) guidelines issued in July 2007 for potentially immunmodulating therapeutics. Fifty-six subjects (eight cohorts of seven individuals; 5 active and 2 placebo) were given a single subcutaneous injection of PI-2301. The first dose was $0.01 \mathrm{mg}$ which is 100 -fold below the Minimal Anticipated Biological Effect Level (MABEL) and 50,000-fold below the No Observed Adverse Effect Level (NOAEL) in the most sensitive animal species tested with PI-2301. The parameters evaluated were safety, monitoring, pharmacokinetics, in vitro T-cell recall responses, antibody response to PI-2301 and changes in serum cytokines and chemokines.

Results: PI-2301 was generally well tolerated through the doses tested thus far ( 6 of the scheduled 8 doses). Evidence of immune priming (as shown by T-cell specific proliferative and cytokine responses) was observed at the projected MABEL dose in humans, i.e., $1 \mathrm{mg}$. Further data collection and analyses are currently underway and will be available in the coming months.

Conclusions: This study represents the initial step in the development of an improved peptide copolymer with immunomodulatory properties for the treatment of multiple sclerosis.

This abstract is submitted by Peptimmune, a privately held company, which plans to commercialize the compound described in this abstract in the future.

\section{P451}

Expression pattern of substance $P$ gene isoforms and peptide production in T-lymphocytes of multiple sclerosis patients

L. Cantalupo, C. Cioni, L. De Santi, P. Annunziata

University of Siena (Siena, IT)

Objectives: Substance P (SP) has been found to be produced by peripheral blood immunocompetent cells involved in neuroinflammation. Recently, a significant association with some haplotypes of the preprotachykinin-A (PPT-A) gene encoding SP precursor was found in multiple sclerosis (MS) families. To date, there is no data on the expression pattern of PPT-A gene isoforms neither on the SP secretion by lymphocytes in MS patients. We analysed the expression of PPT-A isoforms and measured the SP levels released by MS T lymphocytes correlating them with the disease activity.

Methods: Peripheral blood mononuclear cells were obtained from 13 normal healthy adult blood donors and 31 subjects with definite relapsingremitting MS, 23 of which were on clinical relapse. Magnetic resonance imaging (MRI) was performed within a median time of 1 week from the onset of a clinical relapse. SP gene isoform expression pattern was detected in $\mathrm{CD}^{*}$ lymphocytes by RT-PCR assay and single isoforms were quantified by computerized densitometric analysis. SP levels in lymphocyte culture supernatants were measured by a commercial competitive ELISA.

Results: Twenty-seven of $31(87 \%)$ MS patients expressed PPT-A beta isoform compared to 11 of $13(85 \%)$ normal healthy subjects (NHS). Gamma isoform was detected in 23 of $31(74 \%)$ MS patients compared to 7 of 13 (54\%) NHS. Alpha and delta isoforms were detected in $1 \mathrm{MS}$ patient respectively, compared to 1 of $13(8 \%)$ NHS. There was a higher but not significant beta isoform /actin and gamma isoform/actin ratio in MS than in NHS (0.7 vs 0.58 and 0.55 vs. 0.30 respectively). In MS patients, higher levels of SP released from lymphocytes were found to be significantly associated with beta and beta-gamma isoforms than those with gamma isoform expression only $(p=0.017)$. All MS patients showed slightly higher SP levels than those of NHS $(p=0.05)$. Significantly lower levels of released SP were found to be associated either with clinical relapses or presence of active MRI lesions $(p=0.039)$. There was an inverse but not significant correlation of SP levels with EDSS score ( $\mathrm{rs}=-0.26)$.

Conclusions: These findings show that SP is secreted mainly with some gene isoforms and there is also a significant association of reduced peptide release with active stages of MS supporting the hypothesis that SP may play a role in the pathogenesis of disease activity warranting further investigation.

This research was supported by a grant from University of Siena (PAR) to P.A.

\section{P452}

Tactile-associated recruitment of cervical cord is more altered in multiple sclerosis patients with more severe disability

M. Absinta, P. Valsasina, F. Agosta, D. Caputo, M. Filippi

Neuroimaging Research Unit (Milan, IT); Fondazione Don Gnocchi (Milan, IT)

Objectives: Aim of this study was to evaluate the pattern of tactile-associated cervical cord activations in patients with relapsing-remitting (RR) and secondary progressive (SP) multiple sclerosis (MS).

Methods: Cervical cord functional MRI scans were acquired from 15 right-handed healthy controls $(6$ men, 9 women; mean age $=37.8$ years, $\mathrm{SD}=10.6), 22$ RRMS patients ( 9 men, 13 women; mean age $=39.4, \mathrm{SD}=8.9$ mean disease duration $=11.5$ years; median EDSS $=2.5$ ) and 15 SPMS patient ( 5 men, 10 women; mean age $=51, \mathrm{SD}=10.3$; mean disease duration $=$ 22 years; median EDSS $=6.0$ ). Using a block design, subjects were scanned when performing a sensory task, consisting of a tactile stimulation of the palm of the right hand. Using a General Linear Model, fMRI data analysis was performed and statistical maps were generated for all subjects $(p=0.05)$. The presence of activity in the right and left anterior, right and left posterior, and middle cervical cord at different levels was assessed by visual inspection on the activation maps from all subjects. The mean intensity signal change induced by the task was computed for all activated voxels within the cervical cord and compared between controls and MS patients using an ANOVA model. A random effect logistic regression model, with the frequency of fMRI activity as dependent variable and the subject as grouping factor, was used to assess the within-group difference in the occurrence of fMRI activity in right vs. left, and anterior vs. posterior cord.

Results: Average cord mean intensity change was $2.9 \%(\mathrm{SD}=0.8)$ in healthy controls and 3.6\% $(\mathrm{SD}=1.1)$ in MS patients $(\mathrm{p}=0.04)$. Healthy controls showed an higher occurrence of fMRI cord activity in the right than in the left cervical cord $(p=0.001)$, as well as in the posterior than in the anterior cord $(\mathrm{p}=0.043)$. RRMS patients showed an higher frequency of fMRI cord activity in the posterior than in the anterior cervical cord $(\mathrm{p}<0.001)$, but they did not show any significant difference between left and right activity. In SPMS patients, no significant difference was found in the occurrence of activity neither between right and left, nor between anterior and posterior cord.

Conclusions: This study shows an abnormal pattern of activations in MS patients following tactile stimulation, with a progressive over-recruitment of cervical cord and loss of lateralization with increasing disability.

\section{P453}

Intense immunosuppression as the initial disease-modifying therapy in clinically active relapsing MS

O. Khan, J. Perumal, S. Hreha, C. Caon, F. Bao, R. Penmasta, I. Zak, Z. Latif, A. Tselis

Wayne State University School of Medicin (Detroit, US)

Objective: Intense immunosuppression (IIS) is often used as rescue or disease stabilizing therapy in clinically active relapsing MS patients with suboptimal response to immunomodulating therapy (IMT). There is increasing interest in the use of immunosuppressive therapy as the initial disease-modifying therapy (DMT) in relapsing MS. However, there is little data on the use of IIS as initial therapy in MS. We conducted a study to examine the feasibility, efficacy, and safety of IIS as the initial therapy in clinically active relapsing MS.

Methods: We examined the medical records of relapsing MS patients who received IIS as the initial therapy at our Center. None of the patients had received FDA-approved DMT prior to the use of IIS. All but two patients had 
received IV methylprednisolone IVMP) for the treatment of relapses as well as pulsed IVMP to stabilize clinically active disease. All patients had experienced at least 2 relapses in the year prior to therapy with IIS. All patients received monthly IV cyclophosphamide (CTX) for 6 months followed by initiation of therapy with DMT including interferon beta (IFNB) or glatiramer acetate (GA). All patients underwent brain MRI scans prior to IIS and at year 1 i.e. 6 months after the completion of IIS therapy. In 2 patients CTX was continued for an additional 6 months.

Results: 19 consecutive patients with clinically active MS received IV CTX for 6 months. Mean age, disease duration, EDSS, relapse rate, gadolinium enhancing lesions (GEL) per patient at baseline were 34.8 years, 3.08 years, $3.61,3.42$, and 3.55 , respectively. At year 1, mean EDSS, relapse rate, and GEL were significantly reduced to $2.22,0.77$, and 0.33 , respectively. Mean percentage brain volume change at year one was $-2.37 \%$. All patients tolerated IV CTX well and no adverse events including opportunistic infections were seen. Additional data beyond one year of follow up will presented.

Conclusions: This study provides the rationale to design treatment strategies based on using IIS in carefully selected clinically active patients who are treatment naïve and may benefit from IIS before starting IMT with IFNB or GA. Carefully designed studies are warranted to examine the use of IIS as the initial DMT in clinically active relapsing MS patients with longterm follow up including immunologic markers of disease activity.

Wayne State University Neuroscience Program

\section{P454}

Cognitive functions in patients with multiple sclerosis and correlation with number and localisation of brain lesions

P. Kostaras, I. Chatzidakis, M. Tsatsani, P. Stathis, K. Drakatos, M. Maltezou

1st. General Hospital of Social Security Services. (Athens, GR)

Objectives: To test the cognitive functions in patients with Multiple Sclerosis (MS) and correlate the results with the total number of brain lesions and their localization.

Methods: We administered the Brief Repeatable Battery of Neurological Tests (BRB-N) on 65 patients treated for MS, in order to evaluate the level of cognitive functioning and compared our findings with data from recent brain MRI of these patients. Due to the relatively small sample size, we looked only for the total number of lesions and their localization (cortical, subcortical, paraventricular or infratentorial).

Results: $75.6 \%$ of patients had more than 9 brain lesions. The location of the majority of these lesions was subcortical $(87.5 \%)$ and paraventricular $(80.5 \%)$. From the BRB-N, only performance on the Symbol Digital Modalities Test (SDMT) showed a highly significant negative correlation with total lesion number $(p=0.004)$; it also showed a significant correlation with paraventricular location $(\mathrm{p}=0.2)$. The Paced Auditory Serial Addition Test (PASAT) showed a highly significant negative correlation with subcortical location of the lesions $(\mathrm{p}=0.008)$.

Conclusion: Impaired sustained attention and concentration (measured by the SDMT) is related to the total number and to paraventricular localization of brain lesions. Impaired sustained attention and information processing speed (measured by PASAT) is related to the presence of subcortical brain lesions. These cognitive functions are more related to brain lesion number and location in patients with MS, than the other tests included on the BRB-N.

\section{P455}

Quality of life in patients with relapsing multiple sclerosis taking intramuscular interferon beta-1a (Avonex ${ }^{\circledR}$ )

R. Hyde, R.A. Rudick, P. Foulds, X. You

Biogen Idec International (Zug, CH); Mellen Center for Multiple Sclerosis Treatment and Research (Cleveland, US); Biogen Idec, Inc. (Cambridge, US)

Objective: In the pivotal phase 3 clinical trial (Multiple Sclerosis Collaborative Research Group [MSCRG] study) of intramuscular interferon beta-1a (IM IFN beta-1a, Avonex) $30 \mathrm{mcg}$ once weekly, one of the pre-planned objectives was to evaluate the effects of IM IFN beta-1a on patient health-related quality of life (QoL).

Methods: QoL was measured in 275 patients using the patient self-reported Sickness Impact Profile (SIP), administered at baseline and every 6 months. It was hypothesized a priori that patients with poorer baseline QoL would show improvement with IM IFN beta-1a treatment. Hence, patients were stratified into 2 groups according to baseline SIP score (intact QoL [SIP score $<10$ ] or poorer QoL [SIP score $\geq 10]$ ]).

Results: A total of 158 patients (IM IFN beta- $1 \mathrm{a}, \mathrm{n}=81$; placebo, $\mathrm{n}=77$ ) completed the SIP at both baseline and 2 years. Baseline disease characteristics and SIP scores were similar between treatment arms among patients with SIP scores $<10$ (IM IFN beta- $1 \mathrm{a}, \mathrm{n}=51$; placebo, $\mathrm{n}=43$ ) and $\geq 10$ (IM IFN beta-1a, $n=18$; placebo, $n=34$ ). Overall, SIP scores were positively associated with Expanded Disability Status Scale (EDSS) scores; patients with disability progression (defined as an increase in EDSS score of $\geq 1$ point sustained for 6 months) demonstrated a significant worsening in the Physical Dimension of the SIP compared with patients who did not experience disability progression ( 0.06 vs $4.19 ; \mathrm{P}=0.031)$. Similar trends were seen for the overall SIP scores and Psychosocial Dimension scores in relation to progression. In IM IFN beta-1a-treated patients with poorer baseline QoL (SIP $\geq 10$ ), a statistically significant improvement in the Physical Dimension score was seen at 2 years compared with placebo-treated patients (3.57 vs $-3.78 ; \mathrm{P}=0.045$ ), and trends towards significance were also seen for the overall SIP score and the Psychosocial Dimension score.

Conclusion: This analysis provides further evidence that disease severity as measured by the EDSS is associated with poorer QoL and that the physical component of the SIP is more sensitive than the psychosocial component. This analysis also provides supportive evidence for IM IFN beta-1a's effect on physical disability with positive impact on QoL as measured by the SIP in patients with poorer baseline QoL scores.

Study supported by Biogen Idec, Inc.

\section{P456}

Re-expression of the adhesion molecule $\mathrm{N}$-Cadherin in the lesions of experimental autoimmune encephalomyelitis implicates a role in remyelination S. Hochmeister, M. Romauch, T. Seifert, J. Bauer, R. Weissert, C. Linington, H-P. Hartung, F. Fazekas, M.K. Storch

Medical University Graz (Graz,AT); Medical University Vienna (Vienna, AT); University of Tubingen (Tubingen, DE); University of Aberdeen (Aberdeen, UK); University of Dusseldorf (Dusseldorf, DE)

Background and aims: N-Cadherin is known- among other members of the group of the calcium-dependant adhesion molecules-to be involved in regulatory processes of morphogenesis and axon guidance during the development of the nervous system. Several recent reports indicate that $\mathrm{N}$-Cadherin plays a role in certain pathologic conditions in the adult brain as well, such as in tumorgenesis, epilepsy and Alzheimer's disease. The function of $\mathrm{N}$ Cadherin in inflammatory demyelinating disease has so far not been investigated.

In vitro studies suggest a role of $\mathrm{N}$-Cadherin in myelination, while other reports found evidence of $\mathrm{N}$ - Cadherin involvement in the formation of the glial scar, which possibly impedes remyelination.

In our study we investigated the expression pattern of N-Cadherin immunoreactivity (N-Cadherin-IR) in experimental autoimmune encephalomyelitis induced by myelin-oligodendrocyte glycoprotein (MOGEAE), an animal model mimicking multiple sclerosis. The major advantage of this model is to allow thorough examination of different stages of demyelination and remyelination during the course of the disease.

Methods: MOG-EAE was actively induced in DA rats. Immunopathological evaluation was performed with light and confocal microscopy on paraffin-embedded CNS sections sampled at days 20 to 120 post immunization.

Results: In unaffected brain areas IR for N-Cadherin was seen on certain neurons in the spinal cord, brainstem, cerebellar nuclei and hippocampus as well as on fibers of the lamina II and III of the dorsal horn and on ependymal cells and the choroid plexus. In actively demyelinating and inactive lesions N-Cadherin-IR was present on astrocytes and endothelium. Double labeling for confocal microscopy confirmed N-Cadherin-IR on mature oligodendrocytes in late active and inactive plaque areas. There was no IR for N-cadherin in fully remyelinating shadow plaques.

Conclusions: Our study shows that the developmentally downregulated adhesion and signaling molecule $\mathrm{N}$-Cadherin is re-expressed in the lesion of autoimmune encephalomyelitis. This result substantiates the hypothesis that $\mathrm{N}$-Cadherin might act as a important mediator in initiating remyelination.

\section{P457}

A retrospective evaluation of an open-label mitoxantrone treatment programme on active multiple sclerosis

S. Hum, Y. Lapierre

Montreal Neurological Institute (Montreal, CA)

Objectives: To evaluate the safety and efficacy of the mitoxantrone program developed at a multidisciplinary research MS Clinic in patients with active or rapidly progressing Multiple Sclerosis (MS). Patients enrolled in this program were not a candidate for, or had failed to respond to traditional immunomodulatory treatment. Safety profile with respect to cardiac function, liver enzyme profile, and hematology were examined. Efficacy was assessed clinically by EDSS. 
Methods: To date, 107 patients $(\mathrm{RR}=32, \mathrm{SP}=55, \mathrm{PP}=11, \mathrm{PR}=9)$ have entered the open label treatment protocol. A dose of $12 \mathrm{mg} / \mathrm{m}^{2}$ was given at each cycle. Complete treatment consists of monthly infusions for the first 3 cycles followed by additional treatments every 3 months for a maximal dose of $108 \mathrm{mg} / \mathrm{m}^{2}$.

Multiple gated acquisition (muga) scan [Radionuclide angiocardiography] was used to evaluate cardiac function by measuring Left Ventricular Ejection Fraction (LVEF). Abnormal LVEF was defined by either a drop to below $50 \%$ or an absolute drop of $10 \%$ from baseline. Complete blood count (CBC) and liver function test were required before each cycle and needed to be within normal ranges (2 Standard Deviation of the mean).

Data regarding dose regimen, cumulative dose, LVEF, CBC, and liver function from patient medical records were reviewed. LVEF were typically performed at dose $0,48,72$ and $108 \mathrm{mg} / \mathrm{m}^{2}$.

Patient demographics, disease characteristics and EDSS were extracted from the clinic's longitudinal database.

Results: Preliminary results of 45 patients indicate an abnormal LVEF in 15 individuals of which 8 had LVEF $<50 \%$ and 7 had LVEF decrease $>10 \%$. On follow up scans, all but one of these patients (f/u muga pending) demonstrated recovered LVEF above $50 \%$ but remained below baseline in all cases. Final analysis to be completed by March 2008.

Conclusions: Conclusions will be presented.

\section{P458}

Presence of antinuclear antibodies and antiphospholipid antibodies in patients with multiple sclerosis. An epiphenomenon or do they really affect the progression of the disease?

S.P. Gonzalez, J.M. Flores, T. Corona

National Instituteof Neurology (Mexico City, MX)

Multiple sclerosis (MS) is the most commonly demyelinating disease among adults, it is believed that has an autoimmune etiology. Diagnostic criteria are based on the dissemination of the clinical signs in time and space, evoked potentials, MRI, examination of cerebrospinal fluid and exclusion of other diagnoses that can cause same symptomatology. In spite of the difficulty to distinguish Multiple Sclerosis of other inflammatory diseases that predominantly affect the CNS, for example systemic lupus erythematosus (SLE) and Antiphospholipid Syndrome, we have to note that exists reports that have showed also in MS the presence of antinuclear antibodies (ANA) and antiphospholipid antibodies (AP) that are most commonly described in other autoimmune diseases.

Objetive: We want to relate the clinical characteristics of patients with Multiple sclerosis that have ANA and AP elevated and if they influence in the progression of the disease.

Methods: Patients from the demyelinating diseases clinic in the National Institute of Neurology and Neurosurgery of Mexico, with the diagnosed as MS between 1996 and 2007 were included. MS is classified in four types, relapsing-remitting (RR), primary progressive (PP), secondary progressive (SP) and relapsing-recurrent (RR2). Assesing age, gender, age at diagnosis, evolution, first clinical event and clinical curse. To asses the progression of disease we determined the Expanded Disability Status Scale (EDSS) of Kurtzke, relapse rate, comorbidity and treatment received. Laboratory: Oligoclonal Bands (OCB) in Cerebroespinal Fluid (CSF), IgG, IgM, IgA, IgE, complement C3, C4, VDRL, lupic anticoagulant mesured by kaolin clotting. ANA mesured by inmunofluoresence in mice liver and was considered as positive titers above 1:50.

Were excluded the patients who could have high titers of ANA or AP asociated to drugs. Another autoantibodies were assesed, like Anti-Ro-SSA, Anti -La-SSB, anti-ENA, anti SM. Anticardiolipin (ACL).

Results: $75 \%$ of patients with MS, which have determination of autoantibodies, presented at least one positive autoantibody. There was a tendency of IgM autoantibodies to be positive in defined MS relapsing-remitting clinical type.

We are still incluiding patients to determine if in a bigger sample are some significancy in the results.

P459

Matrix metalloproteinase-9 and matrix metalloproteinase-2 in multiple sclerosis

Y. Benesová, M. Beránek, H. Novotná, P. Stourac, M. Hladíková, Z. Kadanka, A. Vasku, J. Bednarík

Masaryk University (Brno, CZ); University Hospital Brno (Brno, CZ)

Objectives: Matrix metalloproteinases (MMPs) are notable contributors to neuroinflammation and blood-brain barrier disruption in multiple sclerosis (MS). The aim of this study was to determine the serum levels of matrix metalloproteinase-9 (MMP-9), matrix metalloproteinase-2 (MMP-2), and their tissue inhibitors (TIMP-1) and (TIMP-2), to investigate possible relation to type and severity of MS.

Materials and methods: The study group consisted of 87 patients, with the median age 39 (range 17-61) years, fulfilling Mc Donald's criteria of MS The control group consisted of 50 healthy subjects, the median age 38 (range 19-62) years. The patients' disability was evaluated with the Expanded Disability Status Scale (EDSS). According to the type of the disease the patients were divided into relapsing-remitting (RRMS), secondary-progressive (SPMS), and primary progressive (PPMS) groups. Serum levels were analysed with the enzyme-linked immunoassay (ELISA) method.

Results: A significant elevation in the MMP-9 serum levels was found in the MS group when compared with the controls $(\mathrm{p}<0.001)$. No difference in the MMP-2, the TIMP-1 and the TIMP-2 serum levels between the whole MS group and the control group was found.

When stratified according to the type of the disease, a significant elevation emerged in the MMP-9 serum levels in the RRMS $(\mathrm{p}<0.001)$ and SPMS $(p<0.001)$ groups when compared with the control group. No difference in the MMP-9 serum levels among the MS groups was found.

Similarly, a significant difference in the MMP-2 serum levels $(\mathrm{p}<0.001)$ among the MS groups was found. A significant elevation occurred in the PPMS $(\mathrm{p}<0.01)$ and the SPMS $(\mathrm{p}<0.05)$ groups when compared with the RRMS group. The increase in the MMP-2 serum levels was also associated with the severity of the disease $(p=0.0001)$. Finally, a significant decrease in the TIMP-2 serum levels in the PPMS group was demonstrated when compared with the RRMS $(\mathrm{p}<0.05)$ group.

In conclusion, we confirmed that the MMP-9, the MMP- 2 and their inhibitors protein serum levels are useful biological markers in MS providing information about the clinical manifestation and severity of the disease.Their impact on the understanding of pathophysiology of MS and on practical management of MS patients, however, remain to be established.

The study was supported by grant NR/8832-4 from the Ministry of Health of the Czech Republic (IGA MZ ER)

\section{Motor neuron disease}

\section{P460}

Accuracy of International Classification of Diseases 9 Clinical Modification code 335.20 in identifying amyotrophic lateral sclerosis patients discharged from university hospital, Undine, Italy

F.E. Pisa, L. Verriello, L.Deroma, D. Drigo, M. Panozzo, P. Bergonzi, G.L. Gigli, F. Barbone

University Hospital of Udine (Udine, IT)

Objectives: Amyotrophic Lateral Sclerosis (ALS) incidence in Europe and North America range from 0.6 to 2.4 per 100.000 person-years (1). Reliable and less variable rates result from prospective population-based registers, based on active surveillance, uniform diagnostic criteria, case ascertainment from multiple sources, including hospital discharge data.

We estimated sensitivity (SE), specificity (SP), positive (PPV) and negative predictive value (NPV), false positive (FPR) and negative ratio (FNR) of 9th International Classification of Diseases Clinical Modification (ICD-9 CM) code of ALS 335.20 in discharge data of University Hospital of Udine, in whose area (529811 persons) 3 municipalities have Standardized Mortality Ratios significantly $>100$ (2).

Methods: We selected data of all inpatients discharged from 01/01/05 to $31 / 12 / 06$ with principal or secondary ICD-9 CM code of ALS, other anterior horn cell disease 335, spinal cord disease 336, peripheral neuropathy 356 , neuropathy 357 , myoneural disorders 358 , muscular dystrophies and myopathies 359. One ALS neurologist and trained medical staff abstracted from clinical records information on upper and lower motor neuron signs and symptoms in bulbar, cervical, thoracic, lumbosacral regions. Revised El Escorial criteria were the gold standard.

Results: Records were reviewed in 511 (97\%) out of 527 discharges: 42 out of 48 with 335.20 met criteria for ALS, 10 suspect and 32 possible, probable or definite. Out of 463 with other codes 3 met criteria for ALS possible, probable or definite. Sensibility was 0.93 (95\% Confidence Interval -95\%CI0.91-0.95), SP 0.99 (95\%CI 0.98-1.00), PPV 0.87 (95\%CI 0.84-0.90), NPV 0.99 (95\%CI $0.98-1.00)$, FPR 0.0065 (95\%CI -0,0005-0,0135), FNR 0.067 (95\%CI 0,0453-0,0887). Excluding suspect ALS, SE was $0.71(95 \% \mathrm{CI}$ 0,67-0,75) and PPV 0,67 (95\%CI 0,63-0,71).

Conclusion: Although El Escorial criteria as gold standard in clinical records review are too restrictive, ICD-9 CM 335.20 shows high SE and SP, similar to studies comparing with registry data, and is useful to identify ALS cases in discharge data of the University Hospital of Udine. 


\section{References}

1. Cronin S, Hardiman O, Traynor B (2007) Neurology 68:1002-1007

2. Uccelli R, Binazzi A, Altavista P, et al. (2007) Eur J Epidemiol 22:781-791

P461

Insulin-like growth factor system in skeletal muscle in amyotrophic lateral sclerosis

C. Lunetta, M. Serafini, A. Prelle, P. Magni, E. Dozio, M. Ruscica, M. Moggio, M. Corbo, V. Silani

IRCCS Istituto Auxologico Italiano, University of Milan (Milan, IT); Foundation IRCCS Ospedale Maggiore Policlinico (Milan, IT); University of Milan (Milan, IT)

Objectives: To investigated the expression of the Insulin-like growth factor1 (IGF-1), IGF-2, binding proteins IGF-BP3, -BP4, -BP5 and IGF-1-receptor (IGF1-R) in skeletal muscle of sporadic ALS (sALS) patients.

Patients and methods: Full clinical, neurophysiological and nutritional assessment of 20 consecutive sALS patients was completed. Muscle biopsies were performed and the samples were compared with specimens with normal hystological exam (no. 15 healthy controls, HC) or non ALS-related neurogenic alteration (no. 12 neurogenic controls, NC). IGF-1, IGF-2, IGF-BP3, BP4, -BP5 and IGF-1 receptors were investigated by immunohistochemistry and Western immunoblotting.

Results: Immunoreactivity for IGF-1 and IGF-BP4 were low and did not show difference with sALS and controls muscles (HC and NC). In HC type II muscle fibers showed decreased immunoreactivity for IGF-2, IGF-BP3 and IGF-BP5 compared with type I muscle fibers. In sALS patients we observed the loss of this specific distribution related to the type grouping. IGF-BP3 and IGF-BP5 in skeletal muscle homogenates were lower in sALS patients compared with HC but not different compared with NC. Skeletal muscle in patients and controls showed immunoreactivity for IGF1-R at level of the sarcolemma. Immunoblotting of homogenates from skeletal muscle showed a band of $95 \mathrm{kD}$ corresponding to IGF1-R with higher expression in sALS patients compared with controls (HC and NC).

Conclusions: ALS is a fatal neuromuscular disorder involving the degeneration of motor neurons accompanied by skeletal muscle atrophy and paralysis. Adult muscle fibers are a source of signals that influence neuron survival, axonal growth, and maintenance of synaptic connections. Among these, IGF-1 has been implicated in anabolism of muscle and nerve tissues, inducing muscle hypertrophy and promoting neuronal survival. To date, very little information is available on the expression of the IGF-I system in the ALS skeletal muscle. Since IGF-1 is a trophic factor to different tissues, including skeletal muscle and nervous system, we can speculate that the up regulation of the IGF-1-R in sALS may reflect a physiological defensive mechanism in response to neural degeneration and muscle atrophy. The activation of IGF-1 system during the course of ALS enforced the therapeutic role of this growth factor.

\section{P462}

Generating a network of intercellular communication in amyotrophic lateral sclerosis by text mining of MEDLINE abstracts

D. Klockenbrink, A. Skusa, G.M. Agena, K. Prank

Medizinische Hochschule Hannover (Hannover, DE); CeBiTec, Bielefeld University (Bielefeld, DE)

Objectives: Four important cell types that are affected in neurodegenerative diseases such as Amyothrophic Lateral Sclerosis (ALS) are motoneurons, astrocytes, microglia, and Schwann cells. Various cell-cell signals occur between the different cell types surrounding motoneurons, such as between astroglia and microglia cells when motoneurons are degenerating. Thus the analysis of all relevant MEDLINE abstracts in the context of the Wobbler mouse model for ALS should lead to new experimentally testable hypotheses from which new insights into ALS should be gained.

Methods: We used a text mining approach on approximately 15 million MEDLINE abstracts to extract triple co-occurrences of:

1) Cell type, intercellular messenger, type of release

2) Receptor, cell type, "containment"-expression

3) Intercellular messenger, receptor and "binding"-expression

The ONtological InNDEXing framework (ONDEX, Koehler et al., 2005) was used in this approach. From the results testable hypotheses of intercellular communication between the four important cell types in the ALS model were generated.

Results: Although the examination of the 347 generated hypotheses was more complicated than expected, the retrieval of approximately $30 \%$ known cell-cell relations in the Wobbler mouse model for ALS is a reasonable result and comparable to other text mining work (Winnenburg et al., 2006).

Conclusion: However, evaluating the resulting hypotheses for the Wob- bler mouse model for ALS remains a problem. In the simplest case, known facts are being reproduced and recognized by biomedical experts. Unfortunately, the remaining network of cell-cell interactions can in most cases not be rated easily since the respective assumption might be true, but has not been experimentally investigated yet. This is the case for the majority of the extracted cell relations. But our approach has big opportunities, since researchers can use the hypotheses, identify the known ones and use the new hypotheses as basis for experiments, to detect these new interactions or to vitiate them.

\section{P463}

Progranulin genetic variability is not associated to sporadic amyotrophic lateral sclerosis in Italian patients

D. Santoro, S. Ghezzi, S. Corti, M.E. Fruguglietti, A. Prelle, M. Mancuso, G. Siciliano, C. Briani, N. Bresolin, G.P. Comi, R. Del Bo

University of Milan (Milan, IT); University of Pisa (Pisa, IT); University of Padua (Padua, IT)

Objectives: To report a systematic mutation screening and association analysis of progranulin (PGRN) in 237 Italian patients with sporadic amyotrophic lateral sclerosis (ALS) and age-matched healthy control subjects, aiming to examine the contribution of genetic variability in PGRN to ALS pathogenesis. Recently, PGRN genetic variability has been described to contribute to ALS in Belgian and Dutch populations.

Methods: All coding exons, exon-intron boundaries and 5' and 3' regulatory regions of PGRN were screened in 60 ALS patients. Subsequently, two case-control studies were performed in 237 unrelated subjects affected by sporadic ALS (64\% males; mean age at onset: $58.1 \pm 12.9$ years) recruited for this retrospective and ongoing study from January 2001 to September 2007. All patients fulfilled the El Escorial criteria for probable or definite ALS. The control group consisted of 250 healthy volunteers matched for ethnic background and age to cases.

Results: No pathogenic mutation was observed in 60 italian ALS patients however, we identified 4 rare coding sequence variants ( 1 missense and 3 silent mutations) that likely represent non-disease-related polymorphisms and 4 more frequent non-pathogenic intronic/3'UTR sequence variants. Two case-control studies, involving IV2+21G >A (rs9897526) and 3'UTR78C $>$ T (rs5848) variants, were performed. Genotype analysis for both variants failed to find different distributions in all ALS patients compared to healthy subjects; the analysis of the age at disease onset according to the different genotypes showed no statistically significant results.

Conclusions: Progranulin mutations are not common cause of ALS; overall, our data argue against the hypothesis of PGRN as a genetic risk factor for motor neuron dysfunction, at least in Italian population.

\section{P464}

Longitudinal study on quality of life in amyotrophic lateral sclerosis patients enrolled and not enrolled in clinical trials

E. Cavallo, P. Ghiglione, A. Guzzo, A. Chiò, A. Calvo, A. Vignola

A.S.O. S. Giovanni Battista - Molinette (Turin, IT)

Background: There are many studies on the quality of life (QOL) in ALS patients, but the relationship between the participation in a clinical trial and the psychological variables is not known.

Objective: To assess the correlation between the involvement in a pharmacological trial and QOL in a series of patients.

Methods: The patients were seen in our ALS center from January 1, 2007 to December 30,2007. QOL, depression, and clinic status were evaluated in 23 patients enrolled in three different randomized double blind placebocontrolled trials and in 23 patients not included in clinical trials (controls). At the first visit and after six months were administered McGill quality of life Scale (MQOL), Zung Depression Scale (ZDS)and ALS Functional Rating Scale Revised (ALS-FRS-R score). Data analysis was performed with SPSS 12.0

Results: The mean age was 55.9 (SD 7.5)years in the study group and 61.2 (SD 12.7)years in the controls, the mean disease duration was respectively 13.5 months(SD 8.1) and 25.3 (SD 15.3). The mean ZDS score was 38.0 (SD 8.2) at the first visit and 39.5 (SD 10.5) after six months in the patients included in the clinical trials ( $\mathrm{p}=\mathrm{ns}$ ) and 42.1 (SD 8.8) and 43.7 (SD 10.0)in the controls $(\mathrm{p}=\mathrm{ns})$. The mean MQOL score was 7.6 (SD 1.0) at the first visit and decries at 6.9 (SD 1.6) after six months in the patients included in the clinical trials and the difference was statistically significant $(p<0,02)$. In the control was 6.7 (SD 1.3) at the first visit and 6,8 (SD 1.0) after six months ( $p=n s$ ). In the study group mean ALS-FRS-R score was 31.2 (SD 7.9) at the first visit and 27.6 (SD 8.9) after six months $(\mathrm{p}=\mathrm{ns})$, in the controls mean mean ALSFRS-R score was 30.8 (SD 8.5) at the first visit and 25.8 (SD 10.0) after six months. 
Conclusions: Our findings indicate that QOL of the patient included in a clinical trial is similar to the controls and decreased after six months more that of the controls. The patient participating in a clinical trial are likely to have excessive expectations of recovery and the progressive worsening of their condition have a greater psychological impact than that observed in patients not enrolled in clinical trials. Depression did not significantly modify in the same time-period.

P465

In vivo longitudinal assessment of grey matter loss in sporadic amyotrophic lateral sclerosis: a tensor-based morphometry study

F. Agosta, M.L. Gorno-Tempini, E. Pagani, S. Sala, D. Caputo, M. Perini, A. Prelle, M.E. Fruguglietti, F. Salvi, I. Bartolomei, M. Filippi

Scientific Institute and University Ospedale San Raffaele (Milan, IT); UCSF (San Francisco, US); Fondazione Don Gnocchi (Milan, IT); Ospedale di Gallarate (Gallarate, IT); Fondazione Policlinico Mangiagalli (Milan, IT); University of Bologna at Bellaria Hospital (Bologna, IT)

Objectives: To assess in vivo longitudinal changes in gray matter (GM) atrophy in patients with sporadic amyotrophic lateral sclerosis (ALS) compared to controls, and to investigate whether GM contraction relates to disease progression rate. Methods: T1-weigthed MRI scans were obtained at baseline and after a median follow up of 9 months from 16 ALS patients (mean age $=$ 56 years, median disease duration $=25$ months) and 10 controls. At each time-point, the ALS functional rating scale (ALSFRS) was used to assess disease severity. Disease progression rate during follow up was calculated as follows: (baseline ALSFRS-follow up ALSFRS)/follow up duration. Patients were classified as showing rapid progression if their progression rate was more than the median value. Tensor based morphometry (TBM) in SPM2 was applied to derive a voxel-wise estimation of GM volume change over time from the deformation field required to warp the follow up scan to the baseline one in each subject. A region of interest analysis was performed based on pathological studies, including frontotemporal cortex and subcortical regions (caudate and putamen) ( $\mathrm{p}<0.005$, uncorrected).

Results: The median ALSFRS were 27 (range $=7-33$ ) at baseline and 25 (range $=6-33$ ) at follow up. The median disease progression rate during follow up was 0.37 units/month (range $=0-2.98$ units/month). Eight patients showed rapid progression. The left premotor cortex and caudate, and the right putamen showed significant volume change in all patients versus controls. ALS patients with rapid progression showed additional progression of atrophy in the left motor and right premotor cortices and postcentral gyrus. An exploratory whole-brain analysis demonstrated that no other area showed significant volume changes overtime.

Conclusions: Motor and premotor cortices showed a significant progression of atrophy over a 9-months period in ALS patients. Faster was the disease progression during follow up greater was the GM loss in these areas. Progression of atrophy in ALS also involved basal ganglia. Tracking longitudinal changes in motor cortical and subcortical regions with TBM may provide an objective marker for monitoring ALS progression and the effects of potentially disease-modifying treatments in clinical trials.

P466

Features of pain in amyotrophic lateral sclerosis patients

I.L. Simone, R. Tortelli, V. Samarelli, M. Sardaro, C. Serpino, E. D'Errico, $R$. Messina, M. De Tommaso

University of Bari (Bari, IT)

Objectives: Pain is a common invalidating symptom in amyotrophic lateral sclerosis (ALS), though its patho-physiological basis are still unclear.The aim of this study is to evaluate clinical and neuro-physiological features of pain in ALS patients.

Methods: Sixty three patients with definite or probable sporadic ALS were examined. Mean age was 63.46 (SD 11.18) years and mean disease duration 48.5 (SD 15.9) months. Fifty five patients had spinal onset. Pain was assessed by Brief Pain Inventory, ranging from 0 (no pain/no interference) to 10 (pain as bad as could be/complete interference). Neurological status(ALSFRS and MMT) and depression (Beck Depression Inventory -BDI) were evaluated. A subgroup of 20 patients were evaluated by Laser evoked Potentials (LEPs) and compared with 40 age and sex matched healthy subjects. LEPs were recorded from five scalp electrodes, positioned on $\mathrm{Fz}, \mathrm{Cz}, \mathrm{Pz}$ and T3, T4 referred to Fz. The CO2 laser stimulus was settled at 7.5 Watts and 25 ms duration in both hands.

Results: Pain was reported by 49 patients $(77.8 \%)$. Mean pain severity was 3.52 (SD 2.5). The most frequent type of pain was cramp (67\%), followed by musculoskeletal pain (53\%); neuropathic pain was uncommon $(14 \%)$. The most frequent pain locations was legs (71.43\%) and arms (40.82\%); 29 patients $(59.18 \%)$ had two or more sites of pain. Ten patients used analgesic drugs (NSAIDs) with a great relief; other drugs did not produce effects. Pain interference in daily living was more frequent for Mobility and Mood items (44.9\% of patients), assuming as pathological score a value $>5$. Pain did not correlate with disease duration, ALSFRS, MMT, BDI, muscle atrophy, spasticity and immobility. The amplitude means of N2-P2 and N1 LEP components were significantly increased in ALS patients compared with controls. A significant N1, N2 and P2 latency prolongation was also found. Eight patients with features of neuropathic pain at the Brief Pain Inventory showed a significant LEPs amplitude increase in comparison with other ALS patients.

Conclusion: Our study indicates that pain is a frequent symptom interfering with daily living in ALS. A possible sensory compensation for motor cortex dysfunction may cause an increase of pain processing in ALS, though early sensory cortical degeneration may subtend the LEPS latency prolongation. Pain in ALS may be a symptom correlated to functional and degenerative changes connected to ALS disease.

\section{P467}

Proteome analysis of cerebrospinal fluid in amyotrophic lateral sclerosis

J. Brettschneider, V. Lehmensiek, H. Mogel, A. Ludolph, H. Tumani University of Ulm (Ulm, DE)

Objectives: Amyotrophic lateral sclerosis (ALS) is the most common form of motor neuron disease characterized by progressive degeneration of spinal and bulbar innervating motor neurons as well as the pyramidal motor neurons. Cerebrospinal fluid (CSF) is a promising source of biomarkers in ALS to advance the understanding of disease pathology as well as to support clinical diagnosis and identification of different subtypes of disease.

Methods: We used the two-dimensional difference in gel electrophoresis (2-D-DIGE) technology to compare CSF samples from patients with ALS $(\mathrm{n}=14)$ with those from neurologically normal controls (CTRL, $\mathrm{n}=\mathrm{v} 14)$. Protein spots that showed a significant difference between patients and controls over three independent 2-D-DIGE gels were selected for further analysis by MALDI-TOF mass spectrometry. For validation of identified spots a western blot analysis was performed.

Results: Analysis of only those spots with a significant difference over all three gels between ALS and controls revealed 6 spots, corresponding to 5 different proteins: 2 proteins were upregulated in ALS as compared to CTRL (alpha-1-antitrypsin precursor, Zn-alpha-2-glycoprotein (ZAG)) and 3 proteins were down-regulated in CSF in ALS (ceruloplasmin precursor (CPP), transferrin, beta-2-microglobulin). Western blot analysis confirmed our finding of elevated CSF ZAG in ALS in 3 of four single patients.

Conclusions: ZAG and CPP have not been implemented in ALS pathology so far. In contrast, several other proteins (transferrin, alpha-1-antitrypsin precursor and beta-2-microglobulin) seem to be unspecifically affected in different neurological diseases and may therefore be of limited value as disease-related biochemical markers in ALS. Though the pathophysiological role of the above mentioned proteins still remains to be further elucidated and additional validation will be needed, our findings may have a relevant impact on the identification of disease-specific markers.

\section{P468}

A German case of Hirayama disease

J.-S. Kang, S. Gawehn, E. Hattingen, H. Steinmetz, F. Trostdorf, R. Hilker, U. Ziemann

Uniklinik für Neurologie (Frankfurt/Main, DE); Uniklinik für Psychiatrie (Frankfurt/Main, DE); Institut für Neuroradiologie (Frankfurt/Main, DE)

Hirayama disease is an initially progressive and then stationary usually monomelic distal upper limb muscular atrophy which occurs predominantly in young men of East Asian origin. The disease is regarded as a variant of motor neuron disease / segmental distal spinal muscle atrophy but its pathogenetic origin remains largely unclear.

We report here a 25-year-old right-handed Caucasian patient of German ancestry with no relevant past medical or familial history who presented with an initially progressive painless atrophic weakness of his left hand with myoclonic jerks and exaggerated muscle tendon reflexes of the left upper limb for 8 years with a stable state for the last 4 years.

T2-weighted cervical magnetic resonance imaging (MRI) showed an atrophy of the caudal cervical spine with a myelopathy signal without any associated compressive cervical vertebral abnormalities. Repeated electromyography (EMG) over the course of nine months showed stable chronic neurogenic changes in left M. abductor pollicis brevis and M. abductor digiti minimi but not in other muscles. Motor neuron disease was not supported by EMG according to the El Escorial protocol. Multifocal motor neuropathy, anterior interosseous or ulnar neuropathy, and brachial plexopathy were excluded by nerve conduction studies. 
Cranial MRI and extended laboratory tests (complete blood count, renal function tests, blood glucose, creatine kinase, immunfixation, electrophoresis, thyroid stimulating hormone, vitamin B6, B12, cerebrospinal fluid, rheumatoid factor, anti-nuclear and anti-neutrophil cytoplasmic antibodies, serum Lyme and lues titre, hepatitis C, human immunodeficiency virus and other neurotrophic viruses, gangliosid GM1-antibodies, and survival motor neuron (SMN) exon 7 and 8 deletion tests) remained all negative.

Given the benign course and the results of this extensive diagnostic work up, which largely excluded relevant differential diagnoses including other forms of motor neuron disease or spinal muscle atrophy we conclude that this is a rare German case of Hirayama disease.

P469

Diagnostic problems and delay of diagnosis in amyotrophic lateral sclerosis M. Buerger, M. Kraemer, P. Berlit

Alfried Krupp Hospital (Essen, DE)

Objectives: Initial symptoms of amyotrophic lateral sclerosis (ALS) mimic spinal cord diseases, mononeuropathies and several neurological syndromes that may decelerate a correct diagnosis. Aim of our study was to investigate if diagnostic and therapeutic parameters have influence on the time of diagnosis.

Methods: We retrospectively reviewed the medical records of 100 ALS patients focusing on clinical and diagnostic data, the timing of diagnosis and treatments attributed to the onset symptoms of ALS. If patients underwent therapy prior to diagnosis of ALS, we explored whether there was an improvement of symptoms due to this treatment.

We compared 3 groups of patients: 1 . patients with bulbar symptoms at the beginning of the disease, 2. those who had surgery or another therapy prior to diagnosis and 3. patients who did not present these criteria. Statistical analysis was done with the Mann-Whitney-Test.

Results: Among 100 patients with ALS, twelve patients (12\%) underwent surgery due to symptoms retrospectively attributable to ALS.

The comparison of duration from first symptoms to correct diagnosis showed a significant difference $(\mathrm{p}=0.01)$ between operated ( 18.44 months) and non operated patients (13.15 months). 34 of all ALS patients ( $34 \%$ ) had bulbar onset symptoms. The mean time from first symptoms to diagnosis was 10.56 months in this group. In patients without bulbar onset it was 16.41 months which also represents a significant difference $(p=0.004)$. In 43 patients $(43 \%)$ other diagnoses were considered and medically treated previous to correct diagnosis, but there was no significant delay of diagnosis $(\mathrm{p}=0.153)$.

Conclusion: Our study revealed that $12 \%$ of our cohort underwent inappropriate surgery prior to the diagnosis of ALS. This is comparable to the data of Srinivasan et al. in 2006 (13.08\%). Bulbar symptoms at onset lead to an abbreviation of time to diagnosis. While medically treated patients with false diagnoses presented no significant delay, patients with surgery showed a delay of correct diagnosis of approximately 6 months. Our study confirms that diagnosis of ALS is still a common clinical problem and shows the need for improvement of diagnostic criteria.

\section{P470}

Glutathione S-transferase pi in motor neuron disease - a possible role in neuroprotection

M. Kuzma-Kozakiewicz, A. Baranczyk-Kuzma, M. Hetman, H. Kwiecinski

Medical University of Warsaw (Warsaw, PL); University of Louisville (Louisville, US)

Glutathione-S-transferase pi (GST pi) is the most important enzyme involved in detoxification of electrophilic and oxidation-derived reactive compounds in the central nervous system. As we reported before, there is a significant decrease of GST pi expression in spinal cord and motor cortex of patients with motor neuron disease (MND). Preventing apoptosis, a process responsible for neuronal loss in neurodegeneration, is currently the main goal in the treatment-directed research. Extracellular signal-regulated kinase (ERK1/2) and phosphatidylinozytol 3-kinase (PI-3K) - the main cellular antiapoptotic pathways are triggered by either neurotrophic or neurotoxic factors. Despite extensive research, the compounds providing neuronal survival within the pathways have not yet been identified. Aim: investigation of GST pi involvement in the pathogenesis of MND and neuronal survival.

Material and methods: GST pi expression was studied in spinal cord of transgenic mice with MND-associated human SOD1 G93A mutation, at presymptomatic and symptomatic stage (age 70 and 140 days, respectively) and in rat brain cortical neurons cultured at the presence and/or absence of neurotrophic (KCl, forskolin) or neurotoxic (glutamate) factors, as well as ERK1/2 and PI-3K inhibitors. GST pi expression was studied using Western blotting and RT-PCR.
Results: Decreased GSTpi expression on mRNA level was found in both groups of transgenic mice. At preclinical stage it accounted for $0.76 \pm 0.08$ in transgenic and $0.92 \pm 0.14$ in wild type animals (control), and at clinical stage it was $0.72 \pm 0.09$ and $0.82 \pm 0.10$, respectively. In cultured neurons all studied factors induced GST pi protein expression in a time-dependant manner. The expression was increased by approximately $235 \%, 220 \%$ and $55 \%$ after a 24hour exposition to forskolin, $\mathrm{KCl}$ and glutamate, respectively. Our experiments also revealed that the $\mathrm{KCl}$, forskolin or glutamate-induced increase of GST pi expression was mediated by either ERK1/2 or PI-3K pathway.

Conclusion: GST pi may be a newly identified target of both ERK1/2 and PI-3K pathways. The decreased expression of GST pi in motor neuron disease may contribute to apoptosis by enhancing the complex process of neurodegeneration.

\section{P471}

Brown Violetto von Laere syndrome or Madras type motor neuron disease? O. Arslan, S. Bek, G. Genc, G. Koc, O. Oz, Z. Odabasi Gulhane Military Medical Academy (Ankara, TR)

Introduction: Brown Violetto Von Laere syndrome (BVVL) which includes sensorineural deafness in the early stage followed or accompanied by lower cranial nerve involvement and Madras Type of Motor Neuron Disease (MMND) which includes pyramidal signs such as pathologic reflexes overlap clinically and electrophysiologically.

Case report: A 14 year-old man who stated that he had had deafness for two years and used a hearing aid device because of sensorineural deafness was admitted to our hospital with dysarthria and unsteadiness of gait lasting for last two months, right facial palsy for one month. Neurological examination revealed hearing loss, tongue fasciculations, right peripheric facial palsy, bilateral foot drop, atrophy of hand intrinsic muscles, bilateral Babinski sign and weak gag reflex. Involvement of anterior horn cell, bilateral facial and lower cranial nerve were observed on electromyography.

Conclussion: BVVL is accepted as a part of family of motor neuron diseases. Sporadic and familial forms of BVVL should be considered as similar but different diseases because the onset age of the disease is later in sporadic forms according to familial forms. Upper motor neuron involvement is rarely reported in BVVL which is accepted as a family of motor neuron diseases. Only 5 cases with pathologic reflex like our case have been reported in the literature. If this clinical finding is present, Madras type of motor neuron disease should be considered. When it was considered it's a special variant to India, sporadic cases have been reported from Thailand and Italy. When we evaluated the literature datas and the characteristics of our case such as being male, presence of pathologic reflex together, we considered our case as MMND. It is a discussion topic if both diseases should be named different because they have different etiopathogenesis even if they are in the same family.

\section{P472}

Bulbar hereditary motor neuropathy type I

O. Arslan, S. Bek, G. Koc, O. Oz, G. Genc, Z. Odabasi

Gulhane Military Medical Academy (Ankara, TR)

Background: Bulbar Hereditary Motor Neuropathy Type I is a rare degenerative disorder characterized by progressive sensorineural deafness, accompanied or followed by a variety of cranial nerve palsies usually involving the motor components of the 7th and 9th-12th cranial nerves, and less commonly spinal motor nerves, and upper motor neurons. We report two brothers who primarily presented with disability of walking, dysphagia, dysarthria and imbalance.

Case presentation: A 20 year-old man who had presented with progressive walking difficulty, dysphagia, dysarthria and imbalance for a few years was admitted to our hospital. Neurologic examination revealed that atrophy of bilateral masseter and tongue muscles, bilateral facial paralysis, nasal speech, bilateral poor gag reflex, dysarthria, hearing loss, bilateral drop foot, decreased deep tendon reflexes (DTR). In his family medical history his older brother, two daughters of mother's uncle and two daughters of mother's maternal aunt have similar complaints. His older brother died at nine year-old and two of his sisters died at infancy because of these complaints. His older brother is 34 year-old. He had developed unsteady gait, hearing loss at eight, and dysphagia, dysarthria and weakness of eyelid at sixteen. He had diagnosis of hereditary polyneuropathy at another centre. Neurologic examination revealed that atrophy of bilateral masseter, tongue muscles, bilateral facial paralysis, nasal speech, poor gag reflex, dysarthria, hearing loss, absent of DTR on lower limbs, bilateral drop foot, atrophy of hand intrinsic muscles. Electrophysiological studies showed bilateral facial and lower cranial nerve involvement and widespread anterior horn cell in- 
volvement on patient and older brother. Both of them showed no pathologic sign in cerebral and spinal imaging. Genetic consultation indicated that type of inheritance might be autosomal recessive.

Conclusion: Choices of treatment is still absent. To date nearly 40 cases of BHMN syndrome have been reported in the literature. The etiopathogenesis is still unknown because of the inadequate datas.

\section{P473}

Benign amyotrophy of hands in non-Asiatics: a long-term follow-up

S. Blumen, M. Sadeh, B. El-Ad, Y. Balash, G. Groozman, N. Blumen, V. Drory Hillel Yaffe Medical Center (Hadera, IL); Wolfson Medical Center (Holon, IL); Maccabi Health Services (Ramat-Gan, IL); Sourasky Medical Center (Tel Aviv, IL); Sheba Medical Center (Tel Hashomer, IL)

Objective: To follow patients presenting with recent onset amyotrophy of upper limbs due to lower motor neuron involvement (LMN) in order to identify features, predicting a benign outcome.

Methods: At presentation all patients underwent neurological and electrodiagnostic evaluation, cervical MRI (in neutral position) and some patients had anti-GM1 titers and GARS gene mutations were looked for. We excluded patients with history of poliomyelitis or radiotherapy, features suggesting mononeuropathies, polyneuropathies, cervical radiculopathies or plexopathies as well as lower limb, bulbar, cerebellar, pyramidal, significant sensory or autonomic involvement. All patients were seen by one of the authors every six months.

Results: After a follow-up of three to eight years, in eleven patients the disease remained restricted to the LMN of one ( 4 cases) or both ( 7 cases) upper limbs. All were males, the disease started before the age of 25 and was sporadic. In 9 patients the distal distribution of muscle weakness and amyotrophy with sparing of brachioradialis suggested typical Hirayama's syndrome; in the other two there was subtle involvement of more proximal muscles. Significant postural and action tremor was found in 7 cases and 6 patients reported cold aggravation. Seven patients (followed for more than five years) felt the disease arrested. Cervical MRI excluded cord compression in neutral position and, when looked for, anti-GM1 antibodies or GARS gene mutations were not found. All but two patients were told by previous consultants that Amyotrophic Lateral Sclerosis is a diagnostic concern.

Conclusions: Hirayama disease and less typical forms of benign juvenile amyotrophy of the hands are not uncommon among non-Asian populations. They are often mistaken for more ominous motor neuron diseases. The importance of gender (male) and early age at onset in reaching the correct diagnosis cannot be overemphasized. Most patients have bilateral features, postural tremor or cold effect.

\section{P474}

Differential diagnosis between oropharynx carcinoma and amyotrophic lateral sclerosis

S. Cammarosano, P. Ghiglione, S. Giacone, A. Pessia, R. Mutani, A. Calvo, A. Chiò

ASO San Giovanni Battista (Turin, IT)

Introduction: We report the case of 74-years-old man, that turned up in October 2006 a severe dysphagia for both liquids and solids; afterwards dysphonia with paralysis of vocal cords. He was also found to be affected by a type II respiratory failure. He was a former-smoker, high-blood pressure sufferer, and positive for HCV antibodies.

During the admmission to hospital in February 07 he underwent chest TC scan (absence of parenchimal or interstitial lesions), EMG (non significant), head MRI (negative), neurological examination; a diagnosis of possible ALS was proposed. The patient showed normal strength of upper and lower limbs, ipercinetic osteotendineal reflexes, moderate spasticity of lower limbs, widespread muscle hypotrophy, altered but intelligible speech; CK level was $61 \mathrm{UI} / \mathrm{L}$. He underwent mechanic ventilation (NIV), with insufficient compliance and he was sent to our ALS Centre.

Since the disturbances didn't worse during the following two months, he underwent a neck MRI that evidenced a thickening of oropharynx posterior part with head-caudal extension of $4.5 \mathrm{~cm}$, lightly disomogeneous structure; there was a moderate enhancement; two lymph-nodes probably reactive laterocervical on the left, were also detected.

On April 2007 he was again admitted to hospital for acute dyspnea, was found with endoscopy stenosis in adduction of vocal cords, that required tracheotomy.

He underwent a biopsy of oropharynx posterior part, that showed the presence of a moderately differentiated squamose keratinized carcinoma, with a superficial invasion of the oropharynx.

Radiotherapy was suggested, while other options (surgery or chemiotherapy) were excluded due to the grading of the lesion.
The patient was treated with radiotherapy and his clinical status remained stationary

Conclusions: the case reported shows the difficulties in doing a correct early interpretation of symptoms, like widespread muscle hypotrophy and ipercinetic reflexes, common in old persons. The diagnosis of ALS remains a matter of exclusion, and in this patient it was initially made because of the clinical featureas and the absence of neuroimaging findings of other disorders that could justify the clinical picture.

\section{P475}

Transplantation of neural stem cells derived from murine embryonic stem cells ameliorates spinal muscular atrophy phenotype

S. Corti, M. Nizzardo, M. Nardini, C. Donadoni, F. Fortunato, F Saladino, N. Bresolin, G.P. Comi

University of Milan (Milan, IT)

Objective: To explore the potential of stem cells to treat Spinal Muscular Atrophy (SMA).

SMA is a devastating genetic motoneuron disease without effective treatment. A potential therapy for SMA could be stem cell transplantation both by neuron replacement and/or neuroprotective effect.

Methods: We generated a homogeneous neural stem cell population (NSCs) from a murine Embryonic Stem (mES) cell line expressing the green fluorescent protein (GFP).

We induced motoneuron differentiation exposing ES-NSC cells to a growth factors cocktail (priming) followed by Retinoic Acid and Sonic Hedgehog. The phenotype was evaluated by morphology and immunocytochemistry. As next step, we transplanted the ES-NSCs, after priming, intratechally in SMA (Smn-/-SMN2+/+SMNDelta7+/+) neonate mice to evaluate their ability to 1) migrate toward the parenchyma 2 ) incorporate functionally within the host environment 3 ) ameliorate the disease phenotype. The fate of transplanted cells was monitored by immunohistochemistry and confocal analysis. Neuromuscular evaluation (hand grip and open field tests) was performed and survival was recorded.

Results: ES-NSCs cells grow as adherent monolayers, are self-renewing and co-express neural stem marker (nestin, SOX1 and LewisX). ES-NSCs can be induced towards a motoneuronal fate as demonstrated by the positivity for HB9, Islet1 and Choline acetyl transferase (ChAT) and formation of neuromuscular junctions in coculture with myotubes. In vivo, the intratechally transplanted NSCs are able to migrate across the meninges, integrate in the parenchyma and robustly differentiate into neuron and motor neurons with axon projecting into anterior roots. In several behavioral tests, transplanted mice exhibited significant functional improvement. Furthermore the survival was significantly extended in comparison to vehicle treated SMA mice ( 19 vs 13 days, $\mathrm{p}<0.001)$

Conclusions: Our data provide the first evidence that NSC derived from ES may exert a significant therapeutic effect on the SMA phenotype opening the path for the development of a combined cellular and molecular therapy for this disease.

\section{P476}

Clinical features and neuropathological findings of familial amyotrophic lateral sclerosis with Gly93Ser mutation in $\mathrm{Cu} / \mathrm{Zn}$ superoxide dismutase S. Ono, M. Suzuki, T. Irie, T. Watanabe, H. Mikami, T. Yamazaki, M. Nomura, K. Oyanagi

Teikyo University Chiba Medical Center (Ichihara, JP); Tokyo Metropolitan Institute for Neuroscience (Fuchu, JP)

Objectives: To report a clinical and neuropathological study of a patient with familial amyotrophic lateral sclerosis (FALS) with Gly93Ser mutation in $\mathrm{Cu} / \mathrm{Zn}$ superoxide dismutase. Patient and methods: The patient's aunt died of ALS at age 67. Our patient developed muscle weakness in the legs, and her gait became disturbed from age 23. A neurological examination at age 24 showed muscle atrophy and weakness in the arms, legs, and neck, predominantly in the distal muscles, and decreased deep tendon reflexes without pathological reflexes. Electromyography showed systemic neurogenic changes with denervation potentials. Complete sequence analysis of the SOD1 CDNA for this patient revealed only a single base pair substitution in exon 4 at codon 93 (GGT - AGT). At age 30, she presented with dysarthria and dysphasia. She became bedridden from the age of 36 years. At the age of 39 years, the patient died of respiratory failure. An autopsy was performed.

Results: In the anterior horns of the spinal cord, there was marked loss of neurons with gliosis, and there was absence of neuronal inclusion bodies like Bunina bodies or Lewy body-like hyaline inclusions (LBHIs) in the remaining cells. The corticospinal tracts in both anterior and lateral funiculi showed a slight degree of myelin pallor. There was myelin loss in the posterior column. There was also a marked myelin loss in the spinocerebellar 
tract. Prominent neuronal loss was noted in the Clarke's nuclei. There was neuronal loss with gliosis in the hypoglossal nuclei, and in the trigeminal motor and facial nuclei. Marked atrophy and myelin pallor were present in the superior cerebellar peduncles. The dentate nuclei of the cerebellum showed neuronal loss accompanied by gliosis and grumose degeneration. There was a moderate neuronal loss with gliosis in the red nucleus.

Conclusion: The clinical characteristics in this case was onset of symptoms in the legs and slow progression. LBHIs in the lower motor neurons are thought to be a significant morphological hallmark of mutant SOD1-related FALS with posterior column involvement, but no inclusion bodies were found in ours. These data suggest that inclusion body formation is not always concerned in the etiology of FALS with SOD1 Gly93Ser mutation.

\section{P477}

Coping strategies and depression in amyotrophic lateral sclerosis and multiple sclerosis

S. Roy-Bellina, C. Almohsen, M.C. Gély-Nargeot, S. Carton, W. Camu

Hôpital Gui de Chauliac (Montpellier, FR)

Objectives: Few studies have analyzed the relationships between depression and neurodegenerative disorders while, conversely, this condition is frequently noted in the affected patients. Coping strategies (CS) may be different between different chronic disorders and a better understanding of specific adaptations in a particular disease could be helpful for patient management.

To compare CS and quality of life (QOL) in multiple sclerosis (MS) and amyotrophic lateral sclerosis (ALS) to determine 1) which CS could be more protective regarding depression; 2) the profile of change in QOL in the course of these diseases.

Methods: There were 14 MS patients, 11 women and 3 men, aged from 32 to $75 \mathrm{yrs}$ (mean $45.6 \mathrm{yrs}$ ) and 14 ALS patients, 3 women and $11 \mathrm{men}$, aged 32 to 78 yrs (mean 63 yrs). Patients with dementia were excluded. They answered to the following questionnaires and tests: coping scale WCC from Lazarus, short version, Beck's scale for depression (BDI-II) and QOL evaluation with NADL.

Results: ALS had the largest impact on activities of daily living (ADL) and instrumental ADL $: p=0.0015$ and $p=0.0014$, respectively. There was, both in ALS and MS, a correlation between the severity of depression and CS focused on emotion $(r=0.557, p=0.0017)$. But, $C S$ are not different between ALS and MS patients. There is a reverse correlation in both diseases between depression and quality of social life $(\mathrm{r}=-0.490, \mathrm{p}=0.0073)$. Depression score is higher in ALS but this does not reach statistical significance.

Conclusion: Severity of depression is similar between ALS and MS, both groups use similar CS. In ALS, loss of autonomy is the major reason for the decrease in QOL. On a daily point of view, spouse and home carers are the most important persons for a patient. In helping him to be an actor of his life and death, they actively contribute to self estimation maintenance.

P478

Executive dysfunction in amyotrophic lateral sclerosis

T. Stojkovic

Institute for Neurology (Belgrade, RS)

Neuropsychological investigations of amyotrophic lateral sclerosis (ALS) patients have revealed cognitive impairment in some non demented patients, characterised mostly by executive diyfunction. The most striking and consistent deficits were found using tests of verbal fluency.

Objective: To investigate the profile of executive dysfunction in non-demented patients with ALS.

Methods: 42 non demented patients with ALS ( 23 females, 19 males) and 18 healthy controls ( 11 females, 7 males) were investigated. The battery off neuropsychological tests, desgined to accommodate the range of physical disability present in ALS, included: Mini Mental State Test; Hamilton Rating Scale for Depression; Scale of Apathy; Test Raven Progressive Matrix; Letter Fluency Test; Category Fluency Test (Animal Naming Test); Boston Naming Test and Cambridge Neuropsychological Test Automated Battery (CANTAB), including: Motor Screening, Spatial Span, Spatial Work Memory and Stockings of Cambridge.

Results: Significant difference beetwen two groups was found on Hamilton Rating Scale for Depression $(\mathrm{p}<0.01)$. Significantly high apathy score was revealed $(p<0.01)$. Also, significant difference was found on Mini Mental State Test, as a sign of cognitive impairment $(\mathrm{p}<0.01)$. The most striking impairments were found on tests of verbal fluency- Letter Fluency Test $(\mathrm{p}<0.01)$, Category Fluency Test (Animal Naming Test) $(\mathrm{p}<0.01)$, indicating executive dysfunction and on Boston Naming Test ( $\mathrm{p}<0.01$ ), as a sign of frontal lobe dysfunction. The differences beetwen groups, found on tests of Spatial Work Memory (strategy score) $(\mathrm{p}<0.05)$, and Stockings of Cam- bridge ((initial thinking time) $(\mathrm{p}<0.01)$ indicate deficits in planning, attention and working memory.

Conclusion: Selective cognitive impairment mostly in the form of verbal fluency deficits, indicating executive disfunction, are present in ALS patients.

\section{Neurorehabilitation}

\section{P479}

Chronic vascular leukoencephalopathy: cinematic and dynamic gait analysis in neuro-rehabilitation outpatients

A. Gagliardo, R. Carrai, C. Falsini, A. Grippo, M. Martini, C. Miotti, S. Verdesca, A. Pizzi

Don Gnocchi Foundation (Florence, IT)

Background: Neuroimaging changes in the cerebral subcortical white matter (WMC) are recognized with the highest frequency in elderly subjects, particularly in those with vascular risk factors. WMC have been consistently reported to be associated with global or selective cognitive deficits, depression, motor and gait impairment (Inzitari D. et al. 2000)

Objectives: To identify an evaluation protocol for the assessment of the distinctive features of the disease and its applicability in neuro-rehabilitation.

Methods: Chronic Vascular Leucoencephalopathy (CVL) outpatients diagnosed by means of brain CT/MR have been recruited at the Neuro-rehabilitation Unit of the Don Gnocchi Foundation.

Patients have been evaluated by means of: Mini Mental State Examination (Folstein MF et al., 1975, Measso G et al., 1993) for a cognitive global level; Geriatric Depression Scale (Brink TL et al., 1982) for mood examination; Functional Indipendence Measure (Guide for the Uniform Data Set for Medical Rehabilitation) for the level of disability and rehabilitation outcome; Nevitt scale (Nevitt et al, 1989) and Berg Balance Scale (Berg K et al., 1989 e 1992) for assessing walking performance and postural balance.

Cinematic and dynamic motor skills during walking, have been evaluated using a Smart-E optoelectronic system (BTS) gait analysis (Davis protocol).

Results: We have recruited 10 patients, $(5 \mathrm{M}, 5 \mathrm{~F}$; mean age 80.8 years range 78-84).

In all patients we observed alterations of both cinematic (knee and hip joint angles) and spatio-temporal parameters (increase of stance and decrease of swing times, decrease of walking velocity, marked increase of stride breadth) in respect to our normative data.

Conclusion: This clinical and instrumental approach seems to be able to assess and quantify a gait pattern alteration in chronic vascular leucoencephalopathy patients. We are going to see if it could be useful for a detection of gait pattern changes after a rehabilitation cycle.

\section{P480}

Effects of modified pen grip and handwriting training on writer's cramp

B. Baur, W. Fürholzer, I. Jasper, C. Marquardt, J. Hermsdörfer

Klinikum München-Bogenhausen (Munich, DE)

Writer's Cramp (WC) is a focal dystonia which can cause heavy problems in daily life. One behavioural treatment approach is the handwriting training developed by Mai and co-workers. We evaluated the use of a modified pen grip (stabilized between index and middle finger) and subsequent handwriting training in $26 \mathrm{WC}$ patients and 14 healthy controls. Handwriting performance was examined once in healthy controls and repeatedly in WC patients (before training, directly after training and after a 3 month follow up period). All subjects wrote a test sentence with their usual and also with the modified pen grip. Writing speed, fluency and pressure were recorded with a digitizing tablet. A sensor foil wrapped around the pen registered grip force exerted on the pen during handwriting. In addition, patients should rate pain after long-time writing and impairment in daily life on visual analogue scales.

Before training WC patients showed increased writing pressure and grip force. Using the modified pen grip caused a decrease of pressure and grip force in all subjects. Seven sessions of handwriting training provoked a further improvement of both parameters in WC patients that remained stable over the follow up period. In contrast, writing speed and fluency were neither influenced by pen grip nor by handwriting training. Pain and impairment in daily life decreased significantly and constantly after training.

Results suggest that WC patients benefit permanently from the use of the modified pen grip in combination with handwriting training. 
Moreover, a current study with 10 patients will show the effects of acoustic grip force feedback in the treatment of WC. Effects of training with and without the acoustic feedback will be compared.

Supported by the Deutsche Forschungsgemeinschaft, DFG HE 3592

P481

Evaluation of neuro-motor deficits in children with cerebral palsy and rehabilitation procedures

C. Bertoncelli

Lenval Hospital (Nice, FR)

Objectives: A correct assessment can help to a wise rehabilitation program of children with cerebral palsy (CP). It is therefore essential to measure the functioning and the development of motor control base mechanisms. Beginning from these data, we have planned a personalized neurorehabilitation - functional training program. We confirmed (Van der Weel F.R., Van der Meer A., Lee D.N, 1996) many perceptive - motor difficulties. Such data could help to verify the diagnosis and may develop appropriate therapeutic procedures.

Methods: Setting is EEAP (Etablissement pour Enfants et Adolescients Polyhandicapés) "H.Germain" of Paediatric Hospital Lenval in Nice (France). We estimated the functionality of motor abilities of 11 patients (6 male, 5 female) with CP. We quantified neurological deficit (scales: Zausman, Samilson, Rancho Los Amigos, Terver, Ashworth modified Bohannon and Smith) and we exactly defined psychomotor development (Gross Motor Function Measure, GMFM, and Brunet-Lézine Test). Based on the outcomes we planned a personalized rehabilitation programme.

Results: 11 children with CP don't suffer of evident muscular tone disturbs (Modified Ashworth Scale) while motor development evaluation (Zausmer) and manual dexterity (Samilson) scales show strong deficiencies. Scheme of Rancho Los Amigos reveals substantial differences among the participants, however they all are strongly diminished during daily life activities (Terver Score). GMFM and Brunet-Lézine Scores specify a motor evolution level among 2 and 12 months (GMFM) and a development psychomotor age among 2 and 48 months (Brunet-Lézine Test). These results may help us to prefer one method through different rehabilitations: neuromotor, psycho-motor or orthopaedic and functional.

Conclusion: Signs that could foretell neurorehabilitation breakthrough are manifold (Wu YW., Day SM., Strauss DJ., Shavelle RM. 2005; Francis K., Bach J., DeLisa J., 1999). Our study quantifies neurological deficits and it estimates psychomotor development, points of departure of rehabilitation program in CP. Verification of neuromotor - orthopaedic disabilities and evaluation of residual psychomotor abilities represent the central pivot of any rehabilitation path in $\mathrm{CP}$.

P482

Challenging FOOD Trial

C. Haider, H. Zauner, K. Schreyer, N. Geringer-Manakanatas, K. Kadar, G. Wood, G. Hasenauer, A. Gassner

Rehabilitation Center (Grossgmain, AT)

Objectives: To prove the hypothesis that patients with neurogenic dysphagia have strongly underestimated resources and show significantly superior improvement in neurorehabilitation under augmented clinical nutrition compared to patients without dysphagia.

Subjects/methods: 127 stroke patients (one dropped) undergoing postacute neurorehabilitation multidisciplinary rehabilitation approach, were routinely screened for neurogenic dysphagia and undernutrition. An aspiration free daily input of 1300-2500 kcal was assured by means of augmented clinical nutrition including changes of food/fluid consistency, feeding assistance, mealtime supervision, therapeutic swallowing manouvers, FEES, ONS, tube feeding, and parenteral nutrition. Outcome was defined as difference of rehabilitation outcome parameters at admission and discharge. Nutritional status was assessed with Innsbruck Nutrition Scale and Nutritional risk screening NRS 2002.

Results: $50 \%$ of the whole sample needed nutritional support. A high amount of cognitive impairment in swallowing compromised patients (56/64) indicates that most of these patients suffered from neurogenic dysphagia affecting the preoral swallowing phase. Only 31/63 of normal swallowing patients were cognitively normal. Swallowing disordered patients exhibited a highly significant better outcome in rehabilitation compared to not swallowing disturbed patients.

Conclusions: Multiple approaches for screening and assessment of dysphagia and undernutrition after stroke and enforced nutrition scenario unmask the underestimated rehabilitation potential of swallowing compromised patients after stroke.
P483

Dysphagia in children and adolescents with severe traumatic brain injury: swallowing assessment and predictive value for weaning

F. Locatelli, S, Strazzer, C. Legnani, G. Rizzi, T. Pigazzini, A. Pavan, C. Malacrida, A. Bardoni, S. Galbiati, F. Formica, E. Beretta, P. Avantaggiato, G. Poggi, V. Amorelli

I.R.C.C.S. E. Medea (Lecco, IT)

Objectives: A major complication commonly seen in persons with severe traumatic brain injury (TBI) is swallowing dysfunction. The aim of this study is to define a quantitative measure for dysphagia in severe TBI, in order to provide a predictive value for weaning.

Methods: Study participants were enrolled among the TBI patients hospitalized at Scientific Institute "E. MEDEA" in the 2002-2006 period. Inclusion criteria were: severe TBI, dysphagia at admission. Clinical history and personal data were registered. Our internal swallowing assessment, named Dysphagia Scale was applied. Each item of the scale was rated on a 1-3 scale value, with 3 being the most unfavourable condition.

Data are reported as mean, standard deviation (SD), median and percentage. Qualitative data are also included. Quantitative variables were analyzed by various statistical methods (i.e. ANOVA, Bonferroni test). The statistical significance of every single test was determined (p).

Results: 280 post-traumatic patients were screened. $102(36.43 \%)$ had dysphagia at admission. 41 had an NG tube, 38 had percutaneous endoscopic gastrostomy (PEG), 8 were fed by combined enteral/oral nutrition, while 15 had oral feeding. 65 patients were included in the study, with mean age at insult of 11.29 years, SD 10.1 years, median 7.83 years. The mean GCS score was of 4.4 (SD 1.6)

At discarge patients were grouped into 3 categories: 35 (53.8\%) were completely weaned, with a mean weaning time being of 29.5 days (SD 30.25 days); 13 (20\%) were partially weaned and had oral feeding and PEG; 17 $(26.2 \%)$ were on enteral nutrition.

The means of the scores at the Dysphagia Scale for the 3 categories were: 15.1 (SD 2.6), 19.3 (SD 2.9), 21.8 (SD 3.2), respectively. The mean of the scores for weaned patients was significantly lower than those of the other 2 categories $(p=0.000)$. In patients achieving oral feeding, the Dysphagia Scale score correlated with weaning time $(r=0.56, p=0.000)$, while it correlated in an inversely proportional manner with GCS score $(r=-0.35 ; \mathrm{p}=0.045)$.

Conclusions: The Dysphagia Scale seems to have prognostic value for weaning, although scores in the middle range have a variable prognosis. This is a retrospective study that needs to be supported by larger samples and prospective studies, ensuring interrater agreement and setting clear cut-offs with sensibility and specificity values. 
P485

BrainStim: cognitive stimulation as preventative method in cognitive aging? I.K. Penner, M. Kobel, K. Opwis, P. Calabrese

University of Basel (Basel, $\mathrm{CH}$ )

Objectives: During aging, cognitive decline is a natural process that primarily affects mental speed, short-term memory and working memory. As cognitive deficits have a negative impact on quality of life, possible approaches for prevention and treatment are of interest. Besides pharmacological treatment, cognitive stimulation of the brain might be one alternative to induce and support plasticity processes and to hereby decelerate the negative effects of cognitive aging.The aim of the present study was to systematically study the hypothesis of induced neuroplasticity in healthy elderly subjects by a specific computerised training of working memory.

Methods: Nine healthy elderly subjects ( 2 female and 7 male) were included in the study. Mean age was 70.11 years $(S D=4.75)$. To determine a cognitive profile at baseline, all participants underwent a comprehensive neuropsychological examination, including memory, working memory, attention, concentration, information processing speed, mental flexibility and intelligence. After the baseline testing, each participant performed the training with the computer program BrainStim four times a week over a period of four weeks at home. When 16 training sessions were completed, each subject was reinvestigated neuropsychologically.

Results: The results document a significant increase in cognitive performance within the training procedure that could be expressed best by an exponential function with steep increase at the beginning and asymptotic course further on. On the neuropsychological outcome measures, only the Paced Serial Addition Test (PASAT) showed a significant change in performance, when simple learning effects from baseline to post-testing were controlled.

Conclusion: There is evidence from several studies that neuroplasticity can be induced by cognitive intervention. The results of our study indicate that working memory performance increased after a four-week computerised training. In this concern, BrainStim seems to positively influence brain functionality in healthy elderly subjects. Whether this intervention has also therapeutical potential in neurodegenerative diseases is subject of ongoing studies.

\section{P486}

Rehabilitation, botulinum toxin and phenol motor nerve block can alter the functional outcome in upper limb spasticity

N. El-Nahas, A. El-Ganzoury

Ain Shams University (Cairo, EG)

Stroke is a common cause of morbidity and lasting disability.

Objectives: To study the impact of combining botulinum toxin type A (BT-A) and phenol (a neurolytic agent) with an extensive rehabilitation program on post-stroke spasticity, functional impairment, and pain.

Methods: We studied 55 patients, six months post-stoke. Only 32 patients completed the study. All were assessed by medical research council paralysis scale (MRC), Ashworth scale, Rivermead Motor scale, Carer burden scale, and the Global pain scale. BT-A was given in the forearm and finger flexors and phenol in the elbow flexor, on individual basis. A specific rehabilitation program was prescribed including splinting, muscle stretching, strengthening, functional electric stimulation and occupational therapy over 6 weeks. Reassessment was done 6 weeks later. Patients were divided into two groups: group I complied to the rehabilitation program (n: 22) and group II did not comply (n: 10).

Results: Group I showed a significant improvement, when comparing before and after treatment, than group II regarding spasticity, functional outcome and carer burden score. While, pain scales showed improvement in both groups. Moreover, a positive correlation was found between the mean change(d) in spasticity score, and the other assessment scales(d)

Conclusion: The overall combined medical and rehabilitation care is likely to be more important in producing functional change than a single specific intervention, such as BT-A injections. Moreover the measurement of treatment outcome should be directed to the level of functional disabilities than basic impairment.

\section{P487}

5000 finger grip movements with a new robotic hand rehabilitation device, a possibility to improve grip abilities and changes on functional MRI

P. Grieshofer

Klinik Judendorf Strassengel (Judendorf Strassengel, AT)

Background: Successful rehabilitation of highly impaired finger and hand movements after stroke often remains unsatisfactory with conventional therapies. Therefore we developed a new mechatronic device for rehabilitation of hand function which allows delivering well characterised, high frequent, repetitive movement sequences in an individualised manner. The goal of the ongoing exploratory study is to use functional MRI to assess potential changes in the central control of movement of the paretic hand associated with such stimulation and to correlate these with performance gains.

Methods: To date, 11 stroke patients with a moderate to high-grade paresis of the upper limb (pinch grip force grades 2 or 3 according to MRC; spasticity according to the Ashworth Scale $<3$ ) have been included. (Before and after three weeks of standardised training using a hand robot (Developed Klinik Judendorf Strassengel), patients were both tested behaviourally and a subset underwent repeated fMRI using an identical paradigm at 3T. The Motricity Index before and after training and force measurements on the robot during each session served to assess gains in functional strength. Results: Subsequent to an average of 5000 grip movements during 15 therapy sessions on the robot additive to conventional PT and ET, patients demonstrated significant improvements in their functional strength, both apparent clinically and by measurements of force using the device.

Whereas fMRI activation patterns with movement of the healthy (nottrained) hand vs. rest did not change after training, significant increases in activation in the cerebellum and basal ganglia were noted with movement of the paretic, trained hand subsequent to therapy. At baseline, movement of the paretic hand vs. rest was associated with activation of a more bihemispheric network with additional recruitment of ipsilateral motor areas in the undamaged hemisphere compared to movement of the unaffected hand.

Conclusion: These preliminary results suggest distinct changes in sensorimotor networks associated with robotic-assisted rehabilitation of hand function in addition to conventional PT after stroke. High frequent afferent stimulation combined with increments in efferent activity might activate networks similar to motor learning in healthy adults. However, these conclusions are limited by the small patient number, the heterogeneity of the cohort, the lack of a control group and the variance in response to therapy.

\section{P488}

Cortical activation changes following botulinum-toxin treatment of poststroke arm spasticity: evidence from functional MRI

P. Hlustik, P. Kanovsky, Z. Senkarova, H. Vranova, P. Otruba, R. Herzig

School of Medicine, Palacky University (Olomouc, CZ)

Objectives: Botulinum toxin (BTX) treatment can relieve focal arm spasticity after stroke, presumably through dynamic changes at multiple levels of the motor system, including cerebral cortex. However, the neuroanatomical correlate of BTX spasticity relief is not known and should be reflected in changes of cortical activation during motor tasks assessed using repeated functional MRI (fMRI).

The goal of our pilot study was to localize with fMRI the changes in sensorimotor system activation induced by botulinum toxin treatment relieving post-stroke arm spasticity.

Methods: Six patients ( 5 males, 1 female, mean age 66.2, SD 9.3 years) with hemiparesis and distal arm spasticity due to chronic middle cerebral artery stroke sparing the motor cortex were studied. Brain fMRI during sequential finger movements of the impaired hand was acquired in two sessions, before and 4 weeks after BTX injection into the spastic arm. Change in arm spasticity was assessed using the modified Ashworth scale (MAS). Motor performance was kept constant across imaging sessions. Following L$\mathrm{R}$ flip of the right hemisphere-lesioned brains, whole-brain fMRI group data were processed with a general linear model, testing for treatment effects with linear contrasts.

Results: BTX treatment decreased arm spasticity across the group (mean MAS change 0.83 , SD 0.41). fMRI data pre- and post-BTX treatment showed activation of the sensorimotor network including contralateral motor cortex, supplementary motor area, bilateral premotor cortices and superior parietal lobule during hand movement. The pre- $>$ post-BTX contrast revealed a significant decrease $(\mathrm{P}<0.05$, corrected $)$ after BTX treatment in activation of a contralateral frontoparietal network with hotspots including (in the order of decreasing significance) dorsolateral prefrontal cortex, primary sensorimotor cortex, inferior parietal lobule and postcentral gyrus.

Conclusion: This pilot study suggests that relief of post-stroke arm spasticity may be associated with changes in activation at several hierarchical levels of the cortical sensorimotor system participating in movement control and planning, including the prefrontal cortex. 


\section{P489}

A comparative study between the aetiology and outcome in patients with severe traumatic brain injury

S. Galbiati, S. Strazzer, A. Bardoni, F. Formica, E. Beretta, F. Locatelli, P. Avantaggiato, G. Poggi

I.R.C.C.S. E. Medea (Lecco, IT)

The severe brain injury is a common cause of disability in children. It results in developmental deficits with motor, cognitive, linguistic and behavioural problems.

Objectives: To assess the outcome of TBI in a group of children and compare it against different etiologies responsible for brain injury.

Methods: We selected a group of patients with severe TBI from the patients hospitalized in our Rehabilitation Institute between 2002 and 2006. The following clinical data were collected: patients' age at insult and sex, Glasgow Coma Scale score (GCS), duration of the unresponsives state, neurological deficits upon awakening, and site of the brain lesion. Each patient received clinical and functional evaluations, specifically: Glasgow Outcome Scale (GOS), Disability Rating Scale (DRS), The Functional Independence Measure for Children (Wee-FIM). All the patients with previous history of brain injury or patients with an associated spinal injury were excluded. We studied 3 groups of patients: post-traumatic children, post-anoxic patients and other etiologies.

Results: We selected 183 patients from a pool of 195 patients with severe brain injury hospitalized between 2002 and 2006. Age at trauma was 7.8 years (SD: 5.4$)$, median 6.7. All the patients ( 115 boys and 68 girls) presented with severe TBI (GCS < 8), their mean GCS score being 4.9 (SD: 1.9). 105 children suffered traumatic brain injury, 31 presented with anoxia, and 47 with other etiology. $57.1 \%$ of the patients showed a multifocal damage, $8.2 \%$ a diffuse axonal damage, $20.1 \%$ a focal damage, $20.1 \%$ showed other lesions, and only $1.6 \%$ of the patients did not show any lesions on the CT or MR. After rehabilitation, the 3 groups of patients showed a significant improvement. Posttraumatic patients show a greater difference between the evaluation at the admission and at the discharge as compared to the other two groups.

Discussion: The severe brain injury in children can lead to multiple impairments and disabilities. Moreover, since children are still developing and their neurological system has not reached full maturation yet, they need an interdisciplinary rehabilitation programme to improve their functional abilities post-insult. There are major differences between etiologies, the postanoxic patients have a more unfavourable prognosis. However, all the groups showed a significant improvement, which supports the validity of an intensive multidisciplinary rehabilitation programme for children.

\section{P490}

Visual disorders and functional recovery in post-traumatic children: evaluation and treatment measures

S. Galbiati, S. Strazzer, A. Bardoni, F. Formica, E. Beretta, F. Locatelli, P. Avantaggiato, G. Poggi

I.R.C.C.S. E. Medea (Lecco, IT)

Objectives: Visual disorders following traumatic brain injury (TBI) are frequent, often multiple, and associated to complex clinical and functional pictures. During childhood and adolescence, they can interfere with development processes, rehabilitation and outcome.

The goal of this study is to show the correlation between visual disorders and functional recovery in a population of post-traumatic children by use of the evaluations at admission and discharge, after motor and neurovisual rehabilitation.

Materials and methods: A total of 95 post-traumatic patients hospitalized in the Acquired Brain Injury Unit of "E. Medea" Institute (Bosisio Parini) were selected, ranging in age between 4 months and 14 years. All the patients had suffered severe TBI (Glasgow Coma Scale GCS $<8$ ). The following data were collected: age at insult, sex, length of coma, neuroradiological imaging. The children received the following evaluations: clinical observation, neurovisual assessment, administration of functional measures (Disability Rating Scale -DRS-, The Functional Independence Measure for Children -FIM-WeeFIM-, Gross Motor Function Measure -GMFM-), cognitive evaluation scales.

Results and conclusion: all the children suffered severe TBI (mean GCS score $=5.73$ ). More than half of the patients $(54.7 \%)$ have visual or oculomotor disorders. All the children underwent intensive motor and neurovisual rehabilitation. They were re-evaluated upon discharge (DRS, FIM, GMFM and IQ), and they showed a significantly clear clinical improvement. The most significant finding is that visual disorders significantly affect the recovery of postural and motor skills as well as recovery of functional independence. Visual acuity deficits are statistically correlated with the GMFM score. An association was found between patients with blindness, hemianopsia, visual deficits and a lower GMFM score. Furthermore, recovery of functional independence and self-sufficiency is statistically influenced by visual deficits, in particular patients with visual acuity deficits show a higher DRS score and a lower FIM score. Equally important is the finding that, patients with visual deficits show a significant improvement on all functional scales after intensive rehabilitation. The association between visual disorders and functional scales can greatly contribute to understanding to what extent visual disorders following TBI are a serious problem in childhood as they affect motor and cognitive skills.

\section{P491}

Electrostimulation of shoulder girdle muscles in facioscapulo-humeral muscular dystrophy patients

S. Sacconi, S. Colson, V. Tanant, C. Benaim, M. Fournier-Mehouas, C. Desnuelle

Fédération des Maladies Neuromusculaires (Nice, FR); UFR STAPS (Nice, FR)

Objectivs: Autosomal dominant Facioscapulohumeral muscular dystrophy (FSHD) is characterized by selective pattern of muscle involvement. Weakness and atrophy typically involve facial and shoulder girdle muscles, then with, the progression of the disease progress to anterior forearm and foreleg muscles and to pelvic girdle muscles.

The physiopatholgical mechanism of this disease, due to a deletion of repeated units named D4Z4 located on 4q35, is still controversial. Up to date, no curative therapy is available for these patients. We proposed in the present study to test feasibility, clinical and biological tolerance and efficacy of shoulder muscle training by electrostimulation in a group of FSHD patients

Methods: 7 patients displaying classical FSHD phenotype participate to this study consisting in daily session of shoulder girdle electrostimulation of 23 minutes for a period of 5 months. We evaluated: clinical tolerance by daily pain and fatigue analogic scales, biological tolerance by measuring CK; feasibility: by measuring the monthly score of participation to sessions; the efficacy by manual muscle testing, quantitative muscle assessment, fatigue severity scale.

Results: Shoulder girdle electrostimulation training was well tolerated. No pain and fatigue after session were reported neither others complications. In particular no sign of rabdhomyolisis, were evident being CK level stable or even reduced. In term of efficacy, a significant improvement of manual muscle testing, quantitative muscle assessment and fatigue severity score was found. Conclusions: Electrostimulation training is well tolerated by patients and is feasible even thought quite constraining. Different level of muscular improvement was seen in different muscles, being the less affected muscles the more responsive. These findings strongly suggest the need of a multicentric study on a largest population of FSHD patients.

\section{P492}

An evaluation of the early rehabilitation programme by Functional Independence Measure Index

E. Vanaskova, D. Krajickova, B. Smidova

University Hospital (Hradec Kralove, CZ)

Objectives: To evaluate the change in activity of acute stroke patients during rehabilitation treatment using Functional Independence Measure (FIM)

Methods: A total of 714 patients (353 male, 361 female) at mean age 72,4 with newly diagnosed acute stroke were admitted to the Stroke Unit Centre, Department of Neurology, University Hospital Hradec Kralove between the years 2003 and 2006. The measurement of the FIM was conducted during 1 week after stroke and 3 weeks later (before and after the end of rehabilitation programme).

Results: The motor subscore of the FIM changed from 34,6 to 51,8 . The cognitive subscore of the FIM changed from 24,4 to 26,3 . The total score of the FIM changed from 58,7 to 78,1 . Distribution by type of discharge: $42 \%$ to community, $30,5 \%$ to long term care facility, $10,5 \%$ to other acute care departements due to complications, $11,3 \%$ to rehabilitation facility, 5,7 \% patients died.

Conclusion: The FIM gain was high in motor subscore and low in cognitive subscore. These changes in subsets of the functional recovery had impact on needs of the subsequent care pattern.

By uderstanding types of impairment clinicans may more precisely design treatment and needs of social support. The FIM is effective in the acute care setting to help determine appropriate discharge status. 


\section{Poster session 3}

\section{Peripheral neuropathy}

\section{P493}

Multifocal motor neuropathy: analysis of prognostic factors

G. Galassi, F. Girolami, A. Ariatti, P. Miceli, M. Stefani, M. De Palma, P. Faglioni

Institute of Neurosciences (Modena, IT)

Objective: Multifocal motor neuropathy (MMN) is chronic disorder evolving throughout decades. Association with antibodies against ganglioside GM1 and response to intravenous immunoglobulins (IVIG) support an autoimmune mechanism. Genetic predisposition related to major human histocompatibility complex (HLA)class I and II encoding antigen presenting molecules to T lymphoctes may play pathogenetic role. Purpose of our study was to define relevance for prognosis of clinical and eletrophysiological features.

Methods: Nine males (mean age 45,2, range 29-63) and five females (mean age 65,2, range 55-73) were studied. Mean age at first evaluation was 51,3 (range 31-77). Duration of illness prior to first evaluation was 34,2 months (range 4-95). Age at onset was 44,71 (range 22-63). Mean duration of follow up was 63,7 months (range11-118). Nine patients received regular IVIG treatment. Impairment and disability measures included grading sum score in six limb muscle pairs (MRC), overall disability status(ODSS), Rankin scale, overall number of conduction block (CBs) among examined nerve segments. Typing of HLA class I and II was performed in MMN patients and in 3,528 healthy bone marrow donors by complement-dependent microcytotoxicity assay, by protein chain reaction amplification with sequence-specific primers or products hybridised with sequence-specific oligonucleotides probes.

Results and conclusions:

1) Effects of gender,age of onset,age of first evaluation,duration of disease, duration of follow up tested on probability of clinical worsening by means of binary logistic regression analyses never reached the onetailed 0.05 significance level

2) Clinical and electrophysiological worsening measured by change scores,i.e, initial MRC-final MRC, final ODSS-initial ODSS, final Rankin-initial Rankin, final CBs/final segments-initial CBs/initial segments resulted significatly (one tailed $\mathrm{P}<0.05$ ) different from zero except for CBs.

3) Worsening in respect of $\mathrm{CBs}$ was significantly (one tailed $\mathrm{P}<0.05$ ) affected by all explanatory variables (MRC, ODSS, Rankin) except by interval between 1st-last evaluation

4) Worsening of ODSS and Rankin was significantly (one tailed P $<0.05$ )affected only by gender

5) Distribution of antigen HLA-A,B and HLA-DRB1*allele was not significantly different between females and males and between controls and MMN patients (chi square 7.44; $\mathrm{df} 14 ; \mathrm{p}$ two tailed asymptotic $=0,9165$; $\mathrm{p}$ two tailed Monte-Carlo $=0,8721$ ) suggesting that MHC does not confer susceptibility to disease process in MMN with or without self gangliosides.

P494

Intraepidermal nerve fibre density did not correlate with heart rate variability and cutaneous silent period in type 2 diabetes

J.H. Lee, K.S. Song, B-J. Kim, A.Y. Lee, E.H. Sohn

Chungnam University Hospital (Daejeon, KR); Korea University Hospital (Seoul, KR)

Objectives: Small fiber dysfunction can not be detected by standard nerve conduction study(NCS). There are several methods to evaluate small fiber function including quantitative sensory testing, analysis of heart rate (HR) variability, assessment of sudo-motor axon reflexes, or quantification of intraepidermal nerve fiber density (IENF). Cutaneous silent period(CSP) is a spinal reflex mediated by A delta cutaneous afferents and is a prospective electrophysiological method for the diagnosis of small fiber dysfunction.

The aims of this study were to determine the clinical significance of IENF and to find the relationship between IENF and HR variability, and CSP in type 2 diabetes.

Methods: Type 2 diabetic patients with putative neuropathy based on clinical symptoms were included prospectively. All patients were given clinical neurological examinations for the determination of small fiber neuropathy and were got NCS for the detecting polyneuropathy. To evaluate small fiber function, heart rate variability according to deep breathing (DB ratio), Valsalva ratio, sympathetic skin response (SSR), CSP, and IENF were done. The results of patients with small fiber neuropathy (SFN) were compared with the results of those with polyneuropathy. We also studied the correlation between IENF and HR variability, and CSP.

Results: IENF density was lower in neuropathy group compared with normal control. Even in SFN only, IENF density was still lower than normal control. IENF density of patients with NCS abnormality was significantly lower than patients with SFN only. IENF density did not correlate with DB ratio, Valsalva ratio, SSR, and CSP latency.

Conclusion: Quantification of IENF density is useful method to detect small fiber dysfunction early. IENF, however, might be other subpopulation of small fiber from cardiovagal or A delta fiber.

\section{P495}

GJB1 gene mutations in suspected inflammatory neuropathies not responding to treatment: report of 3 cases

M. Laura, A.W. Michell, J. Blake, A. Cox, V. Gibbson, M. Davis, N.M. Murray, M.M. Reilly

MRC Centre for Neuromuscular Disease (London, UK); National Hospital for Neurology and Neurosurgery (London, UK); Addenbrooke's Hospital (London, UK)

It is generally accepted that whilst inflammatory demyelinating neuropathies often cause patchy demyelination resulting in conduction block, temporal dispersion and variation in conduction velocities, the demyelinating hereditary neuropathies such as Charcot Marie Tooth disease type 1 (CMT1) are usually characterised by homogeneous conduction slowing. Furthermore, a forearm median motor conduction velocity of $38 \mathrm{~m} / \mathrm{s}$ is generally used as a cut-off to distinguish demyelinating forms of CMT (CMT1) from axonal types (CMT2). It is now increasingly recognized that certain types of genetically defined CMT can have intermediate conduction velocities. X-linked CMT (CMTX) due to mutations in the Gap junction protein âl (GJB1) gene usually presents with a generalised sensorimotor neuropathy which is more severe in affected males. Upper limb nerve conduction velocities (NCV) are in the intermediate range (30 to $40 \mathrm{~m} / \mathrm{s}$ in males and 30 to $50 \mathrm{~m} / \mathrm{s}$ in females) and conduction slowing is less uniform in comparison with CMT1.

Objectives: To describe three cases of CMTX in which the neurophysiological findings initially appeared to support the clinical diagnosis of a demyelinating inflammatory neuropathy.

Methods: Sequencing of GJB1 gene in patients with a demyelinating neuropathy not responding to treatment.

Results: Mutation of the GJB1 gene was found in three patients (two females and one man) with a demyelinating neuropathy. In all three patients neurophysiology was characterised by conduction block and temporal dispersion. They all received immunosuppressant treatment without clinical response.

Conclusions: These reports highlight the possibility that patients with GJB1 mutations might be erroneously diagnosed as having an inflammatory neuropathy and receive unnecessary immunosuppressive therapy. Sequencing of the GJB1 gene should be considered in suspected cases of inflammatory demyelinating neuropathy which fail to respond to treatment. The mechanism by which GJB1 mutations might cause segmental demyelination is unknown.

P496

Clinical outcome of patients with Guillain-Barré syndrome treated in the NICU

M. Nojszewska, A. Opuchlik, M. Lipowska, H. Kwiecinski

Medical University of Warsaw (Warsaw, PL)

Guillain-Barré syndrome (GBS) is an acute inflammatory demyelinating polineuropathy with a potentially serious course. Many patients become unable to walk independently and may require treatment at the intensive care unit due to serious dysautonomia and respiratory failure. Plasma exchange (PE) and intravenous immunoglobulin (IVIg) are effective methods of treatment in rapidly progressing GBS. We undertook a retrospective evaluation of all adult GBS cases admitted to the NICU at our Department over the last 15-year period to determine the rates of severe morbidity and mortality, and to assess functional outcome. In addition, we compared the efficacy of PE and IVIg in the treatment of severe GBS.

Methods: 117 patients with GBS were hospitalised in the years 1992-2007 at our Department serving as a regional referral centre for neuromuscular disorders. The modified Hughes Disability Scale (HDS) was used to evaluate the severity of functional disability.

Results: 57 (100\%) patients (36 men), mean age $50 \pm 18$ years, admitted to the NICU were unable to walk independently ( $\geq 3$ points HDS) or required 
mechanical ventilation. Only 13 (22\%) patients required mechanical ventilation, but in majority $(61 \%)$ of them the duration of mechanical ventilation was shorter than 14 days. Five $(8.8 \%)$ patients underwent tracheostomy. Mortality occurred in only 2 patients $(3.5 \%)$. Almost two-thirds suffered at least one major complication: urinary tract infection $(26 \%)$, pneumonia (16\%), hyponatremia (12\%), deep venous thrombosis (5\%), and pulmonary embolism $(3.5 \%)$.

24 patients were treated with PE, 24 received IVIg, and 4 were treated with both methods. In addition, $43(75 \%)$ patients also received a short course of high dose of corticosteroids.

Hospital stay was a median 27,5 days in the PE group and 35,5 days in the IVIg group. Median duration of mechanical ventilation was 5 days in the PE group and 6 days in the IVIg-treated patients. Most patients from both study groups at discharge had $<3$ points HDS, and there was no significant difference between mean values of HDS.

Conclusion: Many patients with severe GBS can make satisfactory functional recoveries when they are treated in the NICU. The clinical efficacy of $\mathrm{PE}$ and IVIg was comparable in our patients.

P497

Tolerability profile of high-dose $10 \%$ liquid intravenous immunoglobulin in chronic inflammatory demyelinating polyneuropathy administered in a home-based setting

M. Rigas, R. Sterling

Crescent Healthcare, Inc. (Anaheim, US)

Objectives: Intravenous immunoglobulin (IVIG) is considered the preferred treatment for chronic inflammatory demyelinating polyneuropathy (CIDP). In controlled trials IVIG has similar efficacy to plasma exchange and prednisolone, with the added benefit of increased quality of life. However, IVIG is a complex therapy and can be associated with adverse effects (AEs) at both the standard and high-dose regimens. Reported rates of AEs range from 12$23 \%$, and may be as high as $34 \%$ for initial infusions of patients naïve to IVIG therapy. We examined the overall safety and tolerability of high-dose IVIG administered to patients with CIDP in a clinically-defined, homebased and nursing-supervised setting to enhance the knowledge base and decision-making criteria for this uncommon patient population.

Methods: For the period between September 2005 and May 2006, patients with CIDP treated with a liquid $10 \%$ IVIG (Baxter Healthcare, Deefield, IL) were identified via a retrospective chart review at Crescent Healthcare, Inc. (Anaheim, CA). There were a total of 44 patients - 13 were naive to IVIG therapy (received their initial infusion during the study period) while 31 were previously-treated patients. The 44 patients studied received a total of 826 IVIG infusions within the study period with a mean monthly dose of $1.72 \mathrm{~g} / \mathrm{kg}$ of IVIG per patient (median: $1.65 \mathrm{~g} / \mathrm{kg}$; range: $0.15 \mathrm{~g} / \mathrm{kg}$ to $7.0 \mathrm{~g} / \mathrm{kg}$ ).

Results: The rate of serious AEs, such as aseptic meningitis, thrombosis, hemolysis, or renal dysfunction was $0 \%$. The overall rate of non-serious AEs was $4.6 \%$. Of the non-serious AEs $(n=38)$, there were 5 late-occurring effects that could not be definitively linked to the infusions and were therefore classified as "possibly infusion-related" (AE rate is $4.1 \%$ if these are excluded) The most common adverse effect reported was headache $(n=10)$, followed by muscle pain $(n=5)$ and rash $(n=5)$. None of the headaches were reported as serious, severe, or prolonged, and $60 \%(6 / 10)$ occurred in just two patients. The lack of AEs, serious or non-serious, in initial infusions of the IVIG-naïve patients is notable as compared to reported rates $(34 \%)$ in these patients.

Conclusion: The results of this review of a number of high-dose liquid IVIG infusions in patients with CIDP utilizing a closely-monitored, yet highly flexible, home-based therapeutic regimen indicate a very favorable tolerability profile, even in patients who are new to IVIG therapy.

This study was funded by a research grant from Baxter Healthcare Corporation.

\section{P498}

Early predictors of mechanical ventilation in Guillan-Barré syndrome

N. Yildiz, A. Alpay, T. Aydin, N. Isik, I. Aydin Cantürk, F. Candan Goztepe Training Hospital (Istanbul, TR)

Background: Guillain-Barré syndrome (GBS) is characterised by limb weakness and areflexia and the prototype of postinfectious autoimmune diseases

Respiratory failure is the most serious short-term complication of Guillain-Barré syndrome and can require invasive mechanical ventilation in $20-30 \%$ of patients.

Purpose: We sought to identify clinical and electrophysiological predictors of respiratory failure in the disease.

Material and method: Forty three patients of GBS were enrolled over a
6 year period between 2000-2006. Clinical and electrodiagnostic data were evaluated retrospectively. Clinical and elctrodiagnostic data were compared between ventilated (17) and non-ventilated (26) patients. The clinical parameters assessed were median age, gender, antecedent infection, prior lung disease, time to peak disability, bifacial weakness, upper limb weakness, bulbar paralysis, neck weakness and autonomic dysfunction.

Results: Comparing the clinical data in the ventilated and non-ventilated groups, early peak disability, time from onset to admission of $<7$ days, autonomic dysfunction and bulbar weakness,, inability to lift the head predicted the onset of respiratory paralysis.

Increased liver enzyme CSF protein levels, inability to lift the elbows, inability to count up to 20 in one breath were different between two groups and predicted the development of neuromuscular respiratory paralysis in GBS.

Age, gender, areflexia, sensorial loss, electrophysiological predictors or preceding infection did not influence the development of neuromuscular respiratory weakness.

Discussion: It is important to predict early risk factors for mechanical ventilation in GBS. We thougt the evaluation of this group of patients in intensive care unit might reduce mortalite and morbidite.

\section{P499}

Severe multifocal motor neuropathies caused by radicular and peripheral nerve conduction blocks

S. Apostolski, I. Basta, L. Suturkova, S. Lavrnic, A. Poceva, Z. Stevic, A. Nikolic, S. Knezevic-Apostolski, T. Stosic-Opincal, T. Ilic

University of Belgrade (Belgrade, RS); Institute of Neurology, CCS (Belgrade, RS); School of Pharmacy (Skopje, MK); MR Center, CCS (Belgrade, RS); Military Medical Academy (Belgrade, RS)

The objective of this study was to investigate the patients with severe and progressive Multifocal Motor Neuropathy (MMN).

Methods included neurological examination, electromyography, nerve conduction studies (NCS), transcranial magnetic stimulation (TMS), MRI of cervical spine and brachial plexus, serum immunoelectrophoresis and antiganglioside antibodies testing and CSF analysis.

Results: Two male patients, age of 49 and 25, and a 52-years old female patient had history of chronic ( 8,5 , and 4 years, respectively), progressive and severe MMN.In all patients severe asymmetric weakness of muscles inervated by radial, ulnar, median and peroneal nerves was observed and the most striking presentation was bilateral wrist and finger drop. EMG showed denervation, the most severe in distal muscles inervated by radial nerves. NCS showed in all patients persistent partial conduction blocks outside the usual sites of nerve compression in ulnar, median and peroneal nerves. Conduction blocks were not detected in radial nerves. Conventional TMS and triple stimulation technique detected proximal conduction blocks and focal temporal dispersion between cervical root emergence and Erb point (prolonged root conduction time). Proximal conduction blocks in all patients caused weakness of muscles inervated by radial nerves. In all patients T2weighted MRI revealed increased signal intensities in the cervical roots (C5C8) and in the proximal segments of brachial plexus. All patients had high titers of polyclonal IgM anti-GM1 antibodies as well as slightly increased CSF protein content. The response to IVIG therapy was good during the first two years of the disease but later the patients recquired an additional immunosuppression by ciclophosphamide and cyclosporine.

\section{P500}

Epidemiological characteristics of Guillain-Barré syndrome in Ribeirão Preto, São Paulo, Brazil

J.S. Santos, W. Marques Jr., A.A. Barreira

Medical School of Ribeirão Preto -USP (Ribeirão Preto, BR)

Objectives: Although Guilllain-Barré syndrome (GBS) is distributed worldwide, there have been few reports on the epidemiology of the syndrome from the southern hemisphere. The aim of the present study was to conduct a study on GBS epidemiology in Ribeirão Preto, São Paulo, Brazil.

Methods: This is a descriptive, and retrospective study based on the review of the data records from all hospitalized patients with Guillain-Barre syndrome occurring in Riberão Peto inhaitants from January 1987 to December 1997.

Results: Over the 11 years of the study, we found an average annual incidence of 1.06 cases per 100,000 inhabitants, ranging from 0.24 to 1.91 cases per 100,000 inhabitants. There was no seasonal preponderance, although there was a predominance of cases in males (1.5:1) over females. Incidence was greatest in women aged from 41 to 50 years old. The greatest number of cases was found in patients, between ages 31 and 40 years old. An infectious event preceded or was concomitant in $65.7 \%$ of cases. Upper respiratory tract infections and diarrhea were the most frequent. Artificial ventilation 
was necessary in $9.4 \%$ of cases, and mortality was $5.6 \%$. An elevated spinal fluid protein without an increase in cell number was found in $83.7 \%$ of patients, and a demyelinating pattern on EMG was seen in $86.8 \%$. Two thirds of the patients were treated at the University Hospital, the regional tertiary medical center. The incidence of SGB showed a significant linear increase over the years, although we were unable to identify the factor(s) responsible.

Conclusions: The linear increase in GBS incidence does not surpass the expected incidence of GBS seen in other cities worldwide. The electrophysiological and clinical profiles of the GBS patients from Ribeirão Preto are closer to those from northern hemisphere series than to those from southern hemisphere series, or from China, Japan or other Latin American countries where the axonal pattern predominates.

CNPq, FAPESP and FAEPA

P501

MR imaging of nontraumatic brachial plexopathies - useful step in differential diagnosis

S. Lavrnic, D. Lavrnic, T. Stosic-Opincal, M. Gavrilov, V. Peric, D. Grujicic Clinical Centre of Serbia (Belgrade, RS)

Magnetic resonance imaging (MRI) is the method of choice for evaluating patients with nontraumatic brachial plexopathy.

Methods: One-year retrospective study included patients with nontraumatic brachial plexopathy. In all patients typical clinical finding was confirmed by upper limb neurophysiological studies (including paraspinal electromyography to distinguish brachial plexopathy from radicular lesion) and in all of them MRI of cervical spine and brachial plexus were performed by 1.5 T MR scanner.

Results: Seventeen patients (11 men and 6 women) met inclusion criteria. Patients ranged in age from 27 to 71 years, with a median of 47 years. Three patients had multifocal motor neuropathy, 5 had malignant disease ( 1 chronic lymphocytic leukemia, 2 metastases of breast cancer and 2 metastases of lung cancer). Two patients had primary neurogenic tumors of the brachial plexus ( 1 schwanoma and 1 neurofibroma), 4 radiation fibrosis (after radiation therapy) and 3 had neuralgic amyotrophy of ParsonageTurner's type.

Conclusions: Evaluation of the brachial plexus presents a great challenge to the clinican and radiologist. According to our results there are several causes of brachial nontraumatic plexopathy which could be distinguish by MRI. In cases with unclear etiology additional investigations (immunological and other analysis of the blood and CSF, repeated electrophysiological studies, ets) could help in definite and accurate diagnosis.

\section{P502}

Late-onset multifocal motor neuropathy

V. Felix-Torres, P. Schestatsky, A. Finkelsztejn, J. Ehlers, M. Chaves

Hospital de Clínicas (Porto Alegre, BR)

Introduction: Multifocal motor neuropathy (MMN) is a very rare condition, affecting only about 1 per 100.000 people. Most patients are between the ages of 30 and 50 when symptoms are noted, with the average age of onset being 40 years.

Methods: We report a case of a 74-year-old woman who was presented with slowly progressive asymmetrical limb weakness and muscular atrophy in the last year. The physical examination showed fasciculations in both hands and in one of the legs. During this period she was diagnosed as having amyotrophic lateral sclerosis by two different clinical centers.

Results: Nerve conduction studies showed motor conduction block at right ulnar, left median and both peroneal nerves. Sensory nerve conduction studies were normal. CSF examination was unremarkable and elevated serum antibodies to GM1 ganglioside were detected in the serum. She was given intravenous immunoglobulin (IVIg) $0.4 \mathrm{~g} / \mathrm{Kg} / \mathrm{d}$ and showed satisfactory clinical (neurologic disability score) and electrophysiological responses (compound muscle action potential amplitudes and conduction block) after 30 days.

Conclusion: This is the first description of a late-onset MMN. The positive response to IVIg highlights the importance of a careful motor nerve conduction study in elderly patients with lower motor neuron syndrome.
P503

$\mathrm{N}$-hexane neuropathy appearing earlier in a patient with family history of polyneuropathy

Z. Ozozen, Ü.S. Benli, Y. Kaya, U. Can, M. Kilinc Toprak

Baskent University (Ankara, TR)

Objectives: $\mathrm{N}$-hexane which is a toxic agent used in industry may penetrate the body by inhalation or absorption and cause toxic peripheral neuropathy which can be diagnosed by detecting 2,5 -hexanedion which is a major metabolite of $n$-hexane in urine.

Case report: A 20-year-old, female patient presented with bilateral distal weakness and paresthesia of her legs for a month. In the last week she also had weakness and numbness of her arms. In her neurological examination she had bilateral predominatly distal weakness and long glove and stocking sensory loss of her extremites with areflexia. Her electroneuromyography showed diffuse mixt predominantly demyelinative sensory-motor polyneuropathy. Her CSF findings were normal. She had been working in a small shoe-factory for six months. The workplace didn't have appropriate air circulation and she was not using gloves. Her family history revealed, an acute polyradiculopathy in her father and grandmother in their teenage period. Her grandmother died from Gullian-Barre syndrome eight months ago at an age of 64 . Her vitamin B12 level was low and urinary 2,5-hexanedion excretion was positive.

Conclusion: Her clinical manifestations and electrophysilogical abnormalities recovered in a year after the discontinuation of exposure to $\mathrm{n}$ hexane. A few months after her presentation, two workers at the same workplace had similar symptoms and also were diagnosed as toxic neuropathy. We suggest that although all the workers had same exposure to n-hexane, the reason for our patient's earlier and severe presentation than other workers may be her genetic tendency towards neuropathy although it was not proven.

\section{Dementia}

P504

Treatment of dementia with rivastigmine: our experience

B. Potic, N. Despotovic, D. Milosevic, M. Davidovic, A. Potic

Clinical Centre "Zvezdara" (Belgrade, RS); Medical Faculty University of Belgrade (Belgrade, RS)

Introduction: The clinical manifestations of dementia are: a memory loss which is universal and often associated with aphasia, apraxia and agnosia; behavioural disturbances; a loss of the activities of daily living.

Material and methods: We analysed a group of 100 patients with the different types of dementia: $15 \%$ of the patients had Alzheimer's dementia, $25 \%$ of patients had Parkinson's disease dementia and the remaining $60 \%$ of the patients were with the vascular dementia.The group comprised the patients aged $65-85$ years with the gender ratio being: $55 \%$ males and $45 \%$ females. All of the patients presented with the distinctive behavioral disturbances: apathy and agression was recorded among $90 \%$ of the patients; $60 \%$ of the patients had the symptoms of anxiety, the symptoms of depression were observed among the $75 \%$ of the analysed patients. A loss of the daily living activities such are eating, toileting, shopping, was experienced by $35 \%$ of the patients. Within $40 \%$ of the patients the signs of the accompanied neurological dysfunction were detected: rigor, tremor, aphasia, apraxia, agnosia, pyramidal motor deficit. Besides the clinical examination, all the patients were tested with MMSE (mini mental state examination) and the brain computed tomography.The latter disclosed a fronto-temporal and hyppocampal atrophy in $79 \%$ of the patients. All the patients were treated with rivastigmine at the doses of $1-4 \mathrm{mg} /$ day and 6-12 $\mathrm{mg} /$ day during the period of six months. In parallel, all were receiving the additional therapy: statins, NSAID, antioxidans, vasodilatators, antiaggregants, sedatives

Results: MMSE test was repeated after the rivastigmine therapy completion.The group of the patients with the test score of $18-23$ before the treatment, gained the improved test results what correlated with the amelioration in the clinical picture:the improved attention, memory and the other cognitive functions, better quality of the behaviour and the activities of daily living.The group of the patients with the MMSE test results between 0-17 before the therapy, did not show any improvement

Conclusion: The outcome of the dementia management depends on the multiple factors. The milder the patient's functioning impairment upon the therapy initiation is, the better outcome of the applied treatment may be expected.This points to the the importance of the early diagnostic evaluation, treatment and the social support to the patients with dementia. 


\section{P505}

\section{MCI and Alzheimer - type dementia}

C. Maglaras, M. Parava, D. Tsiptsios, P. Beredimas

Psychiatric Hospital of Petra Olympou (Patra, GR); Psychiatric Hospital of Petra Olympou (Thessaloniki, GR); Psychiatric Hospital of Petra Olympou (Katerini, GR)

Introduction: Mild Cognitive Impairment (MCI) represents a diagnostic syndrome which has been introduced in order to determine the transitional stage of cognitive discount between normal aging and dementia. The most widely acceptable termination is "amnestic MCI" characterized by the following diagnostic criteria: memory impairment confirmed by a third observer, normal global cognitive function, objectively confirmed memory failure (psychometric tests), intact every-day activities and absence of dementia.

The aim of this study is to enlighten the prognostic factors that determinate the transition of MCI to Alzheimer Disease (AD).

Materials and methods: 100 patients that fulfilled the criteria of MCI have been examined in the neurologic department of Petra - Olympou Psychiatric Hospital and followed - up for the period of two years in order to detect the transition rate to $\mathrm{AD}$, in relation to the presence of psychiatric disorders. 55 of the examined patients suffered from psychiatric symptoms, whereas the rest 45 were free of any psychiatric condition.

Results: 11 subjects $(20 \%)$ from the group of the MCI - psychiatric patients fulfilled the $\mathrm{AD}$ criteria after a 2 year period, while the respective MCI patients without psychiatric symptomatology were only $3(4,4 \%)$.

Conclusion: The above evidence leads to the conclusion that the presence of psychiatric disorders in patients suffering from MCI represents an aggregating prognostic parameter in the development of $\mathrm{AD}$ at a follow-up of at least two years.

\section{P506}

Centro-conceptual aphasia: nosologic framework, clinical and imagistic description

D. Reisz, M. Simu, D. Chirileanu, A. Bursa

University of Medicine and Pharmacy (Timisoara, RO); County Hospital (Timisoara, RO)

From the first clinical descriptions of aphasic syndroms made by Broca $(1861,1863)$ and Wernicke $(1874)$ attempts have been made to build a neuropsychological explicative model for language. Over the years, many classification schemes and explicative models were developed to reach a more comprehensive model for the complex clinical reality of aphasia.

Objectives: The present paper intends to revisit the Wernicke-Lichtheim schema (1865) as a general neuropsychological model for language. We discuss arguments for the existence of a third center of language, different from the Broca center and the Wernicke center, proposed in the WernickeLichtheim schema as a centro-conceptual center and we attempt the identification of the anatomical location of this center.

Methods: We examined 11 patients that presented a post stroke aphasic syndrom characterized by impairments of language according to a description of centro-conceptual aphasia we have made in previous papers. The patients underwent a free interview followed by an evaluation of the aphasic syndrome using a method adapted to the Romanian language and an imagistic evaluation with MRI.

Results: From the manifold clinical expressions of aphasia there emerges a category of patients that present the characteristics of a unitary aphasic syndrom corresponding to the lesion of the presumed conceptual center of language. This centro-conceptual aphasia is characterised by a thick anomia for nouns that designate tangible objects, an anomia that results both in an impairment of language understanding as well as a non-fluent aphasia with difficulties in remembering and enunciating nominal nouns. The ability to work with numbers is relatively conserved, this performance being constantly better than the degree of aphasia would suggest. We also found a good preservation of operational words, verbs, connector words and the grammar structure of language. The lesions responsible for the appearance of this aphasic syndrom are either large lesions in the temporal lobe, or left parieto-occipital lesions.

Conclusion: The Wernicke-Lichtheim schema and classification prove to be useful instruments for the classification and understanding of language impairment with a large containing power and good clinical use, making a correct identification of the majority of clinical types of aphasia possible. Centro-conceptual aphasia proves the coherence and comprehensivity of the Wernicke Lichtheim model.
P507

Effects of antihypertensive therapy on cognitive decline in Alzheimer's disease

E. Duron, A. Rigaud, H. Lenoir, F. Latour, M. Seux, O. Hanon

Broca Hospital (Paris, FR)

The impact of antihypertensive therapy on the cognitive decline in subjects with already declared Alzheimer's disease (AD) is still unknown.

Objective: To evaluate prospectively, the impact of antihypertensive therapy on the cognitive decline in subjects with already declared $\mathrm{AD}$

Methods: We conducted a prospective study in a memory clinic including 290 outpatients suffering from $\mathrm{AD}$, according to the DSM IV international criteria. Each year, cognitive functions were assessed by the Mini Mental State Examination, during of follow-up period of 3 years. During this follow-up, cognitive functioning was compared considering the use of antihypertensive therapy or not.

All patients were treated with a Cholinesterase Inhibitor, the first line treatment of $\mathrm{AD}$.

Results: Mean age was $78 \pm 7$ years and $68 \%$ of patients (196/290) were female. Fifty-one percent (147/290) of the population used an antihypertensive therapy. At inclusion, the mean MMSE was similar in both groups (antihypertensive therapy versus no antihypertensive therapy $22,45 \pm 4,54$ vs $22,19 \pm 4,82, p=0.56$ ). After a three year follow-up, the cognitive decline was significantly less important among patients using antihypertensive therapy compared to those without antihypertensive therapy (MMSE scores $=$ $22,04 \pm 5,12$ vs $21,27 \pm 5,29$ at 1 year; $20,81 \pm 5,64$ vs $19,56 \pm 5,76$ at 2 years and $18,85 \pm 6,75$ vs $17,69 \pm 6,38$ at 3 years, $p<0.001$ for all periods). These results were observed in multivariate analysis after adjusting for confounding variables as age, sex and educational level.

Conclusion: These results suggest a positive effect of anti hypertensive therapy on cognitive function among patients suffering from $\mathrm{AD}$, by slowing down cognitive decline during a 3 year follow-up.

\section{P508}

Cerebrospinal fluid biomarkers in patients with Alzheimer's disease: Serbian group of patients

G. Mandic, E. Stefanova, I. Markovic

Institute of Neurology (Belgrade, RS); Institute of Biochemistry (Belgrade, RS)

Background: Alzheimer's disease (AD) is the most common form of dementia. Definitive diagnosis of AD can be given only by neuropathological examination of the brain specimen. One diagnostic tool are biomarkers in the cerebrospinal fluid (CSF), which is especially valuable early in the course of the disease. Suggested biomarkers are total tau protein (T-tau), phosphorylated tau protein (P-tau) and 42 amino acid form of amyloid-beta peptide (Abeta42).

Methods: $48 \mathrm{AD}$ patients (the diagnosis of $\mathrm{AD}$ was given following the DSM-IV and NINCSD-ARDA criteria) were compared to 23 healthy controls (the patient who undergone minor surgery under spinal anesthesia. Those who had Mini-Mental State Examination (MMSE) under 28 were excluded). The severity of dementia was evaluated using the MMSE. The duration of disease was estimated as the time since the first noticeable symptomes. CSF T-tau, P-tau 181, Abeta42 were determined using a sandwich ELISA (INNOTEST, Inoggenetics).

Results: Abeta42 was significantly decreased $(\mathrm{p}<0,001)$, P-tau and T-tau were significantly incresaed $(\mathrm{p}<0,001)$ and Abeta42/P-tau ratio was significantly lower $(p<0,001)$ in the AD group compared to healthy controls. There was no significant difference in the level of biomarkers in $\mathrm{AD}$ groups devided by the duration of the disease $(p>0,05)$, yet a significant difference was found in $\mathrm{AD}$ groups devided by the severity of the disease $(\mathrm{p}<0,001)$

Conclusion: Abeta42 was significantly decreased, P-tau and T-tau were significantly incresaed and Abeta42/P-tau ratio was significantly lower in the $\mathrm{AD}$ group compared to healthy controls. Stage specific decrease of Abeta 42 was shown as well as state specific increase of P-tau and T-tau. This suggest that these CSF bomarkers might be good additional biological surrogate markers for making the diagnosis of AD.

\section{P509}

Olfactory dysfunction relative to ApoE epsilon4 status in patients with mild cognitive impairment

H. Magerova, M. Vyhnalek, A. Varjassyova, J. Laczo, M. Bojar, J. Hort

Charles University, 2nd Medical School (Prague, CZ)

Objectives: Patients with Alzheimer's disease (AD) have an impairment of smell identification. This impairment occurs already in the amnestic mild cognitive impairment (aMCI) which precedes AD. People with ApoE ep- 
silon4 allele in genotype are at an increased risk to develop AD. The effect of ApoE epsilon4 allele on olfactory impairment is still uncertain.

Methods: The sample includes $16 \mathrm{AD}$ patients fulfilling the NINCDSADRDA criteria for probable $\mathrm{AD}, 47 \mathrm{MCI}$ patients who were classified as amnestic MCI (aMCI, $\mathrm{n}=37)$ and non-amnestic MCI (naMCI, $\mathrm{n}=10)$ based on Petersen's criteria and a control group $(n=17)$. All subjects underwent a multiple-choice smell identification test developed at our Memory clinic and the University of Pennsylvania Smell Identification Test. Furthermore, epsilon4 status was assessed to characterize the groups: AD/epsilon4- $(n=4)$, AD/epsilon4+ $(\mathrm{n}=12)$, aMCI/epsilon4- $(\mathrm{n}=22)$, aMCI/epsilon4+ $(\mathrm{n}=15)$ naMCI/epsilon4- $(n=6)$ and naMCI/epsilon4+ $(n=4)$. The epsilon4- and epsilon4+ groups did not differ in age and MMSE.

Results: Smell identification was impaired in both $\mathrm{AD}$ subgroups $(\mathrm{p}<0.005)$ and both aMCI subgroups $(\mathrm{p}<0.01)$. There was no significant difference between naMCI/epsilon4-, naMCI/epsilon4+ and controls.

Conclusion: The presence of ApoE epsilon4 allele in genotype does not influence the degree of smell identification impairment among the individuals with the same severity of the disease according to MMSE. The smell identification impairment in aMCI and intact smell identification in naMCI supports the hypothesis that smell identification is impaired early in $\mathrm{AD}$, whereas naMCI typically converts to non-AD dementia.

Supported by grants GAUK 7910/2007, GACR 309/05/0693 and internal grant of Motol Hospital.

\section{P510}

A case of cholinesterase inhibitor responsive posterior cortical atrophy H-M. Park, K-H. Park, S-H. Kim, Y-B. Lee

Gil Medical Center (Incheon, KR)

Objectives: Posterior cortical atrophy(PCA) is a rare, often presenile dementia that presents primarily with signs and symptoms of cortical visual dysfunction, but relatively preserved memory function until late stage of the disease. There are several reports about symptoms, imaging and pathologic findings. But report about treatment results is very rare. We report a case with PCA, confirmed by MRI and F18- FDG PET study, responsed to cholinesterase inhibitor.

Methods and results: A 58-year-old right handed woman presented initially with visual dimness and difficulty to finding stuffs around her. She had partial Balint syndrome, partial Gerstman syndrome, and idiomotor apraxia. She had mild memory disturbance also, but preserved insight of her disease. Neuropsychological evaluation showed decreased bilateral parietal and left temporal functions. Brain MRI and F18- FDG PET revealed typical bilateral occipitoparietal atrophy and hypometabolism. Cholinesterase inhibitor administration led to amelioration of symptoms, especially left temporal function, which we were confirmed by neuropsychological test.

Conclusion: Recently, there are many studies about clinico-pathologic correlations of PCA. They insist that PCA is not a variant of Alzheimer's disease, but a distinctive dementia syndrome. However, the treatment responsiveness of this case may support that PCA is the variant of Alzheimer's disease.

P511

Stroke and vascular cognitive impairment

I. Barbov, S. Vlaski-Jekic, I. Petrov, V. Petrova

Clinic of Neurology (Skopje, MK)

Background: The causes and characteristics of Mild Cognitive Impairment (MCI) after stroke and the risk factors related to it are still not well identified.

Purpose: To evaluate the cognitive capacities in a cohort of ischemic/hemorrhagic stroke survivors and to identify the clinical determinants of vascular MCI (vMCI) and dementia.

Methods: A total of 100 consecutive patients admitted to Neurological Clinic with a ischemic (76) or hemorrhagic (24) stroke were evaluated, 6 and 12 months after the stroke with a neuropsychological battery that included a screening battery (the Mini Mental State Examination - MMSE and the Hachinski Ischemic Score - HIS) and a comprehensive battery administrated to those who had a low score in MMSE. For the diagnosis of vascular MCI we used Petersen criteria. Vascular dementia was defined according to the DSM IV and ICD 10 criteria.

Results: vMCI frequency after 6 months was $40 \%$ and after 12 months was $38 \%$, being really a high percent. Dementia after 6 months developed $6(6 \%)$ and after 12 months, $10(10 \%)$ of patients. Our data suggest that deficits in cognitive domains other than memory (single domain MCI) are the most frequent cognitive decline, registered 6 and 12 months after stroke (22 vs. $19 \%$ ). Risk factors for vascular MCI are almost the same vascular risk factors for development of stroke. The most frequent risk factors in patients with vMCI are hypertension $(80 \%)$ and diabetes mellitus $(60 \%)$. Lesion type, side and localization in our group were heterogeneous.

Conclusion: Our data suggests that patients with stroke develop vMCI in the first 6 months after stroke, and dementia after 1 year. The impairment in cognitive domains other than memory (single domain $\mathrm{MCI}$ ) are the most frequent cognitive decline. vMCI and dementia most frequently appears in patients with multiple bilateral infarctions and in patients with cortical reduced changes and white matter changes

\section{P512}

Olfactory discrimination testing correlates with memory impairment in Alzheimer's disease and mild cognitive impairment but not in vascular dementia

M. Vyhnalek, H. Magerova, J. Laczo, A. Varjassyova, J. Hort

Charles University and Motol Hospital (Prague, CZ)

Background: The early impairment of olfactory identification in Alzheimer disease (AD) is well known and is considered to be caused mainly by degeneration in anterior olfactory nucleus, entorhinal cortex and amygdale which are in close anatomic connection with memory relevant temporal lobe structures. AD is often preceded by Mild cognitive impairment (MCI) and olfactory identification deficit has been found in MCI as well.

The smell identification impairment has been demonstrated also in Vascular dementia (VD). The anatomopathological substrate of this impairment in VD has not yet been determined.

The aim of this study was to evaluate the degree of olfactory dysfunction in $\mathrm{AD}, \mathrm{VD}$ and $\mathrm{MCI}$ and to correlate the data with results of memory and executive function tests.

Methods: 24 patients with mild AD according to NINCDS-ADRDA criteria (mean MMSE $=21), 17$ patients with VD according to NINDS-AIREN criteria (mean MMSE $=22$ ), 30 MCI patients according to Peterson's criteria (mean MMSE $=26$ ) and 18 healthy controls underwent a multiple choice olfactory identification test with 18 different odors developed in our memory clinic and a battery of neuropsychological tests including the Auditory verbal learning test (AVLT), TMT A, B and verbal fluency test.

Results: $\mathrm{AD}, \mathrm{VD}$ and $\mathrm{MCI}$ patients showed considerable impairment in olfactory identification test compared to controls $(\mathrm{p}<0,001)$. The mean scores (and interquartile ranges) for VD, AD, MCI and controls were 9,3 $(5,2)$; $8,9(2,2) ; 10,3(6,2)$ and $15,7(2,5)$, respectively. The results of smell test significantly correlated with AVLT $1-5$ scores in AD and MCI patients $(\mathrm{p}<0,01$, $r=0,58$ and $p<0,01, r=0,53$ respectively). We found no correlation with AVLT in VD patients $(\mathrm{p}=0,34, \mathrm{r}=0,21)$ and no correlation of smell test with executive dysfunction in any of the patient groups.

Conclusion: The olfactory identification is impaired in all 3 patient groups. The correlation with memory test in $\mathrm{AD}$ and MCI patients could reflect the medial temporal lobe degeneration in these diagnoses. There was no correlation with memory in VD contrasting to AD. This demonstrates greater heterogeneity of smell impairment in VD and reflects possible different anatomopathological substrates involved in olfactory impairment in VD patients.

Supported by Charles University grant 7910/2007

\section{P513}

Rapidly progressive dementia: Whipple's disease with isolated involvement of the central nervous system

M.N. Cardoso, J.C. Damasio, J. Chaves, A.P. Correia

Hospital Geral de Santo Antonio (Porto, PT)

Introduction: Whipple's disease is a rare systemic disease caused by Tropheryma whipplei. The common clinical picture reflects preferential involvement of gastrointestinal and osteoarticular systems. Isolated central nervous system (CNS) infection is described only in 32 patients. The most frequent neurologic manifestations are cognitive impairment, neuropsychiatric symptoms, supranuclear palsy, hypothalamic dysfunction, oculomasticatory or oculo-facio-skeletal myorhythmia.

Case report: A 60-years-old woman with progressive cognitive deterioration, neuropsychiatric disturbances (social/feeding behaviour and hallucinatory-delusional activity), and alterations in sleep pattern was evaluated in June 2006. Four months later, a generalized (axial predominance) myoclonus was noted. She was admitted in our hospital in November 2006. The neurological examination showed apathy, fluctuation of attention, delusional speech, visual hallucinations, apendicular and axial rigidity, generalized myoclonic tremor with perioral and eyelid involvement. The diagnostic workup (including brain magnetic resonance imaging, electroencephalogram and a test for 14-3-3 protein in the CSF) was normal or negative. In March 2007, she was readmitted on account of clinical worsen- 
ing, with akinetic mutism, apraxia of lid opening, supranuclear gaze palsy and a more exuberant myoclonic tremor. A brain single photon emission computed tomography (SPECT) showed a left fronto-temporal hypoperfusion. Polymerase chain reaction (PCR) tests for T. Whipplei in cerebrospinal fluid, blood and duodenal mucosa were positive. Duodenal mucosa periodic acid Schiff (PAS) staining was negative. Ceftriaxone was initiated, followed by oral cotrimoxazol. Six months after treatment initiation, she had myoclonus improvement. Nonetheless, cognitive impairment was maintained and behavior alterations worsened with agitation, disinhibition and exuberant grasping.

Comments: Whipple's disease with isolated CNS involvement should be considered in the differential diagnosis of rapidly progressive dementia. Neuropsychiatric symptoms can be prominent at the beginning and may delay diagnosis. Although treatable, prognosis is reserved. Approximately $50 \%$ of patients present clinical improvement, $25 \%$ have severe neurological sequelae and $25 \%$ die. We believe this patient has probable Whipple's disease, given the clinical and laboratory results. The findings of SPECT were described in the literature.

\section{P514}

Early diagnosis of Alzheimer's with biomarkers in cerebrospinal fluid

N. Chandra, A. Singh, V. Kumar Singh

GSVM Kanpur (Kanpur, IN); Medical College (Sikkim, IN)

Objective: Alzheimer's disease (AD) is the most common cause of dementia among population of age 65 and older. The diagnosis of sporadic $\mathrm{AD}$ is based on clinical exclusion criteria and is definitive after autopsy of brain after death. From many years doctors and scientist are working in direction to find marker which could help physician to make early diagnosis.

Methods: At our hospital we studied cohort of $37 \mathrm{AD}$ patients who were on acetylcholinestrases drug. Established biomarkers present in cerebrospinal fluid (CSF), like total tau(T-tau), phosphorylated tau(P-tau) and 42 amino acid isoform of beta-amyloid (Abeta42) were analysed.

Results: Abeta 42 predicted AD with sensivity of $76 \%$ and sensivity of $100 \%$. p-tau predicted sensivity of $71 \%$ and specificity of $85 \%$.

Conclusion: Outcome of study is that combined biomarker are more reliable as compare to single. Neuropsychological assessment and CSF biomarkers as well as neuroimaging findings (both structural and functional) have reported variable accuracy values, but better results have been obtained by combined biomarker approach.

P515

Reversible dementia due to herpes simplex type 2 encephalitis with normal MR imaging

P. Urban, W. Jöhnck, S. Marrakchi, R. Brüning

Asklepios Hospital Barmbek (Hamburg, DE)

Objectives: Herpes simplex type 2 encephalitis is rare outside the neonatal period and has not so far been described in an immunocompetent adult presenting with dementia.

Results: A 78 year old immunocompetent man presented with a one year history of progressive impairment of short term memory and word finding difficulties. On admission he was alert but not oriented in time and situation. Short term memory was decreased. Speech production was decreased and slight word finding difficulties were present. The mini mental test (MMSE) was 21. Neuropsychometry revealed intellectual underfunctioning on tests of sustained attention and concentration, impairment of memory functions, and evidence of visual perceptual and frontal executive difficulties indicative of a considerable cognitive dysfunction. Brain MRI brain was unremarkable. Electroencephalogram showed a $9 \mathrm{~Hz}$ rhythm with intermittent excess of bilateral frontotemporal $3 \mathrm{~Hz}$ activity. Cerebrospinal fluid (table) contained 633 leucocytes $/ \mu l$ ( $99 \%$ lymphocytes), raised protein $(1.339 \mathrm{mg} / \mathrm{l}$, normal: $<500)$, reduced CSF:serum glucose ratio $(35: 103 \mathrm{mg} / \mathrm{dl}$ $=34 \%)$, increased lactate $(3.2 \mathrm{mmol} /$, normal: $<2.1)$, raised IL-6 (195 ng/l, normal: $<8.1$ ). PCR for herpes simplex type 2 was positive. The patient was treated with a ten day course of intravenous aciclovir $(3 \times 750 \mathrm{mg} / \mathrm{d})$. Since the PCR for HSV 2 remained positive in the CSF following aciclovir treatment, a subsequent treatment with valaciclovir $(3 \times 1 \mathrm{~g} / \mathrm{d})$ was started over a period of three months. In a repeat CSF examination after that time the PCR for HSV 2 became negative and antiviral treatment was terminated. MMSE improved to a score of 29 .

Conclusion: Our observation further expands the increasing spectrum of atypical findings in herpes simplex virus encephalitis. HSV 2 encephalitis may present as dementia without MRI abnormalities. Due to the excellent treatment response a HSV 2 encephalitis should be considered in the diagnostic work up of dementia. Even in immunocompetent older patients with dementia the necessity of CSF examination is underlined.
P516

Haemodynamic effects of nilvadipine in an Alzheimer dementia trial

S. Kennelly, F. Crawford, M. Mullan, B. Lawlor, R.A. Kenny

Trinity College Dublin (Dublin, IE); Roskamp Institute (Sarasota, US)

Introduction: The treatment of hypertension with a dihydropyridine calcium channel blocker (DHP-CCB) might reduce the incidence of Alzheimer dementia (AD) by $55 \%$. Nilvadipine is a highly lipophilic DHP-CCB, which crosses the blood brain barrier. It increases cerebral blood flow and may interfere with cerebral Beta-amyloid levels in AD. No study to date has examined the tolerability of Nilvadipine within a non-hypertensive $\mathrm{AD}$ population.

Methods: A ten-week open label study deign was used. Subjects with mild/moderate AD (MMSE 14-27) were enrolled. Subjects with orthostatic hypotension $(\mathrm{OH})$ or on current antihypertensive treatment were excluded. $\mathrm{OH}$ was diagnosed on the basis of a symptomatic drop in blood pressure (BP) on standing, or a fall in $\mathrm{BP}$ of $>20 / 10 \mathrm{mmHg}$ which failed to recover to baseline in 3 minutes. Active stands (AS) were performed using an Amsterdam finometer. Ambulatory BP monitors were performed prior to and following treatment for six weeks. Mean recordings of $>10 \mathrm{BP}$ readings were required for inclusion of data for analysis.

Results: The study group ( $n=32$; mean age 68.1yrs; 15 female; Mini Mental State Examination (MMSE) $19.7 \pm 4.3$ ) received Nilvadipine $8 \mathrm{mg}$ CR daily for 6 weeks. The control group $(n=12$; mean age 72.3 yrs; 6 female; MMSE $23.2 \pm 2.9$ ) received no treatment. There was no significant decline in either systolic or diastolic blood pressure from baseline $(131.6 / 78.8 \mathrm{mmHg})$ to follow up $(132.3 / 77.0 \mathrm{mmHg})$ while on Nilvadipine. No significant increase in the "initial" systolic BP drop in the treatment group (Baseline drop $31.3 \mathrm{mmHg}$ versus $33.3 \mathrm{mmHg}$ (on Nilvadipine) $\mathrm{p}<0.58$ ) was noted on AS. A significant increase in the drop in Diastolic BP was evident (Baseline drop $12.8 \mathrm{mmHg}$ vs $20.6 \mathrm{mmHg}$ (on Nilvadipine) $\mathrm{p}<0.01$ ). This drop was symptomatic in one subject who was diagnosed with $\mathrm{OH}$ following treatment. No significant changes were noted in the BP monitoring or the active stands within the control group. There was no significant change in MMSE $(+0.72$ $\mathrm{p}<0.16)$ or the EXIT $25(-2.03 \mathrm{p}<0.12)$ within the study group. No subject reported symptoms of $\mathrm{OH}$ or falls during the trial period.

Conclusion: Nilvadipine was well tolerated within this group of non-hypertensive patients with AD. No subject was discontinued off the drug. Further randomised, blinded studies are required to examine its efficacy in $\mathrm{AD}$. A correlation of alterations in cerebral blood flow and changes in $\mathrm{CSF} /$ plasma/serum A-beta levels will be performed when the sample size is larger.

\section{Infection}

\section{P517}

Atypical subacute sclerosing panencephalitis. Report of one case A. Lugo-Pong, G. Cárdenas, J.I. Castro-Macias National Institute of Neurology (Mexico City, MX)

Introduction: Subacute sclerosing panencephalitis (SSPE) is a progressive inflammatory disorder of the central nervous system with both poor prognosis and high mortality. The disease has been related to a persistent and aberrant measles virus infection and no effective treatment has been available.

Objective: To emphasize the case of a patient with atypical SSPE.

Case report: A 17 year old male, who had been previously immunized against measles in his childhood. He began his illness 10 months before with generalized seizures and slight behavioural changes at the college, after that his mother realised that he was complaining of progressive leg weakness and non specific visual disturbances. Neurological examination showed bilateral myoclonic jerks, macular swelling, papilledema and severe cognitive deterioration. Electroencephalography showed pseudoperiodic sharp and slow wave discharges. Magnetic resonance showed diffuse T2 signal hyperintensities in both cortico-subcortical and periventricular regions. Highly positive titters of antimeales antibodies. He began treatment with Isoprinosine and intratechal interferon alfa, despite transient subjective improvement; he continued to deteriorate and finally died.

Conclusion: SSPE is a progressive inflammatory disorder of the central nervous system related to a persistent and aberrant measles infection, the main clinical manifestation at the beginning are behavioural changes and in our case the first manifestation was generalized seizures and progressive deterioration in few months and finally he died despite of transient improvement. We should be alert of the possibility that the clinical presentation of SSPE in the context of exposures to measles virus and particularly to measles immunization. 
P518

HIV-1 infection of human neuronal cultures results in aberrant neurotransmitter synthesis

A. Palser, G. Trillo-Pazos, E. Jauniaux, D.H. Miller, R. Weiss

Institute of Neurology (London, UK); University College Hospital (London, UK); Wohl Virion Centre, UCL (London, UK)

Objectives: HIV-1 associated dementia (HAD) affects approximately $25 \%$ of untreated HIV-1 infected individuals and manifests clinically with motor and cognitive impairments $10-25$ years after initial infection. HIV enters the brain early in disease and infects multiple cell types, leading to microgliosis, astrocytosis and neuronal damage. In vitro up to $10 \%$ of neurons can be infected with HIV-1 but little is known concerning the characteristics of these cells or the mechanisms underlying the restricted infection of neurons. We aimed to characterise the vulnerability of different neuronal subtypes to HIV-1 infection using a primary human neuronal culture system.

Methods: We have developed an in vitro human neuronal culture system comprising approximately $70 \%$ neurons, $25 \%$ astrocytes, $5 \%$ oligodendrocytes and no microglia. Cultures were infected with both CXCR4 and CCR5using HIV-1 viruses and expression of neurotransmitter synthetic enzymes for dopamine, 5HT, noradrenaline, GABA and acetylcholine measured by Western blotting and RT-PCR at up to 21 days post HIV-1 infection. Infectious virus production was measured by titration of culture supernatant on chemokine receptor expressing NP2 glioma cells.

Results: Infection of these cultures with HIV-1 is characterised by neuronal apoptosis and astrocytosis. Expression of neurotransmitter synthetic enzymes for dopamine, GABA and serotonin are decreased as a result of HIV- 1 infection with both CCR 5 and CXCR4-using virus at 3 to 21 days post infection. Furthermore, infectious virus is produced at timepoints co-incident with the loss of neurotransmitter synthetic enzymes. In contrast, cholinergic markers are increased at later timepoints following infection. GABAergic neurons appear to be more markedly affected by CXCR4-using virus compared with CCR5-using virus.

Conclusion: These findings suggest that dopaminergic, serotonergic, GABAergic and cholinergic systems are specifically affected by HIV-1 infection. These neurotransmitter systems have been implicated in other forms of dementia, such as Alzheimer's disease. Particular subpopulations of neurons may therefore be a target for the virus in the brain and this may enable development of new therapies to target cognitive impairment.

\section{P519}

Neurological evaluation of the leper king Baldwin IV of Jerusalem

A.L. Guerrero

Hospital Clínico Universitario (Valladolid, ES)

Objectives: Leprosy is an ancient disease, and references to it are found in the Bible. In the medieval period, physicians became more aware of leprosy symptoms and differentiated it from other similar diseases. Baldwin, the leper king of Jerusalem (1161-1186), probably contributed to an increasing interest and tolerance to this disease in medieval Christian states. In this work we review historical descriptions of the neurological manifestations he developed.

Methods: William of Tyre, tutor of Prince Baldwin and out-standing historian, gives us in his Chronicle a description of first symptoms experienced by nine-years-old prince. He observed that, when playing with friends, pinching each other with their nails, the others cried out when they were hurt, whereas Baldwin bore it all with patience. William noticed then that half of his right arm and hand were partially numb. No hypopigmented patches, other skin lesions, or thickened palpable nerves were described. At this moment, records do not mention a definite diagnosis of leprosy, but his father, king Amalric, was concerned about the problems of future kingdom succession. During Baldwin's puberty, cutaneous lesions appeared and diagnosis was evident. His condition became worse and, by his early twenties, muscle weakness made him unable to walk. He got blinded, probably due to keratopathy related to cranial nerves involvement. Repeated attacks of fever led to progressive worsening of his disease, and he died in Jerusalem, aged twenty-five, perhaps because of a septicaemia from infected sores.

Results: The earliest sign of Baldwin's disease was anaesthesia; it is, so, likely, that at this point he had either tuberculoid or pure neural form of leprosy. Over time he downgraded to the lepromatous form. We review therapy available in the crusader period against this disease, and segregation into leprosaria criteria in European medieval states.

Conclusion: Leper king Baldwin biography gives us interesting descriptions of neurological clinical features of leprosy. Besides, it helps us to discover twelfth century medicine knowledge of the symptoms of leprosy, and the general approach of the treatment of those with this disease in this period.

\section{P520}

Acute chorea on reactivation of Epstein-Barr virus infection as the first manifestation of AIDS

C. Castillo, A.C. Fonseca, A. Lobanova, H. Foreid, R. Geraldes, L. Albuquerque Hospital de Santa Maria (Lisbon, PT)

Background: Movement disorders are reported in 2 to $3 \%$ of patients with AIDS, mainly tremor, parkinsonism, hemichorea and hemibalism. Generalized chorea is rarely described in association with infection with toxoplasmosis, progressive multifocal leukoencephalopathy and HIV dementia.

Clinical Case: We report a case of acute chorea on reactivation of Epstein Barr infection as the first manifestation of AIDS.

A 71-year-old woman with a personal history of diabetes and with no familiar history of movement disorders was admitted after the acute onset of generalized involuntary movements of the four limbs. These changes had begun five days before admission and simultaneous with fever, headache, sore throat and non productive cough. Neurological examination showed brief and fast segmentary involuntary movements of the four extremities General observation disclosed fever $-38,5^{\circ} \mathrm{C}$ tympanic temperature, tachypnea and diffuse inspiratory crackles. There was no hypoxemia, hypocalcemia, hyponatremia or blood sugar changes. Chest x-ray showed diffuse interstitial enhancement. Laboratory examinations disclosed lymphopenia, anemia, thrombocytopenia and C-protein of $8,8 \mathrm{mg} / \mathrm{dL}$. CSF had 17 lymphocytes. Blood serologies were positive for IgM and IgG Viral Capside Antigen (VCA), IgG EBV nuclear antigen (EBNA). VIH was positive with a total count of CD4+ of $90 \mathrm{cel} / \mathrm{ml}$. Blood serologies for Cytomegalovirus, Toxoplasmosis, M. pneumonia, C. burnetti and Chlamydia were negative. Antistreptolysin O titers were normal. CSF serologies were negative for Cryptococcus and syphilis. M. tuberculosis, M. pneumonia and JC virus PCR in the CSF were negative. Brain MRI showed unspecific white matter changes. The patient was on haloperidol for 24 hours and was empirically treated with amoxicillin and clavulanic acid. Three days after admission chorea had remitted and C-protein started to decline. A second lumbar puncture performed twelve days after the first had only two lymphocytes.

Discussion: The beginning of neurological symptoms simultaneously with the onset of the respiratory infection and accompanying serologies evocative of reactivation of infection by Epstein Barr Virus suggests that there may be a direct connection between them.

To our knowledge acute reversible generalized chorea in the setting of reactivation of Epstein-Barr in a patient with AIDS has never been reported.

\section{P521}

Microglial activation causes neuronal depolarisation in a glial/neuronal coculture model

D. Hinkerohe, N. Prochnow, A. Haghikia, D. Wolfkühler, U. Schlegel, P. Faustmann, R. Dermietzel

Knappschaftshospital Ruhr-University (Bochum, DE)

Objectives: The balanced presence of microglia and astrocytes is an essential condition for the functional integrity of neurons of the central nervous system in healthy subjects. While astrocytes play a major role in the regulation of ion and neurotransmitter homeostasis, microglia influences specific and unspecific immunoresponses. Proinflammatory conditions like increased percentages of microglia, lead to a functional decoupling and increase in resting potential of cocultured astrocytes in vitro (Hinkerohe et al., 2005).

Methods: In the present study we established a triple coculture model of neurons, astrocytes and microglia to investigate the direct influence of evoked proinflammatory conditions on neurons in an astroglial syncytium. We demonstrate in whole-cell patch clamp recordings that neurons' membrane resting potentials become significantly depolarized, when gap junctional coupling in astrocytes is reduced under microglial activation. In order to check for the astroglial coupling properties Lucifer yellow was added to the recording pipette solution. The degree of fluorescence spread gave rise for the cell to cell connectivity in the syncytium. Light-, Fluorescence imaging and combined immuncytochemistry against $\mathrm{C} \times 36, \mathrm{C} \times 43, \mathrm{ED} 1$, GFAP, glutamate and Calcium binding proteins was used to describe the morphological status of the cocultured neurons, microglia and astrocytes in detail.

Results: Under proinflammatory condition (30\% microglia) $\mathrm{C} \times 43$ expression in astrocytes is reduced significantly $(\mathrm{p}=0.0364$, Man-Whitney-UTest) and microglia is activated. In coincidence neuronal resting potential is depolarized in a statistical strongly significant manner $(p=0.0014$, ManWhitney-U-Test). Possible changes of the neurons' immunohistochemical properties are still analyzed.

Conclusion: We assume the astrocytotic $\mathrm{K}+$ release to be responsible for the neuronal depolarization. The present combination of techniques in a coculture system makes molecular and physiological effects of neuroinflam- 
mation measurable. Neuroinflammation in vivo may be mimicked and treatment effects may be analyzed in such a model in vitro.

\section{P522}

Brain abscesses caused by Listeria monocytogenes in non-imunocompromised host

D. Mon, C. Serrano-Munuera, A. Gasos, P. Comas, M. Alarcon

Hospital Sant Joan de déu de Martorell (Martorell, ES)

Objective: Brain abscess caused by Listeria monocytogenes in a healthy man is reported.

Introduction: L. monocytogenes is a pathogen that causes meningitis, meningoencephalitis, or bacteriemia. It usually occurs in neonates, immunocompromised hosts and elderly adults. Brain abscesses are extremely rare.

Case report: A 52 year-old man with a history of diabetes was admitted in hospital because of left-side focal seizure followed by generalized tonicclonic seizures. No history of fever or headache was reported. Plain brain computed tomography (CT) was normal. Contrast administration revealed three hyperdense cortical lesions, two in the right frontal lobe, and one in the right occipital lobe. Magnetic resonance imaging (MRI) showed a fourth additional lesion in the frontal lobe on T2 and FLAIR-weighted images, with homogeneous contrast enhancement. No edema, hemorrhage or hydrocephalus was reported. No sinusitis, otitis or congenital malformations were found. Cerebrospinal fluid (CSF) culture was positive for L. Monocytogenes. CSF glucose and protein concentrations were normal. CSF cells count and pathological exam excluded malignancy. Blood cultures were negative. Routine laboratory studies were normal. Serology for HIV was negative. Whole body CT was normal. No evidence of right-to-left shunt or valvular vegetations was shown by echocardiography. Electroencephalogram was normal.

The diagnosis of brain abscesses by L. monocitogenes was made and patient was treated with endovenous ampicillin and gentamicin. Seizures were treated with valproate with good response. A new MRI two months later showed no change in lesions size however there was not contrast enhancement.

Conclusion: We present a case of brain abscesses by L. monocytogenes in an inmunocompetent patient. Brain abscesses by L. monocitogenes are infrequent since the most common form of CNS listeriosis is meningoencephalitis. Their localitation is usually subcortical (i.e. thalamus, pons and medulla) and mortality is three times higher than nonlisterial brain abscesses. Neurological sequelae are common among survivors. The patient we present is somehow extraordinaire since he did not suffer from any predisposing condition for such a central nervous system infection. In addition, the abscesses location in frontal and occipital lobes was atypical as well as the benign course that may be explained by his immunocompetent condition.

\section{P523}

Insidious sinusitis leading to catastrophic cerebral aspergillosis in transplant recipients

D. van de Beek, R. Patel, N. Campeau, A. Badley, J. Parisi, A. Rabinstein, E. Manno, E. Wijdicks

Mayo Clinic (Rochester, US)

Objectives: Cerebral aspergillosis, fortunately rare, is an important cause of mortality in organ transplant recipients.

Its clinical recognition can be problematic. We describe two transplant recipients with insidious Aspergillus sinusitis leading to catastrophic CNS infection.

Methods: We present two patients with sinusitis leading to catastrophic cerebral aspergillosis. We measured galactomannan in serum and CSF.

Results: Patient 1: A 56-year old male with a pancreas and kidney transplant for insulin dependent diabetes developed meningoencephalitis and cerebral infarction. He had persistent headaches and fever for six weeks.

Three weeks after admission, his level of consciousness suddenly worsened. CT showed right frontal cerebral hemorrhage with extension to the ventricles, and hydrocephalus. The serum galactomannan antigen test was normal (0.39 index), but elevated in CSF (6.47 index).Autopsy showed cerebral edema with frontal hematomas with extension into the ventricular system resulting in midline midbrain herniation. There were acute and subacute cerebral infarcts in multiple vascular territories. Microscopy showed focal vascular invasion of Aspergillus species. Patient 2: A 62-year old female with kidney transplant secondary to diabetes had a history of headache of six month duration. Twelve days after admission, she suddenly deteriorated. CT demonstrated new subarachnoid hemorrhage. Serum galactomannan antigen titers were normal (0.06 index), but in CSF antigen titers were elevated (2.72 index). Autopsy was consistent with cerebral aspergillosis.
Conclusion: New onset headache in transplant recipients should raise the possibility of sinus aspergillosis that can lead to central nervous system (CNS) involvement with poor outcome. Galactomannan antigen in CSF may be a useful diagnostic test in suspected cerebral aspergillosis, even if serum titers are normal.

P524

A rare case of CNS fusariosis

D.S. Shim, J.H. Seo, S.M. Kim, I.N. Sunwoo

Yonsei University College of Medicine (Seoul, KR)

Objectives: Fusarioum species (spp.) cause localized infection in immunocompetent hosts. It rarely cause systemic infection in immunocompromized hosts, especially the patients with hematologic malignancies, but, CNS infection is very rare and is regarded to be disseminated from the remote source. Here we report a case of CNS fusariosis.

Case presentation: A forty one years old male presented with decreased mentality during the admission in the hospital. He had been diagnosed as hemolytic anemia two years ago and has been treated with immunosuppressants. Three months before the attack, he had admitted to the hospital for fever and cough lasted for a month and for the evaluation of the lung mass revealed by chest computed tomography (CT). There was no evidence of malginancy in whole body bone scan, positron emission tomography, repeated sputum cytology and bronchial washing cytology. Lung aspiration cytology revealed necroinflammatory excudate. Transbronchial lung biopsy (TBLB) showed acute nonspecific suppurative inflammation. He was diagnosed as lung abscess of unknown pathogen. He was discharged with oral roxithromycin. He developed fever, chill, cough, and sputum in 2 months af ter discharge and was re-admitted to the hospital. Chest CT taken at the outpatinet clinic showed that the previous lung mass decreased in the size with cavitation, but, new segmental consolidation developed. Chest CT was followed during the admission and showed aggrevation of the findings. TBLB was repeated only to fail to show the etiology. On the 15th hospital day (HD) high spiking fever developed and he became drowsy in five days. Vital sign was stable. He had no focal neurologic deficit. Brain CT and magnetic resonance image (MRI) manifested multiple rim enhancing lesions in left frontal lobe and basal ganglia with mass effect and subfalcine herniation. He recovered alertness after the drainage of the brain abscess, which was removed surgically subsequently. Brain pathology confirmed invasive fusariosis. Lung tissue was re-evaluated and two PAS-positive banana-shaped fungal organisms were also found. He was treated with intravenous amphothericin $\mathrm{B}$ for 7 days and discharged with oral voriconazole.

Conclusion: We presented a rare case of CNS fusariosis. The diagnosis might be mysterious if not for the brain pathology, even though the cerebral lesions are presumed to be spread from the lung abcess, which was repeatedly biopsied only to fail to establish the etiology.

\section{P525}

Types of respiratory impairment in patients with acute tick-borne encephalitis

E.V. Prazdnichkova, L.I. Volkova

Regional Clinical Hospital 1 (Ekaterinburg, RU)

Middle Urals is an endemic for tick-born encephalitis (TBE) region of Russia, the average morbidity rate being 3 times higher than corresponding rate in Russian Federation. During last years the high TBE morbidity is being registered in Urals (7.6 - 42.1 per 100 thousand population). The frequent occurrence $(7-10 \%)$ of severe focal forms is being noted also with isolate or complex impairment of different central nervous system levels.

Materials and methods: During 7 years (1999-2006) 115 patients with multilevel focal TBE forms were treated in neurology department of Regional Clinical Hospital 1 of Ekaterinburg, Russia. 48 patients $(70.6 \%)$ with the most severe forms were being treated in intensive care unit (ICU). All patients had been transferred to ICU at the $7 \pm 4.5$ day of TBE. $39(81.2 \%)$ patients showed respiratory impairment and had needed ventilation.

Results: We defined 4 types of respiratory impairment in TBE patients:

1. Central type in $19(39.6 \%)$ patients, that developed due to progressing cerebral oedema;

2. Bulbar type in $35.5 \%$ of patients, that developed due to involvement of bulbar muscles and aspiration;

3. Periferal type in $4,2 \%$ of patients due to pareses of respiratory muscles and diaphragm;

4. Combinatory type of respiratory disorder $(20,7 \%)$.

Among 48 patients that had been treated in ICU lethal outcome was registered in 18 patients at $40 \pm 38.8$ day of the disease. Secondary bronchopulmonary infection (in 61.5\%), progressing cerebral and brainstem oedema $(34.6 \%)$, sepsis in $11.5 \%$ and pulmonary embolism (in $3.8 \%$ ) were 
registered to be main causes of death. Favourable outcome was noted in patients, that were started to being ventilated earlier (at $5 \pm 2.7$ days of the disease) in contrast with group of patients with lethal outcome, in which ventilation was being started at $8.8 \pm 5.5$ day of the disease. Also among favourable risk factors we noted high level of consciousness and rate of saturation when ventilation started and earlier tracheostomy (at $3.1 \pm 1.6$ day of the disease).

Conclusion: The routine examination of patients with severe focal TBE forms should be focused at the detection of elementary signs of respiratory impairment, that can be predictors of respiratory failure and include investigation of respiratory rate, bulbar signs, level of consviousness and polyomielitic syndrome.

\section{P526}

Screening of clinical efficiency of albendazole and its association to plasmatic levels

G. Cárdenas, H. Jung, A. Fleury, C. Marquez

National Institute of Neurology (Mexico City, MX)

Introduction: Albendazole (ALB) is currently use for the treatment of neurocysticercosis. Albendazole has been found to be more effective than praziquantel, but it has low solubility and an extent metabolism of first step, it disminishes its absorption and only albendazole sulphoxide (AS), the active metabolite can be detected, according to this much variability exists in intra and inter individual plasmatic levels of AS. Until now there is not information about the minimal effective concentration of AS for the treatment of this illness.

Objective: To determine if there is relationship between the plasmatic levels of AS and clinical- therapeutical response.

Methods: We performed a prospective, longitudinal study with $37 \mathrm{pa}-$ tients with neurocysticercosis, with an age range from 16 to 68 years. All of them received $30 \mathrm{mg} / \mathrm{kg} / \mathrm{d}$ of ALB during 8 days. We determine the plasmatic concentration at eighth day of treatment. In order to classify clinical-therapeutical response we consider reactive and non reactive to treatment, the first were those whose parasites disappeared or were calcified and the second one were all whose parasites suffer no changes.

Results: A total of 24 patients were considered reactive to treatment and 14 non reactive. The main parasite localizations were subarachnoideal space or intraventricular. The intensity of cerebrospinal fluid (CSF) inflammation was associated with non response to treatment. Plasmatic levels of AS showed great variability. In the group of reactive, levels varied between 0.12 and $5.8 \mu \mathrm{g} / \mathrm{ml}(1.34 \pm 1.08)$ and in non reactive levels varied between. 3 y 2.7 $\mu \mathrm{g} / \mathrm{ml}(1.59 \pm 1.5)$, there were no significant differences, $\mathrm{P}=0.62$. Between sex and age there were no differences $(\mathrm{P}=0.68$ and $\mathrm{P}=0.33$ respectively)

Conclusion: We included several physiological variables to the analysis and we did not find any relation between plasmatic levels of AS and clinicaltherapeutical response.

P527

Cerebral toxocariasis - a multifaceted disease

G. Mihailescu, C. Mihailescu, E.I. Davidescu, S. Nica, C. Nuta

UMF Carol Davila Bucharest (Bucharest, RO); Al. OBREGIA Clinical Hospi-

tal (Bucharest, RO); IOMC (Bucharest, RO)

Objective: Toxocariasis is a consequence of human infection by the larvae of Toxocara canis. It can be in most cases asymptomatic. When symptomatic it depends on the invasion of larva migrans visceralis in different organs (liver, lungs, eyes, muscles and central nervous system - brain or spinal cord). It is considered one of the major infectious agents causing epilepsy, but also generating encephalitis, myelitis and vasculitis. The neurological examination may show a great variety of symptoms and signs making the diagnosis very difficult.

Methods: We studied 18 patients ( 8 men and 10 women) over 20 years of age, diagnosed with cerebral toxocariasis, admitted over a period of 5 years in the Neurology Department of the Colentina Hospital in Bucharest, regarding demographic characteristics, symptoms and signs, diagnosis, associated disorders and management.

Results: At admittance, the patients presented with pyramidal syndrome $(n=8)$, cerebellar syndrome $(n=6)$, seizures $(n=5)$, cranial nervs pathology (VII-XII) $(n=5)$, sensitivity disturbances $(n=5)$, intracranial hypertension $(\mathrm{n}=1)$.

The diagnosis was based on: eosinophilia, positive ELISA tests for antitoxocara antibodies in serum and cerebrospinal fluid, cerebral computer tomography and magnetic resonance imaging, histopathological examination showing eosinophil granulomata and vasculitis localised in the white matter and also on the clinical and radiological improvement and normalisation of the cerebrospinal fluid parameters following antihelmintic therapy. As as- sociated pathologies we found cerebral lacunarism $(n=2)$, demyelinating disease $(\mathrm{n}=2)$, astrocytoma $(\mathrm{n}=1)$ and polyradiculoneuritis $(\mathrm{n}=1)$.

Conclusions: In a patient with hypereosinophilia of unknown origin and cerebral granulomatous disease, the differential diagnosis of Toxocara canis infection must not be overlooked. Toxocariasis may be a difficult diagnosis due to the variety of clinical symptoms and signs. In spite of a lower specificity the immunological and imagistic methods are a valuable asset in the diagnosis and treatment of cerebral toxocarosis.

\section{P528}

Clinical and virological observations in acute encephalitic syndrome G. Ngonga, P. Locatelli, L. Signorini, L. Soavi, G. Carosi, A. Padovani Spedali Civili di BS (Brescia, IT)

Objective: To acquire data on the burden of acute encephalitis syndrome with clear cerebrospinal fluid (CSF) in our hospital; define which infectious agents are most frequently found in CSF, and evaluate the clinical outcome of these patients.

Method: We collected the data on patients with acute encephalitis syndrome admitted in our department from November 2006 to December 2007. The patients all underwent lumbar puncture and performed neuroimaging (brain CT scan and/or MRI), electroencephalogram (EEG), in the acute phase of the illness. We performed routine study of CSF (cell count, glucose and protein, viral/Koch bacillus culture and/or PCR detection of DNA/RNA virus, oligoclonal bands). All patients underwent antiviral treatment with acyclovir.

Results: We collected 30 patients, mostly women (18 women:12 men), aged from 24 to 84 year with a mean age of 58 year. The most frequent clinical manifestations were seizures, confusional state, headache and fever. All our patients had EEG alterations. Brain CT scan was negative for infectious alterations in nearly all patients. Brain MRI scan showed lesions suggestive for ongoing encephalitis or inflammation in most patients (12 positive, 8 negative). The infectious agent in CSF was found in only 4 cases, with PCR detection (2 cases of Herpes Simplex Virus type 1,1 case of HSV type 2, and a case of adenovirus). The clinical outcome of our patients was mostly good. They completely or almost completely recovered, and had a Rankin score at dismission $<3$ (range $0-6$ ).

Conclusion: This small group of patients doesn't allow to have statistically significant results but our preliminary data show that in acute encephalitic syndrome it's very difficult to identify the infectious agent, also if CSF is collected in the early stage of the illness. The use of brain MRI scan is a more sensitive diagnostic tool than CT scan. EEG is not useful for ethiological diagnosis, but for the follow up of seizures and cortical dysfunction. The good clinical outcome observed in our patients may be the result of many reasons: early admission to hospital, minor severity of the neuroradiological findings, early beginning of antiviral treatment, or non herpetic nature of the infection.

We stress the importance to establish guidelines in suspected encephalitis in order to perform as soon as possible the study of CSF, neuroradiological investigations and start immediately the antiviral treatment.

\section{P529}

Tuberculous meningitis and subsequent moyamoya disease

H.-A. Yi, H. Lee, E.-J. Chung, I.-M. Kim, S.-D. Yi

Keimyung University School of Medicine (Daegu, KR)

Background and significance: The conditions associated with moyamoya disease include genetic and hematologic disorders, neoplasms, drug abuse and infectious diseases. There have been rare reports of the case with moyamoya disease following tuberculous meningitis (TBM). We experienced a case of moyamoya disease with TBM with short interval between two conditions.

Case: A 23 year-old female presented to hospital with severe pulsatile headache, nausea and vomiting for a week. She didn't have any abnormalities on neurological examination. Brain MRI showed no specific parenchymal abnormalities and no meningeal enhancement. Brain MRA was also normal. CSF examination revealed lymphocytic pleocytosis with positive tuberculous PCR. After starting anti-Tbc medication, she felt better and discharged. Two months later, she visited ER again with febrile and chilling sensation with headache, and confused mentality. CSF examination and brain imaging study were done again. There were tuberculomas at the left sylvian fissure, brainstem, anterior thalamus on the brain MRI. There was slight narrowing of the left middle cerebral artery and it seemed to be caused by compression of the tuberculoma. We changed and elevated the dose of anti-Tbc medication and she recovered. As she complained of intermittent tingling sensation of the left hand after discharge and she had abnormal EEG findings, we cannot help using anti-epileptic agent. After one and half years of 
medication, followed up MRI showed no further meningeal enhancement and tuberculoma like lesions. But MRA showed severe narrowing of the both MCA and ICA. Cerebral angiography revealed the finding of moyamoya disease. As she refused to get any surgical procedure and didn't have any symptoms, we decided to quit taking anti-Tbc medication and continue to observe her symptoms.

Conclusion: Among the well-known vascular complications of posttuberculous meningitis, moyamoya disease can be possible. The pathophysiologic mechanism for large arterial occlusion might be inflammation and subsequent autoimmune response.

\section{P530}

Seizures in encephalitis: predictors and outcome

J. Kalita

Sanjay Gandhi PGIMS (Lucknow, IN)

Objective: To evaluate the frequency and predictors of seizure in encephalitis patients and its effect on outcome.

Methods: In a prospective hospital based study, patients with encephalitis were evaluated clinically and presence of seizure, its type and duration were noted. Patients' consciousness was assessed by Glasgow coma scale (GCS) score and neurological findings were recorded. Blood count, serum chemistry, electroencephalography (EEG), cranial MRI and CSF examination were done. Diagnosis of encephalitis was based on ELISA and PCR and grouped into herpes, Japanese, dengue and nonspecific encephalitis. Hospital mortality and 3 month outcome was noted. 148 patients were included; and their median age was 26 (range 1-75) years. Seizures occurred in 63 $(42.6 \%)$ patients of whom 18 had status. Seizure was more common in herpes $(75 \%)$ followed by Japanese $(54 \%)$ encephalitis.

Results: The predictors of seizure in encephalitis were age, GCS score and cortical involvement on MRI. 61\% children had seizure compared to $36.6 \%$ adults and $54.2 \%$ cortical involvement on MRI had seizure compared to $14.5 \%$ without. Seizure was not related to mortality but was associated with poor outcome.

Conclusion: In encephalitis, seizure occurs in $42.6 \%$ patients especially in children with low GCS score and having cortical involvement on MRI.

\section{P531}

Effects of Toll-like receptor 2 agonist Pam3CysSK4 on inflammation and brain damage in experimental pneumococcal meningitis

J. Sellner, C. Gianinazzi, R. Landmann, S. Leib

Technical University Munich (Munich, DE); University of Berne (Berne, $\mathrm{CH})$; University of Basel (Basel, $\mathrm{CH}$ )

Objectives: Recognition of Gram-positive pathogens by the innate immune system relies on the binding of bacterial lipoproteins to Toll-like receptor 2 (TLR2). The activation of TLR2 was shown to be critically involved in the pathogenesis of pneumococcal meningitis (PM) and might contribute to development of central nervous system inflammation and hippocampal neuronal injury. Here, we evaluated the effect of the TLR2 agonist PamCys3SK4 on mediators of a neuro-inflammatory host response and apoptosis within the subgranular zone of the hippocampal dentate gyrus in experimental pneumococcal meningitis.

Methods: Nursing Wistar rats were infected on postnatal day 11 by direct intracisternal injection with $10 \mu \mathrm{l}$ of saline containing Streptococcus pneumoniae. Saline was given to sham-infected controls. Animals were randomized for intracisternal treatment with Pam3CysSK4 or saline at 12 and $24 \mathrm{~h}$ after infection. At $18 \mathrm{~h}$ after infection, CSF was taken for evaluation of white blood cell (WBC) count, TNF-alpha and MMP-9. Apoptotic damage within the subgranular zone of the hippocampal dentate gyrus was evaluated by histomorphometry at $30 \mathrm{~h}$ after infection.

Results: Compared to saline-treated littermates, Pam3CysSK4 lead to significant alterations of CSF parameters including an increase of WBC counts $(\mathrm{P}<0.0001)$, of TNF-alpha $(\mathrm{P}<0.001)$ and of MMP-9 levels $(\mathrm{P}<0.0001)$ in sham-infected animals. Similar values for WBCs, and MMP-9 were found in animals with PM, while TNF-alpha was significantly higher in PM $(\mathrm{P}<0.0005)$. The neuro-inflammatory mediators were not altered by Pam3CysSK4 in PM. Moreover, Pam3CysSK4 treatment was not associated the induction of apoptosis in sham-infection and did not further increase apoptosis in PM.

Conclusion: TLR2 stimulation by Pam3CysSK4 is sufficient to induce a neuro-inflammatory response in the CSF of infant rats similar to that observed in PM. However, Pam3CysSK4 does not alter the course of PM with regard to neuro-inflammatory mediators in CSF and the occurrence of hippocampal apoptosis. We conclude that TLR2 is not critically involved in the pathogenesis of hippocampal injury in this infant rat model of PM.
P532

The distribution of HSV-1 and T-cells in the three branches of human trigeminal ganglia

K. Hüfner, A. Horn-Bochtler, T. Derfuss, C. Glon, I. Sinicina, V. Arbusow, M. Strupp, T. Brandt, D. Theil

Ludwig-Maximilians University (Munich, DE)

Objectives: After primary infection of the mouth, HSV-1 travels retrogradely along the maxillar (V2) or mandibular (V3) branch of the trigeminal nerve to the trigeminal ganglion (TG), where it establishes lifelong latency. During this latency its latency-associated transcript (LAT) and a chronic T-cell infiltration can be detected in the ganglia. Symptomatic HSV-1 reactivations frequently manifest as herpes labialis (15-30/100 persons in the western population annually). Ocular HSV-1 infections are rare (6-21/100000 persons annually). However, once an episode of ocular disease has occured, recurrences are seen in $10 \%$ of the patients/year. We investigated whether this clinical observation is mirrored by the distribution of HSV-1 LAT and CD3 T-cells in the neurons projecting to the 3 branches, V1 (opththalmic), V2 and V3, of the human TG.

Methods: 12 TG from 8 subjects were analyzed. Latent HSV-1 was detected via LAT in situ hybridization, CD3 positive T-cells and latent VZV via immunohistochemistry. In 8 ganglia neurons were allocated to the different branches of the TG by neurofilament staining of consecutive sections. In selected ganglia the neurons of the V1 and the V 3 branches were identified via post mortem tracing with DiI.

Results: All ganglia showed a positive hybridization signal for LAT. The neurons projecting to the V2 and V3 branches of human TG contained significantly higher numbers of LAT than those of the V1 branch. The same held true for the number of CD3 positive T-cells. LAT and CD3 T-cells were usually found clustered in the V2 and V3 portion of the TG, whereas only single, scattered LAT-positive neurons were found in the V1 and T-cells were sparsely spread among the whole V1 portion. In contrast to HSV-1, VZV was evenly distributed over all branches of the infected ganglia.

Conclusion: The occurrence of LAT and immune cells was more prominent in the V2 and V3 portion of the TG than in the V1 portion. These findings reflect the mode of HSV-1 infection of the TG and the clinically observed frequencies of herpes labialis and herpetic eye disease. As expected, VZV did not abound in either the V2 or V3 but was evenly distributed in the $\mathrm{TG}$, thus reflecting its different route of infection via the blood. However, once herpetic eye disease occurs, it often recurs. In the affected individuals HSV-1 probably establishes latency in the V1 branch in the same manner as it commonly does in the neurons of the $\mathrm{V} 2$ and $\mathrm{V} 3$ branches.

The study was supported by Friedrich-Baur Stiftung.

P533

Demographic aspects of clinical and epidemiological peculiarities of tickborne encephalitis in Middle Urals

L.I. Volkova, O.P. Kovtun, E.V. Prazdnichkova

1st Regional Clinical Hospital (Ekaterinburg, RU); Ural State Medical Academy (Ekaterinburg, RU)

Middle Urals is a highly endemic for tick-born encephalitis (TBE) region of Russia, the average morbidity rate being 3.3 times higher, than average corresponding value in Russian Federation.

The aim of our work is to analyze and compare demographic, clinical and epidemiological peculiarities of TBE in different periods of TBE investigation (in 1953-1960 and in 1992-2006 years).

Materials and methods: The work is based on database of TBE patients that consists of 9674 patients suffered from TBE from 1992 till 2006. Comparative analysis included also 402 cases of TBE from 1945 till 1960.

Results: During last 50 years investigation of TBE in Russia revealed shifting from severe focal to fever forms. Thus, when compared with 1945-1960 data in the period of 1992-2006 we noted significant increase of fever forms ( $13.2 \%$ and $72 \pm 4.7 \%$ accordingly); 3.5 times fold decrease of meningeal forms ( $78.6 \%$ and $22.6 \pm 5.2 \%$ accordingly) and 1.5 fold decrease of focal forms $(8.2 \%$ and $5.3 \pm 0.7 \%$ accordingly).This positive tendency is a result of increased TBE vaccination rate, e.g. the average vaccination rate in population in 2006 was found to be $72 \%$. Changing of patient age was noted also; when compared with 1945-1960 years data, in 2000-2006 period we revealed prevalence of patients aged $>40$ years $(17.1 \%$ and $47.8 \%$, respectively), especially older than 60 years; group of elderly and senile patients was found to be $5.7 \%$. Nevertheless, in spite of global positive tendency, the clinical structure of TBE in children remains relatively severe, disease proceeds as a classic neuroinfection with prevalence of meningeal and focal forms. In elderly and senile patients the clinical course of TBE is also severe with high mortality rate.

Conclusion: The before mentioned demographic clinical TBE aspects in 
endemic region require development of age dependent urgent care guidelines for patients in acute period of the disease, and TBE prevention programme in risk groups for focal and meningeal forms.

P534

The implementation of adjunctive dexamethason therapy in bacterial meningitis: preliminary data of a prospective cohort study

M.C. Brouwer, S.G.B. Heckenberg, J. de Gans, L. Spanjaard, D. van de Beek Academic Medical Center, University of Amsterdam (Amsterdam, NL)

Objectives: Adjunctive dexamethasone should be routine therapy in adults with community-acquired bacterial meningitis in developed countries. We present preliminary results on the implementation of dexamethasone in clinical practice.

Methods: In 2006 we started a new Dutch prospective nationwide cohort study on cerebrospinal fluid (CSF) culture proven community-acquired bacterial meningitis. Outcome was graded with the Glasgow Outcome Scale and a score of 4 or less was considered unfavorable. Multivariate analysis was performed including the 4 most important risk factors for an unfavorable outcome: age $>60$ years, score on Glasgow Coma Scale [GCS], CSF white cell count $<1000 / \mathrm{mm}^{3}$ and dexamethasone therapy. Odds ratios (OR) and $95 \%$ confidence interval (CI) were calculated.

Results: From March 2006 to January 2008, 389 patients were included. Data were complete for 282 patients. CSF culture yielded S. pneumoniae in 193 episodes $(69 \%)$, N. meningitidis in $45(16 \%)$ and other bacteria in 43 (15\%). The standard dose of dexamethasone (10mg QID for 4 days, started before or with first dose antibiotics) was administered in 208 of 274 episodes $(76 \%)$. A different regimen of dexamethasone was given in 33 of 274 episodes (12\%). Outcome was unfavorable in 93 episodes (33\%) including 55 deaths $(20 \%)$. Overall, standard regimen of dexamethasone decreased the rate of unfavorable outcome $(29 \%$ vs. $45 \% ; p=0.01)$. Multivariately, age $>60$ (OR 2.34, CI 1.30-4.22; $\mathrm{P}=0.004$ ), CSF leukocytes $<1000$ (OR 5.26, CI 2.73-10.10; $\mathrm{P}<0.001)$ and GCS score (OR 2.46 CI 1.18-5.13; $\mathrm{P}=0.016)$ were related with unfavorable outcome. In the pneumococcal subgroup, dexamethasone decreased unfavorable outcome in the univariate $(31 \%$ vs. $59 \%$; $\mathrm{P}=0.001)$ and multivariate analysis $(\mathrm{OR} 2.85, \mathrm{CI} 1.27-6.39: \mathrm{p}=0.01)$.

Conclusion: 1. Adjunctive dexamethasone for bacterial meningitis has been successfully implemented in the Netherlands; 2 . The proportion of patients with pneumococcal meningitis has increased to $70 \%$; 3 . Dexamethasone protects against unfavorable outcome in pneumococcal meningitis.

\section{P535}

Chronic progressive multifocal leukoencephalopathy in an immunocompetent person: a case report and review of the literature

P. Patrikios, J. Kril, M. Rodriguez

University of Sydney (Sydney, AU)

Cases of progressive multifocal leukoencephalopathy with a long duration of disease have previously been reported but have hitherto received little attention. Renewed interest in this disease has occurred recently because of its association with monoclonal antibody treatments such as natalizumab and rituximab. We report a case that had a disease duration of 11 years, the second longest reported, in a patient not known to have had any form of immunosuppression. The patient's earliest symptoms were of handwriting difficulties as a result of hand apraxias. The disease course was one of a slowly progressive, predominantly motor, neurodegenerative-like illness. Magnetic resonance imaging showed lesions early on in the disease course, the first time this has been shown in a long-standing case, but the diagnosis was made on post-mortem tissue. We review the literature on this topic and propose that progressive multifocal leukoencephalopathy should be considered in differential diagnoses even when the disease duration is many years long and also when there is no known form of immunosuppression.

P536

Klebsiella pneumonia meningoencephalitis - A case presenting with strokelike symptoms without any meningeal signs

P. Wipfler, G. Pilz, O. Lesicky, G. Ladurner, J. Kraus

Paracelsus Medical University and Salzburger Landesklinken (Salzburg, AT)

Introduction: Klebsiella pneumonia is an opportunistic pathogen that causes infections, mainly in immunocompromised patients and it is an uncommon pathogen of bacterial meningitis in western countries. Under certain certain circumstances differential diagnosis between stroke and meningoencephalitis can be difficult.

Case report: A 37-year-old caucasian male presented with acute symp- toms of right-sided hemiparesis without any signs for meningitis. His past medical history revealed idiopathic thrombocytopenic purpura treated with prednisolone as well as insulin dependent diabetes mellitus type I. Cranial MRI-scan demonstrated multiple diffusion-weighted hyperintense lesions which were indicative for acute cerebral embolism. Transesophageal echocardiogram showed persistent patent foramen ovale. When the patient's vigilance level slowly deteriorated, lumbal puncture was performed revealing a cerebrospinal fluid (CSF) cell count of $2000 / \mathrm{ml}$, as well as elevated lactat and protein. In CSF cultures Klebsiella pneumonia was defined. Further abnormalities indicative for a primary inflammation focus could not be detected.

After initiation of ceftriaxon treatment the patient improved continuously. Serial cranial MRI after 20 days showed a significant reduction in the size and number of lesions presumably indicating inflammatory lesions. After 4 weeks of rehabilitation the patient showed only a right sided dysdiadochokinesia.

Discussion: In our case an early diagnosis of meningoencephalitis has been masked by the accidental coincidence of two clinical constellations: Firstly, the patient presented like an acute stroke without fever, meningism or highly elevated CRP. According to an extensive search in literature septic metastatic focal encephalitis due to klebsiella is a very rare condition, in particular in Western countries.

In conclusion, our case indicates that increased physician awareness allows prompt diagnosis of meningoencephalitis with appropriate medical intervention even in patients presenting without any meningeal signs. Early diagnosis including CSF and rapid onset of treatment are essential for survival.

\section{P537}

Temporary protein 14-3-3 detection in cerebrospinal fluid of a patient with neurosyphilis

R. Crols, S. Engelborghs

AZ Middelheim (Antwerp, BE); Institute Born Bunge (Antwerp, BE)

Background and objective: Neurosyphilis has become an infrequent cause of dementia in the western world. We determined brain specific proteins beta amyloid peptide, total and phospho-tau and 14-3-3 in cerebrospinal fluid (CSF) of a patient with subacute cognitive impairment and behaviour disturbances, caused by neurosyphilis.

Case report: a 54-year old homosexual man was admitted on the neurological department because of progressively increasing confusion, memory problems and bizarre behaviour since one year. The neurological examination displayed mild dysarthria, a discrete left central facial palsy and Babinski sign, a finger agnosia, and positive palmomental reflexes. The patient was sometimes confused and aggressive, especially at night. A MRI of the brain showed 2 small vascular lesions in the right frontal lobe. The blood examination showed a positive HIV and syphilis serology, but no signs of immunodeficiency. A cerebral arteriography was normal. Examination of CSF showed 66 white blood cells (WBC)/mm3, oligoclonal banding and a positive syphilis serology. A diagnosis of neurosyphilis was made and a therapy was started with intravenous penicilline during 4 weeks, with marked amelioration of the cognitive and behaviour problems. CSF was re-examined 3 weeks after the end of the treatment and showed only $1 \mathrm{WBC}$ and persisting oligoclonal banding.

Results: Total tau, phospho-tau, beta amyloid and protein 14-3-3 were measured in both CSF samples. Protein 14-3-3 was positive in the first sample, but negative in the sample after therapy. All the other protein determinations were normal.

Discussion: Protein 14-3-3 is considered as a highly sensitive and specific marker of Creutzfeldt-Jakob disease. However, this high sensitivity and specificity is only achieved when the control group consists of patients with slowly progressive brain degenerative disease, such as Alzheimer's disease. Several case reports exist where 14-3-3 is elevated in other diseases with subacute brain degeneration. For the first time, we report an temporary detection of protein 14-3-3 in a patient with neurosyphilis, with normalisation after therapy.

Conclusion: For the first time, protein 14-3-3 was detected in a patient with neurosyphilis and subacute cognitive decline. Following treatment, protein 14-3-3 could not be detected anymore. Neurosyphilis should be included in the differential diagnosis of patients with subacute cognitive decline and presence of protein 14-3-3 in CSF 


\section{P538}

Late-onset chronic encephalomyelitis due to measles infection

V. Bourg, P. Thomas, S. Chanalet, C. Laffont, C. Lebrun-Frenay

Hopital Pasteur (Nice, FR); Hopital L'Archet (Nice, FR)

We describe 3 case-reports of patients with chronic late-onset encephalitis. Patients characteristics were: women (3), age at onset of neurologic disoders ranged from 16 years to 30 years, no medical history. Involvement was characterised by many relapses of neurologic and psychiatric symptoms. Neurologic symptoms consisted in sensitive or motor deficit, headaches, epileptic seizures, coma. Psychiatric symptoms consisted with delirium, hallucinations and desinhibition. The study of cerebro-spinal fluid showed high cellularity (30-80 lymphocytes), high protein and IgG levels with oligoclonal bands. Brain and spinal MRI showed multiple T2-weighted hypersignals mimicking demyelinating disorders without gadolinium enhancement. In all cases reported, all symptoms improved with corticosteroid treatment. Final diagnosis was ensured by measles serology : very high level of IgG in blood and CSF.

Chronic encephalitis is a very rare complication of measles. It must be clearly and rapidly identified in order to not misdiagnose with multiple sclerosis, Behcet, erythematous disseminated lupus and PESS. It is considered as an inflammatory disease triggered by a reinfestation by the measles virus. Most of the patients had contracted measles before 3 years-old and never had vaccine prophylaxis. The evolution seems to be favorable with long-term low-dose of corticosteroids.

\section{Epilepsy}

\section{P539}

Morphological alterations in the blood-brain barrier, glia and neuron in the hippocampus of patients with mesial temporal lobe epilepsy

C. Gurses, G. Purcu, B. Ahishali, M. Kaya, A. Karasu, A. Sencer, N. Bebek, B. Baykan, A. Canbolat, A. Gokyigit

Istanbul University (Istanbul, TR)

Objectives: Mesial temporal lobe epilepsy (MTLE) is one of the most common human epileptic syndromes, hippocampal sclerosis being its most characteristic pathologic finding. The underlying cause of MTLE still remains unknown. To perform electron microscopy and immunohistochemistry to demonstrate the changes in neuron, glia and endothelial cells from the hippocampal regions of the patients with MTLE.

Method: Our study involves 20 patients $(8 \mathrm{~F} / 12 \mathrm{M})$ with intractable MTLE who had undergone selective amygdalahippocampectomy (7 right, 13 left). Specimens from the patients' hippocampi were obtained between the years 2005 and 2007. The mean age of the patients was $30.2 \pm 8.6$ years. The age at onset of epilepsy was $16 \pm 6.3$ years. The mean duration between the onset of epilepsy and surgery was $13 \pm 10.3$ years. As is common in such studies, all the patients submitted their written consent. We also used c-Fos, one of the immediate early genes important in signal transduction linking environmental stimuli to neuron, immunohistochemistry to examine patterns of neuronal activation and their relationship to seizure expression.

Results: Morphologically, narrowed microvessel lumen, disordered luminal membrane or surface infoldings and swelling of endothelial cells were frequently observed. Endothelial tight junctions were also found to be intact. Ultrastructurally, a network of basal lamina-like electron-dense materials of variable thickness encircled the vessel lumina. Neurons presenting electron microscopic features of necrotic neuronal cell death, including shrunken cell bodies and a slightly darkened and vacuolar cytoplasm with severely swollen mitochondria were detected. The cytoplasm of these astrocytes revealed swelling of mitochondria and many vacuoles. In addition to altered reactive astrocytes, related to perivascular edema swollen endfoot of astrocytes were noticeable. There were particular lipofuscin structures in neuron, glia and also in endothelial cells of BBB. Immunohistochemistry for c-fos showed that TLE neurons in the hippocampal area express $c$-fos in their nuclei.

Conclusion: In this study, we postulate that morphological alterations in neuron and astroglia as well as endothelium could disturb the functioning of the BBB in patients with mesial temporal lobe epilepsy and the disturbed $\mathrm{BBB}$ in hippocampus may alter neuronal environment in this region rendering it liable to epileptogenesis.
P540

Nocturnal epileptic seizures with minor-motor-events: a type of seizure with controversy in its pathophysiology. A case report

C. Tilz, Y. Wang-Tilz, H. Stefan, C. Eggers

Krankenhaus Barmherzige Brueder (Linz, AT); University Erlangen-Nuremberg (Erlangen, DE)

Objectives: Nocturnal seizures are seizures with some difficulties to be correctly diagnosticated, not only because they often remain unperceived, but also because the diversity of sleep-related non-epileptic events. The presented case-report should demonstrate a patient with minor-motor-events, nocturnal seizures with stereotye character and polymorph clinical appearance.

Methods: The 67 year old woman introduced herself in our outpatient service beacuse of sleep-disturbance for 5 years and excessive daytime sleepiness. Pre-existing conditions were atrial fibrillation, but no neurological diseases. The neurological examination was without pathological findings. Because of her history she was already treated with ropinirole without success assumpting that a restless-less-syndrome was the reason of her problems.

Polysomnography (PSG) revealed short stereotype events several times per minute during NON-REM-sleep with with flexion-movements of the arms and the legs and a highly reduced sleeping time. 2-channel-EEG during PSG revealed event-related polyspikewave-complexes.

A standard surface-EEG was without pathological findings, but sleepEEG showed a bifrontal theta-slowing and longtime EEG a seizure onset pattern on F4.

Results: Treatment with gabapentine (1500mg daily dose) made the patient completely seizurefree and also the daytime sleepiness disappeared.

Conclusion: Minor-Motor-Events are stereotype events during NONREM-Sleep with high frequency and short duration (below 4 seconds) in patients with epilepsy. Their pathomechanism is not clear yet: Some authors suggest that they might represent the first clinical manifestation of more complex seizures, others believe that they are non-epileptic events due to an incomplete inhibition of excitatory motorneurons. Our data show event-correlated epileptic discharges suggesting an epileptic origin of those events which indicate an epileptic origin of those events. Making the diagnosis is not always easy, but if correctly diagnosed, they can be well treated.

\section{P541}

Two case of dentatorubropallidoluysian atrophy with progressive myoclonus epilepsy

H-K. Park, J-H. Jung, H-G. Oh, D. Jeong, J-Y. Park, T-W. Kim

Soonchunhyang University Cheonan Hospital (Cheonan-City, KR); Soonchunhyang University (Gumi-City, KR)

Objectives: The dentatorubropallidoluysian atrophy(DRPLA) is a rare autosomal dominant disease that is seen predominantly in the oriental population. This neurodegenerative disorder is related to a trinucleotide(CAG) repeat on chromosome $12 \mathrm{p}$ and a unusual cause of progressive myoclonus epilepsy(PME).

Methods and results: 34-year-old female and 31-year-old male showed progressive myoclonus epilepsy with ataxia, dementia and choreoathetosis. Brain MRI revealed cerebellar and brainstem atrophy accompanied by dilatation of fourth ventricle. The demonstration of expanded CAG repeat in the gene for DRPLA was used to confirm the diagnosis.

Conclusion: We report two case of sporadic DRPLA of early adult type with PME.

\section{P542}

Localisation of executive motor function by cortical electrical stimulation I. Borggräfe, J. Remi, C. Vollmar, P.A. Winkler, S. Noachtar University of Munich (Munich, DE)

Objectives: The lateral and medial aspect of the central and frontal cortex were studied by electrical stimulation in epilepsy surgery candidates in order to determine the topography and somatotopic distribution of areas harboring negative motor areas (NMA). NMA were defined as cortex whose electrical stimulation elicits inability to maintain or initiate voluntary movements.

Methods: Results of electrical stimulation of the cortex were evaluated in a total of 115 patients in whom invasive electrodes were implanted for planning resective epilepsy surgery at the University of Munich Epilepsy Center. Exact localization of the placement of the subdural electrodes was defined by fusion of 3-dimensional reconstructed MRI and CT images in 12 patients and by analysis of plane skull X-rays in another 7 patients.

Results: We obtained negative motor responses in a total of 76 electrodes 
(out of 862) covering the primary somatomotor and supplementary sensorimotor areas in 19 patients. Negative motor responses involved the contralateral arm in $70 \%$, both arms in $15,7 \%$ and the tongue in $14,3 \%$ of the electrodes (the latter could not be lateralized). Stimulation of the lateral fronto-central cortex elicited significantly more frequently contralateral negative motor responses ( $81 \%$ contralateral vs. $19 \%$ bilateral) than stimulation of the mesial fronto-central cortex which lead to more bilateral negative motor responses $(62,5 \%$ bilateral vs. $37,5 \%$ contralateral) (chi-square test, $\mathrm{p}<0.02$ )

Conclusions: Exact localization using 3-D fusion methods reveals negative motor areas widely distributed throughout the precentral gyrus and the mesial fronto-central cortex. Negative motor areas represent most likely areas involved in motor planning and show a somatotopic organization. The lateral NMA serves more contralateral motor planning whereas the mesial NMA bilateral movements.

\section{P543}

Neonatal seizures due to intraventricular/periventricular haemorrhage unfavourable prognostic factor. Retrospective study

M. Stefanescu, D.A. Plesca, D. Dragomir, A. Toma, A. Antrasian, A. Zamfirescu

"Dr. V. Gomoiu" Children Clinical Hospital (Bucharest, RO); "Panait Sarbu” Obstetric-Gynecology Clinical Hospital (Bucharest, RO)

Objectives: Study of prognostic significance of neonatal seizures occurring at newborn infants with intraventricular/periventricular hemorrhage (IVH/PVH).

Methods: The authors analyzed the outcome of 17 children (16\%) with antecedents of IVH/PVH, who experienced neonatal seizures short after birth, from a total of 130 newborn infants with neonatal seizures of different etiology.

Results: IVH/PVH represented the second important cause of neonatal seizures with early onset (first three days of life, with a peak in the second day). Subtle neonatal seizures predominated, but tonic, clonic and myoclonic seizures were also encountered. EEG in neonates with seizures due to IVH/PVH showed, in the great majority of cases, severe backround abnormalities (voltage suppression, electrocerebral silence, localized suppressionburst). Neonates with neonatal seizures due to IVH/PVH developed severe cerebral lesions (porencephalic cysts, obstructive hydrocephaly), demonstrated by neuroimaging investigations (cranial echography, tomography and magnetic resonance). IVH/PVH in premature infants $(<32$ weeks of gestation and very low birth weight) was associated with high rate mortality. Survivors constantly developed severe cognitive and motor impairment (severe or profound mental retardation and spastic cerebral palsy), as well as severe epilepsy and sensorial deficits. Obstructive hydrocephaly was also common.

Conclusion: Neonatal seizures due to IVH/PVH were correlated with high neonatal mortality rate and poor neurological outcome

\section{P544}

Secondary generalisation prolonges seizure duration in temporal but not in frontal lobe epilepsies

Ö. Bilgin, J. Rémi, S. Noachtar

Neurologische Klinik und Poliklinik (Munich, DE)

Ictal epileptic activity tends to spread over the brain and may eventually generalize secondarily. We aimed to evaluate the duration of seizures arising from the frontal and temporal lobe in order to better understand spread and inhibitory mechanisms involved.

We investigated the seizure duration as measured by ictal non-invasive EEG in 233 seizures arising form the temporal $(n=130)$ and frontal $(n=103)$ lobes in 41 patients with frontal $(\mathrm{n}=20)$ and temporal lobe epilepsies $(\mathrm{n}=21)$.

Of the seizures arising from the temporal lobes $13.8 \%$ generalized secondarily compared to $24.3 \%$ secondary generalization in FLE. In TLE patients, the average duration was significantly longer in secondarily generalized seizures (95s) as in non-generalized seizures of TLE patients (62s; $\mathrm{p}=0.002$ ). In frontal lobe epilepsies, the average duration of non-generalized seizures (66s) did not differ from seizures that generalized secondarily $(68 \mathrm{~s})$. The seizures in TLE and FLE did not differ in their average total duration (67s vs. 66s) and duration of the seizures without secondary generalization (62s vs. 66s).

Only 15 of the 233 seizures lasted longer than 120s (8 TLE; 7 FLE).

In conclusion, secondary generalization of ictal epileptic activity prolonges seizure duration in TLE but not in FLE. This observation may be related to the differential action of inhibitory mechanisms between frontal and temporal lobes. Our findings are not supporting the concept that exhaustion is setting an end to epileptic activity. One can speculate, that instead active inhibitory mechanisms are at play in extratemporal lobes and not in the temporal lobes, which need to be activated by the spread of epileptic activity from the temporal lobe to then terminate epileptic activity.

\section{P545}

Vagus nerve stimulation: an increasing effectiveness

P. Myrianthopoulou, A. Dietis, S. Papacostas

Cyprus Institute of Neurology \& Genetics (Nicosia, CY); Nicosia General Hospital (Nicosia, CY)

Objective: This retrospective study documents the outcome of intermittent vagal nerve stimulation (VNS) in 17 patients (pts) with medically intractable epilepsy, the results of seizure frequency (SF) reduction and some complications that occurred.

Method: Medical files of the pts implanted for 1 year or longer were reviewed for changes in seizure rate. The length of the follow-up averaged 37.5 months (range 12 months -5.3 years). The age range was $16-59$ years (mean 37.5 years).

Results: As a result of decrease in SF, the patients were separated into 5 groups: Group 1: $\geq 75 \%$, Group2: $50-74 \%$, Group 3: $25-49 \%$, Group 4: $0-24 \%$, and Group 5: $\leq 0 \%$. At the 1 year follow-up, Group 1 consisted of 0 pts, group 2 of 7 pts, group 3 of 5 pts, group 4 of 5 pts and group 5 of 0 pts. At total follow-up time, $12(71 \%)$ of the 17 pts had a $50 \%$ or more reduction in the number of seizures compared to their baseline. Of these patients, 5 $(31 \%)$ had a $75 \%$ or more reduction. Of the 17 pts, $15(88 \%)$ had $25 \%$ or more reduction of seizure activity. One patient had an $8 \%$ decrease during the first 6-15 months of therapy and then a reemergence of seizure rate occurred which brought him back to his baseline frequency. Another patient had an average of a $10 \%$ decrease in SF in 21 months but afterwards there was a reemergence of SF and worsening. Even though there were good results, one patient decided to have his stimulator removed. The guardians reported an overall shorter duration of seizures and decrease in severity. Complications included sleep apnea, coughing and hoarseness and transient pain at implantation site, but these complications were dealt with or tolerable because of the good results of seizure reduction.

Conclusion: This retrospective study shows that the effects of VNS therapy vary for each patient. On average there was an increase in the effectiveness over time. VNS therapy appears to be a positive adjunct treatment for medically intractable epilepsies.

\section{P546}

Worsening factors in patients with post-traumatic epilepsy

S. Nica, E.I. Davidescu, G. Mihailescu

Carol Davila University of Medicine (Bucharest, RO)

Background: Epilepsy is one of the most common serious neurological disorders in the world. Traumatic brain injury (TBI) accounts for $20 \%$ of symptomatic epilepsy in the general population and for $5 \%$ of all epilepsy. Posttraumatic epilepsy is a serious clinical problem and can occur soon after injury or may develop months to years later, more often in young patients. The general prognosis of posttraumatic epilepsy is favorable. Some of the patients experience an aggravation of seizures after a period of well controlled seizures. This aggravation can be prevented in most cases by a better approach to the patient.

Methods: Patients considered for the study were diagnosed with posttraumatic epilepsy (32 patients; 6 females and 26 males with a mean age of 45.74 years); $27 \%$ with early onset seizures and $73 \%$ with late onset seizures. 10 patients experienced simple (4 patients) or complex ( 6 patients) partial seizures and 22 patients experienced convulsive generalized seizures. All the patients had a normal neurological examination; 19 of them had no structural lesion at brain imaging and 23 had some structural lesions on brain imaging. All the patients received antiepileptic drugs and had a long period of perfect controlled seizures (11 month to 6 years). 29 patients included in the study experienced, afterwards, an aggravation with increased number of seizures per month (the same type of seizures) and 3 of the patients with complex partial seizures evolved into secondary generalized convulsive seizures. We try to find out the main causes for the worsening and the possibility to prevent it.

Results: The alcohol abuse alone or associated with withdrawal from anticonvulsivant drugs, depression and anxiety associated with withdrawal from anticonvulsivant drugs and new brain trauma after seizures were the main causes for aggravation in our study patients. 4 patients have no identifiable worsening factors. There are no difference between the patients who $\mathrm{had} / \mathrm{had}$ not structural lesions on brain imaging.

Conclusions: A better approach and management by combining antiepileptic drugs therapy with antidepressants or anxiolitics if necessary 
and a psychologic support (individual psychotherapy) are the best way to prevent worsening of posttraumatic epilepsy. The patients who experienced generalized convulsive seizures are more susceptible to all this worsening factors. In some cases there are no worsening factors identifiable.

\section{P547}

Antiepileptic drugs and gastrointestinal symptoms

S. Razeghi Jahromi, M. Togha

Tabriz University of Medical Scienses (Tabriz, IR); Tehran University of Medical Scienses (Tehran, IR)

Objective: Gastrointestinal disorders are among the most common side effects of antiepileptic drugs. They might lead to discontinuation or irregular consumption of drugs and can affect the quality of life. Aiming to evaluate the frequency of gastrointestinal side effects of different antiepileptic drugs, a case series study was done in an outpatient group on mono and poly therapy

Method: The study was performed on 105 patients suffering from posttraumatic epilepsy, who referred to a referral epilepsy clinic, where complicated cases have been visited. Data was analyzed in two divided groupsmono and poly (two or more) therapy.

Excluding criteria were primary gastrointestinal problems, receiving toxic dose or irregular use of drugs. Data was analyzed, using chi square test.

Results: Five patients who did not use antiepileptics on regular basis were excluded from the study. Seventy six patients $(72.4 \%)$ were on two or more antiepileptics, and $23(5.7 \%)$ on monotherapy. The most common prescribed drug for monotherapy was carbamazepine and the most frequent combination was phenytoin and carbamazepine.

Reflux (34.6\%), nausea (33.7\%), constipation ( $26 \%$ ), vomiting $(22.1 \%)$ diarrhea $(21.2 \%)$ and dysphagia (19.2\%) were in descending order of frequency.

Nausea was significantly higher in monotherapy with carbamazepine $(\mathrm{P}=0.047)$. Polytherapy associated with significantly higher rate of $\mathrm{GI}$ symptoms, including esophageal reflux, diarrhea and dysphagia in comparison to monotherapy. Polytherapy including phenytoin, gabapentine and valproate had significant relationship with diarrhea $(\mathrm{p}=0.01)$, dysphagia $(\mathrm{p}=0.029)$ and reflux $(\mathrm{p}=0.022)$ respectively. Combinations including gabapantine were the worst one according to GI symptoms (nausea, vomiting, dysphagia, constipation and diarrhea).

Conclusion: Gastrointestinal symptoms as the side effects of antiepileptic therapy are considerable, especially with Polytherapy. Esophageal reflux and dysphagia are frequently neglected, although they are among the most common side effects. Modifying treatment modality or changing dietary pattern may lead to relief or significant reduction of these undesirable complications.

\section{P548}

\section{Quality of life in epileptic patients}

S. Shaafi

Tabriz University of Medical Science (Tabriz, IR)

Background: Epilepsy is a chronic disease, which has a major influence on the quality of life in the epileptic. Considering many aspects of epilepsy one can conclude that it has influential effects in multiple aspects of life in these patients. The aim of this research was to study the quality of life of the epileptic patients in our society, and compare these result with other centers around the world.

Materials and methods: One hundred eighty nine patients were enrolled according to the criteria of the International league Against Epilepsy (ILAE). The patients were interviewed using a standard questionnaire, Quality of life in Epilepsy (QOLIE- 31-P).

Findings: Among 189 patients total quality of life was minimally 16.20 and maximally 87.5 with a mean of ( $5014.59 \mathrm{SD}$ ). The mean score for men was (50 16.11 SD; 16.2-85.35) and for females (49.28 14.47 SD; 24.14-87.5), which showed no significant difference between sexes p Value $=0.549$ ). The mean score in the monotherapy group (52.95 16.22 SD) showed a significant difference in comparison to the poly therapy group with a mean score of $(46.9812 .07 \mathrm{SD}),(\mathrm{P}$ Value $=0.005)$, indicating a favorable profile for the former. There was no significant difference statistically with regard to age groups and educational status, but duration of the epilepsy less than one year had statistically significant better quality of life.

Conclusion: The results of this study were compared with similar studies in other centers all over the world. The findings on the role of the sex of the patients was the same as the studies in the India and Georgian University, but were different to that of a European study of 15 countries. This may stem form cultural differences and beliefs. The care of the epileptic patients must take into account the many factors influencing the quality of life in these patients. Adhering to a monotherapy regimen by the physicians has an important role in improving the quality of life in the epileptics. The epilepsy because of its chronic course has an unfavorable long- term influence on the quality life of the patients.

\section{P549}

Sexual dysfunctions, depression and quality of life in epileptic patients U. Turk Boru, S. Koc, M. Tasdemir

Kartal.Eg.Ar. Has. (Istanbul, TR); Marmara University (Istanbul, TR)

Objectives: There are few studies about associations between sexual dysfunctions, depression and quality of life in epileptic patients under antiepileptic treatment.

In this study, we aimed to evaluate quality of life and sexual function, depression in epileptic patients under antiepileptic treatment and in healthy controls.

Methods: The study included 56 epileptic patients (24F) and 47 healthy volunteers $(24 \mathrm{~F})$ between ages $18-55$, who were married and had no psychiatric or systemic illness. Quality of Life Scale (SF-36), IIEF (International Index of Erectile Function), FSFI (Female Sexual Function Index) and Hamilton Depression Scale were used. Sexual dysfunction and SF-36 and Hamilton Depression Scale scores were compared between patients and controls.

Results: Sexual function scores were significantly lower in epilepsy patients under antiepileptic treatment than healthy controls $(F ; p=0.000, M$; $p=0,006)$. Quality of life scores of epilepsy patients were significantly lower than those of healthy controls (physical health, $p=0.000$; psychological health, $p=0.016$; total score, $p=0.001$ ). There is statistically significant difference in none of the scores between the patients with monotherapy and those with polytherapy $(p>0,05)$

Conclusion: Sexual functions and quality of life were found significantly lower in epileptic patients under antiepileptic therapy. Monotherapy and polytherapy made no difference on functions. Depression was not found in the patients. This may depend on the mood-stabilizing effects of the antiepileptic drugs. Further investigations are required.

\section{P550}

Severe amnesia after temporal status epilepticus: report of 2 cases V. Bourg, B. Bailet, S. Chanalet, P. Thomas

Hopital Pasteur (Nice, FR)

Severe amnesia following partial status epilepticus had been reported in few cases.

We describe 2 patients with severe amnesia after temporal status epilepticus. In the first case (women, 63 years), there was a history of partial posttraumatic epilepsy in childhood. Antiepileptic drug had been stopped for several decades. This patient had been hospitalized in geriatric department in July 2000 for a prolonged confusion syndrome. The EEG showed many temporal seizures localized either on the left or the right side. Brain MRI showed T2-weighted bilateral mesiotemporal hypersignal. The duration of the status epilepticus was estimated at 1 month. When she was addressed in our epileptological unit in May 2002, she presented a severe anterograde amnesia with fabulations. Brain MRI showed bilateral hippocampal atrophy. The second patient was a 67 years-old man hospitalized in our neurologic department in August 2007 for confusional syndrome since 3 weeks. There was a history of remitting similar symptoms one year ago. The EEG found left temporal status epilepticus. Improvement was obtained after Fosphenytoin infusion but anterograde amnesia persisted. Brain MRI showed right hippocampal atrophy and T2-weighted temporal hypersignal. Since this event, there is no cognitive improvement but T2-weighted hypersigna has disapeared.

Severe amnesia is a rare complication after partiel status epilepticus and worsen quality of life. In most reported cases, sequellae occurred after very prolonged status epilepticus mostly because of delayed diagnosis and/or inadequate treatment. In our 2 patients, it is interesting to note that epilepsy was not active prior the occurrence of the status epilepticus. EEG has a major diagnostic value in patients with confusional syndrom.

\section{P551}

Investigation of cognitive function in epilepsy

Y. Zhang, J-H. Chen, K. Dong

Beijing Tiantan Hospital, Capital Medical University (Beijing, CN); Beijing Mining Aggregation General Hospital (Beijing, $\mathrm{CN}$ )

Objective: Epilepsy is a chronic disease of central nervous system, which is described by the presence of repeated unprovoked seizures. Consequences of epilepsy can be as serious as the disorder itself and resulting cognitive 
deficit can be more debilitating for the patient than the actual seizures itself. In this study, we tried to evaluate the cognitive function of patients with epilepsy and to explore the effect of the related factors of epilepsy on cognition in China.

Methods: We selected 100 patients who were admitted at Beijing Tiantan Hospital from Jan, 2006 to Aug, 2007, and 100 normal people as control group, all matched for age, education, and all participants had a normal intelligence level based on the Wechsler Adult Intelligence Scale, and did not take medications other than antiepileptics (AEDs) or have a psychiatric history. We used Mini-Mental State Examination(MMSE), Clinical Dementia Rating (CDR) and Montreal cognitive assessment(MoCA) to exam their cognitive.

Results: Epileptic patients had lower MMSE, MoCA and higher CDR than the healthy controls, with significant difference. MMSE, CDR and MoCA had negative correlation with duration of seizure disorders, and seizure frequency. Suggesting duration as covariant, correlation index of MMSE and seizure frequency was $0.001(\mathrm{P}<0.05)$ while suggesting seizure frequency as covariant, the counterpart was $0.06(\mathrm{P}>0.05)$.

Conclusion: These findings indicate that MMSE, CDR of patients with epilepsy are lower than those of the healthy population and that seizure frequency is the independent risk factor of impaired cognition in epilepsy.

The practice of monitoring antiepileptic treatment limited to seizure control should be revised; cognitive impairments also need to be taken into account The classical principle of treating only seizures needs to be reconsidered.

State Key Laboratory of Cognitive Neuroscience and Learning Open Project Grant and Beijing Superiority talented found (20071D0300400078)

\section{General neurology}

\section{P552}

Bickerstaff's brainstem encephalitis with polyneuropathy

C. Nobrega, S. Nunes Oliveira, R. Almeida

Centro Hospitalar de Lisboa Central (Lisbon, PT)

Introduction: Ophthalmoplegia, ataxia, impaired consciousness and/or pyramidal signs constitute a syndrome described in 1957 and since then known as Bickerstaff's brainstem encephalitis (BBE). Miller-Fisher syndrome (MF) characterized by ophthalmoplegia, ataxia and areflexia is a clinical variant of acute inflammatory polyneuropathy (PNP). We present a case of BBE with PNP. Common aspects of etiopathogenesis and clinical presentation of BBE and postinfectious PNP are discussed and supported with a literature review.

Case report: A 63 years old female presented with vertigo, unsteady gait and paresthesia and reported a respiratory infection the week before. Clinical examination showed an ataxic gait and left gaze nystagmus. Clinical deterioration ensued and 72 hours later stupor, flaccid tetraparesis with preserved reflexes and bilateral Babinski sign, bilateral external ophthalmoplegia and left central facial paresis were present. Later, mechanical ventilatory support was needed. Cerebrospinal fluid and Magnetic Ressonance Imaging were normal. Electromyography showed axonal motor-sensory PNP aspects. Positive antibody anti-Campylobacter jejuni was found. After plasmapheresis, clinical improvement occurred. Forty-five days after admission, the patient was discharged with no signs or symptoms.

Conclusion: This case reports a monophasic central and peripheral nervous system disease with apparent response to plasmapheresis treatment, fulfilling diagnostic criteria of BBE with PNP.

Literature review shows that postinfectious PNP and BBE are probably different expressions of the same entity. This is supported by case reports of clinical overlap and possible common etiopathogenesis.

P553

Adult-onset Krabbe disease: usefulness of diffusion tensor imaging in understanding the pattern of white matter degeneration

D. De Feo, A. Falini, B. Colombo, D. Dalla Libera, P. Annovazzi, V. Martinelli, S. Bianchi, G. Comi

San Raffaele Hospital (Milan, IT)

Introduction: Globoid-cell leukodystrophy (GLD) is a genetic disorder caused by the deficiency of galactocerebrosidase (GALC), mapped on chromosome 14q31. The disease occurs among infants with a rapidly fatal course, but rarer late-onset forms also exist. The clinical phenotype of the later-onset forms is variable. Adult patients more often develop slowly progressive spastic paraparesis. The imaging features in the late onset disease differ from that of the infantile form: in the adult GLD the pyramidal tract and occipital white matter involvement is prominent, whereas cerebellar white matter and deep grey matter involvement is uncommon.

Objectives: We report a case of adult onset Krabbe disease with particular focus on the DTI analysis.

Methods: Patient underwent clinical, neurophysiologic and neuroimaging examination including non conventional MRI studies.

Results: A 25-year-old woman presented with a slowly progressive gait disturbance. Family history was negative for neurological disorders. She began to experience stumbling in walking because of slowly progressive weakness and spasticity of both lower limbs two years ago. Neurological examination revealed selective pyramidal signs with prevalent involvement of the lower limbs. The neuropsychological assessment revealed long term memory loss and visuo-spatial dysfunction. The ophthalmologic evaluation and VEPs were normal, but BAEPs revealed a borderline delay in CCT. EMG resulted negative for PNS affection. EEG presented a normal general organization with burst of irregular polymorphous delta activity that showed a slight prevalence on right posterior regions without paroxysmal discharge. The subcortical white matter of the primary motor area, centrum semiovale, corona radiata and the upper and medial CST showed bilaterally T2 hyperintensity at conventional MRI and significant reduction in FA and an increase in Dav at DTI analysis. CST below this level and the more distal occipital radiations revealed only the reduction in FA. The GALC activity in leukocytes was reduced significantly consistent with the diagnosis of GLD. The molecular analysis for the gene deletion Ex11-17del in GALC gene was negative; further genetic studies are ongoing.

Conclusion: The MRI findings support the inferior bound progression of pathological process in GLD and outline DTI potential in the detection of a praecox involvement of optic radiations in absence of clinical and electrophysiological impairment of visual system.

\section{P554}

Neurosarcoidosis, a rare disease of the CNS. Two case reports

E. Nikolakaki, K. Kalamafkianaki, N. Kouroumalos, M. Kontogiorgaki, D. Andrinos, G. Georgakakis

General Hospital of Chania (Chania, GR); General Hospital "G. Gennimatas" (Athens, GR)

Objectives: Sarcoidosis has a prevalence ranging from 3 to 50 cases per 100000 of population. An insult of the CNS, though quite rare, concerns $1-7 \%$ of the affected population. In almost a fifth of the cases where CNS is affected, neurological manifestations set suspicions for diagnosis of the disease's systemic form One third of those patients, follow a relapsing-remitting course of this chronic disease.

The aim of this two case presentation is to comment on the incidence of Neurosarcoidosis (NSR), a systemic granulomatous inflammatory disease of unknown etiology, but mainly to focus attention on possible diagnostic pitfalls.

Methods: Two case reports:

First case concerns a 22 year-old male patient with a history of sarcoidosis diagnosed 2 years before admission with lymph node biopsy after thoracotomy. His complaint was headache and on neurological examination only positive signs of meningitis were found. Brain contrast- enhanced MRI revealed diffuse leptomeningeal infiltrations.

Second case is a female patient aged 42 also diagnosed with sarcoidosis 2 years before admission using again lymph node biopsy. She came mentioning abnormal sensation on her lower limbs and imbalance. The neurological examination finding was a spinal cord syndrome. Contrast-enhanced MRI revealed foci of abnormal magnetic signal present throughout brain and spinal cord, without any leptomeningeal infiltrations.

Results: Both patients were treated with average doses of methylprednisolone for overall 2 months (including gradual withdrawal). Clinical picture improved much sooner. Symptoms did not reappear upon follow-ups.

Conclusion: NSR usually manifests with cranial nerve palsies, focal neurological signs from the brain or spinal cord, symptoms representing hypothalamic insult, seizures, hydrocephalus or recurrent aseptic meningitis. But it may also appear with atypical clinical signs as in the cases above. As it is a rare disease, misdiagnosis may easily occur. Nevertheless, once the disease is diagnosed, disability may soon be reversed. 
P555

Nucleoplasty: one-year follow-up

F.A. Antochi, M.O. Romanitan, A. Roceanu, O. Bajenaru, B. Dorobat, $R$. Nechifor

Emergency University Hospital (Bucharest, RO)

Objectives: Nucleoplasty is the minimally invasive method of disc decompression, the most advanced form of percutaneous discectomy developed to date. It has few important advantages as compared to other surgical methods such as: it is involving minimal tissue trauma, the recovery time is faster than before, it uses local anesthetic, it has a short duration and it has a painful rehabilitation period. Since the method is newly adopted in Romania, the aim of our study was to centralize the benefits and the side effects of the method in order to disseminate it on the national level.

Methods: A 12-month prospective, nonrandomized, human clinical study was started in the Neurology Department of the Emergency University Hospital, Bucharest, covering one year time interval (December 2006 December 2007). The study included 60 patients presenting lumbar pathology at more than one level. Several inclusion criteria were respected: persistent radicular pain with or without back pain, the persistence of the leg pain, the failure of the conservative therapy, MRI showing herniation less than $6 \mathrm{~mm}$ and preservation of disc height (less than $50 \%$ loss). We excluded patients with no previous conservative therapy for at least 6 weeks, significant neurological deficits, reflex change, patients with bladder/bowel functional loss, The patients with disc extrusion, presence of a disc fragment or with disc height less than $50 \%$ of normal were excluded as well. The used method was the Dekompressor, a highly efficient method for removal of intervertebral disc nucleus pulposus through the smallest available channel under

fluoroscopic control. Periprocedural the patients received antibiotics treatment.

Results: Up to date there were no procedure related complications. An amount of $89 \%$ of patients reported improvement of the simptomatology with almost $60 \%$ the first 6 months post procedure while $92 \%$ of patients were satisfied with results of 12 months post procedure reporting the reduction of the analgesic intake. In $4 \%$ of cases there was no benefit of the method. The follow-up of the patients is still on-going.

Conclusion: The results of our study confirm that Nucleoplasty provides the therapeutic benefits of earlier percutaneous disc decompression techniques without many side effects. Clinical results are very promising when the inclusion criteria are strictly respected and therefore we encourage the patients to expect rapid and sustained pain reduction after the procedure.

P556

Superficial siderosis due to root avulsion

G.K. Papadimas, V. Zouvelou, M. Rentzos, A. Konstantinopoulou, P. Kokotis, I. Evdokimidis

Aeginition Hospital University of Athens (Athens, GR)

Superficial siderosis of the central nervous system is mainly characterized by cerebellar involvement, myelopathy and neurosensory hearing loss. Root avulsion due to traumatic plexus injury is a rare cause of this newly recognized entity. The postulated mechanism is that a severe traumatic injury, such as brachial plexus avulsion can result in a chronic intermittent bleeding of the already fragile and vulnerable medullary and radicular veins.

In the present case, a 49-year-old man presented with a great walking difficulty with spastic paraparesis, cerebellar ataxia, sensorineural deafness and urinary urgency. He also referred some episodes of mild gait instability during the last ten years. Thirty one years earlier, he had a severe motor bike accident resulting in complete paralysis of his right upper extremity attributed to a right brachial plexus injury. The patient was submitted to a thorough work-up. CSF examination showed an increased protein level, xanthochromia and oligoclonal bands. Brain and spinal cord MRI with T2 sequences revealed a dark rim of hypointensity along the surface of the cerebral cortex, brainstem, cerebellum and spinal cord, a characteristic finding of hemosiderin deposition. Gadolinium-enhanced MR imaging demonstrated no additional abnormalities.

The pathogenesis of superficial siderosis remains still obscure. Microglia, the resident immune cells of the central nervous system is the cellular compartment where hemoglobin is converted to heme, iron, ferritin and the hemosiderins. The activation of microglia causes the production of many proinflammatory and neurotoxic factors, possibly responsible for a potential inflammatory and degenerative component in the pathogenesis of superficial siderosis. The demonstration of CSF oligoclonal bands in this case of superficial siderosis due to root avulsion may be explained in the context of an underlying inflammatory process.
P557

Study of neurologic complications in Iranian patients with Behçet's syndrome

H. Ayromlou, A. Ebrahimi, S. Joodi

Imam Reza Hospital (Tabriz, IR)

Objective: Behcet syndrome is an inflammatory disorder that in CNS occurs multifocal ischemic lesions due vasculitis in 5 to 49 percent. Major criterias in Behcet includes oral ulcers and minor criteria include genital ulcers, ocular involvement, skin lesions and positive Pathergy test. The other important signs of the discover are articular, gastrointestinal, vascular and CNS involvement. The most common manifestation of CNS involvement include fever, sever headache, paresis, seizure and delirium. The purpose of this study is survey of neurologic complications in patients with Behcet syndrome.

Methods: In this study, prevalence of syndrome criteria \& neurological symptoms were surveyed in 15 patients for one year. Also, all patients were evaluated with VEP, SSEP and BAEP. Patients, MRI were reviewed as well. Finally, results of neurologic examination, EPs test and MRI were calculated in percent and compared in two normal and abnormal groups.

Results: In this study, the most common criteria were oral aphthus and ocular involvement. The most common findings in CNS involving were paresis, acute severe headache, seizure and increased tendon reflexes. Also arthritis was the most common systemic manifestation. In BAEP, 3 patients had bilateral mild demyelination type lesions in brain stem and 3 ones had axonal type auditory nerve lesion. In VEP, 5 patients had bilateral axonal type, 2 ones had unilateral axonal type and 4 ones had bilateral demyelinating type optic nerves lesions. In SSEP 5 patients had bilateral mild demyelinating type and 7 ones had unilateral mild demyelinating type Somatosensory tracts lesions. MRI showed brain lesions in half of the patients, which mainly involved brain stem and cerebral white matter.

Conclusion: In this study, there was no clear difference in prevalence of age, sex, CNS symptoms and clinical criteria in the patients with Behcet syndrome and other similar studied. BAEP,VEP,SSEP and MRI results were similar to some other studies. Our findings show that both MRI \& Eps techniques are necessary in detecting of CNS involved in patients with Behcet syndrome disease.

\section{P558}

Posterior reversible encephalopathy syndrome - 5-year series, 2003-2007

H. Chua, Y. Sitoh

National Neuroscience Institute (Singapore, SG)

Background: Posterior reversible encephalopathy syndrome (PRES) is a clinicoradiologic entity characterized by headache, altered mental state, seizures and visual loss, associated with imaging findings of bilateral cortical, subcortical and brainstem edema. The precipitants of PRES include acute hypertension, sepsis, multiorgan failure, renal dysfunction, eclampsia and drugs.

Methods: We retrospectively reviewed the Department Registry of records over 5 years. Patients with predominantly white matter edema on CT or MRI brain with subsequent clinical and radiologic recovery were included.

Results: There were 5 patients ( 4 female, 1 male) and ages ranged from 19-53 years. Presentation: headache $(40 \%)$, unconsciousness (40\%), seizures $(20 \%)$, visual loss $(20 \%)$. Predisposing factors: hypertension $(100 \%)$, sepsis $(80 \%)$, multiorgan failure $(60 \%)$, renal dysfunction $(60 \%)$, eclampsia $(20 \%)$. All had neurological deficits ranging from ataxia and weakness to coma and loss of brainstem reflexes. Neuroimaging showed white matter abnormalities: supratentorial (100\%), cerebellum (60\%), brainstem $(40 \%)$. All recovered with treatment of underlying medical conditions with good neurological outcome.

One patient had recurrent PRES: A $44 \mathrm{yr}$ old female was operated for gangrenous jejunum/ileum. Postoperatively she developed multiorgan dysfunction with PRES which resolved with treatment. Four weeks later, PRES recurred due to uncontrolled hypertension. MR angiogram showed diffuse vasospam. Hypertension was treated successfully with intravenous labetalol. Repeat MRI brain and MR angiogram showed resolution of PRES and vasospasm.

Conclusion: Clinicians ought to be vigilant of PRES, a reversible white matter disorder with myriad presentation. The most important predisposing factors are hypertension and sepsis. 
P559

Anti-neuronal antibodies in Hashimoto's thyroiditis patients with central nervous system involvement

H. Durmus, S. Içöz, E. Tüzün, M. Kürtüncü, H. Hanagasi, N. Bebek, B. Baykan, G. Akman-Demir, M. Eraksoy

Istanbul University (Istanbul, TR)

Objectives: Central nervous system (CNS) involvement in Hashimoto's thyroiditis (HT) is speculated to be caused by an autoimmune mechanism. To investigate the role of anti-neuronal autoantibodies in the pathogenesis of HT encephalopathy, cerebrospinal fluid (CSF) and sera of HT patients with or without CNS involvement were tested by serological methods.

Methods: Sera and CSF of 5 HT patients with CNS symptoms (37-58 years old; 4 women, 1 man) and sera of $5 \mathrm{HT}$ patients without CNS symptoms (45-71 years old; all women) were tested for anti-neuronal antibodies by indirect immunohistochemistry and Western blot methods using rat brain and spinal cord samples.

Results: Three female HT patients with CNS involvement presented predominantly with limbic symptoms such as acute behavioral change, seizures, depressed mood and alteration in eating habits, a 37-year-old female patient presented with epilepsia partialis continua and a 47-year-old male patient had progressive pyramidocerebellar syndrome and right oculomotor nerve palsy. All patients had normal CSF cell counts and protein concentrations and 2 patients had CSF oligoclonal bands. Cranial MRI examination showed bilateral white matter lesions in 4 patients. Three female patients demonstrated diffuse slowing in EEG and two patients showed frontoparietotemporal or temporal involvement in PET/SPECT examination. While sera of all HT patients with CNS symptoms reacted with cytoplasms (3 patients with limbic and 1 with pyramidocerebellar symptoms) or nuclei (1 patient with limbic symptoms) of cerebral neurons, CSF of these patients and sera of 5 HT patients without CNS involvement revealed no anti-neuronal antibodies. Serum antibodies of only one patient with limbic symptoms immunohistochemically labeled the nuclei of spinal cord neurons. Sera of patients with CNS involvement but not isolated HT patients reacted with multiple rat brain proteins with variable molecular weights in Western blot studies.

Conclusion: HT patients may occasionally develop CNS symptoms. Serum antibodies of HT patients with neurological findings show clear and robust reactivity against multiple CNS antigens. Selective presence of antineuronal antibodies in sera of HT patients with CNS involvement suggests that anti-neuronal autoantibodies may be associated with the pathogenesis of neurological findings observed in HT patients.

P560

Neuroprotective effects of Resveratrol ${ }^{\circledR}$ in the model of 6-hydroxydopamine lesion in rats

M.T. Joghataei

Iran University of Medical Sciences (Tehran, IR)

Background: Scavenging of the reactive oxygen species (ROS) involved in oxidative stress has been the subject of many recent studies. Increasing evidence show that resveratrol, a polyphenol compound contained in several dietary products, exhibits cytoprotective effects. This study shows the neuroprotetion by resveratrol after the unilateral intrastriatal injection of 6-hydroxydopamine (6-OHDA) in rats, considered as a model of Parkinson's disease(PD)

Methods: Rats were pretreated with resveratrol ( $10 \mathrm{mg} / \mathrm{kg}$; i.p) 1 hour before surgery and treated daily ( $10 \mathrm{mg} / \mathrm{kg}$; i.p) for 14 days. Two weeks after the 6-OHDA lesion, rats exhibit a rotation behavior as a response to the apomorphine, apomorphine- induced rotation and number of nissl-stained neurons of substantia nigra pars compacta (SNC) were counted.

Results: Our results showed significant decrease in the number of apomorphine-induced rotations in resveratrol - treated lesion group, as compared to lesion-operated animals and protect neurons of SNC against 6-OHDA toxicity.

Conclusion: Our data demonstrated beneficial effects of resveratrol in this model of PD.

P561

Entropy analysis of electroencephalogram signals during recovery from coma

O. Gosseries, A. Vanhaudenhuyse, C. Schnakers, A. Walravens, G. Moonen, S. Laureys

Coma Science Group (Liege, BE); Inkendaal Hospital (Brussels, BE); University Hospital of Liege (Liege, BE)

Introduction: With the improvement in reanimation techniques, the number of persons surviving severe brain injury is increasing. These patients in an altered state of consciousness represent a problem in terms of their diagnosis. Indeed, assessing the level and content of consciousness in a non-communicating person is intrinsically difficult and it has been demonstrated that around one patient in three who is clinically diagnosed as "vegetative" is in fact conscious or minimally conscious. It is hence mandatory to study the role of para-clinical markers of consciousness, such as electroencephalography (EEG) entropy, in order to objectively assess patients' consciousness. Here, we reduced the complex information contained in the EEG to state entropy values. The aim of the study was to investigate the utility of EEG entropy calculation to disentangle vegetative state [VS] from minimally conscious state [MCS] patients.

Methods: A multi-centric prospective study recording fronto-temporal artifact and sedation-free EEG recordings in $20 \mathrm{VS}$ ( 8 traumatic) and $20 \mathrm{MCS}$ (12 traumatic) patients. Behavioral (Coma Recovery Scale - Revised [CRS$\mathrm{R}]$ ) and automated entropy monitoring (Datex-Ohmeda S/5, Helsinki, Finland) assessments were obtained in the acute $(n=21)$ and chronic setting $(\mathrm{n}=19)$.

Results: EEG entropy measurements showed a significant correlation with behavioral assessments (CRS-R total scores; $r=0,586$ ). Mean state entropy in VS patients were significantly different from mean values obtained in MCS (38 vs. 71, respectively). ROC analysis showed that a state entropy value cut-off of 48 differentiated VS from MCS with a sensitivity and specificity of $80 \%$.

Conclusions: EEG-state entropy measurements are a promising tool in monitoring consciousness in non-sedated severely brain-damaged patients and permit differentiation of vegetative from minimally conscious states. False positives $(20 \%)$ seem to be due to muscular artifacts. Our findings are encouraging in the search for electrophysiological correlates of consciousness in severe acute brain damage but require further improvements of the employed algorithms in order to be of use in the clinical setting and the individual patient level.

P562

Cervical spinal cord compression caused by a vertebral osteochondroma in hereditary multiple exostosis

A. Rabot

Hopital Pasteur (Nice, FR)

Background: Osteochondroma, common pediatric bone tumor, can be solitary or multiple. Hereditary multiple exostose (HME) is a benign autosomal (resulting from mutation in the EXT1 and EXT2) dominant disorder manifested by the presence of multiple osteochondromas affecting long and flat bones. Vertebral osteochondromas are uncommon localization and may cause neurology compromise. The objectif of this manuscript is to describe a rare case of cervical spinal cord compression due to an exostosis of $\mathrm{C} 7$ and revue literature.

Case desc ription: We describe a case of HME in a 17 year old girl with stiff neck and paraesthesia of the right superior limb due to an osteochondroma arising from the lamina of C7. The patient underwent laminectomy of $\mathrm{C7}$ with total removal of the exostosis and spinal cord decompression. Two months after surgery, patient presented an excellent recovery without paraesthesia and return to college.

Conclusions: Spinal cord compression due to an ostechondroma is an extremely rare and serious complication of HME. An osteochondroma of spine must always be considered in patient with HME who have neural symptoms. Neurosurgical approach should be recommended to realize spinal cord decompression before major neurological damage develops.

P563

Effect of botulinum toxin on skin innervation in patients with essential palmar hyperhidrosis

R. Liguori, F. Falzone, A. Giuliani, L. Calzà, E. Bugiardini, A. Baruzzi, P. Montagna, V. Donadio

University of Bologna (Bologna, IT)

Objectives: Botulinum toxin produces a functional block of sweat glands innervation. The effect of botulinum toxin on the other type of skin fibers has not been extensively reported. Our goal is to evaluate the effect of botulinum toxin on somatic and autonomic skin innervation in patients affected by essential palmar hyperhidrosis in chronic treatment with botulinum toxin.

Methods: We studied 5 patients (mean age $34 \pm 2$ years-old) who underwent skin biopsies. $3 \mathrm{~mm}$ punch skin biopsies were taken from adjacent areas of the third finger: 1) before the toxin injection; 2) after one month from the injection; 3 ) and before the next injection (mean period $7 \pm 3$ months). Biopsies were fixed in Zamboni solution, cut into $60 \mathrm{IIm}$ section using freezing slide microtome, and processed by an indirect immuno-fluorescent technique using pan-neuronal antibodies (protein gene product 9.5-PGP 9.5), 
and specific antobodies for cholinergic (Vasoactive Intestinal Peptide- VIP) and adrenergic (Dopamine beta hidroxilase- $\mathrm{DbH}$ ) innervation. The skin innervation study was performed by a laser scanning confocal microscopy.

Results: After one month from the botulinum toxin injection qualitative immunohistochemical analisis showed an increased density in VIP and PGP immunoreactive innervation around sweat glands. The sweat gland densitiy of the VIP and PGP immunoreactive fibers returned at the baseline level before the next injection, when the clinical effect of the toxin vanished. Furthermore no significant differences were found after the botulinum toxin injection in the innervation of the epidermal PGP-immunoreactive fibers, of the dermal VIP-immunoreactive fibers as well as in the adrenergic innervation evaluated by the $\mathrm{DbH}$ immunoreactive fibers.

Conclusion: Botulinum toxin treatment induces a selective modification of the cholinergic innervation of the sweat glands innervation without affecting other type of skin fibers.

P564

Posterior leukoencephalopathy and brain endothelial cell dysfunction caused by the macrolide roxithromycin

R. Tsabari, S. Tal, D. Nas, R. Aronovich, D. Gurwitz, J. Chapman, I. Blatt

Sheba Medical Center (Tel Hashomer, IL); Tel Aviv University (Tel Aviv, IL)

Objectives: To describe posterior leukoencephalopathy (PL) associated with roxithromycin treatment and to examine its pathogenesis.

Background: PL has been associated the macrolide tacrolimus (FK506) but has not yet been described with the very similar macrolide antibiotic roxithromycin (RXM)

Design/methods: We describe 2 patients in whom clinical and radiological manifestations of PL developed following treatment with RXM. In one case a brain biopsy was obtained. in-vitro experiments were performed measuring the phosphorylation of extracellular signal related kinase (pERK) in rat BVEC (G8) brain endothelial cells exposed to RXM, tacrolimus and cyclosporine.

Results: The patients developed visual field loss, epileptic seizures and typical posterior white matter lesions on MRI in close association with RXM administration. Significant resolution of the signs followed discontinuation of RXM. Histology in one case showed demyelination with macrophage infiltration and erythrophagocytosis compatible with impaired endothelial function. In the brain endothelial cell line experiments, RXM at a concentration of $50 \mu \mathrm{M}$ produced inhibition of ERK phosphorylation similar to concentrations of $20 \mu \mathrm{M}$ tacrolimus and $5 \mu \mathrm{M}$ cyclosporine.

Conclusions: We describe the clinical association of RXM with PL. Similarly to the macrolide tacrolimus, RXM causes inhibition of second-messenger function in brain endothelial cells and probably induces PL through endothelial dysfunction.

\section{P565}

Extensive anterior intradural spinal arachnoid cyst associated with syringomyelia: a case report

S. Ferrari, L. Politi, L. Peruzzotti Jametti, S. Mammi, G. Comi, M. Comola

San Raffaele Hospital (Milan, IT)

Objective: To describe a rare case of anterior intradural spinal arachnoid cyst (ISAC) spreading throughout the spinal cord, associated with syringomyelia.

Methods: A 55-year-old caucasian male presented weakness, numbness and stiffness of his right arm associated with slowly progressive paraparesis. Past history revealed a severe cervical trauma at the age of 16. Magnetic resonance imaging demonstrated a reduced thickness of the whole spinal cord with a focal atrophy at $\mathrm{C} 2 / \mathrm{C} 3$ level. A small syringomyelic cavity was detected at $\mathrm{C} 1 / \mathrm{C} 2$ level, associated with an extramedullary cystic cavity, anterior to the spinal cord, spreading from $\mathrm{C} 2 / \mathrm{C} 3$ to D12. Because of worsening symptoms, the patient underwent a right posterior laminectomy at $\mathrm{C} 3$ level, with the positioning of a small shunt between the cyst and the subarachnoid space. The patient experienced a transitory relief of the symptoms, soon followed by a progressive worsening of his condition. A specific cinematic-MRI (cMRI) was then carried out to assess the dynamic changes of the CerebroSpinal Liquid inside the ISAC. This approach showed a regular sisto-diastolic modulation of flow inside the cyst, normal shunt function and wide communication between the cyst and the subarachnoid space. These results were indeed in great discrepancy with the clinical findings.

Results: In order to define the real dynamic of fluid movement and the opportunity of another surgery intervention, the patient underwent a CTmyelography (CTM). Only a small quantity of contrast was found inside the cyst, thus confirming the clinical diagnosis of a poor communication within the shunt.

Conclusions: Extramedullary Cysts of Spinal Cord (ECSC) are uncom- mon causes of midollar compression. The current classification distinguishes among meningeal extradural cysts (including ISACs), non meningeal epidural cysts and neurenteric cysts. ECSC are indeed rare entities and ISACs are even less common. To our knowledge, only another case is reported in literature with an intradural cyst growing along the entire spinal cord on a ventral position. Moreover the association with a syringomyelic cavity is extremely rare. CMRI may be a useful tool to detect a communication between the cyst and the subarachnoid space, but when the cyst causes marked compression, this approach may not be a valid technique. In our case, MRI established the diagnosis of ISAC, but only CTM led us to define the real dynamics of the fluid components inside the cyst.

\section{P566}

Are direct connections between occipital and frontal lobes unique to the human brain?

S. Forkel, A. Danek, M. Catani

King's College (London, UK); Ludwig-Maximilians University (Munich, DE)

The unique adaptive nature of the brain relies on the ability to modify behavioural responses to the surrounding environment as perceived through our senses. Adaptive behaviour based on visual inputs depends on the mutual interaction between the occipital and frontal lobes (Zeki 1993). Preliminary evidence suggests that a set of direct connections between most ventral occipital and frontal regions may represent a unique feature of the human brain (Catani 2007). Here, we review 200 years of anatomical studies on the connectional anatomy of the occipito-frontal systems in both humans and animals.

In the human brain the existence of direct ventral connections between occipital cortex and orbito-frontal cortex has been demonstrated using post mortem and recent in vivo diffusion tensor tractography dissections. Dorsal occipito-frontal connections have been identified in human brains with agenesis of the corpus callosum (Onufrowicz 1887), but whether an equivalent exists in the normal brain is still debated (Schröder 1901, Türe 1997). In the monkey brain a superior (dorsal) fronto-occipital fasciculus has been identified using axonal tracing techniques, but a ventral direct occipito-frontal system has not been found (Schmahmann 2007).

In conclusion the existence of ventral direct occipito-frontal connections has been widely replicated in human (Curran 1909, Cowley 1982, Bürgel 2005, Lee 2005) but not in monkey brain (Markis 2006, Schmahmann 2007). This suggests that a ventral occipito-frontal network may represent a novel pathway in the evolutionary scale. The functional correlates of the inferior occipito-frontal system are not known, but recent studies suggest that this pathway may be implicated in unique human skills (e.g. reading) or other tasks (e.g. face processing) that facilitated our ability to communicate and interact with others.

\section{References}

Bürgel U (2006) NeuroImage 29:1092-1105

Catani M (2007) Brain 130:602-605

Cowley A (1982) AJNR 4:915-925

Curran EJ (1909) J Comp Neurol Psychiat 19:645-656

Lee SK (2005) RadioGraphics 25:53-68

Markis N (2007) 37:1100-1111

Onufrowicz W (1887) Arch Psychiatrie 18:305-328

Schmahmann JD (2007) Brain 130:630-653

Schröder P (1901) Monatsschr Psychiatrie Neurol 9:81-99

Türe U (1997) Neurosurgery 40:1226-1232

Zeki S (1993) A vision of the brain. Blackwell

\section{P567}

Baclofen neurotoxicity: from encephalopathy with periodic sharp waves to withdrawal delirium

S. Gasverde, C. Baima, A. Franco, P. Gai, D.M. Papurello

Ospedale Ciriè (Ciriè, IT)

Introduction: Baclofen has become the drug of choise for treatment of spasticity. Confusion, drowsiness and stupor have been reported rarely in elderly patients with cerebrovascular diseases.

We describe a patient who experienced an acute baclofen-overdose encephalopathy with unusual EEG findings followed by delirium induced by abrupt drug discontinuation.

Case report: A woman aged 60, with spastic tetraparesis caused by traumatic myelopathy occuring 4 years previously, was evaluated for acute onset of disturbed consciousness. She had received baclofen ( $100 \mathrm{mgldie})$ for more than 2 years to control spasticity.

On admisssion she presented stuporous with diffuse areflexia and hypo- 
tonia. Laboratory tests were normal with the exception for increased creatinine $(1.94 \mathrm{mg} \backslash \mathrm{dL})$; in the past few days she assumed ciprofloxacina for recurrent urinary infections.

Cerebral CT and MRI scans were normal. Study of a CSF sample revealed no abnormalities. EEG showed pseudo-periodic generalized thiphasic sharp waves discharges.

Baclofen was withdrawn, the patient was given supportive treatment with intravenous fluids and creatinine gradually normalized.

Consciousness was restored within 72 hours after baclofen discontinuation but disorientation, anxiety, visual allucination and tremor developed during the following days.

A short-term antipsychotic therapy with concomitant bendodiazepine controlled acute psychiatric symptoms.

Discussion: Baclofen is excreted unchanged by the kidney for more than $85 \%$. The drug might have accumulated to a toxic level because of acute impaired renal function found on admission. The EEG showed pseudo-periodic sharp waves discharges and similar pattern have been described in literature associated with baclofen overdose. In our patient this hypotesis is strenghten by normal neuroimaging and CSF study, absence of other metabolic disturbances and consciousness restoration after drug cessation. Delirium arose secondarily from baclofen abrupt discontinuation as a conseguence of disinhibition of previously suppressed monoamine pathways.

Conclusion: Baclofen neurotoxicity with pseudo-periodic triphasic sharp waves EEG pattern may masquerade as other metabolic-toxic encephalopathies and when recognized early carries an excellent prognosis. Drug discontionuation is deemed and antipsicotic medication may be associated to prevent drug widrawal delirium; concomitant benzodiazepine may be used to mitigate the risk of seizures.

\section{P568}

Fregoli's syndrome and traumatic brain injury

S. Pires-Barata, J. Palma Góis, M.H. Teixeira da Silva

Hospital do Espirito Santo (Évora, PT)

The Fregoli syndrome is a delusional misidentification syndrome. This is a rare syndrome and it is most often seen among psychiatric disorders but also reported in brain diseases. Usually associated with diffuse lesions, right temporal lobe has also been referred as implicated. We report a case of a 19 year old male, who, one year and half after a traumatic brain injury, developed a delusional misidentification syndrome - Fregoli type. The patient was submitted to neuropsychological evaluation. The evaluation revealed memory impairment and executive functions disorders, no impairment was observed in visual recognition. The magnetic resonance study showed lesions in the first frontal lobe circumvolution, bilateral lesions in the basal ganglia and right side of corpus callosum. Although Fregoli Syndrome is rare among acquired brain injuries, it might be important to take it in consideration in order to avoid misinterpretations of psychotic symptoms.

P569

Copper deficiency myelopathy and subacute combined degeneration of the cord: why is the phenotype so similar?

S.R. Jaiser, G.P. Winston

Newcastle General Hospital (Newcastle upon Tyne, UK); National Hospital for Neurology and Neurosurgery (London, UK)

Objectives: Copper deficiency myelopathy (CDM) mimics subacute combined degeneration of the cord due to vitamin B12 deficiency (SCD) both clinically and radiologically. We hypothesised that this phenotypic congruence may be attributable to a shared pathophysiological mechanism, and sought to identify candidate metabolic pathways that depend on both copper and vitamin B12.

Methods: We searched the literature for copper and vitamin B12-dependent enzyme systems in animals and humans, aiming to identify areas of overlap.

Results: In humans, vitamin B12 acts as a cofactor to only two enzymes.

The first enzyme, methylmanoyl CoA mutase, catalyses the isomerisation of methylmalonyl CoA to succinyl CoA. There is no evidence that the enzyme is copper-dependent, or that it has any role in the pathogenesis of SCD.

The second enzyme, methionine synthase, demethylates methyltetrahydrofolate to tetrahydrofolate transferring the methyl group to homocysteine to generate methionine. The methyl group is subsequently transferred to a range of macromolecules via a transmethylation cycle. Decreased activity of methionine synthase has two main effects. Firstly, it leads to reduced levels of tetrahydrofolate, which is required for purine synthesis. Secondly, it causes failure of methylation of lipids, nucleic acids and proteins including myelin proteins and lipids. Copper is thought to modulate the activity of me- thionine synthase and adenosylhomocysteine hydrolase, another enzyme involved in the methylation cycle. Adenosylhomocysteine hydrolase, however, is not known to be vitamin B12-dependent.

Conclusion: We propose that dysfunction of the transmethylation cycle may be central to the pathogenesis of both $\mathrm{CDM}$ and SCD. This cycle has established roles in folate metabolism and myelination, and there is evidence that it contains both vitamin B12-dependent and copper-dependent enzymes.

\section{Multiple sclerosis}

P570

The cost of disability and medically related absenteeism among privately insured United States employees with multiple sclerosis

A. AL-Sabbagh, R. Bennett, H. Birnbaum, J. Ivanova, S. Samuels, M. Davis, A. Phillips, D. Meletiche

EMD Serono, Inc. (Rockland, US); Analysis Group, Inc. (Boston, US); Analysis Group, Inc. (New York, US)

Objective: Chronic diseases, such as multiple sclerosis (MS), are associated not only with direct costs of therapy, but also with indirect costs related to comorbidities and absenteeism from work. This analysis evaluates the annual indirect costs of privately insured United States (US) employees with MS vs matched employee controls.

Methods: A database containing disability data from 17 US private insurers was used to identify employees aged 18-64 years with at least $1 \mathrm{MS}$ diagnosis (diagnosis code ICD-9-CM: 340.x) after January 1, 2002. MS employees were selected regardless of whether they were receiving diseasemodifying drugs or not. Employees with MS were matched by age and sex to an employee control group without MS. All study participants were required to have continuous health coverage 3 months before diagnosis (baseline) and 12 months after (study period). Comparison of baseline comorbidities and differences in measures of indirect resource use (disability and medically related absenteeism) were evaluated using chi-square tests. Wilcoxon rank-sum tests were used for univariate comparisons of mean disability and medically related absenteeism days and associated annual indirect costs during the study period.

Results: 989 privately insured employees with MS met the study criteria (women, 76\%; mean age, 44 years). Compared with employee controls, employees with MS had significantly higher rates of comorbid mental health disorders and other neurological and physical disorders as measured by the Charlson Comorbidity Index. Employees with MS were also more likely to have short- or long-term disability vs controls $(21.4 \%$ vs $5.2 \%$, respectively; $\mathrm{P}<0.0001$ ), resulting in a higher average number of disability days per year ( 29.8 vs $4.5 ; \mathrm{P}<0.0001)$. Employees with MS also had a higher incidence of medically related absenteeism and associated absenteeism days compared to employee controls. Annual costs for disability, on average, were $\$ 3868$ for employees with MS and $\$ 414$ for employee controls $(\mathrm{P}<0.0001)$. Costs for medically related absenteeism were also higher in the group with MS compared with controls ( $\$ 1901$ vs $\$ 1003$, respectively; $\mathrm{P}<0.0001)$. Total indirect costs averaged $\$ 5769$ for employees with MS and $\$ 1417$ for controls $(\mathrm{P}<0.0001)$.

Conclusion: On average, the indirect costs of employees with MS were more than 4 times those of employee controls. Effective treatments may reduce the burden of this disease in the workplace.

This study was supported by EMD Serono, Inc. and Pfizer Inc.

\section{P571}

Paraoxonase 1 activity does not change in stable and progressive type of multiple sclerosis but decreases during relapse of the disease A. Jamroz-Wisniewska, H. Bartosik-Psujek, J. Beltowski, Z. Stelmasiak Lublin Medical University (Lublin, PL)

Paraoxonase 1 (PON1) is an antioxidant enzyme bound in plasma with highdensity lipoproteins that is also present in brain. It plays an important role in organophosphate detoxification and prevention of atherosclerosis. Multiple sclerosis (MS) is a chronic disabling disease that is the most common demyelinating disease of the CNS usually affecting young adults.

Objectives: The aim of our study was to estimate the activity of PON1 in patients with relapsing-remitting (RR) form of MS both during the stable and acute stage of the disease and in patients with progressive form of the disease.

Methods: PON1 activity toward two synthetic substrates and lipid pro- 
file were studied in 137 people with definite MS according to the McDonald's criteria. Depending on clinical symptoms of MS, the patients were divided into three subgroups: $40 \mathrm{RR}$ patients in relapse, (F/M 30/10; mean age: $33.2 \pm 8.0$ years; Expanded Disability Status Scale, EDSS $3.4 \pm 1.3), 42 \mathrm{RR}$ patients in remission, (F/M 31/11; mean age: $32.1 \pm 7.8$ years; EDSS $2.5 \pm 1.0$ ) and 55 progressive MS patients (F/M 41/14; mean age: $39.6 \pm 9.2$ years; EDSS $5.8 \pm 0.9$ ). The control group included 40 healthy people (F/M 30/10; mean age: $36.3 \pm 12.6$ years).

Results: PON1 activity toward paraoxon and phenyl acetate did not differ significantly in MS patients comparing to control group. PON1 activity toward paraoxon in RR patients in relapse was significantly lower in comparison to the other MS groups $(\mathrm{p}<0.01)$. We observed hypercholesterolemia in MS patients comparing to control group.

Conclusion: PON1 activity does not change in the course of stable and progressive type of MS and is decreased by the relapse of the disease.

P572

Anti-inflammatory cytokine gene polymorphisms in multiple sclerosis: a study in a Spanish population

A.L. Guerrero, J.A. Garrote, D. Pérez, A. Mendoza, J.T. López Alburquerque, J.C. Morán, A.B. Guerrero, D. Bernardo, A.B. Caminero, J. Martín-Polo, S. Merino, H. Alonso, C. Jiménez, J.J. Ruíz-Ezquerro, E. Arranz

Hospital Río Carrión (Palencia, ES); Hospital Clínico Universitario (Valladolid, ES); Hospital del Bierzo (Ponferrada, ES); Hospital General (Segovia, ES); Hospital Universitario (Salamanca, ES); Hospital General (Soria, ES); Universidad de Valladolid (Valladolid, ES); Hospital Virgen de Sonsoles (Avila, ES); Hospital Virgen de la Concha (Zamora, ES)

Objectives: Cytokine gene polymorphisms are known to influence susceptibility and disease course of many autoinmune diseases, and previous studies have suggested a role for some of these genes in susceptibility to multiple sclerosis (MS). Dysregulation in the expression of anti-inflammatory cytokines may play a key role in MS development and progression.

Methods: Multicentric, prospective, longitudinal, case-control observational study, in 7 hospitals belonging to the Public Health Network SACYL (Castilla y León Community, Central Spain) Main goal to assess whether cytokine-coding genes have a prognostic value as markers of response to treatment with Interferon beta-1b. Inclusion criteria: diagnosis of Relapsing Remitting MS (RRMS), at least 6 months from first clinical event, age above 18 years, EDSS $0-6.5,2$ or more relapses in the 3 previous years, naïve, beginning treatment with interferon beta $1 \mathrm{~b}$. Comparative control group. In the inclusion visit, the following polymorphisms were determined: Transforming Growth Factor betal (TGFB1) (transition T-C in codon 10 and C-G in codon 25), Interleukin-10 (IL10) (transitions A-G in position -1082, T-C in $-819, \mathrm{~A}-\mathrm{C}$ in -592 , all of its promoter), Interleukin 4 (IL4) (transitions G-T in position -1098, C-T in -590 and C-T in -33 of its promoter) and alfa receptor IL4 (IL4RA) (transition A-G in position +1902) Preliminary data of influence of these genetic polymorphisms on susceptibility to MS have been analyzed for this communication using chi-square and Fisher's exact tests.

Results: 24 patients according inclussion criteria were included in the study, and 70 healthy volunteers in the control group. $65 \%$ patients were female, mean age 33.7 years (22-53), and median EDSS 1.5 (0-3.0) We observed a significant increase in the G/G and T/G genotypes of IL4 -1098 (p: 0.032 ) and in the C/C genotype of IL4 -33 (p: 0.019) (OR: 3.1; IC $95 \%$ (1.1-9.0)) in our MS patients. The G allele at IL4 -1098 was more prevalent in the patients than in the controls ( $\mathrm{p}: 0.011$ ). No significant differences were observed in either of the TGFB1, IL10 or IL4RA polymorphisms investigated.

Conclusion: Although the size of the study group is small, these results indicate that polymorphic variations of anti-inflammatory cytokine IL4 may play a role in MS susceptibility in our population.

Supported by a grant from Bayer Schering

\section{P573}

Postural balance in multiple sclerosis. Is it being assessed properly?

C. Fjeldstad, G. Pardo, C. Frederiksen, D. Bemben, M. Bemben

MS Center of Oklahoma (Oklahoma City, US); University of Oklahoma (Oklahoma City, US)

Compromised postural balance is a common manifestation of Multiple Sclerosis (MS) and can be attributed to specific deficits such as weakness, spasticity and ataxia. There is lack of good quantitative postural imbalance assessment methods that can help the clinician in evaluating progression of this deficit.

Objective: To examine postural balance in a population of MS patients and healthy controls by a standard screening tool, the Berg Balance Scale, and a more technically involved device, the Neurocom Balance Master.

Methods: Ten healthy controls and 17 individuals diagnosed with MS, that were ambulatory without aids, ranged in age from 20 to 54 years and were significantly similar in age, height, weight, and BMI. Each subject performed a series of six balance measures using the Neurocom Balance Master and was assessed with the Berg Balance Scale.

Results: Independent t-tests indicated a significant difference between the Control group and the MS group for the Berg Balance Scale $(\mathrm{p}<0.05)$, with the MS group performing significantly worse. Likewise, there was a significant difference between the two groups on five of the balance measures $(\mathrm{p}<0.05)$, including walk across $(\mathrm{cm} / \mathrm{sec})$, step quick turn ( $\mathrm{sec})$, sensory organizational test (equilibrium score), adaptation test with toes down (score), and directional control (\%). There was a moderate to high positive correlation between the Berg Balance Scale and approximately $70 \%$ of the balance tests performed on the Neurocom for the MS group.

Conclusions: MS patients demonstrated significantly more postural instability compared to the Control group and moderate to high positive correlations $(0.50-0.80)$ between postural assessments by the Neurocom and the standard Berg Balance Scale. This indicates that the Berg Balance Scale remains an effective screening tool for balance, but does not account for imbalance conditioned by the dynamic response to external changes such as surface inclination.

\section{P574}

Apo-E and TNF-alpha gene polymorphisms on patients with primary progressive type of multiple sclerosis in Hungary

E. Losonczi, V. Honti, K. Bencsik, Z. Fricska-Nagy, E. Szalczer, C. Rajda, J. Füvesi, Z. Illés, K. Mátyás, T. Csépány, C. Rózsa, L. Vécsei

University of Szeged (Szeged, HU); Biological Research Center (Szeged, HU); University of Pécs (Pécs, HU); County Hospital (Kecskemét, HU); University of Debrecen (Debrecen, HU); Ferenc Jahn Hospital (Budapest, HU)

Objectives: The genetic background of multiple sclerosis (MS) is unclear but we can consider it as a polygenetic disease. Our aim was to investigate the apolipoprotein E (Apo-E) and the tumor necrosis factor-alpha (TNF-alpha) polymorphisms in primary progressive patients in a multicenter survey.

Methods: Our study was based on 45 primary progressive (PP) patients from 5 Hungarian MS Centers. To the control group we enrolled 45 sex and age matched relapsing-remitting (RR) patients with MS and 45 sex and age matched healthy controls (HC). Specific PCR was carried out on the isolated DNA obtained from blood samples of the groups. The PCR products were respectively digested by Hin6I and TasI restriction enzymes in the case of Apo$\mathrm{E}$ and TNF-alpha. The digestion products were resolved on an $8 \%$ polyacrilamide gel and detected under ultraviolet light after being stained with ethidium bromide. The results were compared with the help of Mann-Whitney U statistical test.

Results: In the PP group the Apo-E E4 allele occurred significantly more frequent than in the RR group and the HC group $(p=0.003, p<0.001)$. The Apo-E E2 allele appeared in the PP group less frequent than in the RR group and in the $\mathrm{HC}$ group $(\mathrm{p}<0.001, \mathrm{p}<0.001)$

In the PP group the TNF-alpha polymorphism was less frequent than in the RR group and in the HC group $(p=0.04, p<0.006)$.

Conclusion: Our study supports the neurodegenerative pathomechanism of PPMS by the more frequent appearance of Apo-E E4 allele and the less frequent of TNF-alpha polymorphism. While the Apo-E E2 allele might be responsible for the development of the inflammation in the RRMS pathomechanism.

\section{P575}

Cognitive and clinical effects of subcutaneous interferon beta-1a in patients with early relapsing-remitting multiple sclerosis: 2 - and 3-year results from the COGIMUS (COGnition Impairment in MUltiple Sclerosis) study

F. Patti, M.P. Amato, M.R. Tola, M. Trojano, P. Ferrazza, O. Picconi, S. Bastianello on behalf of the COGIMUS Study Group

Objectives: To assess the effects over time of two doses of interferon (IFN) beta-1a on cognitive function and clinical parameters in patients with early relapsing-remitting multiple sclerosis (RRMS).

Methods: Patients with RRMS (McDonald criteria) aged $18-50$ years who were naïve to treatment at baseline were recruited to this prospective, multicentre, observational, dose-controlled study. Patients received IFN beta-1a, 22 or $44 \mathrm{mcg}$ subcutaneously three times weekly, and were evaluated clinically, including complete neurological examination, at baseline and every 6 months for 3 years. Rao's battery of seven neuropsychological tests was performed at baseline and every 12 months thereafter.

Results: The study recruited 459 patients for treatment with IFN beta-1a ( $22 \mathrm{mcg}, \mathrm{n}=213 ; 44 \mathrm{mcg}, \mathrm{n}=246$ ). At 2 years, the proportion of patients who 
developed cognitive impairment in two or more tests was significantly smaller with the $44 \mathrm{mcg}$ dose of IFN beta-1a than with the $22 \mathrm{mcg}$ dose $(28.1 \%$ versus $39.1 \%$, respectively, $\mathrm{p}=0.035)$. Preliminary results of a 3 -year efficacy analysis are based on data from 304 of 363 patients completing 3 years of treatment $(22 \mathrm{mcg}, \mathrm{n}=164 ; 44 \mathrm{mcg}, \mathrm{n}=199)$. Over 3 years, 222 of 304 (73.0\%) patients were relapse free, $58(19.1 \%)$ experienced one relapse, 21 $(6.9 \%)$ experienced two relapses, $14(4.6 \%)$ experienced three relapses, 4 $(1.3 \%)$ experienced four relapses and $3(1.0 \%)$ experienced five relapses. The annualized relapse rate was 0.19 . Sustained Expanded Disability Status Scale progression (one-step confirmed over 6 months) occurred in 45 $(14.8 \%)$ patients. Additional data on 3-year cognition will be presented together with additional clinical efficacy and safety data.

Conclusions: A dose-dependent reduction in the progression of cognitive impairment with IFN beta-1a therapy supports early initiation of highdose, high-frequency IFN beta-1a therapy in RRMS.

Study supported by Merck Serono International S.A.

\section{P576}

Neutralising antibodies do not affect efficacy of interferon beta-1b: further analysis of the BEtaferon ${ }^{\circledR}$ in Newly Emerging multiple sclerosis For Initial Treatment study (BENEFIT)

H-P. Hartung, G. Edan, M. Freedman, L. Kappos, D. Miller, X. Montalban, C. Polman, L. Bauer, V. Lanius, C. Pohl, R. Sandbrink

Heinrich-Heine-Universitat (Dusseldorf, DE); Centre Hospitalier Universitaire (Rennes, FR); Ottawa Hospital (Ottawa, CA); University Hospital Basel (Basel, $\mathrm{CH}$ ); National Hospital for Neurology and Neurosurgery (London, UK); Hospital Vall d'Hebron (Barcelona, ES); Vrije Universiteit Medical Centre (Amsterdam, NL); Bayer Schering Pharma AG (Berlin, DE)

Objective: To determine whether neutralising antibodies (NAb) influence the clinical efficacy of interferon beta-1b (IFNB-1b; Betaferon(r)) in patients with a first event suggestive of multiple sclerosis (MS).

Methods: The BENEFIT study is designed to evaluate the impact of early versus delayed initiation of IFNB-1b treatment on disease evolution in patients with a first event suggestive of MS. In the placebo-controlled phase of BENEFIT, patients were randomised to IFNB-1b $250 \mathrm{mcg}$ or placebo subcutaneously every other day for 2 years or until a diagnosis of clinically definite MS (CDMS). Patients who completed this phase were then eligible for enrolment to a follow-up phase in which all were offered IFNB-1b $250 \mathrm{mcg}$ for up to 5 years after randomisation. Primary outcomes include time to confirmed disability progression. NAb titres were measured using the in-vitro MxA assay every 6 months. For neutralising activity three different categories of positivity were evaluated (between $\geq 20$ and $<100$, between $\geq 100$ and 400 and $\geq 400 \mathrm{NU} / \mathrm{ml}$ ). A neutralising activity below $20 \mathrm{NU} / \mathrm{ml}$ was defined as negative.

Results: Data are available for patients 3 years after randomisation. Patients treated early (those initially randomised to IFNB-1b) had a $40 \%$ reduction in risk of confirmed disability progression compared with those originally randomised to placebo $(p=0.022)$. NAbs were measured in $277 / 292$ early-treated patients. Over 3 years, positive NAb titres $(\geq 20 \mathrm{NU} / \mathrm{ml})$ were detected at least once in $88(30.2 \%)$ of theses patients; $41(46.6 \%)$ had reverted to stable NAb-negative status by Year 3 . The respective frequencies for the other titre categories were $7.2 \%$ and $8.6 \%$, reverting to NAb-negative status in $23.8 \%$ and $4.0 \%$, respectively. No effect of NAb positivity, regardless of titre, was found on time to CDMS, or time to confirmed disability progression. Further analyses regarding a potential impact of NAb on other trial outcomes, including magnetic resonance imaging, are currently underway, and will be presented.

Conclusions: NAb positivity, regardless of titre, did not affect the efficacy of IFNB-1b treatment in delaying conversion to CDMS or disability progression over 3 years.

Study supported by: Bayer Schering Pharma AG, Berlin, Germany

P577

Epidemiology of multiple sclerosis in Herzegovina

I. Klupka-Saric, M. Galic

Clical Hospital Mostar (Mostar, BA)

Objective: To determine epidemiological rates of multiple sclerosis (MS) in Herzegovina (southern part of Bosnia and Herzegovina)

Methods: In the period 1996-2006, by the analysis of all available health and medical sources in the investigated area, $106 \mathrm{MS}$ potential patients have been singled out. By the application of diagnostic criteria according to CM Poser on the prevalence day, 31 December 2006., 96 patients ( 33 males, $63 \mathrm{fe}-$ males), clinically certain cases of MS have been confirmed.
Results: Crude prevalence of MS was 30.99/100,000 (95\% CI: 24.8-37.2). Prevalence was highest in the mountainous municipality of Posu?je, 49.6/100.000 and lowest in the coastal municipality of Neum (0 incidence). The mean age of the patients on prevalence day was $41.4 \pm 10.2$. years and the mean age at disease onset was $30.7 \pm 6.4$ years; the mean duration of the disease was $10.7 \pm 7.5$. years. The annual incidence of MS was $1.7 / 100,000(95 \%$ CI, 1.2-2.1). The course of the disease was relapsing remitting in $58 \%$, secondary progressive in $28 \%$, primary progressive in $9 \%$ and progressive relapsing $1 \%$ of MS patients. Sensory (33\%) and motor (24\%) initial symptoms were most common.

Conclusion: Herzegovina is an area of moderate risk for MS. The distribution of the disease is heterogeneous. MS is more common in mountainous, rarer in coastal municipalities. The continuation of the research with the application of more sophisticated methods is necessary for the clarification of this difference.

P578

Bone mineral density, motor function, and vitamin D status in fully ambulatory persons with multiple sclerosis - population-based cross-sectional data

L.H. Steffensen, S.I. Mellgren, M.T. Kampman

University Hospital of North Norway (Tromsø, NO); University of Troms $\emptyset$ (Tromsø, NO)

Objectives: Several studies have shown that bone mineral density (BMD) at the femoral neck decreases with increasing physical handicap (EDSS-score) in multiple sclerosis (MS) patients. Possible explanations are less weightbearing exercise or less UV-exposure resulting in reduced vitamin D generation in the skin. Prevention of osteoporosis is a high priority, because treatment of the established disease remains sub-optimal. We will present baseline data from the randomised controlled trial "Can vitamin D supplementation prevent bone loss in persons with MS?"

Methods/results: 139 patients aged 18-50 years with a diagnosis of MS according to McDonald's criteria and an assumed EDSS score of $\leq 4$ (retrieved or estimated from hospital charts) from Troms and Finnmark counties were invited to participate in the randomised controlled trial. So far the response rate is higher than $60 \%$. Baseline measurements will be performed in the period from 28 January to 7 March 2008.

We will present results on BMD (femoral neck, lumbar spine, both forearms), serum $25(\mathrm{OH})$ vitamin D, EDSS, MSFC, 10 foot tandem walk test and strength of grip (Martin vigorometer). Regression analysis will be applied to estimate the effects of serum $25(\mathrm{OH})$ vitamin D, EDSS, motor function, lifetime corticosteroid treatment, and age on BMD in women and men.

Conclusion: We will present data from a larger number of BMD measurements in fully ambulatory persons with MS than have been included in previous studies. Forearm BMD in persons with MS has not been reported by others. At this site, BMD is independent of ambulatory function.

\section{P579}

Increased production of new T-lymphocytes after Copaxone ${ }^{\circledR}$ therapy M. Chiarini, R. Capra, A. Sottini, C. Ghidini, C. Zanotti, S. Federico, C. Cordioli, M. Rottoli, M. Zaffaroni, L. Imberti

Spedali Civili (Brescia, IT); Multiple Sclerosis Center Ospedale di Montichiari (Montichiari, IT); Multiple Sclerosis Center Ospedale di Bergamo (Bergamo, IT); Multiple Sclerosis Center Ospedale S.A. Abate (Gallarate, IT)

Objective: To evaluate the extent of thymic output in Multiple Sclerosis (MS) patients and the role of Copaxone ${ }^{\circledR}$ in the generation of new T lymphocytes we measured the amount of T-cell receptor excision circles (TRECs) and the number of recent thymic emigrants (RTE) with CD4+CD45RA+CCR7+ CD31+ phenotype.

Methods: One hundred and ninety patients (age from 20 to 65 years) with relapsing-remitting MS and 80 healthy donors were included in the study. Among MS patients, 80 were treated with Copaxone ${ }^{\circledR}(20 \mathrm{mg} / \mathrm{die})$ from at least one year, 80 had received no immunomodulatory treatment ("naïve" patients), and 30 ("treated" patients) have began the treatment with Copaxone $^{\circledR}(20 \mathrm{mg} / \mathrm{die})$ at the moment of the inclusion in the study ("follow-up" patients). In the follow-up patients, sampling was done before the treatment beginning (T0), and then after 4 and 12 months. The EDSS score ranged from 1 to 5,5 .

Thymic output was measured both by Real-Time PCR for the determination of TRECs and by flow cytoflorimetry for the quantification of the RTE with CD4+CD45RA+CCR7+CD31+ phenotype.

Results: We have not found differences in TRECs number in controls, naïve and treated patients, even when samples were divided on the basis of age; the analysis of the correlation between age and TRECs number showed that a growing age was related to a diminishing TRECs number in the 3 
groups of samples. The percentage of RTE decreased in MS untreated patients, while Copaxone ${ }^{\circledR}$ treatment induced a statistically significant rise of this cell population percentage both in regards to untreated MS patients and healthy controls. Furthermore, we observed significant differences in the groups of treated patients with age ranging from 20 to 35 years and from 36 to 50 years, in comparison to untreated patients with the same age. Finally, follow-up patients can be divided in 2 groups, one in which there was an increased percentage of RTE at 12 months (but not at 4 months) after therapy initiation and one with a stable RTE percentage.

Conclusion: The decreased RTE in MS may be responsible of the limited diversity of T-cell repertoire found in these patients. RTE recovery after Copaxone ${ }^{\circledR}$ suggests that this therapy may directly or indirectly induce the generation of new lymphocytes, preferentially in patients under 50 years of age. Therefore, the percentage of RTE, but not the number of TRECs, appears to be a good marker of newly generated T lymphocytes in MS.

P580

Progranulin gene mutation scanning in multiple sclerosis patients with cognitive impairment

M. De Riz, C. Fenoglio, D. Scalabrini, C. Comi, A. Pietroboni, E. Venturelli, F. Cortini, C. Villa, M. Serpente, M. Leone, F. Monaco, N. Bresolin, D. Galimberti, E. Scarpini

Dino Ferrari Center Ospedale Maggiore Policlinico (Milan, IT); Ospedale Maggiore University Amedeo Avogadro (Novara, IT)

Objective: To perform an association study of rs9897526 and rs5848 in the gene encoding for Progranulin (PGRN) in Multiple Sclerosis (MS) patients with and without cognitive impairment as compared with age-matched controls to determine whether the presence of these variants influences the susceptibility to MS or to the development of cognitive impairment during the course of the disease.

Background: PGRN is 593 aminoacid secreted glycoprotein which is expressed in many tissues, including the Central Nervous System. Mutations in PGRN are responsible for familial Frontotemporal Lobar Degeneration with ubiquitin pathology. Progranulin is highly expressed in microglial cells and likely plays a role in neurodegeneration. To date, studies about the role of this protein in cognitive decline occurring in MS patients are still lacking.

Methods: Preliminary case-control study was performed in 154 patients with MS (of which 19 with cognitive impairment, evaluated through neuropsychological testing) and 144 controls for rs9897526 and rs5848 Single Nucleotide Polymorphisms (SNPs) by allelic discrimination using an ABI PRISM 7000 instrument (ABI). Haploview software was used to test for association.

Results: No significant differences were found between patients, either with cognitive decline or not, and controls. However, a tendency towards an increase of the frequency of rs5848 T/T genotype was found in MS patients compared with controls $(16 \%$ versus $7 \%, \mathrm{P}>0.05)$. No differences were found stratifying according to gender or the subtype of MS.

Conclusion: PGRN likely doesnjit influence either the susceptibility to develop MS, or the risk to have cognitive impairment during the course of the disease. However, these results need to be confirmed in a wider population to draw definitive conclusions.

P581

Cognitive functions in patients with multiple sclerosis and correlation with disability status, age and level of education

M. Maltezou, P. Kostaras, G. Cheilakos, P. Karakasis, I. Koutentaki

1st. General Hospital of Social Security Services (Athens, GR)

Objectives: Contemporary data suggest the high impact of cognitive deficits in patients with multiple sclerosis (MS) as a result of brain lesions accompanying the disease. The aim of our study was to investigate in which degree cognitive deficits correlate to illness severity, age and educational level.

Methods: We collected demographic data including age and years of education from 65 hospitalized patients treated for MS, and evaluated the level of cognitive functions, by administering the Brief Repeatable Battery of Neurological Tests (BRB-N). The Expanded Disability Status Scale (EDSS) was used to assess the severity of disability.

Results: In our study group, 39 were women and 26 men. The average age was 42.1 years, and the mean time of education was 11.51 years. The average EDSS score was 3.95. The average score on the Symbol Digital Modalities Test (SDMT), a part of BRB-N that tests sustained attention and concentration, was 36.52. All the tests of BRB-N except Word List Generation, showed a positive correlation with years of education and a negative correlation with age, varying from significant $(\mathrm{p}<0.5)$ to highly significant $(\mathrm{p}<0.001)$. However, only SDMT showed also a highly significant negative correlation with EDSS $(\mathrm{p}=0.002)$.
Conclusion: Age and educational level correlate significantly to the level of cognitive function and must be taken into account before screening for cognitive deficits in patients with MS. Only the SDMT showed a significant correlation with severity of disability, suggesting a more disease-related cognitive deficit.
P583

Long-term evaluation of neuronal metabolism in relapsing-remitting multiple sclerosis: combined results from two cohorts of glatiramer acetate treated patients examined prospectively with serial brain proton magnetic resonance spectroscopy (1H-MRS) for 6

O. Khan, S. Hreha, J. Perumal, Y. Shen, Y. Yang, C. Caon, F. Bao, R. Penmasta, Z. Latif, A. Tselis, I. Zak

Wayne State University School of Medicine (Detroit, US)

Background: Brain 1H-MRS allows in vivo examination of neuronal integrity by quantifying the neuronal mitochondrial marker $\mathrm{N}$-acetylaspartate (NAA), often expressed as a ratio to creatine $(\mathrm{Cr})$. We investigated the effect of disease modifying therapy on neuronal mitochondrial function by performing $1 \mathrm{H}$-MRS in RRMS treated with glatiramer acetate (GA) for 6 years.

Methods: Two cohorts of previously treatment-naïve RRMS initiated therapy with GA, were followed prospective fashion with serial brain $1 \mathrm{H}-$ MRS. Both groups underwent brain 1H-MRS scans using an identical multivoxel technique within a large volume of interest (VOI) located in the central white matter superior to the corpus callosum. Group 1 patients underwent MRS scans at baseline and annually for 6 years, whereas Group 2 underwent MRS scans at baseline and then at years 3 and 6 . We previously reported results of Group 1 MRS data after 4 years of follow up. We now report data from both groups after 6 years of MRS scanning. Data acquisition 
and post-processing was done in a blinded fashion. A small group of RRMS patients who elected to remain at enrollment were also followed with annual MRS. Healthy volunteers were scanned periodically as controls.

Results: A total of 53 patients were studied. Patients in Group $1(n=18)$ had a mean age, disease duration, and EDSS of 43.5 years, 8.2 years, and 2.77, respectively. Baseline mean NAA/Cr in the VOI was $1.97(+0.24)$. After 6 years of follow up, 15 of the 18 patients in the treated group were still participating in the study with a mean NAA/Cr of $2.20+0.16$ or improvement by $11.6 \%$. Patients in group $2(n=31)$ had a mean age, disease duration, and EDSS of 35.1 years, 5.8 years, and 2.53, respectively. Baseline mean NAA/Cr in the VOI was $1.99+0.1$ at baseline and was $2.12+.08(+6.53 \%)$ at year 6 in all 31 patients participating after 6 years. Overall, of the 46 of 49 patients treated with GA, mean NAA/Cr improved from 1.99 at baseline to 2.15 at year $6(+8.04 \%, \mathrm{p}<0.05)$. Untreated patient data $(\mathrm{n}=4)$ and healthy controls $(n=9)$ will also be presented. Furthermore, detailed analysis of normal appearing white matter as well as conventional MRI metrics will be presented.

Conclusions: This long-term H-MRS study supports the use of NAA as a reliable marker for assessing long-term disease progression and therapeutic response. It also confirms our previously published findings suggesting a beneficial effect of GA on neuronal mitochondrial function in RRMS.

Supported in part by a grant from Teva Neuroscience, USA

\section{P584}

Progression of disability over 2 years predicts disability at 8 years in patients with relapsing multiple sclerosis

R. Hyde, R.A. Rudick, J-C. Lee, R.A. Bermel, B. Weinstock-Guttman, D. Bourdette, C. Tornatore, H. Zhang, X. You

Biogen Idec International (Zug, CH); Mellen Center for Multiple Sclerosis Treatment and Research, Cleveland Clinic Foundation (Cleveland, US); Baird Multiple Sclerosis Center (Buffalo, US); Oregon Health \& Science University (Portland, US); Georgetown University Medical Center (Washington, US); Biogen Idec, Inc. (Cambridge, US)

Objective: Disability progression, defined as worsening by ?1 point on the Expanded Disability Status Scale (EDSS) sustained for 6 months, was the primary end point in the pivotal phase 3 clinical trial (Multiple Sclerosis Collaborative Research Group [MSCRG] study) of intramuscular interferon beta-1a (IM IFN beta-1a; Avonex ${ }^{\circledR}$ ). However, there are limited data on the relationship between disability classified in this way and future, clinically relevant disability.

Methods: A post hoc analysis was conducted to assess the relationship between sustained progression in EDSS score over 2 years and EDSS score at 8 years in patients with multiple sclerosis (MS) from the MSCRG study. Positive predictive values (PPVs) were calculated to determine the relationship between progressor status in the MSCRG study and future clinically relevant EDSS milestones.

Results: The analysis included 160 patients who received $\geq 2$ years of treatment in the MSCRG study and who were re-examined $\geq 8$ years after randomisation ( $93 \%$ of potentially eligible patients). Progressors during the clinical trial $(\mathrm{N}=45)$ were significantly more likely than non-progressors $(\mathrm{N}=115)$ to reach EDSS milestones $\geq 4.0, \geq 5.0, \geq 6.0$, and $\geq 7.0$ at 8 -year follow-up (all $\mathrm{P}<0.001$ ), even after adjusting for treatment assignment and baseline EDSS score (all P < 0.001). Compared with placebo, patients treated with IM IFN beta-1a were significantly less likely to progress to EDSS scores $\geq 4.0(\mathrm{P}=0.007)$ or $\geq 5.0(\mathrm{P}=0.010)$. After progression was confirmed, 11 of 45 patients $(24 \%)$ improved to a lower EDSS score. Patients who did not improve demonstrated higher PPVs than those who did, although the overall predictive value of progression remained the same. PPVs of progressor status over 2 years were not different across treatment and baseline EDSS score $>2$ (all P > 0.05)

Conclusion: Progressor status in the MSCRG study was a meaningful predictor of future clinically significant disability. IM IFN beta-1a significantly reduced the probability of reaching clinically relevant EDSS milestone scores of 4.0 and 5.0 at 8 years.

Study supported by Biogen Idec, Inc.

\section{Muscle disorders}

P586

Therapeutic strategies of oxidative stress modulation in mitochondrial myopathies

C. Carlesi, S. Piazza, L. Volpi, E. Molesti, M. Mancuso, M. Franzini, A. Paolicchi, G. Siciliano

University of Pisa (Pisa, IT)

Objectives: Mitochondrial myopathies (MM) represent a group of disorders due to a biochemical defect of mitochondrial respiratory chain that results in reduction of energy cell production. The impaired mitochondrial respiratory function may lead to an excessive reactive oxygen species generation, oxidative stress and cell damage. Aerobic training has been showed to increase muscle performance in patients with $\mathrm{MM}$, although reasons for that are not known. Loss of glutathione (GSH) is associated with impairment of the electron transport chain (ETC) possibly as a consequence of diminished ATP availability or increased oxidative stress. The evidence of GSH low levels in MM patients raise the possibility that treatment strategies targeted at restoring a GSH deficiency should be considered.

Methods: To analyze the exercise-related circulating levels of oxidative stress markers as lipoperoxides, GSH, advanced oxydation protein products and the ferric reducing ability of MM patients in relation to a 10 week aerobic training program and to 30 day administration of a cysteine donor stimulator of endogenous gluthatione synthesis (Prother, AFR, $10 \mathrm{mg} / \mathrm{die}$ ) and to assess the basal level of nuclear DNA damage in terms of chromosome and DNA alterations in leukocytes of 13 patients presenting several forms of MM before and after 30 days of Coenzyme Q10 treatment.

Results: At rest and before training mean blood level of lipoperoxides was increased as compared to controls $(\mathrm{P}<0.05)$. After an aerobic training of 10 weeks the blood level of lipoperoxides decreased at rest $(\mathrm{P}<0.01)$ and during the exercise test $(\mathrm{P}<0.05)$.

After one month of antioxidant treatment, oxidative stress markers were not changed in MM patients. The Comet assay indicated a slightly higher level oxidative DNA damage in MM patients as compared to controls. $\mathrm{Pa}$ tients receiving Coenzyme Q10 showed a significant decrease in frequency of micronucleated cells.

Conclusion: Our data confirm beneficial effects of regular physical activity in MM. After antioxidant treatment oxidative stress markers were not changed in MM patients. However other study, conducted in Myotonic Dis- 
trophy patients and Amyotrophic Lateral Sclerosis patients, show a significantly decrease of AOPP levels. This suggests that different oxidative stress generating mechanisms operate in MM, compared to other neuromuscular disorders. To deepen into these mechanisms would be worthy in order to optimize therapeutic strategies in mitochondrial disorders.

P587

Facioscapulohumeral muscular dystrophy: atypical phenotype in patients with large $4 \mathrm{q} 35$ deletions

C.P. Trevisan, E. Pastorello, C. Angelini, G. Tomelleri, L. Palmucci, G. Siciliano, C. Bruno, F. Comacchio

University of Padua (Padua, IT); University of Verona (Verona, IT); University of Turin (Turin, IT); University of Pisa (Pisa, IT); University of Genua (Genua, IT); Azienda Ospedale - University of Padua (Padua, IT)

Objective: The molecular mutation underlying FacioScapuloHumeral Muscular Dystrophy (FSHD) is characterised by a deletion on chromosome $4 \mathrm{q} 35$, with a residual fragment size ranging $10-38 \mathrm{~Kb}$. In patients with the largest deletions the disease often presents in early childhood with atypical phenotype. We explored frequency of hearing loss and other clinical features in FSHD patients with large 4q35 deletion.

Patients and methods: We considered six subjects with $4 \mathrm{q} 35$ deletions ranging $10-13 \mathrm{~Kb}$. They were three males and three females with a mean age of 43 years. We evaluated their clinical phenotype, with specific consideration of severity of muscle involvement, hearing loss, mental retardation, epilepsy.

Results: Among the six cases, four presented an infantile-onset, with severe or moderate dystrophic phenotype: they were three males, aged 16, 21 and 52 years (4q35 fragment size of 12,10 and $13 \mathrm{~Kb}$ ) and a 40 year-old female $(11 \mathrm{~Kb})$. These four patients showed a clear-cut sensorineural hearing loss, associated to mental retardation in three, and either to epilepsy in two of them. The other two cases presented a moderate muscle involvement: they were two females ( 13 and $12 \mathrm{~Kb}$ ) aged 61 and 67 years with typical adolescence onset of weakness. The auditory ability of these two subjects was mildly affected, mainly involving high frequencies and of subclinical type. None of them presented mental retardation nor epilepsy.

Conclusions: Considering the present cases and those to date reported in the literature, it comes out that the common clinical feature of FSHD patients with large $4 \mathrm{q} 35$ deletion $(10-13 \mathrm{~Kb})$ is represented by sensorineural hearing loss. Moreover, in the cases with a fragment size of only $10-11 \mathrm{~Kb}$, it appears that the disease determines a severe dystrophic phenotype with infantile onset, clear-cut hearing loss and mental retardation, frequently associated to epilepsy. Differently, in the cases with shorter deletion $(13 \mathrm{~Kb})$ the disease shows a milder clinical appearance with infrequent brain involvement, even if with hearing loss in the majority of cases.

\section{P588}

Facioscapulohumeral muscular dystrophy presented with hypertrophic cardiomyopathy: a case study

M. Tsuji, M. Kinoshita, Y. Imai, N. Kohora

Kobe City General Hospital (Kobe, JP)

Objective: Although cardiac involvement is frequently encountered in patients with various types of muscular dystrophy, it rarely occurs in patients with facioscapulohumeral muscular dystrophy (FSHD). To our knowledge, only three FSHD patients have been reported to have cardiomyopathy. The aim of our study is to describe a patient with FSHD who developed hypertrophic cardiomyopathy (HCM) before the onset of muscle symptoms.

Methods: A case report and review of the relevant literature.

Results: A 38-year-old man with bilateral deafness noted at 4 years old was referred to our hospital because of ECG abnormality found at medical check-up. Echocardiogram and left ventriculogram of the patient demonstrated dilatation of the left ventricle and poor contractility. Histopathological examination of cardiac biopsy showed marked disarray and mild fibrosis, indicating a dilated phase of HCM rather than dilated cardiomyopathy. He had no difficulty doing daily and vocational activities at that time. Later he developed muscle weakness in the right arm and was referred to our neurology clinic at the age of 40 . Scapular winging, asymmetrical facial weakness, and right arm muscle atrophy were evident. Electromyographic studies were consistent with chronic progressive myopathy. He also developed visual disturbance due to retinal vasculopathy. We diagnosed him as FSHD with cardiomyopathy, retinal vasculopathy and sensorineural hearing loss. His 12-year-old daughter had symptoms of infantile FSHD, and FSHD was genetically confirmed (informed consent was obtained before the genetic analysis). She had sensorineural hearing loss, however she revealed neither cardiomyopathy nor retinal vasculopathy.
Conclusion: This is the first report of an FSHD patient with biopsyproven HCM. This case illustrates that HCM may develop in patients with FSHD.

\section{P589}

A stop codon mutation in the 5' of the dystrophin gene associated to a Becker muscular dystrophy phenotype

F. Magri, R. Virgilio, R. Del Bo, F. Fortunato, S. Ghezzi, R. Cagliani, M. Sironi, M.G. D’angelo, V. Crugnola, M. Moggio, N. Bresolin, G.P. Comi

University of Milan (Milan, IT); I.R.C.C.S. E. Medea (Bosisio Parini, IT)

Objectives: Duchenne muscular dystrophy (DMD) is caused either by frameshift or by stop-codon mutations along the dystrophin (DYS) gene, while Becker muscular dystrophy (BMD) results from in-frame less deleterious mutations.

To describe a nonsense point mutation in the 5' of the DYS gene associated with clinical and biochemical data suggestive for BMD phenotype.To better understand the molecular basis underlying this atypical clinical presentation through protein expression analysis.

Methods: A 8-year-old boy was investigated for asymptomatic iperCKemia. The parents referred frequent falling and mild running difficulties. The patient underwent to an extensive clinical evaluation, comprehensive of a complete cardiological and respiratory assessment, and to an accurate follow-up. After 3-ys muscular involvement was stable, the patient was still ambulant. Gowers' sign was absent.

Muscle biopsy was obtained from brachial biceps. Dystrophin immunohistochemistry (IHC) and Western blot (WB) analysis were performed using three monoclonal antibodies against mid-rod domain, $\mathrm{NH} 2$ and $\mathrm{COOH}$ epitopes.

DYS gene was analysed through multiple PCR and MLPA; the screening was completed by direct sequencing of all 79 exons, the exon-intron boundaries and the promoter region.

Results: The patient presented with a mild muscular involvement, suggesting a BMD phenotype. Muscle biopsy showed a mild dystrophic pattern. This data was confirmed by IHC analysis that showed a discontinuous labelling with reduced intensity with the three dystrophin epitopes.

No delection/duplicaton was found with multiplex PCR and MLPA analysis. Gene sequencing revealed the presence of the point mutation c.9G $>$ A within exon 1 , affecting the third codon of the protein $(\operatorname{Trp} 3 \mathrm{X})$.

WB analysis provided evidence for a dystrophin product of reduced intensity and smaller molecular weight compared to the controls.

Conclusions: Nonsense mutations usually determine severe DMD phenotype. Here we report a nonsense Trp3X mutation at the 5' of the DYS gene associated with a mild phenotype. In this patient's muscle, a downstream ATG start codon located within exon 6 may be used as an alternative starting site for dystrophin translation, accounting for the production of a shorter protein.

This case might provide further insight into the mechanisms that determine genomic rearrangements in the DMD locus and into the relevance of mRNA processing, in addition to genomic data, in order to predict the clinical phenotype in dystrophinopathies.

\section{P590}

Subtypes of limb-girdle muscular dystrophies in the state of Ceará, Brazil G. Ramos de Oliveira, L.H. Pimentel, C.M. Castro Costa, F.A. Aquino Gondim Universidade Federal do Ceará (Fortaleza - Ceara, BR)

Objectives: The term limb-girdle muscular dystrophy (LGMD) is employed to describe a group of noncongenital muscular dystrophies with progressive proximal weakness not due to dystrophin deficiency. The autosomal inheritance pattern can be dominant or recessive and new distinct forms continue to be described. The aim of this study is to report the clinical and muscle findings of the LGMDs seen in a tertiary care hospital in the state of Ceará, Brazil.

Methods: We studied 41 patients from 34 families with chronic progressive weakness in a limb-girdle distribuition without facial involvement and muscle biopsy features suggestive of dystrophy/myopathy at a tertiary care hospital between May 2005 and December 2007. All patients were born in State of Ceará. Muscle biopsies specimens were immunostained for dystrophin, sarcoglycan, dysferlin, myotilin, merosin and emerin on all cases.

Results: We found a specific protein deficiency in 23 patients $(56 \%)$ from 20 families. Among these patients $11(47.8 \%)$ had sarcoglycanopathy and 12 $(52.2 \%)$ had dysferlinopathy and the pattern of inheritance was autosomal recessive or sporadic. There were 15 female and 8 male patients. Among the sarcoglycanopathy patients, the mean age was $19.3 \pm 11.2$ years and the mean disease duration was $11.9 \pm 7.2$ years. Among dysferlinopathy patients these values were $42.8 \pm 14.7$ and $19.4 \pm 10.3$ years, respectively. The mean creatine 
phosphokinase (CPK) level was 2996.7U/L in affected cases and was elevated in $21(91.3 \%)$ patients. Symptomatic cardiac involvement was not observed, but eletrocardiographic changes were seen in $8(34.7 \%)$ patients with confirmed LGMD and in $6(54.5 \%)$ patients with sarcoglycanopathy. Among patients with LGMD phenotype but with normal immunohistochemistry, two families had an autosomal dominant inheritance.

Conclusion: In our study, almost $44 \%$ of the total muscle biopsies from the LGMD patients did not reveal any protein deficiency after immunostained reaction. This maybe explained by the lack of evaluation of antibodies against other specific proteins, like calpain. Despite of this, immunohistochemistry is very important in the study and classification of LGMD if genetic testing is not available. Despite the referral bias (tertiary center), further studies are necessary to characterize the genetic background of the different LGMD subtypes in the State of Ceará, Brazil, where consanguineous marriages were frequent during the XX Century, specially in the countryside area.

Supported by research grants from UFC, FUNCAP, CNPq and CAPES

\section{P591}

\section{Double heterozygotic patient for both McArdle and dysferlin myopathies}

H. Foreid, R. Santos, T. Evangelista

Hospital de Santa Maria (Lisbon, PT); Instituto de Genética Médica Jacinto de Magalhães (Porto, PT)

Objectives: To report clinical manifestations and muscle biopsy abnormalities in a patient with heterozigoty for two neuromuscular recessive disorders: McArdle's disease and Dysferlin deficient dystrophy; to integrate family genetic data and to hypothesise on this genetic association.

Methods: A 28 years old male with muscle pain after exercise, tiredness, "second-wind" phenomenon and muscular cramps was submitted to neurological evaluation, blood determination of creatine kinase (CK) levels and electromiographic (EMG) studies. Although his father had an established diagnosis of McArdle's disease the absence of familial consanguinity lead to the decision of performing a muscle biopsy to the index case. After muscle biopsy data, patient and first degree relatives were tested for mutations on both myophosphorylase and dysferlin genes.

Results: On neurological evaluation mild proximal weakness of upper limbs was observed. CK levels were 294 UI and EMG disclosed a myopathic pattern. A complete absence of dysferlin was noted at the immunohistochemical study of the muscle sample. Patient's genetic study revealed a compound heterozigoty for myophosphorylase gene (R50X mutation on PYGM gene) and dysferlin gene (c.5509G >A mutation on DYSF gene) with normal cariotype. The father presented the R50X mutation on PYGM gene in homozygoty and the mother was a heterozygote for the c.5509G $>$ A mutation on DYSF gene.

Conclusion: We presented the clinical and histologic phenotype of a patient with a genetic trait for both myophosphorylase and dysferlin deficiencies. Genetic investigation of this family suggests the usual recessive hereditarity of both diseases: myophosphorylase deficiency inherited from the father and dysferlin deficiency from the mother. Although being a heterozygote for these two recessive diseases this patient was not a silent carrier, he had symptoms of a metabolic myopaty and histologic features of dysferlin deficiency. It seems to be an interaction between these two mutations possibly advantageous from a phenotypic point of view when compared with homozygotic patients for each mutation.

\section{P592}

\section{McArdle disease mimicking polymyositis: when to treat}

I. Mahjneh, H. Tuominen

Oulu University and MHSO Hospital (Pietarsaari, FI); Oulu University (Oulu, FI)

Objectives: McArdle's disease has a wide spectrum of clinical presentations. Few cases with McArdle's disease presenting as polymyositis (PM), have been reported in literature. Differential diagnosis between these two diseases is very important in the consideration of possible treatment.

Method: We have studied a 62 years old woman with proximal muscle weakness. The patient underwent clinical exam, CK, EMG. Muscle MRI using T1-weighted and T2-weighted short time inversion recovery (STIR) with six sets of axial slices was obtained. Muscle biopsy was obtained from femoral muscle and processed for routine pathologic, histochemical, immunohistochemical, and ultrastructure studies.

Results: The patient has had during the last 5 years proximal muscle weakness and pains. After accurate anamnesis, second wind phenomenon was referred. The patient showed only slight proximal muscle weakness in the upper and lower limbs. CK level was constantly high. Ischemic exercise test was abnormal. EMG and Muscle MRI were normal. Muscle biopsy showed total absence of myophosphorylase activity. Focal myositis was present. Electron microscopy revealed glycogen accumulations. Corticosteroids have been initiated without any improvement.

Conclusion: The classical form of McArdle can be distinguished from PM. Instead, cases with adult onset with only muscle weakness and increased $\mathrm{CK}$, can mimic PM. In these cases, inflammation is secondary to muscle damage due to metabolic deficiency. We conclude that patients with $\mathrm{McAr}$ dle simulating PM in whom muscle MRI is normal should not undergo corticosteroids or immunosuppression treatment.

\section{P593}

Metformin improves insulin resistance in myotonic dystrophy

H. Takada, S. Kon, Y. Oyama

National Hospital Organization (Namioka, Aomori, JP)

Objectives: A clinical intervention study of metformin on insulin resistance in myotonic dystrophy (MD1) was performed to investigate beneficial effects for treatment against glucose intolerance in MD1.

Methods: Subjects were patients with MD1 who showed fasting or postprandial hyperinsulinemia, and had never been treated by medicine for diabetes. One hundred $\mathrm{mg}$ of metformin per day was administered for twelve weeks. Clinical findings, $75 \mathrm{~g}$ oral glucose tolerance test (OGTT), routine blood tests including lactic acid were examined before and after the trial. All the medicine, diet, exercise, and rehabilitation were fixed similarly during the trial. Sigma serum insulin value (IRI) which was a summation of IRIs during OGTT and sigma blood sugar (BS) summated BSs in the same manner, IRI and BS at 120 minutes during OGTT (IRI-120, BS-120), and the homeostasis model assessment insulin resistance (HOMA-IR) were calculated. Statistical analyses were carried out by Wilcoxon signed-rank test.

Results: Thirteen patients with MD1 were participated in this study $(8 \mathrm{fe}-$ males and 5 males, aged $55.0 \pm 2.7$ years; median value \pm standard error, same as following). The median value of the number of CTG repeat was $1500 \pm 248$. One patient showed diabetes pattern on OGTT, five were IGT pattern of OGTT, and seven had hyperinsulinemia with normal BS pattern on OGTT. Sigma IRI after the trial was significantly reduced than that before $(195.7 \pm 44.8$ vs. $291.0 \pm 48.5 \mathrm{microU} / \mathrm{dl})$. IRI- 120 (17.5 $\pm 6.2 \mathrm{vs.} 53.6 \pm 9.6 \mathrm{mi}-$ croU/dl) and fasting IRI ( $4.5 \pm 0.7 \mathrm{vs.} 6.3 \pm 1.1 \mathrm{microU} / \mathrm{dl})$ after the trial were similarly decreased than those before. On the other hand, there was no significant difference after vs. before the trial for sigma BS (501.0 \pm 32.0 vs. $519.0 \pm 40.0 \mathrm{mg} / \mathrm{dl}), \mathrm{BS}-120(99.0 \pm 14.0$ vs. $116.0 \pm 14.0 \mathrm{mg} / \mathrm{dl})$, and fasting BS $(83.0 \pm 2.0$ vs. $83.0 \pm 3.0 \mathrm{mg} / \mathrm{dl})$. HOMA-IR after the trial showed a tendency of decrease compared before $(0.965 \pm 0.15$ vs. $1.142 \pm 0.23, p=0.0546)$. There was no significant difference between the trial for routine blood tests including lactic acid and body weight. No adverse events were recognized during the trial.

Conclusion: Metformin treatment is useful to improve insulin resistance in MD1 without any adverse events for short period observation of twelve weeks.

National research group for the clinical studies on treatment and medical management of muscular dystrophy supported by the Japanese Ministry of Health, Labour and Welfare

\section{P594}

Sleep disorders in myotonic dystrophy type 1

M.N. Cardoso, E. Santos, J. Ramalheira, J. Lopes, T. Coelho

Hospital Geral de Santo Antonio (Porto, PT)

Background: Myotonic Dystrophy (MD) is the most frequent dystrophy in adults and a multisystemic disease. Daytime hypersomnia is a usual symptom and an important social limitation for those patients. Other sleep disorders have been described. Pathophysiological theories include central regulation sleep dysfunction, respiratory failure and/or apnoea, particularly of central type.

Objective: To describe sleep abnormalities recorded in nocturnal polysomnography (NPSG) and multiple sleep latency test (MSLT) in MD patients.

Methods: Twenty-five patients with clinical, electromyografic and genetic diagnosis of MD type 1 were selected randomly from our neuromuscular outpatient clinics. NPSG followed by MSLT was performed in every patient.

Results: There were 13 women and 12 men, aged between 18 and 65 years (mean 44.4 years)

Twelve patients showed reduced sleep efficiency. Micro-awakenings were the most frequent finding. Fifteen showed $\mathrm{O} 2$ saturation average below $95 \%$ (in 5 below $90 \%$ ) and the minimal nocturnal $\mathrm{O} 2$ saturation ranged from 50 to $93 \%$. Six patients had, while awake, O2 saturation below $90 \%$. Seven pa- 
tients had apnoeas, four of which fulfilled criteria for obstructive sleep apnoea syndrome (OSAS). One patient had apnoea/hypopnoea index of $32 /$ hour. Central type apnoeas occurred in one patient and two had obstructive apnoeas without criteria of OSAS.

MSLT showed an average latency inferior to ten minutes in 13 patients, thus presenting criteria for daytime hypersomnia. Ten patients with hypersomnia showed nocturnal or wake $\mathrm{O} 2$ saturation below $90 \%$.

Discussion: Daytime hypersomnia was a frequent and noteworthy finding, given the patients had been selected in a random fashion. Importantly, although only four patients had criteria for OSAS, low $\mathrm{O} 2$ saturation was frequent, both in wakefulness and sleep.

P595

Aldosterone and excessive daytime sleepiness in myotonic dystrophy

M. Falorni, L. Volpi, V. Calsolaro, E. Caldonazzo, E. Bonanni, F. Monzani, M. Emdin, G. Siciliano

University of Pisa (Pisa, IT)

Objective: Excessive daytime sleepiness (EDS) is common in patients with Myotonic Dystrophy type 1(DM 1) and is a major factor that interferes with the social life of the disease. There are contrasting opinions in literature about the mechanism of EDS in DM1; according to some Authors, EDS could be caused by sleep-disordered breathing, hypercapnia or disturbance of sleep architecture, according to other Authors, it could be due to a central sleep regulation dysfunction, probably modulated from neuroendocrinal factors that govern sleep and awake.

The aim of the present study was to assess the presence of sleep/wake cycle disturbance in a group of MD 1 patients and their possible correlations with hypothalamic-pituitary-adrenal axis dysregulation. The influences of baroceptor and chemoceptor reflexes dysfunctions on EDS were also evaluated.

Methods: Eight MD 1 patients and ten healty age-matched controls were studied by means of an all-night polysomnographic recording, the multiple sleep latency test (MSLT), the evaluation of hypothalamic-pituitaryadrenocrticical activity and baroceptor and chemoceptor reflex study. Moreover in MD patients were administrated the Eworth Slleepiness Scale (ESS) and the Fatigue Severity Scale (FSS).

Results: The mean MSLT value was significantly lower in patients than in controls. Six of the eight patients were found to have pathological EDS, but only one presented nocturnal apnoeas and only one periodic leg movements.

There weren't significantly differences in baroreptor and chemoceptor reflexes, between patients and controls.

Overall, we didn't find an hypotalamic-pituitary-adrenal axis dysregulation in DM1 patients; even thought an inverse correlation was found between aldosterone dosage in clino and orthostatism and MSLT, this indicating that an increase in aldosterone values is correlated with an enhancement of EDS.

Conclusion: Our data suggest that EDS is common in MD 1 patients and that it is probably caused by a primary dysfunction of central sleep regulation, with aldosterone maybe playing an important role in sleep/wake cycle disturbance pathogenesis.

\section{P596}

Cephalalgia, myopathy and familial dementia with CADASIL-like MRI and multiple mtDNA deletions

M. Servida, L. Napoli, D. Ronchi, P. Ciscato, A. Bordoni, A. Prelle, G.P. Comi, M. Moggio, N. Bresolin, M. Sciacco

Fondazione Ospedale Maggiore IRCCS (Milan, IT)

Objective: To describe a patient with cephalalgia, fatiguability, CADASIL-like brain MRI, family history for dementia and no Notch3 mutations, whose muscle biopsy shows signs of mitochondrial dysfunction.

Methods: A 67 years old man came to our observation with a several year history of episodic olocranic cephalagia with nausea and vomiting associated, in the last seven years, with easy fatiguability, myalgias and slight hyperckemia (450-500 U/l). EMG was myopathic. A brain MRI showed multiple confluent hyperintense lesions in the subcortical and deep white matter. Neuropsychological examination was normal. EKG showed anterior left hemiblock with mild heart dilatation and $40 \% \mathrm{EF}$ at echocardiography.

Familiar history was positive for dementia (mother and the 2 maternal aunts). The grand-daughter of one of the two aunts (daughter of a daughter) is affected with schizophrenia. No cognitive problems are reported in the three maternal uncles.

The patient underwent skeletal muscle biopsy of left biceps muscle which we studied by histological, histochemical and immunohistochemical (dystrophin, sarcoglycans, caveolin, merosin) methods. Southern blot analy- sis of the mitochondrial DNA (mtDNA), search for MELAS and MERRF mtDNA point mutations and a genetic test for Notch3 gene (CADASIL) were also performed.

Results: Muscle biopsy only showed some fiber size variability and a few COX-negative fibers, half of which ragged red. Southern blot analysis of mtDNA was positive for multiple deletions, which were confirmed by PCR. A3243G, A8344G and A8356G mtDNA point mutations were absent. No Notch3 mutations were found.

Conclusions: recent studies have demonstrated that Notch3 gene mutations may also manifest as neuropathy and myopathy and, particularly, cooccur with, and, possibly, predispose to, mtDNA mutations (Finsterer, 2007). A mitochondrial encephalopathy with CADASIL-like MRI, no Notch3 mutations and an $8.3 \mathrm{~Kb}$ mtDNA macrodeletion has been described in a patient with typical clinical features of mitochondrial disorder and negative family history (Akhvledani et al,2007). In our case, we found mtDNA multiple deletions, but no Notch 3 mutations in a patient with morphological evidence of mitochondrial dysfunction, but clinical features and family history suggestive of CADASIL. Search for nuclear DNA mutations in genes responsible for mtDNA multiple deletions is underway.

\section{P597}

Late-onset Leigh syndrome in an adult patient with mitochondrial ND3 gene mutation

M. Tchikviladzé, P. Laforet, B. Eymard, M. Vidailhet, F. Delbos, S. Filaut, A. Lombès, C. Jardel

Pitié-Salpêtrière (Paris, FR); APHP Saint-Antoine (Paris, FR)

Introduction: Leigh syndrome (LS) is a subacute necrotizing encephalomyopathy occurring most often in childhood, and frequently associated with complex I deficiency. Recently, a novel mutation m.10197G >A in mitochondrial (mt) DNA-encoded complex I subunit gene ND3 was identified in young children of three unrelated families with LS and dystonia.

Case report: We report here the observation of an adult patient with LS harbouring the same mutation. A 30-year-old man presented at the age of 6 years, with bilateral optic atrophy and horizontal nystagmus. These signs remained stable until the age of 30 when the patient developed a subacute neurological disorder with dysarthria, ophthalmoplegia, and dystonic gait, concomitant with thyrotoxicosis. A partial reversal of the neurological symptoms occurred after treatment of hyperthyroidism, followed by a progressive worsening/decline in the following years.Brain MRI showed bilateral brainstem lesions.CSF and serum lactate concentrations were normal. Histochemical studies performed on skeletal muscle were unremarkable, but the measurement of the respiratory chain complexes activities showed an isolated defect in complex I.

Subsequent analysis of the mt DNA revealed a m.10197G $>$ A mutation in the MT-ND3 gene, resulting in a substitution of hydrophobic alanine to hydrophilic threonine in the highly conserved amino acid 47. Mutant mtDNA was present at different levels of heteroplasmy in all studied tissues: muscle $(100 \%)$, buccal mucosa cells $(70 \%)$ and leucocytes less than $50 \%$.

In conclusion: We report the first case of severe adult LS due to a mutation in MT-ND3 gene with late-onset and severe neurological decline. This neurological severity is not usual with a low amount of blood mutated DNA. The influence of thyroid dysfunction cannot be excluded as worsening factor of the neurological status of the patient. Moreover, this report suggests that mt DNA complex I genes are candidate genes for Leigh syndrome in adults as it is the case in children.

\section{P598}

Peculiar ultrastructural findings in congenital myopathies due to selenoprotein $\mathrm{N} 1$ gene new mutations

M.E. Fruguglietti, R. Cagliani, L. Napoli, A. Berardinelli, M.G D'Angelo, K. Gorni, S. Orcesi, C. Lamperti, E. Signaroldi, R. Tupler, N. Bresolin, M. Moggio, G.P. Comi, A. Prelle

Foundation I.R.C.C.S. Ospedale Maggiore (Milan, IT); I.R.C.C.S. E.Medea Bosisio Parini (Lecco, IT); I.R.C.C.S. Fondazione Istituto Neurologico C. Mondino (Pavia, IT); Università di Modena (Modena, IT)

Objectives: To describe the clinical, histopathological and genetic findings in three Italian families affected with autosomal-recessive congenital myopathies caused by Selenoprotein N1 gene mutations (SEPN-RM).

The SEPN-RM comprises four distinct autosomal-recessive disorders: rigid spine muscular dystrophy (RSMD), the classic form of multiminicore disease $(\mathrm{MmD})$, a desmin-related myopathy with Mallory body like inclusions (MB-DRM), and a form of congenital fiber-type disproportion (CFTD).

Methods: The first proband was a 31-y-o female: she was floppy at birth, then she developed axial weakness and mild lower limb-girdle weakness. 
The second one is a 13-y-o boy with delayed acquirement of motor milestones: at age 11 years, he underwent surgery for a severe scoliosis. The third one was a 8 y-old girl with persistent neck flexor muscle weakness. At age 30 months, she was able to walk with a waddling gait and hyperlordotic posture. Her clinical features showed a relatively benign course, with axial hypotonia, proximal upper and lower limb muscle weakness. Her 3 year-old brother, after a pregnancy complicated by polyhydramnios, showed a hypotonic-hyporeflexic syndrome at birth. In all probands a muscle biopsy was performed and analysed by histochemistry, immunohistochemistry (IHC) and electron microscopy (EM). SEPN1 coding region and the (SECIS) element were sequenced.

Results: Muscle biopsy was normal in all probands at light microscopy. EM examination showed several sarcomeres lacking the Z-line in Patient 1 and some multiminicores in a few muscle fibers in Patient 2 and Patient 3. SEPN1 gene analysis revealed five mutations, three of which are novel. $\mathrm{Pa}-$ tient 1 is a compound heterozygous for the c.1-19_+73del92 and c.1176delA mutations; patient 2 has two frame-shift mutations (c.1-48 +51del99; c.13_22dup10), while the affected children of the third family are homozygous for the c.1388-1 $\mathrm{g}>\mathrm{c}$ splice site mutation.

Conclusions: The genetic investigation of three probands affected with congenital myopathy showed new mutations in the SEPN1 gene. In two probands, the disease was associated with multiminicores at muscle biopsy. Most notably, in proband 1 , the most peculiar pathological feature was a focal $\mathrm{Z}$ line absence. This a novel finding in SEPN1 pathology and may help in the diagnosis in adult patients with a history of congenital myopathy.

\section{P599}

Diaphragm movements in myotonic dystrophy

O. Yahara, T. Kimura, K. Kuroda, K. Fujiwara, H. Aizawa, T. Katayama, J. Sawada, Y. Aburakawa, T. Saitou

NHO Douhoku Hospital (Asahikawa, JP); Asahikawa Medical Colleg (Asahikawa, JP)

Background: In myotonic dystrophy (MD1) patients, respiratory muscles, including diaphragm, weakness develops, contributes to lung infection and hypoventilation may occur. Some patients had respiratory insufficiency from early stage. Some studies showed that diaphragm showed reduced relaxation and myotonic discharges. To investigate the respiratory muscles weakness of MD patients clearly, we studied respiratory muscle movements, especially diaphragm.

Method: We evaluated diaphragm movements in MD patients and control by using the angiography equipment. On supine position, we measured the lateral and antero-posterior diameter during maximal inspiration and expiration movement. Moreover, we drowned the shape of diaphragm between maximal respiration movements and, then using image analysis system, we made the comparison of diaphragm shapes between DM and controls.

Results: In respiratory movement, anterior-posterior diameter of thoracic cage had moved more than lateral. All patients with MD had raised diaphragm in both inspiration and expiration movement. Control had raised diaphragm only in expiration and not in inspiration. There were not almost differences between diaphragm shapes in DM patients during respiratory movement. In contrast, controls had convexity shape in expiration and flat shape in inspiration. Therefore, diaphragms in MD almost did not contract and had weakness.

Conclusion: Diaphragm elevations have seen and diaphragm shape did not change during respiratory movement. Diaphragm weaknesses have been noted in all DM cases.

\section{P600}

Diagnostic relevance of myohistological examination in patients with mitochondrial disorders

P. Tacik, M. Deschauer, S. Zierz

University of Halle- Wittenberg (Halle/Saale, DE)

Objectives: Myohistological mitochondrial changes are an important tool in diagnosing mitochondrial disorders. However, they are also seen in other neuromuscular diseases e.g. in oculopharyngeal muscular dystrophy (OPMD) presenting a similar clinical picture to chronic progressive external ophthalmoplegia (CPEO). The aim of this study was to analyse the diagnostic significance of myohistological examination in patients with the common mitochondrial disorder CPEO. Additionally, data of selected patients with other mitochondrial disorders were presented to highlight the importance of genetic testing.

Methods: A quantitative examination of mitochondrial myohistological changes was performed in patients with following diseases: CPEO (multiple deletions $n=10$, single deletions $n=10,3243 A>G$ mutation $n=2$ ), other mi- tochondrial phenotypes $(3243 \mathrm{~A}>\mathrm{G} \mathrm{n}=4,8344 \mathrm{~A}>\mathrm{G} \mathrm{n}=1)$, OPMD $(\mathrm{n}=4)$, amyotrophic lateral sclerosis (ALS, $n=6$ ), inclusion body myositis (IBM, $\mathrm{n}=6$ ) and in 11 healthy controls. Muscle biopsies were stained for $\mathrm{cy}-$ tochrome $c$ oxidase/succinate dehydrogenase (COX/SDH) to identify COXnegative fibres (CNF) and with modified Gomori's trichrome staining (mGT) to detect ragged red fibres (RRF).

Results: A significant increase of CNF was seen in all CPEO patients (mean $5.63 \%$, range $0.59 \%-20.6 \%$ ) vs. normal controls (mean $0.045 \%$, range $0 \%-0.2 \%$ ). An increase of RRF was noticed in 21/22 CPEO patients (mean $1.46 \%$, range $0 \%-4.6 \%$ ). In patients with single deletions the number of CNF was higher (mean $8.045 \%$ ) compared to those with multiple deletions (mean $2.33 \%$ ).

In patients with other mitochondrial disorders a marginal increase of $\mathrm{CNF}$ was observed in 4 patients (mutation $3243 \mathrm{~A}>\mathrm{G} \mathrm{n}=4$ ), whereas no increase of RRF was detected in 4 patients (mutation $3243 \mathrm{~A}>\mathrm{G} \mathrm{n}=3,8344 \mathrm{~A}>\mathrm{G}$ $\mathrm{n}=1)$. An increase of mitochondrial changes was also seen in patients with OPMD (mean CNF 0.59\%, RRF 0.58\%), ALS (mean CNF 0.16\%, RRF $0.14 \%$ ), and IBM (mean CNF $3.9 \%$, RRF $0.42 \%$ ). Conclusions:

1. COX/SDH staining is a more sensitive method for detecting mitochondrial changes than mGT staining reaching a sensitivity of $100 \%$ in diagnosing CPEO.

2. CNF were more prominent in patients with single deletions compared to those with multiple deletions.

3. Myohistological mitochondrial changes are not pathognomonic of mitochondrial diseases (e.g. they cannot always clearly differentiate CPEO from OPMD)

4. In some patients molecular genetic testing is necessary to establish the diagnosis of mitochondrial disease.

P601

Adult glycogenosis type II (Pompe's disease): clinical course compared with acid alfa-glucosidase activity and histopathological abnormalities in muscle biopsies

R. Rola, E. Lewandowska, A. Lugowska, T. Wierzba-Bobrowicz, E. Pasennik, T. Stepien, D. Ryglewicz

The Institute of Psychiatry and Neurology (Warsaw, PL)

Objectives: Glycogen storage disease type II (GSD II) is an autosomal recessive disease caused by mutations of the acid alfa-1,4-glucosidase (GAA) gene. The pathophysiology of GSD II is associated with a lowered activity of GAA, however, it has not yet been determined whether the level of GAA deficiency is directly proportional to the level of glycogen storage and clinical course of adult type of GSD II. The aim of the study was to compare the clinical course, histopathological abnormalities and GAA activity.

Methods: Clinical involvement was assesed with a routine clinical examination and patient history in three patients with adult type of GSD II. Patient 1, aged 19, as well as patients 2 and 3 (two sisters), aged 31 and 29, respectively underwent elcetrophysiological examintion which revealed the electrophysiological symptoms of muscle involvement. The muscle biopsy was taken in all of the patients. For each examined patient, the activity of lysosomal GAA activity (at pH 3.8) was measured fluorometrically in isolated blood leukocytes (L) and dried blood spots (DBS). The biopsy samples were studied histologically, immunohistologically and ultrastructurally.

Results: All of the patients had symptoms of progressive muscle weakness and respiratory failure. However there were significant differences in the time course of respiratory failure and distribution of muscle weakness. The muscle biopsies displayed similar morphological features of glycogen storage, however, with different intensity.

All patients showed reduced GAA activity (patient $1-0.12$ in DBS and 0.07 in L; patient $2-0.05$ in DBS and 0.07 in L; and patient $3-0.12$ in DBS and 0.09 in $\mathrm{L}$ ).

Conclusions: Based on the study results, we concluded that the level of GAA deficiency in late-onset type II glucogenosis was not directly proportional to the amount of glycogen storage and the disease severity.

\section{P602}

Medical food in mdx mice: genistein and flavocoxid ameliorate muscle pathology and function

S. Messina, A. Mazzeo, A. Bitto, M. Aguennouz, A. Migliorato, M.G. De Pasquale, F. Squadrito, G. Vita

University of Messina (Messina, IT)

Soy isoflavones have been reported to have antioxidant bioactivities, scavenging free radicals and increasing antioxidant protein expression, and also to inhibit the transcription factor NF-kB. We showed in previous studies that 
the inhibition of the transcription factor NF-kB throught drugs with also antioxidant properties, have beneficial effects in $\mathrm{mdx}$ mice. The drugs used are not available for clinical studies.

Objectives: We tested whether genistein and flavocoxid, supplements with known antioxidant and antinflammatory properties readily available for clinical use, could have a beneficial effect on muscle function, morphology and biochemical pattern in mdx mice.

Methods: Five-week old mdx mice received for five weeks either genistein ( $2 \mathrm{mg} / \mathrm{kg}$ i.p. daily), flavocoxid ( $5 \mathrm{mg} / \mathrm{kg}$ i.p. daily) or vehicle.

Results: Flavonoids treatment 1$)$ increased forelimb strength $(\mathrm{p}<0.05)$ and strength normalized to weight $(\mathrm{p}<0.05)$ and decreased fatigue $(p<0.05) ; 2)$ reduced serum creatine-kinase levels $(p<0.01) ; 3)$ increased GPX activity and reduced markers of oxidative stress $(\mathrm{p}<0.05) ; 4)$ blunted NF-kB DNA-binding activity $(\mathrm{p}<0.05) ; 5)$ reduces muscle necrosis $(p<0.01)$ and enhances regeneration $(p<0.05)$ with an augmented number of myogenin-positive satellite cells and myonuclei, and of developmental myosin heavy chain-positive fibers.

Conclusions: Our results suggest that these flavonoids might have a beneficial effect on muscle function and morphology in mdx mice. Further studies are needed to investigate the biochemical substrates of such encouraging preliminary results taking into account that these supplements could be easily introduced in the daily diet of patients with Duchenne muscular dystrophy.

\section{P603}

Cysteine donor enriched dietary intake treatment in mitochondrial myopathies

S. Piazza, C. Carlesi, L. Volpi, M. Lodato, G. Ricci, M. Mancuso, M. Franzini, A. Paolicchi, G. Siciliano

University of Pisa (Pisa, IT)

Objectives: In mitochondrial myopathies (MM) OXPHOS genome mutations generate impairment of respiratory chain, a key event to imbalance intracellular ATP production and trigger apoptotic pathway. In this context, metabolically deranged mitochondria are a potent source of reactive oxygen species (ROS), a process targetable by putative effective therapies. With regard to mitochondrial function, loss of glutathione (GSH), a key intracellular antioxidant, is associated with impairment of the electron transport chain (ETC) possibly as a consequence of diminished ATP availability or increased oxidative stress.

Methods: Aim of the study has been to analyze the exercise-related circulating levels of oxidative stress markers of $10 \mathrm{MM}$ patients in relation to 30 day administration of a cysteine donor stimulator of endogenous gluthatione synthesis. Exercise was performed by a step by step cicloergometer intermittent incremental powerload protocol till $70 \%$ of maximal contraction power output.

Results: Compared to 7 matched controls, MM patients showed significantly $(\mathrm{p}<0.05)$ increased levels of oxidative stress products, as advanced oxidation proteins products, and reduced levels of ferric reducing ability. Aerobic training was able to significantly reduce $(\mathrm{p}<0.05)$ exercise related ROS production, this being modulated by the antioxidant dietary regime utilized. After one month of antioxidant treatment, AOPP levels were decreased, but not significantly and GSH levels were not changed in mitochondrial myopathies patients.

Conclusion: Our results confirm, in vivo, the pathogenetic role of oxidative stress in mitochondrial diseases and indicate possible strategies to revert the deleterious effects of respiratory chain deficiency in these diseases.

\section{P604}

Magnetic resonance imaging of brachial plexus in multifocal motor neuropathy: a case report

T. Stosic-Opincal, S. Lavrnic, M. Gavrilov, V. Peric, S. Apostolski

Clinical Centre of Serbia (Belgrade, RS)

Objectives: Multifocal motor neuropathy (MMN) is an acquired autoimmune demyelinating neuropathy with slowly progressive weakness and fasciculations without permanent sensory loss. Clinically, it may be difficult to differentiating it from amyotrophic lateral sclerosis (ALS) with predominant lower motor neuron involvement. In MMS muscle atrophy and rapid progression are lacking. Unlike ALS, MMN usually respond to treatment with intravenous immunoglobulin even after many years of duration. If nerve conduction study with needle electromyography is nonspecific in MMN, then an alternative or additional method is magnetic resonance imaging (MRI) examination of brachial plexus. Differentiation of MMN from motor neuron disease is important because MMN is a treatable disorder.

Methods: A 50-year-old man was hospitalized to neurology department with severe atrophy of hand, forearms, arms and calves muscles. His com- plaints had started four years before his admittance. Numbness and fasciculations in his right hand were the first symptoms that had slowly progression to atrophy without sensory loss. Magnetic resonance imaging (MRI) examination was performed on $1.5 \mathrm{~T}$ scanner. Following MR sequences and planes were used: saggital T2 weighted (T2W), T1-weighted (T1W) and fat suppression $\mathrm{T} 2 \mathrm{~W}$, axial $\mathrm{T} 2 \mathrm{~W}$ and $\mathrm{T} 1 \mathrm{~W}$ sequences in the level of third cervical to first thoracal vertebral body and post contrast T1W sequence in three planes.

Results: The electrophysiological examination has not revealed persistent conduction blocks in the ulnar nerves regardless the most severe atrophy has been found in muscles innervated by those nerves. MRI examination revealed inflammation of $\mathrm{C} 5-\mathrm{C} 7$ nerve roots.

Conclusion: MRI examination of brachial plexus seems to be very important for detection of nerve roots inflammation in patients with symptoms of multifocal motor neuropathy. The electrophysiological examination method was not sufficient to detect those changes in this case.

\section{P605}

Pathogenic role of ribonuclear inclusions in myotonic dystrophy type 2: myth or reality?

V. Caldiera, R. Cardani, R. Perbellini, P. Tremolada, E. Mancinelli, G. Meola University of Milan (Milan, IT)

Objectives: Myotonic dystrophy type 2 (DM2) is caused by a CCTG expansion in intron 1 of the zinc finger protein 9 gene which generates a toxic RNA implicated in the pathology of the disease. Mutant transcripts are retained in muscle nuclei producing ribonuclear inclusions (RIs), which result in misregulated splicing events that explain many symptoms of DM2, such as myotonia and insulin resistance. Nevertheless the cause of skeletal muscle degeneration, which is a cardinal feature of the disease, remains still unknown. In order to study if RIs and the subsequent nuclear sequestration of MBNL1, a splicing factor, are involved in DM2 muscle wasting, we have correlated the degree of fibres atrophy with the number of RIs or with the dimension of RIs and MBNL1 nuclear foci.

Methods: The study has been carried out on 4 DM2 patients who underwent 2 different biopsies of biceps brachii at different years of age. Fibre diameters have been evaluated on cryostat sections immunostained with fast or slow myosin. Moreover, we have performed FISH in combination with fast myosin-immunofluorescence to evaluate the number of RIs present in nuclei of the two fibre types, or FISH in combination with MBNL1-immunofluorescence to quantify fluorescence intensity and area of RIs and MBNL1 foci.

Results: A preferential type 2 fibre atrophy has been observed in both the biopsies examined from each DM2 patients. The number of RIs appears to be similar in fast and slow fibres indicating that no correlation exists between the degree of fibre atrophy and the number of RIs. A slight increase in fluorescence intensity and area of both RIs and MBNL1 foci is observable in the biopsies of each patient examined. Indeed it has been demonstrated that in DM2 patients expansion size increase with patient's age. Nevertheless this increase is not always accompanied by an increase of the number of RIs or of the degree of fast fibre atrophy.

Conclusions: Our data indicate that higher fast fibre atrophy is not accompanied to a higher number of RIs in this type of fibres as compared to slow fibres. The increase in fluorescence intensity and area of both RIs and MBNL1 foci in the second biopsy seems indicate an increase in expansion size and in MBNL1 sequestration over the time. However this increase is not related to a worsening of histopathological pattern. The number and the dimensions of ribonuclar inclusions and MBNL1 do not seem to be indicators of the severity of muscle wasting.

\section{P606}

Plasma leptin levels in patients with myotonic dystrophy type 1

V. Rakocevic Stojanovic, S. Peric, Z. Stevic, I. Basta, Z. Tasic, S. Popovic, T. Ille, D. Lavrnic

Clinical Center of Serbia (Belgrade, RS); Institute of Endocrinology (Belgrade, RS); Medical Faculty (Belgrade, RS); Institute of Neurology (Belgrade, RS)

Objectives: Myotonic dystrophy type 1 (DM1), the most common adult form of muscular dystrophy, is a systemic disorder in which insulin resistance is well recognized. Insulin resistance is an important determinant of circulating leptin concentrations in humans, but its independent contribution on plasma leptin levels are controversial.

In this study we analysed plasma leptin levels in nondiabetic DM1 patients and in age, sex and body mass index matched healthy subjects and assessed the influence of leptin on insulin resistance and other clinical manifestations of DM1. 
Methods: Body mass index (BMI) was calculated as body weight divided by height squared. Body composition, fasting and post-oral glucose tolerance test, fasting insulin, cholesterol and plasma leptin levels were studied in 18 DM1 patients, 10 women and 8 men, aged 23-60 years. Body composition was measured using a bioelectrical impedance analyzer. Insulin sensitivity was modeled according to a homeostasis model assessment (HOMA) computer-solved model.

Results: Serum leptin levels were higher in patients with DM1 than in healthy controls $(12.1 \pm 8.9 \mathrm{ng} / \mathrm{ml} \mathrm{vs.} 8.4 \pm 7.6 \mathrm{ng} / \mathrm{ml}, \mathrm{p}<0.05)$, and higher in man than in women with DM1 in comparison with healthy controls $(10.4 \pm 7.3 \mathrm{ng} / \mathrm{ml}$ vs. $3.3 \pm 2.6 \mathrm{ng} / \mathrm{ml}$ in men and $13.4 \pm 10.3 \mathrm{ng} / \mathrm{ml}$ vs. $12.5 \pm 7.9 \mathrm{ng} / \mathrm{ml}$ in women, respectively). In our patients with DM1, leptin levels were correlated with BMI, fasting insulin and insulin resistance (HOMA), $\mathrm{p}<0.01$, whereas leptin concentrations were not correlated with age, body fat, the number of CTG triplet repeats, fasting glucose and plasma cholesterol levels.

Conclusions: The pathological significance of leptin overproduction in DM1 patients is unclear at present. We found correlation among leptin levels and insulin resistance in our patients with DM1. It seems that long-term hyperinsulinaemia results in increased leptin production and circulating leptin concentrations in DM1 patients.

\section{Neuro-oncology}

\section{P607}

Unusual extension and characteristics of a ganglion cell tumour: a MRI and MR spectroscopy study

A. Angelou, D. Karacostas, N. Foroglou, E. Tsatsali, G. Karkavelas, I. Milonas, N. Tascos

Aristotle University of Thessaloniki (Thessaloniki, GR); Eurodiagnosis Imaging Center Thessaloniki (Thessaloniki, GR)

Introduction: The incidence of gangliocytomas(GC) has been reported to range from 0.1 to $0.5 \%$ among all CNS tumors. These neoplasms show a predilection for the cerebral hemispheres or the floor of the third ventricle, they are rarely found in the spinal cord, and localization to the cerebellum is considered uncommon. To our knowledge, involvement of the brainstem has not been previously described

Case study: A 36 year old woman presented with 1 year history of slowly progressive gait disturbance. Examination revealed left abducent and peripheral facial paresis, ipsilateral cerebellar dysmetria and mild right spastic hemiparesis. Brain CT showed a hypodensity in the posterior fossa with mild mass effect and no contrast enhancement or calcifications. On MRI, the lesion extended from the higher medulla up to the pons, invading the left cerebellar hemisphere. It depicted a low signal on T1 images, with no gadolinium enhancement and an impressively homogeneous high signal on T2 and FLAIR images

Proton MR spectroscopy provided similar data for both the cerebellar and brainstem lesion. Spectral peaks demonstrated low levels of $\mathrm{N}$-acetylaspartate(NAA), high Choline(Cho) relative to Creatine(Cr)level and increased $\mathrm{Cho} / \mathrm{Cr}$ ratio. After neurosurgical intervention, the lesion could not be completely resected due to the extensive involvement of the brainstem. However, multiple specimens were obtained from the left cerebellar hemisphere. Microscopic examination revealed a significant number of isolated or clustered ganglion cells while there was no evidence of glial neoplastic differentiation. Scattered Rosenthal fibers were also observed. Immunohistochemistry demonstrated ganglion cells negative in GFAP with positivity of some in synaptophysin. The Mib-1/ki67 index was found $<1 \%$. These findings suggested the diagnosis of GC. On follow-up, the patient's neurological condition remains stable 10 months after diagnosis and 2 years after the symptoms' onset.

Discussion: CNS ganglion cell tumors are classified as GC or gangliogliomas depending on the predominance of neoplastic neuronal or glial cells respectively. The predominance of mature ganglion cells, lack of glial neoplastic differentiation and the absence of GFAP immunoreactivity, all suggest a diagnosis of GC as happened in our case. Concerning spectroscopy in $\mathrm{GC}$, although there are no literature data to compare, the results of the present case revealed low NAA levels and high $\mathrm{Cho} / \mathrm{Cr}$ ratio favoring cerebral neoplasia

\section{P608}

Diffuse large $B$ cell lymphoma presenting as a piriformis syndrome

B.S. Ye, D.S. Shim, S.M. Kim, I.N. Sunwoo

Department of Neurology Yonsei University College of Medicine (Seoul, KR)

Introduction: Lymphoma can cause peripheral neuropathy by direct infiltration and compression of nerves, or by paraneoplastic, immunological, metabolic, infectious mechanism, and by the side effects of treatments. Piriformis syndrome is a rare condition characterized by pain and paresthesia located in the buttock, often radiating to the posterior thigh. Clinically, piriformis syndrome should be suspected when a patient has sciatica which is precipitated by the position of flexion, adduction, and internal rotation of the hip and is aggravated by sitting or lifting.

There were several case reports of lymphomatous involvement of sciatic nerves, but to our knowledge, no case presented as piriformis syndrome was reported.

Case report: A 74-year-old male farmer suffered from intermittent pain radiating from posterior thigh to dorsal foot and sole since September 2007. The radiating pain was induced and aggravated by flexion, adduction and internal rotation (FAIR) of the right hip joint. He had sciatica which was aggravated by the hip flexion and sitting, tenderness on the buttock area. With the findings of electrodiagnostic study, presumptive diagnosis of piriformis syndrome was made. Magnetic resonance imaging revealed an infiltrating mass lesion with enhancement, involving the right sciatic nerve and perineural soft tissue between the sciatic nerve and the right piriformis muscle. Surgical release of the right piriformis muscle and the biopsy of the soft tissue mass were done. The radiating pain was relieved after the surgery for a few days. The final histological diagnosis was a diffuse large B cell lymphoma. Postoperative staging revealed diffuse abdominal, pelvic, abdominal wall, and left humerus head involvements without bone marrow infiltration. The patient underwent systemic chemotherapy cycles.

Conclusions: We report a patient with diffuse large B cell lymphoma presenting as piriformis syndrome.

\section{P609}

Vertebral haemangioma causing compressive neurological symptoms

C. Dominguez-Gonzalez, E. De Pablo-Fernandez, L. Ballesteros, R. Gordo, A. Sánchez, S. Moreno, L. Martin, J. Ruiz

Hospital Universitario 12 de Octubre (Madrid, ES)

Objectives: Vertebral hemangiomas are benign developmental hamartomas of vascular tissue that do not possess malignant potential. They have been identified in 10-12\% of postmorten studies and are usually asyntomatic (diagnosed as incidental findings during imaging studies in the routine evaluation of back pain). However, vertebral hemangiomas extention into the spinal canal must be included among the differential diagnoses when back pain and signs of myelopathy are present, particularly in the thoracic spine.

Methods: We report a case of a 62 year old woman presented with a 6-month history of sensory changes and weakness in both lower limbs. Examination revealed a spastic paraparesis with altered sensation to the level of the umbilicus. Magnetic resonance imaging showed that the body of T9 returned hyperintense signal on T1WI and T2WI. There was compression of the thecal sac by soft tissue extension of the lesion into the spinal canal and a few tiny foci of signal void were seen within the intraosseus portion of the lesion. The histological diagnosis was haemangioma of bone.

Results: The clinical presentation of spinal cord compression caused by vertebral hemangioma has characteristically been insidious. Neurologic findings are typically slowly progressive over several weeks to months preceded by increased back pain. The level of this lesion was the lower thoracic spine, wich is consistent with the most common location for symptomatic vertebral hemangioma. Of all lesions with extraosseous protussion, $90 \%$ are found in the thoracic level, $75 \%$ of these are found between $\mathrm{T} 3$ and $\mathrm{T} 9 . \mathrm{Be}-$ cause of its ability to visualize osseus structures, CT is most useful for the intraosseous portions of the lesions and MRI evaluation characteristically highlights the extraosseous portion of the neoplasm, wich yields high-intensity T2 signal, indicative of the vascular soft tissue involvement.

Conclusion: Although the reported incidence of vertebral hemangiomas causing compressive neurological symtoms is infrequent, this condition must be included in the differential diagnosis when one presents with symtoms of a progressive myelopathy. The importance of preoperative diagnosis lies in the requirement for angiography and, if necessary, for embolitation, in that hemorrhage may be life threatening. 


\section{P610}

The role of apoptose-proteins in the evolution of meningiomas

F. Roser, C. Pfister, E. Endemann, H. Pfrommer, M. Tatagiba, A. Bornemann, R. Ritz

University of Tubingen (Tubingen, DE)

Introduction: Survivin is an inhibitor of apoptosis protein that blocks apoptosis by binding to caspases-3 and -9. It is highly expressed in less-differentiated embryonic cells and rapidly dividing tumours, but not in terminally differentiated adult tissues. Elevated survivin levels are found in malignant systemic tumours and are associated with chemo- and radiation resistance and therefore poor prognosis. SMAC/Diablo is a pro-apoptotic protein that inhibits anti-apoptotic proteins such as Survivin. SMAC/Diablo expression in meningiomas has not been previously analysed.

Materials and methods: Over one-hundred meningioma surgical specimens and normal human cortex were immunohistochemically stained and graded for the presence of Caspase 3, Caspase 9, Survivin and SMAC/Diablo. In addition, Western blot and PCR analysis were performed to detect the presence of the apoptotic enzymes in vivo and in vitro.

Results: SMAC/Diablo shows a constant high-level expression in meningiomas in contrast to Survivin, which is expressed throughout the specimens on a low-level. Caspase 3 and Caspase 9 showed ubiquitous expression, with an overall higher level of Caspase 3.

Conclusion: Apoptotic proteins are potential targets for treatment options in apoptosis-down-regulated meningiomas. We demonstrate a high expression of SMAC/Diablo in meningiomas of all grades, which is consistent with the finding of low-level expression of its counterpart survivin. These findings are in contrast to other reports who described an over-expression of survivin in meningiomas.

\section{P611}

Meningioma research - A translational approach from lab to surgery

F. Roser, C. Pfister, M. Tatagiba, A. Bornemann, R. Ritz

University of Tubingen (Tubingen, DE)

Objective: Meningiomas are benign tumours that can be cured by surgical resection. However, recurrence in meningiomas occurs frequently. Longterm management in patients with subtotal tumours resection or with a distinct histology remains controversial and difficult. Adjuvant systemic therapy in these tumours is necessary to achieve long-term control. Therapeutic and experimental approaches are presented and discussed.

Methods and results: Immunohistochemistry with certain indicators like progesterone receptor (PR) status and the Ki-67 proliferation index has shown to predict recurrence. Data of 600 meningiomas was correlated with recurrence on long-term follow-up ( $>65$ month). Combining two factors of proposed prognostic significance in benign meningiomas - proliferation index (Ki-67 LI $<4 \%$ vs. $\geq 4 \%$ ) and PR-Status (neg. vs. pos.) - a significant decreased recurrence free survival could be shown for negative $\mathrm{PR}$-status and $\mathrm{Ki}-67 \mathrm{LI} \geq 4 \%$, whereas proliferation rates and PR status were not age dependent. Further cell culture laboratory investigations (western blot, PCR) demonstrated a high expression of COX-2 and 5-LO receptors in meningiomas as potential targets for new therapeutic strategies. Immortalisation of a benign meningioma cell lineage with blockade of telomerase activity allows cell growth monitoring over multiple passages to perform inhibition assays in order to test chemotherapeutic agents like COX-2 or 5-LO inhibitors.

Conclusion: The routine use of proliferation indices and prognostic markers in benign meningiomas in combination indicate a good correlation to the clinical course. However in histopathological borderline cases a precise prognosis for recurrence in the individual patient is still difficult to accomplish. Current research provides new tools for determination of aggressiveness demonstrating basement membrane interaction and expression of increasing levels COX-2 receptors in high-grade meningiomas. Immortalisation of a benign meningioma cell line provides a reliable tool to study clinical use of systemic therapeutic options in aggressive meningiomas.

\section{P612}

Study of primitive brain tumour metabolism with microdialysis

G. Dran, E. Carré, J.J. Risso, P. Paquis, M. Lonjon

Hôpital Gui de Chauliac (Montpellier, FR); Service de Santé des Armées (Toulon, FR); Hôpital Pasteur (Nice, FR)

Objectives: The primitive tumours of the central nervous system (SNC) constitute, in spite of their relative scarcity, an important source of morbidity and mortality and represent a major problem of public health. Whatever the therapeutic sequence used, combining surgery, radiotherapy and chemotherapy, the prognostic of the glioblastoma (GBL) remains frightening.
A better comprehension of the natural history of these tumours is thus necessary in the sight of the installation of new treatments. Our research project is integrated in the field of fundamental explorations of the malignant glioma and more particularly in the exploration, in vivo, of the malignant gliomas metabolism. Using the technique of microdialysis, we measured concentrations of glucose, lactate, pyruvate, glycerol and glutamate in malignant brain tumors and in the normal peri-tumoral tissue (PTT).

Methods: We carried out a prospective and consecutive trial on the patients admitted to the Neurosurgical Department of Pasteur Hospital in Nice, France. Microdialysis catheters were introduced in a stereotactic way. The first catheter was inserted in tumour and the second in normal peri-tumoral tissue.

Results: Fourteen patients were included in the study

The distribution of the patients according to histological diagnostic of their pathology was as follows: nine glioblastomas $(64 \%)$, three anaplasic oligodendrogliomas (21\%) and two metastases of bronchopulmonary ori$\operatorname{gin}(14 \%)$.

For the whole patient, the rate of glucose was lower in the tumour than in the PTT. This result was found in each histological sub-group with a high significativity $(p=0,01)$ in the sub-group of glioblastomas. The glycerol, glutamate, lactate rates as well as the lactate/pyruvate ratio were on the other hand higher in the tumour than in the PTT.

Conclusion: In the glioblastoma we found a significant reduction in the intra-tumoral rates of glucose and an increase in the lactate rates and lactate/pyruvate ratio compared to adjacent healthy cerebral brain. We also showed a tendency to the increase in the intra-tumoral glycerol and glutamate rates compared to healthy brain. These data already had been shown before, in vitro and also, partly, in human by PET. Neurooncologic microdialysis studies preceding this work were very rare. Our results are very similar besides there, which confirms in an encouraging way the reliability and the reproducibility of this technique.

P613

Paraneoplastic limbic encephalopathy mimicking acute viral encephalitis I. Markakis, E. Alexiou, M. Gerazounis, G. Gkekas

General State Hospital of Nikaea (Piraeus, GR)

Objectives: Paraneoplastic limbic encephalopathy (PLE) is a rare nonmetastatic complication of various tumours that follows a chronic or subacute clinical course and is characterized by personality changes, memory loss, seizures and hallucinations. Early diagnosis is difficult since disease markers are often negative and characteristic symptoms can be mimicked by a variety of conditions. We present a case of PLE, initially presenting as acute herpetic encephalitis.

Case-History: A 56-year old man was admitted to our department with a 4-day history of headache, fever and confusion. On neurological examination he was alert but presented severe disorientation and profound impairment of recent memory. There were no cranial nerve or pyramidal signs, nor evidence of sensory dysfunction. Computed tomography of the brain was normal. CSF analysis revealed lymphocytic pleocytosis, increased protein and normal glucose. Electroencephalography showed diffuse slow activity with paroxysmal slow wave bursts. Acute herpetic encephalitis was suspected and the patient was put on intravenous acyclovir and levetiracetam. Routine laboratory assays were normal. Serological tests and CSF polymerase chain reaction for infectious pathogens were negative, as were onconeural antibodies. Cranial MRI revealed bilateral enhancing lesions of medial temporal lobes with increased T2 signal intensity. On the forth day of hospitalisation, the patient developed aphasia, agitation and generalized seizures. Six days later he became afebrile but his cognitive functions continued to decline. Repeat lumbar puncture revealed normal cell count and protein, with positive oligoclonal bands. A paraneoplastic syndrome was suspected. Computed tomography of the chest showed a right paratracheal lymph node mass, whose biopsy revealed an oat cell carcinoma. The patient was put on steroids and chemotherapy. Six months later, he had complete tumour remission and a marked neurological improvement.

Conclusions: Although PLE usually presents as a chronic or subacute encephalopathy, it can rarely invade acutely, being indistinguishable from herpetic encephalitis. The inclusion of PLE in the differential diagnosis of acute encephalitis is of clinical significance, given that this rare disorder may respond well to immunosuppressive and antineoplastic treatment. 
P614

Paraneoplastic cerebellar degeneration associated with tongue cancer and anti-CV2 autoantibodies

I. Zaganas, E. Saloustros, L. Vamvakas, M. Mavridis

University Hospital of Crete (Heraklion, GR)

Paraneoplastic cerebellar degeneration is associated with a variety of tumors and auto-antibodies against different neuronal antigens. The commonest neoplasms associated with this condition are breast, ovarian and lung cancer.

Here we describe a 60 -year-old patient, who was admitted because of dizziness and gait unsteadiness. Eight months earlier, locally advanced tongue (head-neck) squamous cell carcinoma (c T4N2M0) was diagnosed. She was managed with 2 cycles of induction chemotherapy with docetaxel/cisplatin/5-fluorouracil followed by radiotherapy with concurrent chemotherapy (carboplatine and anti-epidermal growth factor receptor monoclonal antibody cetuximab) and showed complete response of the primary tumor and the affected lymph nodes.

On examination the patient showed nystagmus, dysarthria, limb dysmetria, predominantly on the lower extremities, and severe gait ataxia. Magnetic Resonance Imaging showed mild cerebellar atrophy. Lumbar puncture cytology was negative. A complete screening for autoantibodies known to be associated with paraneoplastic cerebellar degeneration revealed high titer for anti-CV2 antibodies in the CSF. A relapse of the tongue tumor and a second occult tumor was excluded on the basis of extensive imaging and endoscopic studies. The patient received repeated cycles of immunoglobulins without improvement in her neurological deficits.

Anti-CV2 autoantibodies causing cerebellar degeneration have been associated with lung cancer, thymoma and gynecological tumors. On the other hand, squamous cell carcinoma of head-neck has rarely been associated with paraneoplastic syndromes, mainly hyperkeratosis and myopathy. This report extends further the spectrum of paraneoplastic syndromes associated with common tumors, such as head and neck cancer.

\section{P615}

Delta-24-RGD induces autophagy in brain tumour stem cells: implications for glioma therapy

J. Fueyo, H. Jiang, E. White, M.M. Alonso, C. Gomez-Manzano, W.K.A. Yung M.D. Anderson Cancer Center (Houston, US)

Therapy for malignant gliomas is not optima. The discovery of brain tumor stem cells (BTSCs) has introduced a paradigm-shift in the use of pre-clinical models to test anti-glioma therapy. BTSCs exhibit the capacity to repopulate tumors after treatment and therefore their targeting is critical to achieve a bona fide remission of the tumor. BTSCs are resistant to chemotherapy and radiotherapy. In this study, we examined the ability of an $\mathrm{Rb} / \mathrm{E} 2 \mathrm{~F}$ pathway targeting oncolytic adenovirus, Delta-24-RGD, soon to be tested in the clinic, to infect and replicate in brain tumor stem cells. We showed that a panel of BTSCs derived from human malignant glioma specimens expressed high levels of adenovirus receptors. Consistently, treatment of these cells with Delta-24-RGD resulted in decreased viability in a dose-dependent manner. Importantly, Delta-24-RGD infection was followed by the triggering of autophagy as assessed by identification of autophagosomes and autolysosomes using electron microscopy. In agreement with the morphological data, biochemical markers of autophagy including Atg 5 and LC3II were upregulated. Treatment of BTSC-derived xenografts showed significant prolongation of survival $(\mathrm{p}<0.0001)$. Furthermore, infected cells expressed high levels of Atg5 in vivo. Importantly a series of E1A and E1B replication competent adenoviruses induced consistently more autophagy in U-87MG cells, with high basal levels of ATG5, than in U-251 MG cells expressing low levels of ATG5. Importantly, infection of embryonic cells from ATG-null mice resulted in a significant decrease of autophagy. Our data indicate that autophagy is in the pathway of adenoviral replication and place $\operatorname{Atg} 5$ as a required event for the induction of autophagy. These results are relevant for the understanding of the molecular events controlling autophagy in cancer cells and may favor the development of novel therapeutic tools by targeting autophagy in apoptosis-resistant cells. This is the first study showing that oncolytic adenoviruses infect and kill brain tumor stem cells and, by uncovering autophagy, offers a new mechanistic approach to understand the anti-cancer effect of this strategy. As requested, new intriguing unpublished data will be presented.
P616

Unilateral palsy of VIII, IX, X, XI, XII cranial nerves in a 42-year-old patient K. Kioulachidis, I. Xidakis, N. Matikas

Evangelismos Hospital (Athens, GR)

Introduction: Glomus jugulare is a rare tumor, usually benign. Because of its anatomical position and mass it causes a significant injure and paresy of the lower cranial nerves. This tumour is highly vascularised, secrets catecholamines, thus mimicking phaeochromocytoma and can be operational.

Case report: We report a case of a young woman (42-years-old) who was admitted in our hospital due to hoarsenes, dysphagia and pain of right shoulder. Symptoms started with pain of right shoulder, difficulty in speech and, after 5 months, dysphagia. The patient also reports substantial weight loss. The neurological examination showed: Right shoulder drop, right Sternocleidomastoid atrophy, right trapezius atrorhy, tongue deviation to the left in the out position, tongue atrorhy, deviation of the uvula, hearing loss from right ear, dysphonia, difficulty in swallowing, and, vivid tendon reflexes. The patient produced a brain and shoulder CT, with no abnormal findings. The biochemical, haematological and hormonal testing was normal. The CT as well as the MRI of the scalp base indicated a mass $3 \times 3 \mathrm{~cm}$ damaging the jugular foramen and affecting adjacent bones. Angiography indicated a vascularised mass at the base of the scalp that its blood sypply is derived from a bifurcation of the external carotid. Clinical and imaging findings led us to diagnose a glomus tumour. The patient is being treated with gamma-knife radiation and embolism.

Discussion: Glomus jugulare is a rare tumour $(0,6 \%$ of head and neck tumours internationally, and $0,03 \%$ of all tumours), usually benign. It can be one of the causes of significant injure and palsy of the lower cranial nerves, because of its anatomocal position and mass. This tumour is highly vascularised and secrets catecholamines, thus mimicking phaechromocytoma. It is treated with gamma-knife radiation, embolism, surgical excision, or combination of the above. The glomus jugulare has identifiable clinical and imaging characteristics, is treatable, so the possibility to locate it should be always taken into account.

\section{P617}

Toxicity of intrathecal liposomal cytarabine (DepoCyte) in addition to systemic high-dose methotrexate based chemotherapy in primary central nervous lymphoma

K. Specht, G. Ahle, A. Juergens, H. Pels, U. Schlegel

Knappschaftskrankenhaus (Bochum, DE)

Objective: To evaluate the toxicity associated with intrathecal liposomal cytarabine (DepoCyte) in immunocompetent patients with primary central nervous lymphoma (PCNSL) receiving systemic and intrathecal chemotherapy.

Methods: Between 11/2005 and 10/2007 liposomal cytarabine (DepoCyte) was given to 26 patients within a phase II study evaluating chemotherapy without radiotherapy in immunocompetent patients with primary central nervous lymphoma. A high-dose methotrexate (MTX) (cycles $1,2,4,5)$ and cytarabine (ara-C) (cycles 3,6$)$ based systemic therapy was combined with intrathecal liposomal cytarabine (DepoCyte) $50 \mathrm{mg}$ given via lumbar puncture in each treatment course, i.e. 6 times. A total of $105 \mathrm{cy}$ cles was given. CSF pleocytosis was assessed at each course and toxicity according to common toxicity criteria (CTC) was prospectively recorded.

Results: No systemic toxicity was recorded. Five patients (19\%) developed grade II/III neurotoxicity, associated with liposomal cytarabine, including a singler epileptic seizures $(n=2)$, transient lumbosacral pain $(n=3)$; two of the latter with dyaesthesia und one with transient urine incontinence. None of these patients had concomitant pleocytosis in cerebrospinal fluid (CSF). MRI scan of the brain and the lumbosacral spine were negative for arachnoiditis. Eight patients of the series showed at least one episode of an asymptomatic CSF pleocytosis.

Conclusion: In general, intrathecal liposomal cytarabine (DepoCyte) was well tolerated in this study. However, few patients suffered from grade II/III neurologic toxicity. None of the patients suffered from permanent neurotoxicity. 


\section{P618}

The relationship between cognitive function, tumour histology and surgical treatment in patients with primary brain tumours

O. Alexandru, V. Ciubotaru, L. Tataranu, S. Fetea, P. Badea, A. Dricu

University of Medicine and Pharmacy (Craiova, RO); Bagdasar-Arseni Hospital (Bucharest, RO); Karolinska Institute University Hospital (Stockholm, SE)

Objectives: It is yet unknown if the cognitive dysfunction in patients diagnosed with brain tumours is caused by the direct effects of the tumor or by its treatment. Our goal was to investigate the relationship between tumor histology and the effects of surgery on the cognitive function in patients diagnosed with primary brain tumors.

Method: The neuropsychological functioning of 14 adult patients diagnosed with malignant brain tumor(10 glioblastomas and 4 astrocytomas, 8 women and 6 men) was compared with the neuropsychological functioning of 14 adult patients diagnosed with meningioma ( 5 women and 9 men).The groups were matched with respect to patient age, gender, education and tumor location. Cognitive function was evaluated by modified mini- mental state examination (3MS test).

Results: Cognitive function presurgery and postsurgery was normal in patients with malignant brain tumors and meningiomas located in the right brain. The cognitive functions decreased before operation in all patients with malignant brain tumors located in the left brain and they did not normalize after surgery. Cognitive function presurgery was decreased in patients with meningiomas and normalized in some patients after surgery.However the 3MS test did not normalize in the most patients with meningiomas after surgery. The mean test scores of meningioma patient group were superior to the mean scores of malignant gliomas patient group but no significant differences were detected. In these patients the tests of: mental reversal, first recall, writing, temporal orientation and spatial orientation diminished after operation.

Conclusions: The surgical treatment should bring benefits for the cognitive function of patients with brain tumors located in the right brain. For patients with malignant brain tumors located in the left brain one should not expect the improvement of cognitive function after surgery. In patients diagnosed with meningiomas in the left brain is hard to anticipate the evolution of neuropsychological functions. Therefore, the tumor histology is not an accurate predictor of the neuropsychological evolution in adults with brain tumors.These patients should be monitorized by complex teams of specialists capable to decide the most adequate treatments in order to maintain a reasonable quality of life.

\section{P619}

Three patients presenting with spinal cord compression as initial manifestation of non-Hodgkin's lymphoma

P. Bernsen, P. van Vierzen, P. Zoon

St. Jansdal Hospital (Harderwijk, NL)

Spinal cord involvement by metastatic complications is not uncommon in the course of systemic cancer. Metastasis may arise from the posterior aspect of the vertebral body and subsequently invade to the epidural space resulting in spinal cord dysfunction by direct compression or vascular insufficiency. Metastases of the vertebral column most often originate from pulmonary, breast, kidney, or prostate carcinoma. Local compression of cranial nerves, spinal cord, cauda equina, or spinal nerve roots by extradural lymphoma is diagnosed in 5 to $10 \%$ of patients with non-hodgkin's lymphoma (NHL), usually in more advanced stages of disease. Lymphomatous involvement of the spinal epidural space occurs in less than $3 \%$ of all systemic lymphomas. Spinal cord compression as initial manifestation of an underlying $\mathrm{NHL}$ is even more rare. In literature it is associated with aggressive disease. Patients with a primary spinal epidural NHL localisation often present with local back pain which may persist for several months before signs of spinal cord compression develop. Magnetic resonance imaging (MRI) is the best radiodiagnostic procedure to detect tumors and metastasis of the spinal cord and epidural space. We report on the clinical features and results of MRI studies of three patients with NHL who presented with a history of back pain for a considerable time followed by rapidly progressive weakness of the legs and sensory disturbances. In all patients the lesion was localised at the midor lower thoracic level. Compression of the spinal cord was caused by a primary epidural process, not by bony destruction. In two patients initial treatment consisted of laminectomy. One patient received radiotherapy after a diagnostic CT-guided biopsy. Pathological investigation revealed a NHL in all patients. At the time of diagnosis no other localisations of a NHL could be found. As NHL is a very radio- and chemosensitive tumor this is the treatment of choice after a tissue diagnosis has been obtained. In case of progressive neurological impairment a decompressive laminectomy followed by radiotherapy may be needed. Further management should include a thor- ough workup to determine the stage of disease and subsequent treatment regime.

P620

The significance of cerebrospinal fluid variables in paraneoplastic neurological syndromes

P. Stourac, J. Bednarova, M. Hladikova, P. Praksova

Faculty Hospital Brno (Brno, CZ)

Objectives: To determine the prevalence of selected laboratory parameters in cerebrospinal fluid (CSF)and serum (total protein,cytology,onconeural antibodies and oligoclonal IgG bands) in a set of patients with paraneoplastic neurological syndromes (PNS). To characterize the most typical neuroimmunological CSF variables profile in classical PNS.

Methods: For the detection of onconeural antibodies we used indirect immunofluorescence on mouse tissue substrate (MeDiCa,USA).Subsequent confirmation was done by immunoblot (Ravo Diagnostics, Germany).The oligoclonal IgG bands(IgG-OB)were diagnosed by isoelectric focusing on agarose gel followed by immunoblot and immunoenzymatic staining. The clinical and laboratory diagnoses were established according to the "Recommended diagnostic criteria for paraneoplastic neurological syndromes" published in I Neurol Neurosur Psychiatry 2004.

Results: We investigated 19 patients (n-19) with PNS and available CSF data. The positivity of well-defined onconeural antibodies in CSF was as following: anti-Yo(n-8), subacute cerebellar degeneration;anti-Hu(n-4),sensory neuronopathy,encephalomyelitis;anti-Yo+anti-CV2(n-1), subacute cerebellar degeneration;anti-Hu+anti-Yo+anti-Ri(n-2), subacute sensory neuronopathy,encephalomyelitis;anti-Hu+anti-Yo(n-2), predominantly brainstem encephalitis;anti-Ri(n-2),encephalomyelitis.The values of total protein in CSF were in range from $39 \mathrm{mg} / \mathrm{dl}$ to $262 \mathrm{mg} / \mathrm{dl}$, with average value $104 \mathrm{mg} / \mathrm{dl}$.The number of cells was in range from 1 to 96 cells/1ul, with average value 7 cells/1ul. Elevated level of total protein was detected in $17 \mathrm{pa}-$ tients (n-17).Pleocytosis was present in 12 patients(n-12). IgG-OB were positive in 4 samples,resp. patients(n-4)from 6 examined samples(n-6).Three IgG-OB positive cases(n-3) had only CSF positivity,1 patient had so called IgG-OB mirror pattern reflecting systemic immune response.

Conclusion: Neuroinflammation expressed as elevation of protein concentration and/or pleocytosis in CSF was positive in 18 patients, i.e. $95 \%$. Onconeural antibodies were detected in $100 \%$ of patients.Concomitant positivity and various combinations of onconeural antibodies confirm paraneoplastic origin of neuroinflammation. Lesser sensitivity of IgG-OB in CSF likely reflects the only humoral arm of immunity,which needs to be completed by more detailed investigation of $\mathrm{T}$ and $\mathrm{B}$ cells immunity. Calculated sensitivity of the above presented CSF laboratory profile in PNS is $95 \%$.

Supported by MSMT0021622404, QLG1-CT-2002-01756-PNSEURONETWORK

\section{P621}

Biocomputing: computer simulation of microscopic growth of glial cell and comparison of irradiation margins

P.-Y. Bondiau, E. Konukoglu, O. Clatz, H. Delingette, N. Ayache

Centre Antoine Lacassagne (Nice, FR); INRIA (Nice, FR)

Introduction: Recent progress in computer science has made simulations in life sciences possible. The latest research into tumor growth simulation has produced results that can be used for teaching purposes and in clinical practice. The identification of tumor growth parameters and their evaluation are central to developing a digital model. We have conducted a feasibility study aiming to simulate the growth of glioblastoma multiforme (GBM) and compare the results with the findings of medical imaging.

Methods: We designed a digital atlas containing values for elasticity and "resistance to invasion" for each brain structure and for the preferred direction of tumor spread.

Data from two patients were entered into the model, which then simulated microscopic growth mechanisms for GBM.

Results: The results were calculated from various proliferation and diffusion parameters and then compared with actual standard margins implemented for irradiation therapy. For both patients, the simulations showed microscopic invasion outside the irradiation margins, when using diffusion parameters higher than proliferation parameters.

Conclusion:

1. The main challenge was to estimate tumor growth parameters; they may be assessed from pathology results.

The study will be continued to compare the actual site of recurrence with the simulation. 
P622

Isolated central nervous system relapse during cytologic and molecular remission in one patient with acute promyelocytic leukaemia: a case-report R. Vivanco-Hidalgo, A. Galvez, E. Giralt, A. Rodriguez-Campello, T. Gimenez, E. Gimeno

Hospital del Mar (Barcelona, ES)

Objectives: Central nervous system (CNS) involvement was a rare finding in acute promyelocytic leukaemia (APL) before the era of treatment with alltrans-retinoic acid (ATRA). We present a case of isolated CNS relapse in a patient with APL in remission

Case report: Clinical course

A 32 -year-old patient was diagnosed of APL in June 2006. Remission induction chemotherapy with idarubicin and ATRA were administered, achiving complete hematologic, cytogenetic and molecular remission in July 2006. He suffered an ATRA syndrome as a complication. Between September 2006 and December 2006 he received consolidation chemotherapy without any complication. After consolidation period, the patient received treatment with mercaptopurine, methotrexate, and three-monthly ATRA.

In October 2007, myelogram was performed and hematologic, cytogenetic and molecular remission was confirmed. Three days later, the patient went to emergency room explaining backache with cervical irradiation, headache, gait disorder and diplopia for 3 weeks. In physical examination, bilateral VI pc palsy, neck rigidity and ataxia were observed.

Hemogram, coagulation and D-dimer were normal. Magnetic resonance image showed diffuse leptomenigeal enhancement in T2 weighted images. Lumbar puncture was performed showing high pressure. 478 cells were detected in CSF, with normal glucose and proteins. CSF cytology identified atypical promyelocytes, and PML/RARA alfa translocation. Under isolated CNS ALP-relapse diagnosis, intrathecal chemotherapy treatment was started, first with liposomal cytarabine (DEPOCYTE) plus dexamethasone, followed by triple therapy (methotrexate, ARA-C and hydrocortisone, with clinical improvement. Moreover, he received systemic chemotherapy too (methotrexate + ARA-C). Finally, craniospinal radiotherapy was delivered. In the last CSF cytology (december 2007), no atypical promyelocytes were found.

Conclusion: Although extramedullary relapse has increasingly been reported in the ATRA era, CNS relapse in the absence of bone marrow disease is rare in patients with APL and only few cases are reported in medical literature. This situation could be due to the upregulation of cellular adhesion molecules on malignant promyelocytes that may facilitate leukemic infiltration into CFS. Further studies are needed to determine the proper treatment in these cases

\section{P623}

Clinical and magnetic resonance spectroscopy study of a brain tumour in a patient with segmental neurofibromatosis

S. Chebel, S. Ben Yahia, M. Khairallah, M. Frih-Ayed

University Hospital of Monastir (Monastir, TN)

Introduction: Segmental neurofibromatosis 1 (SNF1) is a rare genodermatosis caused by somatic mutations in the NF1 gene. It consists of localized characteristic pigmentary lesions (i.e. café-au-lait spots and freckling) and/or neurofibromas intermingled with areas of unaffected skin, often configuring specific mosaic patterns. Serial study using magnetic resonance imaging (MRI) and magnetic resonance spectroscopy (MRS) of brain tumor in a 16-year-old boy with SNF1 was described.

Case report: A 16-year-old boy with congenital dorsal scoliosis and SNF1was evaluated for bilateral optic atrophy. Neurological examination showed an isolated tetra pyramidal syndrome. The cerebral MRI showed a brain tumor involving bilaterally basal ganglias and temporal lobes. On MRS, the ratios of N-acetylaspartate (NAA)/creatine and that of choline/creatine were each reduced in the brain lesion. In addition, elevation of the lactate/lipid peak was shown.

Commentary and conclusion: In this study, we examined serial MRI and MRS of brain tumor in a patient with SNF1. MRS was a noninvasive technique for detecting the presence of tumors tissue in the brain through its metabolic activity. MRS has an important role in clinical and it can be used to differentiate malignant and nonmalignant brain lesions from normal brain tissue.
P624

Anti-neuronal antibodies in small-cell lung cancer patients without paraneoplastic disorders

S. Içöz, E. Tüzün, M. Kürtüncü, P. Oflazer, G. Akman-Demir

Istanbul University (Istanbul, TR)

Objectives: Small cell lung cancer (SCLC) is frequently associated with paraneoplastic antibodies and various neurological syndromes. Some SCLC patients may also develop serum anti-neuronal antibodies without developing neurological symptoms. In this study, we aimed at investigating the frequency of serum anti-neuronal antibodies in neurologically normal SCLC cases.

Methods: Thirty-three SCLC cases with no neurological signs or symptoms and 25 healthy controls were included in our study. Serum anti-neuronal antibodies were screened with indirect immunohistochemistry using biotin-avidin-peroxidase method.

Results: Anti-neuronal antibodies were detected in sera of $7(21.2 \%)$ SCLC cases. All 7 samples reacted with widespread nuclei of the rat brain. Sera of 25 healthy control cases did not reveal any anti-neuronal antibodies. The difference between the patient and control groups was statistically significant $(\mathrm{p}<0.01)$. Antibody negative and positive SCLC patients did not differ by age or gender.

Conclusion: The anti-neuronal antibody frequency of neurologically normal SCLC patients in our study is in line with previously reported cohorts. Immunohistochemistry is a practical, quick and reliable screening test for SCLC patients and might be useful in determination of patients that need to be further examined with western blot technique.

\section{P625}

Bone marrow stromal cells contribute to neoangiogenesis in malignant gliomas probably by differentiation into pericytes

T. Birnbaum, J. Hildebrandt, C. Schichor, B. Krebs, B. Korte, F. Trillsch, P. Nelson, $R$. Goldbrunner, A. Straube

Klinikum der Universität München (Munich, DE)

Objectives: The use of bone marrow stromal cells (MSC) as cellular vectors is a promising new approach for the treatment of malignant glioma. In previous studies we could demonstrate that MSC are characterized by a strong tropism towards glioma, and that several angiogenic growth factors are involved in this process. In the current study we tested the hypothesis that MSC contribute to neoangiogenesis in malignant gliomas. For this purpose we analyzed 1) the intra-tumoral distribution of human MSC in vivo and 2) the differentiation of MSC under the influence of glioma-conditioned medium in vitro.

Methods: Human MSC were isolated from bone marrow biopsies and characterized by FACS. The differentiation of human MSC under the influence of glioma-conditioned medium was analyzed in vitro using light microscopy and immunofluorescence staining. The in vivo distribution of intravenously administered human MSC was studied using a rat glioma model. The experiments were performed with native MSC and MSC, which were transfected with a plasmid expressing the red fluorescent protein under the control of the Tie2 promoter (RFP-Tie2). Brain sections were analyzed using immunohistochemistry and confocal laser microscopy.

Results: After intravenous administration MSC were found only within the tumor and in the adjacent tissue, but not in the normal brain. The majority of MSC was found in the wall of tumor microvessels. Administration of RFP-Tie2 MSC revealed a vascular specific reporter gene expression, indicating that MSC underwent differentiation during neoangiogenesis. Under the influence of glioma-conditioned medium the morphology of MSC changed from the typical fibroblast-like into a pericyte-like appearance. Immunofluorescence staining of these cells revealed expression of CD151,VEcadherin, alpha smooth muscle actin, desmin, and NG-2 (in contrast to native MSC, respectively), while there was no expression of von Willebrand Factor and myosin detectable.

Conclusion: We could demonstrate that malignant gliomas actively recruit intravenously administered human MSC in vivo. Therefore, these cells could be used as cellular vectors in new anti-glioma therapies. Furthermore, we could show that MSC preferentially incorporate into neoangiogenic vessel walls. Together with the results of the in vitro assays we postulate 1) that MSC actively contribute to neoangiogenesis in malignant gliomas and 2) that this is due to differentiation of MSC into pericytes.

This work was supported by the FöFoLe program of the university of $\mathrm{Mu}$ nich. 
P626

Clinical and neurophysiologic features of spinal cord meningiomas

V. Grincensen, M. Gavriliuc

Institute of Oncology (Chisinau, MD); Institute of Neurology and Neurosurgery (Chisinau, MD)

We present the longitudinal clinical and electrophysiological study of 27 patients with spinal meningioma. All cases were confirmed by MRI and histological examinations. Electrophysiological examination included needle electromyography, sensitive and motor electroneurography, F-wave study, Hoffman reflex, bulbo-cavernosus reflex. Clinical and electrophysiological findings were assessed in each case together with the level of spinal lesion.

Electrophysiological abnormalities were founded in $100 \%$ of cases. Based on statistical analysis of the results, electrodiagnostic criteria were elaborated for the discrimination of each clinical syndrome of spinal meningioma. In addition to this data were founded that compressive damage of spinal cord tissue causes the morphofunctional reorganization of motor units and segmentar neuro-muscular apparatus. Moreover, as a result of neuronal plasticity at this level of nervous system news programs of motor function are established.

The general conclusion of this study is that multimodal electrophysiological investigation as a consciously extension of clinical examination is very important in the positive and differential diagnosis of spinal meningioma. An electrophysiological examination is also useful for the prognosis of tumoral spinal cord process and for the control of neurosurgical treatment efficacy.

Practical recommendations of the study consist from clinical- electrophysiological-neuroimagistical algorithm of evaluation of spinal meningioma.

\section{P627}

Neurofibromatosis type 1. A retrospective study in 42 patients

V. Silva, A. Azevedo, I. Costa, T. Pimentel, J. Nunes, D. Salgado

IPOFG (Lisbon, PT)

Objectives: To determine the clinical features of Neurofibromatosis type 1 (NF-1), its association with primary nervous system tumors and the long term survival, in an oncological hospital series.

Methods: Data were collected retrospectively, by chart review by an independent observer and according to a standardized form, on all patients with the diagnosis of NF-1 followed between January 1985 and February 2007. The long term survival was determined by means of Kaplan-Meier survival curves.

Results: 42 patients fulfill the NF-1 criteria (23 males, 19 females), mean age of 17.4 years, mean time of follow-up of 6.7 years. $18(42.9 \%)$ patients had defined family inheritance and $15(35.7 \%)$ were sporadic cases. Among the major clinical features the most recognized were "café au lait spots" $(37 / 88.1 \%)$ and peripheral neurofibromas $(27 / 64.3 \%)$. UBOs were found in 14 patients $(33.3 \%)$. Association with tumors (excluding cutaneous neurofibromas) occurred in 37 patients (88.1\%): 19 (51.4\%) with optic pathways gliomas, $10(27.0 \%)$ with other central nervous system (CNS) gliomas (4 astrocytomas, 1 oligodendroglioma, 4 glioblastomas and 1 gliomatosis of the cerebellum), $2(5.4 \%)$ with malignant peripheral nerve sheath tumors, 1 $(2.7 \%)$ with a sarcoma, $2(5.4 \%)$ with neurofibromas of the peripheral nerves with aggressive behaviour do to their localization and $3(7.1 \%)$ with probable radicular neurofibromas. In Kaplan-Meier analysis, at 10 years the cumulative survival was $100 \%$ for the optic pathways gliomas and $66.7 \%$ for the other CNS gliomas. All the peripheral nervous system tumors had a cumulative survival at 7 years of $100 \%$, except for the malignant peripheral nerve sheath tumors with a survival rate of $0 \%$ at 15 years.

Conclusion: Despite the shortcomings of a highly chosen population due to a referral bias, this study exemplifies the NF-1 clinical heterogeneity and the variability of its complications. It confirms the important association with CNS tumors, of which optic gliomas were the most frequent, but it also shows relevant association with more aggressive tumors, such as other CNS gliomas and malignant peripheral nerve sheath tumors.

\section{P628}

Intravascular large B cell lymphoma, imaging features and outcome Z. Alkawi, H. Alhindi, H. Murad

KFSH \& RC (Riyadh, SA)

Intravascular Large B Cell Lymphoma (IVLBCL) is a rare malignancy that is difficult to diagnose. We present the clinical, imaging and biopsy findings in a patient with IVLBCLwho had a good short term outcome with emphasis on distinctive imaging findings and their pathological correlates.

IVLBCL is a rare form of extranodal non-Hodgkin lymphoma with predilection to the central nervous system (CNS). Lymphoma cellsform microscopic intravascular masses.Vascular occlusion can lead to multiple infarct-like lesions in the CNS.

The patient underwent CT, MRI, and PET scans followed by a brain biopsy and microscopic examination. Clinical imaging assessments were repeated after treatment.

The patient presesnted with a generalized tonic clonic seizure followed by persistent lethargy. Imaging showed a subcortical non-enhancing low-attenuation lesion on CT. The subcortical lesion had low Ti signal and high T2 signal typical for edema. The overlying cortex had foci of increased T1 signal, and dilated cortical veins became visible with Gadolinium enhancement. Apparent Diffusion Coefficient (ADC) map showed increased values consistent with vasgenic edema without infarct. The lesion was hypometabolic with contralareal cerebellar diaschesis. The diagnosis was made by brain biopsy. Significant clinical and imaging improvement occured after treatment.

The imaging features of multiple white matter edematous changes and engorged cortical veins should raise suspicion of IVLBCL. Biopsy is needed for diagnosis. Early treatment improves both clinical and imaging findings.

\section{Poster session 4}

\section{Cerebrovascular disorders}

P629

Comparison of risk factors between young and old patients with spontaneous intracerebral haemorrhage in Iran

A. Chitsaz, M. Saadatnia

Isfahan University of Medical Sciences (Isfahan, IR)

Background: Intracerebral haemorrhage (ICH) is a bout $13 \%$ of strokes in patients older than 45 years, and $20 \%$ of strokes in younger ones. In developing countries it seems to be more cases of ICH in young patients.

Objective: Purpose of this study was comparison risk factors, location and prognosis of spontaneous intracerebral hemorrhage (ICH) in young and old patient in 163 cases.

Methods and materials: 163 patients with age range 15 to 91 year old, that have spontaneous ICH after undergoing computed tomography scan divided in two groups, 15 to 45 year old and older than 45 year old. In all patients blood dyscrasis, anticoagulant toxicity, toxemia, traumatic hemorrhage, primary subarachnoid hemorrhage, vascular malformation and brain tumor, were excluded. We analyzed differences of risk factors, locations and final outcome with Ranklin scale between two groups.

Results: In this study markedly different risk factors were hypertension ( $P<0.001$, more in olders), alcohol use $(P=0.03$, more in youngs $)$, stroke recurrence $(P=0.01$, more in older $)$, low education state $(P=0.006$, more in olders), mortality and disability after 6 months from ICH onset $(\mathrm{P}=0.04$, more in olders). Location of hemorrhage were significantly different as it was more lobar in youngs and non lobar in olders $(\mathrm{P}=0.03)$

Conclusion: ICH in young people is mainly lobar and result from vascular malformation, hypertension is markedly fewer in youngs.

We must before ICH known previously unnoticed age - related genetic and environmental risk factors for ICH and prevent of damage of arterial wall.

P630

Migrainous incomplete infarction

A. Kunizawa, S. Suzuki, A. Asai, K. Kawamoto, Y. Kitazawa, G. W. Ickenstein, B.K. Siesjo, M. Fujioka

Kansai Medical University (Osaka, JP); Helios General Hospital (Aue, DE); Lund University (Lund, SE)

Object: Migraine is one of the leading causes that destroy the social life with its high prevalence rate of $4.0-8.2 \%$ in men and $11.9-21.9 \%$ in women. The hypothetical mechanism of migraine includes vascular, neuronal, and trigeminovascular theories. These hypotheses involve the cerebral vasospasm/vasodilatation, spreading cerebral hypoperfusion, cortical spreading depression, and/or cerebrovascular inflammation. The migraine seems to increase independently the risk of the stroke in the patients. We describe the case of migraine that resulted in multiple-tandem cerebral vasospasms 
leading to incomplete infarction (selective neuronal death and gliosis but not pannecrosis) and subsequent atrophy of the frontal and insular cortices.

Methods: A 45-year-old woman (3-year history of migraine with aura) suddenly developed visual aura and left arm weakness followed by headache and confusion. We repeatedly performed neuroimaging and neurological studies in the patient within one year after onset.

Results: Repeated CT scans never showed abnormal density in the brain during the study period. She totally recovered within 72 hours after the hemiplegic migraine attack. However, MRA at onset showed transient stenosis of the right internal carotid (C2 portion) and the right middle cerebral (M1 and M2) arteries. The simultaneous diffusion-weighted MRI revealed a hyperintensity of laminar form in the right cerebral frontal and insular cortices in the acute stage ( $<5$ days). MRI (T1-/T2-W and FLAIR) showed slowprogressive atrophy of the right frontal and insular cortices but no signal changes in the brain during the study period. The migraine attack never recurred after the hemiplegic event.

Conclusion: To the best of our knowledge, this is the first report of adultonset hemiplegic migraine case of incomplete infarction due to vasospasm revealed by MRA. We suggest the importance of long-term follow-up study for neurodegeneration after a hemiplegic migraine attack even in the case without acute infarction.

P631

Spontaneous spinal epidural haematoma inducing acute anterior spinal cord syndrome

A. Kunizawa, S. Suzuki, A. Ryu, A. Asai, K. Kawamoto, G. W. Ickenstein, Y. Kitazawa, M. Fujioka

Kansai Medical University (Osaka, JP); Helios General Hospital (Aue, DE)

Objectives: Spontaneous spinal epidural hematoma (SSEH) is very rare. Its etiology remains unclear; however spinal venous stroke seems to be involved. We report the first case of posterior cervical SSEH leading to acute anterior spinal cord syndrome as the false localizing sign.

Methods: A 63-year-old healthy woman suddenly suffered from progressive weakness of the right lower limb. The neurological test 30 minutes after onset revealed complete paralysis of the bilateral lower extremities, paresis of the bilateral hands (right>left), and disappearance of superficial body sensation below the level of C8. However, deep sensation remained completely intact. We repeatedly performed neuroimaging and neurological studies in the patient within one year after onset.

Results: MRI showed acute epidural hematoma light-dorsal to the spine between $\mathrm{C} 3$ and Th 5 levels. The hematoma had its maximum diameter at C7. In addition, MRI revealed a central-type Cervical Intervertebral Epidural Haematoma (CIDH) of $\mathrm{C} 6 / 7$ that had been asymptomatic. Therefore, the spinal cord at $\mathrm{C} 6 / 7$ was remarkably

compressed on the right side by these CIDH and hematoma. The patient underwent the surgical decompression 7 hours after onset. SSEH was diagnosed based on the MRI and intraoperative findings. She completely recovered from the neurological deficits after the operation, except for the slight motor clumsiness of the right $\mathrm{C} 8$ region remaining at the 6th month. The follow-up MRI showed a small cystic change in the spinal gray matter of the C8 segment without any spinal compression.

Conclusion: Our patient with posterior SSEH had acute anterior spinal cord symptoms. This discrepancy between the SSEH location and the neurological localizing sign seem to result from the preexisting CIDH that led to the direct injury of the anterior spinal cord and to anterior spinal ischemia by compressing the anterior spinal artery.

\section{P632}

\section{Life events and stroke}

A. Nassef, A. Abdelsamee

Ain Shams University (Cairo, EG)

Background: On asking about stroke risk factor knowledge in the publics, stress is the most frequently mentioned factor. Many studies tried to examine such relationship, some studies showed that there is a relation and other indicating that there is none.

Aim of the work: This study was designed to examine if there is a relationship between life events and occurrence of stroke (Is stress a risk factor for stroke?). And if yes which type of life events is implicated.

Patients and methods: 60 patients with first ever stroke in their life time were included. All patients were admitted to stroke unit in Ain Shams Specialized Hospital. Control groups consisted of 50 healthy subjects matched for age and sex with patients group and 60 subjects matched for age, sex and risk factors for stroke with the patients group, but without stroke. All patients were subjected to full history taking and neurological examination, CT and /or MRI brain, ECG and Echo and full laboratory investigations. All pa- tients and control subjects were interviewed using Life Events and Difficulties Schedule (LEDS) with its new version (1995) for assessment of life events and difficulties during the six months preceding the onset of stroke (for the patients) / or the interview (for the controls).

Results: When considering severe events and marked difficulties, the patients reported much more than both control groups with highly significant difference $(\mathrm{P}<0.001)$. Events involving humiliation and/or entrapment make the highly significant difference between patients and controls but events involving loss or danger alone showed no significant difference $(\mathrm{P}<0.05)$. The distribution of severe life events along the 6 months before stroke (in patients group) and or before the interview (in the control groups) showed that patients have significant higher rate of reporting 1 day, 1 week, and 6 months before stroke onset than their matched controls during the same timing. We also found that the lesser the load of risk factors of stroke in the patients group, the higher the rate of occurrence of severe events specially those involving humiliation and /and entrapment.

Conclusion: Severe events especially those involving humiliation and/or entrapment may be considered as an independent risk factor for stroke. Such conclusion is worthy as this will be a modifiable risk factor with possibility of preventive actions via improving coping skills and social support.

\section{P633}

Hyperphosphorylation of tau protein indicates Alzheimer-like axonal degeneration in superficial CNS siderosis

D. Kondziella, H. Zetterberg

University Hospital Sahlgrenska (Gothenburg, SE)

Objectives: In superficial CNS siderosis chronic subarachnoidal hemorrhage leads to hemosiderin deposits in the subpial layers of the brain and spinal cord. Many years usually pass between the initial event causing chronic bleedings and the development of cerebellar ataxia, sensory hearing loss and various sensorimotor deficits. The only therapeutic option is to identify and eliminate the bleeding source. Otherwise slow relentless decline to a bedridden state and dementia is usually unavoidable. However, it is not known how precisely leptomeningeal hemosiderin deposits induce progressive neurodegeneration.

Methods: Here we present the first report of a patient with superficial CNS siderosis in whom cerebrospinal fluid biomarkers of brain damage were assessed.

Results: Levels of neurofilament light protein, glial fibrillary acidic protein, total tau protein and, most importantly, hyperphosphorylated tau protein were increased.

Conclusion: The results indicate that in superficial CNS siderosis iron toxicity and oxidative stress lead to hyperphosphorylation of tau and Alzheimer-like neurodegeneration.

\section{P634}

Atrial fibrillation and other risk factors in development of ischaemic stroke G. Pavlic, N. Diaconu, A. Grossu

Institute of Neurology and Neurosurgery (Chisinau, MD); Institute of Cardiology (Chisinau, MD)

Objective: To study the frequency of atrial fibrillation (AF) and cardiovascular diseases in ischemic stroke patients (IS) and to determine the most important factors in its development.

Materials and methods: The study included 735 patients with IS (333 men and 402 women) admitted in the neurology department of a municipal hospital from Chisinau during one year. We have analyzed: anamnesis, presence of cardiovascular diseases, ECG, laboratory data and treatment. Mean subjects age represented $67 \pm 8$ years. Tachyarrhythmia were recorded on ECG in $201(27,4 \%)$ patients, including the chronic and persistent forms of AF in $72,6 \%$, the paroxysmal form-in $23,4 \%$ and atrial flutter-in $5 \%$ of cases. The first study group included subjects suffering from AF $(n=201)$ and the rest of them (534) were included in the second group. The hospital lethality rate was $18 \%$

Results: The mean age of patients with $\mathrm{AF}$ (1st group) was $70,4 \pm 0,64$ and $72 \%$ of them had $>65$ years with slight female preponderance $(58 \%)$. The subjects from the 2nd group were younger, their mean age was $64,3 \pm 0,46$ years ( $p<0,05)$, only $49,8 \%$ of them had $>65$ years, of female gender being $53 \%$.

In general, 695 patients $(94,5 \%)$ suffered from cardiovascular diseases. Ischemic heart disease (IHD) was diagnosed in $65 \%$ from 1st group vs. $44,5 \%$ in the $2 \mathrm{nd}(\mathrm{p}<0,001)$, including myocardial infarction in anamnesis in $22,9 \%$ and $11,8 \%$, respectively ( $<<0,001)$. The rate of arterial hypertension was the same in both groups $(87,5 \%$ vs $89,8 \%)$. Rheumatic valve disease (RVD) was much more frequent in the 1 st group $(9,45 \%$ vs $1,7 \%)$ $\mathrm{p}<0,001$. Past tomboembolic complications were seen with the same fre- 
quency in both groups of subjects $(29,35 \%$ vs $29 \%)$, with slight predominance of repeated tomboembolic events in 1st group ( $6 \%$ vs $2,6 \%) p<0,01$. Clinical signs of heart failure (HF) were more frequent in patients from 1st group $(97,5 \%$ vs. $54,3 \%) p<0,01$. Diabetes mellitus had the same frequency in both studied groups $(29,8 \%$ and $30,5 \%)$. The lethality rate of patients with IS was higher among patients with $\mathrm{AF}(31,3 \%$ and $13, \%) \mathrm{p}<0,001$.

Conclusions: AF was recorded in $27,4 \%$ of patients with ischemic stroke. The hospital lethality rate was higher in this group of patients, being $31,3 \%$. Older age (more than 65 years), female gender, concomitant cardiovascular diseases (IHD, HF, RVD) in association with AF represented the factors determining a higher risk for stroke development.

\section{P635}

Degree of cerebral hypoperfusion in patients with carotid artery stenosis and occlusion

G. Witkowski

Institute of Psychiatry and Neurology (Warsaw, PL)

Background: Perfusion CT is used to visualise the localisation and severity of cerebral infarction in the acute phase of stroke. This method also can be applied to assess the degree of cerebral hypoperfusion in the unilateral stenosis of internal carotid artery (ICA). The aim of this work was to estimate the level of hypoperfusion in groups of patients with symptomatic stenosis, unilateral and bilateral occlusion of ICA.

Methods: Until now 35 patients with TIA were included: 21 with unilateral ICA stenosis, 10 patients with unilateral ICA occlusion, and 4 patients with bilateral ICA occlusion. In all patients perfusion CT was performed together with angio-CT and/or cervical and intracranial Doppler ultrasonography. The degree of ICA stenosis together with collateral circulation was assessed. In group of patients with unilateral occlusion, following causes were present: atherosclerosis (4 patients), occlusion after stent implantation (2 patients) and balloon occlusion of ICA.

Results: In 12 patients about $125(111-148 \%)$ increase in mean transit time (MTT) parameter was observed in the hemisphere on the side of stenosed ICA. Hovewer in 9 patients with carotid artery stenosis no asymmetry in cerebral perfusion was found. No changes in cerebral blood flow $(\mathrm{CBF})$ and cerebral blood volume (CBV) parameter were found. In all patients with ICA occlusion unilateral increase of mean transit time was present. In case of bilateral occlusion MTT disturbances were much more profound and observed in both hemispheres, except area of posterior circulation. Blood flow (CBF) parameter was also decreased, however blood volume (CBV) was normal or slightly increased. In all patients except those with bilateral stenosis, signs of collateral circulation through anterior communicant artery (ACoA) were found.

Conclusion: In majority of patients with stenosis or occlusion of ICA perfusion CT showed abnormalities with different degree - especially in MTT parameter. The most pronounced abnormalities were present in case of bilateral ICA occlusion and absence of collateral circulation though ACoA. The question which remained open is if the degree of perfusion CT abnormalities can help to predict the risk of stroke in patients with carotid artery occlusion. In the next step prospective observation of our patients is planned.

\section{P636}

Helios Neuronet: a new teleneuromedical network of certified stroke units and neuro-intensive care units within the HELIOS hospital group G.W. Ickenstein, U. Müschenborn, J. Klisch, H.P. Vogel, H. W. Kölmel, S. Isenmann

HELIOS General Hospital (Aue, DE); HELIOS General Hospital (Schwelm, DE); HELIOS General Hospital (Erfurt, DE); HELIOS General Hospital (Berlin, DE); HELIOS General Hospital (Wuppertal, DE)

Introduction: Patients with an acute neurological disorder who arrive at the emergency room (ER) of a HELIOS General Hospital without a Neurology Department, now have the opportunity to consult a Stroke Center 24/7 via video-conferencing for further diagnostic and treatment options. The clinical examination is performed together and the "remote" stroke expert has access to the radiological data (CCT, MRI) simultaneously.

Methods: Four Stroke Center of the HELIOS hospital group (Aue, BerlinBuch, Erfurt, Wuppertal) set up a network to offer a teleneuromedical service for patients with acute neurological disorders. The online server of the "HELIOS NEURONET" accumulates data from these patients to ameliorate the quality of patient management. The acute neuromedical service is offered throughout a 24/7 calling system and the on-call responsibility rotates weekly between the Stroke Centers.

Results: In a hospital group the infrastructure of Neuro-ICUs and Stroke Units can be extended by a teleneuromedical service to use this high poten- tial in patient care management. Especially the teleneuromedical network including the specialties neurology, internal medicine, neurosurgery and neuroradiology - leads to a faster and safer quality of care in patient service. New concepts and time saving results will be presented and discussed.

Discussion: The efficiency of teleneuromedical services in acute stroke care in rural areas is confirmed in literature. A teleneuromedical network system with a weekly rotating service between the Stroke Centers has the advantage of an effective management during the on-call-week. The teleneuromedical consultations within the network of NeuroIntensivCare and Stroke Units at the HELIOS hospital group promises an improvement in quality of acute care in rural areas and smaller hospitals without certain specialties.

\section{P637}

Cyclophosphamide-induced reversible posterior leukoencephalopathy syndrome

M.J. Abenza, B. Fuentes, D. Diaz, E. Diez-Tejedor

Hospital Universitario La Paz (Madrid, ES)

Introduction: Reversible posterior leukoencephalopathy syndrome (RPLS) is a clinical radiological syndrome, characterized by acute headache, altered consciousness,seizures, and hypertension. The most frequent causes are hypertensive encephalopathy, eclampsia, and some immunosuppressive therapies. White matter edema can be seen in magnetic resonance imaging (MRI), and resolves in 2 or 3 weeks.

Case report: A 27-year-old male with hypertension and glomerulonephritis caused by Goodpasture syndrome, treated with cyclophosphamide (150 milligrams per day) and prednisone (30 milligrams per day), for the previous month, who developed acute headache, emesis, hypertensive crisis (blood pressure was $205 / 135 \mathrm{mmHg}$ ) and generalized tonic-clonic seizure. Neurological examination only showed agitation and confusion. Biochemical testing showed blood urea nitrogen serum of $149 \mathrm{mg}$ per decilitre and a serum creatine level of $8.50 \mathrm{mg}$ per decilitre. Blood gas analysis showed $\mathrm{pH}$ 6.88. An MRI had edema in the posterior regions of the cerebral hemispheres. Cyclophosphamide treatment was replaced with rituximab. The cranial MRI 3 weeks later showed no disturbances.

Discussion: Only three reported cases of RPLS in patients treated with combination chemotherapy including cyclophosphamide have been reported. Authors could not determine which drug (or combination) was the cause, all cases associated with hypertension too. This could be the first case of cyclophosphamide-induced RPLS.

\section{P638}

Neurological manifestations of the antiphospholipid syndrome: a study from Isfahan, Iran

M. Etemadifar, F. Hajghasemi, A.H. Maghzi, M. Kaji Esfahani

Isfahan University of Medical Sciences (Isfahan, IR); Islamic Azad University of Najafabad (Najafabad, IR)

Background: Antiphospholipid (Hughes) syndrome (APS) is defined by the presence of antiphospholipid antibodies accompanied by venous and/or arterial thrombosis, recurrent fetal loss or thrombocytopenia. APS can be associated with several neurological manifestations including headache, cerebral ischemia, cognitive dysfunction, dementia, psychosis, depression, seizures, chorea and transverse myelopathy.

Objectives: To describe the clinical and demographic characteristics of Persian patients with APS who were referred to Al-Zahra hospital Neurology clinic.

Methods: Patients with the diagnosis of APS who had manifested neurological symptoms who were referred to our neurology clinic were studied. These patients were evaluated and clinical and demographic data were obtained by the neurologist.

Results: Thirty five female patients $(87.5 \%)$ and 5 male patients $(12.5 \%)$ with a mean (SD) age of 34.54 (11.35) for female and 46.6 (17.2) years for male were identified. A female preponderance of 7:1 was observed.APS was associated with Systemic Lupus Erythematosus (SLE) in 9 patients $(22.5 \%)$. Among neurological manifestations cerebrovascular disease were the most frequent events, found in 17 patients (42.5\%) (4 males, 13 females). Other neurological manifestations included: headache which was present in $10 \mathrm{pa}-$ tient $(25 \%)$, epilepsy in $9(22.5 \%)$, Transient Ischemic Attacks (TIA) in 7 $(17.5 \%)$,vertigo in $7(17.5 \%)$, amnesia in $5(12.5 \%)$, loss of consciousness in $3(7.5 \%)$ and Ocular syndromes in 2 patient. Non-neurological manifestation included: fetal loss in 24 patients $(60 \%)$,thrombosis in 12 patient (30\%),pulmonary embolism in 4 patient $(10 \%)$, lividoreticularis in 2 patients (5\%) and Myocardial infarction in one (2.5\%).

Conclusion: Central nervous system involvement is common in patients with APS. Although the mechanism of neurological involvement in patients 
with APS is believed to be thrombotic in origin, a number of other neurological manifestations, including optic atrophy, chronic headache, dementia,chorea and seizures could not be explained solely by hypercoagulability and can have more complex causes. The APS has a wide variety of clinical and immunological manifestations and the ethnic origin as well as environmental and socioeconomic factors can modify the disease expression. In our study Cerebrovascular disease, headache and epilepsy were among the most common neurological manifestations in Persian patients.

\section{P639}

Reversal of MR-DWI lesion in acute stroke after I.V. thrombolysis: a case report

M. Furlan, M.M. Ricca, C. Zat, G. Callari, A. Arrigo, A. Friedmann, C. Serrati Imperia Hospital (Imperia, IT)

Introdution: the aim of thrombolysis is to restore blood flow in the cerebral ischemic tissue. DWI/PWI mismatch1 is the usual standard way to select patient for thrombolysis. We present a case of acute DWI lesion that seems to identify ischemic penumbra and not deat tissue.

Case report: a 66 years old men, previous smoker with hypertension, at 11 a.m. started complaining an acute onset of right hemiparesis and aphasia. At 1 p.m. the patient was hospitalized: the neurological exam showed right sever hemiparesis, right hypoestesia, aphasia and rigth emianopsia suggesting a left MCA stroke. NIHSS was 21. Cerebral MRI revealed a DWI lesion in left MCA territory and MCA(M1) stenosis, confirmed by TCD. RTPA $0.9 \mathrm{mg} / \mathrm{kg} / 60 \mathrm{~min}(10 \%$ bolus $)$ was started at $1.50 \mathrm{p} . \mathrm{m}$. In one hour and half the patient improved and NIHSS was 2. At 3.30 p.m. MRI follow up showed MCA recanalization and disappearance of DWI lesion in left MCA territory.

Conclusion: our case shows that, in acute stroke, DWIlesion can be due to ischemic penumbra and does not correspond to dead tissue. We suggest that MRI perfusion weigted imaging (PWI) with contrast and long post processing elaboration is not necessary to select patients for thrombolisytic treatment in acute stroke. Moreover, patients without PWI/DWI mismatch could be erroneously excluded from thrombolysis, having ischeamic penumbra inside DWI lesion.

\section{References}

1. Schellinger PD MD, Fiebach JB MD, Hacke W MD, PhD (2003) ImagingBased Decision Making in Thrombolytic Therapy for Ischemic Stroke: Present Status. Stroke 34:575-583

2. Sobesky J, et al. (2005) Does the Mismatch Match the Penumbra? Stroke 36:980-985

3. Rivers CS, et al. (2006) Do Acute Diffusion- And Perfusion- Weighted MRI Lesions Identify Final Infarct Volume In Ischemic Stroke? Stroke 37:98-104

\section{P640}

Effects of admission hyperglycaemia on stroke severity and mortality in northeasthern Nigeria

M.M. Watila, Y.W. Nyandaiti, A. Ibrahim

University of Maiduguri Teaching Hospital (Maiduguri, NG)

Background: Hyperglycaemia is associated with worse outcome in both acute ischaemic and haemorrhagic stroke.

Objectives: To determine the prevalence of hyperglycaemia in stroke patients and to determine the relationships between hyperglycaemia with mortality and stroke severity in Subsaharan Africa.

Methods: A prospective study of patients hospitalized with acute stroke at the University of Maiduguri Teaching Hospital, from January 2004 to December $2007(n=93)$. Admission stroke severity using Barthel's ADL index, finger prick blood sugar level where obtained and new diabetes diagnoses were obtained. Hyperglycaemia was defined as admitting random serum BG $>6 \mathrm{mmol}$. The relationship between hyperglycaemia and stroke severity was assessed using the coefficient of correlation.

Results: $50.5 \%$ of patients admitted of all stroke subtypes had a RBS $>6 \mathrm{mmol} .7 .5 \%$ of our patients were diabetic. Hyperglycaemic patients significantly had longer duration of stay $\mathrm{p}=0.015$. Patients with admission hyperglycaemia had worse stroke outcome, with $75 \%$ of our mortalities having a RBS of $>6 \mathrm{mmol}$.

Conclusion: Hyperglycaemia is a common metabolic disorder in all stroke subtypes and is associated with worse clinical outcome. Control of blood sugar is important in acute stroke if patients are to have a favorable outcome.

\section{P641}

Common carotid intima media thickness in brain infarction versus intracranial haemorrhage

M. Saadatnia, F. Fatehi, A. Mousavi, A. Rezaee

Isfahan University of Medical Science (Isfahan, IR)

Introduction: The association of common carotid intima media thickness (CCA-IMT) with stroke has been well established. The aim of this study was to investigate the diagnostic ability of vascular risk factors, including CCAIMT, to distinguish between ischemic stroke types and intracerebral hemorrhage.

Material and methods: Overall, 313 consecutive stroke patients and 94 control subjects hospitalized in neurology departments of two educational hospitals of Isfahan University of Medical Sciences from January 2006 to September 2007 were included. According to TOAST criteria ischemic strokes are classified into the following groups: large artery atherosclerotic stroke (LAA), cardioembolic stroke (CE), lacunars infract (LI), intracrania hemorrhage (ICH). IMT was measured by Carotid duplex for all patients.

Results: Mean (SD) of CCA-IMT in LAA, CE, LI, ICH and control groups were $1.08(0.23), 0.96(0.20), 1.02(0.20), 0.74(0.22)$, and $0.80(0.14)$ respectively. Mean (SD) of CCA-IMT in brain infarct patients (LAA, CE, LI) was 1.04 (0.22). In one-way ANOVA, results revealed that CCA-IMT could differentiate BI from control and ICH group, as well as LAA and LI from control group. There was not significant difference between ICH and control group as well as LAA and LI group.

For each increment of $0.1 \mathrm{~mm}$ in CCA-IMT the probability of suffering from BI versus ICH increased by $37.6 \%$ (95\% CI: $28.15 \%-48.94 \%$, $\mathrm{p}=0.000)$.

Conclusion: Increased CCA-IMT values are a factor favoring BI over ICH patients. The measurement of CCA-IMT may be a useful non-invasive diagnostic tool for the risk assessment of BI with respect to ICH in stroke patients.

\section{P642}

Cardiac arrhythmia in acute stroke Egyptian patients without known cardiac diseases

N. Kitchener, N. Farag, B.E.Z. Fouad

General Organization for Teaching Hosp. (Cairo, EG); Ain Shams Univerity (Cairo, EG); National Heart Institute (Cairo, EG)

Objective: Cardiac arrhythmia and Electrocardiographic changes are well known to appear with acute stroke. The physiological basis for the arrhythmias commonly observed after a stroke is not well understood. So this work aimed to investigate if acute stroke cause cardiac arrhythmia, in the form of QT dispersion (QTd), and if this arrhythmia could be related to lesion localization and/or size.

Method: The study group consisted of 54(31 men and 23 women) patients who had an acute stroke and no history or signs of cardiovascular disease. An age-matched control group ( $n=28,15$ men and 13 women,) free of cardiovascular disease was also included. Simultaneous 12-lead electrocardiograms (ECGs) were recorded within the first 24 hours (24h-ECG) and after 72 hours (72h-ECG) from stroke onset. QT dispersion was assessed both automatically and manually with assessors blinded to the clinical data.

Results: Of the stroke patients, 25 had right-sided and 29 had left-sided lesions. There were 43 ischemic and 11 hemorrhagic strokes. QT dispersion, corrected QTd, and automated QTd were significantly increased in the $24 \mathrm{~h}$ ECGcompared with the 72h-ECG (mean value 63 and range, 29-84 milliseconds versus mean 38 and range, $0-76$ milliseconds, $\mathrm{P}<0.005 ; 56 \pm 19$ versus $36 \pm 21$ milliseconds, $\mathrm{P}<0.001$; and 52 with range, $18-89$ versus 33 with range, $0-83$ milliseconds, $\mathrm{P}<0.005$, respectively). However, QTd in the $72 \mathrm{~h}$ ECG was similar to QTd in the control group. While in the 24h-ECG corrected QTd was significantly greater in patients with large infarcts and large hemorrhages (mean $\pm \mathrm{SD}=74 \pm 19$ versus $53 \pm 21$ milliseconds, $\mathrm{P}<0.05$ ), in the 72h-ECG corrected QTd was greater in patients with right versus left sided lesions ( $52 \pm 17$ versus $24 \pm 18$ milliseconds, $\mathrm{P}<0.05$ ).

Conclusion: QT dispersion is increased in the first 24 hours in patients with acute stroke and no cardiovascular disease compared with the control group. Although this finding seems to be related to the size of the lesion rather than to the localization or type of stroke in the first 24 hours, after 72 hours specific lesion localization influences the QTd.

\section{P643}

Paroxysmal dysarthria and ataxia

S. Bek, O. Arslan, G. Genc, U. Ulas, Z. Odabasi

Gulhane Military Medical Academy (Ankara, TR)

Introduction: Paroxysmal dysarthria and ataxia episodes are typically characterized by sudden onset, brief duration and frequent repetition. There are 
only two case reports in the literature due to vascular events. We report a case with PDA and outline that the symptomatology occurs due to parietal diaschisis.

Case History: A 37 year old right handed male was admitted to the hospital with diplopia and right hemihypoesthesia. Neurologic examination showed diplopia due to oculomotor nerve palsy, Babinski sign and hyperreflexia on the right without any paresis. Cranial magnetic resonance images (MRI) showed a high intensity area in the left midbrain on T2 and diffusion weighted images. MR angiography did not indicate any apparent stenosis in the major vessels. Together with the neurologic findings and MRI signs, midbrain infarction was diagnosed and we administered aspirin $300 \mathrm{mg} /$ day. Two weeks after onset he had no residual symptoms. In his monthly control examination he complained about paroxysmal attacks of slurred speech and incoordination of his right arm. The paroxysms occured with increasing frequency up to 10 times a day and lasted 20 seconds at most. During an episode, ataxia of the arm on the right and dysartria were observed. Single photon emission computerized tomography (SPECT) study was performed while the patient was having frequent paroxysmal attacks and showed marked hypoperfusion in the left parietal lobe. In 4 weeks his attacks ceased. SPECT was normal showing no hypoperfusion in the left parietal lobe.

Discussion: Stroke produces an area of focal damage and distant areas of reduced blood flow and metabolism, termed diaschisis. Since vast number of pathways interconnect brainstem, diencephalic and telencephalic neuronal aggregates, a brain stem vascular lesion might be expected to exert transynaptic effects at many distant loci as can be seen in the case we presented. We hypothesize that PDA and parietal diaschisis does not occur simultaneously but hypoperfusion might be the cause and PDA might be the outcome.

\section{P644}

Susac's syndrome

S. Bek, S. Demirkaya, B. Firtina, G. Genc, E. Eroglu, Z. Odabasi

Gulhane Military Medical Academy (Ankara, TR)

Introduction: Since first reported in 1979, there have been over a hundred cases of SUSAC's syndrome (SS) reported. SS consists of the clinical triad of encephalopathy, branch retinal artery occlusion, and hearing loss. This syndrome has protean manifestations and is often misdiagnosed. We present a case of SS, with typical clinical triad and imaging results, whom was reffered to our department with a diagnosis of multiple sclerosis.

Case report: A 36-year-old woman presented with headache, vertigo, ataxia, vomiting, visual impairment of the right eye, tinnitus and hearing loss on the right side, and attention fluctuating. Neurological examination revealed confusion, upper temporal quadronopsia on the right side, hearing loss on the right side, vertical nystagmus on upgaze, horizontal nystagmus on right gaze, Romberg sign $(+)$. Routine laboratory studies were normal except mild elevated erythrocyte sedimentation rate, Factor VIII, Von Willebrand factor antigen. Cerebrospinal fluid examination showed mild protein level elevation. Her cerebral magnetic resonance imaging (MRI) revealed multifocal supratentorial white matter lesions, including the corpus callosum. The lesions of the corpus callosum were typically small and involved the central fibers with relatively sparing of the periphery. The riddled callosum consisted of small central holes. There were also deep gray matter lesions. Fluorescein angiography of the right eye showed typical temporal arterial occlusion. She was administered $1000 \mathrm{mg}$ /day methylprednisolone IV for 5 days followed by deflazocort $60 \mathrm{mg} /$ day PO, aspirin $300 \mathrm{mg} /$ day PO, nimodipine $90 \mathrm{mg} /$ day PO and hyperbaric oxygen treatment for 10 days. She was discharged with clinical improvement after 15 days with $6 \mathrm{mg} / \mathrm{week}$ tapering dose for steroid. 15 days after discharge she was hospitalised with relapse. Her felazocort dosage was increased to $60 \mathrm{mg} /$ day and she was administered IVIg $0.4 \mathrm{mg} / \mathrm{kg} /$ day for 5 days. She was discharged with significant clinical improvement on 15th day of her second hospitalisation. Monthly IVIg replacements are planned.

Discussion: With typical clinical triad and MR imaging results, SS was established as a diagnosis. Tapering steroid treatment in the early phase resulted with relapse. We suggest that steroid treatment should not be tapered before complete remission and IVIg administration should be applied as soon as the diagnosis is established.
P645

The coexistence of large and small vessel atherosclerosis in stroke: a comparative study based on risk factors

S. Sahin, A. Eren, S. Ayalp, B. Arpaci, S. Karsidag

Maltepe University (Istanbul, TR); Uzunkopru Government Hospital (Edirne, TR); Bakirkoy Mental Health and Neurological Diseases Education and Research Hospital (Istanbul, TR)

Background: Atherosclerosis is the most common cause of ischemia in the periphery, coronary and central nervous system. Ankle/Brachial Index (ABI) is the most important diagnostic test, which indicated the degree of occlusion in peripheral vascular diseases. This study was planned because there were limited number of studies comparing basically intracranial, extracranial (carotid vessels), peripheral vessels and coronary arteries being influenced with risk factors, carotid stenosis and ABI data in stroke case.

Methods: Sixty patients with acute ischemic stroke were investigated prospectively for presence of atherosclerotic risk factors and markers, like hypertension, diabetes mellitus, hyperlipidemia, smoking, ischemic heart disease and claudicatio intermittens, in their medical histories. Carotid atherosclerosis was diagnosed by doppler ultrasonography, whereas intracranial atherosclerosis (leukoaraiosis) was diagnosed by cerebral computed tomography; coronary atherosclerosis was diagnosed by electrocardiography (ECG), and peripheral vessel atherosclerosis was diagnosed by ABI. According to degrees of carotid artery stenosis, patients were classified into three groups: mild, moderate and severe.

Results: Although there was no significant differences in three patient groups, leukoaraiosis was significantly high in the moderate and severe stenosis groups $(\mathrm{p}=0.008)$. Mean age, smoking, ischemic heart disease and peripheral vessel atherosclerosis were confirmed significantly high in oneway analysis performed on severely stenotic group $(p=0.02, p=0.001$, $\mathrm{p}=0.05, \mathrm{p}=0.02$, respectively). There was a high degree of negative correlation between carotid artery stenosis and ABI $(r=0.49)$.

Conclusion: We detected a strong concomitance between carotid artery stenosis and smoking, coronary artery disease, cerebral atherosclerosis in cases with stroke. Also we detected a direct correlation between carotid stenosis and presence of leukoaraiosis, whereas an inverse correlation between carotid stenosis and ABI. These results indicated that large and small vessels might manifest parallel alterations on the same risk factor basis.

\section{P646}

The correlation of ABI values and the risk for silent strokes in 120 patients aged over 60

S.M. Deme, P.D. Nanu, C.D. Jianu, S. Kory-Calomfirescu

West "Vasile Goldis" University of Arad (Arad, RO); Victor Babes University of Medicine (Timisoara, RO); Iuliu Hatieganu University of Medicine (ClujNapoca, RO)

Introduction: Lacunary infarcts and leukoaraiosis are frequently found on CTs and MRIs in apparently healthy elderly persons. The risk for stroke increases 2- to 10-times in patients with one or more silent infarcts. Risk factors for silent strokes are thought to be comparable to those for stroke, and therefore, patients with cardiovascular disease may be at high risk for silent strokes. It is known that an impaired ankle-brachial index (ABI) relates to a higher risk for ischemic stroke.

Objective: The objective of this study is to investigate the prevalence PAD in patients with silent strokes diagnosed incidentally for other cerebral pathology.

Material and method: The study was conducted in the Neurology Clinic of Arad, during 2006-2007. There were included a series of 120, with the age over 60, that were examined in ER by CT scan or MRI for another reason than the susceptibility to have an ischemic stroke. The patients had no known history of PAD, or history of cardiovascular or cerebrovascular.

Results: Of these 80 patients, mostly men (70\%) with an average age of $66 \pm 2.5 \mathrm{yrs}, 35 \%$ presented on the CT scan image or MRI silent strokes, such as lacunary infarctions $(27 \%)$ or leukoaraiosis $(45 \%)$, and both the rest of the patients. In these patients with silent strokes was determined the ABI, as well as those that had no cerebral vascular disease. In patients with silent strokes, 36 patients ( $85 \%$ of 42 ) had an ABI lower than 0.9 , and no history of covert PAD. On the other side, the patients with no vascular disease had in a proportion of $92 \%$ of the cases a normal ABI.

Discussions: Silent strokes and peripheral artery disease share common risk factors. The patients accidentally diagnosed with silent infarctions, most often the cause of the disease is a diffuse small vessel disease, rather than large atherosclerotic vessel disease. When other cardiovascular risk factors are considered, studies show that the correlation between the ABI and the risk or the incidence for silent strokes, or even stroke, is severely modified, the ABI values not playing anymore such an important prediction role.

Conclusion: An altered $\mathrm{ABI}$ in patients with no history of cardiovascular 
(including PAD), or cerebrovascular disease correlates with cerebral silent ischemic vascular disease. ABI values may be in the future recognized as a risk factor for silent stroke, knowing that peripheral or coronary vessel disease preceeds with at least one decade brain's vessel atherosclerotic disease.

\section{P647}

Serial follow-up of MR spectroscopy in reversible posterior leukoencephalopathy syndrome

S-Y. Lee, N-H. Kim, H-J. Baek, S.H. Kim, S.H. Lee, S.S. Kim

Kangwon National University (Chuncheon, KR); Dongguk University (Koyang, KR)

Objectives: To describe the MR spectroscopy (MRS) findings in each phase of reversible posterior leukoencephalopathy syndrome (RPLS) and evaluate whether MRS is helpful to 1) differentiating from other conditions, 2) verifing the pathophysiology, and 3) predicting outcome.

Methods: We performed MRS, diffusion-weighted imaging, and conventional MR during acute, subacute and resolving phases of two episodes in a patient with hypertensive encephalopathy

Results: There were multifocal edematous lesions in bilateral frontal, left temporooccipital and bilateral cerebellum at the first episode, and in bilateral cerebellum and left pons at the second episode. ADC values of the lesions were high. Choline was normal at acute phase and elevated at subacute period. NAA was low even after clinical recovery and disappearance of the lesion on conventional MR, since acute phase. Lactate peak was not detected.

Conclusion: MRS helps differentiate RPLS from demyelinating inflammatory disease, and mitochondrial encephalopathy, but not from viral encephalitis. NAA decrease doesn't predict poor outcome.

\section{Higher function disorders}

\section{P648}

Contribution of visual and somatosensory signals to the perception of the vertical direction in right brain damaged patients

B. Braem, A. Saj, J. Honoré, Y. Coello, M. Rousseaux

CHRU (Lille, FR); Université de Lille (Lille, FR)

Objectives: Physical properties of objects are perceived with a precision that depends on the sensory system at work. When a same property is evaluated through several sensory systems, their relative weights can be computed using a maximum likelihood estimation (MLE model) which takes into account the intrinsic variability of each system. In the present study, we used this model in order to investigate the change induced by hemispheric lesions in the relative weights of visual and somatosensory signals in the estimation of vertical direction. We also assessed the variation of sensory integration resulting from modulation in body positioning.

Methods: Patients with a relatively recent right-hemisphere stroke were recruited. Three were neglect and hemianoptic $(\mathrm{N}+\mathrm{H}+)$, five were neglect without hemianopsia $(\mathrm{N}+\mathrm{H}-)$, and nine were neither neglect nor hemianoptic (N-H-). They were compared with eight healthy controls (C). The participants had to adjust a vertical rod to the direction of gravity, in darkness, and in seated or supine position. In the visual condition, the luminous rod was moved by the experimenter according to the instructions of the participant. In the haptic condition, the non-visible rod was moved by the participant. In the combined condition, the luminous rod was moved by the participant. We measured the error relative to the gravity direction and the relative weight of visual and somatosensory inputs.

Results: A counter-clockwise deviation was found in $\mathrm{N}+\mathrm{H}-\left(-3.9^{\circ}\right)$ and $\mathrm{N}+\mathrm{H}+\left(-3.7^{\circ}\right)$ patients, as compared with $\mathrm{N}-\mathrm{H}-\left(-1.5^{\circ}\right)$ and $\mathrm{C}\left(-0.9^{\circ}\right)$ participants. The error was slightly more severe in the sitting position. The relative weights differed between groups, with a clear superiority of visual inputs in comparison with somatosensory inputs for $\mathrm{C}$ subjects $(0.91 \%)$, which was reduced in $\mathrm{N}-\mathrm{H}-(0.73 \%), \mathrm{N}+\mathrm{H}-(0.77 \%)$ and especially in $\mathrm{N}+\mathrm{H}+$ $(0.59 \%)$ patients. Body positioning had no influence in C subjects, but supine position increased the relative weight of visual inputs in the patient groups.

Conclusion: Patients with right hemispheric lesions relied less on visual inputs than normal subjects do for estimating the direction of gravity. This reduction was amplified by both spatial neglect and hemianopsia, and by sitting position. We suggest that deficit in the integration of visual and vestibular information due to the lesion contributes to the observed effects.
P649

Primary progressive aphasia without dementia in a Chinese male

J.H. Chen, N. Wei, Y. Zhang

Beijing Mining Aggregation General Hospital (Beijing, CN); Beijing Tiantan Hospital (Beijing, CN)

Primary Progressive Aphasia (PPA) is a syndrome of declining language function disorders, and there were many cases of PPA without dementia which have been reported before, otherwise, there are still uncertain questions to be resolved. Only a few cases have been reported in China, We do not know the characteristics of Chinese PPA.

The patient was a 56 years old, right-handeded male, He lived in Shijiazhuang city and was a businessman. He was admitted by the department of Neurology, Beijing Tiantan Hospital on 20th March 2004. His major complaint was progressive deterioration of his language skills for the past three years. He reported those three years ago, he could not name daily objects. He could describe the functions or the attribute of the objects. He was able to select the objects if extra cues were provided. Two years ago, his auditory comprehension skills were still normal. Starting last year, his language skills reduced slightly. He also reported his personality changed, such as becoming more impatient and more irritable. Upon admission, the patient was unable to run his business. He expressed his needs by writing words down or by his body language or gestures. We used the Aphasia Battery of China to evaluate this patient's oral fluency and used Western Aphasia Battery to determine the aphasia type, and the results was that he was Wernicke's aphasia with fluent oral language. His aphasia severity was at the level four as classified by Boston Diagnostic Aphasia Examination. Additionally, the Clincal Scale for Memory was the diagnosis tool used to evaluate the patient's memory, and he had no memory disorders. Finally, the Mini Mental State Examination and Weschsler Adult Intelligence Scale with Chinese modifications were used to evaluate patient's intelligence, and he was intelligent, and MRI indicated that this patient had atrophy in the left frontal lobe and left temporal area.

PPA is a disease with language disorders as its dominant clinical manifestation. The clinical symptoms, the neuropsychological and neuroimaging findings all support the diagnosis of PPA of our case.

State Key Laboratory of Cognitive Neuroscience and Learning Open Project Grant and Beijing Superiority talented fund (20071D0300400078)

\section{P650}

Dysfunction of pentose phosphate shunt contributes to perimidline regionally selective damage of brain in thiamine deficiency

C. Zhong, Y. Zhao, X. Pan, Y. Wang, Z. Hong

Zhongshan Hospital, Fudan University (Shanghai, CN); Shanghai Medical College, Fudan University (Shanghai, CN); Huashan Hospital, Fudan University (Shanghai, $\mathrm{CN}$ )

Objective: To explore the role of transketolase (TK) and pentose phosphate shunt in pathogenesis of perimidline regionally selective damage of brain in thiamine deficiency (TD) and Wernicke encephalopathy.

Methods: TD mouse model was produced by thiamine-depleted diet. Pyruvate dehydrogenase (PDH), alpha ketoglutarate dehydrogenase (KGDH), TK activities and NADP+, NADPH content of brain were determined. TK mRNA expression was investigated through in situ hybrid. The effect of TK siRNA on propagation and differentiation of hippocampal neural stem cell was studied in vitro.

Result: Compared to normal control, the activities of brain PDH of TD9, TD14, and TD21 were $99.63 \pm 1.88 \%, 95.12 \pm 1.22 \%$, and $95.07 \pm 0.76 \%$ respectively and no statistical difference was found $(\mathrm{P}>0.05)$. The activities of brain KGDH of TD9, TD14, and TD21 were $99.20 \pm 1.91 \%, 91.45 \pm 1.24 \%$, and $88.34 \pm 1.53 \%$ respectively. There was a statistical difference between normal control and TD21 $(\mathrm{P}<0.05)$. There was no statistical difference between normal control and TD9 and TD14 (P>0.05). The activities of brain TK of normal control, TD9, and TD14 were $10.90 \pm 0.67,10.46 \pm 0.94$, and $9.19 \pm 1.84 \mathrm{U} / \mathrm{g}$ protein. There was a statistical difference between TD14 and normal control $(\mathrm{P}<0.05)$. The content of brain NADP+ of normal control, TD9, and TD14 was $20.22 \pm 3.67,17.89 \pm 3.54$, and $18.45 \pm 2.54 \mathrm{pMol} / \mathrm{L}$ respectively and no significant difference was found among them. The content of brain NADPH of normal control, TD9, and TD14 was $9.37 \pm 1.57$, $7.17 \pm 0.73$, and $7.43 \pm 1.11 \mathrm{pMol} / \mathrm{L}$ and the rate of NADP+ and NADPH (NADP/NADPH) was $2.17 \pm 0.21,2.49 \pm 0.34$, and $2.49 \pm 0.10$. The content of NADPH and the rate of NADP and NADPH of TD9 and TD14 decreased significantly compared to normal control $(\mathrm{P}<0.05)$. The study of in situ hybrid showed that TK mRNA was mainly expressed in perimidline regions of brain such as dentate gyrus of hippocampus and medial habenular nucleus. TK siRNA significantly impaired the function of proliferation and differentiation of hippocampal stem cell in vitro. 
Conclusion: The dysfunction of pentose phosphate shunt due to decreased activity of transketolase contributes to the pathogenesis of perimidline regionally selective damage of brain in TD and Wernicke encephalopathy.

This study was supported by the funding from Shanghai Science \& Technology Committee(064119536 and 07DJ14005)

P651

Thalamic Gerstmann syndrome or diaschisis?

E.C. Rosca, M. Simu

University of Medicine and Pharmacy (Timisoara, RO)

Objectives: The present paper analyses the neuropsychological impairments induced by a left thalamic hemorrhage in a 65 years old right-handed patient, with 8 years of formal education.

Methods: An extensive neuropsychological examination was performed 12 days after the stroke onset.

Results: The patient presented a mild motor aphasia, constructional apraxia, low visuo-spatial reasoning, agraphia, severe acalculia (consisting in impaired counting, transcoding, naming of arithmetical signs, number comparison, simple arithmetic operations, mental and written calculations, estimation of the result of an operation, fraction comparisons and calculations, digit span, positioning numbers on a scale, general and personal numerical knowledge and time estimation), finger agnosia and right-left confusion.

Conclusion: The pattern of acalculia found in our patient does not correspond to the current cognitive models of number processing which postulate that left thalamic acalculia should be characterized by dissociations with impaired rote arithmetical facts but spared semantic numerical knowledge. Still, the symptoms of Gerstmann syndrome and the impaired semantic number processing might be explained by diaschisis, with implications in the patient's recuperation.

P652

Clinical correlations in acute confusional states

E.I. Davidescu, S.M. Nica, I. Buraga, G. Mihailescu

University of Medicine and Pharmacy (Bucharest, RO)

Objectives: To assess the clinical correlations in an acute confusional state.

Background: Acute confusional state is a transient disorder of cognition and attention accompanied by disturbances of the sleep-wake cycle and psychomotor behavior. In our country, patients with confusional states usually are refered to emergency room within neurology departments.Delirium is a syndrome rather than a disease and its pathophysiology depends largely on the etiology. The symptoms can be attributed to numerous causes, seldom being at interference of specialties.

Methods: We followed up patients with this syndrome, hospitalized in our clinic, between 2006 and 2007. From more than 6500 patients treated in our department in one year, only 60 patients had this diagnosis at admittance, 34 women and 26 men, aged between 25 and 96 years, with a mean age of 68.8 years. More than $60 \%$ of women and almost $40 \%$ of men were aged between 70-79 years old. We tried to undergo investigations to discover the underlying pathology. Most frequent pathological antecedents were arterial hypertension, ischemic heart disease, stroke, atrial fibrillation, trauma and diabetes mellitus. Only $16.66 \%$ patients didn't have any antecedents. All patients performed clinical and neurological examination, blood tests, ECG, chest X-ray, EEG and CT scan. Few (almost 7\%) of them had a cranial MRI and $(20 \%)$ Doppler examination, and some $(8.33 \%)$ had a spinal tap.

Results: The main findings at presentation were:confusion, desorientation, memory loss, headache, and less common, atention loss, agitation, etc. The mean time of hospitalization was 6.43 days and more than $10 \%$ of the patients were hospitalized longer than 2 weeks. Most of the patients $(73.33 \%)$ were from urban zones where accesibility to emergency departments is higher. A positive diagnosis was made in 54 patients and in other 6 of them the underlying disease was a psychiatric one.

Conclusions: We found out that the most frequent underlying pathology in patients confusional state reffered to our neurology clinic was hypertension, followed by atherosclerosis with all its complications as ischemic heart disease, multiple strokes and dementia, and cranial trauma, in association with alcohol abuse, the latter especially in younger patients without pathological antecedents.Promptly treatment of hypertension and association of antiedematous drugs resolved the majority of cases with a remision of symptoms within $24-48$ hours from treatment onset.

\section{P653}

Assessment of cognition in Nigerian chronic alcoholics

F. Salawu, A. Danburam, A. Olokoba, M. Wakil

Federal Medical Centre (Yola, NG); University of Ilorin Teaching Hospital (Ilorin, NG); Federal Neuropsychiatry Hospital (Maiduguri, NG)

Intellectual impairment is a devastating complication of alcohol abuse. The consequences of intellectual impairment are far-reaching, particularly for young people in their most reproductive years.

Objectives: To determine whether there are differences in cognition between Nigerian chronic alcoholics and non-alcoholic controls.

Method: The 4-item CAGE screening instrument for alcohol abuse was administered to alcoholics aged 18 and over interviewed at random at selected beer parlours in the municipal. Any alcoholic who scored two and above in the screening instrument was included in the study. Seventy subjects fulfilled the inclusion criteria. Exclusion criteria included a current or past psychiatric diagnosis, illicit drugs that could affect the central nervous system e.g. amphetamines, cannabis; a history of severe head injury with loss of consciousness, occupational exposure to organic solvents e.g. paint and glue; and individuals suspected to have hepatic or Wernicke's encephalopathy. Randomly selected non-drinking controls from the general population were matched for sex, age to within three years of the most recently studied alcoholic. Seventy non-intoxicated chronic alcoholics and 70 non-alcoholic controls were compared using the Community Screening Instrument for Dementia (CSI-D) to assess language, memory, registration, attention and calculation, recall, praxis and orientation. The alcoholics were classified in two sub-groups based on their consumption of ethanol per occasion on five or more days in the past 30 days: light drinkers $(<5$ alcoholic drinks) $(n=45)$ and heavy drinkers ( $\geq 5$ alcoholic drinks) $(n=25)$.

Results: Two different criteria for impairment were used, one generous and the other more conservative. The generous one denotes overall impairment if a score on at least two domains of the CSI-D battery is one standard deviation below the norm, while the conservative one requires that a score on at least one domain is two or more standard deviations below the norm. The two criteria for abnormality scores yielded markedly different estimates of impairment. Overall, non-alcoholics performed better than alcoholics in most of the entire neuropsychological measures. However, light drinker performed better than heavy drinkers in one function domain (orientation to place $\mathrm{p}<0.0001$ )

Conclusion: Cognitive decline occur in un-intoxicated Nigerian chronic alcoholics and appear to be independent of quantity of ethanol consumed.

Financial support received from Management of University of Maiduguri Teaching Hospital for dissertation submitted to the West African College of Pyhsicians

\section{P654}

Performance of normal Nigerian students on the community screening instrument for dementia

F. Salawu, S. Bwala, M. Wakil, A. Danburam, N. Aqil, A. Olokoba, L. Ahmed Bello

Federal Medical Centre (Yola, NG); University of Maiduguri (Maiduguri, NG); Federal Neuropsychiatry Hospital (Maiduguri, NG); University of Maiduguri Teaching Hospital (Maiduguri, NG); University of Ilorin Teaching Hospital (Ilorin, NG)

Objective: The Community Screening Instrument for Dementia (CSI-D) has been and validated in many populations and its usefulness in Nigerians has been assessed in elderly uneducated subjects. Hence, this cross-sectional study sets out to define the performance of normal Nigerian students on the CSI-D to make its use more relevant.

Methods: One hundred and forty three normal students (62 females and 81 males) attending a conventional secondary school in Maiduguri metropolitan Nigeria were randomly selected and administered the CSI-D under standardized condition viz; in a well lit quiet room in the morning. The administration of the test battery took approximately 20 minutes. Cognition was examined by gender. Subjects with a history of mental impairment, epilepsy, head injury, drug and alcohol abuse were excluded. It is comprised of the following function domains: language expression (naming, definition, repetition and fluency), registration, attention and calculation, recall, orientation to place and time, language comprehension (motor response), memory recall, memory and praxis. To estimate the degree of dysfunction in the study population, we choose a definition of neuropsychological impairment that was a function of each subject's cognitive test scores. Impairment was operationally defined by at least one score two standard deviations below the normative mean for a given test or two or more test scores at least one standard deviation below the mean. Group differences were tested by independent t-test for performance on the CSI-D and Chi square for categorical variables. 
Results: If we consider as cut-off values the mean score for each test less 1 standard deviation for comparison, 12 males and 11 females were classified as neuropsychologically impaired. On the other hand, if we take as normal the mean \pm 2 standard deviation for each test, 32 males and 27 females were impaired. The poorest performance on the various domains of the CSI$\mathrm{D}$ was with language expression (naming), while full scores were obtained in registration. As expected education as expressed in years of school attendance was positively correlated with higher scoring in all function domain and total CSI-D test scores. There was also correlation between age and CSID function domains.

Conclusion: The CSI-D is discriminating for Nigerian students with high and low levels of education and literacy and for subjects from developed and less developed communities.

\section{P655}

Implication of neural network model for prediction of memory deficit: a comparison of neural network model with conventional statistical models G. Saraf, M. Saraf

SAS Institute of Information Technology \& Research (Chandigarh, IN); PGIMER (Chandigarh, IN)

Objectives: Neural network, an emerging approach of cognition science, is thought to play a substantial contribution in understanding of neural circuit and the related neurobiological phenomena such as memory formation. This study was designed to develop the artificial neural network model and compare it with established statistical tools.

Methods: We used the data set obtained after training of 320 animals using Morris water maze model. The mouse was trained many times every day. The normal mouse showed a gradual learning process while amnesic mouse did not learn the given task even after repetitive training. We used this data set for training of model and developed a neural network model using the multi-layer perceptron and the radial basis function neural set. The validity of neural network was also tested with known established data to predict the state of memory. The precision of neural network model compared logistic regression and general linear model.

Results: We computed the accuracy and sensitivity of all models after analysis of data. We found that the accuracy and sensitivity of output were tend to higher in multi-layer perceptron and the radial basis function neural set than general linear model logistic regression and general linear model. The neural network was able to sort out amnesic mouse among normal mice population and could be able to differentiate amnesic mouse out of normal mouse.

Conclusion: The results suggest that neural networks could be a better solution for complex, non-linear medical decision support system than conventional statistical techniques such as logistic regression and general linear model.

\section{P656}

A possible link between cholinergic and GABAergic system in induction of amnesia

M. Saraf, S. Prabhakar, A. Anand

P.G.I.M.E.R. (Chandigarh, IN)

Objectives: The cholinergic system is implicated in pathology of Alzheimer's disease. Benzodiazepines are known to produce amnesia by involvement of GABAergic system. In order to combined with our earlier report of the contribution of Bz/GABA (Benzodiazepine-gammaaminobutyric acid)-ergic system in amnesia, this study was designed to investigate the link between cholinergic and Bz-GABAergic system in amnesia.

Methods: We employed Morris water maze scale to test the amnesic effect of scopolamine (an acetylcholine receptor antagonist) and diazepam (a benzodiazepine receptor agonist). Rotarod test was conducted to screen muscle coordination activity of mice. In order to discern the mechanism of these amnesic agents, we compared the effect of these amnesic agents on downstream molecules of long term potentiation such as cAMP, phosphodiesterase, protein kinase A, protein kinase $\mathrm{C}$, inducible nitric oxide (iNOS), MAPkinase, phosphorylated cAMP response element binding protein (pCREB), total CREB, nitrite, nitrate, total nitrite. Westernblotting and immunohistochemistry were performed for evaluation of expression of $\mathrm{pCREB}$ and iNOS.

Results: Behavioural results demonstrated that scopolamine $(0.1 \mathrm{mg} / \mathrm{kg}$ ip) and diazepam $(1.75 \mathrm{mg} / \mathrm{kg}$ ip) both produced anterograde amnesia in Morris water task. The molecular studies revealed that scopolamine significantly downregulated protein kinase $\mathrm{C}$, phosphodiesterase, iNOS, total nitrite and iNOS without altering cAMP, protein kinase A, MAP kinase, $\mathrm{PCREB}$ and CREB. Calmodulin level was partially suppressed by scopolamine. Moreover, diazepam up-regulated MAP kinase, pCREB and iNOS, while it re- duced nitrite, nitrate, total nitrite, CREB expression, phosphodiesterase, cAMP without affecting calmodulin levels.

Conclusion: These observations suggest that scopolamine induced blockade of an acetylcholine receptors suppress protein kinase $\mathrm{C}$ and calmodulin activity, which could possibly inhibit the excitatory cholinergic system, interfere long term potention and consequently produce amnesia in mice. The modulation of kinases and retrograde messenger can not be ruled out for scopolamine induced amnesia. On the other hand, diazepam induced up-regulation of MAPkinase, pCREB and iNOS could be a consequence of benzodiazepine mediated activation of Bz-GABAergic system. These findings suggests the possible counter regulatory role of cholinergic and GABAergic on memory impairment.

Department of Biotechnology (DBT) New Delhi Research Grant

\section{P657}

Slowly progressive Foix-Chavany-Marie syndrome

M. Otsuki, Y. Nakagawa, A. Ogata, H. Houzen, T. Ozawa, I. Takahashi, K. Sakushima, I. Yabe, M. Nishizawa, H. Sasaki

Health Sciences University of Hokkaido (Sapporo, JP); Hokkaido Neurosurgical Memorial Hospital (Sapporo, JP); Obihiro Kosei Hospital (Obihiro, JP); Niigata University Brain Research Institute (Niigata, JP); Hokkaido University (Sapporo, JP)

Objectives: To investigate the neurological and neuropsychological features of the patients with slowly progressive difficulty in speech output and classify them into sub-groups which could predict the subsequent clinical course.

Methods: Ten right-handed patients with progressive difficulty in speech output ( 7 male, 3 female) without past history of neurological disease participated in this study. Their ages ranged from 55 to 78 years (mean age: 61.3). Various neuropsychological examinations were administered to the patients: digit span forward and backward from the Wechsler Adult Intelligence Scale- Revised, Western Aphasia Battery, Raven Colored Progressive Matrices, Wechsler Memory Scale-Revised, Trail Making Test. The regional perfusion scans were studied using Single- Photon Emission Computed Tomography (SPECT). Each patient also had Magnetic Resonance Imaging (MRI) scans. The follow up studies were done 2 to 5 years after initial evaluation.

Results: The patients could be classified into three sub-groups in terms of clinical findings. Three patients developed initially difficulty in word findings, regardless of the presence of anarthria with aggravation to almost mute within several years after onset. Their general intelligence were preserved within several years, however, they become to exhibit frontal symptoms such as stereotyped behavior at least in five years after the onset. Three patients showed progressive pure anarthria without word finding difficulty, or orofacial apraxia (OFA) or mental deterioration. The other four patients initially manifested pure anarthria with OFA, and shortly followed by dysphagia and suprabulbar palsy. Their symptoms showed automatic voluntary dissociation which become the clinical hallmarks of Foix- Chavany- Marie syndrome (FCMS). Bain MRI of all the patient revealed cortical atrophy predominantly in the left or bilateral frontal regions, and the SPECT images of all the patients showed decline of blood flow in the frontal lobe regions. However, there were no specific findings that contribute the clinical classification into sub-groups.

Conclusion: The patients with slowly progressive difficulty in speech output could be classified into three sub-groups: aphasia with word finding difficulty type, pure anarthria type, and FCMS type. In particular, we designate the third type as slowly progressive FCMS. Adoption of this clinical classification is useful to predict the following clinical prognosis of the patients.

\section{P658}

A case of diffuse axonal injury causing reversible amnesia

M.A. Cakmak, T. Seleker, N. Isik

S.B. Istanbul Goztepe Training Hospital (Istanbul, TR)

This is a case of 28-year-old girl, injured in a motor vehicle accident. On admission, her Glasgow Coma Scale score was 8. Physical examination revealed no evidence of traumatic injury. A computed tomographic(CT) scan of the brain showed no structural central nervous system lesions. At the first hour of admission, the patient was awake but uncommunicative. At the second hour, she was awake and oriented and had no abnormalities on neurologic examination except antero-retrograde amnesia. Her amnesia dissolved in 64 hours after head trauma. Magnetic resonance imaging(MRI) was performed 4 days after trauma. In the right side of body and splenium of the corpus callosum, T1-weighted images showed an expanded area of low signal intensity with an irregular margin; this area was of high signal intensity on T2- 
weighted images. Gadolinium-enhanced T1-weighted MRI disclosed peripheral enhancing of the lesion. On the eighteenth day of the first admission, we used proton magnetic resonance spectroscopy(MRS) to identify the lesion, showed regression of the lesion and diminished NAA/creatine $(\mathrm{Cr})$ levels, suggesting diffuse axonal damage. Also, decreased apparent diffusion coefficient(ADC) confirmed the axonal injury. Follow-up MRI was performed 4 months after injury, T1 and T2-weighted images showed evident regression of the lesion. Even with closed head trauma, brain parenchyma can be severely damaged because of disruption of axonal fibers by shearing forces during acceleration, deceleration, and rotation of the head, well known as diffuse axonal injury(DAI). During head trauma, increasing rotational acceleration of the head causes centripetal extension of the shearing injury from the peripheral areas to the central brain structure. Due to the anatomical location, corpus callosum injury is one of the common components of DAI. It is usually larger and more evident than the other lesions seen on MRI, that makes it easy to use as a diagnostic marker. As conclusion, MRI is useful for differential diagnosis and the detection of callosal lesions. Due to the anatomical shape and location of the corpus callosum, both coronal and sagittal fluid-attenuated inversion recovery(FLAIR) and diffusion tensor imaging (DTI) images are most useful for visualizing lesions. MRS can quantify damage after DAI and NAA levels may be a sensitive indicator of the neuronal damage. In future, diffusion-weighted images may be useful in determining treatment strategies for acute head injury.

\section{P659}

Planning and problem-solving: performance of healthy adults on the tablet PC Tower of London puzzle

N. Kühnpast, A. Faber, F. Sürer, A. Hinz, A. Danek

Ludwig-Maximilians-Universität (Munich, DE)

Objectives: The Tower of London (ToL) puzzle is a popular test to assess planning and problem solving abilities in both clinical and nonclinical populations. However, cross-study comparisons are hardly possible since the ToL has been employed in many variants and with a variety of tasks and performance measures. Therefore, in the present study we used a computerized ToL puzzle with the physical appearance, instructions, and selection of tasks following Shallice's original version.

Methods: We tested 81 healthy subjects (aged 20-70 years) in order to provide normative data of nonclinical controls. In addition to the original set introduced by Shallice we included tasks with 6,7 , and 8 moves that allow a further investigation of specific configurations. A broad set of performance measures was employed including Total Moves as well as Solution Time (composed of Initiation and Completion Time).

Results: We found performance differences related to demographic variables as well as to structural parameters, determined by the specific configuration of the task. Although younger subjects were faster in solving a given task, we found that older subjects only needed more time to execute the movements but did not differ in Initiation Time. Concerning the impact of the planning phase we identified a general relationship between correctness and Initiation Time. This strengthens the idea that longer mental preplanning, in contrast to "online" planning, influences the accuracy of the solution. Furthermore, performance was linked to the start and ending configuration of a task, when comparing tasks with equal numbers of moves. Especially those with a flat-end configuration (one ball on each rod) were more demanding than tower-ending tasks (where all balls are stacked on the largest rod). We also investigated isomorphic problems, i.e. tasks which share an identical sequence of ball moves to solve but differ in the colour of the balls by permutation. Our findings support the suggestion that subjects' performance -although not being aware of iso-problem similarity- significantly improves with experience, which differs from a general learning effect.

Conclusion: The ToL puzzle offers a variety of opportunities for investigators of human planning and problem solving abilities. Results of the present study emphasize the usage of several performance measures and the careful selection of tasks with different configurations as well as further exploration of iso-problems.

\section{P660}

Localisation paradigm for aprosodic speech: three case reports

S. Jensen, D. Benvenuti, V. Pelliccia, D. Damiano, M.E. Verrocchio, A. Iudice University of Pisa (Pisa, IT)

Objectives: Aprosodia is a disruption in the expression or comprehension of changes in pitch, loudness, rate, or rhythm that convey a speech emotional intent. The localization paradigm for prosodic dysfunction refers to injury to the right hemisphere for induction of emotional speech prosody, and to the left hemisphere for propositional prosody. Methods: We assessed the aprosodic speech of three male patients, right-handed native Italian speakers. One subject had undergone surgery for a left frontal prerolandic differentiated astrocytoma, while two patients had a large right temporo-frontoparietal ischemic lesion due to internal carotid or middle cerebral artery occlusion. Patients' injuries resulted in a syntactic aprosodia in the first case, and in an expressive aprosodia in the other two.

Results: Speech and language examination revealed no dysarthria or orolingual apraxia. Naming, repetition, comprehension, writing, and reading were normal. Patients' voice, however, was extremely monotonous. The two patients with affective aprosodia were unable to change a sentence tone according to their mood or to a particular given situation, while the syntactic prosody was preserved. On the opposite, in the propositional aprosodia patient there was no impairment in expressing emotions using prosody, while he was unable to turn declarative speech into questions or commands. The aprosodic disturbances had a quite remarkable impact on patients' daily living and a devastating effect on social interactions in one case. Affective aprosodia was more frustrating than propositional aprosodia, and associated with depression. Review of patients' neuroimaging revealed atrophy of the right frontal lobe in two cases and an involvement of the left rostral prerolandic frontal gyri in the third patient.

Conclusions: A localization paradigm for prosody impairment, i.e. the right frontal lobe being important for the expression of emotional prosody, whereas the left frontal lobe for fluent syntactic/propositional speech, is supported by clinical presentations related to right and left hemisphere lesions, as well as by functional imaging studies.

\section{P661}

Reading numbers in patients with left spatial neglect

Y. Martin, T. Bernati, A. Saj, R. Caumont de la Mesquita, M. Rousseaux Centre l'Espoir (Hellemmes, FR); CHRU (Lille, FR); Université de Lille (Lille, FR)

Introduction: Compared with reading words, reading numbers is more complex. Indeed, the quantity associated with one specific digit varies according to its position in the sequence, and must at time be apprehended by a visual analysis of the following digits, especially for large numbers. This activity has been little studied in spatial neglect. Two single case studies of neglect dyslexia have been reported, which highlighted a dominant proportion of substitution errors on the left side, and this increased with stimulus length. We aimed at investigating this ability in a relatively large series of patients with left spatial neglect.

Methods: We included patients with a relatively recent right hemispheric stroke. Fifteen showed neglect in classical paper and pencil tests (bell test, line bisection, Ogden test), and 10 had no neglect. They were compared with 20 healthy control subjects. An original battery was presented, which consisted in 80 numbers of 1 to 8 identical or different digits. We analysed the number of correct responses and errors (omissions, substitutions, perseverations, addition, duplication, segmentation).

Results: The N+ group showed a significant drop of correct responses $(68 \%)$, in comparison with the $\mathrm{N}-(96 \%)$ and $\mathrm{C}(99 \%)$ groups, which did not differ. The size has no significant effect, but interacted with the group, because short numbers were read better than longer ones. In $\mathrm{N}+$ patients, errors were predominantly omissions, before substitutions and segmentations. They predominated on the left side, especially for longer numbers. In non-neglect patients and C subjects, they were preferentially segmentation errors.

Conclusion: Our study in a large group of neglect patients showed a definite preponderance of omission errors in number dyslexia. We did not confirm the observations of previous authors who suggested predominance of substitutions. In fact, neglect dyslexia for numbers is relatively comparable to word dyslexia, because the main problem is that patients do not take into account left sided information.

\section{P662}

Neuroanatomic correlates of post-stroke aphasias studied with imaging

Z.H. Yang, N. Li, X.Q. Zhao, Y.L. Wang, Y.M. Zhang

Beijing Tian Hospital, Capital Medical University (Beijing, CN)

Background and purpose: Stroke is the most frequent serious neurological disorder in the world and the third leading cause of death in the industrialised contries. Among the diverse cognitive deficits caused by stroke lesions, aphasia is the most devastating, to the extent that some aphasic individuals consider themselves to have lost their personhood.

Recently, most studies believed that lesion sites are decisive to the attack and types of aphasia, which is also in disputation. We observed the lesion sites of aphasia cases, and tried to reveal the relationship between aphasia types and lesion sites. 
Methods: The lesion sites were selected from 1198 patients with cerebral infarction, who were hospitalized in the stroke unit of our hospital between february 2002 and february 2006, and totally 325 patients with aphasia were enrolled.

All patients received MRI examination within 1 week after admission and evaluated with the western battery aphasia to decide what their aphasia type, and classified with the boston diagnostic aphasia examination severity grading standard to show what the language severity.

Results: Of which 1198 cases, there were 5 cases whose Broca's areas and 4 cases whose Wernicke's areas damaged had no language disorder, and aphasia was observed in 325 patients, the aphasia types showed that there were 83 Broca's aphasia cases, 48 Wernicke aphasia cases, 12 conduction aphasia cases, 36 transcortical motor aphasia cases, 17 transcortical sensory aphasia cases, 19 transcortical mix aphasia cases, 58 global aphasia cases and 52 anomic aphasia cases. 240 cases were located classical language centers while 85 cases were located at other sites. In 325 aphasia patients, grade severity were $0,1,2,3,4$ grade had $84,79,77,63,22$ cases respectively and many of 0 and 1 grade were distributed classical language centers.

Conclusions: This study showed 1) that it is possible to draw the anatomy of aphasia with a few number of deviant cases, 2) lesion location is the main determinant of aphasic disorders, and 3) that most lesion locations associated to specific disorders fit classical data in aphasiology, that is, most of aphasia type lesions are meet with classical language centers, other site damaged also produce aphasia., and language disorders of patients whose lesion site at classical language centers were serious

State Key Laboratory of Cognitive Neuroscience and Learning Open Project Grant and 20071D0300400078

P663

Study on motor language centre of Chinese people

X.Q. Zhao, C.X. Wang, Z.H. Yang, Z.H. Han, Y.M. Zhang

Beijing Tian hospital (Beijing, CN); Ministry of Education (Beijing, CN)

Background and purposes: The Chinese is a morpheme character system mainly demonstrated by meaningful signs and phonetic signs. Its form and approach of cognition, the analysis and storage of character meanings all differ from the western phonetic characters. Meanwhile, the Chinese is an intonation language, and has the duality of carrying the language message and possessing variable melody. Based on all listed above, the procession of the Chinese character may be different from the phonetic character.

We tried to locate the motor language centre of healthy volunteers whose native language is Chinese by using magnetoencephalography (MEG) and studied the procession of Chinese.

Methods: Ten healthy volunteers whose native language is Chinese were involved, and the result is all right handedness, among which are six men and four women aged between 28 and 37. The educational background is at least undergraduated without impediment speech and cognitive handicap.

We designed language tasks, adopted methods of reading true characters and false characters (respectively 90,180 in total, all specific names, names of common objects) we also matched strictly the number of names, the time of presenting every name and the internal time between two tasks.

The character image were projected onto the screen of magnetic shielding to be read by subjects, produced language stimulation which induced magnetic activity and through the record and analysis of MEG system, the signal of language induced magnetic activity finally combines with MRI figure, formed language function MEG location image.

Results: After the stimulation of true and false characters on the cerebral hemisphere, all the subjects induced two late magnetic reaction waves, and the polarization of left part is better than that of the right part. The wave shapes of late elements of reaction waves (200-600ms after stimulation), produced after subjects' cerebral hemisphere accepts the stimulation of true and false characters are basically similar. All subjects' motor language center is located in the back of gyrus frontal inferior.

Conclusion: Chinese can activate both hemispheres because they have language sense and also graphic sense and their structures are more complicated than that of phonetic characters, and the left hemisphere of righthanded Chinese people is the dominant hemisphere. The motor language center of normal Chinese people basically located at the classical motor language centre.

Key Laboratory for Cognitive Science and Learning of Ministry of Education and School of Psychology and Nature Science Foundation of Beijing (No. 7052035)
P664

Preliminary study of origins of repetition deficits

Y. Zhang, J.H. Chen, Y. Zhou, K. Dong

Beijing Tiantan Hospital, Capital Medical University (Beijing, CN); Beijing Mining Aggregation General Hospital (Beijing, CN)

Purpose: There are many mechanism of conduction aphasia, such as Wernicke-Geschwind's theory of disconnection, Storch-Goldstein's theory of central aphasia, the defect pattern of Auditory-speech shorten memory and the Bidirectional pattern.

In this study, based on exploring the causes of repetition deficits of two Chinese patients with conduction aphasia, we tried to discuss the characteristics and theories of repetition deficits, and provide more rich objective proof for clinical diagnose and rehabilitation of the disease.

Methods: Two cases with repetition deficits admitted Neurology Department of Beijing Tiantan hospital in Jan, 2006, the results of Western aphasia battery showed that they were conduction aphasia, and we required them to administrate three typical cognitive tests. The tests were phonological comprehensive and recognition test, spoken output test, and short memory test, which investigated the cases' capacity of phonological inner processing, phonological production and short memory, respectively.

Results: The two cases normally performed in all comprehensive and recognition tasks. One case presented the difficulty in all spoken output tasks (54-75\%, correct), in spite of keeping intact short memory capacity (memory span was 3-8). And another case had severe deficits in repeating non-words $(62 \%$, correct) and short memory capacity (memory span was $1-2$ ), although he failed to show cognitive limitation in other phonological output tasks (92-100\%, correct).

Conclusion: Repetition deficits have some different origins. The origins of the deficits of two cases in current study are the impairment at later phonological processing stage, and breakdown for short memory capacity, respectively.

State Key Laboratory of Cognitive Neuroscience and Learning Open Project Grant Superiority talented found (20071D0300400078)

\section{Extrapyramidial disorders: Parkinson's disease}

\section{P665}

Neuroprotective effect of glycyrrhizin in the 1-methyl-4-phenyl-1,2,3,6tetrahydropyridine mouse model of Parkinson's disease

D.E. Kim, S.E. Park, J.H. Han

Seoul Veterans Hospital (Seoul, KR)

Objectives: A licorice compound glycyrrhizin exerts cytoprotective or antioxidant effect in various disease conditions, but its effect against the 1methyl-4-phenyl-1,2,3,6-tetrahydropyridine (MPTP)-induced brain tissue damage remains uncertain. The present study elucidated the protective effect of glycyrrhizin against brain tissue damage in the MPTP mouse model of Parkinson's disease.

Methods: The present study measured the activities of antioxidant enzymes and formation of tissue peroxidation products in brains of MPTPtreated mice. The present study also performed in vitro assay to examine the effect of 1-methyl-4-phenylpyridinium (MPP+) on the mitochondrial respiratory electron flow, membrane potential and cytochrome $c$ release and measure the scavenging action of glycyrrhizin against reactive oxygen species.

Results: MPTP treatment increased activities of total superoxide dismutase, catalase, and glutathione peroxidase and levels of malondialdehyde and carbonyls in the basal ganglia, diencephalon plus midbrain compared to control mouse brain. Co-administration of glycyrrhizin $(16.8 \mathrm{mg} / \mathrm{kg}=$ $20 \mu \mathrm{M}$ ) attenuated the MPTP effect on the enzyme activities and formation of tissue peroxidation products. Glycyrrhizin attenuated the $500 \mu \mathrm{M} \mathrm{MPP}+-$ induced inhibition of electron flow, changes in the membrane potential and cytochrome $\mathrm{c}$ release in isolated brain mitochondria. Glycyrrhizin (1-50 $\mu \mathrm{M})$ showed a scavenging action against superoxide radical, hydrogen peroxide and hydroxyl radicals.

Conclusion: Glycyrrhizin may prevent the toxicity of MPTP against brain tissue by suppressing mitochondrial damage and oxidative tissue damage. Glycyrrhizin seems to attenuate oxidative brain tissue damage occurred in Parkinson's disease through antioxidant action and prevention of mitochondrial dysfunction. 


\section{P666}

Comparison of acute challenge with apomorphine and levodopa in candidates for deep-brain stimulation with Parkinson's disease

E. Trachani, E. Markaki, Z. Kefalopoulou, K. Constantoyannis, J. Ellul, E. Chroni

University of Patras (Patras, GR)

Objectives: The motor response to either levodopa test (dopaT) or apomorphine test (apoT) has been used in selecting patients with Parkinson's disease (PD) for deep brain stimulation (DBS) of subthalamic nucleus. We compared the motor response induced by these two tests and identified pro and cons for their clinical use.

Methods: Nineteen candidates for DBS with idiopathic Parkinson's disease $(29 \%$ females, mean age $64.7( \pm 8.8)$ years and mean duration of disease $11.6 \pm 5.6$ years) were assessed using the Unified Parkinson's Disease Rating Scale (UPDRS) III. The assessments were carried out; 1 . in "off period" - 12 hours after withdrawal of antiparkinsonian medication; 2 . in "on period" 20-30 min following increasing doses of subcutaneous apomorphine (up to $6 \mathrm{mg}$ ); 3. in "on period" - after administration of $150 \%$ of the usual dosage of levodopa combined with carbidopa. Selection criterion for DBS was set at $30 \%$ improvement of their baseline UPDRS III scores.

Results: Two patients did not complete the apomorphine test due to side effects (nausea and vomiting). In the remaining 17 patients was observed a mean reduction of the UPDRS III of $15.3 \pm 9.4$ and $19.8 \pm 10.8$ points in the apoT and dopaT respectively $(\mathrm{p}=0.012)$. Overall, $36 \%$ motor improvement was observed in the apoT compared with $49 \%$ improvement in the dopaT $(\mathrm{p}=0.013)$. The sensitivity and specificity of the apoT compared with dopaT was $67 \%$ and $100 \%$ in selecting PD patients for DBS,

Conclusions: A greater motor improvement was obtained with dopaT compared with apoT and this could have also affected the selection of patients for DBS. It remains, however, to be seen which test predicts better long term outcome after DBS.

P667

The effect of deep-brain stimulation on the sudomotor function in patients with Parkinson's disease

E. Trachani, Z. Kefalopoulou, V. Sirrou, K. Constantoyannis, E. Chroni University of Patras (Patras, GR)

Objectives: To evaluate the potential effect of deep brain stimulation (DBS) on sweating disturbances that constitutes one of the more common non-motor manifestations in Parkinson's disease.

Methods: Seven patients with idiopathic Parkinson's disease were studied. There were 4 men, 3 women with a mean age of $60.1 \pm 2$ years (range 54-70 years) and mean disease duration of $14.7 \pm v 2.9$ years (range 6-27 years). Each patient was assessed under two treatment conditions, the day before surgery during on-stage with regular oral medication and 6 months after DBS during turned on-stage. The assessment included: (i) Unified Parkinson's Disease Rating Scale (UPDRS) motor score; (ii) answering to a semi-structural questionnaire referred to autonomic disturbances including a detailed part related to sweating; (iii) neurophysiological study of the sympathetic skin responses (SSR) from the palm bilaterally and sole unilaterally elicited by distal electrical stimulus. DBS was achieved by implantation of electrodes bilaterally in the subthalamic nucleus in 6 patients and in the global pallidus in 1 patient.

Results: DBS improved motor performance in all patients with a mean pre-post difference of the on-stage UPDRS motor score 15.5 \pm 9.6 . According to the questionnaire's responses prior to DBS 3 patients complained of excessive hyperhidrosis ( 1 in the face, 1 in the truck, 1 in upper and lower limbs) and 1 experienced lower limb hypohidrosis. On the follow-up examination all 4 patient reported considerable improvement of the sweating function. The neurophysiological study disclosed that prior to DBS in the patient with limbs' hyperhidrosis the SSRs were absent throughout, whereas after DBS they were elicited from both palms and sole. Moreover, in two other patients with no sweating symptoms the SSR from the sole was unobtainable pre -operative and was normally recorded post-operative. No change of SSR recordings was revealed in the remained 4 patients.

Conclusion: Although the small sample size does not allow statistical analysis, our data based on the questionnaire responses and the SSR recordings suggested that in patients with Parkinson's disease, DBS in parallel to improvement of the motor control has also a positive effect on the sudomotor regulation.
P668

Assesment of valvulopathy in Parkinson's disease patients on ergot derived dopamine receptor agonists

F. Candan, O. Oztop Cakmak, N. Isik, H. Ciftci, I. Aydin Canturk, C. Basbugu Goztepe Training and Research Hospital (Istanbul, TR)

Objectives: It has been reported that Parkinson's disease (PD) patients on ergot derived dopamine receptor agonists (EDDA) are associated with an increased risk of devoloping cardiac valvulopathies. Our aim is to evaluate Parkinson's disease patients treated with EDDA by echocardiography and investigate the cardiac valv regurgitation and the fibrosis.

Methods: Transtorasic Echocardiography (TTE) was performed on 73 Parkinson's disease patients. 43 patients with Parkinson's disease treated with EDDA (pergolid or cabeser) at least 6 months and 30 never treated with an ergot derived dopamine agonists (age and gender matched controls) were evaluated with TTE. None of them had a history of cardiac disease. The relation between the valvulopathies, drug duration and dosage of mediation is evalueted.

Results: The PD patients treated with EDDA (23 men and 20 women) had a mean age of $61.2 \pm 11.3(26-78)$ years. $81.3 \%(n: 35)$ of them had valvulopathies, significantly higher than the PD patients who had not been treated with EDDA $(\mathrm{p}<0.05)$.

Mean agonist duration was $1.9 \pm 1(1-4)$ years and mean daily dose was $3.3 \pm 1.4(1-6) \mathrm{mg} /$ day for 31 patients on cabergoline. Mild valvuler regurgitation was seen in 27 patients $87.7 \%$, (frequency order; mitral, aort, tricuspit) and in addition fibrotic valve was seen in $933.3 \%$, (frequency order; aort, mitral) of them. One patient had a fibrotic aort valve only.

Mean agonist duration was $5.3 \pm 2.7(3-10)$ years and agonist daily dose was $2.3 \pm 1.1(0.75-4) \mathrm{mg} /$ day for 12 patients on pergolide. Mild valvuler regurgitation was seen in 7 patients, $58.3 \%$ (frequency order; aort, mitral, tricuspit) and fibrotic aort valve was seen in $3,42.8 \%$ of them.

Results: Cardiac valv regurgitation and fibrosis was frequently seen in patients on EDDA. Follow up echocardiographic monitoring should be performed regularly for the valvulopathies in these patients.

\section{P669}

Insufficient information of medicine intake in elderly Parkinson patients I. Magnussen, D. Leer, H. Holst Aalborg Hospital (Aalborg, DK)

Objective: The frequence of orthostatic hypotension defined as a systolic drop of more than $20 \mathrm{~mm} \mathrm{Hg}$ measured in elderly patients in our specialis movement disorder clinic was found to be $40 \%$ which is rather high. The reason could be discrepancy between the reported intake of medicine and the real intake.

Methods: 125 patients more than 65 years of age with Parkinsons disease was consequtively included from the out-patient clinic.

Two specialist nurses visited the patients at home or at the nursing homes obtaining information of the actual intake of parkinson medicine and concomitant medication also from relatives and professionals. We compared the data with the case notes in the clinic.

Results: In $46 \%$ of the patients we found disagreement between the reported intake and the medication known from our case notes.

Conclusion: Treating patients as partners in therapy decisions is known to be crucial to the therapeutic process. Not only the lack of compliance in elderly patients with Parkinsons disease but also considerable lack of information of the total use of medication may influence the outcome of neurological consultation in an out-patient clinic.

P670

Scoliosis in Parkinson's disease

J-S. Baik, S-W. Han, J-Y. Kim, J-H. Park

Sanggye Paik Hospital, (Seoul, KR)

Objective: To investigate the scoliosis changes in idiopathic Parkinson's disease (IPD) and compare clinical characteristics in IPD with scoliosis and without scoliosis.

Background: Abnormal postures frequently present in patient with IPD and often wrongly attributed to orthopedic problems. Scoliosis is common in IPD and occurs more frequently in PD patients than in the general elderly population. The relationship of age, gender, and dopaminergic pharmacotherapy with scoliosis in IPD is poorly understood.

Methods: Eighty patients ( 33 men, 47 women, mean age: $68.6 \pm 8.4$ years old) with IPD from January to December 2007 who were visiting to our clinic, were involved to this study. All of them were taken whole spine scan to evaluate of scoliosis. We got information of clinical characteristics by history taking and reviewed their charts. The degree of scoliosis was measured 
by Cobb's method on imaging. We subdivided them with spinal asymmetry $\left(0^{\circ}-10^{\circ}\right)$ and true scoliosis (over $\left.10^{\circ}\right)$ by degree of scoliosis and compared some clinical characteristics among these groups.

Results: 52 of 80 patients (65\%) had no scoliosis, and 28 patients ( $35 \%)$ had scoliosis. 23 of 28 patients with scoliosis had single lesion and five had double lesions. Most of single lesion was lumbar area. Woman had more in true scoliosis than man and later symptom onset of Parkinson's disease in true scoliosis group than in no scoliosis group. The site of dominant Parkinsonian symptom and scoliosis convexity was same in $57.1 \%$ of true scoliosis patients. There were no significant differences of UPDRS III, duration of Parkinsonian symptoms, mean Hoehn and Yahr stage and presence of back pain between two groups.

Conclusions: Scoliosis in IPD is not uncommon. We didn't find any differences between those with and without scoliosis in IPD.

P671

Inhibition of glycogen synthase kinase-3 reduces L-dopa-induced neurotoxicity

K-Y. Lee, S-H. Koh, H. Kim, Y. Lee, D-I. Chang, J. Kim, H-T. Kim, S. Kim

College of Medicine, Hanyang University (Seoul, KR)

The neurotoxicity of L-3,4-dihydroxyphenylalanine (L-DOPA), used for the treatment of Parkinson's disease, remains controversial. Although there are many reports suggesting that long-term treatment of L-DOPA causes neuronal death, an increasing body of recent evidence has proposed that LDOPA might be neuroprotective rather than neurotoxic. We investigated the effect of L-DOPA on neuronally-differentiated PC12 (nPC12) cells by treating cells with various concentrations of L-DOPA for $24 \mathrm{~h}$. We also studied whether glycogen synthase kinase (GSK)-3 activation is related to L-DOPAinduced neurotoxicity by simultaneously treating cells with several concentrations of L-DOPA and a GSK-3 inhibitor for $24 \mathrm{~h}$. MTT (3-[4,5-dimethylthiazol-2-yl]-2,5-diphenyltetrazolium bromide) assay, trypan blue staining, cell counting kit-8, and DAPI staining all showed that L-DOPA decreased nPC12 cell viability at high concentrations. In addition, $100 \mu \mathrm{M} \mathrm{L}$ DOPA treatment significantly increased the activity of GSK-3 and death signals including cytochrome c, activated caspase- 3 , and cleaved PARP and decreased survival signals, including heat shock transcription factor-1 in a concentration-dependent manner. Treatment with GSK-3 inhibitor VIII or lithium chloride prevented L-DOPA-induced cell death. Together, these results suggest that L-DOPA induces neuronal cell death at high concentrations and that the neurotoxic effect of L-DOPA might be partly mediated by GSK-3 activation.

\section{P672}

Characterisation of the 6-OHDA model of Parkinson's disease using manganese-enhanced MRI

M.A. Rueger, J.P. Munasinghe, A.P. Koretsky, R.D. McKay

Uniklinik Koeln (Cologne, DE); NINDS (Bethesda, US)

Objective: In an animal model of Parkinson's disease, specific loss of dopaminergic neurons is induced by local injection of the neurotoxin 6-hydroxydopamine (6-OHDA) into the striatum of rats, leading to retrograde degeneration of the dopaminergic nigrostriatal tract. This model is routinely used to study neuroprotective or regenerative treatment strategies in a preclinical setting. Location and size of the lesion before and after experimental treatments are usually measured histologically by the quantification of dopaminergic neurons, which requires sacrificing the animals. In the present study, we investigated the use of in vivo Manganese-enhanced Magnetic Resonance Imaging (MEMRI) to characterize and quantify 6-OHDA lesions repetitively over time in the living rat.

Methods: Adult rats received stereotactic injections of 6-OHDA into the right striatum, and saline injections into the left striatum. MR imaging was performed $24 \mathrm{hrs}$ after the lesion, followed by weekly imaging over 7 weeks. Prior to each imaging session, some rats were injected intraperitoneally with $\mathrm{MnCl} 2$ as a contrast agent. Some rats were stereotactically injected with $\mathrm{MnCl} 2$ into the substantia nigra 2 weeks after 6-OHDA lesioning for neuronal tract tracing.

Results: T2 weighted MR images of rat striatum $24 \mathrm{hrs}$ after 6-OHDA lesioning showed significant T2 elongation, while saline injection showed no effect. Systemic application of $\mathrm{MnCl} 2$ as a contrast agent led to T1 shortening in lesioned striatum for up to 7 weeks after the lesion, indicating a persistent loss of blood-brain barrier integrity. $\mathrm{MnCl} 2$ injected into the substantia nigra of intact rats led to the transport of $\mathrm{Mn} 2+$ into the striatum, while no tracing occurred in rats with a lesioned dopaminergic pathway, indicating that only functionally intact neurons promote the transport of $\mathrm{Mn} 2+$ along the nigrostriatal tract.

Conclusion: MEMRI using $\mathrm{MnCl} 2$ as a contrast agent can detect small changes in blood-brain barrier integrity in the striatum caused by 6-OHDA. Moreover, the ability of $\mathrm{MnCl} 2$ to trace functionally intact neuronal pathways can be used to identify rats that have a degenerated nigrostriatal pathway. These techniques allow a non-invasive monitoring of neuroprotective or regenerative treatments in the living experimental animal over time, and can therefore facilitate the preclinical development of novel therapies.

\section{P673}

Non motor symptoms in Parkinson's disease

O. Oztop Cakmak, N. Isik, F. Candan, I. Aydin Canturk

Goztepe Training and Research Hospital (Istanbul, TR)

Objectives: Non motor symptoms (NMS) are one of the major causes affecting quality of life in Parkinson's disease patients and recognition of NMS is important beside the motor symptoms.Our aim is to investigate the presence of NMS and to evaluate the relation between NMS and the demographic and clinical features of Parkinson's disease patients.

Methods: Our study included 102 nondemanted idiopathic Parkinson's disease (IPD) patients (59 males). All of the patients were questioned with the 30 items questionnaire (NMS Quest) screening the presence of the symptoms among the gastrointestinal psychiatric, urinary, sleep, cognition and autonomic and the other systems during the last month. The score was calculated as summing the positive ('yes') responses.

Results: Demographics of IPD study population were; mean \pm SD age 64 . $6 \pm 11.4(24-89)$ years; mean disease duration $7 \pm 5(1-27)$ years; mean disease onset $57.5 \pm 12.5(19-83) ;$ Hoehn \&Yahr median stage $2(1-4)$.

The presence of NMS in all patients was observed. Mean NMS score was $11.5 \pm 5.7(2-23)$. There was no significant relation between the total NMS scores and the demographic and clinical features of the patients. The more prevelant NMS were; 'feeling sad,low or blue' $(73.5 \%$, n: 75), 'a sense of urgency to pass urine' (66.7\%, n: 68) and 'getting up regularly to pass urine' $(62.5 \%, n: 64)$. There was no significant relation between these symptoms and demographic and clinical features of patients.

Conclusion: The presence of NMS symptoms in all patients even at the disease onset and young ages is remarkable. For a comprehensive assessment and a better quality of life of the Parkinson's disease patient, it's important to evaluate the presence of NMS and initiate further investigations and treatment.

\section{P674}

Zonisamide in managing impulse control disorders in Parkinson's disease P.E. Bermejo, R. Velasco-Calvo

Puerta de Hierro University Hospital (Madrid, ES)

Impulse control disorders (ICDs) are a set of behaviors which are now recognized to occur in a subgroup of patients with Parkinson's disease (PD). Their pathophysiology remains unclear although a dopaminergic mechanism seems to be implicated. Zonisamide is a new anticonvulsant that has been suggested to improve motor functions in PD and some studies have proposed its efficacy in treating binge eating disorder and alcohol intake. However, there are no studies that attempt to investigate the potential role of this drug in impulse control behaviors associated with PD. We evaluated the safety and efficacy of zonisamide in four patients with ICDs and PD who did not improve following either a reduction of or switch to levodopa or dopamine agonists.

Four (three men and one women) were enrolled in this study. The average age was 63.2. All of them had been diagnosed with PD for at least three years prior to the emergence of the ICD. Two men had hypersexuality, another had pathologic gambling and the woman had compulsive shopping. Despite their attempts to reduce levodopa or switch dopamine agonists, their ICDs did not improve and motor functions worsened. Zonisamide was started at $25 \mathrm{mg}$ and titrated to $200 \mathrm{mg} /$ day over six weeks. The duration of the treatment was at least 120 days. Severity of the behaviors was assessed by means of the Clinical Global Impression (according to a analogical scale from 0 to 10) and global impulsivity by means of Barratt Impulsiveness Scale. There was an marked reduction in the severity of their impulsive behaviors (mean change from baseline -3.3) and global impulsiveness was also improved (mean change -4.1). Average UPDRS motor score changed marginally $(-0.4)$. The drug was safe and well tolerated.

Although the exact pathogenesis of ICDs is unknown, it has been related to dopamine replacement therapy. Zonisamide has multiple mechanisms of action, including blockage of voltage gated sodium channels, augmentation of GABA and inhibition of the release of glutamate, inhibition of the carbonic anhydrase enzyme and biphasic effects on the dopaminergic system. Despite the fact that the exact mechanisms of action remain unknown, some data suggest that zonisamide may be useful as therapy for patients with a variety of ICDs. Although our study was small, open-label and observational, 
our results suggest that this drug may also play a role when these disorders are associated with PD.

\section{P675}

Helicobacter pylori eradication improves treatment response in advanced Parkinson's disease

R. Borgohain, V.C. Reddy, A.K. Meena, S.A. Jabeen

Nizam's Institute of Medical Sciences (Hyderabad, IN)

Objective of the study: To investigate whether Helicobacter pylori eradication could improve clinical response to L-dopa in patients with advanced Parkinson's disease and motor fluctuations.

Materials and methods: At baseline all the eligible patients were screened for H.Pylori infection with an IgG ELISA serological test. Only H. pylori positive patients were given eradication therapy. All patients were assessed at baseline and immediately, 1 and 2 weeks after treatment (or corresponding times) with the Unified Parkinson's Disease Rating Scale I-III (UPDRS I-III) at the best on condition and at the practically defined off condition. Onset and duration of on time with L-dopa and total daily on time were assesed. On time duration was assessed using the Hauser diary. Finally we compared these parameters in each group and also $\mathrm{H}$. pylori positive vs negative groups at baseline and at $0,1, \& 2$ weeks after treatment.

Results: Twenty-eight patients (15 post deep brain stimulation)with a mean age of 58.8(9.8)years and disease duration of 9.8 (5.7) years were screened. Of these $18(64 \%)$ were positive for $\mathrm{H}$. pylori infection and 10 $(36 \%)$ were negative. There was highly significant improvement $(\mathrm{p}<0.05)$ in treatment group for on stage total UPDRS I-III, UPDRS III, onset of on after levodopa, on time duration and daily total on time duration at $0,1 \& 2$ weeks compared to baseline but off stage total UPDRS I-III \& off UPDRS III did not improved significantly $(p>0.05)$. In the untreated group there was no significant improvement $(\mathrm{p}>\mathrm{v} 0.05)$ for above parameters and there was significant worsening of total on/day compared between 0 week and 3 weeks.

On comparing treated versus untreated groups, no significant difference at baseline for all the above parameters was seen $(p>0.05)$ while there was significant improvement $(\mathrm{p}<0.05)$ in treatment group at 0,1 and 2 weeks for on total UPDRS I-III, on UPDRS III, and onset of on, duration of on and daily total on duration after levodopa. However there was no significant difference for off total UPDRS I-III and off UPDRS III ( $p>0.05$ ).

Conclusion: H. pylori eradication significantly improves the clinical status (on total UPDRS I-III, on UPDRS III, onset and duration of on after levodopa and daily total on duration) of infected patients with PD and motor fluctuations.

\section{P676}

Initial motor symptoms influence cognition in a longitudinal observation of patients with Parkinson's disease

Y. Zhelev, M. Petrova, M. Raycheva, L. Traykov

Medical University (Sofia, BG)

Objective: Parkinson's disease (PD) is often associated with mild cognitive impairment (MCI). Cognitive impairment is common even in early PD. A recent study showed that the conversion rate of PD-MCI to dementia is $15 \%$ per year, or similar to that of Alzheimer's disease. Research has shown that several clinical disease parameters may correlate with cognitive deterioration. The present study examined the association between type of motor symptom at disease onset and cognitive decline in a longitudinally followed group of patients with idiopathic Parkinson's disease.

Methods: We investigated 32 cognitively intact patients with PD (disease onset - after 40 years of age) and 25 controls. All subjects underwent a comprehensive neuropsychological assessment including tests of episodic memory, language, constructional praxis, psychomotor speed, attention and executive functions. PD and mild cognitive impairment was diagnosed according to established criteria. Information concerning type of initial motor symptom was obtained from subject report during clinical interview and from medical documentation.

Results: We assessed the cognitively intact PD patients $20+4$ months af ter the baseline examination. Ten of them progressed to mild cognitive impairment (PD-MCI) over this period of time, showing $31 \%$ rate of progression from cognitively preserved PD to PD-MCI. A statistical significant deference between two groups (PD, PD-MCI) has been demonstrated regarding type of initial motor presentation of disease.

Conclusions: Nearly one third of our PD group developed PD-MCI during the mean two years study period, suggesting that PD population has a high risk of developing MCI. Bradykinesia/rigidity as initial motor presentation of PD has been shown to influence further cognitive deterioration. These findings should be interpreted with caution because of the small number of subjects included, and due to the relatively short period of follow-up.

\section{Epilepsy}

\section{P677}

Malfunction of sexuality in patients with epilepsy

A. Kapidzic, L. Kovacevic, O. Sinanovic

School of Medicine University of Tuzla (Tuzla, BA)

Objective of the study was to determine the existence of hypo-sexuality in the group of patients with epileptic seizures.

Methods: The group tested was consisted of 50 males and 50 females with generalized epileptic seizures of tonic-clonic type, while the control group was consisted of 50 males and 50 females with simple partial epileptic seizures. Patients were of normal neurological status, and both groups were formed on random sample base. The assessment of sexuality malfunction was conducted on base of adequate questions from Hamilton test, where the malfunctions were marked as strong, moderate, or their existence is negative. The gained results were compared to X2 test, and values of $\mathrm{p}<0,005$ were considered as significant ones.

Results: Actual age of subjects was $32.3 \pm 12.1$ in comparison to $31.8 \pm 13.9$ in the group of patients with simple partial epileptic seizures $(p=0.78)$. Sexual malfunctions were denied by 67 subjects, while $33(33 \%)$ expressed as having those. Moderate malfunctions existed at $19(19 \%)$, opposite to $15(15 \%)$ in the group of patients with simple partial epileptic seizures, while the strong malfunctions existed at $14(14 \%)$ subjects, opposite to $6(6 \%)$ in control group $(\mathrm{p}<0,01)$. In both groups the hypo-sexuality was more represented at females, but there were no significant difference.

Conclusion: Patients with generalised epileptic seizures of tonic-clonic type are more affected by sexual malfunctions then those having simple partial epileptic seizures.

Key words: sexual malfunction, epilepsy

\section{P678}

Non-compliance with anti-epileptic drugs at a district hospital in northeastern Nigeria

A. Danburam, F. Salawu, B. Grema, M. Wakil, J. Dikka

Federal Medical Centre (Yola, NG); Aminu Kano Teaching Hospital (Kano, NG); Federal Neuropsychiatry Hosptali (Maiduguri, NG)

With appropriate treatment and lifestyle changes, majority of epileptics will become seizure-free. The effect of non-compliance is enormous and may lead to an increase in seizure frequency and low quality of life.

Objectives: The aim of this study was to determine the knowledge, attitudes, and socio-economic factors of individuals living with epilepsy who presented to the neurology clinic.

Methods: A descriptive study design was employed on 25 consecutive adolescent and adult epileptic patients to assess their perspectives of epilepsy, compliance with treatment and impact of antiepileptic drugs on seizure control. Open and closed ended questionnaire were used as a guide during the interview.

Results: The median age of study population was 26 years. The patients had suffered from epilepsy for a median of three years and visited the neurology clinic for a median of four times a year. The median educational attainment was primary school and a majority were unemployed. Most of the patients did not know the causes of the disease, side effects of the medication and why they should be on medication for a prolonged period. More than a half of the patients indicated that they did not take their antiepileptic drugs regularly. One third had difficulty paying for the drugs. A quarter was satisfied with medical treatment alone, whereas $12 \%$ combined medical treatment with "native medicine" or spiritual church healing.

Conclusion: There is a general lack of understanding of epilepsy by these patients. The patients were poorly informed and the healthcare workers did not spend sufficient time and effort in counselling them. Non-compliance is a complex problem that needs intervention from both the patients and the primary healthcare worker.

\section{P679}

Neurophysiological profile of somatic and autonomic nerve fibres in patients with epilepsies

E. Chroni, V. Sirrou, E. Trachani, G. Sakellaropoulos, P. Polychronopoulos University of Patras (Patras, GR)

Objectives: To detect potential neurophysiological abnormalities of somatic and autonomic nerve fibres and examine their relationship in an unselected group of patients with epilepsy.

Methods: Sixty patients ( $36.7 \pm 12.5$ years, M/F:28/32) of whom 35 suffered generalized and 25 partial seizures for a period varied from 6 months 
to 48 years were enrolled. All patients had no condition related to neuropathy and were under treatment with one (36 patients) or multiple antiepileptic drugs (24 patients). A group of 60 healthy subjects matched for sex and age to the patient group served as control.

Latencies of sympathetic skin responses (SSR), which were elicited by peripheral electrical stimulation and recorded unilaterally from palm and sole, were measured. SSR amplitude measurements, due to their great variability, were not analysed. Parasympathetic activity was evaluated by Valsalva ratio (shortest $\mathrm{RR}$ interval during phase II to longest RR interval during phase IV) and tilt ratio (30/15 beat RR interval). The sural nerve sensory action potential (SAP), a reliable marker of somatic nerve fibre function, was also assessed.

Results: In the patient group SSR latencies were significantly prolonged, whereas Valsalva ratio, tilt ratio and amplitude of sural SAP were significantly lower than in the control group (Mann-Whitney test, $\mathrm{p}<0.004$ ). Based on normal limits appropriately set for each parameter, analysis of individual values in the patients disclosed the following: $20 \%$ of them had at least $1 \mathrm{ab}-$ normal SSR latency value; in addition, 2 SSRs from the palm and 5 from the sole were absent. Abnormal valsalva ratio was evident in $48.3 \%$ and abnormal tilt ratio in $16.7 \%$ of the patients. Sural SAP amplitude was below normal limit in $11.7 \%$. Six out of the 7 patients with abnormally low sural SAP amplitude demonstrated as well abnormal valsalva ratio ( $\mathrm{x} 2$ test, $\mathrm{p}<0.05)$. There was also a tendency for sural SAP amplitude and tilt ratio abnormalities correlation $(p<0.09)$. No such correlation was detected between SSRs and parasympathetic tests or SSRs and sural SAP abnormalities.

Conclusion: Applying easily performed neurophysiological tests a considerable number of abnormalities concerning both somatic and autonomic nerve function were detected in patients with epilepsy. Although a link between parasympathetic and somatic nerve fibres abnormalities appeared to exist, diverse factors influencing nerve activity can better explain our findings.

\section{P680}

Non-epileptic psychogenic seizures: "diagnostic difficulty" - clinical, laboratory, and neurophysiological study

G. Ibrahim

Sohag Faculty of Medicine (Sohag, EG)

Psychogenic nonepileptic seizures (PNES) account for $10-40 \%$ of patients referred to epilepsy centers. Distinguishing between NES and epileptic seizures is a very difficult task facing the clinician.

Patients and methods:This study included 20 epileptic patients and 20 patients suffering from psychogenic nonepileptic seizures. All patients were subjected to the following, Detailed medical and neurological history and examination, psychometric tests including intelligence assessment and Minnesota Multiphasic Personality Inventroy and the following investigations: Routine laboratory investigations, pre and post-ictal Creatinephosphokinase, Routine EEG, Prolonged video EEG recordings with the use of induction technique, Auditory event related potentials (P300) using oddball paradigm done in three sessions, preictal, postictal (within 6 hours from the onset of fits), and interictal (6-48hours from the onset of fit), and CT brain for the epileptic group.

Results: The comparative studies included: demographic and neurological history variables, seizure semiology, psychological testing, serum CPK and event related potentials measurements. Those comparative studies revealed highly significant difference between the two groups as regard age of onset, duration of fits, seizure semiology. MMPI-II and P300 evidenced powerful techniques in differentiation.

Conclusion: The combination of more than one of these variables particularly P300 and MMPI-II raised the diagnostic accuracy of PNES even without video EEG

\section{P681}

Doparesponsive dystonia after lamotrigine use due to epilepsy

H. Ebrahimi

Kerman University (Kerman, IR)

Dopa-responsive dystonia is a broad term used to describe forms of dystonia that respond to a medication called levodopa. Dopa-responsive dystonia typically presents as a dystonic gait disorder that begins in early childhood. The most common type form is called DYST5, which is a dominant inherited condition caused by mutations. A therapeutic trial with levodopa remains the most practical initial approach to diagnosis. We report a case of Doparesponsive dystonia after uses lamotrigine.

Case study: We report a case of Dopa-responsive dystonia induced after receiving lamotrigine $50 \mathrm{mg} /$ daily due to tonic-clonic seizures in 5 years old girl, which begin the seizure attack from two months ego. After starting the lamotrigine the patients was free seizure for one year, but dystonic pasture is seen in lower limb and after a time spread to lumbar spine, and after that to cervical area. The dystonic attacks worse later in the day. Physical examinations were normal. Brain MRI scans was normal, hematologic and serologic laboratory tests were normal. The dystonic pasture did not disappear after lamotrigine discontinued. For patient started levodopa+ carbidopa $50 \mathrm{mg} /$ day, the dystonic pastures after two days disappeared.

Conclusion: this sporadic case of Dopa-responsive dystonia may be an irreversible side effects of lamotrigine (without treatment by drug). Thus need to study about it more.

\section{P682}

Nonconvulsive status epilepticus in a patient treated with carbamazepine I. Ghione, D. Santoro, V. Lucchini, A. Cappellari, A. Prelle, G. Conti, N. Bresolin

Ospedale Maggiore Policlinico di Milano (Milan, IT)

Objectives: To describe how treatment with carbamazepine paradoxically can produce nonconvulsive status epilepticus.

Methods: We report the case of a 77-year-old woman who presented with a sub-acute cognitive decline with drowsiness, confusion, memory impairment, hallucinations, strange behaviours, intermittent periods of unresponsiveness and fluctuating walking difficulties. Before admission to our Hospital she was treated with carbamazepine as a focal secondary epilepsy in a patient with a cerebrovascular encephalopathy. However, after 2 days of antiepileptic treatment she worsened dramatically and she was admitted to our Clinic: At our observation she was drowsy, inappropriate, vain and confabulating. The serum sodium concentration was $119 \mathrm{mmol} / \mathrm{l}$, and an EEG recording showed a pattern consistent with a nonconvulsive status epilepticus. Carbamazepine was interrupted, and serum levels of sodium were gradually corrected.

Moreover, in order to treat the persisting nonconvulsive status, following the ineffective intravenous administration of lorazepam, we prescribed phenytoin in vein, with an immediate EEG improvement, followed by a slow, progressive, resolution of her symptoms within few days.

Results: We describe the case of an old woman in whom the administration of carbamazepine induced a nonconvulsive status epilepticus through hyponatremia.

Conclusion: Treatment with carbamazepine should be considered with caution in elderly patients, who are more susceptible to hyponatremia, because of their impaired ability to keep water and sodium homeostasis in response to dietary and environmental changes.

\section{P683}

Patient with epilepsy therapeutic-resistant to eight anti-epileptic drugs free of seizures with Keppra ${ }^{\circledR}$

I. Stefanova

SBALNP "St.Naum” (Sofia, BG)

Objective: The objective is to present a case with 30 years history of Epilepsy treated with 8 different antiepileptic medications with insofition effect and very good therapeutic results with Keppra add on treatment.

Methods: The patient PDN male 44 years old who has partial motor seizures in his left leg, spreading in the left half of his body seizures sometimes with secondary generalization into tonic-clonic seizures. The seizures are very frequent up to several a day. The evaluation of the patient includes Electhroencephalography / EEG /, Computer Tomography /CT/ of main brain, Electrocardiography / ECG /, accessing of anticonvulsants blood levels, dairy of the seizures. In different periods of this 30 years duration at his epilepsy he was treaten totally with eight antiepileptic drugs as monotherapy or in combination: Phenobarbital $150 \mathrm{mg} / \mathrm{d}$, Carbamazepine $900 \mathrm{mg} / \mathrm{d}$, Gabatril $300 \mathrm{mg} / \mathrm{d}$, Lamictal $300 \mathrm{mg} / \mathrm{d}$, Topamax $300 \mathrm{mg} / \mathrm{d}$, Trileptal $900 \mathrm{mg} / \mathrm{d}$. The efficacy of these treatments was only partial - a little reduction of the frequency.

Results: In 2007 we started combination therapy with Trileptal $3 \times 450 \mathrm{mg}$ and Keppra $2 \times 1000 \mathrm{mg}$. Now the patient is free of seizures and his EEG shows reduction of the abnormal diffuse and focal activity.

Conclusion: Keppra with it's new mechanisms of anti-epileptic activity gives opportunity for good results in the therapy of treatment resistant epilepsies with long duration. 


\section{P684}

Acute stroke-elicited epilepsia partialis continua responsive to intravenous sodium valproate: case report and literature overview

K. Rejdak, E. Papuc, P. Dropko, Z. Stelmasiak

Medical University of Lublin (Lublin, PL)

Objectives: Epilepsia partialis continua has been defined as a partial somato-motor status characterised by clonic muscular twitches repeated at fairly regular, short intervals in one part of the body lasting for a period of days and weeks (Juul-Jensen and Denny-Brown, 1966). Usually it is a late manifestation of focal progressive or non progressive brain lesions (Schomer DL, 1993).

We present an interesting case of epilepsia partialis continua (EPC) as an acute manifestation of cortical ischemia in the course of vasospasm after subarachnoid hemorrhage $(\mathrm{SAH})$ responsive to sodium valproate (VPA) treatment.

Methods and results: A 72-year-old, right-handed woman suffering SAH complicated with intracerebral left hemispheric haematoma was admitted. Five weeks later acute vasospasm developed affecting contralateral hemisphere. EPC ensued as an acute complication, with clonic jerks of the left side of the face and left upper limb. This was related to epileptiform ictal activity, followed by periodic lateralized epileptiform discharges (PLEDs) from the right hemisphere. When the standard treatment with benzodiazepines and phenytoin failed, the infusion of VPA was started, which completely abolished seizure activity giving the improvement of patient's neurological condition.

In conclusion, EPC may be an acute complication of cortical ischemic damage and valproate intravenous preparation is an interesting alternative for the treatment of this condition.

\section{P685}

Utilisation of electroencephalographic service in a tertiary health care institution:a five-year study

M. Wakil, A. Danburam, S. Pindar, O. Beida, H. Mustapha, F. Salawu

Federal Neuropsychiatry Hospital (Maiduguri, NG); Federal Medical Centre (Yola, NG)

The electroencephalograph is the most frequently performed investigation for seizures and epilepsy, and it is valuable in the investigation and diagnosis of many other neurological conditions such as coma, encephalopathy and dementia.

Objective: The utilization of the electroencephalographic (EEG) service in a tertiary health care institution was reviewed to determine the number of EEGs done: constraints that militated against the full utilization; and the usage rate by outside health care facilities.

Methods: This retrospective study of the register of patients who had EEG done at the Federal Neuropsychiatry hospital (FNH) Maiduguri between January 2001 and December 2005 was carried out.

Results: Six hundred and eleven EEG recordings were carried in the 5year study period. About $70 \%$ of the total number of patients was referred from the consultative clinics of the Federal Neuropsychiatry Hospital and $30 \%$ of the number was patients referred from health-care facilities outside of the FNH. The Department of Medicine was the major referral department and the epilepsies or seizure disorders were significantly the major reason for referring patients for EEG. Most EEGs were performed after 48 hours of first unprovoked seizures with practically no ictal EEG recordings. Less than $10 \%$ of the EEGs were performed on paediatric group.

Conclusion: The EEG facility at the FNH was grossly underutilized in the study period as the 611 EEGs done only represented $15 \%$ of the estimated number that could have been done given the available work force; however, there was brevity of equipment. There were only two EEG machines at the FNH and non-availability of consumable items such as recording paper and electrode gel and rising costs of the EEG were identified as the causes of under-utilization.

P686

Efficiency of sleep deprivation in electroencephalography

O. Oz, S. Bek, G. Genc, Z. Gokcil, Z. Odabasi

Gulhane Military Medical Academy (Ankara, TR)

Objective: In this study we aimed to assess the ratio of interictal epileptiform discharges (IEDs) in electroencephalographies (EEGs) after sleep deprivation (SD) with normal first routine EEG in patients with strong epileptic history.

Methods: Patients who referred to our outpatient clinic between January 2006-July 2007 and who had strong history of suspected seizures included.
Ninety eight of them had normal routine EEG. We obtained sleep-deprived EEGs (SDEEG) of these 98 patients after 24 hours sleep deprivation.

Results: Mean age was $25.4 \pm 9.6 .33$ patients $(33.7 \%)$ had IEDs in SDEEG. Fourteen patients had focal abnormalilty (14.3\%), 19 patients had generalised abnormality $(19.4 \%)$ and 2 of them had ictal activity.

Discussion: Only $20 \%$ to $50 \%$ of epileptic patients have IEDs on their first "routine" EEG. If routine EEG fails to reveal epileptic activity, especially in patients with a strong clinical evidence, several activation methods such as SD may increase the diagnostic yield of the EEG. Interictal epileptiform discharges are strongly correlated with epilepsy. In our study SD in patients with epilepsy increased the yield for detection of epileptiform abnormalities. These results suggest that SDEEG should be the next step for supportive confirmation in patients with strongly suspected seizures with normal first routine EEG.

P687

Efficacy and tolerability of topiramate in monotherapy, and adjuvant therapy with carbamazepine, in comparison to carbamazepine monotherapy, in treatment of complex partial and generalised seizures

R. Abolfazli, H. Safaei, G. Esmaeeli Djavid, H. Sikaroodi, S. Samadzadeh Tehran University of Medical Science (Tehran, IR); Academic Center for Education, Culture and Research (Tehran, IR)

This study conducted to assess efficacy \& tolerability of Topiramate (TPM) in monotherapy and adjuvant therapy in comparison to Carbamazepine (CBZ) in refractory generalized tonic-clonic (GTC) and complex partial seizure (CPS) and evaluation of drug interactions between new and old Anti Epileptic Drugs

Methods: Two main groups of CBZ $(n=24)$ and TPM $(n=20)$ of GTC and complex partial refractory epilepsy patients were assessed for efficacy and tolerability of drugs in an open label controlled trial study. TPM group include TPM monotherapy $(n=8)$ and TPM adjuvant therapy with CBZ (TPM$\mathrm{CBZ})(\mathrm{n}=12)$. The patient should have GTC or complex partial epilepsies refractory to the maximally tolerable doses of one AED or insufficient efficacy(at least one period in month),for those who entered TPM adjuvant therapy with CBZ,they must be refractory to CBZ.Patient undergo 6 month treatment period and assessment were taken in pre treatment and 4 sessions during treatment (1st week, 1st month, 3rd month and 6th month). The primary efficacy variables were the seizure reduction rate (SRR), responder rate ( $>50 \%$ reduction in seizure frequency) \& seizure-free rate (SFR)

Results: Median pre-treatment seizure frequency has no significant differences between groups $(p=0.178)$. The Mean percentage of SRR has no significant differences between 3 groups $(P=0.139)$. Although it is lower in TPM-CBZ group. Percentage of responders also shows no significant difference between groups $(\mathrm{P}=0.523)$. SFR had significant difference between three groups, and was very low in TPM-CBZ group $(\mathrm{P}=0.008)$. The incidence of side effects was higher in the CBZ $(33.3 \%)$ than in the TPM or TPM-CBZ groups ( $25 \%$ both), but this shows no significant difference $(\mathrm{P}=0.832)$. Discontinuing treatment rate shows no significant difference between groups, although it was higher in TPM main group $(\mathrm{P}=0.368)$

Conclusion: CBZ and TPM as monotherapy were effective in refractory GTC and CPS. Putting all results together,there is a weak group (TPM-CBZ) in contrast to TPM mono therapy and CBZ mono therapy in efficacy and tolerance variables. The results of this study may be due to drug interaction between hepatic enzyme inducer AED (CBZ) and lower serum concentration and higher urine metabolites of TPM in presence of CBZ.The study results, suggest that it may be, not a good combination therapy for CBZ and TPM together, along with new evidences from pharmacokinetic studies of these drugs that confirm this idea.

\section{P688}

Role of ketogenic diet in treatment of refractory epilepsy in children

S. Ahluwalia, S. Singh, A. Singh, V. Kumar Singh

B.R.D. Medical School (New Delhi, IN); Medical Colllege (Sikkim, IN); GSVM Kanpur (Kanpur, IN)

Objective: In children with intractable epilepsy, the ketogenic diet is often more effective than other therapy.

Methods: The diet is effective in therapy-resistant patients. Although the mechanism by which the diet protects against seizures is unclear, there is evidence that it causes effects on intermediary metabolism that influence the the pattern of protection of the ketogenic diet. During consumption of the ketogenic diet, marked alterations in brain energy metabolism occur, with ketone bodies partly replacing glucose as fuel and responsible for anticonvulsant action of diet. 39 children were identified as surgical candidates, of whom 33 eventually had resective surgery and were followed subsequently. A comparison cohort group was also created of 31 children matched for age 
and seizure frequency that received the diet but were not surgical candidates.

Results: Of the 17 children who received both dietary and surgical therapies, there was a higher likelihood after 3 months of both $>90 \%$ seizure reduction and seizure freedom following surgery, both $p<0.0001$. Similarly, the 31 cohort children who were not surgical candidates were more likely to be seizure-free and remained on the diet for 6 month duration compared to the surgical group.

Conclusions: Children with both surgery and diet has more decreased in seizure frequency as compared to diet alone.

\section{P689}

The effect of oxcarbazepine and carbamazepine treatments on serum lipid levels

S. Kurt, H. Karaer, Y. Kaplan

Gaziosmanpasa University Faculty of Medicine (Tokat, TR); Inonu University, Turgut Ozal Medical Center (Malatya, TR)

Objective: The influence of antiepileptic drug (AED) therapy on serum lipid levels has been demostrated in many studies. There are very few and inconsistent studies each other in respect to the influence of oxcarbazepine on serum lipid levels.We aimed to investigate the effect of oxcarbazepine and carbamazepine on lipid levels.

Methods: In this retrospective study,all epileptic patients who were registered to our outpatient clinic between 1st January 2003-1st December 2007 and treated with oxcarbazepine and carbamazepine monotherapy were reviewed.The patients who had lipid levels were included.The patients data was compared with control group.

Results: The serum levels of LDL were significantly high in the patients who treated with CBZ (36 patients) and OXC (25 patients) compare to control group ( 30 healthy individuals), whereas serum levels of HDL was significantly low in comparison with control group. Statistically significance weren't determined between the levels of total cholesterol and triglyceride. Furthermore between serum LDL and HDL levels in the patients who treated OXC and CBZ monotherapy weren't statistically significance.

Conclusions: Both CBZ and OXC have negative effect on the serum lipid levels.But significantly difference wasn't determined between CBZ and OXC in terms of the effect on lipid levels.

P690

\section{Memory and epilepsy}

S. Shaafi

Tabriz University of Medical Science (Tabriz, IR)

Introduction: Epilepsy is among the most common neurologic disorders and affects over one million Iranian. Epilepsy is a disabling neurologic disorder of repeated seizures caused by excessive or abnormal brain electrical discharges.depending on the area of the brain affected, the seizure may appear in the form of convulsions, muscle jerks, a blank stare, or a loss of awareness. Patients with epilepsy frequently complain of memory difficulties.

The purpose of this study is to investigate compromised memory function of epileptic patients and determine the correlated factors.

Methods and materials: In this study we chose 100 epileptic patients with different type of seizure, 50 generalized tonic clonic seizure, 25 complex partial and 25 mixed seizure. MMSE test was done on each patient. In this study we evluated the prevalence of memory dysfunction and it's severity in epileptics. To finding the etiologic risk factors for development of memory difficulties, we evaluated the correlation between this problem and type of seizure, age of patient, duration of disease, anti epileptic drugs. We also determined the relationship between MMSE score and sex, age of onset of seizure.

Results: The prevalence of memory dysfunction was $83 \%$ (37\% mild, $44 \%$ moderate and $2 \%$ severe) The memory function in this studied group was adversely influenced by early age at onset of seizure, type of seizure, antiepileptic drugs and duration of disease. The scores in mixed seizure was meaningfully lower than generalized tonic clonic seizure. The memory effects on those patients taking carbamazpine -valproat were noticeably worse than those taking carbamazpine alone. Other factors such as sex and seizure frequency were not in correlatoin with severity of $\mathrm{m}$ emory dysfunction.

Conclusion: Early diagnosis of epilepsy and it's control is the important key factor in preventing memory decline. Medication selection and dosage adjustment need to be made that will minimize adverse effects and drug interaction, while maximizing seizure control.

\section{P691}

The efficacy of low-dose lamotrigine monotherpy in the epileptic patient

S.H. Kim, H.S. Kim

Dong-A University Hospital (Busan, KR)

Objective: Lamotrigine, a newer broad-spectrum antiepileptic drug, is widely used in adult and children with epilepsy. This study was designed to evaluate the efficacy and tolerability of low dose lamotrigine monotherapy in patient with newly diagnosed epilepsy.

Methods: We analyzed 61 outpatients with epilepsy, between May 14 2002 and May 25, 2006, who received $100 \mathrm{mg}$ /day lamotrigine as target maintenance dosages. Initiation dasage was started at $50 \mathrm{mg} /$ day for $1-2$ weeks based on tolerability and then maintenance dose of $100 \mathrm{mg} /$ day was continued. Effectiveness was measured by the retention rate at 1 year. In the patients with adverse events or continued seizure, lamotrigine was changed to other antiepileptic drug or increased to current maintenance dosage, respectively.

Results: Retention rate at 1 year was $45.8 \%$ in low dose lamotrigine monotherpy. The lamotrigine-induced rash occurred in 5 patients $(8.5 \%)$, where 3 patients developed a rash associated with the low dose lamotrigine therapy $(100 \mathrm{mg} /$ day $)$. Other adverse events were weight gain, memory disturbance, sedation, skin acne, sexual dysfunction and gastrointestinal discomfort.

Among the patients taken with maintenance dosage $(150 \mathrm{mg}-300 \mathrm{mg} /$ day) lamotrigine, seizure responder rates at 1 year was $31.3 \%$.

Conclusion: The efficacy of low dose lamotrigine monotherapy in the newly diagnosed epilepsy might be effectiveness. But we consider that it will be needed to be confirmed in multi-center and prospective case control studies.

\section{P692}

\section{Non paroxysmal EEG signs of brain damage in epilepsy}

V. Dakukina, N.N. Misiuk, T.V. Dokokina, A.O. Kozmidiadi, I.V. Matveichuk Medical University (Minsk, BY)

Quantitative EEG (QEEG)standards of normal functional state of the brain are: alpha-rhythm mean frequency $\leq 9.25 \mathrm{~Hz}$; alpha-rhythm peak frequency $\leq 9.25 \mathrm{~Hz}$; maximal values of slow-wave activity in delta-1,-2 and tetha-1,-2 diapasons $\leq 29 \%$ under frontal, central, parietal and occipital electrodes; spatial organization of beta frequency without focusing in central and parietal zone, spatial organization of alpha frequency with frontal < occipital gradient.

It is necessary to mark that these QEEG patterns of brain damage doesn't depend from visual pattern of brain electric activity. They are the authentic evidence of pathologic process actuality. They are reversible - any of them may be reduced in the result of correct anticonvulsant and neurometabolic therapy.

Thus, neurophysiologic characteristics of brain functional state may have a great diagnostic value for clinical neurologists occupied with epilepsy.

The aim of the investigation was to find non paroxysmal particularities of brain damage in epilepsy by mean of QEEG.

Objectives: 182 patients with epilepsy aged 18-55 years have undergone detailed clinical examination - psychic, neurological and somatic status was studied accurately. All of them were in active phase of the disease and have been subdivided in three groups according to etiology: symptomatic $(n=108$, idiopathic $(n=26)$ and cryptogenic one $(n=v 48)$.

Methods: Diagnostic QEEGpatterns have been composed of following parameters: alpha-rhythm mean frequency, alpha-rhythm peak frequency, maximal values of slow-wave activity in delta-1, delta- 2 , tetha- 1 and tetha- 2 diapasons,spatial organization of beta frequency,spatial organization of alpha frequency. EEG was performed on 19 channels and analyzed in spectral and period metric regimen by the use of "Mitsar"system.

Results: QEEG revealed patterns of organic brain damage in $81,9 \%$ of cases. Their diagnostic efficacy among groups was: $86.1 \%$ for symptomatic, $69.2 \%$ for idiopathic, $79,2 \%$ for cryptogenic epilepsy. The most sensitive patterns were: slowing of alpha-rhythm mean frequency, exceeding of slowwave activity values and beta-focus.

Conclusion: The data obtained during this study strongly fits the hypothesis that activity of epileptic process may retraced with the help of Quantitative EEG. These characteristics of the brain's functional state are very sensible and neurologists have possibility to take into account this information while prescribing correct therapy or depriving anticonvulsants. 
345) and prescription charge exemption records (017). The cohort was followed from birth until either onset of epilepsy, death, emigration, or $31 \mathrm{De}$ cember 2006. The association between gestosis and risk for epilepsy was analysed with cox proportional model ( $\mathrm{p}<0,05$, two-tailed test).

Results: Eleven individuals of our cohort ( 5 males and 6 females) developed long-term epilepsy. The relative risk of long-term epilepsy in individuals born from women with gestosis was 12,9 (95\% confidence interval, CI: $3,2-51,5)(\mathrm{p}=0,0003)$. The risk was significantly higher in the females: 24,0 (95\%CI: 4,8-118,7) $(\mathrm{p}=0,0001)$.

Conclusion: Gestosis might be a risk factor for long-term epilepsy. Its interaction with female gender suggests a role of hormonal factors in these patients' epileptogenesis.

We acknowledge Regione Autonoma della Sardegna (Assessorato dell' Igiene, Sanitè della Assistenza Sociale) for financial support.

\section{General neurology}

\section{P696}

Gait impairment and hypophosphataemia. A case of acquired hypophosphatemic "oncogenic" osteomalacia in a 40-year-old patient

A. Angelou, T. Afrantou, M. Paschalidou, I. Milonas, N. Tascos

Aristotle University of Thessaloniki (Thessaloniki, GR)

Introduction: Weakness of skeletal muscle with preserved or even brisk tendon reflexes is the most common manifestation of phosphate deficiency. In the majority of cases, hypophosphatemia results from malabsorption or hyperparathyroidism. Herein, we describe a patient who was admitted to the neurological department with a gait disorder and finally was given the diagnosis of acquired hypophosphatemic osteomalacia ( $\mathrm{AHO}$ ), a very rare syndrome which is usually associated with tumors of mesenchymal origin

Case report: A 40year old man was evaluated for progressive difficulty in walking and diffuse pain in his legs of 4 months duration. Examination revealed proximal muscle weakness producing a characteristic waddling gait, and exaggerated knee and ankle jerks. Conduction velocities, EMG study and spinal cord MRI were normal

Results from CSF analysis, routine blood tests and muscle enzymes were within normal range. However, an extensive laboratory workup revealed low phosphorus( $1,1 \mathrm{mg} / \mathrm{dl}, \mathrm{n} . \mathrm{v}: 2,5-4,5 \mathrm{mg} / \mathrm{dl})$ and normal calcium levels, normal levels of 25-hydroxy-cholecalciferole, low levels of 1,25-dihydroxycholecalciferole $(9,8 \mathrm{pg} / \mathrm{ml}, \mathrm{n} . \mathrm{v}: 29,6-65,1 \mathrm{pg} / \mathrm{ml})$, and phosphaturia $(460 \mathrm{mg} / 24 \mathrm{~h}$, n.v: $0-300 \mathrm{mg} / 24 \mathrm{~h})$. Although these findings were absolutely confirmatory for AHO, we carried on with a wide immunological screening, Bence-Jones protein, glucosuria, aminoaciduria, tumor markers, antiHu, antiYo, antiRi antibodies, which all failed to suggest an alternative diagnostic possibility. DEXA displayed low $\mathrm{T}$ and $\mathrm{Z}$ scores indicating osteopenia Octreoscan, usually performed in these cases for detection of possible tumor locations, was negative. Finally, a new spine MRI showed a vertebral hemangioma, but the patient refused to undergo surgical resection. Since then, he receives oral phosphate replacement therapy, and after 2 years of follow up his neurological state seems to be moderately improved

Discussion: AHO is associated with increased renal phosphate clearance and low circulating 1,25-dihydroxy-cholecalciferole. Many patients are shown to have benign tumors with prominent fibrous and vascular characteristics. To date, less than 150 cases of "oncogenic" AHO have been described and none has ever been reported in the neurological literature despite its clinical presentation which is dominated by muscle weakness and diffuse limb pain. Resection of the tumor, which is the treatment of choice, leads to the resolution of symptoms and normalization of laboratory findings.

P. Cossu, M. Pugliatti, A. Balata, T. Riise, G. Rosati

University of Sassari (Sassari, IT); University of Bergen (Bergen, NO)

Objectives: Epilepsy is a serious brain disorder characterised by recurrent, unprovoked seizures. The etiology of epilepsy can be documented in about one-third to one-half of cases. Few studies have evaluated the effect of environmental factors originating in early life. The current literature emphasises increased risk of adverse perinatal outcomes in the presence of severe gestational hypertension. The aim of this study was to estimate the association between gestosis and long-term risk of epilepsy persistent in adult life.

Methods: We carried out a retrospective cohort study of 1641 live newborns at the Sassari University Dept. of Obstetrics and Gynaecology between 1 January 1980 and 31 December 1981 using medical charts compiled by nurses, obstetricians and neonatologists. We obtained information on epileptic patients from the Epilepsy Case Register held at the University Dept. of Clinical Neurology, the Sassari Health District discharge (ICD-9M

\section{P697}

Changes of CSF cytokines levels in neuro-Behcet's disease

A. Borhani Haghighi, H. Ittehadi, J. Rahmati, S. Ghaffari Poorjahromi, B. Pourabbas, A. Ghaderi, A. Safari Shiraz University of Medical Sciences (Shiraz, IR)

Background: Neurological manifestations of Behçet's disease (Neuro-Behçet's disease) present in $5 \%$ to $30 \%$ of patients. Although cytokines play a pivotal role in pathogenesis of Behcet's disease, published studies about the cerebrospinal fluid levels of the cytokines in Neuro-Behçet's disease are scanty.

Methods and material: Nine patients with active parenchymal and one patient with non-parenchymal neuro-Behçet's disease, and six patients with 
headache attributed to Behcet's disease, 13 patients with viral meningitis, and 19 healthy controls were recruited. Cerebrospinal fluid (CSF) interleukin $6,8,10$, tumor necrotic factor-beta, and interferon-gamma were measured using enzyme-linked immunosorbent assay (ELISA) method.

Results: Patients with viral meningitis had significantly higher levels of all investigated cytokines in comparison with the patients with parenchymal neuro-Behçet's disease, headache attributed to Behcet's disease, and controls (all P values <0.05). CSF interleukin 6 was significantly higher in patients with parenchymal neuro-Behçet's disease in comparison with the controls $(\mathrm{P}=0.011)$ and patients with headache attributed to Behcet's disease $(P=0.019)$. CSF levels of investigated cytokines had no significant difference between patients with headache attributed to Behcet's disease and controls.

Conclusion: In spite of some previous studies, our investigation showed loss of analogy between CSF cytokine profiles of patients with parenchymal Neuro-Behçet's disease and viral meningitis. Also we postulated a crucial role for interleukin 6 in immunopathogenesis of neuro-Behçet's disease.

\section{P698}

Clinical heterogeneity of Hashimoto encephalopathy

A. Boutsi, A. Angelou, C. Balla, N. Taskos, M. Paschalidou

Ahepa University Hospital (Thessaloniki, GR)

Introduction: Hashimoto's encephalopathy (HE) is depicted as a rare and controversial clinical expression of autoimmune thyroiditis and is defined by the presence of high serum concentration of antithyroid antibodies and brain dysfunction in the absence of other obvious pathological process. Pathogenesis is unclear and the neurological spectrum is wide: confusion, seizures, myoclonus, tremor, cerebellar signs, stroke-like episodes, hallucinations, dementia. We describe 2 patients with suspected Hashimoto's encephalopathy.

First case: A 58-year-old woman, free of medical history, presented with an acute confusional state, gait disturbance, cerebellar signs and fever with blood leucocytosis. CT-Scan and lumbar puncture (L.P) were normal. Later she developed symptoms of upper respiratory infection but the persistence of pathological neurological symptoms led to further investigations. EEG revealed paroxysmal slowness on the left frontotemporal area and MRI showed mild cortical atrophy. Serum FT3, FT4 were low and TSH was elevated with an increase of thyroid auto-antibodies in serum and CSF. The patient received thyroxin with great clinical and EEG improvement

Second case: A 51-year-old woman with a history of Hashimoto's thyroiditis treated by L-Thyroxin was admitted with generalised status epilepticus. Her neurological examination, L.P and brain MRI were normal. She was treated with antiepileptic drugs being asymptomatic for nine months until she presented again an absence status epilepticus. After a modification in antiepileptics, she recovered but later on she became confused with disorientation, retrograde amnesia and hallucinations while the EEG was normal. MRI, CSF analysis and autoimmune markers were again normal except of an increase of thyroid serum auto-antibodies. The patient received i.v.corticosteroids for three days with complete recovery and continued per os for two months with tapering. After removing corticosteroids she had a relapse with the same encephalopathic symptoms, again with a normal EEG. She was put again on prednisolone $64 \mathrm{mgr}$ per day with a complete remission.

Discussion: The nature and course of Hashimoto's encephalopathy are still debated. The clinical improvement after L-thyroxin or corticosteroid administration in our cases suggests an underlying autoimmune process but there is no specific marker linking directly thyroid dysfunction and encephalopathy.

\section{P699}

Retropective evaluation of 33 patients with blepharospazm treated with botulinum A toxin

B. Arica, A.C. Sarikaya, M.C. Akbostanci, N. Mutluer

Ankara Univercity Medical School (Ankara, TR)

Bleparospasm is a focal dystonia with involuntary continuous or repetitive eye closure. It is a disease with unknown etiology, with female preponderance that is most frequently seen in patients above 50 . Symptoms are generally ameliorated while talking and looking down, and aggravated with walking and bright light. After botulinum A toxin injections, time to first symptom relief, effectiveness of the treatment (by a $0-100$ visual analog scale), duration of effectiveness, and side effects were recorded. There were 33 patients with blepharospasm (20 females) with a mean age of 54,5 (range $30-76$ ), treated in a total of 123 sessions. We analyzed the records of 19 patients who at least had two control visits (19 patients in 109 sessions). Mean time to first symptom amelioration was 6,0 days. Patients reported that they were $66 \%$ better when compared with their preinjection status. Duration of effectiveness was 2,3 months. Side effects were recorded in $33 \%$ of sessions, none of which had lasted more than 3 weeks. Side effects were: Ecchymosis in injection site after 16 sessions, failure to close eyes after 6 sessions, ptosis after 4 sessions, itching or burning eyes after 4 sessions, eye pain after 2 sessions and diplopia after one session. Our findings are in accordance with previously reported recovery (60-93\%) and side effect rates (11-18\%). Botulinum toxin treatment is safe and effective for patients with blepharospasm.

\section{P700}

Tongue pseudohypertrophy in idiopathic hypoglossal nerve palsy

D.N. Holle, O. Kastrup, S.-Y. Sheu, M. Obermann

University Hospital Essen (Essen, DE)

Objectives: Hypoglossal nerve palsy is a common finding in neurological diseases when associated with other cranial nerve palsy or further pathology. In general, hypoglossal

nerve palsy is associated with atrophy of the affected side of the tongue. Most of the them are caused by tumours. Different common causes are trauma, medullary infarctions,

multiple sclerosis, Guillain-Barrè neuropathy and infection. In contrast, isolated hypoglossal nerve palsy is a rare feature.

Case report: Here we present a case of uncommon idiopathic hypoglossal nerve palsy with atypical extensive neurogenic pseudohypertrophy of the tongue. A 42 year-old man

presented with slow progressive swelling of the left tongue over the last months and tongue deviation to the left with sudden onset two years ago. Additionally he had

noticed a difficulty to speak in terms of mild dysarthria. No other neurological deficit was present. His past medical history was unremarkable. Electromyography showed

complex repetitive discharges revealing a neurogenic lesion in the left genioglossal muscle. Magnetic resonance imaging (MRI) and biopsy showed fatty degeneration

and neurogenic atrophy of the left tongue. Malignancy, ischemic cerebral lesion, acute or chronic infection and vessel pathology were ruled out. However, the underlying pathology remained unclear.

Conclusion: Isolated hypoglossal nerve palsy is an uncommon finding.Therefore, the most common reasons are tumours or idiopathic nature but Chiari malformation, dural arteriovenous fistula, encephalomyelitis and pseudianeurysm of the internal carotid artery are also described as underlying conditions of isolated hypoglossal nerve palsy. Additionally, our patient presented with an atypical pseudohypertrophy of the tongue. The reason for this swelling is most probably a marked so called "neurogenic hypertrophy" sometimes seen in leg muscles in patients with chronic rootdamage. Therapy and outcome of isolated hypoglossal nerve palsy mainly depend on the underlying cause.

However, an excellent outcome is reported for idiopathic hypoglossal nerve palsy.

P701

Severe refractory stiff-person syndrome responding to rituximab therapy E. Whetton, J. Ealing, I. Bruce, D. McKee

University of Manchester (Manchester, UK); Hope Hospital Salford (Manchester, UK); Manchester Royal Infirmary (Manchester, UK)

Case report: A 48 year old woman with type 2 diabetes mellitus developed progressive walking difficulties and painful muscle spasms in the legs. On examination she walked with a stiff-legged gait and an exaggerated lumbar lordosis. There was hypertonia of the legs with associated axial rigidity, superimposed upon which bursts of myoclonic-type jerks of the paraspinal and abdominal muscles were observed.

Magnetic resonance imaging of the spine and cerebrospinal fluid examination were normal. Neurophysiological examination showed simultaneous contraction and co-contraction of agonist and antagonist muscle groups typical of stiff person syndrome (SPS). This was confirmed by the presence of very high titres of antibodies to glutamic acid decarboxylase in the serum. Her condition rapidly progressed until she became bedbound with severe rhythmic spasms of the abdominal and thoracic muscles starting to interrupt respiration (video 1 ). Serum creatine phosphokinase levels rose to the degree that rhabdomyolyis was feared and she was transferred to the intensive care unit for therapeutic neuromuscular blockade, intubation and artificial ventilation.

Treatment with steroids, plasmapheresis and benzodiazepines allowed an initial recovery followed by a prolonged period of rehabilitation during which she became independently mobile. Several months later she began to deteriorate with worsening mobility and increasingly severe lower limb muscle spasms. High dose steroid treatment provoked psychosis. Intra- 
venous immunoglobulin therapy was unsuccessful. Further plasmapheresis was complicated by line infection and septic shock. At this point treatment with rituximab was given. Within six weeks she had become independently mobile, and several months later she was able to manage at home without support (video 2). The improvement has been maintained over twelve months with maintenance mycophenolate mofetil (MMF) and diazepam, and on examination there are only minimal signs of SPS.

Discussion: The patient described developed severe acute SPS progressing to incipient rhabdomyolysis and respiratory failure. An initial response to standard therapy was followed by a relapse which proved refractory to treatment until rituximab therapy achieved remission, subsequently maintained by MMF. This case adds weight to a previously published case report that rituximab may be successful in the treatment of otherwise refractory severe SPS.

\section{P702}

Left cerebral hemi-atrophy related to central nervous system vasculitis: a case report

F. Esposito, M. Rodegher, L. Straffi, B. Benedetti, V. Martinelli, W. Brück, G. Comi

San Raffaele Institute (Milan, IT); University Medical Centre (Göttingen, $\mathrm{DE})$

Objective: To report on an unusual case of left hemispheric vasculitis in a patient with progressive cognitive impairment.

Methods: Observational case report.

Results: 4 years ago a 33-year-old female began to complain of language difficulties, mainly characterized by anomias, and mnesic deficit. A brain MRI showed moderate atrophy of the left cerebral hemisphere, with multiple lesions in periventricular region, centrum semiovale and cerebellar hemisphere; all the lesions were located in the left hemisphere and a few of them showed enhancement after gadolinium infusion. Oligoclonal bands were detected in the cerebrospinal fluid and a diagnosis of possible multiple sclerosis was made. The cognitive impairment progressively worsened and after 2 years the patient had reading difficulties, semantic paraphasias and mnesic deficit for recent events. Moreover she became very shy, developed a marked lack of self-confidence and she complained of right hemianopsia and right leg weakness. A new brain MRI demonstrated a worsening of the selective left hemi-atrophy and an increased number of white matter lesions, most of them with enhancement. A brain SPECT study showed a hypoperfusion of the left hemisphere, mainly in the occipital lobe. Lysosomal enzyme test was normal. Some months later the patient complained of sudden hyposthenia at the right arm. She was treated with a high dose of methylprednisolone, with improvement of the weakness. She was subsequently hospitalised in our department, where a brain MRI with spectroscopy revealed an increase in the choline/creatine ratio in the lesions, whereas the $\mathrm{N}$-acetyl aspartate peak was normal. Glutamate receptor antibodies (antiGluR3) were negative. A brain biopsy revealed perivascular lymphomononuclear infiltrates, mostly T lymphocytes and macrophages, and CD20-positive B cells. The neuropathological features confirmed the clinical suspect of vasculitis. According to the rapid progression of the disease and the biopsy result, the patient was treated with alemtuzumab.

Conclusions: This is the first case described in the literature of left cerebral hemi-atrophy related to central nervous system vasculitis. Brain biopsy, even if dangerous and invasive, should be considered as indispensable in patients with clinical aggressive course and atypical MRI features, in order to make a definite diagnosis and start a specific therapy.

\section{P703}

Extensive intramedullary lesion: an uncommon presenting form of neurosarcoidosis

F. Sierra Hidalgo, E. Correas Callero, E. de Pablo Fernández, A. Herrero San Martín, J. Herreros Rodríguez, J.P. Romero Muñoz, A.C. Martínez de Aragón Calvo, T. Moreno Ramos

Hospital Universitario 12 de Octubre (Madrid, ES)

Objectives: Neurological involvement in sarcoidosis, when exists, appears soon in the disease, and it is the presentation form in about $10 \%$ of cases. Spinal sarcoidosis is rare, less than $0,5 \%$ of the cases of neurosarcoidosis. Here we present an uncommon case.

Methods: A 53-years-old woman presented with gait disturbance and several falls appearing progressivelly in the last 2 months. She also complained of cough, asthenia and weight loss of 10 kilograms in the last 8 months. Neurologic examination revealed diminished strenght in both legs, generalized hyperreflexia with bilateral Babinski sign, and spastic gait. She had not sensation or sphincters disturbances, nor meningeal signs. Results: Blood analyses showed raised white cells, erythrocyte sedimenta- tion rate and immunoglobulins. Cerebrospinal fluid analysis showed lymphocytic pleocytosis, elevated proteins and normal glucose, with oligoclonal bands. Microbiologic studies in blood and cerebrospinal fluid were all negative. Citology of cerebrospinal fluid only showed mature T cells. Spinal Magnetic Resonance images showed an extensive longitudinal gadoliniumenhanced intramedullary lesion from cervicomedullary junction to the conus medullaris. Chest X-ray and CT-scan showed bilateral pulmonary infiltrates. A lung biopsy demonstrated non-caseating granulomas. Other causes of lung granulomatosis were excluded, and the diagnosis of neurosarcoidosis was made. Treatment with oral Prednisone (50 miligrams a day) was begun and the patient had a good recovery after 21 days.

Conclusion: Myelitis is a rare form of presentation of neurosarcoidosis. It is important to have a high-grade suspicion and make an early diagnosis in order to improve the prognosis of short-term motor function.

\section{P704}

Primary amyloidoma of the central nervous system H. Foreid, A.C. Fonseca, J. Sá, C. Barroso, T. Evangelista Hospital de Santa Maria (Lisbon, PT)

Objectives: To report a case of a brain amyloidoma including spectroscopic data. Due to the rarity of this disorder we have also performed a review of the literature.

Methods: Presentation of clinical manifestations and aspects of ancillary diagnostic exams found in a case of primary brain amyloidoma. Computed Tomography (CT) scans, Magnetic Resonance Imaging (MRI), histological and immunohistochemical aspects of brain biopsy as well as brain spectroscopy were performed. Comparative analyses of spectroscopic data and a review of the literature were done.

Results: We report on a 51-year-old male, with a 7 years history of epilepsy and progressive functional deterioration that was admitted for a generalized tonic-clonic seizure. Neurological examination disclosed cognitive impairment and aphasia. CT scan revealed periventricular posterior hypodensities that were hypointense on T1-weighted MRI, heterogeneous on T2-weighted MRI, and enhanced with gadolinium. On pathological examination various deposits of an amorphous coalescent material were found in white matter and in the majority of vascular walls. This eosinophilic material was birefringent on Congo Red and stained positively with transthyretin antibody. Investigation for systemic amyloidosis was negative. Spectroscopy revealed increased choline peak, decreased $\mathrm{N}$-acetyl-aspartate (NAA) peak and no lactate doublet.

Conclusion: Nodular deposition of amyloid in the brain is a very rare form of central nervous system amyloidosis, being this case the 18th one reported. Our data is in agreement with previously reported clinical and histological aspects. We have only found one previous description of spectroscopic analysis. When compared, both cases showed increased choline and low NAA peaks that can be interpreted as increased turnover and decreased cellular viability as in neoformative lesions. The only difference was the presence of a lactate doublet, absent in our patient's exam. The lactate doublet seen in the first spectroscopic study of amyloidoma could be attributed to a different metabolic phase with higher anaerobic metabolism and the eventual presence of necrotic and infarcted tissue within the lesion.

\section{P705}

Seizure and psychosis as debut of a HSV-2 encephalitis in an inmmunocompetent woman

J. Fernandez Dominguez, L. Gabaldon Torres, M.J. Aguilar-Amat Prior, M.J. Abenza, F.J. Arpa Gutierrez

Hospital Universitario La Paz (Madrid, ES)

Introduction: Encephalitis is the most common nervous central system (NCS) infection. Herpes simplex virus (HSV) is the most frequent etiological agent, especially HSV-1 in adults, being the type 2 (HSV-2) more common among babies.

Material and methods: We present a 32-year-old inmunocompetent homosexual woman who was accepted in our hospital for a bizarre case of seizures followed by an infantile behaviour that continued during all her stance at the hospital. Blood systematic analysis, EEG, lumbar puncture (LP) and cephalorraquid fluid studies (CFS), as well as neuroimaging techniques (Cranial CT and MRI, PET-CT) were done.

Results: The first MRI developed was normal. The preliminary result of the CFS was unspecific and the serologic screening as well. A second MRI was done due to clinical worsening of the patient, without pathological findings. PCR for the Herpes Virus group was done, with a positive result for HSV-2. Finally a PET-TAC showed a pathological metabolism in the left fronto-parieto-temporal regions.

Conclusions: Even when the behaviour alterations are not the typical de- 
but for an herpetic encephalitis, they are possible. The fact of having two conventional neuroimaging without pathological findings is unusual as well. HSV-2 as pathological agent is mostly seen in children and inmunocompromised adults. The positive result for a HVS PCR in CSF has not only high sensibility but specificity as well. Sometimes a PET-TAC is required to make the correct diagnosis of this disease.

\section{P706}

Pharmacological profile of perampanel: a novel, non-competitive selective AMPA receptor antagonist

N. Tokuhara, S. Hatakeyama, H. Amino, T. Hanada, Y. Nishizawa

Eisai Co., Ltd (Tsukuba, JP)

Objective: To evaluate the in vitro pharmacological profile of perampanel, an oral non-competitive selective AMPA receptor antagonist.

Background: Inhibition of AMPA receptors to reduce glutamate signaling and any associated excitotoxicity offers a potentially unique approach for treatment of certain neurological disorders. Perampanel is a novel noncompetitive selective AMPA receptor antagonist that has shown efficacy in preclinical models of Parkinson's disease and epilepsy.

Methods: The effect of perampanel on AMPA-mediated intracellular $\mathrm{Ca} 2+$ concentration changes in cultured cortical rat neurons was examined with the chelating dye Fura II-AM. The effects of perampanel and talampanel on AMPA-induced currents were measured by whole cell patch clamp recordings in cultured rat hippocampal neurons. Effect of perampanel on radioligand interactions with 63 physiologically relevant receptors, enzymes, and transporters were determined using established in vitro assays.

Results: The IC50 for perampanel inhibition of AMPA-induced elevation of [Ca2+] was $93 \mathrm{nM}$. Perampanel had no physiologically meaningful effect on radioligand binding in any of the assay systems used. Patch clamp studies showed a distinct effect of perampanel $(10 \mu \mathrm{M})$ on AMPA-induced current compared with talampanel $(10 \mu \mathrm{M})$.

Conclusion: These preclinical findings suggest that perampanel, a noncompetitive selective AMPA receptor antagonist, has a unique pharmacological profile. This profile supports perampanel's potential use in diseases associated with excessive glutamate signaling and neurotoxicity, such as epilepsy and Parkinson's disease.

Authors are employed by Eisai Co., Ltd.

\section{P707}

Use of intravenous immunoglobulin in clinical practice: a retrospective claims database analysis

P. Bonnet, H. Friedman

Baxter BioScience (Westlake Village, US); Analytic Consulting, Inc (New York, US)

Background: Intravenous Immunoglobulin (IVIG) is a plasma-derived product used to treat a wide variety of disorders. Dosage and frequency vary depending on the condition being treated. The objective of this study was to determine the most common diagnoses associated with IVIG therapy, and examine frequency of administration and dosing used to treat various disorders.

Methods: Pharmacy claims for IVIG (Gammagard Liquid and Gammagard S/D, Baxter Healthcare Corporation) were extracted from a US homecare company dataset for the year 2006. For every claim, the following information was available: patients' age, dose shipped, frequency of administration and diagnosis. Patient weight was obtained by matching patient age to the corresponding national average weight from the Center for Disease Control and Prevention (CDC). Dose per claim (in mg/kg) and frequency of administration were examined across indications. Descriptive statistics were utilized in this analysis.

Results: The database included 10,167 pharmacy claims from 1,068 patients. Approximately $64 \%$ of these patients were prescribed IVIG for Immune Deficiency -ID-. The other diagnoses found in the database were Chronic Inflammatory Demyelinating Polyneuritis -CIDP- (12\% of the patients), Peripheral Neuropathy -PN- (8\%), Multiple Sclerosis -MS- (5\%), Dermato/Polymyositis -DMPM- (4\%), and Myasthenia Gravis -MG- (2\%). Four patients were prescribed IVIG for Inflammatory and Toxic Neuropathy -ITN- and three for Stiff-man syndrome -SM-. The average doses of IVIG prescribed for ID, MS and MG were 473,799 and $820 \mathrm{mg} / \mathrm{kg}$, respectively. Claims for other neurological indications (CIDP, DMPM, ITN, PN and SM) ranged from 932 to $1212 \mathrm{mg} / \mathrm{kg}$ (median: $1033 \mathrm{mg} / \mathrm{kg}$ ). In the majority of cases, IVIG was administered every 3 to 4 weeks for ID, CIDP, DMPM and SM. IVIG seemed to be administered more frequently (every week or every other week) for PN, MG and MS in this patient population.

Conclusion: This database analysis demonstrated that IVIG is often used in clinical practice for neurological indications and provided insight on commonly used doses.

Dr. Patrick Bonnet is employed by Baxter Healthcare Corporation

\section{P708}

Collet-Sicard syndrome

S. Bek, O. Arslan, G. Genc, U. Ulas, Z. Odabasi

Gulhane Military Medical Academy (Ankara, TR)

Introduction: Multiple cranial neuropathies can be seen quite often in neurological daily practice. The Collet-Sicard syndrome, unilateral lesion of lower four cranial nerves (CNs) (IX, X, XI, XII) without Horner's syndrome, is a rare syndrome. We report an idiopathic recurrent Collet-Sicard syndrome.

Case Report: A 32 year-old dentist had a gradual onset of hoarseness and dysarthria. His complaints got worse in first two weeks and gradually decreased. He has been admitted the neurology department in the fourth week of the symptoms onset. His medical history was unremarkable except, he had the same symptoms 7 years ago. He diagnosed as Collet-Sicard syndrome and prescribed prednisolone $60 \mathrm{mg} /$ day per orally for 3 months. His symptoms had resolved completely in 2 months. On examination, he had a right sided palsies of the four lower cranial nerves, which manifested as a droop of the soft palate to the right, deviation of uvula to the left, loss of gag reflexes on the right, weakness of right upper trapezius and sternocleidomastoideus muscles, deviation of the tongue to the right, slurred speech, hoarseness, difficulty in swallowing and frequent choking. He had neither Horner's syndrome nor taste disturbance. His cervical vertebra and skull base computerized tomography was completely normal. No vascular abnormality was seen in carotid magnetic resonance (MR) angiography. Brain MR was normal except left maxillary sinusitis and no contrast enhancement was seen in cerebrum. As the patient's signs and symptoms were improving on his admission in the fourth week, we decided to observe without any steroid treatment. On his sixth week follow-up, neurological examination was normal without any residual deficit.

Discussion: Multiple cranial neuropathies have been commented as selflimited nature of the syndrome and tendency to reoccur but to our knowledge idiopathic recurrent Collet-Sicard syndrome has never been reported. Multiple cranial neuropathies, accepted as idiopathic, may have viral origin although can not be shown definitely.

\section{P709}

Novel biocompatible nanoparticles for metal chelation therapy in neurological disorders associated with trace metal imbalance

S. Davaran, R. Khandaghi, A. Khani

Tabriz University of Medical Science (Tabriz, IR); Imam Khomeini Hospital (Tabriz, IR); Shahid Beheshti University of Medical Science (Tabriz, IR)

Objectives: The transition metals, such as iron, copper, zinc and aluminum have both functional and toxic roles in the CNS. These metals have been found in high concentrations in the brains of patients with Alzheimer disease $(\mathrm{AD})$. Thus the strategy of lowering brain metal ions and targeting the interaction of beta-amyloid (A beta) peptide and metal ions through the adminstration of chelators has merit. The recent findings that nanoparticle delivery systems can cross the blood- brain barrier (BBB), has led neuroscience researchers to investigate whether chelators conjugated to nanoparticles could act to reveres metal ion induced protein precipitation.

Methods: We have synthesized a novel transition metals chelator that can be used to conjugate with biocompatible polymeric nanoparticles. We have also tested the

metal binding properties of the chelators and some biological properties such as the in vitro ability of metal removal from tissue sections of Alzheimer's disease brain. The novel transition metals chelator was covalently conjugated to poly (N-isopropyl crylamide; PNIPAAm) with functional groups such as thio and carboxyl groups on its surface for covalently bonding with chelators via thioether or ester bonds. Then, we quantitatively measured the amount of chelators that conjugated to the particles and determined the ability of the chelator-particle systems to bind metals such as iron. We have also determined the human-plasma-protein-absorption patterns.

Results: The synthesized chelators removed iron from ferritin and were capable of removing iron from tissue sections of AD brain. Through changing the system-surface properties, the chelator-particle systems can preferentially absorb ApoE.This novel chelator-nanoparticle system, when incubated in human plasma, preferentially absorb ApoAI.

Conclusion: These studies indicate that the novel chelator-nanoparticle systems have the potential to enter brain and bring excess metals out of the 
brain thereby prevent metal-associated oxidative damage. Our results also show the potential to optimize the property of chelator-nanoparticle systems by changing polymer structure, linkages, and nanoparticles with different size. The preferential absorptions of ApoAI.allow the systems to mimic the ApoE or ApoAI nanoparticles and cross the BBB through LDL transport mechanism.

\section{P710}

Misleading One Detail (MODE): a preventable mode of diagnostic error?

S. Khoury, T. Ben Hur, S. Arzy

Hadassah Hebrew University Hospital (Jerusalem, IL)

Objective: Despite advances in our understanding of cognitive biases in clinical practice, little is known about correction or prevention of diagnostic errors. The presence of a single misleading detail may lead clinicians down a cognitive and actual path toward an incorrect diagnosis.

Methods: We surveyed 51 attending physicians in neurology and internal medicine departments, presenting each with 10 clinical vignettes and soliciting their diagnosis of the condition leading to the presentation. Each of the 10 clinical cases included a single misleading detail.

Results: This survey elicited a wrong diagnosis in $90 \%$ of cases, which was reduced to $30 \%$ when omitting the misleading detail from the vignette. Diagnostic accuracy did not improve by warning physicians about potentially misleading information. Asking physicians to identify a leading diagnostic detail and then to formulate an alternative diagnosis after omission of the detail, significantly reduced diagnostic error-rate by nearly $50 \%$.

Conclusion: Systematic re-examination of leading diagnostic clues may help to reduce errors in diagnosis.

\section{Multiple sclerosis}

P711

Cytotoxic T lymphocyte associated antigen-4 (CTLA-4) Exon $1 \mathrm{~A} / \mathrm{G}$ polymorphism in Iranian patients with multiple sclerosis

A. Borhani Haghighi, S. Ghahramani, N. Azarpira, M. Pourjafar, A. Safari

Shiraz University of Medical Sciences (Shiraz, IR)

Background: Cytotoxic T lymphocyte antigen-4 (CTLA-4) is a T cell surface receptor of activated $\mathrm{T}$ cells.

Material and methods: We studied 100 Iranian patients with clinically definite multiple sclerosis (MS) and 100 ethnic, sex and age matched controls. CTLA-4 exon $1 \mathrm{~A} / \mathrm{G}$ polymorphism was compared in patients and controls.

Results: There was no statistically significant difference in the allelic $(\mathrm{P}=0.503)$ and genotypes $(\mathrm{P}=0.66)$ distribution among patients and controls. Also gender, course and progression index did not reveal any statistically significant differences between the groups with different genotypes and alleles.

Conclusion: As a non-European patient population, our results are consistent with the major previous studies showing no significant associations between CTLA4 exon 1 polymorphism and neither MS nor any of its subtypes.

\section{P712}

Not only disability and psychological condition influence on fatigue of patients with multiple sclerosis

A. Gruszczak, K. Pocinska, Z. Stelmasiak, H. Bartosik-Psujek

Clinical Hospital number 4 in Lublin (Lublin, PL)

Objective: Fatigue is one of the most frequent and most disabling symptoms in multiple sclerosis (MS). Because of multidimensional concept of fatigue, physiological, psychological and social aspects should be concerned. The aim of the study was to analyze correlations between the level of fatigue and another parameters of health related quality of life and sociodemografic and clinical data.

Material: Between January and November 2007, 122 inpatients with different type of clinically definite MS were investigated in Department of Neurology, Medical University of Lublin. Mean age was 37,65 $\pm 8,9$ years, mean disease duration 6,68 $\pm 6,3$ years, mean Expanded Disability Status Scale (EDSS) score $4,06 \pm 1,89.67,21 \%$ of patients were women, $32,79 \%$ men, $61,47 \%$ urban dwellers and $38,53 \%$ country dwellers; $66,39 \%$ were married and $33,61 \%$ single; $50,81 \%$ disability pensioners, $35,27 \%$ white collar workers, $10,65 \%$ blue collar workers and 3,27\% unemployed. Multiple Sclerosis
Quality of Life Inventory (MSQLI) was used, which consists of 138 questions organized in 10 scales, generic and specific, but in this study only RAND 36Item Health Survey 1.0 Questionnaire (SF-36) and Modified Fatigue Impact Scale (MFIS) were analyzed. Sociodemografic and connected with disease data were also collected. The disability of patients was estimated using EDSS. The results were statistically analyzed.

Results: All 3 subscales of MFIS significantly correlated with all 8 subscales of SF-36. But the highest Spearman's correlation ratio was obtained for MFIS and Vitality Scale (VT) and Mental Health Scale (MH). Considering subscales of MFIS: for Physical Subscale with Physical Functioning Scale (PF), Role-Physical Scale (RP), Vitality Scale(VT); for Cognitive Subscale with Mental Health Scale (MH); for Psychosocial Subscale with Vitality Scale (VT), Social Functioning Scale (SF). Positive correlation between MFIS and EDSS was obtained (the highest for Physical Subscale) but not with a time of duration and a course of disease, age, sex, domicile, civil state and education. MFIS total score is also significantly correlated with occupational activity and material situation of patients, the disability pensioners obtained higher scores than white collar workers, similarly the poorest person's vs average rich ones.

Conclusions: The results prove that fatigue of MS patients is strictly dependent not only on their disability and psychological condition but also on fulfilled social roles.

\section{P713}

Botulinum toxin type A (Botox $\left.{ }^{\circledR}\right)$ injection in bladder dysfunction in patients with multiple sclerosis (Ain Shams experience)

A. Nassef, T. Osman

Ain Shams University (Cairo, EG)

Background: Bladder dysfunction is very common in patients with multiple sclerosis (MS), affecting up to $60-90 \%$ of patients with MS at any stage during the course of their disease. Bladder dysfunction in MS patients can be classified into (a) Detrusor hyperreflexia or overactivity and (b) Detrusor external sphincter dyssynergia (DESD).

Aim of the study: To evaluate the effect of botulinum toxin type A (BTXA) (BOTOX) in the management of urinary dysfunction in MS patients.

Methods: Fifteen (15) clinically definite MS patients, according to Poser criteria, of the relapsing "Cremitting type(RR) were admitted in Ain Shams University Specialized Hospital (ASUSH) during one year period, from October 2004 to October 2005 and assessed: clinically, neurophysiologically and radiologically by MRI brain and spinal cord. Urodynamic study was done to classify the type of bladder dysfunction. Patient were treated with either 300 units of botulinum toxin type A (BTX-A) (botox) injected in the bladder base and trigone in cases of detrusor hyperreflexia or 100 to 200 units of botox injection divided in equal doses into the four quadrants of the external sphincter in cases of DESD.

Results: Nine patients (60\%) had detrusor hyperactivity (hyperreflexia.) "Group A" and 6 patients $(40 \%)$ had detrusor external sphincter dyssynergia, DESD "Group B". The maximum efficacy of BTX-A injection occurred between 7 and 21 days following the injection. Follow up duration ranged from 3-6 months with continuous symptoms improvement. In bladder wall treatment group (group A) with bladder wall hyperreflexia there were highly significant decrease in the number of micturations per 24 hours and highly significant increase in the cystometric capacity $(p<0.001)$ after injection. In urethral treatment group (group B) with DESD there were highly significant decrease in the mean postvoid residual urine volume and in the maximum voiding pressure $(\mathrm{P}<0.001)$.

Conclusion: Our finding showed that the urodynamic study is an essential tool in diagnosing bladder dysfunction in MS patients and botulinum toxin type $\mathrm{A}$ is a promising tool of management of the urinary dysfunction in patients with MS. More study should be done on the use of BTX-A in other neurological disorder with urinary disturbances.

\section{P714}

Premature ovarian failure prevention during mitoxantrone treatment in multiple sclerosis women

C. Rivoiro, M. Clerico, G. Contessa, A. Tavella, D. Viglietti, P. Ripellino, P. Barbero, L. Durelli

San Luigi Gonzaga University Hospital (Orbassano, IT)

Objectives: Mitoxantrone has been approved for the treatment of worsening progressive Multiple Sclerosis (MS) on the basis of two controlled studies. Premature ovarian failure (POF) with consequent irreversible amenorrhea and infertility is a common long-term consequence of chemotherapics. This is particularly relevant in MS which often affects women of reproductive age and where mitoxantrone have been associated with $10-20 \%$ of secondary irreversible amenorrhea. Preliminary studies on Systemic Lupus Erythemato- 
sus (LES) women treated with cyclophoshamide showed that Gonadotropinreleasing hormone-analogue (GnRH-analogue) treatment significantly prevented POF. To prevent POF in mitoxantrone-treated MS women in reproductive age with $\mathrm{GnRH}$-analogue is the main objective of this study.

Methods: A monthly intramuscular depot injection of $3.75 \mathrm{mg}$ Triptorelin (Gonapeptyl depot sir 3,75mg so Ferring Spa) was administered after informed consent to 12 relapsing or progressive MS women treated with mitoxantrone. Hormonal treatment continued for all the mitoxantrone cycle. Mitoxantrone was given at the dose of $12 \mathrm{mg} / \mathrm{m}^{2}$ every month for 6 months in relapsing-remitting patients or at the dose of $12 \mathrm{mg} / \mathrm{m}^{2}$ every 3 months for 18 months in secondary progressive patients (tailored to individual leukocyte count).To assess the preservation of the ovarian function we measured Follicle-Stimulating Hormone (FSH), Luteinising-hormone (LH), 17beta estradiol and progesterone level every month, before, during and after the injection of $\mathrm{GnRH}$-analogue, during the therapy with mitoxantrone. Diagnosis of POF was based on secondary amenorrhea and on FSH levels $\geq 40 \mathrm{mIU} / \mathrm{m}$ for at least 12 months.Kaplan-Meier survival estimates was used to calculate the cumulative preservation of ovarian function. Significance was tested with a Cox regression"Cbased model and Cox proportional hazards regression was used to estimate the hazard ratio of ovarian failure.

Results: All patients underwent periodical neurological and gynaecological control and no one have any significant side effects from the GnRH-analogue therapy, besides transitory weight increase, flushing and injection site pain.

Six of the patients treated with GnRH-analogue finished the mitoxantrone cycle and returned to normal menstrual cycles, with hormonal test confirming the fertility.

Conclusion: Gonadotropin-releasing hormone-analogue GnRH-analogue treatment in women with MS in therapy with mitoxantrone can prevent POF.

P715

Relationship between NMO-IgG seropositivity and BBB damage in demyelinating diseases

C. Tortorella, M. Ruggieri, R. Leante, G.B. Zimatore, A. Frigeri, A. Lia, E. Di Monte, A. Ghezzi, A. Rizzo, M.P. Amato, B. Hakiki, F. Patti, A. Lugaresi, E. Merelli, P. Livrea, M. Svelto, M. Trojano

University of Bari (Bari, IT); Osp. Gallarate (Gallarate, IT); University of Firenze (Firenze, IT); University of Catania (Catania, IT); University of Chieti (Chieti, IT); University of Modena (Modena, IT)

Objectives: Neuromyelitis optica (NMO) is an inflammatory demyelinating disease that selectively affects optic nerves and spinal cord. It is considered a severe variant of multiple sclerosis (MS), and frequently is misdiagnosed as MS, but prognosis and optimal treatments differ. A serum immunoglobulin $\mathrm{G}$ autoantibody (NMO-IgG) serves as a specific marker for NMO. Here we show a correlation study, in patients with different demyelinating disease phenotypes, between NMO-IgG seropositivity and clinical, cerebrospinal fluid (CSF) and MRI parameters.

Methods: We examined 124 patients with various demyelinating disease phenotypes: 42 definite multiple sclerosis (MS), 19 possible MS, 21 NMO, 11 optic neuritis, 17 myelitis and 14 recurrent form of optic neuritis or myelitis. Seventy-five coeval serum and CSF samples were available. NMO-IgG was detected by indirect immunofluorescence.

Results: Nineteen patients were NMO-IgG seropositive: 9 NMO, 3 myelitis, 2 optic neuritis, 4 recurrent form of myelitis or optic neuritis and one possible MS. The specificity for NMO was $98 \%$ vs MS and $91 \%$ vs other demyelinating diseases; the sensitivity was $43 \%$ vs both categories. No differences were found in demographic and clinical parameters between NMOIgG seropositive and seronegative patients. Mean CSF/serum albumin ratio (QA) and frequency of pathological QA values were significantly higher in NMO-IgG seropositive vs seronegative, irrespective of clinical diagnosis $(p=0.0 .23 ; p=0.009)$. No differences were found in CSF white cells, nor IgGindex. Intrathecal IgG oligoclonal bands (OB) were found with similar frequency in seropositive and seronegative group, when only non-MS patients were considered. Brain MRI was more frequently negative $(p=0.06)$ whereas spinal MRI lesions were more often longer than 2 vertebral segments $(\mathrm{p}=0.003)$ in NMO-IgG seropositive group.

Conclusion: Our preliminary data suggest that NMO-IgG seropositivity identify a sub-group of demyelinating diseases mainly characterized by BBB damage, absence of $\mathrm{OB}$ synthesis and extensive spinal lesions. A pathogenetic role of NMO-IgG in BBB impairment might be speculated.
P716

Higher exposure to interferon beta- $1 b$ is associated with improved longterm outcomes but the effect is substantially reduced by delaying treatment D. Goodin, G. Ebers, A. Reder, T. Traboulsee, D. Langdon, K. Beckmann, J. Jones

University of California (San Francisco, US); University of Oxford (Oxford UK); University of Chicago (Chicago, US); University of British Columbia (Vancouver, CA); University of London (London, UK); Bayer Schering Pharma AG (Berlin, DE); Bayer HealthCare Pharmaceuticals Inc (Wayne, US)

Objectives: Cross-trial comparisons and anecdotal reports suggest that a relationship exists between the timing of exposure to disease-modifying treatment (DMT) in multiple sclerosis (MS) and the relative effect of the DMT. This study utilized the Betaferon(r) 16-Year Long-Term Follow-up (IFNB 1b LTF) study data to investigate the relationship between the timing of drug exposure and long-term undesirable clinical outcomes (secondary progressive MS, wheelchair requirement, or death).

Methods: The details of the IFNB-1b LTF follow-up study are outlined elsewhere. For this analysis, a variety of theoretical weighting schemes were applied to the IFNB-1b drug exposure as measured by a medication possession ratio (MPR; proportion of time on therapy before undesirable event). These schemes either increased or decreased the MPR based on time since first symptom and on Expanded Disability Status Scale (EDSS) score at the start of drug exposure. A recursive partitioning algorithm was employed to: (1) determine the optimal weighing scheme (that which best predicted longterm outcome) and (2) divide the patients into "more" and "less" exposure groups. Adjustment techniques, including propensity scoring, were applied to reduce bias in evaluating the relationship between weighted exposure and outcomes. Extensive sensitivity analyses were run using different definitions of long-term outcome, assumptions about the data, and statistical adjustment techniques.

Results: Both an increase in time between first symptom and starting DMT treatment, and EDSS at the time of DMT initiation substantially reduce effective drug exposure. For example, delaying treatment 2 years from the first reported symptom in a patient with an EDSS $=1.5$ results in an estimated $40 \%$ reduction in treatment effect. After applying the optimal weighting scheme, and using propensity scoring to reduce bias, the "more" exposure group has a significantly lower risk of an undesirable clinical outcome (Cox hazard ratio $=0.420,95 \%$ confidence limits: $0.277-0.636, p<0.001$ ). Sensitivity analyses confirm the robustness of the results.

Conclusions: Increased exposure to IFNB-1b reduces the risk of undesirable clinical outcomes. The magnitude of the effect of delaying treatment (time from first symptom or higher EDSS) on undesirable clinical outcomes is substantial.

This study was supported by Bayer Schering Pharma AG, Berlin, Germany

\section{P717}

Association between adherence with multiple sclerosis disease-modifying therapy and severe relapses using 3 measures of medication adherence D. Meletiche, M. Dickson, C. Kozma, D. Okuda, C. Fincher, R. Bennett, A. ALSabbagh

EMD Serono, Inc. (Rockland, US); University of South Carolina (Columbia, US); University of California (San Francisco, US)

Objective: Adherence to disease-modifying drugs (DMDs) for multiple sclerosis (MS) is an important component of MS treatment and may help prevent relapses. This study evaluated the relationship between 3 measures of medication adherence for MS drug therapy and the likelihood of a severe relapse.

Methods: Patients were selected from the PharMetrics (Watertown, MA) database if they had at least 1 claim for interferon beta-1a intramuscular (IM), interferon beta- $1 \mathrm{~b}$, interferon beta-1a subcutaneous (SC), or glatiramer acetate from January 1, 2000 through December 31,2004. Patients who were continuously eligible for 24 months following their first DMD prescription (index data) and 6 months before the index data (preperiod) were evaluated. Patients who were younger than 18 years of age or older than 65 years of age, had received natalizumab after the index date, used a study drug in a healthcare facility, or resided in a long-term care facility were excluded from the study. A severe MS relapse was defined as an MS-related hospitalization or emergency room visit. Three different methods were used to assess medication adherence: medication possession ratio (MPR), consistence, and persistence. Covariates included age, sex, United States (US) geographic region, treatment status, comorbidities, and use of mono- or multidrug therapy.

Results: 2388 patients met the study criteria. Patients had a mean age of 43.9 years; $76.4 \%$ were women, $76.7 \%$ were new patients, and $8.1 \%$ had at 
least 1 severe MS relapse. A significant association between increased adherence and decreased likelihood of severe MS relapse was observed using each of the 3 adherence measures with odds ratios of 0.921 (MPR), 0.895 (consistence), and 0.946 (persistence). Other significant covariates $(\mathrm{P}<0.05)$ in all models were comorbidity and East region of the United States (using Midwest region as the reference). Age, gender, and the other regions were not significant at alpha $=0.05$.

Conclusion: Increased adherence was significantly associated with a decreased risk of severe relapse as assessed by 3 distinct drug adherence measures. These results are consistent with previously reported findings on maximum gap in drug therapy and risk of severe MS relapse.

This study was supported by EMD Serono, Inc. and Pfizer Inc.

\section{P718}

Selectin gene cluster genetic variation: association study in two independent multiple sclerosis populations

D. Scalabrini, C. Fenoglio, L. Piccio, A. Cross, M. De Riz, E. Venturelli, F. Cortini, C. Villa, M. Piola, B. Parks, J. Rinker, N. Bresolin, E. Scarpini, D. Galimberti

University of Milan, IRCCS Osp Maggiore (Milan, IT); Washington University School of Medicine (St. Louis, US)

Objective: To perform a case-control study in two Multiple Sclerosis (MS) populations for the known Single Nucleotide Polymorphisms (SNPs) reported in selectin gene cluster coding sequences (rs6133, rs4987310 and rs5368 in P-selectin, L-selectin and E-selectin genes respectively) in order to determine whether the presence of these variants influences the susceptibility to MS.

Background: Selectins are a group of adhesion molecules involved in the recruitment of lymphocytes from the blood into the central nervous system.

The selectin cluster maps to 1q23-25. Several regions on chromosome 1 have been associated with MS. The first high-powered admixture scan on African American cases and controls identified a locus in the centromeric region of chromosome 1, while other studies reported the region 1q44 as susceptibility locus for MS. In combination, this evidence makes the selectin genes good positional and functional candidates for involvement in the pathogenesis of MS.

Methods: 165 Italian patients and 122 American cases with MS as well as two series of same-size populations of controls were genotyped for rs6133 (G/T), rs4987310 (C/T) and rs5368 (C/T) SNPs in P-selectin, L-selectin and E-selectin genes, respectively. Allelic discrimination was performed using an ABI PRISM 7000 instrument (ABI). Haplotypes were tested for association by using the Haploview software.

Results: Haploview analysis revealed that only rs4987310 and rs5368 markers were in strong $\mathrm{LD}\left(\mathrm{D}^{\prime}=0.94\right)$ in both populations. A decreased frequency of TC haplotype was found only in the American MS population compared with controls $(3.8 \%$ versus $11.7 \%, \mathrm{P}=0.04)$. Conversely, any difference was found in the frequency of rs6133, rs4987310 and rs5368 between patients and controls in both populations.

Conclusion: The presence of the TC haplotype on chromosome 1 selectin gene cluster is likely to act as protective factor toward the risk of developing MS in American population. These preliminary results suggest that the selectins gene cluster may be the locus responsible for the strong linkage found on chromosome 1 in Americans with MS, whereas it doesn't seem to influence MS susceptibility in the Italian MS cohort. Confirmatory analysis on larger populations is needed to draw definitive conclusions.

P719

Treatment response to interferon beta: cytokine, chemokine, and chemokine receptor mRNA levels in peripheral blood of multiple sclerosis patients correlate with interferon dose

G. Contessa, C. Rivoiro, A. Cucci, M. Clerico, P. Barbero, B. Ferrero, P. Ripellino, D. Viglietti, L. Durelli

Clinical and Biological Sciences-University of Torino (Orbassano, IT)

Objective: MS is thought to be a T helper 1 (Th1) mediated disorder of central nervous system and cytokines and chemokines produced by these cells appear to be involved in the pathogenesis of the inflammatory demyelination. The levels of pro-inflammatory (Th1 type) and anti-inflammatory (Th2 type) cytokines have been, in fact, found to correlate with signs of disease activity. The aim of this study is to assess the correlation between different levels of cytokine, chemokine, and chemokine receptor mRNA in relapsing remitting(RR)-MS patients treated with different doses of interferon(IFN) beta-1b and different treatment responses.

Methods: Real time-PCR (Taqman, ABI PRISMTM 7900 Sequence detection system) in whole blood of 38 RR-MS patients was used to quantify
mRNA levels of 23 immunological molecules (chemokines: CCL3, CCL5, CXCL10, MCP-1; chemokine receptors: CCR1, CCR5, CXCR3; cytokines: IFN-beta, IFN-gamma,IL-10,IL-12a,IL-12b,IL-18,IL-1-b,IL-4,IL-5,IL-8,TNFalpha, TGF-beta;IL-1-receptor- antagonist;MMP-9;TIMP-1;VLA-4). Suboptimal responders were identified during the first 6 months of IFN beta-1b treatment, $250 \mathrm{mcg}$, every other day (EOD), on the basis of persisting MRI activity or of the occurrence of clinical relapses. These patients were thereafter randomised to receive either the increase dose of IFN beta $375 \mathrm{mcg}$ EOD or to continue the standard dose.

Results: Twenty patients had a suboptimal response to IFN beta treatment. mRNA levels of IL-18 $(p=0.02)$, IFN-gamma $(p=0.03)$, CXCL10 $(p=0.05)$, TNF-alpha $(p=0.03)$ were significantly increased, and those of IL$10(p=0.05)$ and TGF-beta $(p=0.05)$ significantly decreased in suboptimal responders with a peak at Month 3 or 6 of treatment. After increasing IFN beta dose, mRNA levels of IL-18, IFN-gamma, CXCL10, TNF-alpha decreased and those of IL-10 and TGF-beta increased in parallel with a significant decrease of the MRI signs of disease activity. The higher IFN beta-1b dose was not associated with an increased frequency of side effects.

Conclusions: Only the patients with an optimal response to IFN beta treatment had decreased level of pro-inflammatory and increased level of anti-inflammatory cytokines and chemokines. In patients with a suboptimal response to IFN beta, the increased of IFN beta-1b dose from 250 to $375 \mathrm{mcg}$ was associated both with decreased MRI signs of disease activity and with a shift of the pro-inflammatory/anti-inflammatory cytokine and chemokine balance toward a Th2 pattern.

\section{P720}

Safety and tolerability of Rebif ${ }^{\circledR}$ New Formulation (interferon beta-1a) in patients with relapsing multiple sclerosis: results from a phase IIIb study G. Giovannoni, O. Barbarash, F. Casset-Semanaz, J. King, L. Metz, G. Pardo, J. Simsarian, P. Soelberg Sørensen, B. Stubinski on behalf of the Rebif ${ }^{\circledR}$ New Formulation Study Group

Objectives: To investigate the safety and tolerability profile of the new formulation of Rebif ${ }^{\circledR}$ (subcutaneous [sc] interferon [IFN] beta-1a; Rebif ${ }^{\circledR}$ New Formulation) in patients with relapsing multiple sclerosis (MS). Data were compared with findings from historical and recent studies - the EVIDENCE (EVidence of Interferon Dose-response: European North American Comparative Efficacy) and REGARD (REbif versus Glatiramer Acetate in Relapsing MS Disease) studies, respectively - with the current Rebif $\oplus^{\circledR}$ formulation.

Methods: Rebif New Formulation ${ }^{\circledR}$ was developed with the aim of improving tolerability and reducing immunogenicity, and is produced without foetal bovine serum and without human albumin as an excipient. This was a Phase IIIb, 96-week, single-arm, multicentre, open-label study of Rebif ${ }^{\circledR}$ New Formulation (44 mcg sc three times weekly) in patients aged 18-60 years with relapsing MS (McDonald criteria; Expanded Disability Status Scale score <6.0). Pre-specified adverse event (AE) categories commonly or occasionally associated with IFN beta-1a were summarized and analysed by combining preferred terms.

Results: Injection-site reactions (ISRs), which included injection-site pain, irritation and erythema, were reported by $30.8 \%$ of patients, versus $85.8 \%$ and $41.2 \%$ in the EVIDENCE and REGARD studies, respectively, and $71.5 \%$ versus $49.0 \%$ and $36.0 \%$ of patients reported flu-like symptoms. Depression/suicidal ideation was reported by $6.5 \%$ versus $22.7 \%$ and $9.4 \%$, respectively. Other pre-specified AE categories were reported by similar proportions of patients in this study as in the EVIDENCE and REGARD studies. The majority of patients experiencing AEs reported mild or moderate events. The incidence of serious AEs (reported by $5.8 \%$ of patients) was similar to that in the EVIDENCE $(8.6 \%)$ and REGARD (7.6\%) studies. There were no unexpected effects on liver enzymes or blood cell counts, and most recorded liver enzyme elevations and cytopenias were of low toxicity grade (based on common terminology criteria for AEs).

Conclusions: These data suggest that, overall, Rebif ${ }^{\circledR}$ New Formulation has a favourable safety and tolerability profile compared with the current formulation of Rebif $\Theta$, particularly with respect to ISRs, which may affect treatment adherence. Local-tissue ISRs are a frequently reported AE associated with sc IFN beta treatment in MS. The tolerability and side-effects of MS treatments, including local-tissue AEs such as ISRs, can influence adherence to therapy.

Study supported by Merck Serono International S.A. 
P721

Early clinical presentation as prognostic factor in multiple sclerosis

G. Iuliano, R. Napoletano

OO.RR. Salerno (Salerno, IT)

Background: Natural history as description of multiple sclerosis (MS) course is being replaced by observation of patients treated as in everyday practice. On our cohort we assess clinical prognostic factors so to find indicators for early therapy.

Patients and methods: 131 patients, $34 \mathrm{~m}$, 97f, age at onset $28.94 \mathrm{yrs}$ (11-50), with complete history of attacks, were included; follow up duration mean 11,6 yrs (2-34). Variables were: Number (N.) of systems at onset: $1=73 ; 2=35 ; 3=3 ; 4=4$; optical neuritis: 29 pts; first inter-attack interval: 27.29 months (1-204); Attacks in the first 2years:mean 1.85 (1-6); Years before DM therapy: $5.42(0-26)$; Therapy; Diagnosis made at onset; outcome variables, EDSS at $0-2$ yrs: $\mathrm{N}=108$; median $=1(0-4.0)$, at $4-6$ yrs: $\mathrm{N}=121$; median $=1,5(0-5.5)$; at $8-10$ yrs: $\mathrm{N}=95$; median $=1.5(0-6.5)$; at $13-15$ yrs: $\mathrm{N}=52$; median $=2.5(0-7,5)$; Shift to secondary progression (SP); time to reach EDSS 3 and EDSS 6 . Statistics were made by Multiple Regression, Kruskal-Wallis, Odds Ratios and Kaplan-Meier curves.

Results: At short time, EDSS at 1yr $(\mathrm{N}=97)$ correlates with the age at onset $(p=0.001)$ and the $N$. of systems at onset $(p=0.000)$. EDSS at 10yrs $(\mathrm{N}=74)$ is in correlation with sex $(\mathrm{p}=0.001)$ optical neuritis $(\mathrm{p}=0.003)$ and attacks in the first two years $(\mathrm{p}=0.008)$ and EDSS at 1 year $(\mathrm{p}=0.001)$. At 15 years EDSS $(\mathrm{N}=37)$ is correlated only with EDSS at $1 \mathrm{yr}(\mathrm{p}=0.000)$. The time to reach EDSS $3(\mathrm{~N}=47)$ is correlated with age at onset $(\mathrm{p}=0.003)$, N.of systems at onset $(p=0.000)$, attacks in the first two years $(p=0.012)$, EDSS at 1 yr $(p=0.000)$, and has direct correlation with the starting of therapy $(p=0.000)$ (longer interval, longer time to reach EDSS 3$)$. Shift to SP is significantly higher in patients with delayed time of diagnosis (Odds Ratio 2.80, $\mathrm{p}=0.03$ )

Discussion: Our data agree with most literature. In short time, older age at onset and higher number of systems interested at onset correlate with EDSS at 1 year. In long time, EDSS at 10 yrs is correlated with sex, optical neuritis, attacks in the first two years, and EDSS at 1 year. At 15 years only early (1 yr) EDSS is correlated with the clinical status. Age and systems at onset, attacks in the first two years, and EDSS at $1 \mathrm{yr}$ shorten the time to reach EDSS 3 . Secondary progression is more common in patients with late diagnosis. We believe it could be useful evaluating, for early treatment, patients with more systems interested at onset, and incomplete recovery after the first attack, in particular if relatively elderly.

\section{P722}

An open-label randomised clinical trial of atorvastatin in combination with IFN Beta 1a in active multiple sclerosis patients: preliminary results G. Orefice, M. Quarantelli, R. Lanzillo, A. Brunetti, P. Salvatore, A. Prinster, G. Vacca, C. Mollica, V. Bonavita, V. Brescia Morra, A. Prinster

Federico II University (Naples, IT); Hermitage Capodimonte (Naples, IT); CNR (Naples, IT)

Background: Statins seem to be with therapeutical potential in immune mediated disorders such as Multiple Sclerosis (MS). Our aim was to determine if treatment with atorvastatin in combination with IFN beta 1a was safe and well tolerated and if it increased therapeutic benefits.

Methods: Relapsing Remitting (RR) MS patients, aged 18-50 years, with clinical or neuroradiological activity on therapy with IFN Beta 1a $44 \mathrm{mcg}$ three times a week for no more then 12 months and at least six months, were randomized in two groups: treatment with IFN alone or in combination with atorvastatin $20 \mathrm{mg}$ per day, for 24 months. Subjects had urine and blood analysis and were assessed by means of Expanded Disability Status Scale (EDSS) every three months. Brain MRI with Gadolinium injection and segmentation post-processing and neuropsychological evaluation were performed at screening and after 12 and 24 months. Primary outcome measure was MRI activity with secondary outcomes of clinical relapses, EDSS variation and safety laboratory data. Pearson test and Fisher test were used when appropriate.

Results: 45 subject were enrolled and randomized to combination therapy (24) of IFN alone (21). At baseline neuroradiological activity, disease duration and EDSS were comparable. Data at 12 months were available for 21 patients in combination arm and 20 patients in IFN alone arm. Data at 24 months were available for 10 patients and 9 patients respectively. Two patients on combination therapy and 3 patients on IFN alone therapy dropped out. Patients with enhancing lesions at MRI decreased from $52 \%$ and $50 \%$ respectively at baseline to $20 \%$ at 12 months and $12 \%$ at 24 months in the first group and to $28 \%$ at 12 months and $14 \%$ at 24 months in second group. Relapses on treatment decrease from a median of 1 relapses per patient to a median of 0 relapses in the second year in both groups, but only in the first group we recorded 0 relapses during the second year while in the second group relapses were 4 over 12 patients. EDSS was stable at 12 and 24 months in both groups. No clinically relevant blood analysis alteration was observed in both groups.

Conclusions: Combination therapy of atorvastatin and IFN Beta 1a was safe and well tolerated. Association therapy is likely to be beneficial especially during the second year on clinical disease activity. Complete clinical, neuropsychological and instrumental data at 24 months will clarify the likely beneficial effect of the association.

\section{P723}

The BENEFIT studies: adherence, tolerability and quality of life with Betaferon ${ }^{\circledR}$ treatment after a first event suggestive of multiple sclerosis

H-P. Hartung, G. Edan, M. Freedman, L. Kappos, D. Miller, X. Montalban, C. Polman, L. Bauer, V. Lanius, C. Pohl, R. Sandbrink

Heinrich-Heine-Universitat (Dusseldorf, DE); Centre Hospitalier Universitaire (Rennes, FR); Ottawa Hospital (Ottawa, CA); University Hospital (Basel, CH); National Hospital for Neurology and Neurosurgery (London, UK); Hospital Vall d'Hebron (Barcelona, ES); Vrije Universiteit Medical Centre (Amsterdam, NL); Bayer Schering Pharma AG (Berlin, DE)

Objectives: To assess adherence to treatment with interferon beta-1b (IFNB$1 \mathrm{~b}$; Betaferon $(\mathrm{r}))$, its tolerability, and quality of life (QoL) in patients with a first event suggestive of multiple sclerosis (MS).

Methods: The BENEFIT (BEtaferon ${ }^{\circledR} /$ BEtaseron ${ }^{\circledR}$ in Newly Emerging multiple sclerosis For Initial Treatment) project is investigating the impact of early versus delayed IFNB-1b treatment on the course of MS over a period of up to 5 years. In the randomised phase of BENEFIT, patients with a first demyelinating event and magnetic resonance imaging (MRI) findings suggestive of MS were treated with IFNB-1b $250 \mathrm{mcg}$ or placebo, subcutaneously, every other day, for 2 years or until diagnosed with clinically definite MS (if earlier). Patients were then eligible to enter the follow-up phase with the option of receiving IFNB-1b for up to 5 years after randomisation. Patients and physicians remain blinded to the original treatment allocation. Adverse events were documented and health-related QoL assessed using the Functional Assessment of MS-Trial Outcome Index (FAMS-TOI) and the EuroQol 5-Dimensional (EQ-5D) questionnaire at clinic visits.

Results: Of the 468 patients randomised to IFNB-1b $(n=292)$ or placebo $(\mathrm{n}=176), 437(93.4 \%)$ completed the double-blind phase, and $418(89.3 \%)$ were enrolled into follow-up. Of these, $378(80.8 \%)$ chose IFNB-1b treatment and $343(73.3 \%)$ were still on treatment at 3 years. Adverse events were within the expected range for IFNB-1b treatment, with no new safety signals arising. FAMS-TOI scores remained high and stable throughout the study and EQ-5D scores 3 years after randomisation favoured the early treatment group $(\mathrm{p}=0.016)$.

Conclusion: IFNB-1b is well-tolerated in patients with a first event suggestive of MS. The high proportion of patients choosing IFNB-1b treatment in the BENEFIT follow-up study and the high adherence rate after 3 years show that patients accepted this treatment. High and stable QoL ratings underline the tolerability of IFNB-1b.

Study supported by: Bayer Schering Pharma AG, Berlin, Germany

\section{P724}

Treatment with glatiramer acetate reduces MRI activity in patients with clinically isolated syndrome suggestive of multiple sclerosis M. Filippi, G. Comi on behalf of the PreCISe study group

Objective: To evaluate the efficacy of early treatment with glatiramer acetate $\left(\mathrm{GA}, \mathrm{COPAXONE}^{\circledR}\right)$ on MRI metrics in patients with clinically isolated syndrome (CIS) suggestive of MS.

Background: GA reduced relapse rate and MRI-monitored disease activity in relapsing-remitting (RR) MS patients. This three year, randomized, placebo-controlled, multicenter trial, assessed the efficacy of GA therapy initiated shortly after the first clinical event in CIS patients.

Design/Methods: Patients at presentation with CIS and at least two T2weighted brain lesions, with a size of at least six mm were enrolled into the study. Only patients with a unifocal disease manifestation were included. They were randomized to receive either sc $20 \mathrm{mg} /$ day GA or placebo. The primary efficacy outcome was time to clinically definite (CD) MS, based on a second clinical attack. MRI metrics served as secondary and exploratory end-points. A preplanned interim analysis was performed on data acquired from approximately $80 \%$ of the three-year study exposure.

Results: A total of 481 patients were randomized to receive GA $(n=243)$ or placebo $(n=238)$. At baseline, the two study groups did not differ in terms of clinical (age, time from first event to randomization, corticosteroid use for first attack, EDSS) and MRI (number and volume of T2 weighted lesions, and number and volume of gadolinium enhancing lesions) metrics. Further to 
the reduction in the risk of developing CDMS by $45 \%$ compared to placebo (hazard ratio of time to CDMS $0.55, \mathrm{p}=0.0005$ ), those patients receiving GA also experienced a reduced number of new T2-weighted lesions by $58 \%$ $(\mathrm{p}<0.0001)$ and of exposure-adjusted T2-weighted lesions volume ( $\mathrm{p}$ value $=0.0002$ ). Analysis of drug effect on the number and volume of enhancing lesions, black holes, and brain atrophy will also be performed and presented at the meeting. GA was well tolerated, with $16 \%$ overall withdrawals to the time of the interim analysis, and a safety profile similar to that observed in RRMS.

Conclusions/relevance: The results establish efficacy of early treatment with GA in both delaying time to CDMS and in reducing MRI activity in patients at presentation with CIS.

The Study was funded by TEVA Pharmaceutical Industries

P725

HLA DR2 \& DQ6 $(D R B 1 * 1501, D Q A 1 * 0102, D Q B 1 * 0602)$ haplotype associated genetic susceptibility in Iranian multiple sclerosis patients

M. Ghabaee, H. Sadeghian, A. Bayati

Iranian Center of Neurological Research (Tehran, IR)

Multiple sclerosis (MS) is protype of inflammatory demyelinating disease of the central nervous system. The etiology of MS remains unclear, but according to current data the disease develops in genetically susceptible individuals and may require additional environmental triggers. The human leukocyte antigen (HLA) class II alleles (DRB1* $\left.{ }^{\star} 1501, \mathrm{DQA} 1^{*} 0102, \mathrm{DQB} 1^{\star} 0602\right)$ is the strongest genetic effect identified in MS. In this study, the role of these alleles were investigated in 183 Iranian multiple sclerosis patients and compared with 100 healthy individuals. HLA typing for $\mathrm{DRB1}^{\star} 1501, \mathrm{DQA} 1^{\star} 0102$, $\mathrm{DQB} 1 * 0602$ was performed by polymerase chain reaction (PCR) amplification with sequence-specific primers (PCR-SSP) method. The results show that, HLA DR $B 1^{\star} 1501$ was significantly more frequent among MS patients ( $46 \%$ vs. $20 \%, \mathrm{PV}=0.0006)$ but $\mathrm{DQA1} * 0102$ haplotype was negatively associated with MS ( $30 \%$ vs. $50 \%, \mathrm{PV}=0.0049)$ and no significant association was found with $\mathrm{DQB} 1 * 0602$ and MS patients in compared with control groups $(24 \%$ and $30 \%, \mathrm{PV}=0.43)$. No significant correlation was observed among these alleles with sex, type of disease; initial symptoms, expanded disability status scale (EDSS), as well as age at onset and familial MS. This study therefore indicates that there is positive association of the HLA * ${ }^{1501}$ haplotype with MS, but none of haplotypes had association with demographic data and clinical symptoms of MS patients which are in line with other studies too.

P726

Absence of anti-myelin antibodies in clinically isolated syndrome indicate a delayed conversion to multiple sclerosis - A 3-year follow-up in a Swiss cohort

O. Findling, I. Greeve, J. Sellner, T. Lauterburg, U. Walker, K.M. Rösler, H.P. Mattle

Inselspital, Berne University Hospital and University of Berne (Berne, $\mathrm{CH}$ )

Background: There is a continuing debate regarding the value of antimyelin-oligodendrocyte-glycoprotein (MOG) and anti-myelin-basic-protein (MBP) antibodies for predicting the conversion from a clinically isolated syndrome (CIS) to clinically definite multiple sclerosis (CDMS). Previously, we reported that the absence of MOG and MBP antibodies is associated with a low risk for CDMS in a Swiss CIS cohort with a mean followup time of 12 months (Acta Neurol Scand 2007).

Aim of the study: We aimed to determine whether anti-myelin antibodies remain a prognostic factor in CIS patients for progression to CDMS during an observation period of 3 years.

Methods: Among the previously reported cohort, 17 of 39 patients had not converted to CDMS during the observation period of 12 month. These patients were evaluated again for conversion to CDMS. The diagnosis of CDMS was established accordingly the McDonald criteria by a second confirmed clinical relapse.

Results: The mean follow up time was 33.2 months $( \pm 7.0)$. Five of the 9 patients who had been negative for both antibodies converted to CDMS (mean time to CDMS $22.9, \mathrm{~b} 9.5$ months). Ten of 11 patients positive for antiMOG and 15 of 19 positive for both anti MOG-and anti-MBP developed CDMS during the observation period (mean time to CDMS $11.9 \pm 8.4$ and $10.6 \pm 9.8$, respectively). There was a significant risk reduction for developing CDMS in patients negative for both antibodies (Breslow generalized Wilcoxon test; $p=0.036$ ). The time to conversion to CDMS was significant longer (Mann-Whitney Test; $p=0.009)$ for the antibody negative patients compared to the patients testing positive for anti-MOG or both anti-MOG and anti-MBP antibodies.
Conclusion: CIS patients who had tested negative for anti-MOG and antiMBP antibodieshad a significantly lower risk to convert to CDMS compared with patients who tested positive for antimyelin antibodies in this 3 year follow-up study. Furthermore the time from CIS to CDMS is significantly prolonged in patients testing negative for both anti-myelin antibodies. Our results show that anti-myelin antibodies may be helpful to select CIS patients for immunomodulatory treatment and determination of individual prognosis.

P727

Parenteral cladribine in patients with relapsing-remitting multiple sclerosis: results of a 2-year, double-blind, placebo-controlled, cross-over study to assess effects on relapses and disease progression

P. Grieb, Z. Stelmasiak, J. Solski, J. Nowicki, B. Jakubowska, M. Ryba

Polish Academy of Sciences (Warsaw, PL); School of Medicine (Lublin, PL)

Objectives: To confirm the efficacy and safety of cladribine in the treatment of relapsing-remitting multiple sclerosis (RRMS), reported previously in an open-label pilot study, in a 2-year, double-blind, placebo-controlled, crossover study.

Methods: Patients with active RRMS (Expanded Disability Status Scale [EDSS] score 1-7) were randomized to receive: cladribine during year 1 and placebo during year 2 (Group A); or placebo during year 1 and cladribine during year 2 (Group B). Cladribine ( $0.07 \mathrm{mg} / \mathrm{kg} /$ day for 5 days) was administered subcutaneously at monthly intervals for 6 months, and at month 9 (cumulative dose: $2.45 \mathrm{mg} / \mathrm{kg}$ ). Neurological examinations and blood counts were performed at baseline and before each treatment course. Steroids were available for the symptomatic relief of severe clinical relapses.

Results: In total, 84 patients (Group A: $\mathrm{n}=40$; Group B: $\mathrm{n}=44$ ) aged 21-51 years were enrolled; 80 patients completed the study. Baseline mean (standard deviation) EDSS scores were 3.86 (1.90) in Group A and 3.98 (1.90) in Group B, and annualized relapse rates were 0.86 and 1.05 , respectively. In year 1, the patient-reported relapse rates were reduced by $73 \%$ (Group A) and $41 \%$ (Group B) versus the pre-study rate. In year 2, relapse rates in Groups A and B were $30 \%$ and $43 \%$ lower than the baseline relapse rate, respectively. The rate of steroid use to treat relapses (number of steroid therapies/patient/year) was lower for patients receiving cladribine than for those receiving placebo (year 1: 0.15 [Group A] and 0.43 [Group B]; year 2: 0.29 [Group A] and 0.21 [Group B]). The average EDSS score in Group A improved during cladribine therapy and regressed towards baseline after crossover to placebo. In Group B, patient EDSS scores remained stable. A total of 57 infectious episodes requiring treatment were noted. The incidence of infections requiring treatment (number of infections/patient/year) was slightly higher during and after cladribine treatment (year 1: 0.40 [Group A] and 0.14 [Group B]; year 2: 0.50 [Group A] and 0.38 [Group B]); however, all events were manageable and non-serious. Reductions in lymphocyte counts to $40-45 \%$ of baseline values were noted after the 2-year study in both groups.

Conclusions: Cladribine achieved a marked reduction in relapse rate; cladribine also reduced the severity of relapses as assessed by the use of steroids.

Supported by the Foundation for Development of Diagnosis and Therapy, Warsaw, Poland

P728

Plasma exchange for severe attacks of CNS inflammatory demyelinating diseases: review of 22 cases

S. Llufriu, Y. Blanco, L. Ramio-Torrenta, J. Lopez, M. Valles, M. Lozano, F. Graus, A. Saiz

Hospital Clinic Barcelona (Barcelona, ES); Hospital Josep Trueta (Girona, ES)

Background/objectives: Previous studies suggest that plasma exchange (PE) could be useful across the full spectrum of severe CNS demyelinating disease. To review our experience with PE for severe attacks of inflammatory demyelinating unresponsive to corticosteroids.

Methods: We reviewed the medical records of 22 patients who had been consecutively treated by PE for severe attacks of inflammatory demyelinating diseases from April 2000 to July 2007 at two university based neurology clinics. Patient diagnoses at onset were established by clinical criteria and some diagnoses were revised based on follow-up. The medical records were analyzed for the patient demographic details, disability at onset, results of the treatment and variables related to the outcome, and the complications of the procedure. Improvement was defined as at least a 1 point decrease in the EDSS (Expanded Disability Status Scale) score.

Results: 13 (59\%) patients were women. The median age at onset was 34 
years (range, 18-51 y). At PE initiation, the syndrome was classified as multiple sclerosis (MS) in 10 cases, acute disseminated encephalomyelitis (ADEM) in 6, transverse myelitis in 2, Marburg variant of MS in 2 and optic neuritis in 2. After follow-up, 1 ADEM was revised to first relapse of MS. Thirteen (59\%) were neurologically normal, and $9(41 \%)$ had a median EDSS of 4.0 (range 1.0-6.0) before the attack. All patients received corticosteroids for their attack before PE, and were severely disabled (median EDSS 6.0, range 3.0-9.5). At discharge 12/22 (54.5\%) patients had improved (median decrease 1 point, range 1-3) and at 6 months 16 (72.7\%) (median decrease 3 points, range 1-6).

One MS patient who had improved at discharge had a relapse 4 months later increasing his EDSS score. PE was well tolerated without major adverse events.

Conclusions: Response to PE was observed in $72.7 \%$ of patients with a variety of acute attack of CNS inflammatory demyelinating diseases, and most of them did so at short- term. PE was well tolerated and should be tried in those patients with persistent severe neurologic deficits following treatment with corticosteroids.

P729

Apoptosis-related molecules in multiple sclerosis during and after pregnancy

S. Rinta, L. Airas, I. Elovaara

University of Tampere (Tampere, FI); University of Turku (Turku, FI)

Objectives: Multiple Sclerosis (MS) ameliorates typically during pregnancy but after delivery the relapse rate often increases. We hypothesize that during MS pregnancy apoptosis of T cells is enhanced. Aim of this study was to examine whether the disease amelioration could be explained by changes in the expression of apoptosis-related molecules in sera.

Methods: Serum samples were collected during third trimester and 3-6 months after delivery from 19 pregnant relapsing-remitting MS patients (RRMS) [age $29.8 \pm 3.0$ years, disease duration $5.6 \pm 3.8$ years and 11 healthy age-matched pregnant women. Among 19 RRMS patients 6 patients were treated with interferon-beta and 3 with glatiramer acetate before gestation. The serum levels of TNF-related apoptosis-inducing ligand (TRAIL) were determined by using ELISA and Fas, Fas Ligand (FasL) and macrophage migration inhibitory factor (MIF) were determined by Luminex suspension array system. The differences between prepartum and postpartum levels in MS patients and controls were compared by the Wilcoxon test and Mann-Whittney U-test.

Results: Postpartum in RRMS patients the median levels of serum TRAIL ( 303.2 vs. $580.7 \mathrm{pg} / \mathrm{ml}, \mathrm{p}=0.01)$ and FasL (113.4 vs. $170.3 \mathrm{pg} / \mathrm{ml}, \mathrm{p}=0.02)$ were significantly higher compared to prepartum values. However, no differences were found between post- and prepartum levels of serum Fas $(4639.0$ vs. $3640.0 \mathrm{pg} / \mathrm{ml}, \mathrm{p}=0.08)$ and MIF $(733.8$ vs. $671.5 \mathrm{pg} / \mathrm{ml}$, $\mathrm{p}=0.12)$. The levels of TRAIL $(637.0$ vs. $1202.9 \mathrm{pg} / \mathrm{ml}, \mathrm{p}=0.04)$ and FasL $(108.2 \mathrm{vs.} 158.8 \mathrm{pg} / \mathrm{ml}, \mathrm{p}=0.03)$ increased also in sera of healthy controls, while the levels of Fas decreased $(4734.2$ vs. $4174.6 \mathrm{pg} / \mathrm{ml}, \mathrm{p}=0.02)$.No differences were found in the levels of serum MIF (664.4 vs. $425.9 \mathrm{pg} / \mathrm{ml}$, $\mathrm{p}=0.06$ ).

Comparison of changes (Delta) in pre- and postpartum levels in MS patients and their controls did not reveal significant differences $(p>0.05)$.

Conclusion: Amelioration of MS disease during pregnancy is not likely to be explained by changes in the levels of TRAIL, Fas, FasL and MIF in sera. However, it seems that these molecules may have an important role for the maintenance of gestation.

P730

Increased expression of apoptosis-related genes in cerebrospinal fluid leukocytes in patients with optic neuritis and lesions on the MRI

T. Rupprecht, J. Waskoenig, N. Goebels

Department of Clinical Neuroimmunology (Zurich, $\mathrm{CH}$ )

Objective: While Optic neuritis (ON) frequently is the first manifestation of multiple sclerosis (MS), it can also have various other neuroinflammatory causes. To get a further insight into the largely unknown pathopyhsiology of $\mathrm{ON}$, we analysed the gene expression pattern of the cerebrospinal fluid (CSF) leucocytes of patients with $\mathrm{ON}$ in a pilot study. We compared the gene expression of ON patients with further demyelinating lesions in the MRI with those ON patients, that are showing no further evidence for inflammation in the brain. None of the patients converted to definite MS during a follow up of 48 months.

Methods: CSF samples from patients with ON $(n=6)$ were lysed in Trizol and stored at $-80^{\circ} \mathrm{C}$. The RNA was isolated using a sample cleanup module, analysed with a Bioanalyser, amplified, and hybridized to a GeneChip ${ }^{\circledR}$ Human Genome U133 Plus 2.0 array. The raw data (approx. 50,000 genes) from each sample were normalized per chip and per gene and then filtered on the expression level. The remaining 19,897 genes were subsequently analysed by ANOVA between those with a normal MRI (MRI neg.) and those with further spots of demyelination in the cerebral MRI (MRI pos.).

Results: While a significant difference in 995 of the expressed genes were expected by chance, 1,536 genes were significantly different between both groups, suggesting a "true" (reliable) difference of gene expression. Thereafter, the genes were filtered on a volcano plot and 214 significant different genes had a more than 2 fold difference in the expression level. Among these, 16 were apoptosis-related genes, which was significantly more than expected by chance from the GO ontology. This hints at differences in the apoptotic process of the CSF leucocytes between MRI neg. and pos. ON patients. The function of several of these apoptosis related genes is reported in the literature. While some of them have been found to play a proapoptotic role in leucocytes (TIA1 and Bcl10), others are elevated in autoimmune processes (TIA1 and GZMB) or their downregulation is associated with a lack of neuroprotection (CLC and SNCA).

Conclusion: The different gene expression could hint at a different pathophysiology or even different subsets of ON. In addition, we found among these different regulated genes a high proportion of those associated with apoptotic processes. This could indicate that even isolated ON might not represent a common disease entity.

\section{P731}

Treatment outcomes in multiple sclerosis patients after high dose immunosuppressive therapy with autologous haematopoietic stem cell transplantation

Y. Shevchenko, A. Novik, A. Kuznetsov, B. Afanasyev, I. Lisukov, O. Rykavicin, A. Myasnikov, T. Ionova, V. Melnichenko, D. Fedorenko, A. Kishtovich, $R$ Kruglina, A. Kartashov, R. Ivanov, G. Gorodokin

Pirogov National Medical Surgical Center (Moscow, RU); Pavlov State Medical University (St. Petersburg, RU); Siberian Branch of Russian Academy of Science (Novosibirsk, RU); Burdenko Central Military Hospital (Moscow, RU); Republic Hospital (Petrozavodsk, RU); Multinational Center of Quality of Life Research (St. Petersburg, RU); New Jersey Center for Quality of Life and Health Outcome Research (New Jersey, US)

During the last decade immunosuppressive therapy (IT) + autologous hematopoietic stem cell transplantation (AHSCT) has been used with increasing frequency as a therapeutic option for MS patients. Comprehensive analysis of long-term outcomes of IT+AHSCT is worthwhile. The goal of our research was to study clinical and patient-reported outcomes in MS patients after early, conventional and salvage/late transplantation.

Fifty-six patients with MS (secondary progressive -27 patients, primary progressive -10 , progressive-relapsing -1 , and relapsing-remitting -18) from 6 medical centers were included in this study (mean age -32.0, range: 17-51; male/female -22/34). Fourteen patients underwent early AHSCT (EDSS 1.0-3.0), 38 patients - conventional AHSCT (EDSS 3.5-6.5) and 4 patients - salvage/late AHSCT (EDSS 7.0-8.5). Median EDSS at base-line was 6.0 (range 1.5-8.0). The median follow-up duration was 18 months (range 6-84 months). Neurological and quality of life (QoL) evaluation was performed at baseline, at discharge, at 3, 6, 9, 12 months, and every 6 months thereafter following IT+AHSCT; MRI examinations - at baseline, at 6, 12 months, and at the end of follow-up.

Notably, no transplant-related deaths or unpredictable severe adverse events were observed. All of 45 patients included in the efficacy analysis experienced improvement $(n=28)$ or clinical stabilization $(n=17)$. Among the patients with improvement there were 20 patients after conventional, 6 - after early and 2 - after salvage HSCT. Among the patients with stabilization there were 15 patients after conventional and 2 - after salvage AHSCT. Two patients (both after conventional AHSCT) deteriorated to a worse score after 18 months of stabilization; 2 others progressed after 12 and 30 months of improvement (early and conventional AHSCT), respectively. All the patients without disease progression were off therapy throughout the post-transplant period. Out of 26 patients included in QoL analysis 24 exhibited QoL response and improved QoL during the follow-up.

In conclusion, IT+AHSCT appears to be an effective treatment for MS both in terms of clinical and patient-reported outcomes. The data obtained point to feasibility of early, conventional and salvage/late transplantation in MS patients. Further studies should be done to establish the best timing for transplantation and to validate IT+AHSCT regimens in patients receiving early, conventional and salvage transplantation. 


\section{Muscle disorders: neuromuscular junction disorders}

\section{P732}

Rituximab $^{\circledR}$ for treatment of refractory myasthenia gravis

C. Lebrun, B. Veronique, N. Tieulie, E. Delmont, A. Samak, F. Sanderson, C. Desnuelle, J. Fuzibet

Hopital Pasteur (Nice, FR); Hopital Archet (Nice, FR)

Objective: MG is an autoimmune neuromuscular disorder for with current therapies carried high risk of side-effects and can be insufficient to stabilize the clinical status. Many therapeutic options can be ruled such as thymectomy, corticosteroids, azathioprine, cyclophosphamide, methotrexate, IgIV or less frequently plasmapheresis.

Methods: We prospectively evaluate the use of rituximab in treatment of generalized refractory myasthenia gravis(MG) after failure of immunosuppressive drugs (IS), intravenous immunoglobulins (IgIV) and plasmapheresis.

We prospectively follow 6 patients with MG who presented a poor response to 2 or 3 lines of immunosuppressive conventional drugs (azathioprine, cyclophosphamide, mycophenolate mofetil) associated with oral corticosteroids. A patient was considered as drug-resistant after 6 months of therapy. IgIV had not improved neurological status and all patient required high doses of cholinesterase inhibitors (from 360 to $440 \mathrm{mg} /$ day) and were bedridden. All but one have AchRAb and one was considered as seronegative (neither AchR nor Musk were detected). Plasmapheresis improved transiently the clinical score (myasthenic score from 40 to 55) but with side effects and no possibility to decrease either corticosteroids or cholinesterase inhibitors.

Results: Rituximab was introduced with a mean follow-up of 1.5 years $\left(375 \mathrm{mg} / \mathrm{m}^{2}\right.$ day $1,8,15,28$ during the first month and then 1 dose each two months). After 2 years of follow-up, all patients stopped corticosteroids and tapered cholinesterase inhibitors from 60 to $180 \mathrm{mg}$ /day (myasthenic score from 90 to 100) without severe infectious event. Rituximab, a chimeric igG k monoclonal antibody that target $\mathrm{CD} 20$ is used for the treatment of relapsing/refractory CD20 positive low-grade non-Hodgkin's lymphoma and other autoimmune neuromuscular diseases. 3 previous reports have described a good response of MG associated with lymphoma requiring rituximab. Recently, 2 case-reports of musk positive $\mathrm{MG}$ and 1 seronegative patient achieved remission after rituximab.

Conclusion: Rituximab is a promising effective drugs for treatment of MG with an important benefit on clinical status and good tolerability in Musk, AchR or seronegative patients.

\section{P733}

Paraneoplastic chronic demyelinating neuropathy and Lambert-Eaton myasthenic syndrome associated with multiple anti-neural antibodies and small-cell lung cancer

C. Rozsa, A. Vincent, Z. Arányi, G.G. Kovacs, S. Komoly, Z. Illés

Jahn Ferenc Hospital (Budapest, HU); John Radcliffe Hospital (Oxford, UK); Semmelweis University (Budapest, HU); National Institute of Neurology and Psychiatry (Budapest, HU); University of Pécs (Pécs, HU)

Objective: Association of small-cell lung cancer (SCLC) with both LambertEaton myasthenic syndrome (LEMS) and paraneoplastic neuropathy is well established. Steroid-responsive chronic inflammatory demyelinating polyneuropathy (CIDP) is mostly autoimmune in origin. Combined paraneoplastic syndromes are a diagnostic challenge. We present a rare case of coexisting LEMS and CIDP of paraneoplastic origin.

Case: A 50-year old smoker female patient developed progressive ascending symmetrical paresthesia and walking difficulties. There was mild weakness in the arms and moderate weakness in the proximal muscles of the legs. Knee and ankle reflexes were absent. All sensory modalities were decreased in the legs. CSF showed elevated protein concentration and oligoclonal gammopathy. Extensive search for malignancy was negative including CT of the chest. Based on clinical and ENG findings CIDP was considered and methylprednisolone (MP) was initiated. The symptoms markedly improved but proximal leg weakness worsened again after 7 months. Dry mouth and constipation also developed. ENG revealed segmental demyelinating features again. Repetitive stimulation indicated LEMS. Serum levels of anti-VGCC antibodies were markedly elevated. Presence of anti-Hu antibody in the serum was also confirmed. This time, she did not respond to MP. Repeated CT scan showed a pulmonary mass lesion and biopsy indicated SCLC. She was treated with radiation and chemotherapy. The neurological signs slowly improved. The patient is on MP therapy, and is fully ambulatory.

Conclusion: The coexistence of paraneoplastic LEMS and CIDP with both anti-VGCC and anti-Hu antibodies has - to our best knowledge - never been reported in a patient with SCLC. In our case the clinical, ENG and CFS findings fulfilled the international criteria for CIDP and LEMS. The close association with SCLC and the marked response to oncotherapy defined the neuropathy and LEMS as paraneoplastic neurological syndromes. The autoimmune origin of both LEMS and CIDP is well established. The co-existence of both diseases, together with a negative chest CT 8 months before the onset of LEMS could have been misleading in our case. We emphasize the importance of the current international guidelines: if the patient is a smoker and older than 40 years, chest CT and search for anti-neural antibodies are warranted in any case of LEMS, even in the presence of another autoimmune disease.

\section{P734}

The comparison between therapeutic efficacy of simplex and extended thymectomy: 15- year follow-up

D. Lavrnic, P. Djukic, V. Rakocevic-Stojanovic, Z. Stevic, A. Nikolic, I. Basta, I. Marjanovic, S. Lavrnic, S. Apostolski

Clinical Centre of Serbia (Belgrade, RS)

The objective of this study was to compare therapeutic efficacy of simplex and extended transsternal thymectomy in patients with generalized mzasthenia gravis (MG).

Methods: The retrospective analysis included 323 patients ( 248 females and 75 males) with generalized MG who underwent transsternal thymectomy over the last 15 years. One hundred seventy six patients underwent «simplex» (ST), and 147 patients underwent «extended» thymectomy (ET).

Results: A significant prevalence of females was observed in both groups of patients (76.1\% in ST and $77.5 \%$ in ET group) and there was no signifficant difference in mean age of onset of the disease (29.8 year for ST and 31.7 year for ET group). The duration of the disease was also similar for both groups of patients (for ST group it was 21.4 months, and for ET group it was 19.8 months). Both groups of patients were treated with similar doses of Mestinon, corticosteroids and/or Azathioprine. In relation to the severity of the disease, mild generalized form (IIA) was present in $36.9 \%$ of ST patients, and $40.8 \%$ ET pateints, while more severe forms (IIB or III) of the disease were registered in $63.1 \%$ of ST and $59.2 \%$ of ET patients. Patients with thymoma were excluded from the study. Good therapeutic outcome was observed in all thymectomized patients (in 71.5\% ST patients, and $88.3 \%$ ET patients). Remission was observed in $31.2 \%$ of ST patients and $46.9 \%$ of ET patients, and significant improvement in $40.3 \%$ and $41.5 \%$ of patients, respectively). Poor outcome (unchanged condition, deterioration of MG and MG related death) was registered in $28.5 \%$ of ST patients and only $10.2 \%$ of ET patients. Frequency of other autoimmune disorders were also similar in both groups of patients (11.4\% and $10.9 \%$ ).

Conculison: Though the large majority of patients improved after thymectomy, therapeutic outcome is better in those who underwent ET. These results could be explained by efficient removal of ectopic minor islands of thymic tissue in mediastinal fat tissue only by extended transsternal thymectomy.

\section{P735}

Chronic progressive external ophthalmoplegia with elevated acetylcholine receptor antibody

J.S. Bae, M.J. Kim, K.M. Kim, H.W. Yang, S.H. Kim, S.J. Kim, O.G. Kim Busan Paik Hospital (Busan, KR)

Background and significance: Distinguishing ophthalmoplegia in mitochondrial myopathies from other neuromuscular diseases, especially ocular myasthenia gravis (MG), can be often difficult. We present a case of chronic progressive external ophthalmoplegia (CPEO) due to mitochondrial myopathy with a diagnostically misleading combination of an elevated acetylcholine $(\mathrm{ACh})$ receptor antibody level

Case: A 51 year-old woman presented with progressive bilateral ptosis and ophthalmplegia for 11 years. She denied a diplopia or fluctuation of symptoms since symptoms onset. There was no family history and previous medical problems. Repetitive nerve stimulation test and Neostigmine test were negative. Needle electromyography showed no myopathic features. Arterial lactate level was upper limit of normal, and lactate / pyruvate ratio was remarkably increased to more than 1:40. The ACh receptor antibody level was also markedly elevated at $14.9 \mathrm{nmol} / \mathrm{L}$ (normal $<0.2 \mathrm{nmol} / \mathrm{L}$ ). Muscle biopsy showed some evidence of a mitochondrial myopathy such as ragged red fibers and myopathic features. Treatment with prednisolone did not lead to objective improvement in either the ptosis or ophthalmoplegia.

Comments: We present a diagnostically confusing case of ophthalmoplegia and ptosis due to mitochondrial myopathy with concurrent elevation of serum ACh receptor antibody. It is unclear whether this elevation represents an epiphenomenon from unknown causes or whether she has both mitochondrial myopathy and ocular MG. 


\section{P736}

\section{HLA typing in myasthenia gravis patients}

M. Al Jumah, A. Hajeer, F. Al Suwaidan, S. Bohlega, A. Al Tahan

King Abdullah International Medical R C (Riyadh, SA); KFSH\&RC (Riyadh, SA); KSU (Riyadh, SA)

Objectives: To study the association of myasthenia gravis (MG) with HLA antigen in Saudis.

Background: MG is an autoimmune disorder involving NMJ receptors. There is a moderate association of MG with the human leukocyte antigen (HLA) system that are coded for by MHC genes. MG associated with HLA system varies in different ethnic groups. In Caucasians, MG associated with the HLA antigens B8, DR3 and DQw2, particularly in young females with thymic hyperplasia. However, HLA association is different in Japanese and French patients. A previous presentation at 2005 ENS meeting our initial study in Saudi Mg patients suggested association with HLA B 8, B18 and DQ5. We present our final results in $109 \mathrm{MG}$ patient and 383 normal controls. Methods: One hundred and nine patients from different families with confirmed diagnosis of MG and 383 normal controls were involved in this study. HLA typing was performed using PCR SSP kit (Dynal). Statistical testing for association was preformed using Epi Info software system. Odds Ratio (OR) was calculated with $95 \%$ confidence interval. $P$ value $<0.05$ is considered significant.

Results: There was significant association in our Saudi MG patients with the following HLA types: HLA B8 (P $<0.00001$, OR 2.51, CI 1.64-3.83), HLA $\mathrm{B} 18(\mathrm{p}<0.02$, OR 2.22 , CI $1.12-4.39$, HLA-A23 (8) $(\mathrm{P}<0.04$, OR 1.83 , CI 1.02-3.11). Significant associations were noted for HLA-DR13 (6) and DR16 (2) as well. Protective significant association was noted with the following HLA types: HLA-B50 (21), HLA DR 10 and DR7. Clinical association was noted between myasthenic crisis and HLA DR 13(6) $(\mathrm{p}<0.02$, OR 3.1.CI 1.48-8.47.

Conclusion: Significant association was noted between HLA type and MG in Saudi patient, mainly for HLA B8, B18, A23 (9), DR13 (6) and DR16 (2). Comparing to other population and ethnic groups, most of this association are new, except for HLA-B8. Myasthenic crisis was associated with HLA DR13 (6), this may suggest increased risk for disease severity. Some HLA types have protective association for MG (HLA-DR 7, HLA B50 (21), and DR10). No significant association could be made between the HLA type and thymus gland pathology, or response to treatment as to the current follow up. Longer follow up is needed to explore the clinical association further.

This study was supported by King Abdullah International Medical Research Center (KAIMRC) and King Abdul Aziz City for Science and Technology (KACST project \#LG P-8-23)

\section{P737}

Tolerability profile of high-dose $10 \%$ liquid intravenous immunoglobulin in myasthenia gravis administered in a home-based setting

M. Rigas, R. Sterling

Crescent Healthcare, Inc. (Anaheim, US)

Objectives: Intravenous immunoglobulin (IVIG) is a complex therapy and can be associated with adverse effects at both standard and high-dose regimens. Reported rates of AEs range from $12-23 \%$, and may be as high as $34 \%$ for initial infusions of patients naïve to IVIG therapy. We examined the overall safety and tolerability of high-dose IVIG administered to patients with myasthenia gravis (MG) in a clinically-defined, home-based and nursingsupervised setting to enhance the knowledge base and decision-making criteria in this uncommon patient population.

Methods: Between September 2005 and May 2006, patients with myasthenia gravis being treated with a liquid $10 \%$ IVIG (Baxter Healthcare, Deefield, IL) were identified via a retrospective chart review at Crescent Healthcare, Inc. (Anaheim, CA). Nine patients were identified: myasthenia gravis (MG) $(n=5)$, myasthenia gravis exacerbation (MGE) $(n=3)$ and ocular myasthenia gravis in conjunction with chronic inflammatory demyelinating polyneuropathy (OMG/CIDP) $(n=1)$. There were 7 females and 2 males with a median age of 64 (range: 42 to 92 ); 4 of the 9 patients were naive to IVIG therapy (received their initial infusion during the study period). A total of 121 IVIG infusions were given within the study period (mean $=13.4$ infusions; range: 2 to 40). The highest number of infusions (40) were received by the OMG/CIDP patient, while the lowest number (2) were received by an MGE patient. The range for the MG patients within the study period was 10-18 infusions. The mean monthly dose of IVIG was $1.53 \mathrm{~g} / \mathrm{kg}$ per patient (range: $0.6 \mathrm{~g} / \mathrm{kg}$ to $2.1 \mathrm{~g} / \mathrm{kg}$ ).

Results: The overall serious adverse effect rate was $0 \%$ and the non-serious adverse effect rate was $3.3 \%$ (experienced in only one patient in the study). One MGE patient experienced a "mild" headache, resolving within approximately one day, following four infusions. The patient also reported that ibuprofen and a slower infusion rate greatly helped relieve her headaches during subsequent infusions. The lack of adverse effects, serious or non-serious, in the initial infusions of the IVIG-naïve patients is notable as compared to reported rates of $34 \%$.

Conclusion: The results of this review of a number of high-dose liquid IVIG infusions utilizing a closely-monitored, yet highly flexible, home-based therapeutic regimen indicate a very favorable tolerability profile in patients with chronic, exacerbated, and ocular presentations of MG, even in patients who are new to IVIG therapy.

Support for this study was funded by a grant from Baxter Healthcare Corporation.

\section{P738}

Rapid involvement of arms and feet should raise suspicion of lung cancer in patients with the Lambert-Eaton myasthenic syndrome

M.J. Titulaer, P.W. Wirtz, J.B.M. Kuks, H.J. Schelhaas, A.J. van der Kooi, C.G. Faber, W.L. van der Pol, P.A.E. Sillevis Smitt, J.J.G.M. Verschuuren

Leiden University Medical Center (Leiden, NL); Haga Hospital (The Hague, NL); University Medical Center (Groningen, NL); Radboud University Nijmegen Medical Centre (Nijmegen, NL); Academical Medical Center (Amsterdam, NL); University Hospital (Maastricht, NL); University Medical Center (Utrecht, NL); Erasmus Medical Center (Rotterdam, NL)

Objectives: Patients with Lambert-Eaton myasthenic syndrome (LEMS) and a small cell lung cancer develop their neuromuscular symptoms more rapidly than LEMS patients without a SCLC. We studied how this clinical information, which is readily available at the first consultation, can be used to predict the presence of SCLC.

Methods: In our first study we collected 38 patients nationwide between 1998 and 2003. Thereafter we collected another 59 Dutch LEMS patients (39 with SCLC and 20 non-tumour patients (NT-LEMS)). We interviewed $40 \mathrm{pa}-$ tients using a structured checklist and reviewed their clinical records in all. We compared frequency and onset of symptoms during the course of LEMS.

Results: Between onset of LEMS and six months SCLC-LEMS patients had developed on average 8 respectively 7 symptoms, while in both cohorts NT-LEMS patients developed only two. Rapid involvement of proximal arm muscles, distal arm muscle, distal leg muscles and the presence of male sexual impotence were found significantly more often in SCLC-LEMS patients in both cohorts $(p<0.00005 ; p=0.0003 ; p=0.0002 ; p=0.0003)$. Cerebellar symptoms, although present in only $10 \%$ of LEMS patients, were almost exclusively related to SCLC-LEMS.

Conclusion: A rapidly progressive course of disease from onset in LEMS patients should raise a high suspicion of SCLC. Special attention should be paid to involvement of upper extremities, involvement of distal arm and distal leg muscles and to male sexual impotence in order to discriminate between SCLC-LEMS and NT-LEMS.

P739

The clinical characteristics and outcome in juvenile-onset Korean patients with myasthenia gravis

S-J. Na, Y-C. Choi

Konyang University Hospital (Daejeon, KR); Yongdong Severance Hospital, Yonsei University College of Medicine (Seoul, KR)

Background and objectives: Juvenile myasthenia gravis (JMG) is an uncommon disease and account for $11-24 \%$ of all patients of myasthenia gravis (MG). Although JMG shares pathogenesis with adult-onset MG, there are differences in gender prevalence, severity, etiology and choice of treatment modality. Unlike adults, clinical characteristics and outcomes of MG are not well studies in children. We investigated the clinical characteristics and outcome in the patients with JMG compared with adult-onset MG.

Method: From January 1995 to January 2007, we retrospectively analyzed the case records of 67 patients with JMG who had symptom onset under the 18 years old. The diagnosis was based on clinical signs of fluctuating weakness of voluntary muscles with fatigability and either a positive response to neostigmine test or the decremental response of more than $10 \%$ in repetitive nerve stimulation test. At the end of the follow-up, the outcome was assessed by a five point scale: asymptomatic (with or without treatment), improvement (partial improvement in symptoms and signs), no change (no objective improvement in MG status), worse, and death.

Results: JMG accounts for nearly $18 \%$ of all patients of MG in this study. Among 67 patients with JMG, 32 are male and 35 are female. The mean age of onset was 8 years, 41 patients were 10 years or below at the onset of myasthenic smptoms. Osserman grades at presentation were as follow: I, 21 patients ( $31 \%)$, IIa, 33 patients (50\%), IIb, 11 patients ( $16 \%$ ) and III/IV, two patients $(3 \%)$. Eighteen patients had disease confined to ocular muscles at a 
mean follow-up was 4.8 years (range 6 month to 10 years). 4 patients had seizure. One patient had associated with other autoimmune disease such as diabetes mellitus. Fourty-one patients $(61 \%)$ received corticosteroids, and azatioprine was added in four patients. Thymectomy was performed in 10 patients, seven below the age of 18 years. Thymic histology was normal in one and showed hyperplasia in eight and thymoma in one. At the end of follow-up, 21 patients were asymptomatic, 23 had partial improvement, and six remained unchanged or worsened and one died. Nine patients showed complete stable remission.

Conclusion: JMG show higher frequency of family history and ocular myasthenia, lack of association of thymoma and other autoimmune disorders, benign course, and better long-term outcome compared to the adultonset MG.

Brain Korea 21 Project for Medicine

P740

Thymoma-associated myasthenia gravis: outcome, clinical and pathological correlations in 221 patients

R. Mantegazza, L. Maggi, F. Baggi, F. Cornelio, C. Antozzi

Fondazione IRCCS Istituto Neurologico C. Besta (Milan, IT)

Objective: Aim of our study was to identify clinical and pathologic variables able to influence the outcome of thymoma-MG (T-MG) patients.

Methods: Complete stable remission (CSR according to MGFA classification) was considered as the primary outcome whereas epidemiological and clinical data were used as stratifying parameters. 221 T-MG patients with at least 1-year of follow-up after thymectomy were included in the study (mean \pm SD: $7.5 \pm 6.1$ years; range: $1.0-31.5$ years). Results were compared with those from a population of 405 non thymoma MG patients (NT-MG) positive for anti-AChR antibodies observed at our Institute. Variables affecting outcome were estimated by life-table analysis, Kaplan-Meier survival curves and Cox proportional hazards regression modeling, calculating the odds ratio (OR) with $95 \%$ confidence interval (CI) in uni- and multivariate analyses. Survival curves were compared using the log-rank test. Chi-square and Student's t-test were used; $P$ values less than 0.05 were considered significant.

Results: $48.9 \%$ of thymomas were invasive; B2 WHO histological type was found in $21.7 \%$ and $A B$ in $19.9 \% .15(6.8 \%)$ patients died because of MG or recurrence of thymoma compared with $3(0.7 \%)$ patients of the NT-MG group. The CSR rate of T-MG patients was $8.6 \%(n=19)$ compared with $23.9 \%(n=97)$ in NT-MG. Pharmacologic remission was recorded in 79 $(35.8 \%)$ T-MG and $101(24.9 \%)$ NT-MG patients, respectively. $189(85.5 \%)$ T-MG patients required prolonged immunosuppression to control the disease, compared with $265(65.43 \%)$ in NT-MG group.

Life table analysis did not show any significant association with clinical variables, except for being ocular at maximal severity $(\mathrm{P}=0.0038)$; furthermore, our analysis did not show any correlation with histological diagnosis and Masaoka staging.

Conclusions: Life table analysis and Kaplan-Meier survival curves on a very large cohort of T-MG patients indicate worse prognosis for T-MG compared with NT-MG over a mean 7 years follow-up.

\section{P741}

Association between ocular myasthenia gravis and Graves' disease

S. Thomann, D. Leifert, M. Christ-Crain, A.J. Steck, A. Czaplinski University Hospital (Basel, $\mathrm{CH}$ )

Myasthenia gravis (MG) is an autoimmune disorder that sometimes occurs in association with other autoimmune diseases. Here we present two cases of ocular myasthenia gravis (OMG) with concomitant Graves' opthalmopathy.

The first patient was a 62-year-old man with a history of right ptosis and double vision since 8 months. Clinically he showed unilateral incomplete opthalmoplegia with exophthalmos and bilateral ptosis. Autoantibodies to acetylcholine receptors (AChR), titin, thyroglobulin (TG), thyroperoxydase (TPO) and TSH receptors (TRAK) were positive. Thyroid hormones were within the normal range. MRI of the orbit showed enlarged eye muscles. He was diagnosed with Graves' disease (GD) and OMG and was treated with pyridostigmin, which lead to a moderate improvement of ptosis and double vision.

The second patient was a 68 year old female patient with a history of double vision since December 2005. Autoantibodies to TPO, TG and TRAK were positive, thyroid function test were within the normal range. MRI of orbit showed thickening of the eye muscles, thus diagnosis of GD was made. After 8 month double vision worsened and the patient developed bilateral ptosis. Neurological examination showed bilateral exophthalmos, complete opthal- moplegia of the left and incomplete opthalmoplegia of the right eye with bilateral ptosis. Autoantibodies to AChR and titin were tested positive and thyroid hormones now showed hyperthyroidism. Treatment with prednison and pyridostigmine lead to a partial improvement of restricted eye movement and ptosis.

Opthalmopathy is one of the triad in GD but also occurs in OMG. $5 \%$ of myasthenic patients have concomitant GD and conversely MG develops in $0.2 \%$ of patients with GD. GD and other thyroid autoimmunity more commonly occur in association with OMG than generalized MG (GMG). Several hypotheses are considered: First, OMG and GMG might represent separate diseases with different spectra of associated diseases, second, an immunological cross-reactitivity against common epitopes on the thyroid and the eye muscles and third, a common genetic background with sharing certain immunogenetic features such as HLA associations.

Conclusion: In the presensce of exophthalmos and extracoular muscle dysfunction due to Grave's disease, myasthenic symptoms may be particulary difficult to elicit and therefore it is important to be aware of this association. The detection of specific antibodies should aid in diagnosing overlapping conditions.

P742

Lambert-Eaton myasthenic syndrome in association with hairy cell leukaemia

T. Zambelis, S. Margeti, D. Mandelos, E. Andreadou

Eginition Hospital (Athens, GR)

Introduction: Lambert-Eaton myasthenic syndrome (LEMS) is a non lifethreatening myasthenic syndrome, affecting mostly proximal muscles, with characteristic autonomic system dysfunction, diminished tendon reflexes and electrophysiological findings that can establish the diagnosis. It is usually associated with small cell lung cancer, although it may accompany other malignancies. A very small portion of cases of LEMS have been reported in the literature in association to lymphoproliferative diseases. As far as it concerns the co-existance with hairy cell leukaemia (HCL), only one case has been published until now.

Methods: (Case report) We present a 57 year old female who had clinical symptoms and electrophysiological findings compatible with LEMS, and a history of hairy cell leukaemia starting 3 years ago. The presenting symptoms were of the kind of autonomic dysfunction (dryness of the mouth to the point to have difficulty to swallow dry food, dryness of the eyes, impotence, postural hypotension). One month later she complained for fatigue, difficulty in arising from a chair, climbing stairs, and walking and dysphonia with nasal speech. Physical examination revealed absent tendon reflexes and mild proximal muscle weakness. Electrophysiological study revealed sensorimotor axonal neuropathy. Repetitive nerve stimulation (RNS) at $3 \mathrm{~Hz}$ in abductor digiti quinti muscle (ADM), Deltoid and Tibialis anterior on the right side showed a decrement of compound muscle action potential (CMAP) amplitude $20-44 \%(5$ th: 1 st CMAP ratio) and at $30 \mathrm{~Hz}$ an increment of 52-100\%, and a three-fold increment in CMAP amplitude after brief maximum voluntary contraction of Tibialis anterior. Extensive imaging studies have not demonstrated any type of carcinoma. A bone marrow biopsy was positive for a small degree infiltration by leukaemic cells. Acetylcholine receptor binding auto antibodies and voltage-gated calcium channels were absent. Anticholinesterase drugs were administered with poor response. Prednisolone resulted in significant amelioration of symptoms.

Conclusions: The electrophysiological findings, the autonomic symptoms and the respond to therapies indicated the diagnosis of LEMS. To our knowledge, this is the second case of LEMS described in association with HCL. 
P743

Phenotypical spectrum of congenital myasthenic syndrome caused by mutations in COLQ gene

V. Mihaylova, J. Muller, J. Vilchez, M. Salih, M. Kabiraj, A. D'Amico, E. Bertini, J. Woelfle, F. Schreiner, G. Kurlemann, V. Rasic, D. Siskova, J. Colomer, A. Herczegfalvi, K. Fabriciova, B. Weschke, R. Scola, F. Hoellen, U. Schara, A. Abicht, H. Lochmüller

Friedrich-Baur-Institute (Munich, DE); University of Newcastle upon Tyne (Newcastle, UK); Hospital Universitari La Fe (Valencia, ES); College of Medicine (Riyadh, SA); Armed Forces Hospital (Riyadh, SA); Bambino Gesu Children's Hospital (Rome, IT); University Children's Hospital (Bonn, DE); University Children's Hospital (Munster, DE); Clinic for Child Neurology and Psychiatry (Belgrade, RS); University Hospital "Thomayerova" (Prague, CZ); Hospital Sant Joan de Deu (Barcelona, ES); Bethesda Children's Hospital (Budapest, HU); University Children's Hospital (Bratislava, SK); Humboldt University (Berlin, DE); Hospital de Clinicas (Curitiba, BR); University of Essen (Essen, DE)

Objective: Here we present the clinical and molecular genetic findings of 22 COLQ-mutant patients.

Background: Congenital myasthenic syndromes (CMS) are clinically and genetically heterogeneous inherited disorders characterized by impaired neuromuscular transmission. Mutations in COLQ cause synaptic basal-lamina associated CMS with endplate acetylcholinesterase deficiency.

Methods: Detailed neurological examination and electrophysiological studies were performed. In all patients the 17 COLQ exons and the flanking intron regions were sequenced.

Results: We identified 20 different mutations of the COLQ gene. We defined additional clinical presentations associated with COLQ mutations that differ from the "classical" phenotypes: Several patients with disease onset at birth or in early infancy presented an unexpected, mild disease course. Moreover, many patients had clinical features reminiscent of limb-girdle CMS with mutations in DOK7 gene, including sparing of eye movements and a predominantly proximal muscle weakness.

There was no long-term objective benefit from esterase inhibitors treatment. Surprisingly, a short-term beneficial effect was observed in 4 patients and a Tensilon test was positive in two. Treatment with Ephedrine was efficient in all five cases where it was administered.

Conclusions: The variability of phenotypes caused by COLQ mutations and an initial positive response to esterase inhibitors in some patients may obscure acetylcholinesterase deficiency as molecular cause of the disease and delay start of appropriate therapy.

P744

Monitoring treatment with cyclosporine microemulsion in myasthenia gravis

Y. Nagane, K. Utsugisawa, S. Suzuki, N. Suzuki

Hanamaki General Hospital (Hanamaki, JP); Keio University School of Medicine (Tokyo, JP)

Objectives: To examine whether monitoring cyclosporine (CsA) blood concentrations can estimate efficacy and safety of treatment with CsA microemulsion pre-concentrate (MEPC) for patients with myasthenia gravis (MG).

Methods: As CsA has a narrow therapeutic window, monitoring blood concentrations is important to predict the effects and side effects of the drug. Although blood concentrations immediately before administration (trough concentrations, $\mathrm{C} 0$ ) have frequently been used to direct dose adjustment of CsA, a stronger correlation between blood concentrations $2 \mathrm{~h}$ after administration (C2) and immunosuppressive effects of the drug was reported in organ transplant patients receiving CsA MEPC. We measured both $\mathrm{C} 2$ and $\mathrm{C} 0$ levels of CsA and examined associations with changes to clinical parameters in $20 \mathrm{MG}$ patients treated with CsA MEPC in an unblinded, 6month prospective open trial. The initial dose of oral CsA MEPC was 4.7 $+-0.5 \mathrm{mg} / \mathrm{kg} /$ day divided into two doses taken $12 \mathrm{~h}$ apart. Dose was maintained for $>1$ month and was subsequently adjusted to maintain morning C0 levels at $<250 \mathrm{ng} / \mathrm{ml}$ and serum creatinine levels at $<1.0 \mathrm{mg} / \mathrm{dl}$.

Results: Initial dose of CsA MEPC provided both high C2 levels (1271.7 $+-327.8 \mathrm{ng} / \mathrm{ml})$ as in organ transplant patients and lower C0 levels (233.1 $+-103.0 \mathrm{ng} / \mathrm{ml}$ ) compared with those in previous trials for MG employing oil-based formulation of CsA. Disease severity, daily dose of prednisolone, acetylcholine receptor-antibody titer levels and levels of interleukin-2 production by peripheral blood mononuclear cells were significantly reduced following treatment with CsA MEPC. A significant correlation existed between C2 levels following the initial dose and clinical improvement in responder MG patients. C0 levels were significantly higher in patients who exhibited increased serum creatinine or hypertension compared with patients free from side effects. Body mass index of individual patients was signifi- cantly correlated with $\mathrm{C} 0$ level, and thus offer a useful marker to predict $\mathrm{C} 0$ levels. High body mass index probably represents an indicator suggesting early dose reduction to avoid side effects.

Conclusion: CsA MEPC was effective at suppressing symptoms and T cell-dependent pathogenesis of MG, and monitoring of both C2 and C0 levels can be more useful than that of $\mathrm{C} 0$ levels alone to estimate efficacy and safety of the drug.

\section{Neuro-opththalmology}

\section{P745}

"Crying for no reason": an unusual case of carotico-cavernous fistula A. Heeney, G. O’Connor, P. Brennan, J. Moroney, N. Tubridy St.Vincents University Hospital (Dublin, IE); Beaumont Hospital (Dublin, IE)

Introduction: A carotico-cavernous fistula (CCF) is a type of dural arteriovenous $(\mathrm{A}-\mathrm{V})$ fistula characterised by abnormal $\mathrm{A}-\mathrm{V}$ shunting within the cavernous sinus and usually manifests clinically with a classical triad of pulsating exopthalmos, conjunctival chemosis and an audible bruit. Case reports of dural A-V fistulae were first published in the 1930s and Barrow et al developed the current classification system of CCF in 1985.

Case: We report the case of a 61-year-old woman who presented with unilateral proptosis and a VI nerve palsy secondary to a spontaneous indirect CCF.

She was well until 3-4 weeks previously when she noticed right eye epiphoria about which she became progressively more embarrassed socially. She was reviewed by a number of ophthalmologists and physicians who reassured her that her fundi and intraocular pressures were normal. She soon, however, developed progressive diplopia. There was no history of trauma. The intraocular pressure was still normal bilaterally and there was no conjunctival injection or bruit.

She underwent CT and MRI brain studies, a CT Angiogram and conventional angiography which confirmed a type C indirect CCF. This involved communication by multiple dural branches of the external carotid artery and the cavernous sinus in a low-pressure system. This contrasts with a direct CCF which is a high pressure system and more commonly secondary to trauma. The hypothesised pathogenesis was nerve compression by an expanding sinus or ischemic neuropathy secondary to venous congestion or arterial steal. This case was unusual as it lacked the classical symptoms usually described and initial imaging was inconclusive. She proceeded to undergo successful obliteration of the fistula via an endovascular approach.

Conclusion: Spontaneous caroticocavernous fistulae are rare and may present with apparently trivial symptoms (in this case excessive lacrimation). This report highlights the importance of the multiple imaging modalities that may be used to identify the fistula and the optimum treatment modalities currently available.

\section{P746}

Asynchronous recovery of vestibular perceptual and oculomotor function following transient complete ophthalmoplegia B.M. Seemungal, P. Massaoutis, D. Green, G. Plant, A. Bronstein Imperial College (London, UK); Moorfields Eye Hospital (London, UK)

The Vestibulo-Ocular Reflex (VOR) is responsible for stabilising gaze during head movements. A brainstem neural circuit called the 'Velocity storage' mechanism optimises the VOR during slow (low frequency) head movements. Velocity storage is so-called since the duration of the signal in the vestibular nerve is prolonged by this neural circuit.

'Velocity storage' is demonstrated by stopping after prolonged wholebody spinning on the spot; the engendered vertigo and nystagmus intensity declines exponentially. 'Velocity storage' prolongs the time constant (Tc) of exponential decline of vertigo and nystagmus from $6 \mathrm{~s}$ (duration of endolymph motion) to $18 \mathrm{~s}$.

In chronic (not acute) external ophthalmoplegia, patients do not complain of head-motion-induced oscillopsia despite excessive retinal slip. Such patients possess deficient 'Velocity storage'. This suppression of the vestibular signal may help to reduce sensations of dizziness from visual or vestibular stimuli. During the evolution of an acute but recovering ophthalmoplegia (from Miller Fisher Syndrome), we serially assessed oculomotor and perceptual vestibular time constants over a period of 2 years and were as follows post-ophthalmoplegia zenith:

Post-recovery(Wks) - PercepTc(s) - EyeTc(s)

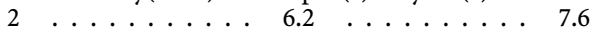

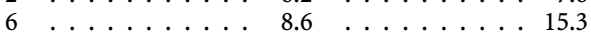




$$
\begin{aligned}
& 7.5 \ldots \ldots \ldots . \ldots 10.6 \ldots \ldots
\end{aligned}
$$

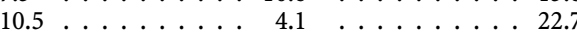

$$
\begin{aligned}
& 13 \ldots \ldots \ldots \ldots \ldots . \ldots \ldots \\
& 15.5 \ldots \ldots \ldots \ldots . \ldots . \ldots 22.7
\end{aligned}
$$

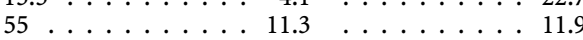

$$
\begin{aligned}
& 82 \ldots \ldots \ldots \ldots 12 . \ldots \ldots
\end{aligned}
$$

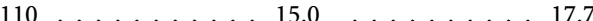

This shows asynchronous recovery of perceptual and oculomotor vestibular mechanisms. Furthermore, the pattern of recovery suggests that plasticity of higher order vestibular mechanisms may play a more important role in vestibular oculomotor recovery than previously thought.

P747

Virtual model of homonymous hemianopia reveals visual search disorders in patients with unilateral occipital stroke

B. Machner, A. Sprenger, D. Kömpf, T. Sander, W. Heide, H. Kimmig, C. Helmchen

Universitätsklinikum SH (Lubeck, DE); Allgemeines Krankenhaus (Celle, DE)

Patients with homonymous hemianopia $(\mathrm{HH})$ are often crucially disabled during self-guided visual exploration of their natural environment. It is a matter of debate as to whether the abnormal visual search behaviour is related to a pure sensory deficit, a deficient spatial organization or individual compensation strategies.

We tested the hypothesis that visual search behaviour in $\mathrm{HH}$ patients is purely determined by the sensory (visual) deficit by comparing them to healthy subjects with a technically simulated, "virtual" $\mathrm{HH}$ (VHH).

Eye movements were recorded during an explorative visual search task in three groups: (i) nine patients with homonymous hemianopia due to unilateral posterior cerebral artery infarctions in their acute stage, (ii) nine agematched healthy VHH subjects, and (iii) nine healthy control subjects. Subjects were asked to search for stationary visual targets on a monitor, which differed in colour and form from similar items serving as distractors. Identified targets had to be marked by a mouse click. The hemianopic field in VHH subjects was elicited by gaze-contingent blanking of one half of the visual hemifield, i.e. always with respect to the actual gaze position.

Scanpath strategies did not differ between $\mathrm{HH}$ and VHH subjects. However, patients showed significantly longer search durations than VHH subjects, which was tightly related to their higher number of fixations and particularly "re-fixations", the repeated scanning of previously fixated items. There was no increase in the number of "re-clicks". Hemifield analyses within both groups, $\mathrm{HH}$ and $\mathrm{VHH}$, did not show any differences concerning number of fixations and re-fixations or amplitude of saccades.

Even patients with small lesions restricted to the visual cortex showed a prolonged search duration and higher re-fixation rate when compared to healthy VHH subjects. Patients' working memory and top-down scanning strategy seemed intact. Due to their acute stage, however, they did not develop adaptive eye movement strategies (no hemifield differences) as described in chronic $\mathrm{HH}$ patients after months.

In conclusion, acute ischemic damage to the occipital lobe leads to visual search disturbances in $\mathrm{HH}$ patients, which the pure visual failure does not fully account for. Rather than a consequence of direct damage to higher cortical areas visual search abnormalities in $\mathrm{HH}$ are more likely to be due to impairment of connecting fibres to cortical (parietal) and subcortical (thalamical) brain regions.

\section{P748}

Isolated transient diplopia: the presenting symptom of an intracranial neoplasm

C. Serrano-Munuera, D. Mon Martínez, P. Comas Bergua

Hospital St. Joan de Déu (Martorell, ES)

Isolated transient horizontal diplopia may be the presenting symptom of a extensive list of conditions including inflammatory and infiltrative orbital diseases, carotid-cavernous fistula, myasthenia gravis, ischemic oculomotor nerve palsy, oculomotor nerve compression from intracranial masses such a neoplasm or aneurism, Fisher's syndrome, stroke, Wernicke's encephalopathy, brainstem encephalitis, paraneoplastic encephalomyelitis, multiple sclerosis, and drug intoxication. It can also be psychogenic.

We report a patient with a short lasting diplopia related to the presence of a clivus chordoma

Case report: A 56yo man complained from sudden horizontal diplopia which last for five days. Diplopia appeared while driving, remained stable for five days and was not associated to other symptoms; especially there was no dizziness, vertigo, headache or weakness. No doctor saw the patient at that point.
His past medical history was only remarkable for smoking cigars (1cigar/day for more than ten years). There was no personal or family history for neoplasm, endocrine, ocular, neurological or autoimmune diseases.

Neurological exam, two months later, was normal, particularly visual acuity, stability of fixation, pursuit movements, saccades convergence, ductions and ocular alignment. Vertical gaze was slightly limited. There was no ptosis and pupils were normal. No fatigability was detected.

Because of the past medical history of smoking, a brain magnetic resonance (MR) was done to rule out transient ischemic attack, although isolated diplopia is not considered a vascular brainstem event for diagnosis purposes. The MRI showed an intracranial and extracranial mass, originated in clivus and extended to the left parasellar region, sphenoidal sinus and superior orbital fissure, suggestive of chordoma. Patient underwent surgery and pathological studies confirmed the diagnosis.

Conclusion: Diplopia is the most common presenting symptom for chordomas. It is usually permanent and associated to extra ocular palsy. Transient diplopia is an uncommon presentation for intracranial o intraorbital neoplasms, including chordoma, and it may be ignored when associated to a normal neurological exam. We strongly recommend performing brain MR in all cases of unexplained transient diplopia when the symptom seems congruent, regardless normal neurological and neuro-ophthalmological exams.

\section{P749}

Upside down reversal of vision in a cerebellar stroke

E. de Pablo Fernández, F. Sierra Hidalgo, E. Correas Callero, J.P. Romero Muñoz, A. Herrero San Martín, J. Herreros Rodríguez, J. Hernández Gallego Hospital Universitario 12 de Octubre (Madrid, ES)

Objectives: Upside down reversal of vision is a rare disorder of perception of the spatial distribution of objects in the visual field. It usually produces 180 degrees rotation in the coronal plane. Other terms have been used for describing this phenomenon: inverted metamorphopsia, room tilt illusion, inverted vision ... It has mainly been described in association with vertebrobasilar ischemia, but many other diverse causes has been reported, including multiple sclerosis, epilepsy, migraine aura, head and neck trauma or peripheral vestibular disorders.

Methods: Case report.

Results: A 47 year-old-man was admitted to the emergency department reporting instability of gait accompanied by nausea and an episode of upside down vision in the coronal plane that lasted about one hour. On neurological examination horizontal rotatory nystagmus on lateral gaze and gait ataxia were observed. One day after admission, a brain diffusion-weighted magnetic resonance image revealed an area of restricted diffusion in the cerebellum that was compatible with an acute ischemic cerebellar infarct in the medial territory of the right posterior inferior cerebellar artery.

Conclusion: The exact pathophysiological mechanism of upside down reversal of vision is still open to debate. The perception of spatial orientation depends on propioceptive, visual and vestibular inputs and their integration in the posterior parietal cortex. Lesions in these pathways or in their integration area would lead to a distortion of spatial perception. In this case, damage in the vestibulo-cerebellar pathways was probably the responsible lesion. Neurologists must be awared of this to make a correct diagnose when upside down reversal of vision is presented.

\section{P750}

Visual search impairment in the elderly: a new cancellation task G. Colazzo, F. Benso, C. Chiorri, F. Bracco, C. Serrati ASL1 Liguria (Imperia, IT); DISA - University of Genoa (Genoa, IT)

Objectives: Traditional cancellation tasks do not discriminate motor speed from visual search, and these two variables overlap in the results. In elderly people this distinction could be clinical relevant to detect early attention impairment, excluding pathological or physiological slowness.

Material: Our test is a paper and pencil cancellation task; visual motor speed is firstly evaluated (time/target); then subject has to complete a visual search for targets without distractors and finally with distractors, in order to isolate pure attention search time. Methods: our test has been included in a complete neuropsychological assessment; 103 consecutive elderly subjects from 60 to 75, spontaneously coming to the hospital for a memory screening have been tested. Subjects have been divided in control group (47), and 3 sub-groups of MCI, according to Petersen's criteria (Petersen '99): MCImild $($ MCIm - $\mathrm{n}=29)$, MCI amnestic (MCIa $-\mathrm{n}=14)$ and MCI multiple domains (MCImd - $n=12)$. Results: Anova between group analyse $(p=0.001)$ and post-hoc Bonferroni's corrections show a significative difference between MCI $\mathrm{md}, \mathrm{MCI} \mathrm{m}$ and control group $(\mathrm{p}=0.01)$. Pure visual search seems to early discriminate MCIm from control group $(p=0.056)$. Pearson's correlation analyse with all cognitive measures shows interesting correlations be- 
tween cancellation task and Rey's copy and TMT. Discussion: results shows an early impairment in visual search that could be an important element to early discriminate a critical and relative pure attention deficit.

Conclusion: Further analyses are necessary to better understand the predictive meaning of this attention impairment; our test seems to be an interesting screening instrument, independent from educational level.

\section{P751}

Evaluation of colour vision in patients with multiple sclerosis

M.A. Sahraian, M. Motamedi, S. Khoramnia, M. Mohammad Ebrahim, S. Jafarian, Z. Moinfar

Tehran University of Medical Science (Tehran, IR)

Introduction: Optic neuritis (ON) is best known for its association with multiple sclerosis (MS). Colour vision deficiency (CVD) has been shown to be sensitive to diagnose ON. Using Ishihara test, we investigated the epidemiological features of CVD among MS patients.

Methods: An observational study was carried out on a series of 110 out of 112 consecutive MS patients in MS Clinic, Sina Hospital, Tehran, Iran, from 21 April to 21 July 2007. Participants with documented congenital CVD were excluded from the study. Moreover, eyes with total blindness were considered to be excluded. Subjects were asked if they had ever had ON or symptoms that might suggest undiagnosed ON. For each patient fundoscopy to assess optic disc paleness and visual field evaluation were performed by a single expert neurologist. Visual evoked potential result was recorded only if a patient was previously undergone the test. The Ishihara series of plates was used for assessment of CVD.

Results: A total of 214 eyes were included for study. Ishihara test was normal in $162(75.7 \%)$ eyes and $52(24.3 \%)$ eyes were shown to suffer from various levels of colour blindness. Among colour deficient eyes 14 (26.9\%) have been considered as SCVD, $21(40.4 \%)$ were Red-Green axis colour deficient, and $17(32.7 \%)$ were diagnosed as total colour blindness or weakness. Based on the logistic regression, Current ON attack (OR $=19.74 ; 95 \%$ CI:7.98 to 48.86; $\mathrm{p}<0.001)$ and Secondary-Progressive type of MS (OR $=4.05 ; 95 \%$ CI:1.52 to $10.75 ; \mathrm{p}=0.005)$ were detected to be independent predictors of CVD.

Conclusion: CVD is a prevalent condition among MS patients and significantly relates to ON and the secondary-progressive type of the MS. On the other hand, CVD, in fewer cases, involved the eyes with no clear history of previous or existing ON. Consistent with previous studies, Red-green axis colour defect was the most common type of CVD between MS patients.

P752

Room tilt illusion as manifestation of left temporo-occipital oligoastrocytoma

M.J. Gallardo, A. Hernández, S. Carrasco Garcia de Leon, J.M. Flores, M.J. Alvarez, J. Vaamonde

Hospital General (Ciudad Real, ES)

Objectives: Room tilt illusion is a spatial perception disorder characterized by a transient rotation of the visual image in different planes, usually in the vertical one. It is a paroxistic phenomenon which presumably transduces a dysfunction of the posterior parietal cortex or its afferences: propioceptive, vestibular or retinian. The etiology more usually involved is vertebrobasilar ischemia. We report a case of this rare disorder with a non-usual etiology and localization of the cause no previously described in the literature.

Methods: A 61-years-old man without medical history referred 7 or 8 episodes of room tilt illusion in the horizontal plane from a period of two months. The ilussion consisted on left-right environment inversion with a 180 degrees distortion, without changes in the size, shape or colour of the objects. They lasted for 3 or 4 minutes and were not followed by any other symptomatology.

Results: Neurological assessment was normal including oculomotor and visual fields examination. Cranial magnetic resonance imaging showed a left temporo-occipital (TO) neoplastic lesion without edema. Electroencephalogram, laboratory data and thoraco-abdominal computerized tomography were normal. Visual episodes disappeared following treatment with carbamazepine. A brain biopsy revealed an oligoastrocytoma.

The patient was treated with radiation and chemotherapy after surgical resection. The outcome was favourable with no recurrence of the visual episodes.

Conclusion: Room tilt illusion has been scarcely reported in association with vertebrobasilar stroke, migraine, multiple sclerosis, labyrinthine disorders, cervical traumatism and epilepsy of the parietal cortex. The pathophysiology has been related to a disorder of posterior parietal cortex where the integration of multiple sensorial stimuli and representation of extra personal space occurs.
In our case, the visual inversion appears as an epileptic phenomenon related to a TO tumour. This localization has not been previously reported as possible origin of tilt illusion. TO areas are association cortices which process stimuli from the ventral visual stream. In man, central vestibular pathways are poorly known. We propose that TO cortex may belong to a network involved in vestibulo-visual integration, as suggests the visual misperception in this case.

\section{P753}

Reversible bilateral sixth cranial nerve palsy, without headache, as a form of presentation of spontaneous intracranial hypotension syndrome P. Pérez, D. Ezpeleta, L. Lillo, P. Fernández, J. Guzmán de Villoria, C. de Andrés

Hospital Gral. Univ. Gregorio Marañón (Madrid, ES)

Objectives: Spontaneous intracranial hypotension (SIH) is a syndrome caused by low Cerebrospinal fluid (CSF) pressure in patients without a history of dural puncture or penetrating trauma. Its most characteristic symptom is orthostatic headache, which is sometimes associated to other symptoms, such as facial numbness, pain or weakness, diplopia or hearing changes (presumably caused by stretching of cranial nerves V, VI, III, IV, VII or VIII), nausea and vomiting, neck stiffness or photophobia.

We describe the clinical and radiological findings of a patient who presented binocular diplopia as the only symptom of SIH.

Methods: A previously healthy 46 year old woman was admitted to the emergency room with a four day history of binocular diplopia.

Clinical examination revealed bilateral sixth cranial nerve palsies. The patient had no nistagmus or signs of fatigability. The rest of the neurological examination was normal.

Results: Blood count, coagulation, serum chemistry, chest X-ray, electrocardiogram and brain and orbits computer tomography (CT) scans were normal. Workup for sarcoidosis, collagen diseases, systemic neoplasy, VDRL, human immunodeficiency virus (HIV), hepatitis B and C were negative. CSF showed an opening pressure of $1 \mathrm{~cm}$ of water during lumbar puncture and was macroscopically clear. Microscopically it revealed no microorganisms, 7 leucocytes $/ \mathrm{mm}^{3}$ and 7 erythrocytes $/ \mathrm{mm}^{3}$. Protein level was $73.3 \mathrm{mg} / \mathrm{dl}$ and sugar level $56 \mathrm{mg} / \mathrm{dl}$ (blood sugar levels of $89 \mathrm{mg} / \mathrm{dl}$ ). Sample was sterile after 48 hours of incubation. Microbiological evaluation of CSF for cryptococcal and borrelian antigen, syphilis and cultures for acid fast bacilli yielded no positive results. Magnetic resonance imaging (MRI) of the brain with gadolinium showed diffuse pachymeningeal enhancement, laminar subdural collections in the brain convexity and signs of downward displacement of the cerebral structures. A medular MRI and isotopic cisternography were performed, but unable to locate the origin of the leak.

Tentative treatment was performed with injection of autologous blood into the epidural space (Epidural blood patch), bed rest, hydration and abdominal binder. Four weeks later, the patient showed a complete remission of the symptoms, with significant improvement in the control brain MRI images.

Conclusion: SIH syndrome should be considered in the differential diagnosis of patients with bilateral sixth cranial nerve palsy, even if characteristic orthostatic headache is not present.

\section{P754}

Herpetic ocular myositis

S. Bek, G. Genc, B. Ozturk, O. Arslan, S. Demirkaya, Z. Odabasi Gulhane Military Medical Academy (Ankara, TR)

Introduction: Painful ophthalmoplegia is one of the most common experienced cases by neurologists and ophthalmologists. There is a long list in differential diagnosis, including neoplasm, inflammation, vascular, infective, rheumatologic diseases and ophthalmologic migraine. Despite improvements in laboratory and imaging techniques, most cases are interpreted as idiopathic.

Case report: A 67-year-old male patient who had complained of double vision and blurring of the left-eye-vision for 25 days and drooping of the left eyelid, loss of motor and visual functions on the left eye for two days was admitted to our hospital. His medical history revealed Type 2 diabetes mellitus for 8 years controlled with oral antidiabetics. His examination showed complete loss of function of left II-III-IV-VI cranial nerves. A 51-year-old male patient who had complained of double vision and drooping of the left eyelid for 3 days was admitted to our hospital. His medical history revealed that same symptoms occurred in 1985 and 1995 on left eye, in 2003 on right eye, and were improved in 2 or 3 months. His examination showed complete loss of function of left III-IV-VI cranial nerves. Both patients did not benefit from the methylprednisolone therapy $1000 \mathrm{mg} /$ day for 5 days. FatSat turbo spin echo MRI showed contrast enhancements spreading from posterome- 
dial sight of periorbital adipose tissue to optic nerve and cavernous sinus in first case, spreading from retroorbital adipose tissue to the anterior wall of maxillary sinus in second case. The biopsy showed focal adipose necrosis, chronic inflammatory adipose tissue. As we performed quantitative PCR for herpes simplex virus (HSV) DNA with the biopsy specimens, we detected HSV DNA (all analysis were performed twice). Patients were treated with acyclovir $4 \mathrm{~g}$ /day for 5 days. In three months follow up, the first patient recovered partially, and the second patient recovered completely.

Conclusion: To our knowledge, these cases are the first cases with ophthalmoplegia due to herpes simplex virus infection. Consequently, we consider that FatSat turbo spin echo MRI sequence should be used in painful ophthalmoplegia routinely and antiviral therapy should be administered if there is any suspicion of myositis and neuritis.

\section{P755}

Radiologic evidence for absence of the abducens nerve in Möbius syndrome S. Bek, G. Genc, E. Eroglu, M. Kocaoglu, Z. Odabasi

Gulhane Military Medical Academy (Ankara, TR)

Introduction: In 1888, Möbius reported patients with congenital, non-progressive, bilateral facial and abducens nerve palsy. Dysfunction of other cranial nerves, orofacial malformations, limb malformations and musculoskeletal system defects are commonly associated features, but they are not necessary for the diagnosis making the syndrome extremely variable in its clinical manifestation. Radiologic studies in patients with Möbius syndrome have been aimed at identifying hypoplasia of the brainstem, and at identifying markers of posthypoxic ischemic damage, such as calcifications in the floor of the fourth ventricle or in the nuclei of cranial nerves. the absence of the facial nerve was shown in only six patients. This is the first report revealing absence of the abducens nerve in Möbius syndrome.

Case report: A 21 year old man was admitted to our department complaining for right pectoral atrophy only. Neurological examination revealed bilateral oculomotor - abducens - facial, left hypoglossal paralysis and right pectoral muscle atrophy. He had no complaints for his cranial nerve palsies. He was diagnosed Möbius syndrome with Poland anomaly. His brain magnetic resonance imaging (MRI) studies revealed pontine atrophy and bilateral absence of abducens nerve.

Conclusion: There are two theories although neither the aetiology nor the pathogenesis of the syndrome have yet been elucidated; a developmental rhombomeric defect including the facial cranial nerve nuclei due to genetic cause or an interruption in the vascular supply of the brainstem resulting in the region of the facial cranial nerve nuclei owing to an environmental, mechanical or a genetic cause. Teratogenicity has been suggested to be an important additional factor in both mechanisms. Although numerous abnormalities were reported in imaging studies of Möbius syndrome. To our knowledge this is the first report revealing absence of the abducens nerve.

P756

Ophthalmoplegic migraine

S. Bek, G. Genc, G. Koc, S. Demirkaya, Z. Odabasi

Gulhane Military Medical Academy (Ankara, TR)

Introduction: Ophthalmoplegic migraine is a rare disorder, with an incidence of approximately 0.7 per million defined as recurrent attacks of headache with migrainous characteristics associated with paresis of one or more ocular cranial nerves (commonly the third nerve) in the absence of any demonstrable intracranial lesion other than MRI changes within the affected nerve. We report a patient with typical clinical features of ophthalmoplegic migraine who had enhancement of the cisternal segment of the oculomotor nerve during the acute phase, followed by resolution as the symptoms resolved.

Case report: 21-year-old man had suffered from right frontal throbbing headache since the age of 8 years, recurring twice a year. His headache lasted for 1-5 days and followed by slight drooping of his eyelid and double vision. He was admitted to our department with slight ptosis and adduction paralysis of the right eye occurring 3 days after his headache ceased. His past medical history was unremarkable. Neurological examination revealed right-side adduction paralysis and ptosis. His brain magnetic resonance imaging (MRI) revealed thickened right oculomotor nerve that enhances intensely after contrast. He was put on methylprednisolone $1000 \mathrm{mg} /$ day IV for 5 days. 3 months after the onset, his ptosis had cleared up and adduction of his right eye was full. We observed that the oculomotor nerve enhancement was lost.

Discussion: Our case was diagnosed as "ophthalmoplegic migraine" according to International Headache Society Classification. Ophthalmoplegic migraine had been considered to have a microvascular, ischaemic aetiology, but more recently it has been reclassified as a demyelinating condition affecting the oculomotor nerves because of the demonstration of swelling and enhancement of the intracisternal portion of the third nerve on MRI scanning in affected patients. Based on the available clinical and radiologic information, the oculomotor nerve enlargement in ophthalmoplegic migraine may be initiated by the trigeminovascular system. With repeated demyelination and remyelination, the oculomotor nerve enlarges focally. To our knowledge, this is the first ophthalmoplegic migraine case presented pretreatment and post-treatment documentation is complete with clinical photographic and MRI and showing the enduring thickening of the oculomotor nerve although symptoms and contrast enhancement were lost.

\section{P757}

Gabapentin and superior oblique myokymia

S. Bek, O. Arslan, K. Hamamcioglu, G. Genc, U. Ulas, Z. Odabasi

Gulhane Military Medical Academy (Ankara, TR)

Introduction: Superior oblique myokymia (SOM) is a rare ocular motility disorder characterized by unilateral paroxysmal oscillopsia and microtremor. Intermittent contractions of the superior oblique muscle cause torsional and vertical eye movements mainly in the primary gaze position and in abduction. The pathophysiology is not clear but recent reports support the neurovascular compression hypothesis. Several medications have shown promise in treating SOM. Only two case reports were published suggesting gabapentin as an alternative medication. This case report outlines a successful symptomatic treatment of SOM with gabapentin when renal eliminated drug is obligatory.

Case History: A 27 -year-old right-handed male presented with an intermittent fluttering sensation of his vision for 10 to 15 seconds per episode occurring 20 to 30 times a day for three months. Slit lamb examination showed intermittent intorsional micro-tremor on the right. Tonic intorsion of the right eye became obvious on abduction. His examination was in normal limits otherwise. Conventional brain magnetic resonance imaging (MRI) showed no abnormality. To evaluate the brainstem and trochlear nerve MRI Fourier transform constructive interference in steady state sequence (CISS) was used. Proximal cisternal segment of the trochlear nerve was seen compressed by right superior cerebellar artery while the opposite counterparts were visible solely. Gabapentin administration was considered which has an advantage of renal elimination mechanism. With a slow titration so as to minimize the sedation side effect, gabapentin dosage was increased to 600 $\mathrm{mg}$ three times daily. Dosage increasing was stoped as $1800 \mathrm{mg} /$ day relieved his symptoms. He is still on gabapentin $1800 \mathrm{mg} /$ day for over a year with no symptoms.

Discussion: Gabapentin efficiency for SOM was reported exceptionally rare with three cases but vascular compression were not documented in those reports. This is the first report case reporting gabapentin efficiency for SOM with a documented vascular compression on imaging. The exact mechanism of gabapentin on SOM is unclear and it is probable that more than GABAergic mechanisms may be involved.

\section{P758}

The neurophysiologic and ocular imaging evaluation of the optic nerve in relapsing-remitting multiple sclerosis

S. Petrescu, D. Stefanescu, C. Panea, L. Voinea, A.M. Dascalu, M. Moldovan, N. Jumara, R. Ciuluvica, A. Stanca

The Emergency University Elias Hospital (Bucharest, RO); The Emergency University Hospital (Bucharest, RO); The Carol Davila University (Bucharest, RO)

Objectives: Demyelination and axonal loss coexist in multiple sclerosis. These processes can be evaluated by non invasive methods.

One of the anatomical parts which could be affected in multiple sclerosis is the optic nerve. Demyelination at this level is measured by the latency of the P100 wave

(reversal pattern visual evoked potential :VEP) and axonal loss can be measured by the retinal nerve fibre layer thickness at the level of the optic nerve head (Heidelberg retinal tomography).

Our goal was to make correlations between the results obtained by functional and anatomical evaluation of the optic nerve with optic neuritis history, visual acuity and the disability status evaluated on expanded disability status scale (EDSS).

Methods: We have studied 20 patients with relapsing remitting multiple sclerosis, for whom we measured the latency of P100 waves, the retinal nerve fibre layer thickness, visual acuity, EDSS score (performed by the same neurologist).

Results: The optic nerve was affected by demyelination in $60 \%$ of cases and axonal loss was evidenced in $35 \%$ of cases. The patients with history of 
optic neuritis presented axonal loss in $20 \%$ cases. Axonal loss evaluated at the optic nerve head correlates with visual acuity changes, also with disability status (EDSS). Detailed results will be present within the session.

Conclusion: There are no clinical and anatomical correlations to measure the disability in multiple sclerosis, but the axonal loss evaluated by retinal nerve layer thickness of the optic nerve head could be a structural biomarker. Axonal loss is thought to cause progression in multiple sclerosis, so the presence and the degree of structural changes of the optic nerve could be a method to evaluate disability, similarly with the diffuse changes noticed on parenchyma of the spinal cord.

\section{Poster session 5}

\section{Cerebrovascular disorders}

\section{P759}

Bilateral paramedian thalamic infarcts

E. Soto-Cabrera, A. Gonzalez, A. Ochoa

National Institute of Neurology (Mexico City, MX)

Introduction: The paramedian thalamic perforating arteries, or Percheron's arteries, usually arise from the first segment of each posterior cerebral artery (PCA). However, they may arise from a single common trunk of PCA. The suggested mechanism of bilateral thalamic infarcts is occlusion of the single common trunk from PCA that supplies the bilateral paramedian thalamic perforating arteries.

Objective: To describe a case of bilateral paramedian thalamic infarct and review the mechanism involved.

Methods: Case report.

82 yo woman with hypertension and dyslipidemia and previous stroke in 1998. In this occasion present with sudden somnolence and weakness. The neurological examination demonstrated somnolence, memory impairment, palsy of third left cranial nerve, left facial weakness, and minimally affected bilateral pyramidal tract. The evolution of patient, was with fluctuation in consciousness, noted for several weeks, with gradual recovery. The neuroimaging studies demonstrated symmetric bilateral hyperintense thalamic lesions on T2-weighted images consistent with ischemic events. Mgnetic Angioresonance was performed, and showed a common trunk irrigating bilateral perforating thalamic arterires.

Conclusion: To our knowledge, there is only one case in the literature where the presence of a single common trunk from 1 PCA was proven radiologically. The natural history of this condition is that patients tend to remain severely disabled. However, recovery has occasionally been reported.

\section{P760}

The Ehlers Danlos connective tissue disorder and cerebrovascular aspects A. Nucera, G. Malferrari, A. Dallari, M. Zedde, N. Marcello

Azienda Ospedaliera Santa Maria Nuova di Reggio Emilia (Reggio Emilia, IT)

Ehlers-Danlos syndrome is a rare disorder of connective tissues that results in unusually flexible joints, very elastic skin and fragile tissues.

A classification in 1997 identified 6 different types of EDS, every one of this represent a singular disease due to a different abnormalities in one of the genes that control the production of connective tissue. There are several variations (with widely varying severity) each affecting a different gene and producing sightly different changes. The results is abnormally fragile connective tissue, which causes problems in joints and bones and may weaken internal organs. The most serious type is vascular type wich reduce life. Because individual are at risk for arterial rupture, aneurysm and/or dissection, gastrointestinal perforation and rupture.

The diagnosis of EDS is based on compatible clinical findings and confirmed by biochemical and/or genetic testing. Biochemical studies in affected individuals demonstrate abnormal electrophoretic mobility and abnormal efficiency of secretion of type III procollagen by cultural dermal fibroblasts. Molecular genetic testing to identify mutations in the COL3A1 gene is available for genetic consuling purposes to individuals with the biochemically confirmed diagnosis of EDS.

We describes two young women with fibromuscolar displasie. The first one made RM because she was affected by TIA. Angiography shown multiples cerebral aneurysm.
The other had a stroke because carotid dissection, with intracranial emorrages (aneurysm formation is found in vertebral arterial, and cerebral media)

According to the presence to some criteria (thin translucent skin, easy bruising, hypermobility of small joints) we suspected the presence of a connettive disorder tissue.

The results of skin biopsy and cultured dermal fibroblasts was compatible with Ehlers Danlos type V, classic.

Conclusion: Elhers Danlos disorder connective tissue is rare, but in front of patient with arterial dissections or intracranial aneurysm which caused stroke, ischemic or emorrages, we can to think to this syndrome

P761

Bilateral buccofacial apraxia due to bilateral infarctions of the premotor cortex

A. Snoha, O. Neuhaus

Kliniken Landkreis Sigmaringen, GmbH (Sigmaringen, DE)

Introduction: Foix-Chavany-Marie Syndrome, also known as bilateral anterior opercular syndrome is a partial paralysis of the face, pharynx and jaw. It was first described in 1926 by Charles Foix, Jean Alfred Emile Chavany and Julien Marie. As a characteristic, there is no paralysis of upper or lower limbs and those affected can still make involuntary movement like smiling, eating or blinking eyes. Symptoms are drooling, trouble talking, jaw jerks and general weakness in the face. The most common cause is stroke, infection of the brain, malformation, degenerative disorder and head trauma.

Case report: An 80-year-old-male with atrial fibrillation presented with abrupt anarthria, dysphagia and a pseudobulbar palsy. He was able to write but not to read. Cough, swallowing, and tongue movements were impaired. Examination revealed a mild right hemiparesis involving the face and arm without sensory deficits. No Babinski sign was present. Brain MRI exhibited two symmetric areas of restricted diffusion in both premotor cortices. Clinically, the symptoms resolved gradually. The patient was put on oral anticoagulants.

Discussion: The simultaneous occurrence of stroke at the premotor cortex on both sides is rarely found in the literature. This case illustrates an uncommon, cardiogenic, strictly symmetric stroke with bilateral buccofacial apraxia.

\section{P762}

The impact of temporary occlusion of external carotid artery on cerebral perfusion and tissue oxygenation

A.A. Divani, R. Pakdaman, T. Berezina, H. Dussa, A.I. Qureshi

University of Minnesota (Minneapolis, US); New Jersey Medical School (Newark, US)

Objective: Hypoperfusion is the main cause of brain injury among stroke patients. Augmenting blood flow to the ischemic tissue to maintain tissue viability can potentially minimize the neurological deficit that may lead to improvement clinical outcomes. The objective of this study was to assess the effect of external carotid artery (ECA) occlusion on ipsilateral cerebral blood flow $(\mathrm{CBF})$ in a preclinical model of cerebral augmented flow.

Methods: Local cerebral hyperperfusion in rats was induced in 12 male wistar rats by ligating the right ECA for two hours. Ipsilateral CBF was determined pre and post-ligation during ECA occlusion using a laser Doppler flowmeter. Sham animals $(n=6)$ were subjected to the craniotomy without ligation of the right ECA. Systemic mean arterial pressure (MAP) was monitored through a cannula in the right femoral artery to ensure that any rise in cerebral perfusion would be not be due to the rise of systemic blood pressure. Blood-brain barrier permeability was evaluated using Evans blue. Cerebral water content was determined using a wet/dry method. In the separate series of rats $(\mathrm{n}=5)$, brain tissue oxygen level (pO2) in the right and left brain hemispheres were determined before and $90 \mathrm{~min}$ after ligation of the right ECA using a tissue oxygenation monitoring unit.

Results: Ligation of ECA resulted in a significant increase in CBF in ipsilateral side compared to the sham animals $(\mathrm{p}<0.0001)$. There was no significant variation in MAP for the treated animals. Vascular permeability and cerebral water content in the right hemisphere after ligation of ECA did not significantly differ from contralateral hemisphere. $\mathrm{PO} 2$ of the ipsilateral hemisphere was significantly higher compared to contralateral area $(\mathrm{p}=0.0012)$ or to the ipsilateral area $(\mathrm{p}<0.0001)$ prior to ligation.

Conclusions: Ligation of the external carotid artery can potentially be a viable option to augment perfusion in the ipsilateral side of the brain where ischemic attack occurs. However, further studies are needed to examine this therapeutic effect on other preclinical models with induction of stroke to better assess beneficial impact of ECA occlusion on stroke recovery. 
P763

Repetitive subarachnoid haemorrhage from a spinal cord arteriovenous malformation

C. Tiu, F.A. Antochi, A. Popescu, R. Nechifor, O. Bajenaru

University Hospital Bucharest (Bucharest, RO)

Objective: The authors present the case of a young woman, 32 years old, who was admitted to the hospital for occipital headache and cervical pain.

Results: Clinical examination was normal excepting a mild nuchal rigidity. She had a history of meningitis in childhood, and of frequent headaches in the adulthood. She had no fever, nausea and vomisments. The cerebral computed tomography showed the presence of the blood in the third and fourth ventricle. Magnetic resonance imaging (MRI) and four vessels cerebral angiography were normal. The evolution was good, with complete remission of pain. She was discharged with the diagnosis of intraventricular hemorrhage. After one year she came again to the hospital for intense left cervical and left arm pain and received a non-steroidal anti-inflammatory treatment. Two days after, she developed a motor deficit in the left arm, followed by pyramidal signs and sensitive troubles in the lower limb, on the left side, and she was hospitalized again. Cerebral computed tomography was normal but the lumbar puncture showed the presence of the blood into the cerebrospinal fluid. A cervical MRI revealed a cervical intramedullary arteriovenous malformation (C7-T1). The spinal angiography confirmed the presence of an arteriovenous malformation type II, (feeding artery from the vertebral artery and rapid venous drainage). The intense neuropathic pain required a combination of gabapentin and oxycodone, for 30 days in order to permit physical therapy.

Conclusion: The authors discuss the importance of checking the spinal cord in cryptogenic subarachnoid hemorrhages, and the prognostic of the case in the particular situation of an inoperable intramedullary, type II, arteriovenous malformation.

\section{P764}

Effect of delayed repeated injection of recombinant human erythropoietin in animal model of stroke

D-I. Chang, S-H. Koh, A. Yoo, S. Oh, Y. Kim, H. Kim, K-Y. Lee, Y. Lee, H-T. Kim, J. Kim, S. Kim

College of Medicine, Kyung-Hee University (Seoul, KR); College of Medicine, Hanyang University (Seoul, KR)

Erythropoietin (EPO) has recently been highlighted as a cytokine with various potent neuroprotective effects. Acute treatment of EPO within $24 \mathrm{hrs}$ after the outbreak of ischemic stroke has been reported to reduce cerebral infarction. In clinical situation, however, early treatment of EPO is not as easy as in vivo study. This study was undertaken to evaluate the effect of delayed repeated treatment of recombinant human EPO (rhEPO) on ischemic stroke animal model. Forty ischemic stroke model rats were divided into 4 groups according to treatment schedule. Ten rats in the first group (E-E) were treated via tale vein with 5 iu of rhEPO per gram once a week after the initial injection of the same dose at reperfusion; 10 in the second group (E-S) with only vehicle once a week after the initial injection of the same dose of rhEPO; 10 in the third group (S-E) with the same dose of rhEPO once a week after the initial injection of only vehicle; and, 10 in the last group (S-S) with only vehicle once a week after the initial injection of only vehicle. Neurobehavioral functions were assessed for 6 weeks after the initial injection. After then, the rats were sacrificed for western blotting and immunohistochemistry. Compared with the S-S group, neurobehavioral function was improved in the E-E and E-S groups, but not the S-E group. Survival signals including pAkt and heat shock transcription factor-1 were increased and death signals including activated caspse- 3 and inflammatory signals including COX-2 were decreased in the E-E, E-S, and S-E groups although infarction volume was decreased only in the E-E and E-S groups. These findings suggest that early treatment of rhEPO is more important in restoring neurobehavioral functions in ischemic stroke but delayed repeated treatment not.

\section{P765}

Effect of hypocalcaemia and hyperglycaemia versus hypercalcaemia and euglycaemia on the infarct size on magnetic resonance imaging diffusion weighted images

I. Ahmed, N. Ahmed

Research Medical Center (Kansas City, US)

Hyperglycaemia is known to be associated with poor outcome in stroke, whereas Hypercalcaemia suppose to have protective effect and associated with smaller infarcts size. We evaluated our cases with Hyperglycaemia and hypocalcaemia on admission and compared with infarct size in patients with Hypercalcaemia and Euglycaemia on the MRI diffusion weighted im- ages (DWI)images in Middle cerebral artery infarcts with similar National Institute of Health Stroke Scale (NIHSS) scores, If it is tue it will have impact on acute treatment of Stroke.

From the stroke registry 65 cases fit the criteria. NIHSS scores where similar in both groups on admission (15-17), Hyperglycaemia (Serum glucose $130-210 \mathrm{mgm} / \mathrm{dl}$, Normal $<120 \mathrm{mgm} / \mathrm{dl}$ ) Hyper calcaemia (Serum calcium 9.5-11.2 mgm/dl,Normal 7.5-8.5 mgm/dl)

27 cases had Hypercalcaemia and Euglycaemic and 38 were Hyperglycaemic and hypocalcaemic. DWI images taken on admission were compared by a Board certified Neuroradiologist. In the group with Hypercalcaemia and Euglycaemia the average infarct size was 6.8 to $10.4 \mathrm{ml}$ whereas in the group with Hyperglycaemia and Hypocalcaemia the infarct size was 11.8 to $15.3 \mathrm{ml}$. Clinicaly at the end of 3 months The NIHSS scores in the first group 8-12 and in second group 13-15.

The above findings suggest that hypercalcaemia may have a Neuroprotective effect wereas combination of Hyperglycaemia and Hypocalcaemia may have adverse effect on the infarct size and outcome at three months.

\section{P766}

Unilateral trismus: a rare manifestation of stroke

L. Alves, P. Alegria, L. Santos, M. Veloso, J. Vale

CHLO (Lisbon, PT)

Objectives: Trismus is defined as tonic contraction of the muscles of mastication. It has been observed in patients with brainstem disorders such as tumours, vascular lesions, multiple sclerosis and degenerative diseases. Vertebrobasilar dolichoectasia (VBD) can originate symptoms and signs due to cranial nerve or brainstem compression, obstructive hydrocephalus or stroke. Our aim is to describe a case of post stroke unilateral trismus (UT) in a patient with VBD.

Methods: The authors reviewed the clinical reports and ancillary tests of a patient with trismus after vertebrobasilar stroke.

Results: A fifty year old male had ictal installation of visual disturbance. His past medical history was relevant for hypertension and TIA resulting in right hemiparesis ten months before. Neurologic examination showed gaze deviation to the right and nystagmus. MRI revealed left pons acute ischemia and VBD. Four days later, he suddenly developed dysarthria, dysphagia and left hemiplegia. Apart from left sensorimotor deficit and right appendicular cerebellar ataxia, examination disclosed bilateral conjugate gaze palsy, right trismus, ipsilateral peripheral facial palsy and anarthria. He was unable to open the mouth or make tongue protrusion. MRI showed acute infarct on the right side of the bulb, pons and cerebellum. Electromyography revealed almost continuous muscular activity in the right temporal and masseter muscles, along with paradoxical activation of both on trying to voluntarily open the mouth. He was treated with botulinum toxin injections into the involved muscles with little improvement.

Conclusion: Trismus after stroke has been described with lesions of the basal ganglia, internal capsules, opercular lesions and brainstem. Post stroke trismus typically develops subacutely along with spasticity. Our patient is unusual in that he developed UT immediately at the onset of the second stroke. The authors would also like to underline the coexistence of "negative" - facial palsy - and "positive" - trismus - signs in this patient as consequences of the same infarct. We speculate that the existence of a compressing deformed basilar, with its presumed irritating effect on the motor part of the trigeminal system, contributed to perpetuate UT.

\section{P767}

Single loading doses of clopidogrel are well tolerated in ischaemic stroke up to 24 hours from onset

M. Awadh, S. Ragab, M. Sherif, H. Afify, H. Aref, R. Moustafa, O. Abdulghani Ain Shams University (Cairo, EG)

Objectives: Few ischemic stroke patients are candidates for IV r-TPA thrombolysis either due to their late presentation or its limitations. Single loading doses of Clopidogrel are approved in per-cutaneous coronary interventions as a pre-procedural prophylaxis against thrombotic coronary and cerebral events with considerable efficacy and safety profiles. A dose-effect relationship exists, with a faster onset and a higher platelet inhibition with higher doses up to $900 \mathrm{mg}$. A useful role might exist in the management of acute ischemic stroke, yet safety remains the major concern.

Methods: 39 consecutive patients ineligible for IV r-TPA \& presented within 24 hours were recruited for this pilot study, excluding lacunar infarctions. Following initial workup, 15 patients received $600 \mathrm{mg}$ (group 1) and 10 received $900 \mathrm{mg}$ (group 2) of Clopidogrel. Both groups were maintained on Clopidogrel daily $75 \mathrm{mg}$ from the next day with full GIT protection. Aspirin $150-300 \mathrm{mg}$ daily was prescribed to 14 sex and age matched controls. According to risk factors, low or full doses of low molecular weight heparin 
(LMWH) were started a day later. NIH-SS was recorded on admission, the second and seventh days. Follow-up imaging (MRI: T1, T2, T2*, FLAIR, diffusion \& MRA, CT on demand) was done within a week.

Results: No significant differences exist among 3 groups in mean age, sex and stroke subtypes. Mean NIH-SS scores at admission, $2 \& 7$ days were: 12.9 , $11.3 \& 8.4$ respectively in group 1 , and $17.3,11.9 \& 6.7$ in group 2 and 10.9 , $9.1 \& 7.6$ in the control group. Significant difference in improvement ( $\mathrm{P}$ value $<0.05)$ exists between group 2 and both group 1 and controls.

4 patients developed self-limiting peripheral bleeding ( 2 epistaxis, 1 subconjunctival haemorrhage, 1 haematuria). 3 patients in group 1 and a patient in group 2 had hemorrhagic transformations of their infarcts in follow up imaging, but none in the control group. All 4 patients had received full-dose LMWH, with only a single clinically manifest cerebral haemorrhage in group 1 , developed beyond the half-life of loading Clopidogrel ( 5 days). This patient had malignant left middle cerebral artery total occlusion with severe vasogenic oedema. Subsequent sub-falccine herniation was imaged before the haemorrhagic transformation.

Conclusion: Loading doses of Clopidogrel appear to be safe in acute management of acute ischemic stroke unless combined with full-dose LMWH, providing a probable clinical improvement in larger doses.

\section{P768}

Correlation between the ultrasonographic and perioperative macroscopic finding in the differentiation of acute internal carotid artery occlusion M. Kral, R. Herzig, D. Skoloudik, D. Sanak, P. Bachleda, P. Havranek, P. Utikal, S. Burval, J. Chmelova, I. Vlachova, P. Kanovsky

Palacky University and University Hospital (Olomouc, CZ); University Hospital Ostrava (Ostrava, CZ)

Objectives: Acute ischemic stroke (AIS) may occur both in the acute and chronic internal carotid artery occlusion (ICAo). Thus, it is important to assess the character of ICAo when considering the recanalization method. The aim was to assess the correlation between the ultrasonographic (US) and perioperative macroscopic finding in patients with AIS with acute ICAo, undergoing an emergent carotid endarterectomy.

Methods: In a retrospective, hospital-based, two-center study, the set consisted of 36 patients (27 males; age 49-79, mean 63.9 \pm 9.1 years). ICAo character was classified as an acute thromboembolus either isolated or in combination with atherosclerotic plaque using the US (B-mode) and the macroscopic perioperative evaluation. Cohen's Kappa was applied when assessing statistical significance.

Results: An acute ICAo character diagnosed by US was confirmed by the perioperative macroscopic evaluation in all cases. US and perioperative macroscopic findings were consistent in 31 (atherosclerotic plaque+acute thromboembolus in 27; acute thromboembolus in 4) and discrepant in 5 (US finding of atherosclerotic plaque + acute thromboembolus classified as acute thromboembolus in perioperative macroscopic evaluation in 2; US finding of acute thromboembolus classified as atherosclerotic plaque+acute thromboembolus in perioperative macroscopic evaluation in 3) cases. The correlation between both methods in the classification of acute ICAo was $86.1 \%$ (95\% CI: 74.8-97.4\%), Kappa $=0.531(\mathrm{p}=0.001)$.

Conclusion: US is a reliable method in the diagnostics of the acute character of ICAo and it has a moderate correlation with perioperative macroscopic finding regarding a differentiation of atherosclerotic plaque and fresh thromboembolus. Thus, US can be used when considering the various recanalization methods.

\section{P769}

Warning signs and symptoms of subarachnoid haemorrhage

M. Togha, M. Sahraian, M. Khorram, P. Khashayar

Tehran University of Medical Scienses (Tehran, IR)

Objectives: Cerebral aneurysms and arteriovenous malformations (AVM) are responsible for more than $95 \%$ of spontaneous subarachnoid hemorrhage (SAH) cases. Eighty to ninety percent of cerebral aneurysms have been diagnosed only after being ruptured, however some warning signs and symptoms can be used as diagnostic keys to suspect presence of a cerebral aneurysm days before spontaneous SAH happens. The reported incidence of warning symptoms in SAH ranges from $13.5 \%$ to $60 \%$. The main objective of this study was to assess the warning signs and symptoms in patients with spontaneous subarachnoid hemorrhage (SAH). The second evaluated variable was the frequency of possible risk factors for SAH.

Methods: All cases of spontaneous subarachnoid hemorrhage admitted in neurology and neurosurgery department of an university hospital, from April 2004 till April 2005 SAH was defined, according to standard criteria, as an abrupt onset of severe atypical headache and/or loss of consciousness, with or without focal neurological signs, with CT, or lumbar puncture evi- dence of focal or diffuse blood in the subarachnoid space. We excluded patients in whom the hemorrhage was found definitely to originate from trauma or intracerebral hemorrhages, and also those who were dead before an accurate diagnosis was made. The data was gathered via a pre prepared questionnaire from the results of the interview with the patient or his relatives. The dataset was entered in SPSS version 11. A T-test was used to test for the proportion of warning signs and symptoms among spontaneous $\mathrm{SAH}$

Results: Overall 28 cases of spontaneous SAH were identified during the study period. The history of headache as the most frequent warning symptom was present in $64.3 \%$ of the cases. Transient loss of consciousness $(42.8 \%)$, difficulty in walking $(21.4 \%)$, hemiparesia $(14.2 \%)$, ocular signs $(14.2 \%)$ and seizure $(3.6 \%)$ followed the headache respectively. Hypertension and smoking, the most frequent risk factors for $\mathrm{SAH}$, were found in 39.3 and $32.1 \%$ of the cases, respectively.

Discussion: Despite recent advances in neurosurgical techniques, the overall outcome after aneurysmal SAH remains poor. Further reductions of the possible risk factors, as well as earlier diagnosis on the basis of warning symptoms and treatment, are desirable. Sudden sever onset headache with or without any neurologic deficits is an important warning symptom for spontaneous subarachnoid hemorrhage.

Iranian Neurologic Research Center, Medical Sciences, University of Tehran

\section{P770}

105 patients with subarachnoid haemorrhage: a retrospective study

M.A. Cakmak, N. Isik, I. Canturk, R. Sari, N. Yildiz, I. Elmaci

S.B. Istanbul Goztepe Training Hospital (Istanbul, TR)

Objective: Subarachnoid hemorrhage (SAH) may arise due to trauma or spontaneously, and is a medical emergency which can lead to death or severe disability even if recognized and treated in an early stage.

In this study $105 \mathrm{SAH}$ patients, consulted and diagnosed by neurology and neurosurgery clinics in our hospital between January 2005 and January 2008 , evaluated to determine etiopathologic, sociodemographic and clinical manifestations.

Materials and methods: Clinical records, neuro-imaging and CSF reports of patients were reviewed. Cranial CT scanning performed in all patients at administration. For further evaluation cerebral anjiography used in $96 \mathrm{pa}-$ tients $(91.4 \%)$, and lumbar puncture in 3 patients.

Results: We detected intracranial aneurysms as the most common pathology $(86.6 \%)$, trauma $(6.6 \%)$ and arteriavenous malformations (AVM) $(4.7 \%)$ consecutively. In 2 patients $(1.9 \%)$ no significant reason have been found. The most frequent complaints were, headache ( $88.5 \%)$, nausea and vomitting $(61.9 \%)$, unconsciousness $(9.52 \%)$, motor disturbance $(9.52 \%)$, and seizure $(0.95 \%)$. The patient population consisted of 59 women and 46 men with an average age of 53.3 years (range, 11-82 years)

SAH severity was assessed according to Hunt-Hess stroke scale, 2 patient Grade $1(1.9 \%), 69$ patients Grade $2(65.7 \%), 24$ patients Grade $3(22.8 \%), 5$ patients Grade $4(4.7 \%), 5$ patients Grade $5(4.7 \%)$. We found out middle cerebral artery aneurysm in 41 patients $(39 \%)$, anterior communican artery aneurysm in 25 patients $(23.8 \%)$, internal carotis artery aneurysm in 9 patients $(8.6 \%)$, posterior communican artery aneurysm in 7 patients $(6.6 \%)$, anterior cerebral artery aneurysm in 5 patients $(4.7 \%)$, basillary artery aneurysm in 3 patients $(2.85 \%)$, anterior coroidal artery aneurysm in 1 patient $(0.95 \%)$. The overall mortality was $9.5 \%$.

Conclusions: Our study confirms earlier studies reporting the most common cause of SAH is intracranial aneurysms $(86.6 \%)$ The diagnosis of SAH usually depends on a high index of clinical suspicion combined with radiographic confirmation via CT scan without contrast. After the diagnosis of $\mathrm{SAH}$, further imaging should be performed to characterize the source of the hemorrhage. This effort can include standard angiography, CT angiography, and magnetic resonance (MR) angiography. If the diagnosis of SAH is yet unclear, lumbar puncture (LP) should be performed.

P771

Gammagard liquid, intravenous immunoglobulin, provides protection against cerebral ischaemia in mice independent of antibodies against human b-amyloid peptide

S. Boman, V. Ciofoaia, H.P. Schwarz, A. Weber, W. Teschner, T. Ahtoniemi, H. Tanila, J. Koistinaho, M. Koistinaho

University of Kuopio (Kuopio, FI); Baxter AG (Vienna, AT); Medeia Therapeutics Ltd (Kuopio, FI)

Objectives: Previous studies indicate that overexpression of human amyloid precursor protein (APP) results in an increased susceptibility to ischemic cerebrovascular lesions in vivo. We therefore hypothesized that b-amyloid 
(Ab), a cleavage product of APP associated with Alzheimer's disease (AD), mediates this susceptibility and that counteracting the effects of $\mathrm{Ab}$ with intravenous immunoglobulin preparation (Gammagard Liquid), which also contains anti- $\mathrm{Ab}$ antibodies, would reduce the susceptibility to ischemic lesions.

Methods: Adult C57Bl/6J wild-type and transgenic mice carrying APPswe and presenilin 1 delta E9 mutations (APdE9 mice) were subjected to permanent middle cerebral artery occlusion. Gammagard Liquid, saline or Gammagard Liquid depleted of human anti-Ab antibodies were administered intravenously at $10 \mathrm{ml} / \mathrm{kg} 1 \mathrm{~h}$ before occlusion onset. $24 \mathrm{~h}$ later ischemic lesions in coronal brain sections stained with 2,3,5-triphenyltetrazolium chloride were measured using image analysis.

Results: Significantly larger cerebral infarcts developed in APdE9 than in wild-type mice, as expected. Blood gases and glucose of the mice were unaffected by the treatments. Importantly, treatment with Gammagard Liquid decreased ischemic lesion sizes in wild-type and showed a trend toward decrease in APdE9 mice. However, anti-Abeta-depleted Gammagard Liquid also protected both wild-type and APdE9 mice from brain ischemia.

Conclusion: The data show increased ischemic susceptibility in double transgenic APP and presenilin 1 mice further confirming that AD-associated pathophysiological changes are linked to neuronal vulnerability following ischemic events. However, cerebral protection from brain ischemia by Gammagard Liquid is not dependent on anti-Ab antibodies. The beneficial effect of intravenous immunoglobulin treatment in this in vivo model calls for further research especially on the mechanism of action and interventional therapeutic potential in stroke.

Financially supported by Baxter AG

P772

Occurrence of capillary telangiectasia, cavernous angioma and developmental venous anomaly (venous angioma) in a single patient

S. Erimaki, E. Papadaki, M. Tzagournissakis, A. Plaitakis

Medical School, University of Crete (Heraklion, GR); University Hospital of Heraklion (Heraklion, GR)

Objective: To report the case of a patient with the simultaneous presence of capillary telangiectasia, cavernous angioma and developmental venous anomaly (venous angioma) in different brain territories.

Background/methods: Capillary telangiectasias, cavernous angiomas and developmental venous anomalies, are disorders of the capillary-venous side of the cerebral circulation. These disorders sometimes occur in pairs, however, the occurrence of all three in a single patient is extremely rare and few reports exist in the literature. Herein, we present the clinical and MRI findings of a 35-year-old patient who was evaluated because of long-term headache and was found to present all the above vascular malformations.

Results: Physical examination of the patient showed the presence of a large cafe-au-lait spot on the lateral aspect of the left arm. Neurologic examination was essentially normal except for gaze evoked nystagmus and impaired vestibulo-ocular reflex suppression. Brain MRI revealed a 4-mm pontine lesion, with nodular enhancement on T1-weighted post-gadolinium images, consistent with capillary telangiectasia. In the right frontal lobe, a second $1-\mathrm{cm}$ subcortical lesion was revealed that was hypointense on gradient echo sequence; T2-weighted sequence showed a hyperintense center surrounded by a peripheral hypointense ring due to hemosidirin deposition, which is typical for cavernous angioma. Finally, after gadolinium administration, a venous angioma adjacent to the right occipital horn was revealed.

Conclusion: The coexistence of all three types of malformations in a single patient suggests that a common pathogenetic mechanism may be operational. Moreover, the remote locations of the lesions raise the possibility that a developmental/genetic defect inducing dysregulation of the signal pathway during angiogenesis may be involved.

P773

Disorders of consciousness in stroke: prevalence and impact on functional outcome

S. Kobierowski

Medical University of Gdansk (Gdansk, PL)

Objectives: The aim of this study was to assess the prevalence of disorders of consciousness (DoC) in stroke, its type, correlation with localization of lesion and impact on functional outcome.

Methods: We have analyzed retrospectively medical documentation of 100 stroke-survivors hospitalized in 2006 in Department of Adults' Neurology, Medical University of Gdansk, Poland. Data on localization of lesion, type of consciousness disorders and functional outcome of the patients were captured. The groups were compared with Chi2 test.
Results: Disorders of consciousness were present in $32 \%$ of the subjects. Qualitative disorders were observed in 6\%, qualitative and quantitative in $7 \%$ and quantitative in $19 \%$ of the subjects. DoC were more common in patients with lesions localized in temporal $(\mathrm{p}<0.05)$ and in occipital $(\mathrm{p}<0,01)$ lobes. The following conditions were statistically more frequent in subjects with DoC: multiple ischemic lesions, previous ischemic lesions, atrial fibrillation, stroke in history, coronary heart disease. Presence of cortical-subcortical atrophy were significantly more frequent in patients without DoC. Functional outcome was much better in patients without DoC: $16 \%$ of them were dependent at the discharge compared with $53 \%$ of patients wit DoC $(\mathrm{p}<0,00001)$.

Conclusion: Disorders of consciousness are frequent problem in ischemic stroke. Their presence depends of comorbidities and on type and localization of stroke. Presence of DoC is prognostic factor of worse functional outcome.

P774

Natural processing of myelin basic protein peptides by antibody-proreases: pathogenic and clinical implications

S.V. Suchkov, V. Mysikov, A. Gabibov, T. Schmidt, N. Ponomarenko, D. Thomas

IM Sechenov Moscow Medical Academy (Moscow, RU); IMONIKI - Moscow Reginal Clinical Research Institute (Moscow, RU); Moscow State University (Moscow, RU); Institute of Bioorganic Chemistry (Moscow, RU); University of Cambridge (Cambridge, UK)

The progress achieved in designing artificial abzymes has evoked the era of clinical abzymology. Recently, we reported that anti-myelin basic protein (MBP) serum Abs from multiple sclerosis (MS) may function as the specific Ab-proteases. MBP-derived recombinant "epitope library" covering the entire molecule of anti-MBP AB-proteases has been constructed. We used ELISA and PAGE/surface-enhanced laser desorption/ionization mass spectroscopy assays to define the epitope binding/cleaving activities of autoAbs isolated from MS sera and healthy controls. The levels of autoAbs to MBP fragments 48-70 and 85-170 as well as to whole MBP and myelin oligodendrocyte glycoprotein (MOG) molecules were significantly higher in MS sera than in those of healthy controls. Patients with MS (77\% of progressive and $85 \%$ of relapsing-remitting) and no healthy controls were positive for catalysis, showing pronounced epitope specificity to the encephalitogenic MBP peptide 81-103. This peptide retained its substrate properties when flanked with two fluorescent proteins, providing a novel fluorescent resonance energy transfer approach for MS studies. Thus, anti-MBP autoAb-mediated, epitope-specific binding and cleavage may be regarded as a specific feature of MS compared with healthy donors and may serve as valuable serum biomarker of the disease progression.

\section{P775}

Early neurological deterioration following intravenous recombinant tissue plasminogen activator therapy in patients with acute lacunar stroke

Y. Hwang, J-G. Seo, H-W. Lee, S-P. Park, C-K. Suh

Kyungpook National University Hospital (Daegu, KR)

Objectives: The aim of this study was to find the rate of early neurological deterioration (END) and favorable outcomes after the acute lacunar stroke with the assumption that the use of intravenous recombinant tissue plasminogen activator (IV rtPA) lacking lysible clots may be associated with adverse events related to the neurotoxic properties of rtPA.

Methods: We reviewed acute lacunar stroke patients within 12 hours of symptom onset during a one year period $(n=76)$. END was defined as a minimum change of either one point on the motor subitems of National Institute of Health Stroke Scale (NIHSS) score or two points on other NIHSS subitems during the first 72 hours after symptom onset. A favorable outcome was defined using $\mathrm{mRS}$ responder analysis. Patients were classified into two groups: those who received (group $\mathrm{A} ; \mathrm{n}=\mathrm{v} 29$ ) or did not receive (group B; $\mathrm{n}=47$ ) IV rtPA. The rate of END and favorable outcomes were compared between groups.

Results: A total of $17 / 76$ patients (22\%) showed ENDs, with 7 (24\%) and $10(21 \%)$ patients in groups A and $B$, respectively $(\mathrm{p}=0.77)$. In a subgroup analysis of the etiologic subtype of small vessel occlusion, the proportion of patients showing ENDs was 4/12 (33\%) in group A and 4/26 (15\%) in group $B(p=0.207)$. The favorable outcome as defined by $m R S$ response, was $9 / 29$ $(31 \%)$ patients in group A and $11 / 47(23 \%)$ in group B $(0.463)$. Within the subgroup of small vessel occlusion, $33 \%$ and $19 \%$ of patients in groups A and $B$, respectively, showed a favorable outcome $(p=0.343)$.

Conclusions: This study showed that the use of rtPA in the acute lacunar stroke did not affect END and favorable outcomes. We suggest that the use of rtPA in cases of no lysible clots might not affect the END and favorable 
outcomes. The treatment strategies in cases of acute lacunar syndrome We propose that the approach of acute lacunar syndrome should be differentiated from the other subtypes of stroke. Comprehensive workups, including multimodal MRI, can be considered before rtPA infusion in cases of acute lacunar syndrome.

\section{P776}

Characteristics of post-stroke depression and the relative factors Y. Zhang, J.H. Chen, Y. Zhou, K. Dong

Beijing Tiantan Hospital, Capital Medical University (Beijing, CN); Beijing Mining Aggregation General Hospital (Beijing, CN)

Objective: Post-stroke depression (PSD) is a very frequent and important consequence of stroke, and the prevalence of major or minor depression among hospitalized patients following a stroke is $22 \%$ and $17 \%$, respectively, and the proportion of patients suffering from major depression increased from $9 \%$ to $16 \%$ during the follow-up, whereas psychological methods of treatment of patients and their families have not yet given conclusive results. In this study, we tried to inverstigate the characteristics of poststroke depression and the relative factors in order to give some instruction for PSD treatment.

Methods: We selected 280 post-stroke patients admitted in Neurology department, Beijing Tiantan Hospital from March, 2006 to July, 2007, informed consent was provided by each of them. We used Hamilton depression Scale (HAMD) to measure the level of depression of these post-stroke patients. The neurological deficits scores were assessed in the patients.

Results: Among 280 post-stroke patients, there were $51.2 \%$ were identified to have PSD. The main manifestations of depression were anxity, retardation, dysfunction of cognition, hopelessness and insomia. Scores of HAMD, retardation and hopeless factor were significantly higher in the patients whose focus were in cortex and hypohloeodal than those in cerebal and brain stem. The level of depression positively correlated with the degree of the neurological deficits, and it had no relation with age, sex and education.

Conlusion: PSD has been considered as the most frequent and important neuropsychiatric consequence of stroke, and our results also showed that the occurrence of PSD in the present series was higher. The damaged lesion different, the characterstics of depression different. The patients whose damaged lesion were in cortex and hypoloeodal are more likely to get depression, The depression degrees are closely related to the degree of

State Key Laboratory of Cognitive Neuroscience and Learning Open Project Grant

\section{Dementia}

P777

Cognitive dysfunction in patients with chronic hepatitis $\mathrm{C}$

A. Nassef, A. Abdelsamee, S. Khalil

Ain Shams University (Cairo, EG)

Background: Patients with chronic hepatitis C virus (HCV) infection frequently describe neuropsychological symptoms and there is a growing body of literature on cerebral dysfunction occurring at an early stage of chronic HCV infection.

Aim of the work: The aim of the present study was to elucidate whether patients' subjective impression of cognitive impairment was accompanied by objective evidence of cerebral dysfunction.

Methods: 12 Patients with chronic hepatitis $\mathrm{C}$ virus infection with compensated liver function were recruited from Ain-Shams university hospitals. Another 8 healthy controls matched for age and sex were recruited from available hospital staff. Patients with potential risk factors for cognitive impairment were excluded. All patients and controls underwent clinical assessment, laboratory test, abdominal ultrasound, neuropsychological assessment, digital electroencephalography (DEEG), brain MRI and MR spectroscopy (MRS).

Results: Psychometric assessment revealed that attention and sustained attention was affected in 6 patients $(50 \%)$, short term memory was affected in 4 patients (33.5\%), psychomotor speed and learning ability was affected in 4 patients $(33.5 \%)$ and immediate verbal and visual memories were affected in 6 patients $(50 \%)$. On intermediate echo sequence of MRS, the HCV group showed significant decrease in $\mathrm{N}$-acetylaspartate/creatine ratios (NAA/Cr ratio) in frontal white matter $(\mathrm{P}<0.05)$. Mild, statistically non significant, elevation of choline/creatine ratios predominantly in basal gan-
glionic(BG) region was noted on both short and intermediate echo sequences. On short echo sequence of MRS, the HCV group showed a mild, statistically non significant, increase in myoinositol/creatine in frontal white matter region as compared to controls. Thus, there is a suggested reduction of normal neurons and accentuation of gliosis in frontal white matter together with membrane degradation in BG in patients with hepatitis $C$ virus infection. Agreement between psychometry and MRS revealed a significant agreement between decreased $\mathrm{NAA} / \mathrm{Cr}$ and impaired attention and sustained attention, immediate verbal memory and immediate visual memory $(\mathrm{P}<0.05)$.

Conclusion: HCV-infected individuals may have deficits in cognitive functions such as attention, working memory, and speed of information processing. They also have abnormalities on MRS that favor the organic explanation of the patients` mental symptoms.

\section{P778}

Abeta affinity-isolated naturally occurring human IgGs specifically crossreact with novel Abeta oligomer and fibril epitopes

A.D. Williams, B. O'Nuallain, L. Acero, A. Weber, H.J. Ehrlich, H.P. Schwarz, A. Solomon

University of Tennessee Medical Center (Knoxville, US); Baxter BioScience (Vienna, AT)

Objectives: To investigate the specificity of Abeta affinity-isolated naturally occurring human IgGs for Abeta conformers (fibrils, oligomers, and monomers), and to determine whether these antibodies and those produced in mice by Abeta vaccination recognize the same epitopes. This study was motivated by our observation that intravenous immune globulin preparations (IGIV) contain amyloid fibril-reactive antibodies that have therapeutic potential for patients with Alzheimer's disease (AD) (O'Nuallain, B. et al. [2006] J. Immunol. 176, 7071-7078).

Methods: Abeta-reactive IgGs were isolated from IGIV by Abeta fibrils and cross-linked oligomers (CAPS) attached to sepharose beads via primary amine coupling chemistry. Antibody-binding curves were generated against Abeta-conformers using a sensitive europium-linked immunosorbant assay (EuLISA). The specificity of Abeta conformer-reactivity was determined using as competitors, Abeta fibrils, CAPS, endogenous serum molecules, and non-amyloidogenic protein aggregates. Competition binding curves were also generated to determine if the reactivity of anti-Abeta antibodies contained in IGIV was against the same epitopes that were bound by antibodies isolated from mice immunized with fibrils or CAPS.

Results: Anti-Abeta fibril and CAPS affinity purified antibodies gave 20-30 fold stronger binding (EC50, 40 nM) to the Abeta conformer used for isolation than unfractionated IGIV. The enriched antibody preparations also cross-reacted with Abeta fibrils or oligomers, (EC50, 100 nM), but not with the monomeric form. Furthermore, passage of IGIV 3-4 times through an Abeta fibril or CAPS column virtually depleted its Abeta-reactivity, indicating that binding was against a discrete number of epitopes present on both fibrils and oligomers. Notably, competition studies involving IGIV antiAbeta IgGs and those isolated from the serum of mice vaccinated with fibrils or CAPS revealed that the two antibody sources recognized different Abeta epitopes.

Conclusion: We have established that Abeta binding by naturally occurring human IgGs present in IGIV is specifically directed against epitopes that are common on fibrils and oligomers. Furthermore, this reactivity differs from vaccine-derived murine anti-Abeta antibodies. These findings provide a basic understanding of anti-Abeta reactivity in IGIV - a preparation that in phase I and II clinical trials has shown promise for treating patients with $\mathrm{AD}$.

This study has been supported by Baxter BioScience.

\section{P779}

Distribution of ApoE alleles in different kinds of mild cognitive impairment C. Lovati, G. Cislaghi, D. Galimberti, P. Bertora, C. Fenoglio, E. Scarpini, C. Mariani

L.Sacco Hospital (Milan, IT); Ospedale Policlinico (Milan, IT)

Background: Alzheimer's disease (AD) is a common cause of dementia among the elderly. A strong association between $\mathrm{AD}$ and presence of epsilon 4 allele (E4) of apolipoprotein E (ApoE) gene has been reported. Mild Cognitive Impairment (MCI) is considered to be the early stage of dementia with single or multiple deficits, non interfering with daily activities. MCI patients may be divided in three mayor groups: (1) Amnestic-MCI (aMCI), which is considered the very early stage of $A D$, with memory impairment with or without other neuropsychological deficits; (2) non-amnestic-MCI (naMCI), that is thought to be the onset phase of other degenerative de- 
mentias different from $\mathrm{AD}$, in which memory is preserved but one or more different cognitive impairments are present; and (3) Vascular Cognitive Impairment (VCI), with single or multiple cognitive disturbances caused by cerebrovascular damage.

Objective: To further study the role of ApoE alleles in $\mathrm{AD}$ pathogenesis, we evaluated their distribution among individuals with different kinds of MCI.

Methods: ApoE aplotype was determined in $46 \mathrm{aMCI}, 21 \mathrm{naMCI}, 25 \mathrm{VCI}$, and 71 non demented healthy controls (HC). Chi square test was applied to compare allelic frequencies.

Results: Among aMCI patients we found $2 \mathrm{E} 2$ alleles (2.2\%), $64 \mathrm{E} 3$ alleles $(69.6 \%)$ and $26 \mathrm{E} 4$ alleles $(28.2 \%)$; among naMCI patients there were $2 \mathrm{E} 2$ alleles $(4.7 \%), 34 \mathrm{E} 3$ alleles $(81 \%)$ and $6 \mathrm{E} 4$ alleles $(14.3 \%)$; in the VCI group $3 \mathrm{E} 2$ alleles $(6 \%), 41 \mathrm{E} 3$ alleles $(82 \%)$ and $6 \mathrm{E} 4$ alleles $(12 \%)$ were found; and among controls we observed $9 \mathrm{E} 2$ alleles $(6.3 \%), 124 \mathrm{E} 3$ alleles $(87.3 \%)$ and $9 \mathrm{E} 4$ alleles $(6.4 \%)$. The distribution of ApoE alleles was significantly different comparing groups (chi square test 3 columns $\times 4$ lines; $p=0.0008$ ). The MCI group was the only that showed a significant difference with respect to HC (chi square test 3 columns $\times 2$ lines; $p=0.00002$ ) after the crossed comparison among groups. It was observed that the difference between aMCI and $\mathrm{HC}$ was sustained by the increased frequency of E4 allele.

Conclusions: The observation that aMCI is the only type of MCI differing from controls for ApoE distribution and that this difference is sustained by the increased frequency of E4 allele (as in $\mathrm{AD}$ ) reinforces the hypothesis that aMCI is in effect the initial clinical step of $\mathrm{AD}$.

\section{P780}

Analysis of the prevalence of behavioural and psychiatric disorders among Alzheimer's disease patients with and without PLAU1 polymorphism

C. Lovati, M. Pesaresi, F. Clerici, D. Albani, D. Galimberti, E. Venturelli, G. Forloni, C. Mariani

L.Sacco Hospital (Milan, IT); Mario Negri Institute (Milan, IT); Policlinico Hospital (Milan, IT)

Background: Urokinase-plasminogen activator (PLAU) gene maps on Ch 10q24, a region linked with Late Onset Alzheimer Disease (LOAD). PLAU is involved in the amyloid metabolism. It was observed that a polymorphism of PLAU gene (PLAU1, on exon 6,RS2227564)was more frequent among LOAD patients than controls and that it may be considered as a possible risk factor for AD.

Aim of the study: To compare prevalence of behavioural and psychiatric disorders (BPSD)among LOAD patients with and without PLAU1 polymorphism.

Methods: PLAU 1 polymorphism was determined in $71 \mathrm{LOAD}$ patients older than 65 years at disease onset and with an history of AD longer than 2 years. Presence of BPSD was clinically obtained. The chi square-test was applied to compare the prevalence of BPSD among patients grouped by PLAU1 polymorphism.

Results: Patients heterozygote or homozygote for PLAU1 polymorphism were 21 and 50 did not have the polymorphism. Among the 21 PLAU- 1 carriers, 15 (71\%) showed BPSD whilst out of the other 50 patients these symptoms were present in 21 subjects $(42 \%)$. Frequence of BPSD resulted higher among PLAU-1 carriers ( $\mathrm{p}=0,026$ by chi $\tilde{2} 2$-test).

Discussion: PLAU-1 polymorphism seems to increase the occurrence of BPSD among LOAD patients.

\section{P781}

Disentangling brain hypoperfusion heterogeneity in frontotemporal dementia: evidence for a brain reserve mechanism

E. Premi, C. Agosti, B. Paghera, M. Plebani, S. Lucchini, B. Borroni, A. Padovani

University of Brescia (Brescia, IT); Brescia Hospital (Brescia, IT)

Objective: Frontotemporal Dementia (FTD) is an heterogeneous condition. Recent literature data have shown that FTD patients may be subgrouped according to the presence or the absence of clear-cut frontotemporal atrophy. The clinical and neuropsychological correlates and disease progression of these two entities are still unknown.

The aim of the present study was: i) to verify the brain perfusion heterogeneity in FTD, ii) to evaluate the related-demographic and clinical correlates, iii) to follow the different disease progression.

Methods: Thirty-five behavioural variant FTD (bvFTD) and 13 temporal variant (tvFTD) patients were enrolled. Each patient underwent clinical, neuropsychological and behavioural assessment, and re-evaluated at 1-year follow-up. At follow-up, FTD diagnoses were confirmed in all the cases. All FTD patients and 14 age-matched healthy controls underwent 99mTc-ECD SPECT scan.
The pattern of brain hypoperfusion in each patient as compared to controls was evaluated by means of voxel-by-voxel statistical analysis (statistical parametric mapping, SPM2). FTD patients were grouped according to the presence (SPECT + ) or the absence (SPECT-) of frontotemporal hypoperfusion at the pre-established threshold $(\mathrm{P}<0.001$ and $\mathrm{P}<0.005)$, and the differences between groups tested.

Results: At baseline, amongst bvFTD patients, 18 belonged to SPECT+ and 17 to SPECT-. The two groups did not differ for demographic characteristics, but for family history for dementia ( $64.7 \%$ vs $23.5 \%, P=0.02)$, and were comparable in respect to global cognitive deficits, behavioural disturbances and functional impairment, but for language and executive functions. SPECT+ patients showed worse scores at fluency, token test and trail making test. At 1-year follow-up, SPECT+ progressed faster than SPECTFTD patients.

All tvFTD patients showed the same pattern of hypoperfusion, involving left temporal lobe.

Conclusions: Despite comparable overall clinical disease stage, bvFTD is highly heterogeneous, and a brain reserve mechanism may be put forward. Genetic background, as underscored by family history for dementia, may be strictly related to a greater brain reserve. tvFTD can not be considered as heterogeneous as bvFTD is.

\section{P782}

Progranulin gene mutation scanning in Alzheimer's disease and frontotemporal lobar degeneration: functional and phenotypic correlations

E. Venturelli, C. Fenoglio, F. Cortini, D. Scalabrini, C. Villa, I. Guidi, A. Mandelli, A. Marcone, L. Perini, S. Pomati, F. Clerici, S. Cappa, C. Mariani, N. Bresolin, E. Scarpini, D. Galimberti

University of Milan, Ospedale Maggiore (Milan, IT); San Raffaele Scientific Institute (Milan, IT); University of Milan, Ospedale L. Sacco (Milan, IT); VitaSalute San Raffaele University (Milan, IT)

Objective: To perform an extensive mutation analysis together with a functional analysis of Progranulin in sporadic Alzheimer's disease (AD) and Frontotemporal Lobar Degeneration (FTLD).

Background: Progranulin (PGRN) is a pleiotropic molecule widely expressed in many tissues, including the central nervous system. Recent findings show that PGRN constitutes a major locus for FTLD with ubiquitin inclusions mapping to chromosome 17q21. Furthermore, it was shown that PGRN expression is increased in activated microglia in several neurodegenerative diseases including AD.

Methods: The coding region for PGRN was screened by direct sequence in 50 patients with $\mathrm{AD}$ and 50 with FTLD. Subsequently a case-control study was performed in $145 \mathrm{AD}$ patients, $100 \mathrm{FTLD}$ and 144 controls for the variants IVS2+7G/A and IVS7+7G/A by allelic discrimination. The effect of these variants on mRNA was investigated by transcription analysis. Haploview 3.1 software was used to test for association. SIFT, PolyPhen and spliceview software were employed for the in silico analysis.

Results: A novel allelic variant (g100169G $>$ A), leading to an aminoacidic change (p.Gly35Arg) was observed in a patient with AD. Polyphen analysis predicted that this variant damages the structure and function of the protein. A further variant (g100165C $>\mathrm{T})$, leading to a silent mutation (p.Asp31Asp) was found in a patient with Semantic Dementia and in another with AD. Both variants were absent in 144 controls. Two rare variants in PGRN splice-site regions (IVS2+7G/A and IVS7+7G/A) were detected by sequencing and then extensively studied by allelic discrimination. The association study demonstrated they are not risk factors for AD or FTLD. Transcription analysis revealed that both polymorphisms don't affect exon splicing.

Conclusions: A novel PGRN mutation, p.gly35Arg was observed in a patient with $A D$ and was absent in additional 268 subjects screened. This variant is predicted to be damaging, thus further investigation is needed to clarify its possible role in neurodegeneration. A silent mutation, p.Asp31Asp, was observed in a patient with SD and in a patient with early onset AD. Interestingly, all these patients had cancer, supporting a role of progranulin variants in cell proliferation. It is conceivable that this effect of progranulin in proliferation together with cell cycle re-activation could have a role also in neurodegeneration. However, further extensive analysis is needed to draw definitive conclusions. 
P783

Neurofunctional correlates of semantic memory in patients with mild cognitive impairment: impact of the genetic risk factor apolipoprotein $\mathrm{E}$ epsilon4

G. Frank, K. Fast, H.H. Klünemann, M.W. Greenlee

University of Regensburg (Regensburg, DE); LMU Munich (Munich, DE)

Objectives: Mild cognitive impairment (MCI) represents in many cases an early stage of Alzheimer's disease (AD). The present functional MRI (fMRI) study deals with the neurofunctional correlates of an important aspect of semantic memory, the recognition of famous faces in patients with amnestic MCI (aMCI) and age-matched controls. It is well known that Apolipoprotein E epsilon4 (ApoE epsilon4) is an important genetic risk factor for the development of AD. Recent investigations reveal that genetic risk translates into reduced or compensatory increased functional brain activity even before alterations can be detected at the behavioural level. Due to the fact that the earliest pathological alterations in $\mathrm{AD}$ occur in medial temporal lobe, a main focus of the study lies on the impact of the genetic status in activation patterns of this cortical structure.

Methods: The present study examined the effects of ApoE genotype on blood oxygen level dependent (BOLD) responses in aMCI-patients and healthy controls during a semantic memory task, which is an experimental version of the Famous Faces Test (Fast et al.,2008) by conducting a fMRI experiment (Siemens Allegra, 3Tesla-scanner). Functional data were processed using SPM5 (Statistical Parametric Mapping 2005). We investigated 15 healthy volunteers, carrying the ApoE epsilon 4 and 15 healthy subjects without the ApoE epsilon 4 gene. In addition, 30 aMCI-subjects were investigated, with half of them ApoE epsilon4-carrier and half non-carrier. The groups of healthy controls and MCI-patients differed significantly in measures of cognitive performance, but were matched according to age, gender and education. Average age of the study participants was 59 years.

Results: The regional patterns of brain activation were similar between groups. However, the group of healthy subjects with ApoE epsilon4 bilaterally showed hypoactivation in medial temporal lobe (MTL) structures during the famous faces task compared to the healthy group without ApoE epsilon4 despite identical task performance. In both aMCI-groups we found significantly reduced MTL activation compared to the control groups.

Conclusion: Our results demonstrate that also semantic memory tasks can be an effective marker for the detection of changes of brain function in MCI subjects. The genetic status influences activation in critical brain regions also in persons with high genetic risk for development of AD long before manifestation of clinical symptoms.

P784

The assessment of beta amyloid, tau protein and cystatin $\mathrm{C}$ in the cerebrospinal fluid laboratory markers of neurodegenerative diseases

J. Mares, R. Herzig, K. Urbanek, P. Hlustik, H. Vranova, V. Sladkova, $R$ Opavsky, B. Lubenova, K. Kollarova, R. Obereigneru, J. Sklenarova, D. Stejskal, J. Vavrouskova, J. Zapletalova, P. Kanovsky

Faculty Hospital (Olomouc, CZ); Hospital (Sternberk, CZ)

The aim of our study was to assess the role of tau protein, beta amyloid and cystatin $\mathrm{C}$ in the diagnosis of Alzheimer dementia (AD) and other neurodegenerative diseases (ND).

At present, many authors focus on new possibilities in the diagnosis of ND. Cerebrospinal fluid (CSF) diagnosis of AD is based on assessment of CSF levels of beta amyloid and tau protein as assessed by ELISA method and fluorescence correlation spectroscopy. Beta amyloid protein is a part the amyloid plaque accumulating in the brain. Tau protein occurs in the skeleton of the central nervous system cells, which stabilizes the axonal system. Its serum and CSF concentrations in healthy individuals are low. Cystatin C is a promising marker, which can be used in the diagnosis of ND.

The levels of tau protein, beta amyloid and cystatin $C$ were assessed in the set of $69 \mathrm{ND}$ patients ( 33 males, 36 females, aged 22-90, mean $60.5 \pm 16.1$ years), and in a control group of 69 subjects without affection of the central nervous system (33 males, 36 females, aged $20-91$, mean $60.5 \pm 16.0$ years).

When compared to the CG subjects, a statistically significant tau protein level decrease was found in ND, tau protein level increase in $\mathrm{AD}$ and cystatin $\mathrm{C} \mathrm{CSF}$ /serum index increase in ND + AD.

Our study confirmed a previously reported results only in part. While tau protein seems to be quite a reliable marker of $\mathrm{AD}$, the role of beta amyloid in $\mathrm{AD}$ diagnosis remains at least questionable. In the case of cystatin $\mathrm{C}$, our results rather confirm the views of some authors, that cystatin $\mathrm{C}$ is not likely to become a marker contributing significantly to differential diagnosis of $\mathrm{AD}$ and ND. $3 / 2003$

This project was supported by the IGA MH CR grant number NF7480 -
P785

Effects of vitamin B6 on age-associated changes of rat brain glutamate metabolism

M. Messripour

Islamic Azad University, Khorasgan Branch (Isfahan, IR)

Introduction: Glutamate and gmma-aminobutyric acid (GABA), act as excitatory and inhibitory neurotransmitters in CNS respectively. Pharmacology of N-methyl-D-aspartate (NNDA) indicated a hyperactivity of glutamate receptors in aging brain, while GABA has been found to be disrupted. Excessive levels of extracellular glutamate in the nervous system are excitotoxic and lead to neuronal death. Glutamate metabolism, mainly by the enzymes glutamate decarboxylase (GAD) which catalyzes the conversion of glutamate to GABA, glutamate pyrovate transaminase (GPT) and glutamate oxaloacetate transaminase (GOT) which catalyze transamination of glutamate to alphaketoglutarate are thought to be important for maintaining extracellular glutamate concentrations below excitotoxic levels in the CNS. However, the metabolism of glutamate in aged brain has not been well elucidated. Because these enzymes are pyridoxal phosphate dependent enzymes, this study was undertaken to investigate the effects of vitamin B6 administration on age related changes in the metabolism of glutamate in rat brain.

Methods: Male Wistar rats (3 and 30 months old) were used Animals were maintained in animal house until the desired age was attained. The male animals were injected intraperitoneally with 10,100 , and $1000 \mathrm{mg}$ vitamin B6/kg body weight /day for 30 days. Animals were killed by decapitation. Brains were homogenized in phosphate buffer ( $\mathrm{pH} \mathrm{7.4)}$ and the activity of GAD, GPT and GOT were measured in the supernatant.

Results: The enzymes activities in aged rat's brain were significantly lower as compared to those of young animals. Vitamin B6 induced activation of the brain enzymes in both ages, however, the differences were significantly pronounced in aged animals.

Conclusion: Significant activation of GAD, GPT and GOT by vitamin B6 in aged rat brain may be resulted from either lower availability of vitamin B6 in aged animals, or;lower affinity of the enzymes for pyridoxal 5-phosphate, which is likely to be related to conformational changes of the enzymes in aging.

Ii is suggested that vitamin B6 may restore the activity of these enzymes in aged brain. Therefore glutamate metabolism might be considered as a therapeutic target for prevention of neurodegenerative disorders and age related symptoms.

\section{P786}

Impact of apoE genotype on soluble adhesion molecules in Alzheimer's patients

M. Reale, C. Iarlori, L. Velluto, D. Gambi

University G. d'Annunzio (Chieti, IT)

Objectives: Multiple genetic and environmental factors are likely to contribute to the development of Alzheimer's disease (AD). The most important known risk factor for $\mathrm{AD}$ is the presence of the $\mathrm{E} 4$ isoform of apolipoprotein $\mathrm{E}$ (apoE) contributing to amyloid-beta deposition, tangle formation, oxidative stress, lipid homeostasis deregulation, synaptic plasticity loss and cholinergic dysfunction. Senile plaques and neurodegenerative changes in $\mathrm{AD}$ could result moreover from endothelial dysfunction. associated with increased expression of cytokines and other factors such as cell adhesion molecules.

Whilst ICAM-1 is expressed on a broad variety of cells, the expression of VCAM-1 is more restricted to endothelial cells. Since adhesion molecules reflect activation of their originating cells, quantification of these molecules in the circulation/CSF is the only method to obtain information about the activation of the adhesion cascade in vivo.

The purpose of this study was to determine and compare the levels soluble adhesion molecules ICAM-1, VCAM-1 and E-selectin in patients with $\mathrm{AD}$ in comparison with age and gender-matched healthy controls and to determine whether there are associations between these molecules and ApoE4 allele.

Methods: 20 AD patients with clinical diagnosis of according to DMS IV$\mathrm{R}$ and NINCDS-ADRDA and $10 \mathrm{HC}$ subjects, matched for sex and age were enrolled. Plasma levels of sICAM-1 and VCAM-1 and E-selectin were measured with commercially available ELISA kits. INNO-LiPA ApoE is used for the identification of apolipoprotein E genotypes e2, e3, and e4 in blood samples

Results: We found that plasma concentrations of sICAM-1 and VCAM-1 were higher in $\mathrm{AD}$ patients versus healthy controls. In contrast, plasma concentrations E-selectin did not differ among the groups. When comparing ApoE4 carriers to non-carriers, in the $\mathrm{AD}$ group we find that ApoE4 carriers have higher plasma sVCAM-1 ICAM-1 and E-selectin levels than non-carriers. However, in the $\mathrm{AD}$ group, both ApoE4 non-carriers and ApoE4 carriers exhibited higher sICAM-1, VCAM-1 and E-selectin levels than HC group. 
Conclusions: The presence of increased s-ICAM, VCAM levels in AD patients may be related to immunological processes involved in pathogenetic mechanisms of AD. The not statistically significant values of s-E selectin, a glycoprotein considered an exclusive marker of endothelial activation, seem to suggest that other cell produce and secrete inflammatory markers.

P787

Occludin and claudins in Alzheimer's disease and vascular dementia

M.O. Romanitan, B. Winblad, O. Bajenaru, N. Bogdanovic

Emergency University Hospital (Bucharest, RO); Karolinska Institute (Stockholm, SE)

Objectives: The tight junctions (TJs) contain integral membrane proteins: occludin, claudins and junctional associated molecules. Both occludin and claudins participates in sealing the paracellular space, occludin anchoring the claudins to the cytoskeleton and the plasma membranes of adjacent cells. In brain, claudin $5(\mathrm{Cl} 5)$ is expressed in the endothelial cells of all the vessel segments, claudin $11(\mathrm{Cl} 11)$ is important in the formation of myelin sheaths while claudin $2(\mathrm{Cl} 2)$ is a major structural component of TJ strands expressed by the epithelial cells.

The aim of this study is to analyze the expression of occludin (Occ) and claudins in cerebral microvasculature and frontal cortex in control ageing brains, Alzheimer's disease (AD) and Vascular Dementia (VD).

Methods: The brain material (cerebral frontal cortex) was obtained from the Huddinge Brain Bank, Stockholm. The study was based on 19 cases: 5 ageing control, $4 \mathrm{AD}$ and $6 \mathrm{VD}$ post-mortem human brains. Immunostaining with rabbit polyclonal antibody against Occ (dilution 1:100), against $\mathrm{Cl}$ 2 (dilution 1:50), $\mathrm{Cl} 5$ (dilution 1:100) and against $\mathrm{Cl} 11$ (dilution 1:200) from Zymed Laboratories was performed on paraformaldehyde-fixed embedded sections (thin sections of $7 \mathrm{Im}$ ) followed by quantitative approach using stereological principles. The neurons, astrocytes and oligodendrocytes were quantified. The stained vessels were appreciated only qualitatively.

Results: Our first observation was the significant increase in ratio of Occ and $\mathrm{Cl} 11$ expressing neurons, glial cells or vessels in frontal cortex in both $\mathrm{AD}$ and VD as compared to controls. The stained neurons were predominantly pyramidal and the predominant stained type of glial cells was astrocytes. At the brain microvasculature level in AD and control brains we observed that Occ expression localized selectively in some endothelial cells while in VD the distribution was in most of the vessel wall. As for the claudins the endothelium was stained both in white and gray matter.

Conclusion: The studies sustains the idea of occludin and claudins expression in human brain neurons, glial cells and vessels suggesting that there is an increase of all protein's expression, especialy occludin and $\mathrm{Cl} 11$, in pathological conditions such as AD and VD compared to controls. Our findings could reveal new pathogenic pathways in dementia disorders and open a new horizon for the functioning of the TJs proteins in the central nervous system.

P788

An Alzheimer disease with dementia with Lewy bodies patient presenting progressive supranuclear palsy-like clinical manifestations and radiological atrophy of midbrain tegmentum. A clinical and neuropathological study N. Kasahata, N. Arai, Y. Makita

Makita General Hospital (Tokyo, JP); Tokyo Metropolitan Institute of Neuroscience (Tokyo, JP)

Objective: To study clinically and neuropatholgically, an Alzheimer disease (AD) with dementia with Lewy bodies (DLB) presenting progressive supranuclear palsy (PSP) like symptoms and radiological atrophy of midbrain tegmentum.

Methods: Clinical study (history taking and neurological examination), radiological study (magnetic resonance imaging (MRI) and MIBG myocardial scintigraphy) and neuropathological study.

Results: The patient was 75-year-old male. At 68 year, he showed difficulty climbing stairs and became easy to fall. At 69 year, he showed mild walking dificulty and used wheelchair. At 71 year, he became unable to walk. At 74 year, he was absent-minded, slept daytime and he showed appetite loss.

Neurological examination showed 1) dementia, 2) supranuclear gaze palsy, 3) neck dorsiflexion, 4) right side dominant plastic rigity and 5) akinesia.

L-DOPA therapy did not improve his clinical symptoms.

MRI demonstrated midbrain atrophy like hummingbird and bilateral frontal and teporal dominant atrophy. MIBG myocardial scintigraphy showed normal findings: $\mathrm{H} / \mathrm{M}$ ratio $=3.38$

Neuropathological examination revealed 1) AD pathology (plaque: Braak C, tangle: Braak VI), 2) Lewy body pathology (DLB limbic form) and
3) mild PSP pathology (neurofibrillary tangles of midbrain tegmentum and grumose degeneration of dentate nucleus).

Conclusion: We think it important that AD with DLB patient may show PSP like clincal features (dementia, supranuclear gaze palsy, neck dorsiflexion and L-DOPA nonresponsive parkinsonism), atrophy of midbrain tegmentum and negative result of MIBG cardiac scintigraphy.

\section{P789}

Effects of memantine treatment on discrete cognitive domains in patients with moderate to severe Alzheimer's disease

P. Mecocci, M. Emre, K. Stender

University of Perugia (Perugia, IT); Istanbul Faculty of Medicine (Istanbul, TR); H. Lundbeck A/S (Copenhagen, DK)

Objectives: Alzheimer's disease (AD) is a neurodegenerative disorder with cognitive, functional, and behavioural symptoms. The objective of this analysis was to evaluate the effects of the NMDA receptor antagonist memantine on specific cognitive domains in patients with moderate to severe AD.

Methods: Data from six randomised, double-blind, placebo-controlled 6month studies with memantine were pooled. The effect of memantine treatment was assessed on three constructed cognitive clusters in 1826 patients (memantine: 959; placebo: 867) with a Mini Mental State Examination (MMSE) score below 20 at baseline. Individual items from the Alzheimer's Disease Assessment Scale cognitive subscale (ADAS-cog) and the Severe Impairment Battery (SIB) were combined into three clusters representing the cognitive domains: language, memory, and praxis, using a face valid approach. Post hoc responder analyses were performed using three cut-offs: any improvement (ADAS- $\operatorname{cog}<0$; SIB $>0$ ), no change or any improvement (ADAS-cog $\leq 0$; SIB $\geq 0$ ), and any worsening (ADAS- $\operatorname{cog}>0$; $\operatorname{SIB}<0$ ) from baseline. No change or any improvement and any worsening are complementary cut-off categories. The number of responders in each domain was compared between treatment groups using Chi-squared test both for observed cases (OC) at Week 12 and Week 24 as well as for the last observation carried forward (LOCF) cohort at Week 24.

Results: The mean baseline ADAS-cog and SIB scores were similar for the memantine and placebo-treated patients. At Week 24 (LOCF), a significantly higher proportion of memantine compared with placebo-treated patients showed any improvement on language $(39 \%$ vs. $32 \%, \mathrm{p}<0.01)$, memory ( $47 \%$ vs. $39 \%, \mathrm{p}<0.001)$, and praxis $(34 \%$ vs. $27 \%, \mathrm{p}<0.01)$. Similar statistically significant results were shown for Week 12 and 24 (OC). Likewise, a significantly higher proportion of memantine-treated patients showed no change or any improvement on the language, memory, and praxis clusters at Week 12 (OC) and 24 (OC and LOCF), compared with placebo-treated patients. The reverse was shown for the any worsening cut-off; a significant smaller proportion of memantine-treated patients showed any worsening on any of the three clusters for all time points (OC and LOCF).

Conclusion: This analysis revealed that memantine treatment provides significant benefits on the language, memory, and praxis domains of cognition in patients with moderate to severe $\mathrm{AD}$.

Profs Mecocci and Emre have been consultants, received research funding, and honoraria from companies in the field of dementia incl Lundbeck A/S, Novartis, Pfizer, Myriad, and Eisai, and Dr Stender is a full-time employee of H Lundbeck A/S

\section{P790}

Anodal transcranial direct current stimulation improves recognition memory in Alzheimer patients

R. Ferrucci, F. Mameli, S. Mrakic-Sposta, M. Vergari, S. Marceglia, M. Fumagalli, F. Cogiamanian, I. Guidi, E. Scarpini, A. Priori

Fondazione IRCCS Ospedale Maggiore Policlinico (Milan, IT)

Objective: Alzheimer disease $(\mathrm{AD})$ is a progressive neurodegenerative disorder characterized by a selective hypoactivation of temporo-parietal (T-P) areas, normally involved in recognition memory. Transcranial direct current stimulation (tDCS) is a non-invasive technique for focal modulation of brain that induces persisting excitability changes of the human cerebral cortex. The aim of this study is to evaluate the cognitive effect of tDCS over the T-P areas in patients with probable AD.

Methods: To assess the cognitive effect of tDCS ( $1.5 \mathrm{~mA}, 15$ minutes) in 8 patients with probable AD, we delivered anodal tDCS (AtDCS), cathodal tDCS (CtDCS) and sham tDCS (StDCS) over the T-P areas bilaterally (P3-T5 left side; P6-T4 right side) in three different sessions, at intervals of at least 1 week. In each session recognition memory was tested at baseline (T0) and 30 minutes after tDCS ended (T1) with a Word Recognition Task (WRT). The accuracy is the number of correct responses from 24 -words list. 
Results: Anodal tDCS at T1 improved the accuracy of the WRT ([mean \pm SD] anodal: T0 $15 \pm 2.83$ vs T1 17.57 $\left.\pm 3.10, p=0.0076^{*}\right)$, while cathodal and sham tDCS failed to induce significant changes.

Conclusion: Anodal tDCS, over the T-P cortex, improves recognition memory in subjects with AD. Because tDCS is simple, safe and inexpensive, our finding prompts studies using repeated tDCS treatment, in conjunction with other therapeutic interventions for the management of $\mathrm{AD}$ patients.

\section{P791}

Cognitive dysfunction after lacunar infarcts

R. Chirileanu, M. Simu, D. Reisz, S. Males, R. Popa, R. Tudor, C. Cirlig, C. Rosca

University of Medicine and Pharmacy (Timisoara, RO)

Aim of the study: Cerebral white matter changes (WMC) and lacunar infarct are both believed to be consequence of small vessel disease. It seems that subcortical lesions may influence cognitive performances evan at early stages of cognitive impairment. We intent to evaluate the cognitive performances of patiens with lacunar stroke

Methods: We recruited 78 consecutive patients who were admitted to the hospital, because of lacunar infarcts documented through MRI. WMC were measured qualitatively and quantitatively. We wanted to describe the frequency and distribution of the lacunar infarcts, and to test their association with cognitive characteristics in MCI.

Rezults: Among the 78 included patients with acute lacunar infarcts, those patients $(n=25) 32 \%$ within the highest quartile of WMC were older, had more lacunar infarcts, more severe stroke, and lower prestroke cognitive function compared with those with less WMC. In addition, their performances in psychometric tests were significantly more impaired. 15 patients (19\%) presented MCI, 18 patients $23 \%$ presented prestroke dementia, and 20 patients $(25 \%)$ with vascular cognitive decline, with poor performances at the executive functions.

Conclusions: Extent of WMC appears to be associated with executive dysfunction in stroke patients with lacunar infarcts. Further large prospective studies with extensive scales of executive function testing are required to confirm this issue. In patients with lacunar stroke, presence of leukoaraiosis is associated with worse performance in multiple cognitive domains. These findings suggest lacunar infarcts plus leukoaraiosis is a common etiology for vascular dementia.

\section{P792}

Cognitive performance in patients with Alzheimer's disease

R. Obereigneru, K. Kollarova, A. Cinova, P. Kanovský

University Hospital (Olomouc, CZ)

Dementia refers to a syndrome that is characterized by progressive deterioration of cognitive functions. Alzheimer disease (AD) is the most common neurodegenerative disorder accounting for at least $50 \%$ of all cases. The aim of the study was to find a neuropsychological method, that helps easily assess cognitive performance in patients with Alzheimer disease. We used Short test for general intelligence (Kurztest für Allgemeine Intelligenz, KAI) and Wechsler Adult Intelligence Scale - Revised (WAIS-R, Wechsler, 1981). Both methods we compared and findings were correlated.

22 patients were studied (12 males, 10 females, mean age 71, SD 6.2 years) with mild-to-moderate AD. The Wechsler intelligence scales are individually administered, composite intelligence tests in a battery format. They assess different areas of intellectual abilities and create a situation in which aspects of personality can be observed. WAIS-R provides three different IQ scores: an overall or Full Scale IQ, a Verbal IQ, and a Performance IQ. WAIS-R was standardized for Czech population. The time for administration is approx. 90 minutes.

KAI is a performance test for adults, the main usage areas are diagnostics of intelligence and findings about the general psychical performance.

The time for administration is approx. 10 minutes.

KAI measures:

A; basic characteristics of information processing: speed of information processing, and "moment of presence", both indices represent the capacity of short-term memory (auditive component, memory span) B; an actual level of overall intelligence (factor "g")

There are significant $(\mathrm{p}<0.01)$ correlations between KAI scale and WAIS-R scales. The highest value of correlation coefficient is for the Full scale WAIS-R and KAI, $\mathrm{r}=0.638$. The lowest value of the correlation coefficient is between Performance scale WAIS-R and KAI, this is caused by higher value of standard deviation in the Performance scale of WAIS-R $(S D=3.61)$.

This pilot study suggests that using KAI as an alternative method for the brief assessment of cognitive functions is meaningful for the practical application.
However, although KAI may offer potentially useful information, it is not considered to be as effective as WAIS-R or other methods explicitly designed for neuropsychodiagnostic purposes. Regarding repeatability, KAI satisfies our requirement, the significant value of the correlation is sufficient evidence for the practical application.

\section{Extrapyramidal disorders}

\section{P793}

Hypoceruloplasminaemia-related movement disorder without KayserFleischer rings: atypical Wilson disease or beyond? C. Zhong, L. Jin, H. Zhang, J. Jin, G. Fei, Y. Zhang, X. Pan, X. Zheng, Y. Mao, Y. Ma, J. Chen, N. Zhao

Zhongshan Hospital, Fudan University (Shanghai, CN)

Objective: To investigate clinical and molecular features and diagnosis of hypoceruloplasminemia related movement disorder (HCMD) without Kayser-Fleischer rings as well as the effect of penicillamine.

Methods: Clinical feature, serum CP, serum copper, urine copper, ATP7B gene sequence, and Apo E genotypes were investigated in 29 patients with HCMD. Seventeen patients with HCMD received voluntarily penicillamine therapy and 11 patients were followed up.

Results: Serum CP in HCMD group $(0.179 \pm 0.029 \mathrm{~g} / \mathrm{L})$ was lower than health control group $(0.291 \pm 0.049 \mathrm{~g} / \mathrm{L}, \mathrm{p}<0.05)$. One homozygous mutation of single nucleotide acid in ATP7B gene $(2467 \mathrm{C}>\mathrm{G}$, Leu823 $>$ Leu) was found and met the DNA diagnostic criteria of Wilson disease in one patient. Four single nucleotide polymorphisms (SNPs) were found and their frequencies were not different between health control group and HCMD group. Six patients exhibited consistent sequence of ATP7B gene listed by GeneBank without any mutation and SNP. The difference of the frequency of non-3/3 genotype was not significant between the two groups. After the D-penicillamine treatment, symptoms were completely removed in one patient, ameliorated in $8 / 11$ patients, and unrelieved in only $2 / 11$ patients.

Conclusion: Only a few HCMD patients were atypical Wilson disease and most HCMD patients might have other types of metabolic disorder of ceruloplasmin. Further investigation of molecular biology and trial of mental chelating treatment on non-WD part should be encouraged.

\section{P794}

Braak's staging in the clinical practice: neurodegenerative markers in cerebrospinal fluid and motor impairment in Parkinson's disease H. Vranova, P. Kanovsky, M. Nevrly, J. Mares, I. Nestrasil, D. Stejskal University Hospital (Olomouc, CZ); Department of Laboratory Medicine (Sternberk, CZ)

Objective: Parkinson disease (PD) is a progressive degenerative illness of the nervous system manifesting itself clinically after the pathology already has reached an advanced stage. According to the Braak's staging the degree of brain pathology in Parkinson disease (PD) is correlated with the motor impairment assessed by Hoehn-Yahr scale. The severity of neurodegeneration should correlate with neurodegenerative markers CSF levels. To assess tauprotein, beta-amyoid(1-42) and cystatin C CSF levels in patients suffering from $\mathrm{PD}$ and in the control group and to compare CSF levels between these two groups and correlate to the both PD duration and severity of motor impairment.

Methods: Tau-protein, beta-amyoid (1-42) and cystatin C in CSF were assessed in 32 patients suffering from PD (23 males, 9 females, aged 37-73, mean $59.9 \pm 11.96$ years), and in a control group (CG) of 20 patients (11 males, 9 females, aged $35-75$, mean $51.2 \pm 10.74$ years). Hoehn-Yahr scale was used for the assessment of the severity of motor manifestation.

Mann-Whitney test and Spearmann correlation were used when assessing statistical significance of the results (CG vs. PD, PD with duration less than 2 years vs. PD with duration more than 2 years, correlation with $\mathrm{H} \& \mathrm{Y}$ stage).

Results: The following statistically significant differences in the CSF were found: higher tau-protein levels in PD patients versus CG $(p=0.05)$, higher tau-protein levels $(p=0.03)$ and tau-protein/beta-amyoid $(1-42)$ ratio $(\mathrm{p}=0.04)$ in PD patients with duration less than 2 years vs. PD with duration more than 2 years. No significant correlation was found between the tauprotein CSF levels and the severity of motor manifestation of PD. No difference in levels of beta-amyoid(1-42) and cystatin C in CSF was found in the CG and PD patients groups.

Conclusion: The study did not approve any correlation between neurodegenerative markers CSF levels and severity of motor manifestation of 
PD. Tau-protein CSF levels were higher in the group of PD patients with disease duration less than 2 years than in the group of PD patients with duration more than 2 years. It can be assumed that the maximum of neurodegenerative changes in PD patients is happening in the first two years after the disease onset.

P795

The phenotype of extrapyramidal tauopathies and the prion protein gene polymorphisms

K. Sabova, D.R. Zimmermann, J.M. Burgunder, H.H. Jung

University Hospital Zurich (Zurich, CH); University Hospital Berne (Berne, $\mathrm{CH})$

Objectives: To determine if polymorphisms in the prion protein gene (PRNP) are associated with particular phenotypes of extrapyramidal tauopathies.

Methods: To date, we studied 24 patients with progressive supranuclear palsy (PSP; $\mathrm{N}=15$ ) and corticobasal degeneration (CBD; $\mathrm{N}=9)$. Review of medical records, structured interview, neurological examination and DNA extraction were performed in all patients. The single exon of the PRNP gene was entirely sequenced for the presence of mutations and determination of known polymorphisms in all patients. Further structured clinical evaluation including assessment of cognitive (MMS, FAB, ADL-FAQ) and movement disorders (UPDRS, WHIGET tremor scale, PSP scale) scores were performed in a subset of 15 patients.

Results: Most sensitive predictors of the phenotype were pronounced vertical gaze palsy in PSP and apraxia as well as cortical sensory loss in CDB. Molecular genetic analysis revealed no PRNP mutations in the cohort. PSP and CDB patients homozygous for Met at the codon $129 \mathrm{Met} / \mathrm{Val}$ polymorphism tended to have an earlier disease onset compared to Patients with Met/Val or Val/Val. PSP patients with Met/Val tended to have a better response to L-Dopa and less cognitive deficits compared to PSP patients with Met/Met.

Conclusions: Although these data have to be confirmed in a larger cohort in this ongoing study our preliminary results suggest that PRNP genotype might be associated with specific phenotypic features in extrapyramidal tauopathies.

This study was supported by ENS Grant in 2007.

\section{P796}

Canadian survey to assess regional differences in the diagnosis and management of movement disorders responsive to botulinum toxin type- $\mathrm{A}$ M. Jog and The Canadian Movement Disorder Study Group

Objective: To assess diagnostic and treatment pathways of movement disorders responsive to botulinum toxin type-A (BoNTA) by geographic region and to review their relative frequency.

Methods: Patients with BoNTA responsive movement disorders completed a 19-question survey developed by the Canadian Movement Disorder Survey Group in 13 Canadian centres. The survey included demographics, time to diagnosis, number of physicians seen and wait times.

Results: This interim analysis includes 698 patients. $72 \%$ were female. The average age was $57 \mathrm{yrs}, 29 \%$ being over 65 . Average travel was $74 \mathrm{kms}$ one-way, ranging from 44 (Quebec) to $99 \mathrm{kms}$ (Atlantic provinces). Common diagnoses included cervical dystonia $(53 \%)$, hemifacial spasm $(20 \%)$, and blepharospasm (10\%). The average number of physicians seen prior to diagnosis was 3.2, ranging from 3.0 (Ontario) to 4.0 (Atlantic provinces). Average time from onset of symptoms to diagnosis was $4.7 \mathrm{yrs}$, ranging from 2.7 (Western provinces) to $6.2 \mathrm{yrs}$ (Quebec). $94 \%$ of patients were treated with BoNTA following diagnosis. Average waiting time to treatment was 3.2 months. Common reasons for delay were physician waiting lists (50\%) or insurance paper work $(20 \%)$

Conclusion: Regional disparity in diagnosis and treatment timelines in Canada may improve with selective awareness programs coupled with additional trained injectors in targeted regions.

\section{P797}

Irreversible parkinsonism due to acute organophosphate intoxication

S. Jung, J-K. Kwak, S-B. Kwon, S-H. Hwang, K-H. Kwon, B-C. Lee

Hallym University College of Medicine (Seoul, KR)

Background and significance: A few cases of parkinsonism following organophosphate insecticides poisoning were reported in the world. Their symptoms fully disappeared within 6 months at longest and brain imagings were normal.
Case: A 55-year-old female developed parkinsonian symptoms such as bradykinesia, shuffling gait, rigidity, and freezing, etc. after acute organophosphate intoxication for suicidal attempt. The brain MRI was done 4 years after onset of parkinsonian symptom. Bilateral basal ganglia lesions (round in globus pallidus and linear in putamen and caudate nucleus) was revealed as low signal intensity on T1-weighted image and high signal intensity on T2-weighted image. Her parkinsonian symptoms were not responsive to conventional antiparkinsonian medication and still continued without change.

Conclusions or commentes: We suggest that brief, large amount of organophosphate intoxication can produce irreversible parkinsonism according to individual susceptibility. In further study including insecticides as environmental factor of parkinsonism, neuroimaging must be done.

\section{P798}

A systematic review of functional imaging in vascular parkinsonism

S. Kalra, D.G. Grosset, H.T.S. Benamer

New Cross Hospital (Wolverhampton, UK); Southern General Hospital (Glasgow, UK)

Background: Vascular parkinsonism (VP) can be clinically confused with other types of parkinsonism. Functional imaging might potentially assist in diagnosing VP. We undertook a systematic literature review of functional imaging studies in the differential diagnosis of VP and idiopathic Parkinson's disease (PD).

Methods: Medline, Embase, Cinahl and PsycINFO databases were searched using a combination of key words. References in all relevant papers were reviewed for additional publications. Papers published before December 2007 and written in English were included.

Results: Seven studies were identified. Three studies looked at the striatal dopamine transporters (DAT) in VP using 123I-b-CIT SPECT (single photon emission computed tomography) or 123I FP-CIT SPECT (DaTSCAN, GE Healthcare). One study reported preserved or mild reduction in the striatal 123I-CIT binding and the putamen/caudate ratio in $13 \mathrm{VP}$ patients compared with 30 normal controls, but a marked reduction in striatal 123I-b-CIT binding in $20 \mathrm{PD}$ patients, especially in putamen. In another study, the mean asymmetry index was significantly lower in $13 \mathrm{VP}$ patients compared to PD using 123I FP-CIT SPECT. In a third report, 20 VP patients were compared to 20 healthy controls with 123I FP-CIT: 9 patients had normal striatal binding comparable with those of the controls. In contrast, 11 patients had significantly diminished striatal binding when compared to controls.

A single study tested both presynaptic DAT and postsynaptic (D2 receptor) striatal activity using 123I FP-CIT and IBZM uptake, but no definitive conclusions were drawn due to the sample size being only 4 cases. Another study showed significant reduction and asymmetry of the uptake of $99 \mathrm{mTc}-$ TRODAT-1 putamen/occipital and striatal/ occipital uptake ratios in PD but not VP. Proton magnetic resonance spectroscopy (MRS) showed no evidence for existence of ischemia or cell loss in patients with VP when compared with PD. Cardiac 123I Metaiodobenzylguanidine (MIBG) uptake showed significantly higher uptake in $19 \mathrm{VP}$ compared to $30 \mathrm{PD}$ patients.

Conclusions: Functional imaging studies of VP are all small and show inconsistent results. Larger studies are needed, to determine their role in guiding the diagnosis and treatment of VP.

\section{P799}

Transcranial direct current stimulation over the motor-premotor cortex in two patients with Tourette's syndrome

S. Marceglia, S. Mrakic-Sposta, F. Mameli, R. Dilena, R. Ferrucci, M. Fumagalli, S. Barbieri, N. Bresolin, A. Priori

Fondazione IRCCS Ospedale Maggiore (Milan, IT)

Objective: Bacause transcranial direct current stimulation (TDCS) induces persistent changes of cortical excitability in humans and functional neuroimaging studies consistently showed abnormalities in frontal cortex of patients with Tourette syndrome (TS), we tested tDCS over the motor-premotor cortex (M-PM) in two patients with TS.

Methods: The patients' motor state was evaluated by tic counting on 10 minutes-videotape. The experimental protocol comprised two sessions (one for cathodal and one for sham-placebo tDCS) each consisting of 5 consecutive days of treatment. Cathodal tDCS $(2 \mathrm{~mA}, 15 \mathrm{~min}$, daily for five consecutive days) was delivered over M-PM cortex monolaterally. In each session, patients were assessed twice, before the beginning of the treatment (day 1) and after 5 consecutive days of treatment (sham or cathodal tDCS).

Results: Whereas sham tDCS left the number of tics unchanged, cathodal tDCS decreased motor and phonic tics (patient 1: motor tics $-21 \%$; phonic tics $-34 \%$; patient 2 : motor tics $-26 \%$; phonic tics $-46 \%$ ).

Conclusions: In our TS patients cathodal tDCS decreased the number of 
motor and phonic tics. Our results prompt further controlled studies for the evaluation of $\mathrm{tDCS}$ as a treatments for TS patients.

\section{P800}

Spasmodic dysphonia may respond to bilateral thalamic deep brain stimulation

V. Evidente, M. Lyons, S. Bansberg, W. Woods, K. Mcnett, R. Hillman, M. Wheeler, C. Adler

Mayo Clinic (Scottsdale, US)

Objective: To describe the effects of bilateral thalamic deep brain stimulation (DBS) surgery in a patient with spasmodic dysphonia (SD) and essential tremor (ET)

Background: There are scant reports of thalamic DBS improving voice tremor in ET patients. There are no published reports of DBS improving SD or dystonic tremor associated with SD. We report a patient with hand tremors due to ET and a voice disorder consistent with adductor SD with a vocal dystonic tremor who responded to bilateral thalamic DBS.

Methods: case description

Cace: A 72 yo woman was seen at Mayo Clinic with a 30-year history of hand tremors from ET. Her hand tremors worsened during the last decade, being refractory to medications including beta-blockers, gabapentin, clonazepam, mirtazapine, and primidone. She also developed adductor type SD at age 64, and received regular botulinum toxin type A (BoNT) injections with good results. In the past year, she claimed that the chronic BoNT injections have made her voice severely hypophonic and high-pitched, and that she did not want further injections. On examination last year, she had a moderate postural tremor and mild resting tremor of both hands, and moderate SD with vocal dystonic tremor. Bilateral thalamic DBS was performed, and at 6 months post-DBS, she rated her hand tremors almost $100 \%$ improved, and her SD about $75 \%$ improved. Voice/laryngovideostroboscopic examinations were done, comparing stimulators ON versus stimulators OFF (rater being blinded). With stimulators OFF, the voice was perceptually rated as moderate SD with moderate-severe vocal tremor; videostroboscopy showed intermittent aperiodicity with laryngeal tremulousness and intermittent incomplete vocal cord closure with sustained phonation. With stimulators $\mathrm{ON}$, the voice was perceptually rated as mild SD with no vocal tremor; videostroboscopy showed no laryngeal tremulousness and complete closure of the vocal cords with sustained phonation. Pulse generator settings at 6 months were $3+, 0,2.3$ volts, 90 milliseconds, and $130 \mathrm{~Hz}$ for the right side; for the left generator, settings were 2+, 0-, 3.2 volts, 90 milliseconds, and $130 \mathrm{~Hz}$. Since DBS, patient has not needed any tremor medications nor BoNT injections.

Conclusion: This case demonstrates improvement not only of tremor but also of spasmodic dysphonia in a patient who underwent bilateral thalamic DBS. Further similar cases may need to be followed closely to see if thalamic DBS may be a viable option for patients with SD.

\section{Preclinical neurology}

P802

Stiff person syndrome: intrathecal passive transfer to the rat with anti-amphiphysin antibodies

C. Geis, A. Weishaupt, S. Hallermann, C. Wessig, T. Wultsch, A. Reif, N. Byts, M. Beck, S. Jablonka, M.K. Boettger, N. Üceyler, W. Fouquet, A.-L. Sirén, S.J. Sigrist, K.V. Toyka, M. Heckmann, C. Sommer

University of Wurzburg (Wurzburg, DE)

Objectives: Paraneoplastic Stiff person syndrome (SPS) with autoantibodies against the intracellular protein amphiphysin is a rare CNS disorder. We investigated the pathogenic role and the specificity of these autoantibodies (Abs).

Methods: Affinity-purified anti-amphiphysin Abs of a patient with SPS were injected intrathecally (i.th.) into rats and their behaviour was analysed. Electrophysiologically, alterations of the H-Reflex and GABAergic dorsal root potentials (DRP) were measured in vivo. IgG binding was localised in the spinal cord with standard immunofluorescence and high-resolution STED (Stimulation Emission Depletion) microscopy. Antigen specificity was tested in in vitro assays by substrate competition and antigen preabsorption using dissociated hippocampal neuron cultures.

Results: I.th. injection of specific anti-amphiphysin Abs induced symptoms of SPS in rats including stiffness, spasms, decreased motor activity and reduced exploration in the elevated plus maze suggesting increased anxietyrelated behaviour. Specific depletion of the anti-amphiphysin Abs from the patient IgG by affinity chromatography abolished these effects. Physiological post-activation depression and D1/D2 inhibition of the H-Reflex were both significantly lowered in animals treated with anti-amphiphysin Abs. Dorsal root potentials were reduced after single and repetitive volleys to $43 \%$ and $51 \%$, respectively, indicating reduced GABAergic inhibition. Immunofluorescence of spinal cord sections revealed colocalization of the injected Abs with presynaptic proteins. Incubation of hippocampal neurons with tagged anti-amphiphysin Abs lead to an amphiphysin-epitope-specific binding and internalization of Abs.

Conclusion: Autoantibodies against the intracellular synaptic protein amphiphysin reproduced the disease-defining phenotype of SPS in an i.th. passive transfer model. Spinal GABAergic presynaptic inhibition was markedly reduced, leading to an increased motor output consistent with neurological symptoms observed in the behavioural analysis. Molecular specificity was shown in-vivo and in-vitro. According to the wide-spread distribution of amphiphysin, also supraspinal functions were altered.SPS is the first CNS disorder with a proven antibody-mediated mechanism caused by autoantibodies against an intracellular protein antigen.

\section{P803}

Grey matter dysfunction in acute and chronic hydrocephalus

D. Kondziella, E. Eyjolfsson, U. Sonnewald, O. Risa

University Hospital Sahlgrenska (Gothenburg, SE); Norwegian University of Science and Technology (Trondheim, NO)

Objectives: Hydrocephalus is one of the few reversible causes of dementia and recent epidemiological studies have shown that normal pressure hydrocephalus (NPH) has a far higher prevalence than earlier estimated. Since dementia is an increasing socioeconomic problem, NPH and other forms of chronic hydrocephalus have become a hot topic in clinical neurology. However, in contrast to earlier doctrines, chronic hydrocephalus is now considered as much a disorder of cellular metabolism as of cerebrospinal fluid (CSF) dynamics. Although paraventricular white matter is mainly affected, also gray matter metabolism is probably disturbed. Yet these disturbances have received little interest so far. We therefore assessed gray matter metabolism in experimental acute and chronic hydrocephalus using in vivo Magnetic Resonance Imaging (MRI) and 1H Magnetic Resonance Spectroscopy (MRS).

Methods: 27 rats were made hydrocephalic by injection of $0.1 \mathrm{ml}$ kaolin into the cisterna magna. 10 rats served as controls. Using a 7-tesla magnet, in vivo MRI and $1 \mathrm{H}$ MRS were performed longitudinally two, four and six weeks later. Volumes of interest included the gray matter regions cortex, thalamus and hippocampus.

Results: In hydrocephalic animals, $1 \mathrm{H}$ MRS revealed decreased glutamate levels in all examined areas at all time points. Moreover, in the acute period levels of $\mathrm{N}$-asparate, creatine, inositol and taurine were reduced in the hippocampus, and taurine was also decreased in the cortex. In addition, T2weighted images showed increase of free water in the hippocampus. A clear lactate peak was detected on spectra from CSF.

Conclusion: It is concluded that gray matter metabolic disturbances indeed exist in hydrocephalus. We hypothesize that decreased cortical gluta- 
matergic control of subcortical structures may contribute to cognitive decline and motor symptoms in hydrocephalus.

\section{P804}

Engraftment of donor cells in CNS depends on proper timing of bone marrow transplantation after preconditioning with cyclophosphamide

P. Jiroutek, L. Š efc

1 st Faculty of Medicine (Prague, CZ)

Objectives: The presence of donor bone marrow (BM) derived cells in CNS seems to have neuroprotective effect in animal models of various neurological disorders. The positive effect of BM transplantation or human umbilical cord blood transplantation was observed in mice model of amyotrophic lateral sclerosis, ischemic brain lesion and traumatic spinal cord lesion. We believe that the crutial factor in the process of neuroprotection madiated by $\mathrm{BM}$ derived cells is the long term survival of the donor cells in CNS. We studied the factors that affect the long term survival of BM derived donor cells in host CNS after BM transplantation. We have tested the hypothesis that the successful BM engraftment is one of the most important features that increses the pool of donor cells in host CNS.

Methods: Three gopups of C57BL/6 mice were irradiated (4Gy, whole body irradiation) and transplanted with green fluorescent protein positive $($ GFP+) BM (4*106 nucleated cells). Group CY2 was preconditioned with cyclophosphamide (CY) $(135 \mathrm{mg} / \mathrm{kg}$ body weight) two days before the BM transplantation (BMT) and group CY5 five days before the BMT. No CY was administrated in the control group. We evaluated the time course of BM chimerism and the number of GFP+ cells in the host CNS twelve month after BMT. The changes of microenvironment in BM after CY administration were analysed properly.

Results: The number of colony forming units (CFU-S) in BM after CY administration fell rapidly to its minimum of $10 \%$ on day 1 and raised again to the local maximum of $70 \%$ on day 3 and than decreased slowly. Percentage of CFU-S in S phase increased to its maximum (52\%) on day 2 and reached the minimum on day 5 , where almost none (1\%) CFU-S in S phase were detected.

The BM chimerism reached $87 \%$ in CY5 group, $25 \%$ in CY2 and $29 \%$ in the control group

In the CNS, the density of GFP+ cells $\left(10-8 / \mathrm{um}^{2}\right)$ was $8,98(\mathrm{p}<0.001)$ in CY5 group, 1,44 in CY2 group and 1,60 in the control group.

Conclusion: The timing of BMT after preconditioning with CY strongly influence the $B M$ engraftment. The microenvironment of high proliferating CFU-S on day 2 after CY administration probably interferes with the ability of the donor cells to keep in the BM in contrast to the BMT performed on day 5 . The degree of $\mathrm{BM}$ chimerism strongly correlates with the long time presence of donor cells in CNS. Successful BM engaftment seems to be an important factor for maintaining the donor cells pool in the host CNS after BMT.

\section{Supported by GA UK 118907}

\section{P805}

Transgenic BDNF expression ameliorates the course of experimental autoimmune encephalomyelitis

S. Dhib-Jalbut, J. Chen, N. Ito, P. Casaccia-Bonnefil, K. Ito

UMDNJ-Robert Wood Johnson Medical School (New Brunswick, US)

Objective: To examine the pre-clinical effect of brain-derived neurotrophic factor (BDNF) on the course of a neurodegenerative model of experimental autoimmune encephalomyelitis (EAE).

Methods: EAE was induced in C57BL/6 mice by the adoptive transfer of myelin oligodendrocyte glycoprotein (MOG 35-55) specific T-cells. This model is characterized by a monophasic inflammatory process followed by a chronic phase with axonal and neuronal degeneration. The course of EAE was compared in human BDNF-transgenic (Tg) mice to that of wild type (WT) mice. BDNF expression in mouse brain was analyzed by quantitative RT-PCR. EAE clinical severity was assessed on a scale of 1-5.

Results: BDNF transcripts were 2.3 fold higher in the brain of BDNF-Tg mice compared to their wild type littermates. EAE was induced in both groups (5 mice per group) and were followed up clinically for 115 days. The human BDNF transgene did not seem to have an effect on the onset or severity of the acute phase. However, recovery from EAE was more efficient in the Human BDNF-Tg group. Mean EAE clinical score in BDNF-Tg mice was consistently lower than that in the WT-mice at all time points between days 20 and 115 following EAE induction. Mean clinical EAE score at day 115 was $0.3+0.27$ compared to $2.6+1.9$ in the WT-mice (p-value: 0.0182 ). Pathological analysis of CNS tissue from both groups is being examined and will be presented.
Conclusion: The results indicate that over-expression of BDNF in transgenic mice is beneficial for recovery from EAE. Consequently, therapies that enhance BDNF expression in the CNS should be considered in the treatment of neurodegenerative diseases associated with CNS inflammation such as Multiple Sclerosis.

Supported by funds from the National MS Society, USA.

\section{General neurology}

\section{P806}

Focusing attention during writing - A reliable method to change the level of automation

A. Nolte, M. Wiesmann

Inn-Salzach-Klinikum (Munich, DE); Ludwig-Maximilians-Universität München (Munich, DE)

Introduction: Writing can be differentiated in two distinct movement patterns: Automated writing also known as open-loop writing and non-automated writing or closed-loop writing. Up today this determination is made by considering the number of inversions in the velocity curve (NIV) with a kinematical analysis. A NIV close to 1 defines the movement as automated, while a NIV significantly greater than 1 is considered as non-automated movements.

Objectives: Up to now the literature mentions several methods to modify the automation level of writing movements. All these methods have in common that more than one parameter of the movement is varied; hence the comparability of the resulting movements by these methods can not be guaranteed sufficiently in respect to their sole effect upon the level of automation. Due to the fact that comparability of results is one key-role of research, a reliable method to modify the automation level meanwhile keeping all other motion parameters unchanged is a pre-requisite. Additionally to this the targeted method should fulfil the following requirements: securely reproducible, with a defined trigger, independent of the subject and the executed motion pattern, and feasible for functional imaging as well as for kinematical analysis.

Methods and results: This work researches whether focusing the attention to the executed writing movement leads to a shift from automated to non-automated writing. The used kinematical analysis together with the results of the functional magnetic resonance imaging (fMRI) revealed clearly that the shift of the attention focus is correlated to a significant change in the level of automation. The common observation that non-automated writing is slower than automated writing was made in accordance with the literature. A controlling study revealed that this slowing down did not affected the level of automation at all. Therefore the conclusion is obvious that the observed change of the level of automation is only a result from the shift of attention.

Conclusion: The shift of attention during writing proves as a reliable method to alter the level of automation for movements. Even more this method allows to keep all other motion parameters constant, is securely reproducible, is simple to trigger, is applicable by almost every subject and with every motion task, and is compatible with kinematical analysis and functional imaging.

\section{P807}

Seizures, subacute dementia and ear inflammation

A. Oliveira, V. Cruz, P. Coutinho

Hospital S. Sebastião (Santa Maria da Feira, PT)

Background: Relapsing polychondritis (RP) is a rare connective tissue disease defined by episodic inflammation of cartilaginous structures and less frequently other tissues with a common antigen. The CNS manifestations occur in $3 \%$ of patients and although their mechanisms are not well understood the relation with a vasculitis phenomenon is possible.

Case report: A 50-year-old man was admitted because of clonic movements of his right hand followed by secondary generalization. A diagnosis of bipolar disease was made years ago with a prescription of mood stabilizers. Six months before admission he began to have de novo short-term memory and naming difficulties with a progressive worsening since then. Two years earlier he started to have episodes of ocular and ear inflammation with spontaneous remissions and relapses. On neurological examination he had an important cognitive impairment: motor transcortical aphasia, shortterm memory deficit and ideomotor apraxia. The remaining exam was unremarkable except for a bilateral Babinski. A general physical examination 
showed inflammation in both ears with sweeling and tenderness. The erythrocyte sedimentation rate was $35 \mathrm{~mm} /$ hour. CSF examination showed a clear fluid, 16 cells $/ \mu \mathrm{L}$ (mostly lymphocytes), proteins $85.3 \mathrm{mg} / \mathrm{dL}$, normal glucose and without microorganisms or malignant cells at microscopical study. EEG showed slow background rhythm and left fronto-temporal continuous slowing. Cranial MRI showed bilateral subcortical white matter hyperintensities on T2-weighted images. A biopsy of the right pinna was performed. The histologic examination showed a marked inflammatory infiltration (predominantly lymphocytes) in the cartilage and the chondrocytes appeared altered. Methilprednisolone and monthly pulsed doses of cyclophosphamide were administered. A dramatic clinical improvement was noted.

Conclusion: This patient was admitted because of seizures and its investigation brought to clinical attention other previous symptoms: relapsing episodes of cartilaginous inflammation and a cognitive impairment thought to be due to the previous psychiatric disorder. Characteristic histologic changes of RP were found after biopsy of the pinna. Clinical improvement after immunosuppressant treatment supported the association between the neurologic and systemic manifestations. The relation between the former and this rare disease was then possible. These very uncommon features of $\mathrm{RP}$ were probably the result of a CNS vasculitis.

\section{P808}

Outcomes of joint neurology-urology clinic in the management of urogenital symptoms in patients with chronic neurological conditions

B. Menezes, C. Young, G. Singh

University Hospital Aintree (Liverpool, UK); The Walton Centre for Neurology and Neurosurgery (Liverpool, UK); Southport General Hospital (Southport, UK)

Background: The joint Neurology-Urology clinic in Liverpool is the only one of its kind in the UK dealing with urogenital disturbances in patients with chronic neurological conditions. This audit was performed to evaluate its management outcomes.

Methods: Records of all patients attending this clinic over a 4 year period were reviewed. Follow-up information of those attending one clinic was retrieved from local Trusts. Outcomes were measured as: significantly positive (prolonged symptomatic resolution), some positive (early symptomatic recurrence) and no positive outcome (no symptomatic benefit).

Results: 109 patients (mean age at presentation: 42.95 years) attended the clinic during the specified period. $60 \%$ of patients had multiple sclerosis while vascular/traumatic/post-operative causes accounted for primary diagnoses in nearly $25 \%$. Common symptoms included frequency, incontinence, urgency, recurrent cystitis, catheter-related difficulties and sexual dysfunction.

Investigations included bladder scan (52\%), urodynamics (28\%), radiological investigations (12\%) and flexible cystoscopy (12\%). A large proportion of these yielding abnormalities, prompt interventions and specialist referrals were offered. Interventions included drug changes, catheter-related interventions, 7 referrals for surgical procedures, bladder training/stimulators, botox injections and vacuum devices/rings/caverneject.

$83 \%$ had significantly positive outcome while some positive outcome was noted in another $11 \%$. Common reasons for lack/absence of benefit included poor compliance/refusal of recommended interventions and disease progression. $86 \%$ of those attending only one clinic had experienced a significantly positive outcome.

Conclusions: The Neuro-Uro clinic is an efficient method of managing urogenital symptoms in patients with chronic neurological disorders. A nation-wide implementation of such a dual-disciplinary strategy may yield prompt and cost-effective results in this patient group.

\section{P809}

Implementation of standard operation procedures into an interdisciplinary neuro-intensive care unit

C. Melzer-Gartzke, K. Angstwurm, G-H. Schneider, M. Deja, C. Spies

Charité Universitätsmedizin (Berlin, DE)

Setting: 15 bed interdisciplinary Neuro Intensive care unit run by neurologists, neurosurgeons and anaesthetists in a university hospital.

The relevance of standard operation procedures (SOPs) as implementation tool of evidence-based medicine in clinical routine is proven in many ICU settings (Pronovost, The New Yorker, 12.2007). The costs for sedative drugs and blood products are considered key process indicators in our ICU to evaluate the implementation rate of sedation guidelines and transfusion protocols. The aim of this study was to evaluate the cost of sedative drugs and blood transfusions after implementation of the SOPs.

Intervention: By introducing a quality management system according to
DIN ISO 9001 in November 2006 standard operation procedures (SOP) were developed for the interdisciplinary intensive care unit. Clinical SOPs were adapted from evidence based literature, national and international guidelines and modified to the specific local situation. Data provided from the institutional medical controlling were analyzed comparing January to December 2006 with January to December 2007. Statistics: paired t-test.

Results: Comparing 2006 and 2007 the interdisciplinary Neuro-Intensive Care Unit treated a constant number of patients $(1678 ; 1744 ;+3,9 \%)$ with an identical length of stay $(4,26 ; 4,23,-0,7 \%)$. All patients were comparable with regard to Apache II (97917; 97000; $-0,9 \%)$, SOFA (26557; 25936; -2,3\%), SAPS II $(196833 ; 197318 ;+0,25 \%)$ Scores and TISS-28 (167446; 168193; $+0,45 \%)$ reflecting a stable clinical workload.

A cost reduction for sedative drugs by $11.8 \%$ and a $47 \%$ reduction in costs of blood transfusions were achieved with a guideline implementation rate of $35 \%$ before and $70 \%$ after SOP introduction.

Conclusion: The implementation of guidelines was achieved by SOP implementation on an interdisciplinary Neuro Intensive Care Unit was accompanied by reduced costs for sedation and transfusion.

\section{P810}

Cognitive gating of cerebellar motor control: a case mimicking psychogenic ataxia

E. Reinhart, F. Salih, D. Harnack, K.-T. Hofmann, C.J. Ploner

Charité Campus Virchow Klinikum (Berlin, DE)

Objective: Recent studies have shown that the cerebellum is not only involved in motor coordination but also controls non-motor functions such as cognition and emotion. Functionally, the integration of the cerebellum into the respective motor and non-motor cerebral networks has been assumed to depend on a segregated arrangement of cerebello-thalamo-cortical pathways within closed loops. Here however, we provide some clinical evidence that post-lesional plasticity allows the cerebellum to induce significant interactions between motor and cognitive cerebello-thalamo-cortical circuits.

Case report: A 59-year-old female patient presented with a 4-year history of right-sided limb ataxia, predominantly affecting the leg. MR-imaging of the brain revealed a T2-hyperintense lesion affecting exclusively the most rostral part of the right dentate nucleus and the adjacent segment of the superior cerebellar peduncle (Figure 1A). Examination of cerebral spinal fluid showed oligoclonal banding but no active inflammation. Serum levels for antinuclear antibody-titer was elevated $(1: 640)$ but further autoimmunologic testing was normal. Paradoxically, ataxia subsided when the patient was asked to perform cognitive tasks like word generation or arithmetic tasks. Results from extended standardized cognitive testing were within normal limits.

Discussion: In summary, the conclusions of this case are threefold: 1.) This case represents rare clinical evidence for functional distinction and somatotopy of the dentate nucleus. 2.) The current model of a parallel and segregated organization of motor and cognitive cerebellar circuits is not sufficient to explain the full spectrum of clinical findings with cerebellar disease. 3.) The significance of cognitive distraction as a diagnostic criteria to distinguish organic from psychogenic cerebellar disorders should be reevaluated.

\section{P811}

All abstracts in one: characteristics of abstracts presented in ENS meeting G. Giaglis, K. Mantziava

Aristotle University of Thessaloniki (Thessaloniki, GR)

Objective: To describe the characteristics of abstracts accepted for oral and poster presentation in the 16th Meeting of the European Neurological Society.

Methods: A total of 127 abstracts accepted for oral presentation and 471 abstracts for poster presentation, written by 2372 authors coming from 48 different countries, were analyzed. Elements regarding authorship, study design and sample as well as abstract form were assessed.

Results: Mean number of authors for each abstract was 5.69 (range: $1-20$ ). Scientists from multiple institutions cooperated in $42.7 \%$ and from different countries in $13.5 \%$ of the studies. Germany, Switzerland and Italy accounted together for the $37.1 \%$ of the total number of presentations. 55 $(9.2 \%)$ of the abstracts concerned laboratory experiments, article reviews and theoretical presentations, while the rest $543(90.8 \%)$ investigated human subjects. $132(24.3 \%)$ of these were case studies of 1 to 11 patients. The rest 411 trials were quantitative and concerned a median of 61 subjects ranging from 5 to 39256 . More than half of these studies (54.3\%) were focused on one patient group, while the rest compared different patient groups and/or healthy controls. 114 (27.7\%) trials had a longitudinal design and 71 $(17.3 \%)$ evaluated one or more treatments. $56(13.6 \%)$ of the abstracts de- 
scribed their results without using any numbers, while 167 (40.6\%) simply reported p-values and only $69(16.8 \%)$ mentioned values of specific statistical tests. 114 abstracts were supported by (at least) a grant. MS and Peripheral Neuropathy studies were supported the most $(30.7 \%$ and $14.9 \%$ of the grants, respectively) and in general laboratory experiments and therapeutical assessments. Genetics and neuro-ophthalmology received no grants. Finally, oral presentations tended to have significantly more authors $[\mathrm{t}(596)=5.24, \mathrm{p}<0.001]$, collaboration of more institutions $[\mathrm{t}(577)=3.06$, $\mathrm{p}<0.01$ ], of a larger number of countries [ $\mathrm{t}(577)=2.47, \mathrm{p}<0.05]$, and more often a grant [Fisher's exact, $\mathrm{p}=0.005$ ] than poster presentations; there was no difference regarding the number of patients or patient groups studied.

Conclusion: Even though the most important asset of a study is the dedication and effort of its contributors, issues like financial support, cooperation of many scientists and institutions, a carefully planned design, an adequate sample size and a clear and informative presentation can play a crucial role in predicting the study's usefulness and potential impact.

\section{P812}

Severe hyponatraemia during combined therapy of diuretics and selective serotonin reuptake inhibitor

G. Giannikopoulos, M. Zorzou, A. Sideratou, N. Pantelaros, P. Sitaras, M. Karamouzos, P. Fexi, I. Ydreos, K. Dabos

General Hospital of Chios (Chios, GR)

Objectives: To present a rare case of severe hyponatraemia due to SIADH during combination therapy with selective serotonin reuptake inhibitor (SSRI)s and diuretics and to clarify the possible effect of diuretics on pre-existing sodium levels through a rechallenge use of these agents.

Methods: Thiazide diuretics and SSRIs have been associated with the development of hyponatraemia via different mechanisms for each medication. SSRIs cause hyponatraemia through the syndrome of inappropriate ADH release. The frequency of hyponatraemia from SSRIs in elderly woman is 8 per 1000 , and typically occurs within 2 weeks of initiating therapy. The known risk factors include advanced age, diuretic therapy, preceded low sodium serum concentration and low BMI.

Results: An 80-year-old woman was evaluated for fatigue, anorexia and nausea over the last 3 days and a 24 hour history of headache, dizziness and confusion. Past medical history included arterial hypertension and dyslipidaemia. She was on hydrochlorothiazide/amiloride $(50+5) \mathrm{mg} / \mathrm{day}$ and simvastatin $20 \mathrm{mg} /$ day. Over the last 15 days she was treated with fluoxetine $20 \mathrm{mg} /$ day for depression. The physical examination revealed a feverless euvolaemic patient. BP 135/85 mmHg, HR 78/min, SatO2 $96 \%$, ECG sinus rhythm, GCS14, reduced muscular tone of upper and lower extremities and she was Laboratory findings: $\mathrm{Na}+99 \mathrm{mmol} / \mathrm{L}, \mathrm{K}+4.75 \mathrm{mmol} / \mathrm{L}$, urea $32 \mathrm{mg} / \mathrm{dl}$, creatinine $0,7 \mathrm{mg} / \mathrm{dl}$, glucose $90 \mathrm{mg} / \mathrm{dl}$, uric acid $3.4 \mathrm{mg} / \mathrm{dl}$, plasma osmolality $215 \mathrm{mOsm} / \mathrm{kg}$, urine sodium concentration $60 \mathrm{mmol} / \mathrm{l}$, urine osmolality $316 \mathrm{mOsm} / \mathrm{kg}$, CRP $0.17 \mathrm{mg} / \mathrm{dl}$, cortisol $25 \mathrm{ig} / \mathrm{dl}$, TSH $0,281 \mathrm{IU} / \mathrm{ml}$ The diagnosis of hyponatraemia due to SIADH was established, the SSRI and diuretics discontinued, and the patient was treated with $1 \mathrm{~L}$ Nacl $0,9 \%$ IV per day, furosemide $20 \mathrm{mg}$ IV twice a day and water restriction $(500-750 \mathrm{ml} /$ day). The reinstitution of normal sodium levels was followed by remarkable clinical improvement $(\mathrm{Na}+134 \mathrm{mmol} / \mathrm{L}$ on exit). In the above case, SSRI added to long term diuretic therapy appears to be the cause of SIADH. To determine whether hyponatraemia caused by diuretics existed prior to administration of SSRI, we started the same diuretics with close monitoring of sodium concentration. Two months later, serum sodium concentration was $142 \mathrm{mmol} / \mathrm{L}$.

Conclusion: Physicians must be aware of the possible development of hyponatraemia when patients prescribed both an SSRI and a thiazide diuretic, and serum sodium concentrations should be carefully monitored for early detection of this potential fatal complication.

\section{P813}

Acute idiopathic longitudinal myelitis with rapid improvement

I.H. Son, S. W. Kang, S.G. Lee, S.J. Han, J.S. Cheong

Sanbon Medical Center, Wonkwang University (Gunpo-si, Gyeonggi-do, KR)

Backgrounds: Acute longitudinal myelitis is a group of disorders characterized by multifocal or long segmental inflammation of the spinal cord, rapidly evolving paraparesis or paraplegia, a sensory level on the trunk, and bilateral Babinski signs.

Case: A 30-year-old man presented with progressive paraparesis which began 4 days before admission. His neurological deficits occurred abruptly and showed rapid progression to paraplegia (MRC grade 1), neurogenic bladder only within 2 days. The sensory deficit was occurred below T4 level. Labotary data showed a white blood cell count of $8,900 / \mathrm{uL}, \mathrm{ESR}$ of $3.0 \mathrm{~mm} / \mathrm{hr}$ (normal $<20$ ). Antinuclear antibody (ANA) titer was negative and anti-ds-
DNA antibody, anti-Smith antibody, anti-phospholipid antibody, anti-cardiolipin antibodies (IgG,IgM), anti-RNP antibody, anti-SSA/SSB antibodies was normal. Serum complement levels was C3 of $128 \mathrm{mg} / \mathrm{dl}$ (normal 90 180) and $\mathrm{C} 4$ of $33 \mathrm{mg} / \mathrm{dl}$ (normal 10 40). And the VDRL test were all negative. Aspirated cerebrospinal fluid contained leukocyte of $432 \mathrm{HPF}$, protein 92.2 $\mathrm{mg} / \mathrm{dl}$ (15 45), and glucose $69 \mathrm{mg} / \mathrm{dl}$ (50 75). Subsequently, the Gram stain and culture of CSF were found to be negative. (IgG index was 0.8) Somatosensory evoked potential findings were suggestive of central conduction defect in bilateral posterior tibial nerve stimulated SSEP pathway.

MRI of the whole spinal cord showed multifocal high-signal intensities involving the cervical, thoracic, and lumbar spinal cord. Brain MRI was nonspecific findings. After prompt administration of intravenous methylprednisolone for 5 days, paraparesis and sensory deficits have recovered to almost normal state over 2 weeks. But voiding difficulty has not improved. Follow up MRI performed one month later revealed markedly decreased high signal intensities in the previous lesions.

Conclusion: We report an unusual case of acute idiopathic longitudinal myelitis which was not accompanied by other connective tissue diseases such as SLE or Sjogren's syndrome and showed dramatic response to steroid therapy.

\section{P814}

Multisystemic atrophy at a university hospital, Madrid

J. Fernandez Dominguez, L. Gabaldon Torres, M.J. Abenza, M.J. AguilarAmat Prior, F.J. Rodriguez de Rivera, T. Ferrer, M. Gutierrez Molina, C. Morales, F.J. Arpa Gutierrez

Hospital Universitario La Paz (Madrid, ES)

Introduction: Multisystemic atrophy (MSA) is a degenerative disease characterized by impairment of several systems: extrapiramidal, cerebellum, pyramidal and autonomic, with multiple possible combinations. Graham and Oppenheimer in 1969, made the first description. Gliosis and neuronal loss are observed in several areas of the Central Nervous System (CNS).

Material and methods: We review the casuistic of the MSA in our hospital between 1993 and 2007. Demography, clinical debut, impairment of the Autonomic Nervous System (ANS) and pathology (in those cases with necropsy) are discussed.

Results: 48 cases were diagnosed as MSA, 2 of them diagnosed by autopsy. $29 \%(\mathrm{n}=13)$ were classified as type OPCA, $53 \%(\mathrm{n}=26)$ as SND, $14 \%$ $(\mathrm{n}=7)$ as Shy-Drager syndrome (SDS) and $4 \%(\mathrm{n}=2)$ received the diagnosis of MSA, due to their advanced evolution. $56 \%$ were women $(n=27)$. The debut mean age was $59.62 \pm 9.81$. In our serie, a significative statistic difference was observed in the age of debut between women and men, being those ones younger (53 yo as mean aged of debut in men). Mean temporal evolution was of $6.77 \pm 3.9$ years. $40 \%$ had impairment of the speech by means of dysarthria. 40 patients had study of the ANS, and in $80 \%$ of them it was impaired. Among those ones with Autonomic disease, the most common manifestation were bladder dysfunction and orthostatic hypotension $(n=25$ in each group), followed by constipation $(n=19)$. A completed study of the evolution was done.

Conclusion: Eventhough nowadays two types of MSA are considered (MSA-cerebellar and MSA-parkinson), in our serie the old Shy-Drager type is consistent itself to be considered as a type. We have found statistical significance between men and women in order to the age of debut of the disease. Mortality and dependence are earlier in the SND (actually MSA-p), having the OPCA (MSA-c) type more "benign". The subgroup with a SDS debut is the one with more mortality in our serie.

\section{P815}

Pneumocephalus: an unusual complication of lumbar puncture M.J. Abenza, B. Fuentes, P. Martinez, M.A. Ortega-Casarrubios, F. Vivancos, M.J. Aguilar-Amat Prior, E. Diez-Tejedor

Hospital Universitario La Paz (Madrid, ES)

Introduction: Pneumocephalus, the introduction of air into the cranial cavity, is a rare complication that may occur when air is used to identify the epidural space (loss of resistance to air technique). The incidence is unknown. Symptoms are acute headache and emesis. The prognosis is usually benign.

Case report: We present a 33-year-old primipara, at 31 weeks' gestation, who suddenly developed a severe headache, without other symptoms, during an epidural anaesthesia for premature birth. Neurological examination revealed no deficits. Emergent computerized tomography of the cranium showed pneumocephalus in lateral ventricles. With conservative management, the headache resolved within a few days.

Discussion: Pneumocephalus is an unusual complication of epidural procedures. 


\section{P816}

Acute idiopathic transverse myelitis: analyses of 43 patients

O. Arici Duz, I. Aydin Canturk, N. Isik, T. Aydin, F. Candan, N. Yildiz

S.B.Istanbul Goztepe Training Hospital (Istanbul, TR)

Objective: Acute idiopathic transverse myelitis may be the first isolated demyelinating event in patients who will eventually develop multiple sclerosis (MS). In this study we aimed to describe prognostically and clinically relevant factors in patients with acute idiopathic transverse myelitis as converting to MS by using Magnetic Resonance Imaging (MRI) and cerebrospinal fluid (CSF) findings.

Method: 43 patients who have been followed up in our Multiple Sclerosis (MS) clinic between 2000 and 2007 years were analyzed. Patients' neurological examination, medical history, CSF and MRI findings were investigated. Recovery ratio and initial Rankin Scale Score were evaluated in all of the patients.

Results: $65 \%$ of the patients were women and mean age was 45.07 $( \pm 13.85)$. According to the medical history of the patients; nine of them had infection history and one of them had pregnancy. The rest of the patients' history was not remarkable. The initial complaints were numbness $(53 \%)$, weakness $(49 \%)$ and urinary symptoms ( $9 \%)$. The effects of CSF biochemical characteristics, Ig G G index and oligoclonal band on the prognosis of the disease were evaluated at the patients whom we could get the CSF findings. $46 \%$ of lesions were located in cervical spinal cord while $54 \%$ of them were located in thoracal spinal cord on MRI. The mean Initial Rankin Scale score of the patients was 2.5. All of the patients were treated with $1000 \mathrm{mg}$ intravenous metilprednisolone throughout seven or ten days. Recovery ratio was $75 \%$ in patients whose initial symptom was numbness, $53 \%$ in weakness and $25 \%$ with urinary symptoms. 5 patients' CSF glucose level were low and there was no recovery in these patients. $60 \%$ of patients who have high CSF protein levels have no improvement while $30 \%$ of them have partial improvement. There is no significant relationship between mean initial Rankin Scale score and clinical state.15 of 43 patients were converted to MS. The mean conversion duration was $3.3( \pm 2.5)$ year. The conversion percent was $13 \%$ in thoracal lesions while it was $40 \%$ in cervical ones.

Conclusion: Our findings suggest that CSF glucose and protein levels and initial complaints of transverse myelitis seems to be valuable in determining the prognosis of the disease, and localization of the lesions might be important to anticipate the conversion ratio to MS.

\section{P817}

Quantitative differences in subjective dizziness and trunk sway in patients with and without mild traumatic brain injury after a whiplash accident O. Findling, C. Schuster, J. Sellner, T. Ettlin, J.H.J. Allum Inselspital, Berne University Hospital (Berne, $\mathrm{CH}$ ); Reha Rheinfelden (Rheinfelden, $\mathrm{CH}$ ); University Hospital (Basel, $\mathrm{CH}$ )

Background: The incidence of whiplash injuries (WI) to the cervical spine is increasing. Presumably people suffering WI frequently have in addition mild traumatic brain injury (MTBI). Dizziness and unsteadiness are common in WI patients and patients suffering MTBI. There is also some evidence, that patients suffering WI and MTBI perceive dizziness differently from those with WI alone.

Aim of the study: In a prospective study we assessed whether there is a quantitative difference between the self-perceived handicap during daily activities due to dizziness in patients suffering from WI alone (WI-group) and those with WI combined with MTBI (MTBI-group). Further this was correlated with instability of trunk sway.

Methods: We used the Dizziness Handicap Inventory (DHI) to evaluate the self perceived handicap due to dizziness in both groups of patients and measured trunk sway during stance and gait tests to quantify balance instability. For comparison with sway of healthy controls we used a reference database based on previous studies.

Results: Eighty patients (24 male, 56 female, WI-group $n=36$, MTBIgroup $n=44$ ) were included in our study. Patients in the MTBI-group perceived significant $(p=0.032)$ higher dizziness and unsteadiness during daily activities than patients with WI alone. Compared to healthy subjects, both groups had significant higher trunk sway during stance tests and the MTBIgroup had significantly increased sway compared to the WI-group. A significantly increased sway was measurable in the MTBI-group during walking tests compared to the healthy control group.

Conclusion: Our findings show that the MTBI-group had a significantly higher subjective balance impairment than the WI-group which was accompanied by increased trunk sway. We detected a different more unstable pattern of balance impairment in the MTBI-group compared to the WI-group, possibly due to cerebral damage following MTBI.
P818

The restless legs syndrome in patients undergoing dialysis therapy

P. Robowski, M. Sieminski, W. Nyka

Medical University of Gdansk (Gdansk, PL)

Objectives: The Restless Legs Syndrome(The Ekbome Disease) is a disease which concerns the compulsion of legs moving and unpleasant sensations in lower limbs(sometimes also in upper limbs) that intensify in the evenings and during nights. Symptoms impede falling asleep and lead to insomnia. The cause of the disease remains unknown. The aim of the research was to estimate the rate of occurrence of the Restless Legs Syndrome in patients that have undergone the dialysis.

Methods: The research concerned the questionary among the group of 66 dialysed patients. It consisted of 20 questions mainly about the principal symptoms that appear in Restless Legs Syndrome and completed with the laboratory tests results: Rbc,Hb, Iron, Ferritin, Tibc,Tsat, serum calcium, phosphate, kalium, cholesterol, triglyceride, HDL and LDL.

Results: In the group of 66 patients there were 26 women (average age 57,5 ) and 40 men (average age 53,5). The undergone intervention was the haemodialysis. In the analysed group 2 patients was dialysed 4 times a week and 64 patients was dialysed 3 times a week.The average time of the therapy of female was 4,4 years and 6,05 of male patients. In this research it was estimated that 59,1 percent of patients presented the symptoms of the Restless Legs Syndrome. The laboratory tests made in all examined group of patients with RLS symptoms : RBC-mean value 3,67 (minimum value 2,55- maximum value 4,98)T/L, Hb-11,07 (6,8-15,2)g/dl, iron-63,85 (19-187)ug/dl, ferritin-599 (28,97-2000)ug/dl, Tibc-224 (144-316)mg/dl, Tsat-29,5 $(9,69-98,94) \%$, calcium in serum-9,4 $(7,39-13,25) \mathrm{mg} / \mathrm{dl}$, phosphate-5,24 $(2,35-8,06) \mathrm{mg} / \mathrm{dl}$, potassium-5,29 $(3,76-6,57) \mathrm{mg} / \mathrm{dl}$, cholesterol-199,69 (86-428)mg/dl, triglyceride-203,61 (63-503)mg/dl, HDL-57,74 (12-235)mg/ dl, LDL-108,49 (10-400)mg/dl.

Conclusions: In the group of patients undergoing the dialysis therapy the RLS symptoms occur very often. It has been pointed that in the examined group of patients abnormalities in iron metabolism and probably abnormalities in fat metabolism and electrolyte imbalance may effect in increasing the rate of RLS occurrence.

\section{P819}

Central nervous system toxicity after cytosine-arabinoside treatment: case report

P. Tortorella, M.E. Biancardi, M. Guido, C. Avolio, L.M. Specchio University of Foggia (Foggia, IT)

Background: Central nervous system (CNS) toxicity has been well described in patients treated with high-doses of cytosine-arabinoside (ARA-C). However, reports pointed out cerebellar damage without changes in other part of CNS. We described a young patient affected by progressive CNS disorders occurred after treatment with ARA-C.

Case history: A 37 years old man (F.G.) suffered from acute myelogenous leukemia in august 2005. He was treated with chemotherapy based on Idarubicine, ARA-C, VP16 (Etoposide), Cyclosporine A before and after the allogenic transplantation of peripheric staminal cells. ARA-C was administered in 2 phases: induction phase ( $220 \mathrm{mg}$ b.i.d. for 7 days), and, after 19 days, rescue phase (6,3 gr b.i.d. for 4 days). In January 2006 the patient showed tremors in limbs, which progressively worsened to develop, in the next 2 months, a complex neurological feature including ataxia, ideational and motor apraxia, nystagmus, oculomotor impairment, diplopia, dysarthria, spasticity so that he become unable to daily living activities. The patient referred to our Clinic in February 2007. The neurological examination revealed: ataxia, diplopia, inexhaustible nystagmus, dysarthria, dysphagia, bilateral dysmetria, postural, kinetic and rest tremor in arms, paraparesis, right Babinski's sign, normal sensibility, and well functioning sphincters. Severe global cognitive impairment, particularly visual-spatial and attentional tasks, and speech production were found. EEG (aspecific and diffuse slow discharges), EMG (sensory-motor neuropathy), and brainstem evoked potentials (normal) were performed. Brain MRI showed a diffuse and severe atrophy of brain, cerebellum, and brain stem, and, in T2 and PD scans, a diffuse hyperintense signal of bilateral periventricular white matter, cerebral peduncles, and, particularly, midbrain. A MRI performed 6 months before was completely normal. CSF analysis was normal, and the Creutzfeldt-Jacob disease was excluded. He underwent 5 cycles of plasmapheresis without efficacy.

Conclusion: Cerebellar toxicity has been described in several reports after high-doses of ARA-C but none of them revealed damage of brainstem and/or brain. In our case a rapid, unexplained and unpredictable degeneration of CNS structures occurred. Nevertheless, this case suggests the possibility that ARA-C could play a role in affecting, with unknown mechanism, cerebellar, cerebral and brainstem neurons and white matter. 


\section{P820}

Extreme intracranial hypertension without hydrocephalus in meningeal carcinomatosis

S. Carrasco Garcia de Leon, A. Hernández, J. Borrás, M.J. Gallardo, J.M. Flores, J. Vaamonde

Hospital General (Ciudad Real, ES)

Objectives: Meningeal carcinomatosis (MC) develops after the invasion of the subarachnoide space by neoplastic cells. MC may be the initial presentation of an unknown tumour requiring a high index of suspicion for the diagnosis because its protean manifestations. Cerebrospinal fluid (CSF) is initially normal in only $3 \%$, although the findings may be non-specific and the identification of malignant cells elusive. We report a case of MC whit manifestations non-previously described, related to extreme intracranial hypertension (ICH)

Methods: A 70-year-old woman whit no significant past history, referred widespread weakness and falls, diplopia, progressive visual loss and headache from the last 2 months. She was admitted because transitory episodes of agitation, unmotivated aggressiveness, headache, vomiting, incontinence, flaccidity and decreased level of consciousness. Neurological assessment revealed right amaurosis, left minimal visual perception, bilateral papilledema, ophthalmoplegia and tetraparesis with generalized hyporeflexia.

Results: CT scan and MRI were unremarkable, such as an extensive systemic study. The CSF exam revealed an opening pressure of $700 \mathrm{~mm} \mathrm{H}_{2} \mathrm{O}$ with normality of biochemical markers. Intracranial pressure monitoring through an intraventricular catheter showed ICH (baseline between 300 and $500 \mathrm{~mm} \mathrm{H}_{2} \mathrm{O}$ ), and increases exceeding $1000 \mathrm{~mm} \mathrm{H}_{2} \mathrm{O}$. These extreme peaks of ICH concurred whit the transient clinical episodes described. CSF cytology was suspicious of malignancy, and $\mathrm{MC}$ of an unknown adenocarcinoma was confirmed in serial lumbar punctures. A ventriculoperitoneal shunt was implanted whit partial improvement.

Coclusion: ICH is described in MC associated or not to hydrocephalus, and may be the unique altered parameter in the CSF exam. Therefore we emphasize the need to measure the CSF pressure in diagnostic lumbar punctures. In our case, we remark the presentation with fluctuating episodes of behaviour and consciousness alterations, related to the extreme increases of intracranial pressure leading to critical cerebral perfusion and consequent hypoxic-ischemic encephalopathy. A palliative shunt can improve the symptoms and the quality of life in these patients.

\section{P821}

Fulminant acute inflammatory demyelinating polyradiculoneuropathy mimicking clinical brainstem death

S. Khoury, S. Arzy, P. Petrou, T. Ben-Hur

Hadassah Hebrew University Hospital (Jerusalem, IL)

Background: The premise for diagnosing brainstem death is the demonstration of irrevocable absence of function of the brainstem, despite the artificial maintenance of ventilation and circulation. Prerequisites include identifying the proximate cause of brain injury and excluding reversible causes of coma (e.g. metabolic, toxic).

Fulminant acute inflammatory demyelinating polyradiculoneuropathy (AIDP) variants may result in a clinical state of quadriplegia, respiratory failure and abolition of brainstem reflexes. In such cases, central nervous system (CNS) disorder may be postulated and a diagnosis of brainstem death may erroneously be made.

Case: We report a young, previously healthy female patient that presented with a rapidly progressive course of neurological deterioration, lapsing into a clinical state resembling brainstem death; patient was apparently comatose, unresponsive, with complete ophthalmoplegia, absent brainstem reflexes and flaccid areflexic quadriplegia. Laboratory studies revealed a previously unrecognized systemic lupus erythematosus (SLE).

The lack of an unequivocal cause of brainstem death prompted yet further investigation, which resulted in the electrophysiological diagnosis of fulminant peripheral polyneuropathy.

Following steroid, plasma exchange and eventually Rituximab therapy, complete recovery was achieved.

Conclusions: Severe neuropathy with complete de-efferentation, can simulate "brain death". Determination of brain death, albeit clinically evident, should not be done where a cause is not clearly determined. Thence, investigations (radiological, electrophysiological and functional) and careful history taking, where clinical evidence of CNS function is unobtainable, are of paramount importance.
P822

Isolated generalised anhidrosis induced by postganglionic sudomotor nerve fibre degeneration: a Ross syndrome minor?

V. Donadio, P. Cortelli, F. Falzone, A. Giuliani, C. Misciali, L. Calzà, E. Bugiardini, P. Montagna, A. Baruzzi, R. Liguori

University of Bologna (Bologna, IT)

Objectives: Ross syndrome is a degenerative disorder characterized by tonic pupil, areflexia and anhidrosis and the underlying lesion affects the postganglionic skin sympathetic nerve fibers. Isolated generalized anhidrosis (GA) induced by a postganglionic sympathetic nerve fibers degeneration has not been previously described.

Methods: We describe a 46 years-old man who lost the ability to sweat all over his body. He complained anhidrosis since adolescence when this problem was limited to one arm only. Neurological examination was normal and the thermoregulatory sweating test (TST) disclosed generalized sweat failure except two small skin areas located in the right palm and left neck. To better specify the sudomotor lesion site the patient underwent: 1) skin biopsy both in the anhidrotic and in the sweating (right palm) skin areas. Skin samples were analyzed by immunofluorescence to study the skin innervation by means of the pan-neuronal marker protein gene product 9.5 (PGP 9.5) and specific markers for the autonomic cholinergic (vasoactive intestinal peptide-VIP) and noradrenergic (dopamine beta hydroxylase$\mathrm{D} ß \mathrm{H})$ fibers and by histopathology to study sweat gland morphology; 2) microneurography from the left Peroneal nerve.

Results: The immunofluorescence analysis disclosed: a) in the anhidrotic skin a reduced adrenergic innervation (DßH immunoreactive fibers). No VIP immunoreactive cholinergic fibers were found around sweat glands; $b$ ) in the sweating skin a reduced autonomic innervation. However sparse cholinergic VIP immunoreactive fibers were still evident around sweat glands.

Sweat glands in the anhidrotic skin appeared substantially normal without microscopic abnormalities or infiltrates on histopathological analysis. Microneurography was unable to record skin sympathetic nerve activity whereas muscle sympathetic nerve activity was recorded showing normal characteristics.

Conclusion: Our patient presented GA induced by a postganglionic autonomic skin nerve fibers degeneration as reported in the Ross syndrome although his clinical picture was incomplete lacking of tonic pupil and areflexia.

\section{Multiple sclerosis}

\section{P823}

Isolated cranial nerve impairment as the presenting syndrome of multiple sclerosis - a case series

A. Focke, F. Then Bergh

Universitätsklinikum Leipzig (Leipzig, DE); Universität Leipzig (Leipzig, DE)

Objectives: Various brainstem syndromes occur in multiple sclerosis (MS), including as its presenting manifestation. By contrast, apart from optic neuritis, dysfunction of a single cranial nerve is far less common. We present a series of consecutive patients in whom an isolated cranial nerve lesion was the presenting symptom of eventually definite MS.

Method: Case series.

Results: Five patients are described: one presented with abducens nerve palsy, two with peripheral facial nerve palsy, and two with the classical syndrome of peripheral vestibular neuritis including the typical findings at caloric stimulations. In all cases, careful examination excluded clinical signs of other cranial nerve dysfunction or long tract lesions in the brainstem. In all patients, MRI including T1 post-Gd images was unremarkable in the brainstem, but showed multiple lesions typical for MS in the periventricular white matter. CSF showed chronic inflammatory changes (minor or absent pleocytosis, IgG synthesis or/and OCB) and excluded borreliosis and other infectious causes. Evoked potentials showed mild subclinical abnormalities localized outside the brainstem in some but not all patients. On detailed questioning, two patients recalled an episode with sensory deficits in the past, which had resolved spontaneously and for which they had not sought medical help. Clinical and MRI follow-up established a diagnosis of MS in all patients.

Conclusion: Especially in younger adults, differential diagnosis and diagnostic evaluation of patients with isolated cranial nerve lesions should include MS, even if the initial course does not suggest additional CNS involvement. 
P824

Performance of automatic white matter lesion segmentation in multiple sclerosis depends on choice of image processing workflow

D. Garcia-Lorenzo, S. Prima, L. Parkes, J.-C. Ferre, N. Roberts, J. Y. Gauvrit, G. Edan, C. Barillot, S.P. Morrissey

INSERM/INRIA/IRISA (Rennes, FR); MARIARC (Liverpool, UK); University Hospital (Rennes, FR)

Objective: To develop an automatic Magnetic Resonance Imaging (MRI) processing workflow for segmentation of White Matter Lesions (WML) and the Normal Appearing Brain Tissues (NABT) in patients with Multiple Sclerosis (MS).

Methods: Over the last 25 years, MRI has been increasingly used for diagnosis, prognosis and as a surrogate marker in MS trials. Manual segmentation has been used to compute the total lesion load in MR sequences but with the major drawbacks: this method is very time consuming and has large intra- and inter-operator variability. Semi-automatic methods tend to reduce this variability, but there is a great promise that fully automatic methods will improve lesion segmentation reproducibility, which is of critical importance when processing huge amounts of MR images, as in large multicenter clinical trials. STREM (Spatio Temporal Robust Expectation Maximization) is a parametric algorithm for longitudinal segmentation of NABT and WML. Two modifications are proposed to improve computation time, accuracy and robustness: (1) to change the initialization method and (2) to use spatial constraints to reduce the number of misclassified voxels. In addition, five different workflows are defined to assess the impact of two preprocessing modules: intensity inhomogeneity correction (IIH) and denoising (DEN). Automatic segmentations are compared with manual segmentations made by an expert. Different MR protocols are used: 3T Siemens, $1 \mathrm{~mm}$ 3D T1-w, 3-mm slice thickness Dual Echo and 3-mm slice thickness FLAIR; 3T Philips, $1 \mathrm{~mm}$ 3D T1-w, 3-mm slice thickness Dual Echo and 3-mm axial slice thickness FLAIR; 3T Siemens, $1 \mathrm{~mm}$ 3D T1-w, 1mm 3D T2-w and 1mm 3D FLAIR.

Results: Adding DEN followed by IIH as a preprocessing workflow before the STREM algorithm improved WML and NABT segmentation results as compared to the other four workflows. Compared with STREM, the observed improvements are reflected in the higher Kappa values, and the reduced processing time (up to 4 times faster).

Conclusion: We have defined a fully automated WML segmentation workflow and demonstrate that segmentation depends on whether or not image preprocessing is performed, and on the choice of the preprocessing methods used. Furthermore, optimizations of the preprocessing methods and their order have a great impact on the WML and NABT segmentation results. Further studies are necessary to address a number of open questions, such as comparison of different algorithms for the same preprocessing task.

DGL is supported by ARSEP France.

P825

Herpesvirus presence in the CSF of multiple sclerosis patients from Northern Greece

D. Koptides, M. Paschalidou, N. Taskos, I. Milonas, A. Constantinou, I. Eleftheriou, M. Gelagoti, C. Christodoulou

Cyprus Institute of Neurology \& Genetics (Nicosia, CY); AHEPA University Hospital (Thessaloniki, GR)

Objectives: The aetiology of Multiple Sclerosis (MS) remains unknown. Several epidemiological studies emphasize the importance of environmental factors, and/or genetic factors. Recently, the hypothesis of a virus able to remain in latency has appeared. Possible common epitopes between viral and cell protein(s) (i.e. myelin) could provoke an autoimmune mechanism (i.e. against myelin) each time that virus is reactivated.

We report our findings concerning herpesvirus presence in patients $₫$ samples during the first episode of MS. For this study we analysed samples from MS patients, collected within six months from the beginning of their first attack. We collected samples from 40 patients from Northern Greece presented at the clinic during 2005-2006 and were diagnosed with possible MS. MRI was performed and blood with CSF samples were collected for evaluation and diagnosis. As control group, samples from 10 patients with other Neurological Disorders (intracranial hypertension, polyneuropathy, stroke, migraines) were used. Our objective was to investigate possible correlation of herpesvirus infection and 1st MS symptoms in patients.

Methods: We analysed serum and CSF samples for the presence of $5 \mathrm{com}$ mon herpesviruses - Herpes simplex virus 1 (HSV1), and 2 (HSV2), Epstein Barr virus (EBV), Cytomegalovirus (CMV) and Varicella Zoster virus (VZV). For viral antibody studies commercial ELISA kits were used. Presence of viral DNA in the samples was examined using molecular methods as PCR Southern blot and hybridization to detect very low amount of viral copies.
Results: The IgG prevalence for the 5 viruses was $10-87 \%$ and IgM $5-15 \%$. Molecular analysis results showed that in $40 \%$ of CSF samples we detected one of the 5 viruses.

A control group of 10 patients with other ND was also investigated. Antibody presence in this group was similar to that observed in MS patients, while viral DNA was detected only in $10 \%$ of samples.

Conclusions: Our results show high percentage of herpesvirus presence in the CSF of MS patients during the 1stepisode. Significant difference in presence of viral DNA in CNS of MS patients compared to control group can suggest a role of herpesviruses in aetiology of MS. A virus could be a trigger leading to creation of autoantibodies against basic CNS proteins with mechanism based on molecular mimicry. These results are an encouragement for further and more extensive studies regarding the role of these viruses in MS

\section{P826}

Relationship between severe relapses and adherence to disease-modifying therapy in multiple sclerosis patients

D. Meletiche, C. Kozma, R. Bennett, A. AL-Sabbagh

EMD Serono, Inc. (Rockland, US); University of South Carolina (Columbia, US)

Objective: Disease-modifying therapy (DMT) can reduce the frequency and severity of relapses in multiple sclerosis (MS) patients. Few data regarding the association between DMT adherence and incidence of MS relapses have been reported. This study evaluated the relationship between treatment adherence and the incidence of severe relapses using medication possession ratio (MPR).

Methods: For this retrospective study, the PharMetrics (Watertown, MA) Patient-Centric database was used to identify patients with at least $1 \mathrm{MS}$ diagnosis or at least 1 DMT-prescription claim (interferon beta-1a, beta-1b, or glatiramer acetate) between July 1, 2000 and December 31, 2004. Continuously eligible patients were evaluated for 6 months before (preperiod) the date of first DMD prescription (index date) and 24 months after the index date (follow-up period). A severe relapse was defined as evidence of at least 1 MS-related hospitalization or emergency room visit. Medication possession ration (MPR) was used to categorize patients as adherent (MPR ?80\%) or nonadherent (MPR $<80 \%$ ). Covariates included age, drug, gender, and treatment history (naive vs previously treated). Previously treated patients had evidence of at least 1 DMT prescription in the preperiod.

Results: 2400 subjects met the selection criteria and were included in the study. Subjects had a mean (standard deviation) age of 43.9 (9.4) years, and $76.3 \%$ were women. Most patients were enrolled in commercial plans $(77.8 \% ; \mathrm{n}=1867)$ and $38.6 \%(\mathrm{n}=927)$ participated in a health maintenance organization and $40.8 \%(n=978)$ in a preferred provider organization. 197 patients $(8.2 \%)$ had evidence of a severe relapse during the evaluation period. The age and gender of patients with severe relapse closely reflected that of the entire sample. More patients with evidence of a severe relapse were classified as nonadherent vs adherent $(61.4 \%[\mathrm{n}=121]$ vs $38.6 \%$ [n=76], respectively). A logistic regression for MPR, age, gender, drug, and patient type, using severe relapses as the dependent variable found nonadherent patients twice as likely to have experienced a severe relapse $(\mathrm{OR}=1.976 ; 95 \%$ CI, 1.462-2.686) vs adherent patients. Age, gender, monotherapy drug groups, and patient type were not significant (alpha level $=0.05$ ).

Conclusion: In this study, an increased risk of severe relapse was significantly associated with nonadherence to therapy.

This study was supported by EMD Serono, Inc. and Pfizer Inc.

\section{P827}

Clinical and electronystagmographic evaluation of the balance system in people suffering from multiple sclerosis

E. Koziorowska-Gawron, R. Podemski, S. Budrewicz, M. Koszewicz, W. Gawron, M. Koziorowska

ASK im. Mikulicza-Radeckiego (Wroclaw, PL); PSK (Wroclaw, PL)

Multiple sclerosis (MS) is an autoimmunological disease of central nervous system, which is one of the most common neurological cause of disability in young people. Electronystagmography, which is based on the analysis of visual-oculomotor and vestibulo-oculomotor reflexes, is an objective method of the balance system examination. The aim of the study was an electronystagmographic evaluation of the balance system in patients with MS.

The material consisted of 59 patients with MS diagnosed according to Mc Donald's criteria, the average age was 36 , the course of the disease was relapsing - remitting. Neurological examination with an evaluation of the pyramidal system, cerebellum, brainstem, sensory system and visual organ condition in Functional Systems Scale (FS) was performed. General effi- 
ciency according to Expanded Disability Staus Scale (EDSS) was estimated in all the cases. Electronystagmographic tests such as spontaneous nystagmus (with opened and closed eyes), smooth pursuit (peduncular and triangular test) and saccadic eye movements, optokinetic and caloric nystagmus were registered.

Spontaneous nystagmus with opened eyes was found in $20 \%$ of the patients, with closed eyes - in $27 \%$. Abonormal smooth pursuit eye movements concerned $39 \%$ of patients in peduncular test, $37 \%$ - in triangular test. Incorrect saccadic eye movements were observed in $86 \%$ of patients with MS. Optokinetic tests revealed significantly higher values of asymmetry of optokinetic nystagmus in patients with MS than in the control group. Patients with MS compared to the control group had significantly higher values of the canal paresis and the directional prepordance in caloric tests. The correlation between the dizziness and the presence of spontaneous nystagmus, incorrect smooth pursuit, saccadic eye movements and optokinetic nystagmus was found. The spontaneous nystagmus, abnormal results of optokinetic and caloric tests correlated with the neurological deficits evaluated in FS. There was strong correlation between certain electronystagmographic data and the age of onset, the character of first symptoms, the duration and course of the disease.

The dysfunction of the balance system in patients with MS is determined by abnormal visual - oculomotor and vestibulo - oculomotor reflexes, what may be objectively revealed in smooth pursuit, saccadic eye movements, optokinetic and caloric tests.

\section{P828}

Assessment of posterior fossa involvement using MRI and correlation with clinical exam in Iranian multiple sclerosis patients

E. Rahimian, R. Abolfazli, M. Tahsini, S. Samadzadeh

Athari Neuroimaging Center (Tehran, IR); Tehran University of Medical Science (Tehran, IR)

Objectives: Our purpose was to describe the frequency of MS plaques in different parts of infratentorial region and to explore their relation with clinical symptoms.

Materials and methods: We reviewed MRI and medical exam of 32 proved cases of multiple sclerosis ( 24 female, 8 male) between 15 -51 years old. According to Mac Donald's criteria and clinical exam 31 patients were relapsing remitting and one was secondary progressive. The MRI was performed with using a 1.5 Tesla Signa infinity MRI unit (GE Medical Systems, Milwaukee, WI), with and without injection of contrast media. We defined the boundaries of infratentorial region from the level of superior colliculi in the midbrain superiorly till medulla inferiorly and any typical T2 hyper intensity was considered abnormal. Prevalence rate of each cerebellar and brainstem manifestation and frequency and distribution of MRI lesions in these patients were studied.

Results: From the cerebellar and brain stem symptoms and signs the most common clinical symptom was vertigo $(68 \%)$ followed by nystagmus $(40 \%)$, facial hypoesthesia $(25 \%)$ Cerebellar ataxia (12\%), abducens palsy, INO, limb ataxia (9\%),Ataxic dysartherai (6\%), facial paresthesia and facial nerve palsy, Hearing impairment (3\%).

None of the cases had involvement of oculomotor, trochlear nerves, Facial neuralgia or bulbar palsy.

All the patients represented at least one plaque in the MRI of infratentorial white matter. The most common involved region was pons $(96 \%)$ followed by Medulla ( $78 \%$ ), Cerebellum (59\%), Midbrain (50\%).

Pyramid was the most common involved area in whole infratentorial region. The most common cranial nerve was 8th followed by 5th and 7th nuclei.

The most common involved region in the pons was Middle cerebellar peduncles.

There was high correlation between involvement of mid brain and nystagmus $(\mathrm{p}=0.05)$

As well as Facial hypoesthesia and between involvements of Middle cerebellar peduncles and MLF with INO. There was no correlation between cerebellar plaques with cerebellar symptoms and signs except nystagmus

Conclusion: In our study there was high correlation between plaques distributed in pons and mid brain with Nystagmus, INO, Facial hypoesthesia but there was no correlation between cerebellar plaques and cerebellar clinical symptoms and signs except nystagmus.

\section{P829}

Oral cladribine in relapsing-remitting multiple sclerosis: study design of the 2-year, Phase IIIb CLARITY (CLAdRIbine tablets Treating multiple sclerosis orallY) extension study

G. Giovannoni, S. Cook, S. Greenberg, P. Chang, G. Comi, P. Rieckmann, P. Soelberg Sørensen, P. Vermersch on behalf of the CLARITY (CLAdRIbine tablets Treating multiple sclerosis orallY) Study Group

Objectives: To investigate the long-term safety, tolerability and clinical benefit of oral cladribine tablets in patients with relapsing-remitting multiple sclerosis (RRMS).

Methods: Eligible patients from the 2-year CLARITY study will enter this Phase IIIb, double-blind, placebo-controlled, multicentre, parallel-group, 2 -year extension study with a pre-study evaluation period, and two 1-year treatment periods. Randomization to the three treatment arms for the 2-year extension study will depend on the initial treatment allocated in the first 2 years of the CLARITY study: patients previously randomized to placebo will receive two courses per year of cladribine tablets throughout the 2-year extension trial; patients who previously received cladribine will be randomized (2:1) to receive two courses per year of cladribine tablets or placebo in their respective groups. Rescue treatment with interferon beta-1a $(44 \mathrm{mcg}$ subcutaneously three times weekly) or another disease-modifying drug will be available. The extension will be blinded for the 2-year study period.

Results: Primary analyses will be based on safety endpoints. These will include assessment of patients for at least one grade 4 CTCAE toxicity for measures of haematological and hepatic function; proportion of patients with grades 3 or 4 adverse events (AEs) for haematological or hepatic indices; incidence of all treatment-emergent AEs and serious AEs; incidence of infections, infection-related AEs and malignancies; time to recovery from haematological and liver toxicity; time to nadir of absolute lymphocyte count and time to recovery to normal values; and changes in QTc interval from baseline. Efficacy endpoints will include the proportion of relapse-free patients, relapse rates, and measures of disability progression at 1 and 2 years. Magnetic resonance imaging endpoints will include measures of: total burden of disease, transformation of T1 gadolinium (Gd)-enhancing lesions to hypointense lesions, brain volume changes, and new T1 Gd-enhancing and T2 lesions. Health-related quality of life, pharmacoeconomic and pharmacogenetic/genomic endpoints will also be assessed.

Conclusions: This study is designed to provide information on the longterm safety, tolerability and clinical benefit of extended administration of oral cladribine tablets for up to 4 years in patients with RRMS.

Study supported by Merck Serono International S.A.

\section{P830}

Central noradrenaline, dopamine and serotonin turnover in relapsing-remitting multiple sclerosis

G. Koutsis, M. Markianos, M. Evangelopoulos, D. Mandellos, G. Karachalios, C. Sfagos

University of Athens (Athens, GR)

Objective: To investigate CSF levels of the main metabolites of noradrenaline, dopamine and serotonin (MHPG, HVA and 5-HIAA respectively) and their relation to disease progression in patients with relapsing-remitting multiple sclerosis (RRMS).

Methods: We estimated levels of CSF MHPG, HVA and 5-HIAA by highpressure liquid chromatography in 33 patients with RRMS and 42 controls. Clinical variables including the expanded disability status scale (EDSS) and the multiple sclerosis severity score (MSSS) were measured in all patients.

Results: RRMS patients had lower levels of MHPG, HVA and 5-HIAA compared to controls, but differences were not statistically significant. MHPG was negatively correlated to the duration of illness ( $p 0.008$ ) and the number of relapses ( $\mathrm{p}$ 0.009). 5-HIAA displayed a negative correlation to EDSS ( $p$ 0.017) and a strong negative correlation to MSSS ( $p$ 0.0001). HVA did not correlate significantly to any of the clinical variables studied.

Conclusion: Disease progression in RRMS has specific effects on neurotransmitter activity. The strong negative correlation of 5-HIAA to the MSSS suggests that this metabolite may be used as a putative biomarker of disease progression in RRMS.

\section{P831}

Role of genetic variation tumour necrosis factor alpha - $\mathbf{3 8 0}$ in Iranian multiple sclerosis

H. Ebadi, M. Shahbazi

Golestan University of Medical Science (Gorgan, IR)

Introduction: Multiple sclerosis (MS) is a polygenic inflammatory autoimmune disorder associated with progressive demylination in central nervous 
system. Tumor necrosis factor-alpha (TNF-a), a proinflammatory cytokine, is believed to play an important role in MS pathogenesis. polymorphism in the (TNF-a -308 G/A), has been reported to influence levels of TNF-a production. The objective of this study was to determine whether sequence variation in the TNF-a gene at position-308 is associated with Iranian MS patients and healthy individual controls.

Material and methods: Genomic DNA samples were prepared from whole blood of 126 MS Patients and 138 control subjects. Patients were divided into two groups according to their expanded disability status scale (EDSS) with mild to moderate or severe disease. The genotypes were determined by SSP-PCR. Student $t$ test and X2 analyses were applied to determine differences between groups.

Result: Frequencies of genotypes and alleles were significantly different between MS and Control subjects $(\mathrm{X} 2=8.92, \mathrm{p}=0.02 ; \mathrm{OR}=1.56 ; 95 \%$ $\mathrm{CI}=1.05-2.33)$. Whereas, there were no statistical differences between early and late onset of MS. Moreover, there were no statistically significant differences between types of MS.Nevertheless, we have not found any statistical significant differences between affected patients in comparison with mild to moderate MS patients.

Discussion: Our results have shown that TNF-a 308 (G/A) polymorphism is strongly associated with development of multiple sclerosis in this population.It seems that high production of TNF-a predicts susceptibility to MS in Iranian population. Likewise, it may be a considerable genetic predisposition for MS disease.

Vice chancellor for research of Golestan University of Medical Sciences for Funding

\section{P832}

Evaluation of the myocardial performance-index for early detection of anthracycline-induced cardiotoxicity in patients with multiple sclerosis: a two-year follow-up

I. Pesci, A. Guareschi, L. Manneschi, P. Pattoneri, E. Montanari Civil Hospital (Fidenza, IT)

Introduction: Mitoxantrone (MTX) was approved by the FDA in 2000 for the treatment of worsening RRMS, SPMS and PRMS. One of the most serious adverse events associated with treatment is cardiac toxicity. The potential congestive heart failure is the factor limiting the total dose in MS patients. It was said that the risk for myocardial toxicity increases with cumulative doses $>100 \mathrm{mg} / \mathrm{m}^{2}$ and the maximal approved dose is $140 \mathrm{mg} / \mathrm{m}^{2}$. We had already demonstred on 21 patients with MS treated with MTX (mean cumulative dose $27,81 \pm 17,09 \mathrm{mg} / \mathrm{m}^{2}$ ) compared to healthy controls that the mean value of MPI was 0,55 $\pm 0,11$ in MS patients compared to 0,39 $\pm 0,05$ in the control group $(p<0,001)$. A significant correlation between the given cumulative dose of MTX and MPI $(r=0,64, p<0,001)$ and mitral deceleration time $(\mathrm{r}=0,50, \mathrm{p}<0,001)$ respectively were demonstrated. The conclusion was that MPI represent an index of combined systolic and diastolic myocardial performance,correlated with the given cumulative dose of MTX.Objective:The purpose of this study is to evaluate the early alterations follow-up in left ventricular(LV)systo-diastolic function in patients with multiple sclerosis (MS) receiving MTX, by the use of this new index.

Materials and methods: The MPI is a non-invasive Doppler measurement of global (systolic and diastolic) ventricular function.It is defined as the sum of isovolumic contraction time and isovolumic relaxation time divided by ventricular ejection time. MPI $>0,47$ is a marker of LV dysfunction. We are following-up the same patients just studied; in particular, at the moment, we had obtained data from ten of them (mean cumulative dose $35.6 \pm 10.3 \mathrm{mg} / \mathrm{m}^{2}$ of MTX), during a monitoring time of $16.3 \pm 10.6$ months.

Results: The trend of mitral flow pattern showed a significant decrease of Epv and Etvi in these MS patients. Neverthless, no-significant decrease of Epv/Apv and Etvi/Atvi were observed. In addition, the mitral deceleration time was significantly increased. The mean value of MPI was $>0,47$ in all patients. One patient had finished the therapy by 2005: his MPI index is nevertheless pathological.

Conclusion: The MPI represent an index of combined systolic and diastolic myocardial performance, strongly correlated with the given cumulative dose of MTX. Once more significant correlation between the given cumulative dose of MTX and MPI and mitral deceleration time respectively were demonstrated. A complete MPI patients' evaluation will be done to confirm this parameter trend.
P833

Experiences in the daily practice with natalizumab

J. Haas, U. Mueller, S. Asimiadou

Jewish Hospital Berlin (Berlin, DE)

Introduction: Natalizumab is licensed for relapsing remitting MS in Germany since July 2006. The guide line of the Expert board of the German MS Society is to use Natalizumab as an escalating therapy in active relapsing remitting MS where interferon beta and or glatirameracetate failed and as a first line therapy in severe exacerbations.

Methods: From July 2006 to Dezember 2008 in our MS center 1239 MS patients were seen. The recommended escalating therapy for RRMS in Germany is mitoxantrone or natalizumab. Patients were informed about the risk profile of natalizumab and mitoxantrone respectively. Meanwhile 91 patients $(6,5 \%)$ were treated with Natalizumab. Concerning safety all patients received a cerebral MRI within the last 3 months before start of therapy and laboratory data to exclude immunodeficiency. The data of all MS patients were documented prospectively in our data base MUSIS.The Berlin MS Register MUSIS meanwhile included 4272 MS patients.

Results: The mean age of the 91 patients is $41 \mathrm{ys}$ (15-63), mean duration 13 ys (0-37), mean EDSS 4,3 (1,0-8,0), mean exacerbation rate the year before strat of treatmen 2.2 and $60 \%$ of the MRIs revealed Gadolinium enhancing lesions $(1-40)$. All patients were pretreated. The mean number of pretreatments was 2.8.21 Patients had only one, 22 two, 24 three and 24 more than 3 pretreatments. In 35 patients cytotoxic drugs failed to stabilize the course and Natalizumab was recommended after an interval of 6 months, in very aggressive MS after at least 3 months. 8 Patients were treated with plasmapheresis due to non remitting exacerbations.

Meanwhile 47 patients were at least 6 months on therapy. The annual relapse rate was reduced from 2,2 to 0.13 . 6 patients stopped therapy: 3 supposed inefficacy, 1 allergic reaction, 1 infection, 1 mamma carcinoma.

Conclusions: Compared to the pivotal trial our patients were older $(40 \mathrm{y}$ versus $35 \mathrm{y}$ ), mean duration of disease was 13 y versus $5 \mathrm{y}$, mean EDSS 4,3 compared to 2,3 and there was a high number of pretreatments. Despite these obvious differences the efficacy in these more advanced MS population with $100 \%$ pretreatment failure is as expected concerning freedom of exacerbation $(91 \%)$ and reduction of relapse rate within the first six months. We observed no severe side effects until now. Especially in young adults we prefer Natalizumab concerning compliance, side effect profile and no risk of infertility in female patients compared to mitoxantrone.

\section{P834}

Intrathecal synthesis measured by the IgG Index and oligoclonal bands in patients with multiple sclerosis. A correlation study

J. Mares, R. Herzig, K. Urbanek, V. Sladkova, R. Opavsky, B. Lubenova, K. Kollarova, P. Hlustik, H. Vranova, J. Sklenarova, V. Bekarek, P. Schneiderka, J. Zapletalova, P. Kanovsky

Faculty Hospital (Olomouc, CZ)

This study tested the correlation between IgG index values and the number of the oligoclonal IgG bands (OCB) in the cerebrospinal fluid (CSF) of patients with multiple sclerosis (MS) and patients without CNS affection.

The detailed assessment of the CSF is currently a part of multiple sclerosis diagnostics. In all stages of this disease, we can find increased IgG levels in CSF. It is possible to demonstrate it as oligoclonal bands.

The set of 150 MS patients consisted of 41 males and 109 females (aged 18-68, mean 36.610 .1 years) and the set of 171 patients without CNS affection consisted of 78 male and 93 female (aged 19 83, mean 46.0 14,1 years). The CSF collected by a lumbar puncture was examined evaluating intrathecal synthesis using the IgG index and determining OCB. The number of alkaline OCB in the CSF was assessed using the method of isoelectric focusation. Pearson s correlation analysis, homogeneity 2 test, Mann-Whitney test, paired-sample t-test (parametric) and Wilcoxon signed-ranks test (nonparametric) were used to evaluate the statistical significance of the results.

No positive correlation between the IgG index and the number of OCB in the group of MS patients was found. Mann-Whitney test also did not demonstrate any significant difference of the IgG index values in patients both with the OCB number 2 and $<2$. Nonparametric Spearman s correlation analyses showed a weak negative correlation between values of IgG index and OCB in patients without CNS affection. Mann-Whitney test showed significantly decreased values of IgG index in patients without CNS affection and with OCB 2.

This study did not confirm the correlation between the IgG index values and the OCB number in the CSF of MS patients. These results support the importance of both parameter of the intrathecal syntesis in MS diagnostics. 
P835

SepINRIA v1.7.1: a free software for lesion segmentation and atrophy evaluation dedicated to multiple sclerosis brain MRI

J.C. Souplet, C. Lebrun, G. Malandain

INRIA (Sophia-Antipolis, FR); CHU Pasteur (Nice, FR)

Objectives: To provide clinicians with a free software allowing them to visualize, to compare, to segment Multiple Sclerosis (MS) brain MRI. To focus this analysis on two criteria: lesion load and brain atrophy which are currently used in MS research to evaluate the burden of disease.

Methods: SepINRIA has different functionalities. Two images (either of the same sequence or of different sequences) can be aligned to be compared. A fully automatic lesion segmentation can be obtained from T1, T2-FSE, T2PD and T2-FLAIR sequences. Neurologists can also create a segmentation of reference or modify the automatic segmentation thanks to manual segmentation or semi-automatic segmentation. Quantitative comparison and analysis (number of lesions, volume) of the segmentations are also available. In addition to these functionalities already existing in earlier version of SepINRIA, atrophy evaluation methods have been added. They can be manual or fully-automatic. In the manual mode, the user has to identify specific points. Then distances between these points are computed and gives linear measures as the width of the brain, corpus callosum and third ventricle. In the automatic mode, the different sequences (T1, T2, PD) are co-registered and skull-stripped. Intra-image and inter-image intensity inhomogeneities are corrected. Then, the brain parenchymal fraction is computed from an automatic segmentation of the brain taking into consideration partial volume effects.

The use of SepINRIA has been simplified. Users can easily import images from DICOM files into a database. All parameters have also been fixed. MRI sequences as well as lesions can be visualized in 2D and 3D.

Results: SepINRIA is available on Linux, MacOsX, Windows and can be freely downloaded at:

http://www-sop.inria.fr/asclepios/software/SepINRIA/

Conclusion: We developed a free software for MS brain MRI analysis. Allowing to visualize images in the same framework, SepINRIA can already be used in clinical context. Automatic lesion segmentation and evaluation of brain atrophy are still belonging to the research field but this can evolved thanks to the availability of these algorithms. Future work will introduce lesion sub-classification, new criteria to compare two segmentations.

\section{P836}

NMO-IgG in idiopathic inflammatory demyelinating disorders among Hong Kong Chinese

K H.Chan, D B. Ramsden, A.C.Y. Chu, K.K.H. Kwok, S.L. Ho

The University of Hong Kong (Hong Kong, HK); University of Birmingham (Birmingham, UK)

Objective: Neuromyelitis optica (NMO) is characterized by monophasic or recurrent attacks of acute transverse myelitis (ATM) and optica neuritis (ON). $73 \%$ of Caucasian NMO patients are seropositive for the NMO-IgG which distinguishes NMO from classical multiple sclerosis (CMS). The NMO-IgG autoantigen is aquaporin-4 water channel. We aim to study the seropositivity rate of NMO-IgG in local patients with idiopathic inflammatory demyelinating disorders (IIDD)

Methods: Local IIDD patients (CMS, ATM [single or recurrent] without ON, ON [single/recurrent] without ATM, ATM with ON [monophasic or recurrent], acute disseminated encephalomyelitis [ADEM]) had sera taken at onset. Control sera were from healthy subjects, and patients with other neurological disorders (OND: stroke, Gullain-Barre syndrome, polymyositis, paraneoplastic neurological disorders). CMS was diagnosed according to revised McDonald's criteria. Patients were followed up for at least 1 year. Sera from IIDD patients and controls were tested for NMO-IgG by indirect immunofluorescence using slides containing Monkey cerebellum and mouse gut tissues. Rabbit anti-aquaporin 4 antibody (Sigma) was used for positive control.

Results: Three of $5(60 \%)$ non-CMS patients with ATM and ON were NMO-IgG positive. All 5 had extensive ATM, 4 of the 5 had severe visual impairment or paraplegia. Two of the 5 had brainstem dysfunction clinically with brainstem abnormalities on magnetic resonance imaging (MRI) brain; MRI brain was otherwise normal for all 5 . All 5 were negative for cerebrospinal fluid oligoclonal bands. One of 25 (4\%) CMS patients was seropositive for NMO-IgG. Among 5 patients with recurrent ATM without ON, 3 were NMO-IgG positive and all 3 had recurrent extensive ATM ( $>3$ vertebral segments on MRI spine); one of the 2 NMO-IgG negative patients had recurrent extensive ATM with brainstem involvement, and one had recurrent ATM extending $<3$ vertebral segments. None of patients with a single attack of ATM (8) or single/recurrent ON (11)were seropositive for NMO-IgG. None of the healthy subjects or patients with OND was NMO-IgG positive.
Two patients with paraneoplastic cerebellar ataxia and breast cancer were seropositive for the Purkinje cell antibody type 1 (anti-Yo).

Conclusion: $60 \%$ of local patients with NMO or recurrent extensive ATM without ON were seropositive for NMO-IgG, while $4 \%$ of CMS patients were seropositive. NMO-IgG is useful for diagnosis of NMO and recurrent extensive ATM without ON.

\section{P837}

Clinical outcome of relapsing-remitting multiple sclerosis among Hong Kong Chinese

K.H. Chan, K.L. Tsang, W. Mak, G.C.Y. Fong, R.T.F. Cheung, S.L. Ho

The University of Hong Kong (Hong Kong, HK)

Objective: To study the long-term clinical outcome of local relapsing remitting multiple sclerosis (RRMS) patients.

Methods: Records of local patients with RRMS for at least 10 years were retrospectively reviewed. All patients had at least two magnetic resonance imaging (MRI) brain scans and MRI spinal cord, and cerebrospinal fluid (CSF) analysis for oligoclonal bands (OCB). All patients fulfilled revised McDonald's criteria for RRMS. Clinical, neuroradiological and serological data were studies, the latest Expanded Disability Status Scale (EDSS) score and EDSS score at 10 years after onset were abstracted. Patients lost to follow-up had latest EDSS score evaluated via telephone interviews with the patients by a neurologist.

Results: 50 RRMS patients were studied. 40 (80 \%) were female. The mean onset age was 26.7 years (range 12-43 years) and the mean duration of disease was 17.8 years (range $10-31$ years). 44 ( $88 \%$ ) were CSF OCG positive. 10 $(20 \%)$ patients received azathioprine (AZT) for a mean duration of 10 years (range 5-13), 24 (48\%) patients received beta-interferon (bIFN) for a mean duration of 8 years (range 1-12 years); one patient received AZT for 10 years then bIFN for 10 years. At 10 years after onset, $10(20 \%)$ had EDSS of 2 or less, $24(48 \%)$ had EDSS $>2-4.0,16(32 \%)$ had EDSS $>4$ and $12(24 \%)$ had EDSS of 6 or more (range 6-7.0). The latest EDSS score at a mean disease duratiion of 17.8 years (range 10-31 years), 10 (20\%) had EDSS of 2 or less, 12 $(24 \%)$ had EDSS $>2-4.0,28(56 \%)$ had EDSS $>4$ and $24(48 \%)$ had EDSS of 6 or more (range $6-8.0$ ). There is no significant difference in the clinical, radiological, serological and treatment characteristics between patients in different clinical outcome groups according to the EDSS score. $14(28 \%)$ patients developed secondary progression at 10 years after onset, and $20(40 \%)$ developed secondary progression at the latest follow-up of mean duration 17.8 years. None of 10 patients with EDSS of 2 or less at 10 years had EDSS $>2.0$ upon the latest follow.

Conclusion: $32 \%$ and $56 \%$ of local RRMS patients had significant neurological disability (EDSS $>4$ ) at 10 years and a mean of 17.8 years after onset respectively, despite that $68 \%$ of patients received either bIFN ( $48 \%)$ or azathioprine $(20 \%)$ for years. Local RRMS patients tend to develop significant long-term neurological disability even with conventional immmunomodulatory therapies. Early novel or combination therapies need to be considered for our RRMS patients.

\section{P838}

Interferon beta and lovastatin affect the phenotype and interleukin-12 level of monocyte-derived dendritic cells in multiple sclerosis patients K. Pociñska, H. Bartosik-Psujek, A. Gruszczak, Z. Stelmasiak Clinical Hospital Number 4 (Lublin, PL)

Objectives: Multiple sclerosis (MS) is an inflamatory demyelinating disease of the central nervous system. Dendritic cells(DCs)are professional antigenpresenting cells and play a major role in the immune system. Recent studies have demonstrated that except of interferon beta, oral cholesterol-lowering HMG-CoA reductase inhibitor may be potent immunomodulatory agent. To explore the influence of interferon beta (IFN beta) and HMG-CoA reductase inhibitor on dendritic cells, we analyzed their effects on the differentiation and maturation of monocyte-derived dendritic cells and we also analyzed DCs effects on the production anti- inflammatory cytokines, such as IL-10 and proinflammatory cytokines (IL-12).

Methods: Peripheral blood mononuclear cells were isolated from twelwe patients (10 female and 2 male) with relapsing-remitting multiple sclerosis. Monocyte-derived dendritic cells were generated in vitro and incubated with IFN beta and different concentrations of lovastatin. The dendritic cells differentiation and maturation were evaluated based on surface phenotypic changes and the expression of CD83, CD1a, CD80, CD86 was analyzed by FACS. The samples for analysis were centrifuged and supernatantas were removed and frozen at $-80^{\circ} \mathrm{C}$ until analysis. The levels of the IL-12, IL-12p40 and IL-10 cytokines were determined in supernatants. All assays were determined in duplicate by enzyme - linked immunoassay (ELISA) acording to the manufacturer's instructions (Quantikine, R\&D Systems). 
Results: The results reveal that IFN beta and lovastatin affect the dendritic cells phenotype. Both agents decrease the expression of CD1a and CD83, which indicates the inhibition of DCs maturation. IFN beta causes essential up-regulation whereas high doses of lovastatin cause down-regulation expression of CD86, which results in biased Th cell responses in MS. Both interferon beta and statins suppress of proinflamatory cytokine secretion by DCs and don't affect significantly on level IL-10.

Conclusions: Our findings indicate that IFN-beta and lovastatin affect the dendritic cells phenotype by inhibition of their maturation. Moreover they regulate cytokine production profile, resulting in the decreased production of proinflamatory IL-12 cytokine. The beneficial action of IFN-beta and statins may be associated with their impact on dendritic cells and balance Th1/Th2 cytokine. The study has provided a new insight into mechanisms of immunomodulatory treatment of multiple sclerosis.

\section{P839}

Isolated occipital neuralgia as multiple sclerosis relapse: a clinical-radiological study of two cases

L. De Santi, L. Monti, E. Menci, P. Annunziata

University of Siena (Siena, IT)

Objectives: Occipital neuralgia determines jabbing pain with or without sensory decrease or dysaesthesia in the distribution of the greater or lesser occipital nerves or of the third occipital nerve. This disorder is mostly idiopathic. However, it may be related to traumatic, compressive or inflammatory injuries at occipital nerve or $\mathrm{C} 2$ radicular level as well as to $\mathrm{C} 1-\mathrm{C} 2 \mathrm{de}-$ generative arthrosis. Furthermore, it can be seldom observed with cervical spinal cord lesions including cavernous angioma, neurosyphilis and inflammatory lesions of myelitis.

Methods and results: We report here two relapsing-remitting multiple sclerosis (MS) patients presenting isolated occipital neuralgia as a MS relapse. In both cases, cervical spinal cord magnetic resonance imaging (MRI) revealed a new $\mathrm{C} 2$ area of increased $\mathrm{T} 2$-weighted signal with enhancement after gadolinium administration. Symptomatology reversed almost completely after treatment with i.v. high dose metylprednisolone.

Discussion and conclusion: Spinal cord, especially at cervical and thoracic levels, is commonly interested in MS pathology and cord lesions can be detected by conventional MRI techniques in up to $90 \%$ of patients with definite MS. Despite the frequency of cervical lesions and their tendency to be symptomatic, occipital neuralgia has been rarely reported in MS. In addition, the criteria used in previous studies for the diagnosis of occipital neuralgia are not specified and neuroimaging data are lacking. In our patients, a new active demyelinating lesion at $\mathrm{C} 2$ level was demonstrated and patient pain meets the International Headache Society criteria for occipital neuralgia. Furthermore, the pain distribution and cervical lesion localization allow us to exclude alternative paroxysmal syndromes attributed to MS such as great auricolar neuralgia and facial pain. We conclude that isolated occipital neuralgia can occur as relapse in MS. Its response to steroid therapy is usually satisfactory.

\section{P840}

T-helper 17 lymphocytes increase during multiple sclerosis relapses and are inhibited by interferon beta

L. Durelli, L. Conti, M. Clerico, D. Boselli, G. Contessa, C. Rivoiro, B. Ferrero, D. Viglietti, P. Ripellino, A. Ghezzi, M. Zaffaroni, F. Novelli

Universita' di Torino (Turin, IT); Ospedale Civile di Gallarate (Gallarate, IT)

Objectives: Balance between interferon (IFN)- $\gamma$-producing helper type 1(Th1) and interleukin-17(IL-17)-producing helper type 17(Th17) lymphocytes in active MS(AMS),IFN receptor expression, and sensitivity to IFN.

Methods: Untreated relapsing-remitting (RR) MS patients either during a relapse(AMS) or with MRI-confirmed inactive MS (IMS). The same patients retested after 6-12 months. Cytokine production and type 1 IFN receptor chain1 (IFNa/_R1) or type 2 IFN receptor chain2 (IFN $\gamma$ R2) expression by CD4+ lymphocytes analysed by FACS on peripheral blood mononuclear cells (PBMC) stimulated for 5 hours with PMA, ionomycin and brefeldin A.Percent producing IFN $\gamma$ or IL-17 evaluated by FACS on RRMS PBMC cultured with anti-CD3 and anti-CD28 with or without IFN,-1a or IFN,-1b increasing concentrations $(10-1000 \mathrm{U} / \mathrm{mL})$. Percent expressing IFN / R1 or IFN $\gamma$ R2 evaluated by FACS on PBMC from healthy donors(HD) activated for 5 days with anti-CD3 and anti-CD28 with or without IL-12 or IL-23 and with or without a mix of IFN?R1- and IFN?-blocking antibodies. STAT1 phosphorylation analysed by Western blotting with anti-phospho-Tyr(701)-STAT1 antibodies on PBMC from HD activated for 5 days as described above and, then, stimulated with IFN $\gamma(1000 \mathrm{U} / \mathrm{mL})$ or IFN, $(1000 \mathrm{U} / \mathrm{mL})$ for $15 \mathrm{~min}$.

Results: Compared to HS $(\mathrm{N}=22)$, percent Th17 was 7 -fold increased in $\operatorname{AMS}(\mathrm{N}=24, \mathrm{p}<0.0001)$ and 2 -fold in IMS $(\mathrm{N}=22, \mathrm{p}=0.0078)$. Percent Th1 was unchanged. The longitudinal comparison of a group of patients $(\mathrm{N}=8)$ confirmed these results. IFN, reduced in a dose-dependent manner IL-17producing CD4+ cells, and not that of IFN $\gamma$-producing cells. Percent Th17 was also reduced in MS patients 3-6 months after starting IFN, treatment $(\mathrm{N}=5, \mathrm{p}=0.02)$. Percent expressing IFN $\alpha /$ R 1 was greater $(\mathrm{p}<0.005)$ in Th17 than Th1 without difference between AMS and IMS. IFN $\alpha /$ R1 expression was high on resting PBMC. During the first days of differentiation in culture IFNal_R1 sharply decreased in both Th subsets. Then it remained low $(1-2 \%)$ on Th1, whereas it progressively increased (up to $12-15 \%$ ) on Th17. Th17 displayed a much more robust ability than Th1 to develop IFN,dependent STAT1 responses. IFN beta showed a dose-dependent inhibition of Th-17 and not of Th1 in vitro proliferation.

Conclusions: An expansion of PB Th17 occurs during relapses. The increased expression of IFN $\alpha /$ R 1 and the selective sensitivity to IFN, of Th17 compared to Th1 cells might explain one of the mechanisms of action of IFN, in MS.

\section{P841}

IgE sensitisation and adverse reactions to natalizumab

M. Bouktsi, A. Manikas, I. Milonas

Interbalkan European Medical Centre (Thessaloniki, GR)

Objectives: Natalizumab is a selective adhesion molecule inhibitor for the treatment of relapsing multiple sclerosis (MS). As other biological agents a great variety of adverse side effects may occur during treatment with natalizumab. Most of them related to the pharmacologic activity of the drug while others are immune mediated and therefore are not predictable. The incidence of hypersensitivity reaction in patients receiving Natalizumab is reported to be $1,9-4 \%$ and $1 \%$ for the anaphylactic reactions. In order to identify which of the hypersensitivity reactions are allergic in nature and thus predictable we studied 10 patients receiving natalizumab.

Methods: Skin prick test (SPT) and intradermal (ID) test (allergy tests) using the drug were done before each drug administration. Additional eosinophils count was recorded before each infusion. Serum tryptase level was measured after a reaction. Patients (2) suffering from chronic urticaria were not excluded from the study but pretreatment with antihistamine and corticosteroids was given.

Results: In a total of 49 administrations only one episode of severe adverse reaction was recorded. Chest tightness and blood pressure drop occurred ten minutes after starting the i.v. infusion. In this patient SPT and ID tests done before the infusion were negative. Eosinophils count and serum tryptase are within normal limits. Allergy tests were negative in every case. Patients with transient eosinophilia did not show any adverse reaction during natalizumab administration.

Conclusion: Given the small number of patients we studied we can assume that the rate of life threatening reactions is too low. The severe adverse reaction that occurred in one of our patients does not seem to be IgE mediated. Considering the value of allergy tests we can also assume that there is no IgE sensitization after natalizumab treatment. Therefore hypersensitivity reactions during natalizumab administration cannot be predicted based on the skin tests. Transient eosinophilia is not related to any adverse reaction. In patients with a history of urticaria pretreatment is necessary to avoid discontinuation of therapy due to an event of urticaria not related to natalizumab.

\section{P842}

Patients with relapsing-remitting multiple sclerosis with negative brain and spinal MRI for more than 2 years: a dilemma in diagnosis and treatment approaches

M. Eraksoy, G. Akman-Demir, M. Kürtüncü, M. Mutlu, E. Tüzün, H. Özcan Istanbul University (Istanbul, TR)

Although MRI detects the white matter lesions of multiple sclerosis (MS), a minority of patients have normal brain and spinal MRI, but positive oligoclonal bands in cerebrospinal fluid (CSF).

We described $7(5$ F,2M)patients presented with relapsing-remitting diplopia $(n=3)$, sensory-motor hemiparesis $(n=3)$ and sensory-motor paraparesis $(n=1)$ and negative brain and cervical/dorsal spinal MRI. MRIs were obtained with 1.5 tesla machines. Five of 7 patients had oligoclonal bands in CSF at early stages of clinical picture.Other diagnoses were eliminated.Disease duration ranges from 2 yrs to 15 yrs. Clasic MS lesions were seen in $1.5 \mathrm{t}$ MRI, mean 6 years later in 3 patients, a few white matter lesions appeared in 4 patients, mean 2 years later.Three patients of the last group had relatives with MS.All the patients had relapsing-remitting course. Two patients were followed 17 years and developed severe disability (EDSS $6.5,9.0$ ) after $15 \mathrm{yrs}$ of onset.Three patients received disease modifying treatment, 2,10 and 12 years after the onset of disease. 
In conclusion, relapsing-remitting course and oligocloal bands in CSF without white matter lesions can occur within first 5 years of MS. Further, prospective studies designed both $1.5 \mathrm{t}$ and 3.0t MRI would be informative about this issue.

\section{P843}

A pilot trial of low-dose naltrexone in primary progressive multiple sclerosis

M. Gironi, F. Martinelli-Boneschi, P. Sacerdote, C. Solaro, R. Cavarretta, L. Moiola, M. Zaffaroni, V. Pilato, M. Cursi, M. Radaelli, S. Bucello, V. Martinelli, R. Nemni, G. Comi, G. Martino on behalf of the Italian Federation of Multiple Sclerosis

Introduction: Naltrexone is an orally semi synthetic opiate antagonist licensed, in a $50 \mathrm{mg}$ dose, for the treatment of alcohol and heroin addiction, while at lower doses it is supposed to exert an agonistic activity triggering a prolonged up regulation of ,-endorphins (BE), an endogenous opioid with immunomodulatory functions. A symptomatic effect on spasticity, pain and fatigue of low dose naltrexone (LDN) in Multiple Sclerosis (MS) has been reported in anecdotic observations.

Methods: A six-month pilot multicentre open-label trial with LDN (5 $\mathrm{mg} / \mathrm{die}$ ) has been carried out in 40 patients (pts) affected by primary progressive (PP)MS. Safety and efficacy of LDN on spasticity, pain, fatigue and depression were the major outcome measures of the study. Clinical and biochemical evaluations were serially performed at follow-up visits. BE levels in peripheral blood mononuclear cells (PBMC), as well as allelic variants of the gene coding for the mu opioid receptors (OPRM1) and its mRNA levels were also measured. Validated scales of measures (Ashworth, Visual Analogue Scale, Beck, Fatigue Severity Scale and SF36) were used for testing spasticity, pain, depression, fatigue and quality of life.

Results: Five drop-outs and two unrelated serious adverse events occurred during the study. Transitory haematological abnormalities (increase of liver enzymes), urinary tract infections, mild agitation and sleep disturbance were the commonest adverse events. The PBMC BE levels increased in all patients since the 3rd month of therapy and were still raised 1 month after the end of therapy discontinuation at a statistically significant levels. No change was found for mRNA levels of OPRM1 and no correlation between allelic variants and clinical responsiveness. According to pre-defined criteria of responsiveness for spasticity, fatigue, pain or depression, more than half of patients reported an improvement in at least one of these secondary outcomes. As regards quality of life, there was a general trend of improvement between final and baseline evaluation. Only 3 patients experienced EDSS clinical progression of the disease during the study.

Conclusion: Our findings support a safe profile for LDN in PPMS, further studies should be considered in order to explore the potential efficacy of the drug in MS patients.

\section{P844}

BG00012 (dimethyl fumarate) for the treatment of multiple sclerosis: evidence for a distinct dual neuroprotective and anti-inflammatory therapeutic modality

M. Lukashev, W.Zeng, S. Ryan, K. Dawson, G.N. O'Neill, S.E. Goelz, D-H. Lee, R. Linker, B. Drukarch, A-M. Van Dam, R. Gold

Biogen Idec, Inc. (Cambridge, US); University of Bochum (Bochum, DE); VU University (Amsterdam, NL)

Objectives: To define further the mechanism by which BG00012 (dimethyl fumarate [DMF]) exerts its therapeutic effects in multiple sclerosis (MS).

Methods: Western blotting, immunohistochemistry, and reporter assays were used to determine the effects of BG00012 on the stability and function of Nrf2. Mouse and rat experimental autoimmune encephalomyelitis (EAE) models were used to assess the effects of BG00012 on disease and central nervous system (CNS) integrity.

Results: In vitro, DMF and its primary metabolite monomethyl fumarate stabilised Nrf2, stimulated Nrf2-dependent transcriptional activity, and increased expression of the prototypical Nrf2 target gene product NQO1. In vivo, increased levels of Nrf2 and NQO1 activity were detected in the CNS of DMF-treated animals. DMF inhibited disease progression and inflammatory CNS infiltration in the acute mouse and chronic relapsing rat EAE, and improved myelin and axon preservation in late chronic mouse EAE. In a phase $2 \mathrm{~b}$ study of patients with relapsing MS, BG00012 $240 \mathrm{mg} 3$ times a day reduced the number of new or enlarging T2 lesions by $48 \%(\mathrm{p}<0.001)$ and the number of new non-enhancing T1 black holes by $53 \%(\mathrm{p}=0.014)$ at 6 months compared with placebo.

Conclusions: BG00012 is an activator of Nrf2, a transcriptional regulator central to oxidative stress response, essential for immune homeostasis, and identified as a major cytoprotective and neuroprotective mechanism. Taken together, the ability of BG00012 to activate Nrf2, its biological effects observed in EAE, and strong effect on non-enhancing T1 black holes in MS suggest that BG00012 may provide a distinct dual therapeutic modality by targeting an important neuroprotective and anti-inflammatory pathway.

Study supported by Biogen Idec, Inc.

\section{P845}

Simvastatin treatment in multiple sclerosis patients receiving Avonex ${ }^{\circledR}$ : a double-blind randomised control trial

M. Togha, M. Nabavi, M.H. Harirchian, N. Beladi moghadam, S. Ahmadi karvigh, A. Enzevaie, M.A. Sahraian, H. Ghanaati, K. Firooznia, A. Noorian, A. Jannati, M. Shakiba

Tehran UMS (Tehran, IR); Shahed UMS (Tehran, IR); Shahid Beheshti UMS (Tehran, IR)

Objectives: Due to their anti-inflammatory and neuroprotective effects, statins seem to be useful in the treatment of Multiple Sclerosis (MS); besides, the simultaneous application of statins and interferon beta might be more effective. The aim of this double blind randomized control trial is to compare Simvastatin with the placebo in Relapsing -Remitting Multiple Sclerosis (RRMS) patients receiving interferon beta (IFNb 1-a). This is a primary report of the ongoing study on 70 patients who have so far completed the protocol.

Methods: Seventy RRMS patients (72.9\% female) who were receiving IFNb 1-a (Avonex), having the EDSS score of less than 5.5 and at least 1 attack in the past 6 months were included and randomized into the 2 groups of simvastatin and placebo. Thirty five individuals were assigned to receive $40 \mathrm{mg} /$ day of Simvastatin. The neurological examinations were performed every 3 months. Laboratory tests and MR imaging were obtained every 6 months. Patients who developed drug complications or the ones who had more than 2 attacks during the study were excluded. The main outcome measures were total attack numbers, EDSS scores, the number of gadolinium-enhancing and new T2 lesions in MR imaging after a 1-year follow up. The means were analyzed by independent and paired T-test.

Results: The total attack number in Simvastatin group $(0.53 \pm 0.84$ mean $\pm S D)$ was less than the placebo group $(1.00 \pm 1.01$, mean $\pm S D)$ with a $\mathrm{p}$-value of 0.04 . The final EDSS scores were significantly $(\mathrm{p}$ value $=0.03)$ lower in Simvastatin group $(1.1 \pm 1.5$, meanv \pm SD) than the placebo group $(1.9 \pm 1.4$, mean \pm SD). In Simvastatin group, the mean and SD of gadolinium-enhancing and new T2 lesions were $0.5 \pm 1.5$ and $2.7 \pm 3.0$, respectively (comparing with $1.2 \pm 1.6$ and $4.1 \pm 3.7$ in the placebo group). Although there was a decreasing trend in MR lesions, this difference was not statistically significant ( $\mathrm{p}$ value $=0.20,0.12$ ); the reason may be the small sample size $(n=50)$ due to our financial limitations in obtaining MR imaging. Drug complications were not detected among our patients.

Conclusion: It is likely that using Simvastatin as an adjuvant therapy may be beneficial in patients with RRMS.

\section{P846}

Automated DTI analysis of multiple sclerosis lesions and their contralateral regions of interest using the mid-sagittal plane as a reference - a comparison study of different automated image preprocessing methods

N. Wiest-Daessle, S. Prima, J.-C. Ferre, J.Y. Gauvrit, G. Edan, C. Barillot, S.P. Morrissey

INSERM/INRIA/IRISA (Rennes, FR); University Hospital (Rennes, FR)

Objectives: First, to estimate the influence of different preprocessing steps for the calculation of different diffusion tensor variants (fractional anisotropy (FA), relative anisotropy (RA) and mean diffusivity (MD)). Second, to estimate their impact on calculations FA, MD and RA values in MS lesions and their contralateral regions of interest (ROI). Third, to determine contralateral (CL) ROIs of MS lesions fully automatically with computation of the mid-sagittal plane.

Background: We propose automated tools for the exact comparison of DTI invariants between MS lesions and their CL ROI in the normal appearing white matter (NAWM).

Patients and methods: Axial ( $2 \mathrm{~mm}$ slice thickness) DW MRIs were acquired on a 3T (Philips) with 15 directions. 5 patients with MS were matched to 5 control subjects. Automated tools were applied for the exact comparison of FA, MD and RA between lesions and their CL ROIs in the NAWM. 3 different methods of DT estimation and also 3 different image distortion corrections in DTI were used. MS lesions masks were automatically aligned with the images of control subjects using classical linear registration methods. DT estimation was performed with standard least square (LS), weighted least square (wLS) and constrained non linear least square (CNLS) methods. Correction of eddy current distortion was performed with linear, polyno- 
mial second order and polynomial third order transformations, and no distortion correction on DTI invariants of MS Lesions and their CL counterparts. The CL ROIs were automatically determined by computation of the mid-sagittal plane as a reference. FA, MD and RA were calculated in MS lesions, their CL ROIs, and in addition compared with "MS lesions masks" automatically projected onto matched normal subjects. Results: In control subjects comparing FA values of "projected" MS lesions with their CL ROIs only CNLS yielded almost identical FA values. In MS patients a statistical difference between lesions and their CL ROIs was found for the FA and the RA (but no for the MD), regardless of the automated image processing methods used.

Conclusions: Accurate calculation of DT variants depends on preprocessing steps of DTI MR images: CNLS for eddy current distortion clearly out-performed other methods. As DT invariants are increasingly used in Neuroscience for a variety of pathologies, accurate and robust DTI image analysis is a prerequisite for assessing group differences or changes over time.

NWD is supported by ARSEP France

\section{P847}

Influence of natalizumab treatment on a panel of cell surface bound adhesion molecules

P. Wipfler, G. Pilz, S. Afazel, E. Haschke-Becher, M. Huemer, M. Jakab, M. Ritter, G. Ladurner, J. Kraus

Paracelsus Medical University and Salzburger Landesklinken (Salzburg, AT); Kardinal-Schwarzenberg'sches Krankenhaus (Salzburg, AT); Paracelsus Medical University (Salzburg, AT)

Introduction: Natalizumab (TysabriTM) treatment is the first monoclonal antibody therapy approved for the treatment of multiple sclerosis (MS). Therapeutic mechanisms of the humanized monoclonal antibody Natalizumab blocking the adhesion molecule (AM) a4-integrin are well known. Blockade of a4-integrin leads to an inhibition of immune cell extravasation and the consecutive inflammation within the central nervous system. However, the effect on other components of the immune system during natalizumab treatment remains only partially clear. Understanding of these effects on further components of the immune system may help to prevent potential side effects or to determine patients who are non-responders early during or even before onset of treatment.

Methods: Quantitative expression levels of cell surface bound intercellular adhesion molecule-1,-2,-3 (ICAM-1,-2,-3), leukocyte function antigen1 (LFA-1) and a4-integrin on mononuclear cells (CD3+ T-cells, CD 19+ Bcells, CD 14+ monocytes/macrophages) in the peripheral blood of MS patients were measured by two colour flow cytometry (Beckman Coulter) right before the first and the fourth infusions of natalizumab. In this ongoing study, we have currently analysed the data of six patients from whom we have investigated the AM profiles at least two times.

Results: We found a significant decrease of unblocked a4-integrin cell surface expression on all investigated mononuclear subsets (B-cells $-59 \%$, T-cells $-52 \%$, monocytes/macrophages $-28 \%$ ) in the blood of MS patients after three months of natalizumab treatment as compared to baseline levels. Moreover, we obtained a consistent increase of the expression of cell surface bound ICAM-1 on mononuclear cells (B-cells 6\%, T-cells $13 \%$, monocytes/ macrophages $6 \%$ ) after three months of natalizumab treatment.

Discussion: Although the actual sample size of investigated patients is relatively small, we obtained impressively consistent results of a sustained decrease of unblocked a4-integrin expression not only in all patients but also in all investigated leukocyte subgroups. These findings indicate that a significant blockade of cell surface bound a4-integrin can be found four weeks after the last natalizumab infusion. Moreover, according to data of previous studies from our laboratory the increase of the expression of cell surface bound ICAM-1 may also contribute to the beneficial effects of natalizumab treatment in MS.

This study was supported by Biogen Idec

\section{P848}

Safety and utilisation of natalizumab in patients with relapsing multiple sclerosis: update from the TYGRIS and TOUCH (TM) programmes

R. Hyde, G. Belcher, C. Bozic, R. Kim, F. Lynn, M.A. Panzara, M. KooijmansCoutinho

Biogen Idec, Inc. (Cambridge, US)

Objectives: Natalizumab (TYSABRI ${ }^{\circledR}$ ), an alpha-4-integrin receptor antagonist, is the first adhesion molecule inhibitor approved for the treatment of relapsing multiple sclerosis (MS). Natalizumab monotherapy significantly reduced the annualised relapse rate by $68 \%$ and the 24 -week sustained dis- ability progression by $54 \%$, and was generally well tolerated, in a pivotal phase 3 clinical study in patients with relapsing MS. The rare risk of progressive multifocal leukoencephalopathy (PML) in patients treated with natalizumab prompted the initiation of 2 ongoing risk management programmes to evaluate the long-term safety of natalizumab: the TYSABRI Global ObseRvation Program In Safety (TYGRIS) and the TYSABRI Outreach: Unified Commitment to Health (TOUCH) Prescribing Program. Updated exposure and safety data from these programmes is released at regular intervals.

Methods: TYGRIS is a voluntary global observational study evaluating the long-term safety of natalizumab in approximately 5000 patients, including patients from TOUCH. Patients will be evaluated in the clinical practice setting every 6 months for up to 5 years, including patients who discontinue natalizumab treatment. Patients will be assessed for medical/MS history and for prior use of natalizumab or immunomodulatory, anti-neoplastic, or immunosuppressive agents, and will be monitored for serious adverse events, including serious opportunistic infections such as PML and malignancies. In the United States, all prescribers, infusion sites, and patients are required to enroll in the TOUCH Prescribing Program, which was established to ensure the appropriate and informed use of natalizumab. In TOUCH, the incidence of serious opportunistic infections including PML, deaths, and treatment discontinuations is assessed.

Results: By mid-December 2007, approximately 30,900 patients had been treated with natalizumab in clinical trial and post-marketing settings. The most current exposure and safety data will be presented.

Conclusion: Collectively, TYGRIS and TOUCH represent the largest long-term follow-up undertaken by any sponsor for a single MS therapy. To date, a favourable benefit-risk profile of natalizumab is supported by preliminary data from these programmes. Our understanding of the long-term safety of natalizumab in relapsing MS will be extended as clinical experience continues to accumulate.

Study supported by Biogen Idec, Inc. and Elan Pharmaceuticals, Inc.

\section{P849}

Cytokines regulate brain derived neurotrophic factor in central nervous system mixed glial cell cultures at the level of gene and protein expression R.P. Lisak, J.A Benjamins, B. Bealmear, D. Studzinski, L. Nedelkoska Wayne State University (Detroit, US)

Objectives: There are studies on the direct effects of cytokines (Cyt) produced by infiltrating inflammatory cells (IC) in the development of lesions in patients with multiple sclerosis (MS)and potential roles in recovery and regeneration.These include production of neurotrophins (NT), including BDNF, by IC, but little on the ability of IC Cyt to regulate expression and production of NT by glia.Our objective was to determine if mixtures of different Cyt regulate brain BDNF at the level of gene and protein expression.

Methods: Mixed rat central nervous system (CNS) glial cell cultures were incubated with Cyt mixtures typical of Th1 and Th2 lymphocytes and monocyte/macrophages $(\mathrm{M} / \mathrm{M}$ ) or additional medium (unstimulated cultures;UC) and examined gene expression at 6 and 24 hours and 3 and 5 days by quantitative real time polymerase chain reaction (QRT-PCR).Expression of a transcript for BDNF as well as to low affinity receptor for nerve growth factor (NGFRp75), a receptor for BDNF, was compared with that of a housekeeping gene, GAPDH. Proteins were extracted from cultures at 24 hours, 3 and 5 days.Proteins were quantitated employing Western blots and compared to levels of beta actin, a housekeeping protein.

Results: There was upregulation of message for BDNF induced by Th2 Cyt at 6 and 24 hours, a return to baseline at 3 days and a subsequent increase at 5 days.M/M Cyt induced transient downregulation of gene expression for BDNF and Th1 Cyt downregulated expression of the gene.There was no effect of any of the Cyt mixtures on expression of the gene for NGFRp75 at any time points.For levels of protein, Th1 Cyt induced a decrease in expression of BDNF at 3 days compared to UC; there was an increase induced by M/M Cyt.There was a decrease in BDNF in UC at 5 days compared to 3 days. BDNF levels at 5 days in response to all 3 Cyt mixtures were higher than 5 day UC.No changes in expression were seen of level of NGFRp75 at 3 or 5 days when compared to UC in agreement with PCR results.

Conclusion: Th1, Th2 and M/M Cyt are capable of regulating levels of gene expression for BDNF in glia confirming effects on gene expression at 6 hours employing microarray technology (Lisak et al 2007). In some instances these effects on gene transcription are associated with changes in levels of protein expression. Studies underway are exploring additional time points, differential effects on different glial cell types and repeated stimulation with Cyt.

Funded by Teva Neuroscience and the Parker Webber Neurology Endowment of Wayne State University/Detroit Medical Center 


\section{P850}

Health-related quality of life in multiple sclerosis: the predictive value of disease-related and psychosocial factors

S. Schipper, M. Wirtz, J. Kugler

German Multiple Sclerosis Association NRW (Dusseldorf, DE); Dresden Medical School (Dresden, DE)

Objectives: It is well known that health-related quality of life (HRQoL) is reduced in patients with multiple sclerosis (MS), not only in comparison to the general population but also compared to patients with other chronic diseases. However, reports in the literature on the influence of psychosocial and disease-related factors on the HRQoL are partially inconsistent. Our aim was to better identify these determining factors in a large, representative study.

Methods: An anonymous questionnaire was sent to 7050 members of the German Multiple Sclerosis Association, Section North Rhine-Westphalia. The questionnaire contained sociodemographic data, disease-related topics, the Multiple Sclerosis Impact Scale (MSIS)-29 (Hobart et al., 2001), the Multiple Sclerosis Quality of Life (MSQOL)-54 Instrument (Vickrey et al., 1995), the Fatigue Severity Scale (FSS, Krupp et al., 1989) and the Freiburg Questionnaire on Coping with Illness (FKV-LIS, Muthny, 1989). Response rate was $44.8 \%(\mathrm{~N}=3157)$.

Results: Our MS patients had significantly lower HRQoL scores compared to the general population in West Germany as evaluated by the federal health survey. This was valid even in patients with short disease duration and mild impairment. As evaluated by different stepwise linear regression analyses fatigue, depression and disability level proved to be the strongest predictors of physical HRQoL. The main determinants of mental HRQoL were depression and passive avoidant coping strategies. Explanation of variance was good, the regression models for the composite scores accounted for $70 \%$ - $81 \%$ (MSIS-29 mental score - MSQoL-54 physical score) of the variance. Further analyses, e.g. cross-validation, confirmed our results.

Conclusions: The impact of fatigue, depression and passive avoidant coping strategies on HRQoL in MS patients further emphasizes the importance of an integrated medical and psychosocial therapeutic approach.

\section{Pain and headache}

\section{P851}

Short-term restoration of facial sensory loss by motor cortex stimulation in peripheral post-traumatic neuropathic pain

D. Fontaine, J.L. Brunetto, P. Paquis, M. Lanteri-Minet

Centre Hospitalier Universitaire de Nice (Nice, FR)

We report a case in which Motor Cortex Stimulation (MCS) improved neuropathic facial pain due to peripheral nerve injury and restored epicritic and thermal sensory loss.

A 66 years old man developed intractable trigeminal neuropathic pain after trauma of the supraorbital branch of the Vth nerve, associated with epicritic and thermal sensory loss in the painful area. MCS was performed using neuronavigation and transdural electric stimulation to localise the upper facial area on the motor cortex. One month after surgery, pain was decreased from 80/100 to 20/100 on Visual Analogic Scale, and sensory discrimination improved in the painful area. Quantitative sensory testing showed the normalisation of thermal detection thresholds.

This case showed that MCS could restore discriminative and thermal sensory loss, resulting from peripheral nerve injury. Although the mechanisms leading to this effect remain unclear, this observation enhanced the hypothesis that MCS acts through modulation of the sensory processing.

\section{P852}

Exploring the relationship between quality of life, disability and pain in patients with failed back surgery syndrome

E. Buchser, A. Manca, S. Eldabe, K. Kumar, R.S. Talyor

Morges Hospital (Morges, CH); University of York (York, UK); James Cook University Hospital (Middlesbrough, UK); Regina General Hospital (Regina, CA); Peninsula Medical School (Plymouth, UK)

Objectives: Neuropathic pain patients appear to experience very low healthrelated quality of life (HRQoL). However, little is known about the impact of pain and functional disability on HRQoL. Research to date often uses disease-specific quality of life measures and not generic (non disease specific) measures such as Short Form 36 (SF-36) or EuroQol 5D (EQ-5D). This research investigates the relationship between pain, disability and HRQoL based on data from a recent randomized controlled trial of neuropathic pain patients with failed back surgery syndrome.

Methods: The PROCESS trial randomized 100 patients from 12 centres to spinal cord stimulation (SCS, $\mathrm{n}=52$ ) plus conventional medical management (CMM) or CMM alone $(n=48)$. Patients' pain, functional capacity and HRQoL were assessed at baseline and at 3 and 6 months as measured by leg and back pain visual analog scale (VAS), Oswestry (ODI), SF-36 and EQ-5D, respectively.

Results: At 6 months, statistically significant differences between the SCS and CMM groups were observed for the EQ-5D, ODI, leg VAS and the physical component score of the SF-36 (all p $<=0.001$ ). Further, greater levels of leg and back pain were associated with lower levels of HRQOL as measured by the EQ-5D, the mental and the physical component scores of the SF-36. Similarly, greater functional disability, as measured by ODI, was negatively correlated with higher HRQoL as measured by EQ-5D.

Conclusion: Pain, disability and HRQoL were highly correlated in this population of neuropathic pain patients. Further evidence of the relationship between these types of outcome measures is needed.

This study was sponsored by Medtronic Europe sàrl.

P853

Effects of fluoxetine on sodium currents in rat sensory neurons

J.H. Han, D.E. Kim

Seoul Veterans Hospital (Seoul, KR)

Objectives: Fluoxetine, a widely used antidepressant drug, has been described as a selective serotonin reuptake inhibitor. In addition to its antidepressant action it has been demonstrated to be effective in alleviating pain associated with various diseases. Dorsal root ganglion (DRG) neurons are primary sensory neurons and transmit peripheral information to central nervous system. Two types of sodium channels are expressed in DRG neurons based on their sensitivity to tetrodotoxin. They are involved in the generation and conduction of nociception. The effects of fluoxetine on sodium currents in DRG neurons were examined to elucidate the analgesic mechanism of the drug.

Methods: DRG neurons were acutely dissociated from rats (2 6 days postnatal) by enzymatic digestion. The whole-cell configuration of patch clamp technique was used to record tetrodotoxin-sensitive (TTX-S) and tetrodotoxin-resistant (TTX-R) sodium currents.

Results: Fluoxetine inhibited TTX-S and TTX-R sodium currents with $\mathrm{Kd}$ values of $60 \mu \mathrm{M}$ and $59 \mu \mathrm{M}$, respectively, at the holding potential of -80 $\mathrm{mV}$. For both types of sodium channels the steady-state inactivation curves were shifted in the hyperpolarizing direction and the conductance-voltage relationship curves were shifted in the depolarizing direction by fluoxetine. These effects combined together would greatly reduce the neuronal excitability.

Conclusion: The blockade of sodium currents in sensory neurons is considered as a possible mechanism for the analgesic action of fluoxetine.

\section{P854}

Central neuropathic pain induced by transverse myelitis

K.D. Park, J. Kim, K-S. Lee

Ewha Womans University Hospital (Seoul, KR); The Catholic University of Korea (Seoul, KR)

Background: Central neuropathic pain is a consequence of numerous neurologic diseases such as stroke, spinal cord injury, or peripheral nerve disorder. Transverse myelitis is a focal inflammatory disorder of the spinal cord, resulting in many neurologic dysfunctions. Although many patients with myelitis suffer from unbearable pain, the neuropathic pain induced by transverse myelitis doesn't arouse neurologist's interests so far. We investigated the characteristics and medical treatments of neuropathic pain of transverse myelitis.

Methods and subjects: Thirty-one patients who were diagnosed with transverse myelitis at Department of Neurology, Ewha Womans University Mokdong Hospital between 2001 to December, 2007 were recruited. Retrospective clinical data were collected from chart review and from the history obtained at the time of evaluation.

Results: The age at onset was $42.6 \pm 18.7$ years. $61.3 \%(19 / 31)$ of the patients were men and $38.7 \%(12 / 31)$ were women. $71.0 \%(22 / 31)$ of the patients had monophasic transverse myelitis. In $29.0 \%(9 / 31)$, antecedent immunization or upper respiratory tract infection were reported. The most involved site of spinal MRI was thoracic cord (thoracic $45.2 \%$, cervical $16.1 \%$, lumbar $3.2 \%$, cervical \& thoracic $22.6 \%$, thoracic \& lumbar $6.5 \%$ ). $67.7 \%(21 / 31)$ of the patients became aware of paresthesia, sensory loss, or numbness as the initial symptoms. Among these patients, $76.2 \%(16 / 21)$ 
complained of painful sensory symptoms such as, pins and needles, tingling, tightening, bursting, cutting, burning \& hot, electric shock-like sensation, or allodynia.

In $80.6 \%(25 / 31)$ of cases, intravenous steroid pulse therapy was identified as an acute treatment. The treatments for pain control were required in $77.4 \%(24 / 31)$ of patients, except seven patients including three children out of thirty-one. Polytherapies using more than one drug were reported in $79.2 \%(19 / 24)$ of cases. Gabapentin was the most used for pain control $(83.3 \%, 20 / 24)$, the second was amitryptiline $(50.0 \%, 12 / 24)$, third were benzodiazepines $(29.2 \%, 7 / 24)$, and the forth was carbamazepine $(25.0 \%, 6 / 24)$.

Conclusions: As this study showed, many patients with transverse myelitis experienced unbearable sensory symptoms as initial symptoms. Most of them $(79.2 \%, 19 / 24)$ required polytheraphies for pain control. Transverse myelitis is one of the main causes of central neuropathic pain and deserves further evaluation for the pain management.

\section{P855}

Comparison of mental stress- and thermoregulatory-induced sympathetic activation on acute experimental pain in healthy subjects

M. Fechir, S. Balon, N. Pfeifer, S. Kritzmann, F. Birklein

Neurologische Klinik (Mainz, DE)

Objective: Former studies investigating the interaction of the sympathetic nervous system (SNS) and pain have come to ambiguous results. This is probably caused by a high prevalence of SNS disturbances in chronic painful conditions. Therefore, an appropriate approach to reveal the mechanisms of the interplay between the SNS pain consists of examining healthy subjects in an experimental pain model.

Methods: We therefore analyzed the influence of differentiated sympathetic activation on electrically-induced pain in healthy subjects. We chose two means to activate the SNS: on the one hand by mentally-induced stress (Stroop task, mental arithmetic), and on the other hand by thermoregulatory stimulation using a water-perfused thermal suit $\left(7^{\circ} \mathrm{C}, 32^{\circ} \mathrm{C}\right.$, or $\left.50^{\circ} \mathrm{C}\right)$. Using easy control tasks (i.e. congruent version of the Stroop task, easy mental arithmetic) we tried to control attentional effects of the these tasks.

Results: Both, stress and thermoregulatory stimuli, strongly activated SNS parameters. However, the patterns of activation were different. While mental stress co-activated heart rate, blood pressure, peripheral vasoconstriction, and sweating, thermal stimulation either increased blood pressure (cold) or heart rate and sweating (warm). Only SNS activation during mental stress led to a significant reduction of perceived pain. The control tasks neither significantly activated the SNS nor altered pain perception. The increase of heart rate in mental stress co-varied with analgesia.

Conclusion: Our results suggest that the pattern of sympathetic activation is critical for its effect on acute pain perception in healthy humans. Further studies on the exact mechanisms behind this phenomenon will enhance our understanding of stress and pain and improve therapeutic approaches in chronic pain patients.

Supported by Deutsche Forschungsgemeinschaft (DFG-Bi 579/1-3)

\section{P856}

Comparison of the effects of sodium valporate supplementation with fish oil and sodium valporate alone in migraine prevention

M. Saadatnia, K. Basiri, M. Sohelipour, V. Shaygannejad, A. Ghorbani

Isfahan University of Medical Science (Isfahan, IR)

Introduction: Omega- 3 polyunsaturated fatty acids (PUFA) have beneficial effects on both specific and non-specific inflammatory reactions; the aim of this study was to evaluate the effect of dietary supplementation with fish oil in migraine prevention.

Patients and methods: A 12-week, randomized, single-blind, clinical trial was conducted from October 2006 to June 2007. A total of 67 patients ( $52 \mathrm{fe}-$ male, 15 men) with migraine headache were randomly allocated to the 2 groups. The first group 38 patients ( 30 females, 8 males with a mean age of $35 \pm 9$ year) received Na-valproate $400 \mathrm{mg}$ daily and the second group $29 \mathrm{pa}-$ tients (22 females, 7 males with a mean age of $36 \pm 9$ years) received Na-valproate $400 \mathrm{mg}$ daily with fish oil supplementation $(180 \mathrm{mg})$. Response to treatment was assessed at $0,1,2$, and 3 months after start of therapy.

Result: A significant decrease in duration, monthly frequency, and severity of headache after month 1,2 , and 3 in compared with month 0 occurred in both groups. There was a significant reduction in headache severity $(P=0.046)$ and frequency $(P=0.044)$ in sodium valporate with fish oil supplementation group after month 1 in comparison with sodium valproate alone. In contrast, there was no significant difference between two treatment groups in duration of the headache after month 1 . Mean intensity, mean du- ration and mean frequency of the attacks after month $2 \& 3$ were not significantly different between the two groups.

Conclusions: This study demonstrates that sodium valporate with fish oil supplementation significantly reduce migraine headache better than sodium valporate alone at the beginning of treatment.

\section{P857}

Intravenous immunoglobulin treatment reduces cerebrospinal fluid and serum nitric oxide metabolite levels in chronic widespread pain syndromes M. Stock, H. Vogel, A. Petzold, C. Melzer, M. Rott, G. Keir, R. Schedel, G. Sprotte

University of Wurzburg (Wurzburg, DE); Institute of Neurology, University College London (London, UK)

Objective: The pathogenesis of chronic widespread pain syndromes (CWP), such as Fibromyalgia, myofascial (MFS) or complex regional pain syndrome (CRPS) is to date unknown. Recent data suggest a potential role of nitric oxide (NO) as an important factor in their pathogenesis.

Since low-dose IVIg has previously been demonstrated to be effective in the treatment of those conditions, we monitored NOx levels in serum and cerebrospinal fluid (CSF) of CWP patients before and two weeks after IVIgtreatment and in controls without chronic pain.

Methods: 30 patients suffering from CWP and 15 non-neurological controls were included. Following an initial acquisition of Serum and CSF, 28 of the 30 CWP patients received $30 \mathrm{~g}$ IVIg and agreed to undergo a second withdrawal of serum and CSF 14 days later. Visual analogue scale (VAS) was used to assess pain severity. Samples were analysed blinded for NOx using a vanadium-based assay.

Results: NOx levels before treatment were significantly elevated in CWP patients compared to controls in CSF (6.96 vs. $4.45 \mathrm{uM} ; \mathrm{p}=0.012$ ) and serum (56.91 vs. $47.40 \mathrm{uM} ; \mathrm{p}=0.025$ ). Following IVIg treatment, NOx levels decreased significantly in CSF of CWP patients (6.96 vs. $5.70 \mathrm{uM} ; \mathrm{P}=0.036)$ and non-significantly in serum (56.91 vs. $52.42 \mathrm{uM} ; \mathrm{p}=0.072)$. Importantly, 25 of the 28 CWP patients $(89.3 \%$ ) improved clinically after IVIg (mean VAS levels before and after IVIg: 68.82 vs. $33.21 ; \mathrm{P}<0.0001$ ).

Conclusion: Our data support the concept of an involvement of NO in CWP and suggest that the treatment effect of IVIg may partly be explained by the down-regulation of an NO-mediated inflammatory process.

\section{P858}

Painful neuropathy associated with psoriatic arthritis

O.J.M. Nascimento, M.R.G. Freitas, T.M. Escada, R.R. Silveira, G. Quintanilha, G. Lacerda

Universidade Federal Fluminense (Rio de Janeiro, BR)

In recent years, many reports have suggested an active role of an immune inflammatory process in PA. Some anecdotal case reports describe a possibly noncasual association between peripheral nerve involvement and psoriatic arthritis. A predominant sensory neuropathy was observed in these clinical and electrophysiological studies.

Objective: The aim of this study is to report the clinical, electrophysiological and hystopathological findings in patients with psoriatic arthritis (PA).

Patients and methods: Three right-handed female patients (39,52 and 73 years old, respectively) and a male (53 years old) fulfilling clinical characteristics of PA were referred complaining of paresthesia and burning pain in the four limbs extremities. Nerve conduction and needle EMG studies were performed. A sural nerve biopsy was performed in all patients. HIV infection, chronic hepatitis, diabetes, alcoholism, mixed connective tissue disease, collagen vascular disorders, hematological disease and malignancies were ruled out, clinically and laboratorially. Patients were not in treatment with hydroxychloroquine, etretinate or infliximab.

Results: Presenting symptoms (numbness and tingling in the distal lower and upper limbs with painful burning feet) were sensory in all cases. These symptoms developed in about two years. Motor symptoms were not present. Distal cramps were common in all patients. Pinprick and temperature sensations were involved in a stocking and glove distribution. Ankle jerks were decreased. Evidences of autonomic or cranial nerve involvement were not found. Electrophysiological tests were within normal limits in two patients. Sural nerve biopsy disclosed a reduction of small fiber density in all cases with axonal degeneration and regeneration. Mononuclear cells was seen in the epineurium in two cases.

Conclusion: A mostly painful small fiber polyneuropathy can be seen in patients with PA. This association seems to be more than a mere coincidence. 


\section{P859}

A neurophysiologic study of pain in patients with meralgia paresthetica P. Schestatsky, E. Lladó-Carbó, J. Casanova-Molla, S. Álvarez-Blanco, J. VallsSolé

Hospital Clínic Barcelona (Barcelona, ES); Hospital Universitari Vall d'Hebron (Barcelona, ES)

Introduction: Patients with meralgia paresthetica (MP) usually experience not only paraesthesias and decreased tactile sensation, but also painful dysesthesias in the distribution of the lateral femoral cutaneous nerve (LFCN). We aimed at assessing the nociceptive pathway in patients with MP.

Methods: We carried out a clinical, psychophysical and neurophysiologic study in 14 patients with MP and 14 healthy control subjects. We assessed pain in the last two months, thermal thresholds and small fibres conduction by using a visual analogue scale (VAS-pain), quantitative sensory testing (QST) and contact heat-evoked potentials (CHEPs), respectively. Data were grouped for control subjects, non-affected side and affected side of patients with MP.

Results: Patients marked a VAS-pain of $4.3 \pm 1.5$. In the affected side, thresholds for warm and heat pain sensations were elevated and the amplitude of CHEPs was reduced in comparison to the non-affected side and controls (Bonferroni's test; $\mathrm{p}<0.001$ for all comparisons). The amplitude of CHEPs correlated inversely with duration of the symptoms ( $\mathrm{r}=-0.57$; $\mathrm{p}=0.002)$, as well as with heat pain thresholds $(\mathrm{r}=-0.18 ; \mathrm{p}=0.01)$. No significant correlations were found between CHEPs and VAS-pain ( $p>0.05$ for all correlations).

Conclusion: Besides the involvement of large myelinated fibres, partial loss of function in small fibres may also account for the painful symptoms of patients with MP, especially in those with longer disease duration.

\section{P860}

Spinal cord simulation compared to conventional medical management for neuropathic pain in patients with failed back surgery syndrome. A cost consequence analysis of the PROCESS randomised controlled trial

R.S. Taylor, S. Eldabe, S. Thomson, E. Buchser on behalf of the PROCESS Study Group

Objectives: Healthcare policy makers and payers are increasingly requiring evidence on costs and health related quality of life (HRQoL) impact of new and existing therapies. Few trials in chronic neuropathic pain have collected such data. This study assesses the costs and HRQoL consequence of spinal cord stimulation in patients with neuropathic pain secondary to failed back surgery syndrome (FBSS).

Methods: A total of 100 FBSS patients were randomised to either spinal cord stimulation plus non-surgical conventional medical management (SCS group) versus non-surgical conventional medical management alone (CMM group). Healthcare resource consumption relating to screening, the use of the implantable generator in SCS patients, associated hospital stay, drug and non-drug pain-related treatment were collected prospectively. Resource consumption was costed at 2005-2006 prices and expressed in Euros. HRQoL was assessed using the EuroQol-5D (EQ-5D). Between group differences were adjusted for baseline values and selected baseline characteristics.

Results: The gain in HRQoL seen in the SCS group was considerably greater than the CMM group with a mean between group EQ-5D difference of 0.25 ( $95 \%$ CI: 0.12 to $0.37, \mathrm{p}<0.001)$ and 0.21 (95\% CI: 0.09 to 0.33 , $\mathrm{p}<0.001$ ) at 3 and 6 months respectively. At 6 -months the overall healthcare cost in the SCS group was $€ 12,653 /$ patient compared with $€ 2,594 /$ patient in the CMM group $(\mathrm{p}<0.0001)$.

Conclusion: Although more costly in the short-term, the addition of SCS therapy to the conventional treatment of FBSS provides important improvements in patient HRQoL. This cost consequence analysis shows SCS to be a good value for money treatment in selected patients with chronic neuropathic pain.

This study was sponsored by Medtronic Europe sàrl.

\section{P861}

New perspectives on Raeder's syndrome: 4 new cases

A. Radojicic, J. Zidverc-Trajkovic, A. Sundic, N. Sternic

Institute of Neurology, Clinical Center (Belgrade, RS)

Objectives: The combination of pain, ipsilateral oculosympathetic defect (ptosis and miosis), and ipsilateral trigeminal dysfunction constitutes Raeder's syndrome. In 1918, Raeder reported the relationship between "paralysis of the oculopupillary sympathetic nerve" and pain in the trigeminal nerve distribution implicating the paratrigeminal area of the middle fossa of the cranium. Later cases of Raeder's syndrome were divided into those with lesions of the paratrigeminal area and those of uncertain site and benign course. Most reports of Raeder's syndrome during the past decade suggested that the site of the lesion was "pericarotid and not paratrigeminal."

Methods: We present 4 patients with similar clinical picture-oculosympathetic paralysis and ipsilateral head pain, diagnosed as Raeder's syndrome, treated at the Institute of Neurology, Clinical Center of Serbia from November 2006 to January 2007. All presented patients had different underlying couse and outcome.

Results: A 38-year-old woman with dissection of internal carotid artery, and a 54-year-old woman with cervical spondylitis fully recovered, while nasopharyngeal carcinoma spreading in middle cranial fossa was discovered in a 55-year-old man. Concerning the fourth patient all additional diagnostic procedures were within a normal range and according to ICHD-II the diagnosis of probable trigeminal autonomic cephalalgia (ICHD-II 3.4) was established.

Conclusions: Differential diagnosis of Raeder's syndrome should include various intracranial and cervical disorders, therefore an early and extensive evaluation of such patients is recommended.

\section{P862}

Pseudotumour cerebri as a reversible side-effect of all-trans retinoic acid treatment in acute promyelocytic leukaemia

B. Machner, B. Neppert, M. Paulsen, C. Hofmann, T. Sander, C. Helmchen Universitätsklinikum SH (Lubeck, DE)

We report on a pseudotumor cerebri syndrome (intracranial hypertension) in a young woman suffering from promyelocytic leukaemia during treatment with all-trans-retinoic acid (ATRA). Clinical signs were headaches associated with blurred vision. Examination revealed bilateral papilloedema with retinal haemorrhages and increased CSF opening pressure while CSF composition and MRI brain scan were normal.

Headache diminished after lumbar puncture with removal of excessive CSF and acetazolamide treatment. Despite persisting signs of intracranial hypertension but still preserved visual acuity, ATRA therapy had to be continued due to the leukaemic haemogram. After termination of ATRA therapy, headache and bilateral papilloedema completely disappeared.

Implications are twofold: 1) Contrary to previous case reports ATRA therapy was continued in our patient despite intracranial hypertension. With careful monitoring (visual acuity) we could show that signs and symptoms were reversible after treatment termination. 2) This case supports previously proposed concepts of vitamin A involvement in the pathomechanism of pseudotumor cerebri as his compounds e.g. ATRA might mediate intracranial hypertension.

\section{P863}

Chronic daily headache with concurrent overuse of ergotamine and triptans E. Nikolakaki, M. Kontogiorgaki, N. Kouroumalos, D. Andrinos, K. Kalamafkianaki, T. Kostas, G. Georgakakis

General Hospital of Chania (Chania, GR); General Hospital "G. Gennimatas" (Athens, GR); Heraklion University Hospital (Heraklion, GR)

Objectives: Study focuses on medication misuse and resulting problems. Ergotamine (EG) is used to treat or prevent migraine (M). Triptans (TR) are considered treatment of choice during $M$ attacks. Due to both agents' vasoconstrictive action, their concurrent use on the same day is strongly contraindicated.

Method: Case presentation: A 40-year-old female was admitted complaining of chronic daily headache $(\mathrm{CDH})$ who was also diagnosed for generalized angiopathy. History is significant for $\mathrm{M}$ starting 20 years ago complicating with overuse of both EG and TR. She initially started using EG gradually increasing its dose. 5 years ago she started using TR. Specifically, during the last year she was using daily: a combination preparation containing $2 \mathrm{mg}$ of EG and $100 \mathrm{mg}$ of caffeine (3 times), with either $5 \mathrm{mg}$ naraTR or $200 \mathrm{mg}$ sumaTR or $20 \mathrm{mg}$ rizaTR or $5 \mathrm{mg}$ frovaTR, or more than one. Headache $(\mathrm{H})$ intensity was changing in a manner dependant on the specific anti-M medication. Other complaints were numbness and weakness, initially on lower limbs. Abnormal sensation, cyanosis and muscular cramps later added to the picture, involving upper limbs as well. Digital Subtraction Angiography showed occlusions of the external iliac, superficial femoral and popliteal artery (AR) on both lower limbs and also the right humeral and ulnar AR. Stenosis also appeared in other vessels as seen in occluding AR disease due to egotism. Two years ago due to stenosis of both renal AR, she underwent balloon angioplasty of the right one. While in our department, brain MRI revealed ischemic lesions on cerebral hemispheres, cerebellar peduncles and medulla oblongata on both sides. MR Angiography revealed occlusions reaching $(50 \%)$ of the left internal carotid AR, milder were present 
among anterior, middle and posterior cerebral and basilar AR, as usually seen in fibro-muscular dysplasia after prolonged use of EG.

Results: Upon admission, medication overuse was interrupted. Oral naproxen, topiramate and amitryptiline were given. A week later, patient was discharged from hospital free of $\mathrm{H}$. Five months after upon follow-up, patient mentions rare $\mathrm{M}$ episodes of mild intensity, responding to oral naproxen. Blood supply of upper and lower limbs improved significantly. Cyanosis, numbness and cramps resolved and limbs' temperature became normal.

Conclusion: No citations were traced reporting cases with simultaneous overuse of TR and EG. Treatment should rather be guided by specialists or harmful consequences may result.

\section{P864}

Effectiveness of amisulpride in chronic daily headache

E. Nikolakaki, N. Kouroumalos, M. Kontogiorgaki, D. Andrinos, K. Kalamafkianaki, L. Karatzaferis, C. Labiris, G. Georgakakis

General Hospital of Chania (Chania, GR); General Hospital "G. Gennimatas" (Athens, GR)

Objectives: Neuroleptics have been used in treating acute headache, mainly because of the drugs' actions in monoaminergic neurotransmission. Their actions in these pathways have not yet been thoroughly clarified. With atypical neuroleptics coming into play and having a better adverse effect profile, even patients with Chronic Daily Headache (CDH) may benefit.

In this study, the effectiveness of low dose amisulpiride in treating patients with $\mathrm{CDH}$ is approached.

Methods: A prospective study was performed. Sample consisted of $18 \mathrm{pa}-$ tients treated with amisulpiride at 25,50 or $100 \mathrm{mg}$ /day and followed up for 3 months. Criteria of sample selection were: a) experiencing $\mathrm{CDH}$ b) being negative for a psychiatric DSM-IV diagnosis and c) having previously failed treatment with at least 3 different agents approved for treating CDH. Effectiveness of treatment was approached by comparing (using a t-test) mean values concerning headache a)days per month, b)hours per day, and c)intensity (using a scale). They were obtained one month before and three months after treatment initiation, in all 18 cases. Side effects were also noted (mentioned either spontaneously or after questioning).

Results: Treatment with amisulpiride resulted in a statistically significant reduction in headache days per month, hours per day, as well as intensity (in the scale used), in 12 out of 15 cases. Insomnia, anxiety and weight gain were reported in three cases.

Conclusion: Though studies with atypical neuroleptics (such as olanzapine, ziprasidone, aripiprazole) in the treatment of $\mathrm{CDH}$ exist, no information concerning use of amisulpiride was retrieved. Possibly, amisulpiride's action on D2 and D3 receptors which also interfere with the pathomechanism of headache may play a part. Moreover, the drug's effectiveness in suppressing anxiety might explain its therapeutic result in $\mathrm{CDH}$. Further studies with placebo controlled trials are needed to confirm amisulpiride's adequacy as a second line agent in treating $\mathrm{CDH}$, along with other neuroleptics.

\section{P865}

Efficacy of venlafaxine for the prophylaxis of migraine: an open label study F. Mayda Domaç, F. Mayda Domaç, H. Misirli, M. Türker, T. Adigüzel Haydarpasa Numune Training and Research Hospital (Istanbul, TR)

Objective: Venlafaxine is selectively effective on the serotonergic and noradrenergic mechanisms. Our aim was to assess the efficacy of venlafaxine in the prophylaxis of severe and frequent migraine attacks.

Methods: Patients with the diagnosis of migraine without aura according to the International Headache Society criteria were included in the study. The frequency, the severity and the duration of migraine attacks, and analgesic use were monitored every 2 weeks for 3 months. Adverse events and patient satisfaction were also evaluated during these visits. Patients were divided into 2 groups according to visual analogue scale (VAS) scores. Treatment with venlafaxine was initiated at $37.5 \mathrm{mg} /$ day for the patients with VAS scores between 50-75 units (group 1) and $75 \mathrm{mg} /$ day for the patients with VAS scores between 76-100 units (group 2). The daily dose was doubled at 4th week up to $75 \mathrm{mg} /$ day for group 1 and $150 \mathrm{mg} /$ day for group 2 . All of the patients have completed the trial. Patients with symptoms of pain rating of $<50$ units on the VAS and infrequent migraine attacks and patients with depression that was observed by Beck depression inventory were excluded.

Results: Fourty seven patients ( 4 men, 43 women) were examined. The mean age was 31.5 years.. There were 8 patients in group 1 and 39 patients in group 2 . There was a significant improvement in the VAS score in both of the groups. The mean VAS score dropped from 68.1 to $35.62(p=0.001)$ in group 1 and from 91.79 to $58.85(\mathrm{p}=0.006)$ in group 2 . The mean frequency of attacks showed a reduction from 13.1 to 7.2 per month $(\mathrm{p}<0.001)$. When the global efficacy was considered, $87.5 \%$ of patients in group 1 and $89.7 \%$ of the patients in group 2 evaluated treatment benefits as either good or very good.

Conclusions: Venlafaxine may be considered for the prophylaxis of migraine in the patients that have frequent attacks because of its high efficacy with low and/or tolerable side effect properties.

P866

Migraine prevalence is increased in patients with ulcerative colitis but not in Crohn's disease

G. Ramos de Oliveira, A. Carvalho Costa, P. Aquino, L. Pinho, S. Silva Jr, L. Braga, J.W. Lima, C.M. Castro Costa, F.H. Rola, F.A. Aquino Gondim Universidade Federal do Ceará (Fortaleza - Ceara, BR)

Objective: We have previoulsy observed that several neurological conditions (including headaches) are common in patients with inflammatory bowel disease - IBD (Oliveira, Inflamm Bowel Dis, 2007 epub ahead). In this study, we evaluated the differences in the prevalence of various headache subtypes in patients with ulcerative colitis (UC), Crohn's disease (CD) and gastritis/dyspepsia.

Methods: After Institutional Review Board approval for a cohort study, patients with Crohn's disease (CD,N $=33$ ), ulcerative colitis (UC, N =60) and gastritis/dyspepsia ( $\mathrm{N}=50$, disease controls) seen at the Gastroenterology Division underwent a standard neurological evaluation, which included a questionnaire to describe the characteristics and severity of their headache, according to the diagnostic criteria from the The International Classification of Headache Disorders, 2nd edition. Descriptive statistics, One-Way ANOVA, Chi-square and risk assessment analysis were used for comparisons.

Results: The overall prevalence of headache (including all differente types) was not significantly different in patients with CD versus UC and controls $(\mathrm{P}>0.05)$. No case of pseudotumor cerebri was diagnosed. The 3 groups had also similar gender (54.5, 48.3 and $38 \%$ of women, respectively) and age distribution $(43.5+2.4,47.8+2.0$ and $42.3+2.2$ years, respectively, $\mathrm{P}>0.05)$. However, a higher prevalence of migraine and/or migraine+possible migraine was evidenced in the UC group (53.3\%), compared to controls ( $32 \%$, $\mathrm{P}=0.05$ and 0.019 , respectively) with a prevalence ratio of $1.48(95 \% \mathrm{CI}$, [1.04-2.66]). Migraine or migraine+possible migraine prevalence was not higher in $\mathrm{CD}$ versus controls $(\mathrm{P}=0.7$ and 0.76 , respectively). Migraine or migraine+ possible migraine was more prevalent in $\mathrm{UC}$ versus $\mathrm{CD}(\mathrm{P}=0.03$ and 0.01 , respectively). The prevalence of tension headache and other headache subtypes was not significantly different among the 3 groups $(P>0.05)$. The overall impact of all headache subtypes on daily activities(number of working days lost or with reduced activity) was not significantly different in patients with $\mathrm{UC}$ versus $\mathrm{CD}(\mathrm{P}>0.05)$. The number of days with headache over a 3-month period was also similar among the 2 groups: $17.8+5.6(\mathrm{CD})$ vs 15.7+3.7 ( $\mathrm{P}>0.05)$.

Conclusions/relevance: Migraine is more prevalent in patients with UC than $\mathrm{CD}$ or gastritis/dyspepsia patients. The mechanisms underlying this association are not clear but deserve further evaluation given the potential drug interaction between the different headache therapies and IBD treatment.

Study supported by: UFC, DECIT/SCTIE/MS, Fundo Setorial de Saúde (CTSaúde/CNPq) and CAPES

\section{P867}

The interrelations of migraine, vertigo and sex

H. Neuhauser, A. Radtke, M. von Brevern, T. Lempert Robert Koch Institut (Berlin, DE); Charité (Berlin, DE)

Objective: Vestibular disorders related to migraine such as migrainous vertigo, BPPV or Menière's disease show a female preponderance. We aimed to investigate if the magnitude of the epidemiological link between dizziness and migraine differs between men and women.

Methods: Nation-wide telephone survey of a representative sample of the general population in Germany $(n=7341)$. Participants were asked about moderate/severe dizziness/vertigo in the past 12 months, spinning vertigo and headaches with dizziness/vertigo. Diagnosis of migraine based on IHS criteria was assigned if respondents reported 1) severe headache, 2) unilateral or pulsatile pain or pain aggravation on physical exertion, 3) nausea/ vomiting or photo- and phonophobia and 4) duration $4-72 \mathrm{~h}$. The link between dizziness and headache was examined separately in men and women. Probability of concurrence of the two symptoms statistically expected if they were not interrelated was calculated as the product of their prevalences and compared to the observed probability of concurrence. Association of sex 
with different types of dizziness among migraineurs was calculated using logistic regression.

Results: There was a female preponderance for dizziness (factor 1.8) both rotational (2.3) and non-rotational (1.5), as well as for headaches (1.3) and migraine (3.0). However, the magnitude of the more-than-chance concurrence of dizziness and headache was lower in women than in men, as reflected by a lower observed than expected female preponderance for concurring dizziness and headache (women:men 0,84). Logistic regression analyses showed that male migraineurs were more likely to have dizziness than female migraineurs. This finding, based on an analysis of 184 male and 584 female migraineurs, has only moderate power, however, and was marginally non-significant (OR $1.37, \mathrm{p}=0.087)$. There was also a tendency for men with migraine to have rotational dizziness $(\mathrm{OR} 1.49, \mathrm{p}=0.089)$ and rotational dizziness with headaches (OR 1.59, $\mathrm{p}=\mathrm{v} 0.079$ )

Conclusions: Men are less likely to suffer from dizziness, headaches and migraine than women. However, men with migraine may be more likely to suffer from dizziness, especially rotational dizziness, than female migraineurs. These findings are (marginally) non-significant but we hypothesize that this may be a power problem. Due to the lower baseline prevalence of dizziness and migraine in men, most vestibular disorders related to migraine remain less common in men than in women.

\section{P868}

Benign paroxysmal positional vertigo and 2 kinds of headaches

K. Takagi, K. Yamazaki, S. Nojima, H. Kobayashi, Y. Ito, T. Nakamura

Tokyo Medical University Kasumigaura Hospital (Inashikigun, Ibaraki, JP)

Objectives: We sometimes experience that patients with benign paroxysmal positional vertigo (BPPV) complain about neck pains and/or headaches. However, international classification of headache disorder 2(ICHD-2) doesn't classify secondary headaches accompanied with BPPV. Previously we investigated the frequency and character of headaches accompanied with BPPV. This time we studied other kinds of headaches accompanied with BPPV. We compared their characteristics.

Methods: We studied the records of patients in our hospital from September 2002 to October 2004. We revealed the accompanying frequency and characteristics (location, duration and intensity) of the headaches associated with BPPV. Then we studied 10 patients with other kinds of headaches related to BPPV.

Results: We analyzed 387 cases of BPPV. Of these cases 67 (17\% of all BPPV cases; 22 males $/ 45$ females, average age of 55.4) felt a headache a few days before or after they felt vertigo. All headaches stopped a few days before or after vertigo went away. All of them satisfy the diagnostic criteria of secondary headache in ICHD-2. Frequently their chief complaint was vertigo, so we asked if they felt a headache. Most of them complained of a dull neck pain, dull forehead headache or whole head headache. The headache was very mild and without laterality like a tension type headache (TH). Furthermore, 19 of the 67 cases originally had a TH, while 48 cases had not experienced it prior to a BPPV attack.

The 10 patients in our outpatient clinic had a headache without BPPV symptoms. But all of them experienced a BPPV attack at the beginning of their headache. After BPPV symptoms stopped, their headaches continued for several months. The headaches were characteristically very similar to those in our former study. They are also similar to TH. Frequently they forgot the initial BPPV attack. Therefore we had to ask about their clinical history more carefully.

Conclusion: A headache accompanied with BPPV is relatively common. The former headaches are very mild headaches and hidden by severe vertigo symptoms. Sometimes we overlook the headaches accompanied with BPPV. Additionally, in our latter study we saw that sometimes the initial BPPV attack can be overlooked. Headaches accompanied with BPPV haven't been well established as a concept of a secondary headache. To avoid misdiagnosing these headaches as primary $\mathrm{TH}$, we recommend that investigators pay careful attention to the relationship between BPPV and headaches.

\section{P869}

Cluster-like headache related to idiopathic intracranial hypertension: a case report

L. Testa, D. Mittino, E. Terazzi, F. Monaco

Amedeo Avogadro University (Novara, IT)

Introduction: Although cluster headache $(\mathrm{CH})$ is assumed to be a primary condition, symptomatic cluster-like headaches have been described. Idiopathic Intracranial Hypertension (IIH) is a secondary headache disorder characterized by headache and visual symptoms. Both papilloedema and $\mathrm{IIH}$-related headache are typically bilateral, but asymmetrical or even unilateral presentations are reported.
Case report: A 28-year-old woman presented with severe left periorbital headache, associated to restlessness and ipsilateral nasal obstruction, conjunctival injection, tearing and eyelid oedema. Attacks, lasting on average 30 minutes, persisted from 4 months, with a frequency of 2-4 attacks/day, 5 days/week. Physical examination and medical history were unremarkable apart from a severe obesity. Neurological examination revealed bilateral papilloedema, more marked in left eye, with initial reduction of visual acuity. Visual field study showed bilateral enlargement of the blind spot and concentric constriction on the left. Brain MRI, angio-MRI and venography were normal. Lumbar puncture in lateral decubitus revealed an opening pressure of $480 \mathrm{mmH} 2 \mathrm{O}$. Cerebrospinal fluid examinations, as well as blood tests, did not show any abnormalities. In the hypothesis of IIH we prescribed acetazolamide and weight loss. Headache episodes gradually decreased, and completely ceased in 4 weeks. Papilloedema disappeared and visual field normalized in 3 months. At the follow-up (10 months) no further headache episodes occurred.

Discussion: The patient fulfilled both the diagnostic criteria for $\mathrm{CH}$ according to the International classification of Headache Disorders (IHCD-2) and the IHCD-2 and Friedman criteria for IIH. Although an accidental comorbidity cannot be excluded, in our opinion both the concomitance of papilloedema with headache in a previously headache-free patient, and the headache improvement with acetazolamide, suggest that here cluster headache has been caused or triggered by IIH. Cranial venous outflow obstruction has been demonstated in IIH, and attributed to compression by the elevated intracranial pressure. Cavernous sinus is known as likely site of involvement in $\mathrm{CH}$ pathogenesis. Whe hypotesize that, in this case, asymmetrical presentation of headache and papilloedema correlated to a non-trombotic left cavernous sinus hypertension. To our knowledge, this is the 2 nd reported case of cluster-like headache due to IIH, and the first one in Europe.

\section{P870}

Spontaneous cerebrospinal fluid hypotension after a sneeze

M. Bernard, Z. Lugo, F. Klapczinski, F. Beroud, A. Ameri

CHG Meaux (Meaux, FR)

A 44 years old woman developped after a sneeze a quickly increasing headache, maximal in $15 \mathrm{mn}$, with an orthostatic component. Cerebral computerized tomography was normal. Cerebro spinal fluid (CSF) examination showed 3 lymphocytic cells $/ \mathrm{mm} 3$ with a major increase in csf protein at 280 $\mathrm{mg} / \mathrm{dl}$ and normal level of glucose. The cerebral magnetic reasonance imaging (MRI) showed minor subdural blood collection in right parietal area, with diffuse pachymeningal gadolinium ehancement. The clinical examination was normal and severe headache completely resolved with strict recombency without a pillow. Medullar MRI showed an extensive epidural CSF collection. So, typically postural headache and these MRI abnormalities were constistent with the diagnosis of spontaneous intracranial hypotension.

Orthostatic headaches relatives to spontaneous CSF leak must be recognized to avoid unnecessary invasive investigations.

\section{P871}

Syndrome of transient headache and neurological deficits with cerebrospinal fluid lymphocytosis: is it auto-immune?

M. Kurtuncu, E. Tuzun, S. Icoz, B. Baslo, D. Kaya, H. Durmus, M. Eraksoy, G. Akman-Demir, M. Ertas

Istanbul Faculty of Medicine, Istanbul University (Istanbul, TR); Acibadem Kozyatagi Hospital (Istanbul, TR)

Objectives: Syndrome of transient headache and neurological deficits with cerebrospinal fluid lymphocytosis (HaNDL) is characterized by headache and episodes of neurological deficits associated with cerebrospinal fluid (CSF) lymphocytic pleocytosis which remits usually within 3 months. Exclusion of other neurological disorders with similar clinical and laboratory findings is sine qua non in the diagnosis. Typical presenting symptom is marching hemi-sensory/motor deficits coincident with migraine-like headaches. Patients may also present with some rare features, like episodic diplopia, confusion, or psychosis. The etiology of HaNDL still keeps its mystery with some theories on para-infectious, auto-immune or epileptic pathophysiology.

Methods: Clinical and demographic data of 11 HaNDL patients was collected. Five patients' sera and CSF samples during relapse were used to detect anti-neuronal antibodies by using indirect immunohistochemistry on rat brain sections. Non-neuronal binding was investigated using monkey intestine and HEP cells. Western immunoblot was assayed using rat brain and human neuroblastoma cell line (SH-SY5Y). Specific binding on western blot membrane was assessed along with other patients' sera with well-known anti-neuronal antibodies. 
Results: Some uncommon clinical features were observed. Two out of eleven patients had relapses after several years. One patient had a psychotic episode, 2 had confusion during attacks and other 2 had bilateral papilloedema between attacks. One patient's MRI revealed meningeal enhancement in the symptomatic hemisphere. All patients had lymphocytic pleocytosis and 4 had elevated CSF pressure. Only 1 out of 4 had CSF oligoclonal bands. Anti-epileptics, calcium channel blockers and steroids were used with considerable success. Three out of five patients' sera and CSF had antibodies to nuclei of brain cells but not non-neuronal tissue. Sera/CSF of 2 sporadic hemiplegic migraine patients did not show any autoantibodies. Western immunoblot assays revealed multiple bands not related to known neuronal nuclear proteins.

Conclusion: The demonstration of anti-neuronal antibodies in patients sera and CSF provide support to the hypothesis that HaNDL may have an auto-immune etiology. In this syndrome probable target antigens might be channel-proteins, explaining good response to anti-epileptics and calcium channel blockers. However, this should be assessed further in larger cohorts.

\section{P872}

Migraine, white matter lesions and subarachnoidal haemorrhage: analysis of one large pedigree

M. Poyraz, Z. Matur, K. Aydin, H. Kayserili, B. Baykan

Sadiye Hatun Medical Center (Istanbul, TR); Istanbul University (Istanbul, TR)

Patients with migraine are at increased risk for white matter hyperintensities detected on magnetic resonance imaging (MRI) and these lesions are common especially in female migraine patients with aura (MA). The pathophysiology and long-term consequences of these lesions are unknown, but there were speculations of an ischemic origin.

In this study we reported an index female patient who has MA with white matter lesions on MRI and her family suffering from migraine and four members from subarachnoidal hemorrhage (SAH).

A 46 year-old woman was admitted to our department for the evaluation of her headaches, paresthesia on the perioral region and hands. She had a history of MA for 20 years. Her neurological examination was normal. Three years ago, bilateral hyperintense lesions in centrum semiovale, posterior corono radiata, frontal white matter and periatrial regions were seen on her T2 and FLAIR weighted cranial MRI. Her markers of vasculitis and procoagulant factors were all negative; echocardiography and Doppler ultrasound of the carotids and vertebral artery were normal. Her past medical history was not relevant except depression. In her repeated cranial MRI did not reveal any new white matter lesions. Her cerebrospinal fluid examination was normal and oligoclonal bands were not detected. No mutation was identified in the NOTCH3 gene with screening of exons 3 and 4 . Eleven members of her family had history of migraine. Similar brain MRI lesions were also observed in her son with migraine and 4 members of the family have had SAH, interestingly.

MRI studies in migraine patients have demonstrated white matter lesions of unknown significance. But, there were no adequate knowledge about examination plan, follow-up and treatment of these patients. Association of this condition with familial SAH was reported firstly in this study in a large pedigree. Advanced epidemiologic and genetic studies are required about this association.

\section{P873}

Familial idiopathic intracranial hypertension

S. Chebel, A. Boughammoura-Bouatay, M. Ammar, M. Frih-Ayed

University Hospital of Monastir (Monastir, TN)

Introduction: Idiopathic intracranial hypertension is a syndrome defined by an increased intracranial pressure of unknown etiology. Idiopathic intracranial hypertension is labeled as "idiopathic" because it has no detectable cause. Rare cases have genetic component were reported.

Objective: To describe two sisters affected by idiopathic intracranial hypertension associated in one of them to a type 1 Chiari malformation.

Patients: Two sisters (aged 34 and 27 years) developed idiopathic intracranial hypertension respectively at ages of 33 and 26 years. Neuroimaging was normal in the second case, while idiopathic intracranial hypertension was associated to a type 1 Chiari malformation.

Discussion: At our knowledge, only few cases of familial presentation of idiopathic intracranial hypertension have been reported so far. Some of them emphasized autosomal dominant, whereas others reported autosomal recessive transmission of the disease and postulated multifactorial causes and genetic mechanism of idiopathic intracranial hypertension.

Conclusion: The reported cases suggest implication of genetic factors in the development of idiopathic intracranial hypertension with possible vari- ation of genes expression among the family's members. Further research in genetic aspects of idiopathic intracranial hypertension is needed in the future.

\section{P874}

Metoprolol for prevention of hypobaric hypoxia-induced headache in individuals with exaggerated sympathetic activity: randomised double-blind clinical trial

S. Jafarian, A. Jahanshahi, J. Lotfi

Sina Hospital (Tehran, IR); Tochal Clinics (Tehran, IR); Shariati Hospital (Tehran, IR)

Objectives: High-altitude headache (HAH) is the most common hypobaric hypoxia-induced symptom experienced by newcomers to high-altitude areas. Metoprolol, a selective blocker of the beta 1-adrenoreceptors, was proven to be effective in migraine prophylaxis. We conducted a controlled trial to assess the efficacy of metoprolol in prevention of HAH.

Methods: A placebo-controlled randomized trial was performed at an altitude of 3500 meters. Participants were included if had a higher systolic blood pressure of $20 \mathrm{mmhg}$ or a higher heart rate of 20/minute than their similar measured index at the altitude of 1500 meters. Exclusion criteria were certain history of cardiac, cerebral, or pulmonary disease and uncontrolled hypertension Twenty nine unacclimatized 20 to 66 years old (mean age $[ \pm S D], 39.9 \pm 13.3)$ participants were randomly assigned to oral bid $50 \mathrm{mg}$ of metoprolol or identical placebo. HAH incidence was measured as primary outcome and Lake Louise acute mountain sickness (AMS) score was considered as secondary.

Results: HAH incidence was not significantly different between subjects under metoprolol (5[27.8\%]) compared with placebo (7[41.2\%]; $\mathrm{p}=0.40)$. Moderate/severe $\mathrm{HAH}$ incidence in metoprolol group (3[16.7\%) versus placebo group $(3[17.6 \%])$ showed that metoprolol also could not reduce HAH intensity $(\mathrm{p}=0.94)$. No difference in AMS score was detected between two intervention groups.

Conclusion: Metoprolol could be the beta blocker of choice in hypoxic conditions since its adverse effects are minimal. However, our findings showed that metoprolol was not effective for prevention of HAH. Ineffectiveness might be resulted from the difference in pathophysiology between hypoxia-induced headache and migraine.

\section{P875}

Hemiplegic migraine associated with recurrent apnoea. Case report

U. Kallweit, G. Egli, P.S. Sándor

University Hospital Zurich (Zurich, $\mathrm{CH}$ )

Hemiplegic migraine (HM) is a rare and severe subtype of migraine with aura. Aura symptoms include transient hemiparesis, and at least one other aura symptom (e.g. sensory, speech, visual). Atypical attacks with encephalopathy, cerebral edema, stroke, fever, coma, seizures and even death can occur. Apnoea (without brain edema) has not been described.

We report a 59-years-old man with transient hemiparesis and nystagmus who developed recurrent episodes of apnoea. Brain MRI (incl. DWI) was unremarkable and cerebrospinal fluid (CSF) analysis was normal. No other neurological or medical causes for coma or apnoea than HM were found. Past medical history included eight emergency admissions over 30 years, with left- or right-sided weakness and, twice, with apnoea, always with complete recovery. The patient's father had migraine and died supposedly from a heart attack at the age of 54 .

We diagnosed a sporadic hemiplegic migraine. A familial form of hemiplegic migraine (FHM) was also considered possible, but no causative gene mutation for FHM could yet be identified. We initiated a therapy with flunarizine $(10 \mathrm{mg} / \mathrm{d})$. No more relapses of hemiplegic migraine or any apneic event occurred for so far 24 months.

Aura in HM might include basic life control systems as respiratory control in the lower brainstem. Respiratory arrest seems to be a second cause of death in HM patients, and might explain some of the yet unexplained deaths. 


\section{Sleep disorders}

\section{P876}

Emotional and reward processing during game playing in narcoleptic patients: a functional MRI study

A. Ponz, R. Poryazova, E. Werth, R. Khatami, P. Boesiger, C.L. Bassetti, S. Schwartz

University of Geneva (Geneva, CH); University Hospital Zurich (Zurich, $\mathrm{CH})$; Swiss Federal Institute of Technology (Zurich, $\mathrm{CH}$ )

Objectives: Narcolepsy with cataplexy (NC) is characterized by excessive daytime sleepiness, cataplexy episodes, caused by hypocretin/orexin (HCRT) deficiency. This hypothalamic neuropeptide is involved in sleepwake and feeding regulation and, as recently described, in incentive motivation. Although NC-patients often report cataplexy episodes when playing games, it is unknown whether hypocretin depletion in humans might selectively affect neural activity in reward systems.

Methods: We acquired event-related fMRI data on 12 NC-patients and 12-matched controls while they performed an incentive delay task. On each trial, subjects were presented with a cue displaying the amount to be won/lost, followed by a target. Subjects pressed on a key as quickly as possible during target presentation. Immediate feedback notified current gain. FMRI data were acquired on a 3-T Philips INTERA system, pre-processed and analyzed using SPM2.

Results: Regions involved in reward processing (ventral striatum, cingulate), motor preparation and visual attention were more activated for larger cues in both groups. However, ventral midbrain, including the VTA, was modulated by the size of the cue presentation in controls but not in NC patients. Prefrontal cortex and nucleus accumbens were more activated during winning compared to losing in the controls only. Importantly, NC patients showed increased amygdala response selectively to large positive reward.

Conclusion: HCRT-deficiency is associated with a lack of modulation in ventral midbrain regions for reward preparation, as well as an increase in bilateral amygdala activity in response to successful trials outcome. Our fMRI findings provide evidence for altered limbic-striatal responses in hypocretin-deficient patients during reward processing.

This work is supported by the Swiss National Science Foundation - Grant \# 3200B0-104100

\section{P877}

Clinical and neurophysiologic evaluation of hypersomnolence in a clinical case of bilateral paramedian thalamic stroke

A.C. Fonseca, R. Geraldes, J. Pires, F. Falcão, C. Bentes, T. Pinho e Melo

Hospital de Santa Maria (Lisbon, PT)

Background: Normal sleep architecture and arousal require an intact thalamus. Thalamic vascular lesions, particularly in the paramedian region may cause arousal disturbances and hypersomnolence. We describe clinical and neurophysiologic aspects of hypersomnolence in a clinical case of bilateral paramedian thalamic stroke.

Clinical case: A 39-year-old man with a personal history of hypertension, smoking and moderate alcohol intake was admitted after sudden conscience impairment preceded by an abrupt headache. Neurological examination showed impaired consciousness with reaction to painful stimuli only, paresis of upward gaze and of the left VI nerve, right ataxic hemiparesis and sensory loss. Brain MRI showed a paramedian bilateral thalamic stroke with mesencephalic and left capsular extension. Cerebral angiography revealed stenosis of the medial and upper third of the basilar artery with occlusion of the left posterior cerebral artery. During hospital stay hypersomnolence was noticed, spending the patient an average of twenty hours per day sleeping. Polysomnography (PSG) on day 3 had a loss of differentiation of sleep stages, with phase 1 NREM in most of the PSG recording, no sleep spindles were registered. A progressive diminution of somnolence was noted after hospital discharge, with no therapy needed. PSG done three months after stroke showed sleep spindles and phase 3 and 4 of NREM sleep. The patient at this point now spent an average of twelve hours sleeping as registered in sleep diaries. This period of sleep was distributed in to patterns. This is, the patient could have uninterrupted sleep from $9 \mathrm{pm}$ to 9 am or sleep time was distributed from $9 \mathrm{pm}$ to 6 am and then three hours after lunch.

Discussion: PSG evaluation is useful for the differential diagnosis between coma and other sleep-arousal changes. There are only few cases of bilateral thalamic stroke with neurophysiologic evaluation described in literature, being usually associated with a impairment of phase 2 NREM with diminish or absent sleep spindles, as present in this case. After acute stroke phase hypersomnolence improved and sleep spindles reappeared as well as phase 3 and 4 of NREM sleep.
P878

Prevalence of cataplexy and excessive daytime sleepiness

C. Falup-Pecurariu, A. Orha, O. Falup-Pecurariu, A. Balescu, R. Mircea, M. Tecau, S. Paripas, M. Palasan

Transilvania University (Brasov, RO)

Background: Cataplexy is a unique event in the biology encountered only in narcolepsy. It is a key symptom in the diagnostic of this disease. In the literature there is a large difference between prevalence studies mainly due to the different diagnostic criteria's.

Objectives this study was to asses the prevalence of cataplexy symptomatology and excessive daytime sleepiness.

Methods: Prospective study on 400 consecutive volunteers with the main age 39,34 years (limits 18-65). They completed a face-to-face interview using demographics variables, Cataplexy section of the Stanford Center for Narcolepsy sleep inventory [Sleep, 22, 199: 77-87] which is a validated questionnaire for epidemiological studies, Epworth Sleepiness Scale (ESS), Pittsburgh Sleep Quality Index (PSQI), Fatigability Impact Scale (FIS), Hamilton Anxiety and Depression Scale.

Results: There were 280 females (70\%). 98/400 (24,5\%) volunteers presented muscles weakness. The majority of these episodes were due to athletic activities $(18,25 \%)$, after athletic activities $(17 \%)$, when the patients were stressed $(11,75 \%)$. Eight patients reported episodes of fallen to the ground and 1 reported that he was unable to move. There was a direct correlation between excessive daytime sleepiness (EDS) and obesity. We divided by ESS? 10 the "sleepy group" which comprised 64/400 patients $(16 \%)$ and ESS $<10$ group B $(84 \%)$. There were a higher incidence of the muscle weakness in the Group A ( $p<0,01)$.

Conclusions: There is a high prevalence of cataplexy-like symptoms in the general population, even in those who did not exhibit EDS. There is need for a careful evaluation for such patients.

P879

The association between sleep questionnaires and obstructive sleep apnoea severity

H-M. Park, K-H. Park, Y-B. Lee, Y-H. Sung, D-J. Shin

Gil Medical Center (Incheon, KR)

Objectives: The obstructive sleep apnea(OSA) syndrome is a potentially disabling condition characterized by excessive daytime sleepiness, disruptive snoring, frequent arousals and nocturnal hypoxemia. Self-reported sleep scales and questionnaires are easy to perform and useful method to evaluate the OSA patients. Each sleep scale and questionnaires are reported as a good screening tool for detection of OSA before polysomnographic evaluation. But there were no report about correlation between sleep scale and severity of OSA patients. We studied each sleep scales and questionnaires to confirm the correlation between each sleep questionnaire and the severity of OSA patients.

Methods: Retrospectively consecutive 147 patients who carried out overnight polysomnogram from January 2005 to June 2007 were enrolled.All of the patients data were reviewed their sleep questionnaires which had done before polysomnographic evaluation. Sleep questionnaires were consisted of Epworth Sleepiness Scale (ESS), Stanford Sleepiness Scale (SSS), Insomnia Severity Index(ISI), Berlin questionnaire, Pittsburgh Sleep Quality Index(PSQI) and Beck Depression Index(BDI). OSA patients were divided into 3 groups according to severity by their respiratory disturbance index (RDI) score.

Results: The OSA patients divided into normal, mild, moderate, severe group by their RDI score; 25 normal patients, 23 mild OSA patients, 29 moderate OSA patients, and 72 severe OSA patients. Among sleep questionnaires, Berlin questionnaire and BDI had significantly correlated to OSA severity $(\mathrm{P}<0.05)$. Between them, Berlin questionnaire showed most positive correlation to OSA severity by RDI score after simple, adjusted statistical analy$\operatorname{sis}(\mathrm{P}<0.005)$.

Conclusion: Each sleep questionnaires such as ESS, SSS, ISI, Berlin questionnaire, PSQI, and BDI are known as a good screening tool for detection of OSA patients. Our results revealed that Berlin questionnaire is a most significant scale for prediction of severity of OSA.

P880

Correlation of bispectral index values with regional cerebral blood flow during sleep

Q. Noirhomme, M. Boly, C. Phillips, P. Peigneux, A. Luxen, P. Maquet, S. Laureys

University of Liège (Liège, BE); Université Libre de Bruxelles (Brussels, BE)

Objectives: We studied changes in regional cerebral blood flow (rCBF), as measured by Positron Emission Tomography (PET), during natural sleep 
monitored with Bispectral index scale (BIS) (1), a well-known monitor of anesthesia, and classical polysomnography.

Methods: Data were obtained from six young, healthy, righthanded, male volunteers (20-30 years old) using the $\mathrm{H} 2 \mathrm{O} 15$ infusion method (2). Each subject spent three consecutive nights in the PET scanner at usual sleep time. During the third night, PET scans were performed both during various stages of sleep when polysomnography showed steady characteristic sleep patterns and during waking at rest with eyes closed in complete darkness. At least two waking, two stage 2, two stages 3-4 and three REM sleep scans were obtained in all subjects. Sleep scoring followed standard international criteria. BIS values were monitored continuously with a portable EEG monitor (A-2000, Aspect Medical Systems, Newton) and recorded at the time of every PET scan. PET data were acquired on a Siemens CTI 951 R 16/31 scanner in 3D mode and analysed with statistical parametric mapping (SPM5). The correlation between BIS and $\mathrm{rCBF}$ was estimated according to the general linear model at each voxel. The results were corrected for multiple comparison (false discovery rate corrected $\mathrm{p}<0.05$ ).

Results: Positive correlations were detected between rCBF and BIS values in dorsolateral prefontal cortices, parietal cortices, anterior and posterior cingulate cortices, the precuneal cortex, the mesofrontal cortices, the mesiotemporal and the insulae. These areas constitute a frontoparietal network known to be related to awareness (3) and to be most active in resting non-stimulated conditions. These areas are known to be involved in attention, memory and conscious sensory perception. BIS values also positively correlated with activity in the brainstem and the thalami, both structures involved in arousal.

Conclusion: BIS values correlating with sleep depth co-vary not only with areas involved in arousal but also with areas involved in awareness of the environment.

\section{Bibliography}

1. Johansen JW, Sebel PS (2000) Development and clinical application of electroencephalographic bispectrum monitoring. Anesthesiology 93: $1336-1344$

2. Maquet $P$ (2000) Functional neuroimaging of normal human sleep by positron emission tomography. J Sleep Res 9:207-231

3. Laureys S (2005) The neural correlate of (un)awareness: lessons from the vegetative state. Trends Cogn Sci 9:556-559.

This work was supported by the Fonds National de la Recherche Scientifique de Belgique, the Fondation Médicale Reine Elisabethe, the Mind Science Foundation (Texas), the ARC de la Communauté française de Belgique, the European Funds Discos and Mindbridge

\section{P881}

Cyclic alternating pattern in narcolepsy patients and healthy controls after partial and total sleep deprivation

R. Poryazova, E. Werth, L. Parino, M.G. Terzano, C.L. Bassetti

University Hospital (Zurich, CH); University of Parma (Parma, IT)

Objectives: Cyclic Alternating Pattern (CAP) is a way to describe the arousability state during sleep. Different parameters can be extracted. In narcolepsy patients reduced CAP time, CAP rate, number of CAP cycles and phase A subtypes (especially subtypes CAP A1) were observed during baseline night time sleep in comparison to healthy controls. Total CAP time, CAP time in slow wave sleep and all CAP rates in healthy subjects were higher in morning recovery sleep and lower in night-time recovery sleep after sleep deprivation. Our aim was to investigate the regulation NREM-sleep at baseline sleep and in morning recovery sleep after partial and total sleep deprivation using CAP-parameters.

Methods: Daytime sleep under either increased (no sleep in the previous night) or decreased sleep pressure (allowing $4 \mathrm{~h}$ of sleep from 23:00-3:00 in the previous night) was recorded in ten drug-free, HLA-positive patients with NC with low/undetectable CSF-hypocretin and ten age, gender and body mass index matched healthy controls. CAP parameters were scored and analyzed for each subject. Repeated measures ANOVA was used for the statistical analysis.

Results: Narcolepsy patients had significantly lower CAP rate, CAP index, CAP time, number of CAP cycles, A1 index and number of A1 cycles in comparison to healthy controls at baseline as well as after partial and total sleep deprivation. In both narcolepsy patients and healthy control subjects there was a significant decrease in these parameters after partial and total sleep deprivation but the changes followed a similar pattern. A2 and A3 subtypes were not significantly different between patients and controls at baseline sleep. Significant decrease in A3 index and number of A3 cycles was observed in both patients and controls after total sleep deprivation but not in A2 index and number of cycles. We could not confirm the earlier findings of increase in total CAP time and all CAP rates in healthy subjects in morning recovery sleep after sleep deprivation.

Conclusion: The persistence of baseline differences between CAP parameters and their similar behaviour after partial and total sleep deprivation suggests similar homeostatic NREM regulation in narcolepsy patients and healthy controls but on a different level.

\section{P882}

Triggers for cataplexy - orgasmolepsy

R. Poryazova, R. Khatami, E. Werth, C.L. Bassetti

University Hospital (Zurich, $\mathrm{CH}$ )

Objective: Strong positive emotions such as joking, laughing and elation are specific triggers for cataplexy in patients with narcolepsy-cataplexy (NC). Cataplexy during sexual intercourse and orgasm (orgasmolepsy), though less often reported, can be a serious problem in NC patients. We describe the frequency and features of loss of muscle tone during sexual intercourse in a series of NC-patients, patients with mixed sleep disorders and healthy controls.

Patients and methods: Review of sleep questionnaires and Stanford cataplexy questionnaires of 75 subjects ( 29 with NC, 26 with mixed sleep disorders and excessive daytime sleepiness (EDS) /fatigue and 20 healthy controls) followed by an interview with specific focus on muscle loss during sexual activity in suspicious cases.

Results: Muscle weakness during sexual intercourse, was reported by three NC patients (two female, age 23 and 24 years, one male, age 25 years), one male patient with behaviorally induced sleep insufficiency and cataplexy-like symptoms and none of the healthy controls. In four NC patients and three patients with other sleep disorders and EDS the occurrence of muscle weakness during sexual intercourse remained uncertain. For the two female NC-patients this specific type of cataplexy occurred by each sexual intercourse as complete bilateral loss of muscle tone. The male patient reported complete bilateral loss of muscle tone during sexual intercourse only when in a relationship involving emotional commitment and trust. In the patient with behaviorally induced sleep insufficiency and orgasmolepsy, cataplexy-like symptoms affected one or the other upper or lower limb and were usually triggered by negative emotions and sports activities.

Conclusion: We suggest that cataplexy during sexual intercourse is a distinct feature in NC patients, but may also occur in other sleep disorders. Deficient arousal and reward dysregulation due to hypocretin deficiency may contribute to emotional motor dysfunction in orgasmolepsy. EDS may represent a gating mechanism for emotional muscle dyscontrol in orgasmolepsy and cataplexy-like symptoms.

\section{P883}

Emotional motor control in narcolepsy-cataplexy

S. Tartarotti, S. Schwartz, J. Sarnthein, E. Werth, C.L. Bassetti, R. Khatami Zurich University Hospital (Zurich, CH); University Medical Centre (Geneva, CH)

Background: Narcolepsy with cataplexy (NC) is a chronic sleep-wake disorder presenting with excessive daytime sleepiness and cataplexy. Cataplexy consists of a sudden loss of muscle tone triggered by intense emotions suggesting the possibility of an abnormal emotional motor control in these patients. Our previous data using functional magnetic resonance (fMRI) and electrophysiology demonstrate an amygdala dysfunction in narcoleptic patients.

Objectives: To test the effect of emotions on voluntary motor control, we developed an emotional version of a stop-signal task. This task provides a measure of inhibitory motor control, which is independent of motor execution and other unspecific cognitive functions, in particular from speed of reaction time.

Design and methods: Seven drug free HLA-DQB1 ${ }^{\star} 0602$ positive NC patients (mean age: $37.3 \pm 11.1$ years, hypocretin-1 deficient in $4 / 4$ tested) and five controls (mean age: $34.8 \pm 7.2$ years) performed an emotional version of stop signal task. Reaction times to "go" stimuli and the latencies needed to stop a motor response (SSRT=stop signal reaction time) were measured under different emotional conditions (neutral and fearful stimuli). SSRT was determined by a dynamic tracking algorithm which converges to a $50 \%$ probability of successfully suppressed motor response. Data were analyzed using ANOVAS with EMOTION as within-subject repeated factor and GROUP as between-subject factor.

Results: Reaction times to Go trials were slower for NC patients for emotional and neutral stimuli (all $\mathrm{p} \leq 0.05$; emotional NC: $713 \pm 111 \mathrm{~ms}$ vs. C: $587 \pm 78 \mathrm{~ms}$; neutral NC: $708 \pm 106 \mathrm{~ms}$ vs. C: $572 \pm 81 \mathrm{~ms}$ ). SSRT measures tended to be faster for emotional stimuli in controls but not in patients (GROUP by EMOTION interaction ( $p<0.08)$; emotional NC: $273 \pm 136 \mathrm{~ms}$ 
vs. C: $263 \pm 57 \mathrm{~ms}$; neutral NC: $247 \pm 137 \mathrm{~ms}$ vs. C: $268 \pm 83 \mathrm{~ms}$ ). As required for a valid SSRT calculation, the probability of inhibition was 0.5 for each group and in each condition.

Conclusions: Although NC patients were generally slower than controls during simple Go trials, they showed effective inhibitory motor control. Critically, motor inhibition was differentially affected in both populations. These preliminary data suggest abnormal influence of emotions on motor inhibition in narcolepsy-cataplexy.

Study supported by the Swiss National Foundation

\section{P884}

Characteristics of nocturnal position change in patients with obstructive sleep apnoea-hypopnoea syndrome

W.C. Shin, S.H. Bu, Y.H. Lee, S.J. Byun

Kyung Hee Medial School (Seoul, KR); Icheon Medical Centre (Icheon, KR)

Objective: It is well known that respiratory disturbance index (AHI) in sleep with lateral position is on average about $40-50 \%$ lower than during sleep supine in patient with obstructive sleep apnea-hypopnea syndrome(OSAS). We investigated that the pattern of nocturnal body position change in patient with OSAS differs among patient with OSAS and control.

Methods: We reviewed the polysomnographic finding of 188 patients with OSAS. We assessed the frequency, sleep stage that positional change occurred, sleep events that could cause the positional change according the severity apnea-hypopnea index and positonal apnea-hypopnea index difference.

Results: There were tendency that positional change during sleep were frequent in patients with high apnea-hypopne index than in patients with lower apnea-hypopnea index and in positional OSAS than non-positional OSAS. Compare with control, respiratory events caused the positional change in patients with OSAS. In relation with sleep stage, the positional changes were noted in all stage including REM sleep, there were no difference in sleep stage in OSAS group.

Conclusion: Nocturnal positional change is common phenomenon in control and patients with OSAS. In patient with OSAS, respiratory events could provoke the positional change during sleep.

\section{P885}

Beneficial effect of piracetam on obstructive sleep apnoea syndrome in patients with multiple system atrophy

Y. Nakamura, H. Sakamoto, I. Yamada

Sakai Hospital (Osaka, JP)

Objective: Patients with multiple system atrophy (MSA) are frequently known to develop obstructive sleep apnea syndrome (OSA) and/or laryngeal stridor with disease process and the tracheostomy or nasal continuous positive air pressure is often needed. Recently EMG investigation showed that the mechanism for stridor is dyskinesia or dystonia of laryngeal muscles. Evidence of the laryngeal dystonia could suggest a new choice of treatment for laryngeal narrowing in MSA. Now, there is no accepted pharmacological treatment of laryngeal narrowing in MSA. Piracetam is a low molecular weight derivate of GABA and have a significant beneficial effect of anti-myoclonus drug. The purpose of this report is to evaluate the effect of piracetam on OSA /laryngeal narrowing in MSA.

Subjects and methods: Three patients with MSA were enrolled. They were all male and their average age was $60.9 \pm 5.7$ years old and the duration of disease was $3.3 \pm 1.2$ years. They had probable cerebellar type (MSA-C). All patients had the polysomnographic study (PSG) and laryngeal endoscopy. In PSG, routine record including EEG, respiration and oxyhaemoglobin saturation $(\mathrm{SaO} 2)$ were measured. The average number of apnea-hypopnea episodes per hour of sleep was apnea-hypopnea index (AHI). OSA was defined as a cessation of airflow for over 10 seconds despite persistent respiratory effort with an $\mathrm{AHI}$ more than 5 . The criteria of piracetam treatment was an AHI above 20/h. They had $12 \mathrm{~g} /$ day of piracetam for two weeks. They were evaluated before and after treatment.

Results: 71-year old male had showed stridor during night. His PSG showed OSA with $46 / \mathrm{h}$ of $\mathrm{AHI}$ and $86 \%$ in average $\mathrm{SaO} 2$. After piracetam treatment, effect of piracetam on apnea was observed (AHI was 16/h and average $\mathrm{SaO} 289 \%$ ). In case 2, his PSG showed $54.3 / \mathrm{h}$ of $\mathrm{AHI}$ and $75 \%$ in average SaO2. After treatment, effect of piracetam on apnea was also observed (29.2/h in AHI and $88 \%$ in average $\mathrm{SaO} 2)$. Case 3 also showed improvement after piracetam (23/h to $12.7 / \mathrm{h}$ in $\mathrm{AHI}, 86 \%$ to $89 \%$ in average $\mathrm{SaO} 2)$.

Conclusions: Piracetam therapy was demonstrated to be effective on OSA and was well tolerated in patients with MSA. To our knowledge, the beneficial effect of piracetam on OSA was not previously reported. OSA in MSA could be caused by laryngeal narrowing with dystonia of laryngeal muscles.
Our findings suggested piracetam has a potential effective drug on OSA and/or laryngeal narrowing in MSA.

\section{P886}

Tracing the dopaminergic borders of periodic leg movements during sleep L. Ferini Strambi, M. Manconi, M. Zucconi, R. Ferri, G. Plazzi Università Vita-Salute S. Raffaele (Milan, IT)

Objective: Periodic leg movements during sleep (PLMS) are repetitive tibialis anterior jerks lasting from 0.5 to 5 seconds, separated by interval ranging from 5 to 90 seconds, organized in series of 4 or more consecutive leg movements (LM). Although the pathophysiology of the PLMS/RLS is unknown, there is increasing evidence of an involvement of dopaminergic neurotransmission. The aim of this study was to define the criteria for PLMS using a new pharmacological approach to recognize a sub-group of LM group with a common dopaminergic origin.

Methods: A single-blind placebo-controlled study in 43 consecutive idiopathic RLS de-novo patients with a PLMS index greater than 10 was carried out. Patients underwent a clinical and neurophysiological evaluation with hematological screening and two consecutive full-night polysomnographic studies (PSG). During the second PSG, all patients were randomized to receive $0.25 \mathrm{mg}$ of pramipexole or placebo. An off-line analysis was conducted on LM parameters such as duration, amplitude, interval, and periodicity.

Results: Compared to placebo, pramipexole significantly $(\mathrm{p}<0.01)$ reduced PLMS while increasing sleep efficiency. Specifically we observed a significant $(\mathrm{p}<0.01)$ reduction in LM ranging between 2 and $4 \mathrm{~s}$ in duration and an inter-movement interval between 6 and $64 \mathrm{~s}$ and a significant decrease in the periodicity of events. No effect of pramipexole was observed on isolated LM. Pramipexole significantly increased the entropy of LM compared to the placebo.

Conclusions: These results strongly support a heterogeneous basis for LM in RLS patients in which there may be a non-dopaminergic in isolated LM and a dopaminergic component in patients classified with PLMS with the current criteria. Therefore, more restrictive scoring criteria for PLMS might be indicated.

\section{P887}

Brain morphology in obstructive sleep apnoea patients and normal controls L. Ferini Strambi, C. Castronovo, M. Aloia, N. Canessa, A. Falini, S. Cappa, D. Perani

Università Vita-Salute S. Raffaele (Milan, IT); National Jewish Medical and Research Center (Denver, US)

Objective: Neurocognitive deficits found in obstructive sleep apnoea (OSA) patients are thought to result from either directly sleep fragmentation or intermittent hypoxemia or their interaction that cause a possible damage or alteration of neural structures. Aim of the study was to assess structural brain changes in severe untreated OSA $(\mathrm{AHI}>=30)$ patients.

Methods: Voxel-based morphometry (VBM) was used to assess patterns of grey matter atrophy in a group of 17 patients with OSA(mean age $42.7 \pm 7.8$ ) compared to a group of 15 healthy controls. The two groups were age- and gender-matched. VBM data were collected using a 3 Tesla scanner (Philips Achieva). Statistical analysis was performed using SPM5 software. In order to collect valid results, an analysis with a correction based on cluster extension was used. Furthermore, age and total intracranial value (TIV) were introduced as covariates.

Results: Two clusters of grey matter loss in OSA patients compared to normal controls have been found in the right superior frontal gyrus and in the left superior parietal lobule, respectively. The reverse comparison (controls vs. patients) did not reveal significant regional differences.

Conclusion: The few VBM studies of OSA patients showed inconsistent results. In particular, some investigations found no evidence of grey matter changes in OSA patients; other studies showed a regional grey matter loss in the frontal cortex, parietal cortex, temporal lobe, anterior cingulate, hippocampus, and cerebellum. Different data analysis methods may explain some of these inconsistencies. The present findings confirm the existence of a grey matter lost in OSA patient with a conservative statistical analysis. The evaluation of treatment effects will clarify the actual impact of gray matter changes in relation to cognitive dysfunction.

This research has been supported by a grant from Respironics Foundation, USA 


\section{Author index}

Abbas, M.A . . . . . . . . . . P238

Abd El Hady, G . . . . . . . . . . . . . . O O108

Abdelsamee, A . . . . . . . P632, P777

Abderrahim, $\mathrm{H} \ldots \ldots \ldots \ldots \ldots$. . . . O999

Abdulghani, O … P279, P637, P705, P814, P815

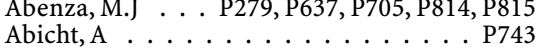

Abolfazli, R . . . . . . . . . . P P687, P828

Abrahams, S . . . . . . . . . O208

Abrams, C . . . . . . . . . . . O88

Abramsky, O . . . . . . . . . . . O193

Absinta, M . . . O O54, O55, P294, P349, P452

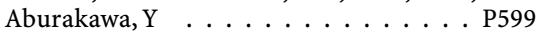

Acero, $L \ldots \ldots \ldots \ldots \ldots$. . . . . . . . . . . . . .

Adamu, A . . . . . . . . . O O103

Adiel, A . . . . . . . . . O O135

Adigüzel, $\mathrm{T} \ldots \ldots \ldots \ldots \ldots \ldots \ldots$

Adler, C . . . . . . . . . O141, P399, P800

Adobbati, L . . . . . . . . . P365

Afanasyev, B . . . . . . . . . . P731

Afazel, $\mathrm{S} \ldots \ldots \ldots \ldots \ldots \ldots$. . . . . . . . . . . . .

Afify, H . . . . . . . . . . . . . . . P P767

Afrantou, $\mathrm{T} \ldots \ldots \ldots \ldots \ldots$. . . . . 696

Afsar, $N \ldots \ldots \ldots \ldots \ldots$ P243

Agena, G.M . . . . . . . . . . P462

Agnelli, G . . . . . . . . . . . O O120

Agosta, F . O165, O167, O209, P342, P452, P465

Agosti, C . . . . . . . . . . P781

Aguennouz, M . . . . . . . . . . P602

Aguilar-Amat Prior, M.J ‥ . P279, P705,

P814, P815

Ahishali, B . . . . . . . . . . . . P539

Ahlborn, M . . . . . . . . . . . P392

Ahle, $G \ldots \ldots \ldots \ldots$. . . . . . . . . . P617

Ahluwalia, $s \ldots \ldots \ldots \ldots \ldots$. . . . P688

Ahmadi karvigh, $\mathrm{S} \ldots \ldots \ldots \ldots$. . P845

Ahmed, I . . . . . . . . . . . . . . . . P765

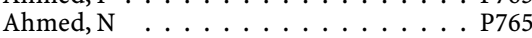

Ahmed Bello, L . . . . . . . . . . P654

Ahtoniemi, $\mathrm{T} \ldots \ldots \ldots \ldots \ldots$. . P771

Airas, L . . . . . . . . . . . . . . . . . . . P P729

Aizawa, $\mathrm{H}$. . . . . . . . . . . . P599

Akbari, R . . . . . . . . . . . . . P320

Akbostanci, M.C . . . . . . . . . . . P699

Akdal, G . . . . . . . . . . . . . . . . O O109

Akgul, E . . . . . . . . . P417

Akhtar, $\mathrm{N} \ldots \ldots \ldots \ldots$. . . O O131

Akman-Demir, G . . . . O 226, P559, P624,

P842, P871

Aktan, S . . . . . . . . . . . . P243

Al Abdulkareem, I . . . . . . . . . . P277

Al Blawi, M . . . . . . . . . . . . . . P277

Al hail, $H_{1} \ldots \ldots \ldots \ldots$. . . . . . O131

Al Jumah, A $\ldots \ldots \ldots \ldots \ldots \ldots$ P277

Al Jumah, M $\ldots \ldots \ldots$ P277, P437, P736

Al Mubarak, B . . . . . . . . . . . . P285

Al Sharif, L . . . . . . . . . . . . P285

Al Suwaidan, $\mathrm{F} \ldots \ldots \ldots \ldots \ldots \ldots \ldots$

Al Tahan, A . . . . . . . . P P736

Al Tassan, N . . . . . . . . . . . P285

Al Traif, I . . . . . . . . . . . . . P277

Al Zaben, A . . . . . . . . . . . . P277

AL-Sabbagh, A . . . . . P P21, P323, P445, P570, P717, P826

Alarcon, M . . . . . . . . . . P522

Albani, D . . . . . . . . . . . . . . P780

Albert, $N \ldots \ldots \ldots \ldots \ldots$

Albuquerque, $\mathrm{L} \ldots \ldots \ldots \ldots \ldots$. . . P520

Aldenkamp, A . . . . . . . . P413

Alegria, P . . . . . . . . . . . P766

Alexandru, $\mathrm{O} \ldots \ldots \ldots \ldots$. . . . . . . .

Alexiou, E . . . . . . . . . . . P308, P613

Alexopoulos, $\mathrm{H} \ldots \ldots \ldots$. . . . . O O79

Alfano, B . . . . . . . . . . P333
Algra, A . . . . . . . . O62, O64, O65

Alhindi, $\mathrm{H} \ldots \ldots \ldots \ldots \ldots \ldots$. . . . . . 628

Alkawi, Z . . . . . . . . . . . . . . . . . . . P628

Alkhairallah, $\mathrm{T} \ldots \ldots \ldots \ldots$

Allum, J.H.J . . . . . . . . . . P817

Almeida, R . . . . . . . . . . . . P552

Almohsen, C . . . . . . . . O O211, P477

Aloia, M . . . . . . . . . . . . O O181, P887

Alonso, $\mathrm{H}$. . . . . . . . . . . . . P572

Alonso, M.E . . . . . . . . . . . . . O84

Alonso, M.M . . . . . . . . . . O91, P615

Alonso de Leciñana, M . . . . . . . . . . O168

Alpay, A . . . . . . . . . . . . . P498

Altenkämper, $S \ldots \ldots \ldots \ldots$. . . . . . . . O225

Alvarenga, $\mathrm{M} \ldots \ldots \ldots \ldots 150$

Alvarez, M.J _ . . . . . . . . . . P752

Alvarez, S . . . . . . . . . . . . . . . O O 272, P306

Álvarez-Blanco, S . . . . . P255, P263, P859

Alvarez-Suero, J . . . . . . . . . . O170

Alves, L . . . . . . . . . . . . . P766

Alves-Leon, $\mathrm{S} \ldots \ldots \ldots$. . . . . . . . . . . O O150

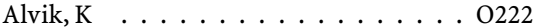

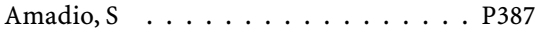

Amann, M . . . . . . . . . P449

Amantini, A . . . . . . . . . . . P379

Amato, M.P . . . . . . . . P575, P715

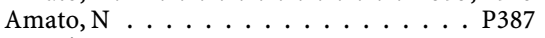

Ameri, A . . . . . . . . . P870

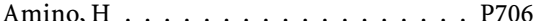

Amiri, S . . . . . . . . . . P284

Ammar, M . . . . . . . . . . . . .

Amorelli, V . . . . . . . . . . . P483

Amouri, R . . . . . . . . . . . P274

Anagnostouli, M . . . . . . . . P400

Anand, A . . . . . . . . . P656

Anastasiadou, M . . . . . . . . . . P423

Anderson, M . . . . . . . . . . . P443

Andrade, R . . . . . . . . . O O138

Andreadou, E . . . . . . . . P400, P742

Andrinos, D . . . . . . . P554, P863, P864

Angelini, C . . . . . . . . . . . . . . . P587

Angelou, A . . . . . . . . P607, P696, P698

Angstwurm, K . . . . . . . . . . . . P809

Anlar, $\mathrm{O} \ldots \ldots \ldots \ldots \ldots$. . . . . . . . . . . .

Annovazzi, $\mathrm{P} \ldots \ldots \ldots \ldots \ldots \ldots$

Annunziata, P . . . . . . . . P451, P839

Antalffy, B . . . . . . . . . O O166

Antochi, F.A . . . . . . . . . P555, P763

Antoniuk, S.A . . . . . . . . . . P293

Antony, D . . . . . . . . . . . . O96

Antozzi, $\ldots \ldots \ldots \ldots \ldots \ldots$ P740

Antrasian, A . . . . . . . . . . . P543

Aparicio, J . . . . . . . . . . . . . P255

Apostolski, $S \ldots \ldots$ P499, P604, P734

Aqil, $N \ldots \ldots \ldots \ldots \ldots \ldots$. . . . . . . . 654

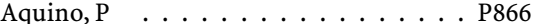

Aquino Gondim, F.A $\ldots \ldots \ldots$ P590, P866

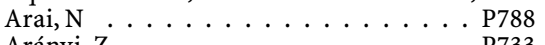

Arányi, Z . . . . . . . . . . . P733

Araujo, H . . . . . . . . . . . . . P368

Arbizu, T . . . . . . . . . . . . . O O193

Arbusow, $\mathrm{V} \ldots \ldots \ldots \ldots \ldots$. . . P532

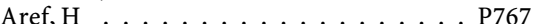

Argentiero, $\mathrm{V} \ldots \ldots \ldots \ldots \ldots$. . . . . . . . .

Argyriou, A $\ldots \ldots \ldots \ldots \ldots$ P377, P378

Ariatti, A . . . . . . . . . P307, P493

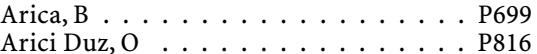

Arnason, B.G.W $\quad \ldots \ldots$ O58, O158, P447

Arnold, M . . . . . . . . . . O68, 0115

Aronovich, R . . . . . . . . . . . P564

Arpa Gutierrez, J . . . . . P279, P705, P814

Arpaci, B . . . . . . . . . . . . P645

Arranz, E . . . . . . . . . . P572
Arrigo, A . . . . . . . . . . . . P639 Arslan, $\mathrm{O} \ldots \ldots$ P416, P471, P472, P643, $\mathrm{P} 708, \mathrm{P} 754, \mathrm{P} 757$

Arzy, $\mathrm{S} \ldots \ldots \ldots \ldots$. . . P710, P821

Asadollahi, M ............ P320

Asai, A . . . . . . P339, P630, P631

Asimiadou, $\mathrm{S} \ldots \ldots \ldots \ldots \ldots \ldots$. . . . P833

Asztely, F . . . . . . . . . . . . O881

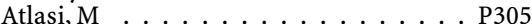

Atzori, M $\ldots \ldots \ldots \ldots \ldots \ldots 187,0 \ldots$

Aufm Kampe, K . . . . . . . . . . . P324

Augustyniak, $\mathrm{M} \ldots \ldots \ldots \ldots$. . P450

Austinat, M.L . . . . . . . . . . . O O204

Avantaggiato, P . . . . P P 483, P489, P490

Avolio, C . . . . . . . . . . . . . P819

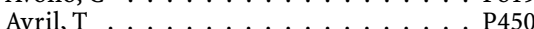

Awadh, M . . . . . . . . P296, P767

Ayache, $N \ldots \ldots \ldots \ldots$. . . . . P621

Ayalp, S . . . . . . . . . . . . . . P P645

Ayarza, A.C . . . . . . . . . . . P352

Aydin, K . . . . . . . . . . . . P872

Aydin, T . . . . . . . . . . P498, P816

Aydin Cantürk, I … P498, P668, P673, P816

Ayromlou, $\mathrm{H} \ldots \ldots \ldots \ldots \ldots \ldots$. . . . . P557

Azarpira, $N \ldots \ldots \ldots \ldots \ldots$. . . . . . . .

Azevedo, A . . . . . . . . . . . . P627

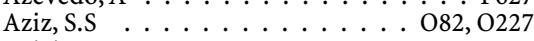

Azizi, A $\ldots \ldots \ldots \ldots \ldots \ldots$ O201, P237

Babron, M-C . . . . . . . . . . . O218

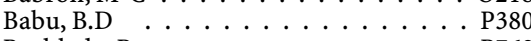

Bachleda, P . . . . . . . . P768

Bachmann, G . . . . . . . . . O200

Bacigaluppi, M . . . . . . . P238

Back, $\mathrm{T} \ldots \ldots \ldots \ldots$. . . . . O66

Badea, $\mathrm{P} \ldots \ldots . . . . . . . . . . .148$

Bader, B . . . . . . . . . . . O O143

Badley, A . . . . . . . . P523

Bae, J.S . . . . . . . . . P735

Baek, H-J . . . . . . . . . . . . . P647

Baggi, F . . . . . . . . . . . . P740

Bahadori, M . . . . . . . P305

Baicus, C . . . . . . . . . . O61

Baier, B $\ldots \ldots \ldots \ldots$. $\ldots \ldots 137$, P249

Baik, J-S . . . . . . . . . . . . P670

Bailet, B . . . . . . . . . . P550

Baima, C . . . . . . . . . . P567

Bajenaru, O . . . P421, P555, P763, P787

Bajko, Z . . . . . . . . . . P366, P393

Bakheet, D . . . . . . . . . . P285

Bal, A . . . . . . . . . . . . . . P359

Bal, S . . . . . . . . . . . P367

Balash, $\mathrm{Y} \ldots \ldots \ldots \ldots \ldots . . . . . . . .247$

Balata, A . . . . . . . . P695

Balescu, A . . . . . . . . P878

Balestrini, M.R . . . . . . . . O O75, P271

Balla, C . . . . . . . . . . . . . . P698

Ballesteros, L . . . . . . . . . . . . . P609

Balon, S . . . . . . . . . . P855

Baña, M . . . . . . . . . . P411

Bansberg, S . . . . . . . . . P800

Bao, F . . . . . . . . . . P453, P583

Baranczyk-Kuzma, A . . . . . . . P P470

Baratta, S . . . . . . . . . O86, P281

Barbarash, $\mathrm{O} \ldots \ldots \ldots \ldots$. . . . P325, P720

Barbero, P . . . . . . . . . P714, P719

Barbetta, L . . . . . . . . . . . . P283

Barbieri, $S \ldots \ldots \ldots \ldots \ldots \ldots$ P231, P799

Barbone, F . . . . . . . . . . . P460

Barbov, I . . . . . . . . . . . . P419, P511

Bardoni, A . . . . . . P483, P489, P490

Barillot, C . . . . . . . . . P824, P846

Barkhof, F . . . . . O54, O186, O191, P342

Barreira, A.A . . . . . . . . . . P500 


\begin{tabular}{|c|c|}
\hline rreto, $\mathrm{J}$. & $\begin{array}{l}\text { Berger, } \mathrm{W} \\
\text { Bergonzi, } \mathrm{P}\end{array}$ \\
\hline $\begin{array}{l}\text { O148, P704 } \\
\text { O209 P465 }\end{array}$ & $\begin{array}{l}\text { Bergonzi, } \mathrm{P} \\
\text { Bergui, } \mathrm{M}\end{array}$ \\
\hline $\begin{array}{l}\ldots \mathrm{O} 209, \mathrm{P} 465 \\
\mathrm{P} 446, \mathrm{P} 571, \mathrm{P} 712, \mathrm{P} 838\end{array}$ & $\begin{array}{l}\text { 3ergui, M } \\
\text { 3erheim, } J\end{array}$ \\
\hline$\ldots \ldots$. . $5563, \mathrm{P} 822$ & O $118, \mathrm{P} 469$ \\
\hline P668 & P398, P674 \\
\hline P856 & mel, R.A \\
\hline P871 & nard, C.C.A. \\
\hline . O180, P876, P881, P882, P883 & ernard, $\mathrm{M}$ \\
\hline$\ldots \ldots \ldots$ P499, P606, P734 & $\mathrm{O} 187, \mathrm{O} 220$ \\
\hline stianello, S & ardo, D \\
\hline stien, D & O194, P661 \\
\hline atlle i Nadal, J & ernsen, $\mathrm{P}$ \\
\hline attaloglu, E . . . . . & eroud, $F$. \\
\hline auer, J & ertini, $\mathrm{E}$ \\
\hline$\ldots \ldots$ O188, P576, P723 & O156, O189, O221, P441 \\
\hline$\ldots \ldots \ldots 0180$ & $\ldots \ldots \ldots \ldots \mathrm{P} 481$ \\
\hline 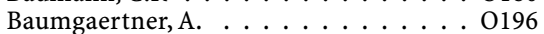 & . . . . . P779 \\
\hline aumgartner, $\mathrm{R} \ldots \ldots \ldots$ & ertotti, A \\
\hline $\mathrm{P} 480$ & $\ldots \ldots$ \\
\hline P725 & ken, $\mathrm{T}$ \\
\hline . . . . . . . P539, P559, P872 & P367 \\
\hline$\ldots \ldots \ldots \ldots$ P396 & O212, P397 \\
\hline$\ldots \ldots$ & . . . . . P819 \\
\hline O141 & P553 \\
\hline . . . . . . . . . O997, P849 & \\
\hline$\ldots \ldots \ldots \mathrm{O}_{0}$ & andani, $S \ldots$ \\
\hline . . . . . . P $539, \mathrm{P} 559$ & $\ldots \mathrm{O} 110$ \\
\hline . P802 & P322 \\
\hline P295 & \\
\hline P323 & O196 \\
\hline P716 & $\mathrm{er}, \mathrm{N}$ \\
\hline . . . O O69, P459 & 122, O125, O126, P855 \\
\hline$\ldots \ldots \ldots$ P 620 & $\ldots \ldots \ldots$ P570 \\
\hline $\mathrm{P} 413$ & P625 \\
\hline $\mathrm{P} 420$ & P346 \\
\hline P685 & $\mathrm{P} 602$ \\
\hline . P410, P416, P417, P471, P472, & $\mathrm{O} 88$ \\
\hline $\mathrm{P}$ & . O $\mathrm{O} 153, \mathrm{O} 200, \mathrm{O} 225$ \\
\hline P754, P755, P756, P757 & \\
\hline$\ldots \ldots \ldots$ & P728 \\
\hline$\ldots \ldots \ldots$ & \\
\hline $\mathrm{P} 412$ & \\
\hline$\ldots \ldots \ldots$ & O184 \\
\hline $\mathrm{O} 219$ & P473 \\
\hline$\ldots \ldots$ & P473 \\
\hline & . . O $058, \mathrm{O} 158, \mathrm{P} 447$ \\
\hline$\ldots \ldots \ldots \ldots \mathrm{P} 443, \mathrm{P} 573$ & . . . . . P293 \\
\hline . . P443, P573 & 060 \\
\hline P295 & P876 \\
\hline & $\mathrm{P} 802$ \\
\hline P374 & $\mathrm{O} 142$ \\
\hline $\mathrm{P} \epsilon$ & vic, $\mathrm{N}$. \\
\hline P8 & \\
\hline $\mathrm{P} 4$ & . . O $58,0158, \mathrm{P} 447$ \\
\hline H.T.S & P285, P736 \\
\hline $\mathrm{O}$ & $\mathrm{O} 214$ \\
\hline P5 & $\mathrm{O} 144$ \\
\hline & P381 \\
\hline . O $221, \mathrm{P} 441, \mathrm{P}$ & \\
\hline $\mathrm{P} 4$ & O193 \\
\hline $\mathrm{O} 97, \mathrm{P} 8$ & O87, P509 \\
\hline P246, $\mathrm{P}$ & erti, L \\
\hline$\ldots \mathrm{P} 321, \mathrm{P} 323, \mathrm{P} 4$ & $\mathrm{O} 123, \mathrm{O} 140, \mathrm{O} 179, \mathrm{P} 880$ \\
\hline P570, P717, & \\
\hline$\ldots \mathrm{O}$ & \\
\hline P750 & 0165 \\
\hline P8 & P283 \\
\hline $\mathrm{D}$ & P722 \\
\hline $\mathrm{P} 4$ & \\
\hline P300, P598 & 0110 \\
\hline O $74, \mathrm{O} 147, \mathrm{O} 164, \mathrm{O} 213, \mathrm{P} 312$ & P379 \\
\hline P366 & P707 \\
\hline & P272, P283, P300, P596 \\
\hline P383, P5 & P396 \\
\hline P489, P490 & P542 \\
\hline P762 & P380, P675 \\
\hline O67 & Haghighi, A \\
\hline Berger, M.M & P610, P611 \\
\hline
\end{tabular}

Borrás, J . . . . . . . . . . . P820 Borroni, B . . . . . . . . . P781

Bosca, M . . . . . . . . . . O215

Boselli, D . . . . . . . . . . . . P840

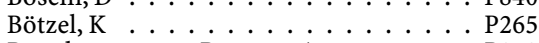

Boughammoura-Bouatay, A . . . . . P873

Bouhlal,Y . . . . . . . . . . P274

Bouktsi, $M \ldots \ldots \ldots \ldots \ldots$ P841

Bourdette, D . . . . . . . . . . . P448, P584

Bourg, V . . . . . . . . P P442, P538, P550

Bousser, M.G . . . . . . . . O68

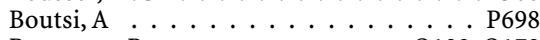

Boveroux, P . . . . . . . . . . O O123, O179

Boxer, A . . . . . . . . . . O165

Bozic, C $\ldots \ldots \ldots \ldots \ldots \ldots \ldots \ldots$

Bozzolo, E . . . . . . . . . . P442

Bracco, F . . . . . . . . . . . P750

Bradac, G.B . . . . . . . . . . . O89

Braem, B . . . . . . . . P648

Braeuninger, $\mathrm{S} \ldots \ldots \ldots \ldots \ldots \ldots$. . . . O204

Braga, L . . . . . . . . . . P866

Bragato, C . . . . . . . . . O O85

Brandt, T . . . . . . . O O176, P532

Brassat, D . . . . . . . . . . . . O218

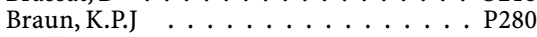

Brédart, $S \ldots \ldots \ldots \ldots \ldots \ldots$. . . . . . .

Bremerich, A . . . . . . . . . P392

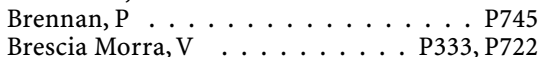

Bresolin, N . . O O197, P272, P283, P286, P300, P463, P475, P580, P589, P596, P598, P682, P718, P782, P799

Brettschneider, J . . . . . . . . . . P467

Briani, C . . . . . . . . . . . P463

Bril, V . . . . . . . . . P P427, P439

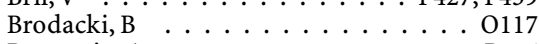

Bronstein, A . . . . . . . . . P746

Brouns, R . . . . . . . . . . . O O173

Brouwer, M.C . . . . . . . . P534

Brown, P . . . . . . . . . . . . . O O224

Bruce, I . . . . . . . . . . . . P701

Bruck, I . . . . . . . . . . . . . P293

Brück, W . . . . . . . . . . . . P702

Brunel, $\mathrm{H} \ldots \ldots \ldots \ldots \ldots$. . . . . . . 211

Brunetti, A . . . . . . P333, P722

Brunetto, J.L $\ldots \ldots \ldots \ldots \ldots \ldots$ P851

Brüning, R . . . . . . . . P515

Bruno, C . . . . . . . . . P587

Bruno, M-A . . . O O111, O112, O113, O123,

$\mathrm{O} 140, \mathrm{O} 155, \mathrm{O} 179$

Bruschi, A ....................... Bu, S.H $\ldots \ldots \ldots \ldots \ldots \ldots$
Bucello, $\ldots \ldots \ldots$ P353, P884

Buchser, E . . . . . . . . O121, P852, P860

Buckley, C . . . . . . . . . O O216

Budrewicz, S . . . . . . P386, P827

Buerger, M . . . . . . . . . . . . P469

Buffon, $\mathrm{F} \ldots \ldots \ldots \ldots \ldots$. . . . . . . . . .

Bugiani, $M \ldots \ldots \ldots \ldots$ P271

Bugiani, $O \ldots \ldots \ldots \ldots \ldots$. . . . . . 281

Bugiardini, E . . . . . O 71, P266, P563, P822

Bunten, S . . . . . . . . . . . P392

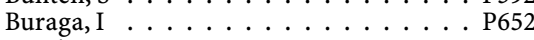

Burcin, C ............ O61

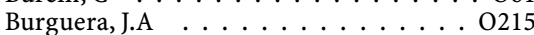

Burgunder, J.M . . . . . . . P795

Burlina, A . . . . . . . . . O71

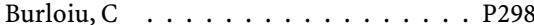

Burns, A . . . . . . . . . P334

Burrows, $G \ldots \ldots \ldots \ldots \ldots 1$

Bursa, A . . . . . . . . P506

Buruiana, $\mathrm{F} \ldots \ldots \ldots \ldots$. . . . P297

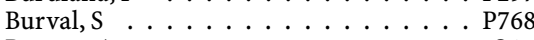

Busson, A . . . . . . . . . . . O O95

Butzkueven, $\mathrm{H} \ldots \ldots \ldots \ldots \ldots$. . . . O101

Bwala, S . . . . . . . O80, O103, P654

Byts, N . . . . . . . . . . . P802

Byun, S.J . . . . . . . . . . . . P353, P884 


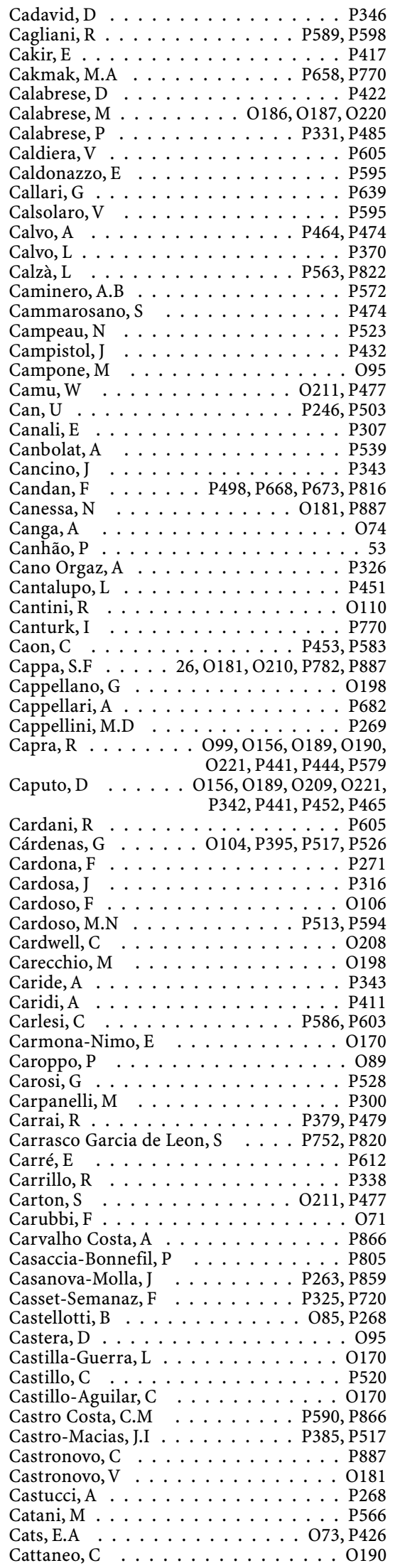

Caumont de la Mesquita, R . . . . . . P661 Cavallaro, T . . . . . . . . . . . . . . O O75 Cavallo, E . . . . . . . . . . . . P464 Cavarretta, R . . . . . . . . . . . . . P843

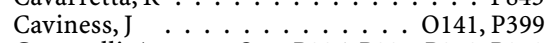
Ceccarelli, A . . . O57, P336, P337, P348, P349 Cercignani, M .......... . O60 Cereda, C . . . . . . . . . . . . . P409 Cerqueira, $\mathrm{R} \ldots \ldots \ldots \ldots \ldots \ldots$. . . . . . . . O Cerri, F . . . . . . . . . . . . . . . . . P387 Chan, K.H $\ldots \ldots \ldots \ldots$ Chanalet, $S \ldots \ldots \ldots$ P442, P538, P550 Chand, S . . . . . . . . . . . P334

Chandra, N . . . . . . . . . . . . . P514 Chang, D.I $\ldots \ldots \ldots . . .20$ P229, P671, P764 Chang, $\mathrm{P} \ldots \ldots \ldots \ldots \ldots \ldots$. . . . . . P829 Chang, $\mathrm{T} \ldots \ldots \ldots \ldots \ldots$. . . . . . O224

Chapman, J . . . . . . . . . . . . P P564 Chatel, M . . . . . . . . . . . . . . . . . P299

Chatzidakis, I . . . . . . . . . . . P454

Chau Huu, Y . . . . . . . . . . . . . . O116

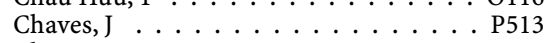

Chaves, M.L . . . . . . . . . . . P502

Chebel, S . . . . . . . . . . P623, P873

Cheilakos, $G \ldots \ldots \ldots \ldots \ldots \ldots \ldots$. $\ldots \ldots \ldots 1$

Chelu, G . . . . . . . . . . . . . . . . . P421

Chen, Jie. . . . . . . . . . . . . . . . . . . P793

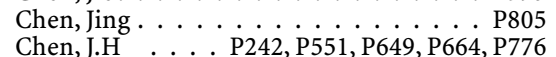

Cheong, J.S . . . . . . . . . . . . P813

Cheung, R.T.F . . . . . . . . . . . . P837

Chiapparini, L . . . . . . . . . . . . P281

Chiarini, M . . . . . . . . . . . . . . P579

Chieffo, R . . . . . . . . . . O O139, P256

Chiò, A . . . . . . . . . . . P464, P474

Chiocchetti, A . . . . . . . . O O198

Chiorri, C . . . . . . . . . P P750

Chirileanu, D . . . . . . . . . . P506

Chirileanu, R . . . . . . . . . . . . . . . P791

Chitsaz, A . . . . . . . . . . . . P629

Chmelova, J . . . . . . . . . . . P768

Cho, A-H . . . . . . . . . . . . P340

Cho, E.M . . . . . . . . . . . . . . P288

Cho, J.H . . . . . . . . . . . . P304

Cho, S.Y $\ldots \ldots$. P288, P303, P309, P317

Choi, B.O . . . . . . P288, P303, P309, P317

Choi, J . . . . . . . . . . . . O76

Choi, K.G . . . . . . . . . . . . P303

Choi, S-A . . . . . . . . . . . . P304

Choi,Y-C . . . . . . . . . . . . . . P739

Chovancová, Z . . . . . . . . . . . P355

Christ-Crain, M . . . . . . . O O67, P741

Christodoulou, C . . . . . . . . P825

Chroni, E . . . . . . . . . P666, P667, P679

Chu, A.C.Y . . . . . . . . . . P836

Chua, $\mathrm{H} \ldots \ldots \ldots \ldots \ldots \ldots \ldots$ P558

Chung, E-J $\ldots \ldots \ldots \ldots$. $\ldots \ldots \ldots$ P288, P303, P309

Chung, P.W $\ldots \ldots \ldots \ldots \ldots \ldots$ P241

Ciammola, A . . . . . . . . . . . . . P365

Cianchi, $\mathrm{C} \ldots \ldots \ldots \ldots$. . . . . . . . . . . . . . . .

Ciccarelli, O . . . . . . . . . O54, O60

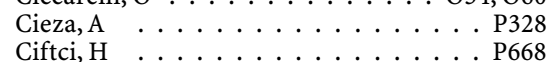

Cinova, A . . . . . . . . . . . . P792

Ciofoaia, $\mathrm{V} \ldots \ldots \ldots \ldots \ldots \ldots \ldots$ P771

Cioni, $\mathrm{C} \ldots \ldots \ldots \ldots \ldots \ldots$. . . . . 451

Cirlig, C . . . . . . . . . . . P791

Ciscato, P . . . . . . . . . . . P596

Cislaghi, G . . . . . . . . . . . . . . . . . . . P779

Citrigno, $\mathrm{f} \ldots \ldots \ldots \ldots$. . . . . O63

Ciubotaru, $\mathrm{V} \ldots \ldots \ldots \ldots \ldots \ldots$. . . P618

Ciuluvica, R . . . . . . . . . . P758

Claeys, K . . . . . . . . . . . . . . . O74

Clanet, M . . . . . . . . . . . O218

Clark, D . . . . . . . . . . . . . . . . . O96

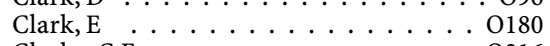

Clarke, C.E . . . . . . . . . . . . O216

Classen, J . . . . . . . . . . . . . . . . . . P324
Clatz, O . . . . . . . . . . P621 Clerget-Darpoux, F . . . . . . . . . . O O218 Clerici, F . . . . . . . . . . . P780, P782

Clerico, M . . . . O O160, P714, P719, P840 Coelho, T . . . P318, P319, P432, P436, P594 Coello, Y . . . . . . . . . . . . . P648

Coenen, M . . . . . . . . . . . . P328

Cogiamanian, $\mathrm{F} \ldots \ldots \ldots \ldots$ P231, P790

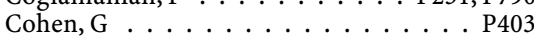

Cojocaru, I . . . . . . . . . . . O661

Colazzo, G . . . . . . . . . . . P750

Coles, A . . . . . . . . . O O192

Colesniuc, R . . . . . . . . . P421

Collado, A . . . . . . . . . . . . . P263

Collimedaglia, $\mathrm{L} \ldots \ldots \ldots \ldots$. . . O99

Collins, K . . . . . . . . . . . . P450

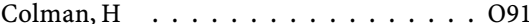

Colombo, B . . . . . . . O O99, O136, P553

Colombrita, C . . . . . . . . . . . O85

Colomer J . . . . . . . . . . P743

Colson, $\mathrm{S} \ldots \ldots \ldots \ldots \ldots \ldots$. . . P491

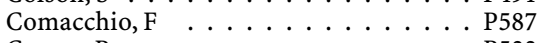

Comas, P . . . . . . . . . . . P522

Comas Bergua, P . . . . . . . . . P748

Combarros, O . . O74, O147, O164, O213, P312

Comi, C . . . . . . . . . . . O198, P580

Comi, G . . . 29, 47, O55, O57, O58, O59, O99, O136, O139, O156, O158, O189, O193, O221, P238, P256, P294,

P336, P348, P349, P441, P447, P553, P565, P702, P724, P829, P843

Comi, G.P … P272, P283, P286, P300, P463, P475, P589, P596, P598

Comola, M . . . . . . . . . . P565

Compston, $\mathrm{A} \ldots \ldots \ldots \ldots \ldots \ldots 46$

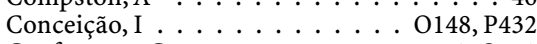

Confavreux, C ........... . 48, O159

Conforti, F.L . . . . . . . . . O63, P270

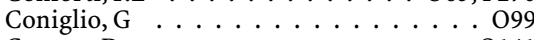

Connor, D . . . . . . . . . . O O141

Consonni, M . . . . . . . . . . . . O210

Constantinescu, A . . . . . . . . P440

Constantinescu, E . . . . . . . O661

Constantinescu, I . . . . . . . . . . . P254

Constantinescu, V . . . . . . . . P440

Constantinou, A . . . . . . . . P825

Constantoyannis, K . . . . . . . . P666, P667

Contessa, G . . . . . . . . . P714, P719, P840

Conti, G . . . . . . . . . . . P283, P682

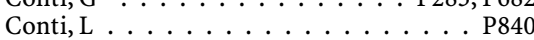

Cook, S.D . O58, O158, P320, P346, P447, P829

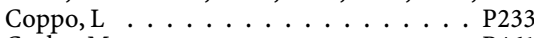

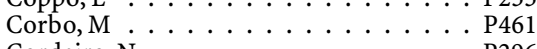

Cordeiro, $\mathrm{N} \ldots \ldots \ldots \ldots \ldots$. . . . . 296

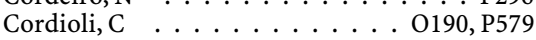

Corea, $\mathrm{F} \ldots \ldots \ldots \ldots \ldots \ldots$ P238

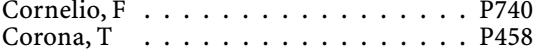

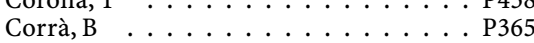

Correas Callero, $\mathrm{E} \ldots \ldots \ldots \ldots$ P703, P749

Correia, A.P . . . . . . . . . . . P513

Correia, $\mathrm{C} \ldots \ldots \ldots \ldots \ldots$ P436

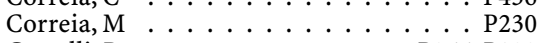

Cortelli, P . . . . . . . . . . . P266, P822

Corti, S . . . . . P283, P286, P463, P475

Cortini, F . . . . . . O O197, P580, P718, P782

Cossu, P . . . . . . . . . . . . . P695

Costa, I . . . . . . . . . . . . . P P627

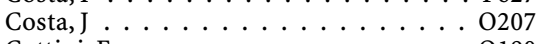

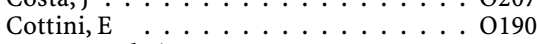

Cournu-Rebeix, I . . . . . . . . . . O O218

Coutinho, P . . . . . . . . . . . P807

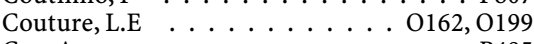

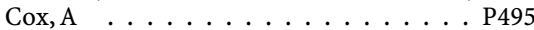

Cramer, J . . . . . . . . . . . . . P443

Crani, $\mathrm{T} \ldots \ldots \ldots \ldots \ldots \ldots$. . . . . 425

Crawford, $\mathrm{F} \ldots \ldots \ldots \ldots \ldots$. . . . . . .

Cretu, L . . . . . . . . . . . . . . . P297

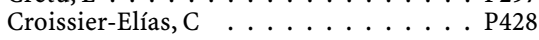




\begin{tabular}{|c|c|}
\hline rols, $\mathrm{R}$ & P537 \\
\hline Cros, D & , P432 \\
\hline Cross, A & P718 \\
\hline Crugnola, $\mathrm{V}$ & P589 \\
\hline Cruz, V & P807 \\
\hline Cruzeiro, M.M & P314 \\
\hline Csapó, K & P393 \\
\hline Csépány, T & P574 \\
\hline Csiba, L . . & P393 \\
\hline Cucci, A & P719 \\
\hline Cucurella, G & 0114 \\
\hline Cuesta, $M$ & $\mathrm{O} 203$ \\
\hline Cursi, M & P843 \\
\hline Cury, $\mathrm{H}$ & P302 \\
\hline Czaplinski, A & P741 \\
\hline
\end{tabular}

D'Agata, F . . . . . . . . . . . . . . . . O89

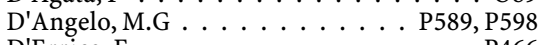
D'Errico, E . . . . . . . . . . P466 D'Onofrio, $\mathrm{H} \ldots \ldots \ldots$. . . . P343, P411 D'souza, A . . . . . . . . . . . O O131 D'Souza, M . . . . . . . . . P262 D'Amico, A . . . . . . . . . . P743 D'Arcangelo, G . . . . . . . . . . O O166 Dabos, K . . . . . . . . . . . . . P812 Dainese, F . . . . . . . . . . . . . O O152

Dakukina, $\mathrm{V} \ldots \ldots \ldots \ldots \ldots \ldots$

Dalakas, M . . . . . . . . P427, P439

Dalla Libera, $\dot{D} \ldots \ldots \ldots \ldots$ O . . . . . 136, P553

Dalla Valle, E . . . . . . . . . . . . O197

Dallari, A . . . . . . . . . . . . . . P760

Damas, P . . . . . . . . . . . . O140

Damasceno, A . . . . . . . P302

Damasio, J.C . . . . . . . . . . . . P513

Damiano, D . . . . . . . . . . P660

Danburam, A . . . O103, O132, O135, P653,

P654, P678, P685

Danek, A . . . . . O O143, O196, P566, P659

Daniels, C $\ldots \ldots \ldots \ldots \ldots$ O $\ldots 142$

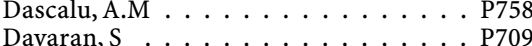

Davidescu, E.I $\ldots \ldots \ldots$ P527, P546, P652

Davidovic, M . . . . . . . . P504

Davies, P . . . . . . . . . . . . O216

Davis, Mary . . . . . . . . . . . . . P495

Davis, Matt . . . . . . . . . . . P570

Dawson, K . . . . . . . . . . P844

de Andrés, C . . . . . . . . . . . . P753

De Biase, I . . . . . . . . . . . . . . . O84

de Carvalho, M . . . . . . . . . O207

De Deyn, P. P . . . . . . . . . . . O O173

De Feo, D . . . . . . . . . . . . . P553

de Freitas, M . . . . . . . . . . . O106

de Gans, J . . . . . . . . . . . . . P P534

de Jong, B.M . . . . . . . . . . . O O144

De Jonghe, $\mathrm{P} \ldots \ldots \ldots \ldots \ldots \ldots$ O74

De Juan-Hernández, $\ldots \ldots \ldots \ldots \ldots . . .9428$

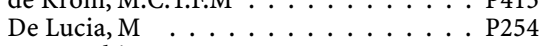

De Marchis, G.M . . . . . . . O68, 0115

de Pablo Fernández, E . . . . P609, P703, P749

De Palma, M . . . . . . . . . . . P493

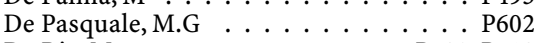

De Riz, M . . . . . . . . . . . P580, P718

De rossi, N . . . . . . . . . . . . O O190

De Santi, L . . . . . . . . . . . . P P451, P839

de Scisciolo, M . . . . . . . . O O110

De Stefano, N . . . . . . . . O O54, O186, P342

De Tommaso, M . . . . . . . . P466

de Vries, P.M . . . . . . . . . . . . . . O144

Debski, M ............ P359

Deguchi, K . . . . . . . . . . . . . O O166

Deja, M . . . . . . . . . P P809

Del Bo, R . . . . . . . . P P286, P463, P589

Del Carro, U . . . . . . . . . . . . . P387

Delbos, F . . . . . . . . . . . . . P597

Deleu, D . . . . . . . . . . . O O131

Delingette, H . . . . . . . . . . . . P621

Dell'Anno, B . . . . . . . . . . . O O110
Delmont, E . . . . . . . . . . . . P732

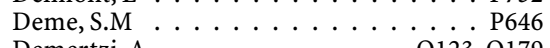

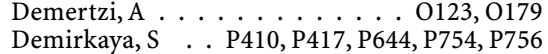

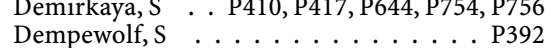

Deng, C . . . . . . . . . P427, P439

Derfuss, $\mathrm{T} \ldots \ldots \ldots \ldots \ldots$. . . . P532

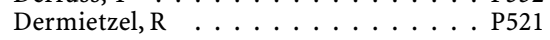

Deroma, L . . . . . . . . . . . . P460

Deschauer, M . . . . . . . . P600

Desnuelle, C . . . . . . . . O146, P491, P732

Despotovic, N . . . . . . . . . P504

Deuschl, G . . . . . . . . . . O142, P351

Deymeer, F . . . . . . . . . . . . . P257

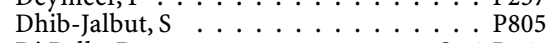

Di Bella, D . . . . . . . . . . . . . . . O86, P269

Di Donato, S . . . . . . . . . O886, P273

Di Fonzo, A . . . . . . . . . . . P272

Di Monte, E $\ldots \ldots \ldots \ldots \ldots$

Di Stasi, V . . . . . . . . . . . . . . . O71

Diaconu, N . . . . . . . . . . . . P634

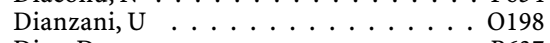

Diaz, D . . . . . . . . . . . . P637

Diaz-Otero, F . . . . . . . . . . O O168

Dickson, M . . . . . P321, P717

Dieterich, M . . . O122, O137, O175, P249

Dietis, A . . . . . . . . . . P545

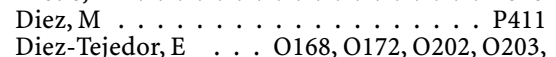

Diez-Tejedor, E $\ldots$ O168, O172, O202, O203,
P370, P637, P815

Dikka, J . . . . . . . . . . . . O103, P678

Dilena, R . . . . . . . . . . . . . . . . P799

Dimas, S . . . . . . . . . . . . P429

Dimitraka, M . . . . . . . P360

Divani, A.A $\ldots \ldots \ldots$ P320, P363, P762

Djukic, P . . . . . . . . . . . . . P734

Dobricic, V . . . . . . . . . . P415

Dobson-Stone, C . . . . . . . . O O143

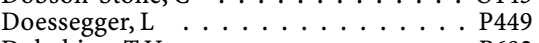

Dokokina, T.V . . . . . . . . . . . . P692

Dominguez-Gonzalez, C . . . . . . . . P609

Domínguez-Perles, R . . . . . . . . . O O147

Donadio, V . . . . . O O71, P266, P563, P822

Donadoni, C . . . . . . . P283, P286, P475

Donaghy, C . . . . . . . . . O O208

Dondelinger, $\mathrm{R} \quad \ldots \ldots \ldots \ldots$ O $\ldots 179$

Dong, K . . . . . P242, P551, P664, P776

Dönmez, B . . . . . . . . . . . . . . . O109

Donofrio, P . . . . . . . . . . . . P427, P439

Dorobat, B . . . . . . . . . . P555

Dos Santos, L.H.C . . . . . . . . . . P293

dos Santos, V . . . . . . . . . . O106

Dozio, E . . . . . . . . . . . P461

Dragomir, D . . . . . . . . . P543

Drakatos, K . . . . . . . . . P454

Dran, G . . . . . . . . . . . . . . P612

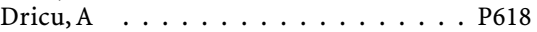

Drigo, D . . . . . . . . . . . P460

Driver-Dunckley, E $\ldots \ldots \ldots \ldots$

Dropko, P . . . . . . . . . . . . . P684

Drory, $\mathrm{V} \ldots \ldots \ldots \ldots \ldots$. . . . P473

Drukarch, B . . . . . . . . P844

Drulovic, J . . . . . . . . . . . . . . . P327

Duarte, J . . . . . . . . . . P343

Duarte, $S \ldots \ldots \ldots \ldots \ldots$. . . . O . . . . . O

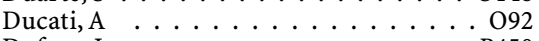

Dufour, I . . . . . . . . . P450

Dujmovic-Basuroski, I . . . . . . . . . P327

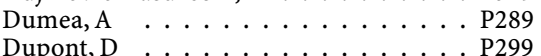

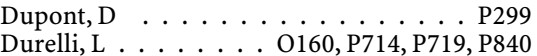

Durmus, $\mathrm{H} \ldots \ldots \ldots \ldots \ldots$. . . . . . . . . 5599, P871

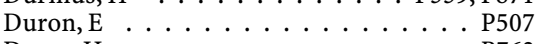

Dussa, $\mathrm{H} \ldots \ldots \ldots \ldots$. . . . P762

Duyar, $\mathrm{H} \ldots \ldots \ldots$. . . . . P449

Ealing, J . . . . . . . . . P701

Eastman, E . . . . . . . . . . . P322

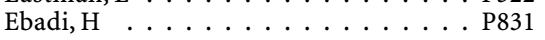

Eberle, $\mathrm{T} \ldots \ldots \ldots \ldots \ldots$. . . . O 125

Ebers, ${ }_{\text {G }} \ldots \ldots \ldots \ldots \ldots \ldots$ P716

Ebner, F.H . . . . . . . . . . O O154

Ebrahimi, A . . . . . . . . P433, P557

Ebrahimi, $\mathrm{H} \ldots \ldots \ldots \ldots \ldots$. . . . . .

Eckhardt-Henn, A . . . . . . . . . O O175

Edan, G . . . . . . . . . O188, O218, P576, P723, P824, P846

Eggers, C . . . . . . . . . . . P540

Eggleton, P . . . . . . . . . . . P330

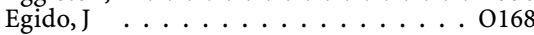

Egli, $G \ldots \ldots \ldots \ldots \ldots \ldots$ P875

Ehlers, J . . . . . . . . . . . . P502

Ehrlich, H.J . . . . . . . . . . P778

El Assawy, N . . . . . . . . . . . . . P259

El Euch-Fayache, G . . . . . . . P274, P374

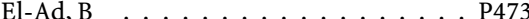

El-Ganzoury, A . . . . . . . . P486

El-khayat, N . . . . . . . . . . . . P362

El-Nahas, $N \ldots \ldots \ldots \ldots \ldots$. . . . . . 4866

Elam, M . . . . . . . . . . P266

Eldabe, $S \ldots \ldots \ldots \ldots$ O121, P852, P860

Eleftheriou, I . . . . . . . . . . . . P825

Elias, W . . . . . . . . . . . P329

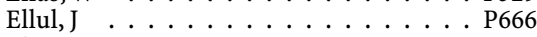

Elmaci, I $\ldots \ldots \ldots \ldots \ldots$

Elovaara, I . . . . . . . . . . P729

Emdin, $\mathrm{M} \ldots \ldots \ldots \ldots \ldots$. . . . . 595

Emre, $M \quad \ldots \ldots \ldots \ldots \ldots \ldots$ P789

Emsley, H.C.A . . . . . . . . . . P414

Endemann, E . . . . . . . . . P610

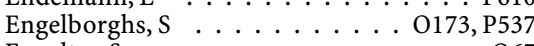

Engelter, S . . . . . . . . . . . . . . . . . . . O667

Enzevaie, A . . . . . . . . . . P845

Enzinger, $\mathrm{\ldots} \ldots \ldots \ldots \ldots$. $\ldots \ldots_{4}$

Epure, D . . . . . . . . . . . . . . . P297

Eraksoy, M . . . . . . . O226, P559, P842, P871

Eren, A . . . . . . . . . . . P P645

Erimaki, $\ldots \ldots \ldots \ldots \ldots \ldots$ P772

Eroglu, E . . . . . . . . . . . P644, P755

Ertas, M . . . . . . . . . P871

Escada, T.M $\ldots \ldots \ldots \ldots \ldots$ P315, P858

Esmaeeli Djavid, G . . . . . . . . P687

Esposito, F . . . . . . . . . . . O99, P702

Etemadifar, M . . . . . . . P320, P638

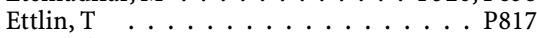

Evangelista, T . . . . . O O148, P591, P704

Evangelopoulos, M . . . . . . . . P830

Evdokimidis, I . . . . . . . . P400, P556

Evidente, V.G . . . . . . . . . . . . . P399, P800

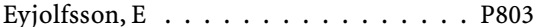

Eymard, B . . . . . . . . . . . . P597

Ezpeleta, D . . . . . . . . . . . P753

Faber, A . . . . . . . . . . . O196, P659

Faber, C.G $\ldots \ldots \ldots \ldots \ldots$

Fabriciova, K . . . . . . . . . . P743

Fabrizi, G.M . . . . . . . . . O O75, P422

Fael, W . . . . . . . . . . . . . P311

Faggiano, $\mathrm{F} \ldots \ldots \ldots \ldots \ldots$

Faglioni, P . . . . . . . . . P493

Fahmy, N . . . . . . . . . . P362

Faist, $M \ldots \ldots \ldots \ldots \ldots \ldots \ldots$

Falautano, M . . . . . . . . . P348

Falcão, F . . . . . . . . . . . . . P877

Falini, A . . . . O O181, P336, P349, P387,

P441, P553, P887

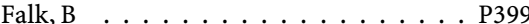

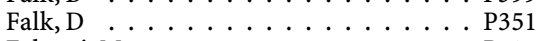

Falorni, $\mathrm{M} \ldots \ldots \ldots \ldots \ldots \ldots \ldots$. $\ldots \ldots 595$

Falsini, C . . . . . . . . . P479

Falup-Pecurariu, C . . . . . . . P878

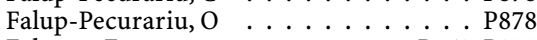

Falzone, F . . . . . . . . . . . . P563, P822

Fancellu, R . . . . . O O86, P268, P269, P281

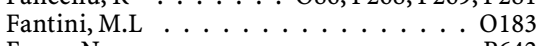

Farag, N $\ldots \ldots \ldots \ldots \ldots \ldots \ldots$

Farina, $\mathrm{f} \ldots \ldots \ldots \ldots \ldots \ldots$. . . . . . . . . . .

Farnikova, $\mathrm{K} \ldots \ldots \ldots \ldots \ldots$ P404 
Farra, $\mathrm{N} \ldots \ldots \ldots \ldots \ldots \ldots$. . . . O 145

Farrell, $M \ldots \ldots \ldots \ldots$. . . . . . . . . . .

Farrell, R . . . . . . . . . . . O96

Fassone, E . . . . . P P272, P283, P300

Fast, $\mathrm{K} \ldots \ldots \ldots \ldots \ldots \ldots \ldots \ldots \ldots \ldots$
Fatehi, $\ldots \ldots \ldots \ldots$

Fauchoux, $\ldots \ldots \ldots \ldots \ldots \ldots \ldots \ldots$ O $\ldots \ldots \ldots$

Faustmann, $\mathrm{P} \ldots \ldots \ldots \ldots \ldots$ P521

Favaretto, A . . . . . . . O O187, O220

Fazekas, F . . . . . . . P342, P456

Fazio, R . . . . . . . . . . . . P387

Fechir, M . . . . . . . O122, O125, P855

Federico, S . . . . . . . . . . P579

Fedorenko, D . . . . . . . . P731

Fei, G . . . . . . . . . . . . . P793

Fekete, $\mathrm{K} \ldots \ldots \ldots \ldots \ldots$. . . . P366

Feldman, $\mathrm{H} \ldots \ldots \ldots \ldots \ldots 27$

Felix-Torres, $\mathrm{V} \ldots \ldots \ldots \ldots$. $\ldots$ P502

Fenoglio, C . . . . . . O O197, O198, P580,

P718, P779, P782

Fereshtehnejad, S.M . . . . . . . . P P P71

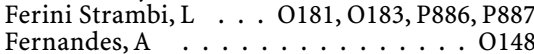

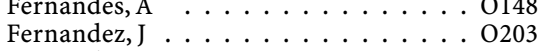

Fernandez, O . . . . O O150, O151, P344

Fernández, P . . . . . . . . . . . . . P753

Fernandez Dominguez, J . . . . . P P705, P814

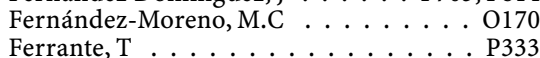

Ferrari, S . . . . . . . . . . P565

Ferrazza, P . . . . . . . . . . P575

Ferre, J.-C $\ldots \ldots \ldots \ldots \ldots$ P824, P846

Ferrer $\mathrm{T} \ldots \ldots \ldots \ldots \ldots \ldots$. . . . . . . . . . . . .

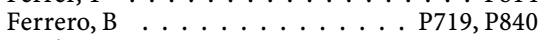

Ferri, R . . . . . . . . . O O183, P886

Ferrucci, R . . . . . . . . P231, P790, P799

Fetea, $\mathrm{S} \ldots \ldots \ldots \ldots \ldots \ldots$. . . . . . . 618

Fexi, $\mathrm{P} \ldots \ldots \ldots \ldots \ldots \ldots \ldots$ P812

Filaut, S . . . . . . . . . . . P597

Filipov, V . . . . . . . O58, O158, P447

Filippi, $M \ldots$ O54, O55, O57, O58, O59, O156, O158, O186, O187, O189, O193, O209, O220, O221, P294, P336, P337, P342, P348, P349, P441, P447, P452, P465, P724

Filipponi, D . . . . . . . P411

Fincher, $\mathrm{C} \ldots \ldots \ldots \ldots \ldots \ldots \ldots \ldots \ldots \ldots \ldots \ldots$

Findling, $O \ldots \ldots \ldots \ldots$ P726, P817

Finkelsztejn, A . . . . . . . . . P502

Firooznia, K . . . . . . . . . P845

Firtina, B . . . . . . . . . . . . P644

Fisniku, L $\ldots \ldots \ldots \ldots \ldots$ O96, 0186

Fieldstad, C . . . . . . . . . P443, P573

Flachenecker, P . . . . . . . . . P329

Fleury, A $\ldots \ldots \ldots \ldots \ldots$ O $\ldots \ldots 4$, P526

Flores, J.M … . . . P P458, P752, P820

Florio, C . . . . . . . . . . . P333

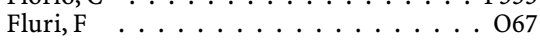

Focke, A $\ldots \ldots \ldots \ldots \ldots \ldots$ P823

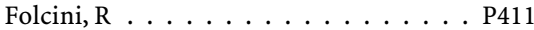

Fondel, R . . . . . . . . . O O122

Fong, G.C.Y $\ldots \ldots \ldots \ldots \ldots$ P P P P P

Fonseca, A.C . . . . . P P520, P704, P877

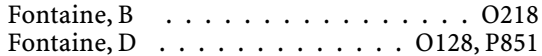

Foreid, $\mathrm{H} \ldots \ldots \ldots \ldots$ P520, P591, P704

Forkel, $\mathrm{S} \ldots \ldots \ldots \ldots \ldots \ldots$. . P566

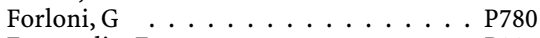

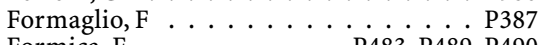

Formica, F . . . . . . P483, P489, P490

Foroglou, $\mathrm{N} \ldots \ldots \ldots \ldots \ldots$. . . P607

Fortunato, F $\ldots \ldots \ldots \ldots$ P286, P475, P589

Fossi, S . . . . . . . . . . . P379

Fotiou, D . . . . . . . . . . . P383

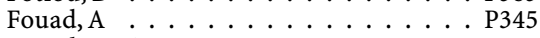

Fouad, B.E.z $\ldots \ldots \ldots \ldots \ldots$ P642

Foulds, P . . . . . . . . . . P455

Fouquet, $\mathrm{W} \ldots \ldots \ldots \ldots \ldots$ P802
Fournier-Mehouas, M . . . . . . . . P491

França-Jr, M . . . . . . . . . . . . . . P302

Francis, G . . . . . . . . . . . . O102

Franco, A . . . . . . . . . . . P567

Frank, G . . . . . . . . . . . . P783

Frank, $S \ldots \ldots \ldots \ldots \ldots \ldots$. . . . P310

Franssen, $\mathrm{H} \ldots \ldots \ldots \ldots$ O73, O206, P426

Franzini, M $\ldots \ldots \ldots \ldots \ldots$. . . . . . .

Frasineanu, A . . . . . . . . . . . . . . . . O661

Frederiksen, C . . . . . . . . . . . . P573

Freedman, M . . . . . . O188, P576, P723

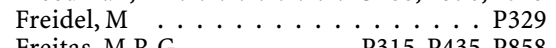

Freitas, M.R.G . . . . . . P315, P435, P858

Frenay, M . . . . . . . . . . O O95

Fricska-Nagy, $z \ldots \ldots \ldots \ldots \ldots \ldots$

Friedman, H . . . . . . . . . . . . . . . . P707

Friedmann, A . . . . . . . . . P639

Frigeri, A . . . . . . . . . . . . P715

Frih-Ayed, $\ldots \ldots \ldots \ldots \ldots$ P623, P873

Frijns, C.J.M . . . . . . . . . . . O64

Fruguglietti, M.E $\ldots$ O209, P463, P465, P598

Fuentes, B . . . . O O168, O172, O202, O203,

P370, P637, P815

Fueyo, J . . . . . . . . . . . . . . O O91, P615

Fuglsang-Frederiksen, A $\ldots \ldots \ldots \ldots$. . . . P260

Fugmann, E.A . . . . . . . . . . . . . P316

Fühlhuber, $\mathrm{V} \ldots \ldots \ldots \ldots \ldots$. . . . O225

Fuhr, P . . . . . . . . P262, P310, P438

Fujioka, M . . . . . . . P339, P630, P631

Fujiwara, K . . . . . . . . . . . . . P599

Fumagalli, F . . . . . . . . . . . P238

Fumagalli, $M \ldots \ldots \ldots \ldots$. . . . . . P790, P799

Furdea, A . . . . . . . . . . . . . 4

Fürholzer, $\mathrm{W} \ldots \ldots \ldots \ldots \ldots$. . . P480

Furlan, M . . . . . . . . . . . P639

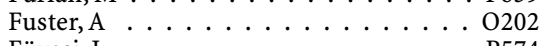

Füvesi, J . . . . . . . . . . . . . P574

Fuzibet, J . . . . . . . . . . . . P732

Gabaldon Torres, L . . . . . . P279, P705, P814

Gabibov, A . . . . . . . . . . . . P774

Gabova, A $\ldots \ldots \ldots \ldots \ldots$

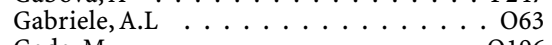

Gade, M . . . . . . 0196

Gagliardo, A . . . . . . . . . . P379, P479

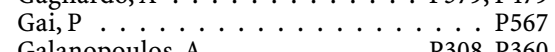

Galanopoulos, A . . . . . . . P308, P360

Galantucci, S . . . . . . . . . . . . . P238

Galassi, G . . . . . . . . . . . . . P307, P493

Galbiati, S . . . . . . . . P P483, P489, P490

Galea, M.P . . . . . . . . . . . . . . O101

Galeote, G . . . . . . . . . . . P370

Galetta, S.L . . . . . . . . . . . . O O159

Galic, M . . . . . . . . . . . . . P577

Galimberti, D . . . . O197, O198, P580, P718,

P779, P780, P782

Gallagher, A . . . . . . . . . . . P412

Gallardo, Elena. . . . . . . . . O74, O147, P312

Gallardo, Eduard . . . . . . . . . . . . . . O147

Gallardo, M.J . . . . . . . . . . P P752, P820

Gallo, O . . . . . . . . . . . P P270

Gallo, P . . . . . . O O186, O187, O220

Galvez, A . . . . . . . . . . . . P622

Gambi, D . . . . . . . . . . . . . P786

Gammans, R . . . . . . . . . . . . . O191

Gandhi, $S \ldots \ldots \ldots \ldots$. . . . . . . . P397

Gandon, G . . . . . . . . . . . . . . P450

Ganesharajah, T . . . . . . . . . . . P397

García, A . . . . . . . . O O74, O147, P312

García-Barredo, R . . . . . . . . . . . . . O147

Garcia-Caballero, J . . . . . . . . . . . O202

García-Gorostiaga, I . . . . . . . . . O213

Garcia-Lorenzo, D . . . . . . . . . . . . P824

Garrote, J.A . . . . . . . . . . . . P572

Gasco, M . . . . . . . . . . . . . . P259

Gasos, A . . . . . . . . . . . . . . P522

Gass, A . . . . . . . . . . . . . . . O 54 , P342, P449

Gassner, A . . . . . . . . . . . . P482

Gasverde, S . . . . . . . . . . . . P567
Gatti, R . . . . . . . . . . . P337

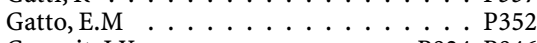

Gauvrit, J.Y . . . . . . . . . . P824, P846

Gavric, M . . . . . . . . . . . . . P327

Gavriliuc, M . . . . . . . P425, P626

Gavrilov, M . . . . . . . P501, P604

Gawehn, S . . . . . . . . . . P468

Gawron, $\mathrm{W} \ldots \ldots \ldots \ldots \ldots$

Geber, C . . . . . . . . . . . . . O122

Gebhardt, A . . . . . . . . . O214

Gebruers, $\mathrm{N} \ldots \ldots \ldots \ldots \ldots$. . . . . . . .

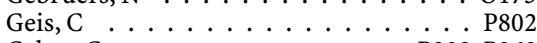

Gekas, G . . . . . . . . . . P308, P360

Gelagoti, M . . . . . . . . . . . . . P825

Gellera, C . . . . . . . . . O 885, O86, P268

Gély-Nargeot, M.C . . . . . . O O211, P477

Gemignani, $\mathrm{F} \ldots \ldots \ldots \ldots \ldots \ldots$. . . . . 422

Genc, G . . . P P410, P416, P417, P471, P472, P643, P644, P686, P708,

Genova, M . . . . . . . . . . . P450

Gentner, R . . . . . . . . . . . . . . P324

Georgakakis, G . . . . . . P P554, P863, P864

George, M.S . . . . . . . . . . . . . O O144

Georgescu, C . . . . . . . . . P250

Georgescu, D . . . . . . . . . . . . . P250

Georgescu, L.A . . . . . . . . . . P250

Geraldes, R . . . . . . . . . P520, P877

Gerazounis, $M \ldots \ldots \ldots \ldots$. . . . P613

Geringer-Manakanatas, $\mathrm{N} \ldots \ldots . . . \mathrm{P} 482$

Gerriets, T . . . . . . O O153, O200, O225

Geusens, $\mathrm{P} \ldots \ldots \ldots \ldots \ldots \ldots \ldots \ldots$

Ghabaee, $M \ldots \ldots \ldots \ldots$. . . . . . . . .

Ghaderi, A . . . . . . . . . . . . . P697

Ghaffari Poorjahromi, S . . . . . . P P697

Ghahramani, S . . . . . . . . . . . P711

Ghanaati, $\mathrm{H} \ldots \ldots \ldots \ldots$. . . . . P845

Gharegozli, K .......... P320

Ghezzi, A ‥ O55, O99, O156, P294, P715, P840

Ghezzi, S . . . . . . . . . . . . P463, P589

Ghidini, C . . . . . . . . . . . . . P579

Ghiglione, P . . . . . . . P464, P474

Ghione, I $\ldots \ldots \ldots \ldots \ldots \ldots \ldots \ldots$ P682

Gholamzadeh, S . . . . . . . . . P264

Ghorbani, A . . . . . . . . . P856

Giacino, J . . . . . . O $112,0113,0155$

Giacone, S . . . . . . . . . . . P474

Giaglis, G . . . . . . . . . . . . . . P811

Gianinazzi, $\mathrm{C} \ldots \ldots \ldots \ldots \ldots \ldots$. . . . . . . .

Giannikopoulos, $\mathrm{G} \ldots \ldots \ldots$. . . . P812

Gibbson, $\mathrm{V} \ldots \ldots \ldots \ldots \ldots$. . . . . P495

Gibson, J.M $\ldots \ldots \ldots \ldots \ldots$

Gibson, L.M $\ldots \ldots \ldots \ldots \ldots \ldots$

Gigli, G.L . . . . . . . . . . O O152, P460

Gil, A . . . . . . . . . . . O202

Gil-Nuñez, A . . . . . . . . . . . . O O168

Gimenez, T . . . . . . . . . . . . P622

Gimeno, E . . . . . . . . . . . . . . P622

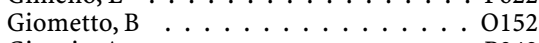

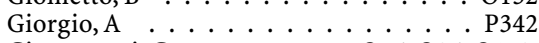

Giovannoni, G . . . . . . . O56, O96, O159,

P325, P720, P829

Giralt, E . . . . . . . . . . . O114, P622

Giraud, A.L . . . . . . . . . . O O163

Girolami, F . . . . . . . . . P307, P493

Gironi, $M \ldots \ldots \ldots \ldots \ldots \ldots$. . . . . . .

Gitman, A . . . . . . . . . . O661

Giuliani, A . . . . . . . . . . . P563, P822

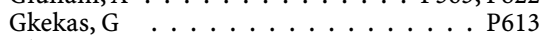

Glon, $\mathrm{C} \ldots \ldots \ldots \ldots \ldots \ldots \ldots \ldots$

Gnezditski, V . . . . . . . . . P247, P248

Goate, A.M . . . . . . . . . . . . O197

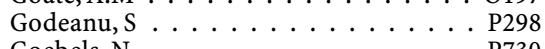

Goebels, N . . . . . . . . . . . P730

Goelz, S.E . . . . . . . . . . . . . P844

Gokcil, Z . . . . . . P410, P416, P417, P686

Gokyigit, A . . . . . . . . . . . . . P539

Golba, A . . . . . . . . . . . . . P359

Gold, R . . . . . . . . . . . O193, P844 


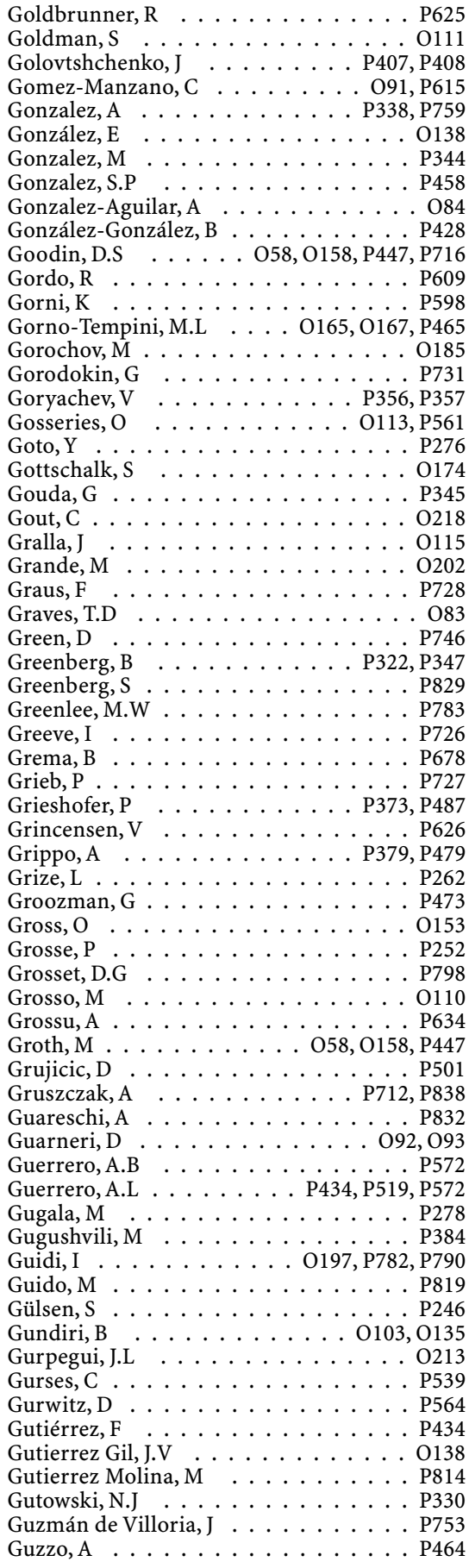

Haas, J . . . . . . . . . . . P329, P833

Haberlova, J . . . . . . . . . . . . . . . O87

Haghighat-Shoar, $M \ldots \ldots \ldots$. . . . .

Haghighi, $M \ldots \ldots \ldots \ldots \ldots \ldots$

Haghikia, A . . . . . . . . . P521

Hahn, M . . . . . . . . . . O O106

Hahnen, E $\ldots \ldots \ldots \ldots \ldots$ O $\ldots 90$, P275

Haider, C . . . . . . . . . . . . P482

Haimov, I . . . . . . . . . . . . . . . O184

Hajebrahimi, Z . . . . . . . P284

Hajeer, A . . . . . . . . . . P736

Hajghasemi, F . . . . . . . . . P638

Hakiki, B . . . . . . . . . . P715
Halacoglu, F . . . . . . . . . . . P372

Halatek, T . . . . . . . . . . . . . . P253

Halder $S \ldots \ldots \ldots \ldots \ldots$

Haliba, Y . . . . . . . . . . . . . O O184

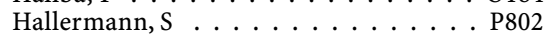

Hamamcioglu, K . . . . . . . . . P757

Han, J.H . . . . . . . . . . P665, P853

Han, S-W $\ldots \ldots \ldots \ldots \ldots$

Han, S.J . . . . . . . . . . . . . . . . . P813

Han, Z.H ............. P376, P663

Hanada, T . . . . . . . . . O133, P706

Hanagasi, $\mathrm{H} \ldots \ldots \ldots \ldots \ldots$. . . . . . . . . . . . . . .

Haneef, Z . . . . . . . . . . . . . . O201, P237

Hanna, K . . . . . . . . . . P427, P439

Hanna, M.G . . . . . . . . . . . . O83

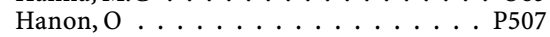

Happe $S$. . . . . . . . . . . . . P392

Hardiman, $\mathrm{O} \ldots \ldots \ldots \ldots \ldots$. . . . . . O208

Harigaya, Y . . . . . . . . . . . . P354

Harirchian, M.H . . . . . . . . . . P845

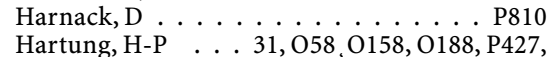

Hartung, H-P . . 31, O58, O158, O188, P427,

Haschke-Becher, E . . . . . . . . P847

Hasegawa, Y . . . . . . . . . . . . . . P267

Hasenauer, G . . . . . . . . . . . . . . . P482

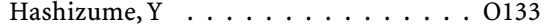

Hatakeyama, S . . . . . . . . . . . P706

Hattingen, E . . . . . . . . . . . P P468

Hauke, J . . . . . . . . . . . . O990, P275

Havranek, $\mathrm{P} \ldots \ldots \ldots \ldots$. . . . . P768

Havrdova, E . . . . . . . O O159, O193

Haybäck, J . . . . . . . . . . . . . . O180

Hebebrand, J . . . . . . . . . . . . . O O66

Heckenberg, S.G.B . . . . . . . . . . P534

Heckmann, M . . . . . . . . . . P802

Heeney, A . . . . . . . . . . . . P745

Heide, $W \ldots \ldots \ldots \ldots \ldots$ O178, P747

Helle, $M \ldots \ldots \ldots \ldots \ldots \ldots \ldots$. . . . . . . . . .

Helmchen, C . . . . O124, O174, P747, P862

Hemmer, B . . . . . . . . . . . . O O157, O188

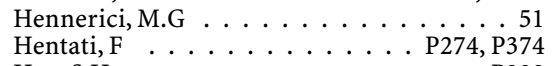

Heo, S.H . . . . . . . . . . . P229

$\begin{array}{lllllll}\text { Herczegfalvi, A } & \ldots & \ldots & \ldots & \ldots & \ldots & \text { P743 } \\ \text { Hermsdörfer, J } & \ldots & \ldots & \ldots & \ldots & \ldots & \text { P480 }\end{array}$

Hernández, A . . . . . . . . . . P752, P820

Hernández, E . . . . . . . . . . . P P434

Hernández Gallego, J . . . . . . . . . . . P749

Herrero San Martín, A … . . P703, P749

Herreros Rodríguez, J . . . . . . . P703, P749

Herscovici, S . . . . . . . . . . . . . O O185

Herzig, R . . . . . . . . P488, P768, P784, P834

Herzog, J . . . . . . . . . . . . P351

Hestvik, A . . . . . . . . . . . O222

Hetman, M . . . . . . . . . P470

Hetzel, A . . . . . . . . . . . . . . . O O119

Hildebrandt, J . . . . . . . . . . . . P625

Hilker, R . . . . . . . . . . . . . . . . O O142, P468

HIllman, $\mathrm{R} \quad \ldots \ldots \ldots \ldots \ldots$

Hinkerohe, D . . . . . . . . . . P521

Hinson, V.K . . . . . . . . . . . . . O144

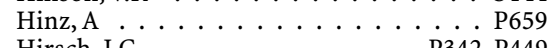

Hirsch, J.G . . . . . . . . . . . P342, P449

Hladíková, M . . . . . . . . . P459, P620

Hluštík, P . . . . . P355, P488, P784, P834

Ho, S.L . . . . . . . . . . . . P836, P837

Hochmeister, S . . . . . . . O O145, P456

Hodapp, M . . . . . . . . . . . . . . P388

Hoellen, $\mathrm{F} \ldots \ldots \ldots \ldots \ldots \ldots \ldots \ldots$ P743

Hoffmann, S . . . . . . . . . . . O O134

Hofmann, C . . . . . . . . . P862

Hofmann, K.-T $\quad \ldots \ldots \ldots \ldots \ldots$. . P810

Hohlfeld, R . . . . . . . . . . . . 32

Holle, D.N . . . . . . . . . . . P700

Holley, J.E . . . . . . . . . . . P P330

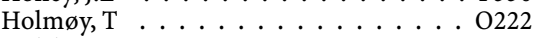

Holsboer, F . . . . . . . . . . O218

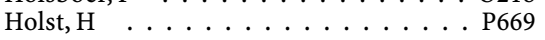

Holton, J.L . . . . . . . . . . . P397

Hong, S.C $\ldots \ldots \ldots \ldots \ldots \ldots$. P240

Hong, Z . . . . . . . . . . . . . P P650

Honoré, J . . . . . . . . . . . O O194, P648

Honti, V . . . . . . . . . . . P574

Hori, K . . . . . . . . . . . . . P267

Horn-Bochtler, A . . . . . . . . . P532

Horozoglu, $\mathrm{H} \ldots \ldots \ldots \ldots \ldots$. . . . . . .

Horrocks, I . . . . . . . . . . . . . . . . . P296

Hort, J . . . . . . . . . . . P509, P512

Horta, I . . . . . . . . . . . P429

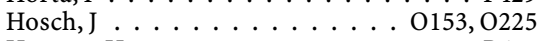

Houzen, H . . . . . . . . . . . P657

Hreha, S . . . . . . . . . . . P453, P583

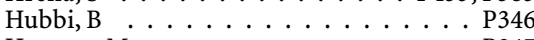

Huemer, $\mathrm{M} \ldots \ldots \ldots \ldots$. . . P847

Hüfner, $K \ldots \ldots \ldots \ldots \ldots \ldots$. . . . . . . . . . . . . . . .

Hughes, R . . . . . . . . . P P427, P439

Hühne, K . . . . . . . . . . . . . . O O87

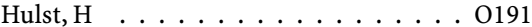

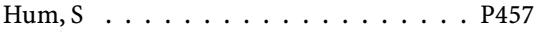

Hutchinson, $M \ldots \ldots$ O159, 0177

Hwang, J.H . . . . . . . . . . . . P288

Hwang, S-H . . . . . . . . . . . . P797

Hwang, S.J . . . . . P288, P303, P309, P317

Hwang, Y . . . . . . . . . . . . . . P775

Hyde, R . . . . . . O O159, P455, P584, P848

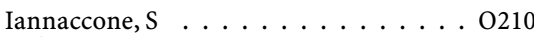

Iarlori, C . . . . . . . . . . . P P786

Ibrahim, Ahmed . . . . . . . . . . . . P345

Ibrahim, Abdullahi . . . . . . . . . O880, P640

Ibrahim, G . . . . . . . . . . . . . P680

Ickenstein, G.W . . . P P39, P630, P631, P636

Içöz, S . . . . . . . . . . . P P559, P624, P871

Iglesias, $\mathrm{F} \ldots \ldots \ldots \ldots \ldots \ldots \ldots$

Ignjatovic, $\mathrm{P} \ldots \ldots \ldots \ldots \ldots \ldots$. . . . . . . . . . .

Iijima, M . . . . . . . . . . . . . P402

Ilic, T . . . . . . . . . . . . . . . P P499

Iliescu, C . . . . . . . . . . . . . P289

Illa, I . . . . . . . . . . . . . . . . . O O147

Illarioshkin, ${ }^{\prime} \ldots \ldots \ldots \ldots \ldots \ldots$ P287

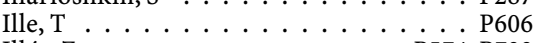

Illés $\mathrm{Z} \ldots \ldots \ldots \ldots$. . . . . . P574, P733

Imai, $N \ldots \ldots \ldots \ldots \ldots$ P234

Imai, $\mathrm{Y} \ldots \ldots \ldots \ldots \ldots \ldots \ldots$

Imberti, L . . . . . . . . . . P579

Imrie, J . . . . . . . . . . . . . . . P295

Inácio, N.M $\ldots \ldots \ldots \ldots \ldots$. . . . O 107

Infante, J . . . . . . . . . O 164, O213, P312

Inoue, $\mathrm{K} \ldots \ldots \ldots$. . . . . . . O166, P276

Inoue, $\mathrm{N} \ldots \ldots \ldots \ldots \ldots \ldots$ P276

Inshasi, $J \ldots \ldots \ldots \ldots \ldots$ P236, P245

Inuggi, A . . . . . . . . . . O O139, P256

Inzitari, $D \ldots \ldots \ldots \ldots \ldots$. . . . . . . . . . .

Ionova, $\mathrm{T} \ldots \ldots \ldots \ldots \ldots \ldots$ P731

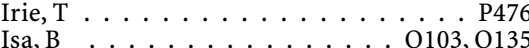

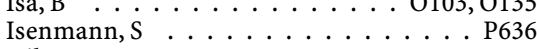

Isik, $\ldots \ldots \ldots$ P498, P658, P668,

. . . . . . . . P673, P770, P816

Ising, $M \ldots \ldots \ldots \ldots \ldots \ldots$ O218

Ito, $K_{\ldots} \ldots \ldots \ldots \ldots \ldots \ldots \ldots \ldots$

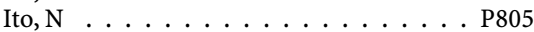

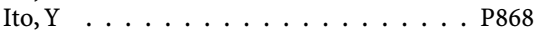

Ittehadi, $\mathrm{H} \ldots \ldots \ldots \ldots \ldots \ldots \ldots$

Iudice, $A \ldots \ldots \ldots \ldots \ldots$

Iuliano, $\mathrm{G} \ldots \ldots \ldots \ldots \ldots$. . . . . . . . . . . . . . . . . . .

Ivanov, $\mathrm{R} \ldots \ldots \ldots \ldots \ldots$. . . . . . . . . . . .

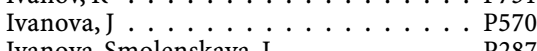

Iwata, M . . . . . . P402, P406, P430, P431

Jabeen, S.A $\ldots \ldots \ldots \ldots \ldots \ldots$. . . P675

Jablonka, S . . . . . . . . . . P802

Jacques, L … . . . . . . . . . . . O O127

Jafarian, $\mathrm{S} \ldots \ldots \ldots \ldots \ldots \ldots$

Jahanshahi, A . . . . . . . . . . P874

Jain, D.C . . . . . . . . . . . P420 


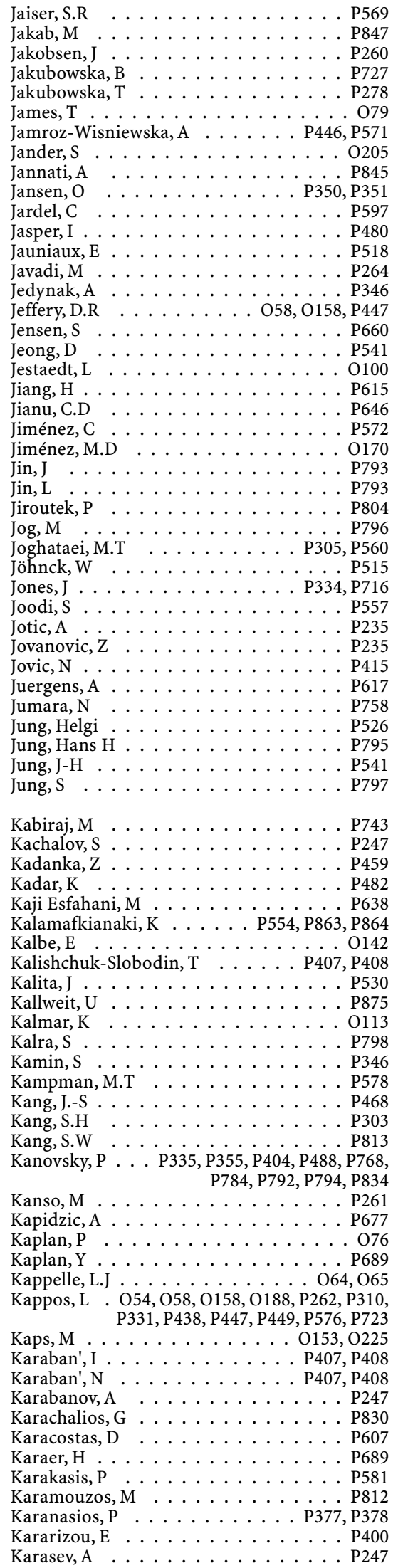

Karasu, A . $\ldots \ldots \ldots$

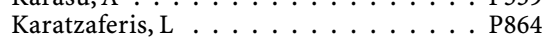

Karkavelas, G . . . . . . . . . . . P607

Karkherian, S . . . . . . . . . . . P320

Karlovasitou, A . . . . . . . . . . . . P383

Karnezis, T . . . . . . . . . . O O157

Karpova, E . . . . . . . . . . . . . . . P287

Karsidag, S . . . . . . . . . . . . . . . . . P645

Kartashov, A . . . . . . . . . . . . P731

Karydas, A . . . . . . . . . . . . . . . O O165

Kasahata, N . . . . . . . . . P788

Kastrup, O . . . . . . . . . . . . . P700

Katan, M . . . . . . . . . . . . . O O67

Katayama, T . . . . . . . . . . P599

Katzberg, H . . . . . . . . . . . . . . P439

Kaufmann, $\mathrm{H} \ldots \ldots \ldots \ldots 138$

Kaussner, Y . . . . . . . . . . . . . O134

Kauwe, J.S.K . . . . . . . . . . . . O O 197

Kawamoto, K . . . . . . . P339, P630, P631

Kay, C.S . . . . . . . . . . . P293, P316

Kaya, D . . . . . . . . . . . . . P871

Kaya, M . . . . . . . . . . . P539

Kaya, Y . . . . . . . . . . . . P246, P503

Kayserili, H . . . . . . . . . . . P872

Kazibutowska, $z \ldots \ldots \ldots \ldots$

Keckarevic, D . . . . . . . . . . . P P313, P415

Keckarevic-Markovic, M . . . . . P313, P415

Kecmanovic, M . . . . . . . . P313, P415

Kefalopoulou, Z . . . . . . . . . . . P666, P667

Kefi, M . . . . . . . . . . . P274

Keir, G . . . . . . . . . . . . . . P857

Kell, C.A . . . . . . . . . . . . . . . . . . . . O163

Kellam, P . . . . . . . . . . . . O219

Keller, M . . . . . . . . . . . . . . O180

Keller, O . . . . . . . . . . . . . . . . . O887

Kennelly, $\mathrm{s} \ldots \ldots \ldots \ldots \ldots \ldots$. . . . . . . .

Kenntner-Mabiala, R . . . . . . . . . . . O134

Kenny, R.A . . . . . . . . . . . . . . . P516

Kerr, D . . . . . . . . . . . . . . . . . . . . . P282

Kerr, R.S.C . . . . . . . . . . . . O662

Kersten, B . . . . . . . . . . . . . O214

Kesselring, J . . . . . . . . . . . . . . . P328

Khachidze, I . . . . . . . . . . . . . . . P384

Khairallah, M . . . . . . . . . P623

Khaleeli, Z . . . . . . . . . . O60, O96

Khalil, S . . . . . . . . . . . . P777

Khan, A . . . . . . . . . . . . O219

Khan, O . . . . . . . . . . . P453, P583

Khandaghi, R . . . . . . . . . P709

Khani, A . . . . . . . . . . . . . . . . . . . P709

Khashayar, P . . . . . . . . . . P769

Khatami, R . . . . . . P876, P882, P883

Khodapanahandeh, F . . . . . . . . . . . O228

Khoma, A . . . . . . . . . P356, P357

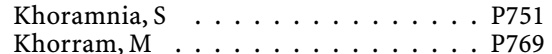

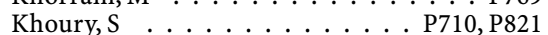

Khurana, D . . . . . . . . . . . P367

Kilinc Toprak, M . . . . . . . . . . . . P503

Kilpatrick, T.J . . . . . . . . . . . . . O101

Kim, B-J . . . . . . . . . . . . . . P494

Kim, B.S . . . . . . . P340, P341, P418

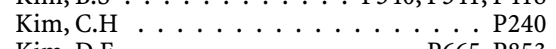

Kim, D.E . . . . . . . . . . . . P665, P853

Kim, D.G $\ldots \ldots \ldots \ldots \ldots$

Kim, G.S . . . . . . . . . . . . P304

Kim, Hyun Sook. . . . . . . P304, P375, P691

Kim, H.T . . . . . . . . . P671, P764

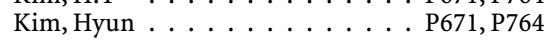

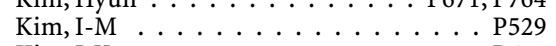

Kim, J-Y . . . . . . . . . . . P670

Kim, Jee . . . . . . . . . . . . . . . . . . P854

Kim, Juhan . . . . . . . . . . . . . P671, P764

Kim, K.H . . . . . . . . . . . . . . . P317

Kim, K.M . . . . . . . . . . . . . P735

Kim, Min Jung . . . . . . . . . . . . P735

Kim, Min-Jeong. . . . . . . . . . . . . . P258

Kim, M.K . . . . . . . . . . . . . . . . . . P229

Kim, N-H . . . . . . . . . . . . . . P647
Kim, O.G . . . . . . . . . P735

Kim, O.J $\ldots \ldots \ldots \ldots \ldots \ldots \ldots$ P375

Kim, $\mathrm{R} \ldots \ldots \ldots \ldots \ldots$. . . . . 848

Kim, S.B . . . . . . . . . . P303

Kim, Sung-Hoon . . . . . . . . . P341, P735

Kim, Su-Hyun. . . . . . . . . . . . . . . P510

Kim, Seong-Hoon. . . . . . . . . . . . P418

Kim, Soyeon. . . . . . . . . . . . . . . P346

Kim, Seung . . . . . . . . . . . P671, P764

Kim, Seung Hyun . . . . . . . . . . . . . . . P229

Kim, Sang Ho . . . . . . . . . . . . P691

Kim, Seo Hyun . . . . . . . . . . . . . P304

Kim, Sung Hun . . . . . . . . . . . . . P647

Kim, S.J . . . . . . . . . . . . P735

Kim, S.M $\ldots \ldots \ldots \ldots$ P524, P608

Kim, S.S . . . . . . . . . . P647

Kim, Tae-Won. . . . . . . . . . . . . . P340

Kim, Tae-Woong . . . . . . . . . P P541

Kim, W . . . . . . . . . . . . . . P375

Kim, Yoon . . . . . . . . . . . . . . P764

Kim, Y.B . . . . . . . . . . . P241

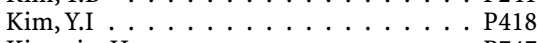

Kimmig, H . . . . . . . . . . P747

Kimura, T . . . . . . . . . . . . P599

King, J . . . . . . . . . . . . . . . P325, P720

Kinoshita, M $\ldots \ldots \ldots \ldots \ldots \ldots$ P588

Kioulachidis, $\mathrm{K} \ldots \ldots \ldots \ldots$. . . P616

Kirawa, U . . . . . . . . . O O103, O135

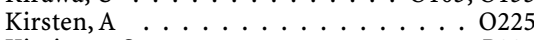

Kirzinger, $S \ldots \ldots \ldots \ldots \ldots$. . . . 3334

Kishtovich, A . . . . . . . . P731

Kitazawa, Y . . . . . . . P P39, P630, P631

Kitchener, N . . . . . . . . . . . O82, O227, P642

Klapczinski, F . . . . . . . . . . . P870

Klatt, J . . . . . . . . . . . . . . . . O O134

Kleinschnitz, C . . . . . . . . O204

Klisch, C . . . . . . . . . . . . P388

Klisch, J . . . . . . . . . . . . . . . P636

Klockenbrink, $\mathrm{D} \ldots \ldots \ldots \ldots \ldots$. . . . . . . .

Klünemann, H.H $\ldots \ldots \ldots \ldots$. . . P783

Klupka-Saric, I . . . . . . . . . . . P577

Klyushnikov, S . . . . . . . . . . . . . P287

Knehans, A . . . . . . . . . . P443

Kneifel, N . . . . . . . . . . . . O153

Knezevic-Apostolski, S . . . . . . . . P499

Knop, M . . . . . . . . . . . . O218

Kobari, M . . . . . . . . P234

Kobayashi, H . . . . . . . . . . . . . P868

Kobayashi, M . . . . . . P430, P431

Kobel, M . . . . . . . . . . . . . . . P485

Kobierowski, S . . . . . . O171, O182, P773

Koc, G . . . . . . P P416, P471, P472, P756

Koc, $s \ldots \ldots \ldots \ldots \ldots \ldots \ldots$

Kocaoglu, M . . . . . . . . P755

Koch, C . . . . . . . . . . . . . P P350

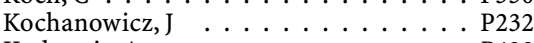

Kodounis, A . . . . . . . . . . P423

Koh, S-H . . . . . . . . . . . . P671, P764

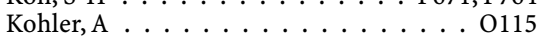

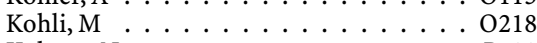

Kohora, N . . . . . . . . . . . P P588

Koistinaho, J . . . . . . . . . . P771

Koistinaho, M . . . . . . . . P771

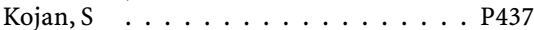

Kokotis, P . . . . . . . . . . . . P556

Kollárová, K . . . P P335, P784, P792, P834

Kölmel, H.W . . . . . . . . . . . . . P636

Komoly, S . . . . . . . . . . . . O193, P733

Kömpf, D . . . . . . . . . . . . . . P747

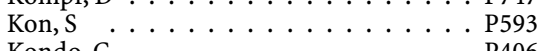

Kondziella, D . . . . . . . O81, P633, P803

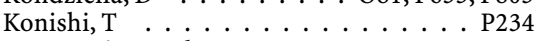

Konstantinopoulou, A . . . . . . . . . P556

Kontaxis, T . . . . . . . . . . . . . P400

Kontogiorgaki, M . . . P554, P863, P864

Konukoglu, E . . . . . . . . . . . . P621

Kooijmans-Coutinho, M . . . . . . P848

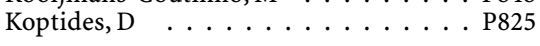




\begin{tabular}{|c|c|}
\hline y,A.P & \\
\hline$\ldots \ldots$ & O92, O93, O94 \\
\hline orteweg, $\mathrm{T}$ & aherrán, E \\
\hline alomfirescu, S & P235 \\
\hline stanjsek, $N \quad \ldots \ldots \ldots \ldots$ & mantea, $\mathrm{E}$ \\
\hline P454, P581 & nont, B \\
\hline P863 & mperti, C \\
\hline P327 & \\
\hline P386, P827 & P332 \\
\hline$\ldots \mathrm{O} 117$ & \\
\hline nos, $\mathrm{E}$ & ndmann, $\mathrm{R}$ \\
\hline alos, N f . . . . . P554, P863, P864 & $\mathrm{O} 78, \mathrm{O} 79, \mathrm{O} 224$ \\
\hline 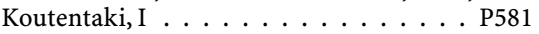 & on, $\mathrm{D}$ \\
\hline P830 & O188, P576, P723 \\
\hline P677 & $\mathrm{O} 112, \mathrm{O} 155$ \\
\hline P733 & Minet, $M \ldots \ldots$. . . \\
\hline P450 & P392 \\
\hline P533 & P270 \\
\hline P291 & P333, P722 \\
\hline ska, M & \\
\hline ska-Gawron, E & P260 \\
\hline$\ldots \ldots$. . P321, P445, P717, P826 & \\
\hline di, A.O . . . . . . . . . . P P692 & P412 \\
\hline $\mathrm{W} \ldots \ldots \ldots \ldots$ & 3, P583 \\
\hline $\mathrm{O} 142$ & P507 \\
\hline P469 & 27, P439 \\
\hline P492 & P495 \\
\hline$\ldots \ldots \ldots$ & $0111,0112,0113,0$ \\
\hline$\ldots \ldots \ldots$ & $\mathrm{O} 155, \mathrm{O} 179, \mathrm{P} 561, \mathrm{P} 880$ \\
\hline$\ldots \ldots \ldots$ O 72, P306 & 75, P269 \\
\hline$\ldots \ldots \ldots 0153$ & urg, $\mathrm{T}$ \\
\hline P536, P847 & P501, P606, P734 \\
\hline$\ldots \ldots \ldots \ldots 0142$ & $\ldots$. . P499, P501, P604, P734 \\
\hline$\ldots \ldots \ldots \ldots$ O76 & 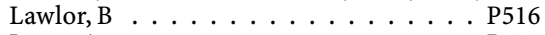 \\
\hline es & P297 \\
\hline$\ldots \ldots \ldots \ldots$. . . . 0184 & P419 \\
\hline 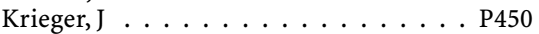 & $\mathrm{O} 217, \mathrm{P} 405$ \\
\hline$\ldots$. . P535 & . .0146 \\
\hline P855 & P715 \\
\hline la, $M \ldots \ldots \ldots \ldots$. . . . . P383 & Frenay, C $\quad$ O95, P442, P538, P732, P835 \\
\hline - & Ledoux, D . . . . . . . . O111, O112, O123, \\
\hline$\ldots \ldots \ldots \ldots$. . . & O140, O155, 0179 \\
\hline$\ldots \ldots \ldots \ldots$ & $\ldots \ldots$ \\
\hline$A \ldots \ldots \ldots \ldots \ldots$ & P797 \\
\hline$\ldots \ldots \ldots \ldots$. . . & $\ldots \ldots \ldots$ \\
\hline$\ldots \mathrm{O} 188$ & P775 \\
\hline P659 & $\ldots \ldots \ldots$ \\
\hline . P738 & \\
\hline , D.M $\ldots \ldots \ldots$ & $\ldots \ldots$ \\
\hline$\ldots$. . P852 & $\ldots \ldots \ldots$ \\
\hline P514, P6 & Hong. . . . . . . . \\
\hline $\mathrm{A} \quad \ldots \ldots \ldots$. . . P339, P630, P6 & 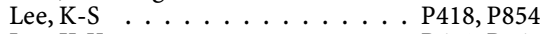 \\
\hline$\ldots \ldots \ldots \mathrm{O} 142_{2}$ & . . . P671, P764 \\
\hline , G . & $\ldots \mathrm{P} 258$ \\
\hline . . P599 & $\ldots \ldots \ldots \ldots$ P258 \\
\hline & . . . P375 \\
\hline , M $\quad \ldots$ O226, P559, P624, P842, P871 & $\mathrm{O} 223$ \\
\hline$\ldots \ldots \ldots$ P406 & $\ldots \ldots \ldots$ \\
\hline P417 & $\ldots \ldots \ldots \ldots$ \\
\hline zakiewicz, M & \\
\hline$A \ldots \ldots \ldots$ & P340 \\
\hline & \\
\hline P797 & $\ldots \ldots \ldots$. . P671, P764 \\
\hline $\mathrm{P} 470, \mathrm{P}$ & . P510, P879 \\
\hline & P353, P884 \\
\hline$\ldots \mathrm{P}$ & .0144 \\
\hline & 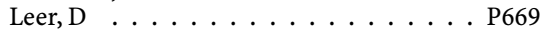 \\
\hline & P397 \\
\hline \multirow[t]{2}{*}{ Kwon, S-B } & O120 \\
\hline & \\
\hline $\mathrm{e}, \mathrm{R}$ & \\
\hline & ek, V \\
\hline P8: & P531 \\
\hline 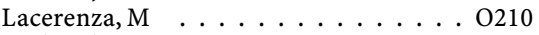 & P741 \\
\hline 0116 & F.S.S \\
\hline & \\
\hline $\mathrm{O} 1$ & 078,07 \\
\hline , 10 & \\
\hline P538 & nesiou, A \\
\hline
\end{tabular}

Lempert, T . . . . . . . . . . . . . P867 Lenart-Jankowska, D . . . . . . . . . . . O O169 Lenoir, H . . . . . . . . . . . . . P507 Leocani, L . . . . . . . . O O139, P256, P387 Leone, $\mathrm{M} \ldots \ldots \ldots \ldots \ldots \ldots$. . . . . . . . . . . . . . . . . Lepore $\mathrm{F} \ldots \ldots \ldots \ldots$. . . . . . P412

Leroux, J . . . . . . . ...... P412 Lesicky, $\mathrm{O} \ldots \ldots \ldots \ldots \ldots$. . . . . . . P536 Lewandowska, E . . . . . . . . . . P601

Leyendecker, $\mathrm{S} \ldots \ldots \ldots \ldots$. . . . . . . . O124

$\mathrm{Li}, \mathrm{N} \ldots \ldots \ldots$. . . . . . P242, P662

Lia, A . . . . . . . . P715

Liao, K-K . . . . . . . . . . . . P394

Liguori, M . . . . . . . . . . . . . . O63

Liguori, R . . . . . O O71, P266, P563, P822 Lillo, L . . . . . . . . . . . . . . P753

Lima, J.W . . . . . . . . . . . . P886

Limborska, S . . . . . . . . . . . . P287

Linington, $\mathrm{C} \ldots \ldots \ldots \ldots 157 \ldots \ldots$

Linker, $\mathrm{R} \ldots \ldots \ldots \ldots \ldots \ldots$. . . . . . . . . . . . . . . . .

Linseisen, $\mathrm{J} \ldots \ldots \ldots \ldots$. . . . . . O66

Lipowska, M . . . . . . . . . . P496

Lisak, R.P . . . . . . . . . . . 49, O97, P849

Lisnic, $\mathrm{V} \ldots \ldots \ldots \ldots \ldots$. . . . . . P425

Lisukov, I . . . . . . . . . . . . . . . P731

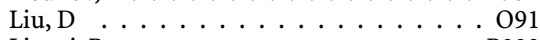

Liuzzi, $\mathrm{R} \ldots \ldots \ldots \ldots \ldots$. . . . 333

Livrea, $\mathrm{P} \ldots \ldots \ldots \ldots \ldots$. . . . P715

Lladó-Carbó, $\mathrm{E} \ldots \ldots \ldots \ldots$. . . . . . . 859

Llorente, C . . . . . . . . . . . . . O202

Llufriu, S . . . . . . . . . . . . . P728

Lobanova, A . . . . . . . . . . P520

Locatelli, F . . . . . P286, P483, P489, P490

Locatelli, P . . . . . . . . . . . . . P528

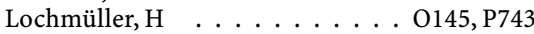

Lodato, $M$. . . . . . . . . . . P603

Lohberger, B . . . . . . . . . . . . O145

Lombès, A . . . . . . . . . . . . . P P597

Lonati, L . . . . . . . . . . . . . P365

Lonjon, M . . . . . . . . . P612

Lopes, J . . . . . . . . . . . . . . . . . . P594

Lopez, J . . . . . . . . . . . . . . P728

López Alburquerque, J.T . . . . . . P P572

López-Chozas, J.M . . . . . . . . O O170

López-Poma, J.C … . . . . . . O215

Lorenzoni, P.J . . . . . . . . . P293, P316

Löscher, W.N . . . . . . . . . O O145

Losonczi, E . . . . . . . . . . . . . P574

Lotfi, J . . . . . . . . . . . . . . . . . P874

Lovati, C . . . . . . O197, P779, P780

Lozano, M . . . . . . . . . . . . P728

Lubenova, B . . . . . . . . . . P784, P834

Lubenová, B . . . . . . . . . P335

Lublin, F.D . . . . . . . . . . . O O159

Luca, D . . . . . . . . . . . . . O O61

Lucchini, ${ }_{\mathrm{S}} \ldots \ldots \ldots \ldots \ldots \ldots \ldots$ P781

Lucchini, V . . . . . . . P272, P300, P682

Ludolph, A . . . . . . . . . . . . . . P467

Ludwig, $S \ldots \ldots \ldots \ldots \ldots$

Lugaresi, A . . . . . . . . . . . P715

Lugo, A . . . . . . . . . . . . P347

Lugo, $Z \ldots \ldots \ldots \ldots \ldots$. . . . . P870

Lugo-Pong, A . . . . . . . . . P395, P517

Lugowska, A . . . . . . . . . . . P601

Lukashev, M . . . . . . . . . . . . P844

Lunardelli, A . . . . . . . . . . . . O196

Lunetta, C . . . . . . . . . . P461

Lupski, J.R . . . . . . . . . . P276

Lüscher, $N \ldots \ldots \ldots \ldots \ldots$

Lutz, S . . . . . . . . . . . . . O218

Luxen, A . . . . . . . . . P880

Luzeiro, I . . . . . . . . . . . . P361

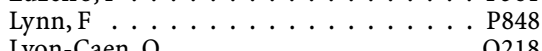

Lyons, M . . . . . . . . . . . . . . . P3999, P800

$\mathrm{Ma}, \mathrm{Y} \ldots \ldots \ldots \ldots$. . . . . . P793

Mabrouk, $\mathrm{T} \ldots \ldots \ldots \ldots \ldots$. . . P374

Machner, B . . . . . . . O O174, P747, P862 
Mackowiak Cordoliani, M.A . . . . . O O195

Maeda, Y . . . . . . . . . . . . . . . . P267

Maegli, J . . . . . . . . . . . . . P343, P411

Magalhães, R . . . . . . . . . . . . P P230

Magariello, A . . . . . . . . . O O63

Magerl, $\mathrm{W} \ldots \ldots \ldots \ldots$. . . . . . O122

Magerova, H . . . . . . . . . . P509, P512

Maggi, L . . . . . . . . . . P740

Maggiore, $\mathrm{C} \ldots \ldots \ldots \ldots$. . . O56

Maghzi, A.H . . . . . . . . . . P638

Maglaras, C . . . . . . . . . P383, P505

Magni, $\mathrm{P} \ldots \ldots \ldots \ldots \ldots$. . . P461

Magnussen, I . . . . . . . . . . . . . . P669

Magri, F . . . . . . . . . . . . P P589

Magureanu, S . . . . . . . . P289, P298

Magyar, M . . . . . . . . . . . P393

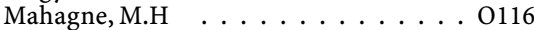

Maher, K . . . . . . . . . . . P345

Maheshwar, U . . . . . . . . P380

Mahjneh, I . . . . . . . . . . . . P592

Mahmoud, A . . . . . . . . . . . P290

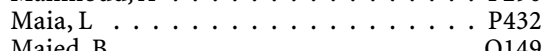

Majerus, $\mathrm{S} \ldots \ldots \ldots \ldots$ O $\ldots 123,0140$

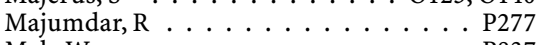

Mak, W . . . . . . . . . . . . . . . . . . . P837

Makita, Y

Makridou, A . . . . . . . . . P377, P378

Makris, $N \ldots \ldots \ldots \ldots$ P377, P378

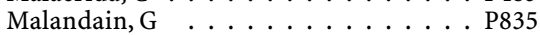

Malas, $\mathrm{S} \ldots \ldots \ldots \ldots \ldots$. . . . . O 153

Males, S . . . . . . . . . . P791

Malferrari, G . . . . . . . . . . . P760

Mall, V . . . . . . . . . . P388

Maloletnev, V . . . . . . . . P384

Maltezou, M . . . . . . P423, P454, P581

Mameli, F . . . . . . . . P231, P790, P799

Mammi, $\mathrm{A} \ldots \ldots \ldots \ldots \ldots \ldots$. . . . . 565

Manca, A . . . . . . . . . . P852

Mancinelli, E . . . . . . . . . . . P605

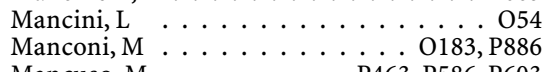

Mancuso, M . . . . . . P463, P586, P603

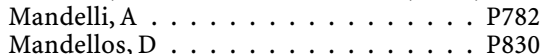

Mandelos, D . . . . . . . . . . . . P742

Mandic, G . . . . . . . . . . . P508

Mangels, I . . . . . . . . . . . O O124

Manikas, A . . . . . . . . . . . P P841

Manneschi, L . . . . . . . . . . P832

Manno, E . . . . . . . . . . . . P523

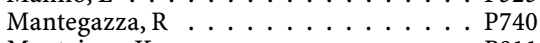

Mantziava, K . . . . . . . . . . P811

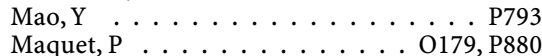

Marceglia, $\mathrm{s} \ldots \ldots \ldots$ P231, P790, P799

Marcello, N . . . . . . . . . . . . . . P760

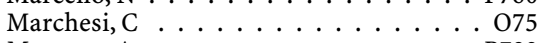

Marcone, A . . . . . . . . P782

Marelli, C . . . . . . . . . . . . P271

Marelli, S . . . . . . . . . . . . . O O183

Mareš, J . . . . . . . P335, P784, P794, P834

Margeti, S . . . . . . . . . . P742

Mariak, Z . . . . . . . . . . . P232

Mariani, C . . . . . O O197, P779, P780, P782

Mariani, G . . . . . . . . . . O O110

Marín-Martín, J . . . . . . . . . . O O170

Marino, S . . . . . . . . . O O54, P342

Mariotti, C . . O O86, P268, P269, P281

Marjanovic, I . . . . . . . . . . P327, P734

Markaki, E . . . . . . . . . . . . . P666

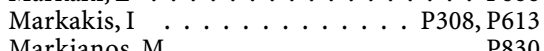

Markianos, M . . . . . . . . P830

Markovic, I . . . . . . . . . . . . . . P508

Maroco Cruzeiro, M.M $\ldots \ldots \ldots$. . . P315

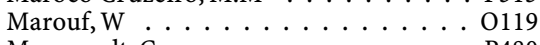

Marquardt, C . . . . . . . . . P P480

Marques Jr., W . . . P P315, P316, P500
Marquez, C . . . . . . . . . . . P P526

Marrakchi, S . . . . . . . . . . . . . . P515

Marrero-Abrante, R . . . . . . . . . . P428

Martin, C.L . . . . . . . . . . . O101

Martin, F . . . . . . . . . . . . . . . . . . . . . . O O157

Martin, L . . . . . . . . . . . . . P609

Martin, V . . . . . . . . . . O91

Martin, Y . . . . . . . . . . . . . P661

Martín-Polo, J . . . . . . . . . . . . . . P434, P572

Martín-Serradilla, J.I . . . . . . . . . . P434

Martinelli, V . . O99, O136, O156, O189, O221, P441, P553, P702, P843

Martinelli Boneschi, F . . . . . . . O O99, P843

Martínez, P . . . . . . . . . . P370, P815

Martínez de Aragón Calvo, A.C . . . . P703

Martínez Poveda, R . . . . . . . . . P326

Martínez-Sanchez, P . O168, O172, O202, O203

Martini, A . . . . . . . . . . . P269

Martini, M . . . . . . . . . . . . . . . P479

Martini, R . . . . . . . . . . . O 72

Martino, G . . . . . . . . . . P843

Martinovic, $Z$. . . . . . . . . . P694

Martins, O . . . . . . . . . . . O103

Martins da Silva, A . . . . . . . . . P432

Mascaro, $\mathrm{S} \ldots \ldots \ldots \ldots \ldots$

Mascioli, E . . . . . . . . . . . . P450

Masjuan, J . . . . . . . . . . . . . . . . . . . . O O168

Massaoutis, P . . . . . . . . P746

Mateo, I . . . . . . . . . . O O164, O213, P312

Matheisel, A . . . . . . . . . . . . O O129

Matikas, N . . . . . . . . . . . P616

Matteoli, M . . . . . . . . . . . . . P272

Matthews, P.M $\ldots \ldots \ldots \ldots$. . . . . . . O

Mattioli, F . . . . . . . . . . . . P444

Mattisi, I . . . . . . . . O187, O220

Mattle, H.P $\ldots \ldots \ldots \ldots . .6 \%$ O68, O115, P726

Matur, Z . . . . . . . . . . . P257, P872

Matveichuk, I.V . . . . . . . . . . P692

Mátyás, K . . . . . . . . . . . . . P574

Mauro, A . . . . . . . . . . . . P259

Mavridis, M . . . . . . . . . P614

Mayda Domaç, $\mathrm{F} \ldots \ldots \ldots \ldots$. $\ldots \ldots 65$, P865

Mayo, K . . . . . . . . . . . . . . . . O O197

Mazanec, R . . . . . . . . . . . . . . . . O O87

Mazurkiewicz-Beldzinska, M . . . . . O129

Mazzei, R . . . . . . . . . . . . O63, P270

Mazzeo, A . . . . . . . . . . P602

Mazzeschi, E . . . . . . . . . . . . P379

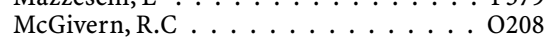

McKay, R.D . . . . . . . . . . . . P672

McKee, D . . . . . . . . . . . . O223, P701

Mcnett, K . . . . . . . . . . . . . P800

McWilliams, $\mathrm{R} \ldots \ldots \ldots \ldots \ldots$

Mecocci, P . . . . . . . . . . . . . . P789

Medeiros, E.B _ . . . . . . . . . . P293

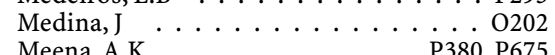

Mehdizadeh, M ........... P305

Mehdorn, M.-H $\ldots \ldots \ldots \ldots \ldots$. . . . . . . .

Meletiche, D . . . . . . . P321, P323, P445,

Mellgren, S.I _ . . . . . . . . . . . P5778

Melnichenko, $\mathrm{V} \ldots \ldots \ldots \ldots \ldots$

Melzer, C . . . . . . . . . . . . . . P857

Melzer-Gartzke, C . . . . . . . . . . . . . P809

Menci, E . . . . . . . . . . . . . . . . . . . P839

Mendoza, $\mathrm{A} \ldots \ldots \ldots \ldots$

Menezes, B . . . . . . . . . . . . 0102, P808

Meola, G . . . . . . . . . . P605

Merelli, E . . . . . . . . . . . . . . . P P715

Merino, S . . . . . . . . . . . P

Merkies, I . . . . . . . . . . . . P427, P439

Mesaros, $S$. . . . . . . . O O156, O189

Mesraoua, B . . . . . . . . . O O131

Messina, $\mathrm{R} \ldots \ldots \ldots \ldots$. . . . P466

Messina, $S \ldots \ldots \ldots$. $\ldots \ldots 02$

Messripour, $\mathrm{M} \ldots \ldots \ldots \ldots \ldots$

Metz, L . . . . . . . . . . . . P325, P720

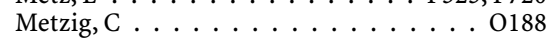

Miall, R.C . . . . . . . . . P403

Miceli, $\mathrm{P} \ldots \ldots \ldots \ldots \ldots \ldots . . .2493$

Michael, N . . . . . . . . . . O O82

Michalak, S . . . . . . . . . . O169

Michell, A.W . . . . . . . . P495

Midi, I . . . . . . . . . . . . . . P243

Migliaccio, R . . . . . . . . . . O O 0167

Migliorato, A . . . . . . . . . P602

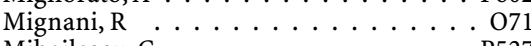

Mihailescu, C . . . . . . . . . P527

Mihailescu, G . . . . . . P527, P546, P652

Mihaylova, $\mathrm{V} \ldots \ldots \ldots \ldots \ldots \ldots$. . . . . . . . . . . .

Mijajlovic, M . . . . . . . . . . . P235

Mikaeili, H . . . . . . . . . . . . P433

Mikami, H . . . . . . . . . P476

Mikhailov, A . . . . . . . . . . . . O145

Miklina, ${ }^{2} \ldots \ldots \ldots \ldots \ldots \ldots$. . . . . 287

Milani, M . . . . . . . . . . O O75

Milia, P . . . . . . . . . . . . O O120

Milic-Rasic, V . . . . . . . . . P291, P313

Miller, B.L . . . . . . . . O O165, O167

Miller, D.H $\ldots$ O56, O60, O96, O186, O188,

O219, P518, P576, P723

Milonas, I . . . . . . P607, P696, P825, P841

Milosevic, D . . . . . . . . . . . . P504

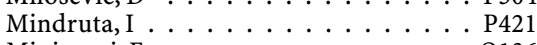

Minicucci, F . . . . . . . . . . . . O136

Mintun, M.A . . . . . . . . O O162, O199

Miotti, C . . . . . . . . . P P479

Mircea, R . . . . . . . . . . . . . P878

Miró Andreu, G . . . . . . . . . P P326

Misciali, C . . . . . . . . . . P822

Misirli, H . . . . . . . . . . . . . . . . P865

Misiuk, N.N . . . . . . . . . . . P692

Mittino, D . . . . . . . . . . . . P869

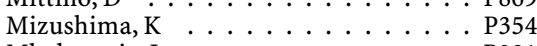

Mladenovic, J . . . . . . . . . . . P291

Mogel, H . . . . . . . . . . . . P P467

Moggio, M . . . . P283, P300, P461,

P589, P596, P598

Mohammad Ebrahim, M . . . . . . P751

Mohr, C . . . . . . . . . . . . O124

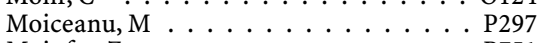

Moinfar, Z . . . . . . . . . . . P751

Moiola, L . . . . . . . . O55, O99, P294, P843

Moldovan, Mircea . . . . . . . . . P758

Moldovan, Mihai . . . . . . . . . . O72, P306

Molesti, E . . . . . . . . . . . . P586

Molina, G . . . . . . . . . . . . P368

Mollica, C . . . . . . . . . P722

Mollo, A . . . . . . . . . . . P270

Molnár, S . . . . . . . . . . . . P393

Molyneux, A.J . . . . . . . O O62

Momose, M . . . . . . . . P402, P406

Mon, D . . . . . . . . . . . . . . P P522, P748

Monaco, A . . . . . . . . . O143

Monaco, F . . . . . O O198, P233, P580, P869

Montagna, P . . . . O O71, P266, P563, P822

Montalban, X ․ O54, O186, O188, P576, P723

Montanari, E . . . . . . . . . . P832

Monti, A . . . . . . . . . . . P231

Monti, L . . . . . . . . . . . P839

Montoya, A . . . . . . . . . . . . . . P391

Monzani, F . . . . . . . . . . . . . P595

Moon, H.S $\ldots \ldots \ldots \ldots \ldots \ldots$ P241

Moonen, G $\ldots \ldots$, O111, O113, O123,

O140, O179, P561

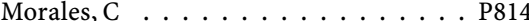

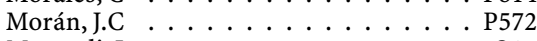

Morandi, $\ldots \ldots \ldots \ldots \ldots \ldots$ O $\ldots \ldots \ldots$

Moraru, E . . . . . . . . . . . . O661

Morbin, $\mathrm{M} \ldots \ldots \ldots \ldots \ldots$. . . P281

Moreira, E . . . . . . . . P230

Moreira, $\mathrm{T} \ldots \ldots \ldots \ldots \ldots$. . . . . . .

Moreno $S \ldots \ldots \ldots \ldots \ldots$. . . . P609

Moreno Ramos, $\mathrm{T} \ldots \ldots \ldots \ldots$. . . P703

Morgenthaler, $\mathrm{N} \ldots \ldots \ldots \ldots$. . . . . . . . .

Moroney, J . . . . . . . . . . . . . P745

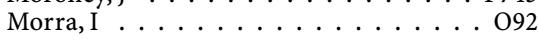




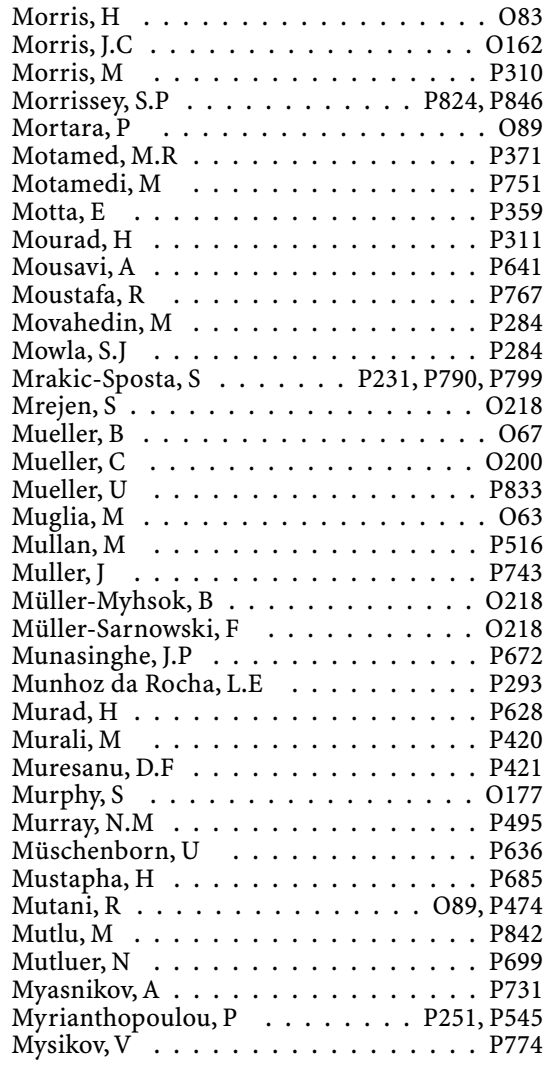

Na, S-J . . . . . . . . . . . P739

Nabavi, M . . . . . . . . . . P845

Nacci, A . . . . . . . . . . . O O110

Nagane, $Y \ldots \ldots \ldots \ldots \ldots$ P744

Nagels, G . . . . . . . . . . . . . O O173

Nagy, $N \ldots \ldots \ldots \ldots$. . . . . . . . . . . . . . . . .

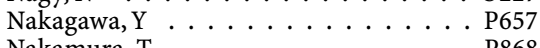

Nakamura, T . . . . . . . . . . . . P868

Nakamura, Y . . . . . . . . . . . . . . P885

Nanetti, L . . . . . . . . . . . . . P269

Nanu, P.D . . . . . . . . . . . P646

Naoumis, D . . . . . . . . . . . P423

Napoletano, R . . . . . . . . P721

Napoli, L . . . . . . . . . . . . . . . P P596, P598

Nardi, K . . . . . . . . . . . . . . . O120

Nardini, M . . . . . . . . . P286, P475

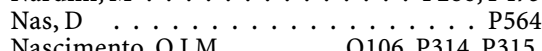

Nascimento, O.J.M … O O

Nassef, A . . . . . . P362, P632, P713, P777

Natalwala, A . . . . . . . . . . . . O216

Navajas-Carasa, D . . . . . . . . . . . P428

Nechifor, R . . . . . . . . . P555, P763

Nedelkoska, L . . . . . . . . . . O97, P849

Nedelmann, M . . . . . . . . . O200

Nedeltchev, K . . . . . . . O68, 0115

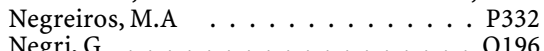

Nehrych, T . . . . . . . . . P356, P357

Nelson, P . . . . . . . . . . . . . . P625

Nemni, R . . . . . . . . . . P843

Neppert, B . . . . . . . . . . . P862

Nessler, S . . . . . . . . . . . . . O188

Nestrasil, I . . . . . . . . . . . . P404, P794

Neuhaus, O . . . . . . . . . . P761

Neuhauser, $\mathrm{H}$. . . . . . . . . . . P867

Neumann, G . . . . . . . . . . . . . O O174

Neumann, K . . . . . . . . . . . . . . O O163

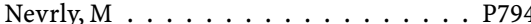

Ngonga, G . . . . . . . . . P528
Nguyen, D . . . . . . . . . . . . P412 Nica, S.M $\ldots \ldots \ldots \ldots$ P527, P546, P652

Nichol, M . . . . . . . . . . . . P334

Nicola, S . . . . . . . . . . . . . . . . . . O198

Nicolau, A . . . . . . . . . . . . . . . . . . O661

Nielsen, J.F . . . . . . . . . . . . . . P260

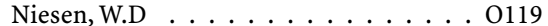

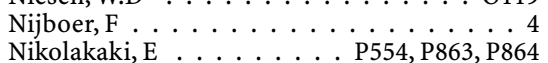

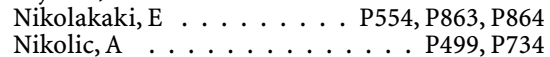

Nishizawa, $\mathbf{M} \ldots \ldots \ldots \ldots \ldots \ldots$

Nishizawa, $Y \ldots \ldots \ldots \ldots$ O133, P706

Nizzardo, M . . . . . . . . . P286, P475

Noachtar, S . . . . . . . . . . P542, P544

Nobrega, C . . . . . . . . . . . . . . P552

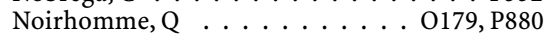

Nojima, S . . . . . . . . . . . . . . P868

Nojszewska, M . . . . . . . . . . P496

Nolte, A . . . . . . . . . . . . . . . P806

Nomura, M . . . . . . . . . . . . P476

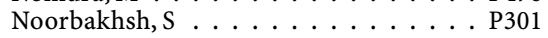

Noorian, A . . . . . . . . . . . . . . . . . P845

Novelli, F . . . . . . . . . . . . . . P840

Novik, A . . . . . . . . . . . . P731

Novotná, H . . . . . . . . . . . . . P459

Novy, J . . . . . . . . . . . . . . . . . . O O130

Nowicki, $\ldots \ldots \ldots \ldots \ldots \ldots \ldots \ldots$. . . . . . . . . . . . . . . .

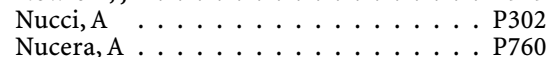

Nucera, A . . . . . . . . . . . P760

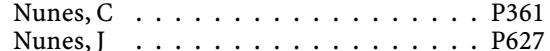

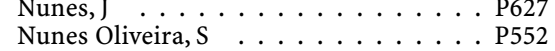

Nuta, C . . . . . . . . . . . . . . . . P527

Nyandaiti, Y.W . . . . . . . . . . O80, P640

Nyka, $W \ldots \ldots \ldots \ldots$. . . . . . P818

O'Brien, M . . . . . . . . . . . O O177

O'Connor, G . . . . . . . . . . . . . . P745

O'Connor, P.W . . . O O58, O158, O159, P447

O'Neill, G.N . . . . . . . . . . . P844

O'Nuallain, B . . . . . . . . . P778

O'Regan, M.E $\ldots \ldots \ldots \ldots \ldots \ldots$ P296

Obereigneru, $\mathrm{\ldots} \ldots \ldots \ldots$ P784, P792

Obermann, $\mathrm{M} \ldots \ldots \ldots \ldots \ldots$ P700

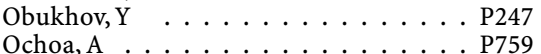

Odabasi, Z . . P410, P416, P417, P471, P472, P643, P644, P686, P708, P754, P755, P756, P757

Odainic, O . . . . . . . . P425

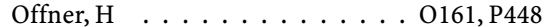

Oflazer, P . . . . . . . . . P257, P624

Ofner, $L \ldots \ldots \ldots \ldots \ldots \ldots$

Ogasawara, A . . . . . . . . O133

Ogata, A . . . . . . . . . . . P657

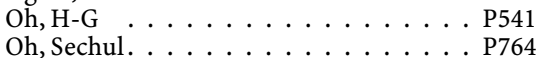

Oh, Seunghun. . . . . . . . . . . . P375

Ohyama, $\mathrm{T} \ldots \ldots \ldots \ldots \ldots$ P276

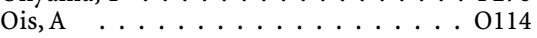

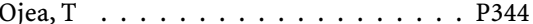

Okuda, D . . . . . . . . . . . . P717

Olivares, A . . . . . . . . . . . O O138

Oliveira, A . . . . . . . . . P807

Ollari, J . . . . . . . . . . . P411

Olokoba, A . . . . . O O103, P653, P654

Olszewska, A . . . . . . . . . . . . O129

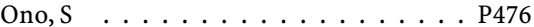

Ono, T $_{2} \ldots \ldots \ldots \ldots \ldots \ldots \ldots$ P267

Opavsky, R . . . . . P335, P784, P834

Oprisan, A . . . . . . . . O661

Opuchlik, A . . . . . . . . . P496

Opwis, K . . . . . . . . . . . P331, P485

Orcesi, S . . . . . . . . . . . . P598

Orefice, G . . . . . . . . . P333, P722

Orha, A . . . . . . . . . . P878

Orsi, L . . . . . . . . . . . . . . . O889

Ortega-Casarrubios, M.A $\ldots \ldots$ O 172, O203,
Orthmann-Murphy, J . . . . . . . O88

Osawa, M . . . . . . . . . . . . P402

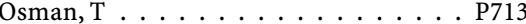

Otruba, P . . . . . . . . . . P488

Otsuki, $M \ldots \ldots \ldots \ldots \ldots$ P657

Ott, S . . . . . . . . . . . P292

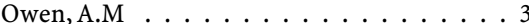

Oyama, Y . . . . . . . . . . P593

Oyanagi, K . . . . . . . . . . P476

$\mathrm{Oz}, \mathrm{O} \ldots \mathrm{P} 410, \mathrm{P} 416, \mathrm{P} 417, \mathrm{P} 471, \mathrm{P} 472, \mathrm{P} 686$

Ozawa, T . . . . . . . . . . P657

Özcan, ${ }_{\text {O }} \ldots \ldots \ldots \ldots \ldots$ O226, P842

Ozozen, Z . . . . . . . . . . . P246, P503

Oztop Cakmak, O . . . . . . . . . P668, P673

Ozturk, B . . . . . . . . . . . P410, P754

Pace, Amy. . . . . . . . . . . . . . . . . . O159

Pace, Andrea . . . . . . . . . . . . . . . . . O94

Paciaroni, M . . . . . . . . . . . O O120

Packman, J . . . . . . . . P P318, P319, P432

Padovani, A . . . . . . . . P528, P781

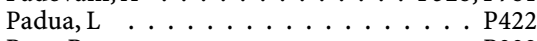

Paes, R . . . . . . . . . . . . P332

Pagani, E . . . . . O O156, O189, P342, P465

Paghera, $\mathrm{B} \ldots \ldots \ldots \ldots \ldots \ldots$ P781

Pakdaman, $\mathrm{H} \ldots \ldots \ldots \ldots \ldots \ldots \ldots$

Pakdaman, R . . . . . . . . P363, P762

Palace, J . . . . . . . . . . . . . O160, P342

Palasan, M . . . . . . . . P878

Palma Góis, J . . . . . . . . . . . . . . . P568

Palmerini, F . . . . . . . . . . . O O120

Palmucci, L . . . . . . . . . . . . . . . . . . . P P587

Palser, A . . . . . . . . . . . . P518

Pan, X . . . . . . . . . . P650, P793

Panea, C . . . . . . . P758

Panozzo, $M \ldots \ldots \ldots \ldots \ldots \ldots$

Pantelaros, N . . . . . . . . . . . P812

Pantzaris, M . . . . . . . . . P251

Panzara, M.A $\ldots \ldots \ldots \ldots$ O159, P848

Paolicchi, A . . . . . . . . P586, P603

Papacostas, S . . . . . . . . P251, P545

Papadaki, E . . . . . . . . P772

Papadimas, G.K . . . . . . . . P556

Papadimitriou, D . . . . . . . . . P286

Papais-Alvarenga, $M \ldots \ldots$. . . . O O151

Papais-Alvarenga, R . . . . O O150, O151, P332

Papathanasiou, E . . . . . . . . P251

Papuc, E . . . . . . . . . . . . . . P446, P684

Papurello, D.M . . . . . . . . . . . P567

Paquis, P . . . . . . . . O O128, P612, P851

Parati, G . . . . . . . . . P365

Parava, $M \ldots \ldots \ldots$ P383, P505

Pardo, G $\ldots \ldots$ P325, P443, P573, P720

Pareyson, D . . . . . . O75, O88, P268, P271, $\mathrm{P} 273, \mathrm{P} 281, \mathrm{P} 422$

Parino, L . . . . . . . . . . P881

Paripas, S . . . . . . . . . . . . P878

Parisi, $\ldots \ldots \ldots \ldots \ldots \ldots \ldots$. . . . . . . . . . . . . .

Parisi, V.L . . . . . . . . . P352

Parissis, D . . . . . . . . P382

Park, H-K . . . . . . . . . P541

Park, H-M . . . . . . . . . . P510, P879

Park, J-H . . . . . . . . . . P670

Park, J-Y . . . . . . . . . . . . . . P541

Park, Kee . . . . . . . . . . . . . . P854

Park, K.H $\ldots \ldots \ldots \ldots \ldots \ldots$ P510, P879

Park, Ki-Duk . . . . . . . . . . . P303

Park, S-P . . . . . . . . . . . P775

Park, S.E . . . . . . . . . . . . . P665

Park, S.W . . . . . . . . . . . P317

Parkes, L.M _ . . . . . . . . . P414, P824

Parks, B . . . . . . . . . . P718

Parman, $Y \ldots \ldots \ldots \ldots \ldots$ P257

Parreira, E . . . . . . . . . . . O O107

Parrilla, P . . . . . . . . . . O202

Paschalidou, M . . . . . P696, P698, P825

Pascual Pascual, I . . . . . . . . . P279

Pasennik, E . . . . . . . . . . P601

Pasquier, F . . . . . . . . . . . O 0195 
Pastorello, E . . . . . . . . . . P587

Patarroyo, J.C … . . . . . . . . O O157

Patat, A . . . . . . . . . P450

Patel, R . . . . . . . . . . . . P523

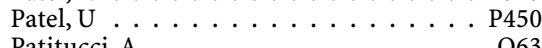

Patitucci, A . . . . . . . . O663

Patrikios, $\mathrm{P} \ldots \ldots \ldots \ldots \ldots$
Patterson, $\mathrm{V} \ldots \ldots \ldots \ldots \ldots$

Patti, F . . . . . . . . P575, P715

Pattoneri, $\mathrm{P} \ldots \ldots \ldots \ldots \ldots \ldots$. . . . . . 832

Paulsen, $\mathrm{M} \ldots \ldots \ldots \ldots$. . . P862

Pavan, A . . . . . . . . . P483

Pavan, G . . . . . . . . . . . . O O136

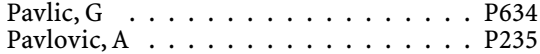

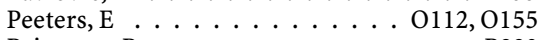

Peigneux, $\mathrm{P} \ldots \ldots \ldots \ldots \ldots \ldots$. . P880

Peiris, $\mathrm{Y} \ldots \ldots \ldots \ldots \ldots \ldots$. . . . P403

Pekmezovic, $\mathrm{T} \ldots \ldots \ldots \ldots \ldots \ldots$. . . . . . 327

Pellas, F . . . . . . . . . . . O O111

Pelletier, I . . . . . . . . . . . P P412

Pelliccia,,$\ldots \ldots \ldots \ldots \ldots \ldots$. . . P660

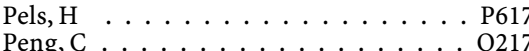

Peng, C $\ldots \ldots \ldots \ldots \ldots \ldots$
Penmasta, $\quad \ldots \ldots \ldots \ldots \ldots$

Penner, I.K $\ldots \ldots \ldots \ldots$ P331, P449, P485

Perani, D . . . . . . . . . . . O181, P887

Perbellini, R . . . . . . . . . P605

Perego, E . . . . . . . . . . . . . . P342

Perelman, $S \ldots \ldots \ldots \ldots \ldots$. . P299

Pérez, D . . . . . . . . . . . P572

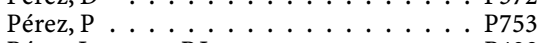

Pérez-Lorensu, P.J $\ldots \ldots \ldots \ldots \ldots \ldots$. . . P428

Pérez-Pérez, H . . . . . . . . . . . P428

Pérez-Saldaña, M.T … . . . . O O215

Peric, . . . . . . . . . . . . P606

Peric, $\mathrm{C} \ldots \ldots \ldots \ldots$ P501, P604

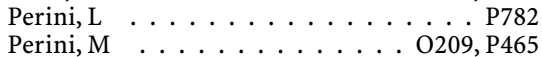

Perini, P . . . . . . . . . . . . . O187, O220

Peris, A . . . . . . . . . . . P379

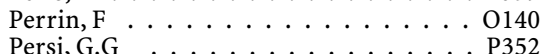

Perumal, J . . . . . . . . . . . . P453, P583

Peruzzotti Jametti, L $\ldots \ldots \ldots \ldots \ldots$. . . P565

Pesaresi, M . . . . . . . . . P780

Pesci, I . . . . . . . . . . . P832

Pesella, F . . . . . . . . . . . O O110

Pessia, A . . . . . . . . P474

Petek, E . . . . . . . . . . . O145

Petrescu, S . . . . . . . . . . P758

Petrou, $\mathrm{P} \ldots \ldots \ldots \ldots \ldots \ldots$. . . . P821

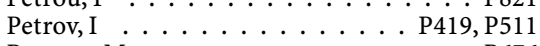

Petrova, $M \ldots \ldots \ldots \ldots$. . . . . . . . .

Petrova, $\mathrm{V} \ldots \ldots \ldots \ldots \ldots$ P511

Petzold, A $\ldots \ldots \ldots \ldots \ldots \ldots$ P857

Pfister, C . . . . . . . . . . . P610, P611

Pfrommer, $\mathrm{H} \ldots \ldots \ldots \ldots \ldots \ldots \ldots$ P610

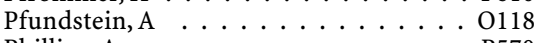

Phillips, A . . . . . . . . . . P P570

Phillips, B.A . . . . . . . . . O101

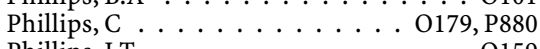

Phillips, J.T _ . . . . . . . . . . O159

Piazza, S . . . . . . . . . . . . . . P586, P603

Piccio, $\ldots \ldots \ldots \ldots \ldots \ldots$ P718

Piccolo, $G \ldots \ldots \ldots \ldots$. . . . . . . . . .

Picconi, O . . . . . . . . . . P575

Pichonnier-Cassagne, $\mathrm{V}$. . . . . . . O149

Pietroboni, A . . . . . . . . . . . P580

Pigazzini, T . . . . . . . . . P483

Pilato, $\mathrm{V} \ldots \ldots \ldots \ldots \ldots$. . . . P843

Pillar, G . . . . . . . . . . . O O 185

Pilz, G . . . . . . . . . . P536, P847

Pimentel, L.H . . . . . . . . . . . . P590

Pimentel, Tiago . . . . . . . . . . . P230

Pimentel, Teresa . . . . . . . . . . . P627

Pindar, $S \ldots \ldots \ldots \ldots \ldots \ldots$. . . . . 685

Pineda, $M \ldots \ldots \ldots \ldots \ldots$. . . . . . . 295
Pinho, L . . . . . . . . . . . . . P866

Pinho e Melo, $\mathrm{T} \ldots \ldots$. . . . . . . P877

Pinnock, R . . . . . . . . . . . . O208

Pinto, Anabela . . . . . . . . . . . . . O207

Pinto, Amélia N . . . . . . . . . . . . . . O O107

Pinto, F . . . . . . . . . . . . P379

Pinto, $5 \ldots \ldots \ldots \ldots$. . . . . . . O 207

Piola, $\mathrm{M} \ldots \ldots \ldots \ldots \ldots$. . . . . . . .

Pires, J . . . . . . . . . . . . . . P877

Pires, L.A . . . . . . . . . . . . . . P314

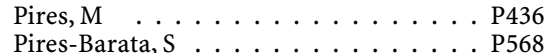

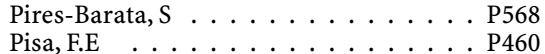

Pitschnau-Michel, D . . . . . . . . . . . P329

Pizzi, A . . . . . . . . . . . . . . . . . . . . P479

Plaitakis, A . . . . . . . . . . P772

Plant, G . . . . . . . . . . . . . . P746

Planté-Bordeneuve, $\mathrm{V}$. . . P P18, P319, P432

Plazzi, G . . . . . . . . . . . . . P886

Plebani, M . . . . . . . . . . . . . P781

Plesca, D.A $\quad \ldots \ldots \ldots \ldots \ldots$ P297, P543

Ploner, C.J $\ldots \ldots \ldots \ldots \ldots \ldots$

Pluderi, $\mathrm{M} \ldots \ldots \ldots \ldots$. . . . . P300

Plumari, M . . . . . . . . . . . . O O85, P269

Poceva, A . . . . . . . . . . . . . . . . . . . . . P499

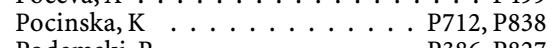

Podemski, R . . . . . . . . . . . P386, P827

Poggi, G . . . . . . . . . P483, P489, P490

Pohl, C . . . . . . . O O188, P447, P576, P723

Politi, L . . . . . . . . . . . . P565

Polman, C.H . O O159, O186, O188, P576, P723

Polo, J.M . . . . . . . . . . . . . . . . O O164

Polychronopoulos, $\mathrm{P} \ldots \ldots \ldots \ldots$. . . . P679

Pomati, S . . . . . . . . . . P782

Poniatowska, $\mathrm{R} \ldots \ldots \ldots \ldots \ldots$. . . P278

Ponomarenko, $\mathrm{N} \ldots \ldots \ldots \ldots \ldots$. . . . . . . . .

Pont, C . . . . . . . . . . . . . . . O O114

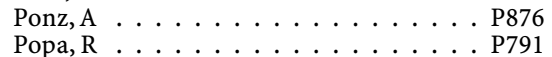

Popescu, A . . . . . . . . . . . . P763

Popescu, B.O . . . . . . . . . . . . . P421

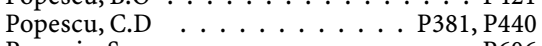

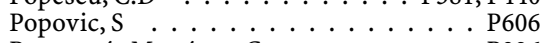

Portugués Martínez, G . . . . . . . . . P326

Poryazova, R . . . . . . P876, P881, P882

Potic, A . . . . . . . . . P291, P504

Potic, B . . . . . . . . . P291, P504

Pourabbas, B . . . . . . . . . . . P697

Pourjafar, M $\ldots \ldots \ldots \ldots \ldots \ldots$

Pourtois, G . . . . . . . . . . . . . P254

Pouwels, P . . . . . . . . . . . P342

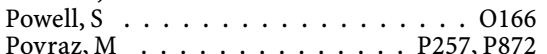

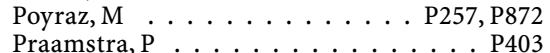

Prabhakar, S . . . . . . . . P367, P656

$\underset{P}{\text { Praksova, } \mathrm{P}} \ldots \ldots \ldots \ldots \ldots \ldots$. . . P620

Prank, K . . . . . . . . . . . . . . . P462

Prasad, V.V.S.R.K _ . . . . . . . . P380

Prazdnichkova, E.V . . . . . . . . P525, P533

Prelle, A . . . . . O 209, P461, P463, P465,

P596, P598, P682

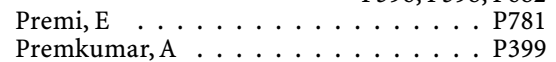

Priano, L . . . . . . . . . . . . . . . . P259

Prima, S . . . . . . . . . . . . P824, P846

Prinster, A $\ldots \ldots \ldots \ldots$ P333, P722, P722

Priori, A . . . . . . . . . . . . . P231, P790, P799

Probst, C . . . . . . . . . . . . O153

Prochnow, $\ldots \ldots \ldots \ldots \ldots \ldots$. . . . . . . .

Prod'homme, $\ldots \ldots \ldots \ldots \ldots$. . . . . . O157

Pruissen, D.M.O $\ldots \ldots \ldots$. . . . . O64, O65

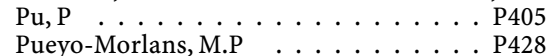

Pugliatti, M . . . . . . . . . P695

Pulizzi, A . . . . O O186, O189, O221, P441

Purcu, G . . . . . . . . . . . P539

Quarantelli, M . . . . . . . . . . . P722

Quasthoff, $5 \ldots \ldots \ldots \ldots \ldots \ldots$. . . . . . . . . . .
Quattrone, A ........... P422

Quigg, $\mathrm{M} \ldots \ldots \ldots \ldots \ldots \ldots \ldots$

Quintanilha, G . . . . . P315, P435, P858

Qureshi, A.I . . . . . . . . P363, P762

Rabinstein, A . . . . . . . . . P523

Rabot, A . . . . . . . . . . . . P562

Radaelli, M . . . . . . . . . . P843

Radojicic, A . . . . . . . . . P861

Radtke, A . . . . . . . . . . . . P867

Radue, E.W . . . . . . . . . P449

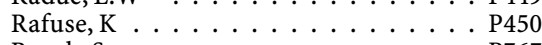

Ragab, S . . . . . . . . . . . . P767

Raggi, A . . . . . . . . . . . O210

Raghavan, $\mathrm{S} \ldots \ldots \ldots \ldots \ldots$. . . P420

Rahimian, E . . . . . . . . P828

Rahmati, J . . . . . . . . . . . P697

Rahnama, S . . . . . . . . P264

Raichle, M.E $\ldots \ldots \ldots \ldots$ O $\ldots \ldots 2,0199$

Rajda, C . . . . . . . . . . . . . . P574

Rakocevic Stojanovic, $\mathrm{V} \ldots \ldots$. . P606, P734

Rakowicz, M . . . . . . . . . P278

Ramalheira, J . . . . . . . . . . . . . P594

Rambold, H . . . . . . . . . . . . O O174

Ramio-Torrenta, L . . . . . . . . . P728

Ramón, C . . . . . . . . . . . . . . P312

Ramos de Oliveira, G . . . . . . . . P590, P866

Ramos Pereira, L . . . . . . . . P280

Ramsden, D B . . . . . . . . . . . P8836

Raschillà, R . . . . . . . . . . O O110

Rascovsky, K . . . . . . . . . . . . . . . O167

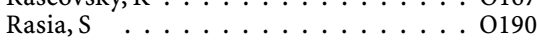

Rasic, $\mathrm{V} \ldots \ldots \ldots \ldots \ldots$. . . . . . P743

Rasmussen, A . . . . . . . . . . . . . O O84

Rattanatharn, R . . . . . . . . P390

Ratti, A . . . . . . . . . . . O 85

Rautenstrauss, B . . . . . . . . . . O887

Ravagnani, M . . . . . . . . P P233

Ravey, E . . . . . . . . . . . . O161

Raycheva, M . . . . . . . . P676

Razeghi Jahromi, S . . . . . . . . . P547

Reale, M . . . . . . . . . . P P786

Reddy, V.C . . . . . . . . . . P675

Reder, A . . . . . . . . . P716

Regaieg, A . . . . . . . . P374

Reggiani, M . . . . . . . P233

Reif, A . . . . . . . . . P802

Reiff, J . . . . . . . . . . . . O142

Reilly, M.M . . . . . . . . . . P495

Reinhart, E . . . . . . . . . . . P810

Reisz, D . . . . . . . . . . . . P506, P791

Rejdak, K . . . . . . . . . . . . P P684

Remi, J . . . . . . . . . . . . . . P P P42, P544

Renaud, S . . . . . . . . P310, P438

Renné, T . . . . . . . . . . . . . O204

Rentzos, M . . . . . . P400, P556

Renz, N . . . . . . . . . O115

Repousis, P . . . . . . . . . . . . P308

Revesz, T . . . . . . . . . . . . . . P397

Reynolds, $\mathrm{R} \ldots \ldots \ldots \ldots$ O56, 0219

Rezaee, A . . . . . . . . . . . . . P P641

Ricca, M.M . . . . . . . . . . . . P639

Ricci, G . . . . . . . . . . . . . P603

Riccitelli, G . . . . . . . . . O57, O156

Rice, G . . . . . . . . . . . . . O160

Ridzon, $\mathrm{P} \ldots \ldots \ldots \ldots \ldots$. . . . . . O . . . . . .

Rieckmann, $\mathrm{P} \ldots \ldots$ O218, $\mathrm{P} 324, \mathrm{P} 329, \mathrm{P} 829$

Rigas, M . . . . . . . . . . . P497, P737

Rigaud, A . . . . . . . . . . . . . . . . . P507

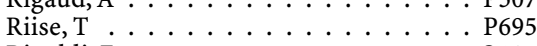

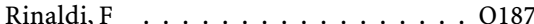

Rinaldi, L . . . . . . . . . . O187, O220

Ringleb, A . . . . . . . . . . . O O66

Rinkel, G.J.E . . . . . . . . . . . . O62

Rinker, $J \ldots \ldots \ldots \ldots \ldots \ldots$ P718

Rinta, S . . . . . . . . . . . . . . P729

Ripellino, P . . . . . . P714, P719, P840

Ripke, $\mathrm{S} \ldots \ldots \ldots \ldots \ldots \ldots$. . . . . O218

Risa, $0 \ldots \ldots \ldots \ldots \ldots$. . . . . . . . . . 


\begin{tabular}{|c|c|}
\hline , J.J & $\mathrm{z}, \mathrm{D}$ \\
\hline itschel, N & ykavicin, $\mathrm{O}$ \\
\hline itter, M & yu, A \\
\hline P610, P611 & yu, J.Y \\
\hline$\ldots \ldots \ldots$ & \\
\hline voiro, C . . . . . . . . . . P714, P719, P840 & tros \\
\hline P483 & adany, W \\
\hline P715 & adatnia, M . . . . . P P629, P641, P856 \\
\hline zuto, $\mathrm{N}$ & bbagh, $M$ \\
\hline ins, T.W $\ldots \ldots \ldots$ & $\ldots \ldots \ldots$ \\
\hline oberts, C & O146, P491 \\
\hline rts, $\mathrm{N}$ & P843 \\
\hline bowski, $\mathrm{P}$ & ian, $\mathrm{H}$ \\
\hline$\cdots \ldots$ O O54, O55, O57, P294, & \\
\hline P336, P337, P3 & $\ldots \ldots$ \\
\hline ceanu, A & $\ldots \ldots \ldots$ \\
\hline . . O57, O99, P336, P348, P702 & P697, P711 \\
\hline es, A.M $\ldots \ldots \ldots \ldots \ldots$ P . . . . P364 & P645 \\
\hline$\ldots \ldots \ldots$ & $\ldots \ldots$. . P751, P769, P845 \\
\hline$\ldots \ldots \ldots$ & \\
\hline uez, $\mathrm{M}$ & lour, D \\
\hline ez de Rivera, F.J & st, $\mathrm{M}$. \\
\hline npello, A & P599 \\
\hline iguez-Rodriguez, $\mathrm{E} \quad \ldots \mathrm{O} 164, \mathrm{O} 213, \mathrm{P} 312$ & P728 \\
\hline$\ldots$ & . . . . O194, P648, P661 \\
\hline$\ldots \ldots \ldots \ldots \mathrm{P} 428$ & \\
\hline P866 & P267 \\
\hline $\mathrm{P} 278, \mathrm{P} 601$ & \\
\hline $\mathrm{P} 313,1$ & oulos, G \\
\hline hitan, M.O $\quad \ldots \ldots \ldots$. . . P555, P787 & P276 \\
\hline$\ldots \ldots$ & $\ldots$ \\
\hline P456 & $\mathrm{O} 209, \mathrm{P} 465$ \\
\hline & \\
\hline P703, P749 & P286 \\
\hline $\mathrm{la}, \mathrm{J} \ldots \mathrm{.}$ & . . . . O103, O132, O135, $\mathrm{P} 653$, \\
\hline . . . P272, P283, P300, P596 & P654, P678, P685 \\
\hline$\ldots \ldots \ldots$ & 0107 \\
\hline . O $054, \mathrm{P}$ & \\
\hline$\ldots \ldots \ldots \ldots$ & P252, P810 \\
\hline P695 & \\
\hline & P614 \\
\hline E.C & . O88, P268, P273 \\
\hline aal, F.R $\ldots$ & P333, P722 \\
\hline$\ldots \ldots \ldots$ & O209, P465 \\
\hline . O154, P610, $\mathrm{F}$ & P687, P828 \\
\hline $\mathrm{C}$ & P732 \\
\hline K.M & $\mathrm{P} 466$ \\
\hline$\ldots \ldots \ldots 0130, \mathrm{P} 409$ & \\
\hline$\ldots \ldots \ldots \ldots$ & P370 \\
\hline $\mathrm{O}$ & \\
\hline $\mathrm{OS}$ & P609 \\
\hline ll, P.M & arranco, $\mathrm{F}$ \\
\hline P85 & $\mathrm{O} 164, \mathrm{O} 213$ \\
\hline$\dot{M}$ & intana, $C \ldots$. \\
\hline . . . O O194, O195, P648, P661 & $\mathrm{k}, \mathrm{R} \ldots \ldots \ldots \mathrm{O} 188, \mathrm{P} 576, \mathrm{P} 723$ \\
\hline$\ldots \mathrm{O} 156, \mathrm{C}$ & O174, P747, P862 \\
\hline & \\
\hline 0540 & \\
\hline $\mathrm{gh}, \mathrm{R}$ & $\ldots \ldots \ldots \ldots \mathrm{P} 463, \mathrm{P} 682$ \\
\hline P318, P319, P432 & P422 \\
\hline ellina, $s \ldots .$. & P361 \\
\hline$\ldots \ldots$. P356, P357, P407, P408 & \\
\hline P733 & P500 \\
\hline Q 02 & \\
\hline$\ldots \ldots \ldots$ O $\ldots 159$, P455, P584 & P591 \\
\hline M.A & ambrano, J \\
\hline P715 & $\ldots \ldots \ldots$ \\
\hline C.A $\ldots \ldots \ldots \ldots$ & $\ldots$. . P655, P656 \\
\hline P609 & P466 \\
\hline P2 & \\
\hline querro, J.J . . . & P699 \\
\hline O196 & \\
\hline M.M . . . . . . . . O O162, O199 & P657 \\
\hline$\ldots$. . P730 & \\
\hline P461 & P287 \\
\hline P3. & O88, P271 \\
\hline $\mathrm{P} 232$ & P599 \\
\hline P8 & \\
\hline P727 & O197, P580, P718, P782 \\
\hline
\end{tabular}

Scammell, T.E $\ldots \ldots \ldots \ldots \ldots$. . . O 180

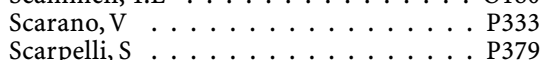
Scarpini, E . . . . . . 28, O197, O198, P580, P718, P779, P782, P790

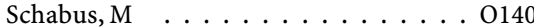

Schara, U . . . . . . . . . . . . . . P743

Schedel, R . . . . . . . . . . . P857

Schelhaas, H.J . . . . . . . . . P738

Schenone, A . . . . . . . . . . P422

Scheper, G.C . . . . . . . . . . P271

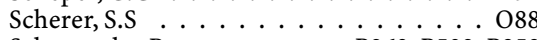

Schestatsky, P . . . . . . . . P263, P502, P859

Schiavone, $\mathrm{V} \ldots \ldots \ldots \ldots \ldots \ldots$. . . P333

Schichor, C . . . . . . . . . . . . . P625

Schiefelbein, R . . . . . . . . . P343

Schiff, $N \ldots \ldots \ldots \ldots \ldots \ldots . \ldots . \ldots . \ldots . . . \ldots$

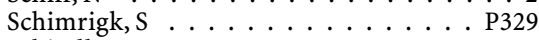

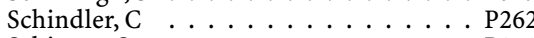

Schipper, . . . . . . . . . . . P850

Schlaeger, R . . . . . . . . . . P262

Schlegel, $\mathrm{A} \ldots \ldots \ldots$ P331, P521, P617

Schlereth, $\mathrm{T} \ldots \ldots \ldots \ldots \ldots \ldots$. . . . O

Schlindwein, P . . . . . . . . O O137

Schmidt, H . . . . . . . . . . P432

Schmidt, T . . . . . . . . . . . P774

Schnakers, C . . . . O111, O112, O113, O123,

O140, O155, O179, P561

Schneider, G-H . . . . . . . O142, P809

Schneider, S.A . . . . . . . . . O212, P397

Schneiderka, P . . . . . . . . . . P834

Schnitzler, A . . . . . . . . . . . . O O142

Schorge, $S \ldots \ldots \ldots \ldots \ldots$. . . . . O 83

Schoser, B .............. O145

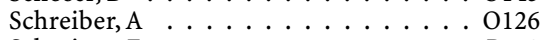

Schreiner, $F \ldots \ldots \ldots \ldots \ldots \ldots$ P743

Schreyer, K . . . . . . . . . . . P482

Schroth, G . . . . . . . . . . . O O115

Schuetz, P . . . . . . . . . . O67

Schuster, C . . . . . . . . . . . P817

Schwartz, S . . . . . . . P254, P876, P883

Schwarz, H.P _. . . . . . . P P771, P778

Schwarzbraun, $\mathrm{T} \ldots \ldots \ldots \ldots$. . . . . . . . .

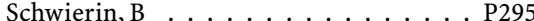

Sciacco, M . . . . . . . . P300, P596

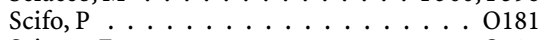

Sciutto, E . . . . . . . . . . . . . O104

Scola, R.H . . . . . . . . P293, P316, P743

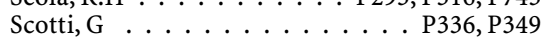

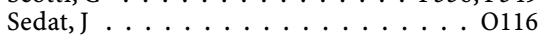

Seeck, M . . . . . . . . . P254

Seeman, $\mathrm{P} \ldots \ldots \ldots \ldots$. . . . . . O

Seemungal, B.M $\ldots \ldots \ldots \ldots$ P746

Šefc, $L \ldots \ldots \ldots \ldots \ldots \ldots \ldots$. . . . . . . . . . . . . . .

Seifert, T . . . . . . . . . . P456

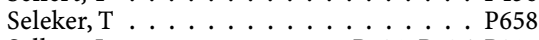

Sellner, J _ . . . . . . . . P531, P726, P817

Selmaj, K.W . . . . . . . . . . . O O193

Semana, $G \ldots \ldots \ldots \ldots$ O . . . . . . O218

Sencer, A . . . . . . . . . . . . P539

Senkarova, Z . . . . . . . . . . P488

Senouci, K . . . . . . . . . . . . O149

Seo, B.C . . . . . . . . . P241

Seo, J.G . . . . . . . . . . . . . P775

Seo, J.H . . . . . . . . . . . . . P524

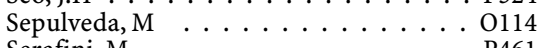

Serafini, $M \ldots \ldots \ldots \ldots$. . . . P461

Serizawa, M . . . . . . . . P234

Serpente, M . . . . . . . . . P580

Serpino, C . . . . . . . . . . P466

Serra Prat, M . . . . . . . . . . . P326

Serrano-Munuera, C . . . . . P P522, P748

Serrati, C . . . . . . . . . P639, P750

Servida, M . . . . . . P272, P300, P596

Servillo, P . . . . . . . . . . . . . O63

Seufert, $\mathrm{C} \ldots \ldots \ldots \ldots \ldots \ldots$ O $\ldots \ldots$

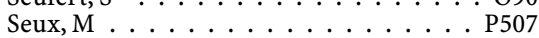

Sève, A . . . . . . . . . . . . . . O O195

Sfagos, $C \ldots \ldots \ldots \ldots \ldots \ldots$ 
Shaafi, S . . . . . . . . . P548, P690

Shadrina, $M \ldots \ldots \ldots \ldots \ldots$. . . . P287

Shahbazi, M ........... P831

Shaheen, I . . . . . . . . . . . . . . . . . . . O O108

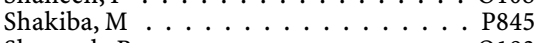

Sharrack, B . . . . . . . . O O193

Shashidharan, P . . . . . . . . . . P397

Shaygannejad, V . . . . . . . P856

Shea, $\ldots \ldots \ldots \ldots \ldots \ldots$ P323

Sheffy, K . . . . . . . . . . . . O O185

Shen, Y . . . . . . . . . . . . P583

Sherif, $M \ldots \ldots \ldots \ldots \ldots$. . . . . . . . . . .

Sheu, S.-Y . . . . . . . . . . . P700

Shevchenko, Y . . . . . . . . . P731

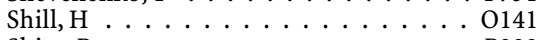

Shim, B . . . . . . . . . . P323

Shim, D.S . . . . . . . . . . P524, P608

Shin, D-J . . . . . . . . . . . . P879

Shin, J.S $\ldots \ldots$. P288, P303, P309, P317

Shin, W.C . . . . . . . . P353, P884

Shon, Y-M . . . . . . . . P P40, P341, P418

Siciliano, G . . . P463, P586, P587, P595, P603

Sideratou, A . . . . . . . . P812

Sieminski, M . . . . . . O O171, O182, P818

Sierra Hidalgo, F … . . . P703, P749

Siesjo, B.K . . . . . . . . . . P339, P630

Signaroldi, E . . . . . . . . . . . . P598

Signorini, L . . . . . . . . . . P528

Sigrist, S.J $\ldots \ldots \ldots \ldots \ldots \ldots$ P802

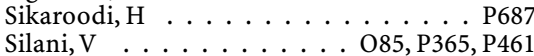

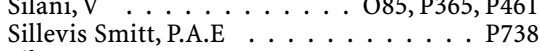

Silva, C . . . . . . . . . . P230

Silva, V . . . . . . . . . . O O107, P627

Silva $\mathrm{Jr}, \mathrm{S} \ldots \ldots \ldots \ldots \ldots$. . . . . P866

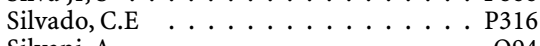

Silvani, A . . . . . . . . . . O994

Silveira, R.R . . . . . . . . . P P858

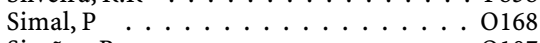

Simões, R . . . . . . . . . . O O107

Simon, J . . . . . . . . . . . . . . . . O995

Simone, I.L . . . . . . . . . . . . P466

Simsarian, J $\ldots \ldots \ldots \ldots \ldots$ P $\ldots \ldots \ldots 25$, P720

Simu, M . . . . . P250, P506, P651, P791

Sinanovic, O . . . . . . . . . P677

Sinczuk-Walczak, H . . . . . . . . P253

Singh, A . . . . . . . . . . P514, P688

Singh, G . . . . . . . . . . . P808

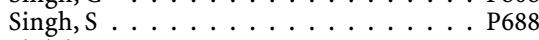

Sinicina, I $\ldots \ldots \ldots \ldots \ldots \ldots$ P532

Sirén, A.-L . . . . . . . . . . . P802

Sironi, M . . . . . . . . . . . . P589

Sirrou, $\mathrm{V} \ldots \ldots \ldots \ldots \ldots$ P667, P679

Siskova, D . . . . . . . . . . . P743

Sitajayalakshmi, S . . . . . . . . P380

$\underset{\text { Sitaras, P } \ldots \ldots \ldots \ldots \ldots \ldots . . . P 812}{\text { Sitoh }}$

Sklenarova, J . . . . . . . . . . . . P784, P834

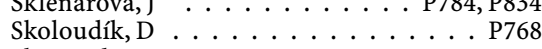

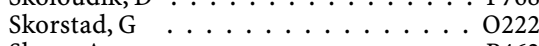

Skusa, A . . . . . . . . . . . P462

Sládková, V . . . . . . . P335, P784, P834

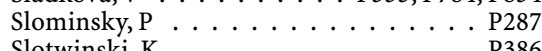

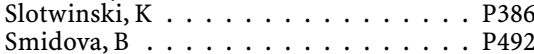

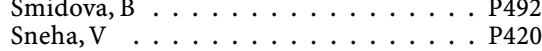

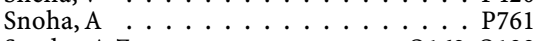

Snyder, A.Z . . . . . . . . . . O162, O199

Soavi, L . . . . . . . . . . . . . . P528

Soelberg Sørensen, P . . . P P325, P720, P829

Soffietti, R . . . . . . . . . O92, O93, O94

Sohelipour, $\mathrm{M} \ldots \ldots \ldots \ldots \ldots \ldots$. . . . . . . .

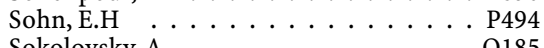

Sokolovsky, A . . . . . . . . . O O185

Solari, A . . . . . . . . . . . . P269, P422

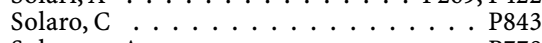

Solomon, $\mathrm{A} \ldots \ldots \ldots \ldots \ldots \ldots \ldots$. $\ldots \ldots 778$

Solomon, $\mathrm{T} \ldots \ldots \ldots \ldots$. . . . O102

Solski, J . . . . . . . . . . . . . . . . . . P727
Solsona Durán, J.F . . . . . . . . . . . P326

Solsona Perlasia, M . . . . . . . . . . . . P326

Soltész, P . . . . . . . . . . . . . . . P393

Sombekke, M . . . . . . . . . . O186

Sommer, C . . . . . . O O69, O125, P802

Son, I.H . . . . . . . . . . . . P813

Song, H.S . . . . . . . . . . . . . P241

Song, K.S . . . . . . . . . . . . . . P494

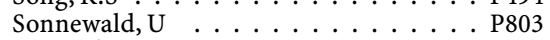

Sorgenfrei, $H_{2} \ldots \ldots \ldots \ldots \ldots$

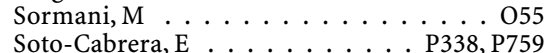

Soto-Cabrera, E . . . . . . . . . . . . P338, P759
Sottini, A . . P579

Sottini, C . . . . . . . . . . . . P444

Souplet, J.C $\ldots \ldots \ldots \ldots \ldots$

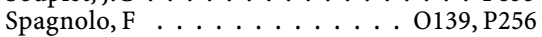

Spanjaard, L . . . . . . . . . . . . P534

Specchio, L.M . . . . . . . . . . . P819

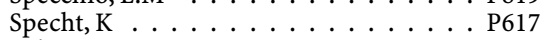

Spies, C . . . . . . . . . . . . . P809

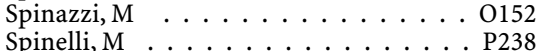

Sprenger, A . . . . . . . . . O O174, P747

Sprotte, $\mathrm{B} \ldots \ldots \ldots \ldots \ldots \ldots$. . . . . . .

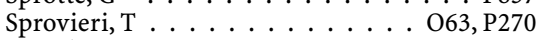

Squadrito, F . . . . . . . . . . P602

Stampatori, C . . . . . . . . . . P444

Stanca, A . . . . . . . . . . P758

Stancea, I . . . . . . . . . . . . . . . . . . . P297

Stapf, C . . . . . . . . . . . O68

Staszewski, J . . . . . . . . . . O O117

Stathis, $\mathrm{P} \ldots \ldots \ldots \ldots \ldots \ldots$. . . . . . . . . . . . . . .

Steck, A.J _ . . . . . . . . . O67, P741

Stefan, $\mathrm{H} \ldots \ldots \ldots \ldots \ldots \ldots$

Stefan, K . . . . . . . . . . . . . P324

Stefanescu, $\mathrm{D} \ldots \ldots \ldots \ldots \ldots \ldots$ P758

Stefanescu, M . . . . . . . . P543

Stefani, M . . . . . . . . . . P493

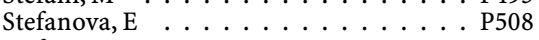

Stefanova, I . . . . . . . . . . P683

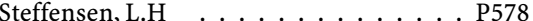

Steinheimer, S . . . . . . . . P252

Steinmetz, H . . . . . . . . . . . . . P468

Stejskal, D . . . . . . . . . . . . P P784, P794

$\begin{array}{lllll}\text { Stellmann, J-P } & \ldots & \ldots & \ldots & \ldots\end{array}$. . . P292

P712, P727, P838

Stemper, B . . . . . . . . O O58, O158

Stender, K . . . . . . . . . . . . . . . . . . . . . P789

Stengel, C . . . . . . . . . . . . . O O87

Stepien, A . . . . . . . . . . . . O O117

Stepien, $\ldots \ldots \ldots \ldots \ldots$ P601

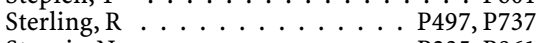

Sternic, $\mathrm{N} \ldots \ldots \ldots \ldots$ P235, P861

Stevic, Z . . . . . . . . . . P499, P606, P734

Stock, M . . . . . . . . . . . . P857

Stoeter, P . . . . . . . . . . . . O O137

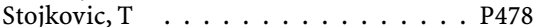

Stojsavljevic, $\mathrm{N} \ldots \ldots \ldots \ldots$. . . . P327

Stoll, $\mathrm{G} \ldots \ldots \ldots \ldots \ldots$

Stolz, E . . . . . . . . . . . . . . O200

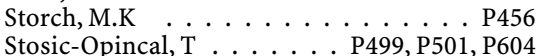

Stourac, P . . . . . . . . . . P459, P620

Straffi, L . . . . . . . . . . . O O139, P256, P702

Strand, C . . . . . . . . . . . . . . . . P397

Strandberg, J . . . . . . . . . . O O81

Straub, V . . . . . . . . . . . O145

Straube, A . . . . . . . . . . P625

Strazzer, S _. . . . . P286, P483, P489, P490

Stromillo, M.L . . . . . . . . . . . O O186

Struck, J . . . . . . . . . . . . . . . O O667

Strupp, M . . . . . . . . . . O O176, P532

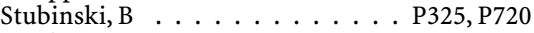

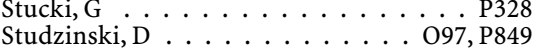

Stuke, $\mathrm{K} \ldots \ldots \ldots \ldots \ldots$

Stupp, R . . . . . . . . . . . O O130

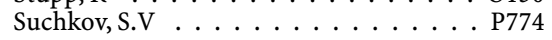

Sue, $\mathrm{L} \ldots \ldots \ldots \ldots \ldots$. . . . . . . O141

Suh, C-K $\ldots \ldots \ldots \ldots \ldots \ldots \ldots$ P775

Suhr, O . . . . . . . . . . . P319, P432

Suissa, L . . . . . . . . . . . . O116

Sulek, A $\ldots \ldots \ldots \ldots \ldots \ldots$ P278

Sundic, A . . . . . . . . . . . . P861

Sung, Y-H . . . . . . . . . . P879

Sunwoo, I.N $\ldots \ldots \ldots \ldots$ P524, P608

Sürer, F . . . . . . . . . . . . . . . . P659

Suri, M.F.K . . . . . . . . . . . . . .

Sussman, J . . . . . . . . . . . . . . . O223

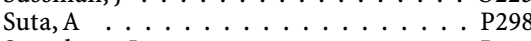

Suturkova, L . . . . . . . . . . . P499

Suzuki, Megumi. . . . . . . . . . . . . P476

Suzuki, Miki . . . . . . . . P402, P430, P431

Suzuki, N . . . . . . . . . . P744

Suzuki, Satishi .............. P339

Suzuki, Satoshi . . . . . . . . . . P630, P631

Suzuki, Shigeaki … . . . . . . . P744

Svelto, M . . . . . . . . . P715

Swanton, J . . . . . . . . . . . . . . . O996

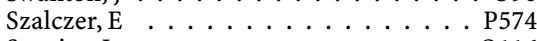

Szapiro, J . . . . . . . . . . . . . O116

Szatmari, $S \ldots \ldots \ldots \ldots \ldots$ P366

Szekeres, C . . . . . . . . . P366, P393

Szirkowiec, $\mathrm{w} \ldots \ldots \ldots \ldots \ldots$. $\ldots \ldots$ P278

Szmuda, M . . . . . . . . . . . . . . O129

Szocs, I . . . . . . . . . . . P366

Tacik, P . . . . . . . . . . . P600

Tagliavini, F . . . . . . . . . P273

Tahsini, M . . . . . . . . . . P828

Taillandier, L . . . . . . . . . . . . O995

Taipa, $\mathrm{R} \ldots \ldots \ldots \ldots \ldots$. . . . . 4336

Takada, $\mathrm{H}$. . . . . . . . P593

Takagi, K . . . . . . . . . . . P868

Takahashi, I . . . . . . . . . . . P657

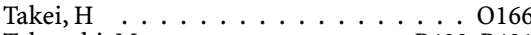

Takeuchi, $M \ldots \ldots \ldots$. . . . . . 4430, P431

Tal, S . . . . . . . . . . . . . P564

Talebi, M . . . . . . . . . P389

Talyor, R.S . . . . . . . . . . P852

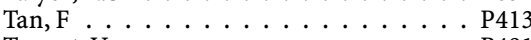

Tanant, V . . . . . . . . . . . . P491

Tanasescu, $\mathrm{R} \ldots \ldots \ldots \ldots$. . . O661

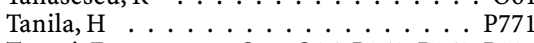

Taroni, F . . . . . O75, O86, P268, P269, P281

Tartarotti, S . . . . . . . . P883

Tascos, $\mathrm{N} \ldots \ldots \ldots \ldots \ldots$ P607, P696

Tasdemir, M . . . . . . . . P549

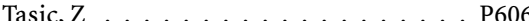

Taskos, $\mathrm{N} \ldots \ldots \ldots$ P382, P698, P825

Tatagiba, M . . . . . . . . O154, P610, P611

Tataranu, L . . . . . . . . . . . . . P618

Tavares, $\mathrm{P} \ldots \ldots \ldots \ldots$. . . . . O O148

Tavella, A . . . . . . . . P714

Taylor, R.S . . . . . . . . . . . . O O121, P860

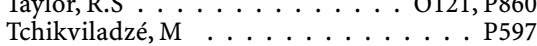

Tecau, M . . . . . . . . . . . . P878

Teixeira da Silva, M.H . . . . . . . . . . P P568

Tejeda, R . . . . . . . . . . . P391

Tejero, M.A . . . . . . . . . . . . P434

Teleanu, R . . . . . . . . . . . . P297

Terazzi, E . . . . . . . . . . . . . . . P869

Terzano, M.G $\ldots \ldots \ldots \ldots \ldots \ldots$. . . . . .

Teschner, $W \ldots \ldots \ldots \ldots \ldots$ P771

Testa, D . . . . . . . . . O O85, P271, P281

Testa, L . . . . . . . . . . . . . . . . . . . P869

Thakre, M . . . . . . . P236, P245

Theil, D . . . . . . . . . . . . . . P532

Then Bergh, $\mathrm{F} \ldots \ldots \ldots \ldots \ldots \ldots$. . . . . . . . . . . .

Theologos, G . . . . . . . . . . . P308

Therimadasamy, A . . . . . . . . P424

Thomann, S . . . . . . . . . P438, P741

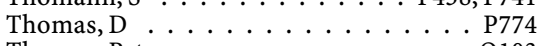

Thomas, Peter . . . . . . . . . . . . . O103

Thomas, Pierre . . . . . . . . . . P538, P550

Thomas-Sukur, $\mathrm{P} \ldots \ldots \ldots \ldots$. . . . . . . . . 


\begin{tabular}{|c|c|}
\hline $\begin{array}{l}\text { Thompson, A.J . } \\
\text { Thomson, S }\end{array}$ & $\cdots \mathrm{O} 54, \mathrm{O} 60, \mathrm{O} 96$ \\
\hline $\begin{array}{l}\text { Thomson, S . } \\
\text { Thorlin, } T\end{array}$ & O121, 0127, P860 \\
\hline Thuler, L. & O151 \\
\hline icmeanu, $M$ & \\
\hline icozzi, $\mathrm{N}$ & $\mathrm{O} 85$ \\
\hline ur & P732 \\
\hline $\mathrm{ilz}, \mathrm{C}$ & P540 \\
\hline erman, V & \\
\hline birana, $\mathrm{M}$ & $\mathrm{O} 160$ \\
\hline M.J & P738 \\
\hline iu, C & P763 \\
\hline dorovic, S & P313 \\
\hline Togha, M & P547, P769, P845 \\
\hline okuhara, $\mathrm{N}$ & P706 \\
\hline$\ldots$ & P575 \\
\hline Tolnay, M & $\therefore \mathrm{O} 180, \mathrm{P} 438$ \\
\hline Toma, & . . . . . P543 \\
\hline kiewicz, K . & 0117 \\
\hline $\mathrm{i}, \mathrm{G} \ldots$ & \\
\hline Ton & P327 \\
\hline oni, $\mathrm{H} \ldots$ & P301 \\
\hline Гӧр1 & P292 \\
\hline Tor & \\
\hline Torj & $\mathrm{O} 222$ \\
\hline$\ldots$ & \\
\hline Tort & P466 \\
\hline , C . . & P715 \\
\hline,$P \ldots$ & P819 \\
\hline$\ldots$ & $\ldots \mathrm{O} 100, \mathrm{P} 324, \mathrm{P} 802$ \\
\hline Toy & .070 \\
\hline $\mathrm{T} \ldots$ & P716 \\
\hline Trac & . . . P P666, P667, P679 \\
\hline Tra & $\ldots \ldots$ \\
\hline A.M . . & P421 \\
\hline$\ldots$ & P676 \\
\hline & $\mathrm{O} 122$ \\
\hline Tre & P412 \\
\hline & P605 \\
\hline Trevisan, C.P . . . . & P587 \\
\hline$\ldots$ & O92, O93, O94 \\
\hline Trigo, & . $\mathrm{P} 432$ \\
\hline $\mathrm{os}, \mathrm{G} \quad \ldots$ & $\ldots$ O $556, \mathrm{O} 219, \mathrm{P} 518$ \\
\hline$\ldots$ & $\ldots \ldots \ldots \ldots$ P 625 \\
\hline & $\mathrm{P} 420$ \\
\hline$[\ldots$. & . . . . . P P \\
\hline & P468 \\
\hline$\ldots$ & O173 \\
\hline Tsa & P564 \\
\hline Tsa & P837 \\
\hline & $\mathrm{P} 607$ \\
\hline Tsa & P454 \\
\hline & O175 \\
\hline sch, $M \ldots$ & $\ldots \mathrm{O} 153, \mathrm{O} 200, \mathrm{O} 225$ \\
\hline & . P P453, P583 \\
\hline $\mathrm{a}, \mathrm{L}$ & $\mathrm{O} 179$ \\
\hline Tsip & . P383, P505 \\
\hline & \\
\hline Tul & O 177, P745 \\
\hline & . P791 \\
\hline Tul & $\mathrm{P} 46$ \\
\hline & \\
\hline $\mathrm{n}, \mathrm{H}$ & P592 \\
\hline & O146, P598 \\
\hline & P346 \\
\hline & P232 \\
\hline Tur & P5 \\
\hline Türker, M & P865 \\
\hline Tüzün, E & P559, P624, P842, P871 \\
\hline Tzagournis & P77 \\
\hline
\end{tabular}

Üceyler, $\mathrm{N} \ldots \ldots \ldots$. . . . . . . . P802 Uchiyama, $\ldots^{2} \ldots \ldots \ldots \ldots \ldots$ P430, P431 Uchiyama, Y . . . . . . . . . . P406 Ueno, M . . . . . . . . . . . . O133 Uhr, M $\ldots \ldots \ldots \ldots$ Ulas, U . . . . . . . . P417, P643, P708, P757 Ulmer, $\mathrm{S} \ldots \ldots \ldots \ldots$. . . . . . . P350, P351 Ungaro, $C \ldots \ldots \ldots \ldots \ldots$ O63, P270
Urban, $\mathrm{P}$

Urbanek, K $\ldots \ldots \ldots \ldots \ldots$

Urbanska, J . . . . . . . . . . . . . O171, O182

Ure, J . . . . . . . . . . . . P411

Utikal, $\mathrm{P} \ldots \ldots \ldots \ldots$

Utsugisawa, K . . . . . . . . . . . . P744

Uziel, G . . . . . . . . . . . . . O88, P271

Vaamonde, J . . . . . . . . . . . P752, P820

Vacca, G . . . . . . . . . . . . P722

Vadas, L . . . . . . . . . . . . . . . . . O184

Vaishnavi, S.N . . . . . . . O O162, O199

Vale, J . . . . . . . . . . . . . . P766

Valko, P.O . . . . . . . . . . . . . . . O180

Valles, M . . . . . . . . . . . . . . P728

Vallet, M . . . . . . . . . . . . . . O 195

Valls-Solé, J . . . . . . . . . P255, P263, P859

Valsasina, P . . . . . O O54, O209, P349, P452

Vamvakas, L . . . . . . . . . . . . P614

Van Dam, A-M . . . . . . . . . . . . P844

van de Beek, D . . . . . . . . . P523, P534

van den Berg, L.H . . . . . O O73, O206, P426

van den Berg-Vos, R.M . . . . . . O73, P426

van den Bergh, W.M . . . . . . . . O62, P280

van der Knaap, M.S . . . . . . . . . P271

van der Kooi,A.J $\ldots \ldots \ldots$

van der Pol, W.L . . . . . . . . . O O73, P426, P738

van Doorn, P . . . . . . . . . P427, P439

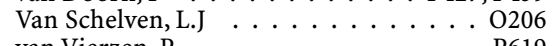

van Vierzen, P . . . . . . . . . . . . . . . P619

Vanaskova, E . . . . . . . . . . . . . P492

Vanbellingen, $\mathrm{T} \ldots \ldots \ldots \ldots$. . . . . O214

Vandenbark, A . . . . . . . . . . . O161, P448

Vanderheyden, J-E . . . . . . . . . . P401

Vandvik, B . . . . . . . . . . . . O O222

Vanhaudenhuyse, A . . . O O111, O113, O123,

O140, O179, P561

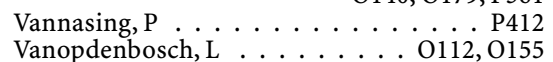

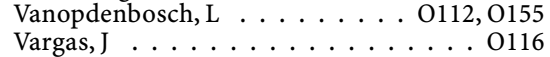

Vargas, S . . . . . . . . . . . . . . P429

Varjassyova, A . . . . . . . . . . P509, P512

Vartdal, F . . . . . . . . . . . . O222

Vasconcelos, C . . . . . . . . . . P332

Vasku, A . . . . . . . . . . . . . P459

Vavrouskova, J . . . . . . . . . . . P784

Vazquez, G . . . . . . . . . . . . . P320

Vazquez,J.L . . . . . . . . . . . . . . . . . . . O164

Vécsei, L . . . . . . . . . . . . . . . P574

Vedders, L . . . . . . . . . . . . O O141

Vega, R . . . . . . . . . . . . . . P429

Velasco-Calvo, $\mathrm{\ldots} \ldots \ldots \ldots$ P398, P674

Velin, P . . . . . . . . . . . . . . . . . . . P299

Velluto, L . . . . . . . . . . . . . P786

Veloso, M . . . . . . . . . . . . . P766

Vendrame, $M \ldots \ldots \ldots \ldots \ldots$ O201, 2237

Ventura, M . . . . . . . . O112, O155

Venturelli, E . . . O197, P580, P718, P780, P782

Verdesca, S . . . . . . . . . . . . . P479

Vergari, M . . . . . . . . . . P231, P790

Vermersch, P . . . . . . . . . . . . P829

Veronique, B . . . . . . . . . . . . P732

Verriello, L . . . . . . . . . . . . . . P460

Verrocchio, M.E . . . . . . . . . . P660

Verschuuren, J.J.G.M _ . . . . . . . . . P738

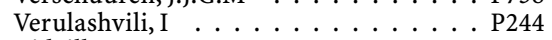

Vidailhet, M . . . . . . . . . . . . P597

Videla, H . . . . . . . . . . P343, P411

Viglietti, D . . . . . . . P714, P719, P840

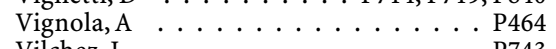

Vilchez, J . . . . . . . . . . . . . . P743

Villa, C . . . . . . . O197, P580, P718, P782

Vilquin, J.T … . . . . . O146

Vincent, A . . 30, O78, O79, O152, O224, P733

Vincent, J.B . . . . . . . . . . . . . O O145

Virgilio, R . . . . . . . . . . . . . . P272, P283, P589

Visconti, D.F . . . . . . . . . . . . . . . . P352

Visioli, F . . . . . . . . . . . . . P422

Visser, N.A $\ldots \ldots \ldots \ldots \ldots$
Vita, G . . . . . . . . P P422, P602

Vitaliani, $\mathrm{R} \ldots \ldots \ldots \ldots \ldots \ldots$. . . . . . . . . .

Vivanco, R.M . . . . . . . . . . O114, P622

Vivancos, F . . . . . . . . . . . P815

Vivekanandhan, $\mathrm{S} \ldots \ldots \ldots \ldots \ldots$ P . . . . . . . .

Vlachova, I . . . . . . . . . . . . . P768

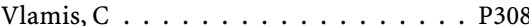

Vlaski-Jekic, $S \ldots \ldots \ldots \ldots$. . . P511

Vlassenko, A.G . . . . . . . . . . . . O162, O199

Vlckova, $\mathrm{E} \ldots \ldots \ldots \ldots \ldots \ldots$

Vogel, $\mathrm{H} \ldots \ldots \ldots \ldots \ldots$. . . . . . . . . . . . . .

Vogel, H.P . . . . . . . . . . . P636

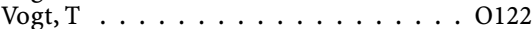

Voinea, L . . . . . . . . . . . . . . P758

Volk, K . . . . . . . . . . . . . . . . . . O200

Volkova, L.I . . . . . . . . . . P525, P533

Vollmar, C . . . . . . . . . P542

Volpi, L . . . . . . . . . P586, P595, P603

von Brevern, $M \ldots \ldots \ldots \ldots$. . . . P867

Vos, H.L . . . . . . . . . . . . . . O664

Vossel, K . . . . . . . . . . . . . . O165

Voustianiouk, A . . . . . . . O O138

Vranova, H . . . . . . P488, P784, P794, P834

Vyhnalek, M . . . . . . . . . . . P509, P512

Vyhnalkova, E . . . . . . . . . . O O87

Waddington-Cruz, M . . . . . . P318, P432

Wagner, $\mathrm{K} \ldots \ldots \ldots \ldots \ldots$. . . . O145

Wakil, M . . O 132, P653, P654, P678, P685

Walberer, M . . . . . . . . . O200

Walker, R . . . . . . . . . . O O143

Walker, $\mathrm{U} \ldots \ldots \ldots \ldots \ldots \ldots$. . . . . . . . .

Walravens, A . . . . . . . . . . . P561

Wang, C.X . . . . . . . P P358, P376, P663

Wang, $\mathrm{Y} \ldots \ldots \ldots \ldots \ldots \ldots$. . . . 6550

Wang, Y.J $\ldots \ldots \ldots \ldots \ldots \ldots$

Wang, Y.L . . . . . . . . . . P662

Wang-Tilz,Y . . . . . . . . . . . P P540

Waskoenig, J . . . . . . . . . . P730

Watanabe, $\ldots \ldots \ldots \ldots \ldots$. . . . P476

Watila, M.M . . . . . . . . . P640

Weber, A . . . . . . . . . P771, P778

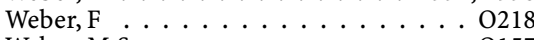

Weber, M.S . . . . . . . . . O157

Wegner, C . . . . . . . . . . . . O54

Wegner, $M \ldots \ldots \ldots \ldots$ P276

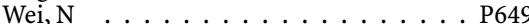

Weinstock-Guttman, B . . . . . . . P584

Weishaupt, A . . . . . . . . . . . P802

Weiss, R . . . . . . . . . . . O219, P518

Weissert, R . . . . . . . . . . P456

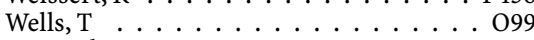

Werneck, L.C $\ldots \ldots \ldots \ldots$ P293, P316

Werth, E . . . . . . P876, P881, P882, P883

Weschke, B . . . . . . . . . . . . P743

Wessig, C . . . . . . . . . . . P P802

Whatmore, J.L $\ldots \ldots \ldots \ldots \ldots$ P399, P800

Whetton, E $\ldots \ldots \ldots \ldots$ O223, P701

White, $\mathrm{E} \ldots \ldots \ldots \ldots \ldots \ldots \ldots$. . . . . . .

Wierzba-Bobrowicz, $\mathrm{T} \ldots \ldots \ldots \ldots$ P601

Wiese, C . . . . . . . . . . P350

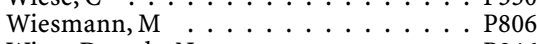

Wiest-Daessle, $\mathrm{N} \ldots \ldots \ldots \ldots$. . . P846

Wijdicks, E . . . . . . . . . . . P523

Wilder-Smith, E . . . . . . . P424

Willemart, T . . . . . . O112, O155

Willems, C.R.B . . . . . . . . . . P280

Williams, A.D . . . . . . . . P778

Winblad, B . . . . . . . . . . . . . . . . P787

Windpassinger, C . . . . . . . O O145

Winkler, P.A . . . . . . . . . P P542

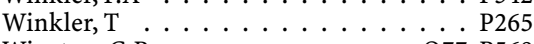

Winston, G.P . . . . . . . . . . O77, P569

Winter, $\mathrm{Y} \ldots \ldots \ldots \ldots \ldots$. . . O66

Wipfler, $\mathrm{P} \ldots \ldots \ldots \ldots$ P536, P847

Wirth, B $\ldots \ldots \ldots \ldots \ldots \ldots$ P275

Wirtz, $\mathrm{M} \ldots \ldots \ldots \ldots \ldots$. . . . 8550

Wirtz, P.W . . . . . . . . P738 
Witkowski, G . . . . . . . . . . P635

Witt, K . . . . . . . . . . . . O O142

Woelfle, J . . . . . . . . . . . . . P743

Wojcik, J . . . . . . . . . . . . . . . . O O99

Wojtecki, $\ldots \ldots \ldots \ldots \ldots \ldots \ldots$. $\ldots \ldots 142$

Wokke, J.H.J ․ . . . . . . O73, P280, P426

Wolansky, L . . . . . . . . . . . P346

Wolfkühler, D . . . . . . . . P521

Wood, F . . . . . . . . . . . . P323

Wood, G . . . . . . . . . . . . P482

Wood, N.W $\ldots \ldots \ldots \ldots \ldots \ldots$. . . P397

Woods, $\mathrm{W} \ldots \ldots \ldots \ldots \ldots$. . . . P800

Wraith, J . . . . . . . . . . . P295

Wu, Z-A . . . . . . . . . . P394

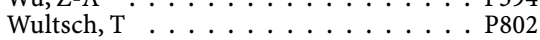

Xidakis, I . . . . . . . . . . . . . . P616

Xifaras, $M \ldots \ldots \ldots \ldots$. . . P360

$\mathrm{Xu}, \mathrm{J} \ldots \ldots \ldots \ldots \ldots \ldots \ldots$

Yabe, $\mathrm{I} \ldots \ldots \ldots \ldots \ldots \ldots$. . . . . . . . . . . . . .

Yagi, $\mathrm{N} \ldots \ldots \ldots \ldots \ldots \ldots$

Yahara, O . . . . . . . . . . . P599

Yamada, I . . . . . . . . . . . P885

Yamamoto, $\mathrm{R} \ldots \ldots \ldots \ldots$ O $\ldots \ldots 66, \mathrm{\ldots} 276$

Yamane, $\mathrm{K} \ldots \ldots \ldots \ldots \ldots \ldots$

Yamazaki, K . . . . . . . . . . . . . . . . P888

Yamazaki, T . . . . . . . . P476

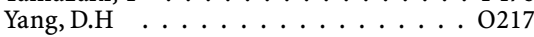

Yang, D.W $\ldots \ldots \ldots$. . . P340, P341, P418

Yang, H.R . . . . . . . . P P288, P309, P317

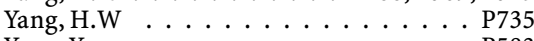

Yang, Y . . . . . . . . . . . P583

Yang, Z.H . . . . . . . P358, P376, P662, P663

Yaouanq, J . . . . . . . . . O218

Yapici, Z . . . . . . . . . . . . . O226

Yazdchi Marandi, M . . . . . . . . . . P433
Ydreos, I . . . . . . . . . . . . . . P P812

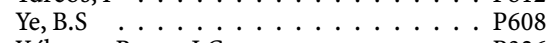

Yébenes Reyes, J.C . . . . . . . . . . P326

Yescas, P . . . . . . . . . . . . . . . . O O84

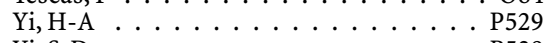

Yi, S-D . . . . . . . . . . . . . . . . . P529

Yildiz, N . . . . . . . P498, P770, P816

Yoo, A . . . . . . . . . . . . P764

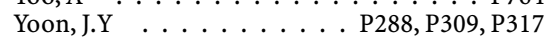

You, $\mathrm{X} \ldots \ldots \ldots \ldots \ldots$. . . . . . . . . . . . . . . P584

Young, C $\ldots \ldots \ldots \ldots \ldots$

Yu, L-H . . . . . . . . . . . . . . P276

Yung, W.K.A $\ldots \ldots \ldots \ldots$ O . . . . . P615

Zaffaroni, M . . . . . . O O189, O221, P441, P579, P840, P843

Zaganas, I . . . . . . . . . . . . . . . P614 Zago, $S \ldots \ldots \ldots \ldots \ldots \ldots \ldots$. . . . . . . . . . .

Zak, I $\ldots \ldots \ldots \ldots \ldots \ldots$ P453, P583

Zambelis, T . . . . . . . . . P742

Zamboni, M . . . . . . . . . . . O210

Zamfirescu, A . . . . . . . . P543

Zamvil, S.S . . . . . . . . . . . . . O O157

Zanelli, E ............... 4450

Zangl, M . . . . . . . . . . P254

Zanotti, C . . . . . . . . . . P579

Zanotti, D ............... P444

Zapletalova, J . . . . . . . . . P784, P834

Zarabi, V . . . . . . . . . . . . . . P301

Zaremba, J . . . . . . . . . . . P278

Zat, C . . . . . . . . . . P P639

Zauner, $\mathrm{H} \ldots \ldots \ldots \ldots \ldots \ldots \ldots$. . . . . . . . .

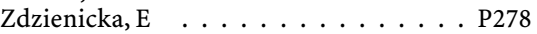

Zedde, M . . . . . . . . . . . P760

Zeller, D . . . . . . . . . . . . . P324

Zeng, W $\ldots \ldots \ldots \ldots \ldots \ldots$. $\ldots \ldots 44$

Zephir, $\mathrm{H} \quad \ldots \ldots \ldots \ldots$. . . . . O149
Zerbi, A . . . . . . . . O136 Zetterberg, $\mathrm{H} \ldots \ldots \ldots \ldots \ldots \ldots$. $\ldots \ldots 33$

Zettl, U . . . . . . . . . . . . . . P329

Zeviani, M . . . . . . . . . O88, P271

Zhang, Hao . . . . . . . . . . . . . P584

Zhang, Hua . . . . . . . . . . . . . P793

Zhang, M . . . . . . . . . . P267

Zhang, Yuhao . . . . . . . . . . . P793

Zhang, Y.M . . . . P P358, P376, P551, P649,

P662, P663, P664, P776

Zhao, N . . . . . . . . . . . . . . P793

Zhao, X.Q . . . . P358, P376, P662, P663

Zhao, Y . . . . . . . . . . . P650

Zharikova, A . . . . . . . . P248

Zhelev, Y . . . . . . . . . P676

Zheng, X . . . . . . . . . . . . . P793

Zhong, C . . . . . . . . . . . . . P650, P793

Zhou, D . . . . . . . . . . O O188

Zhou, $\mathrm{Y} \ldots \ldots \ldots \ldots \ldots$ P664, P776

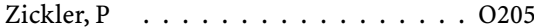

Zidverc, J . . . . . . . . . . . . . P235

Zidverc-Trajkovic, J . . . . . . . . . . P861

Ziemann, $\mathrm{U} \ldots \ldots \ldots \ldots \ldots$. . . . P468

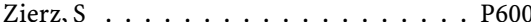

Zimatore, $\mathrm{G} . \mathrm{B} \ldots \ldots \ldots \ldots \ldots$. . . . . . . . .

Zimmermann, D.R $\ldots \ldots \ldots \ldots \ldots$. . P795

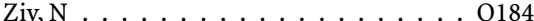

Zoccarato, M . . . . . . . . O152

Zoon, P . . . . . . . . . . . . . P619

Zorzou, $\mathrm{M} \ldots \ldots \ldots \ldots \ldots$. . . . . . . .

Zouari, M . . . . . . . . P274, P274

Zouvelou, $\mathrm{V} \ldots \ldots \ldots \ldots \ldots$. . . . 556

Zuberi, S.M . . . . . . . . . . . O O83, P296

Zucconi, M ............ O183, P886

Zuliani, L . . . . . . . . . . O O152

Zweifel, C . . . . . . . . . . . . O67

Zwergal, A . . . . . . . . . O O176 